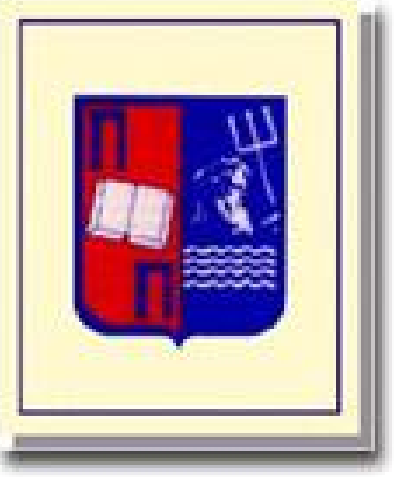

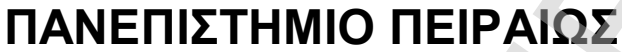

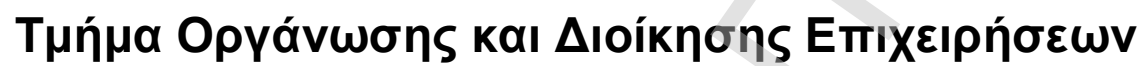

ЕФАРМОГЕ

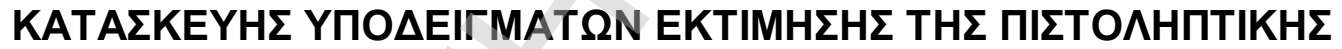

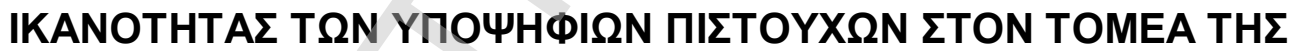

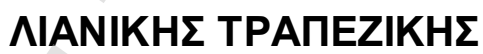

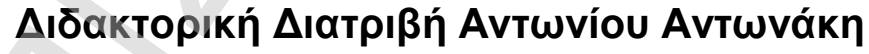


AФIEP $\Omega \Sigma H$

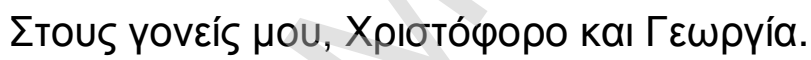




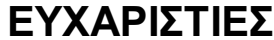

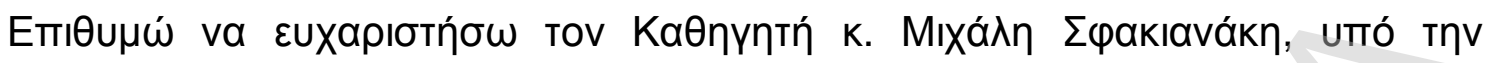

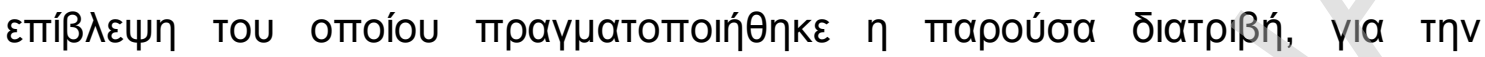

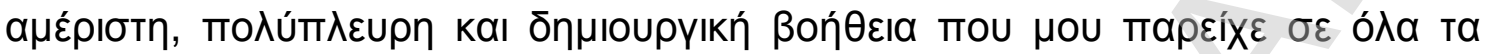

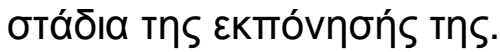

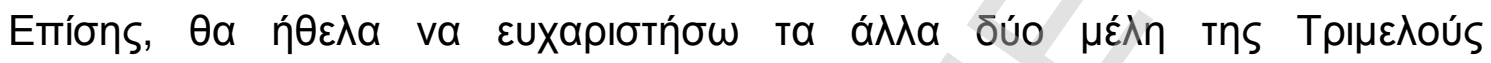

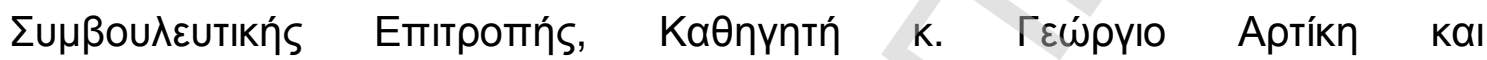

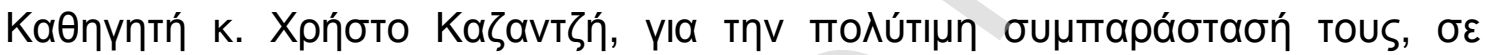

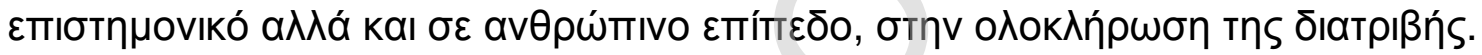

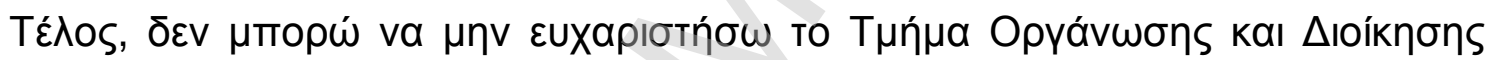

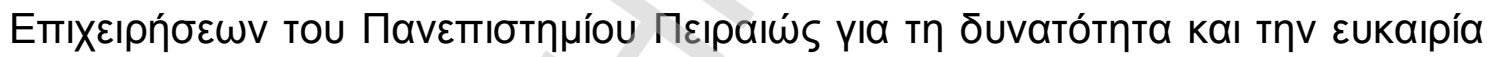

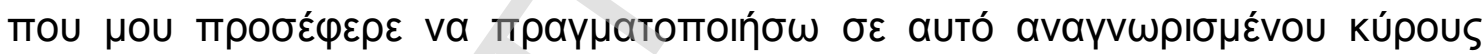

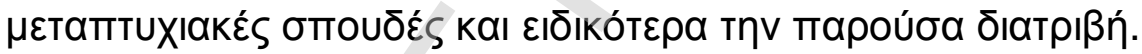

Avtúvios Avtwvákns 
$\Sigma \varepsilon \lambda$.

$\Sigma$ YNTOMОГРАФІЕ $\Sigma$

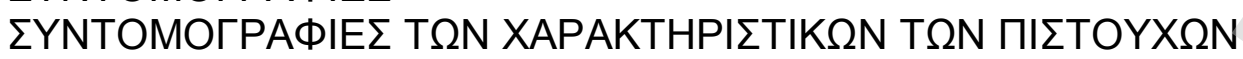

ПOY XPH $\Sigma I M O \Pi O I O Y N T A I ~ \Sigma T H N ~ A N A \wedge Y \Sigma H$ T $\Omega N \Delta$ E $\Delta O M E N \Omega N$

$\Sigma$ HMANTIKOI OPOI

$\Sigma Y N O \Psi H$

KATA $\Sigma T A \Sigma H \Pi I N A K \Omega N$

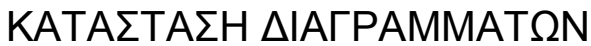

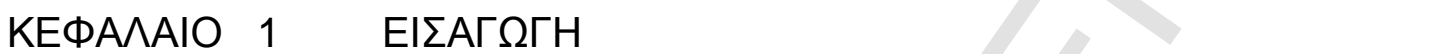

$1.1 \sum$ TóXOI tns $\Delta$ เатрıßńs 1

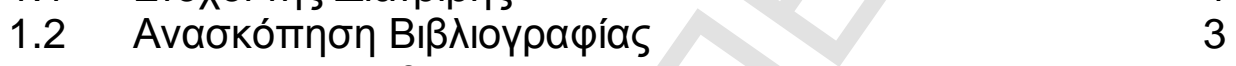

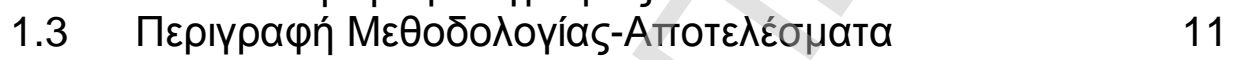

$1.4 \Delta$ ouń tns $\Delta$ เатрıßńs 18

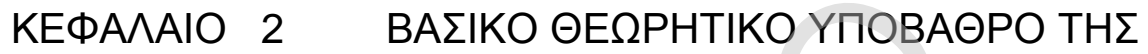

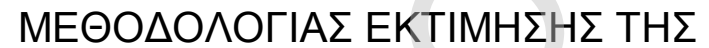

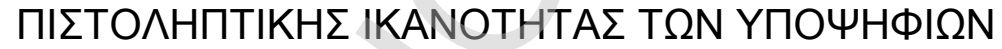

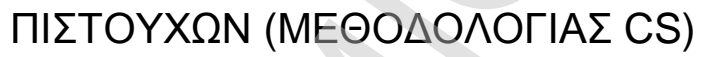

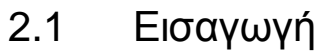

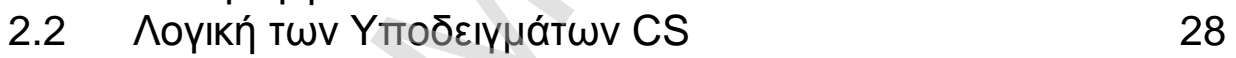

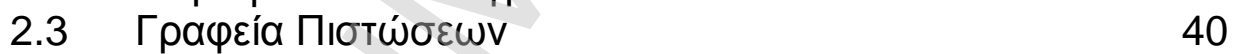

2.3.1 Eıбaүwүń 40

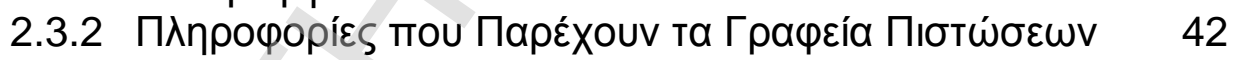

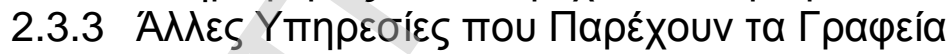

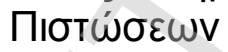

2.3.4 Твıргбías

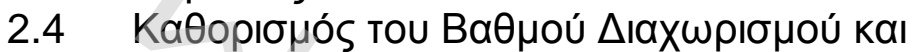

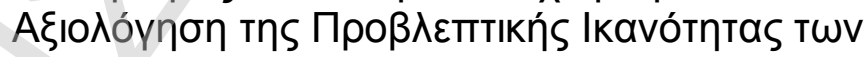

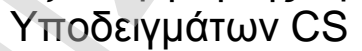

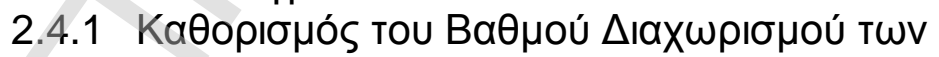

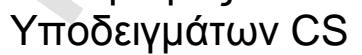

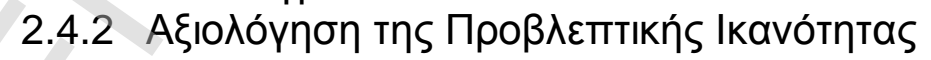

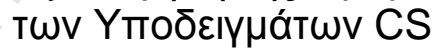

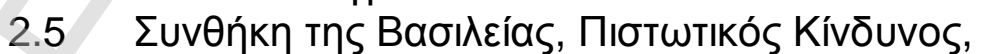

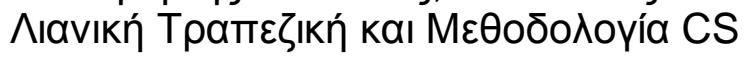

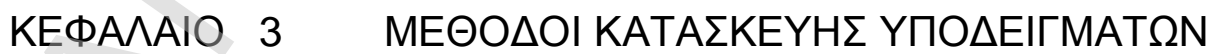

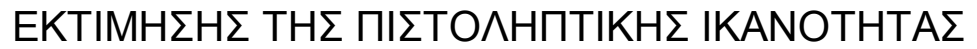

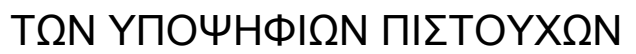

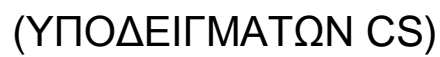

3.1 Eıбaүwүń

3.2 Kavóvas tou Aтגoú Bayes 69

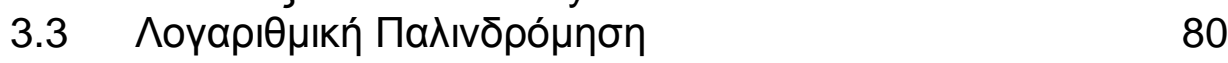

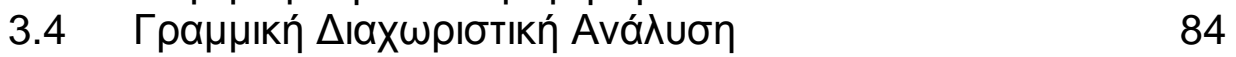

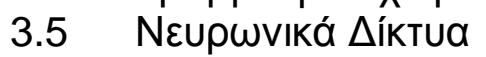




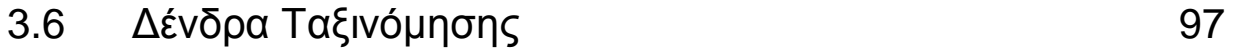

3.7 M

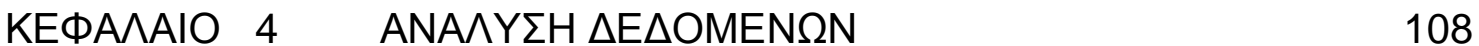

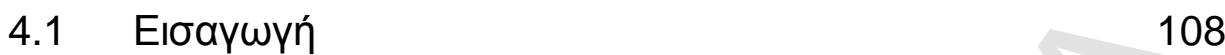

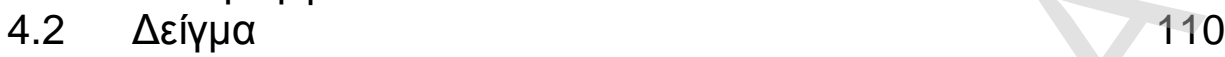

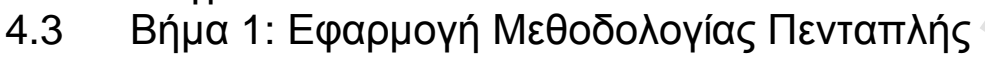

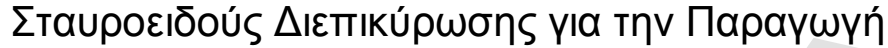

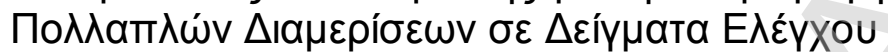

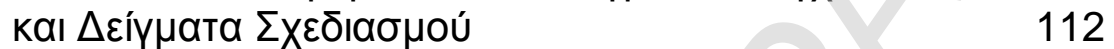

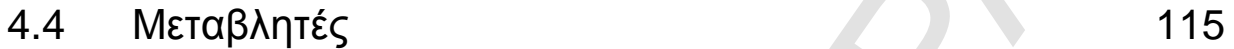

4.4.1 Катохи́ Autokivńtou (CAR) 119

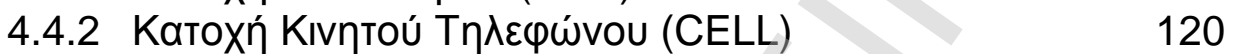

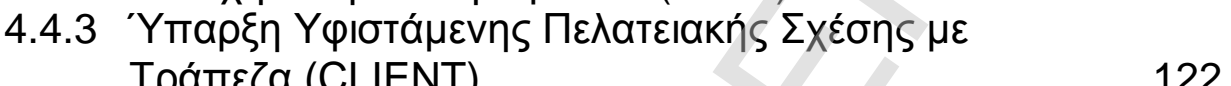

4.4.4 Фúlo (GENDER) 123

4.4.5 Катохи́ Пıбтштাкńs Káptas (CRD) 124

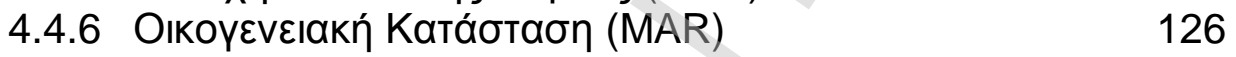

4.4.7 Túmо Katoıkías (HOME) 127

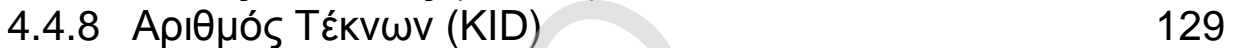

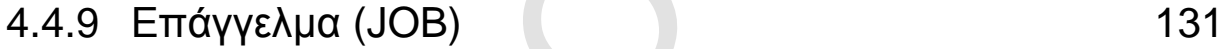

4.4.10 H入ı́́a (AGE) 133

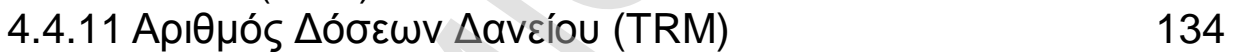

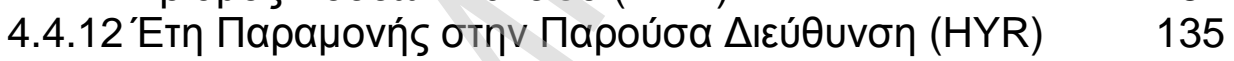

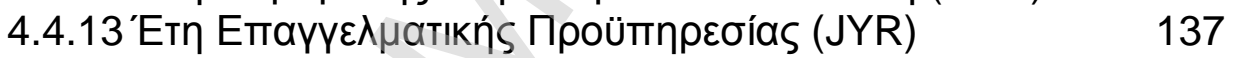

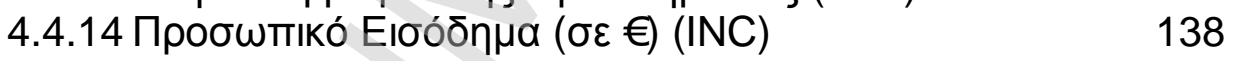

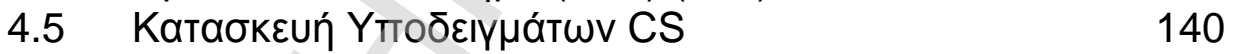

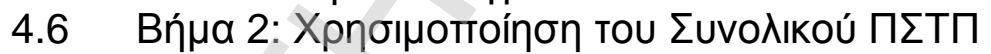

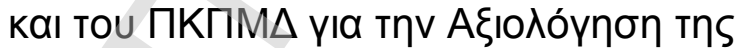

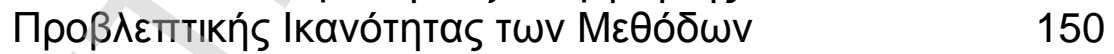

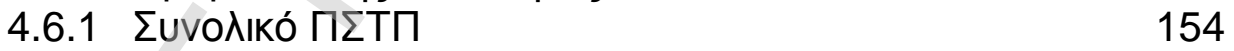

4.6.2 ПКПМ 159

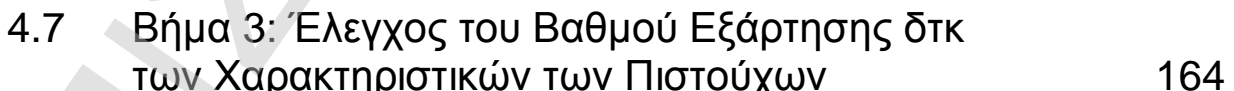

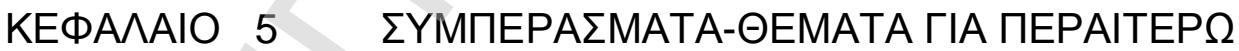
EPEYNA 180

$\begin{array}{ll}\text { ВІВАІОГРАФІА } & 184\end{array}$

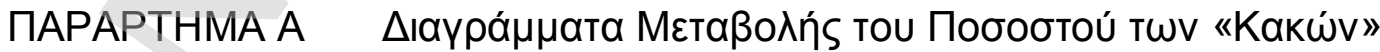

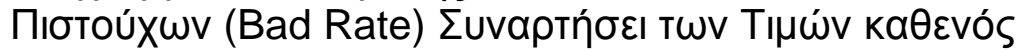

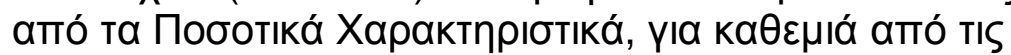

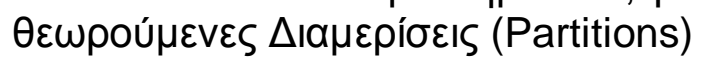

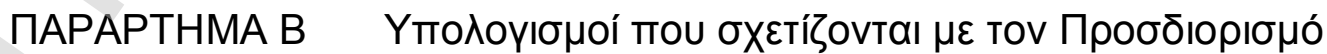

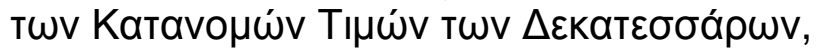

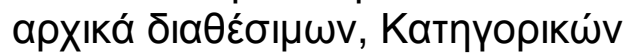

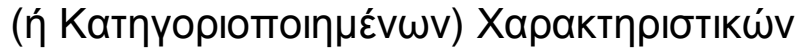




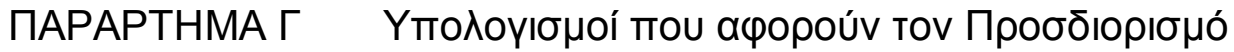

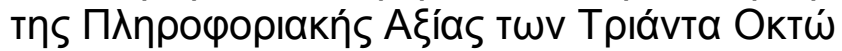
$\Psi \varepsilon \cup \delta о \mu \varepsilon т \alpha \beta \lambda \eta т \omega ́ v$

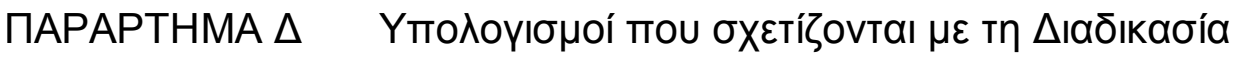

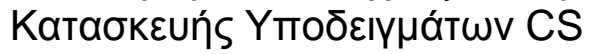

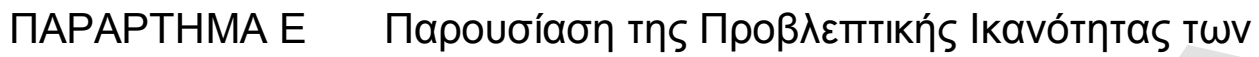

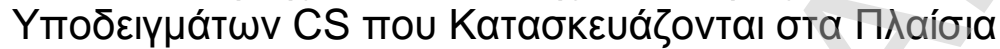

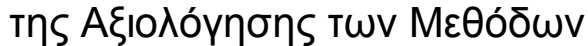

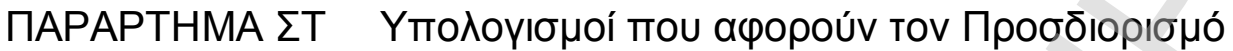

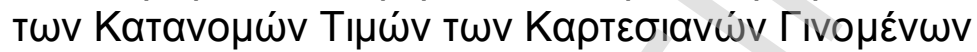

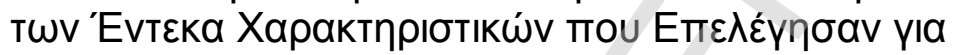

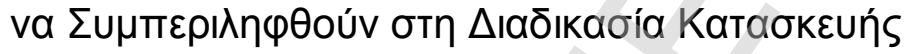

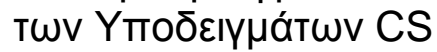




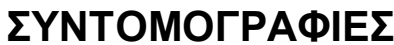

- CS: Credit Scoring

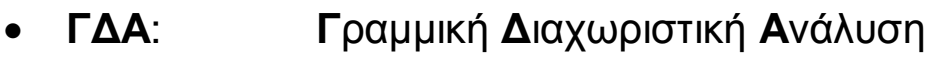

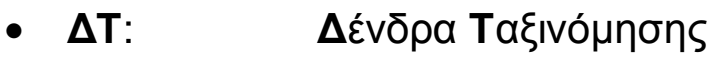

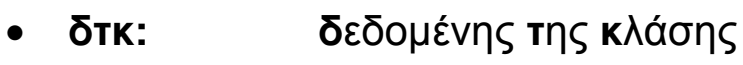

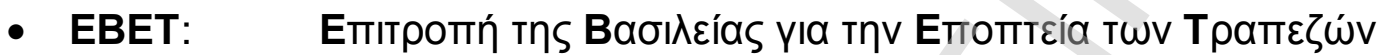

- KAB: Kavóvas tou Atinoú Bayes

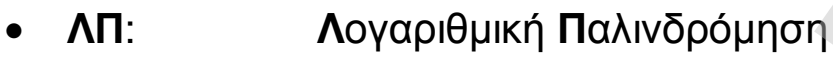

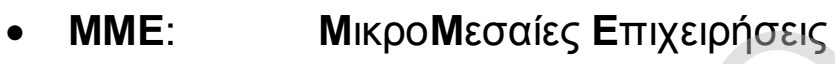

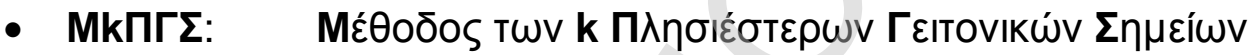

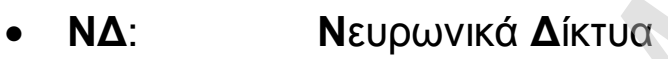

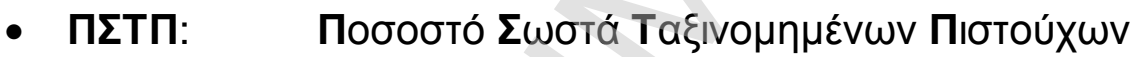

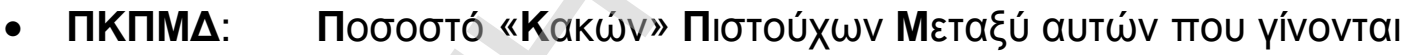
$\Delta \varepsilon$ KTOÍ 


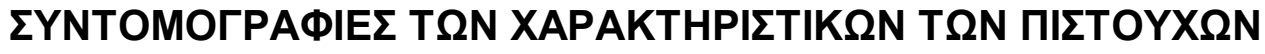

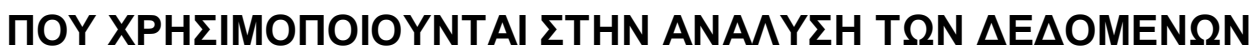

- AGE: H入ıкía

- CAR: Katoxń Autokivńtou

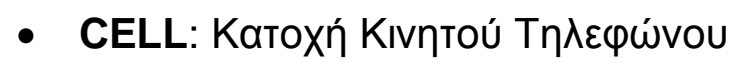

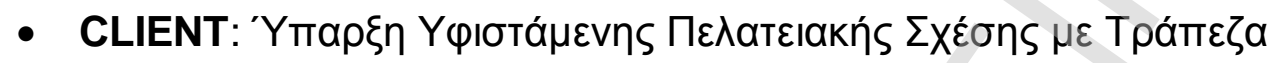

- CRD: Katoxń Пıбтwтikńs Káptas

- GENDER: Фúৗo

- HOME: Tútros Katoiкías

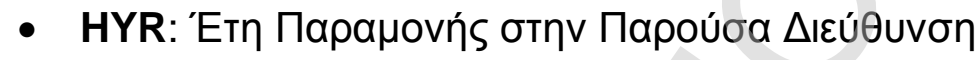

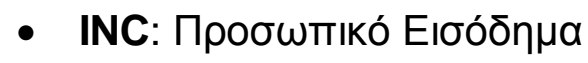

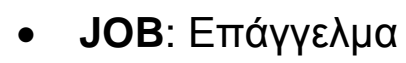

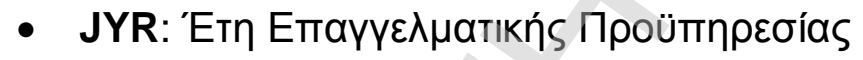

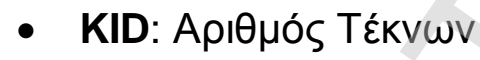

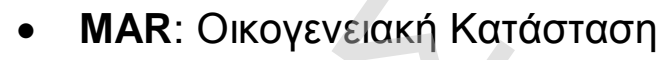

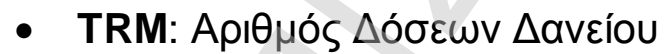




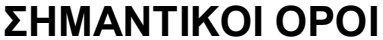

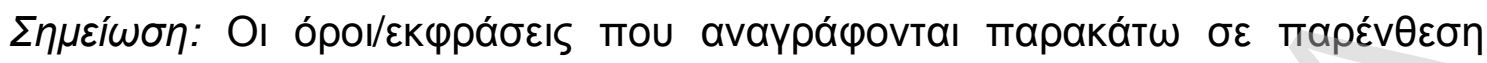

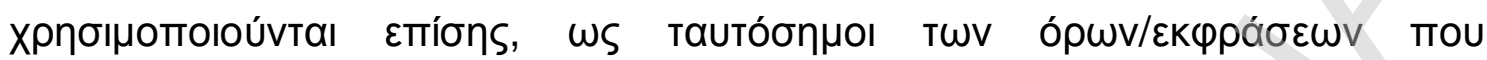

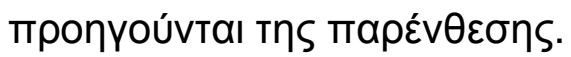

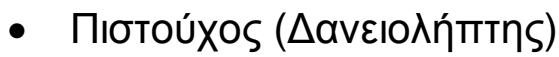

- $\quad \Delta a ́ v \varepsilon ı a(X o p \eta ү n ́ \sigma \varepsilon ı \zeta)$

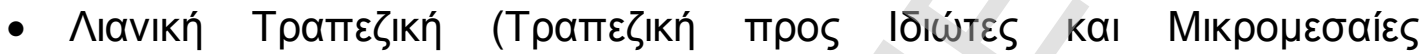

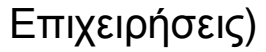

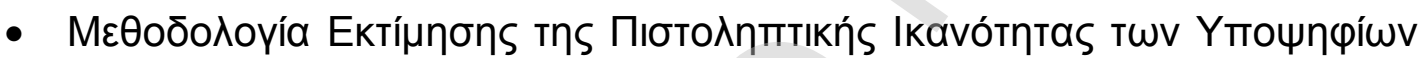

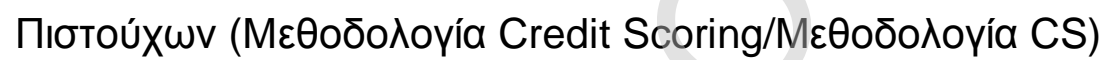

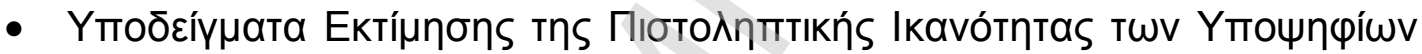

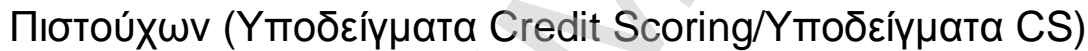

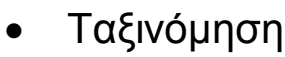

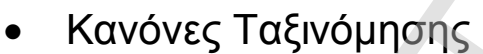

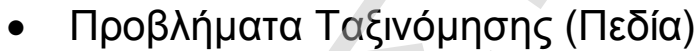

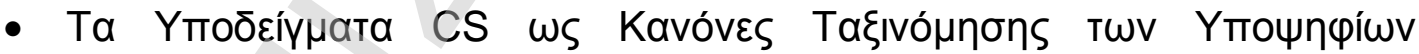
Пıотоúxwv

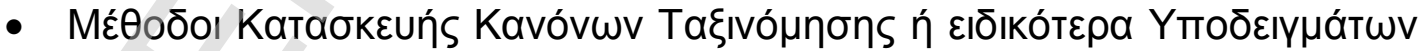
CS

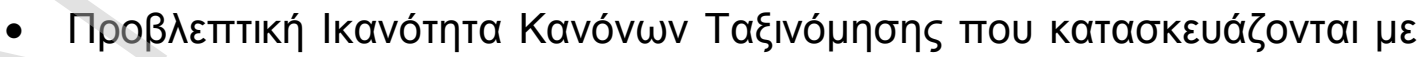

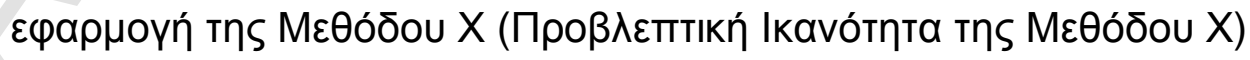




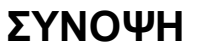

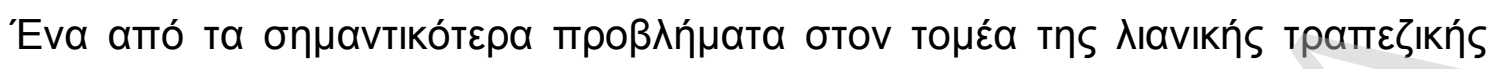

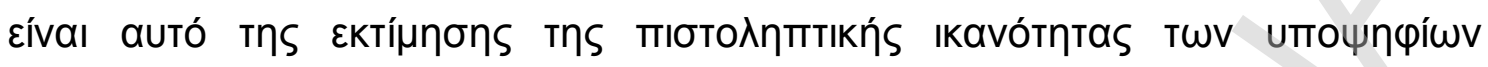

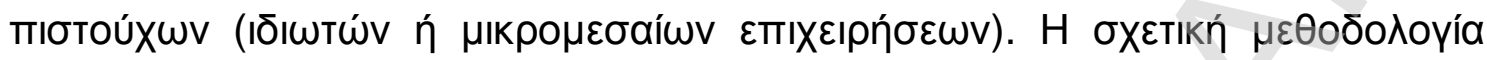

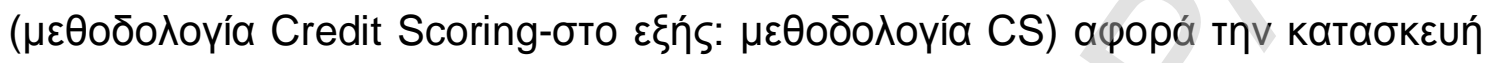

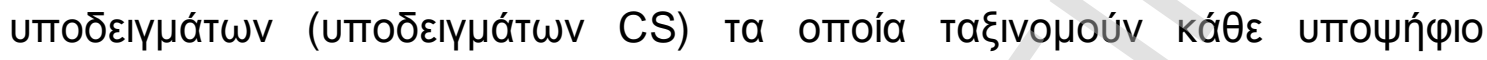

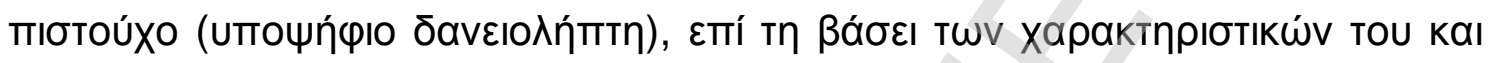

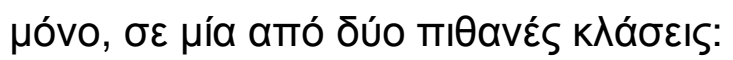

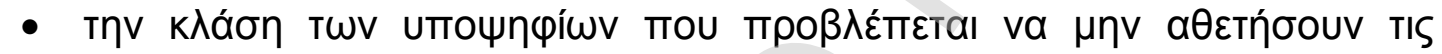

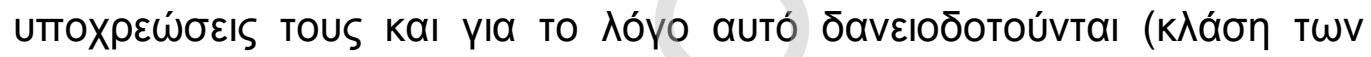

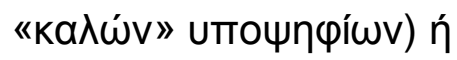

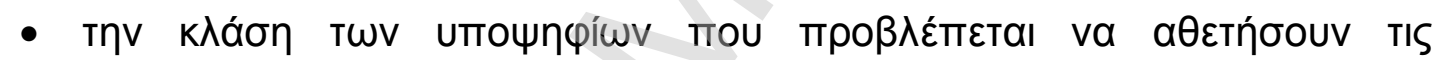

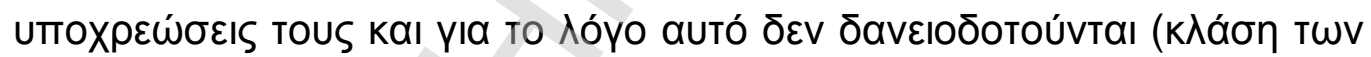

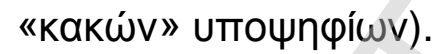

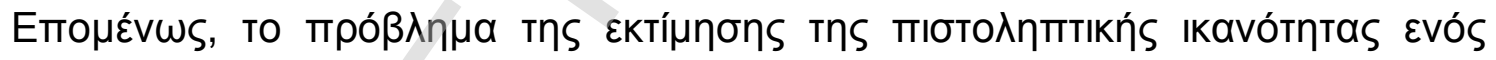

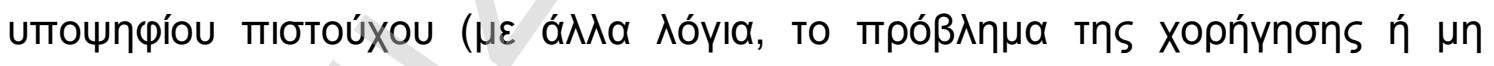

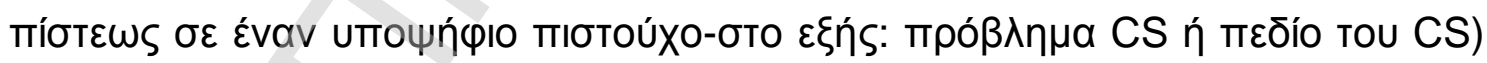

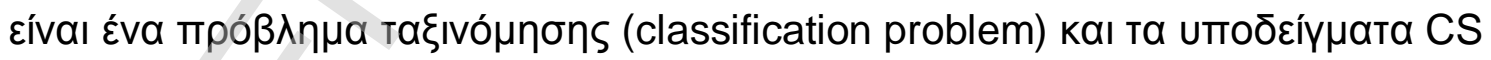

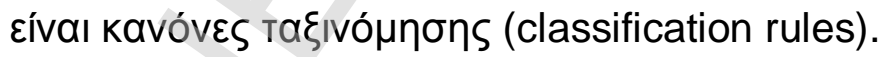

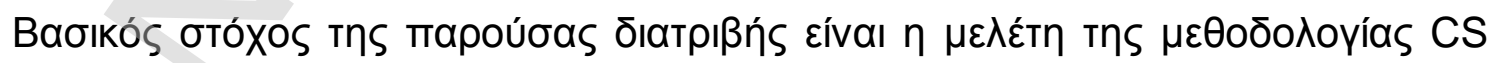

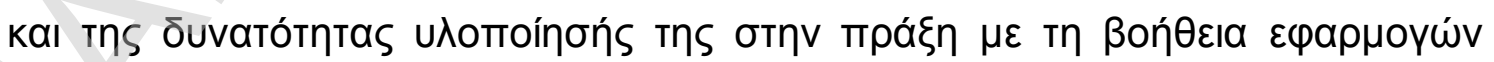

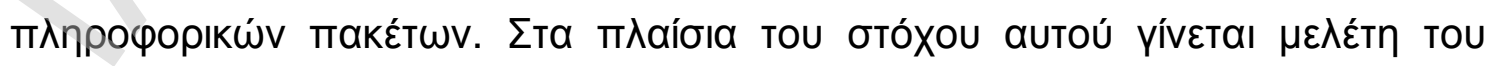

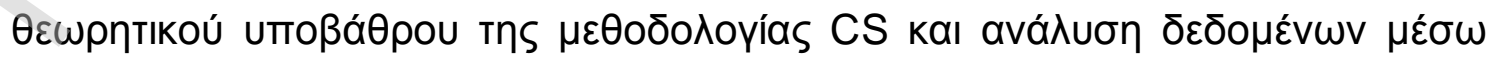

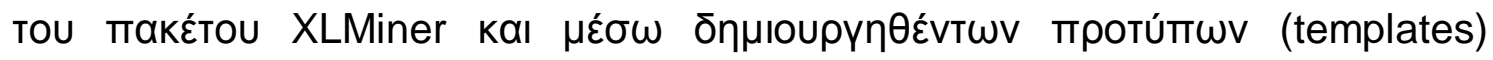




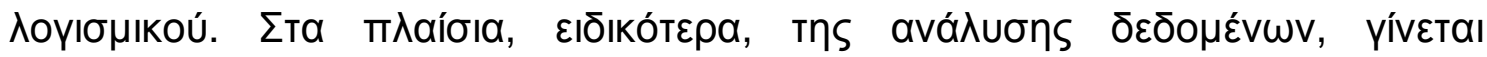

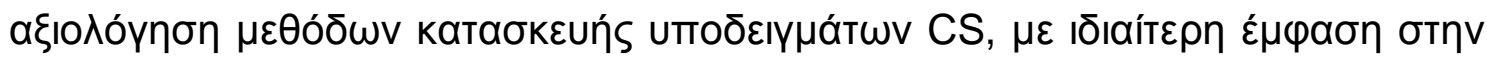

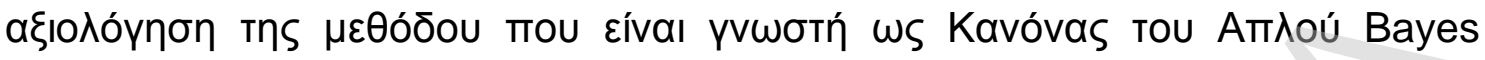

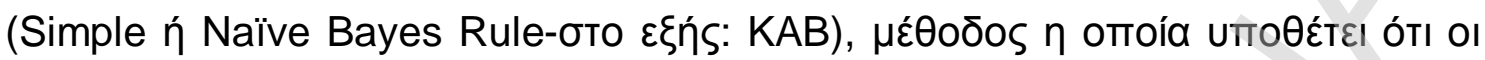

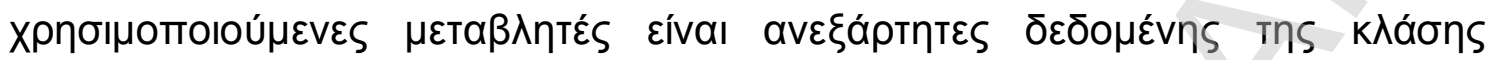

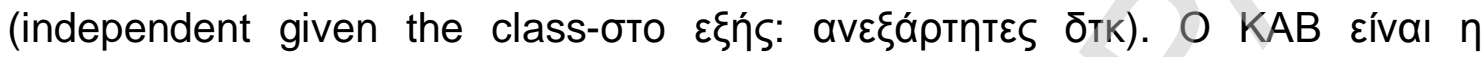

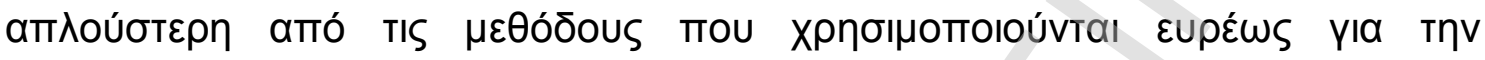

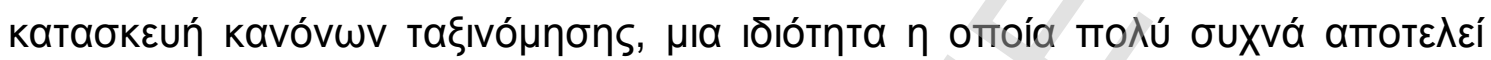

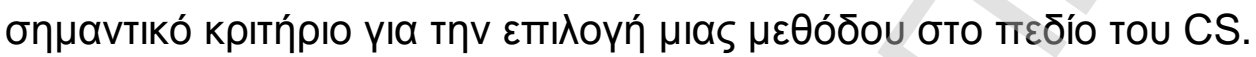

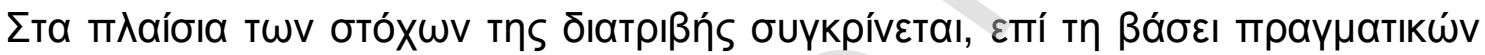

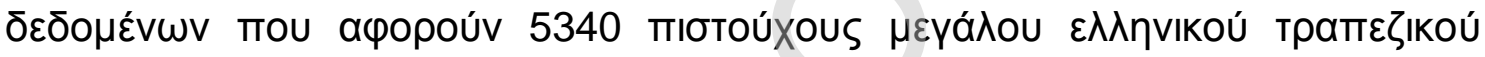

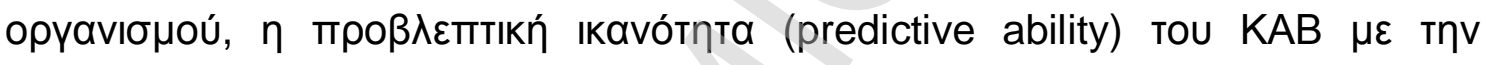

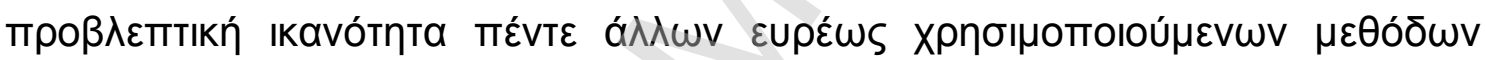

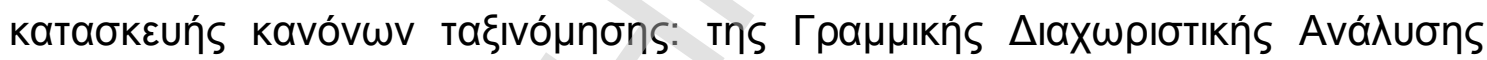

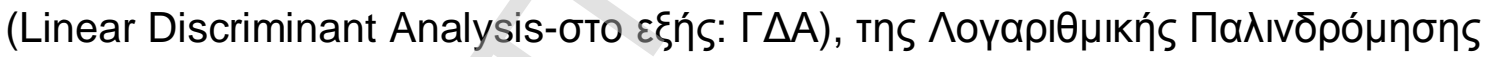

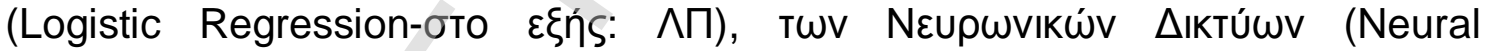

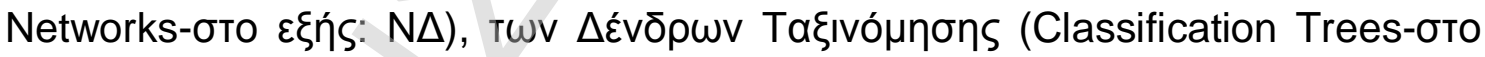

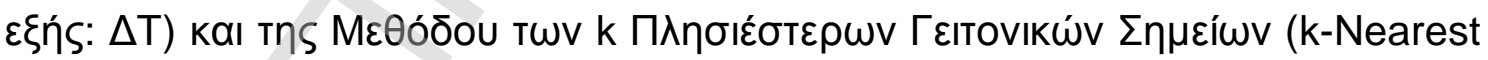

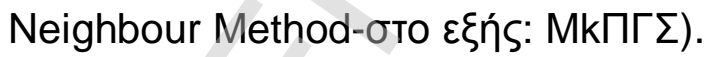

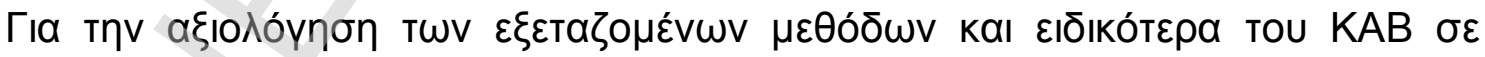

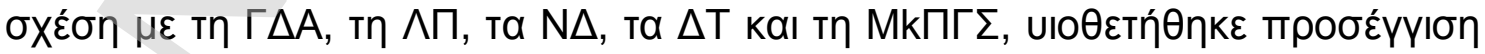

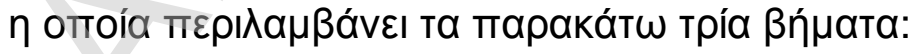

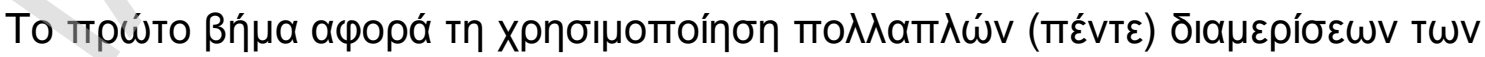

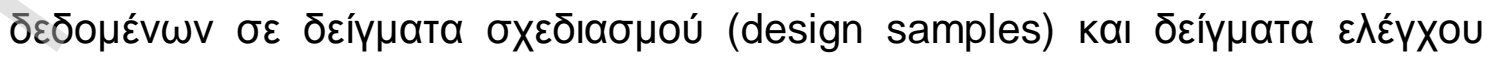

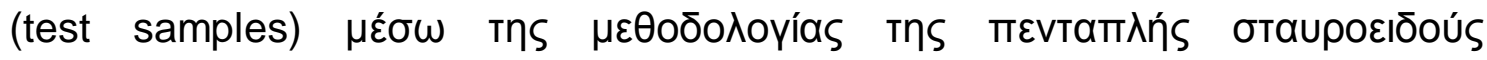




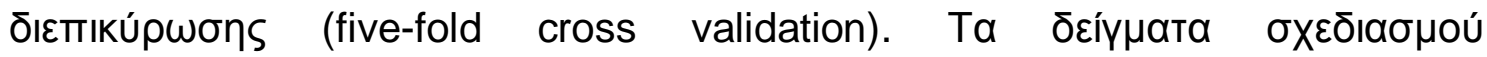

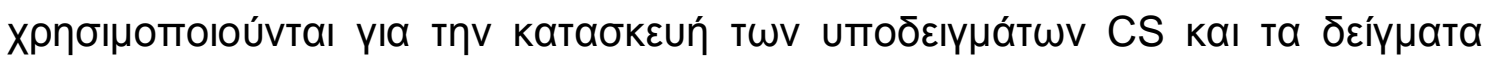

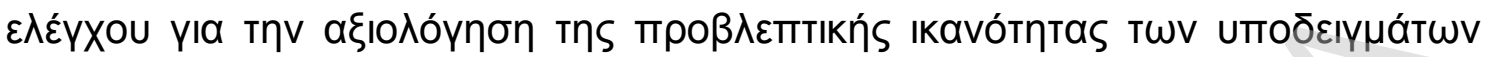

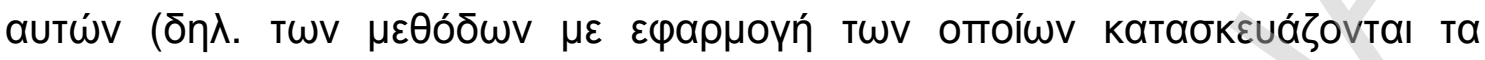

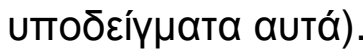

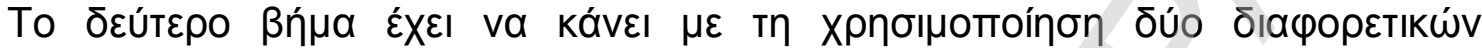

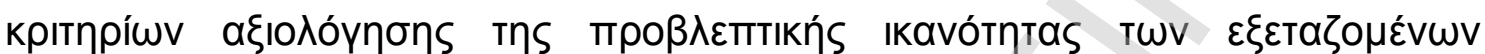

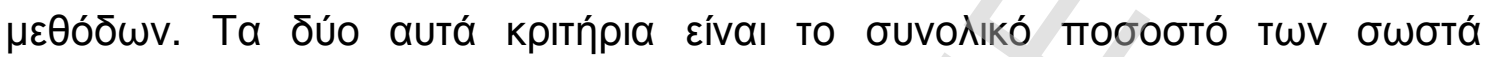

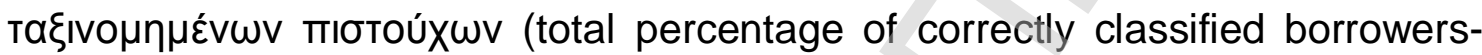

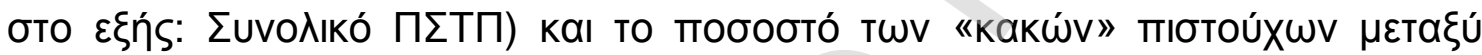

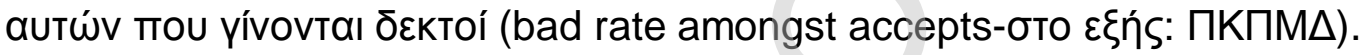

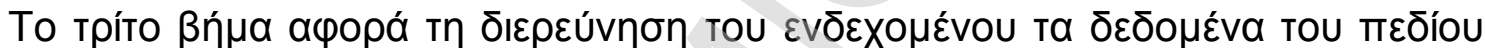

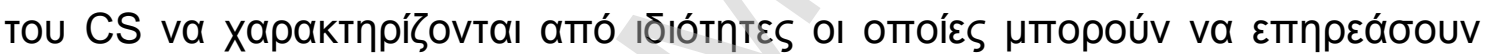

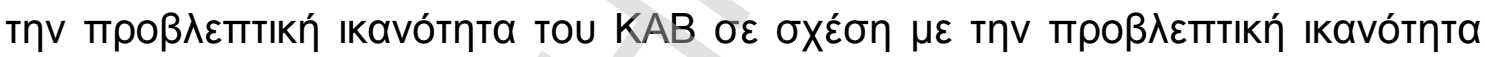
á $\lambda \lambda \omega v \mu \varepsilon \theta o ́ \delta \omega v$.

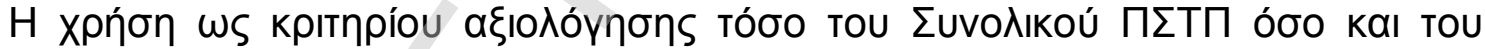

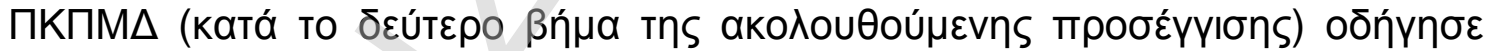

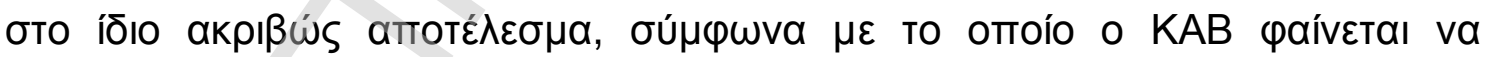

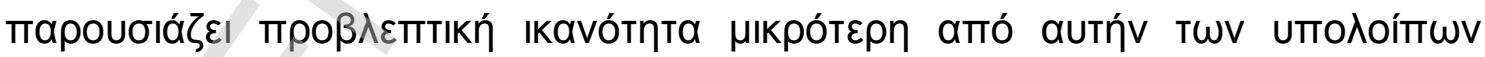

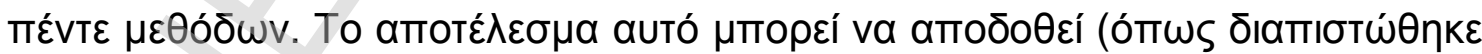

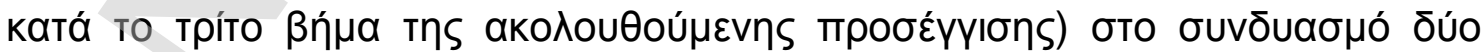

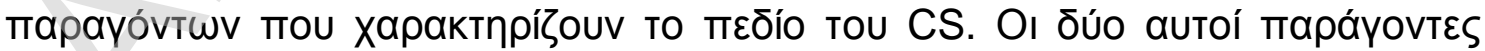
Eívaı: 


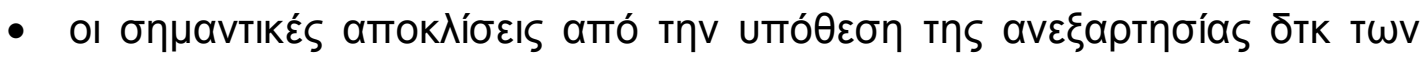

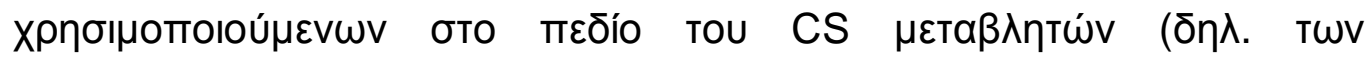

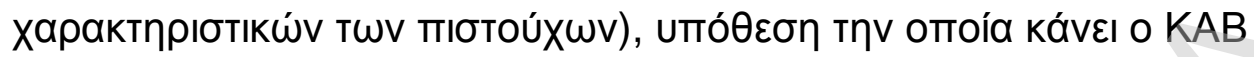

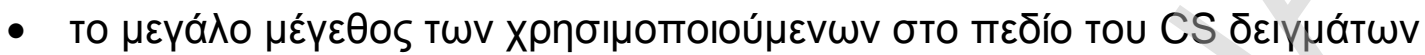
$\sigma \chi \varepsilon \delta \overline{\prime \alpha \sigma \mu \rho}$

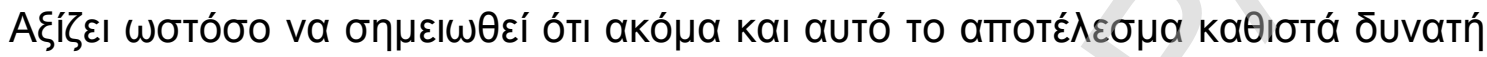

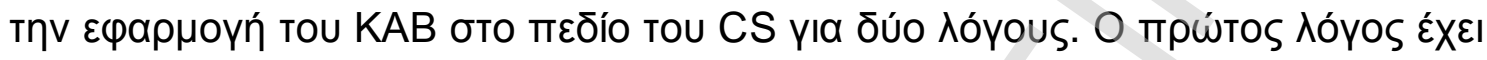

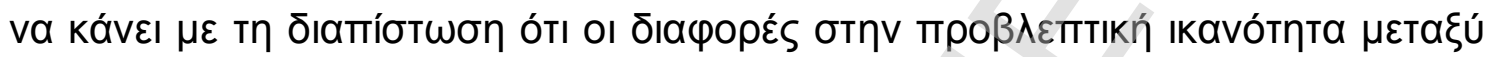

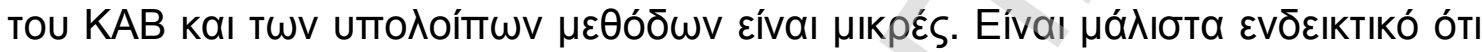

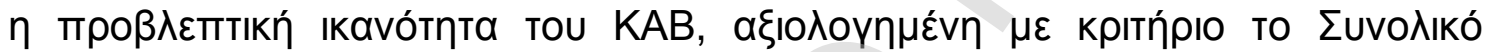

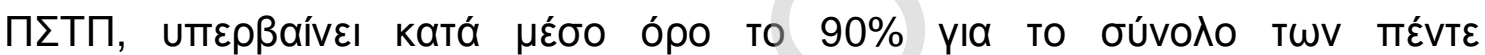

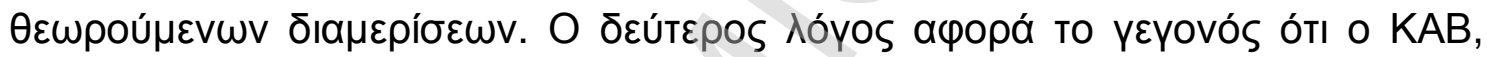

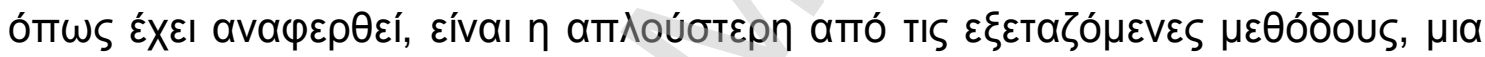

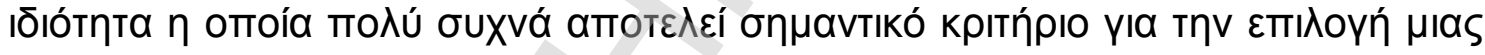
$\mu \varepsilon \theta$ 


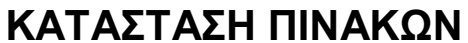

$\Sigma \varepsilon \lambda$.

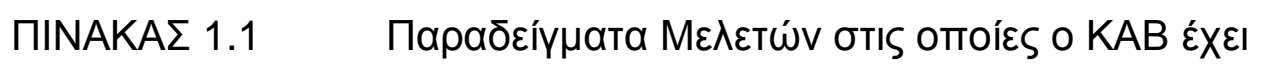

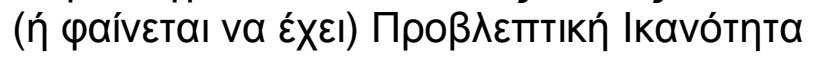

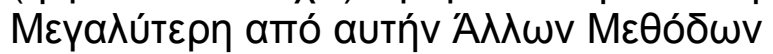

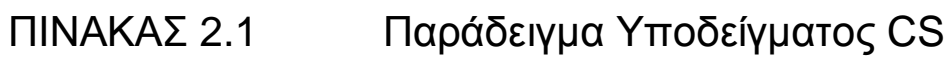

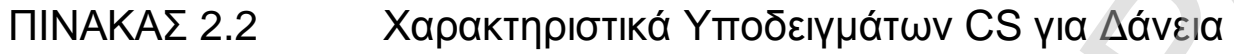

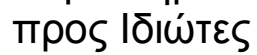

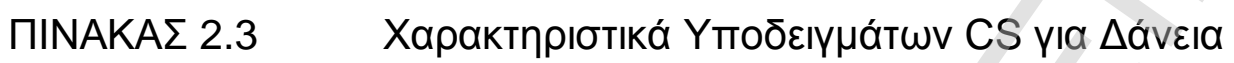
трољ MME

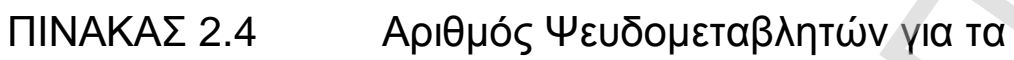
Характпрібтіка́ тоu Пívaka 2.1

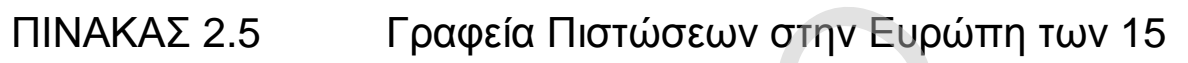

ПINAKA $\Sigma 2.6$

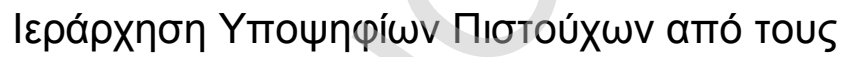

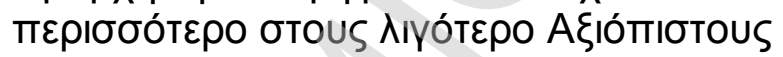

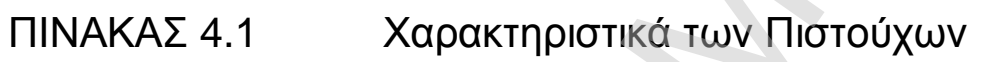

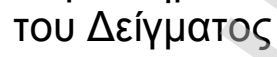

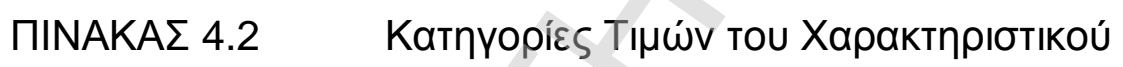
«Катохń Autokivńtou»

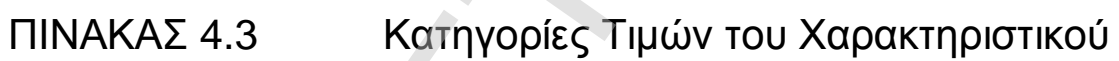

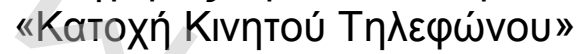

ПINAKA $\Sigma 4.4$

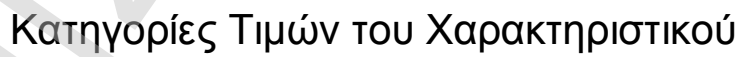

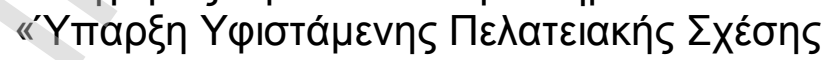

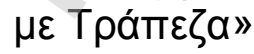

ПINAKA $\Sigma 4.5$

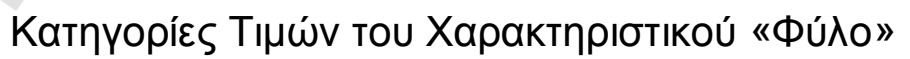

ПINAKA 24.6

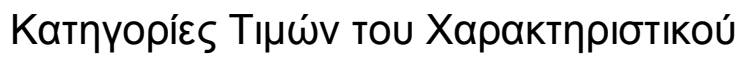

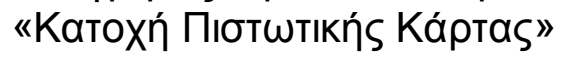

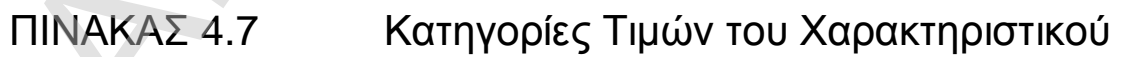

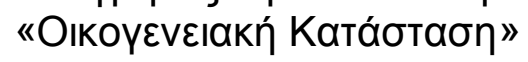

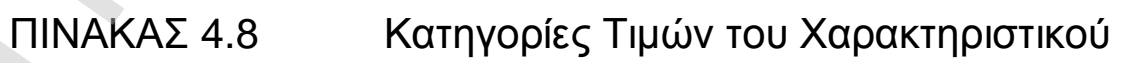
«Tútros Katoıkías"

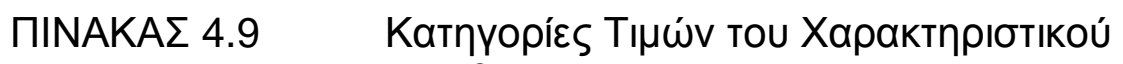

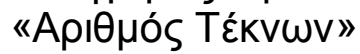




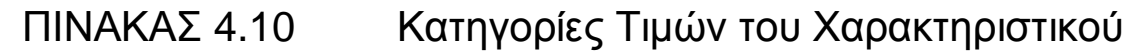

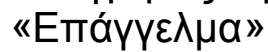

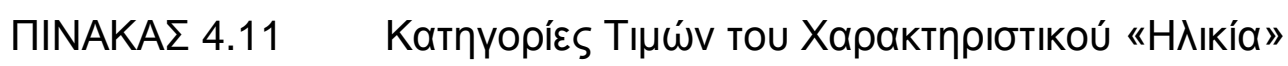

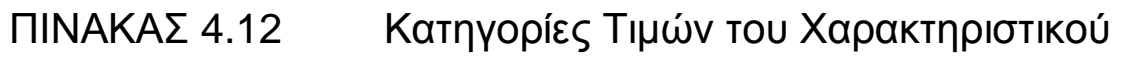

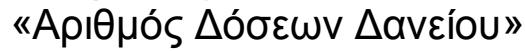

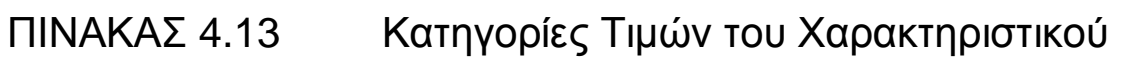

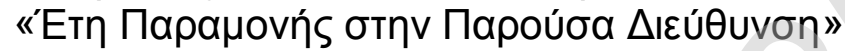

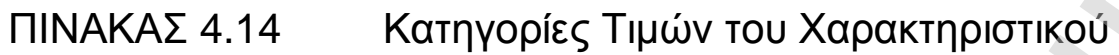

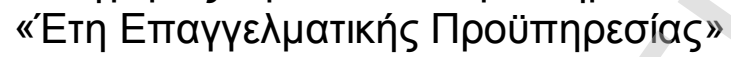

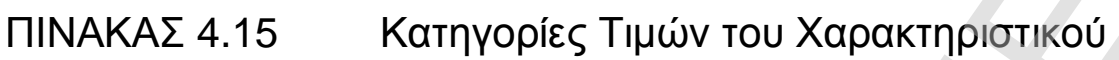

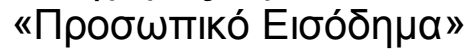

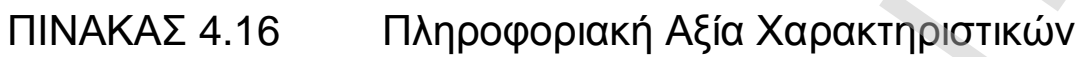

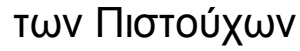

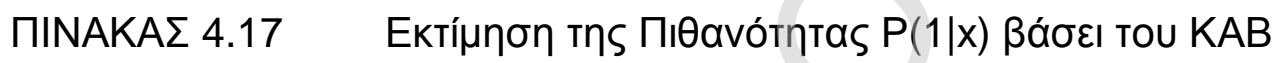

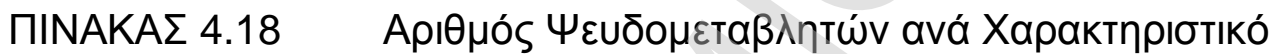

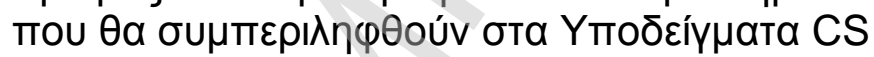

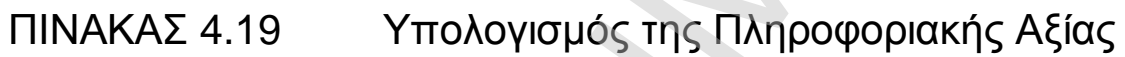

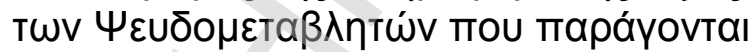

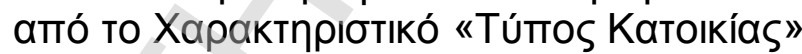

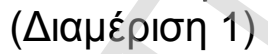

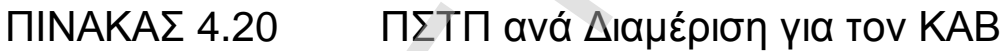

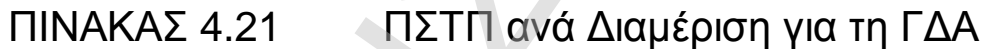

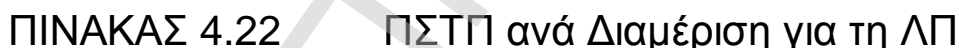

ПINAKA $\Sigma 4.23$

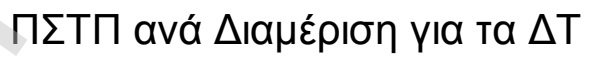

ПINAKA $\Sigma 4.24$

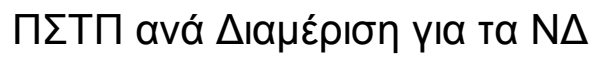

ПINAKA $\Sigma 4.25$

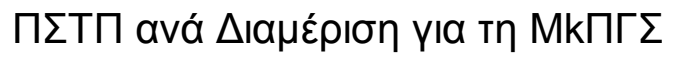

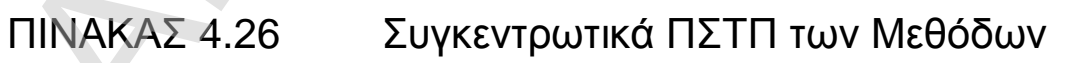

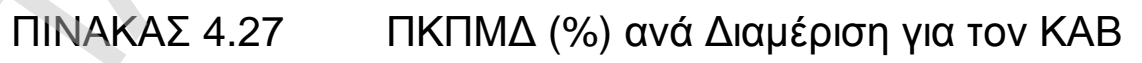

ПINAKA 2.28 ПКПM 


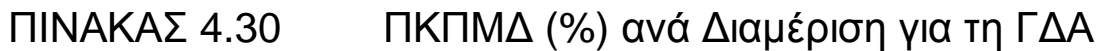

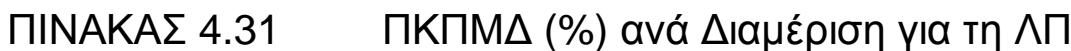

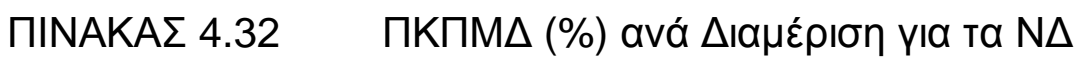

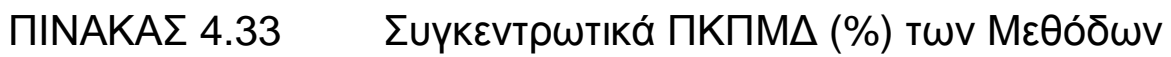

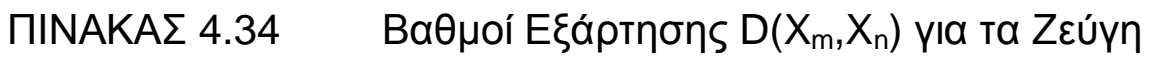

Характпрібтіки́v $X_{m}, X_{n}$

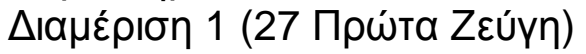

ПINAKA $\Sigma 4.35$

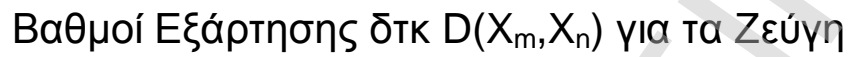

Характпрібтіки́v $\mathrm{X}_{\mathrm{m}}, \mathrm{X}_{\mathrm{n}}$

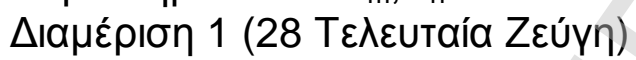

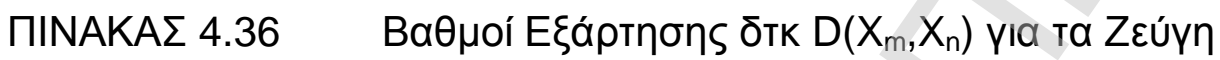
Характпрібтіки́v $\mathrm{X}_{\mathrm{m}}, \mathrm{X}_{\mathrm{n}}$

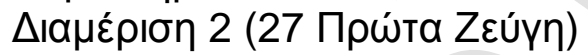

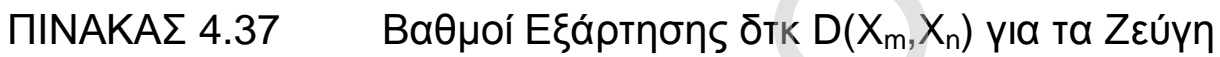

Хapaktnрібтікúv $\mathrm{X}_{\mathrm{m}}, \mathrm{X}_{\mathrm{n}}$

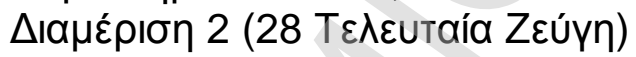

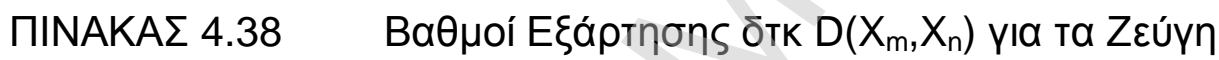
Хaрактпрібтікúv $\mathrm{X}_{\mathrm{m}}, \mathrm{X}_{\mathrm{n}}$

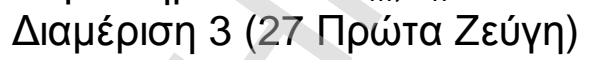

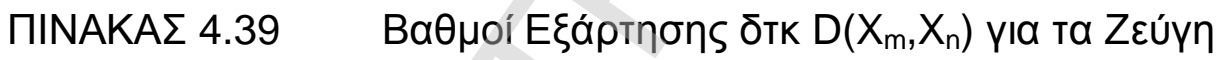
Хapaktпрібтікúv $\mathrm{X}_{\mathrm{m}}, \mathrm{X}_{\mathrm{n}}$

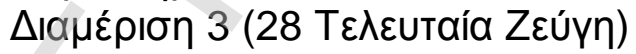

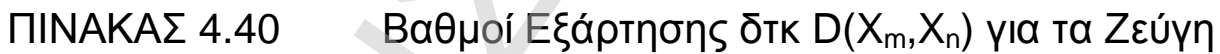
Характпрібтіки́v $\mathrm{X}_{\mathrm{m}}, \mathrm{X}_{\mathrm{n}}$

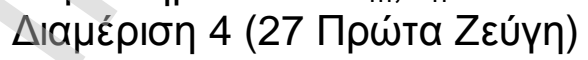

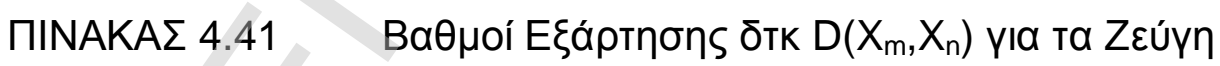
Хaрактпрібтіки́v $\mathrm{X}_{\mathrm{m}}, \mathrm{X}_{\mathrm{n}}$

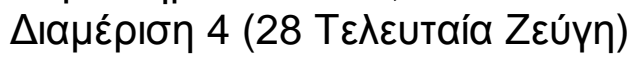

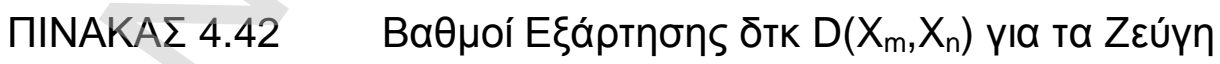

Хaрakтпрібтіки́v $\mathrm{X}_{\mathrm{m}}, \mathrm{X}_{\mathrm{n}}$

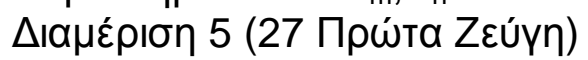

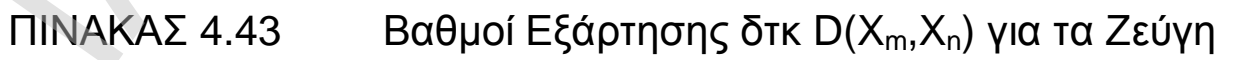
Хaрaктпрібтікúv $\mathrm{X}_{\mathrm{m}}, \mathrm{X}_{\mathrm{n}}$

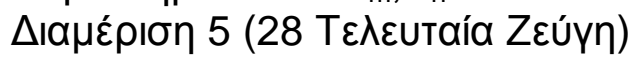




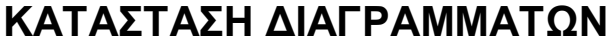

$\Sigma \varepsilon \lambda$.

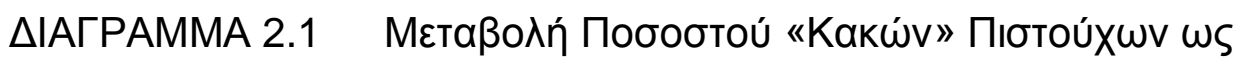

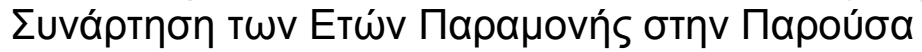

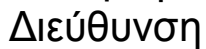

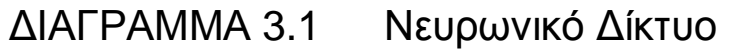




\section{KEФA^AIO 1}

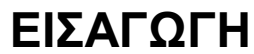

\subsection{ETóXol tns $\Delta$ IatpIßńs}

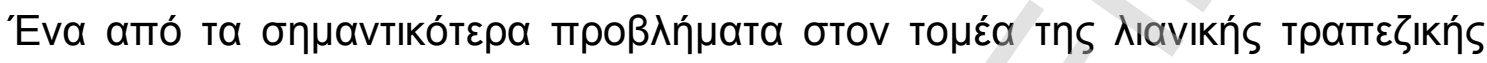

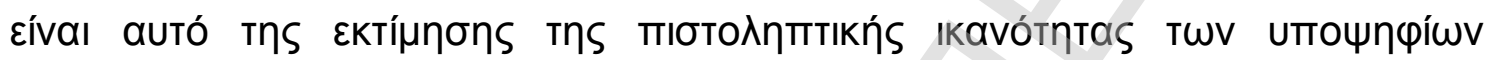

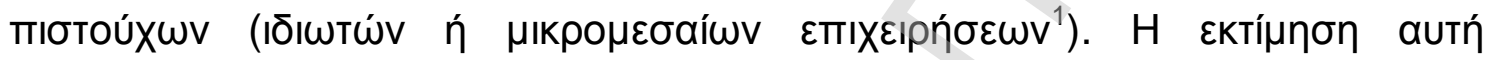

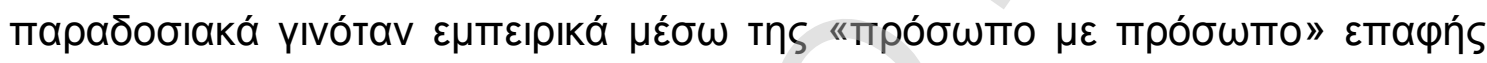

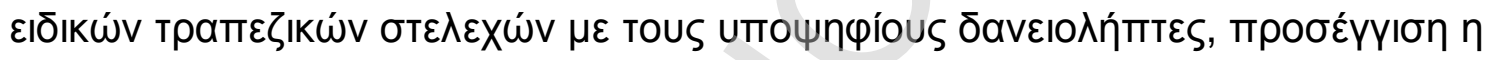

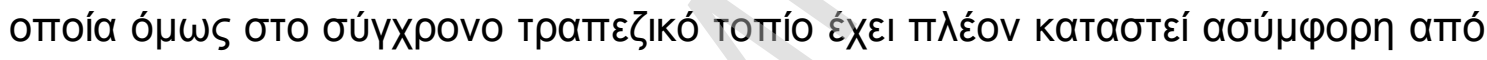

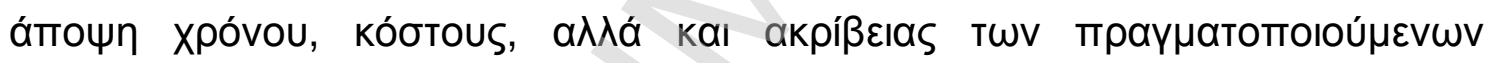

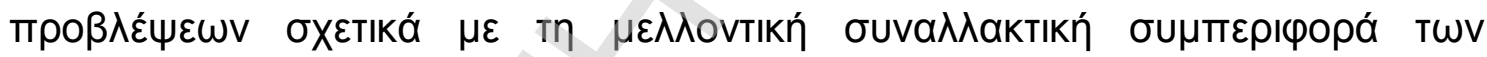

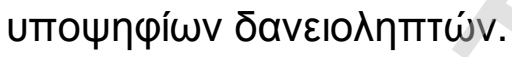

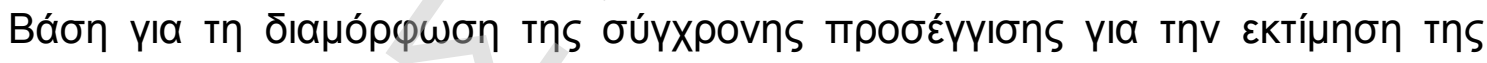

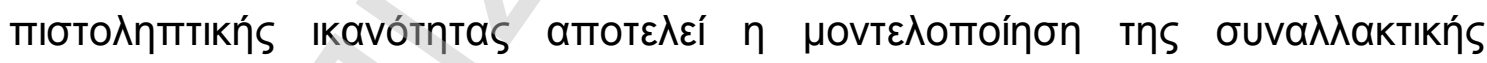

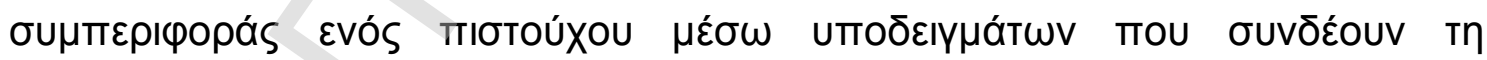

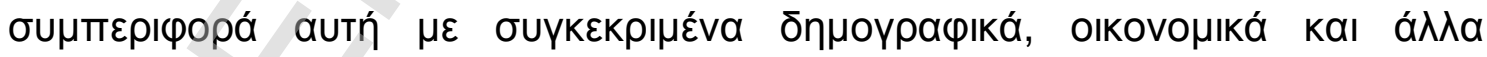

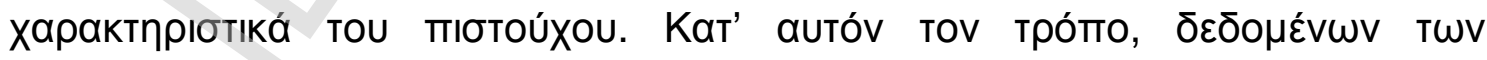

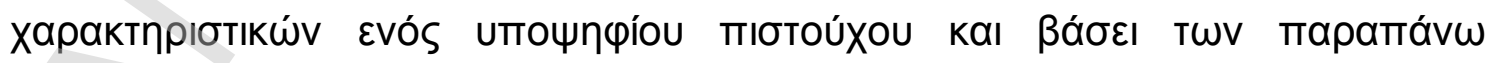

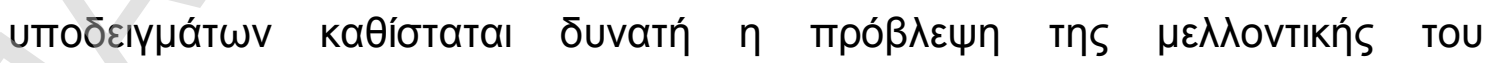

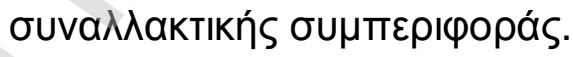

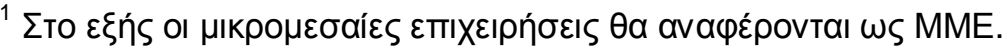




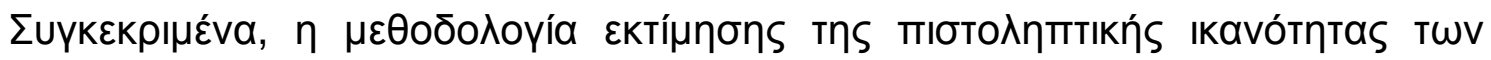

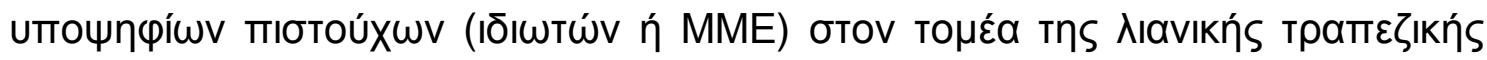
(

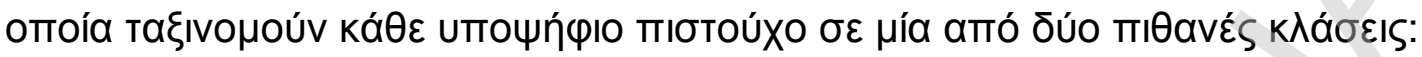

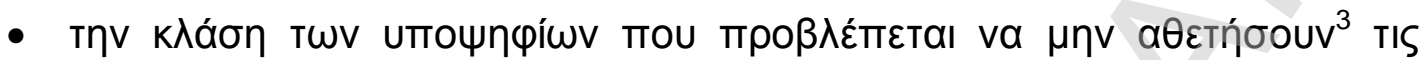

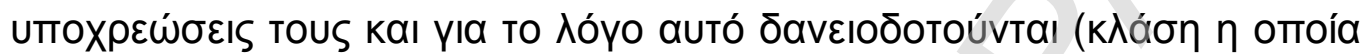

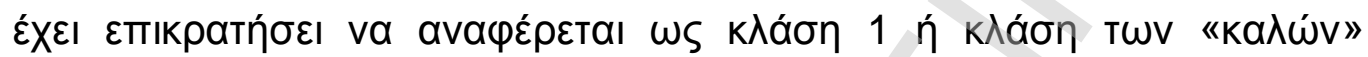

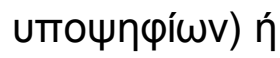

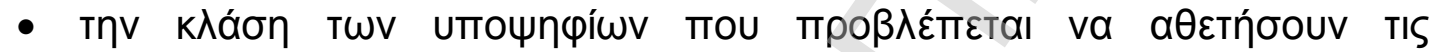

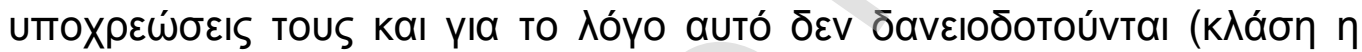

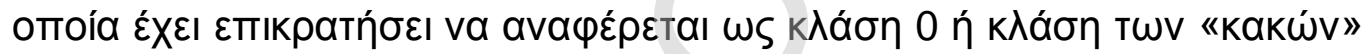
uточчпі́́wv).

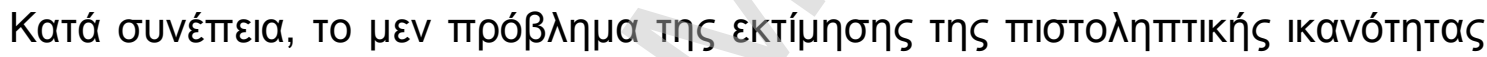

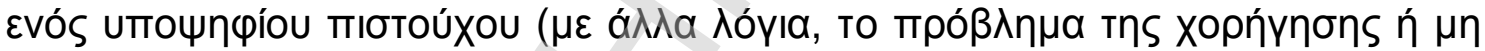

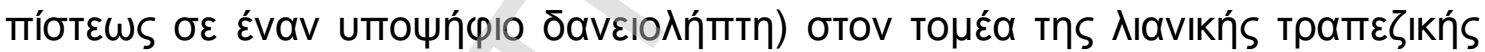

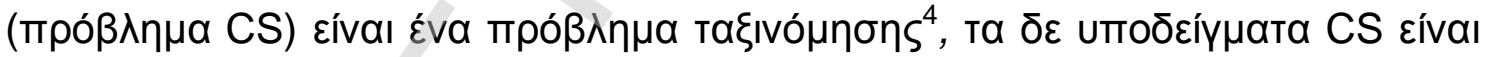

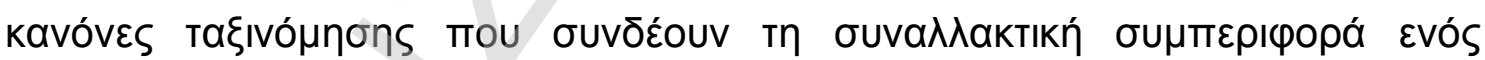

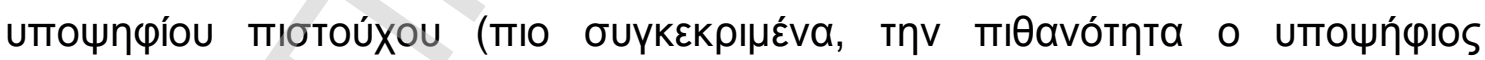

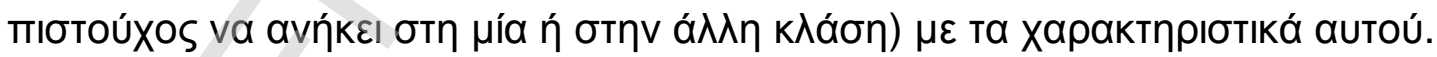

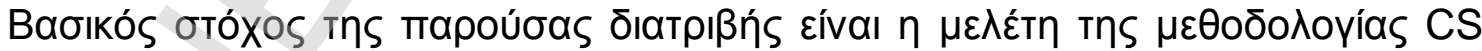

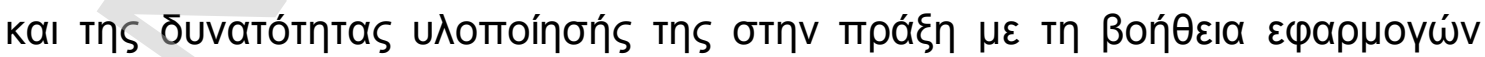

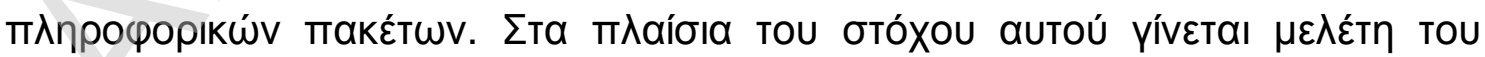

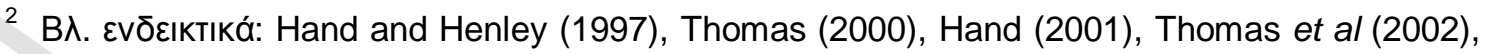
Thomas et al (2004), Hand (2005).

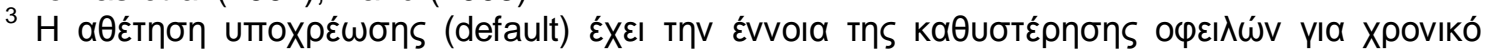

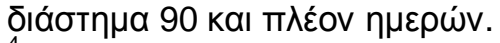

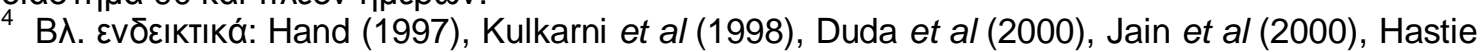
et al (2001), Webb (2002). 


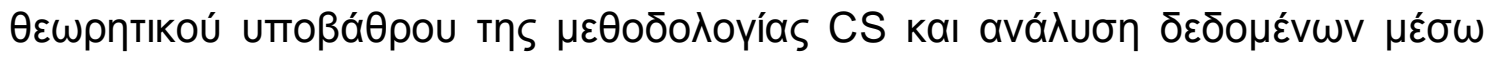

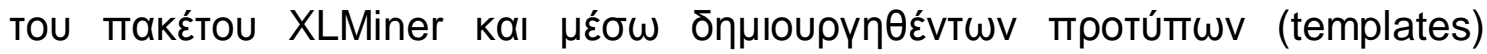

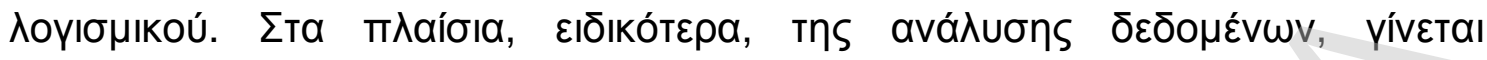

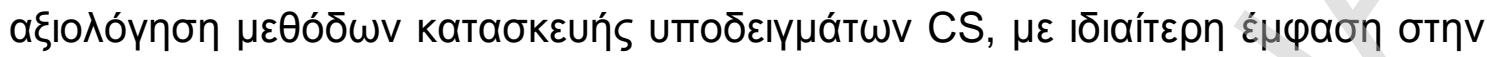

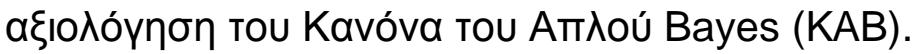

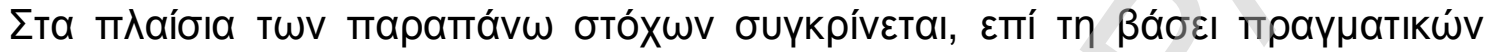

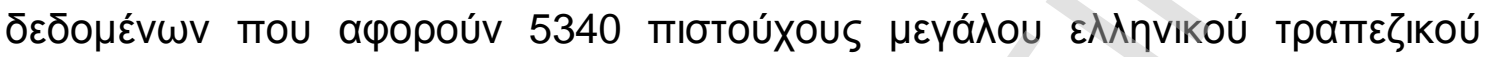

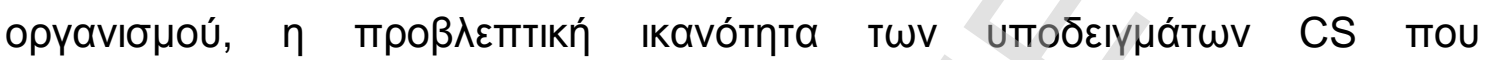

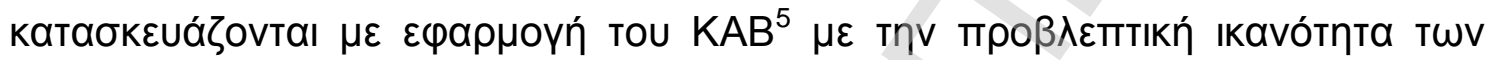

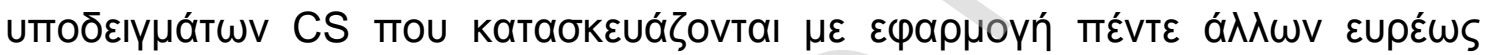

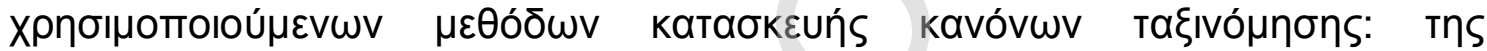

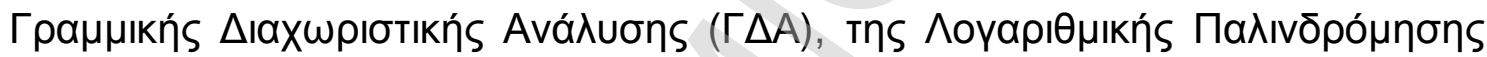

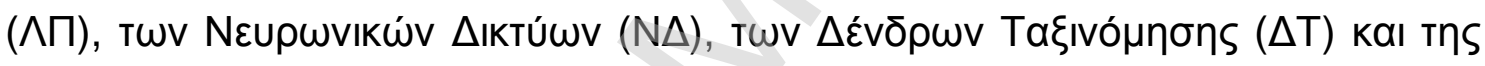

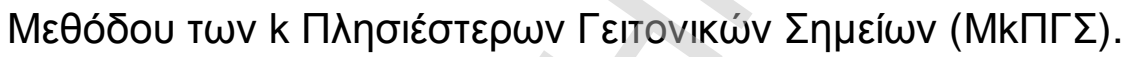

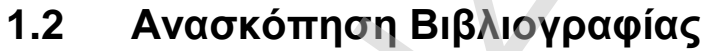

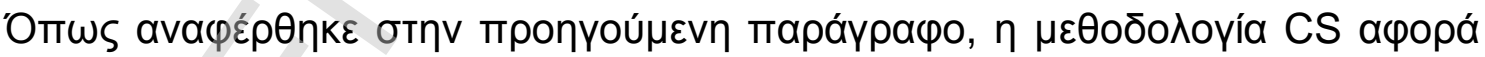

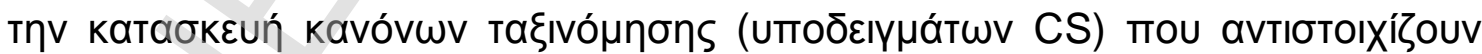

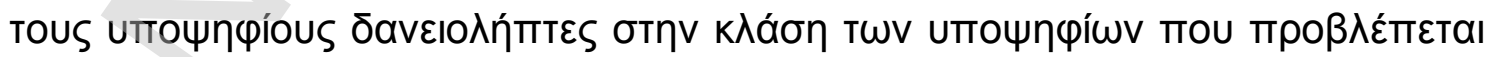

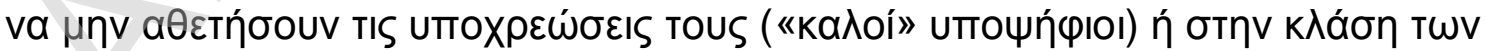

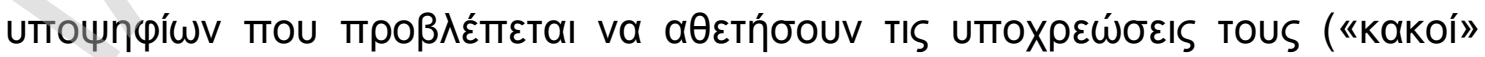

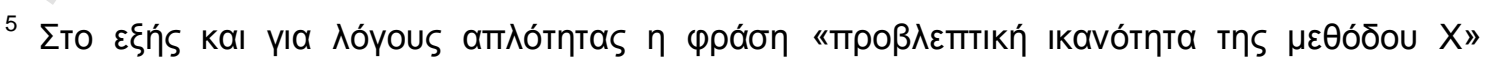

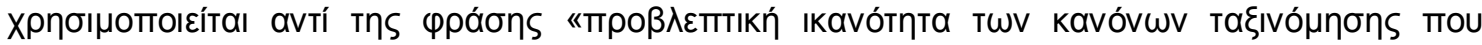

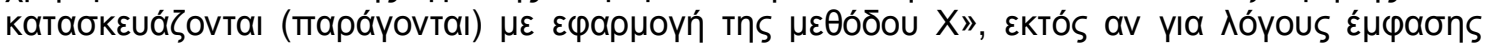

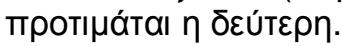




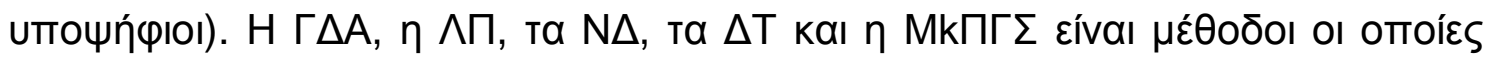

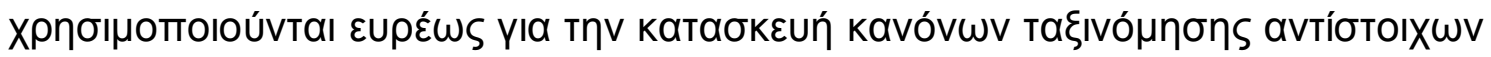

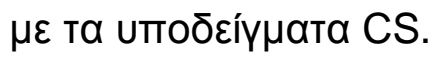

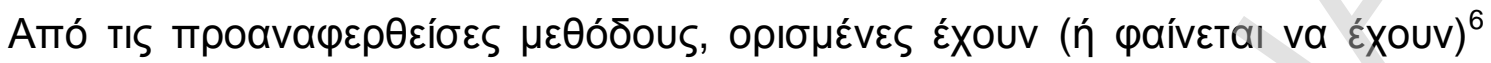

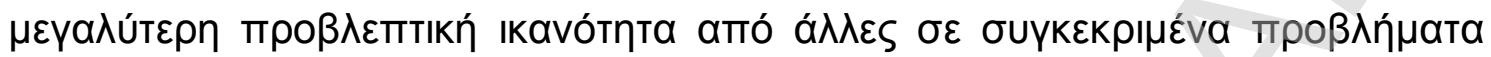

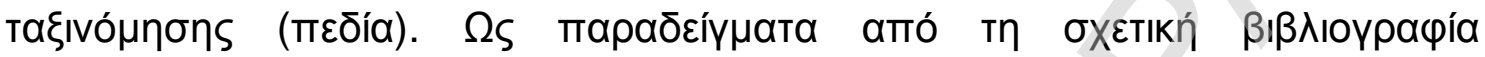

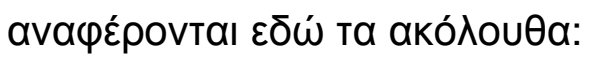

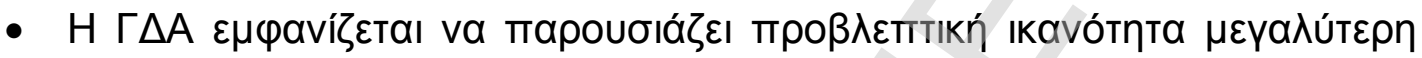

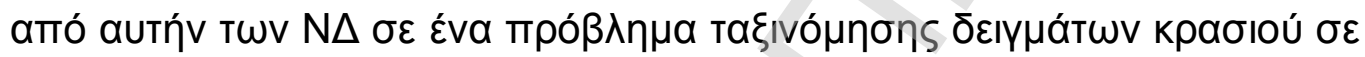

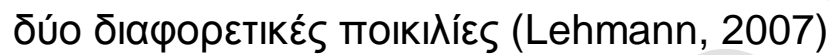

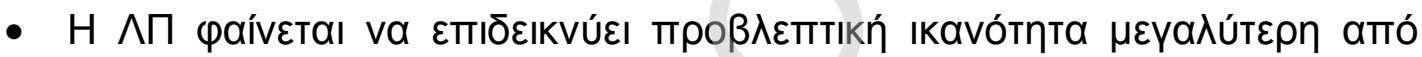

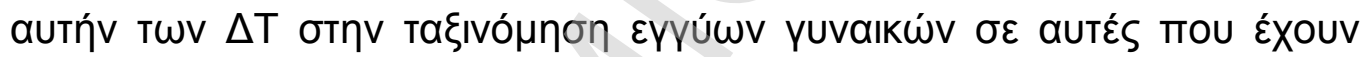

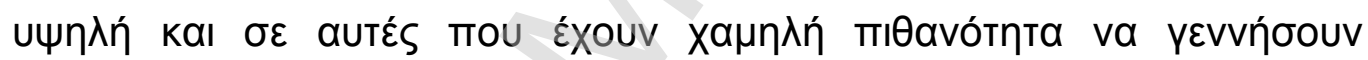

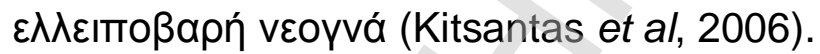

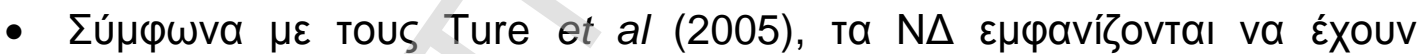

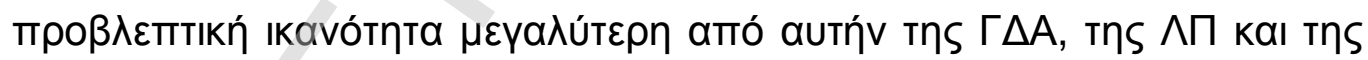

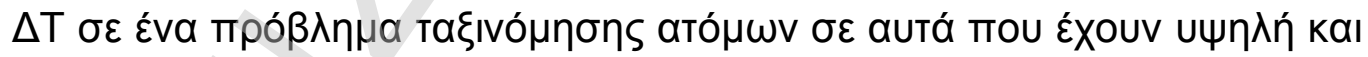

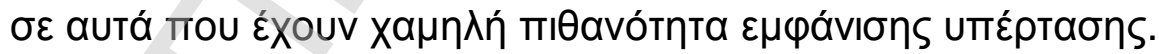

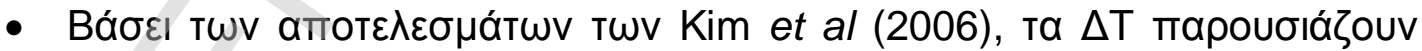

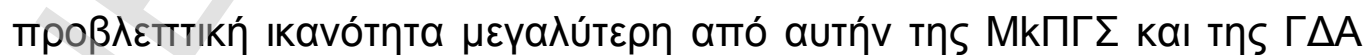

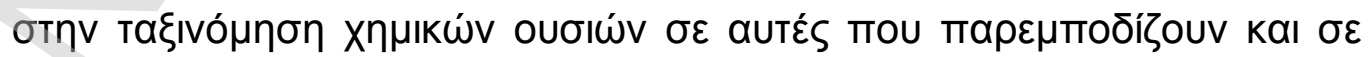

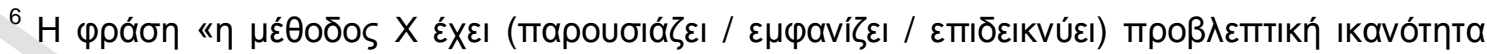

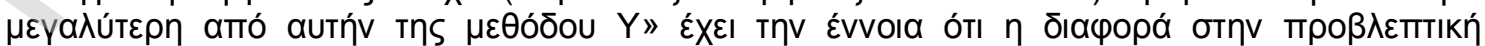

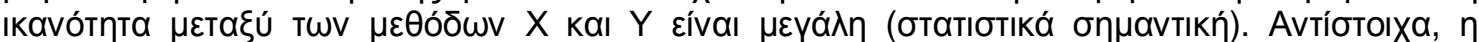

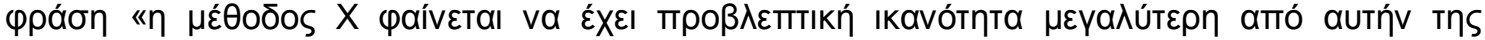

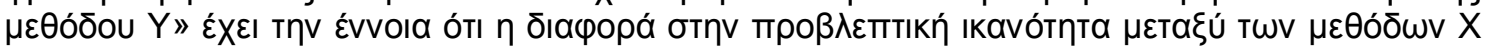

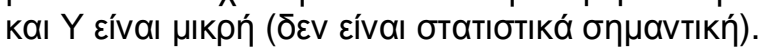




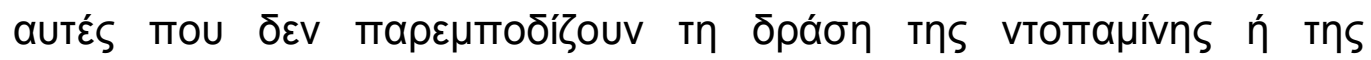

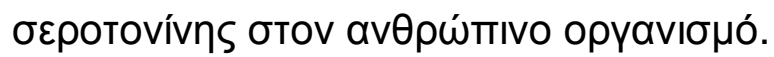

- H MkПГ

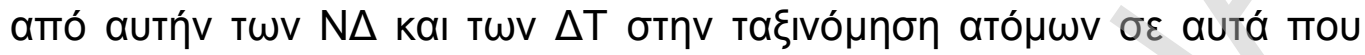

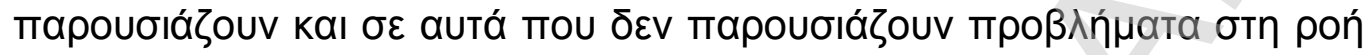

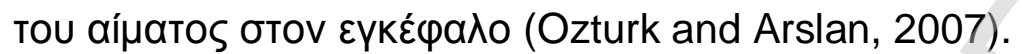

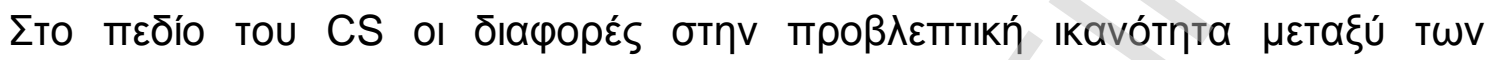

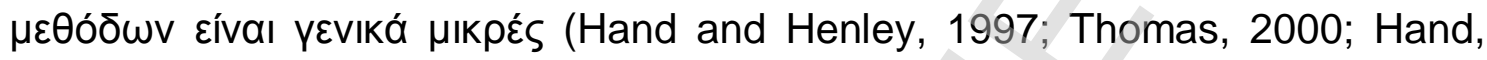

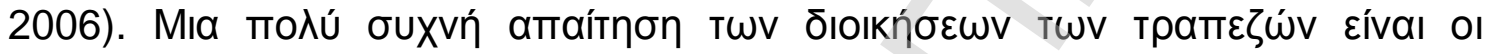

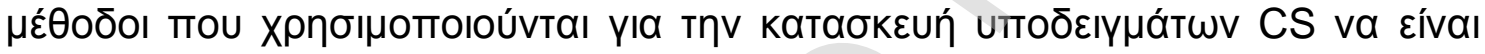

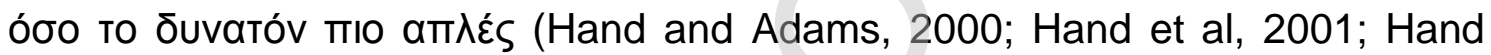

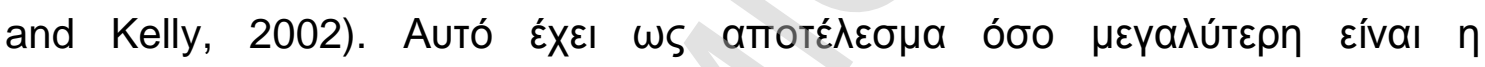

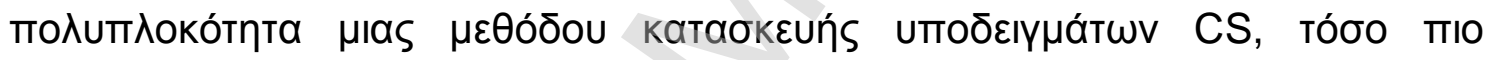

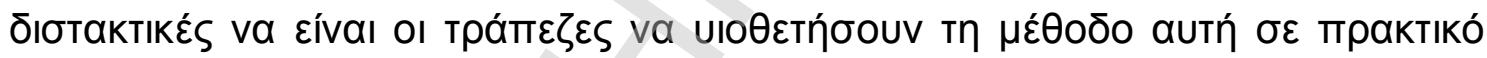

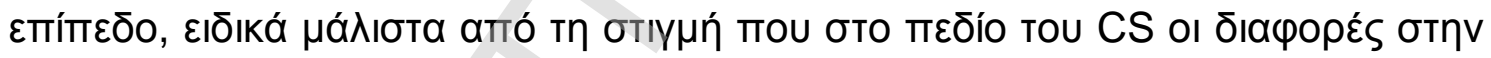

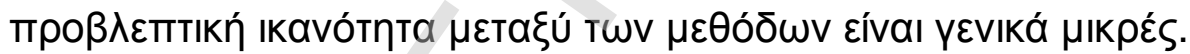

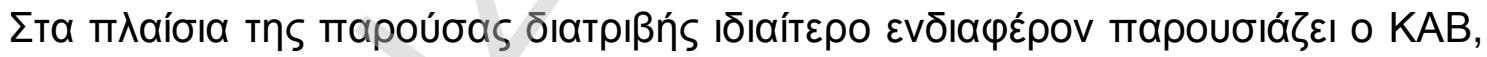

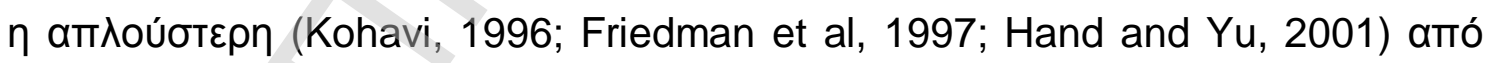

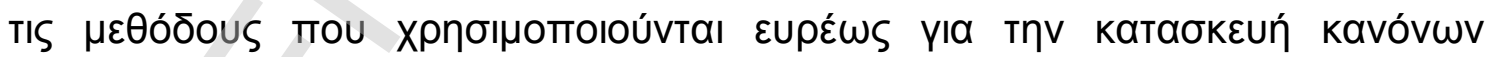

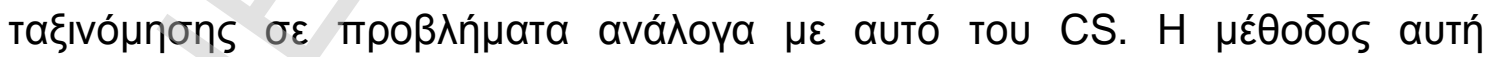

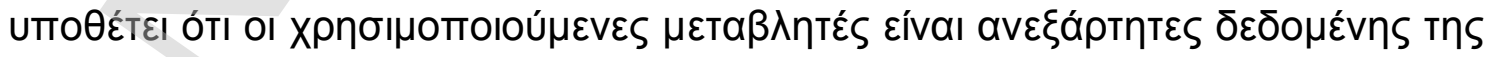

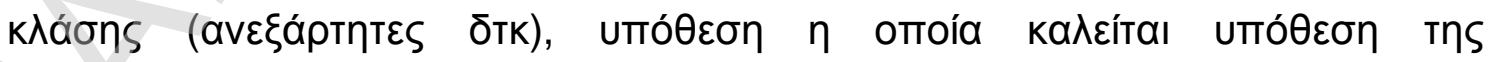

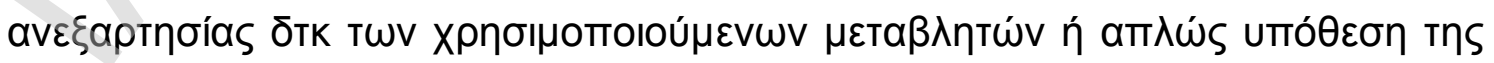

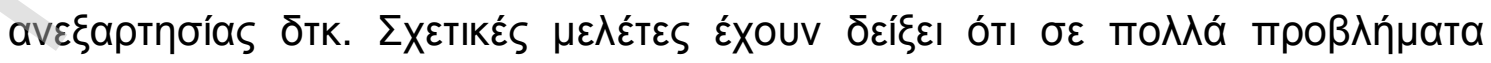

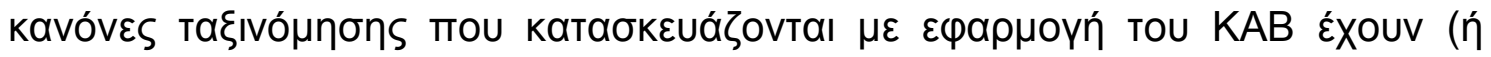




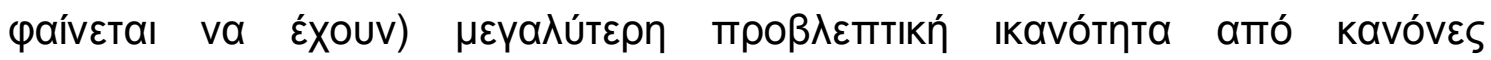

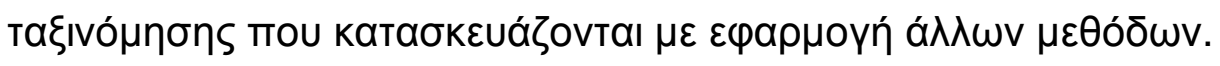

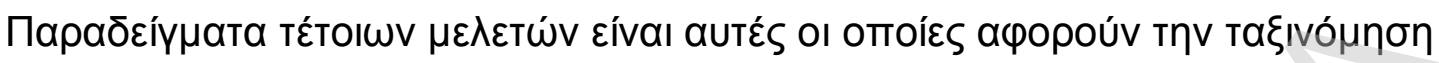

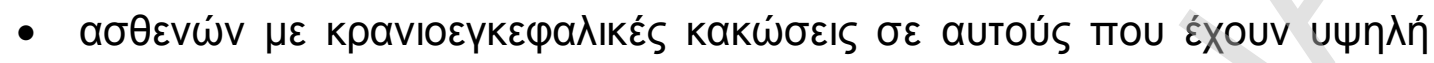

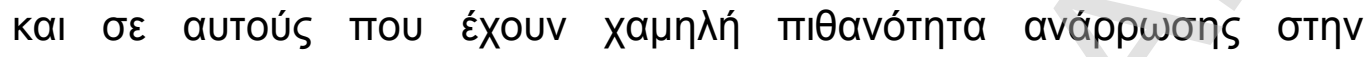

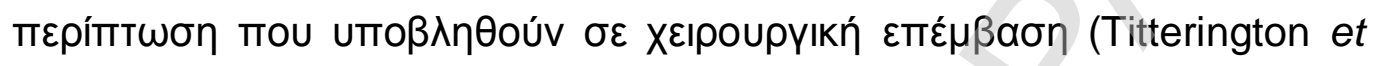
al, 1981)

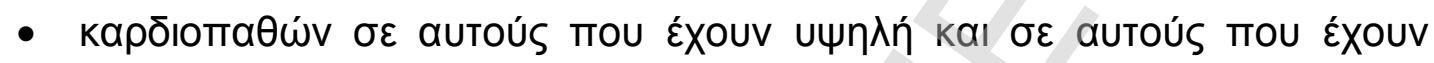

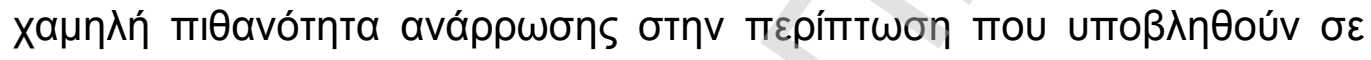

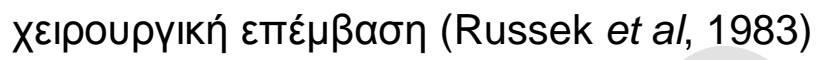

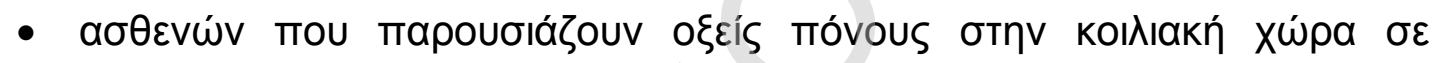

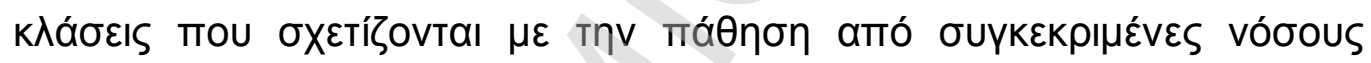
(Gammerman and Thatcher, 1991; Ohmann et al, 1996)

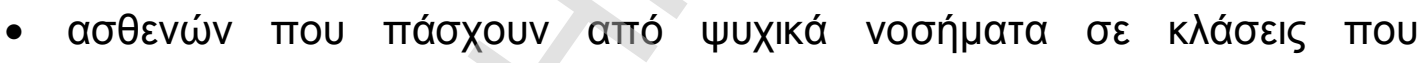

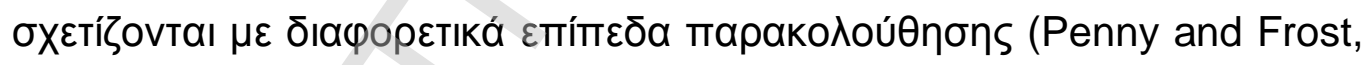
1997)

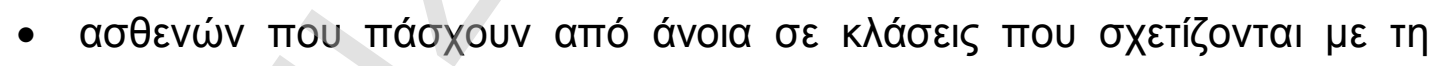

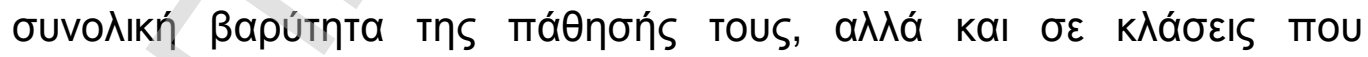

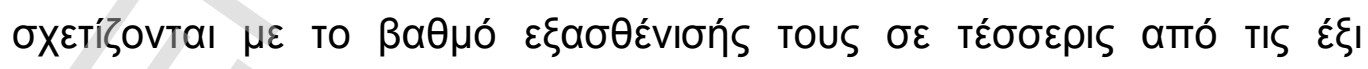

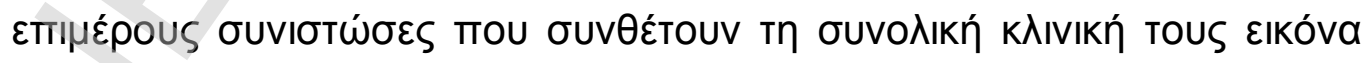
(Mani et al, 1999)

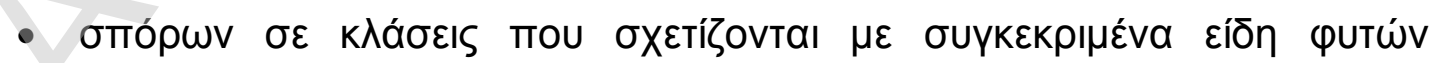
(Granitto et al, 2002; Granitto et al, 2005)

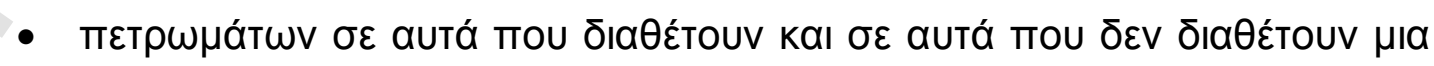

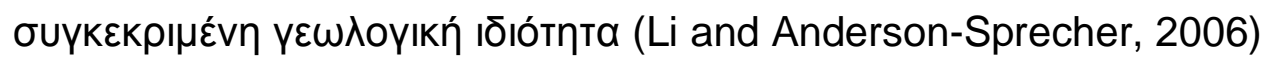




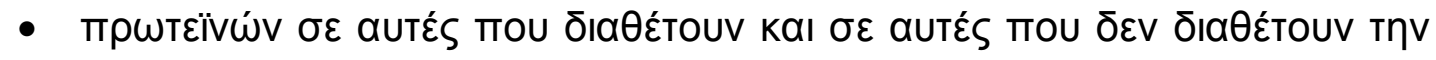

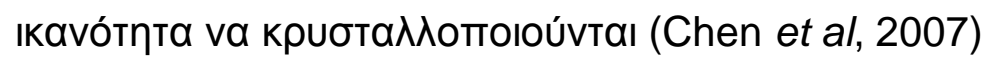

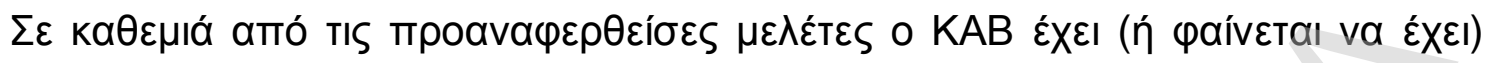

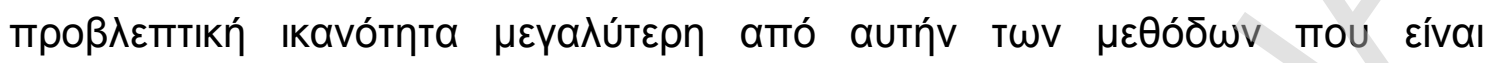

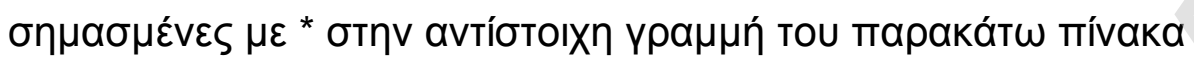

ПINAKA 1.1

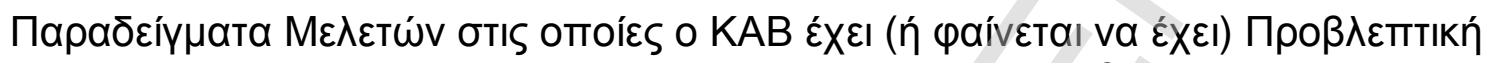

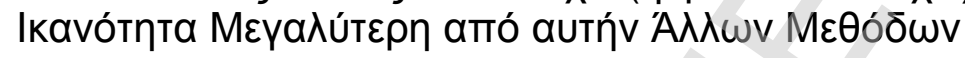

\begin{tabular}{|c|c|c|c|c|c|}
\hline & $\Gamma \Delta \mathrm{A}$ & $\Lambda \Pi$ & $\mathrm{N} \Delta$ & $\Delta \mathrm{T}$ & $\mathrm{Mk} \Gamma \Gamma$ \\
\hline Titterington et al (1981) & $*$ & $*$ & & & \\
\hline Russek et al (1983) & $*$ & & & & \\
\hline $\begin{array}{c}\text { Gammerman and Thatcher (1991) } \\
\text { Ohmann et al (1996) }\end{array}$ & & & & $*$ & \\
\hline $\begin{array}{c}\text { Penny and Frost (1997) } \\
\text { Mani et al (1999) }\end{array}$ & & $*$ & $*$ & & $*$ \\
\hline $\begin{array}{c}\text { Granitto et al (2002) } \\
\text { Granitto et al (2005) }\end{array}$ & & & $*$ & & \\
\hline Li and Anderson-Sprecher (2006) & $*$ & & & & \\
\hline Chen et al (2007) & & $*$ & & $*$ & \\
\hline
\end{tabular}

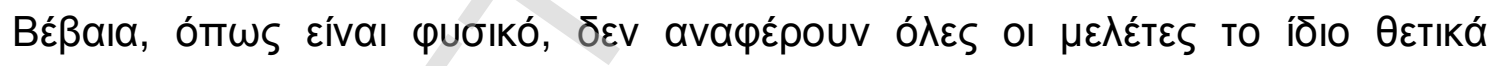

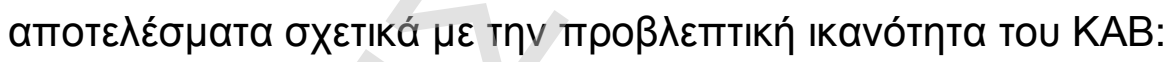

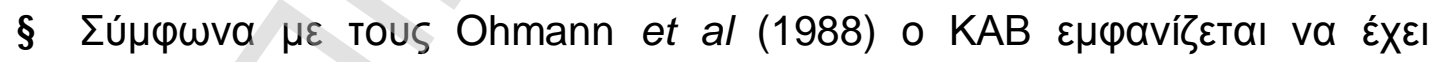

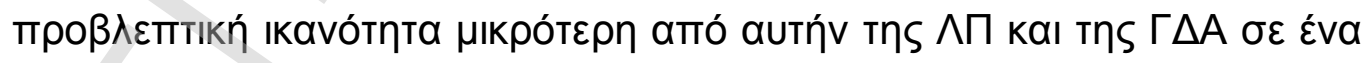

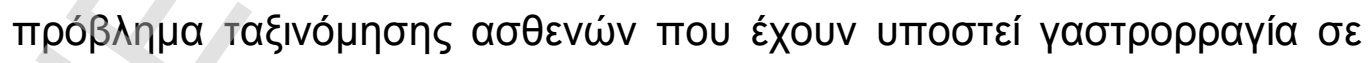

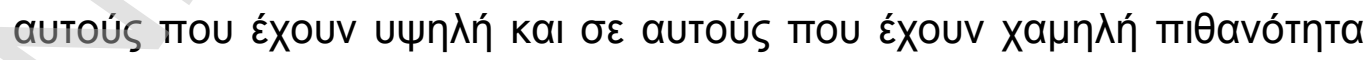

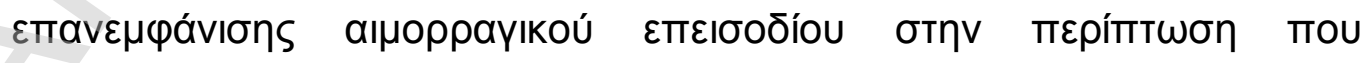

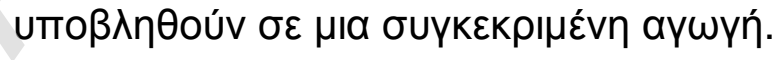

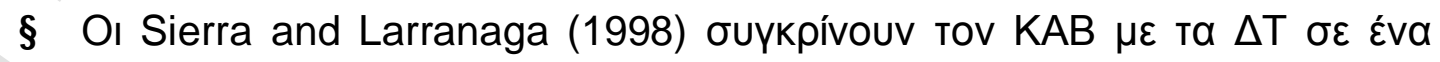

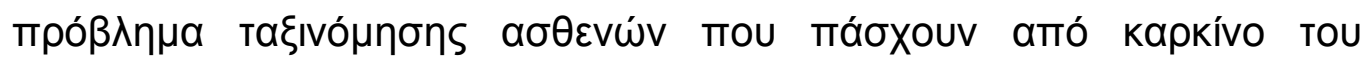

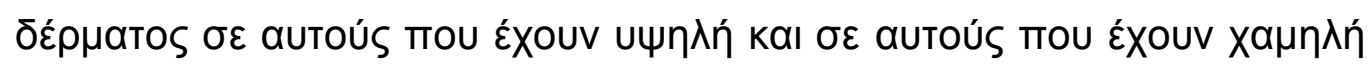




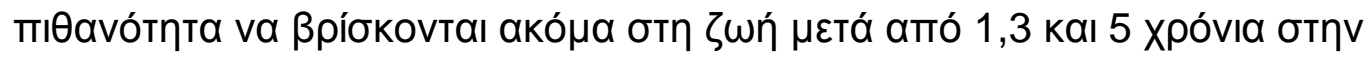

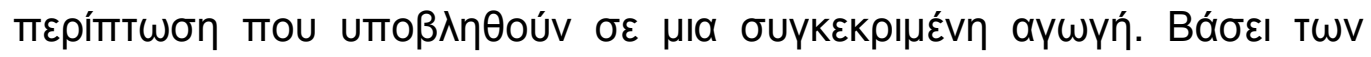

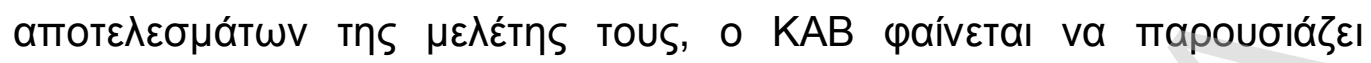

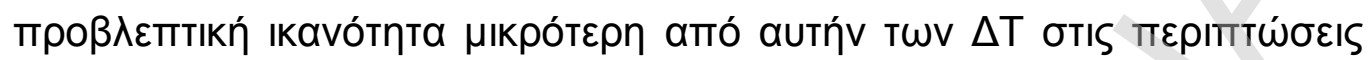

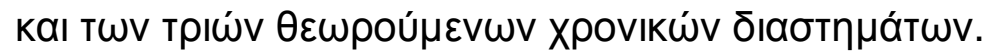

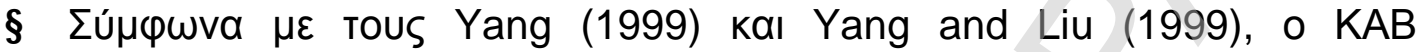

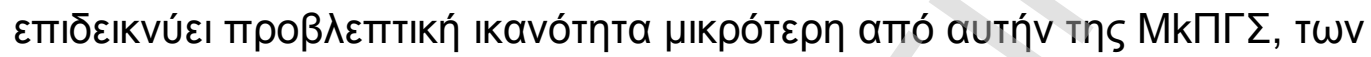

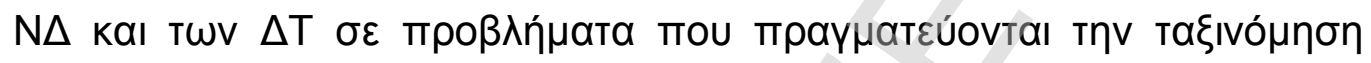

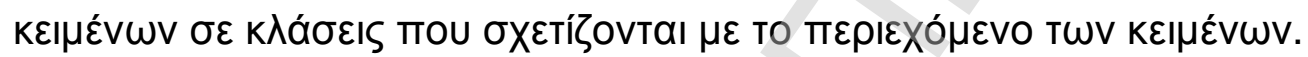

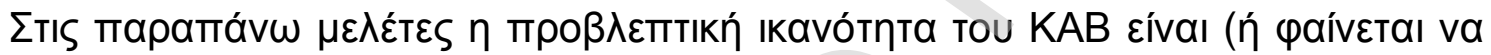

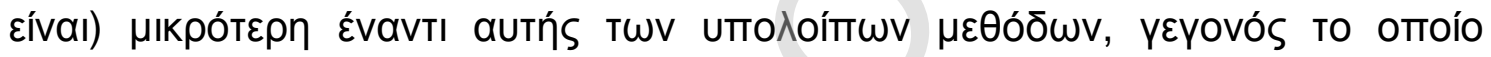

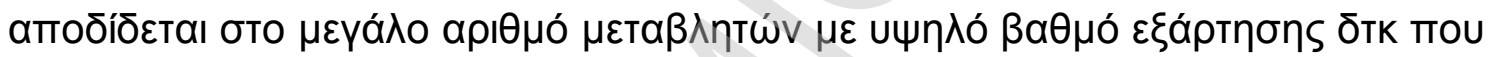

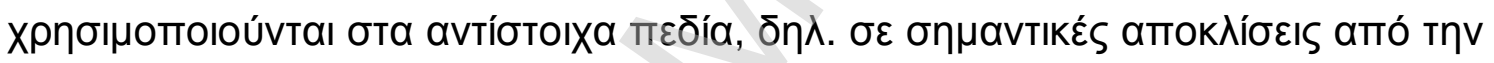

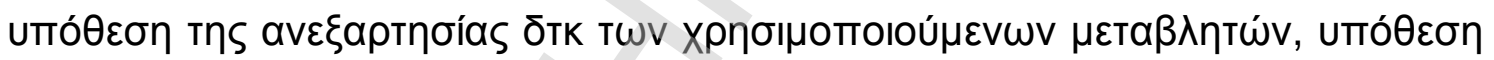

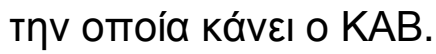

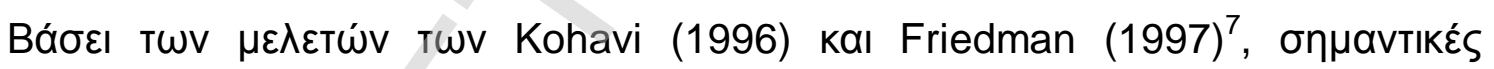

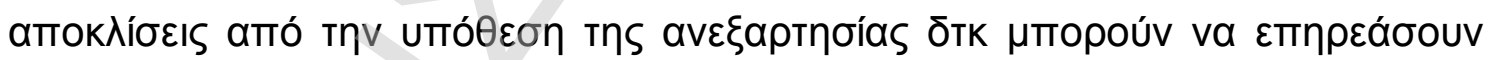

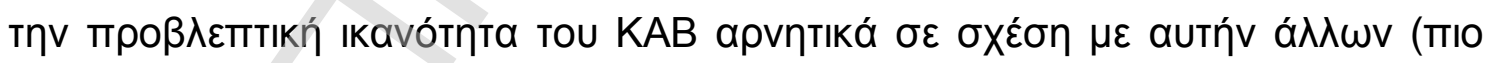

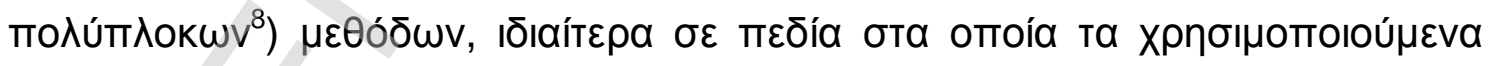

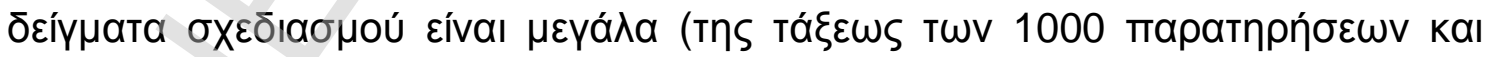
ávw).

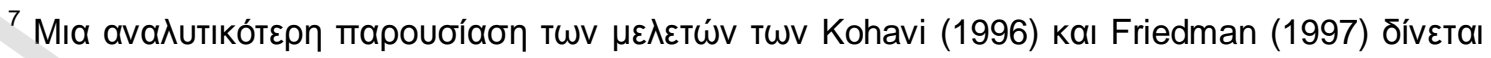

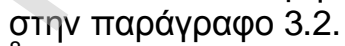

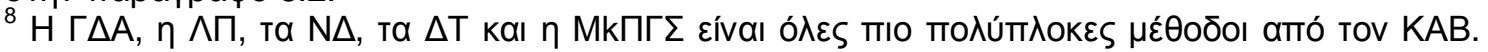

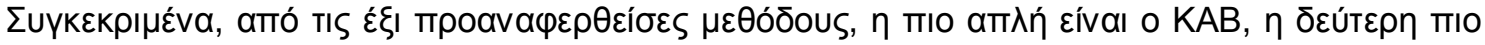

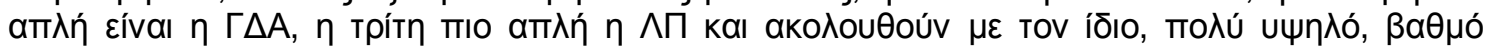

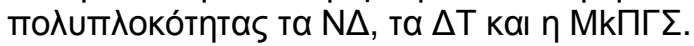




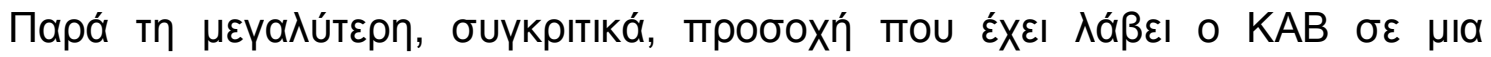

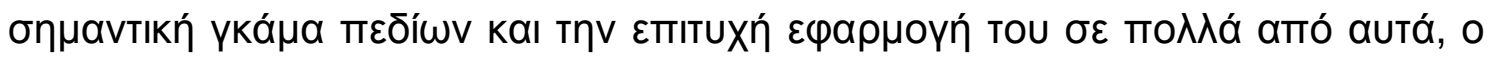

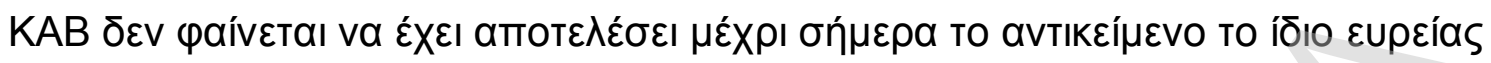

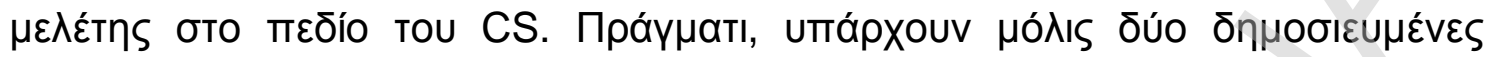

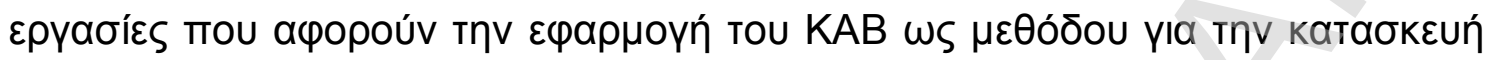

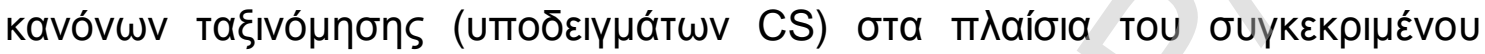

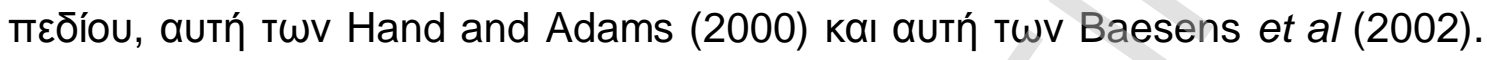

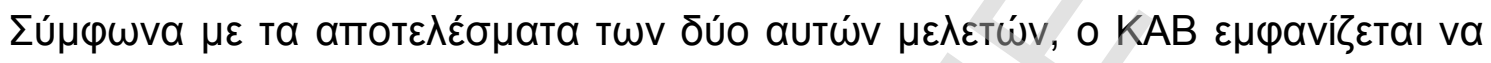

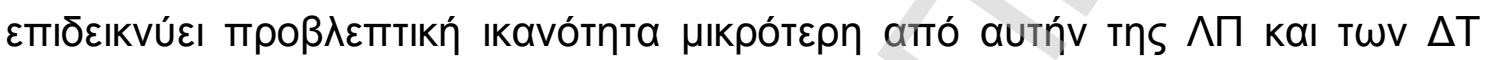
avtíotolxa.

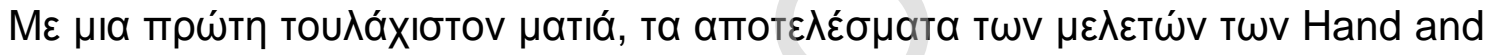

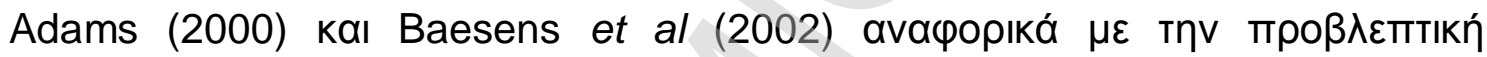

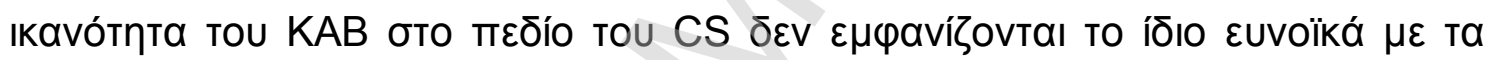

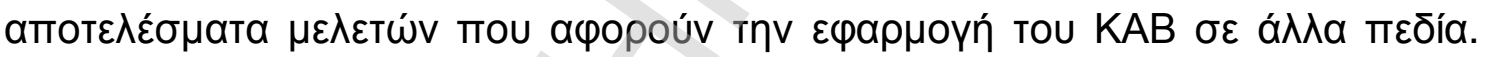

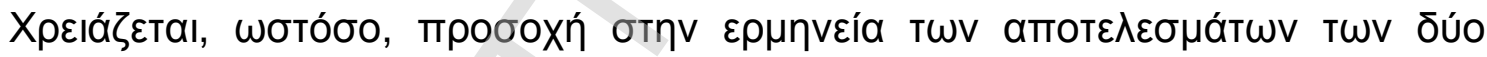

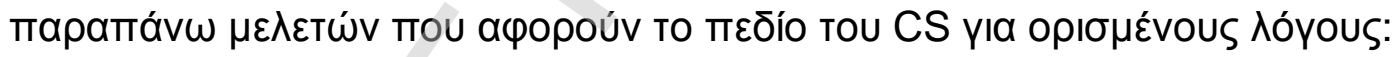

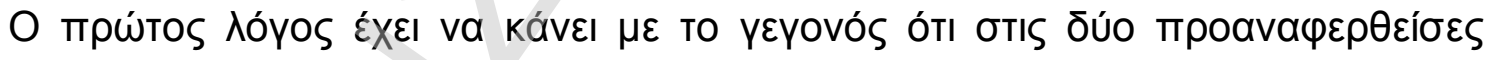

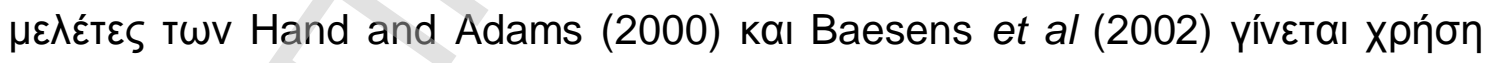

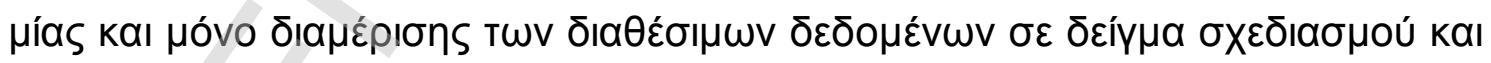

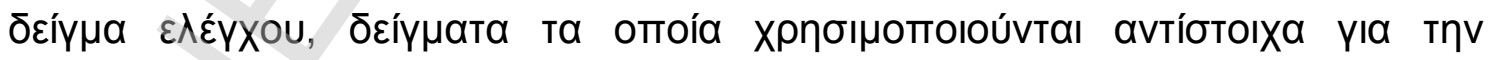

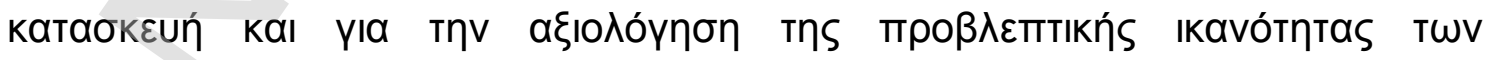

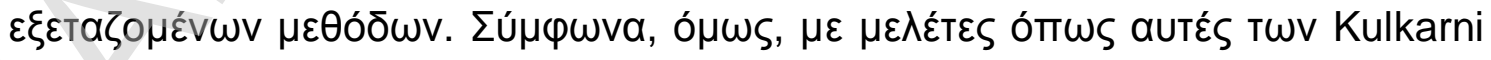
et al (1998), Zhang et al (1999) kaı Jain et al (2000), $\eta$ хрпбıнoтоínon

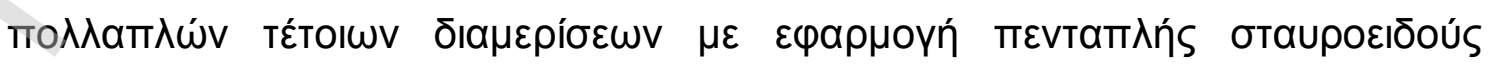

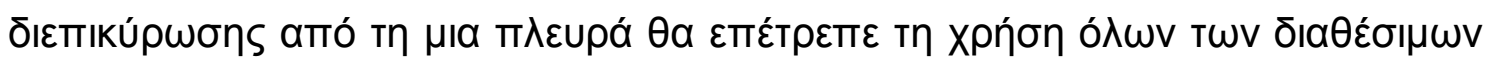




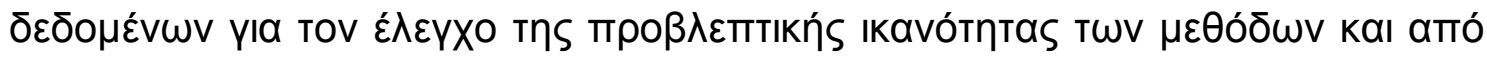

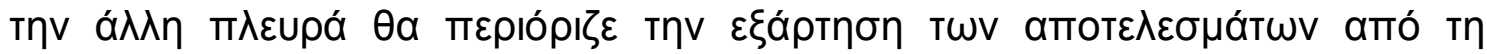

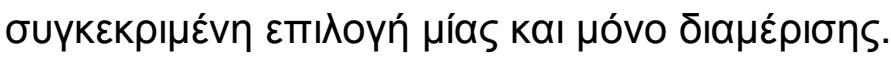

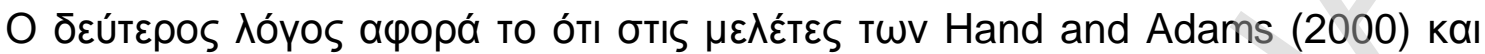

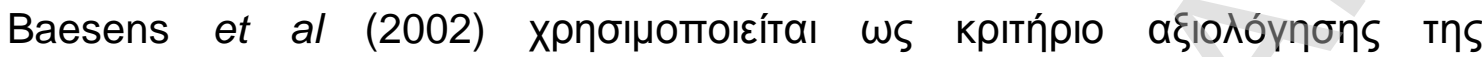

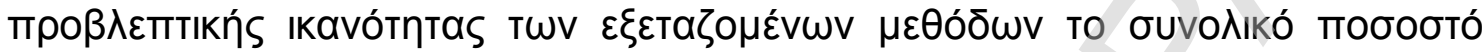

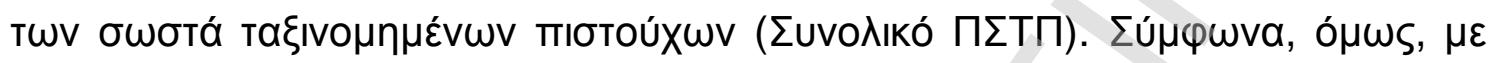

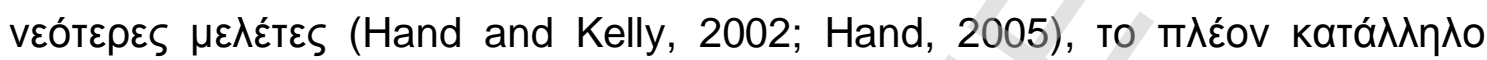

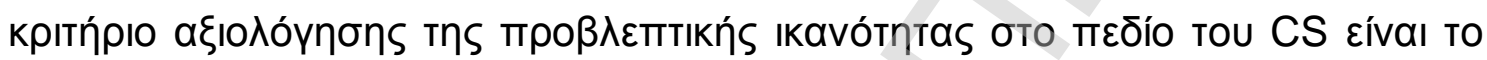

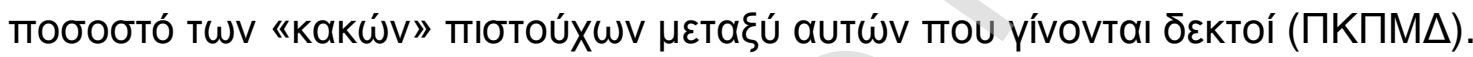

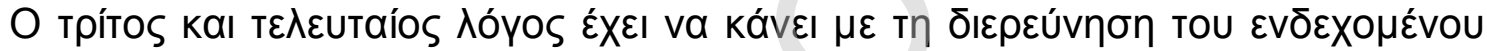

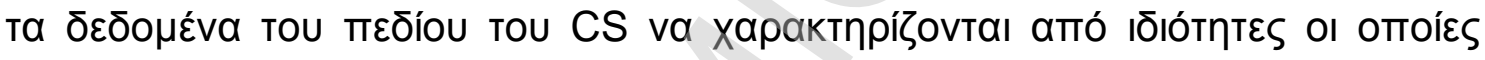

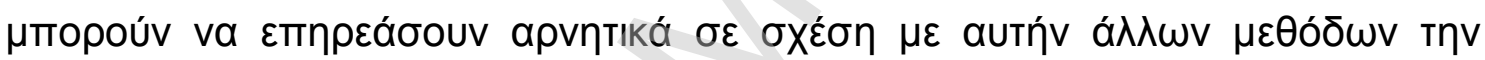

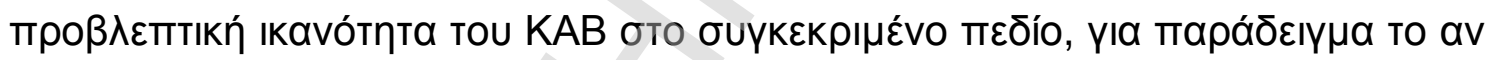

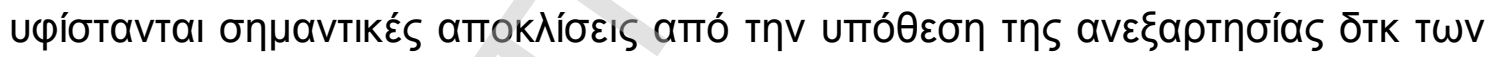

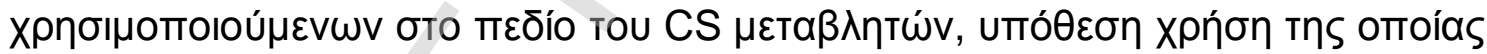

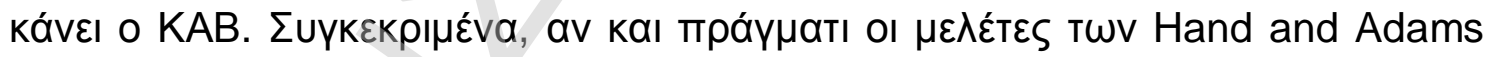

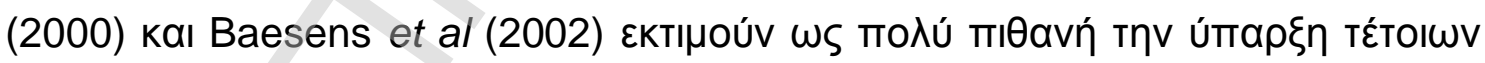

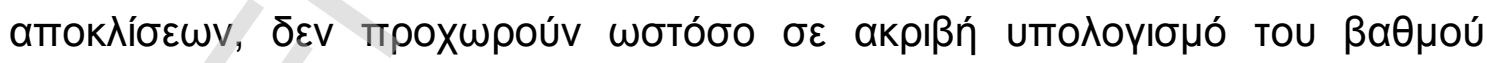

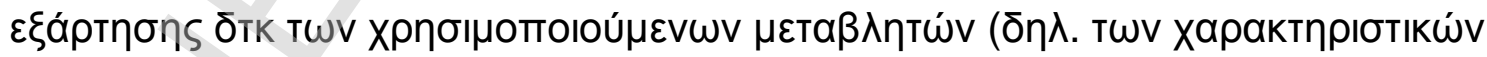

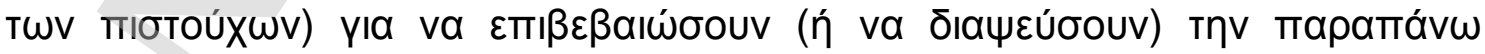
EKTífnon. 


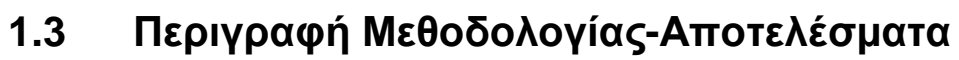

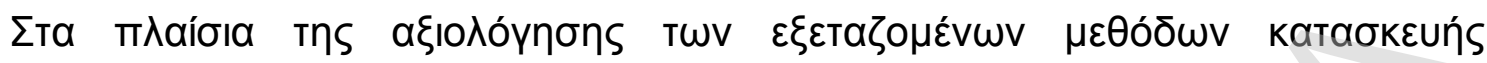

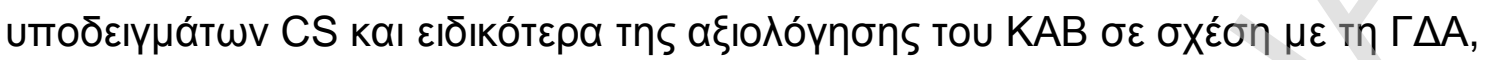

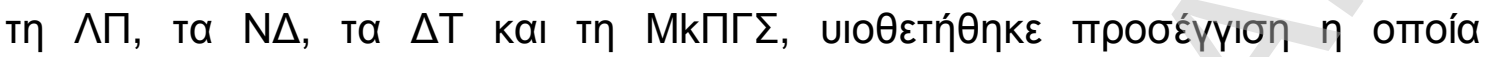

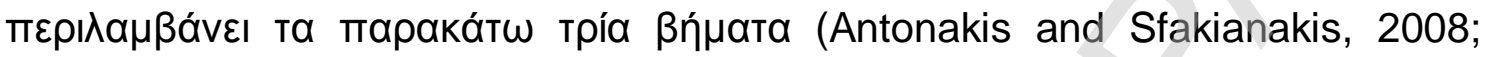
Antonakis and Sfakianakis, 2009A):

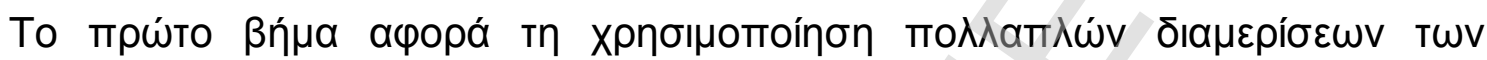

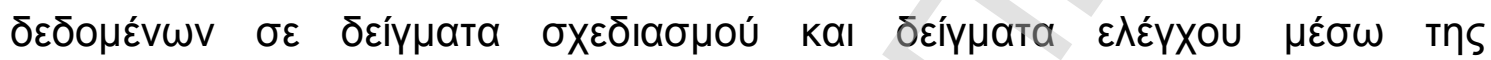

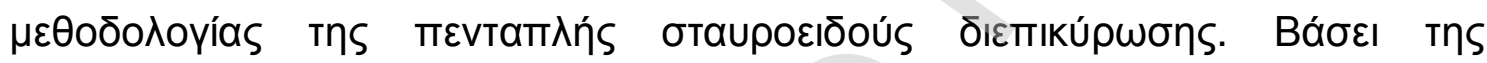

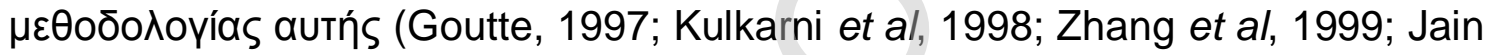

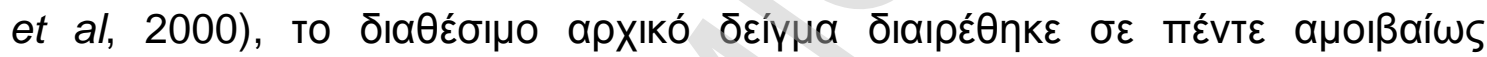

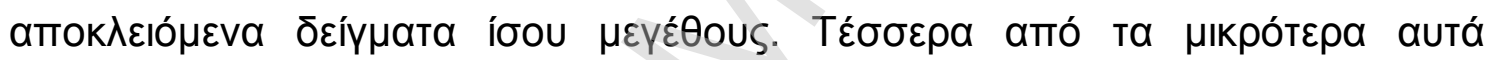

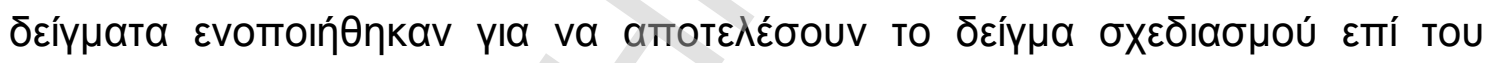

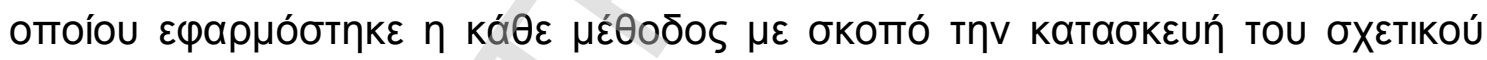

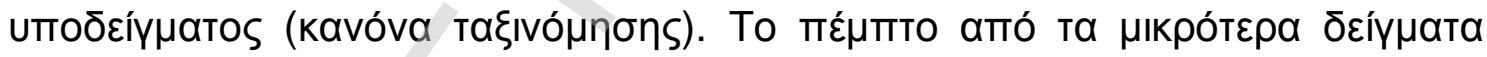

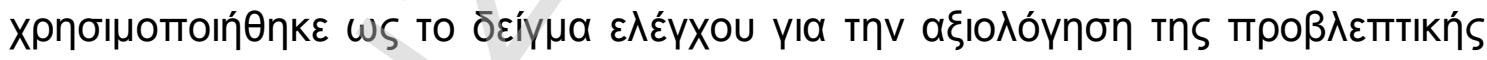

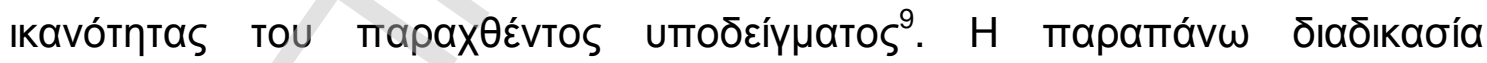

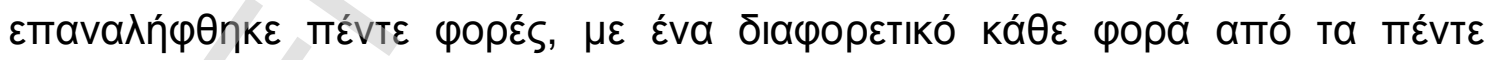

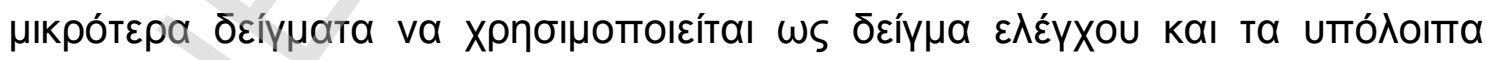

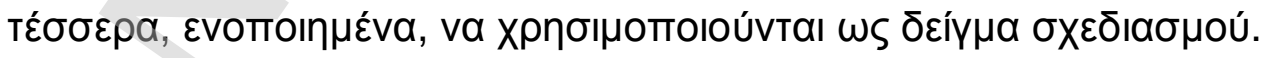

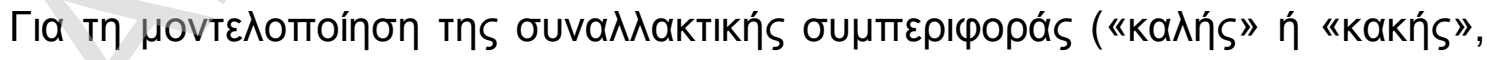

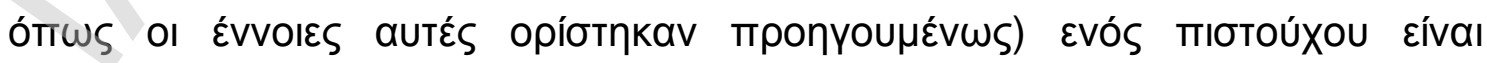

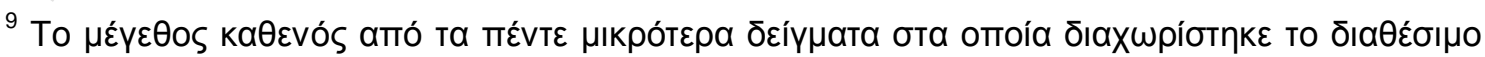

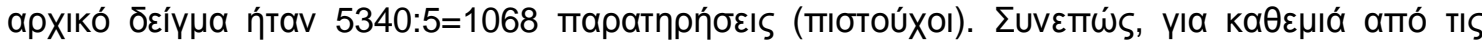

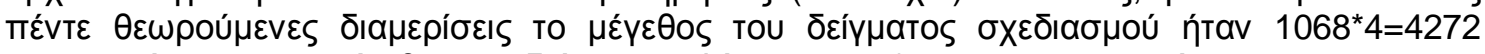

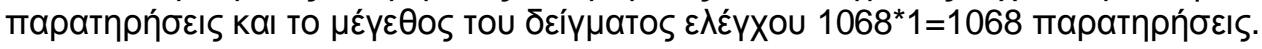




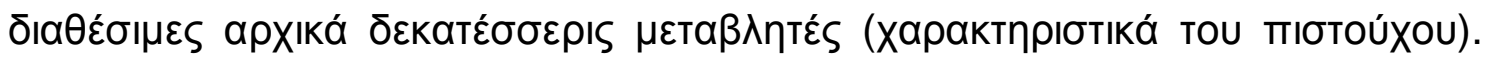

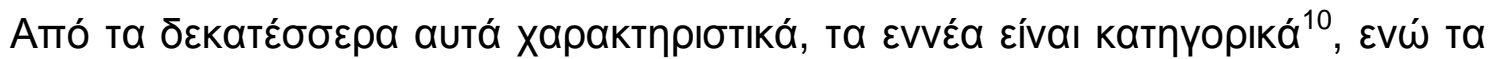

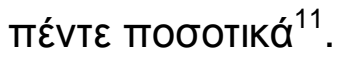

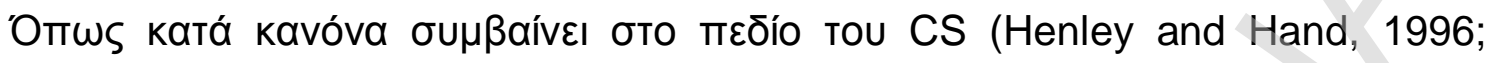

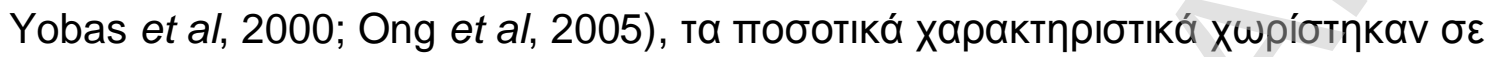

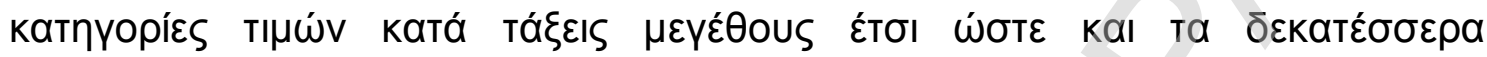

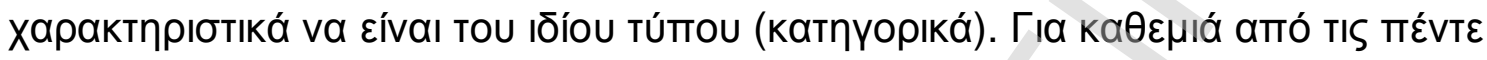

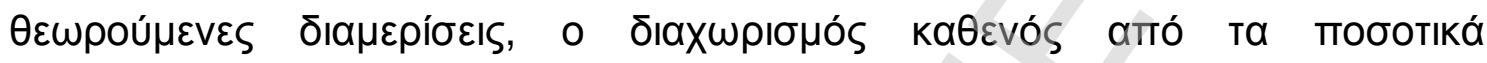

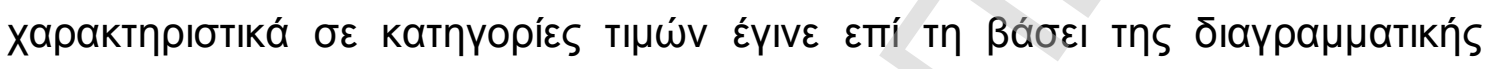

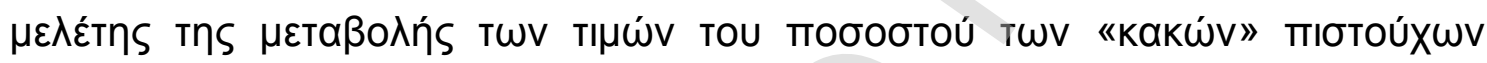

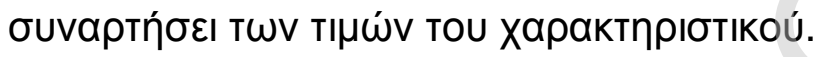

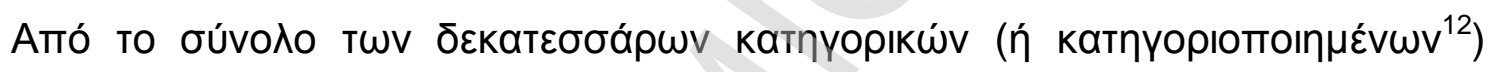

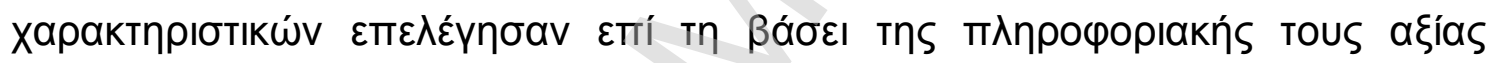
(information value) (Hand and Henley, 1997; Thomas, 2000; Hand, 2001) yıa va

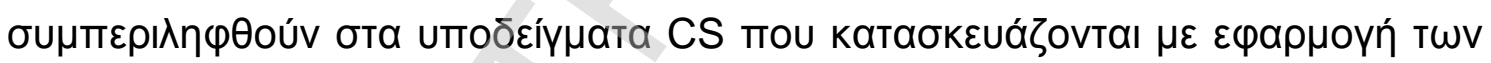

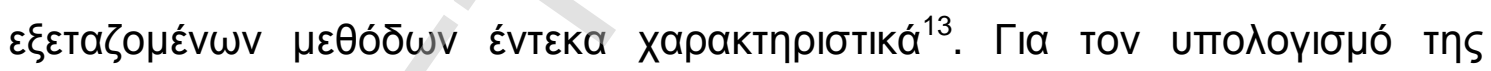

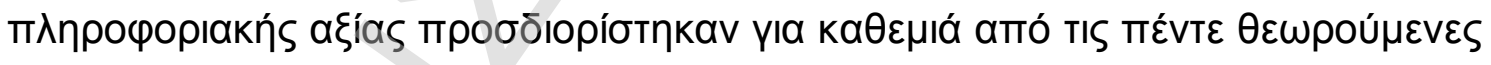

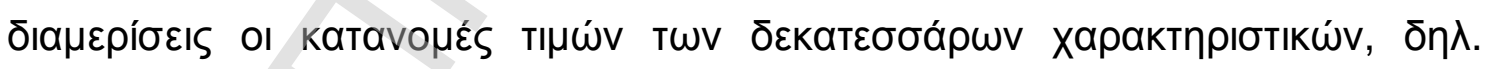

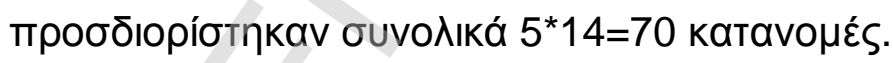

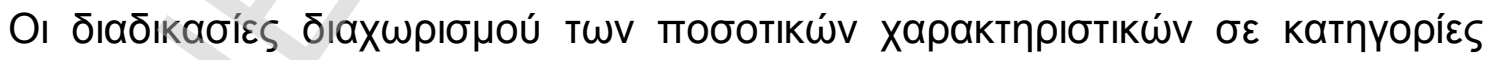

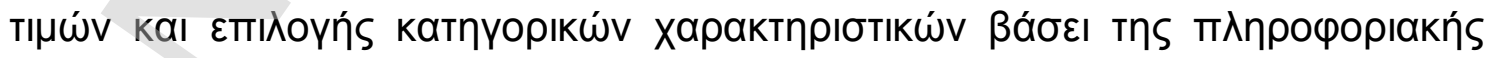

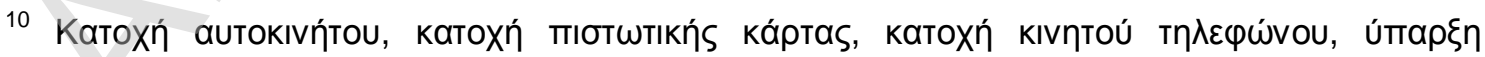

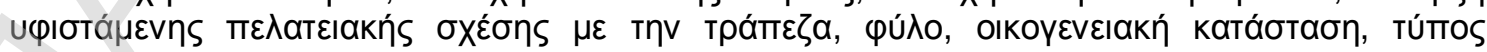

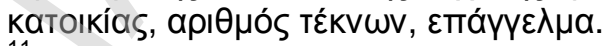

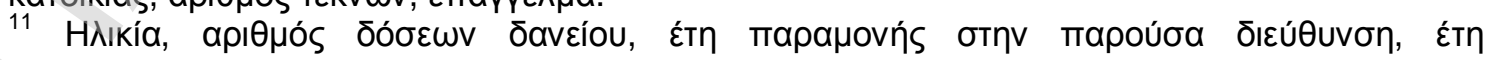

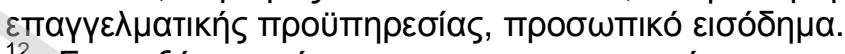

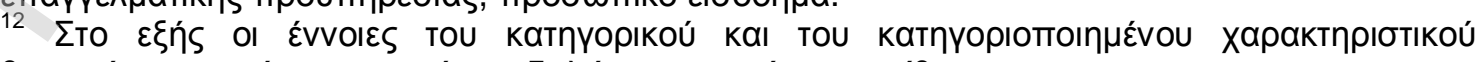

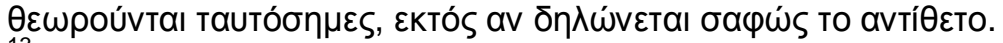

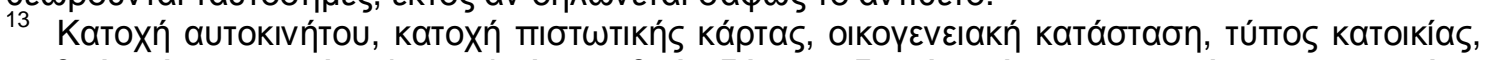

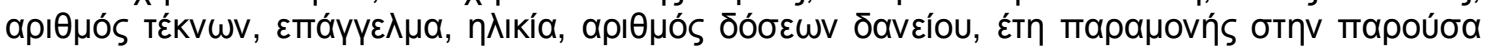

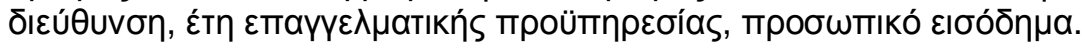




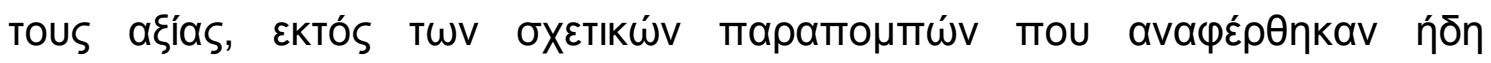

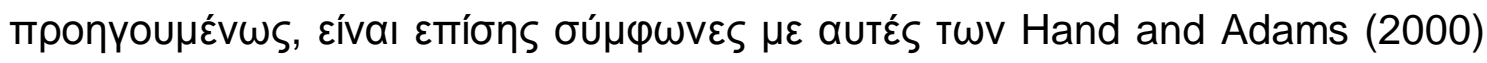
kal Baesens et al (2002).

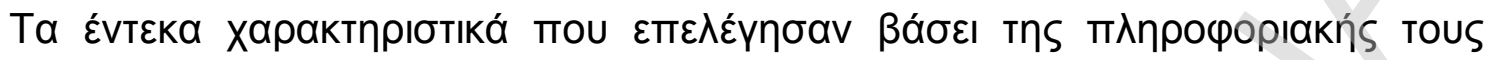

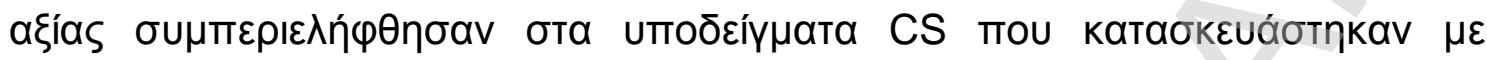

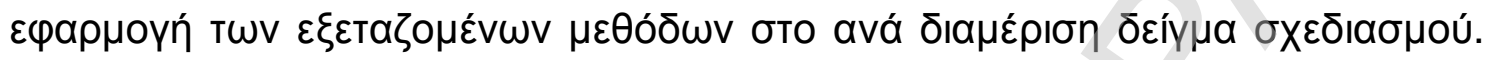

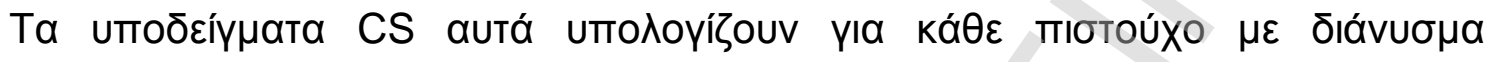

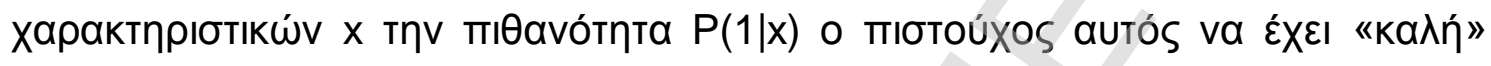

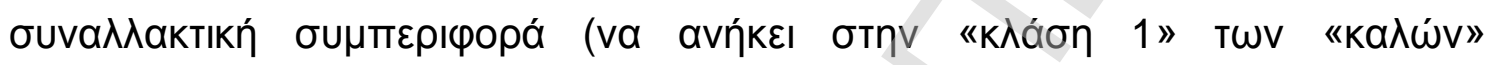

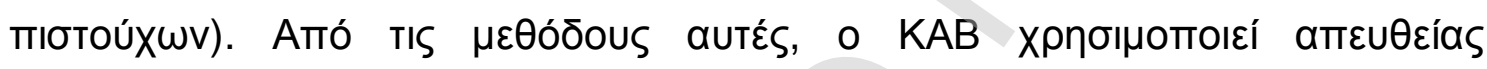

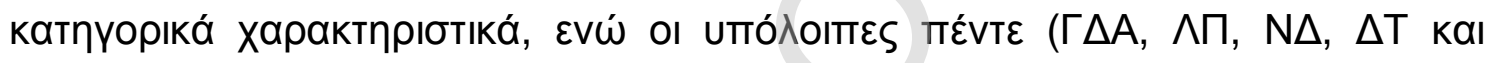

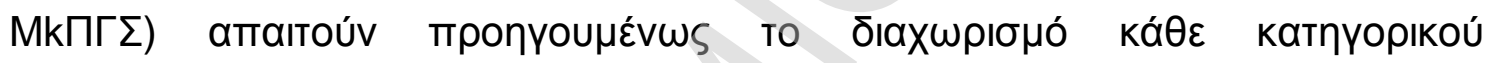

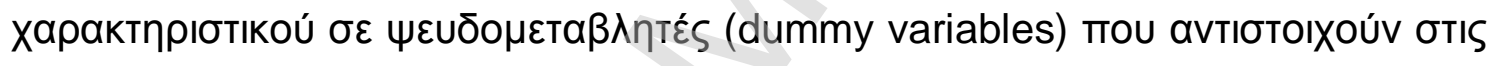

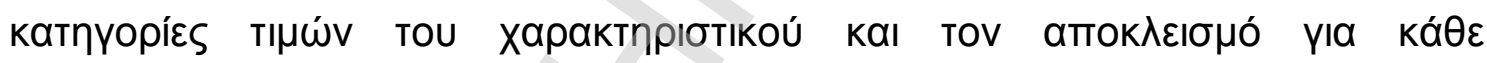

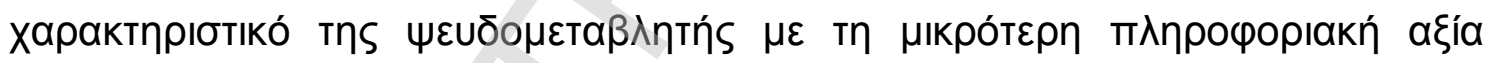
(Hand and Henley, 1997; Thomas, 2000; Hand and Yu, 2001).

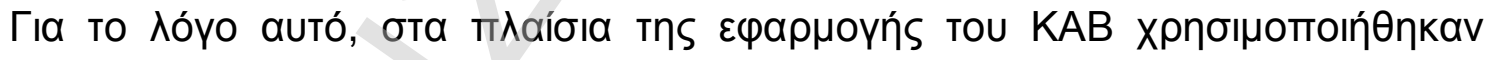

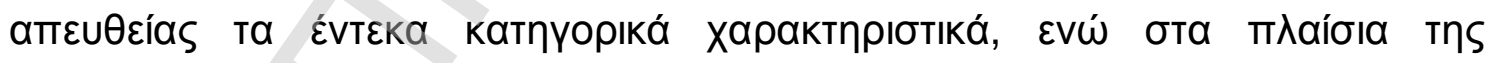

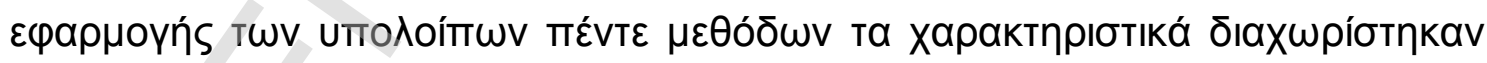

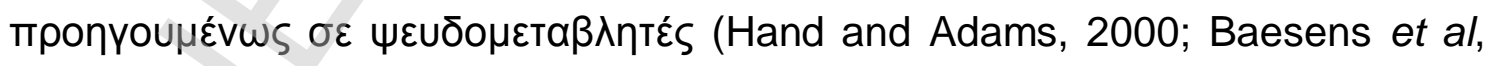

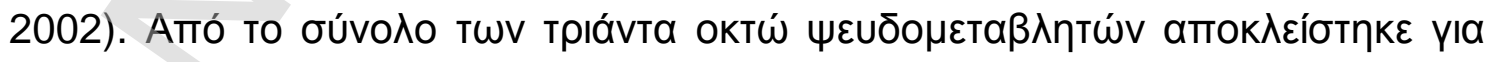

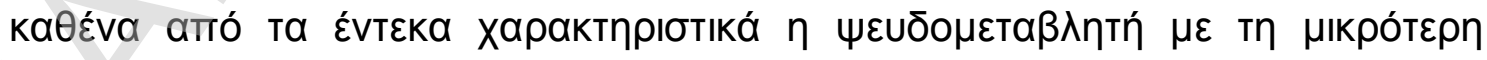

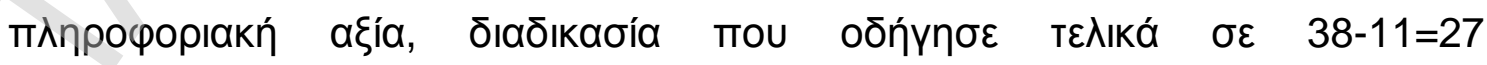

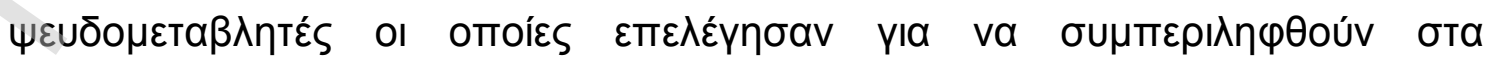

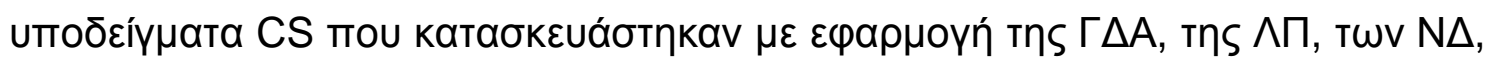




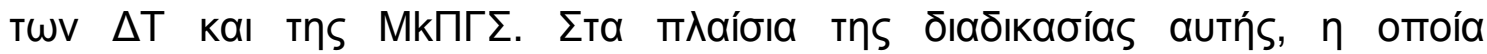

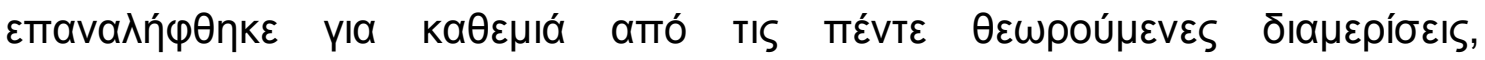

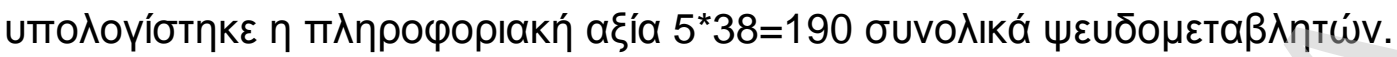

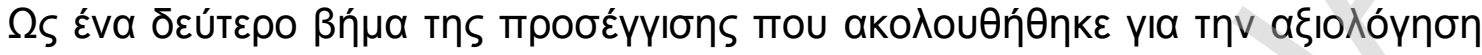

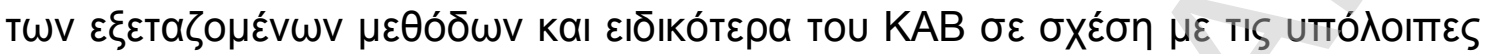

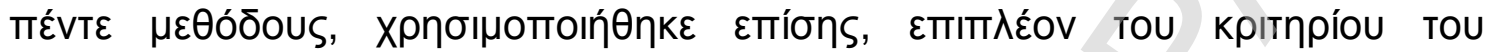

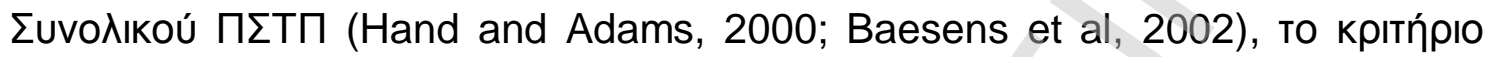

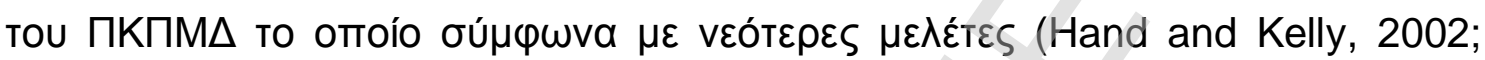

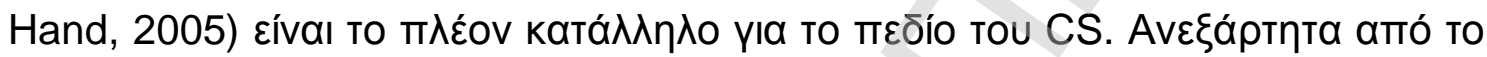

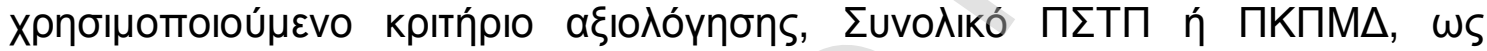

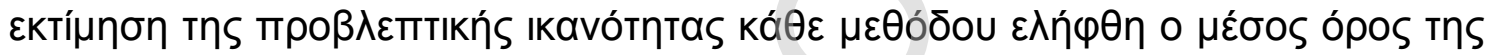

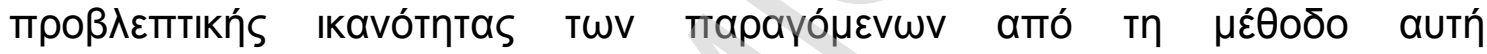

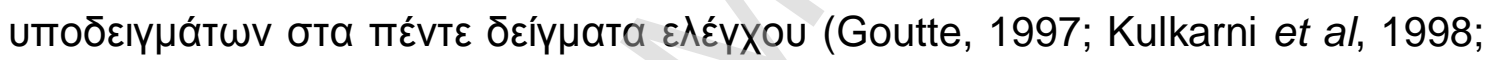
Zhang et al, 1999; Jain et al, 2000).

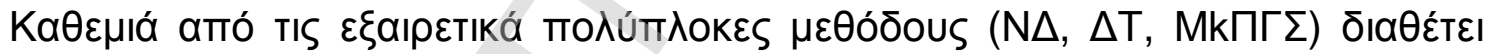

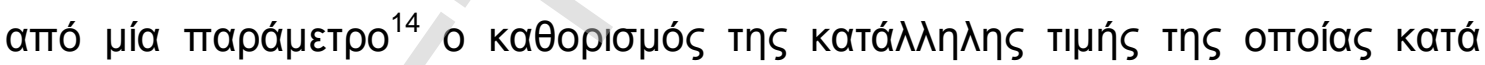

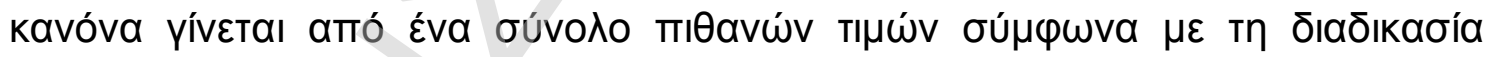

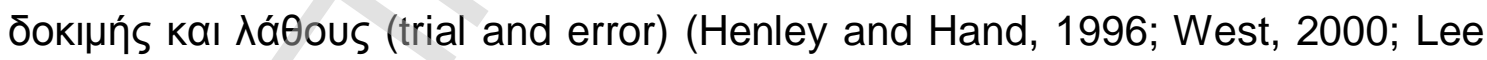

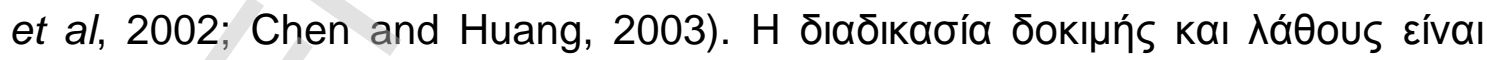

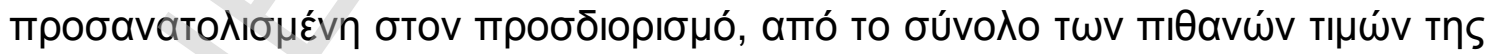

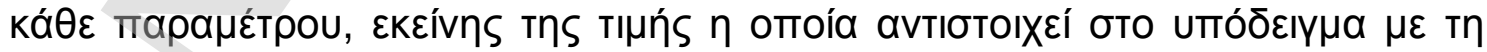

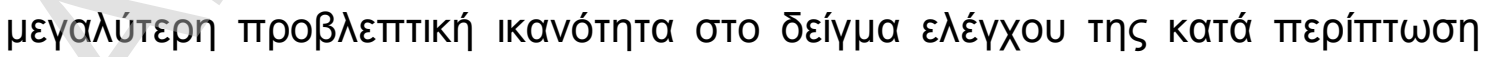

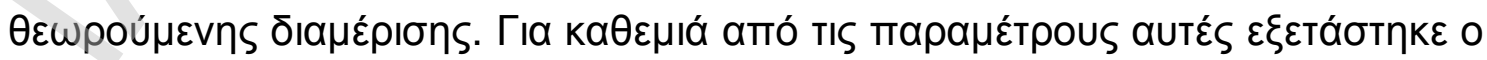

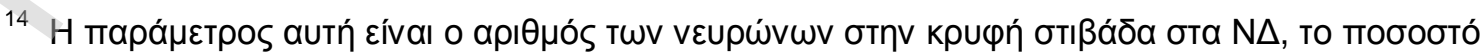

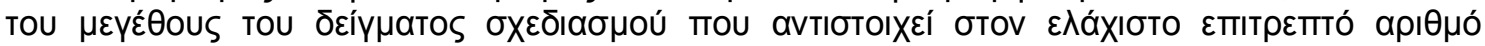

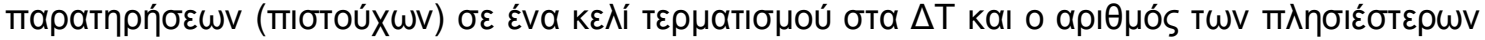

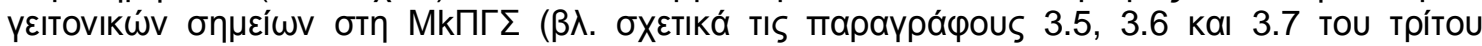

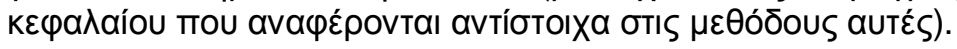




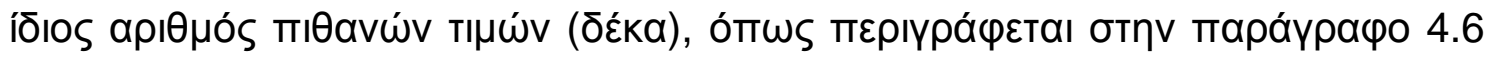
(Antonakis and Sfakianakis, 2008; Antonakis and Sfakianakis, 2009A). Bó́øı

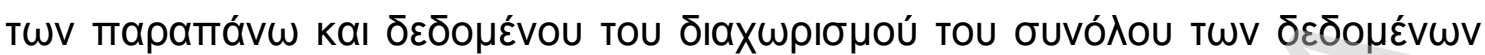

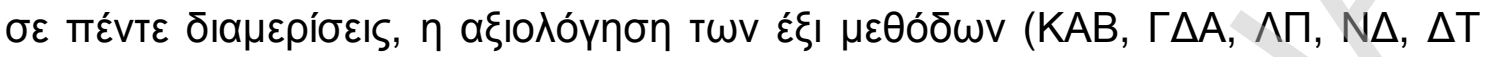

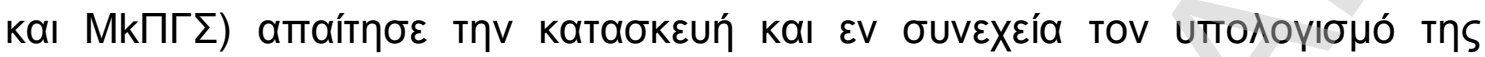

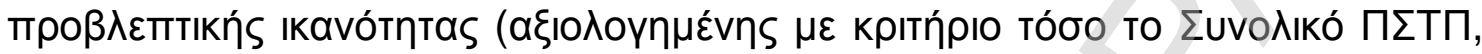

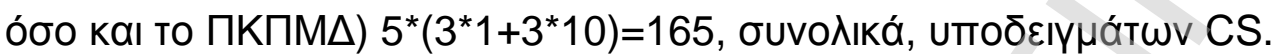

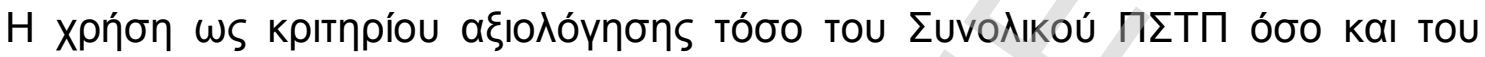

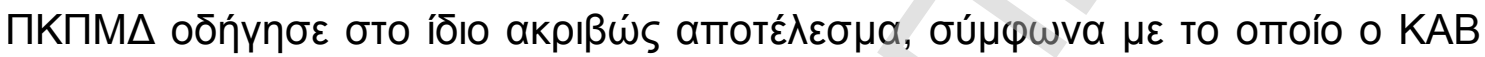

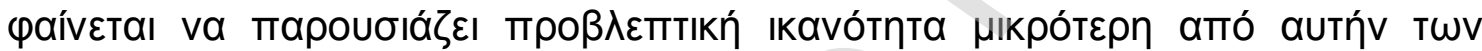

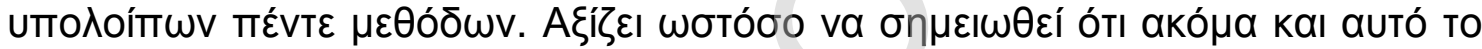

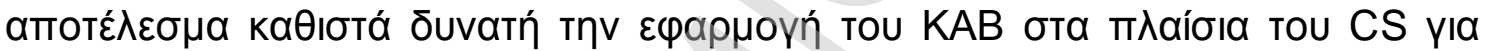

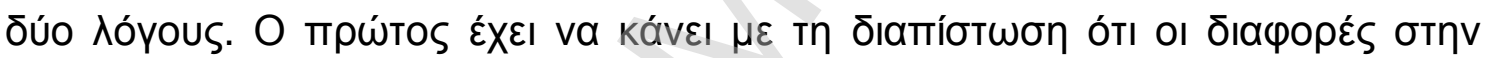

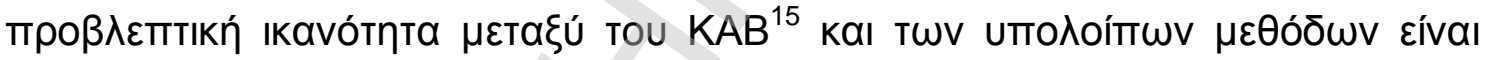

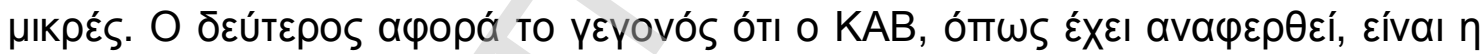

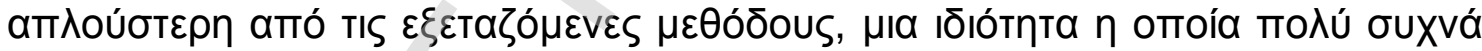

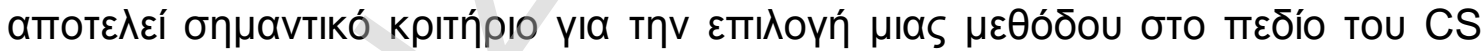
(Duin, 1996; Hand et al, 2001; Hand, 2006).

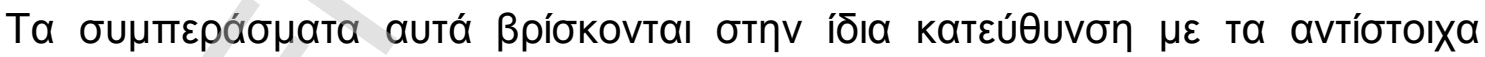

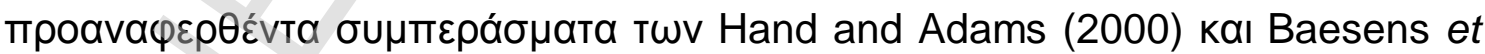

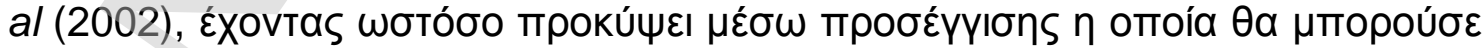

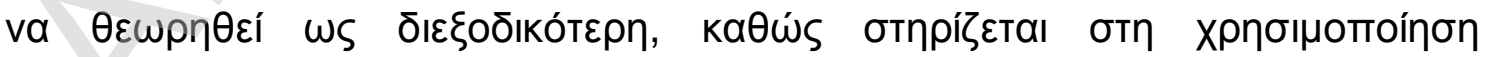

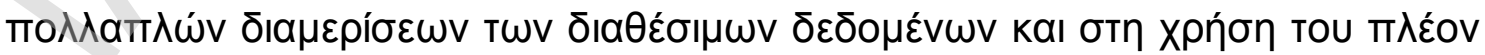

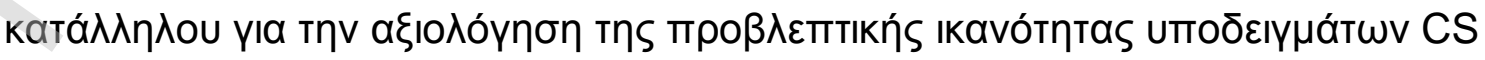

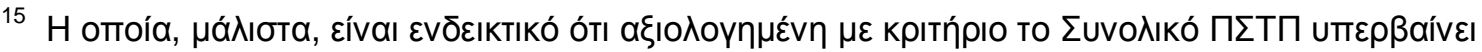

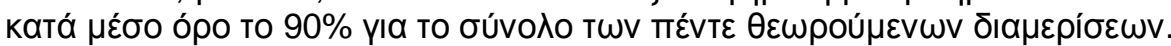


крітпрíou tou ПКПМ ПГТП.

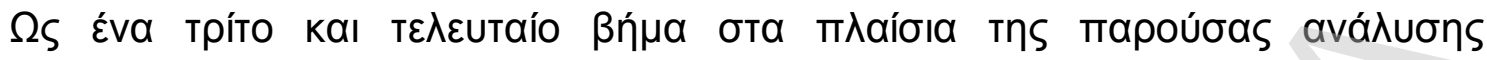

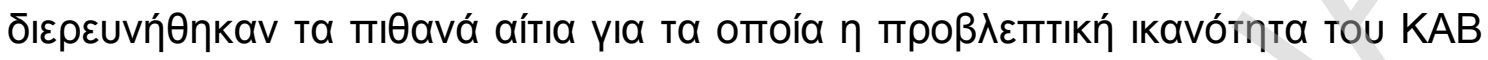

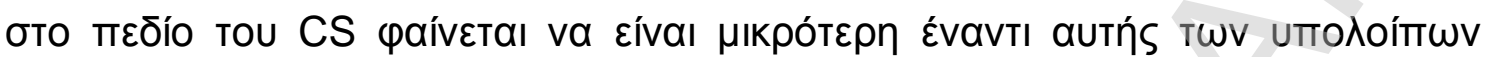

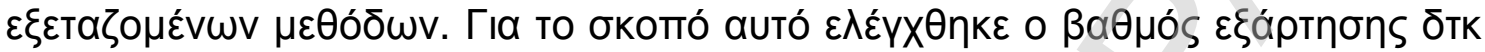

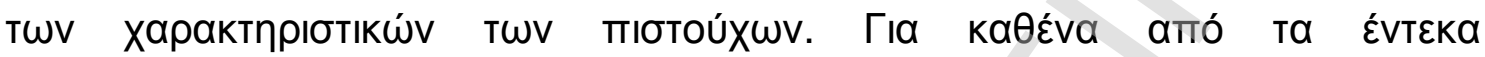

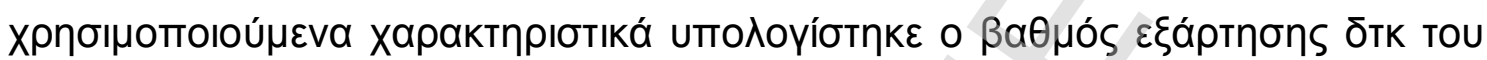

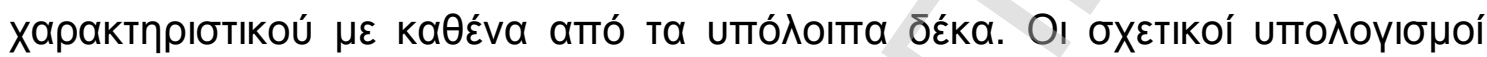

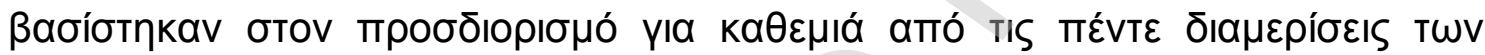

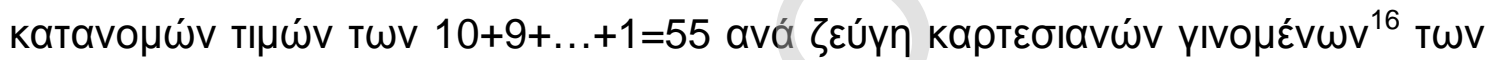

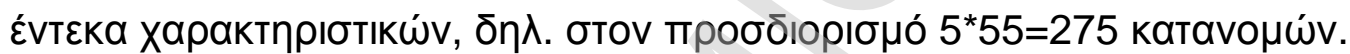

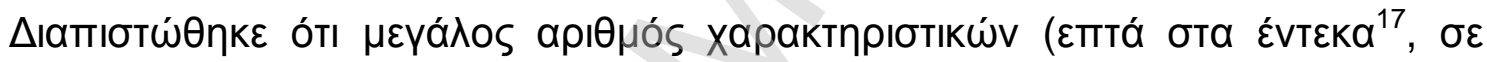

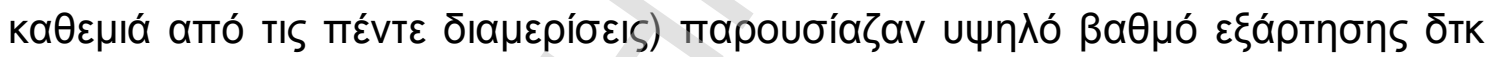

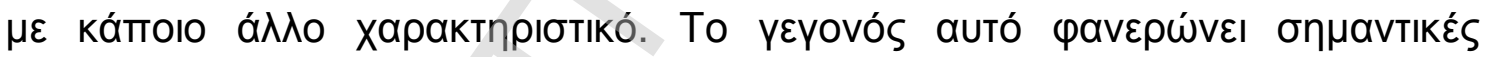

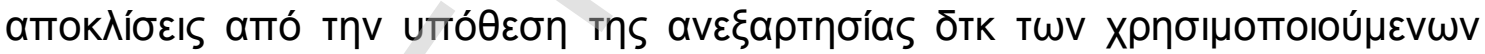

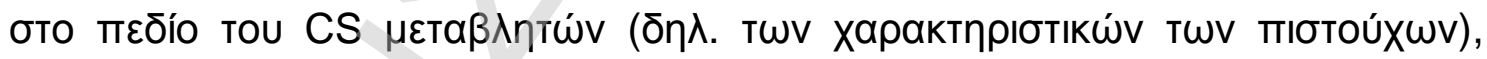

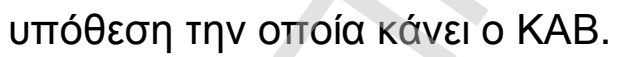

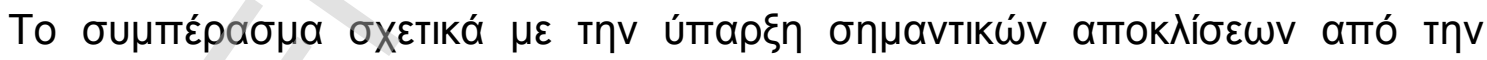

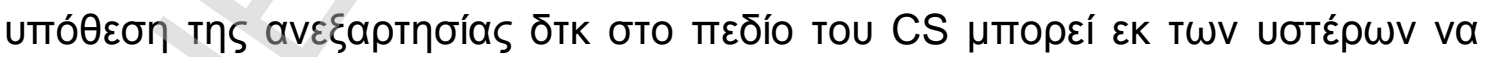

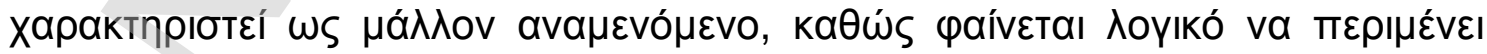

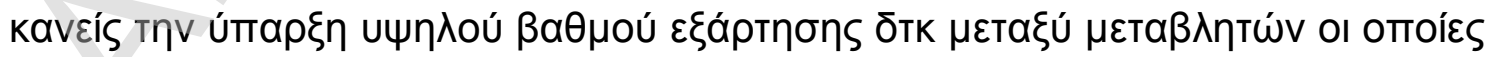

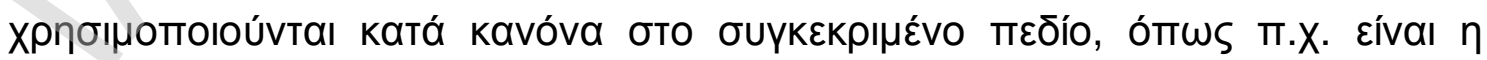

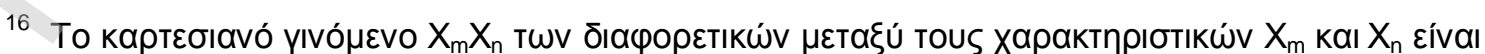

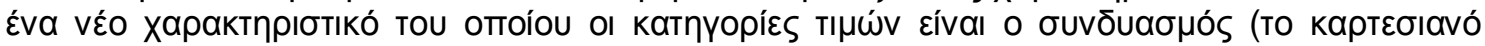

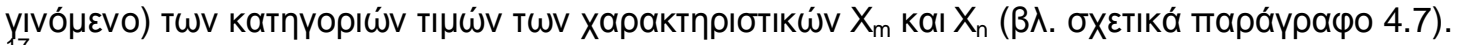

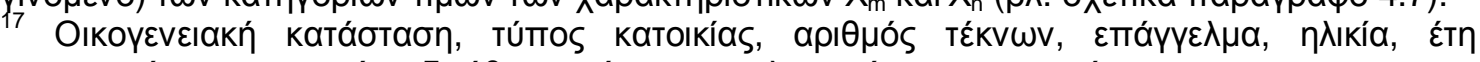

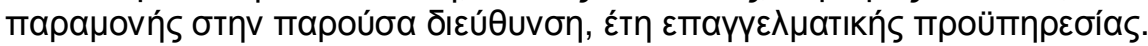




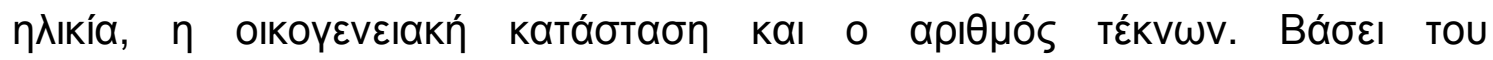

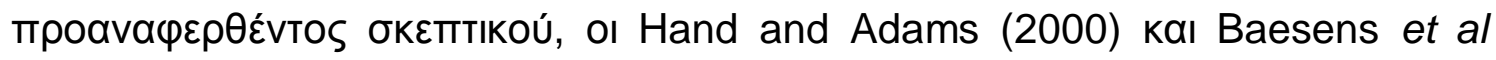

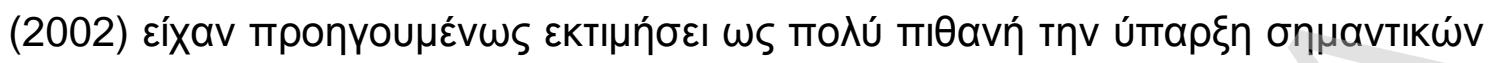

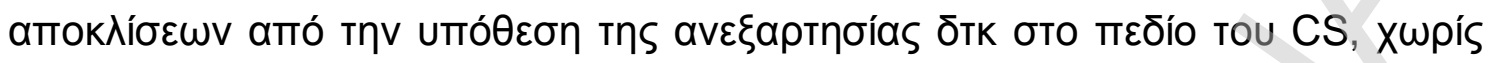

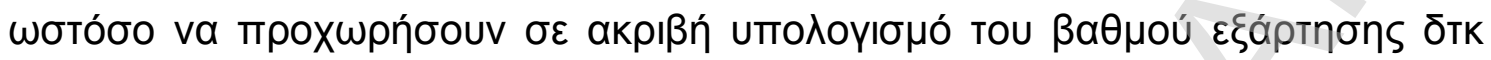

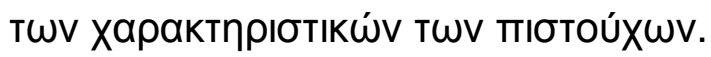

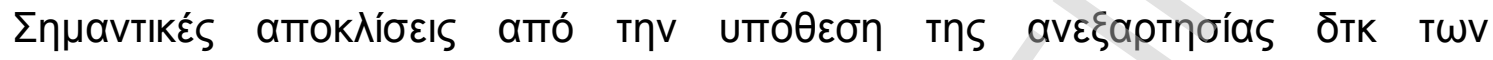

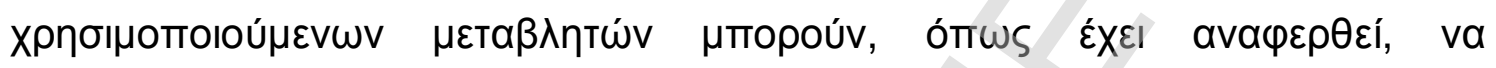

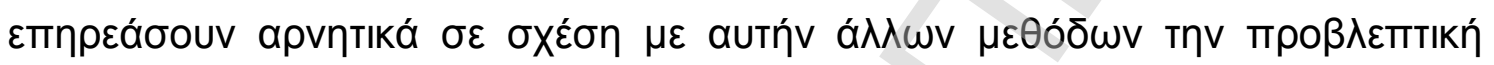

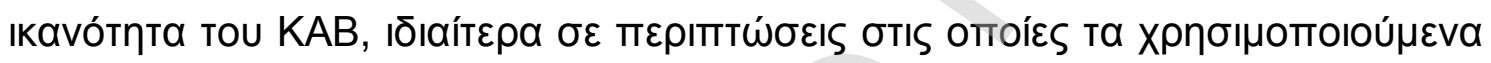

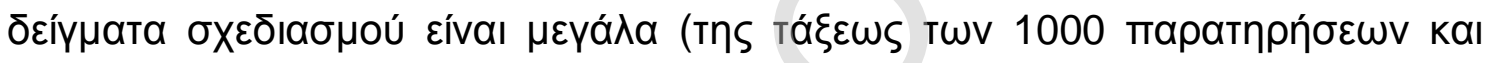

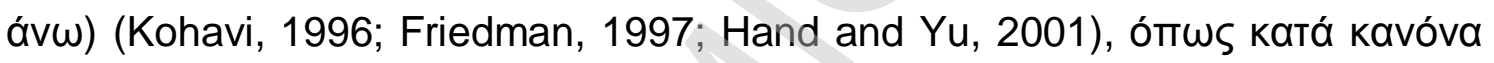

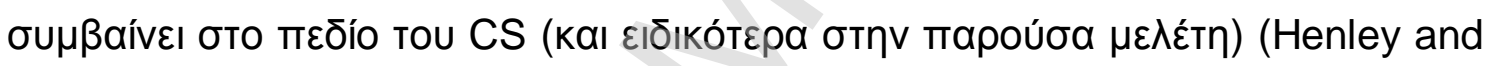

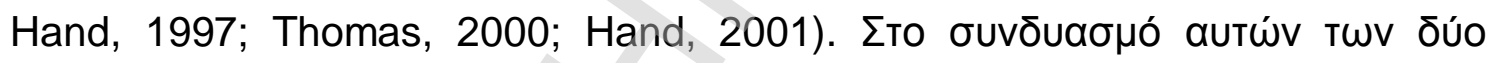

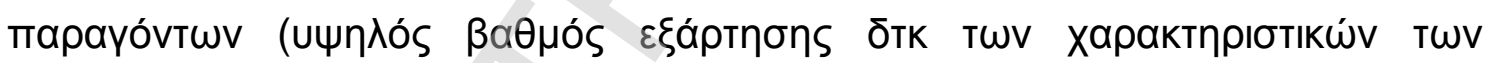

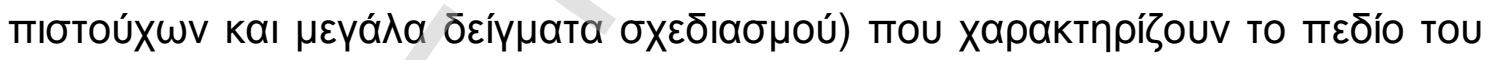

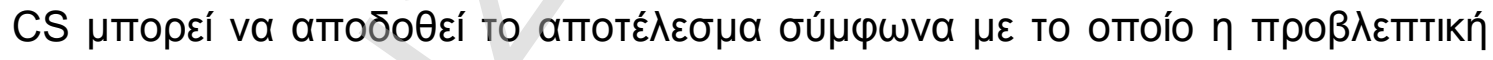

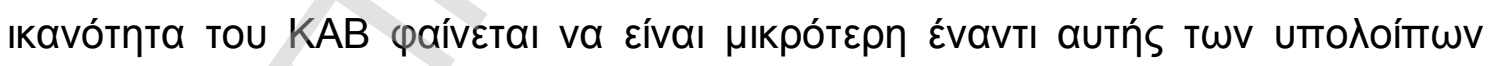

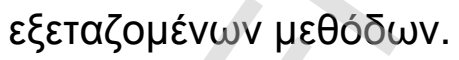

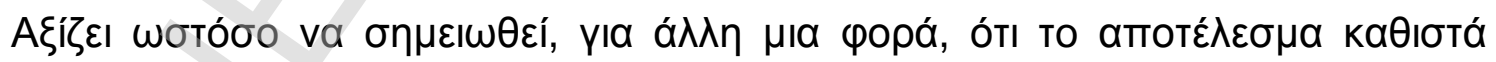

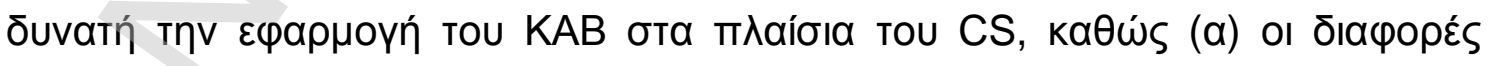

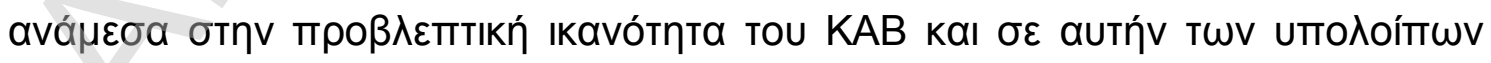

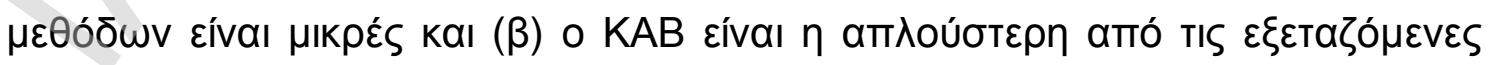

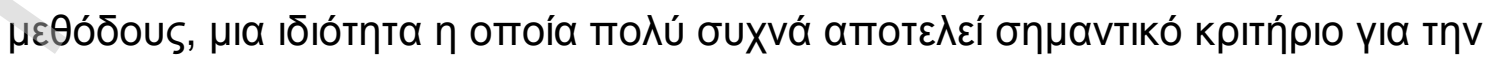

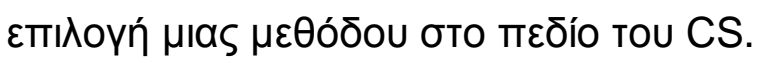




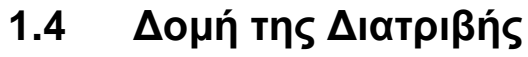

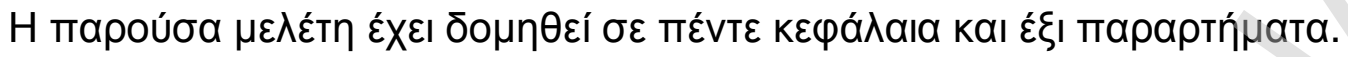

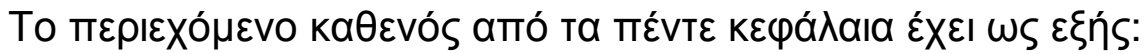

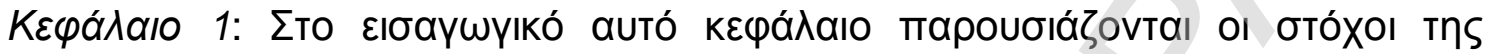

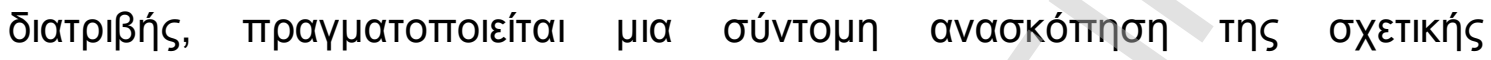

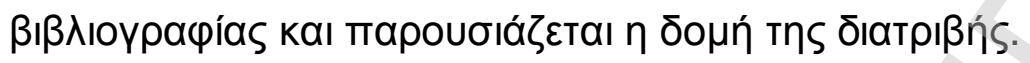

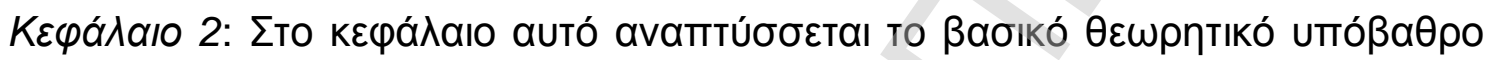

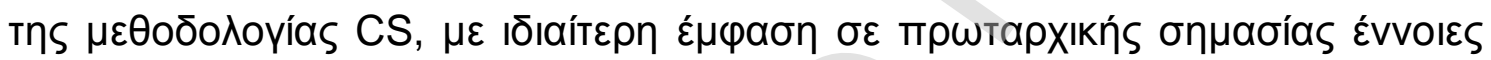

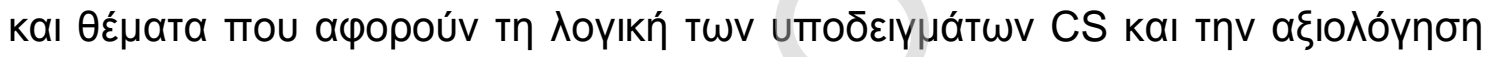

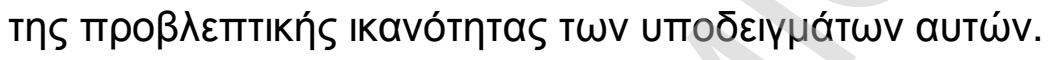

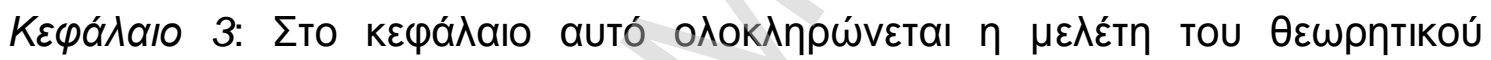

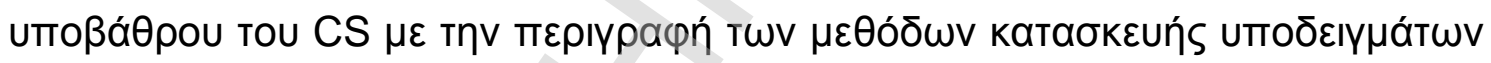

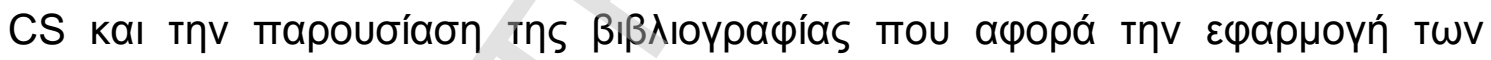

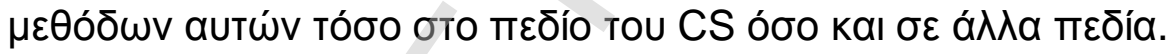

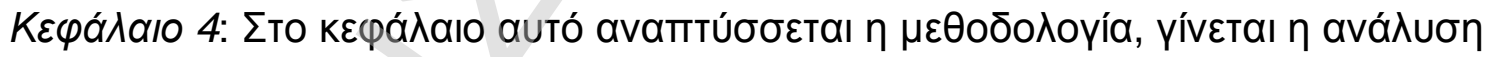

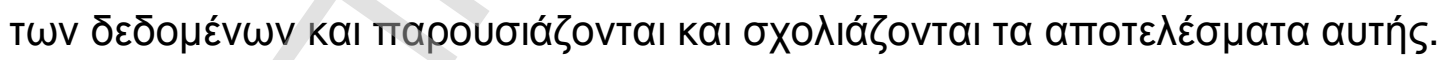

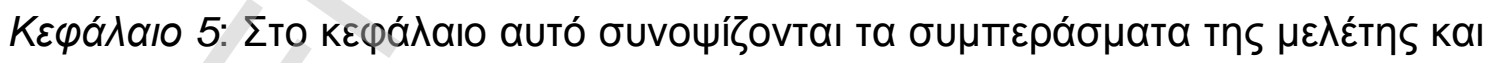

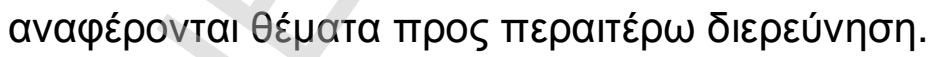

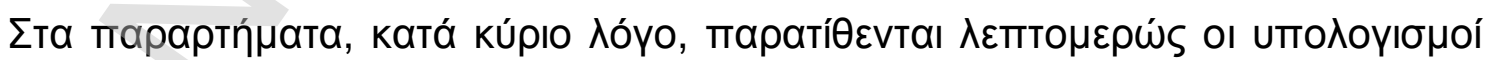

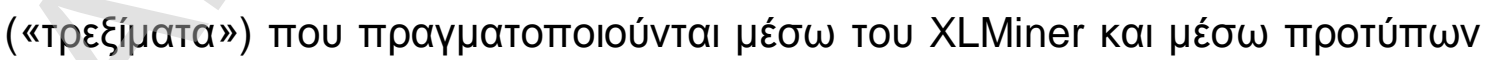

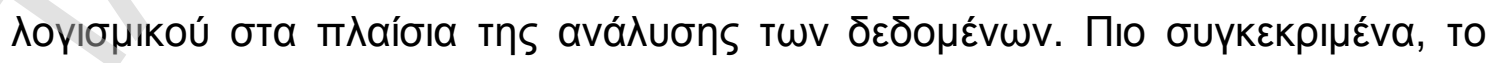

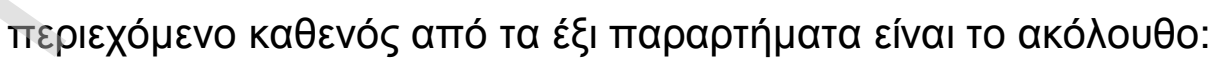




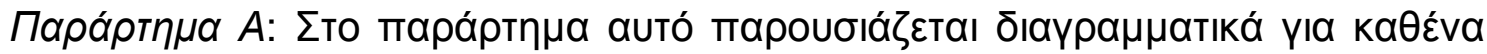

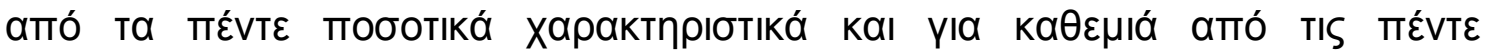

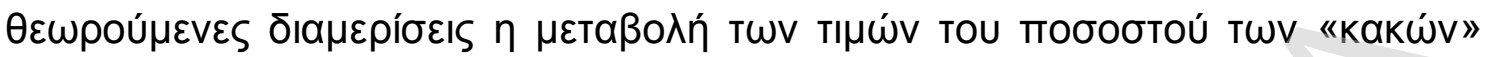

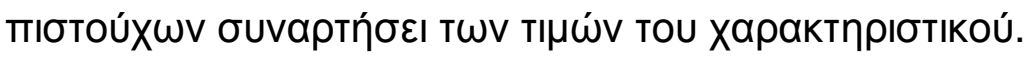

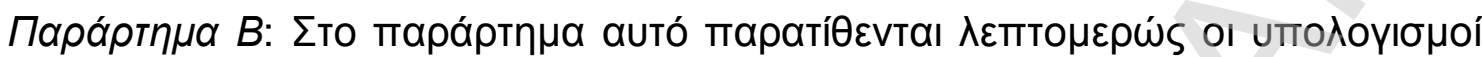

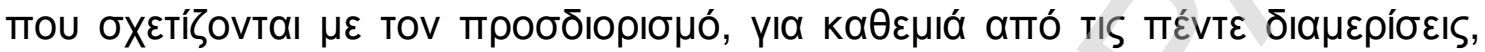

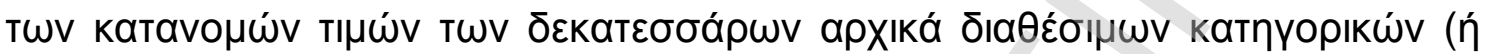
катпуорıттопнц́์

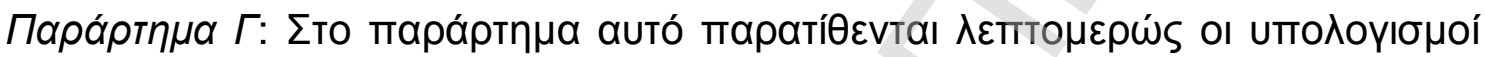

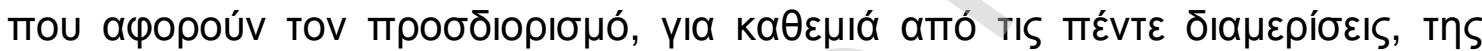

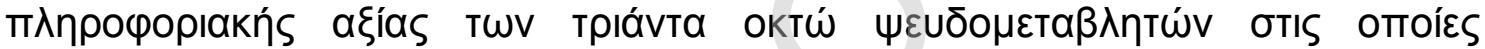

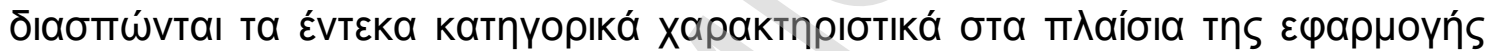

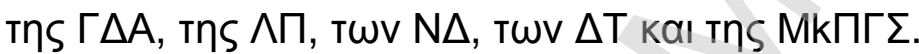

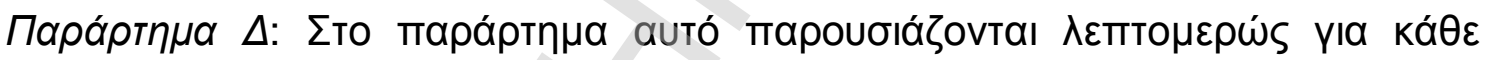

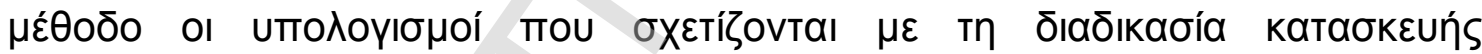

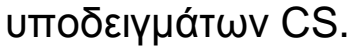

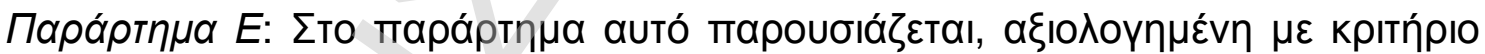

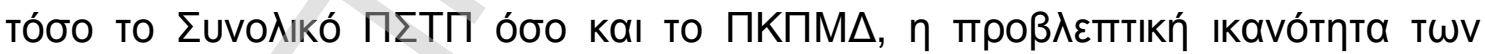

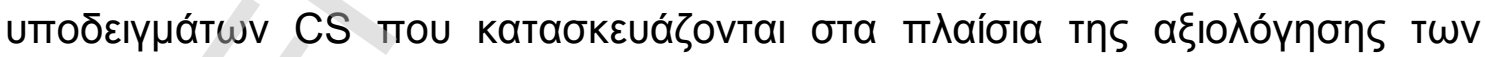
$\mu \varepsilon \boxminus o ́ \delta \omega v$.

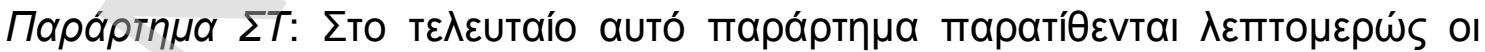

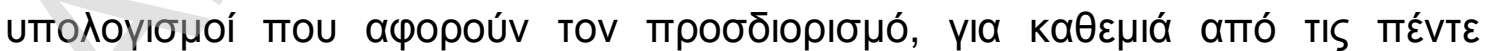

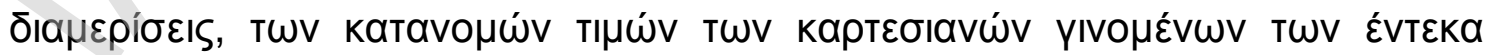

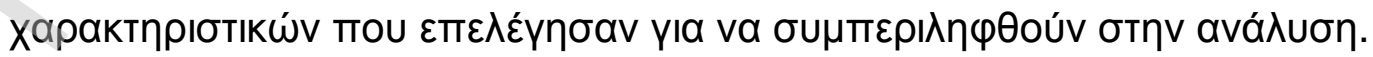




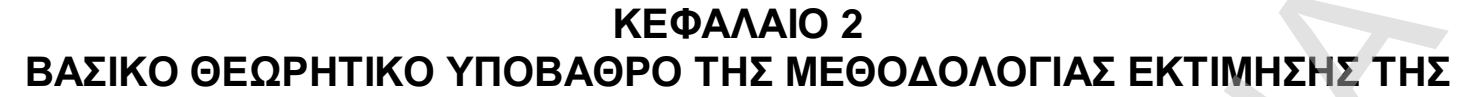

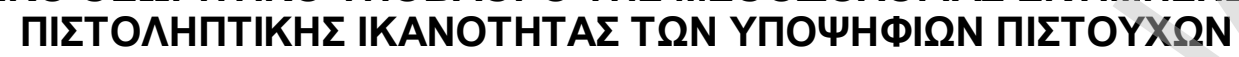

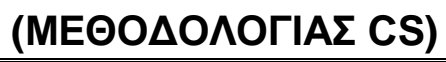

\subsection{Eıбaywyń}

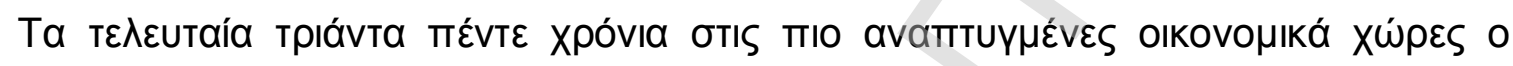

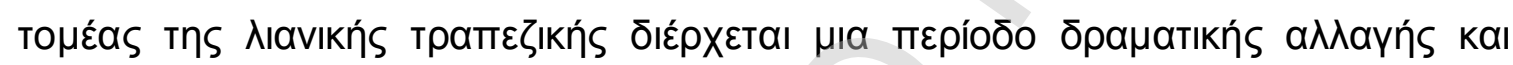

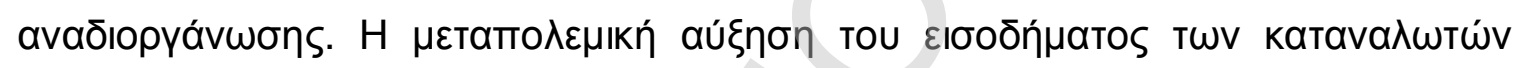

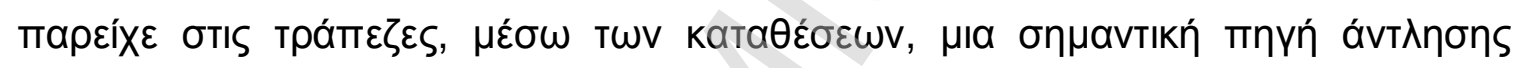

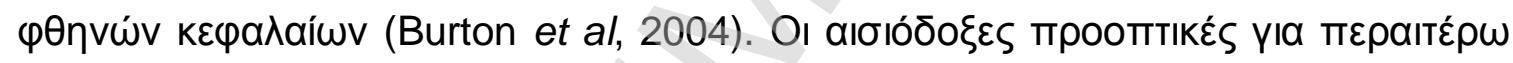

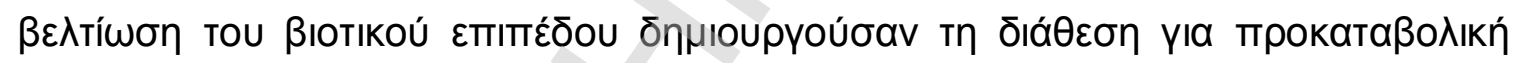

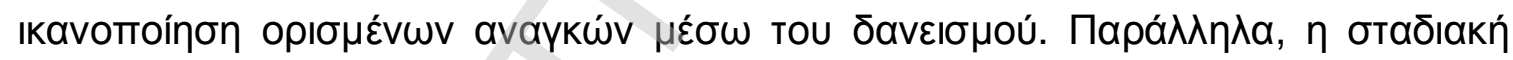

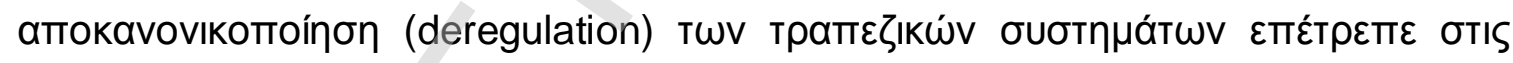

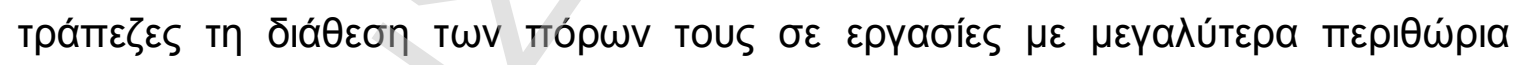

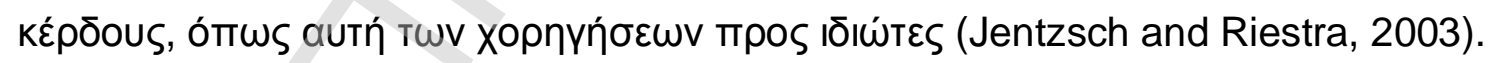

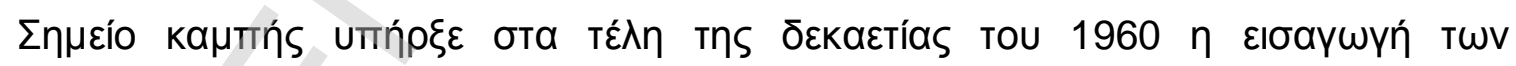

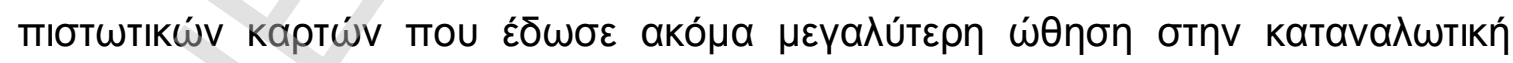

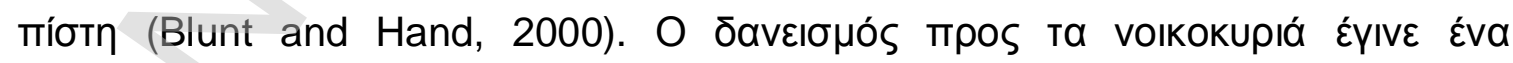

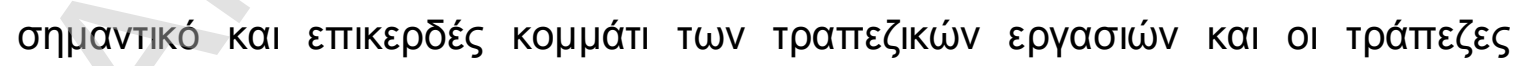

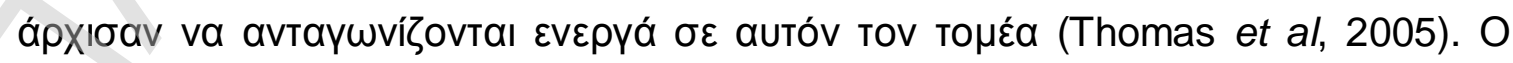

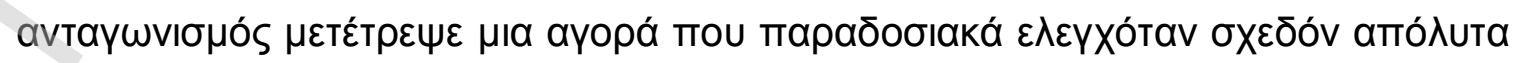




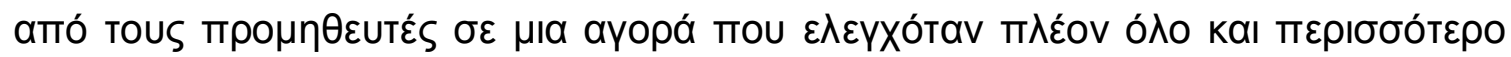

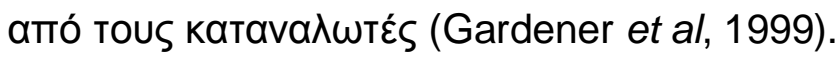

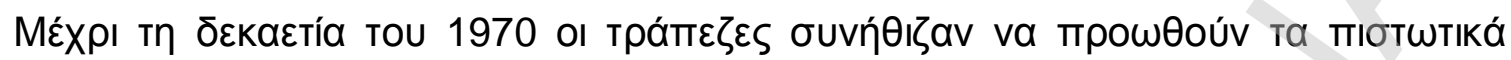

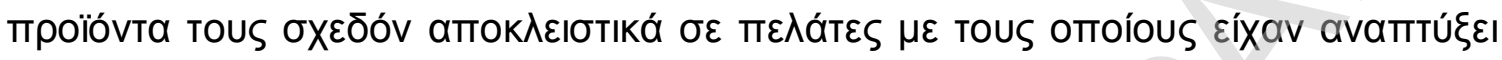

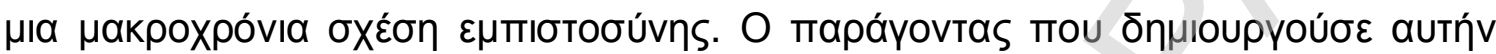

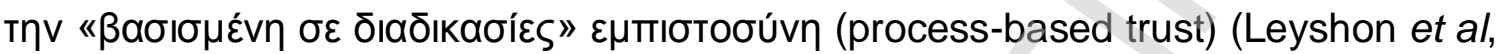

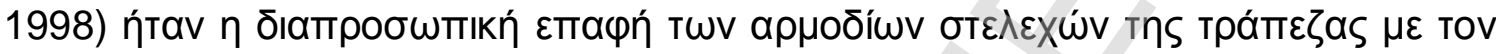

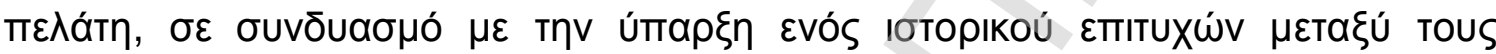

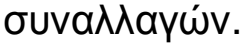

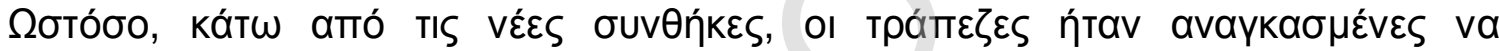

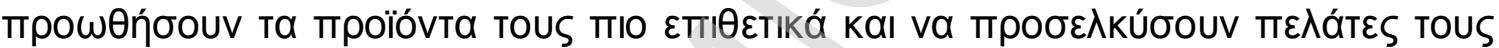

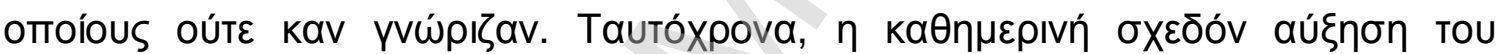

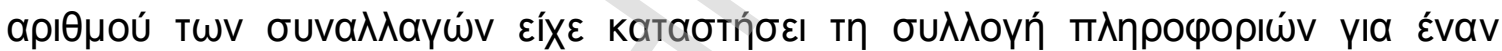

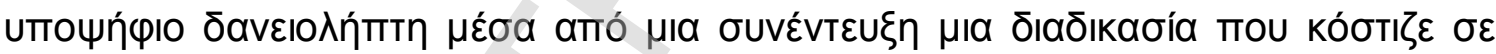

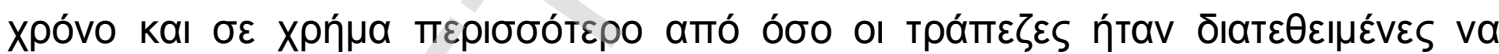

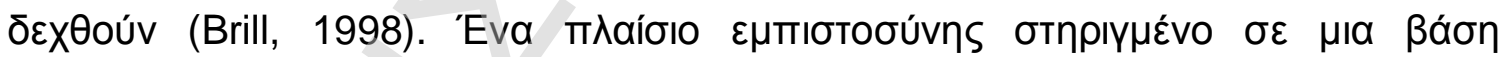

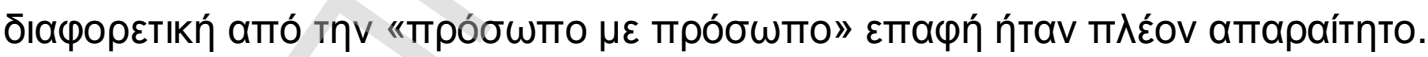

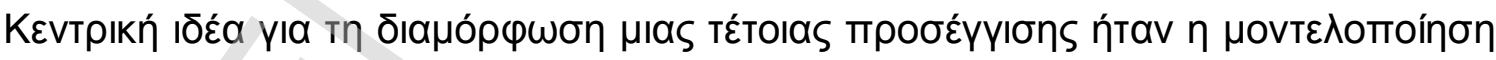

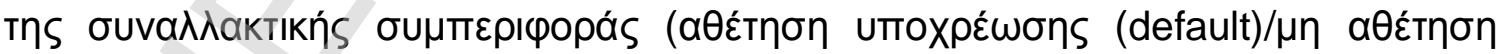

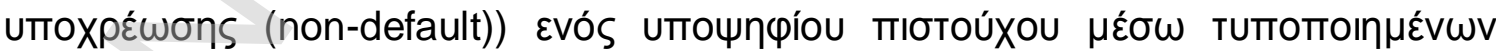

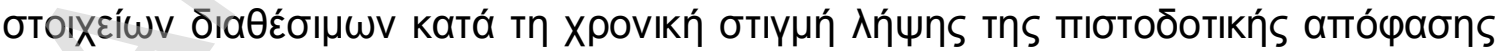

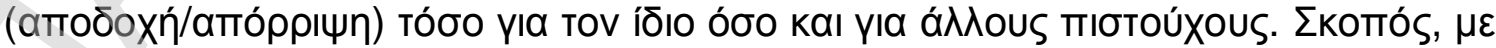

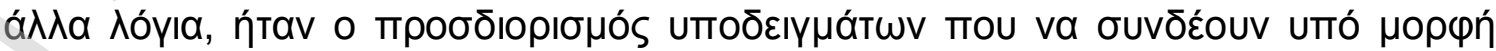




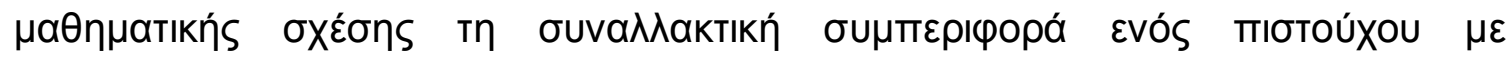

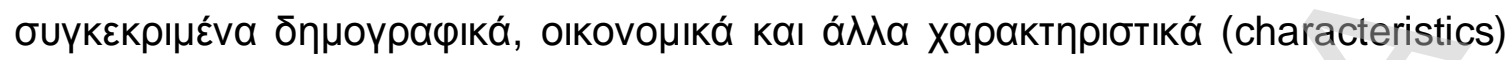

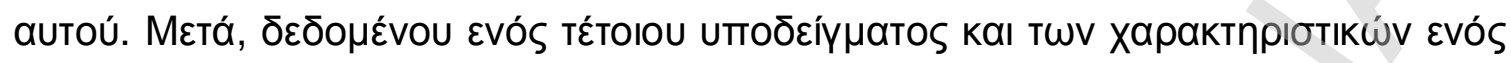

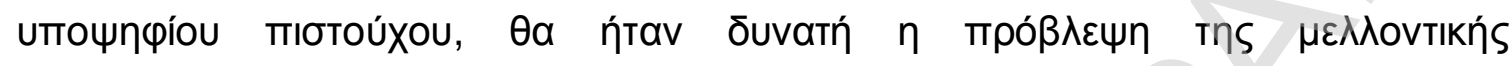

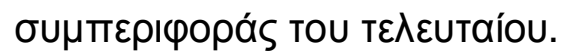

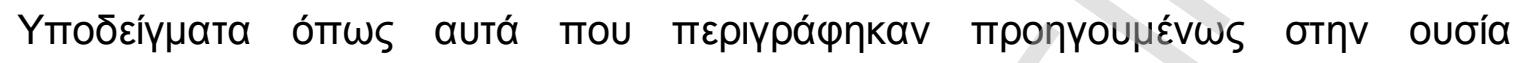

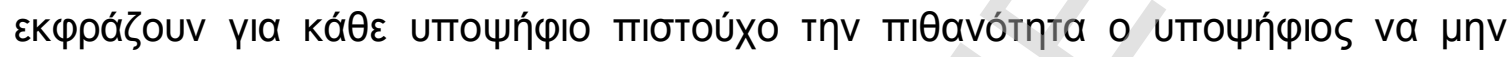

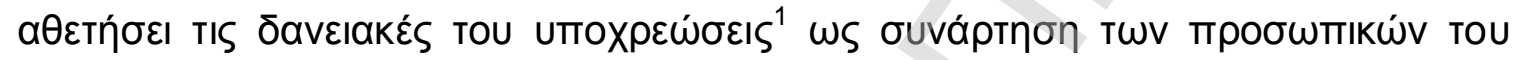

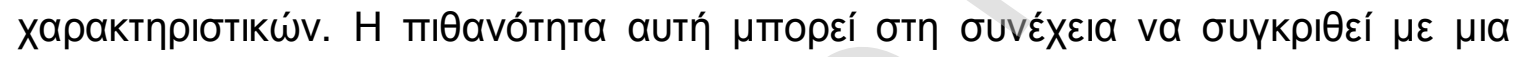

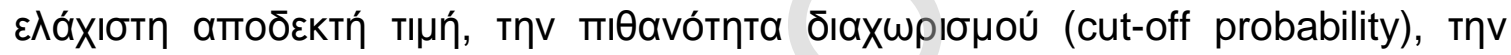

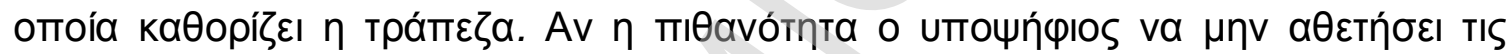

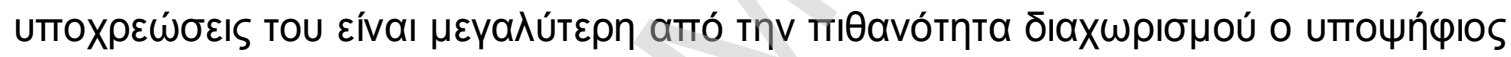

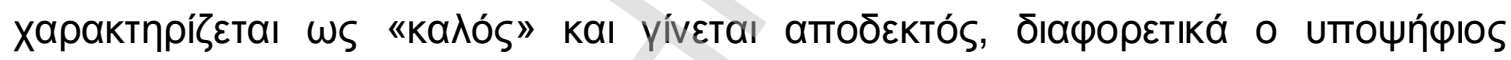

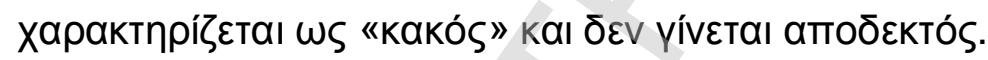

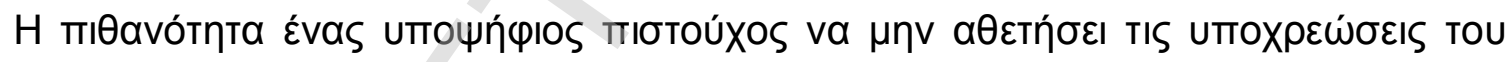

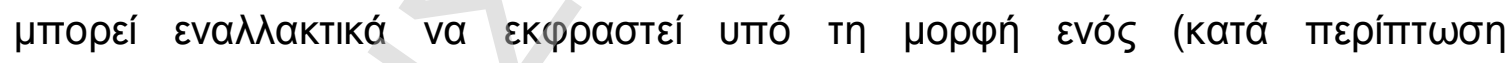

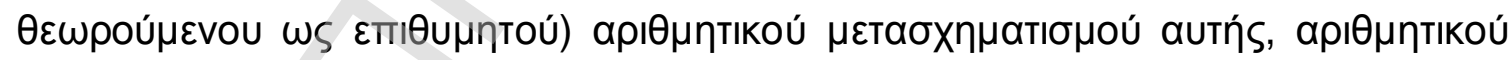

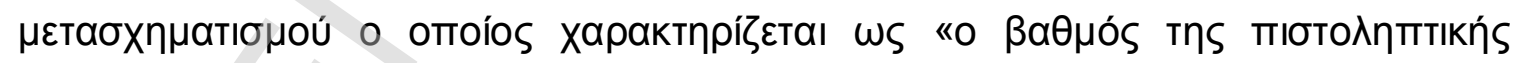

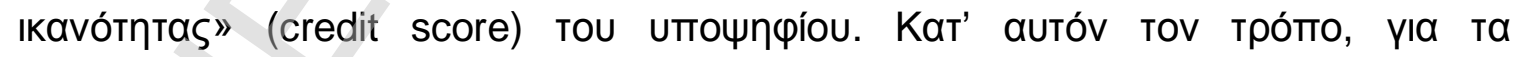

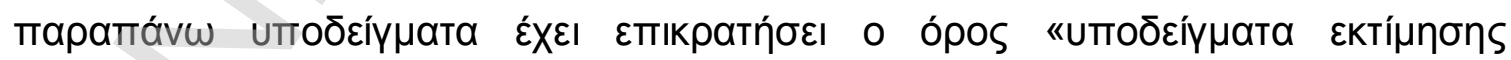

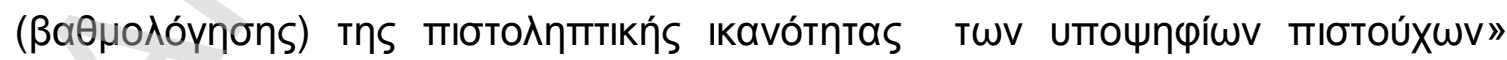
(credit scoring models).

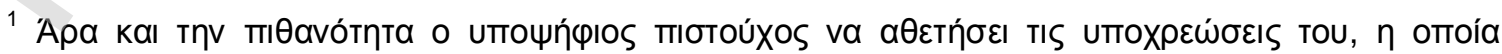

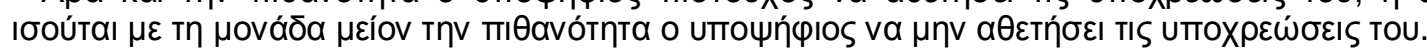




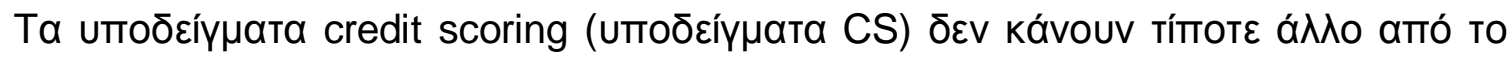

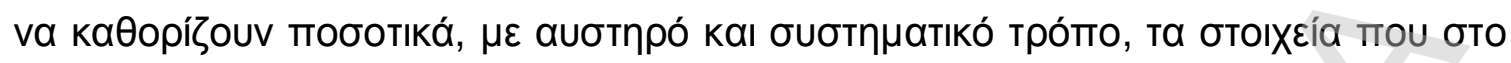

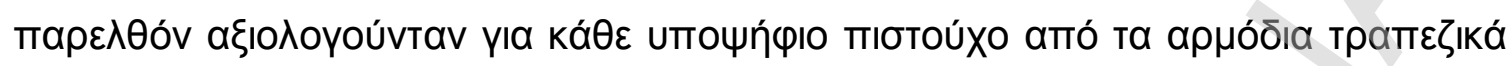

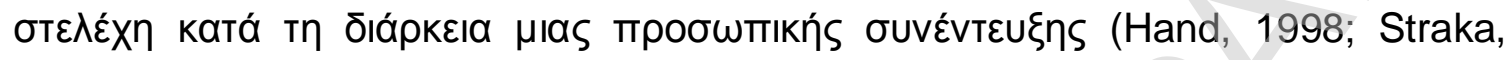

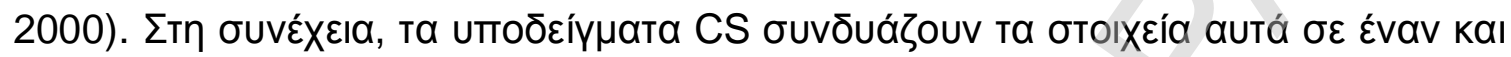

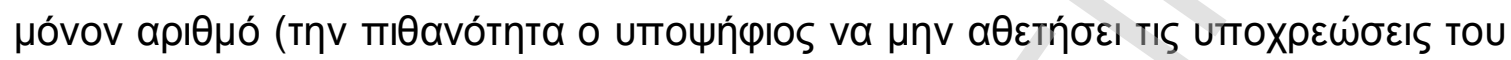

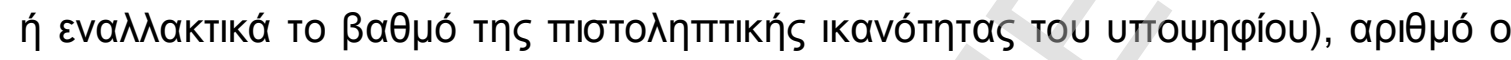

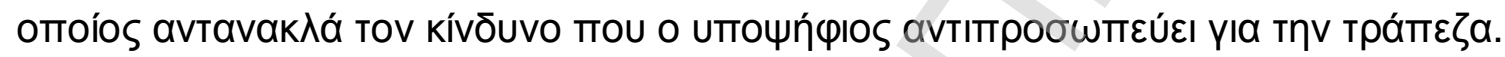

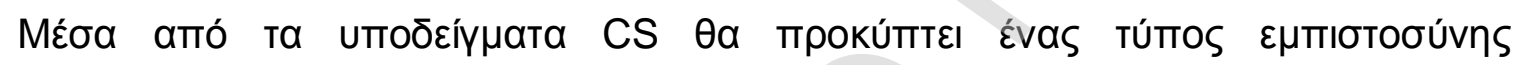

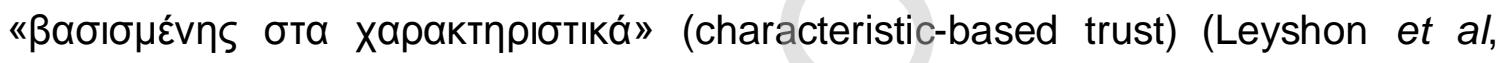

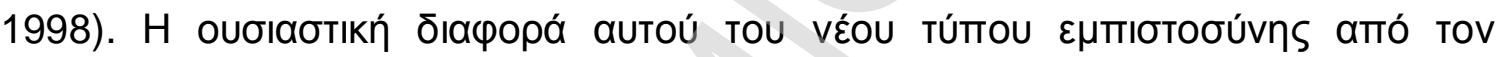

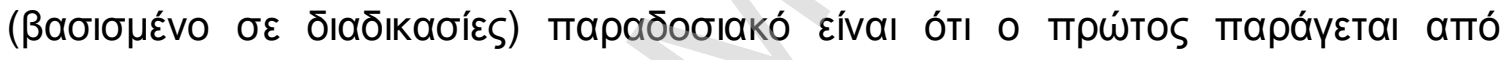

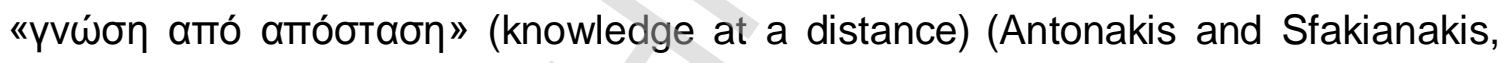

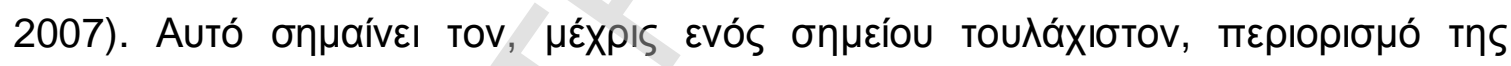

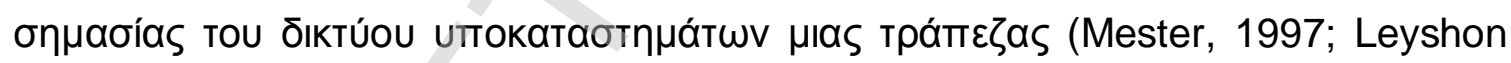

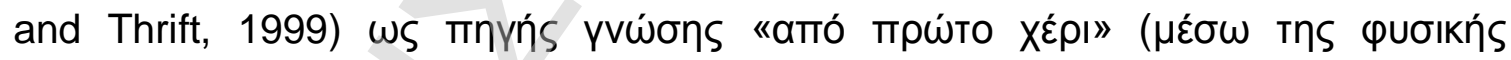

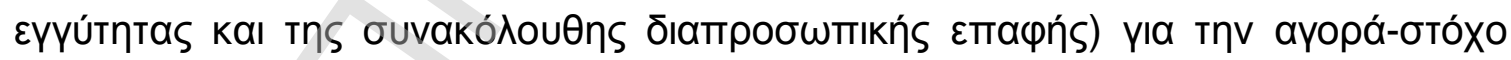

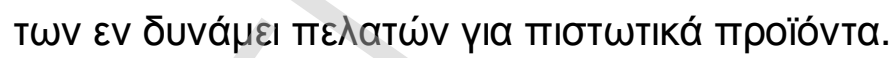

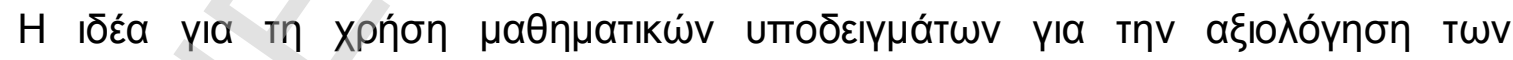

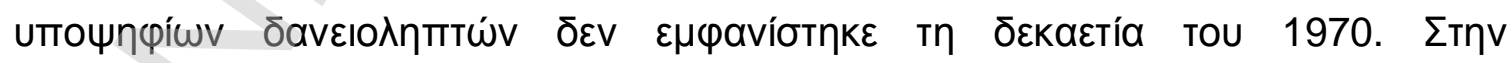

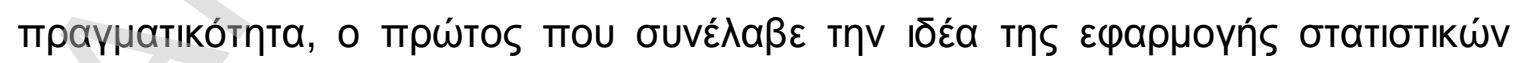

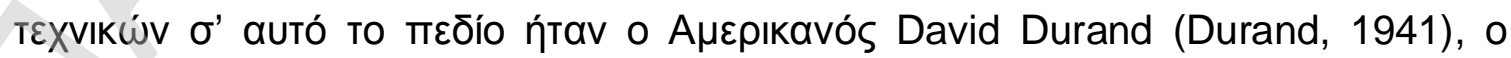

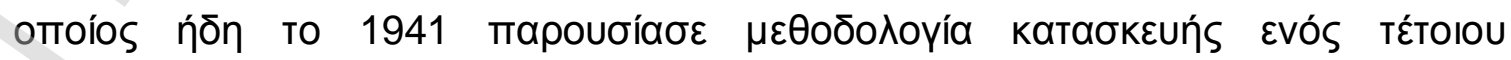




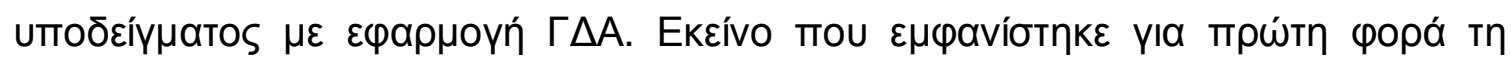

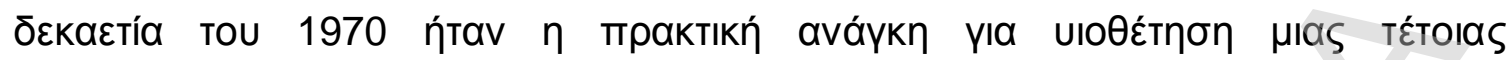

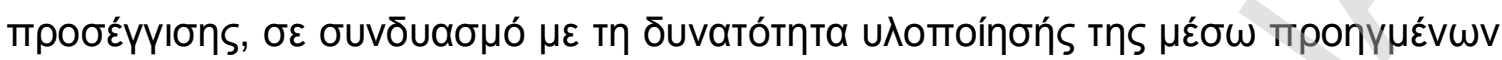

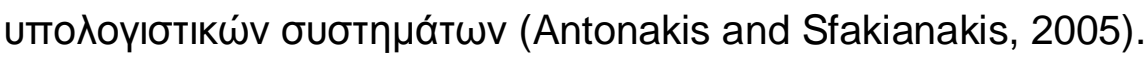

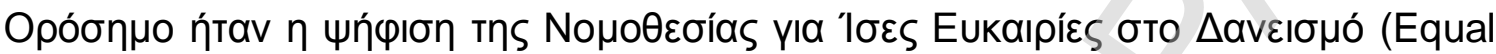

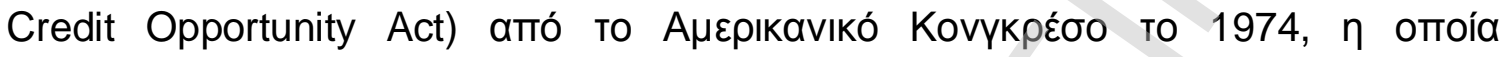

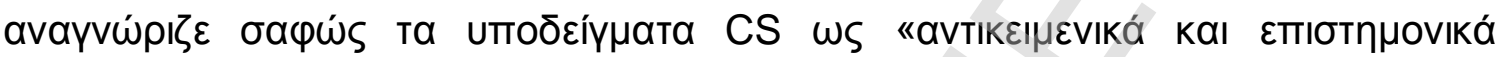

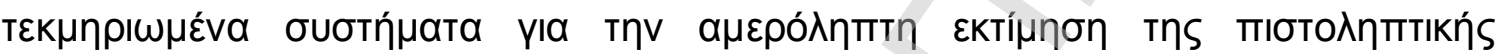

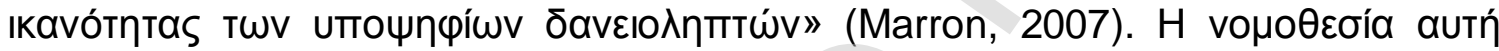

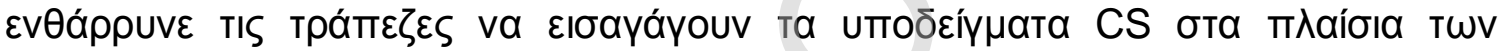

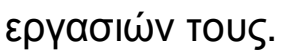

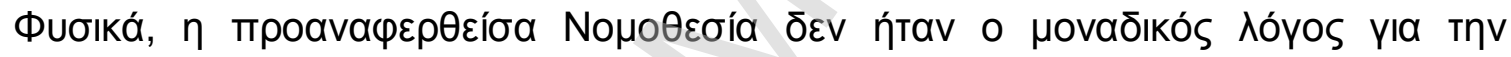

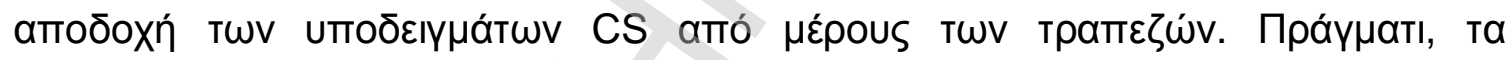

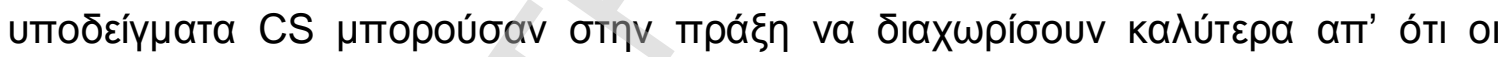

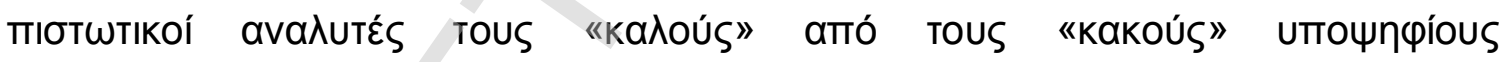

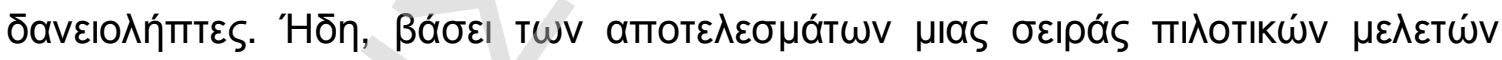

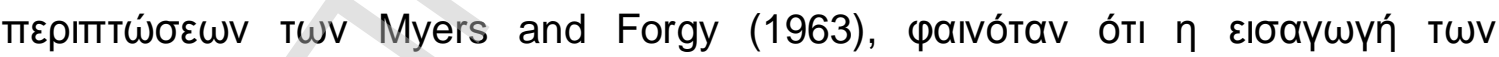

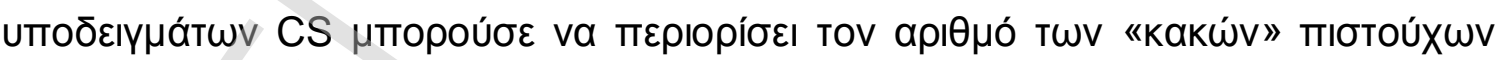

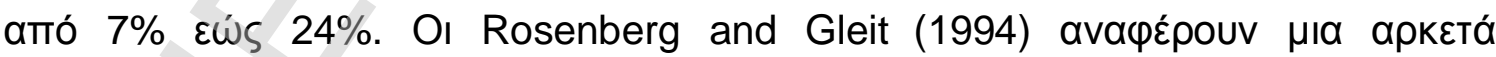

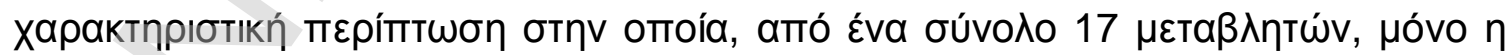

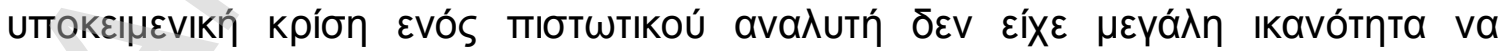

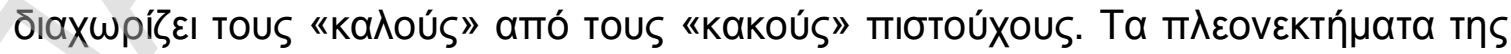

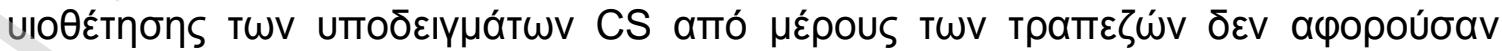




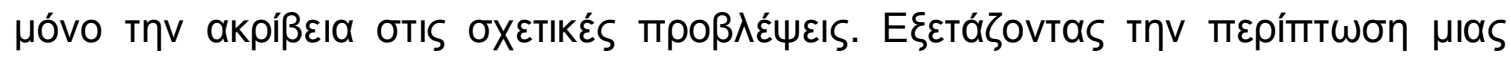

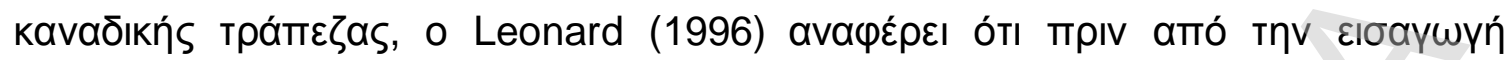

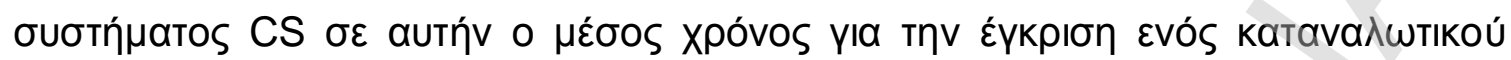

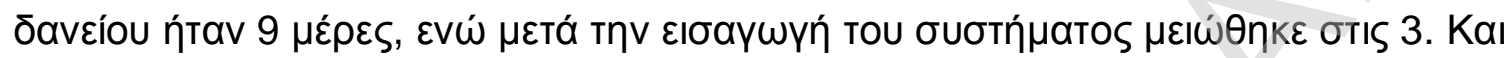

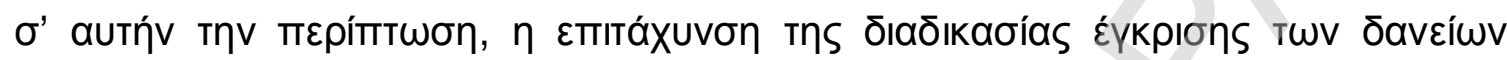

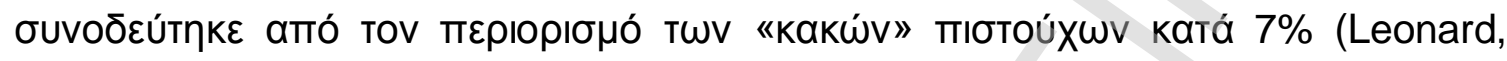
1995).

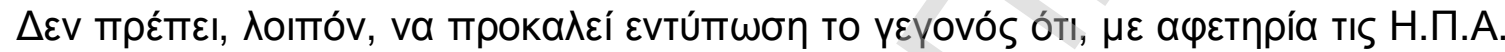

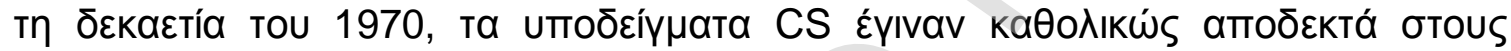

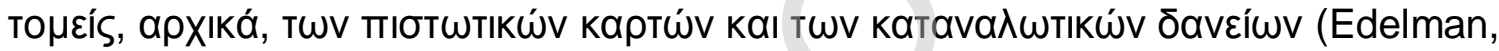

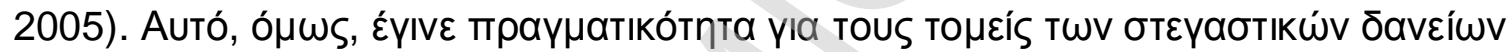

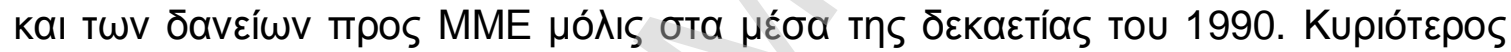

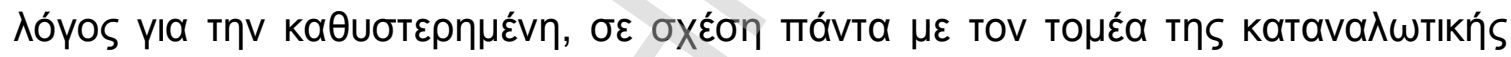

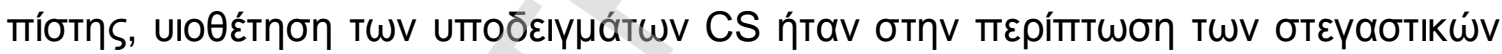

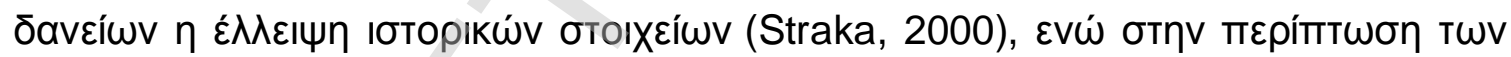

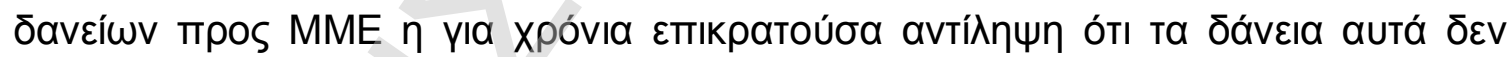

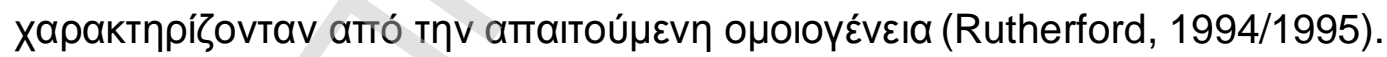

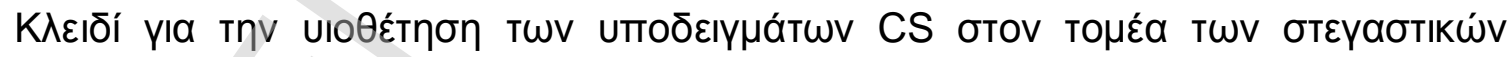

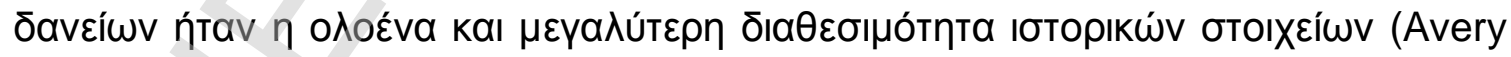

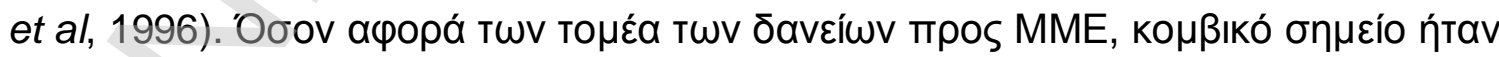

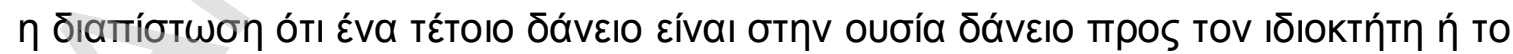

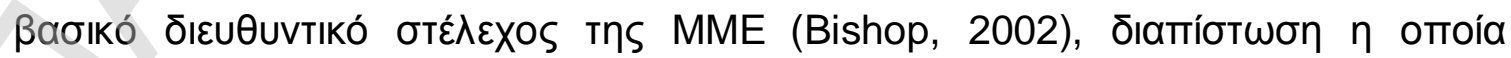




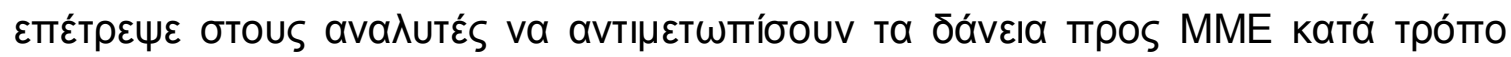

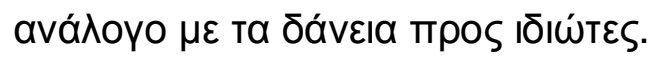

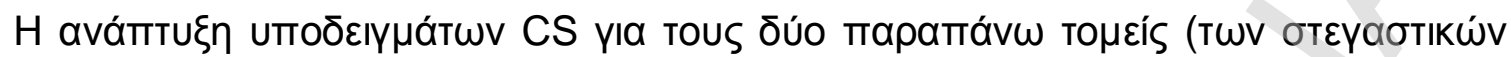

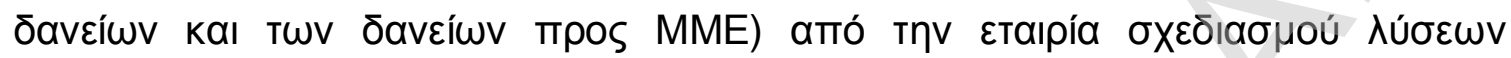

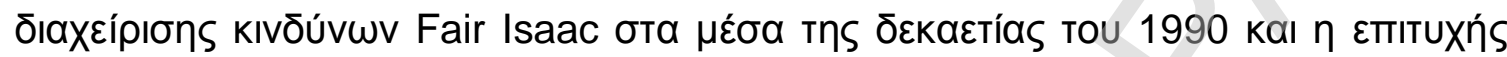

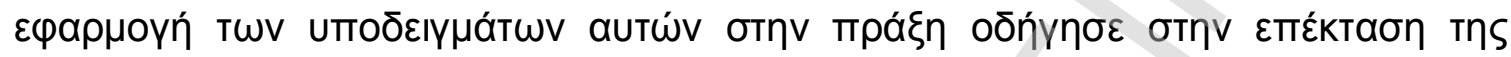

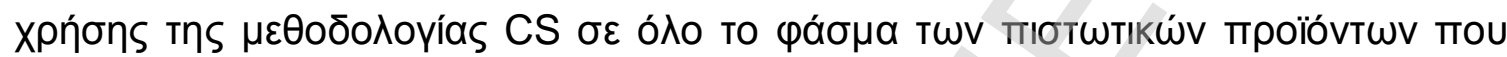

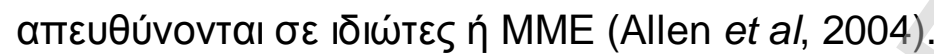

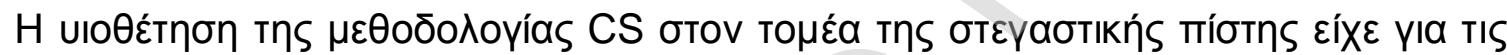

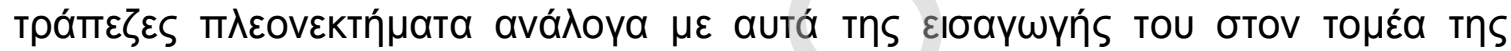

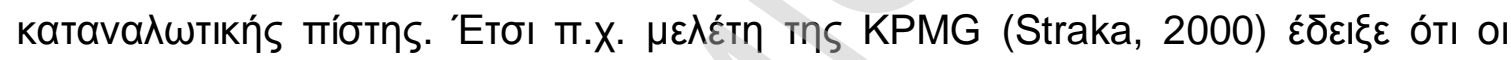

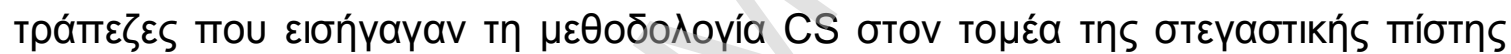

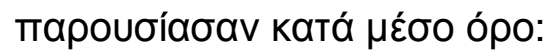

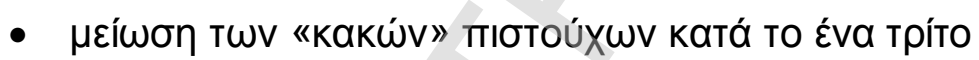

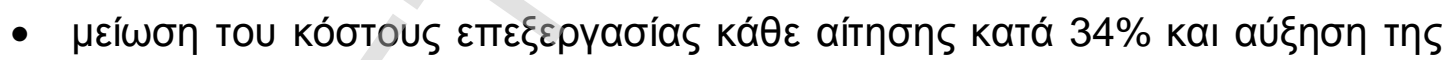
тараүшүıко́тптаৎ ката́ 37\%

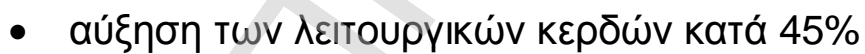

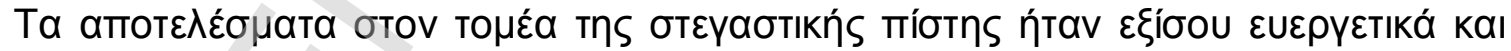

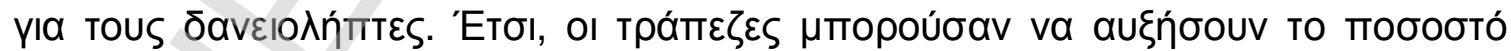

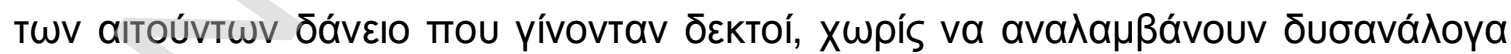

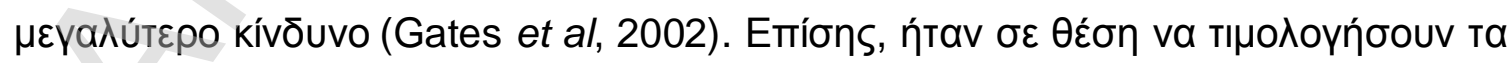

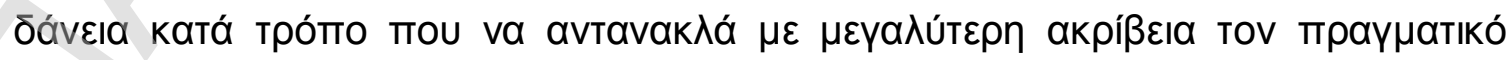




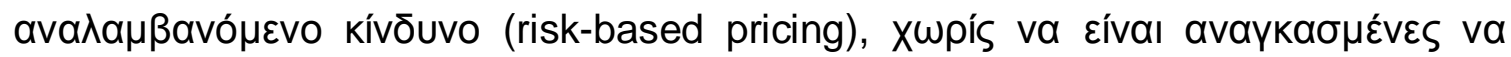

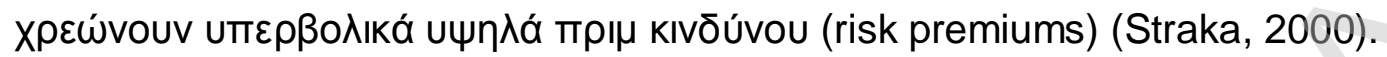

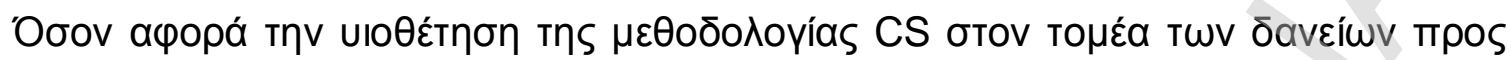

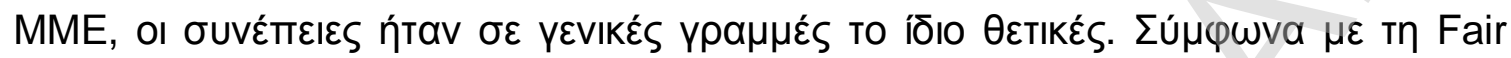

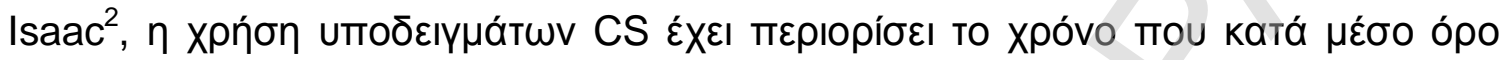

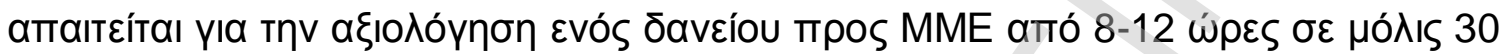

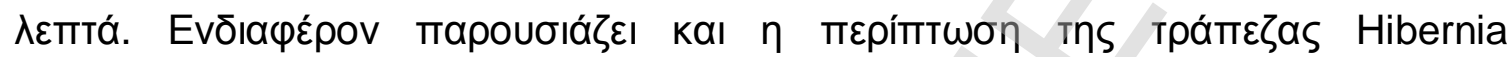

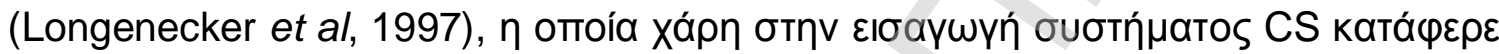

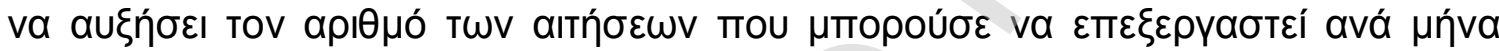

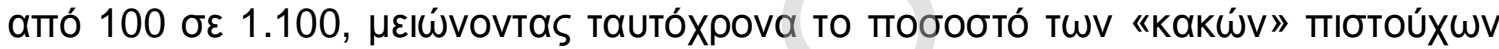

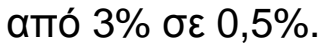

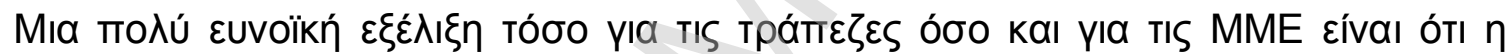

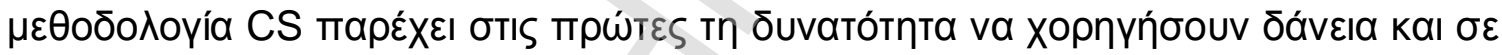

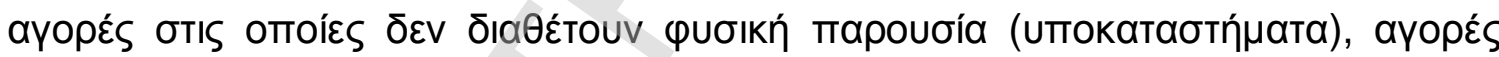

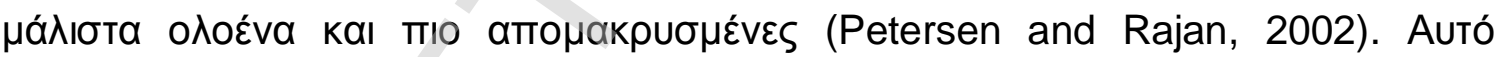

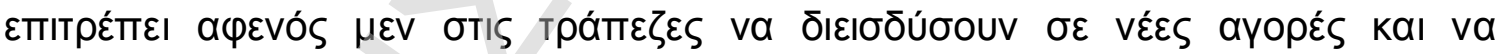

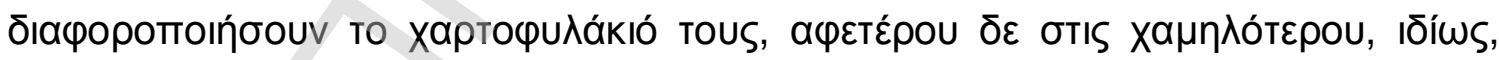

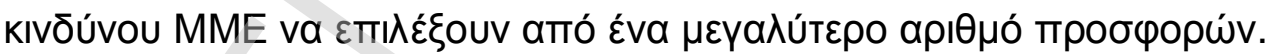

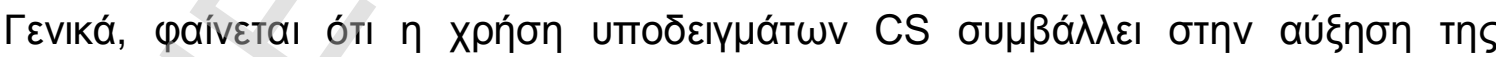

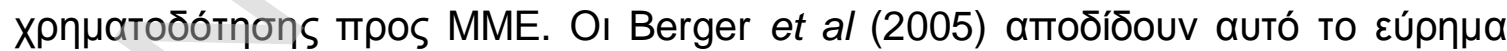

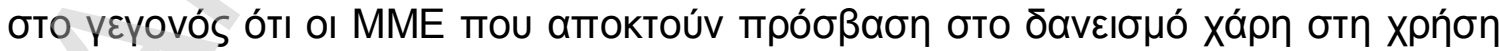

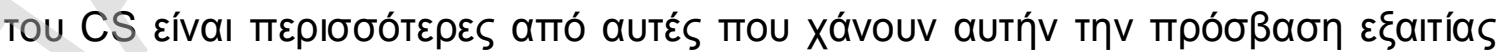

\footnotetext{
${ }^{2}$ Fair Isaac (1995). First pooled-data scorecards available for small-business lenders. ViewPoints Newsletter, 19.
} 


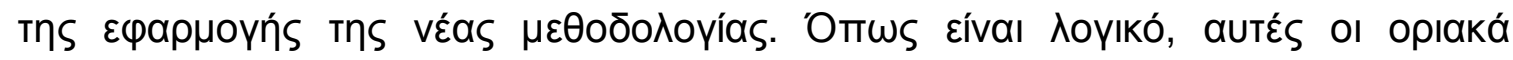

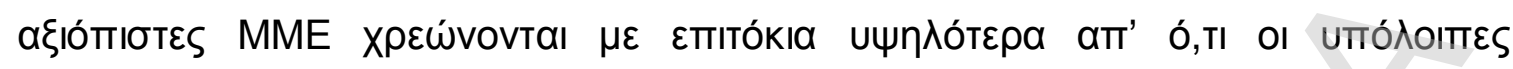

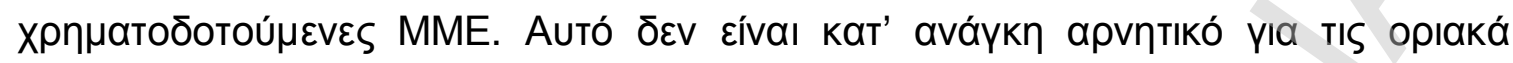

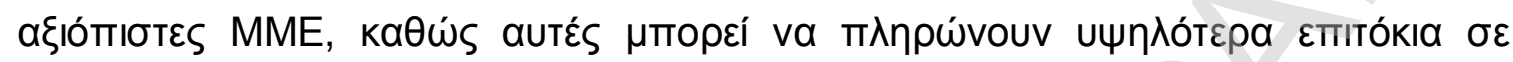

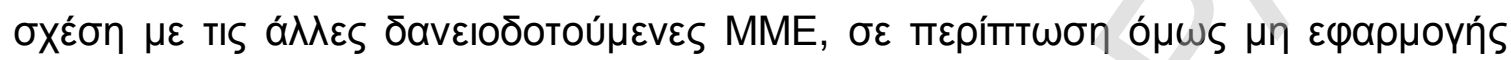

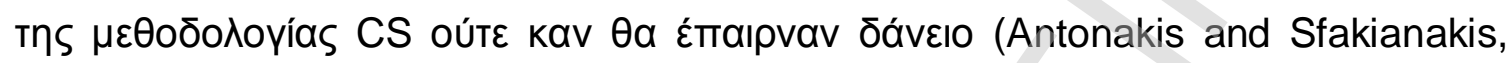
2009B).

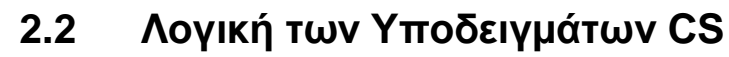

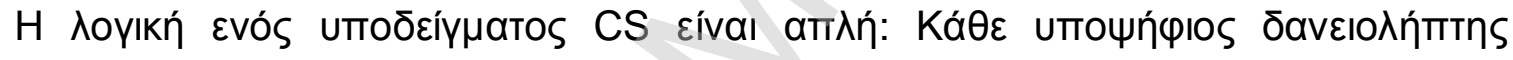

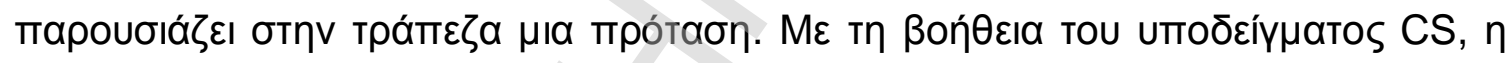

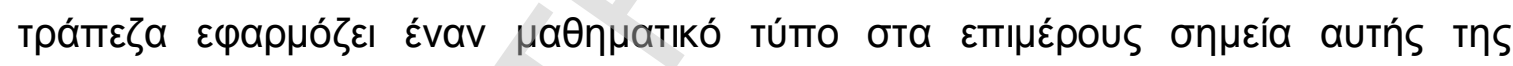

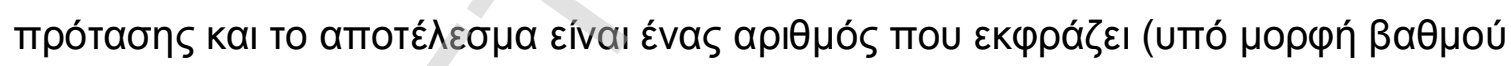

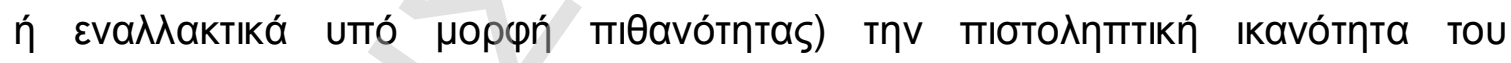

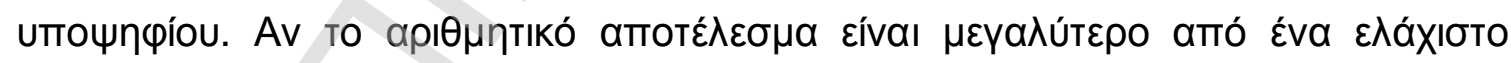

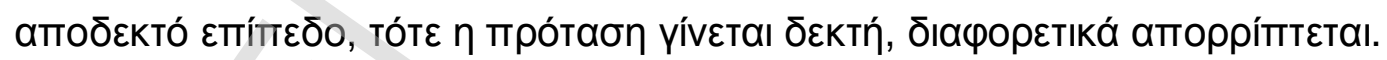

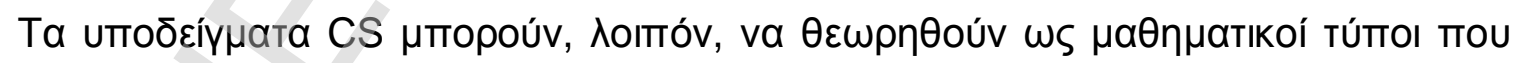

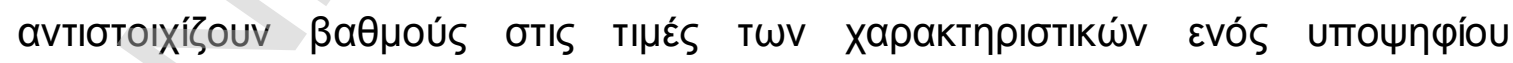

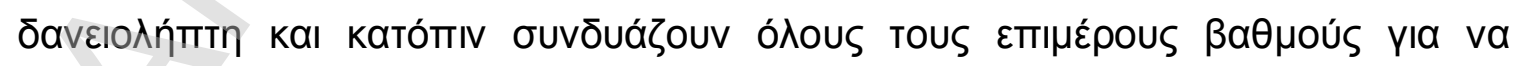

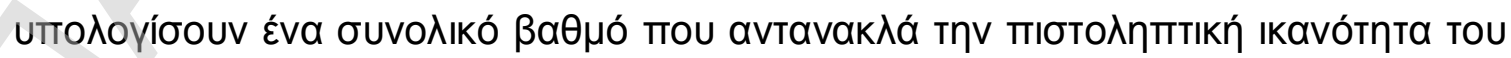
uாочпүíou. 


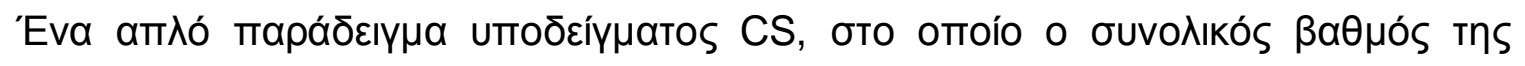

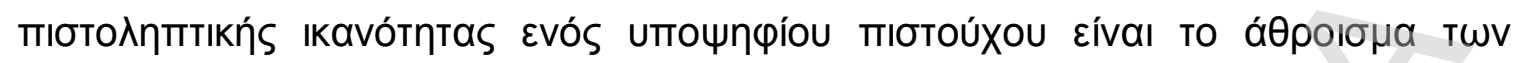

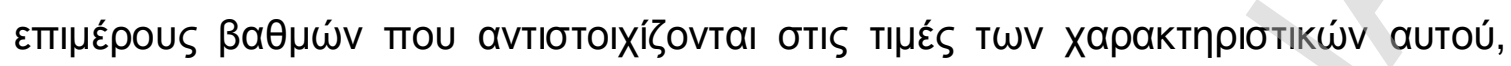

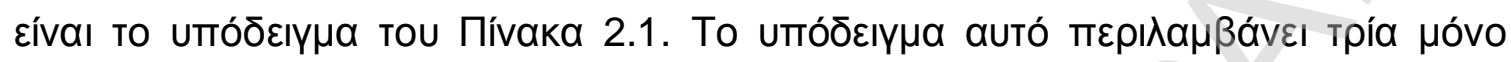

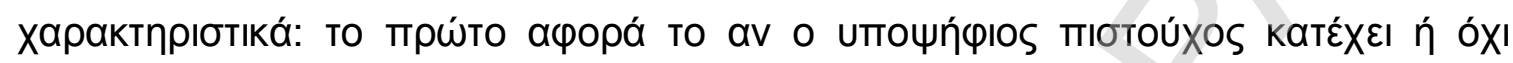

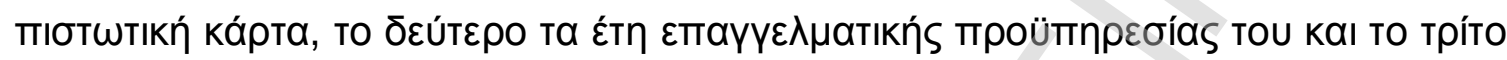

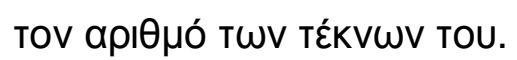

ПINAKA $\Sigma 2.1$

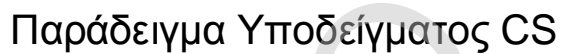

\begin{tabular}{|c|c|c|c|c|}
\hline ХарактпрІбтіка́ & 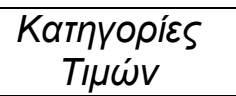 & 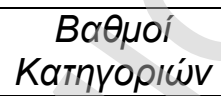 & 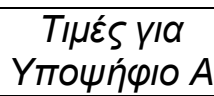 & 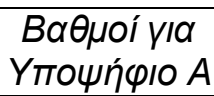 \\
\hline \multirow{2}{*}{$\begin{array}{l}\text { Катохи́ } \\
\text { Пıбтштікńs } \\
\text { Кápтаs }\end{array}$} & OXI & -7 & OXI & -7 \\
\hline & $\mathrm{Nal}$ & 22 & & \\
\hline \multirow{4}{*}{ 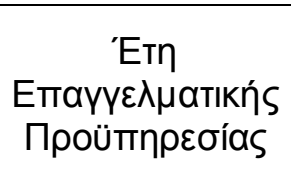 } & $1-3$ & -12 & & \\
\hline & $4-8$ & -5 & & \\
\hline & $9-12$ & 2 & & \\
\hline & $13+$ & 16 & 15 & 16 \\
\hline \multirow{4}{*}{ 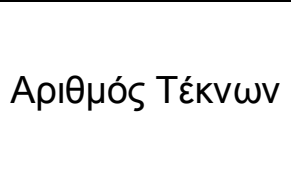 } & 0 & -10 & & \\
\hline & 1 & 32 & & \\
\hline & 2 & 7 & 2 & 7 \\
\hline & $3+$ & -4 & & \\
\hline 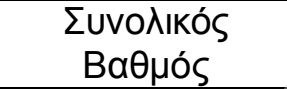 & & & & 16 \\
\hline
\end{tabular}

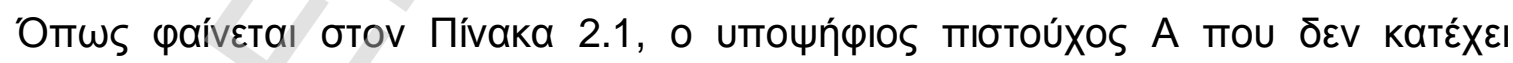

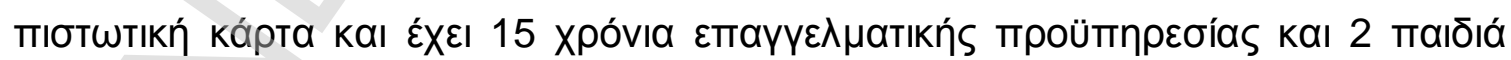

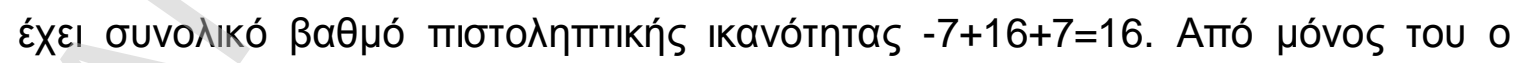

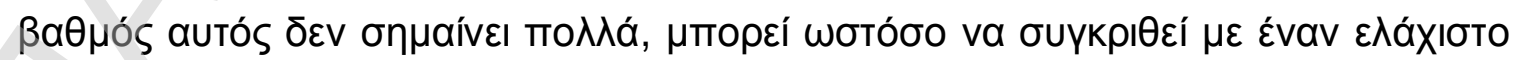

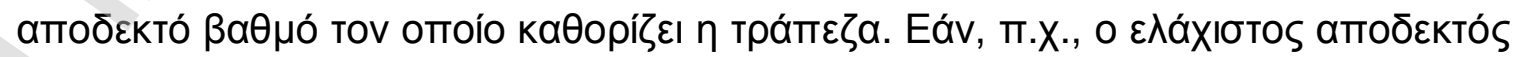

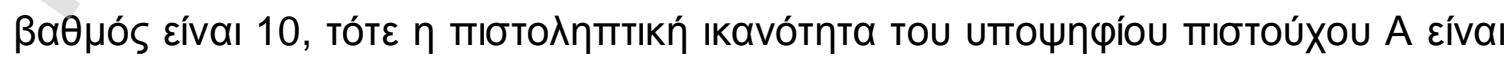




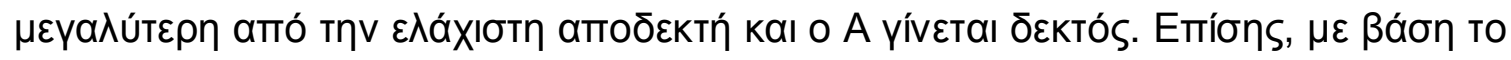

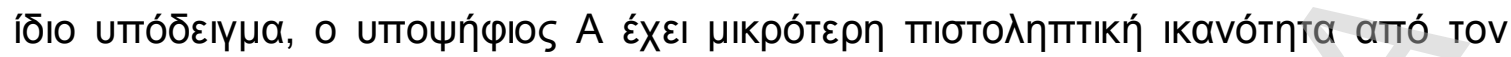

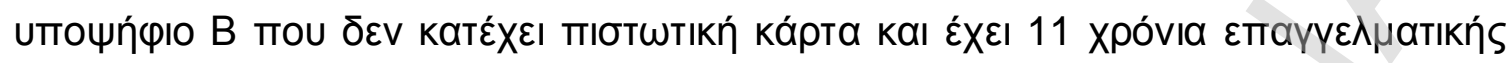

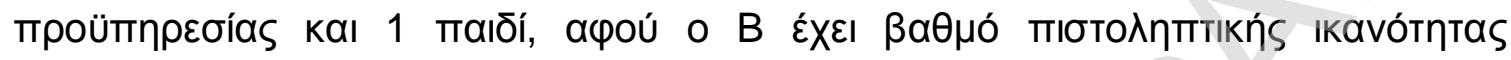
$-7+2+32=27$.

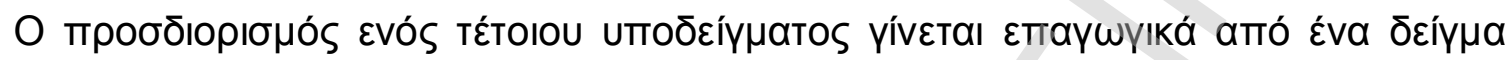

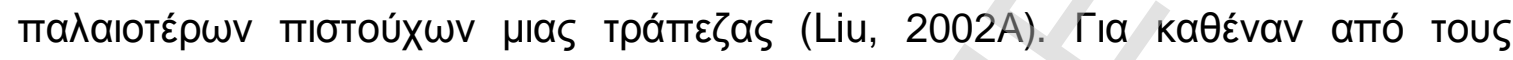

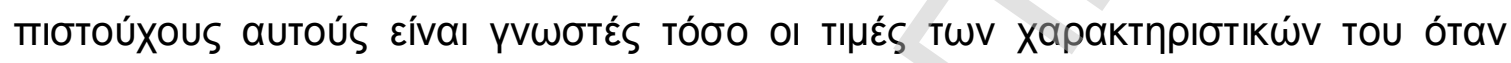

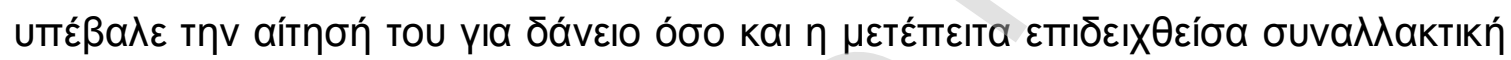

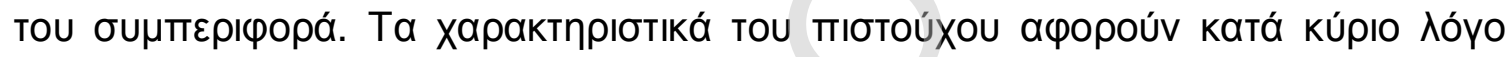

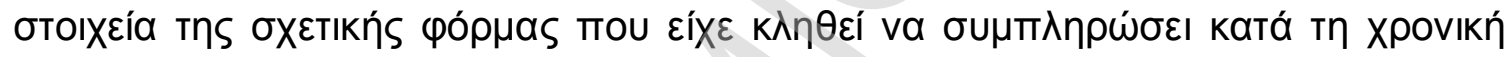

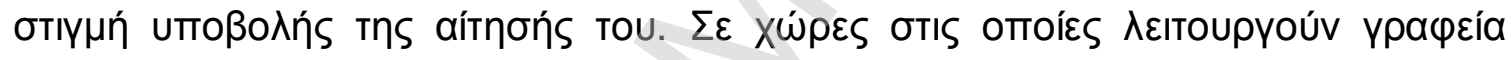

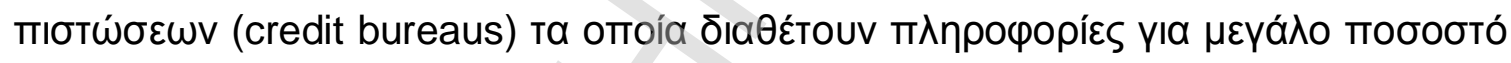

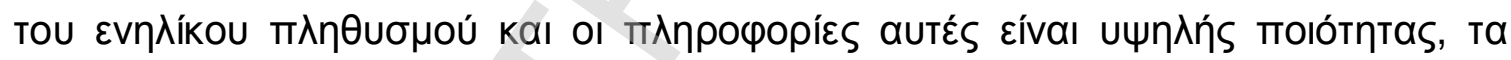

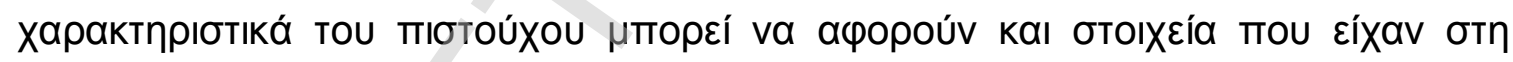

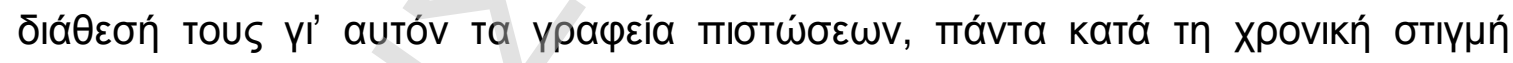
uтоßoגńs tns aítnońs tou (Hand, 2001; Thomas et al, 2002). To mapamávw

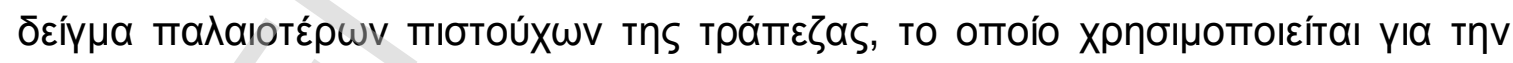

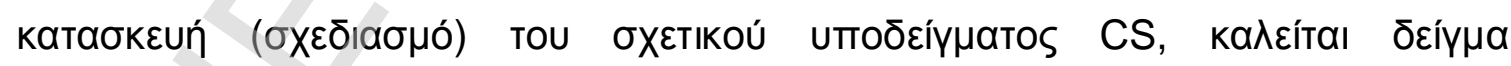

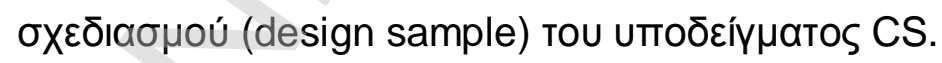

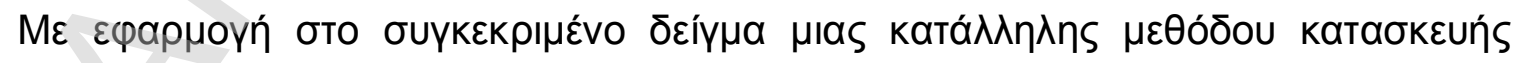

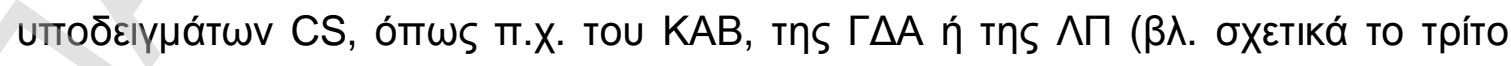

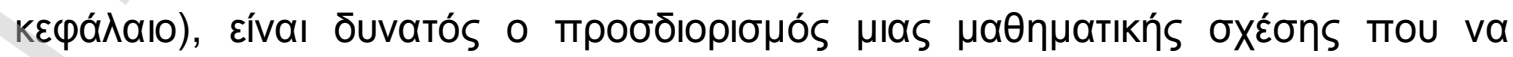




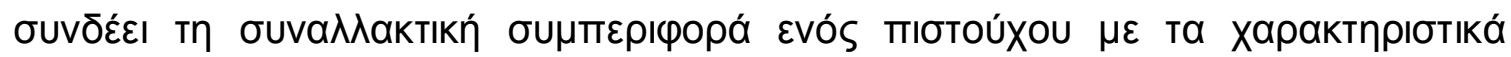

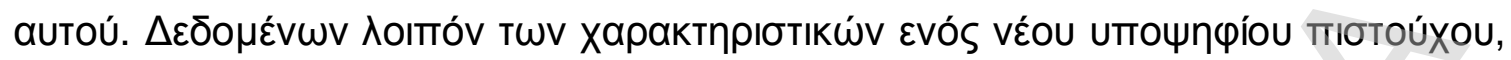

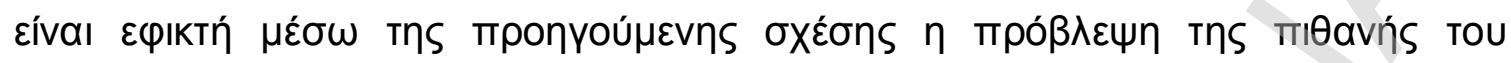

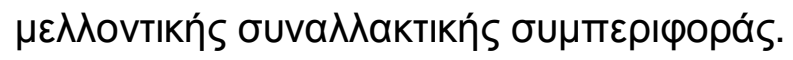

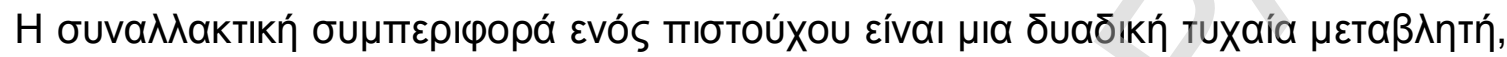

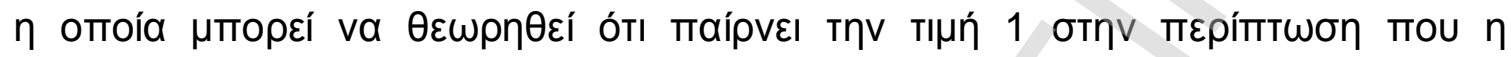

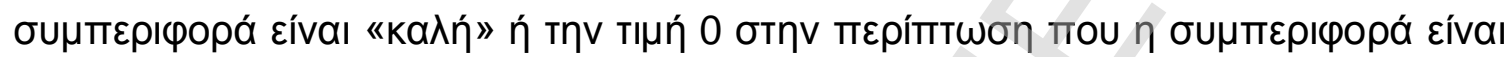

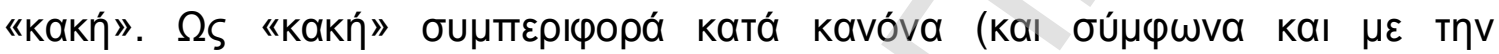

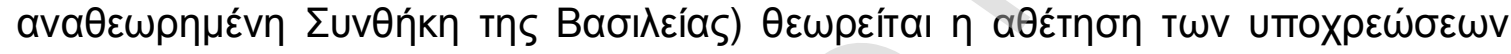

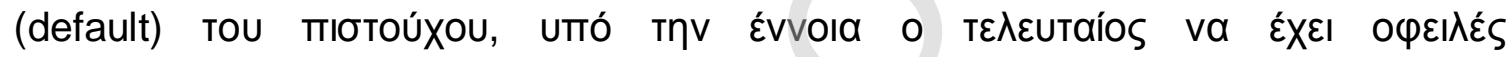

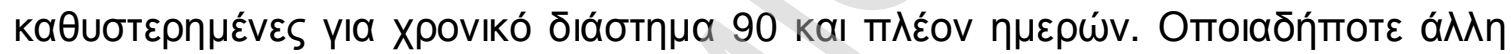

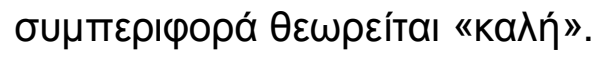

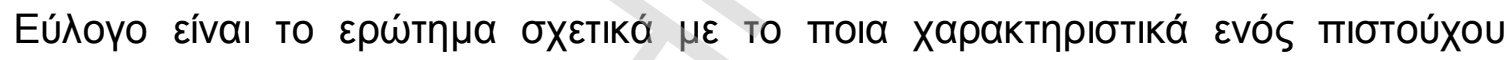

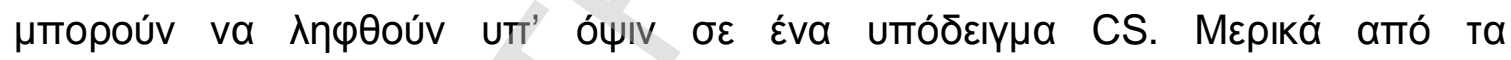

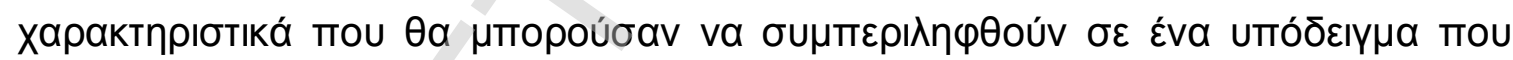

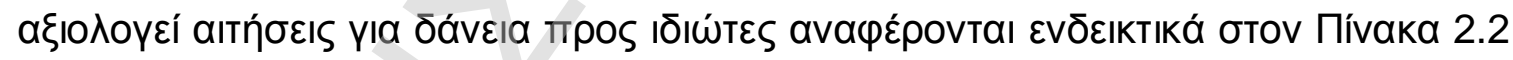

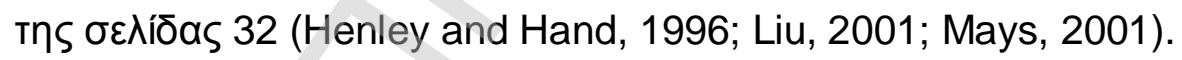

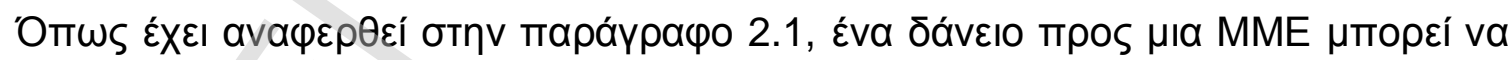

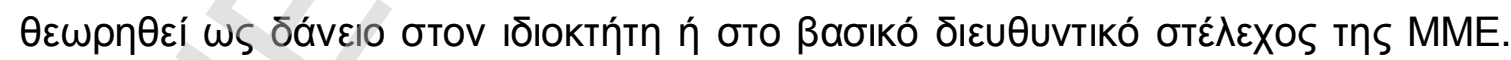

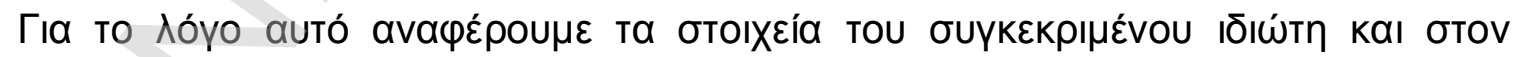

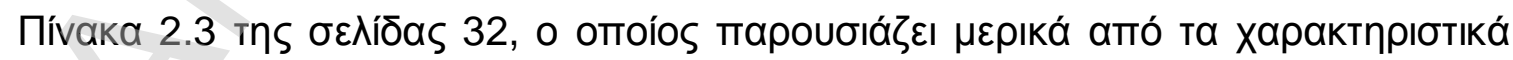

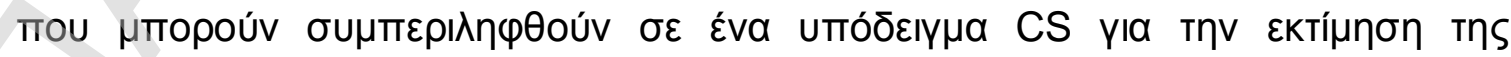
пıбтоגпптікńs ıкаvóтптаs MME (Liu, 2001; Behr and Guettler, 2007). 
ПINAKA $\Sigma 2.2$

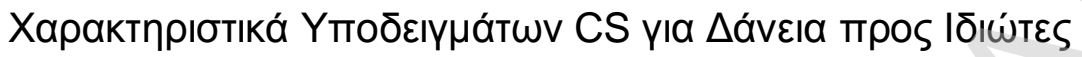

\begin{tabular}{|c|c|}
\hline Túmо Хар Хактпрıбтıки́v & 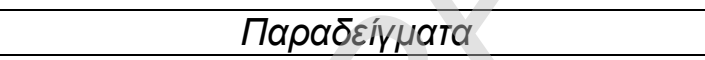 \\
\hline 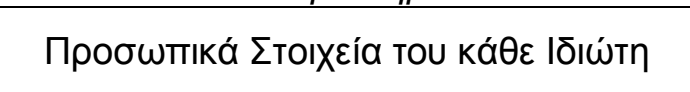 & 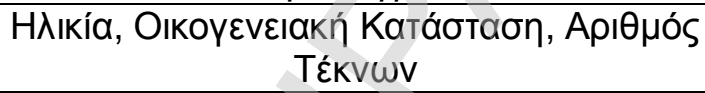 \\
\hline 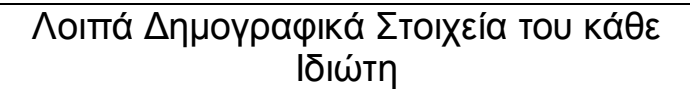 & 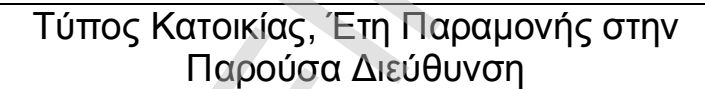 \\
\hline 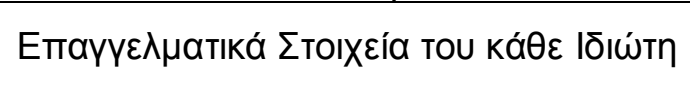 & 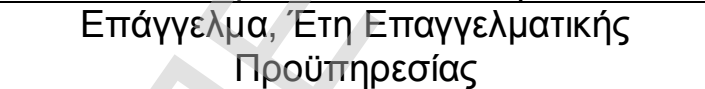 \\
\hline 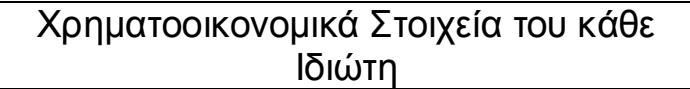 & 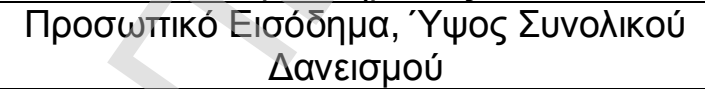 \\
\hline 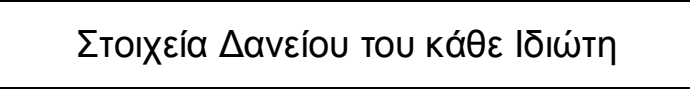 & 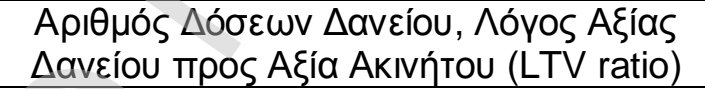 \\
\hline 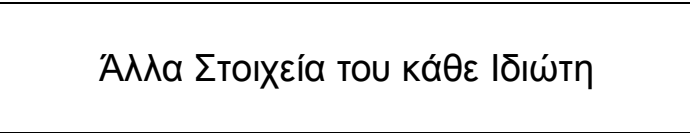 & 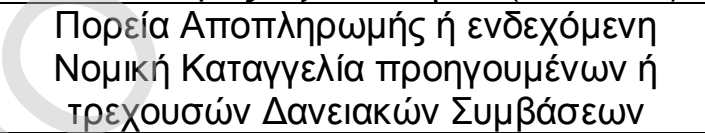 \\
\hline
\end{tabular}

ПINAKA 2.3

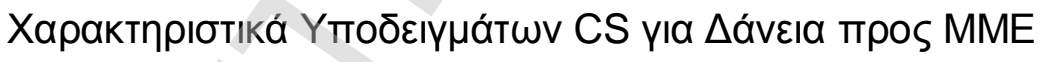

\begin{tabular}{|c|c|}
\hline Túmоо Характпрıбтıки́v & 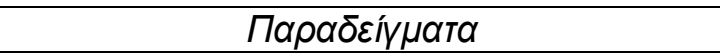 \\
\hline 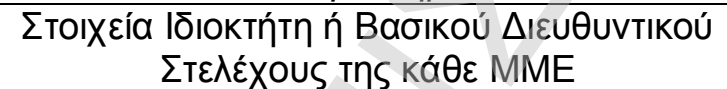 & 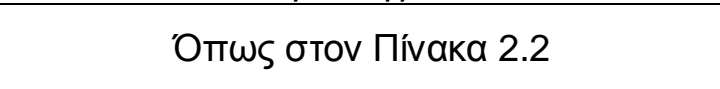 \\
\hline 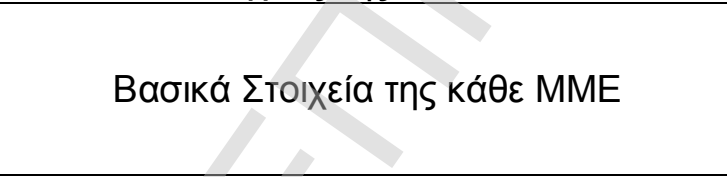 & 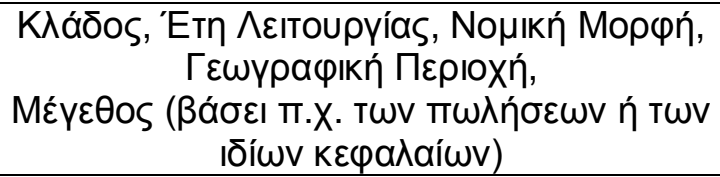 \\
\hline 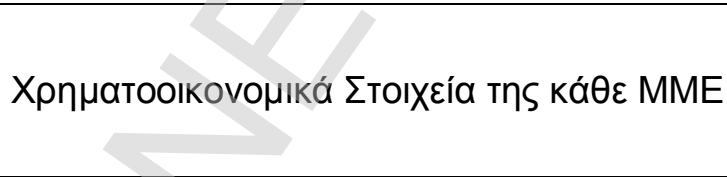 & 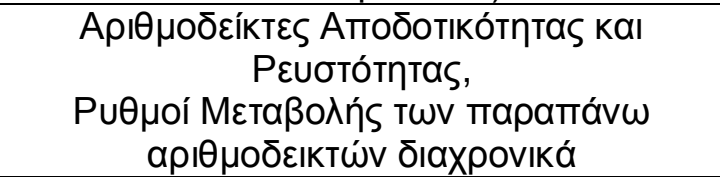 \\
\hline Al & 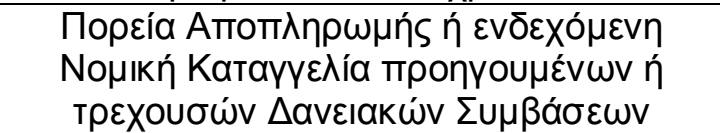 \\
\hline
\end{tabular}




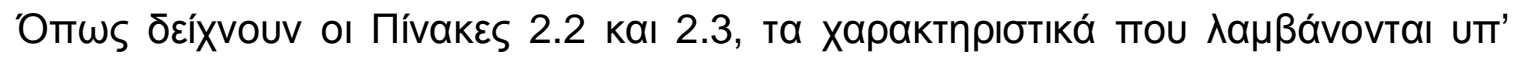

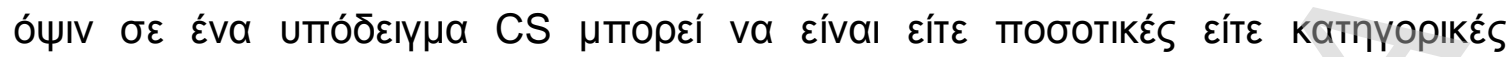

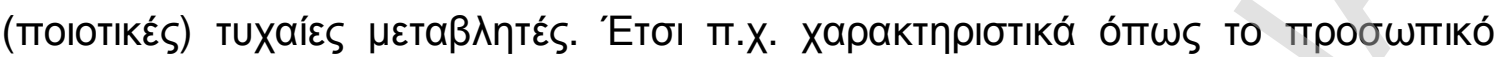

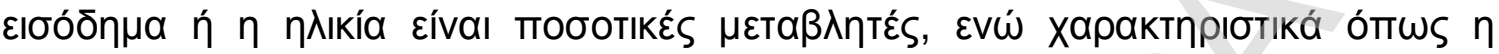

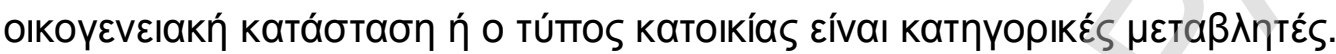

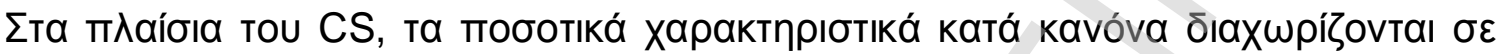

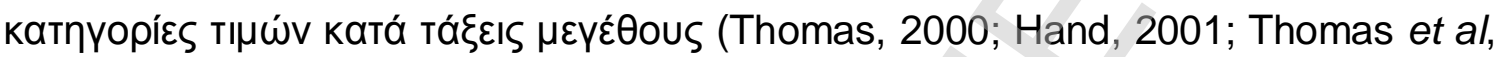

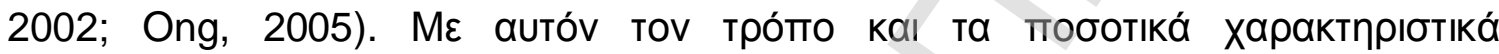

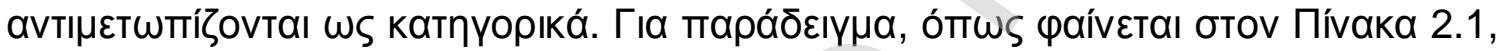

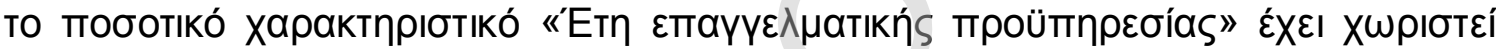

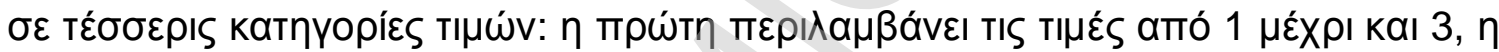

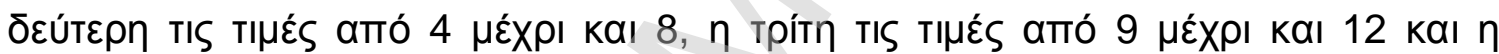

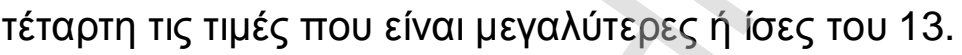

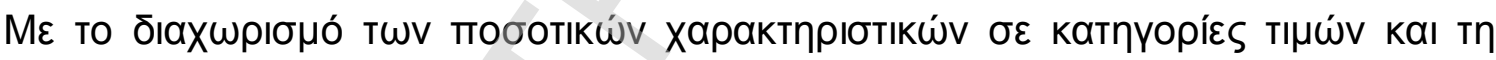

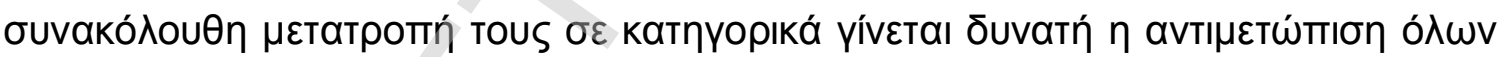

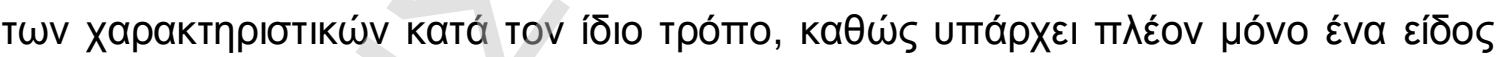

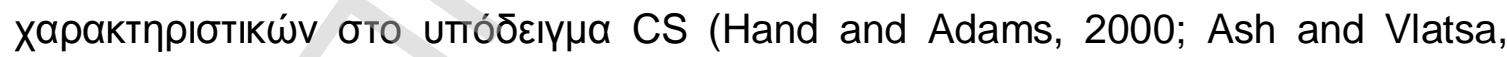

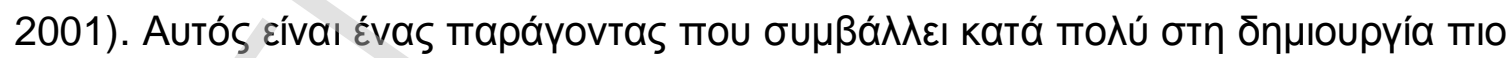

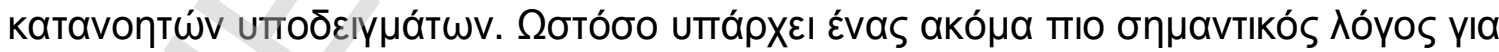

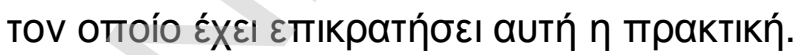




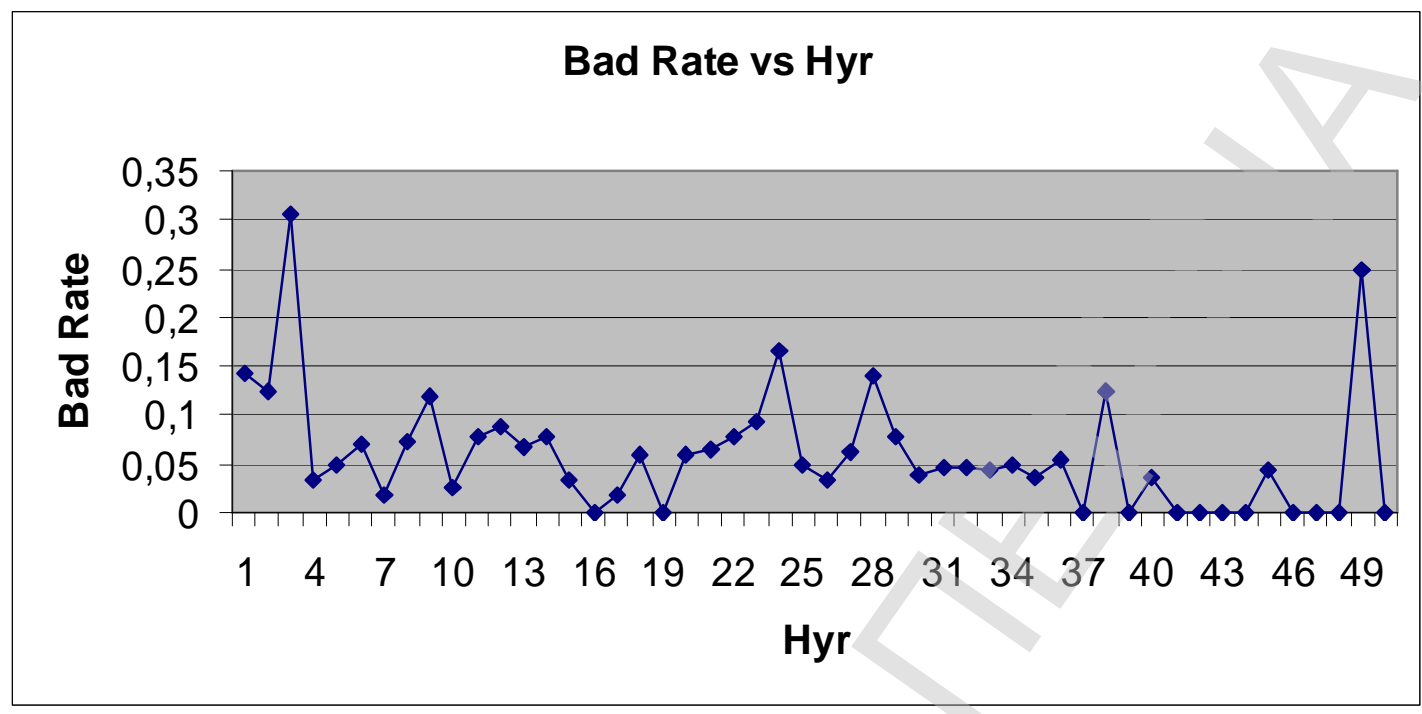

$\triangle$ ІАГРАММА 2.1

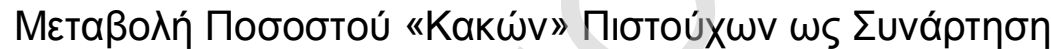

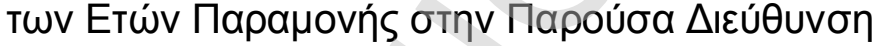

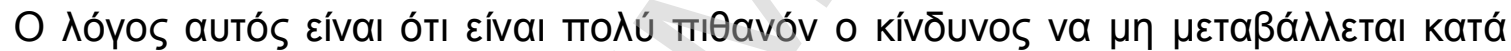

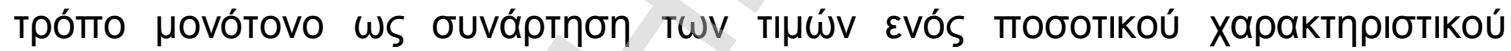

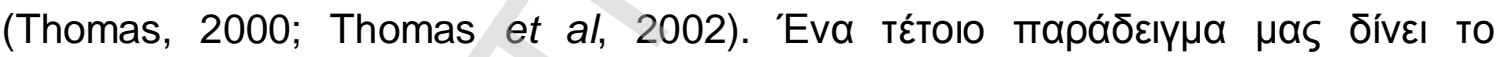

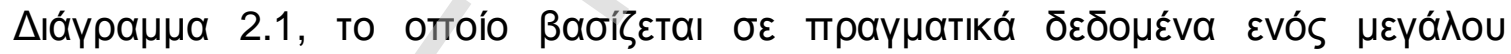

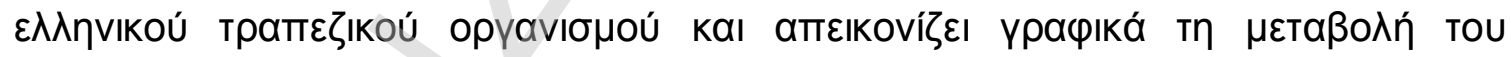

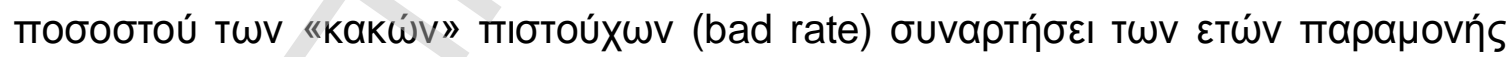

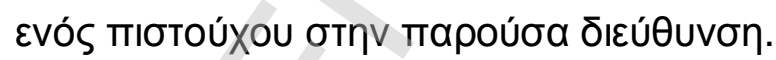

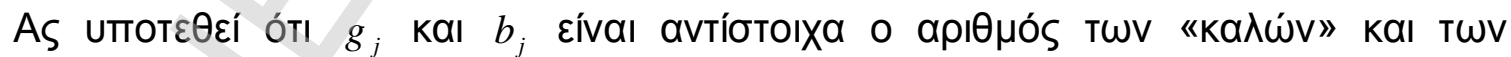

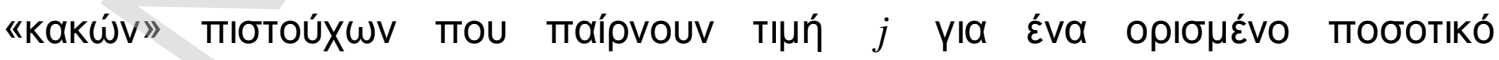

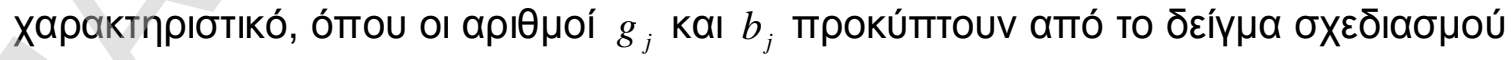

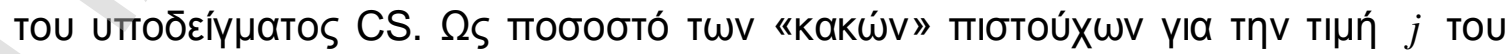

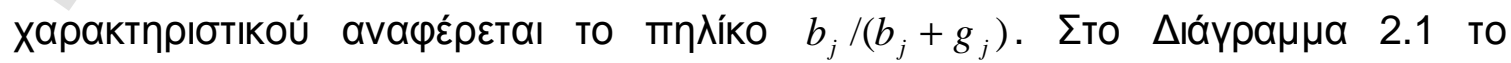




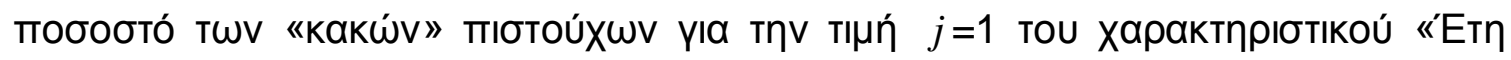

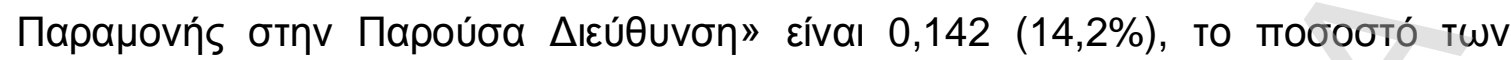

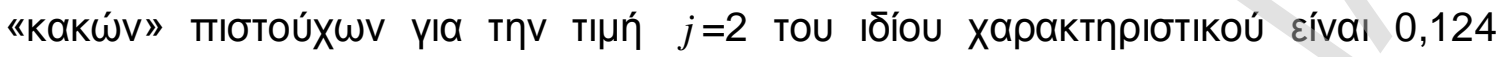

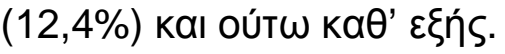

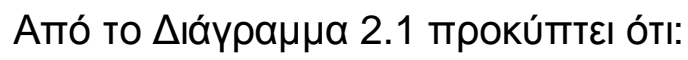

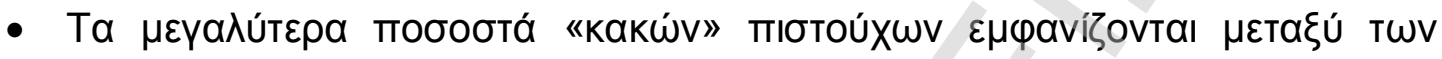

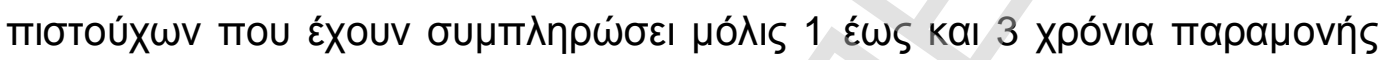

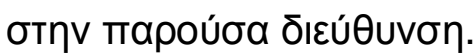

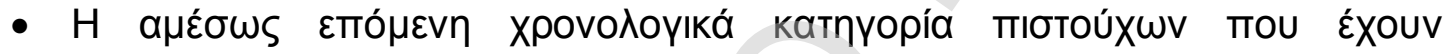

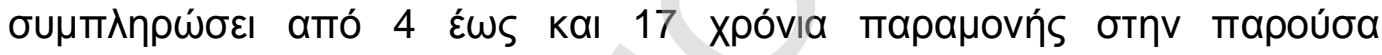

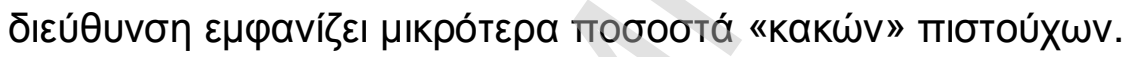

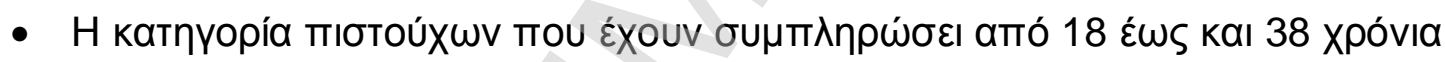

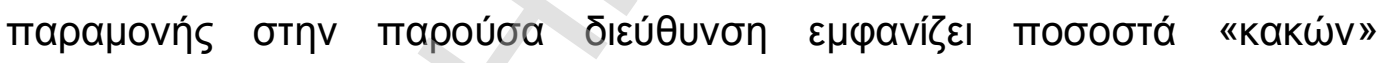

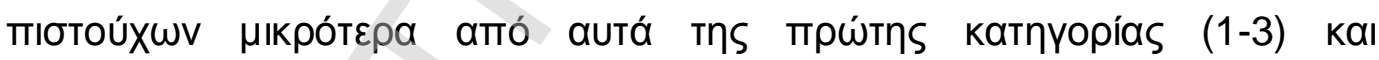

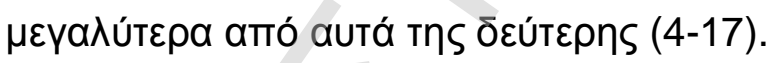

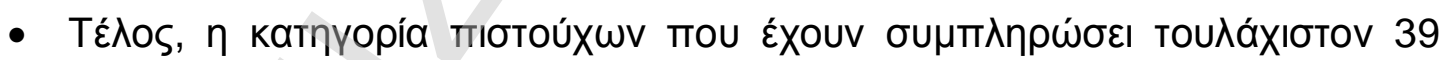

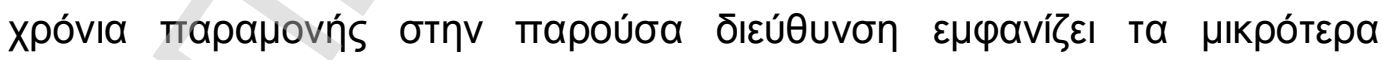

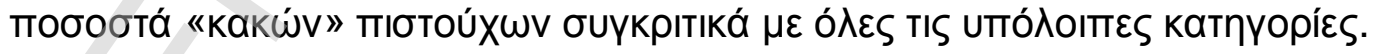

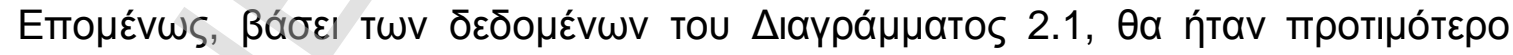

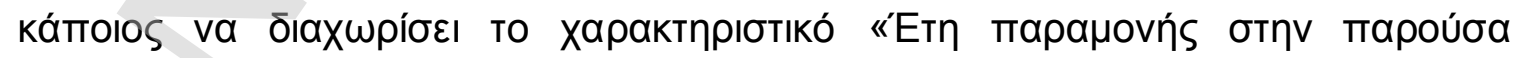

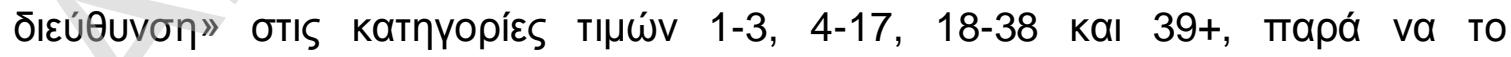

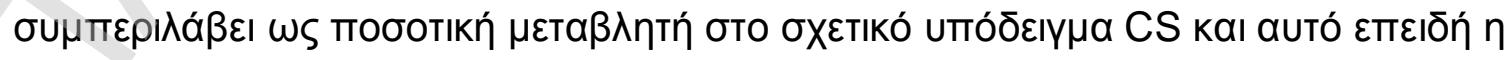




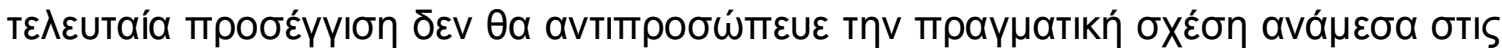

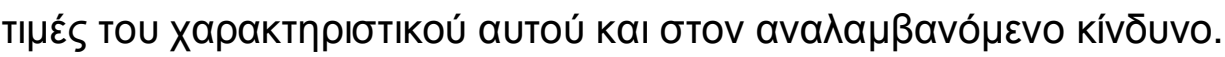

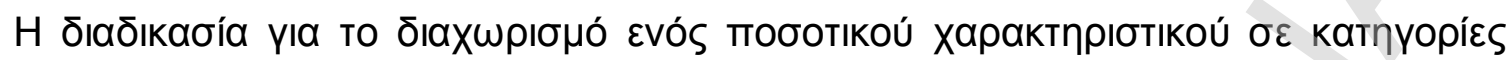

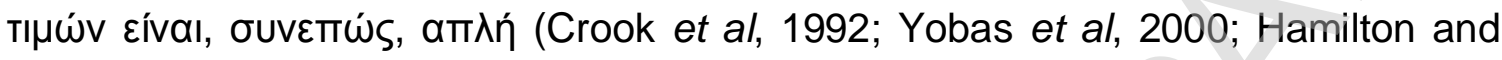

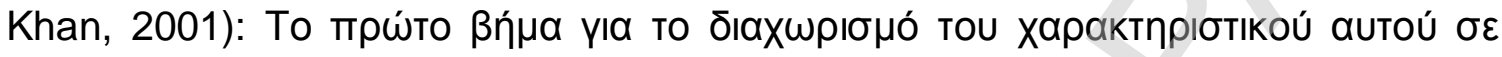

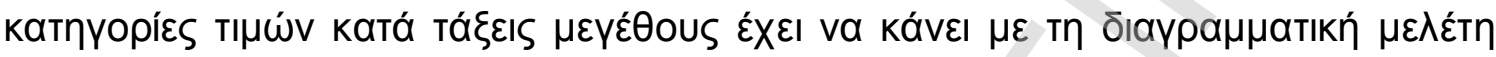

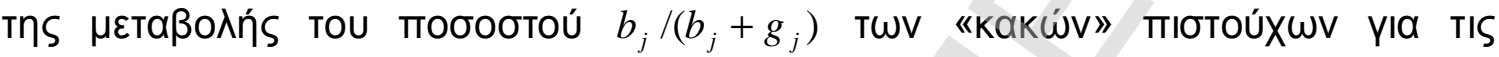

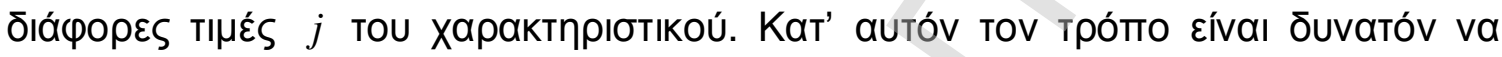

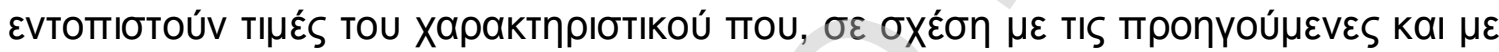

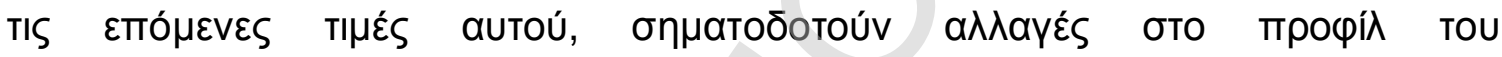

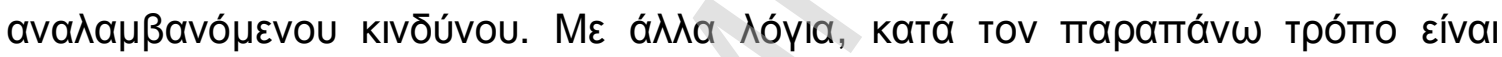

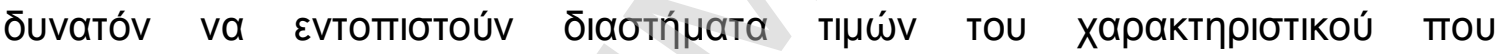

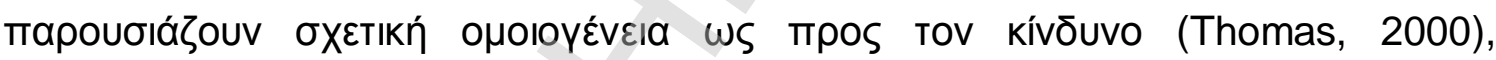

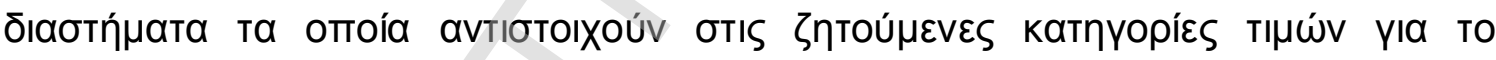

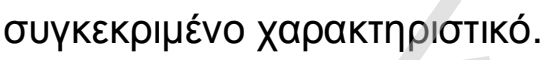

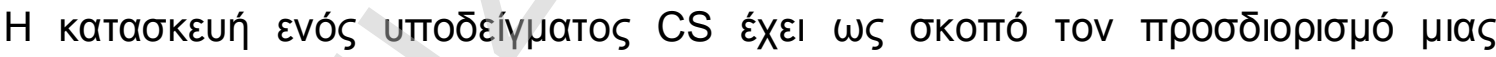

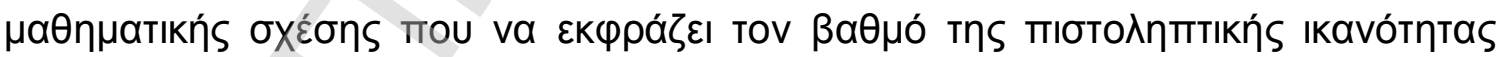

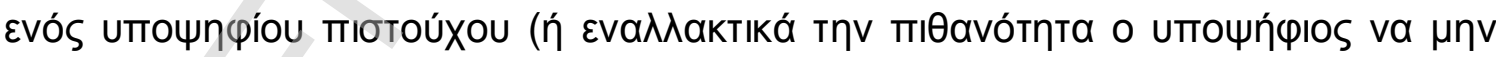

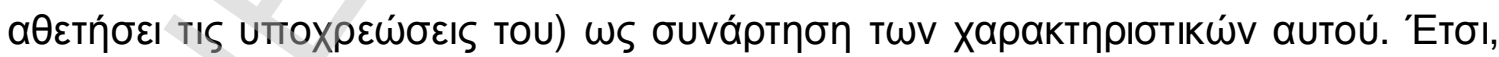

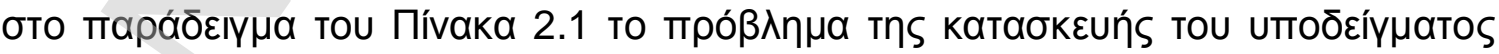

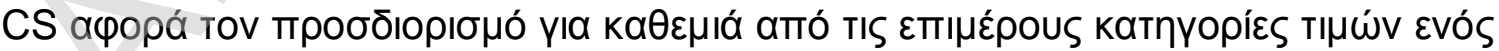

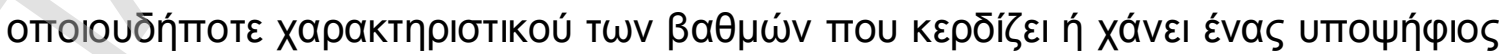

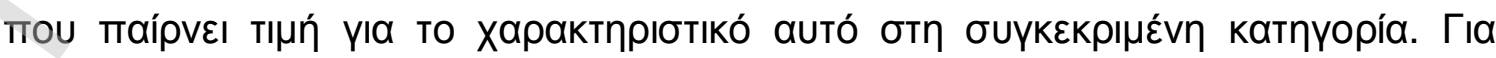




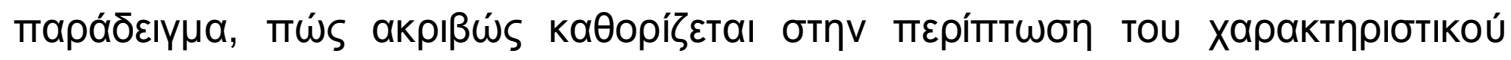

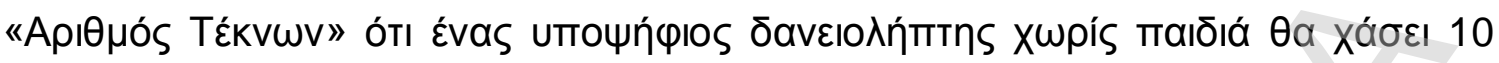

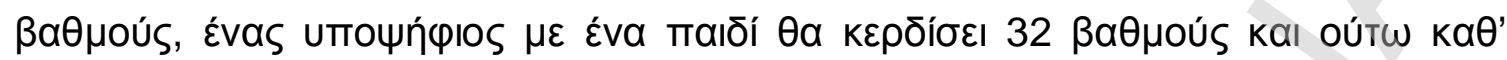

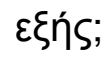

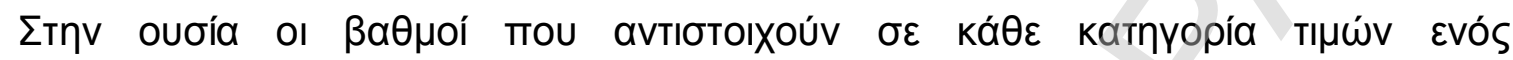

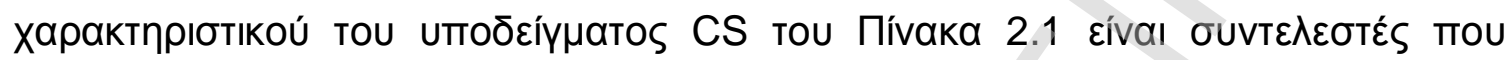

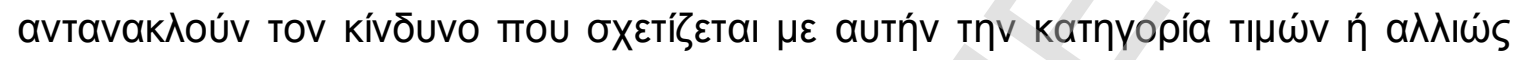

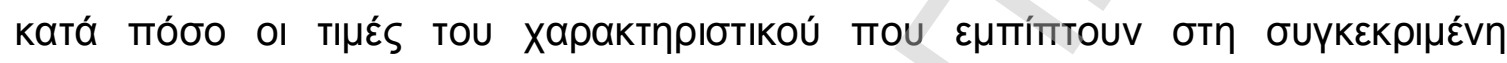

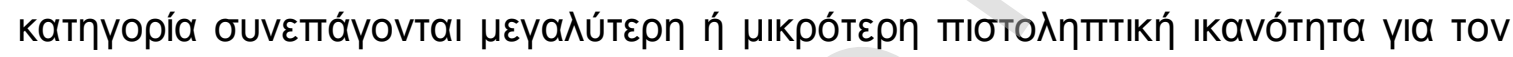

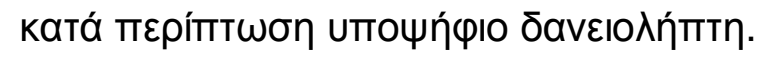

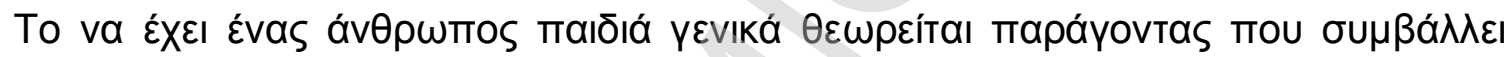

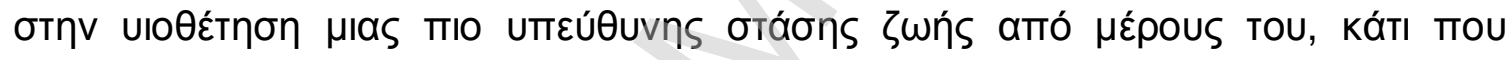

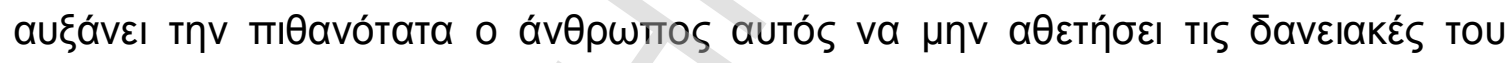

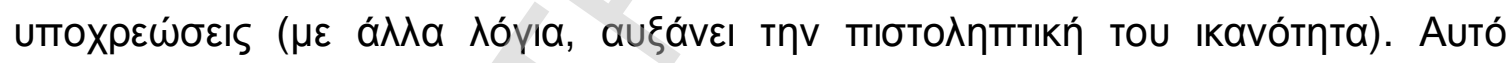

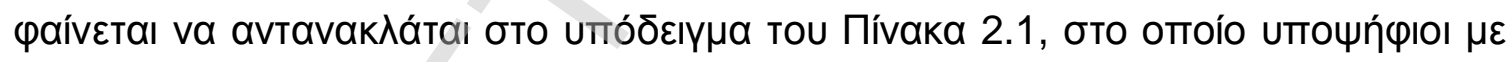

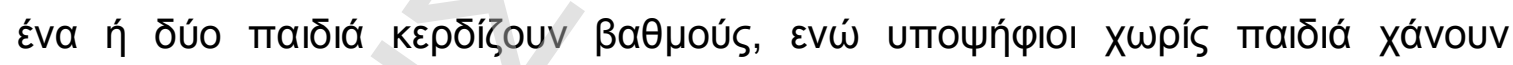

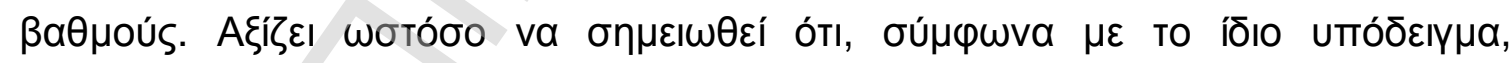

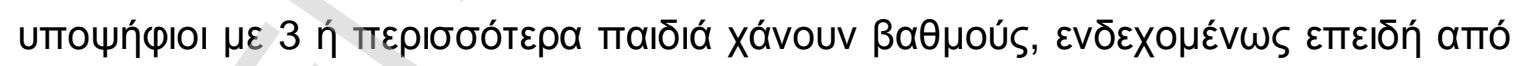

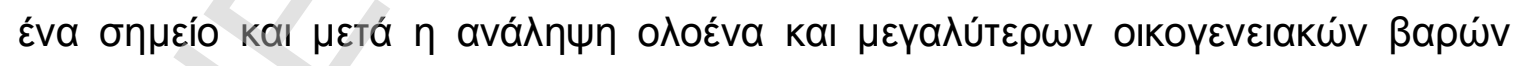

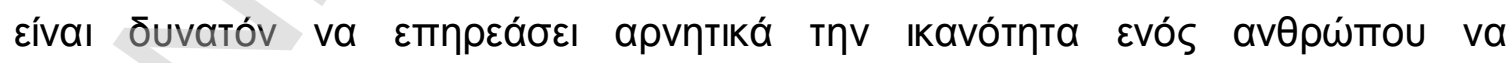

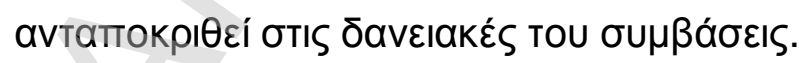

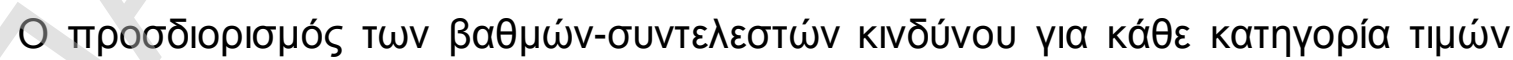

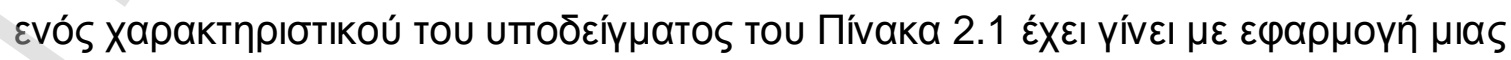




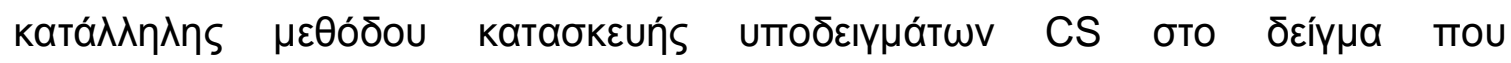

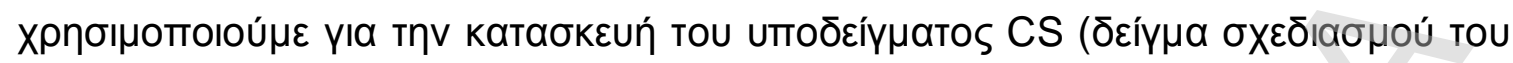

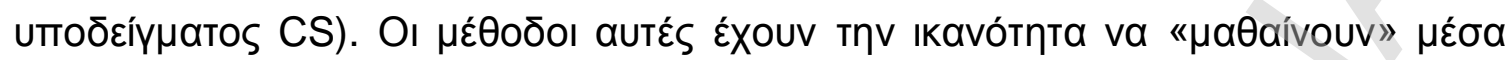

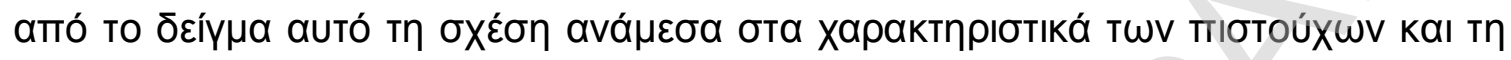

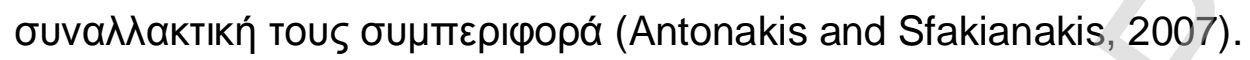

$\Delta \varepsilon \delta$

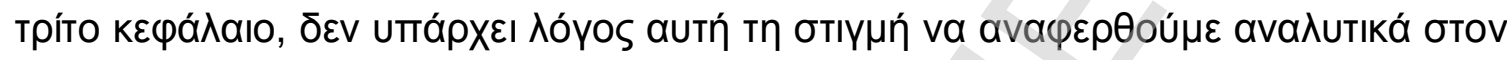

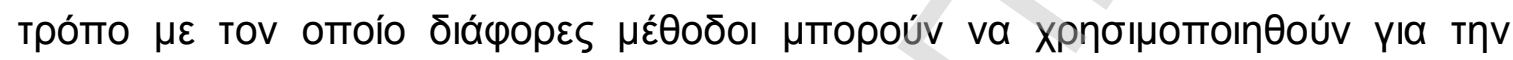

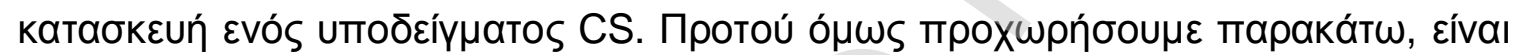

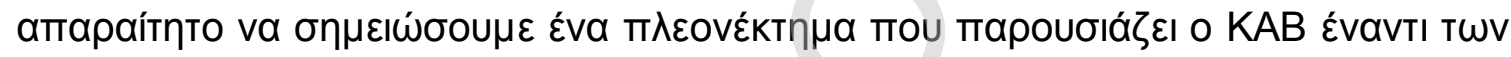

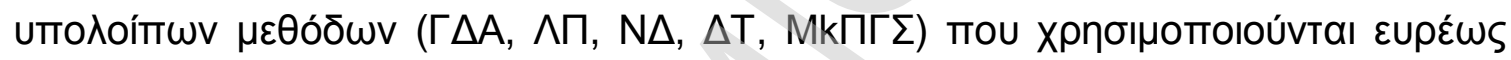

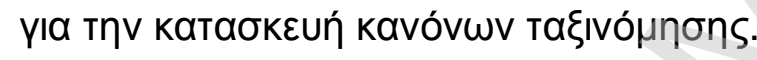

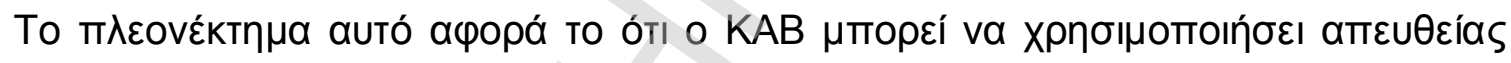

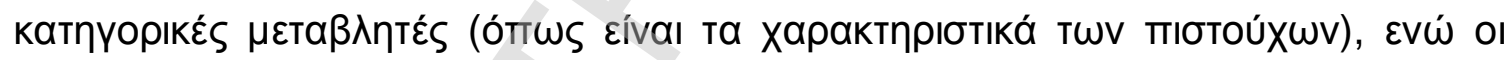

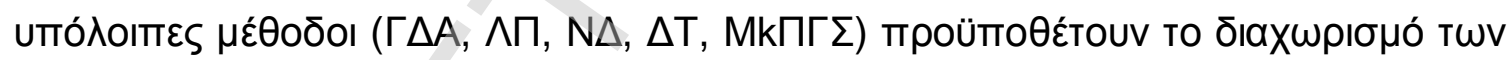

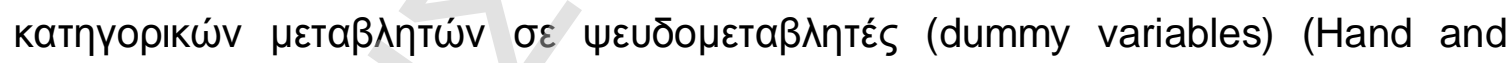

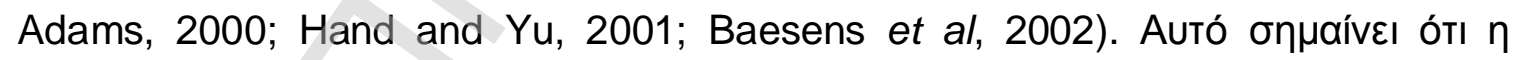

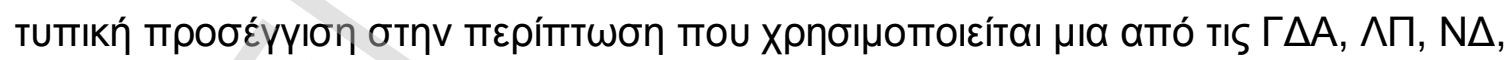

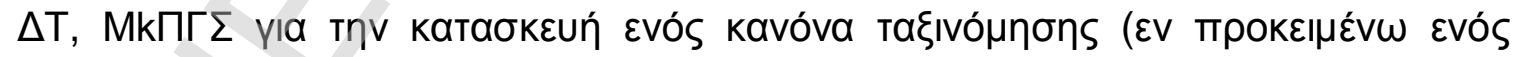

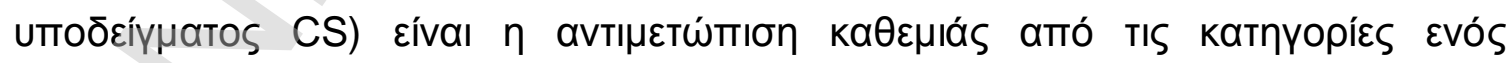

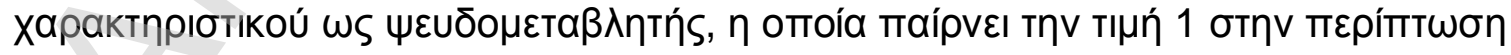

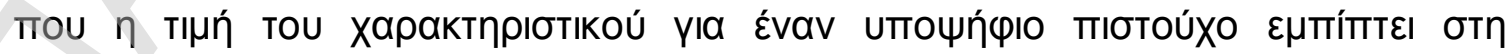




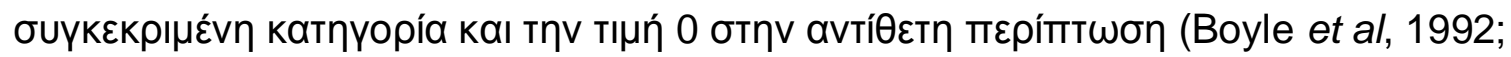
Hand and Henley, 1997; Thomas, 1998).

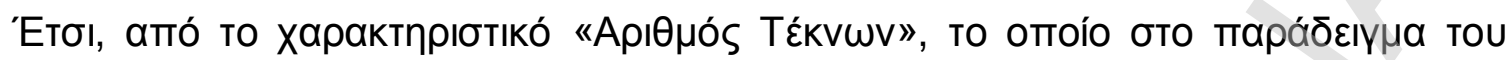

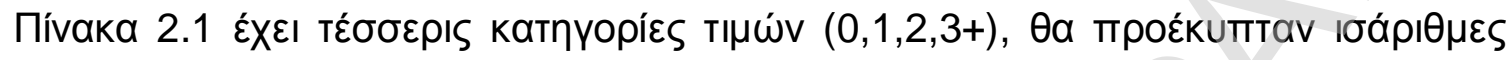

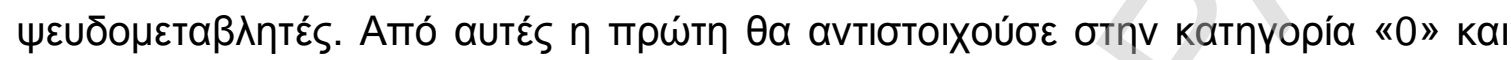

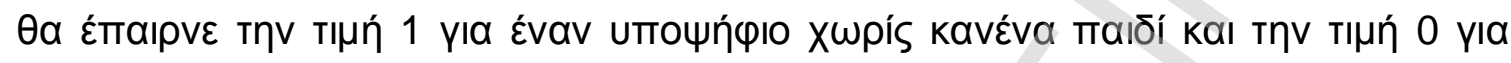

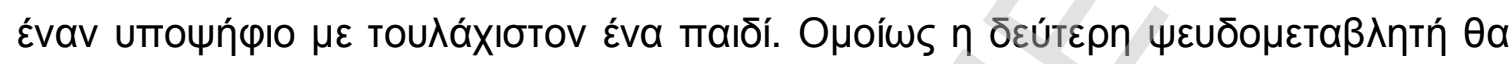

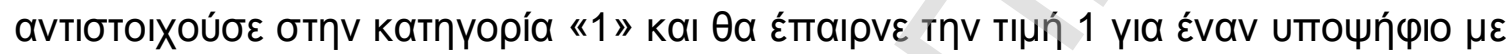

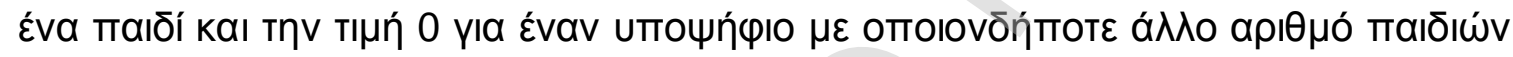

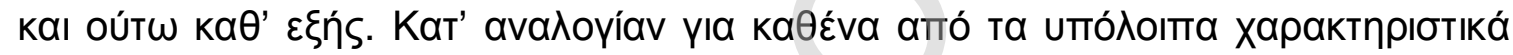

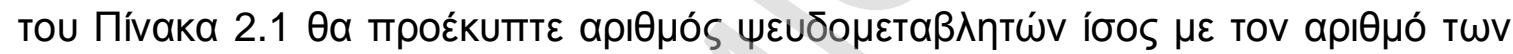

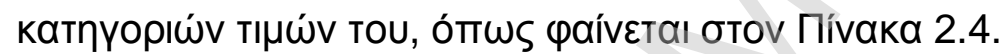

ПINAKA 2.4

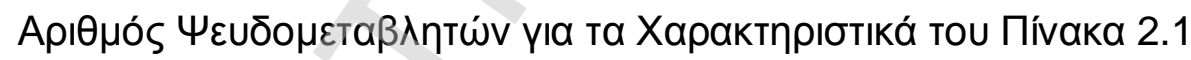

\begin{tabular}{|c|c|c|c|}
\hline ХарактпрІбтіко́ & 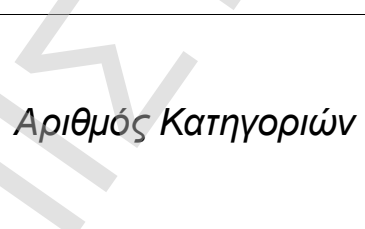 & 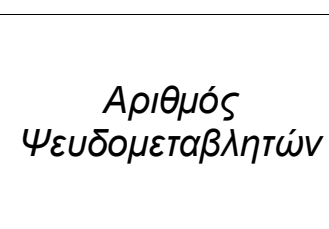 & 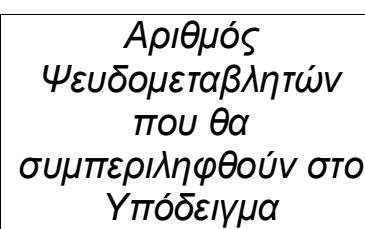 \\
\hline 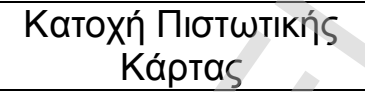 & 2 & 2 & $2-1=1$ \\
\hline 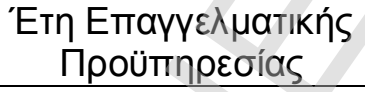 & 4 & 4 & $4-1=3$ \\
\hline 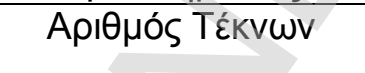 & 4 & 4 & $4-1=3$ \\
\hline
\end{tabular}

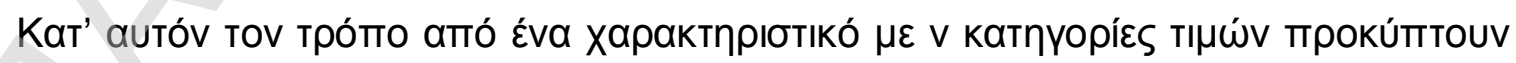

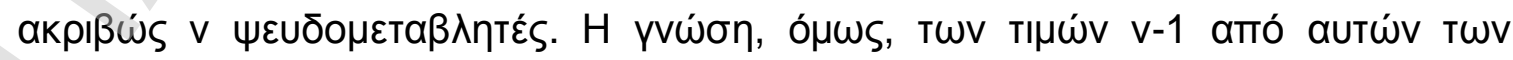

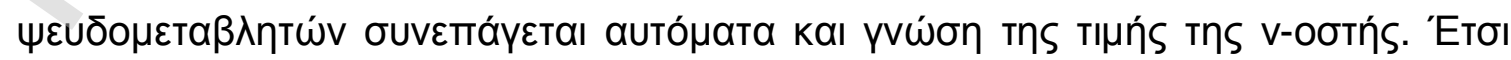




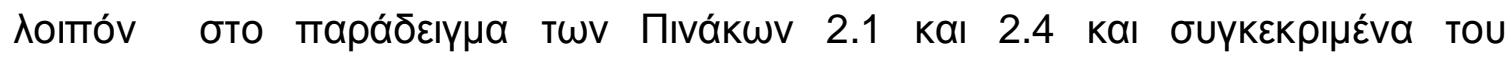

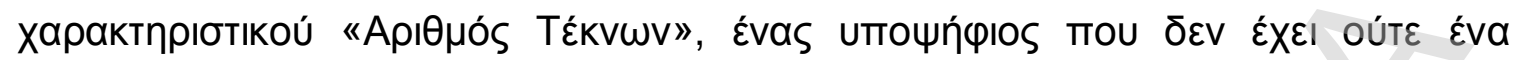

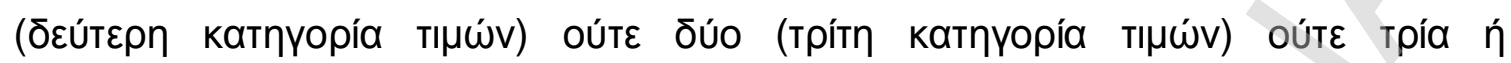

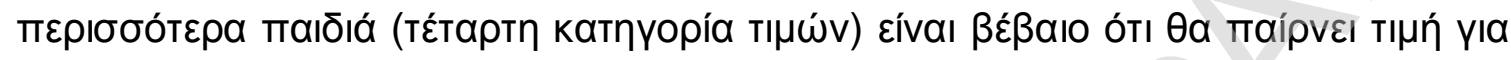

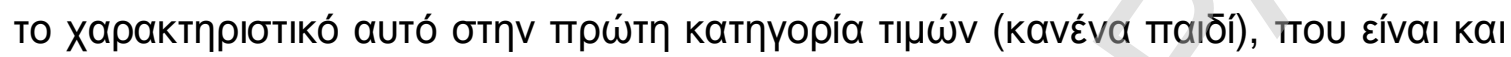

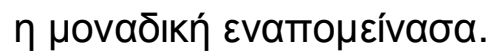

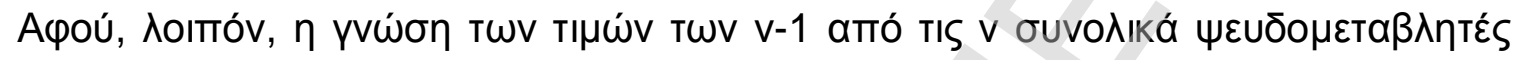

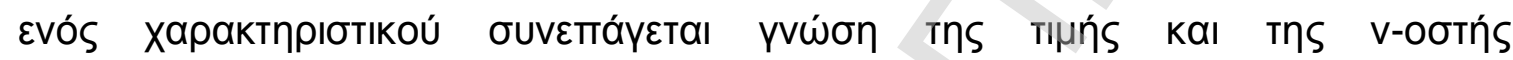

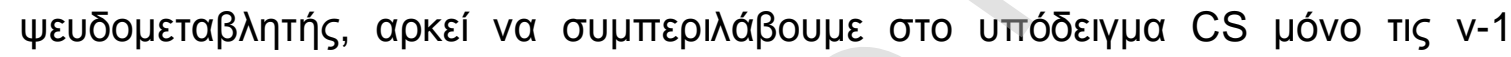

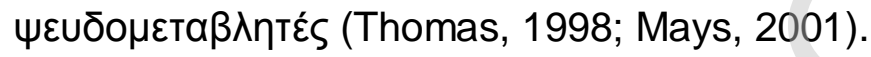

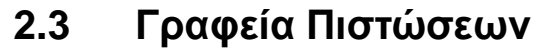

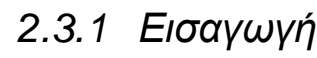

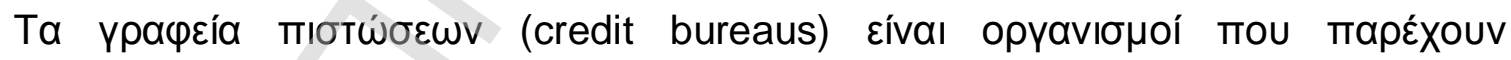

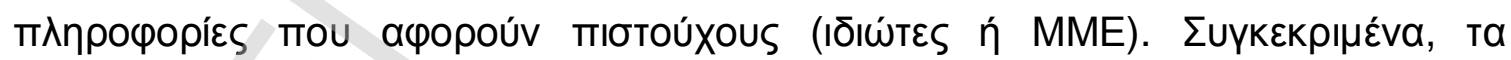

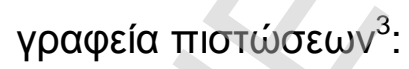

\footnotetext{
${ }^{3}$ International Finance Corporation (2006A). Credit Bureau Knowledge Guide. Washington, DC:
} World Bank Group. 


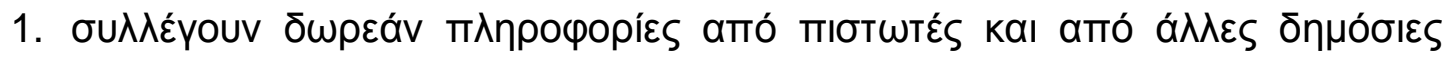

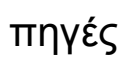

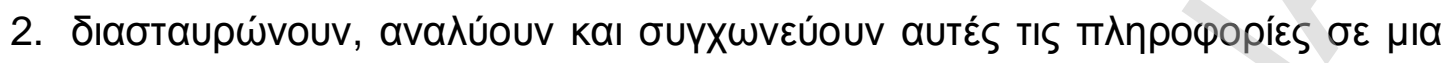

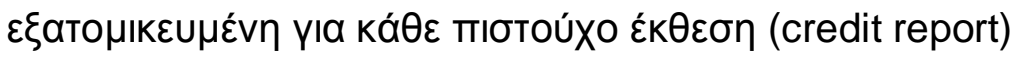

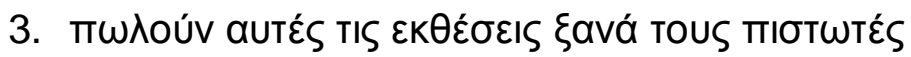

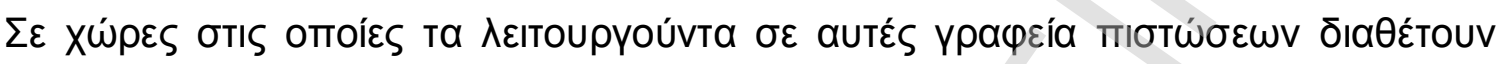

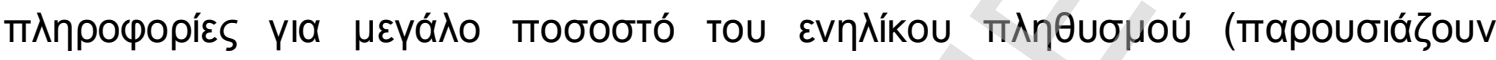

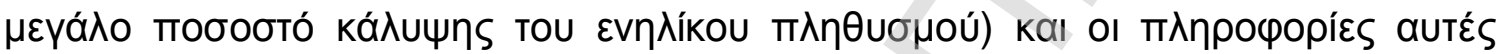

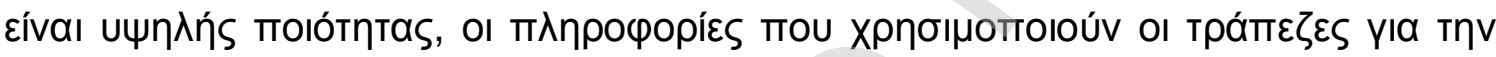

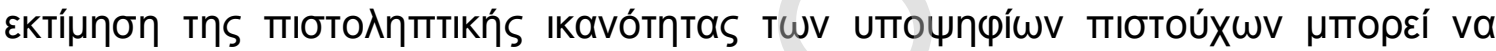

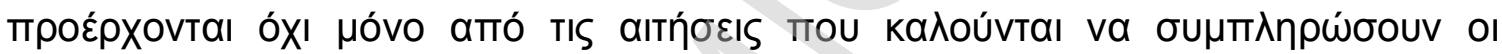

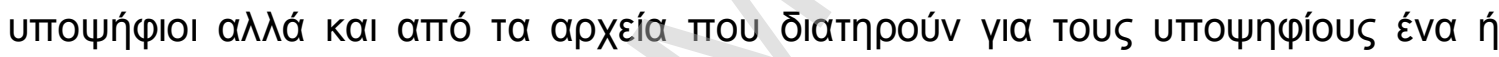

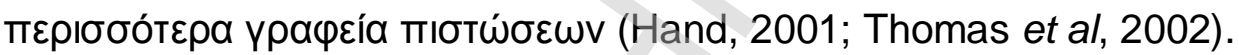

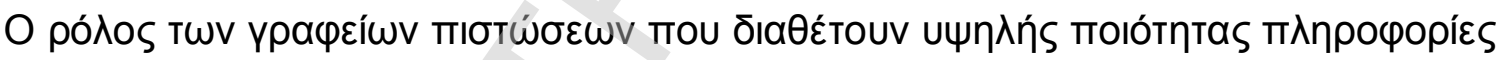

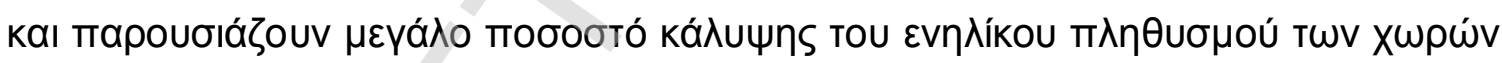

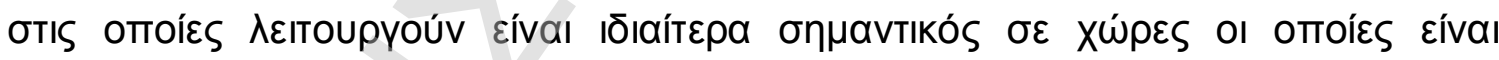

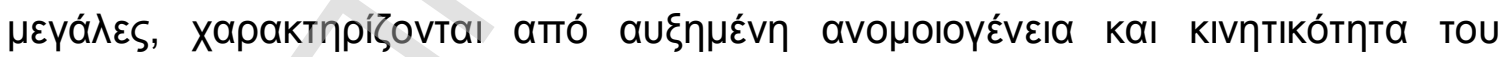

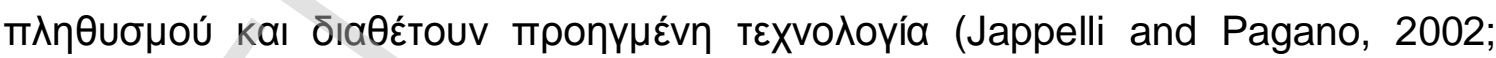
Miller, 2003).

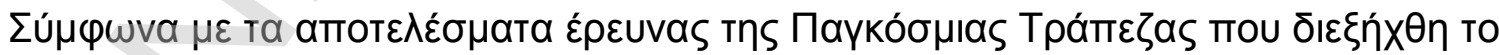

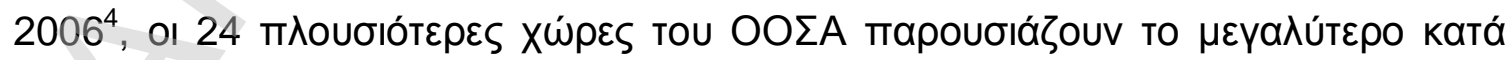

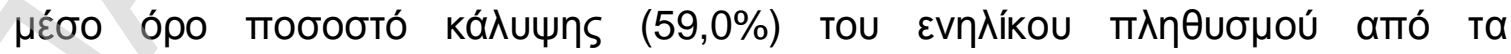

\footnotetext{
${ }^{4}$ International Finance Corporation (2006B). Doing Business in 2006. Washington, DC:,World Bank Group.
} 


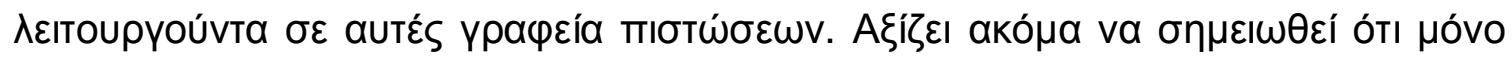

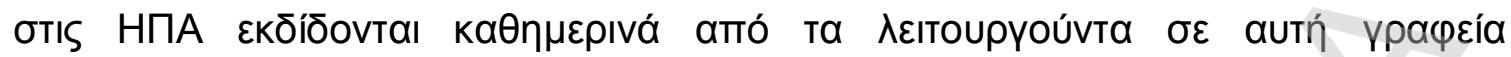

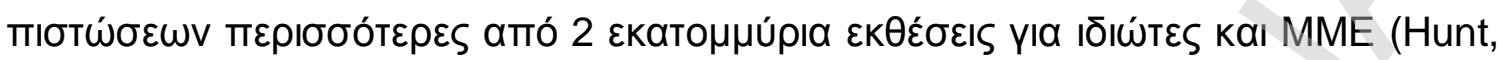
2003).

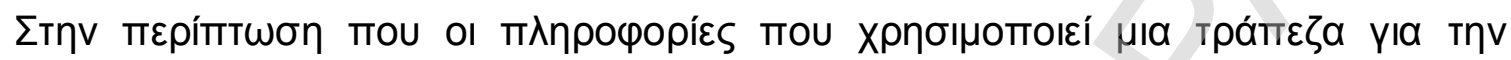

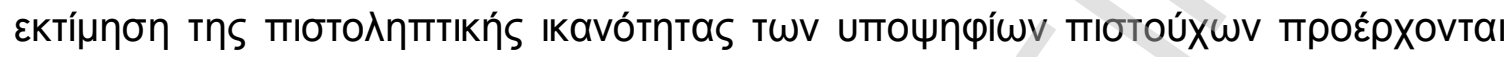

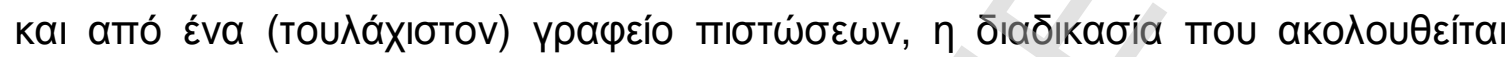

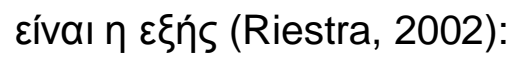

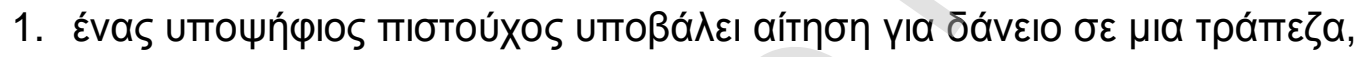

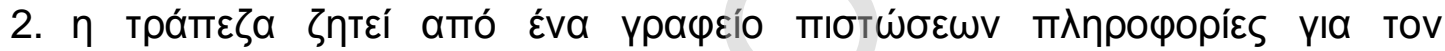

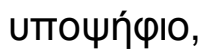

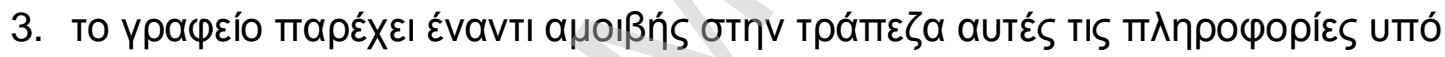

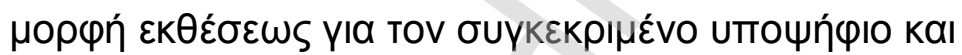

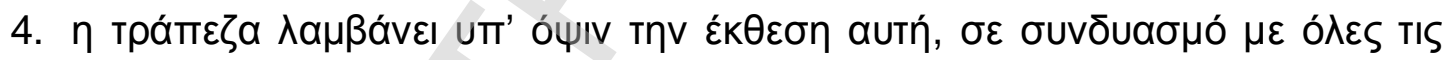

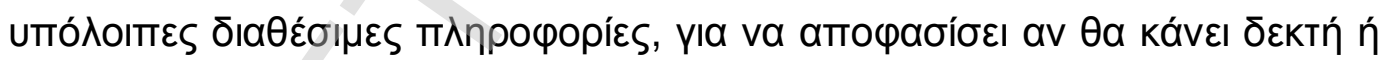

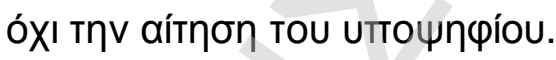

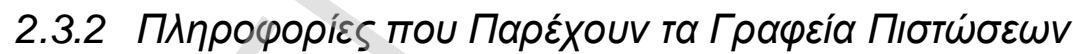

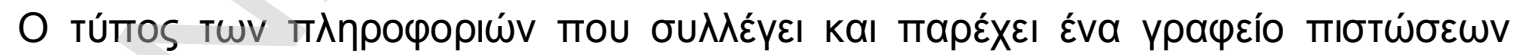

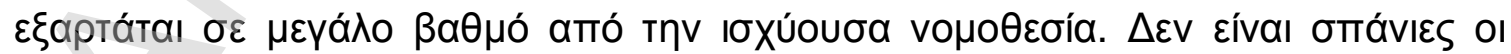

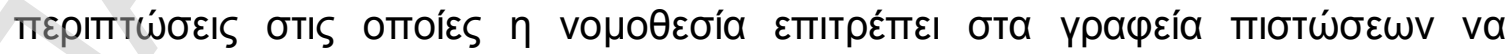

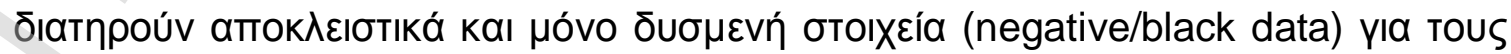




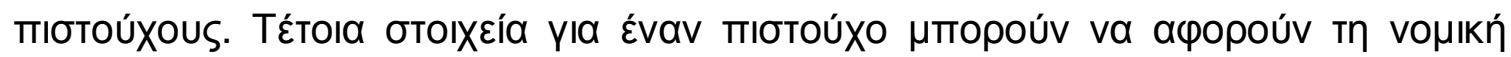

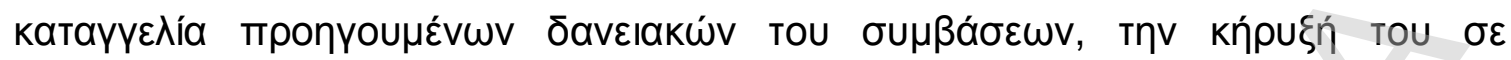

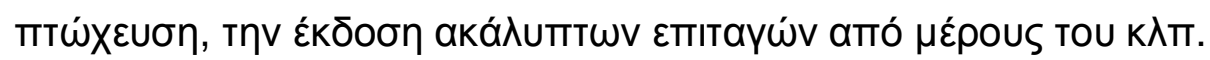

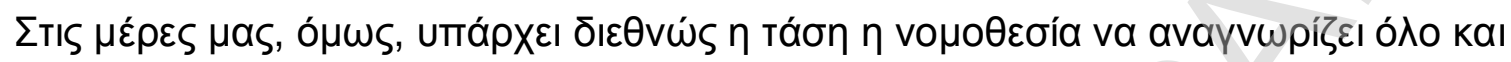

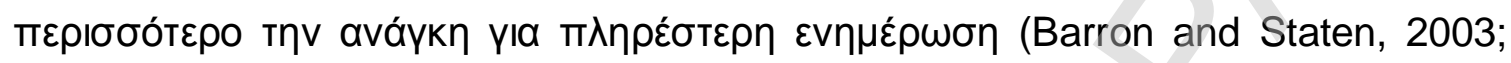

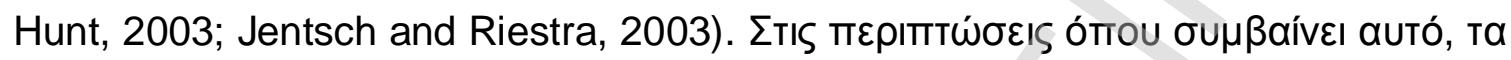

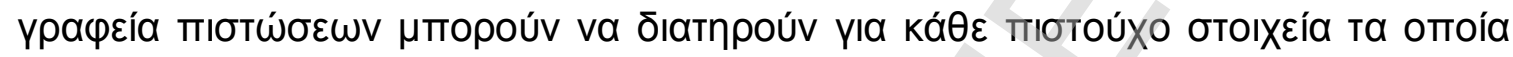

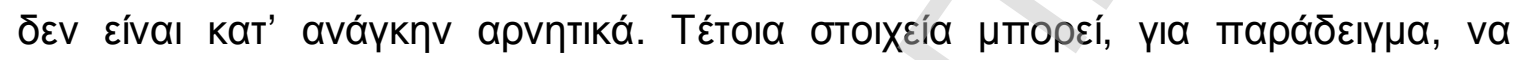

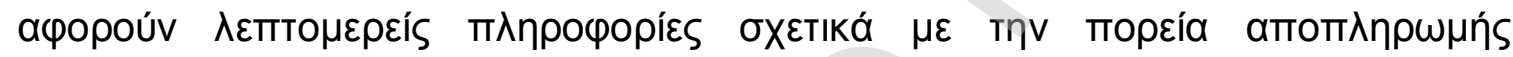

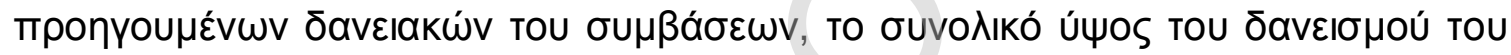

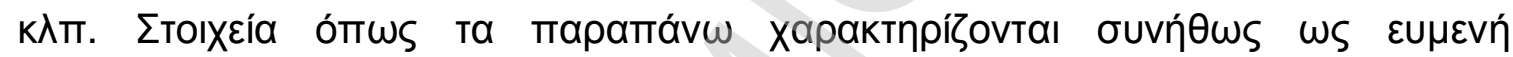
(positive/white data).

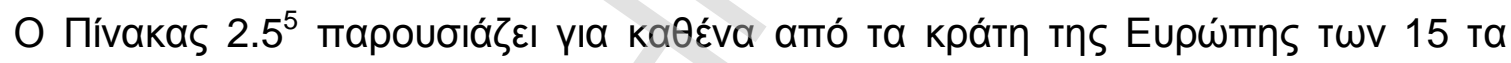

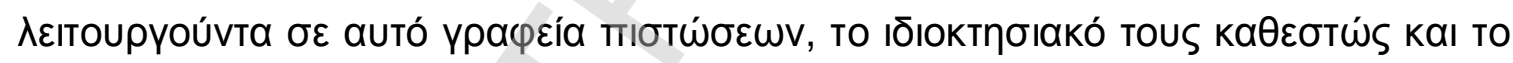

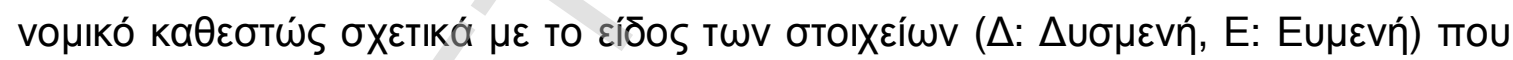

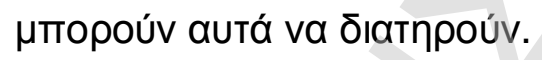

${ }^{5}$ Oxera Consulting Ltd. (2008). Accentuating the Positive: sharing financial data between banks. Agenda: Advancing Economics in Business, December, 1-4. 
ПINAKA $\Sigma 2.5$

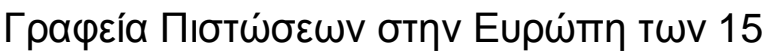

\begin{tabular}{|c|c|c|c|}
\hline KمáTOS & 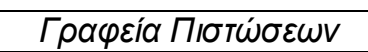 & 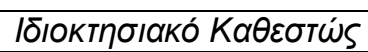 & 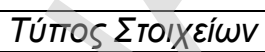 \\
\hline Аибтрі́a & KSV & 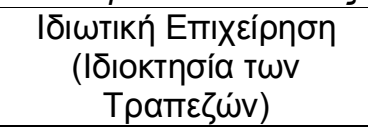 & $\Delta+E$ \\
\hline 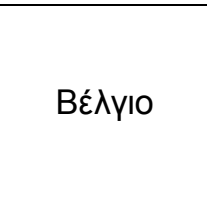 & NBB, UPC & 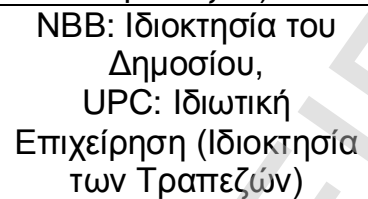 & $\Delta+E$ \\
\hline 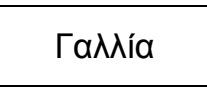 & 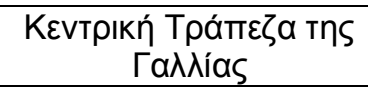 & 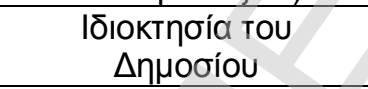 & $\Delta$ \\
\hline 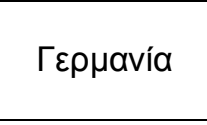 & Schufa Holding & 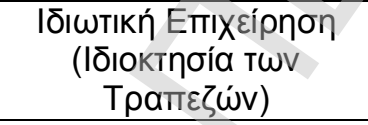 & $\Delta+\mathrm{E}$ \\
\hline$\Delta$ avía & RKI & 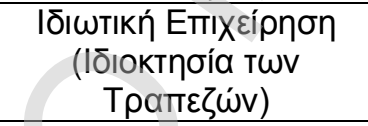 & $\Delta$ \\
\hline 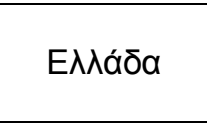 & Tદıрદoías & 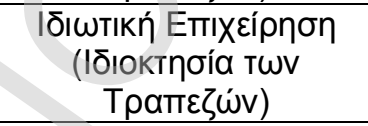 & $\Delta+\mathrm{E}$ \\
\hline I $\rho \lambda \alpha v \delta i ́ a$ & Irish Credit Bureau & 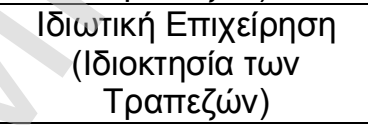 & $\Delta+\mathrm{E}$ \\
\hline lomavía & 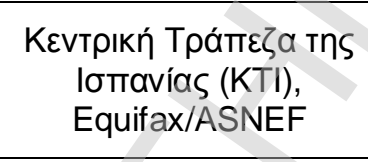 & 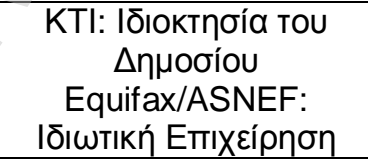 & $\Delta+\mathrm{E}$ \\
\hline Iтa入ía & CTC, CRIF & 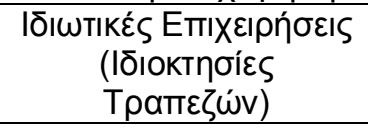 & $\begin{array}{l}\text { CTC: } \Delta, \\
\text { CRIF: } \Delta+E\end{array}$ \\
\hline M. Bрєтаvía & $\begin{array}{l}\text { Equifax, Experian, Call } \\
\text { Credit }\end{array}$ & 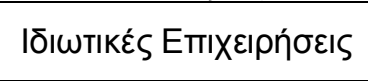 & $\Delta+\mathrm{E}$ \\
\hline Oגরavסía & $\begin{array}{c}\text { Bureau Krediet } \\
\text { Registratie }\end{array}$ & 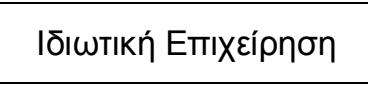 & $\Delta+\mathrm{E}$ \\
\hline Портоуаגía & 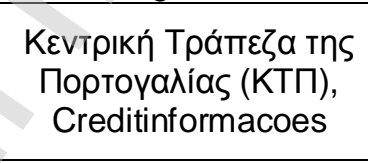 & 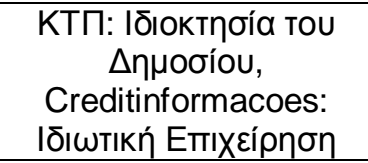 & $\Delta+\mathrm{E}$ \\
\hline Eounōía & UC AB & 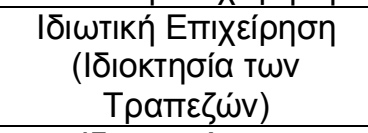 & $\Delta+\mathrm{E}$ \\
\hline ФIv^avסía & Suomen Asiakastieto & 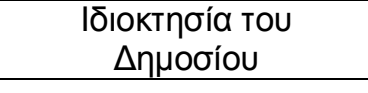 & $\Delta$ \\
\hline
\end{tabular}




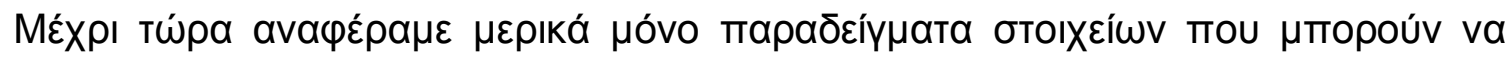

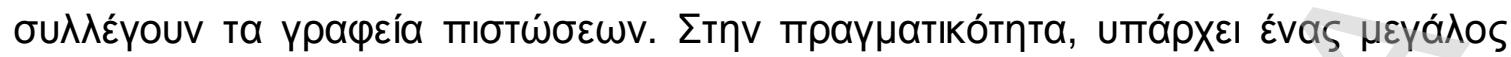

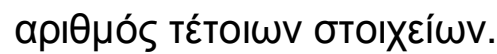

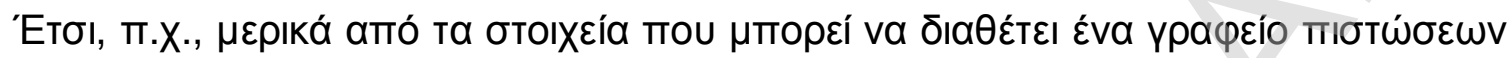

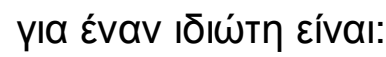

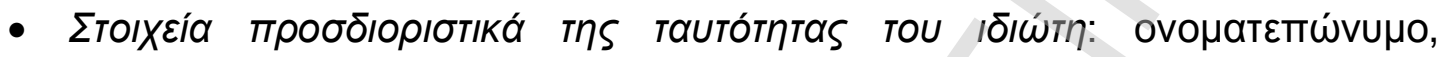

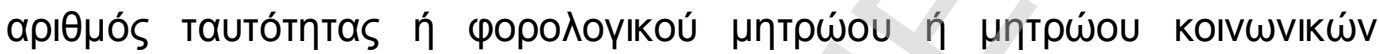

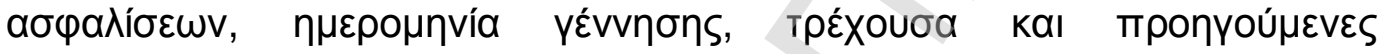

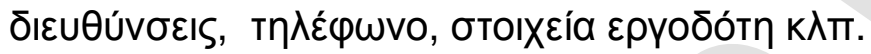

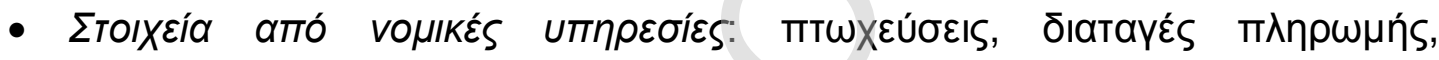

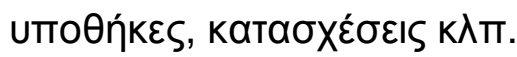

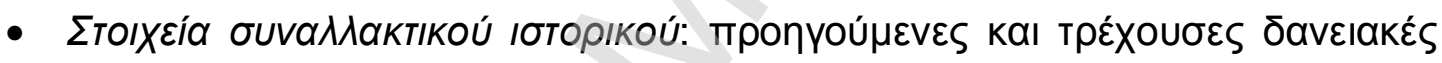

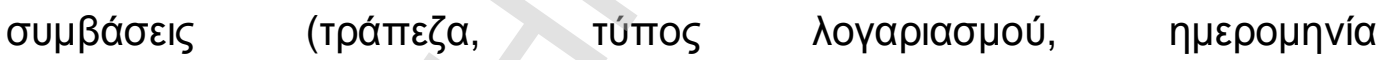

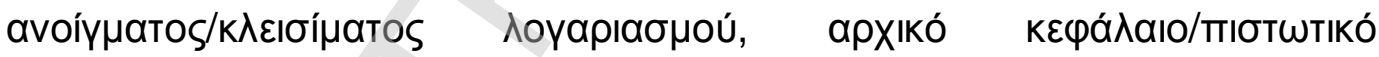

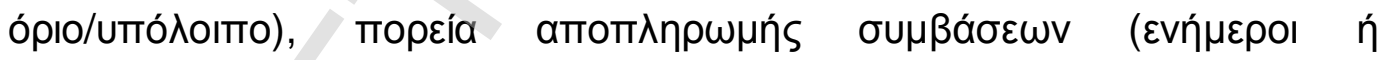

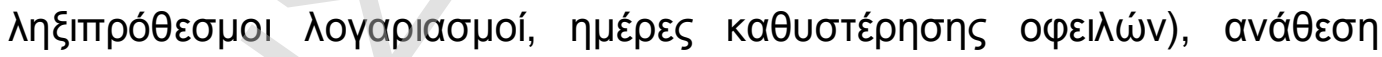

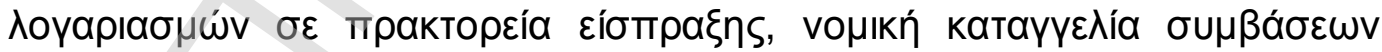
$\kappa \lambda \pi$.

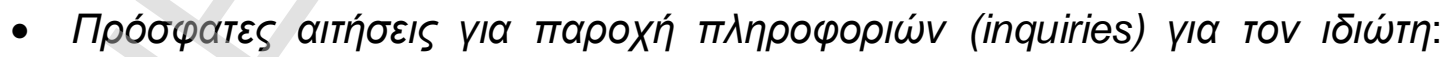

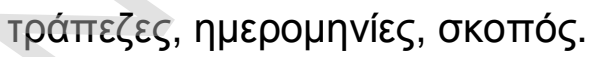




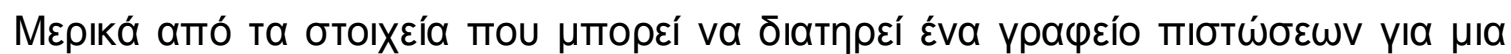
MME عíval:

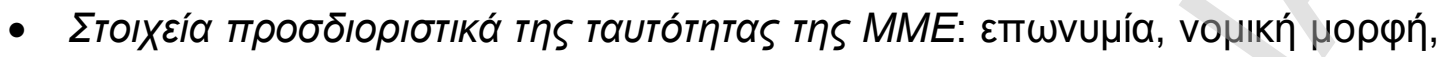

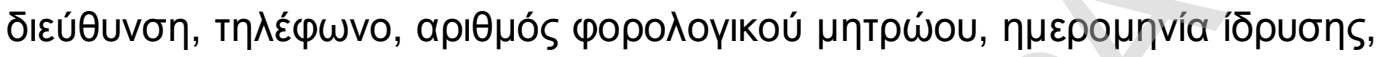

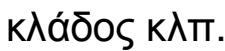

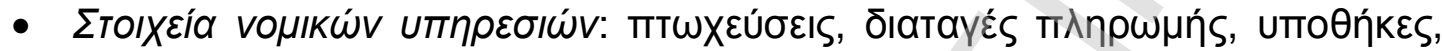

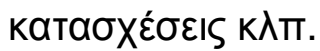

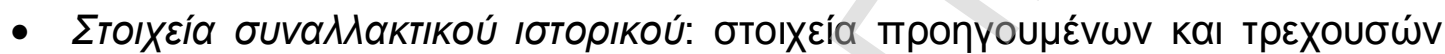

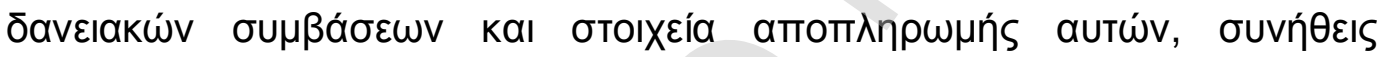

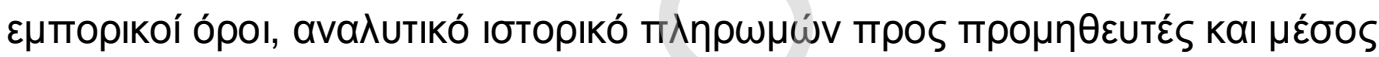

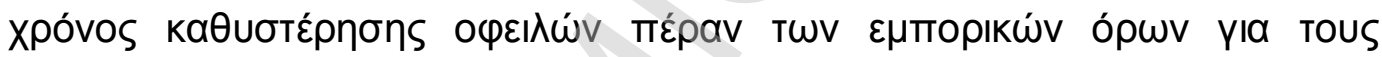

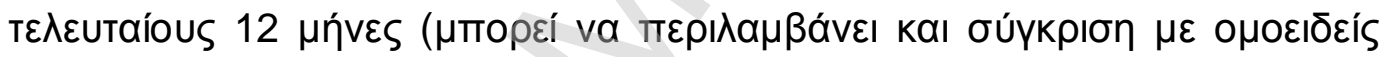

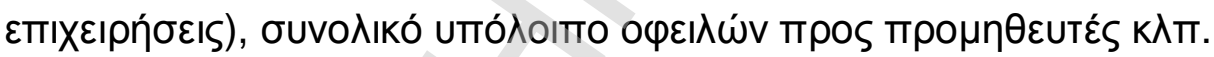

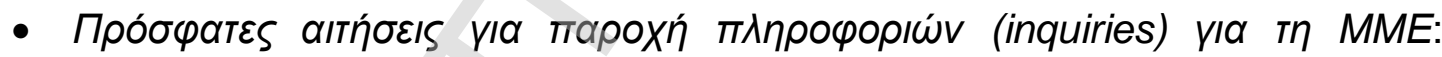

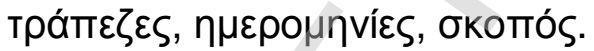

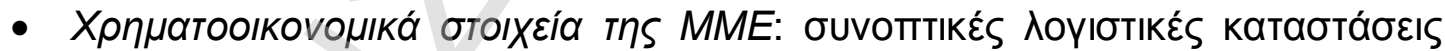

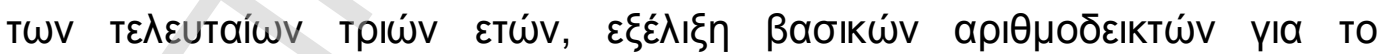

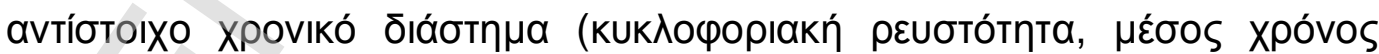

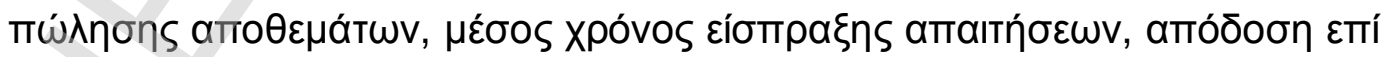

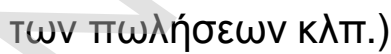

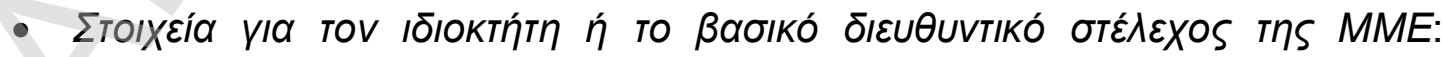

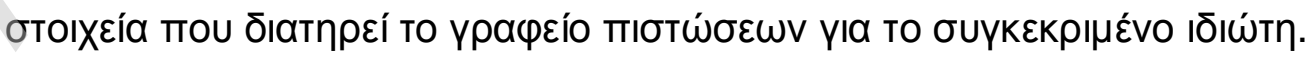




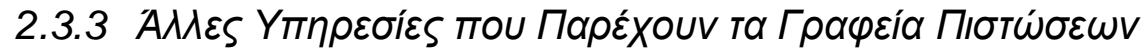

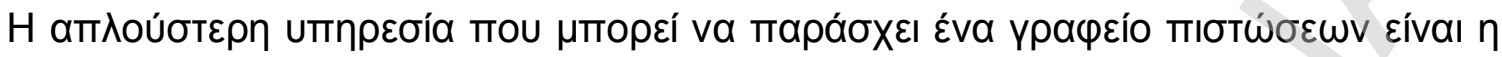

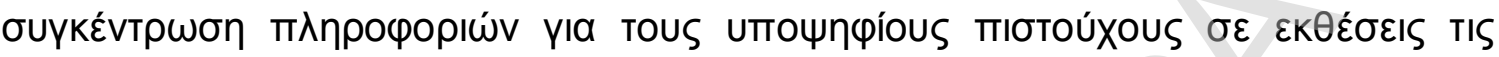

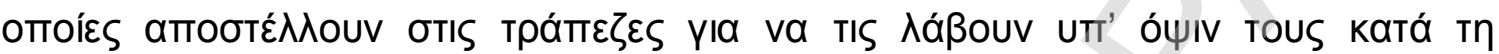

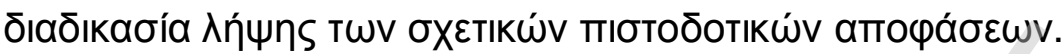

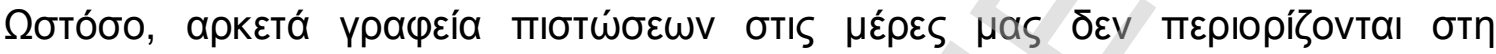

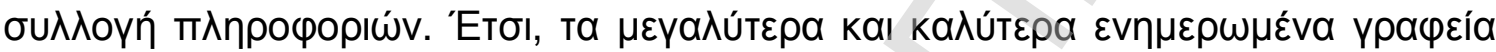

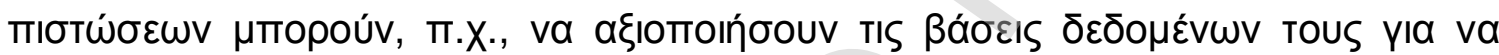

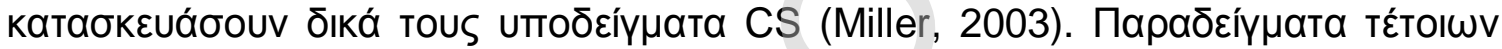

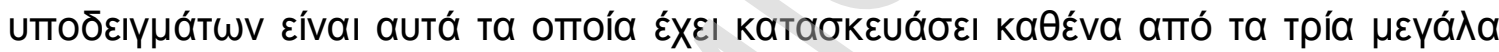

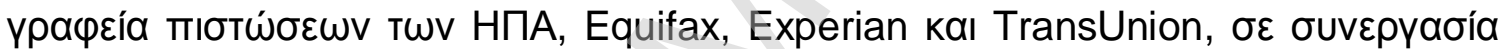

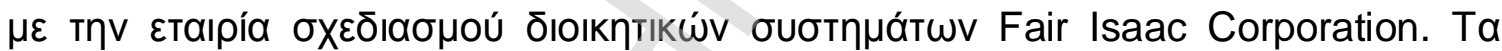

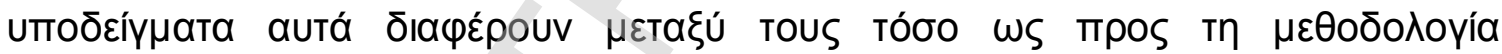

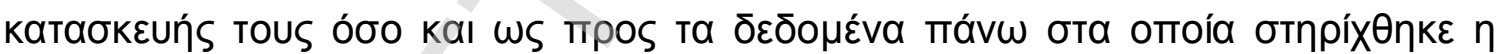

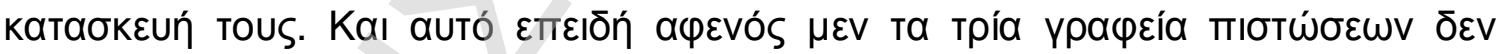

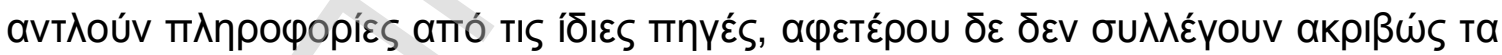

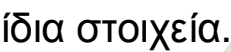

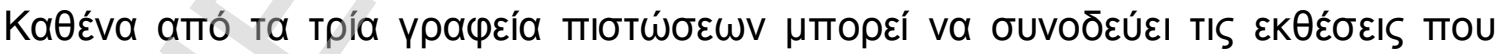

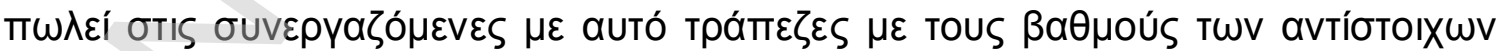

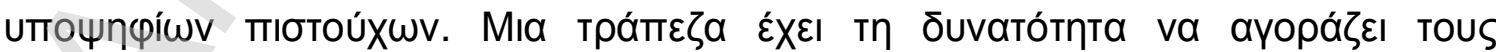

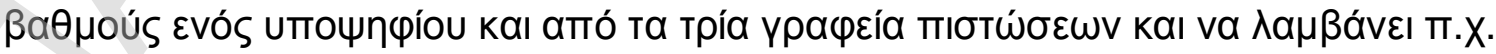




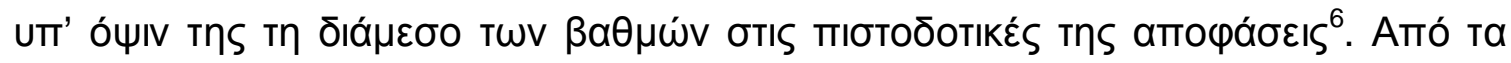

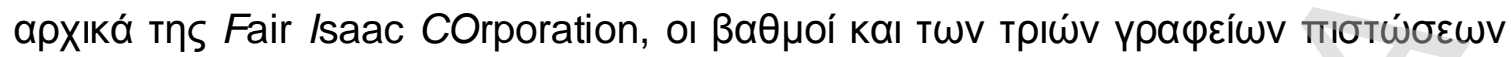

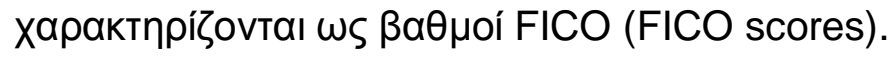

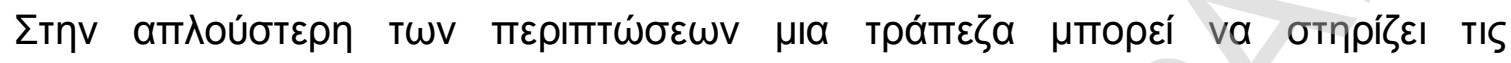

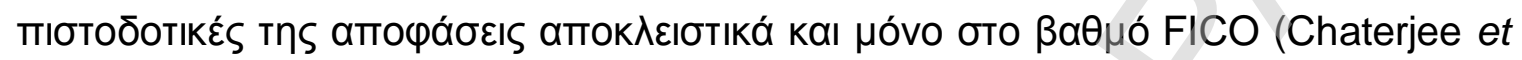

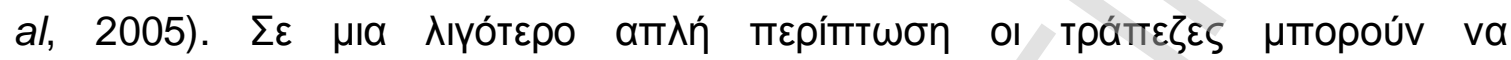

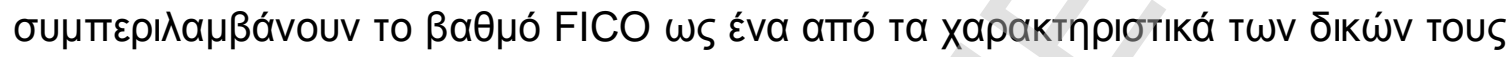

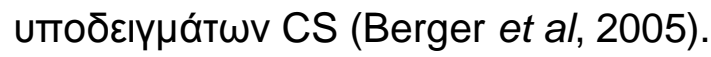

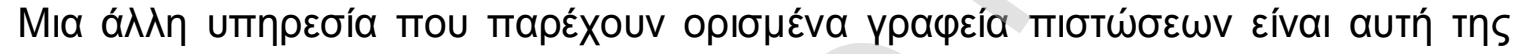

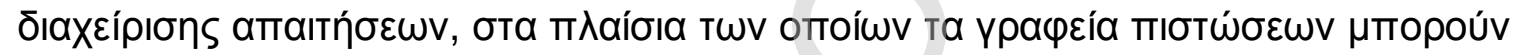

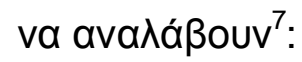

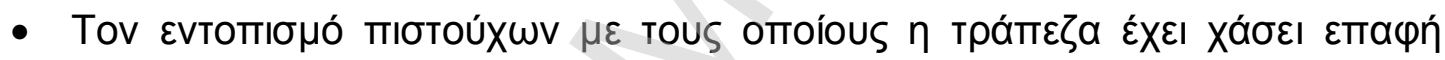

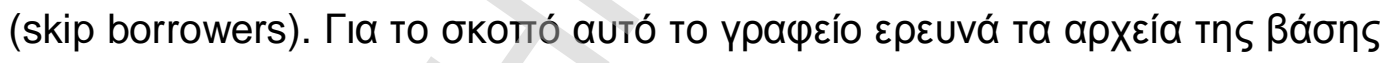

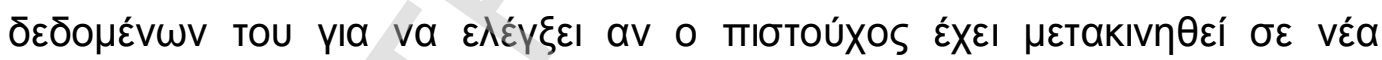

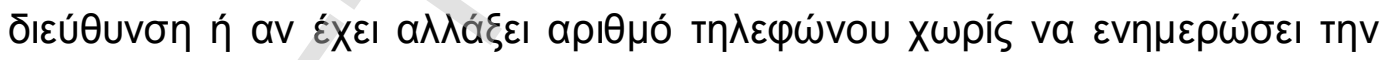

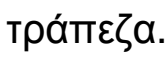

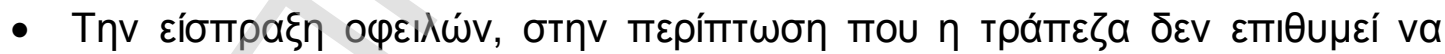

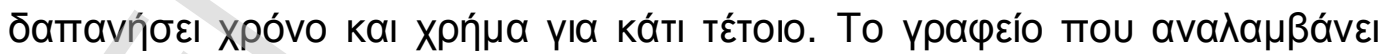

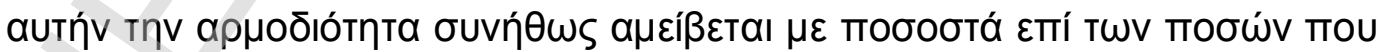

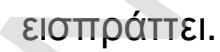

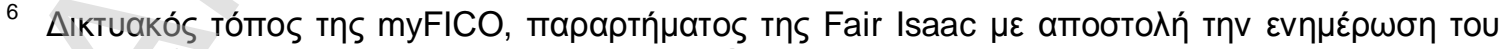

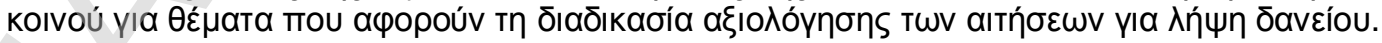

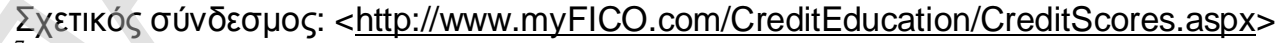

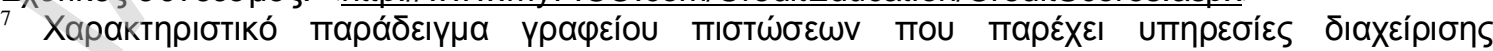

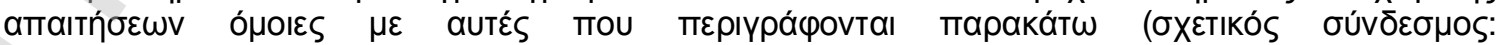
$<$ http://www.baycorp.com.au/services-for-customers/services-for-customers.aspx >) cival to Baycorp

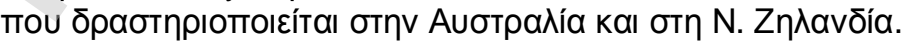




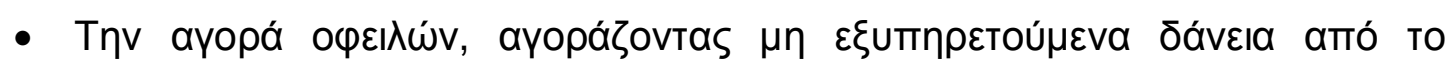

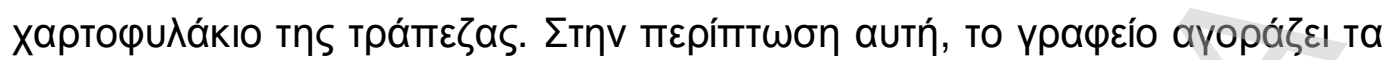

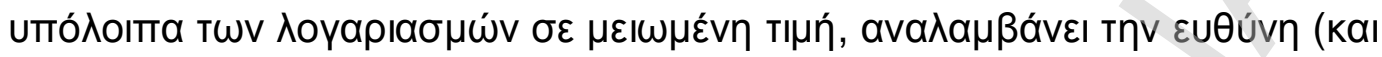

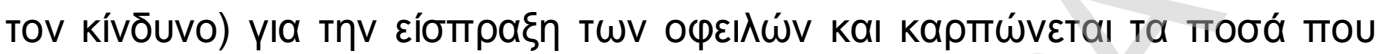

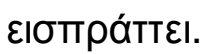

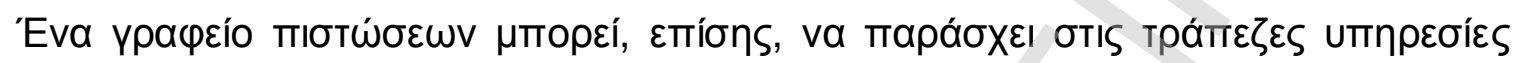

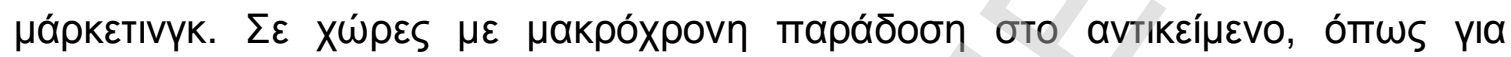

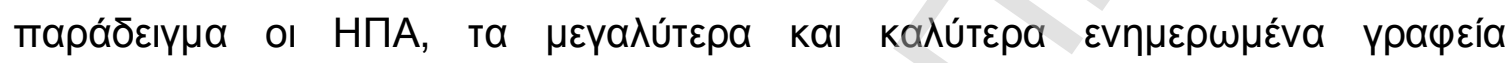

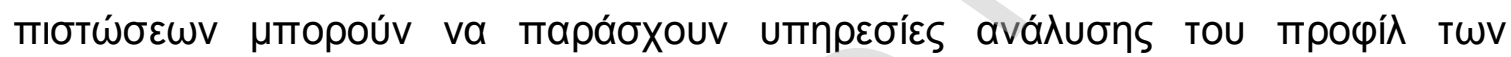

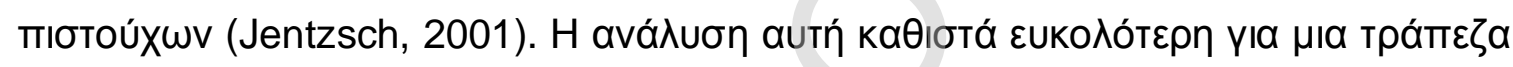

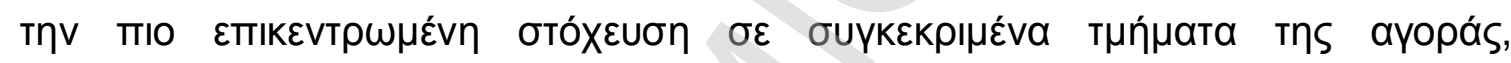

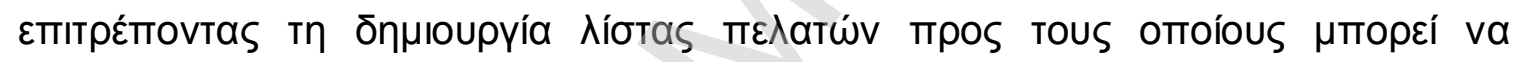

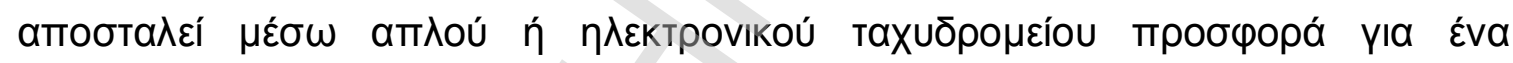

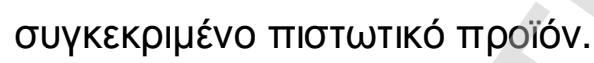

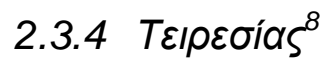

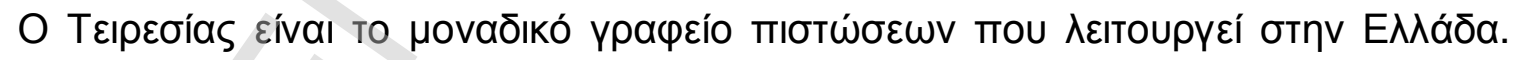

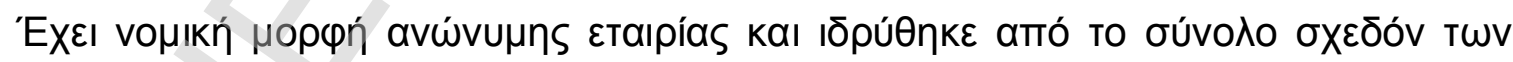

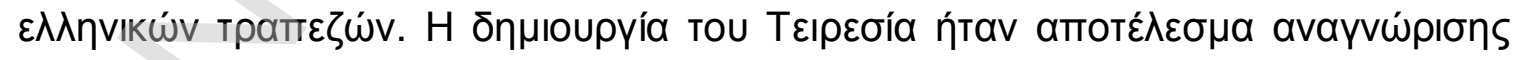

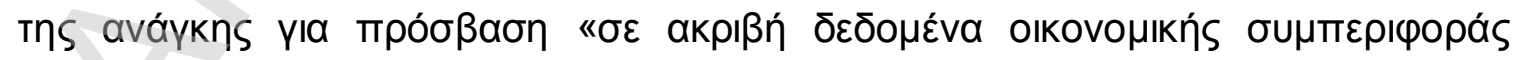

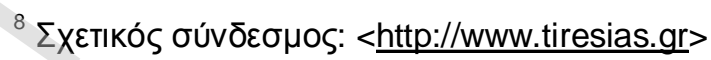




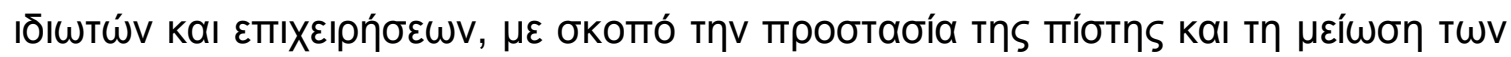
$\varepsilon \pi ı \varphi \alpha \lambda \varepsilon \mid \omega ́ \mathrm{~V}$.

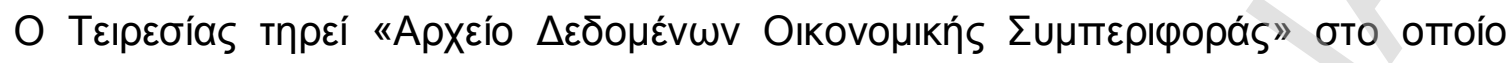

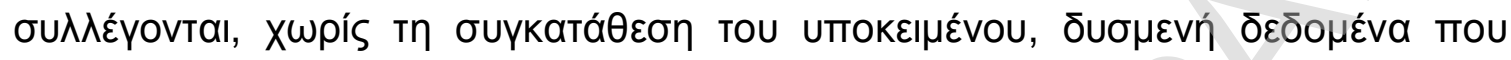

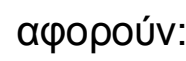

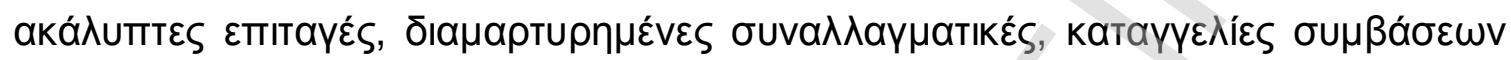

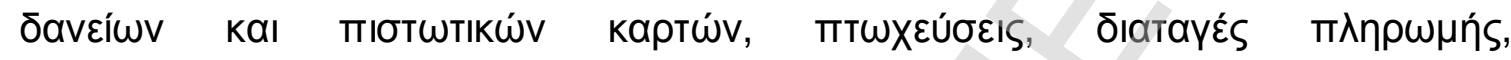

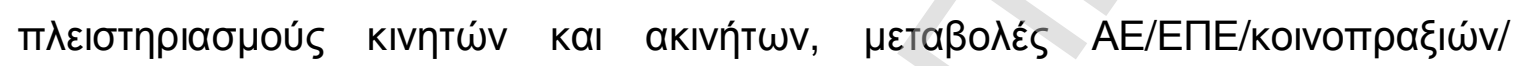

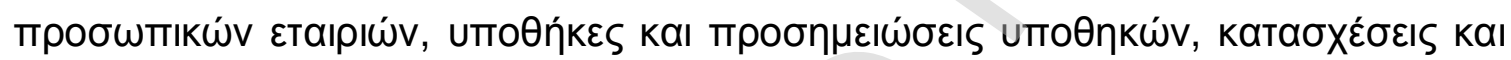

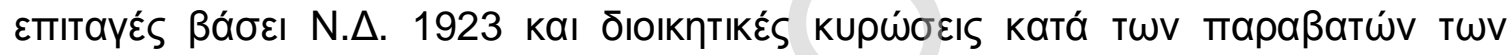

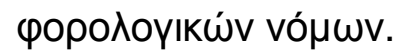

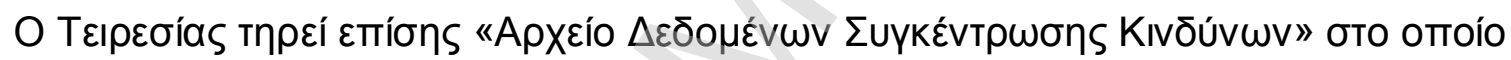

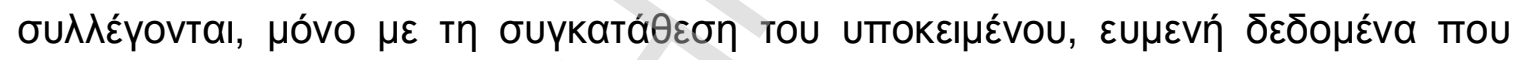

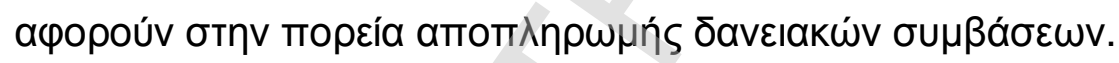

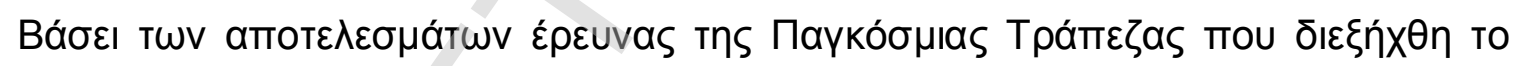

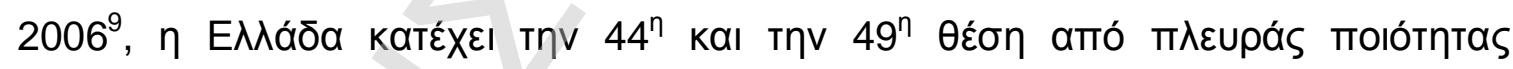

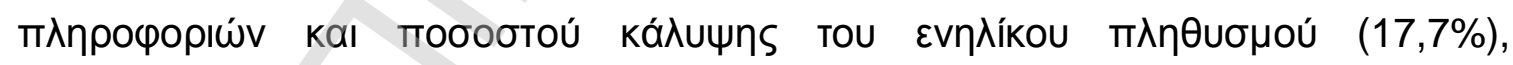

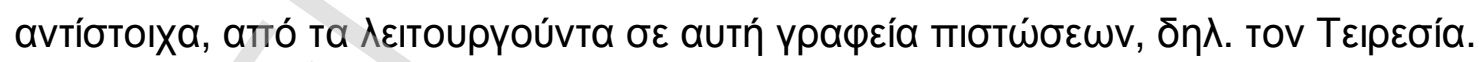

${ }_{9}^{9}$ International Finance Corporation (2006B). Doing Business in 2006. Washington, DC:,World Bank Group. 


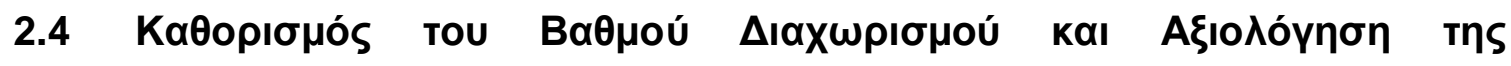

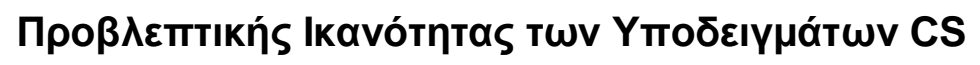

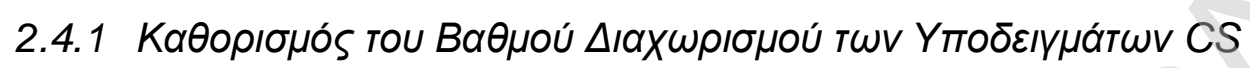

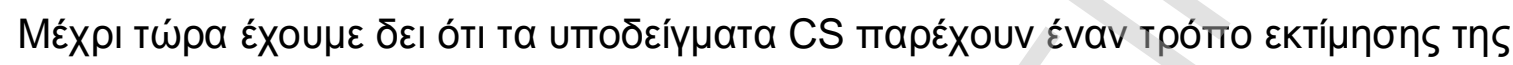

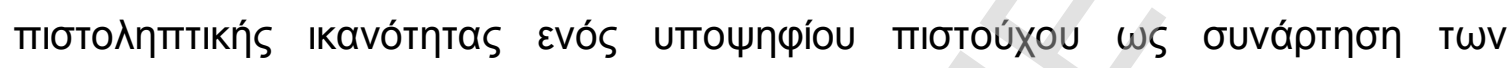

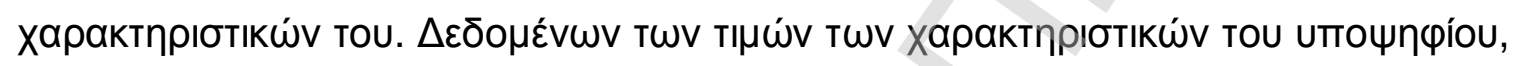

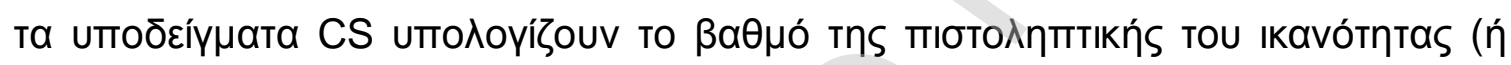

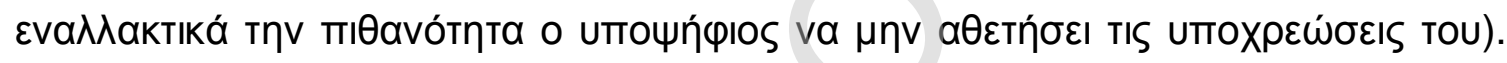

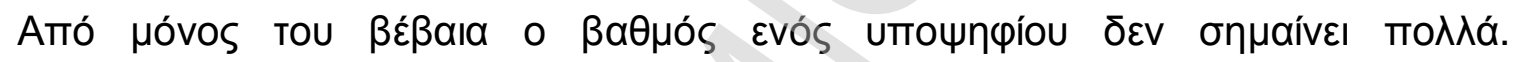

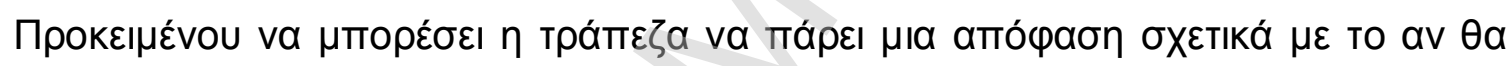

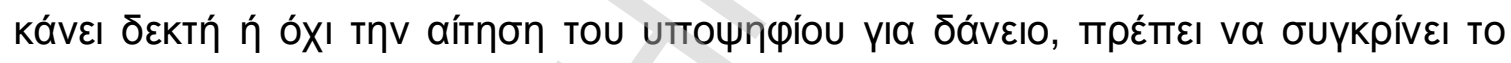

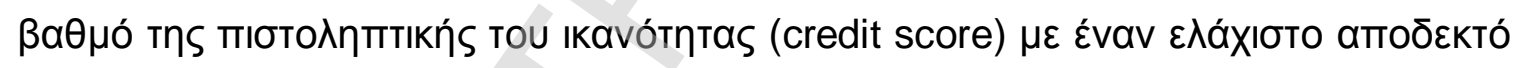

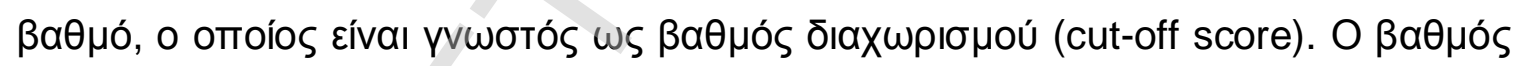

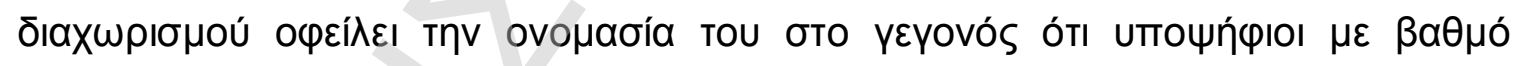

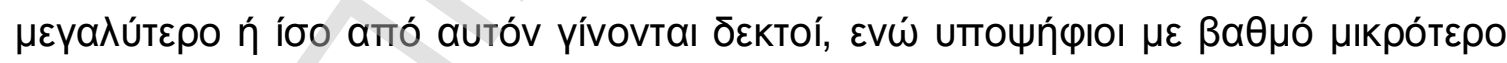

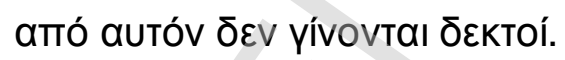

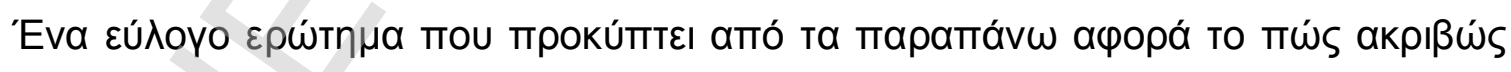

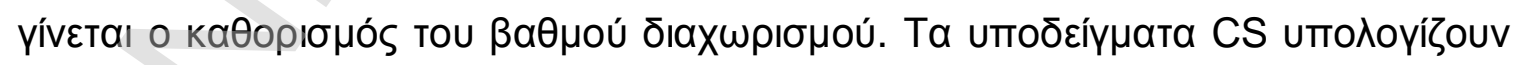

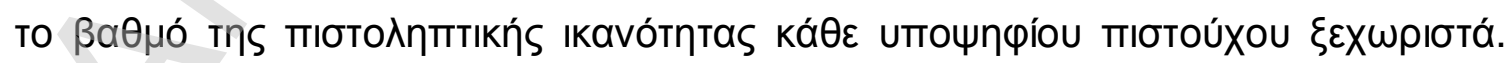

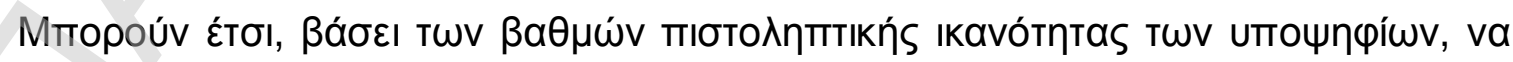

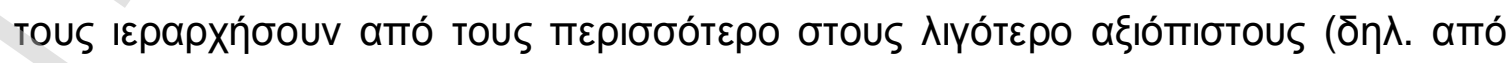




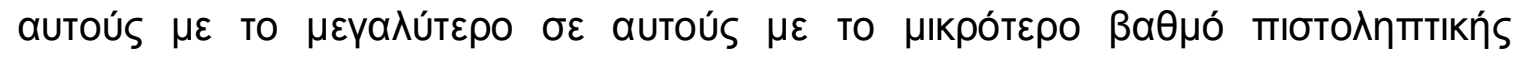

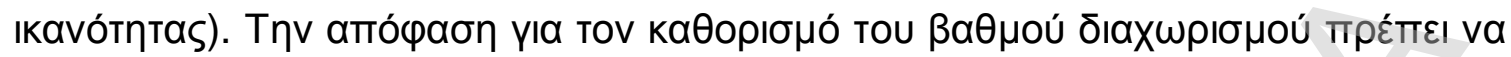

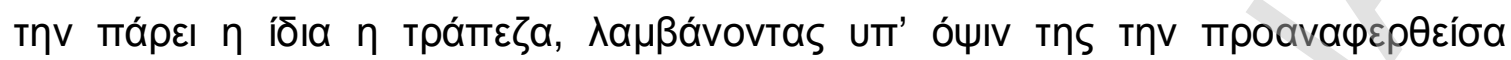

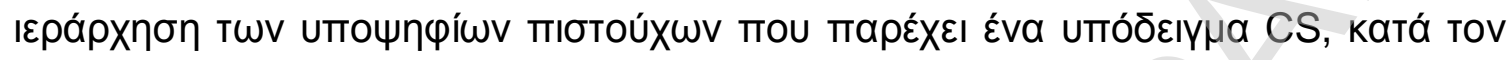

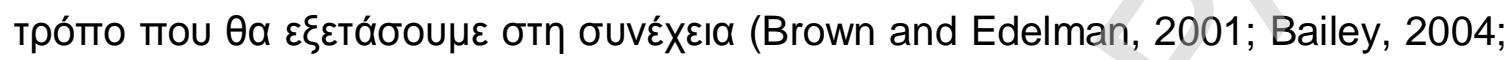
Mays, 2004).

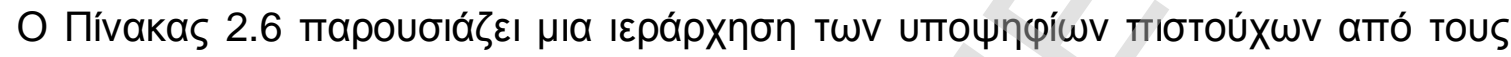

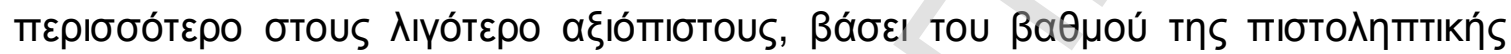

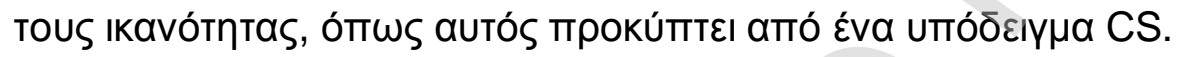

ПINAKA 2.6

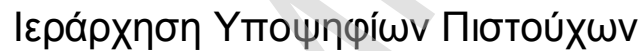

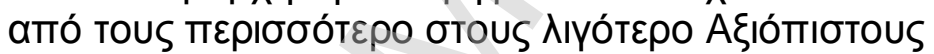

\begin{tabular}{|c|c|c|}
\hline 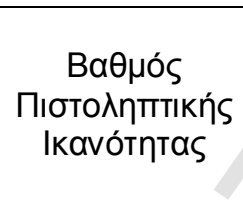 & 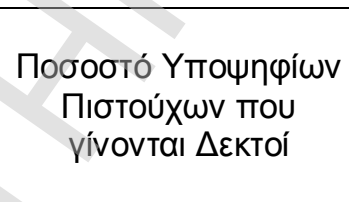 & 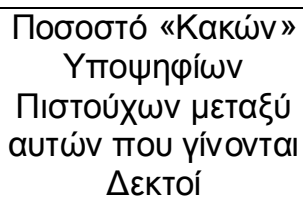 \\
\hline$\ldots$ & 8 & $\ldots$ \\
\hline 278 & $74,5 \%$ & $1,45 \%$ \\
\hline 277 & $75,0 \%$ & $1,50 \%$ \\
\hline 276 & $75,7 \%$ & $1,55 \%$ \\
\hline 275 & $76,2 \%$ & $1,60 \%$ \\
\hline 274 & $76,8 \%$ & $1,65 \%$ \\
\hline 273 & $77,7 \%$ & $1,70 \%$ \\
\hline 272 & $78,2 \%$ & $1,75 \%$ \\
\hline 271 & $78,9 \%$ & $1,80 \%$ \\
\hline 270 & $79,5 \%$ & $1,85 \%$ \\
\hline 269 & $80,0 \%$ & $1,90 \%$ \\
\hline 268 & $80,6 \%$ & $1,95 \%$ \\
\hline$\ldots$ & ... & .. \\
\hline
\end{tabular}

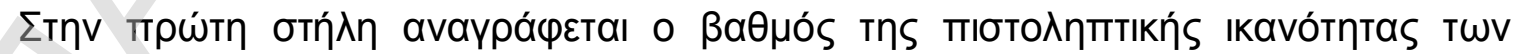

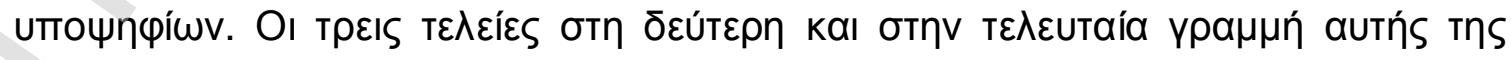




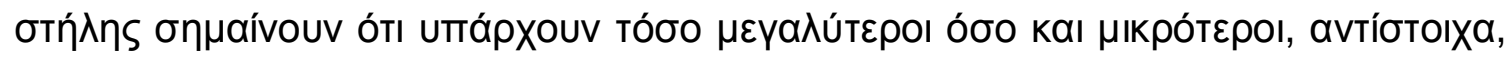

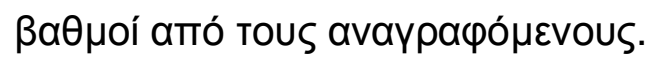

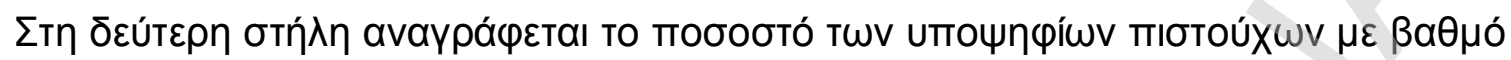

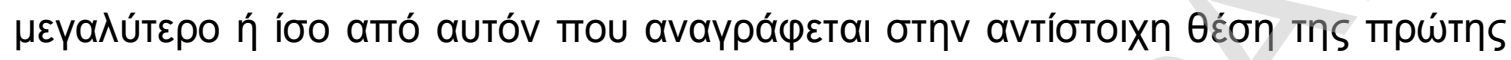

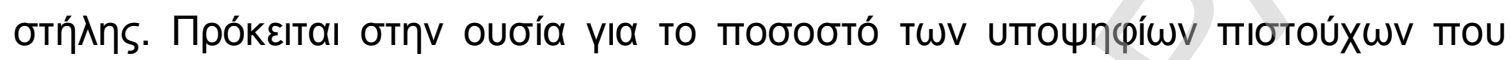

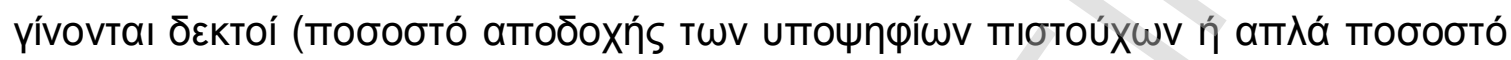

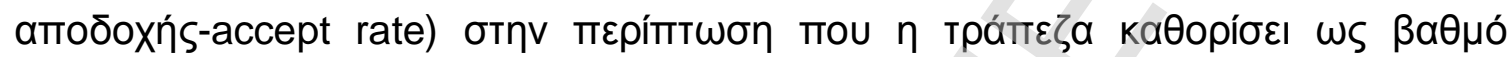

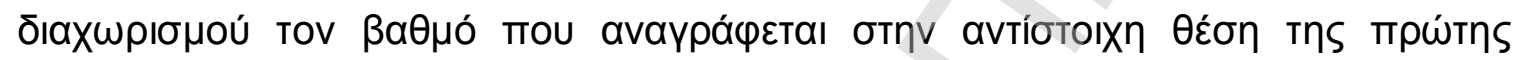

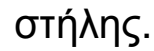

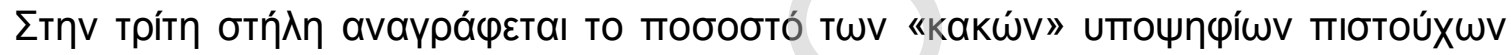

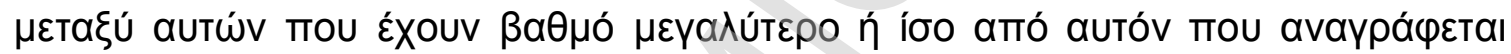

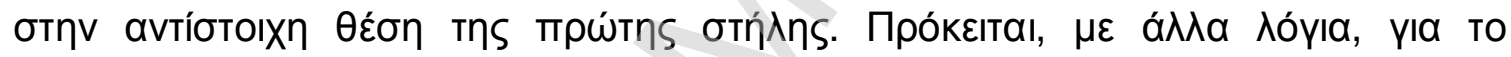

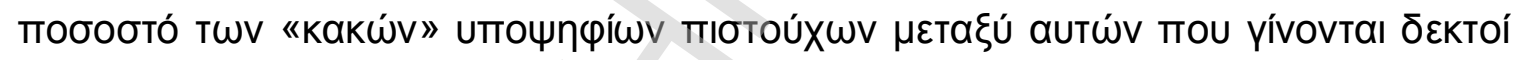

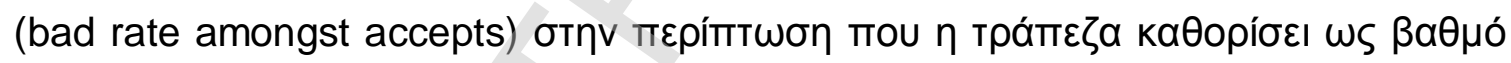

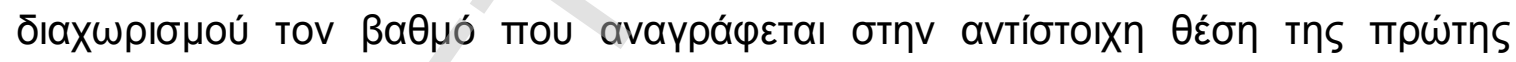

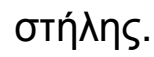

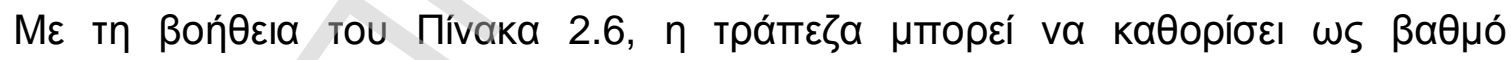

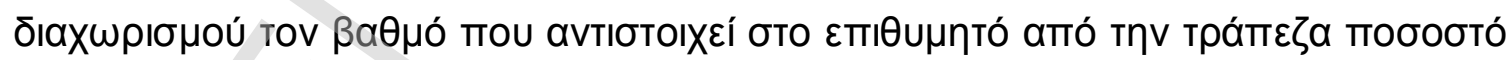

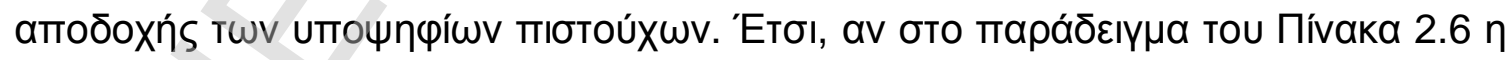

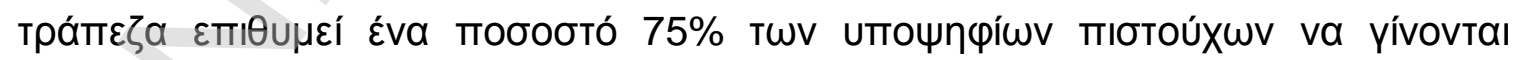

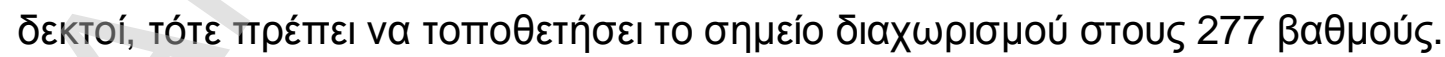




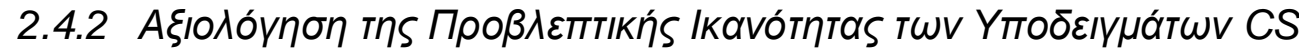

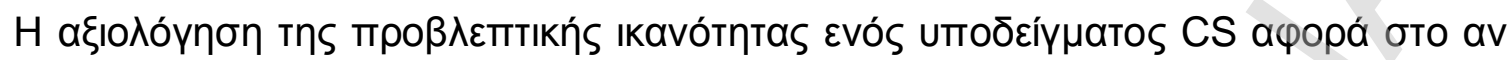

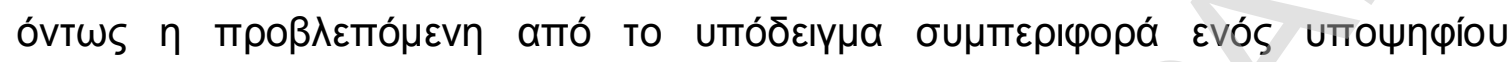

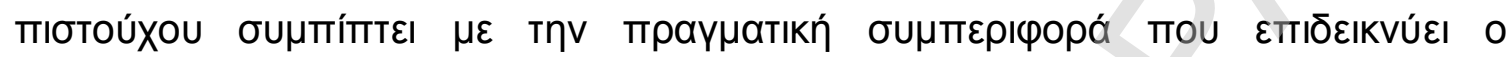

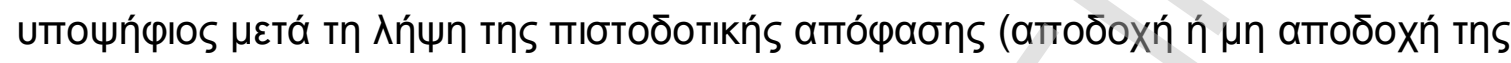

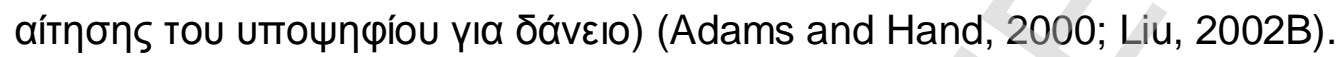

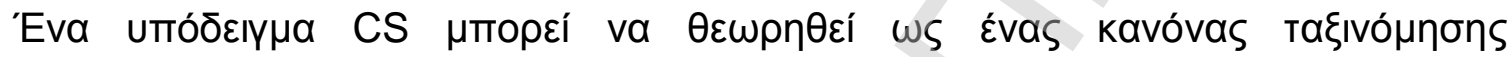

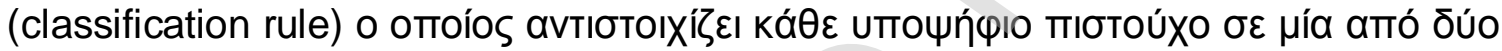

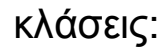

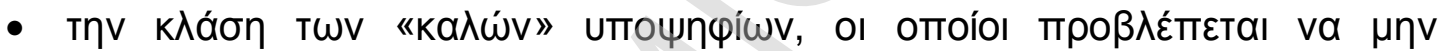

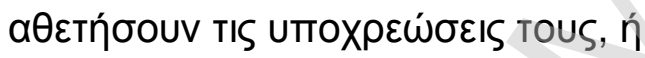

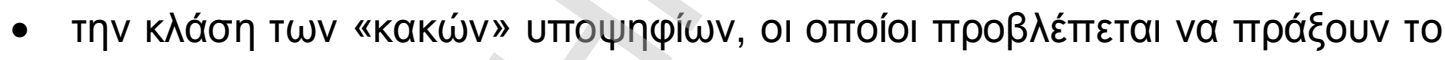
avтí日eto

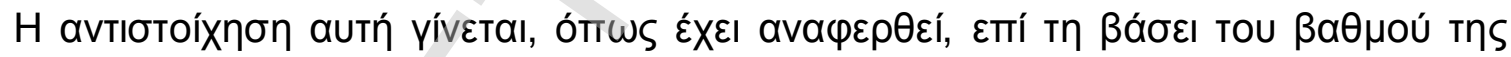

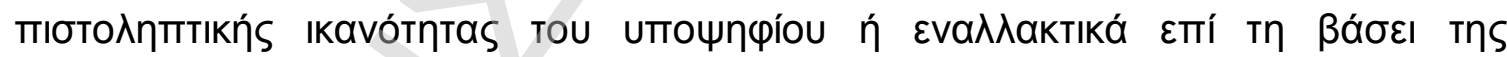

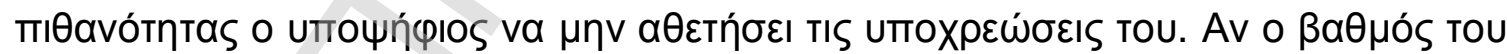

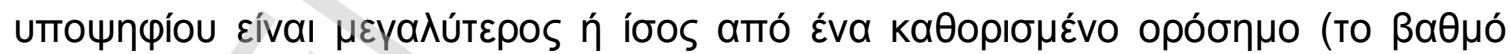

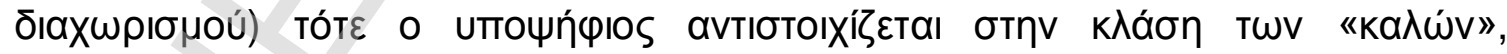

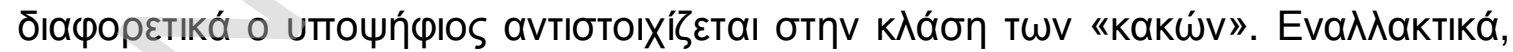

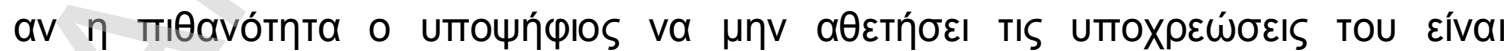

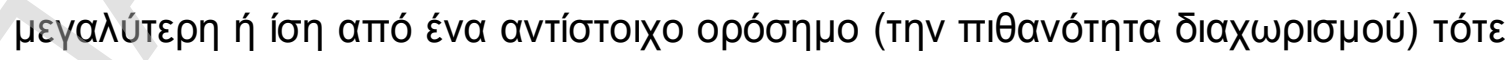




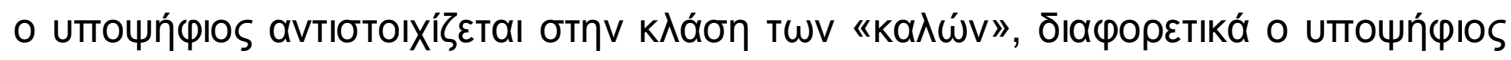

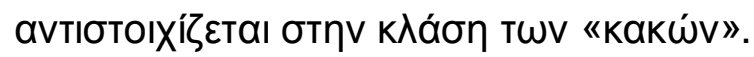

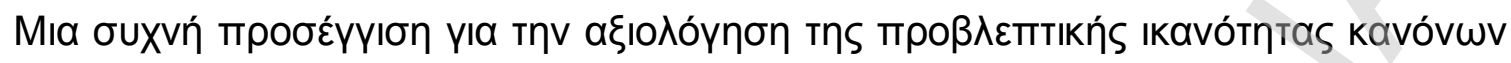

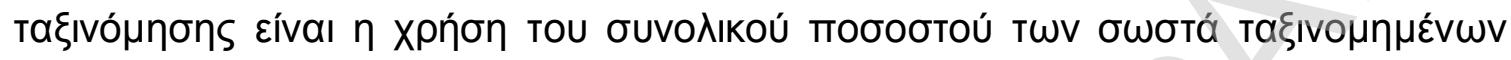

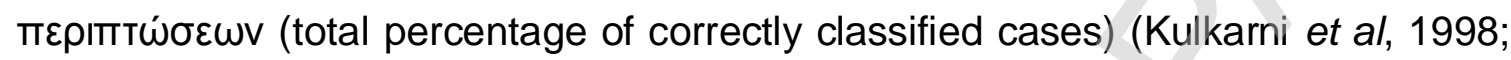

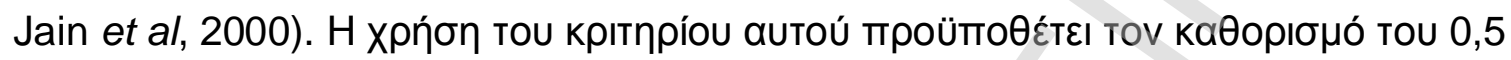

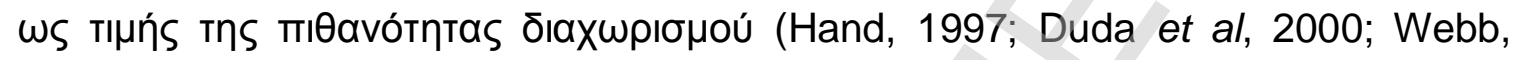

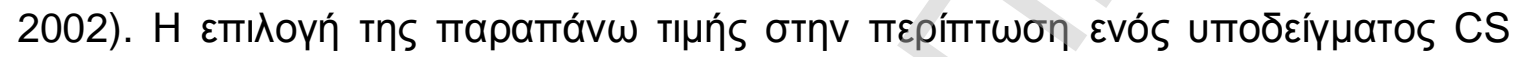

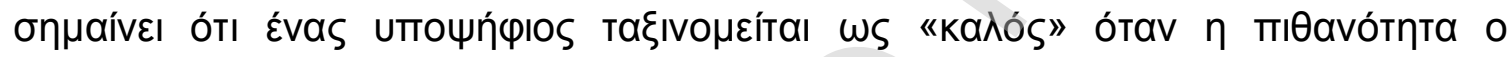

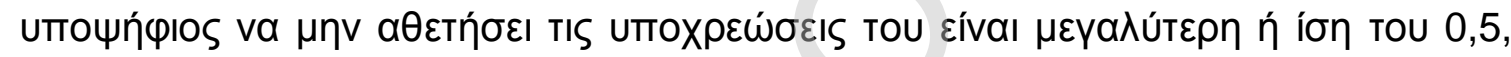

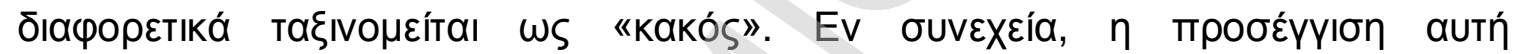

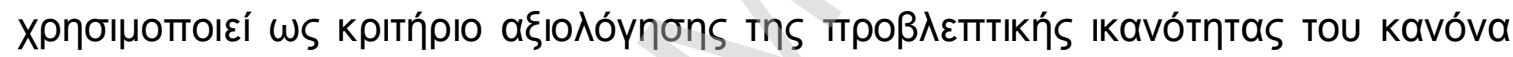

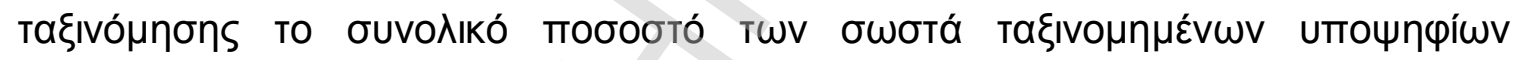
пıбтои́X $\omega v^{10}$

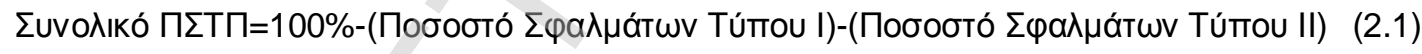

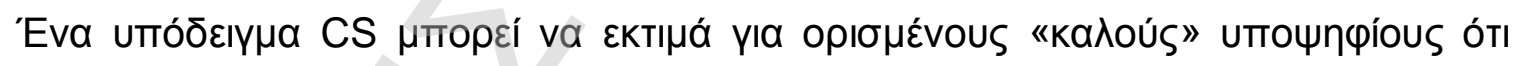

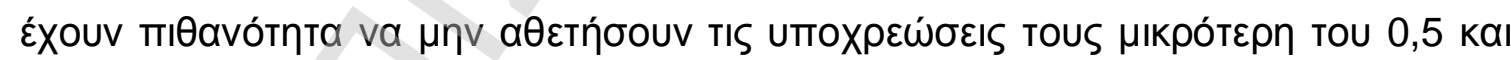

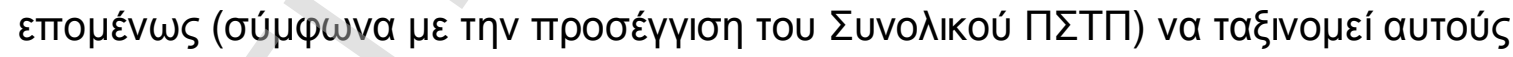

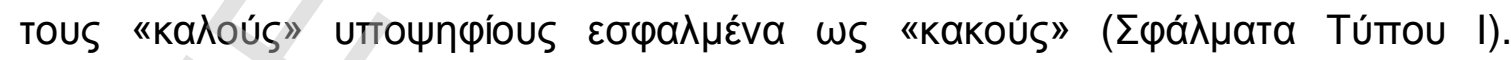

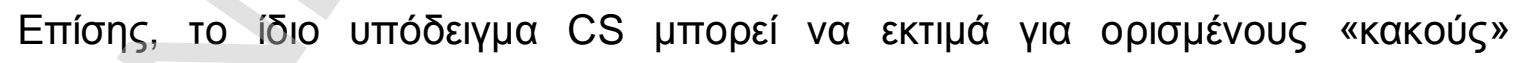

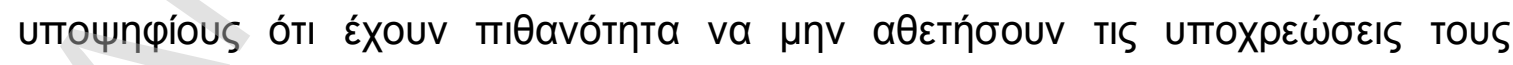

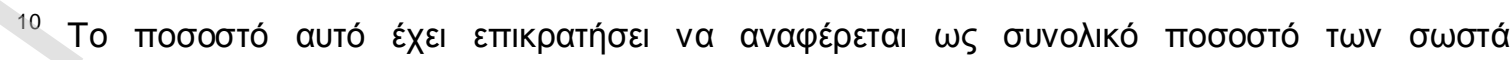

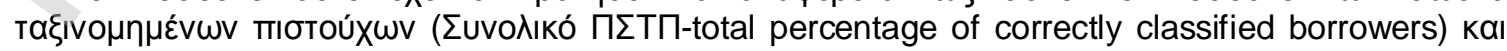

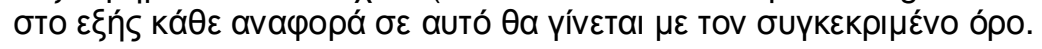




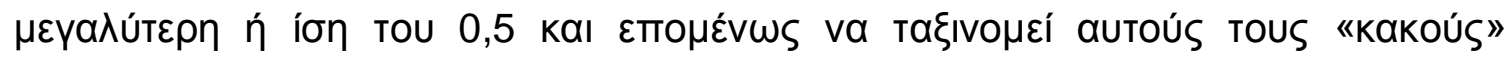

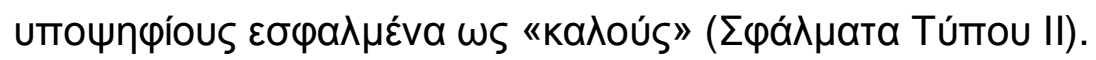

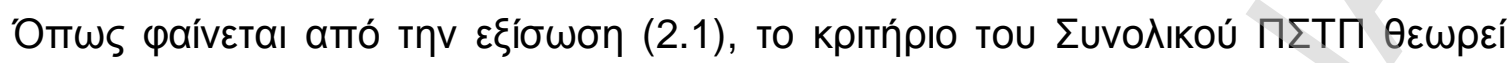

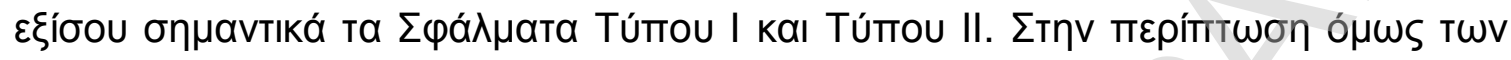

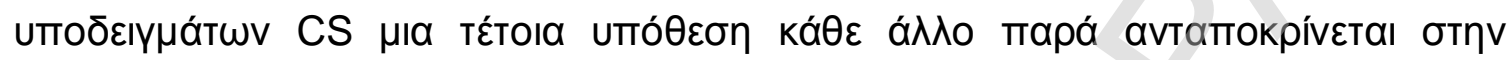

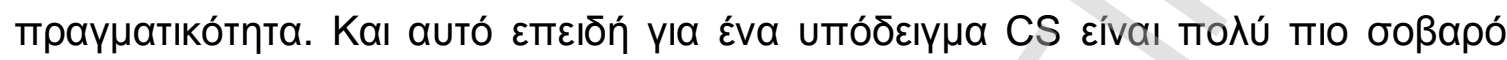

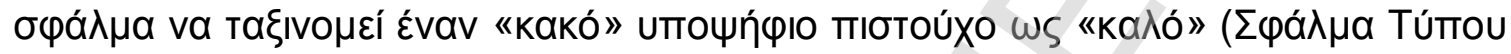

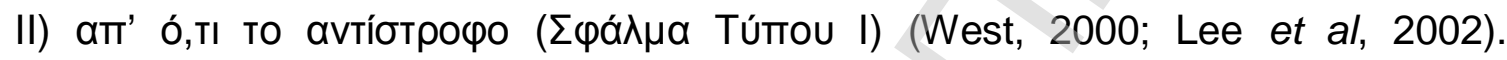

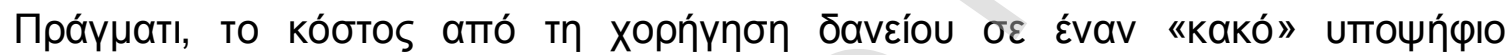

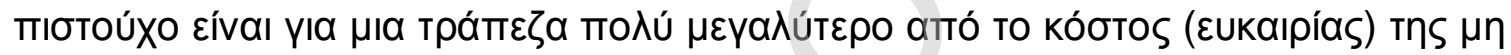

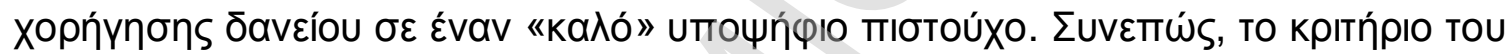

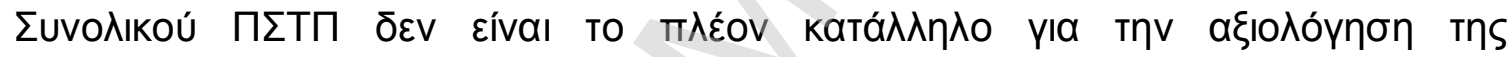

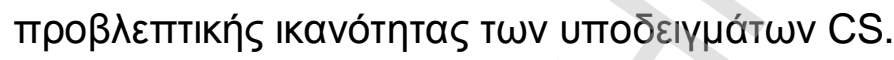

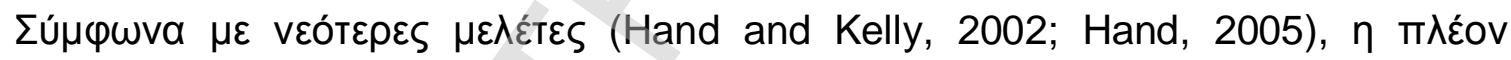

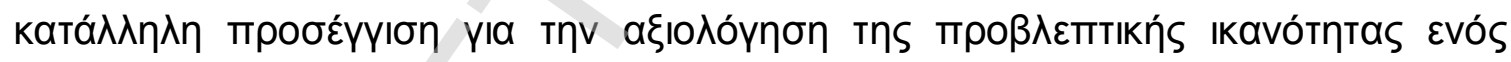

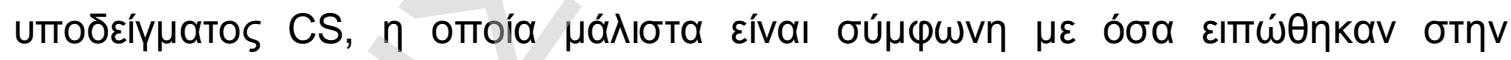

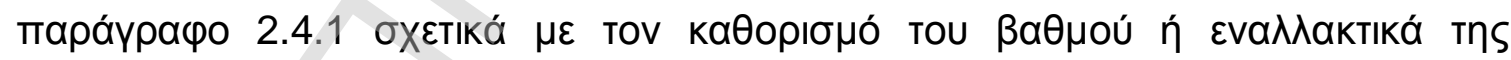

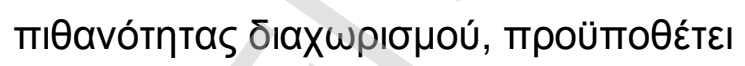

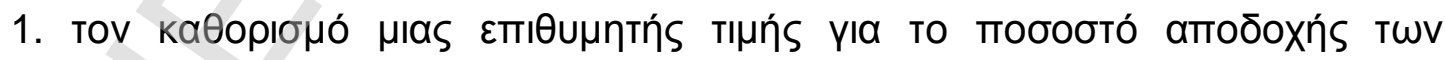

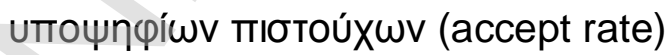

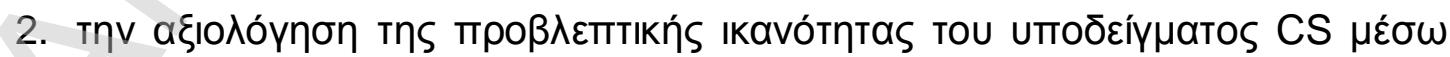

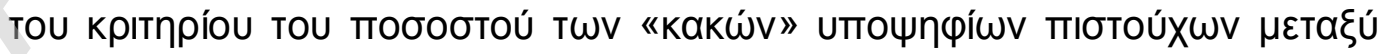




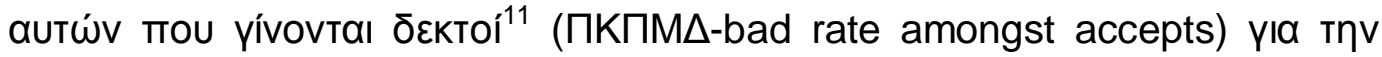

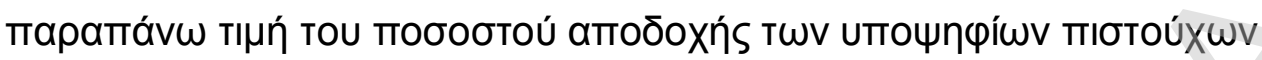

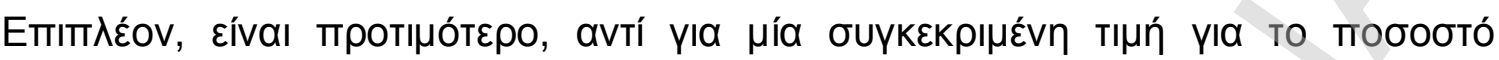

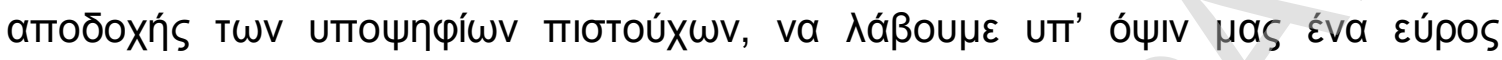

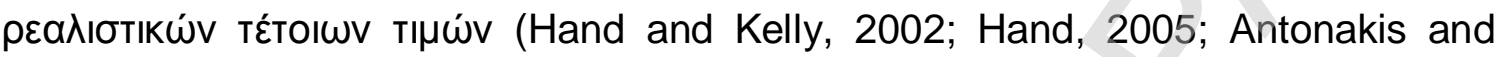
Sfakianakis, 2008; Antonakis and Sfakianakis, 2009A).

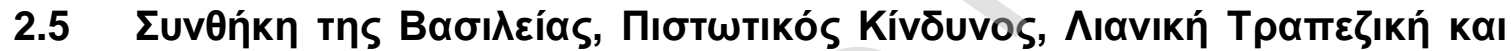

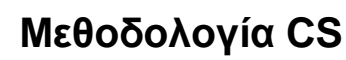

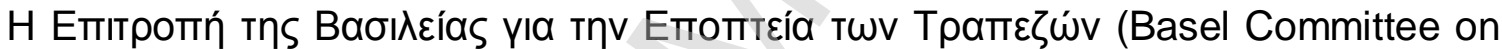

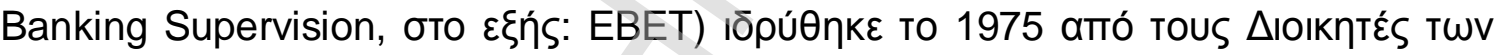

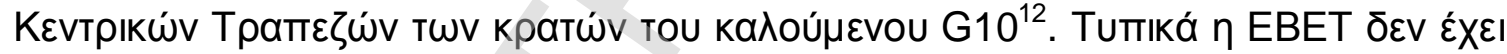

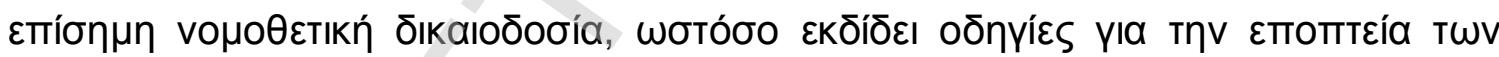

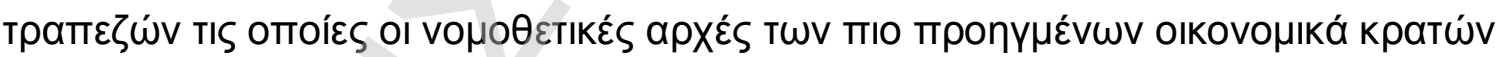

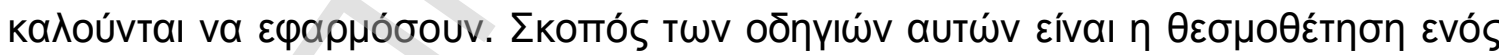

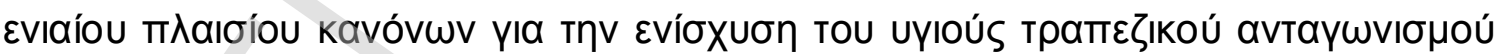
(Marshall, 2003).

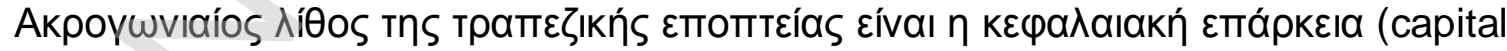

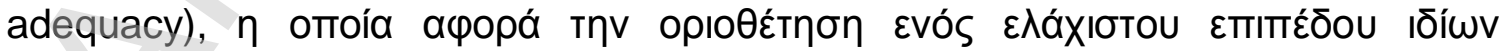

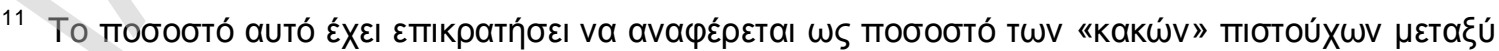

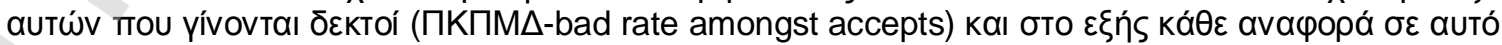

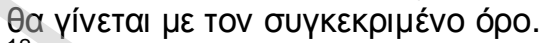

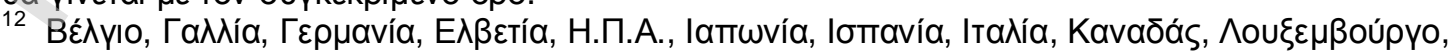

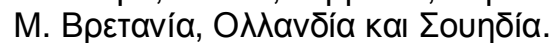




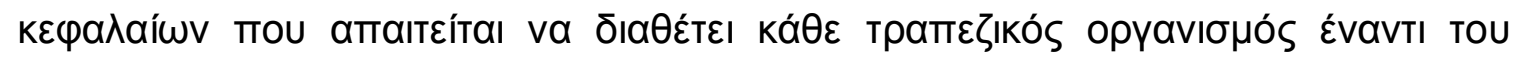

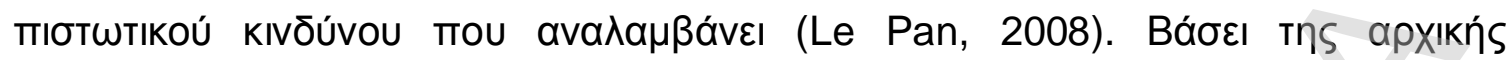

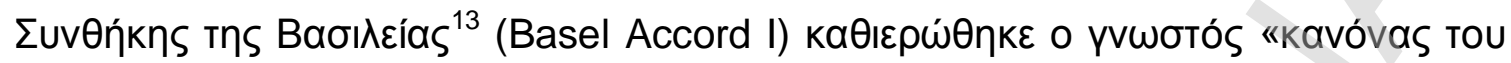

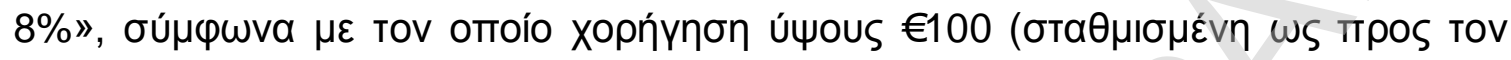

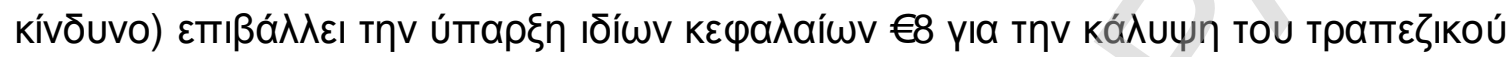

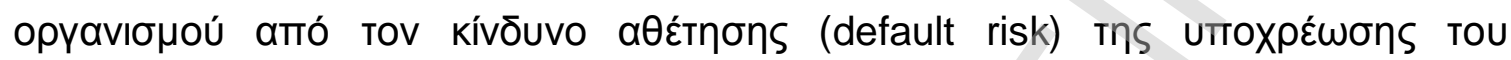

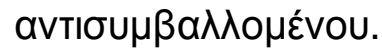

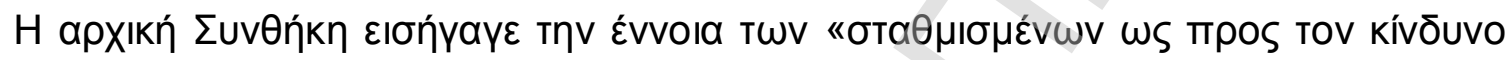

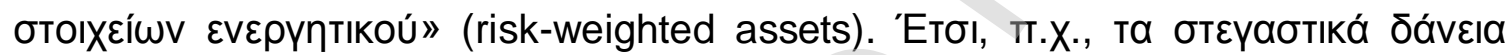

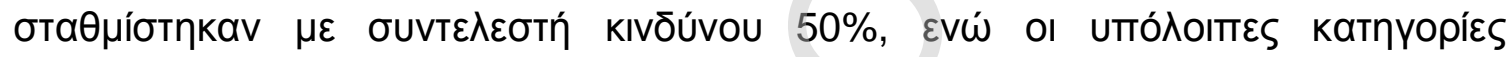

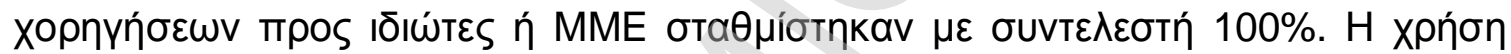

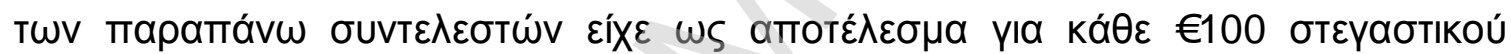

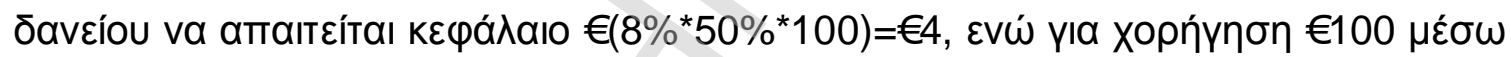

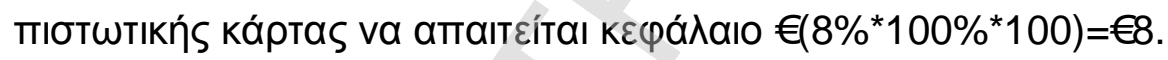

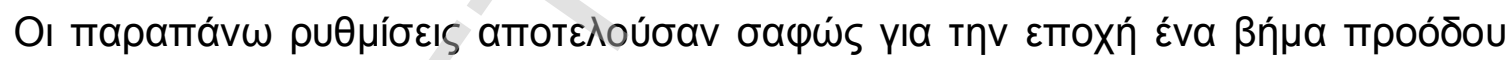

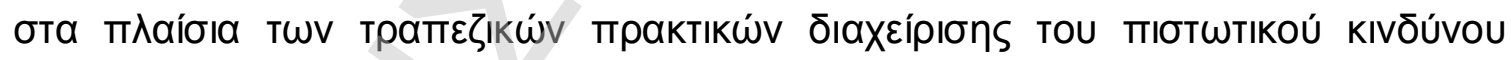

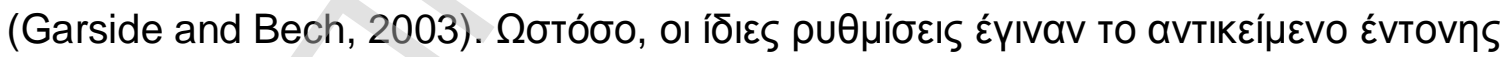

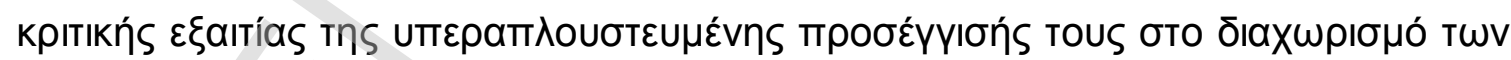

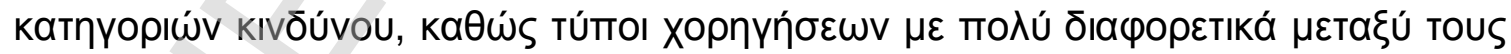

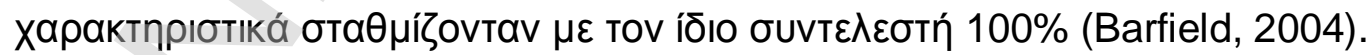

${ }^{13}$ EBET (1988). International Convergence of Capital Measurement and Capital Standards. 


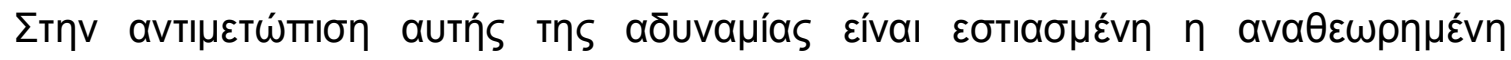

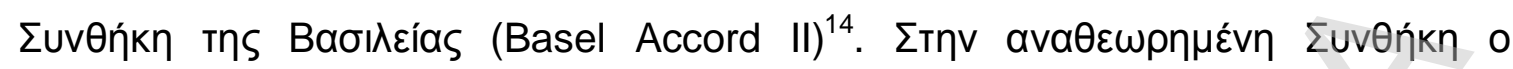

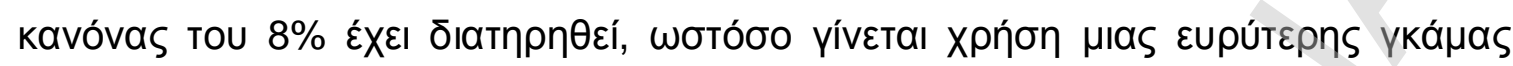

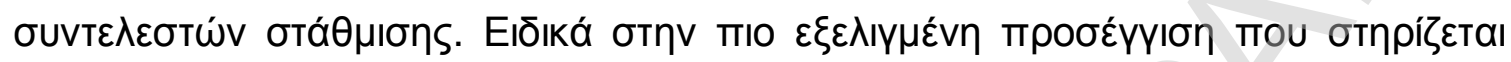

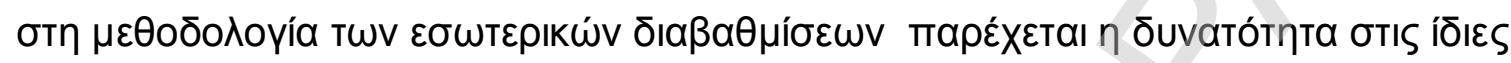

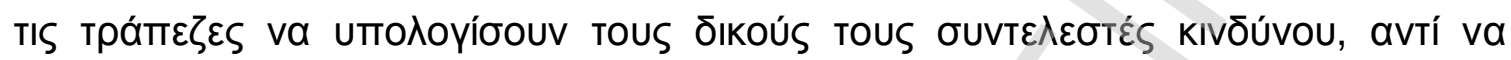

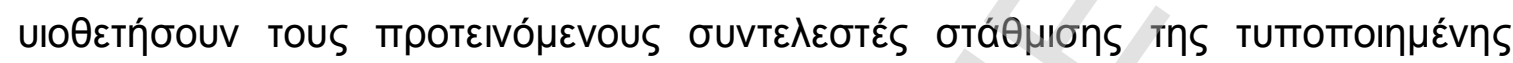

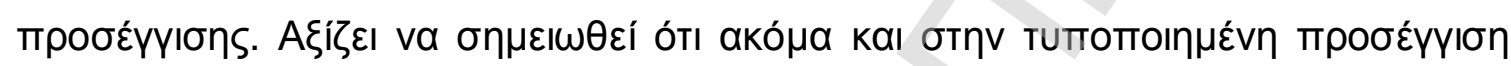

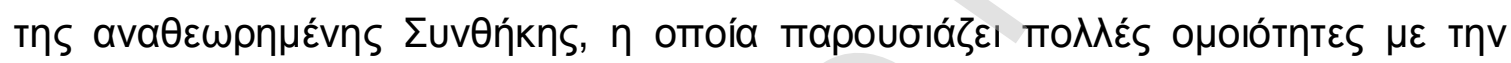

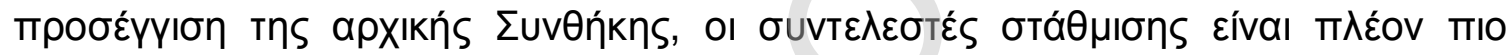

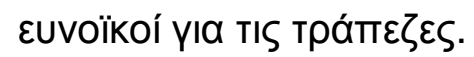

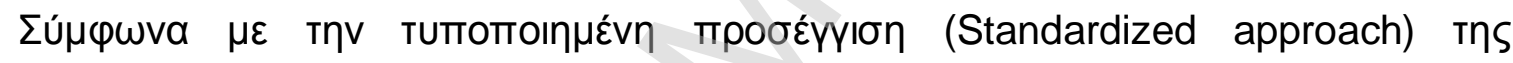

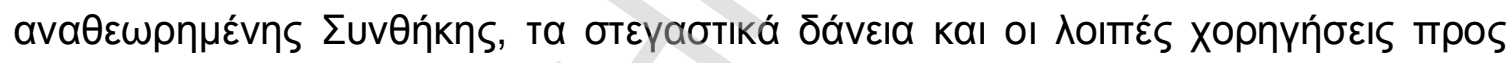

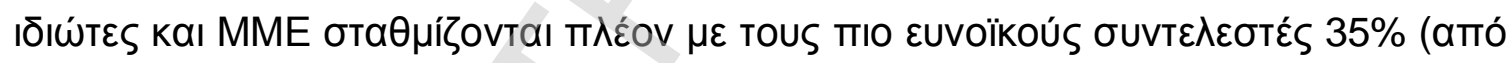

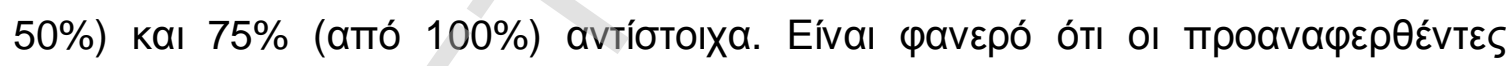

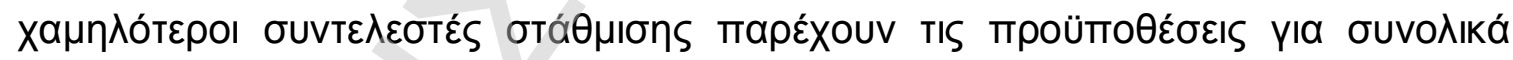

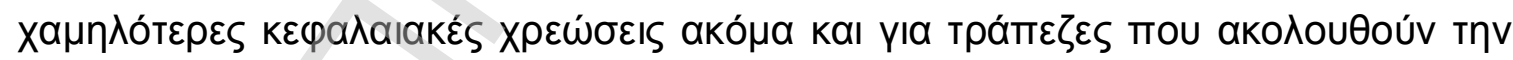

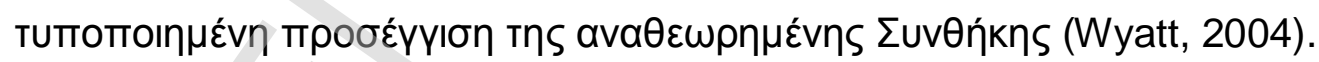

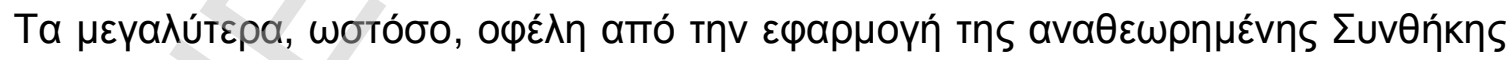

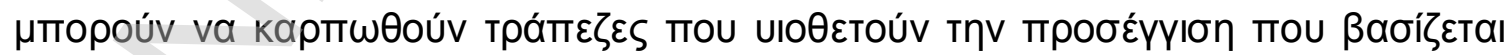

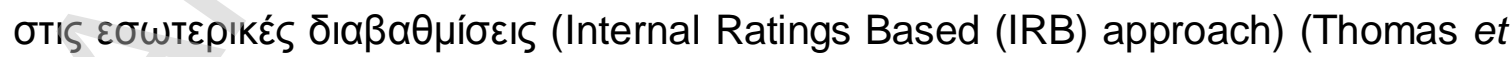

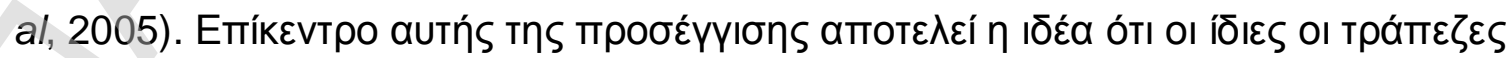

${ }^{14}$ EBET (2005). International Convergence of Capital Measurement and Capital Standards: A Revised Framework. 


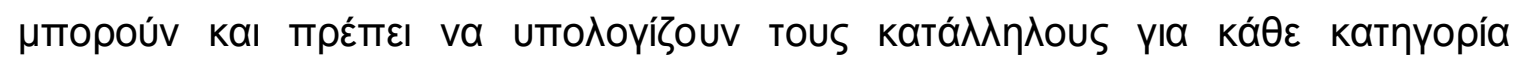

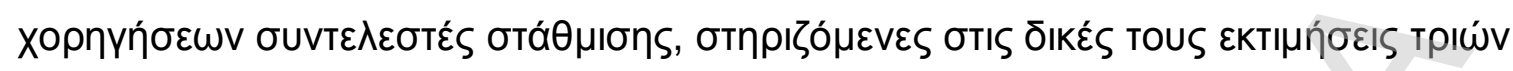

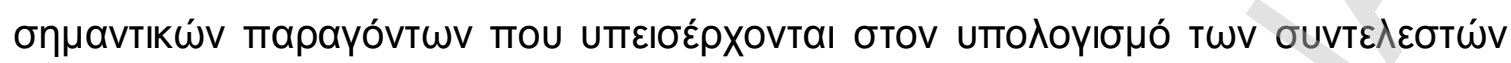
autẃv.

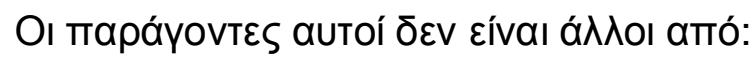

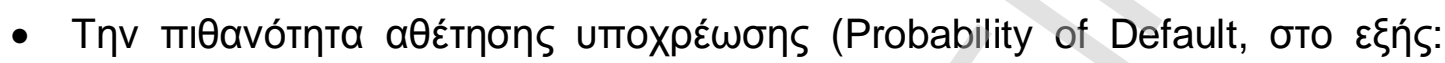

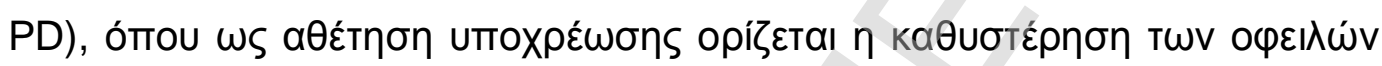

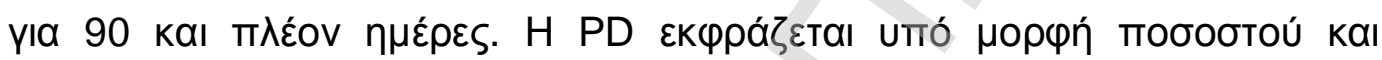

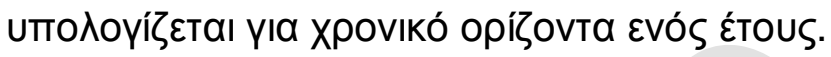

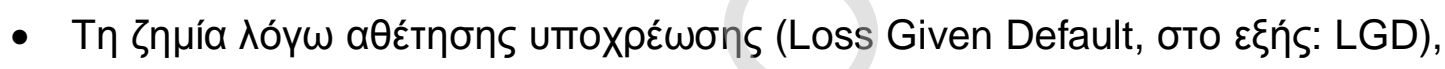

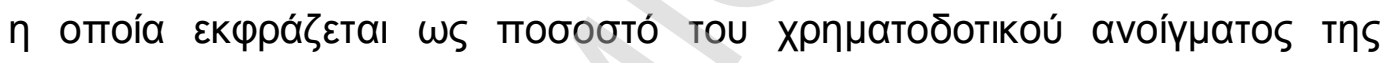

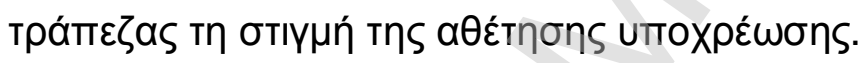

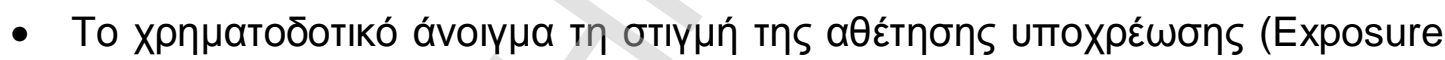

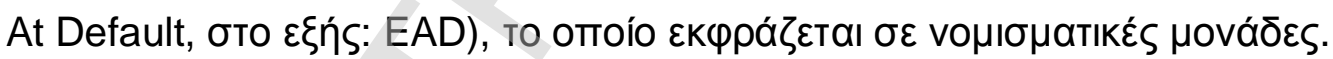

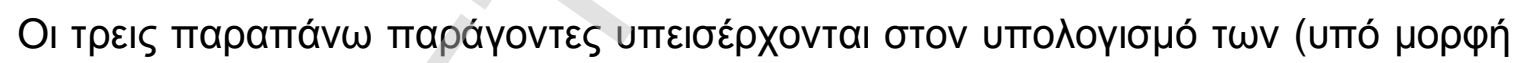

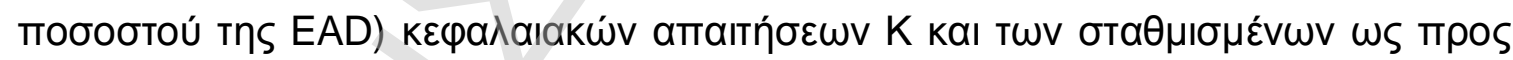

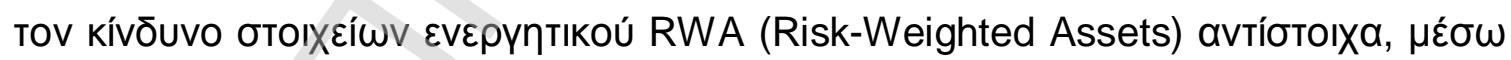
TWV túmWv (Gordy, 2003; Perli and Nayda, 2004)

$$
K=L G D * N\left(\left(\frac{1}{1-R}\right)^{1 / 2} N^{-1}(P D)+\left(\frac{R}{1-R}\right)^{1 / 2} N^{-1}(0.999)\right)-L G D * P D
$$

kaı

$$
R W A=12,5 * K * E A D
$$




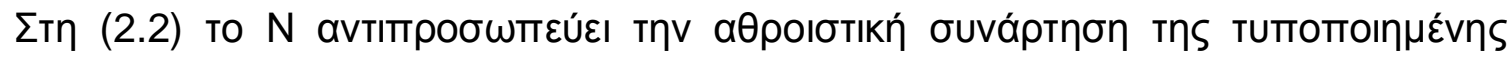

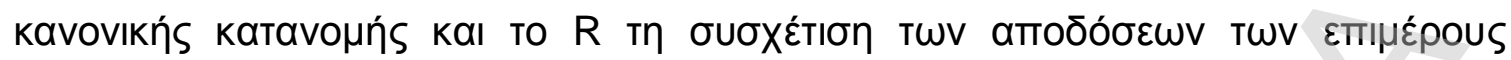

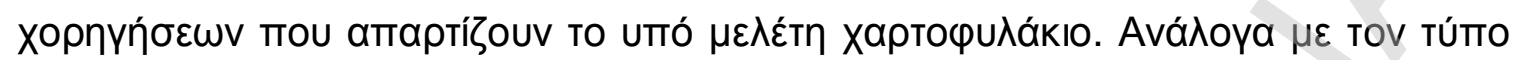

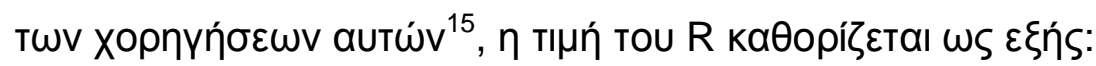

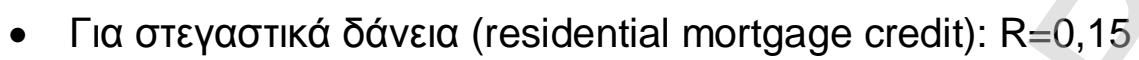

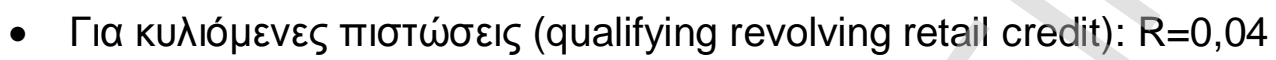

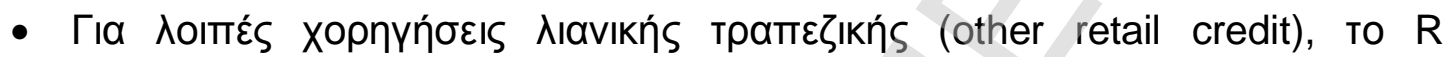

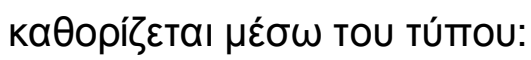

$$
R=0,03 * \frac{1-e^{-35^{*} P D}}{1-e^{-35}}+0,16 *\left(1-\frac{1-e^{-35^{*} P D}}{1-e^{-35}}\right)
$$

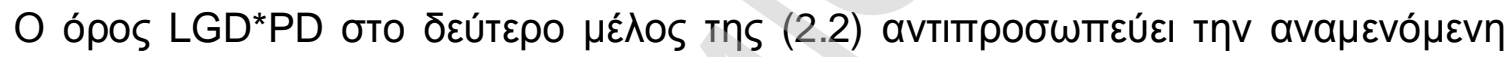

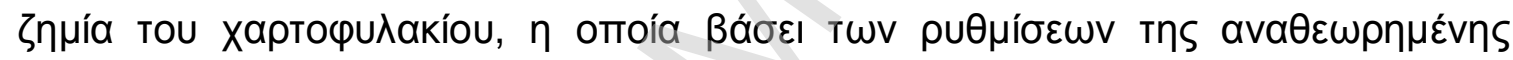

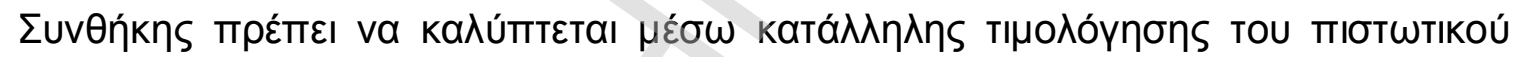

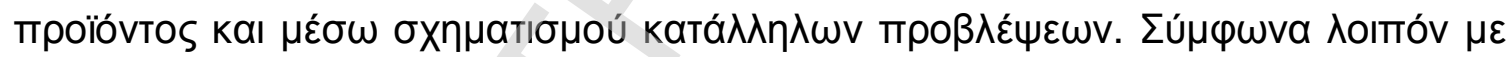

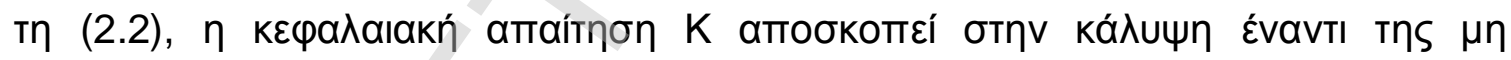

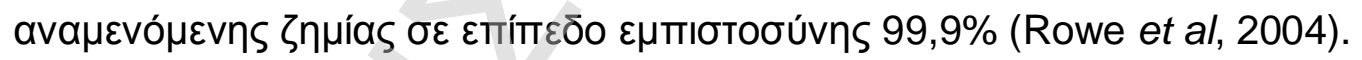

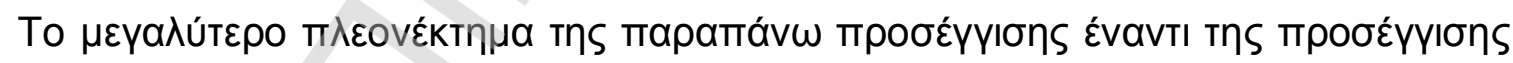

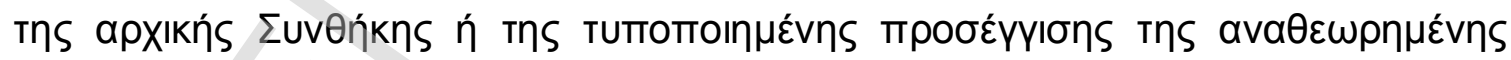

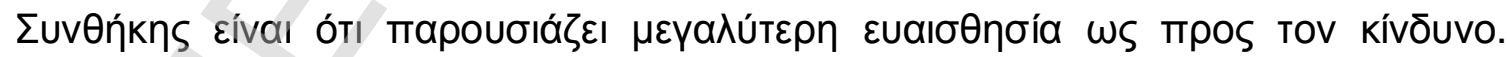

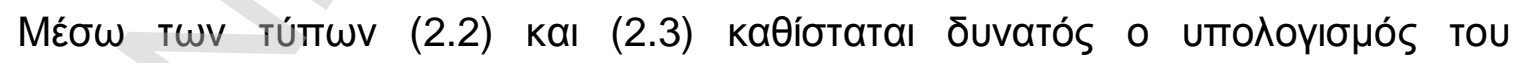

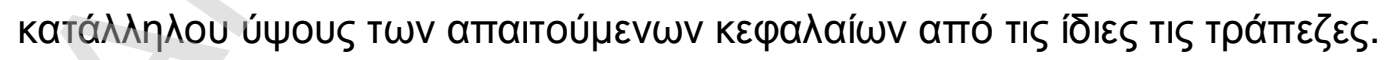

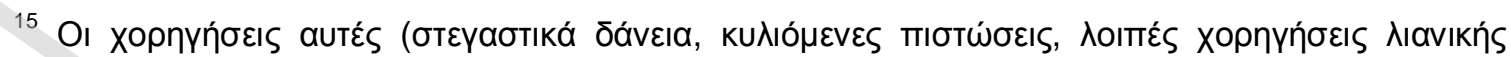

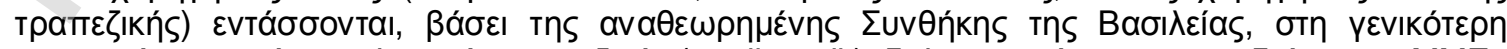

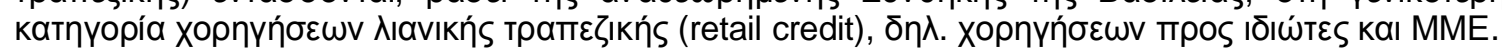




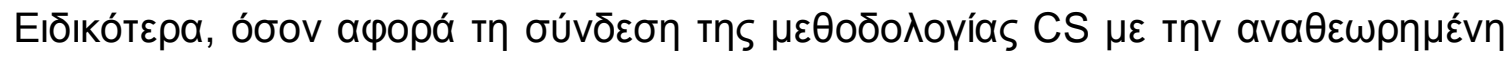

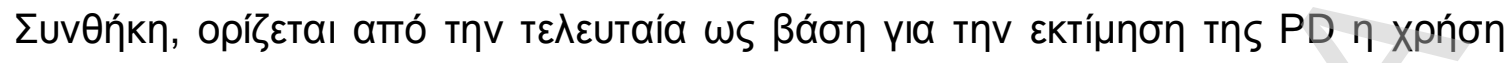

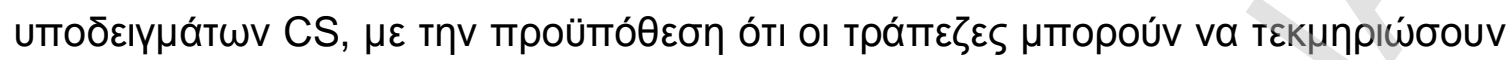

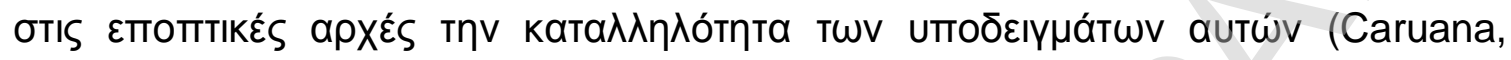

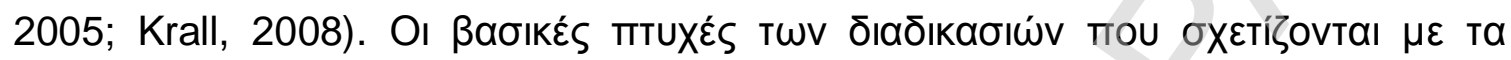

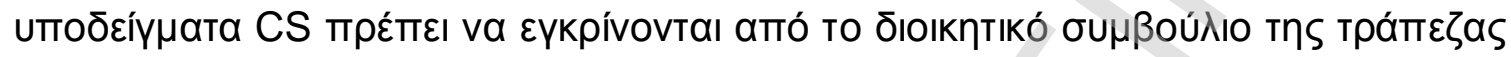

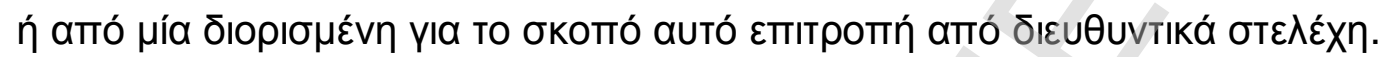

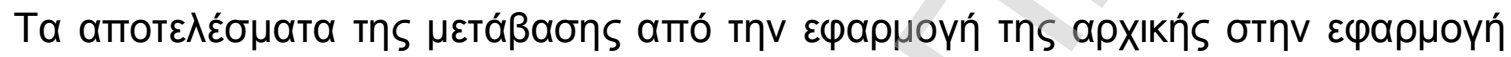

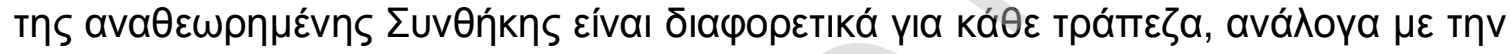

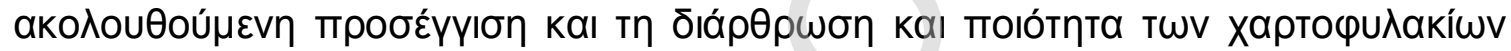

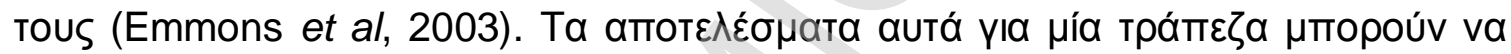

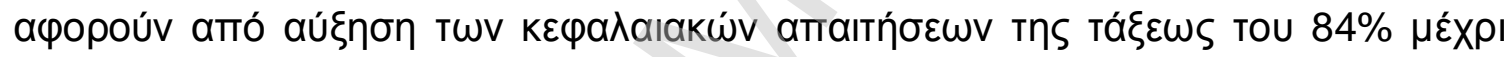

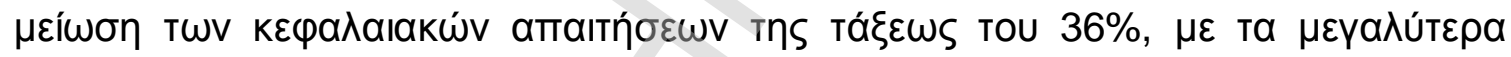

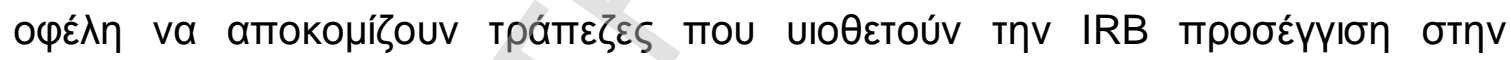

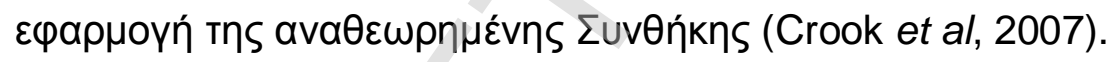




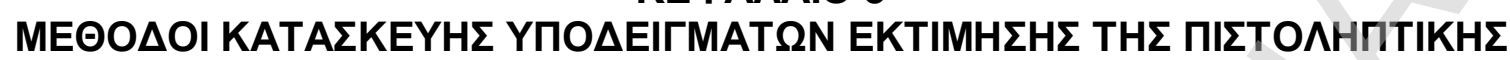

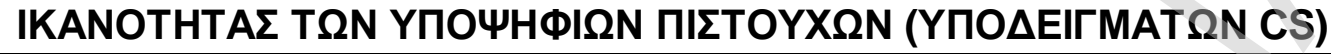

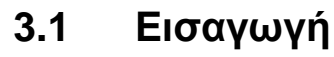

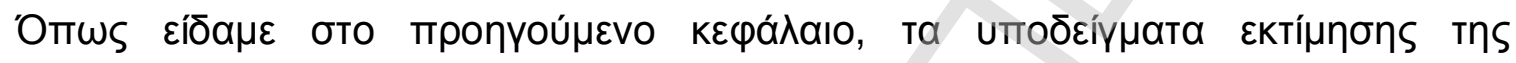

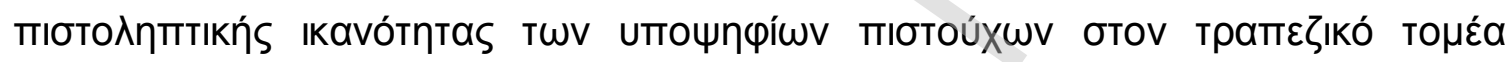

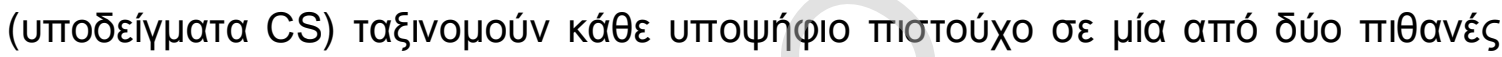

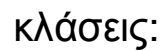

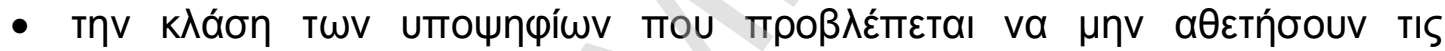

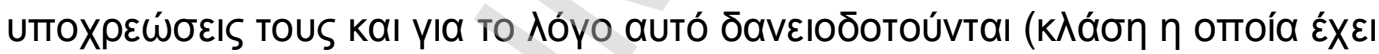

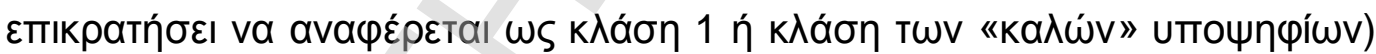
ń

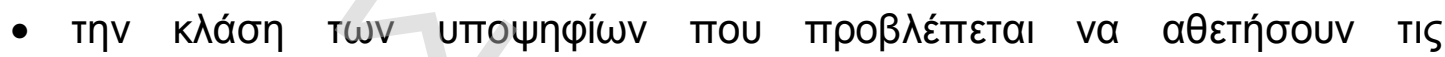

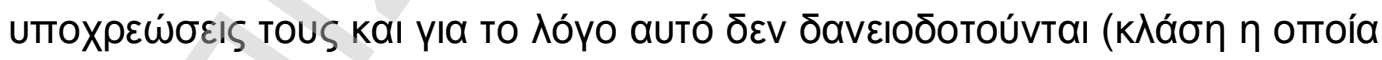

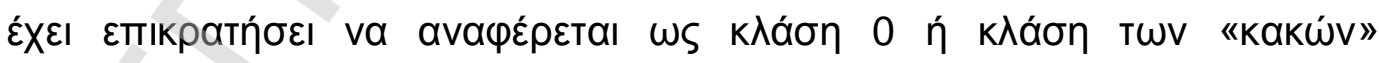

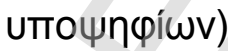

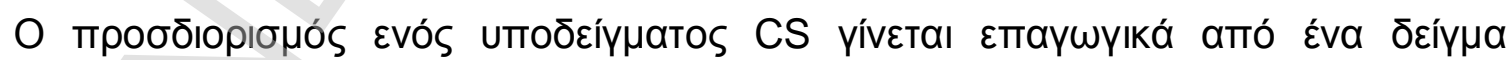

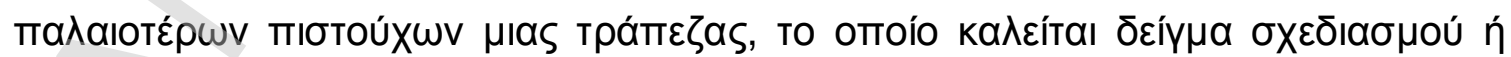

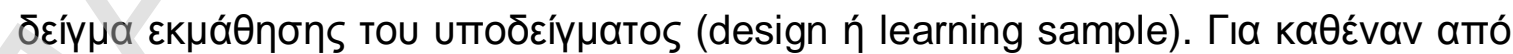

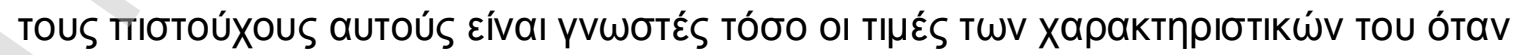

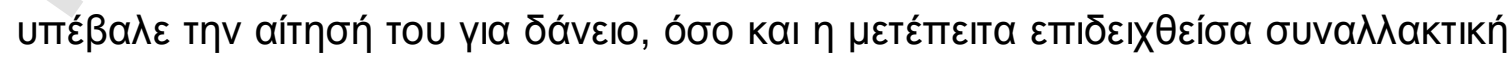




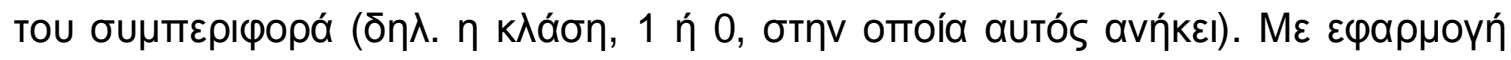

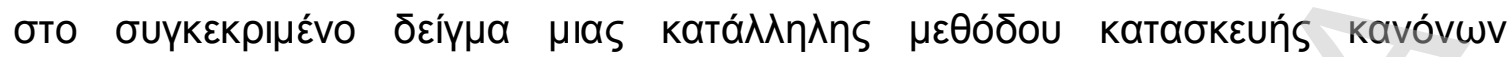

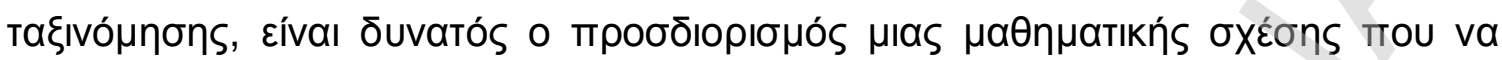

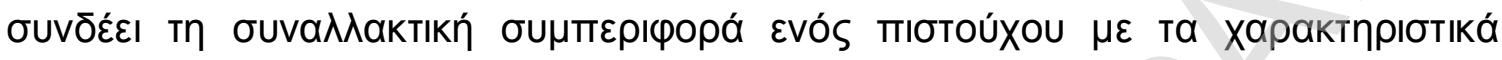
aUtoú.

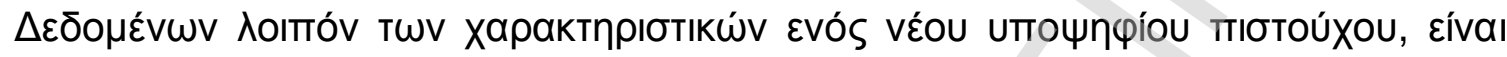

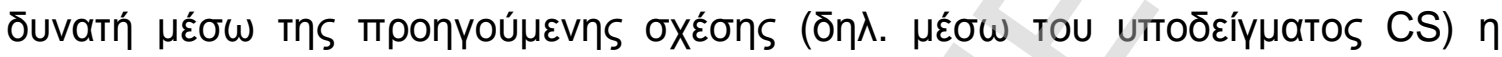

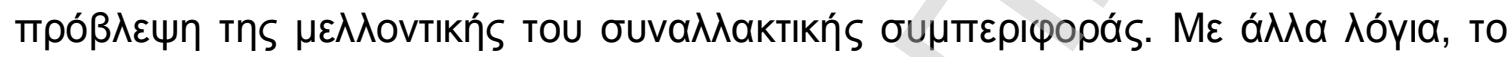

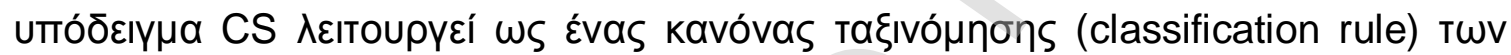

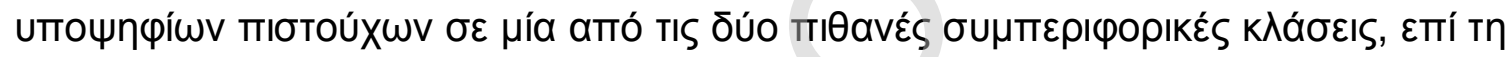

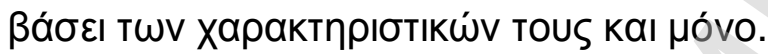

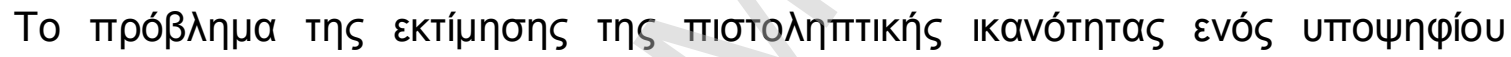

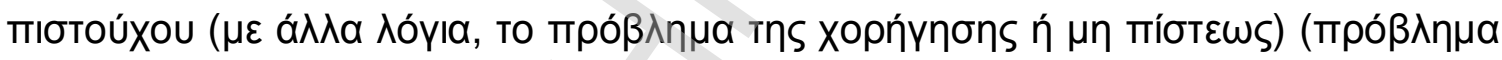

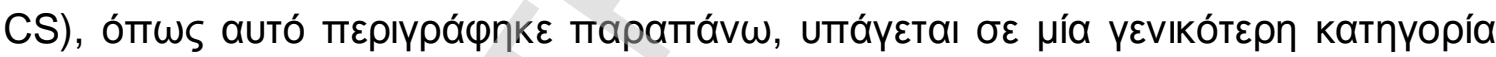

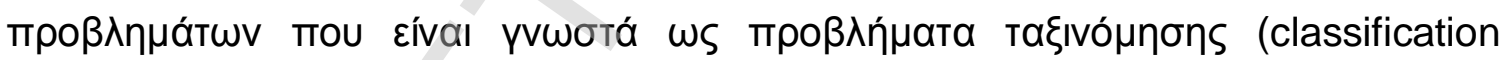
problems).

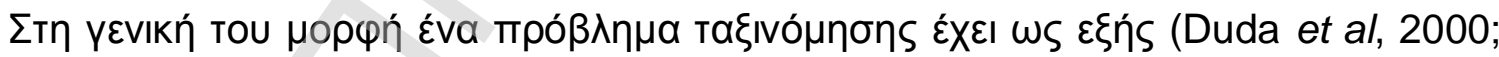
Webb, 2002):

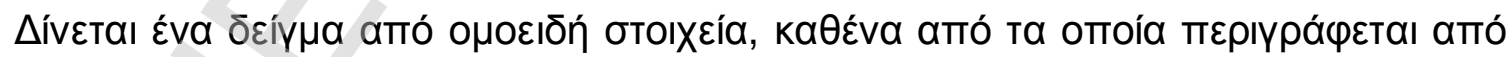

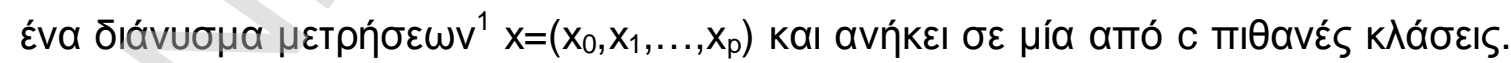

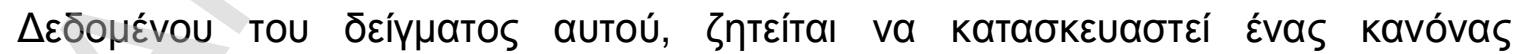

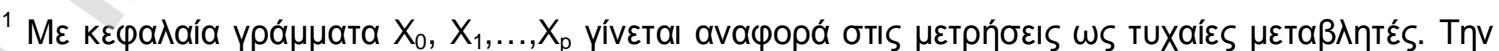

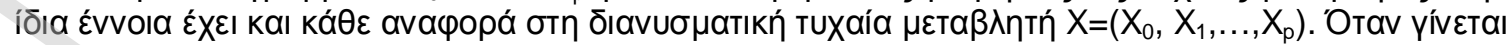

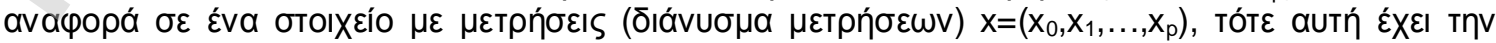

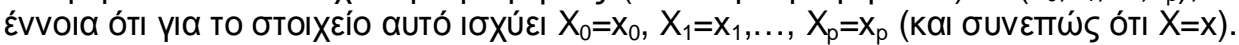




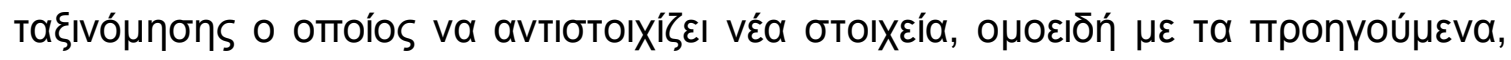

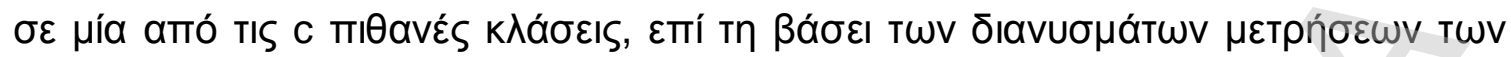

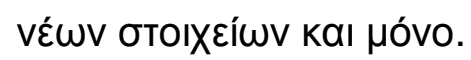

$\Delta \varepsilon \delta$

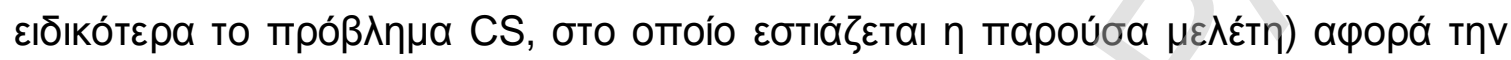

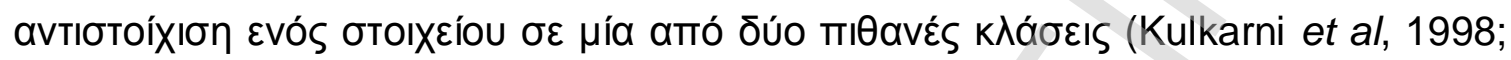

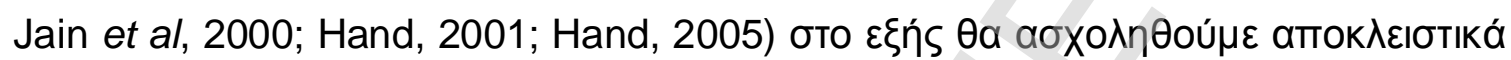

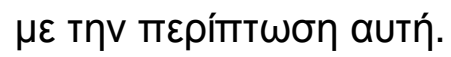

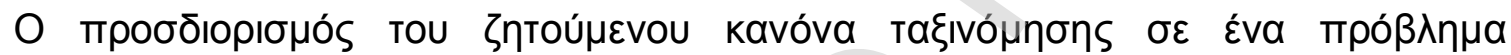

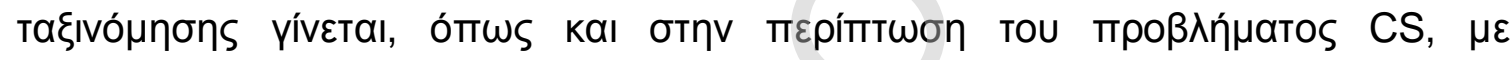

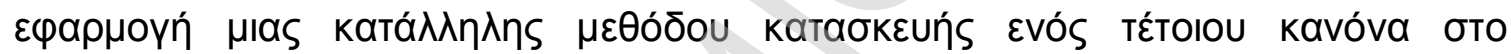

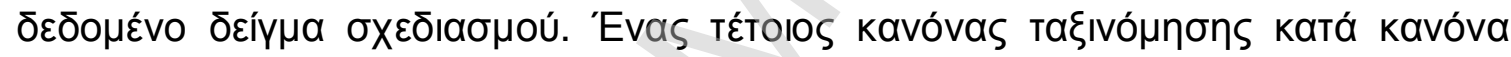

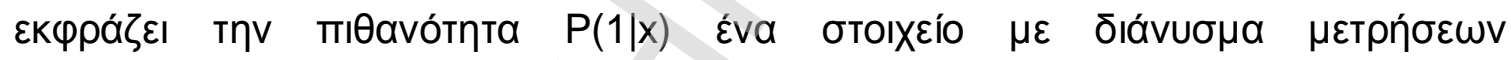

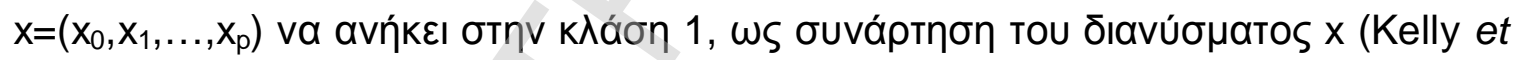
al, 1999; Hand and Yu, 2001).

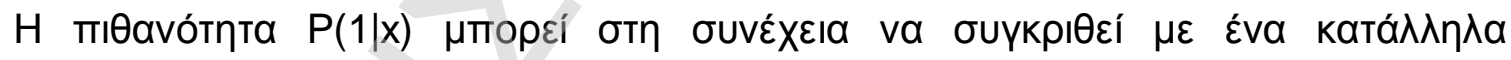

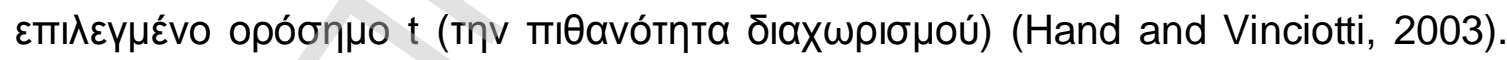

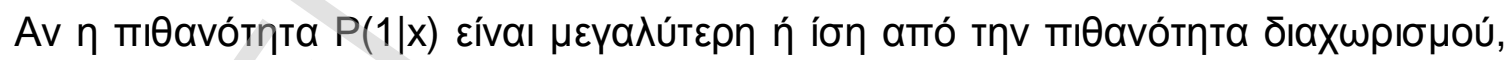

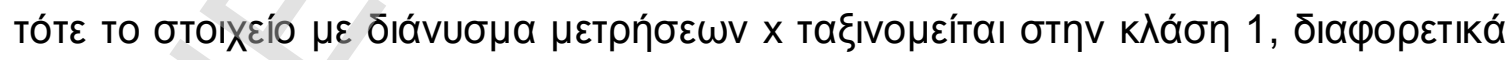

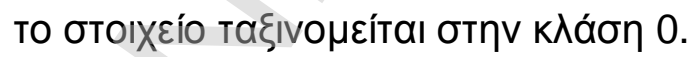

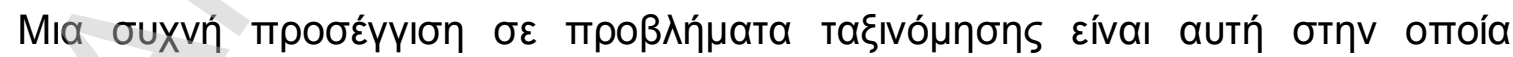

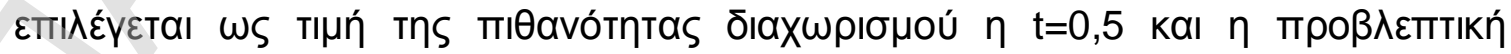

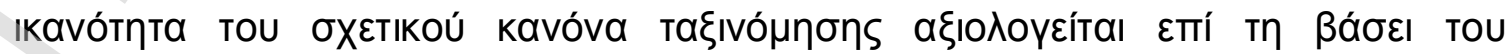




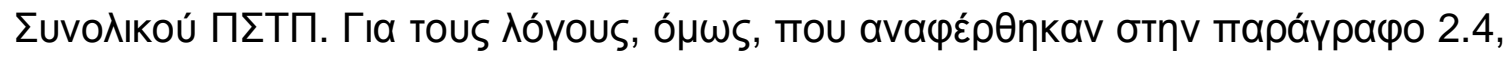

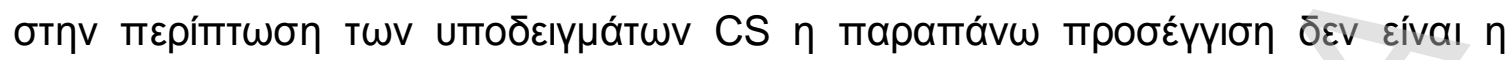

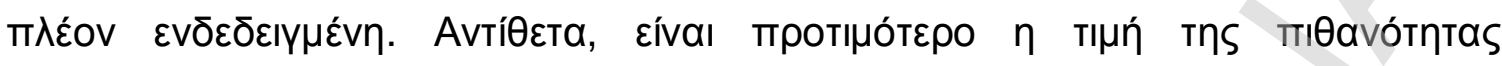

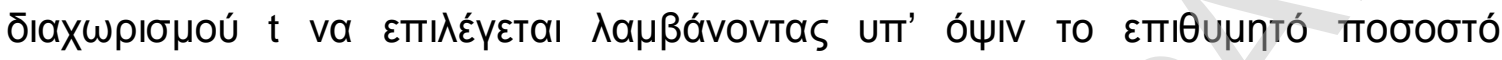

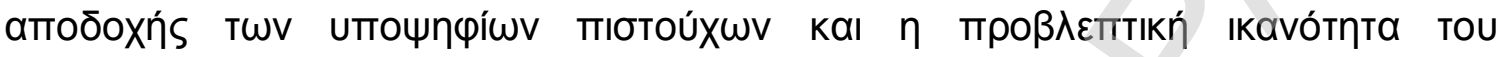

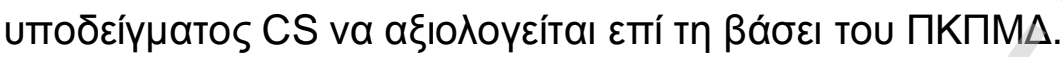

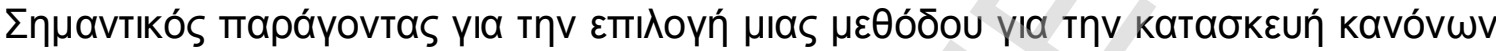

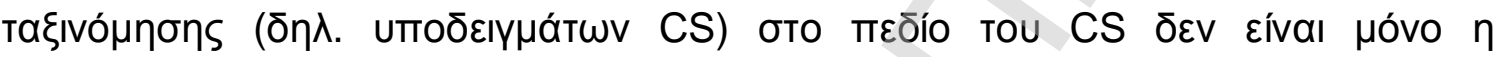

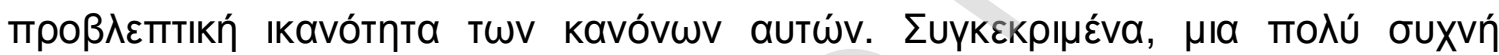

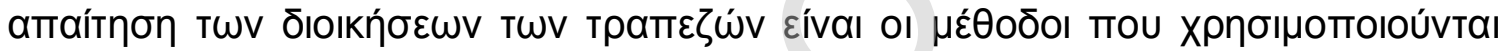

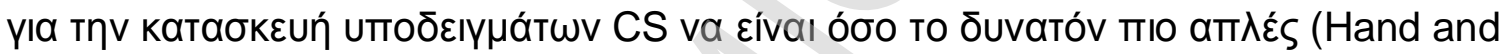

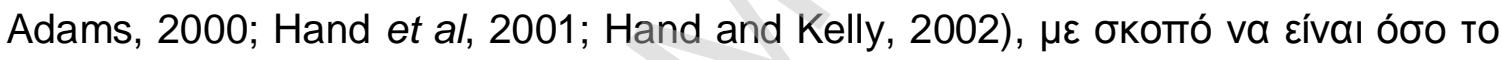

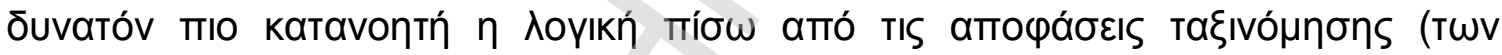

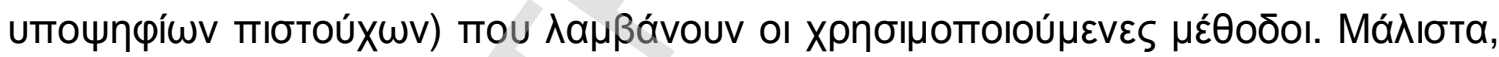

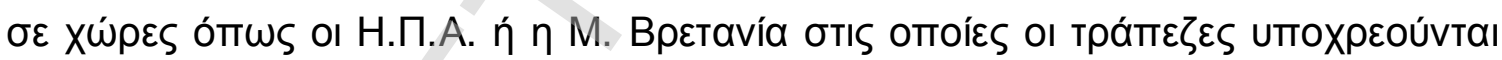

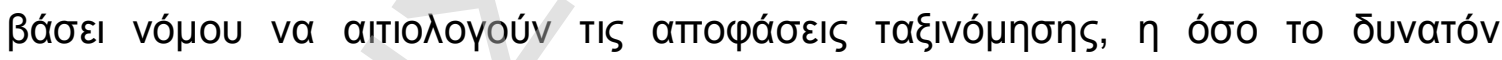

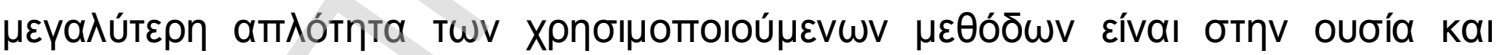

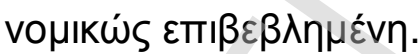

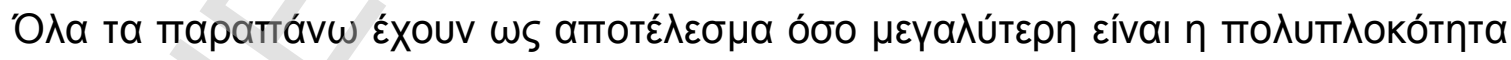

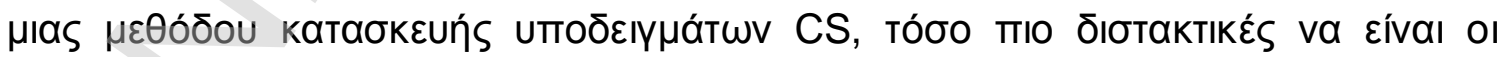

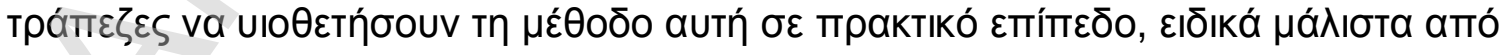

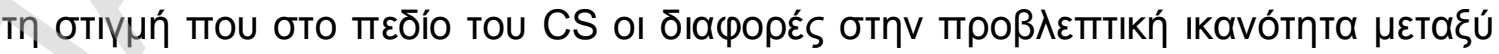

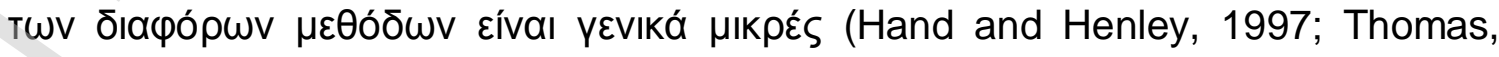




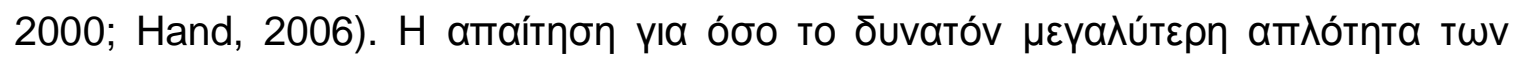

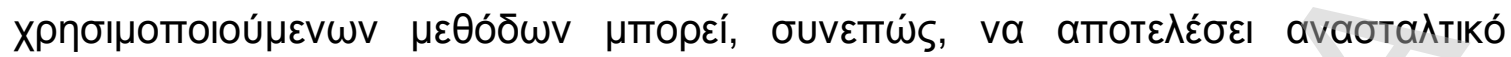

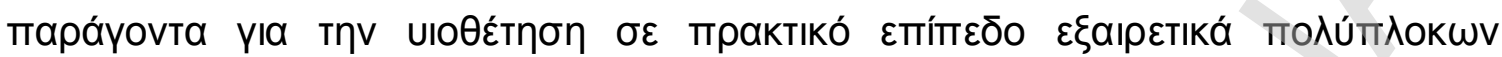

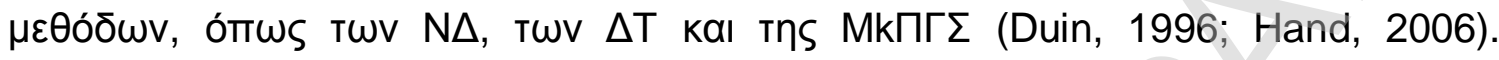

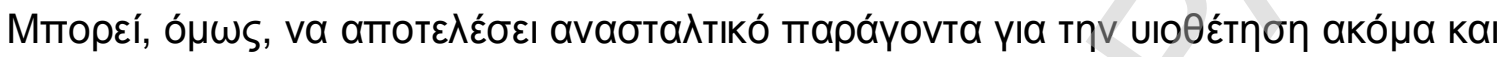

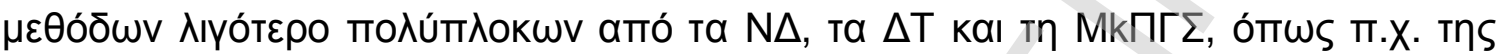

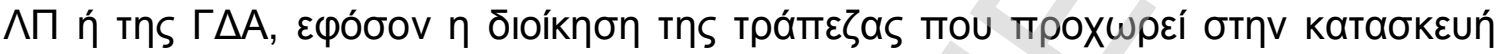

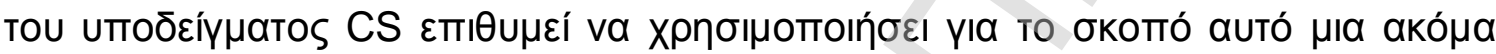

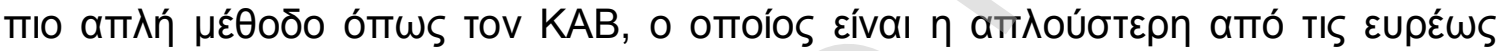

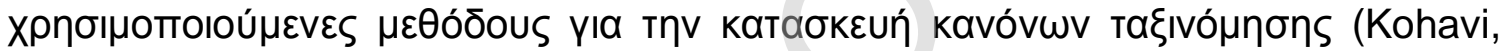
1996; Friedman et al, 1997; Hand and Yu, 2001).

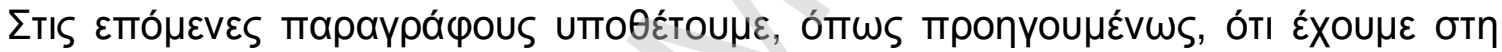

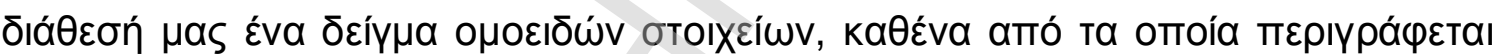

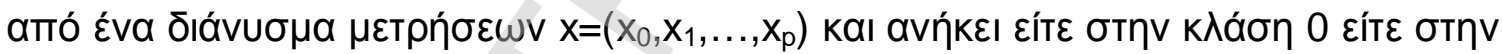

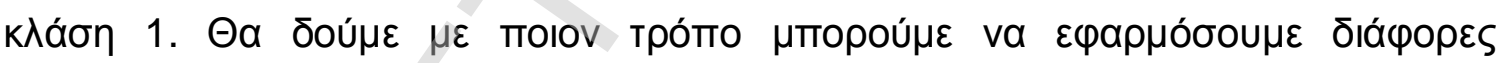

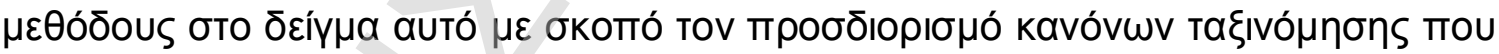

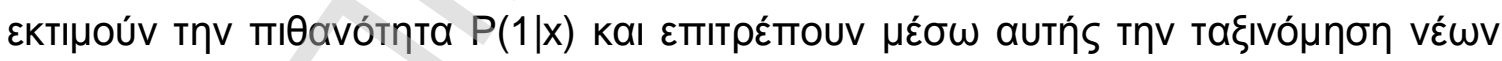

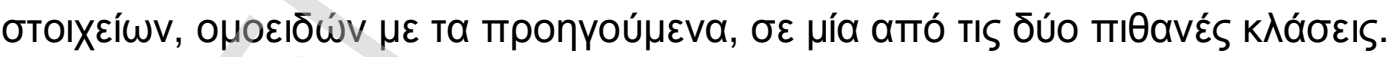

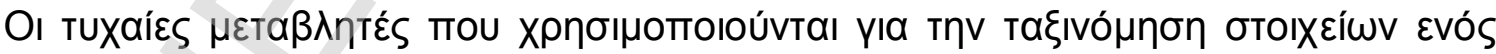

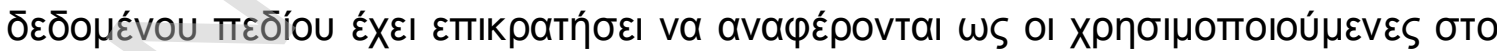

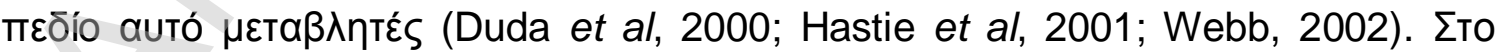

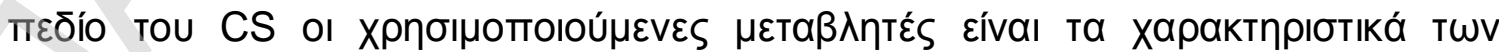
пібтои́х 


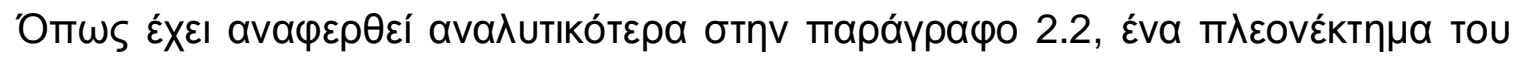

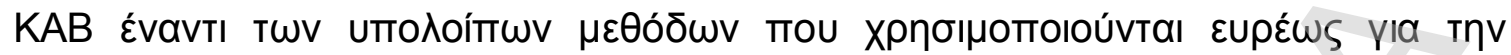

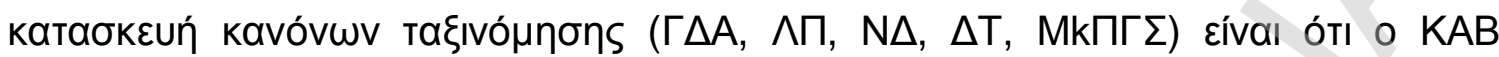

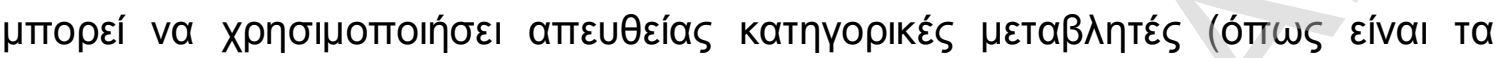

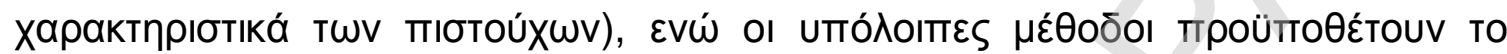

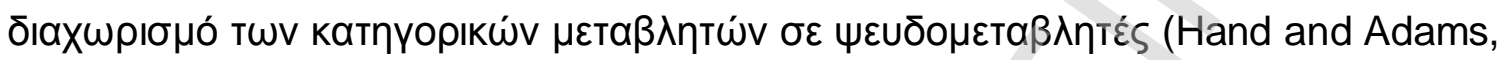
2000; Hand and Yu, 2001; Baesens et al, 2002).

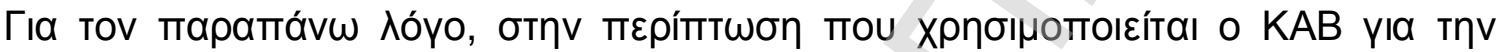

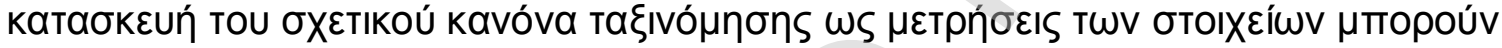

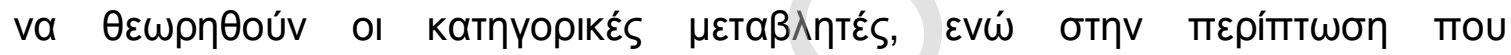

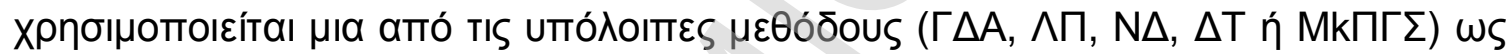

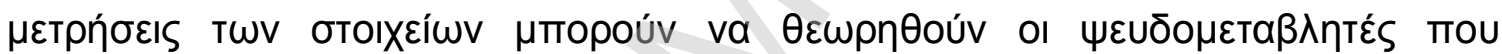

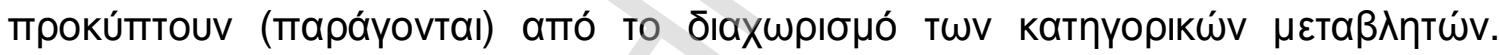

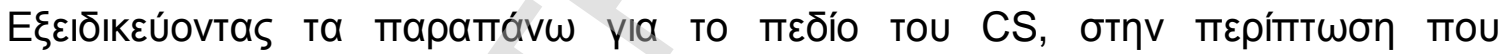

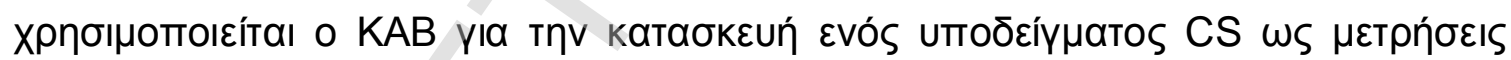

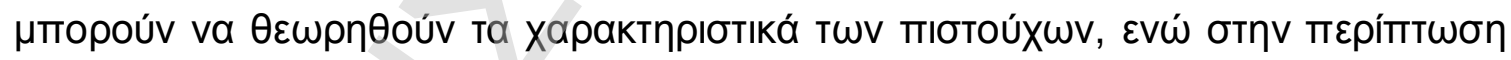

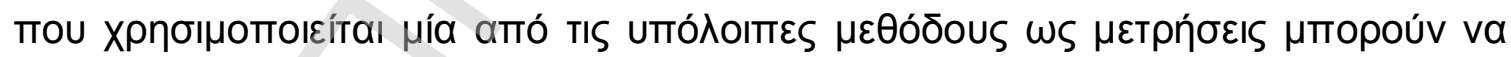

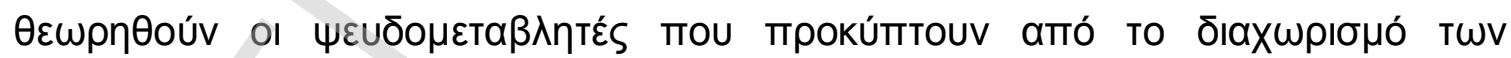

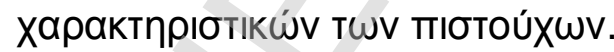




\subsection{Kavóvas tou AтrAoú Bayes}

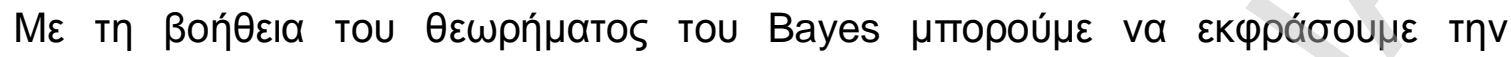

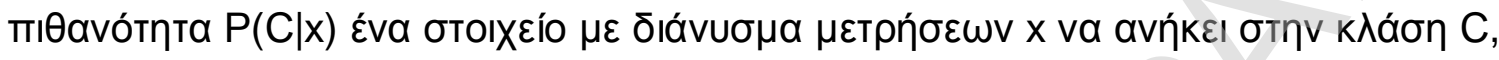

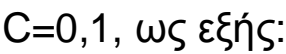

$$
P(C \mid x)=f(x \mid C) P(C) / f(x)
$$

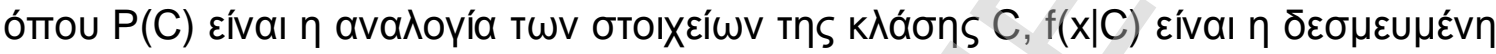

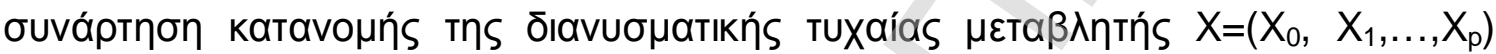

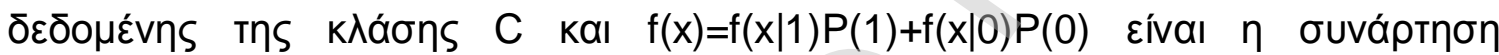
катаvouńs тns X.

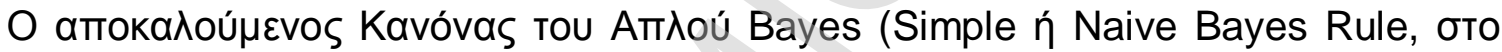

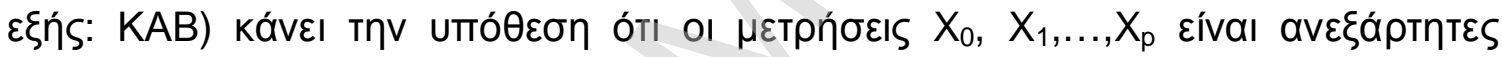

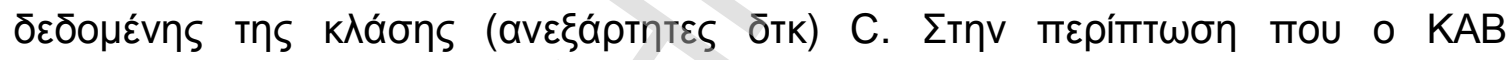

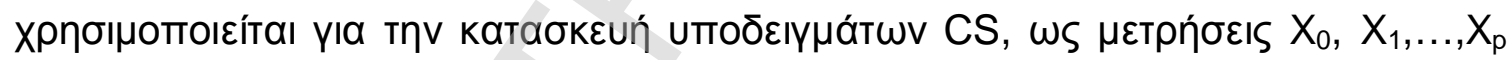

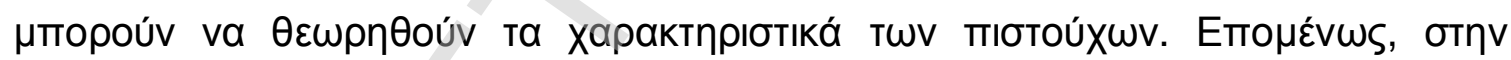

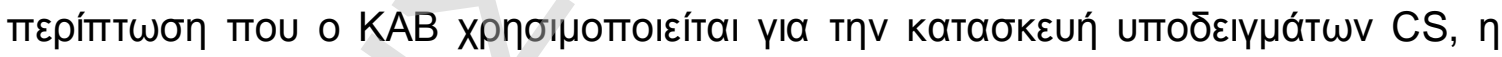

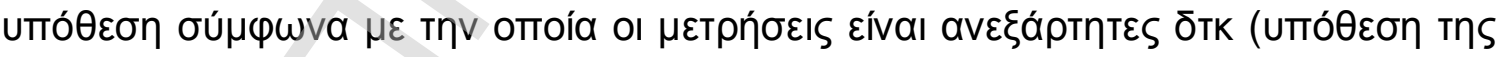

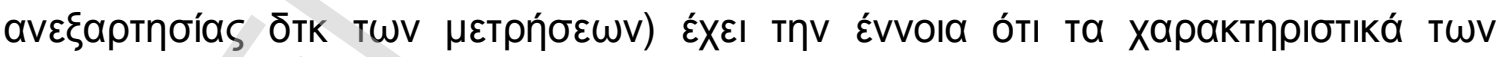

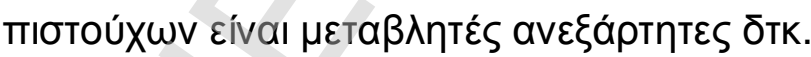

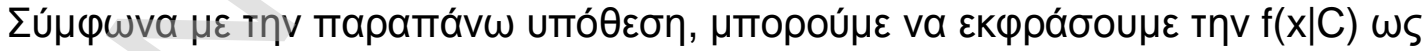

$$
f(x \mid C)=\Pi f_{i}\left(x_{i} \mid C\right)
$$

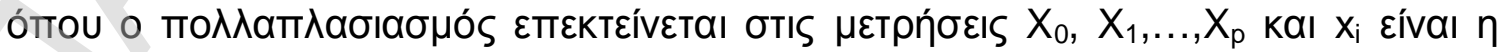

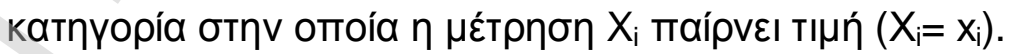




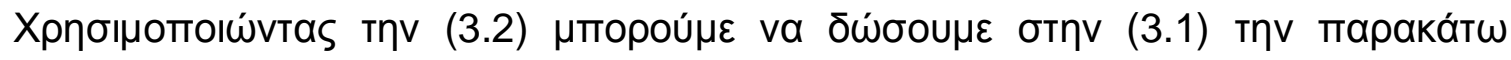
норфи́:

$$
P(C \mid x)=\Pi f_{i}\left(x_{i} \mid C\right) P(C) / f(x)
$$

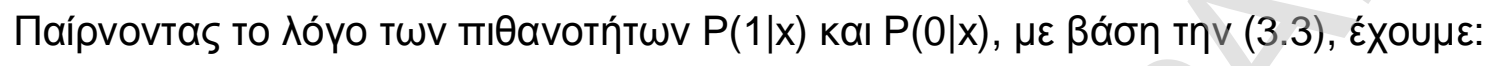

$$
\frac{P(1 \mid x)}{P(0 \mid x)}=\frac{P(1)}{P(0)} \Pi \frac{f_{i}\left(x_{i} \mid 1\right)}{f_{i}\left(x_{i} \mid 0\right)}
$$

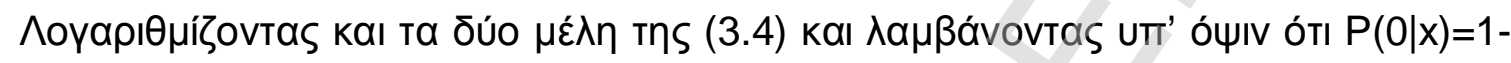
$P(1 \mid x), \varepsilon ́ X O u \mu \varepsilon:$

$$
\ln \frac{P(1 \mid x)}{1-P(1 \mid x)}=\Sigma w_{i}\left(x_{i}\right)+b
$$

о́тоо

$$
w_{i}\left(x_{i}\right)=\ln \frac{f_{i}\left(x_{i} \mid 1\right)}{f_{i}\left(x_{i} \mid 0\right)}
$$

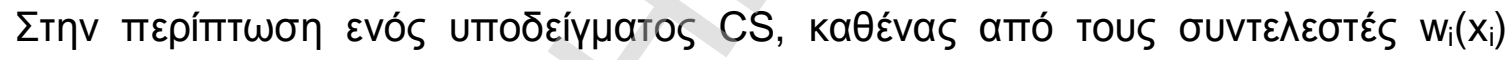

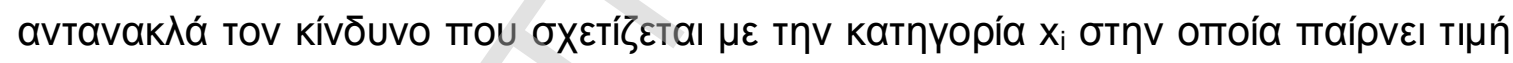

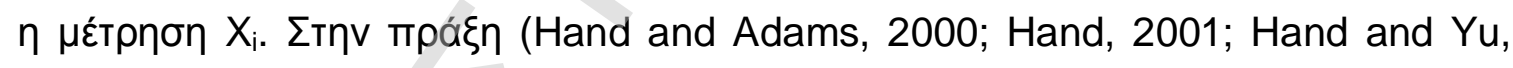

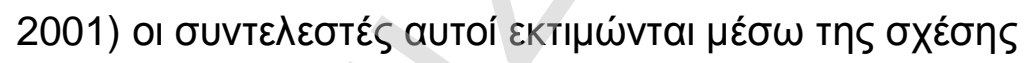

$$
f_{i}\left(x_{i} \mid C\right)=\frac{n_{C}\left(x_{i}\right)+1 / 2}{N_{i C}+1}
$$

о́тоо

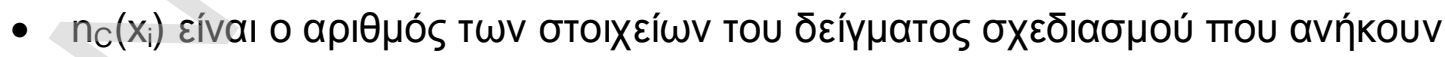

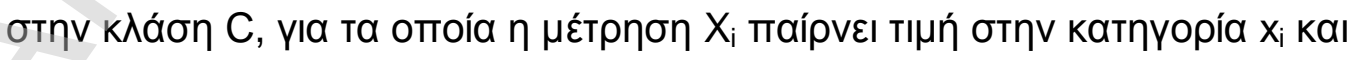

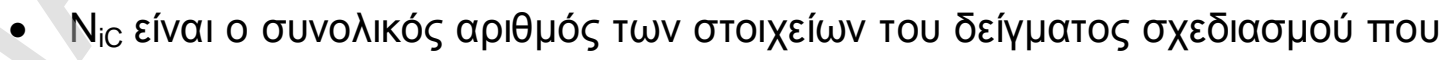

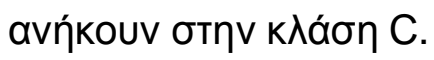




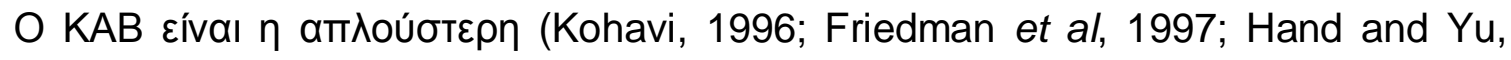

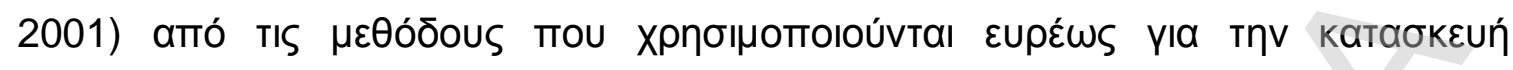

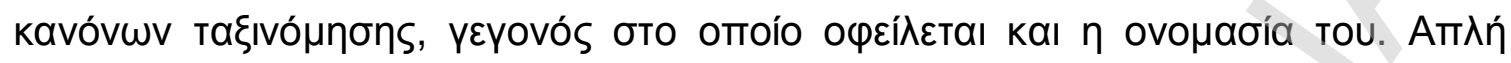

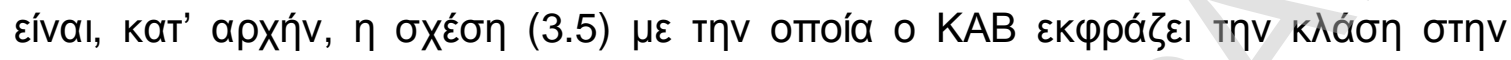

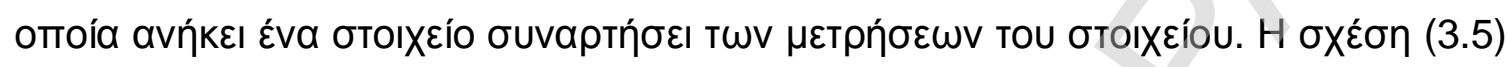

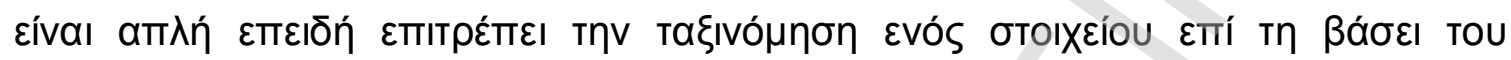

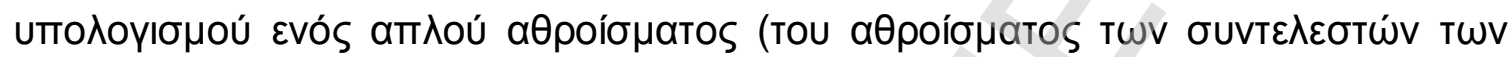

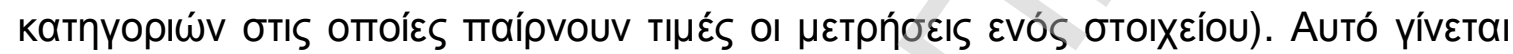

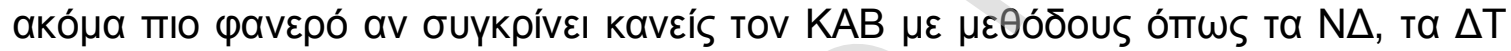

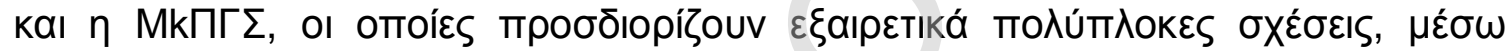

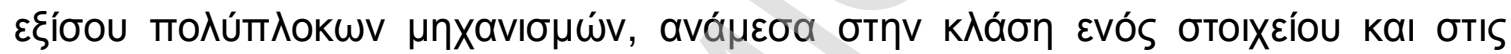

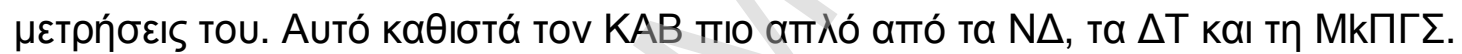

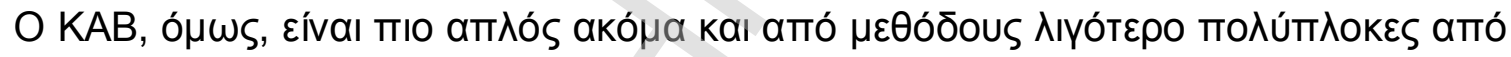

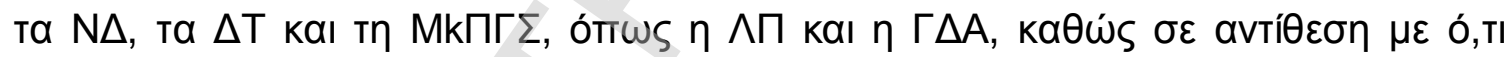

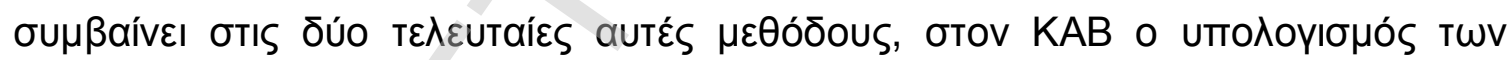

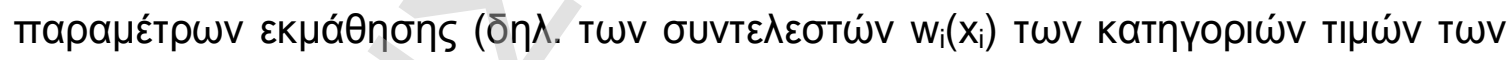

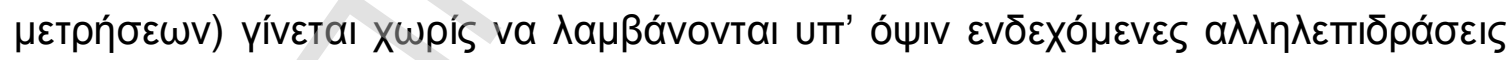

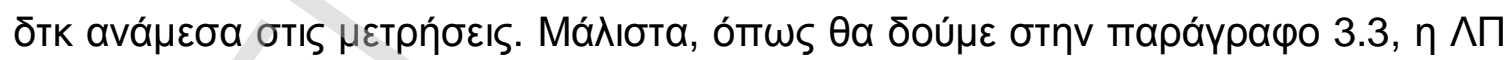

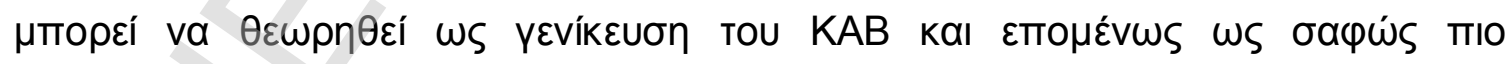

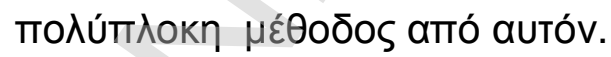

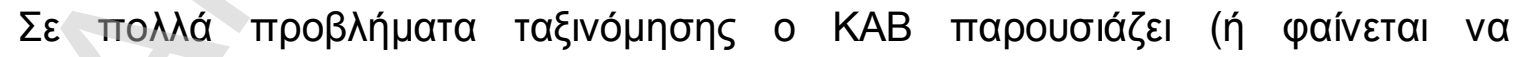

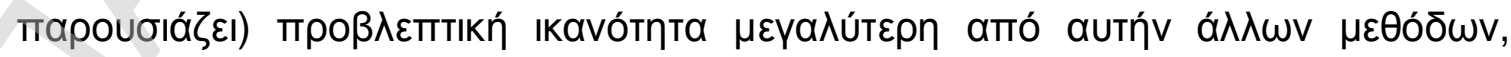

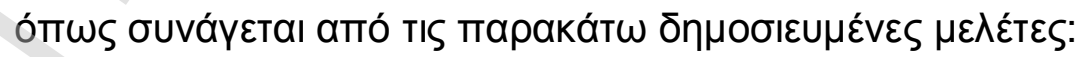




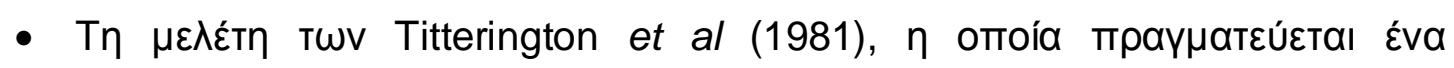

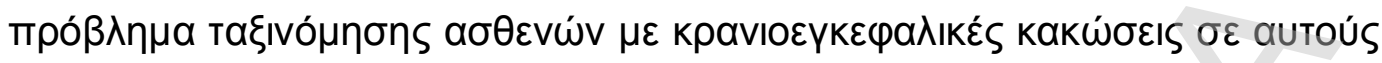

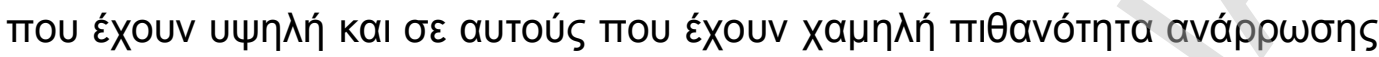

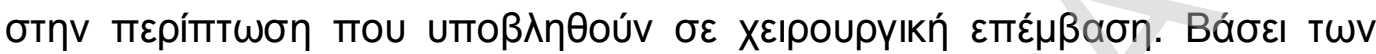

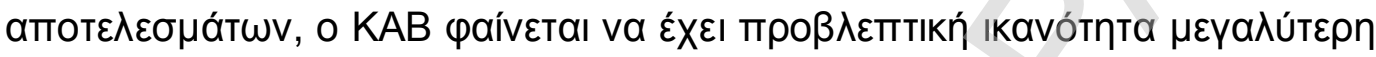

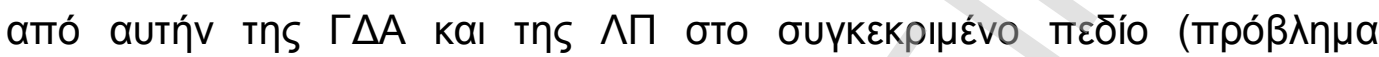

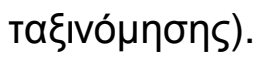

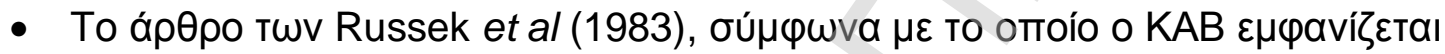

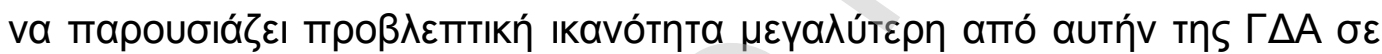

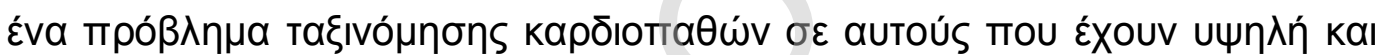

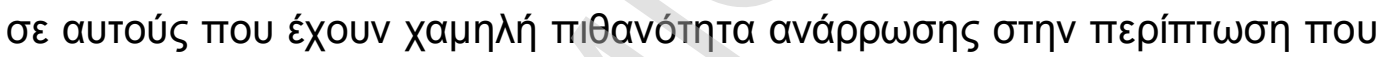

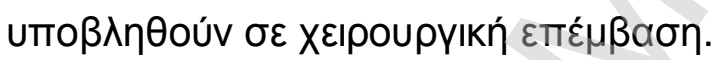

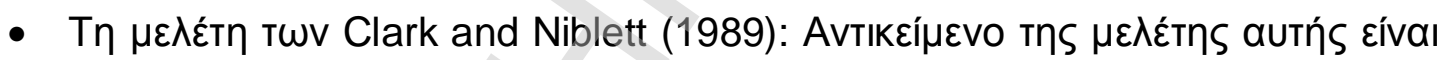

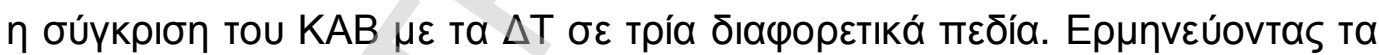

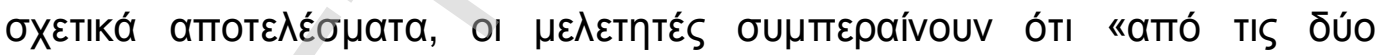

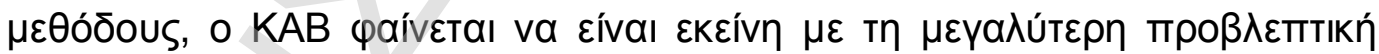

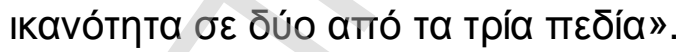

- Tis epyaoírs twv Gammerman and Thatcher (1991) kaı Ohmann et al

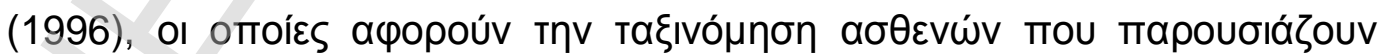

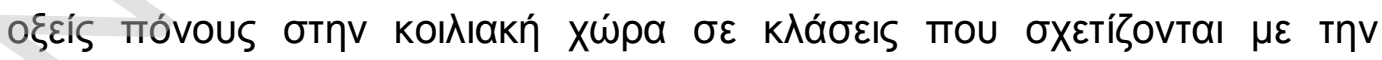

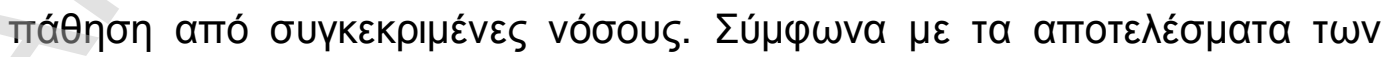

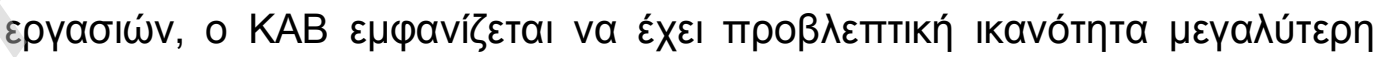

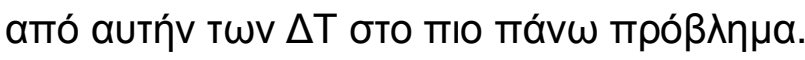




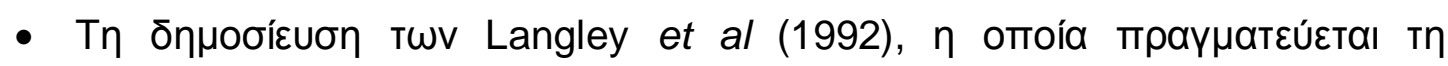

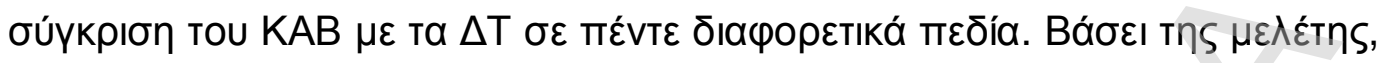

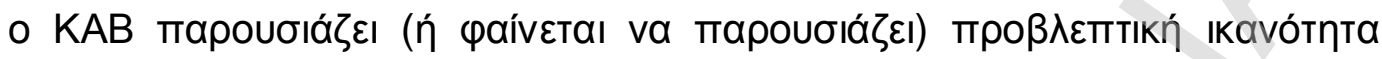

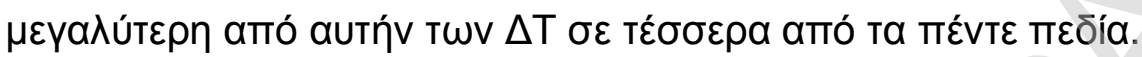

- Ta ápӨpa twv Domingos and Pazzani (1997) kaı Friedman et al (1997), та

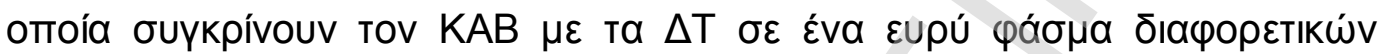

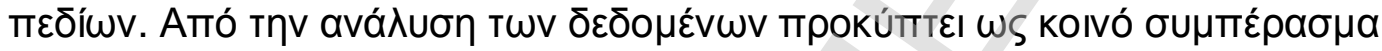

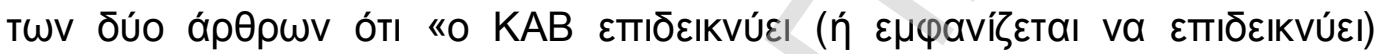

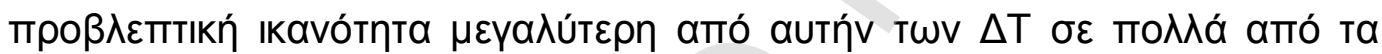
пєठі́́⿱ ».

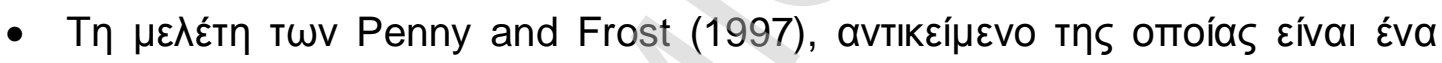

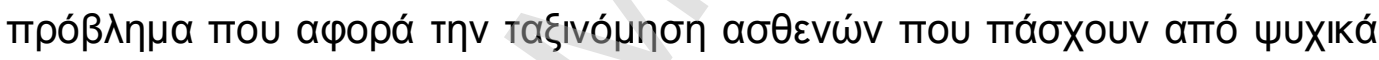

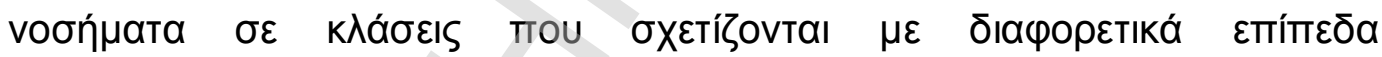

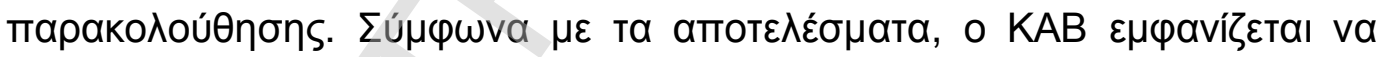

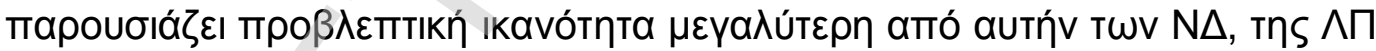

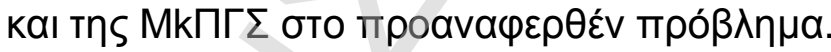

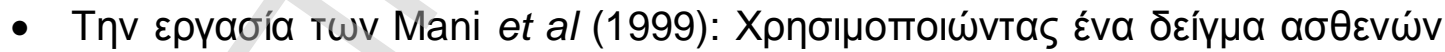

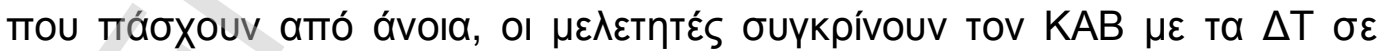

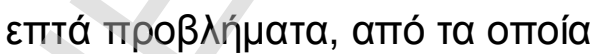

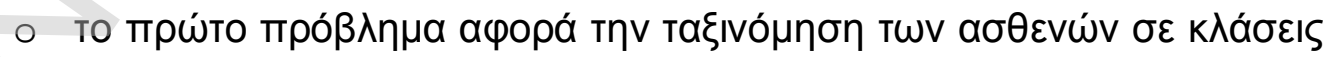

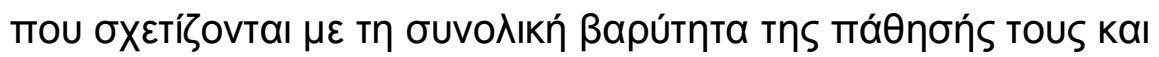

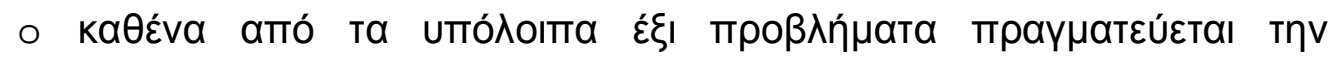

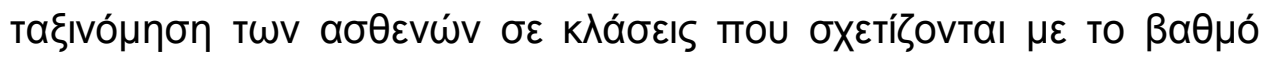




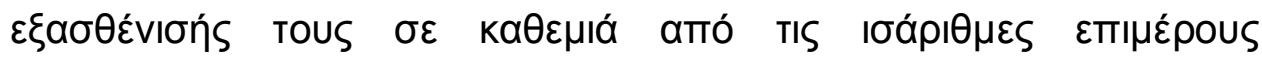

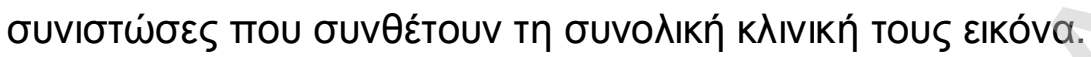

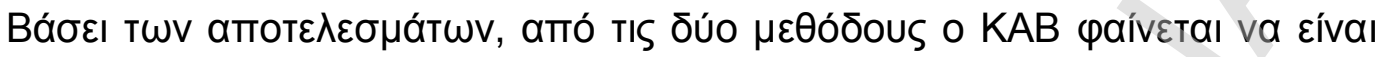

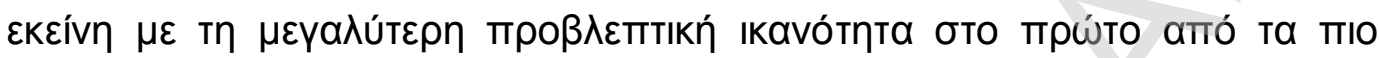

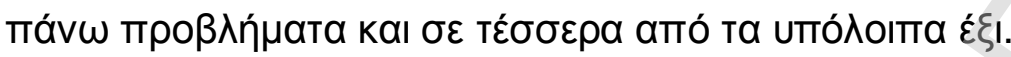

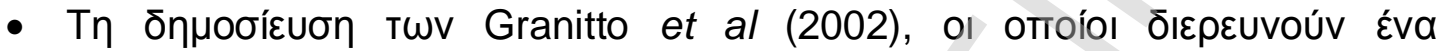

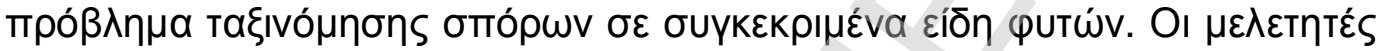

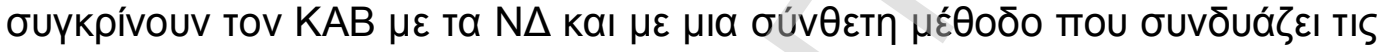

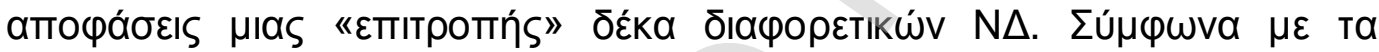

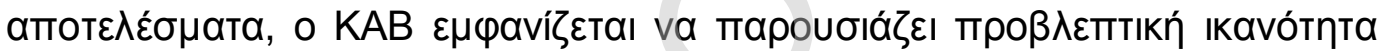

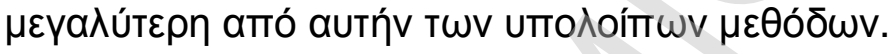

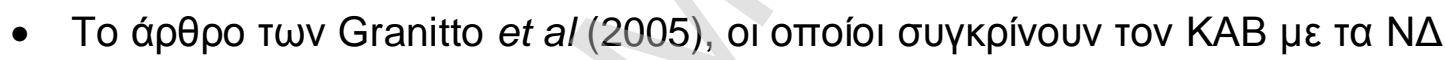

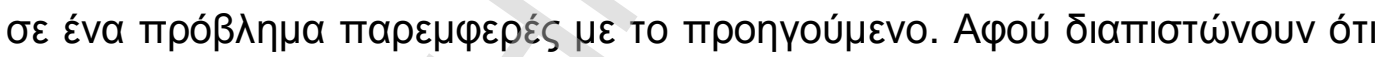

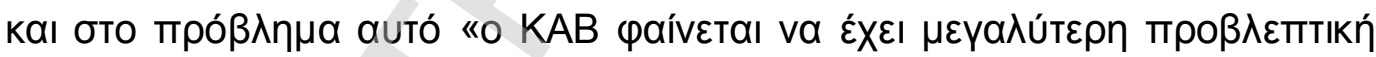

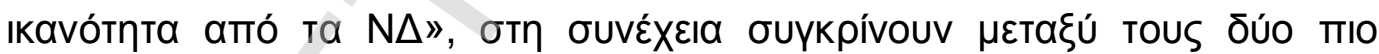

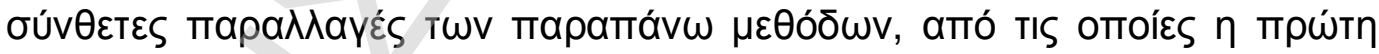

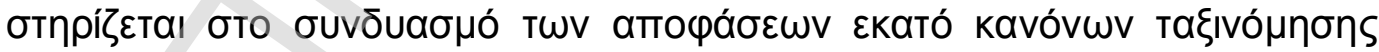

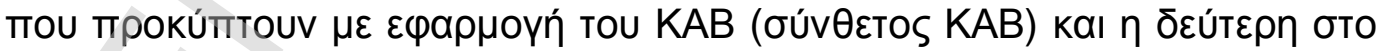

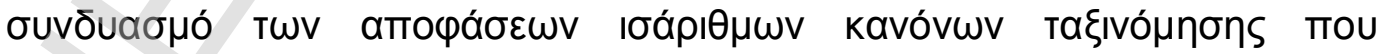

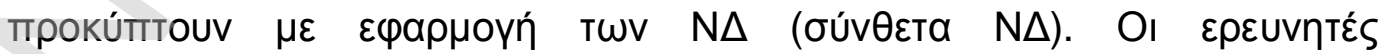

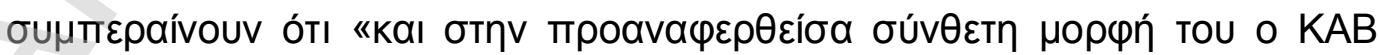

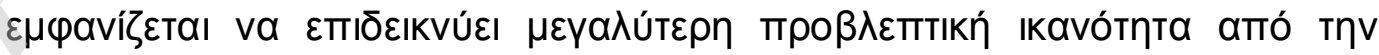

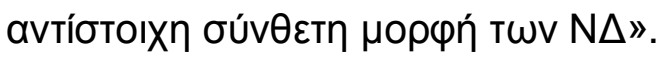




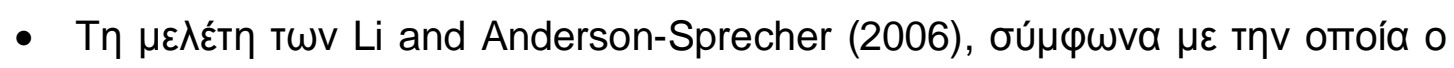

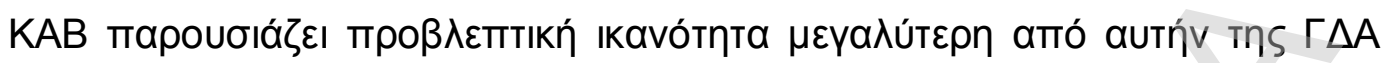

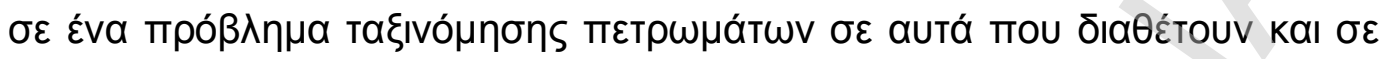

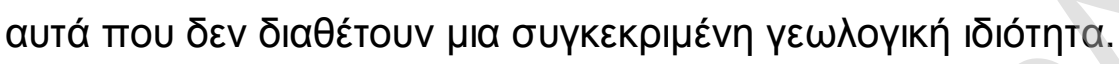

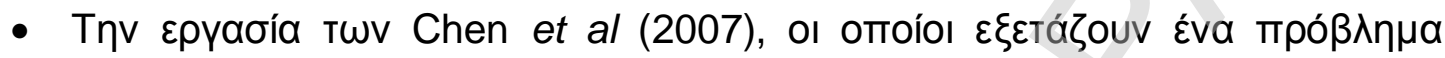

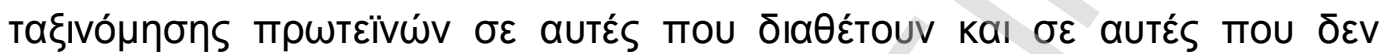

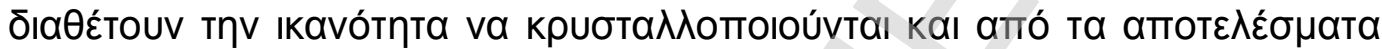

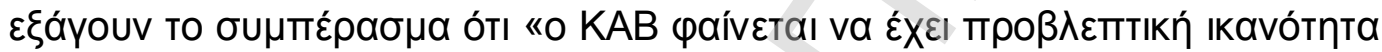

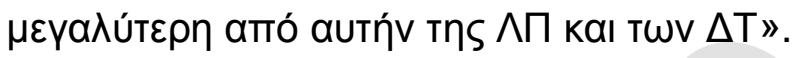

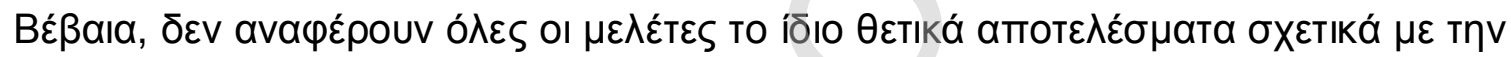

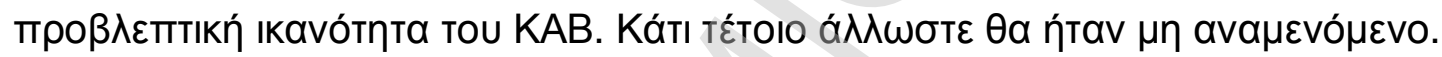

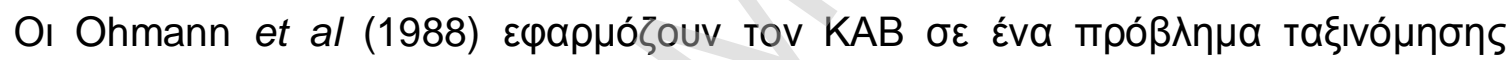

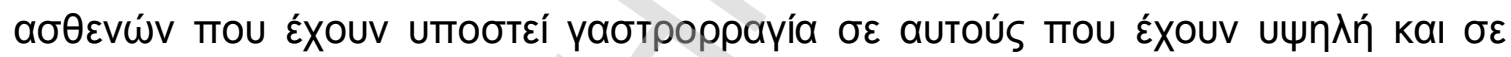

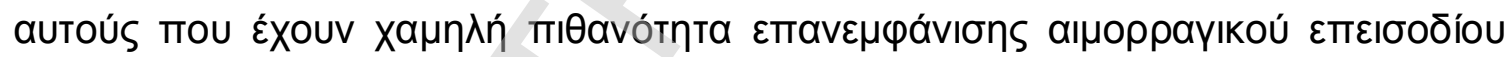

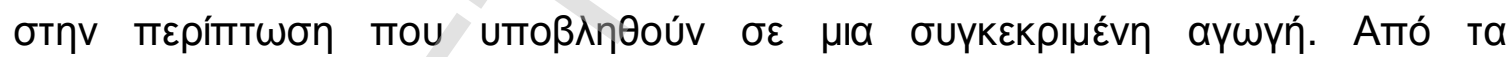

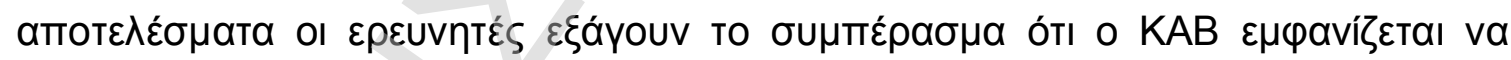

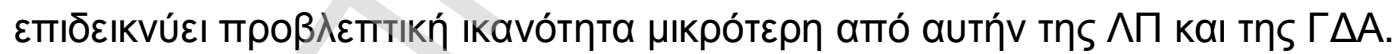

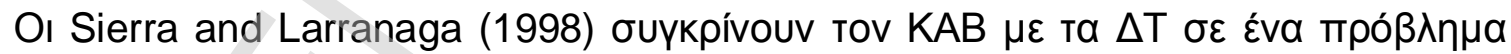

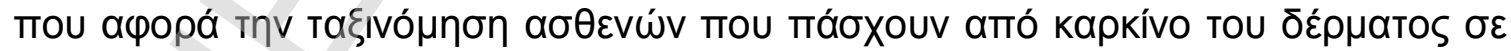

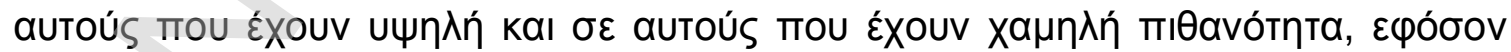

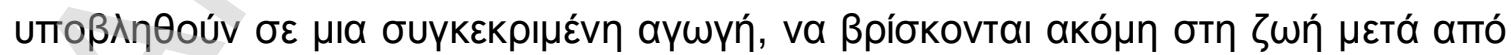

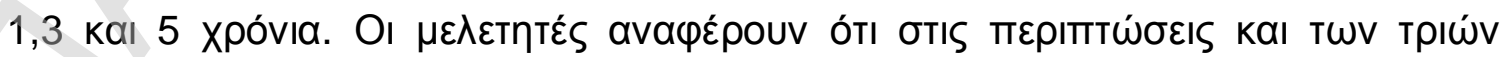




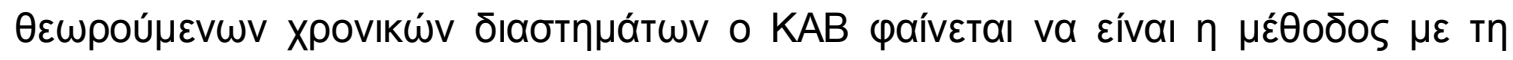

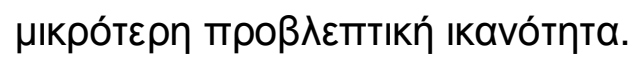

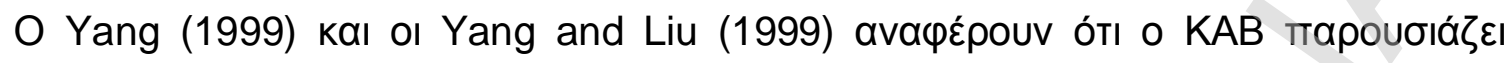

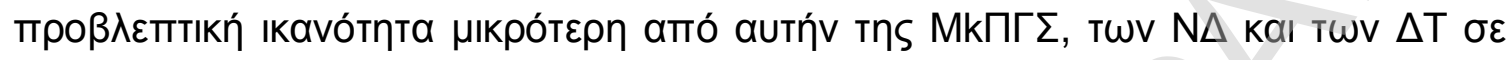

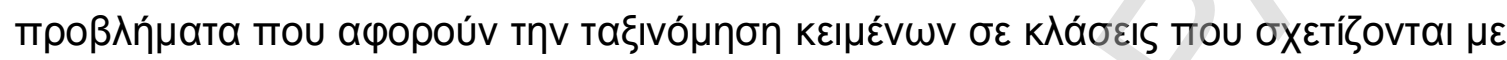

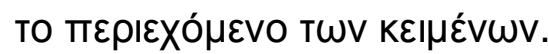

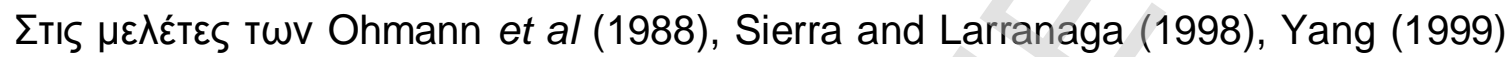

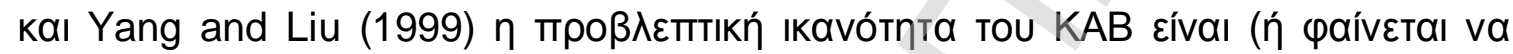

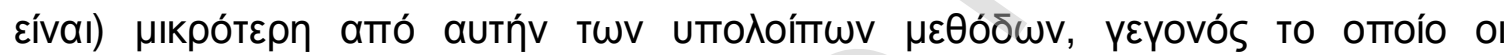

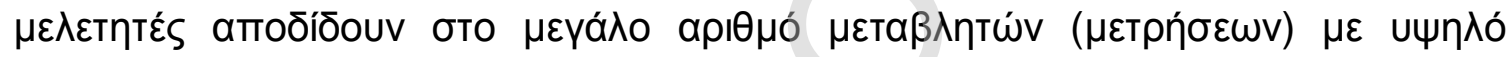

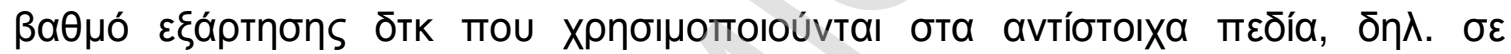

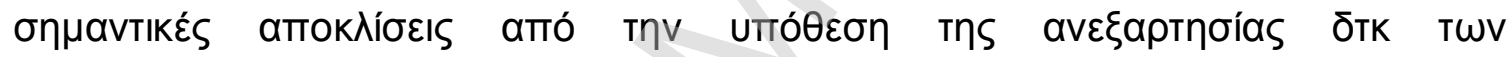

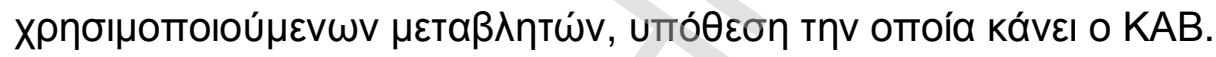

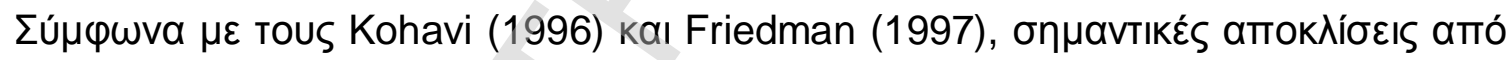

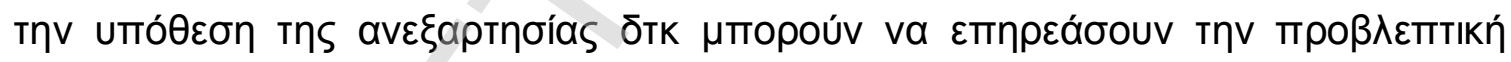

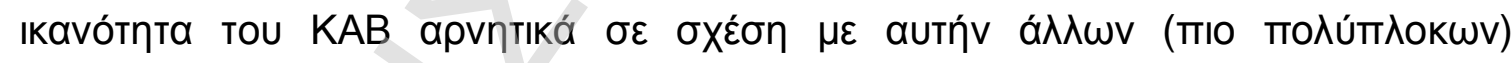

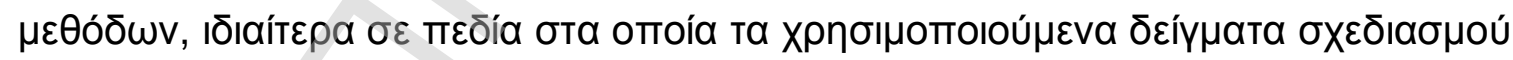

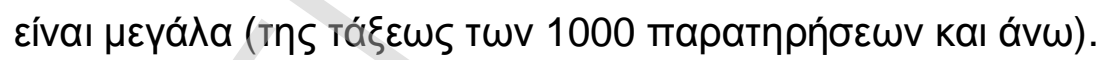

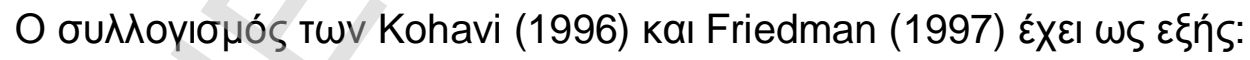

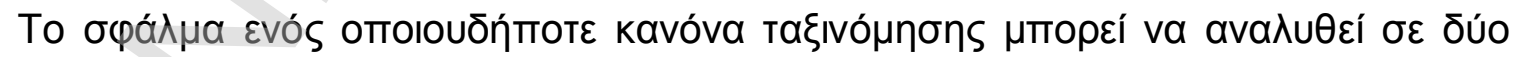

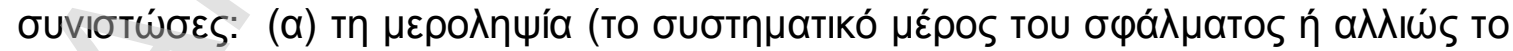

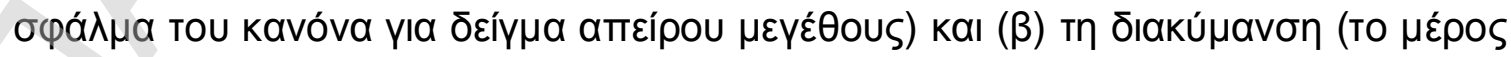




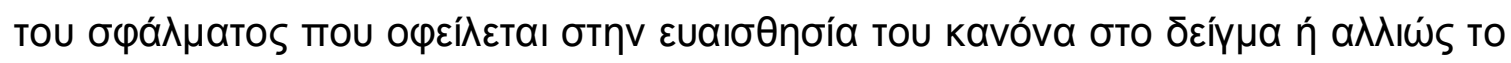

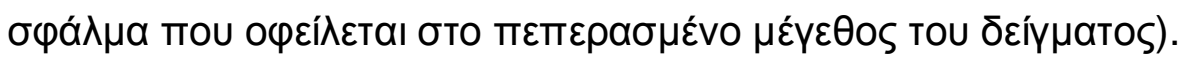

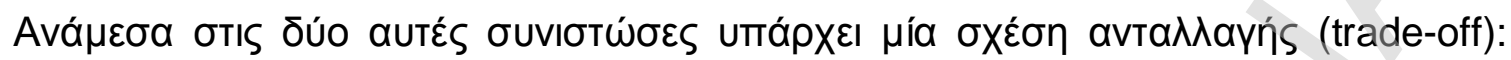

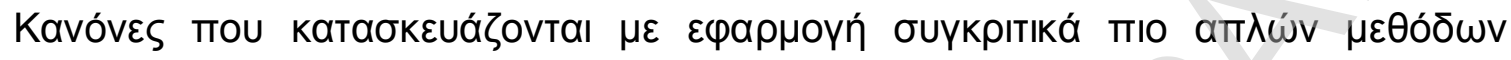

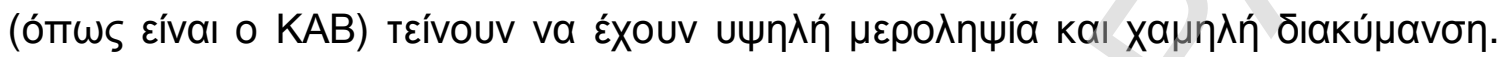

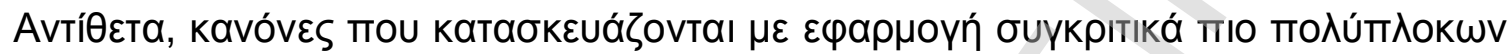

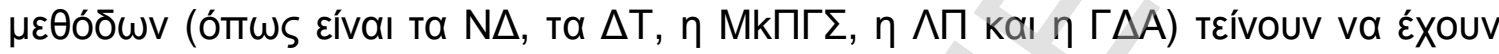

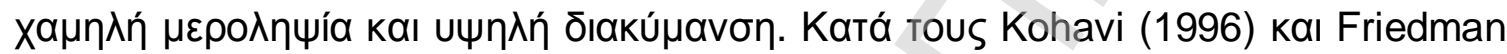

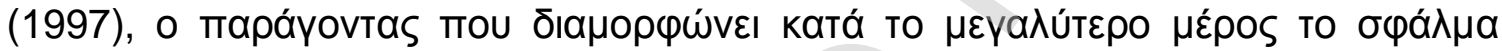

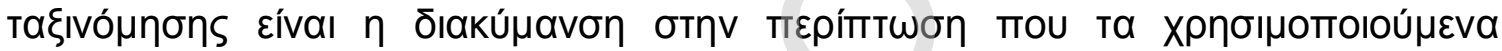

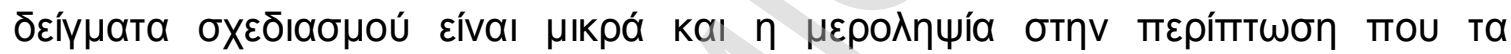

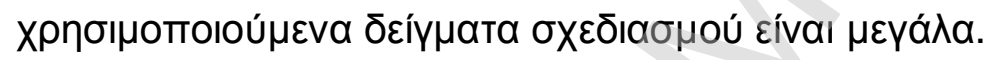

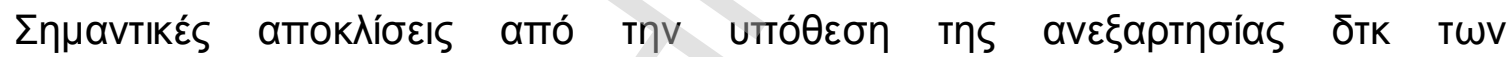

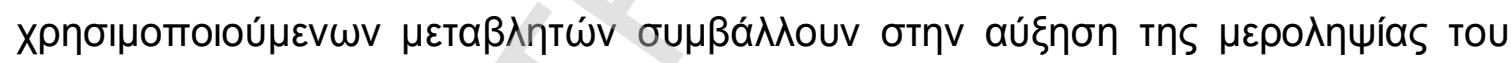

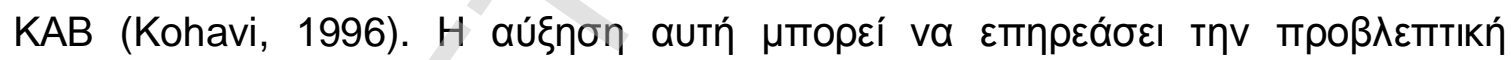

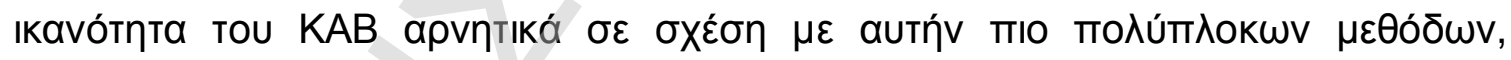

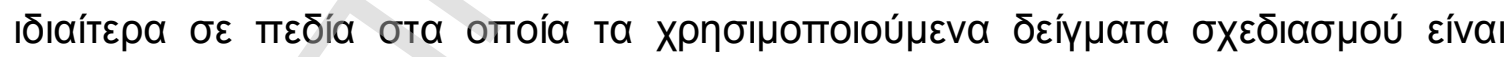

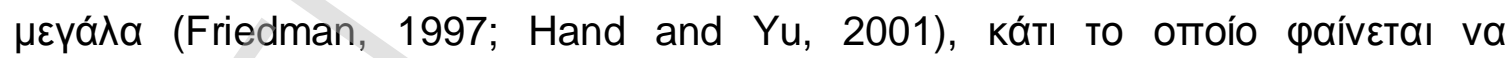

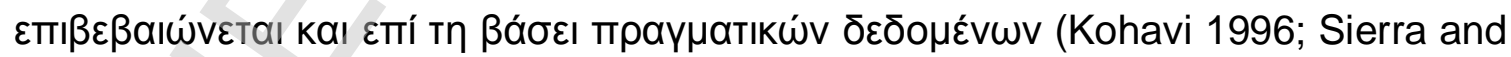

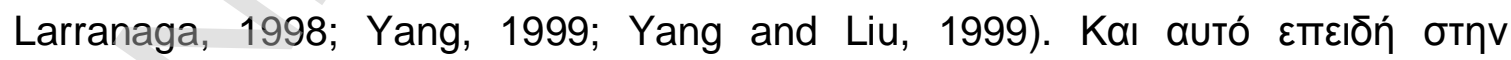

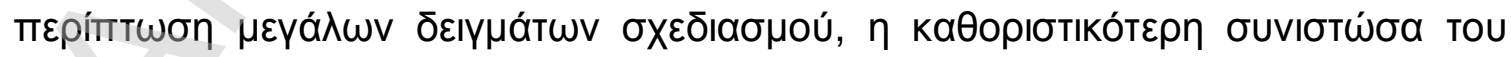

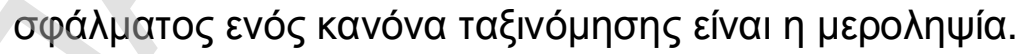




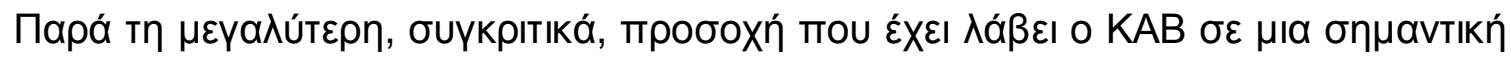

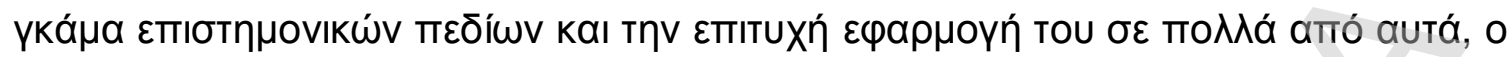

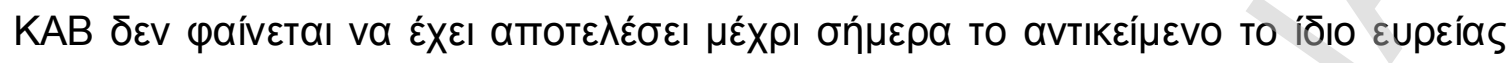

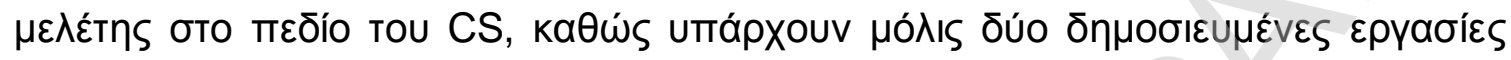

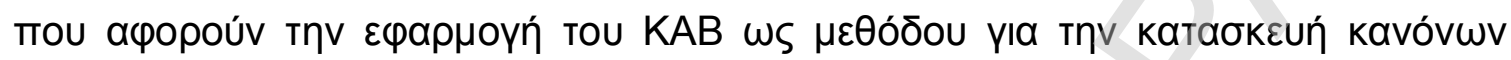

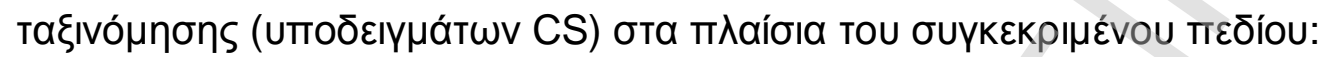

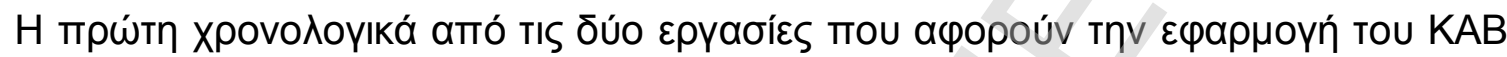

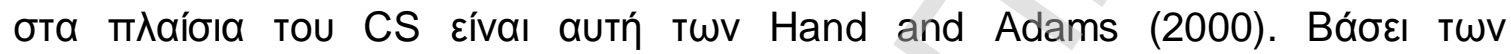

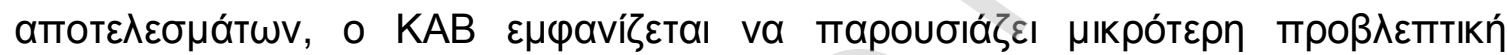

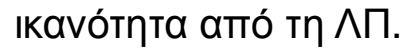

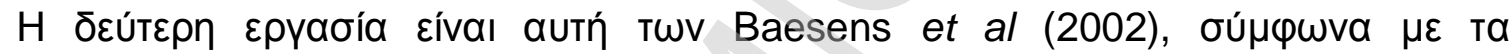

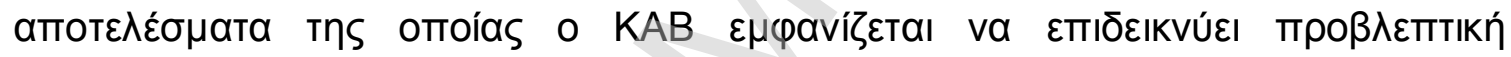

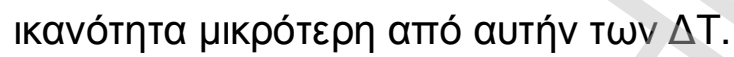

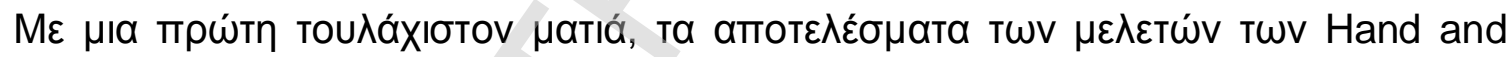

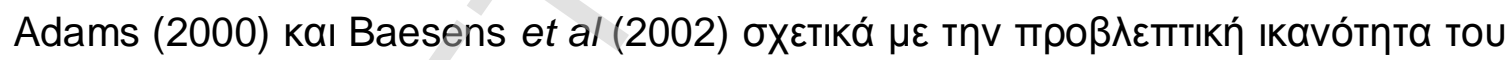

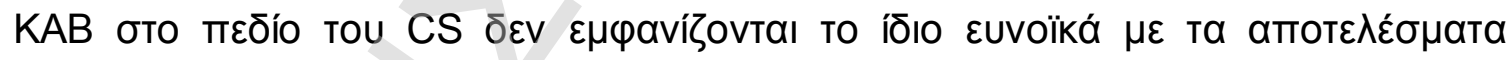

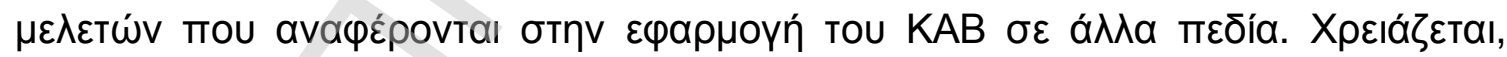

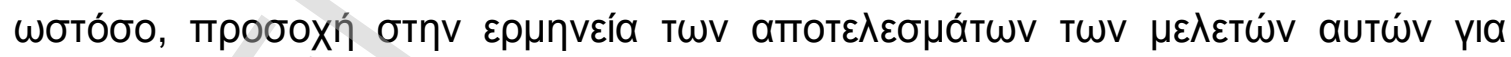

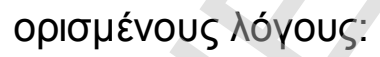

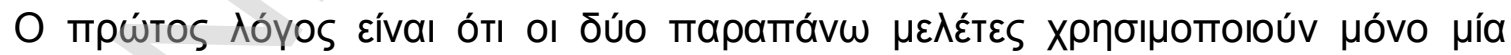

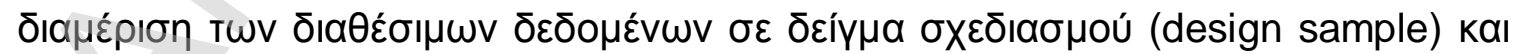

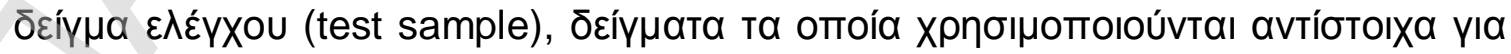

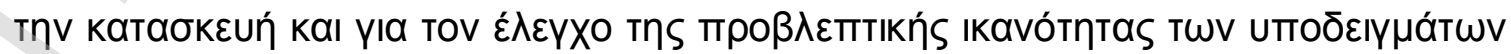




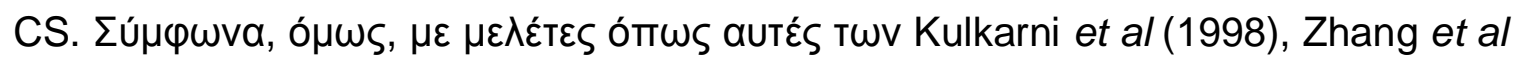

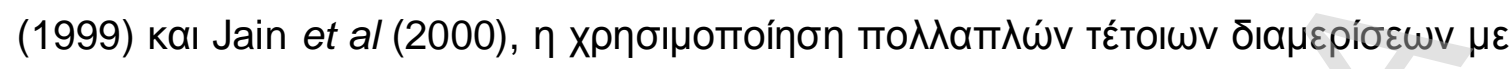

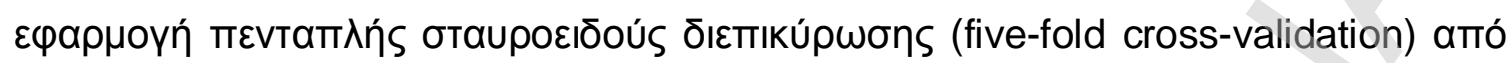

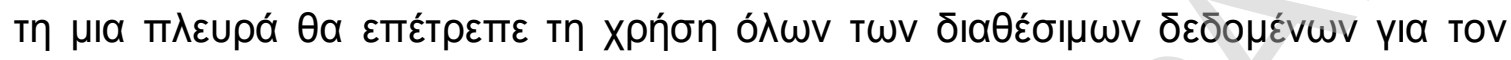

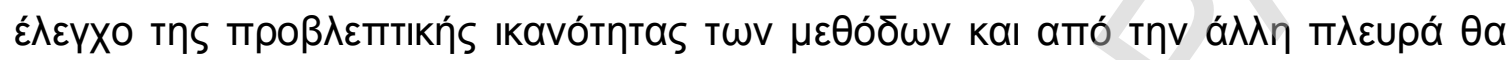

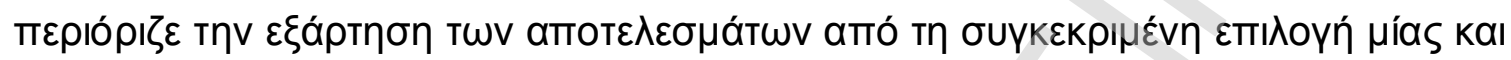

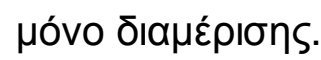

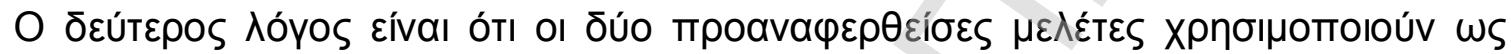

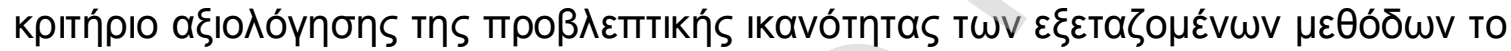

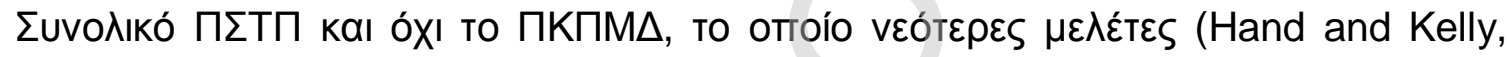

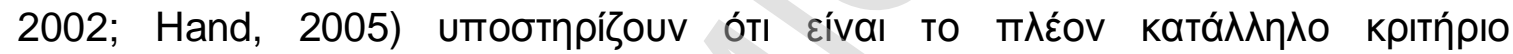

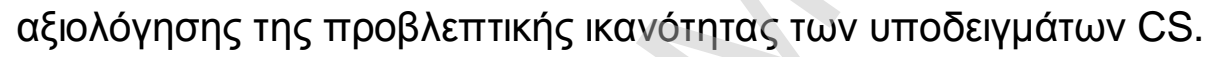

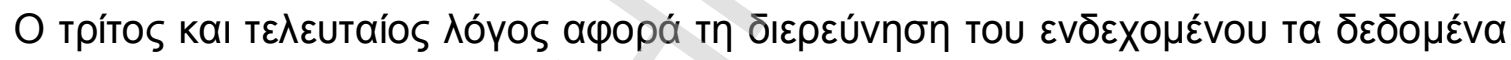

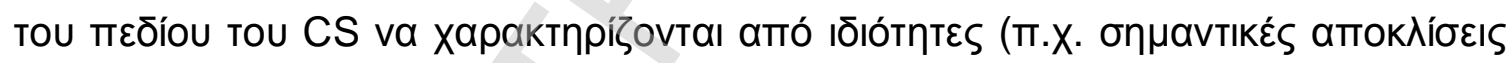

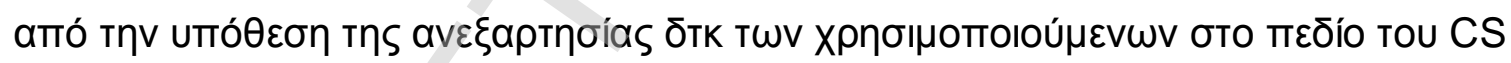

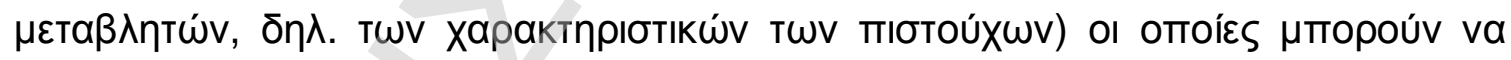

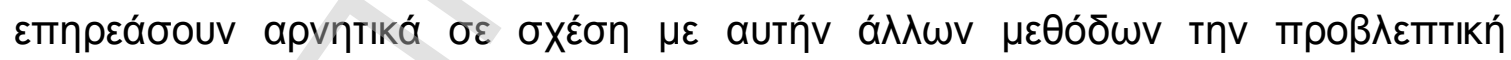

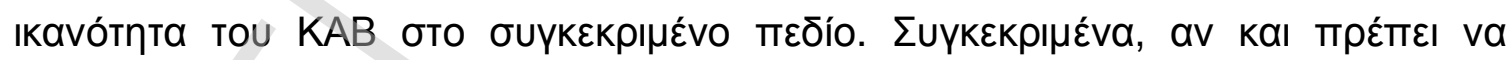

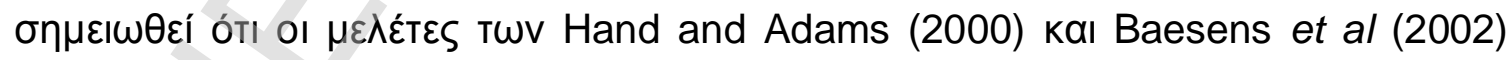

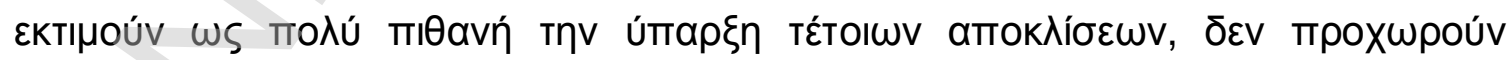

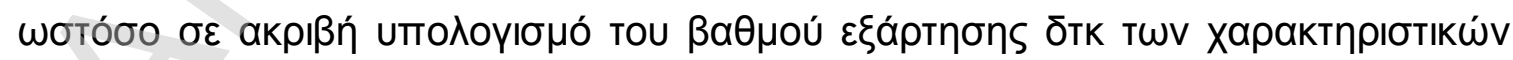

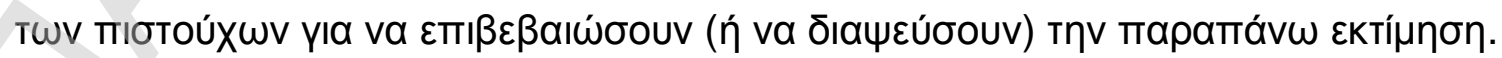




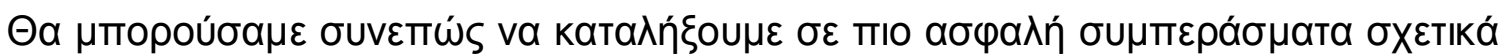

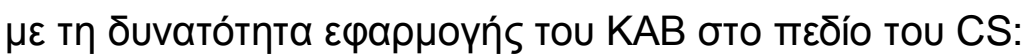

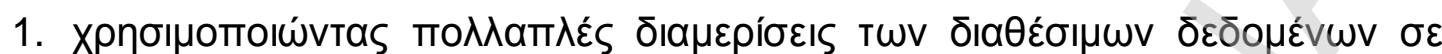

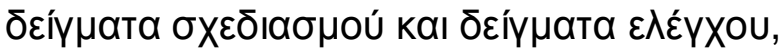

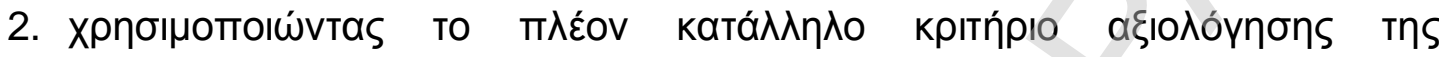

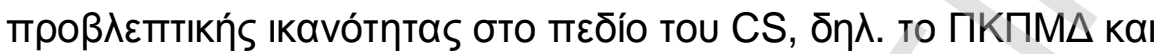

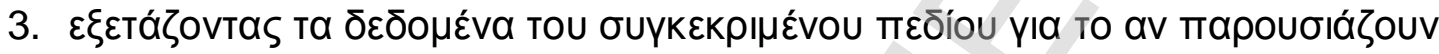

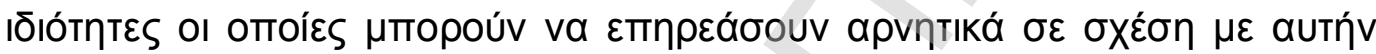

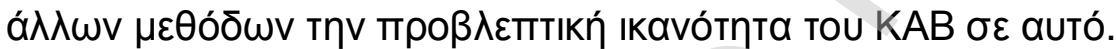

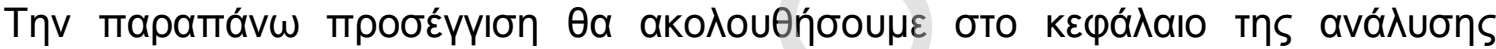

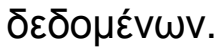

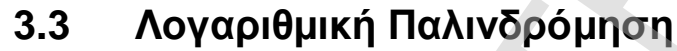

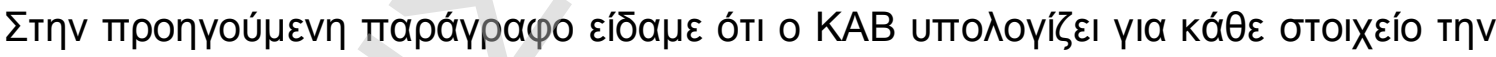

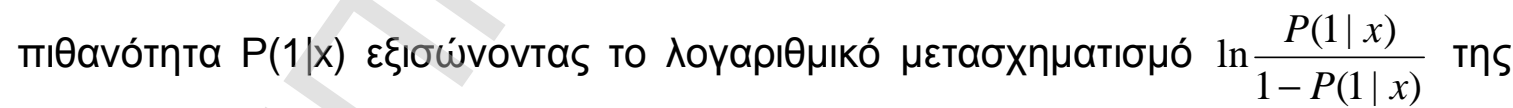

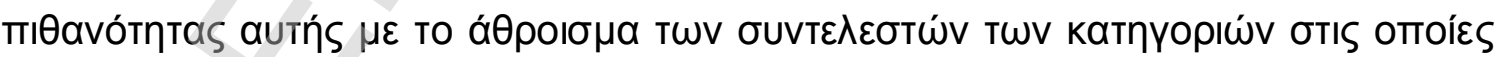

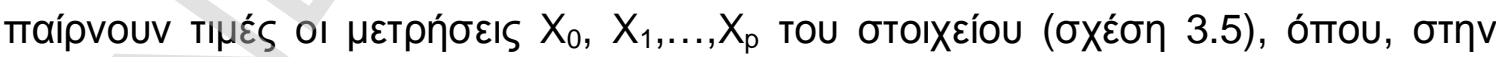

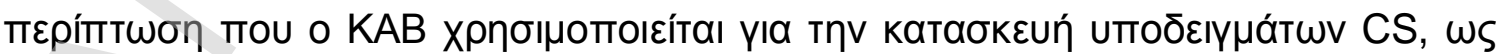

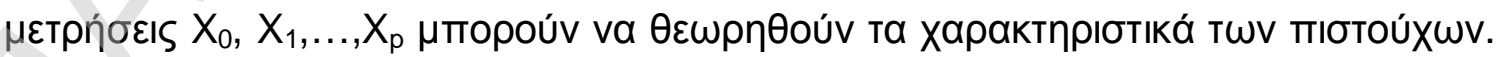

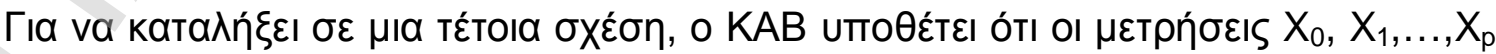

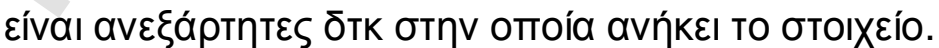




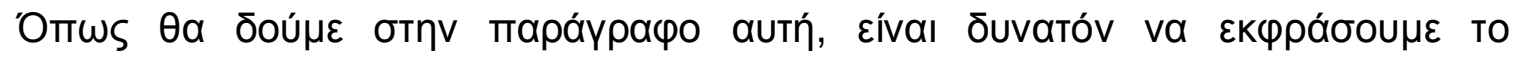

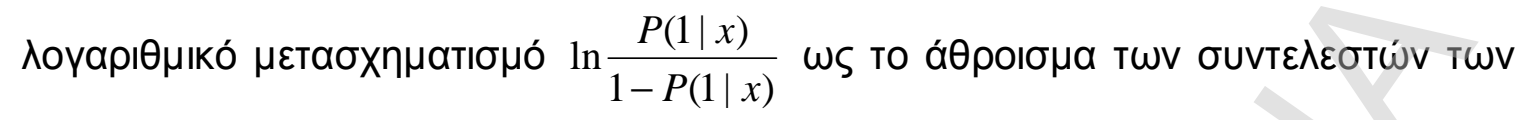

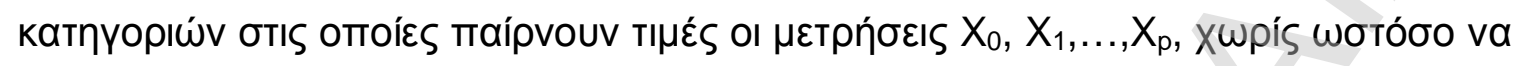

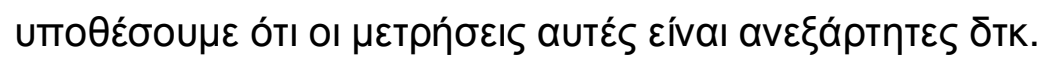

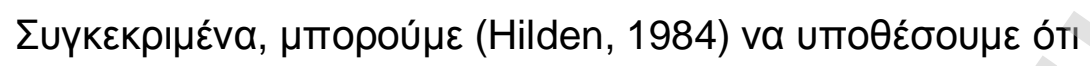

$$
f(x \mid C)=h(x) \sqcap g_{i}\left(x_{i} \mid C\right)
$$

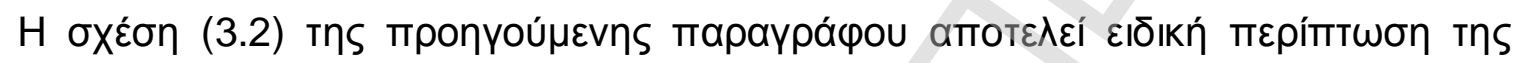

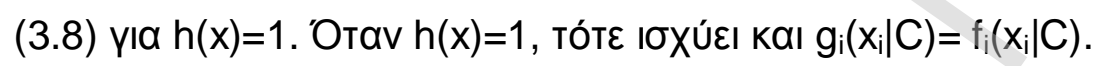

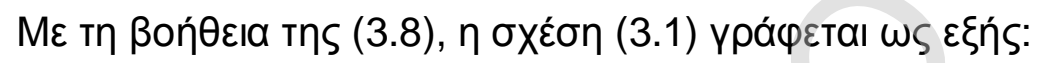

$$
P(C \mid x)=h(x) \sqcap g_{i}\left(x_{i} \mid C\right) P(C) / f(x)
$$

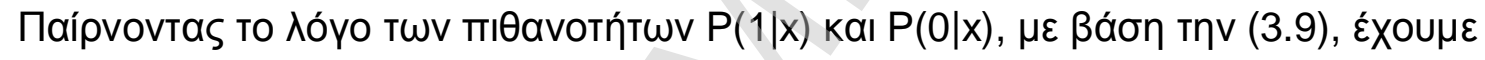

$$
\frac{P(1 \mid x)}{P(0 \mid x)}=\frac{P(1) \Pi g_{i}\left(x_{i} \mid 1\right)}{P(0) \Pi g_{i}\left(x_{i} \mid 0\right)}
$$

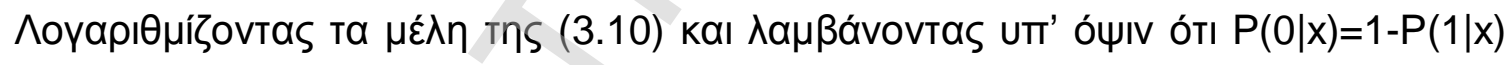
прокÚттвІ о́т।

$$
\ln \frac{P(1 \mid x)}{1-P(1 \mid x)}=\Sigma u_{i}\left(x_{i}\right)+b
$$

о́тоо

$$
u_{i}\left(x_{i}\right)=\ln \frac{g_{i}\left(x_{i} \mid 1\right)}{g_{i}\left(x_{i} \mid 0\right)}
$$

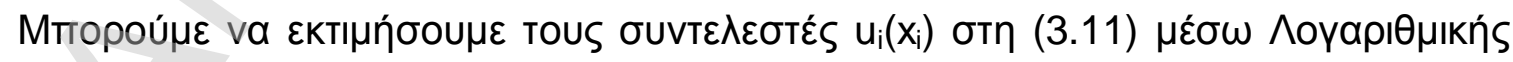

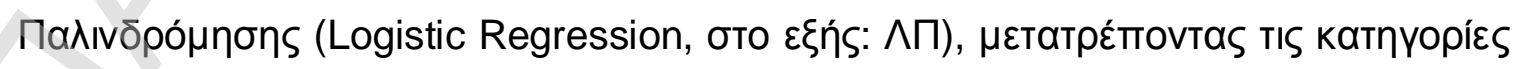

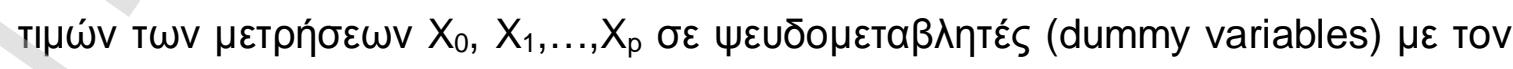




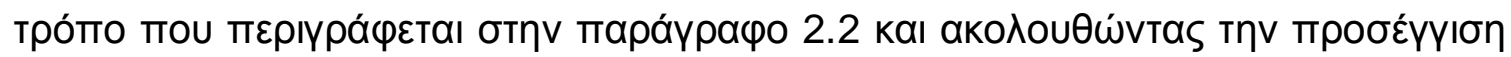

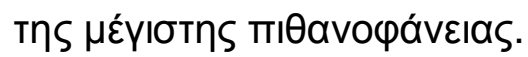

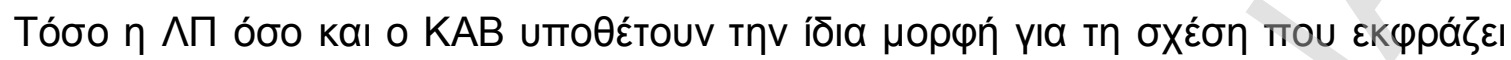

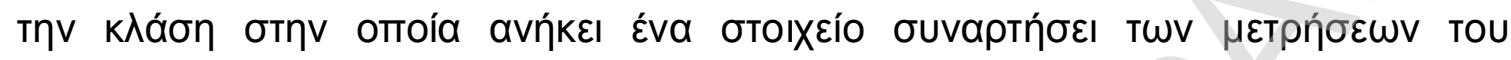

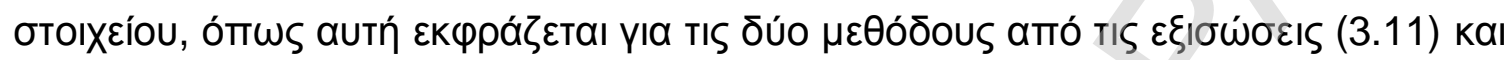

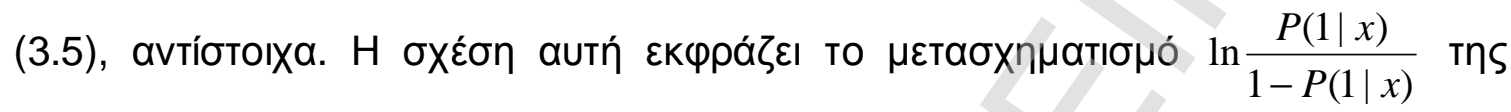

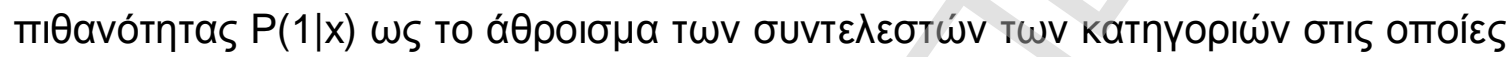

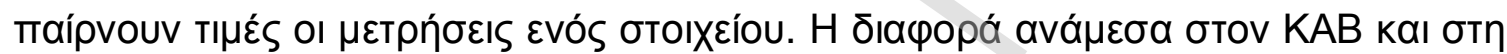

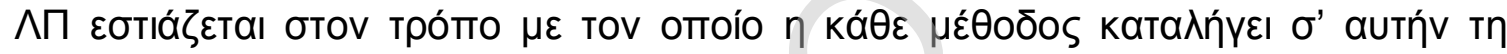

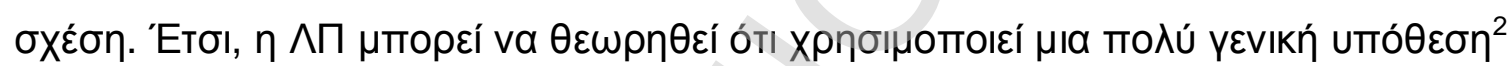

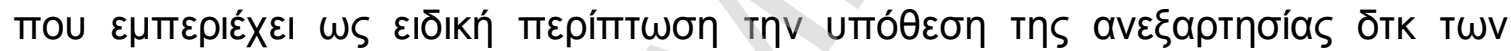

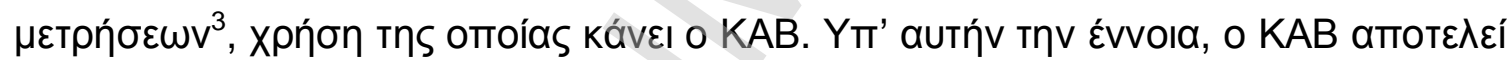

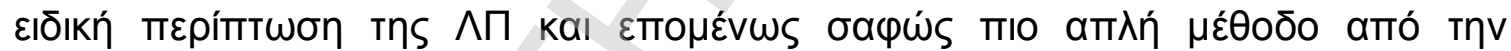

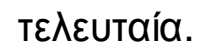

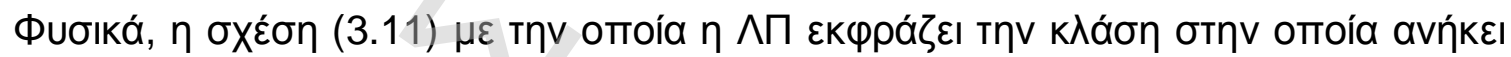

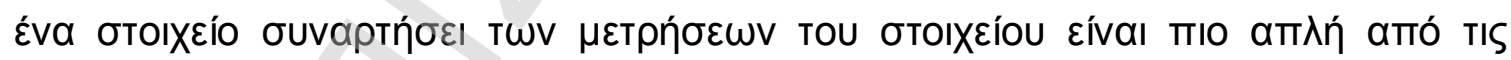

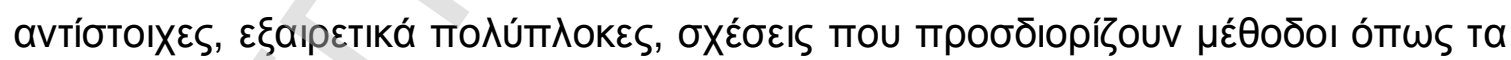

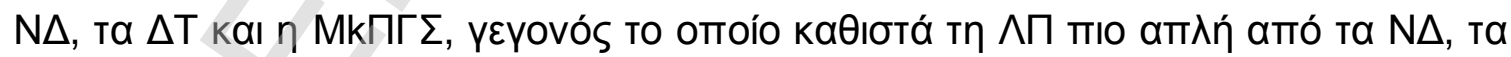

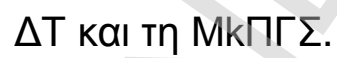

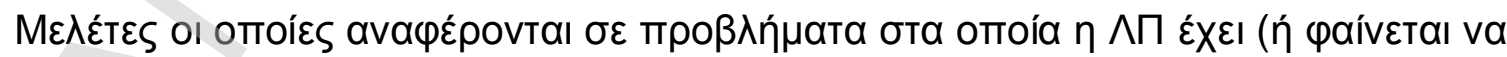

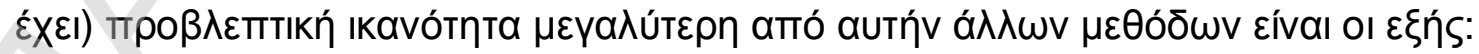

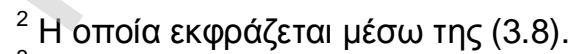

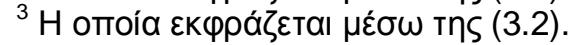




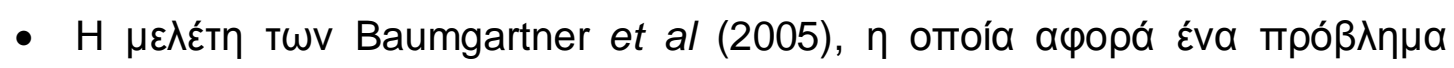

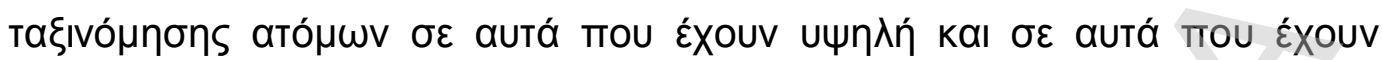

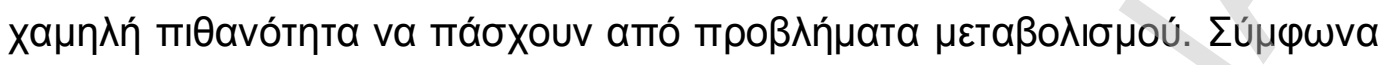

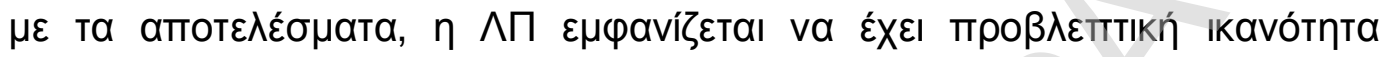

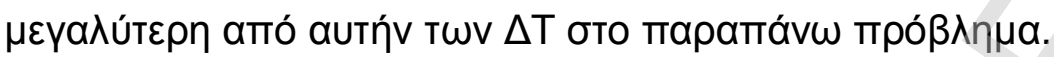

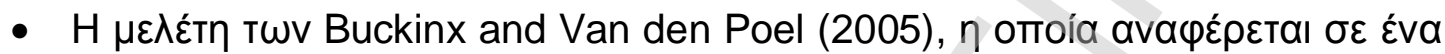

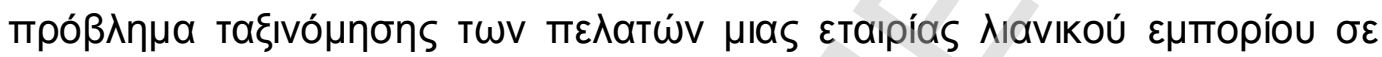

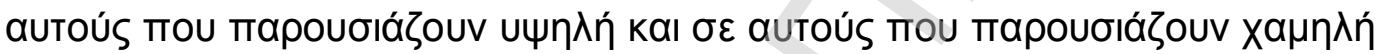

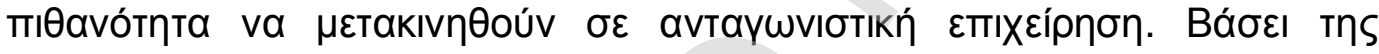

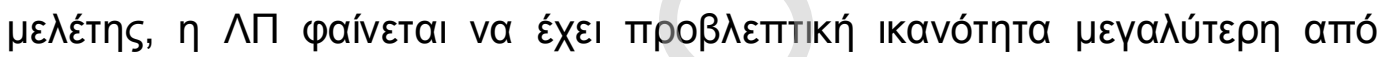

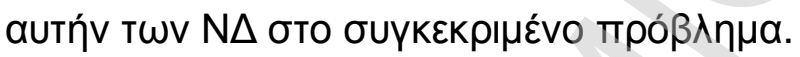

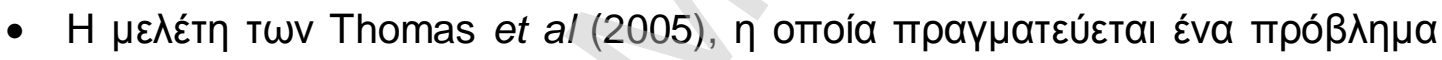

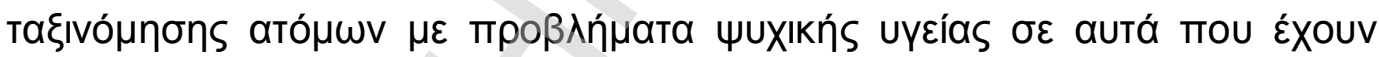

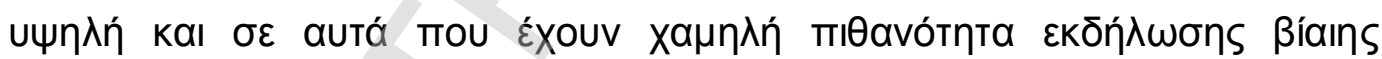

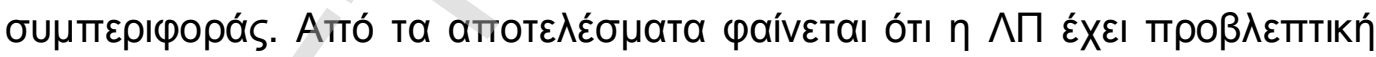

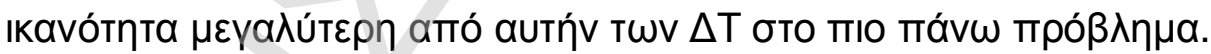

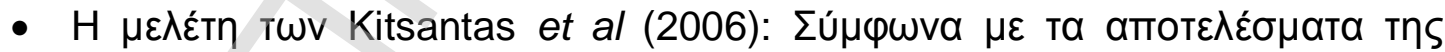

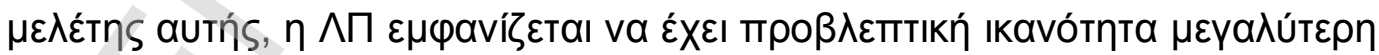

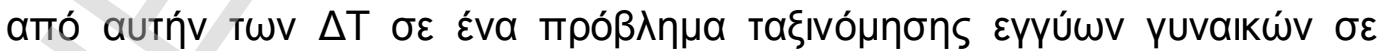

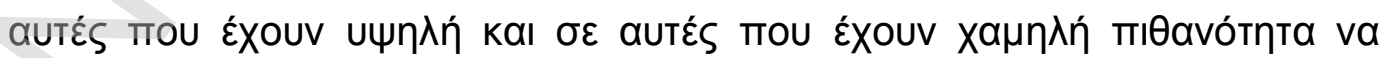

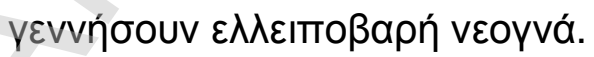




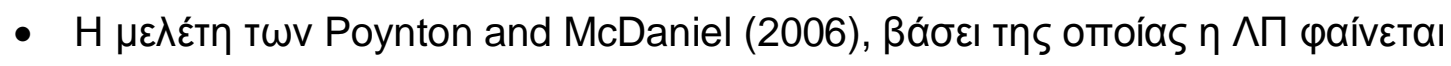

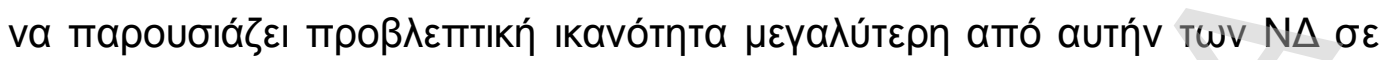

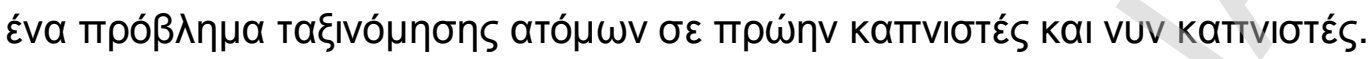

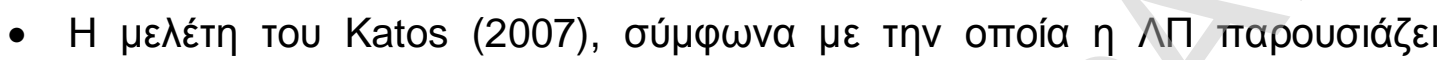

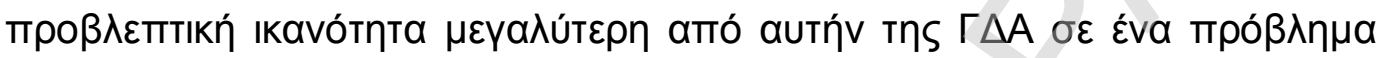

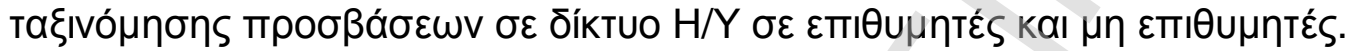

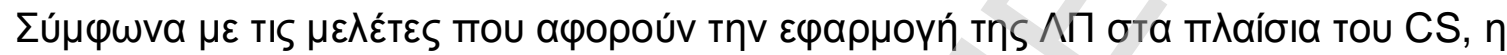

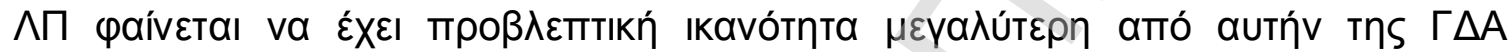
(Henley and Hand, 1996; West, 2000) kal TOU KAB (Hand and Adams, 2000).

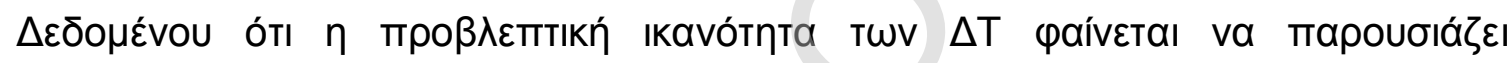

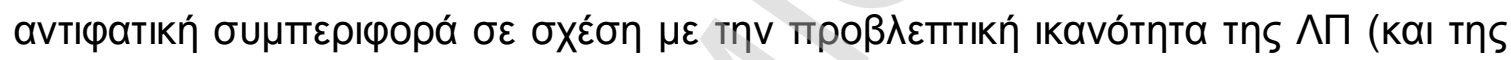

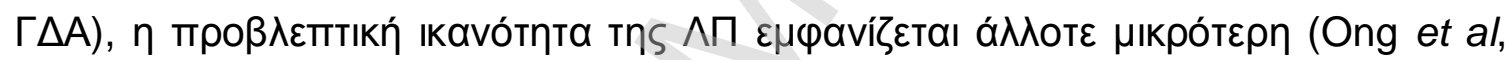

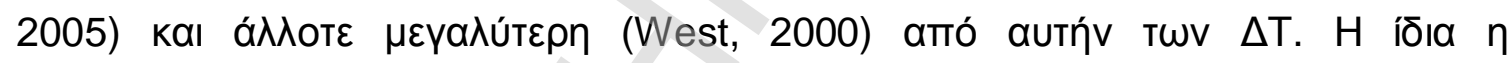

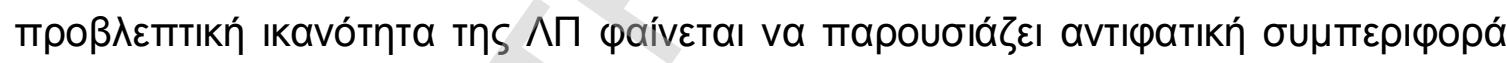

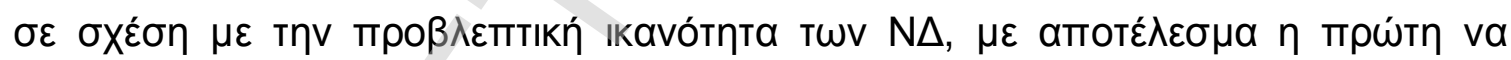

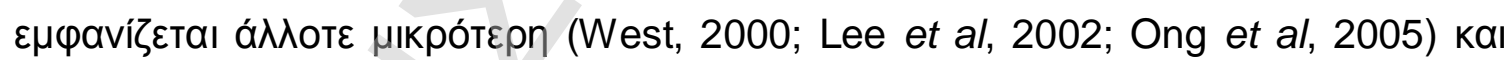

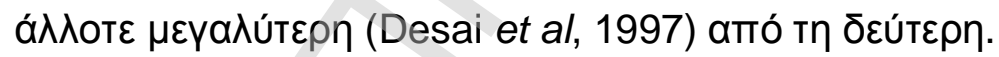

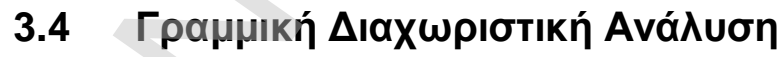

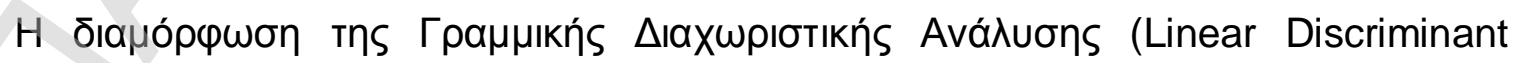

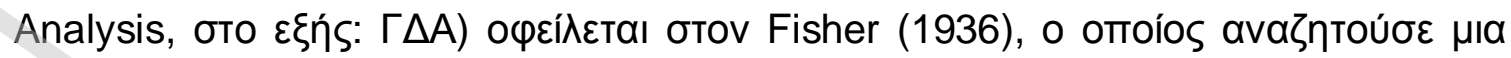




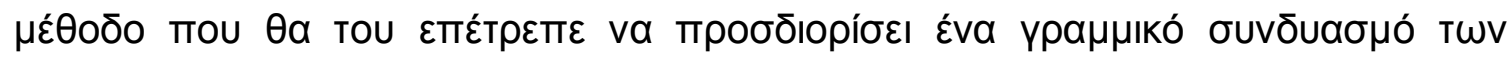

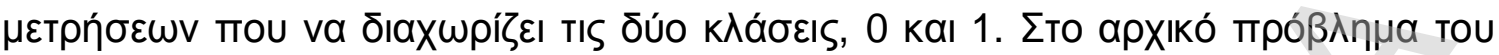

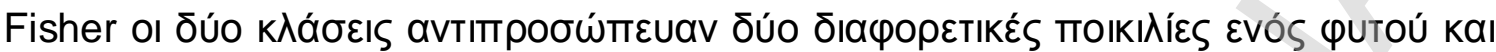

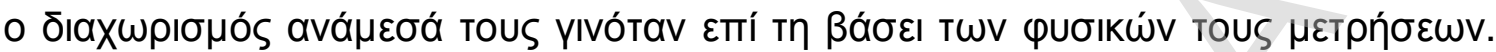

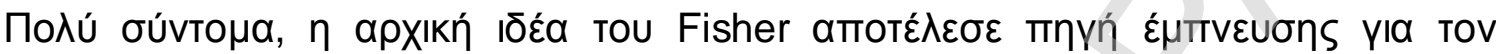

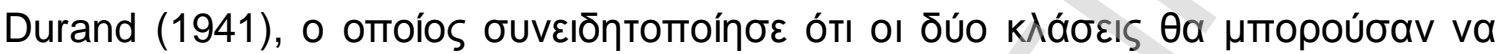

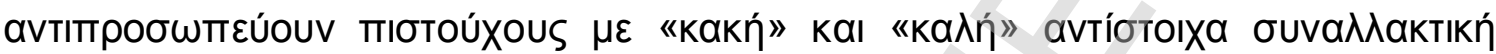

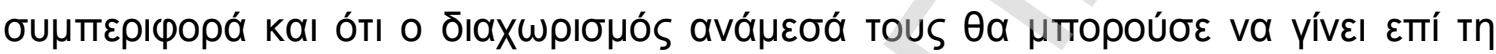

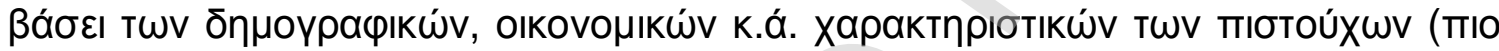

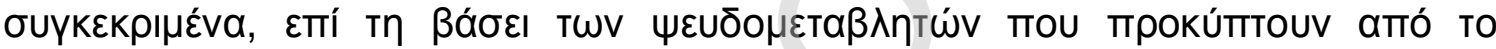

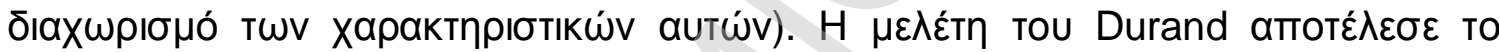

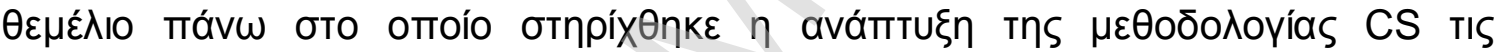

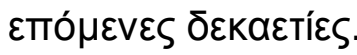

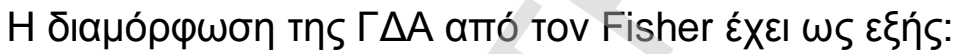

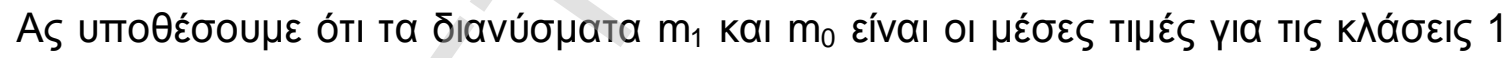

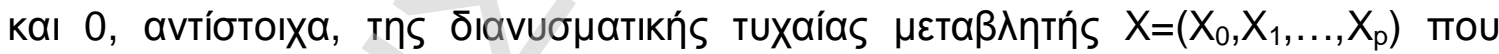

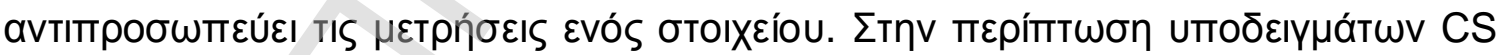

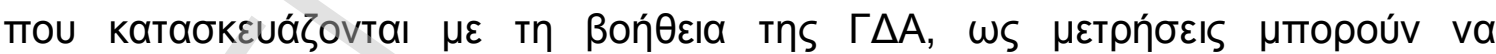

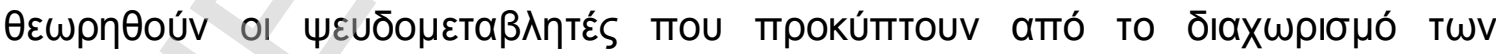

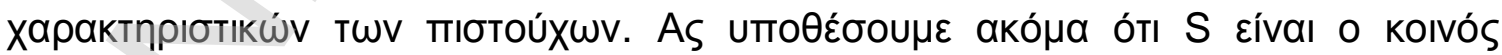

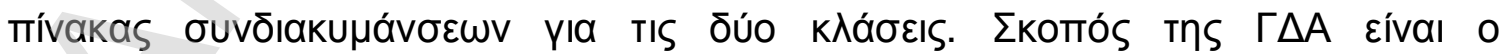

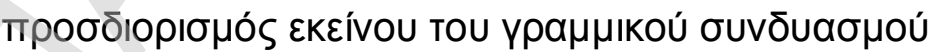

$$
Z=\sum_{i=0}^{p} w_{i} X_{i}=w X
$$




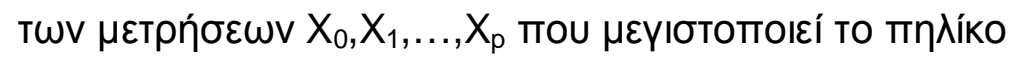

$$
M=w^{T} \frac{m_{1}-m_{0}}{\left(w^{T} S w\right)^{1 / 2}}
$$

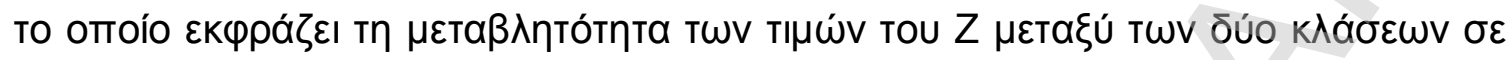

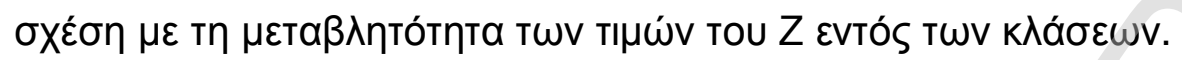

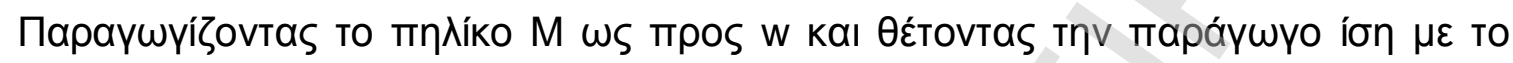

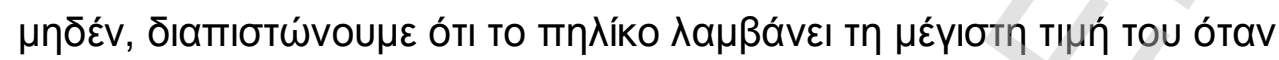

$$
\left(m_{1}-m_{0}\right)\left(w S w^{T}\right)=\left(S w^{T}\right)\left(w\left(m_{1}-m_{0}\right)^{T}\right)
$$

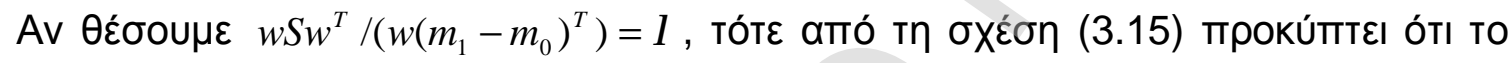

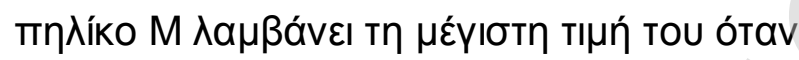

$$
w^{T}=\lambda S^{-1}\left(m_{1}-m_{0}\right)^{T}
$$

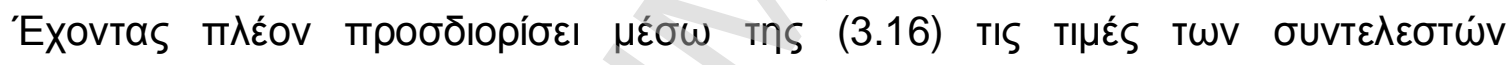

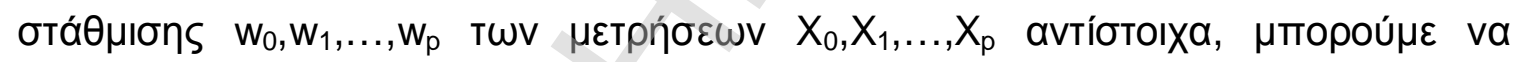

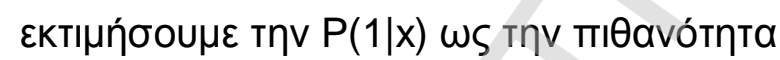

$$
P(1 \mid Z)=P(Z \mid 1) P(1) /\left(\sum_{j=0}^{1} P(Z \mid j) P(j)\right)
$$

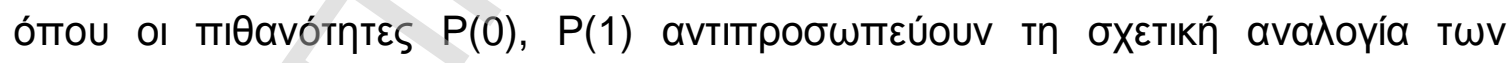

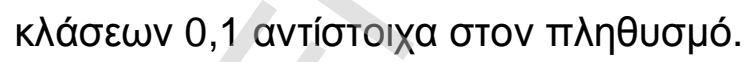

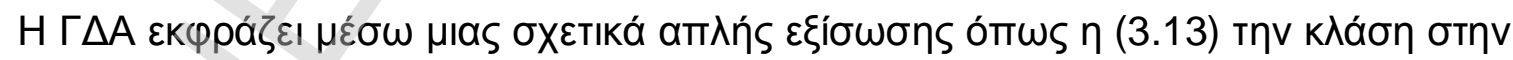

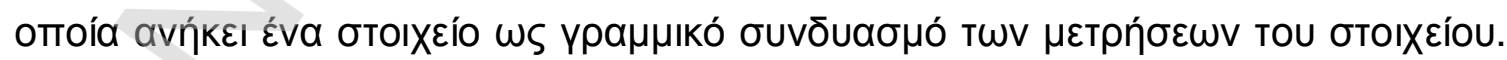

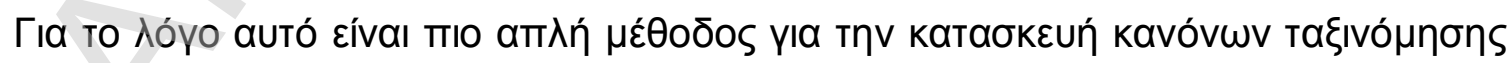

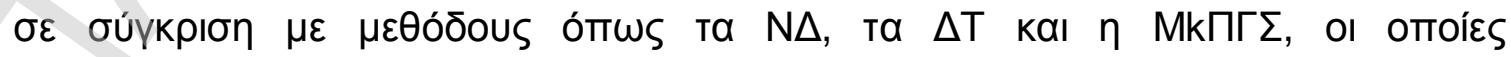

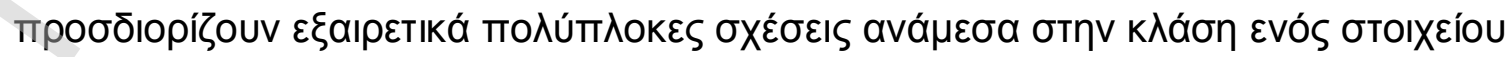




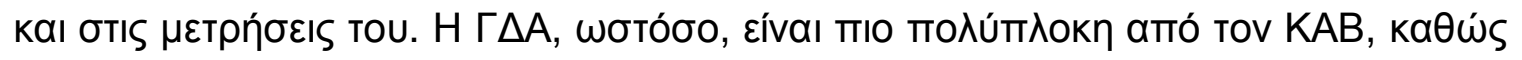

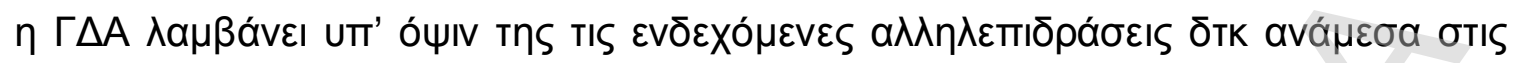

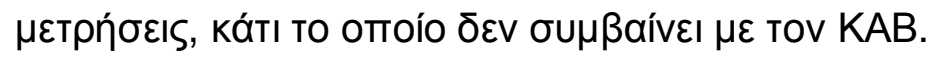

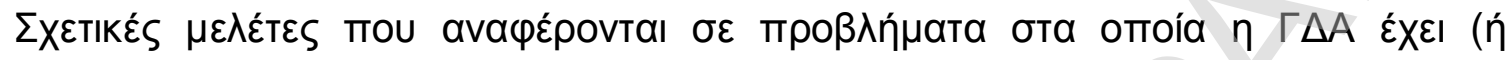

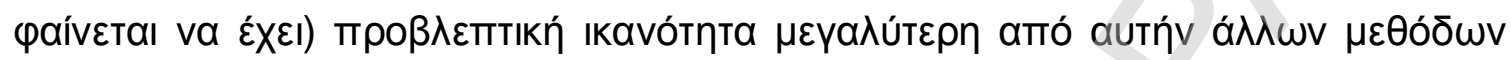

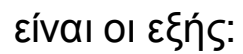

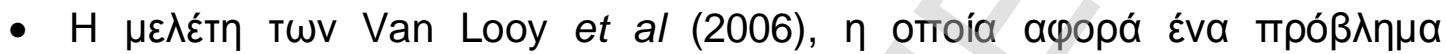

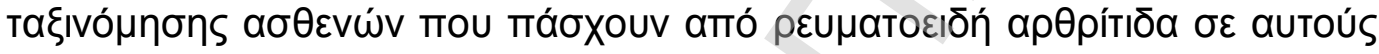

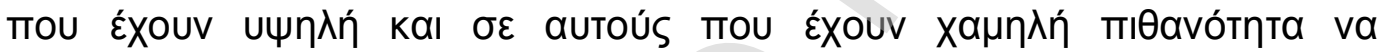

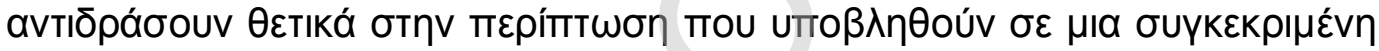

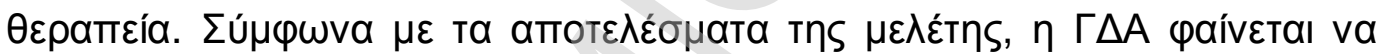

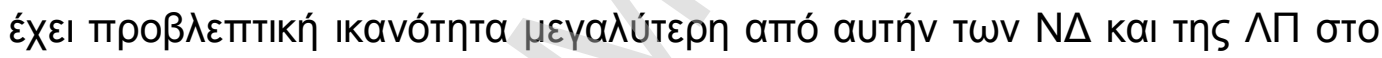

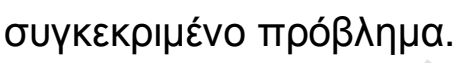

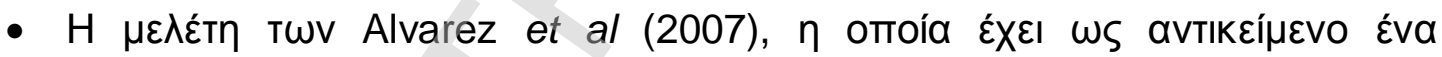

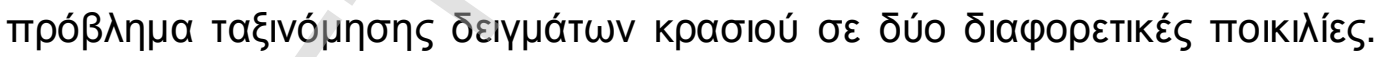

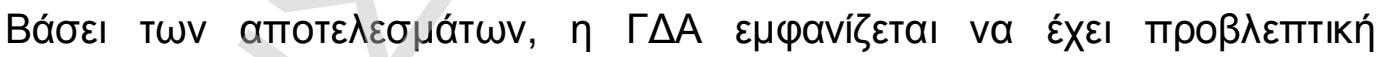

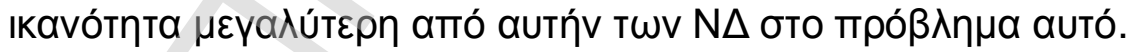

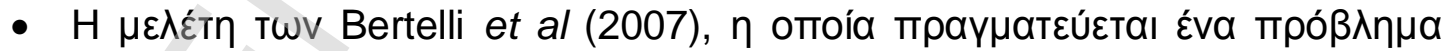

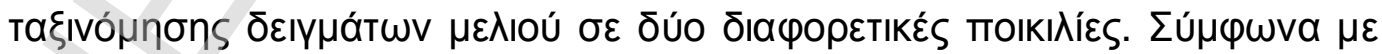

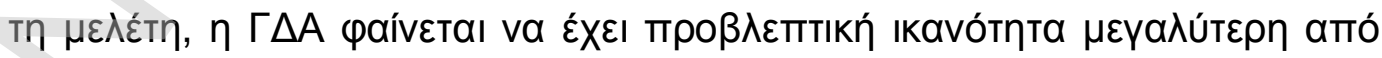

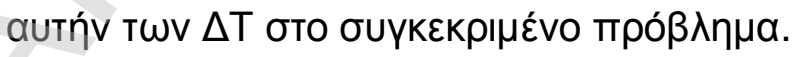




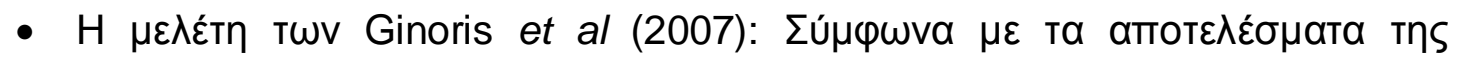

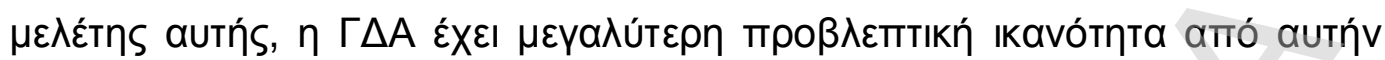

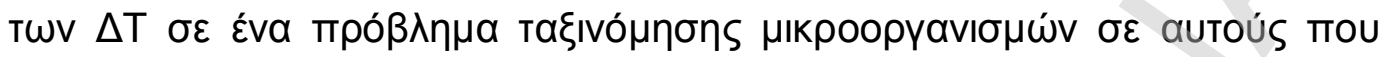

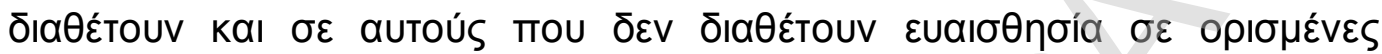

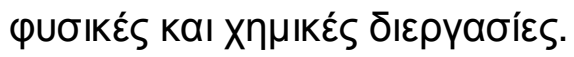

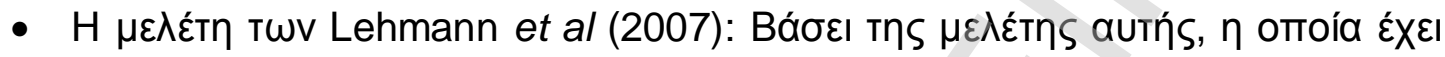

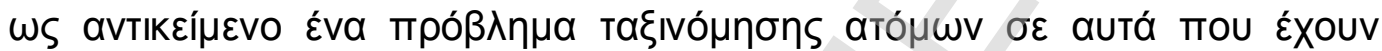

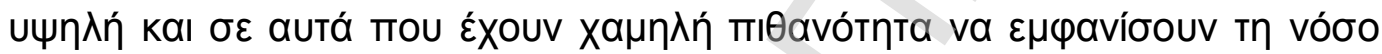

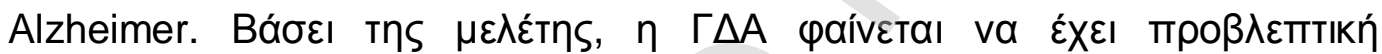

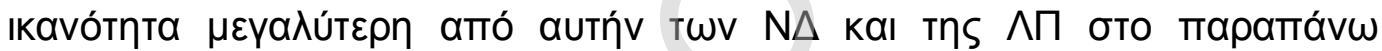

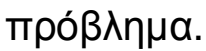

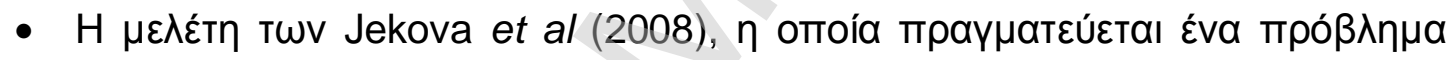

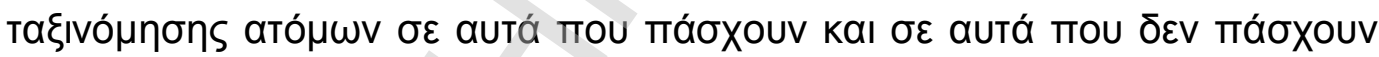

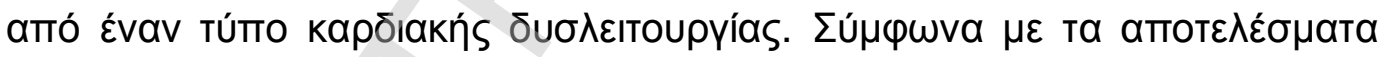

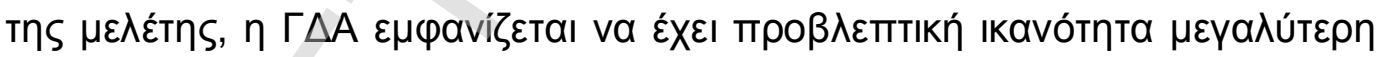

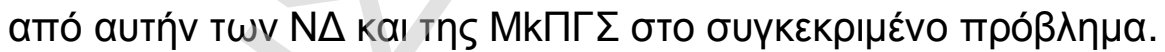

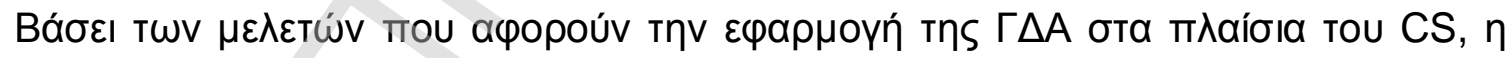

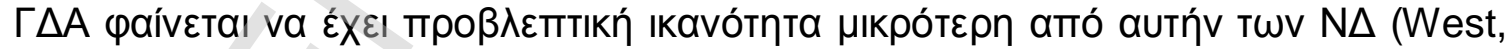
2000; Lee et al, 2002; Chen and Huang, 2003; Malhotra, 2003) kaı amó autńv tns

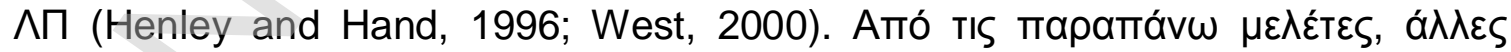

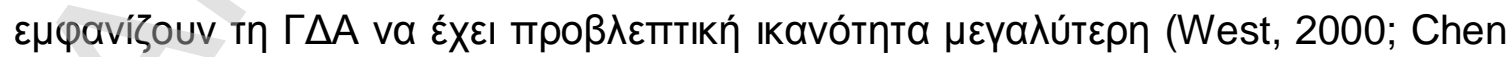

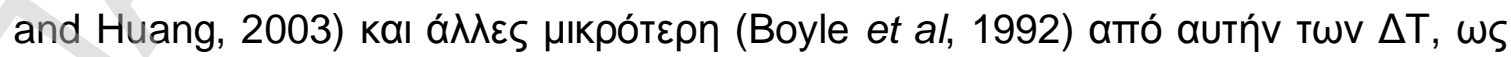

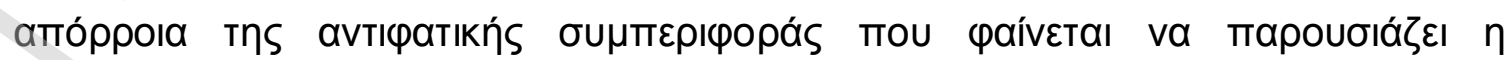




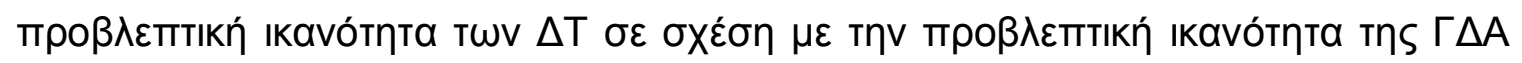
(каı тпऽ $\wedge П)$.

\subsection{NeupwViká $\Delta$ í́ktua}

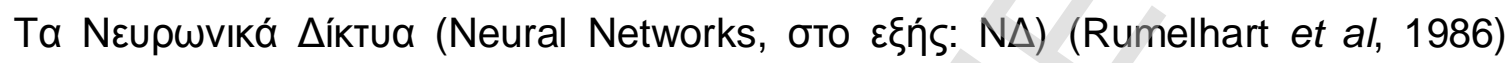

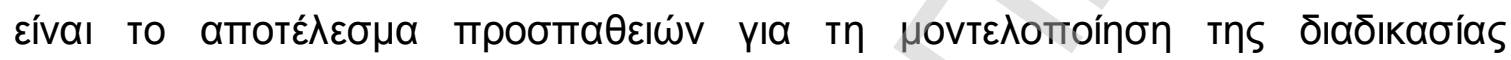

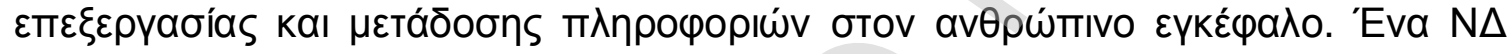

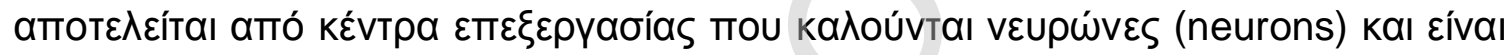

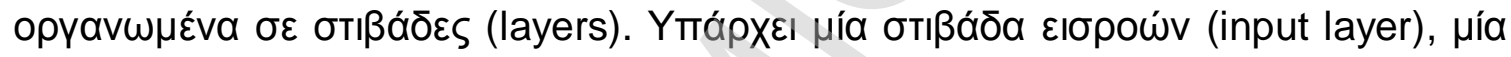

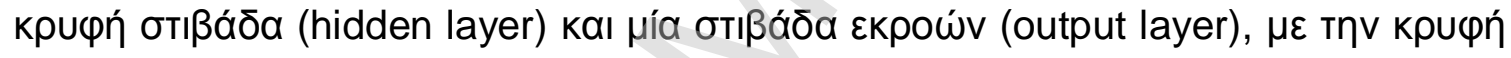

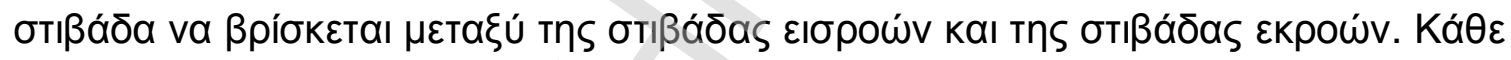

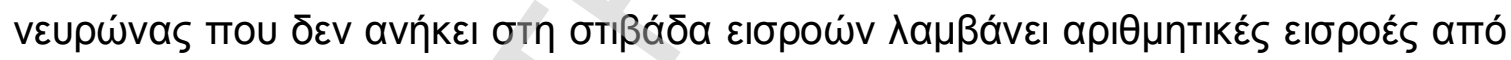

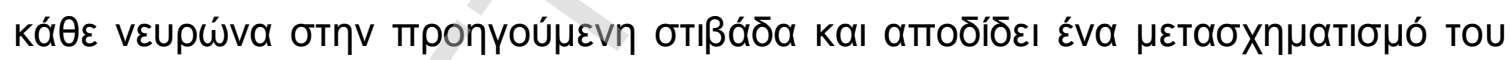

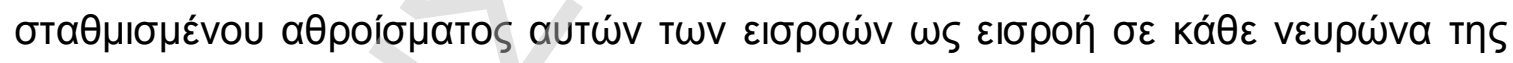

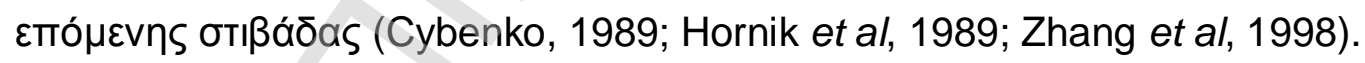




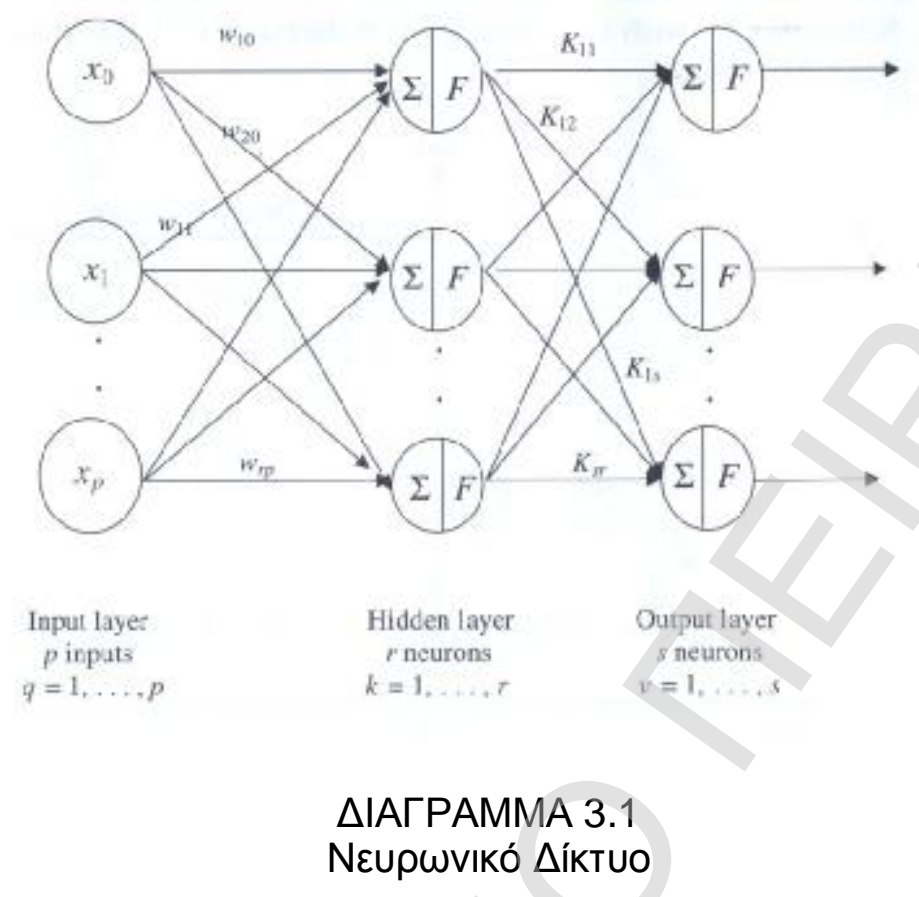

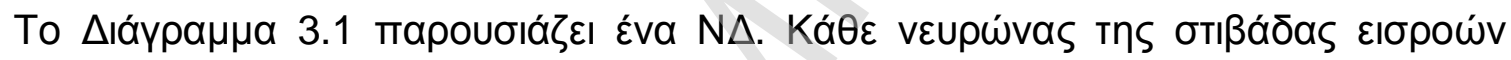

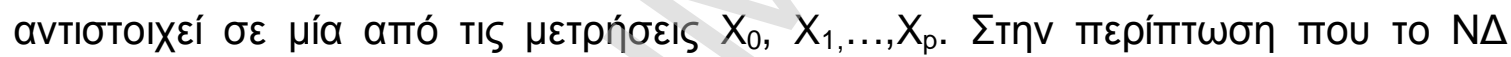

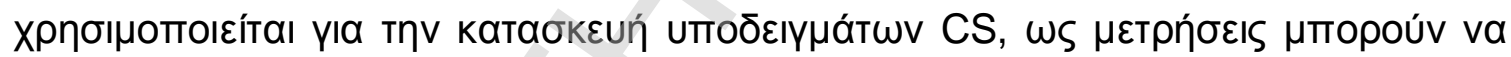

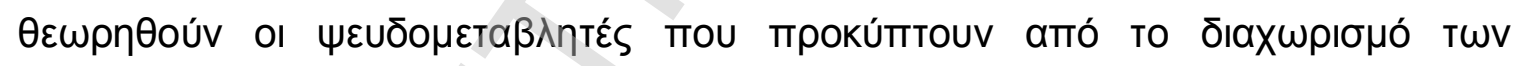

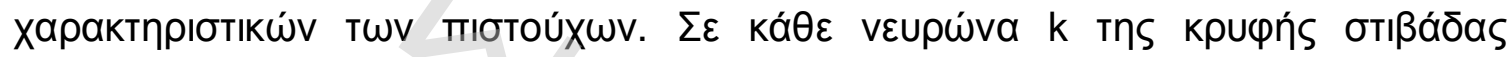

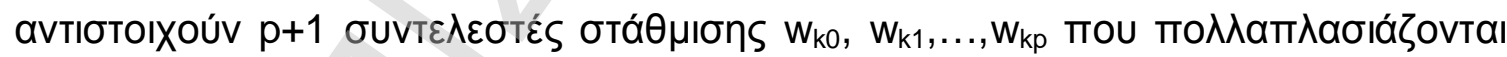

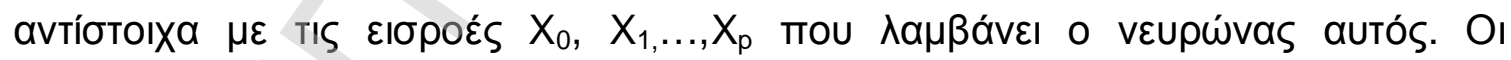

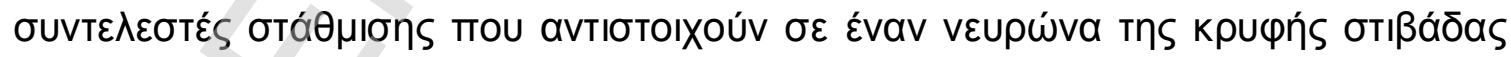

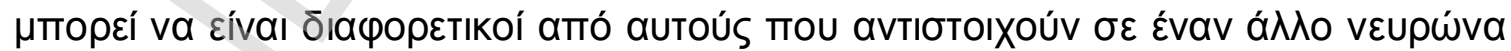

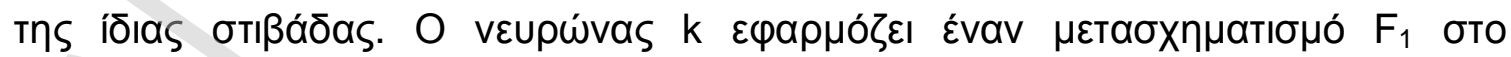

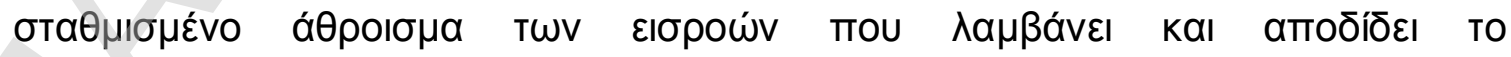

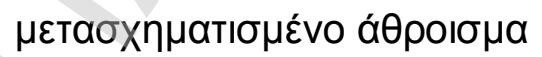




$$
Y_{k}=F_{1}\left(\sum_{q=0}^{p} w_{k q} X_{q}\right)
$$

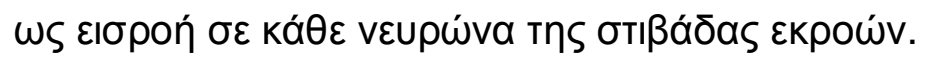

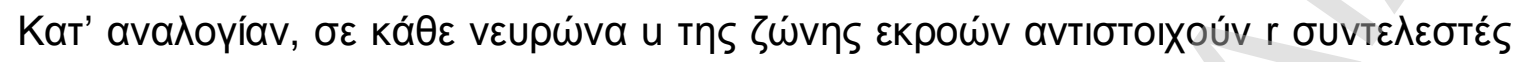

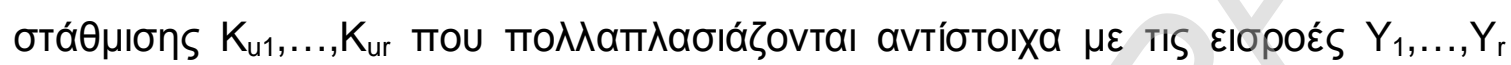

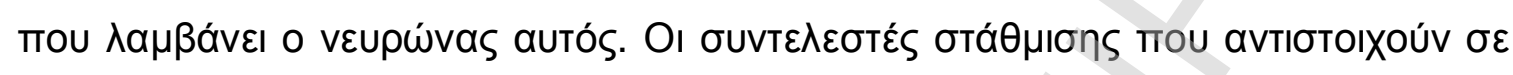

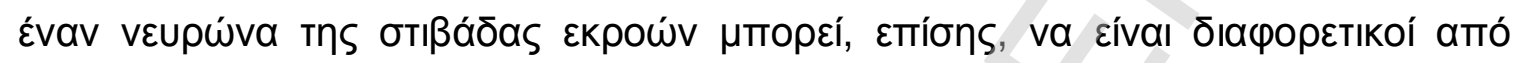
aUtoú

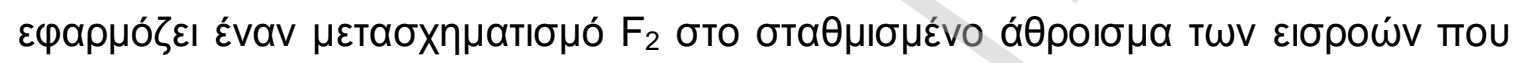

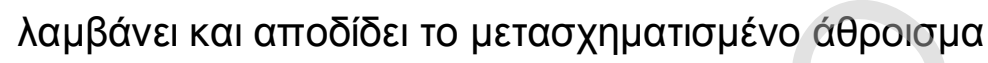

$$
Z_{u}=F_{2}\left(\sum_{k=1}^{r} K_{u k} Y_{k}\right)
$$

wऽ عкроп́.

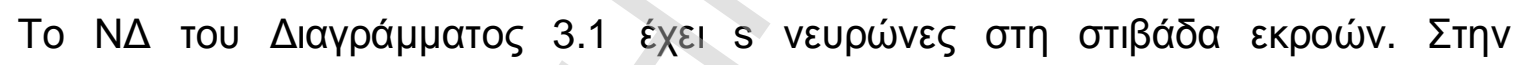

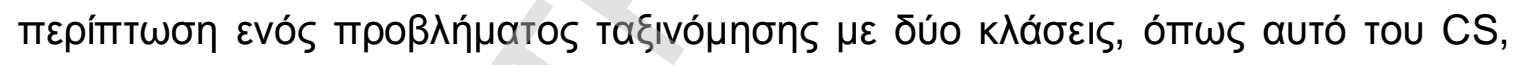

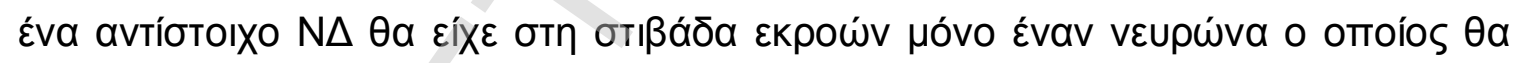

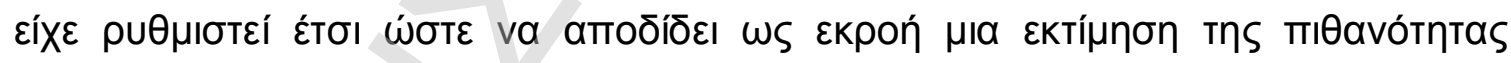

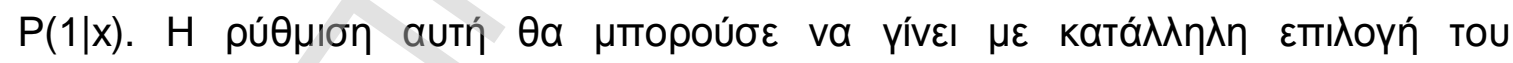

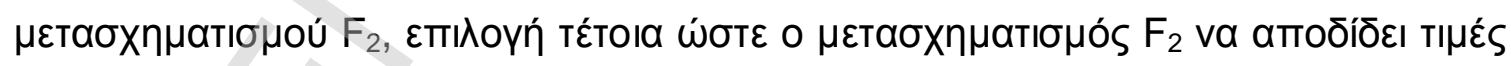

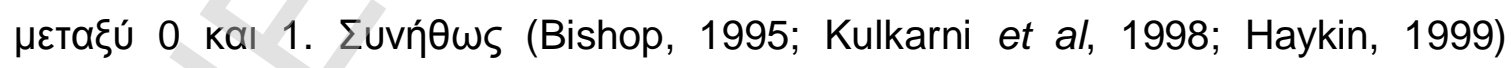

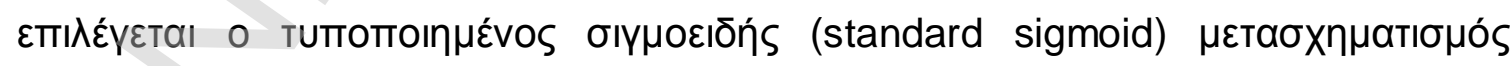
$F_{2}(\tau)=1 /\left(1+e^{-\tau}\right)$. 


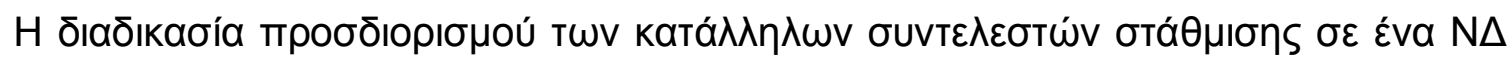

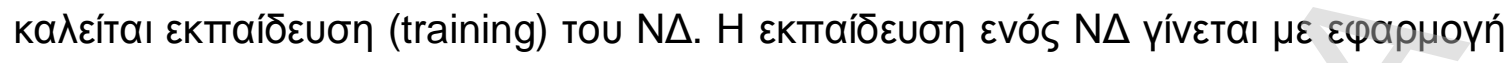

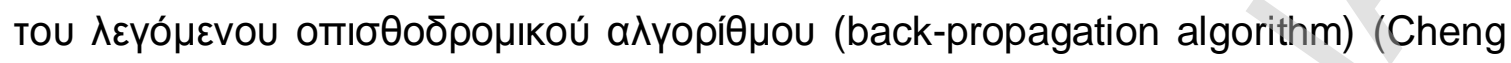
and Titterington, 1994; Michie et al 1994; Malhotra and Malhotra, 2003). ApxIKó

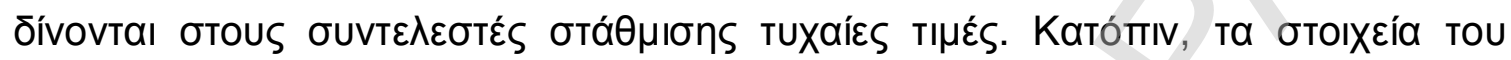

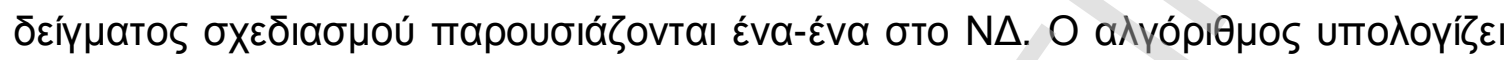

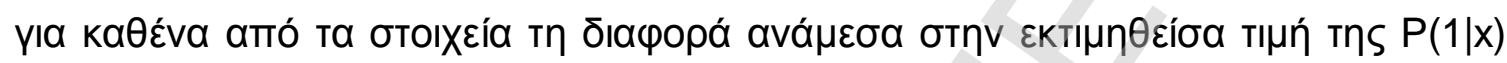

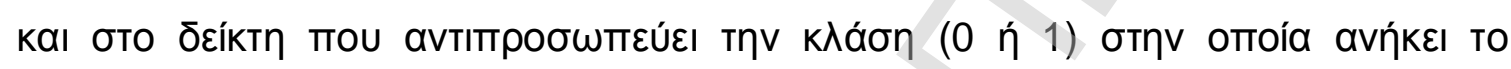

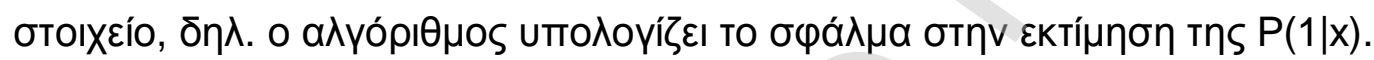

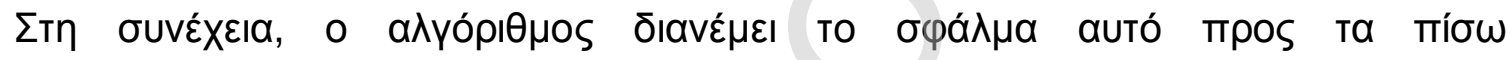

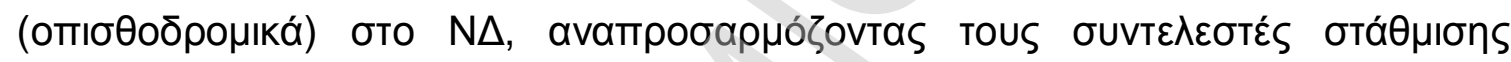

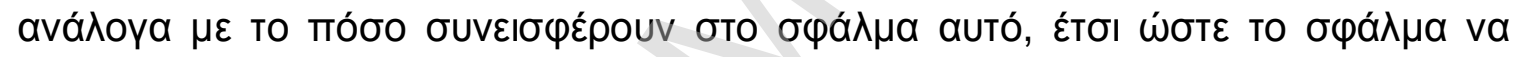

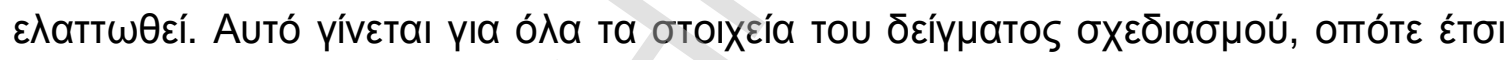

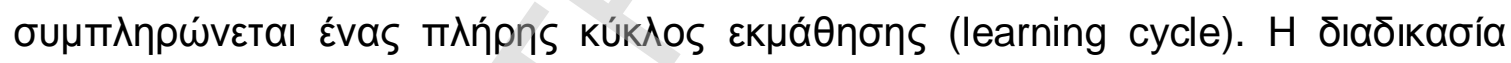

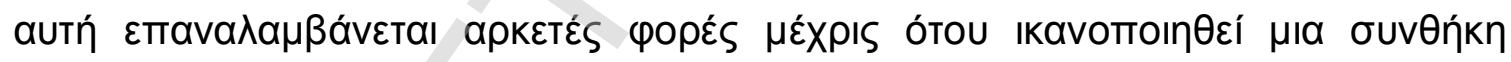

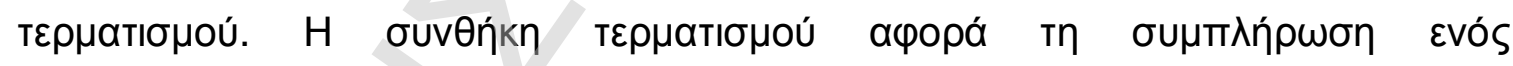

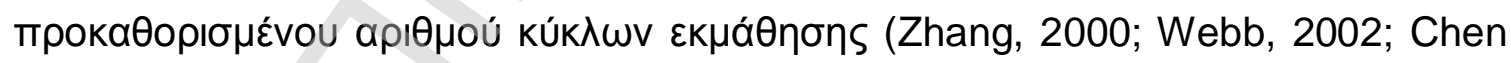
and Huang, 2003).

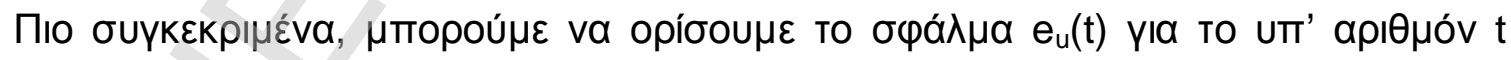

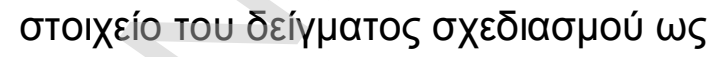

$$
e_{u}(t)=O_{u}(t)-Y_{u}(t)
$$

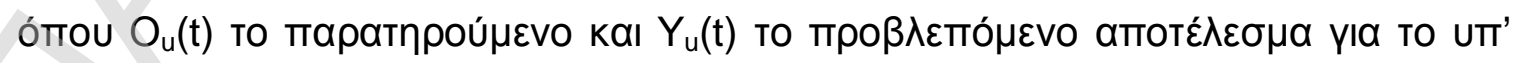

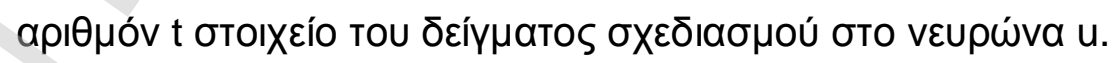




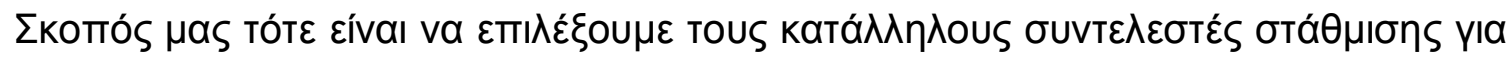

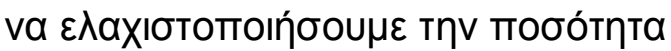

$$
E(t)=(1 / 2) \sum_{u=1}^{s} e_{u}^{2}(t)
$$

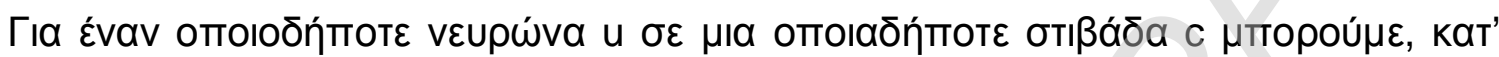

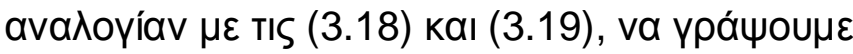

$$
L_{u}^{[c]}=\sum_{k=0}^{r} w_{u k} Y_{k}^{[c-1]} \text { (3.22) каı } Y_{u}^{[c]}=F\left(L_{u}^{[c]}\right)
$$

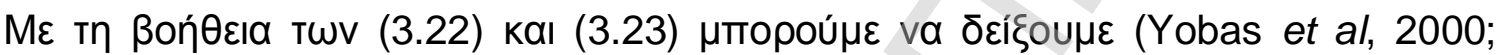

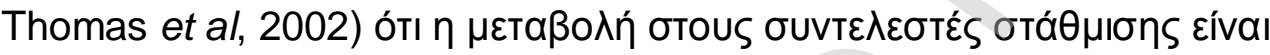

$$
\Delta w_{u k}(t)=-n \frac{\partial E(t)}{\partial w_{u k}(t)}=n e_{u}(t) F^{\prime}\left(L_{u}(t)\right) Y_{k}(t)=n \delta_{u}(t) Y_{k}(t)
$$

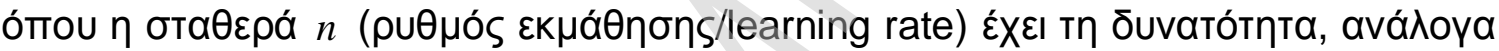

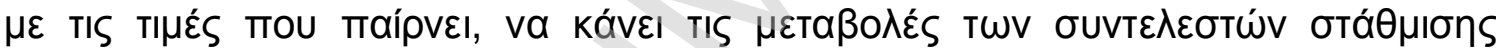

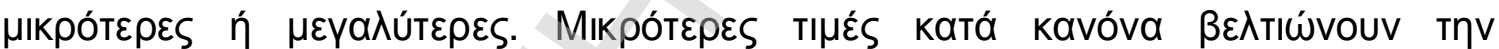

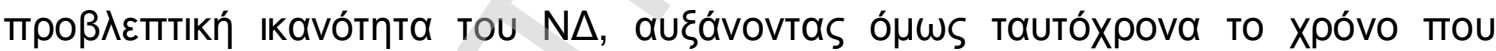

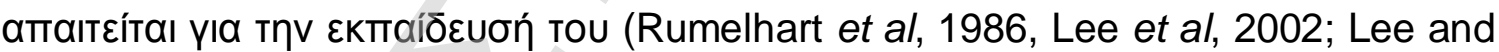
Chen, 2005).

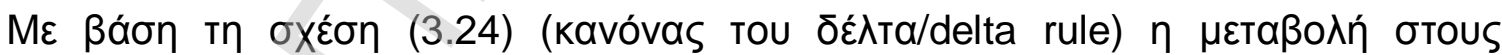

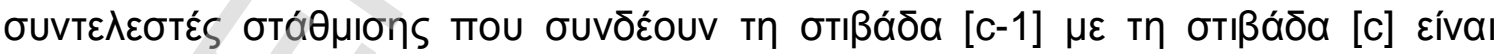

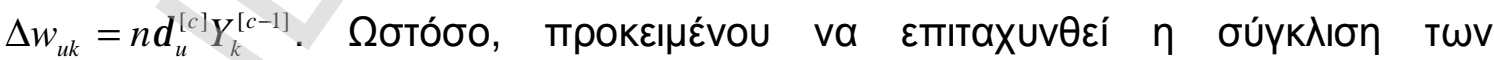

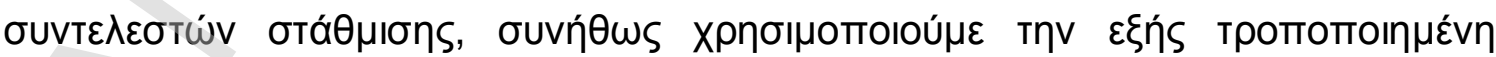

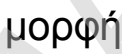

$$
\Delta w_{u k}(t)=a \Delta w_{u k}(t-1)+n \delta_{u}^{[c]} Y_{k}^{[c-1]}
$$




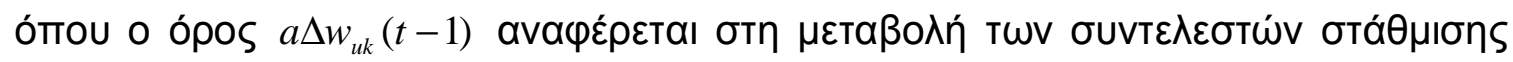

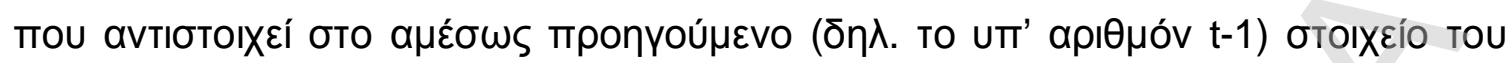

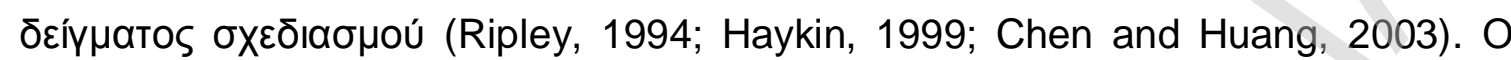

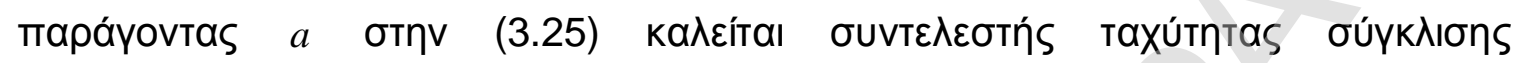
(momentum).

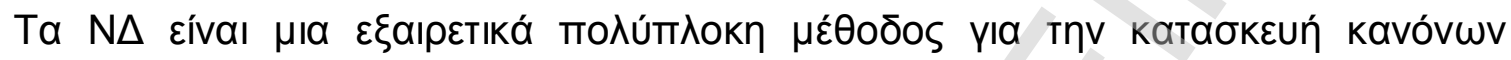

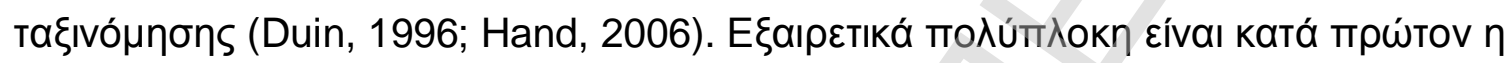

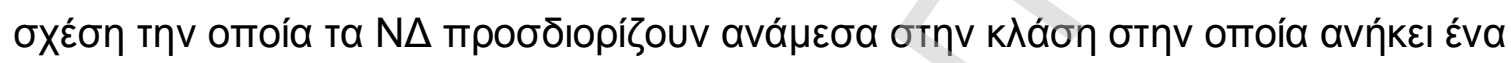

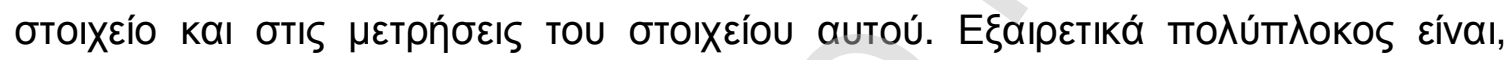

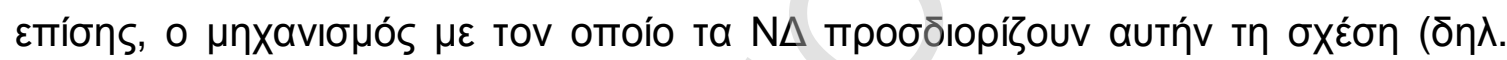

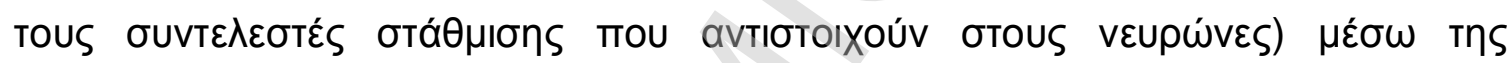

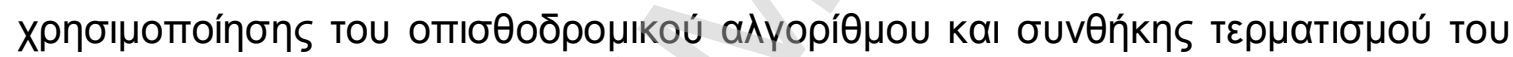

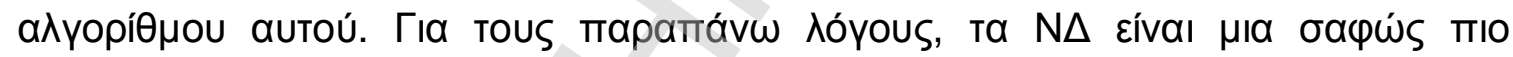

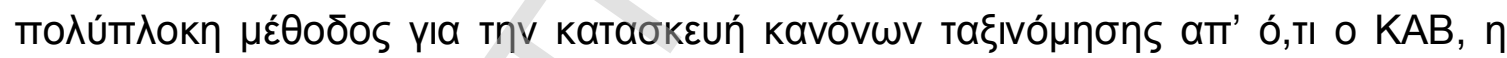

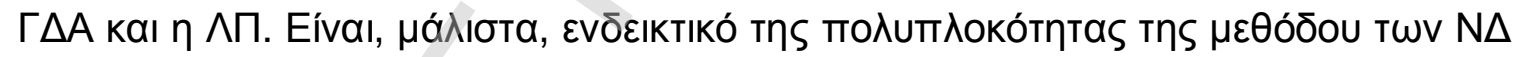

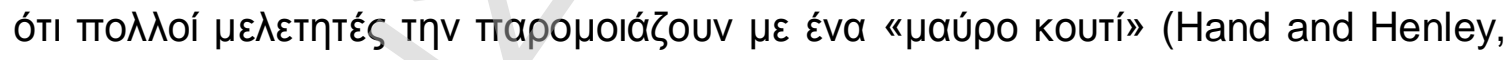
1997; West, 2000; Chen and Huang, 2003).

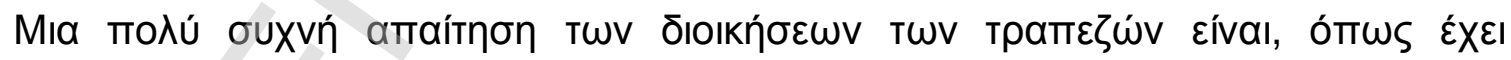

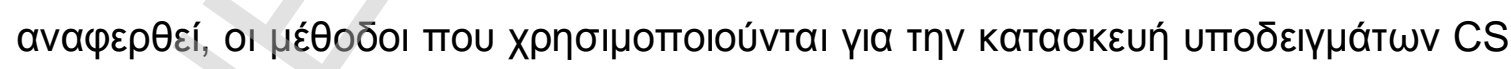

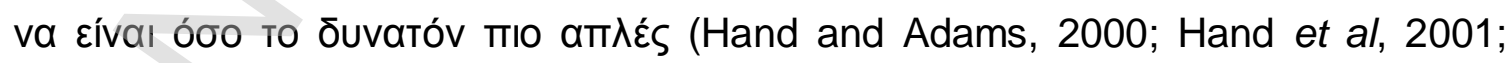

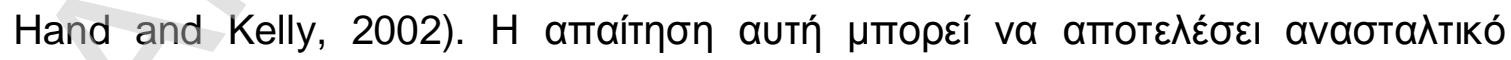

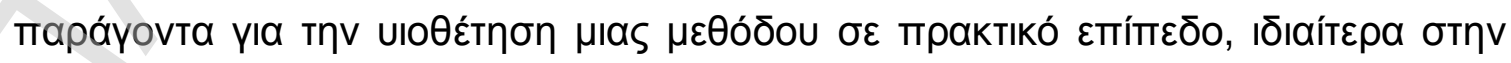




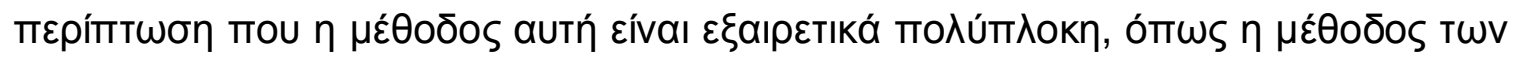
$N \Delta$.

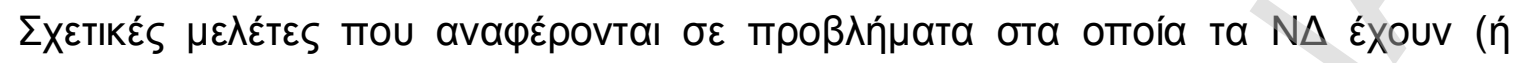

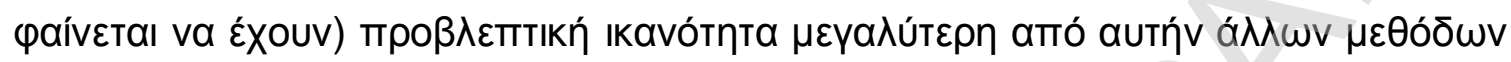

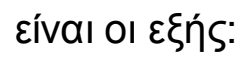

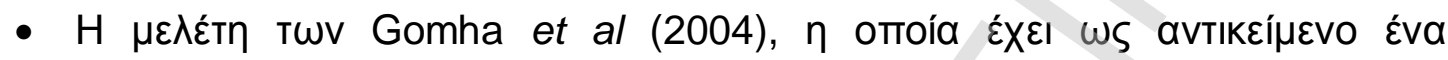

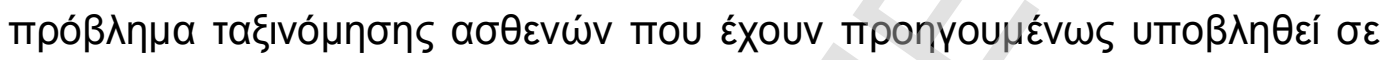

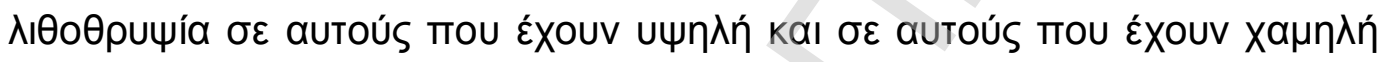

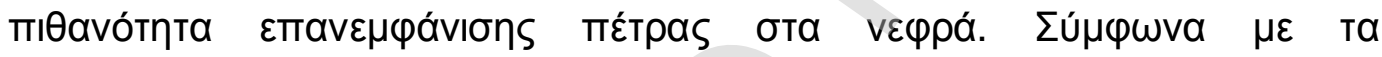

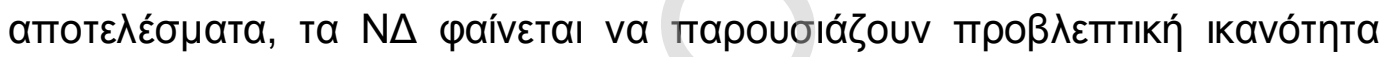

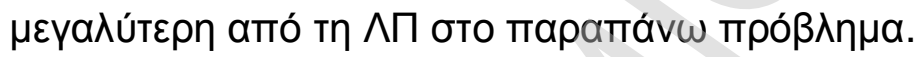

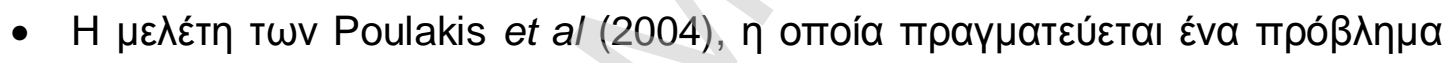

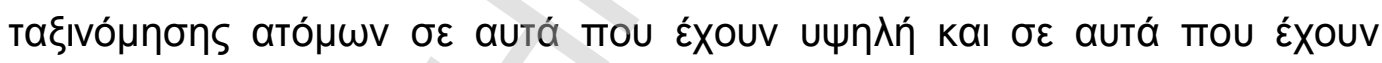

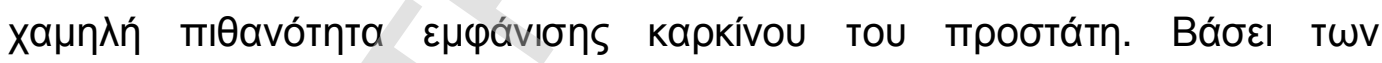

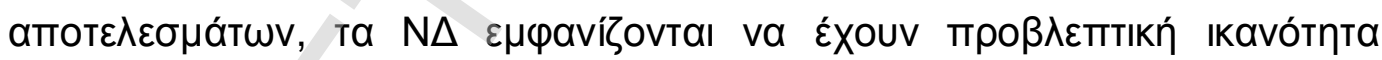

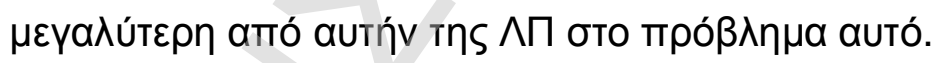

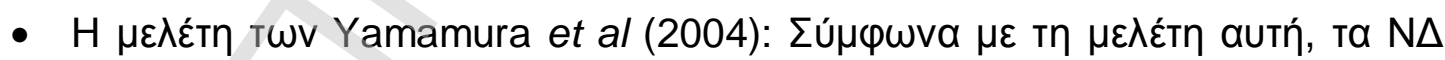

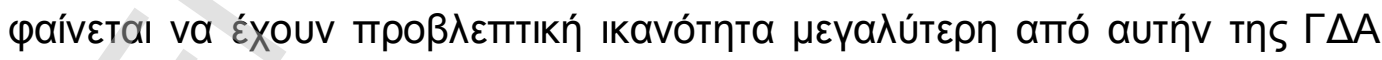

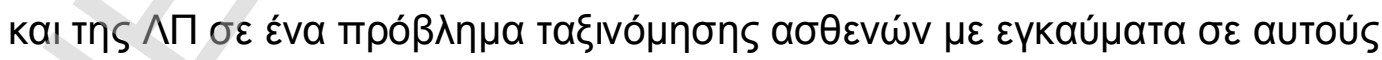

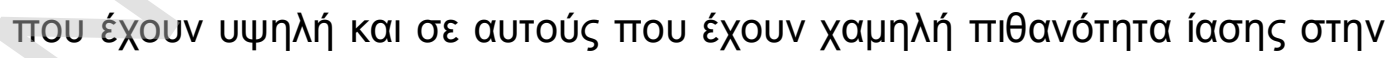

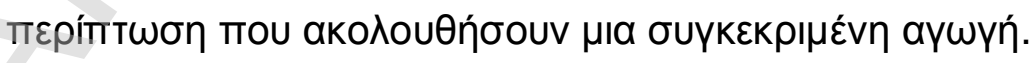

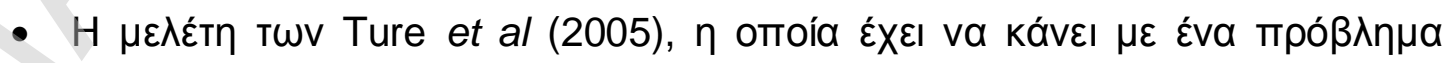

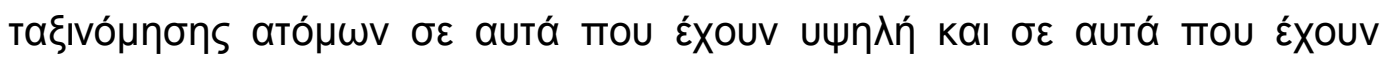




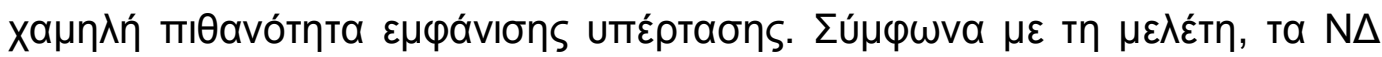

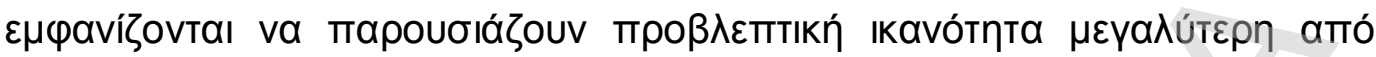

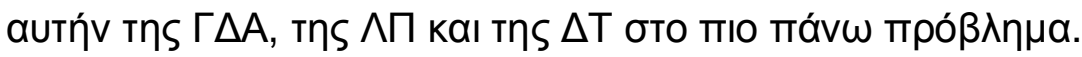

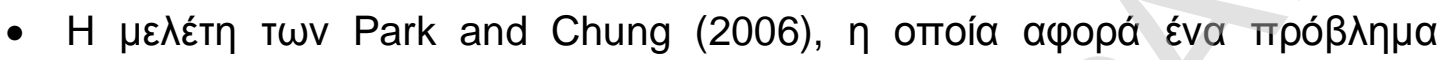

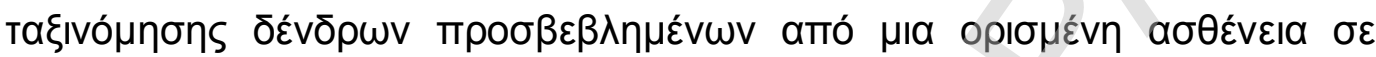

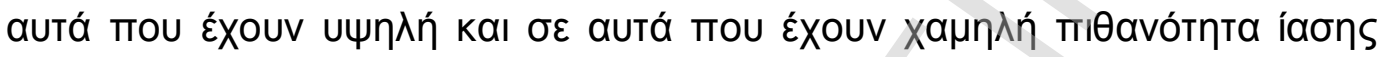

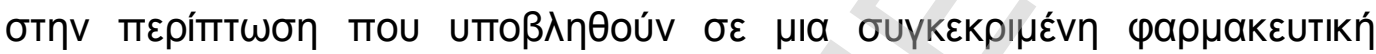

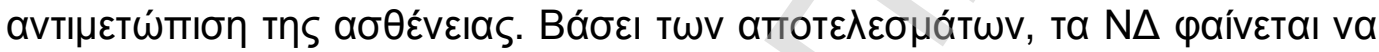

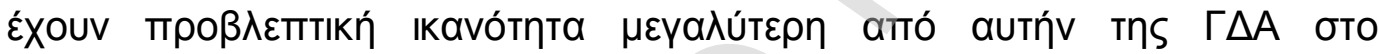

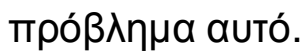

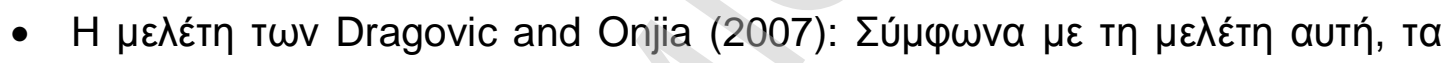

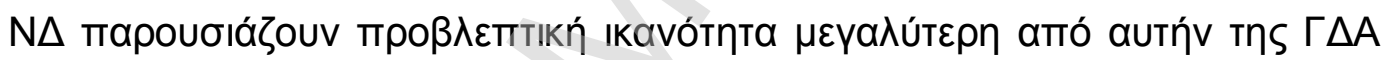

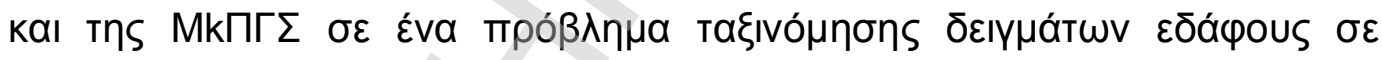

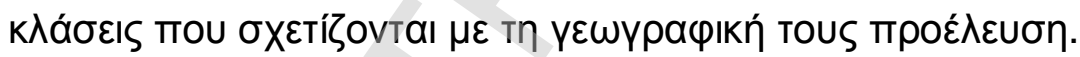

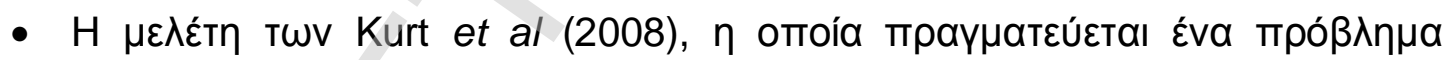

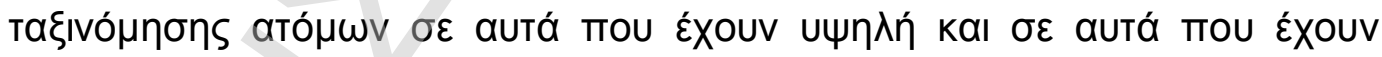

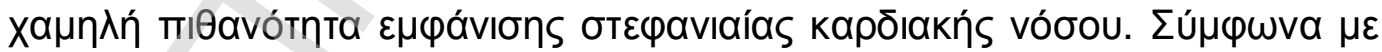

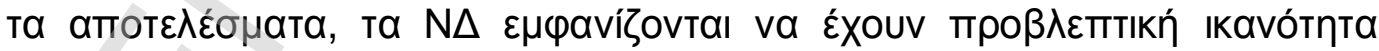

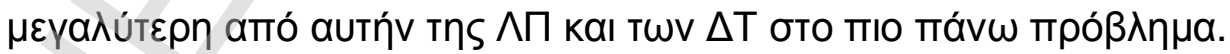

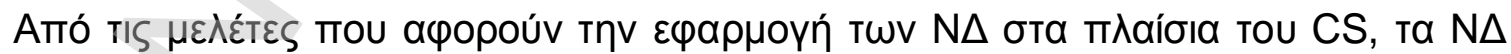

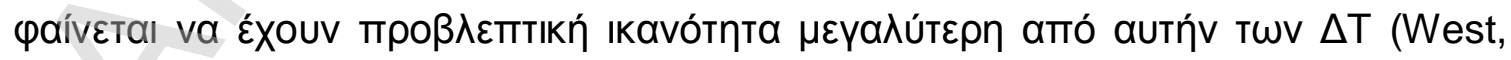
2000; Chen and Huang, 2003; Ong et al, 2005) kaı тnऽ Г $\Delta \mathrm{A}$ (West, 2000; Lee et al,

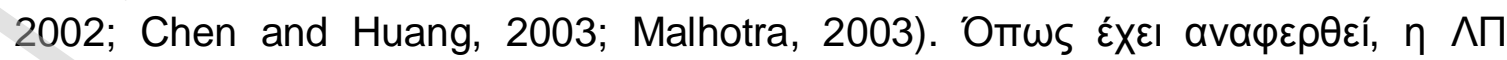




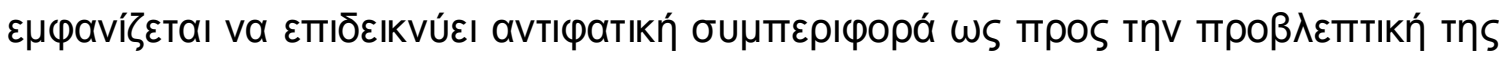

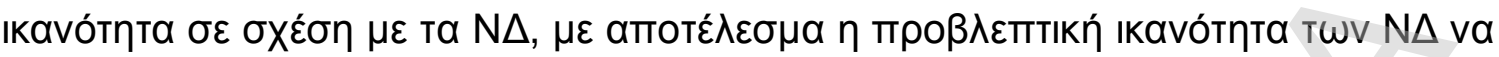

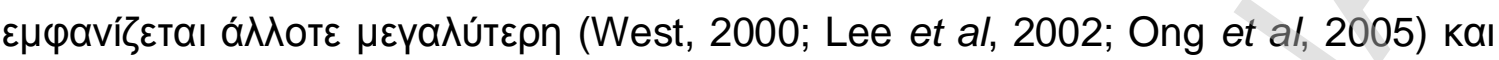

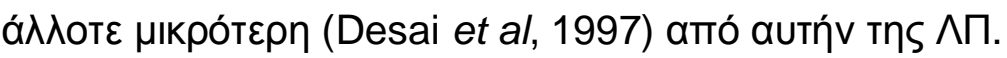

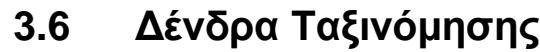

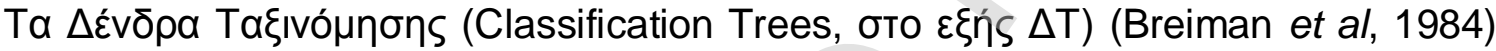

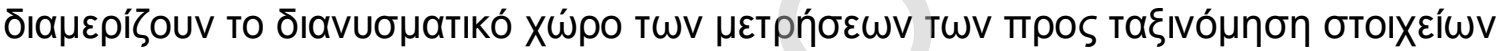

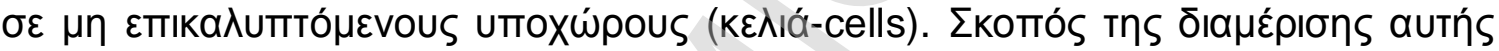

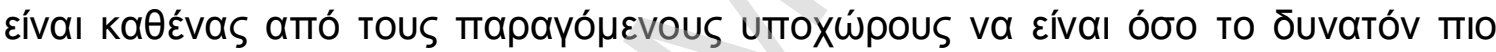

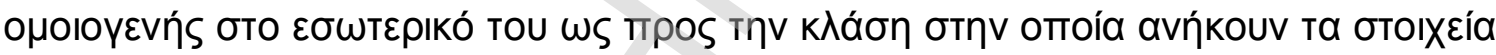

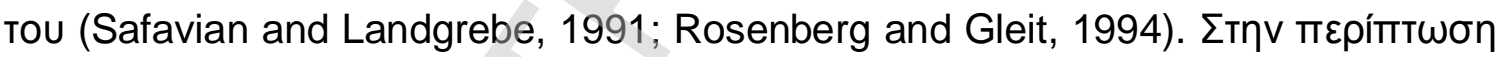

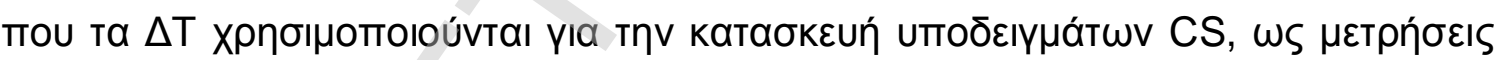

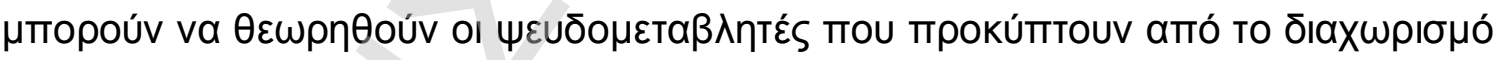

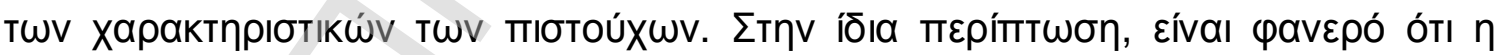
o

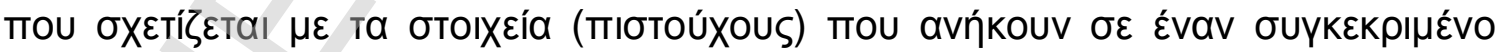
UTóxwpo (Thomas, 2000).

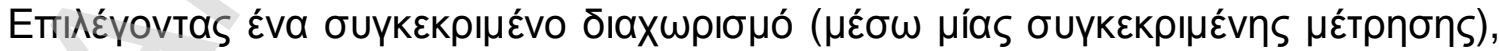

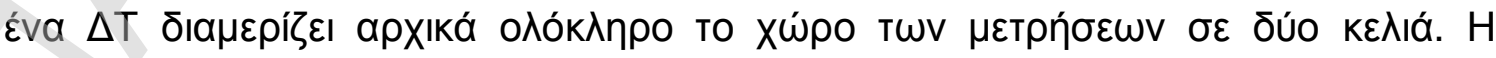

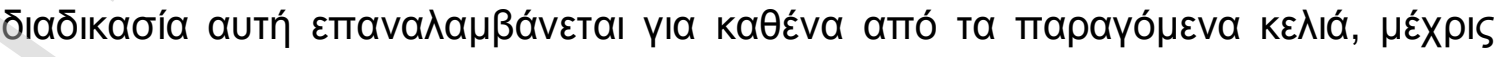




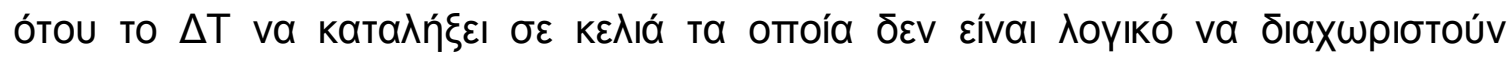

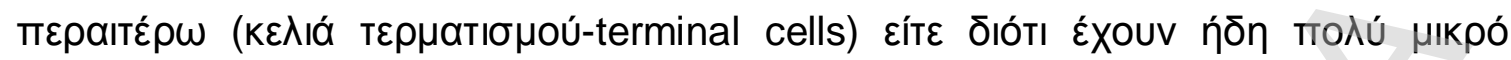

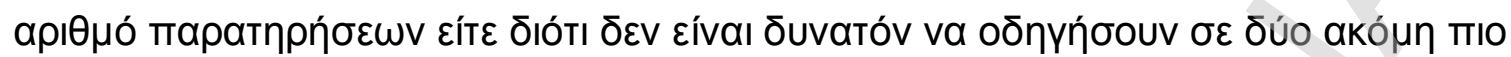

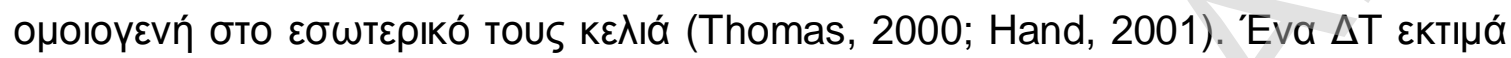

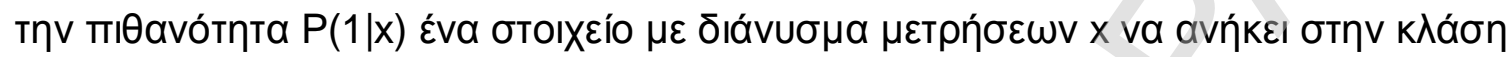

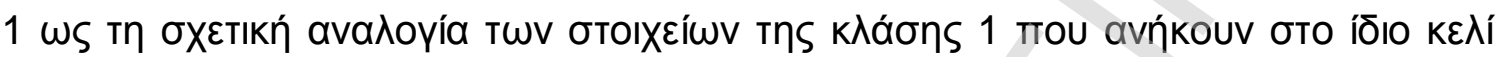

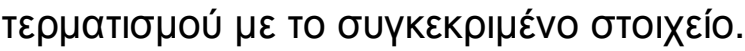

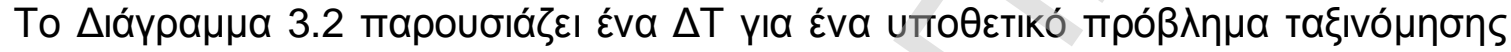

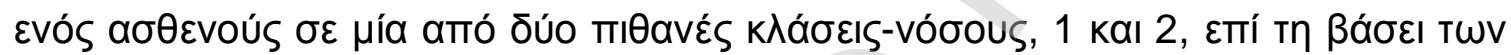

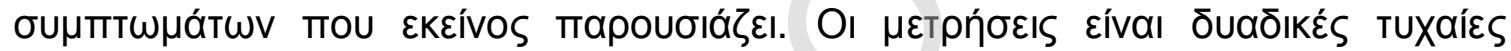

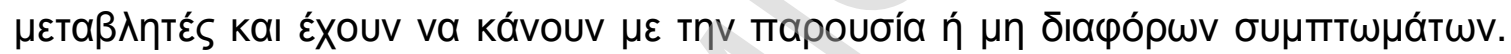

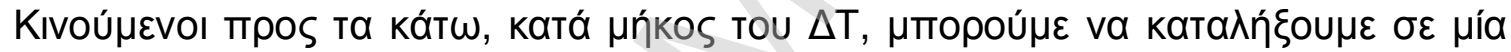

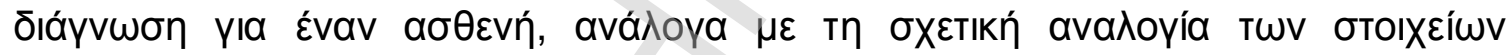

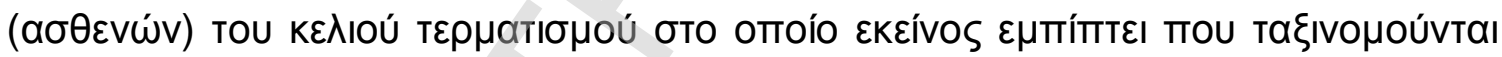

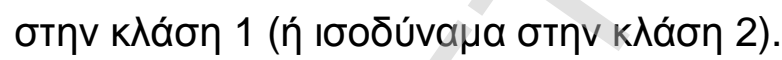




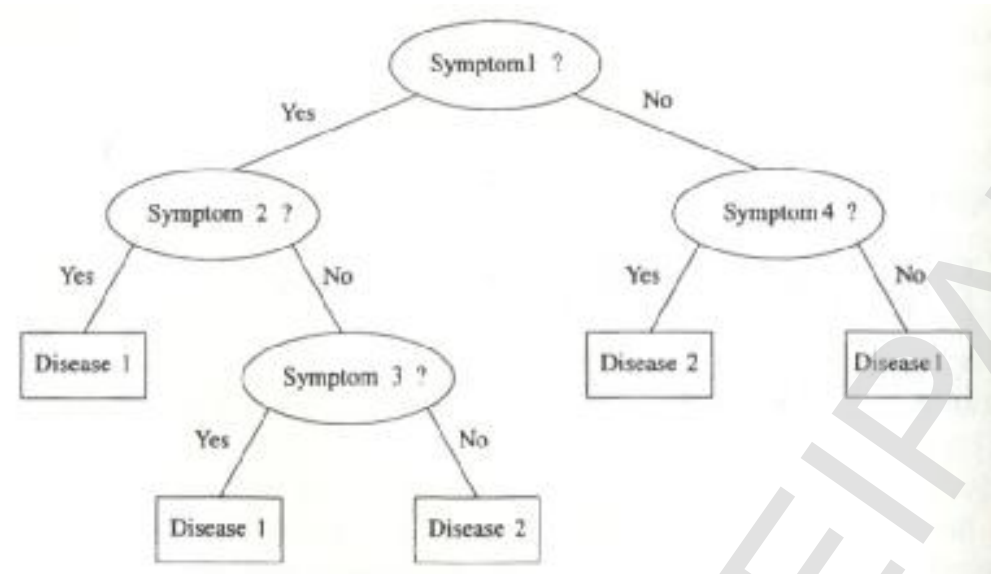

$\triangle$ МАГРАММА 3.2

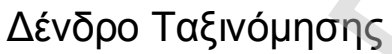

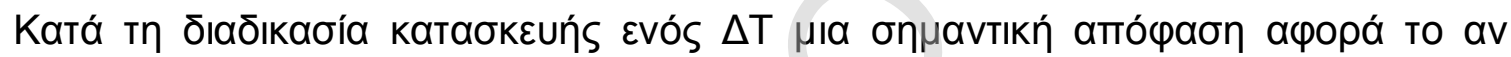

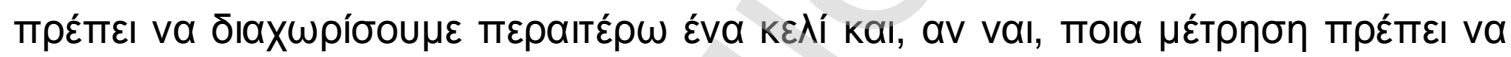

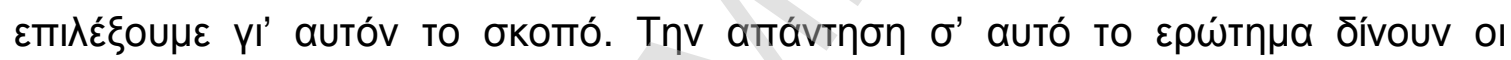

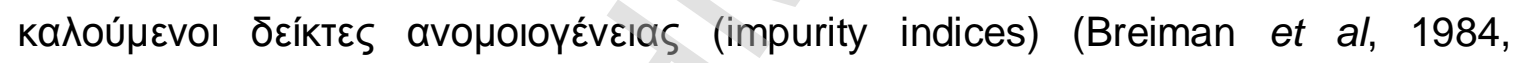

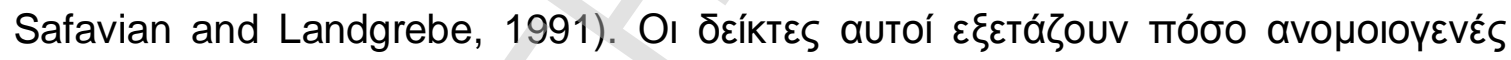

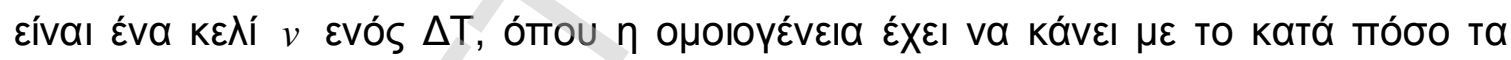

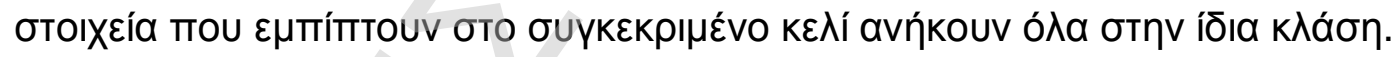

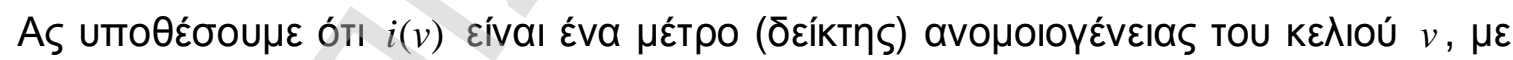

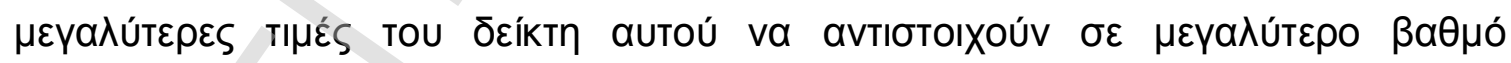

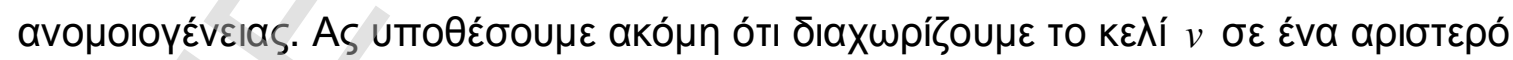

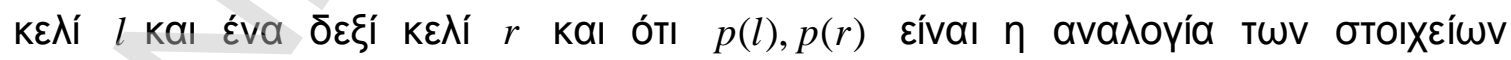

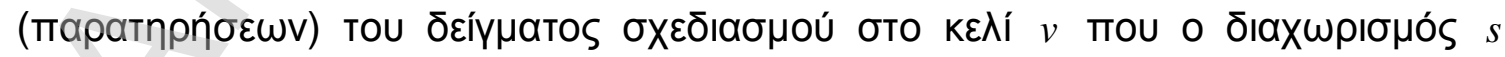

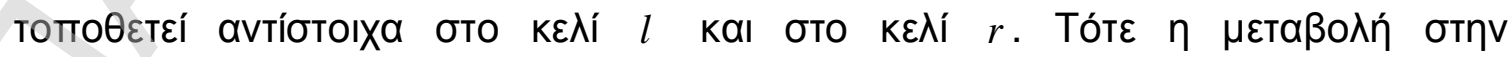




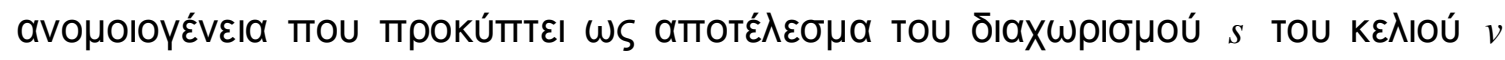
Eívaı

$$
\Delta i(s, v)=i(v)-p(l) i(l)-p(r) i(r)
$$

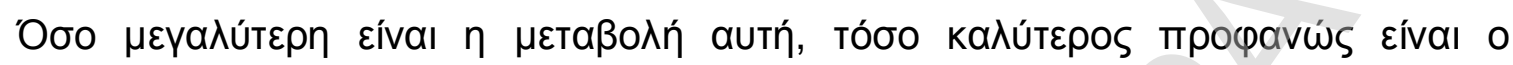

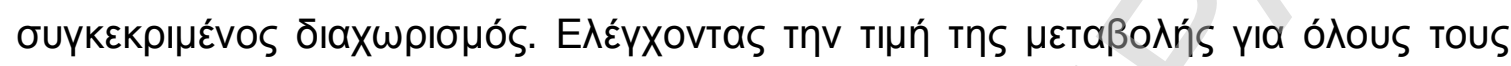

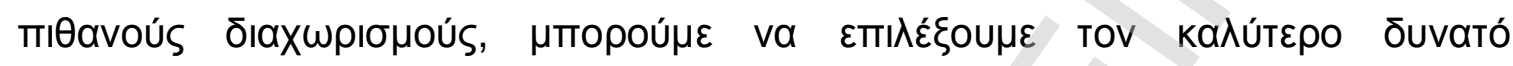

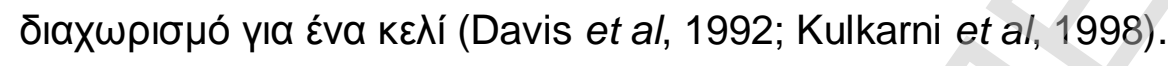

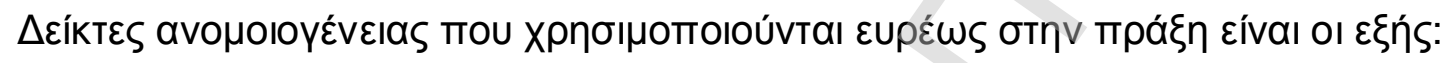

$$
\begin{aligned}
& i(v)=1-\max _{j}\{p(j \mid v)\}=\min \{p(1 \mid v), p(0 \mid v)\} \\
& i(v)=\sum_{j=0}^{1} p(j \mid v)[1-p(j \mid v)]=p(1 \mid v) p(0 \mid v) \\
& i(v)=2 p(l) p(r) \sum_{j=0}^{1}|p(j \mid l)-p(j \mid r)|
\end{aligned}
$$

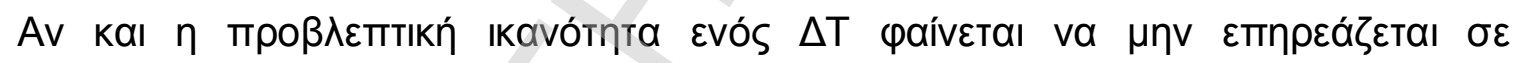

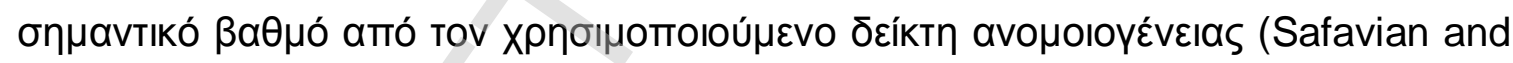

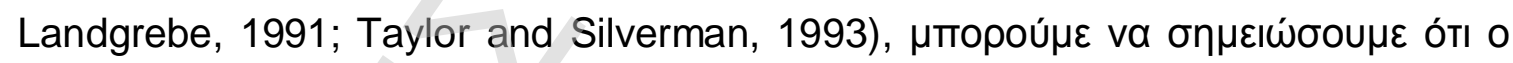

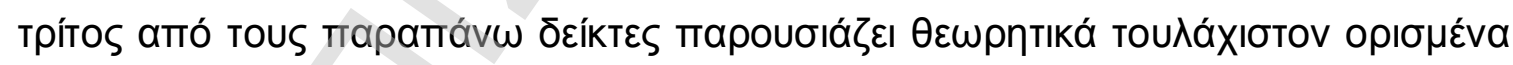

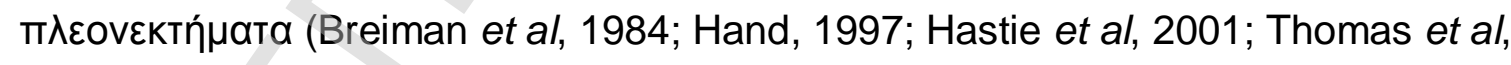
2002):

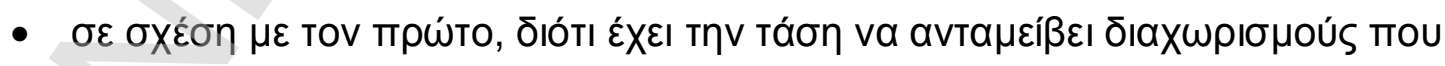

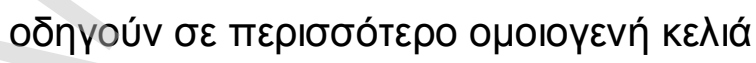

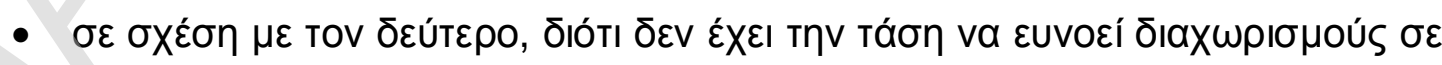

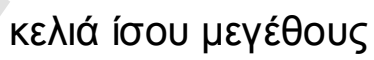




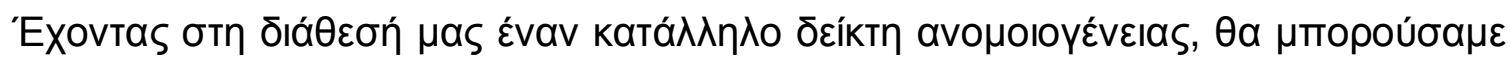

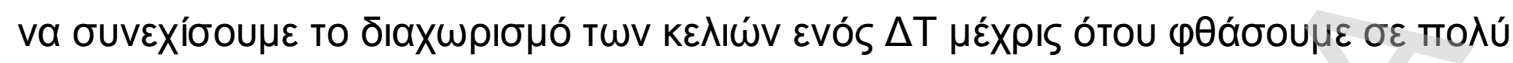

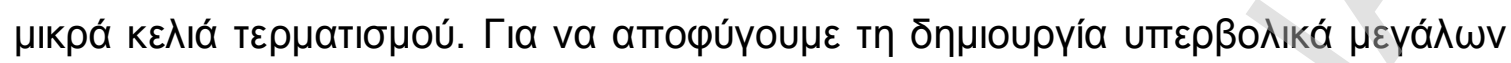

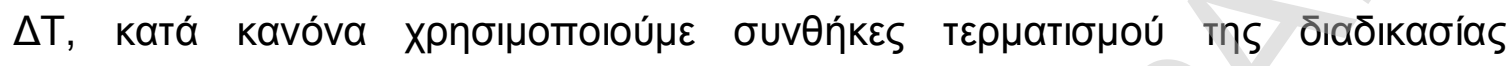

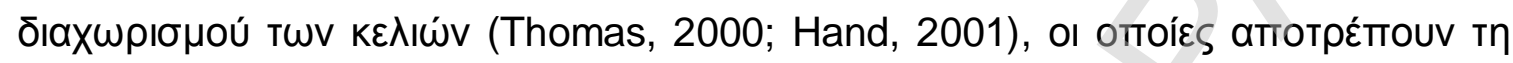

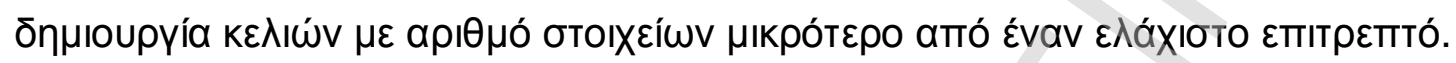

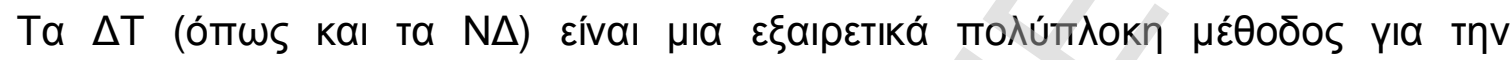

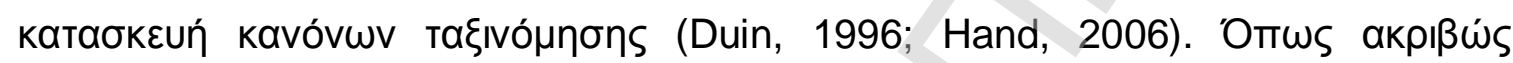

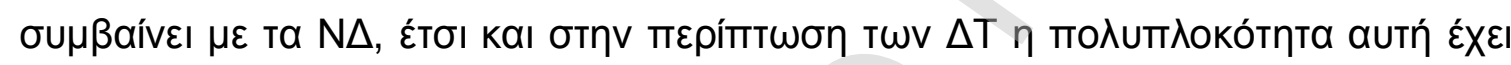

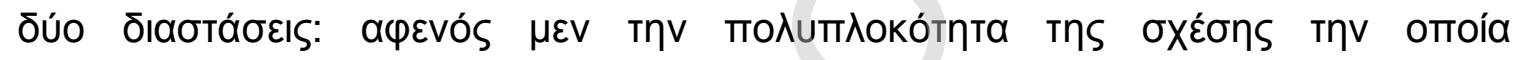

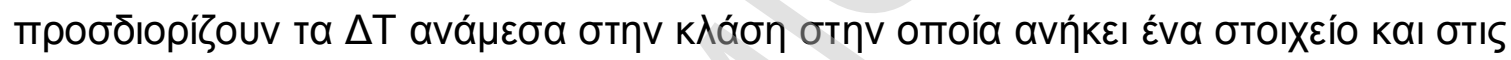

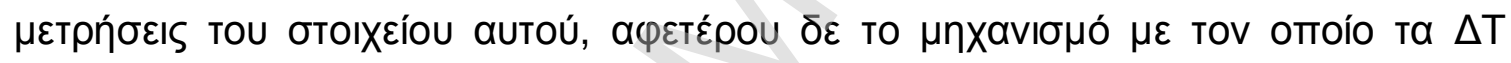

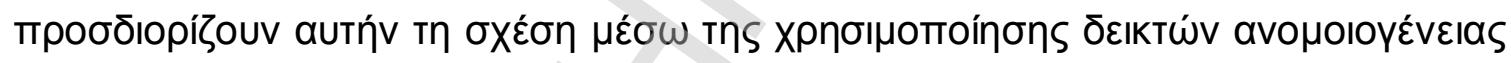

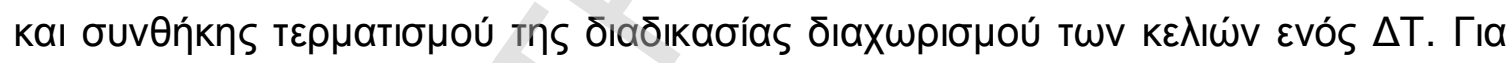

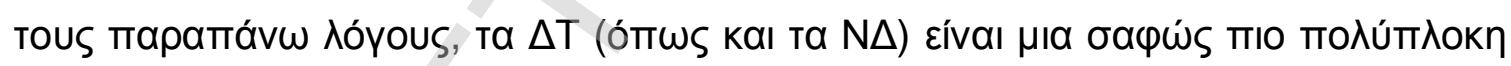

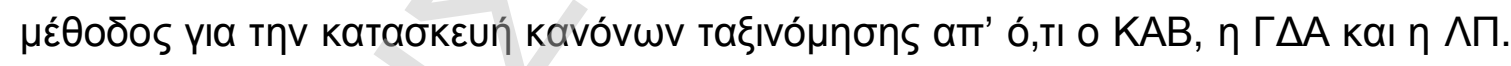

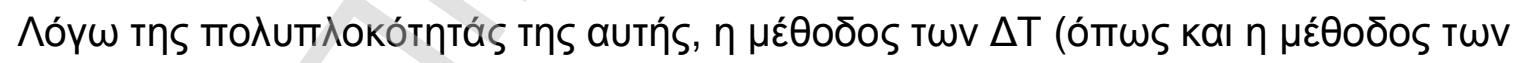

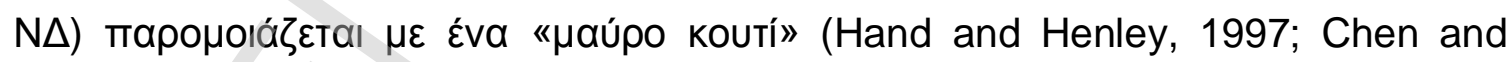
Huang, 2003).

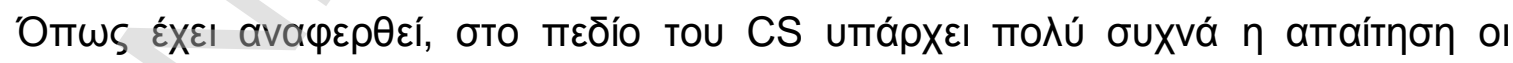

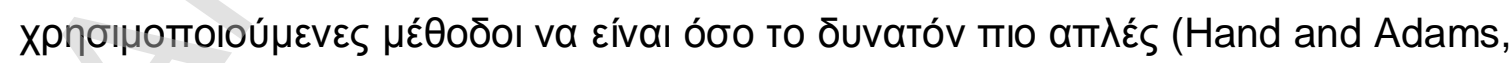

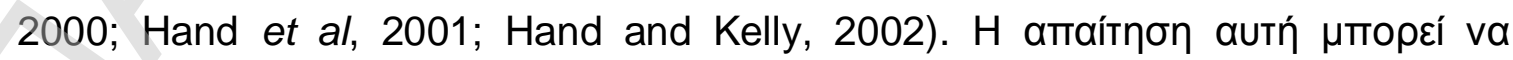

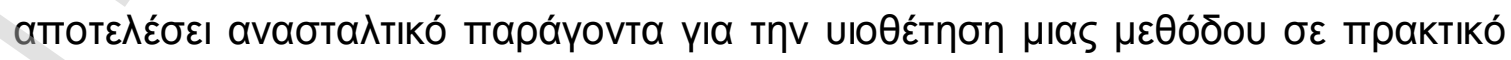




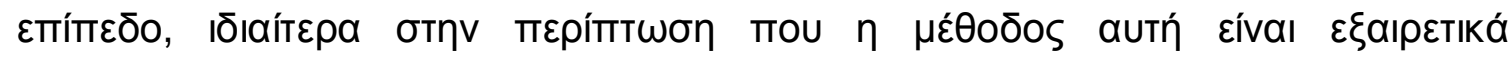

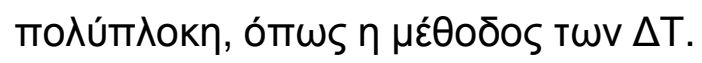

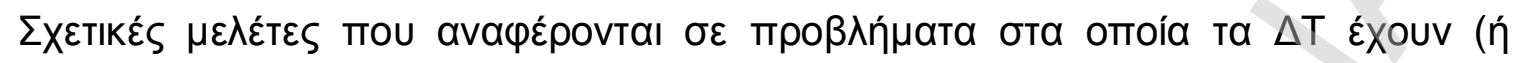

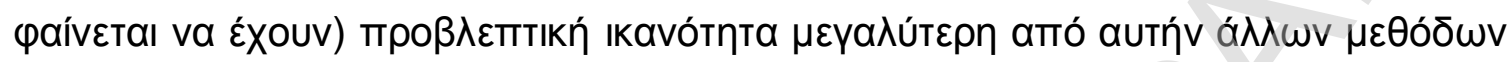

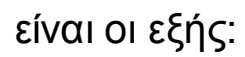

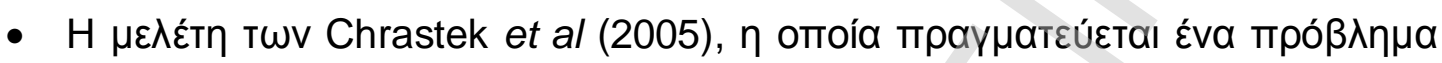

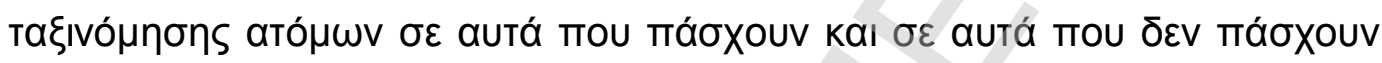

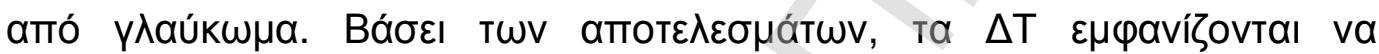

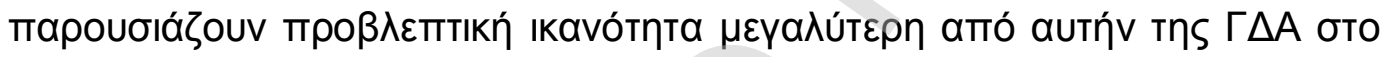

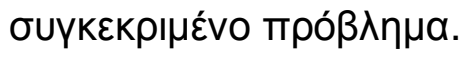

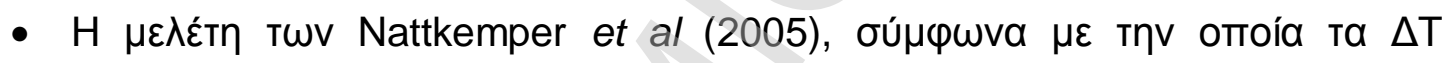

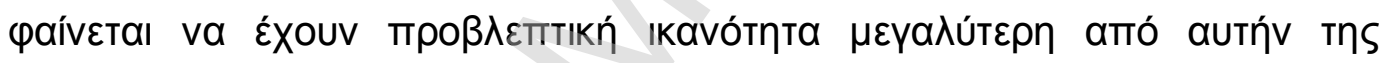

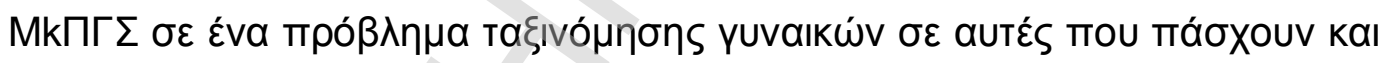

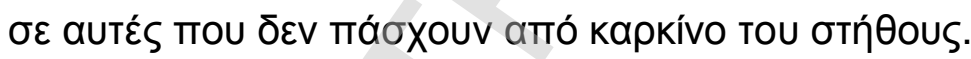

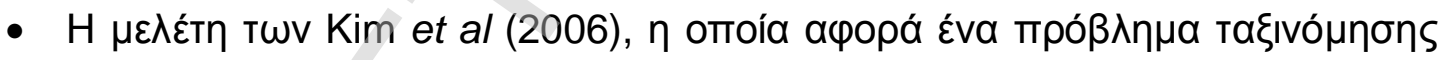

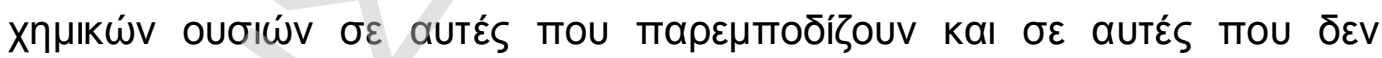

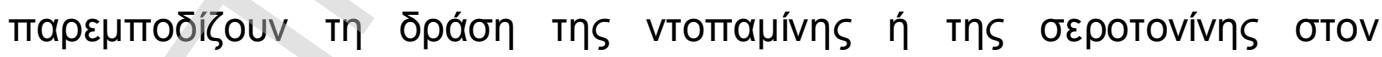

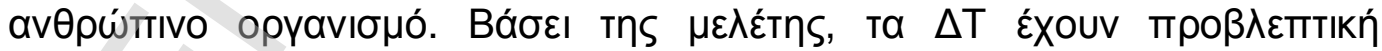

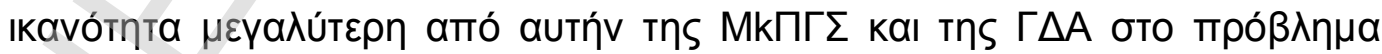
aUTó. 


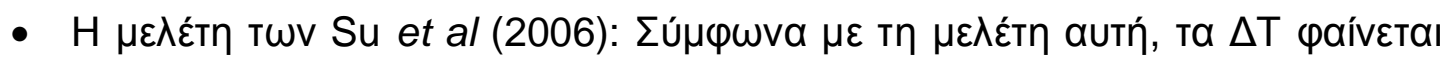

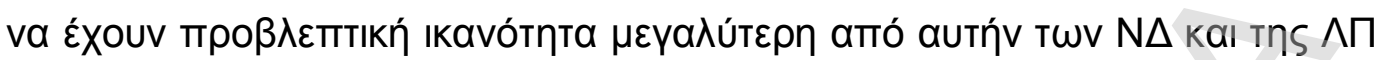

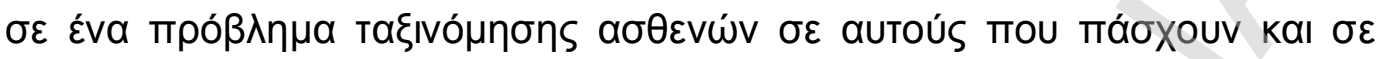

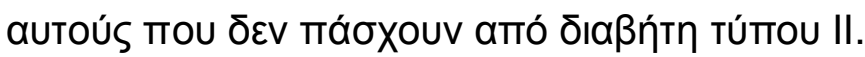

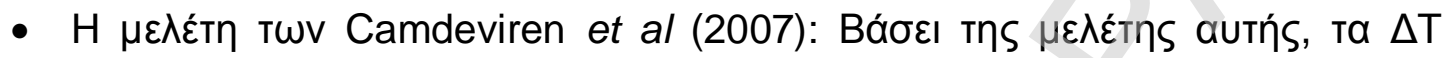

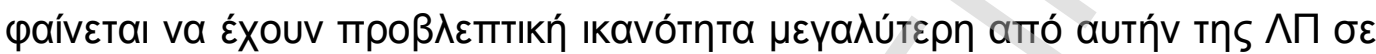

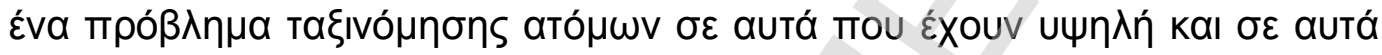

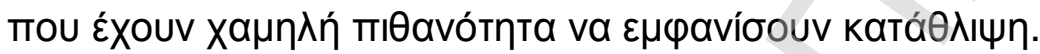

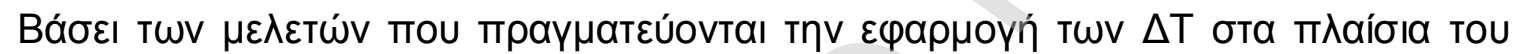

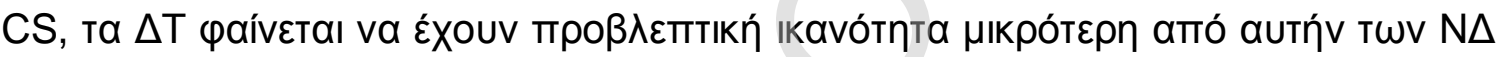

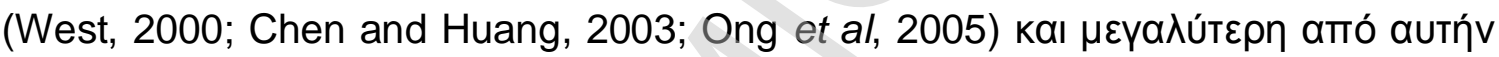

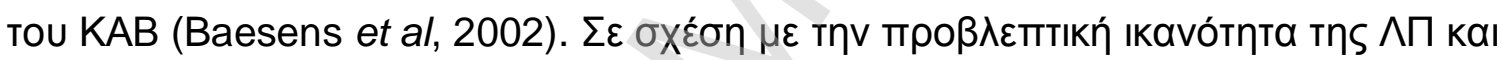

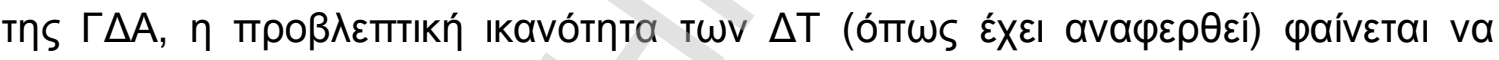

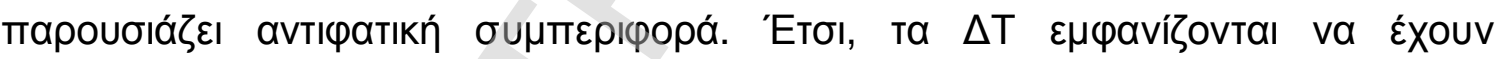

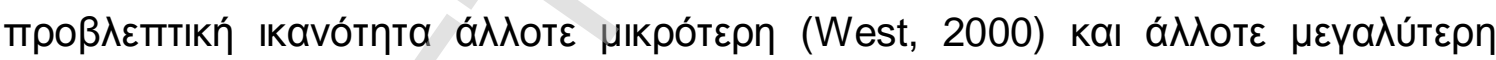

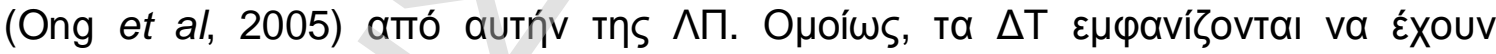

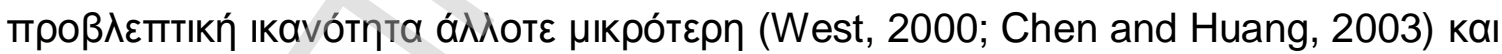

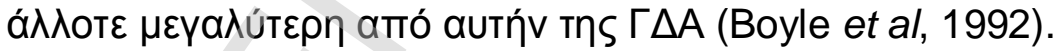




\subsection{M}

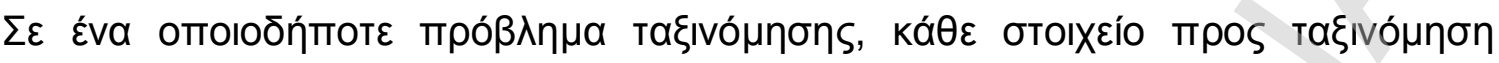

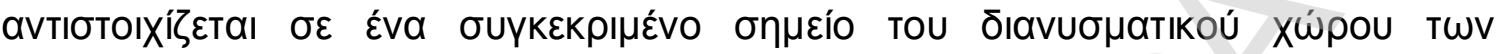

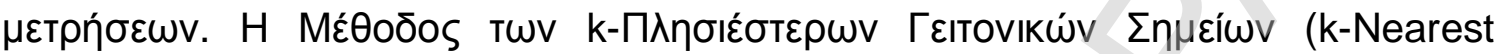

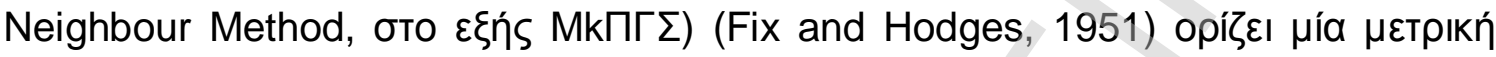

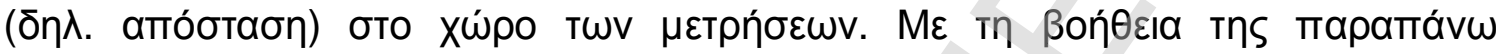

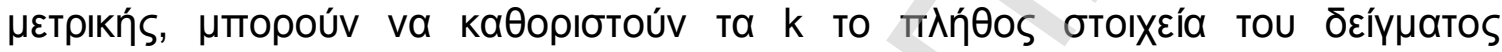

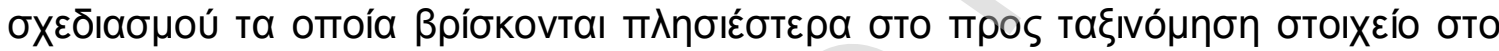

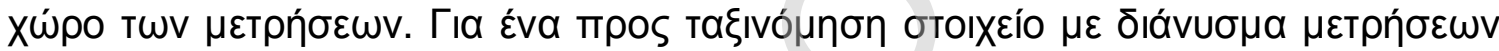

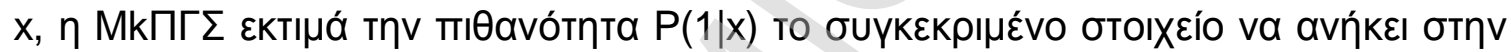

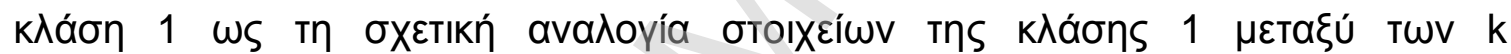

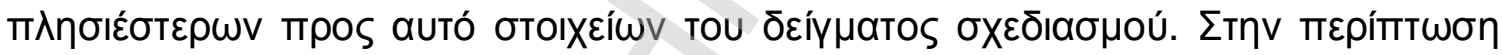

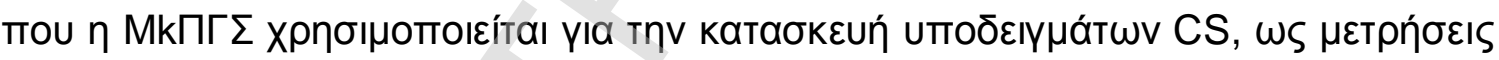

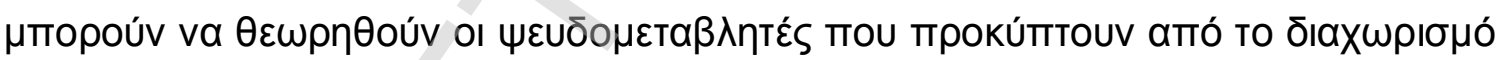

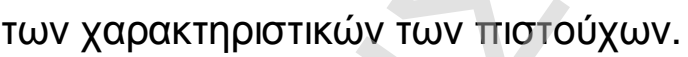

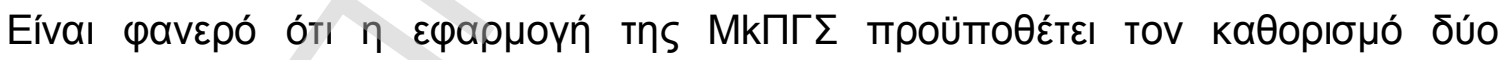

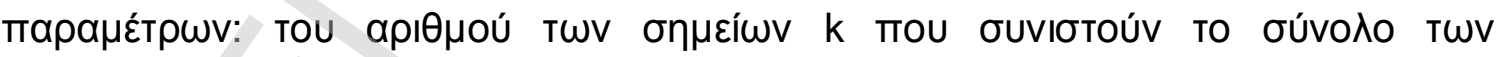

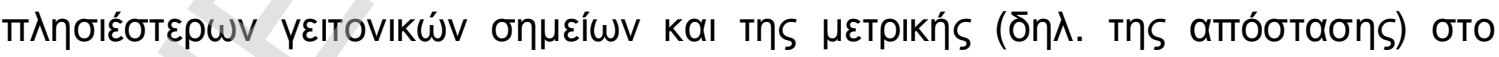

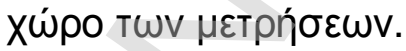

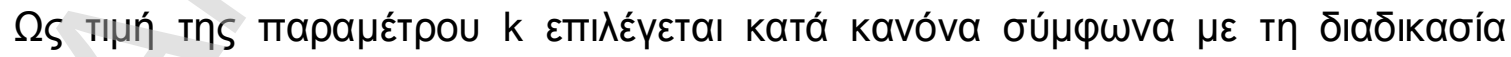

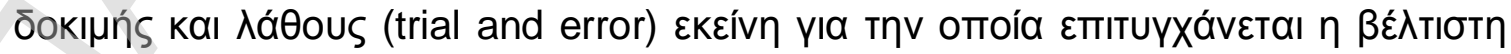

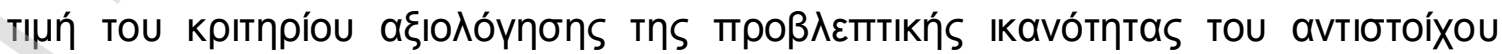




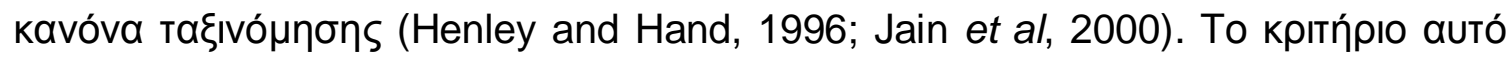

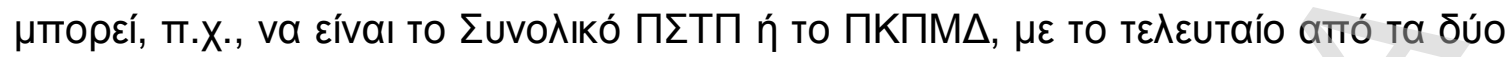

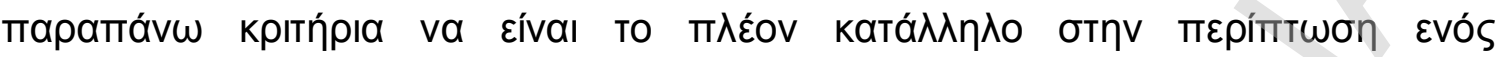
троßли́натољ CS.

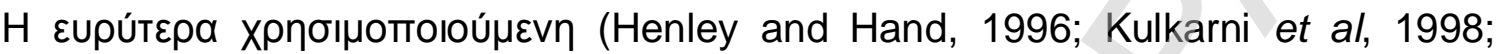

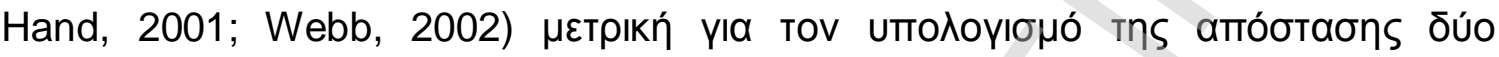

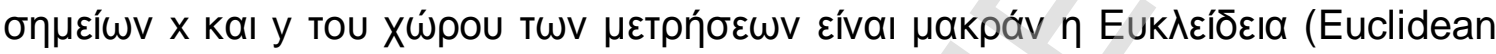
metric):

$$
d(x, y)=\left\{(x-y)^{T}(x-y)\right\}^{1 / 2}
$$

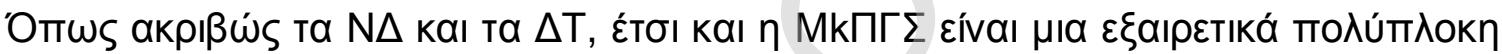

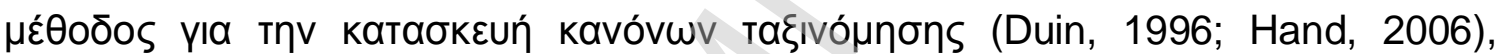

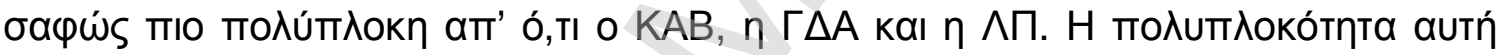

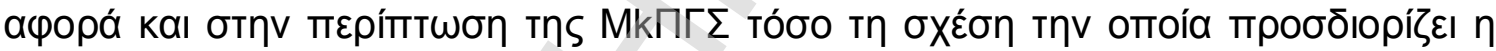

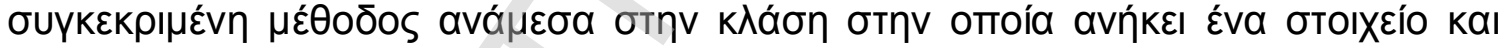

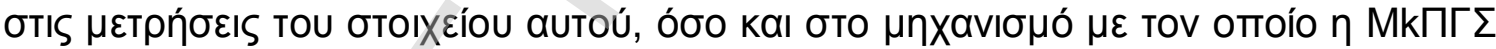

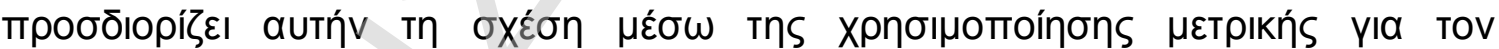

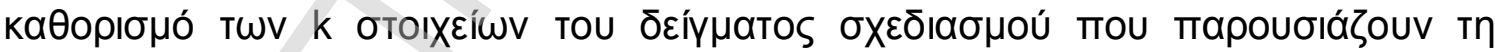

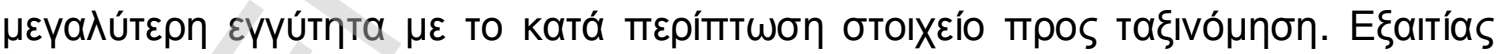

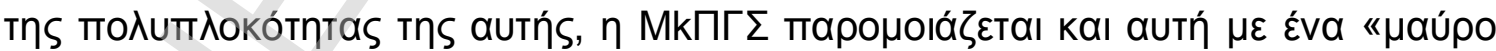
Koutí» (Hand and Henley, 1997; West, 2000; Chen and Huang, 2003).

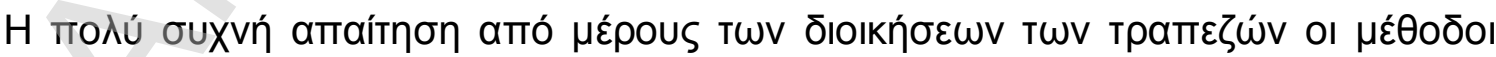

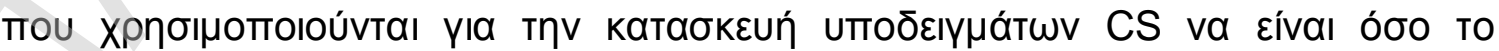

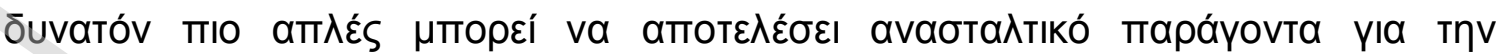




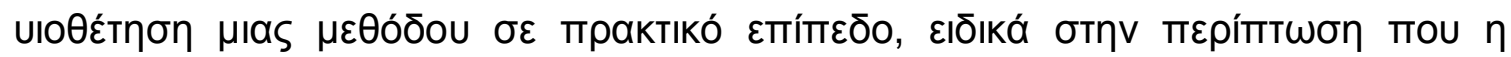

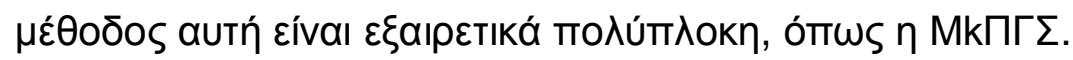

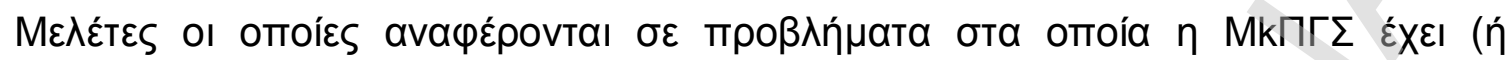

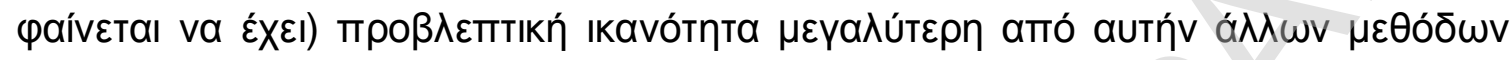

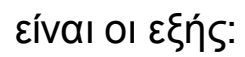

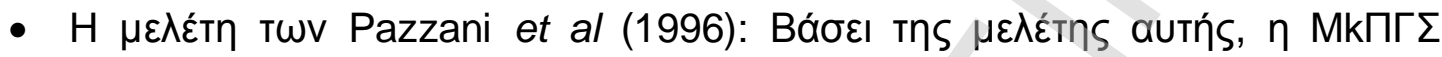

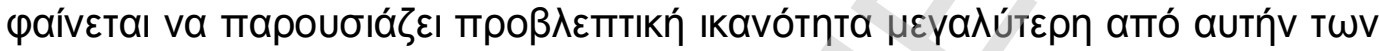

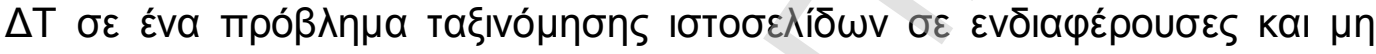

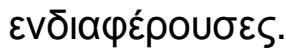

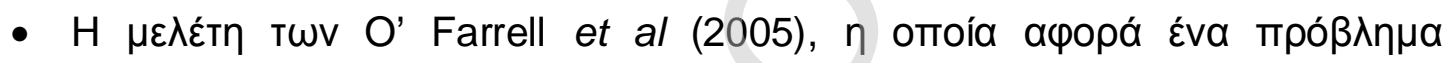

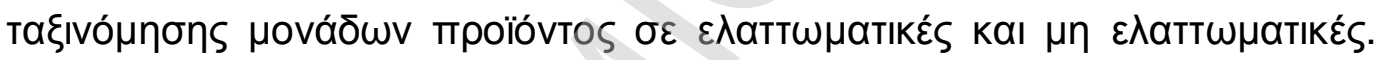

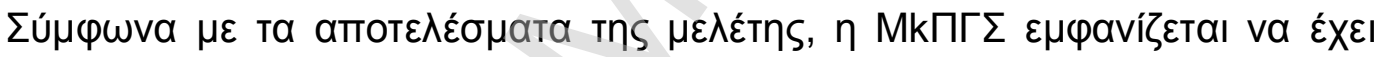

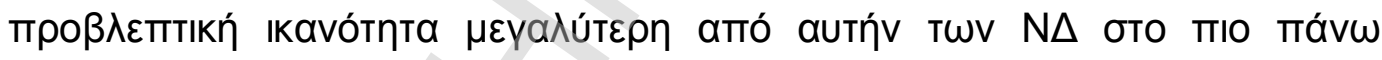

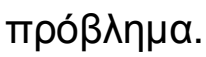

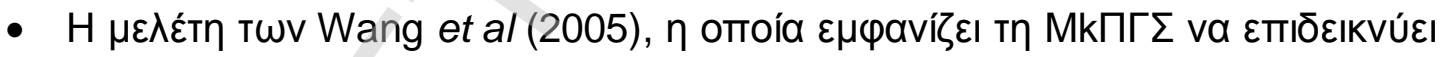

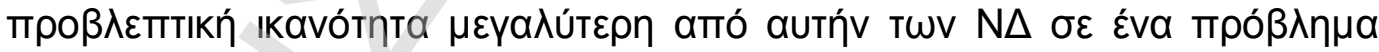

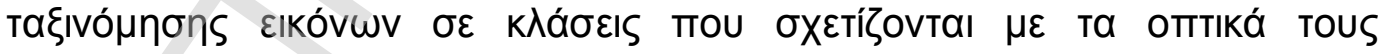
характпрібтіка́.

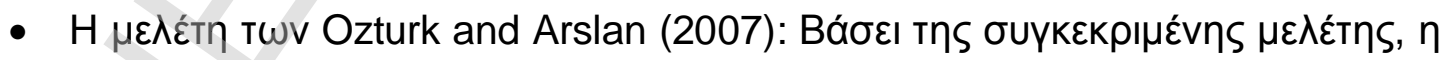

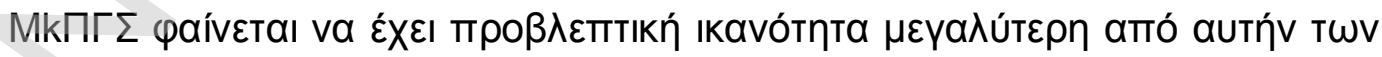

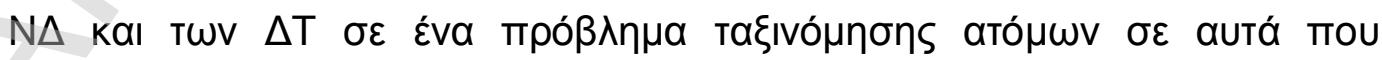

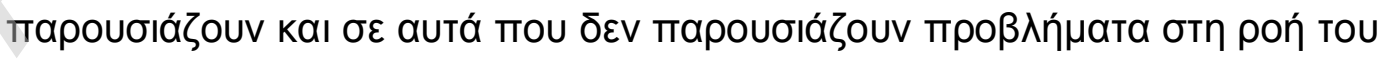

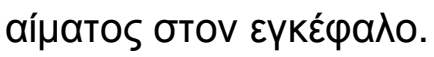




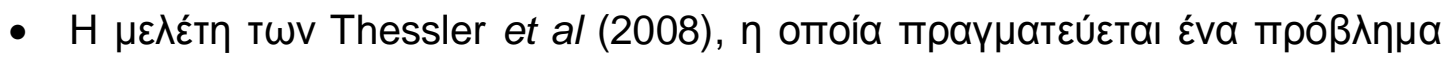

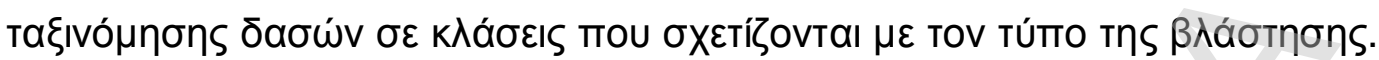

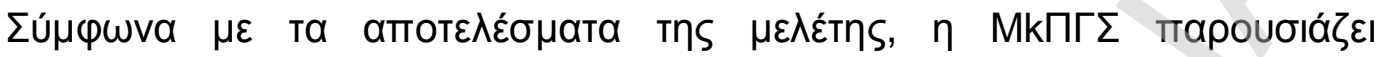

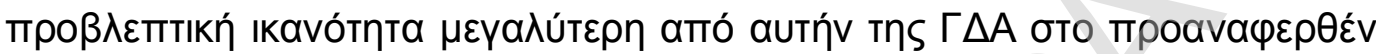

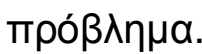

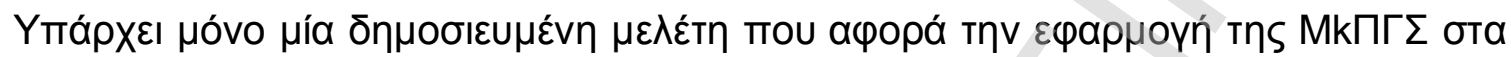

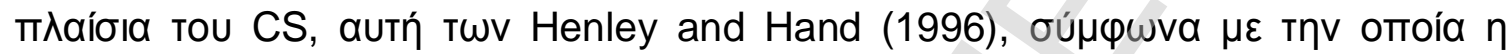

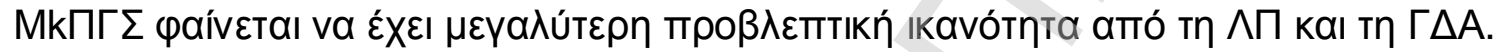




\section{KEФA^AIO 4 \\ ANA $\Lambda$ YH $\triangle E \Delta O M E N \Omega N$}

\subsection{Eıбaywyń}

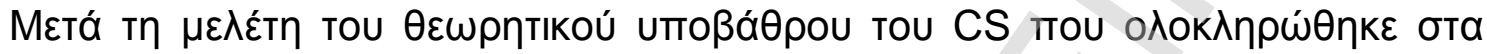

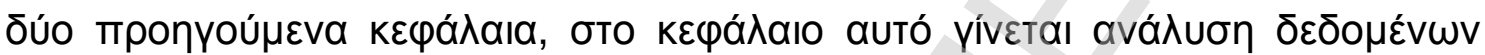

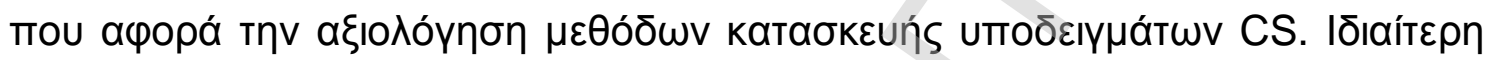

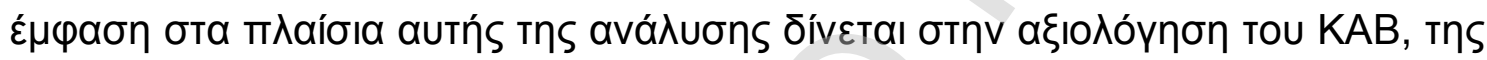

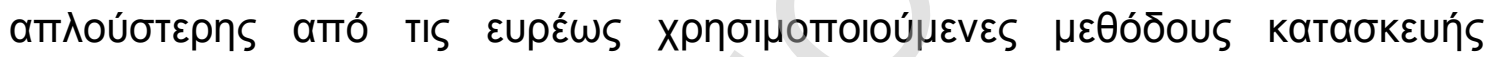

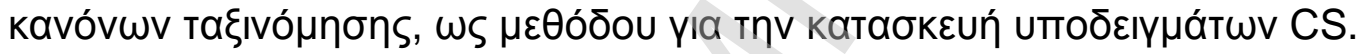

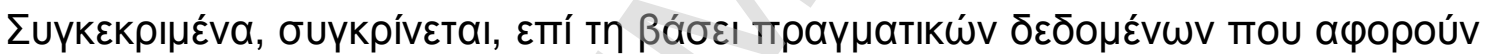

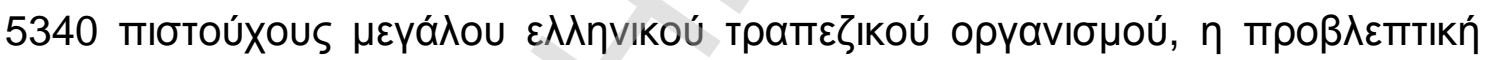

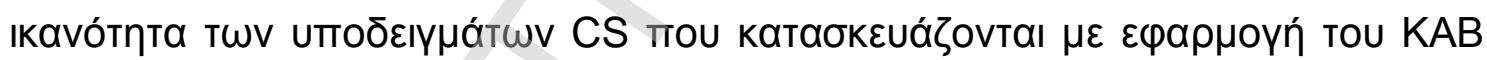

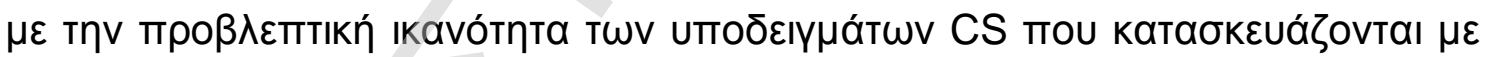

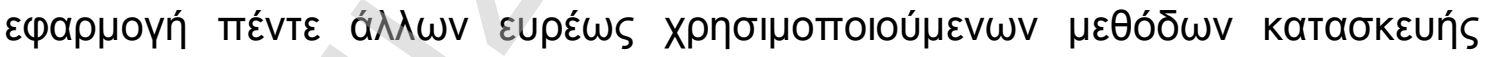

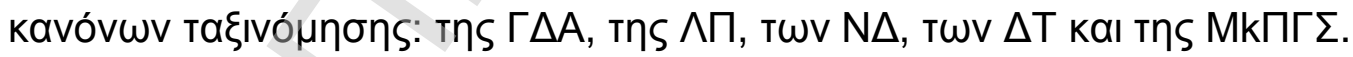

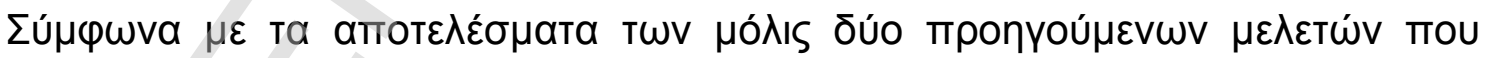

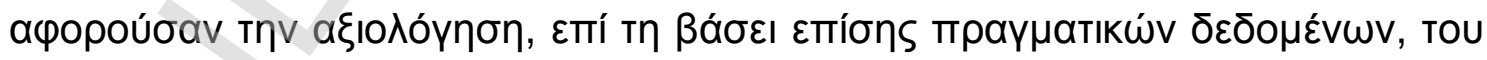

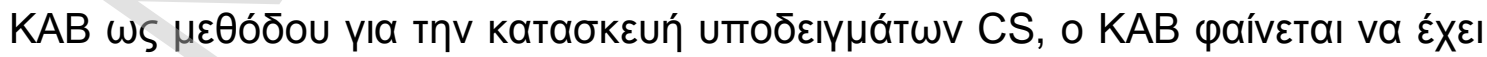

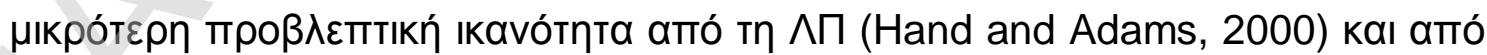
та $\Delta \mathrm{T}$ (Baesens et al, 2002). 


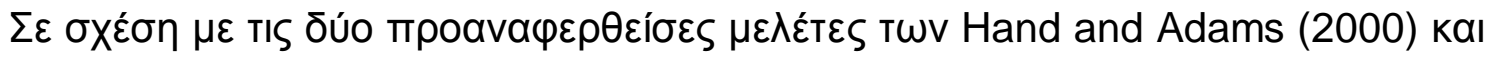

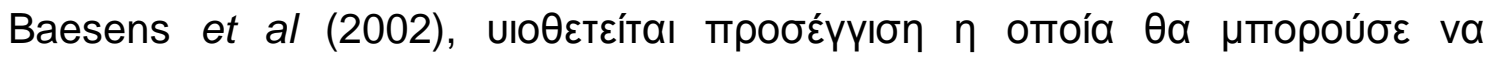

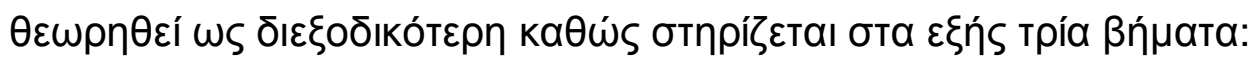

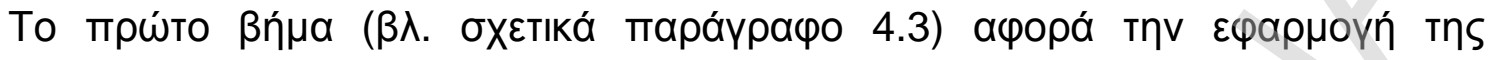

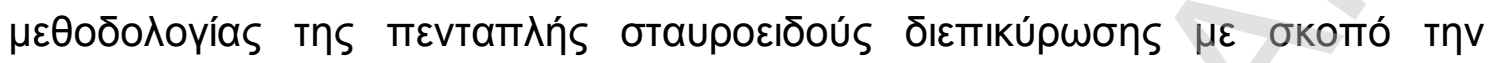

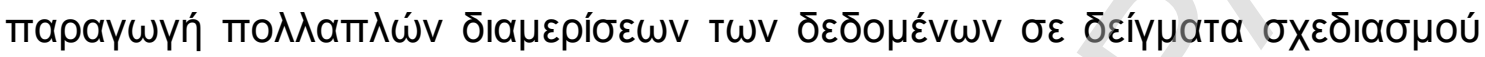

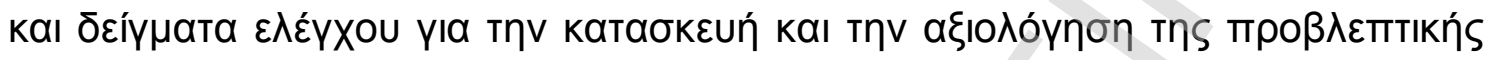

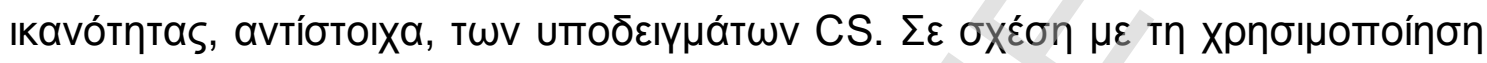

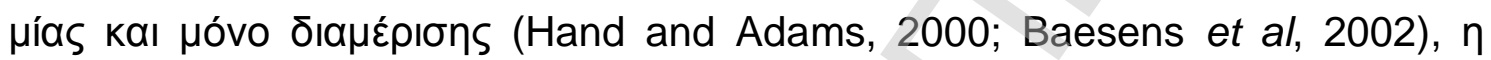

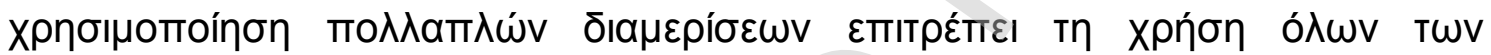

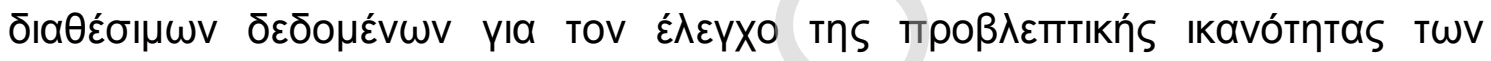

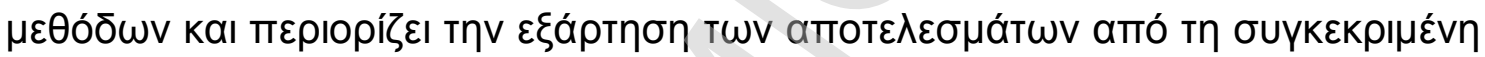

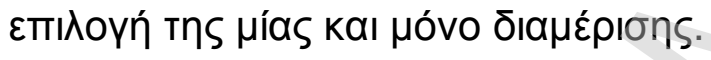

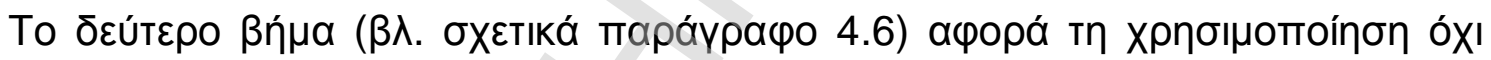

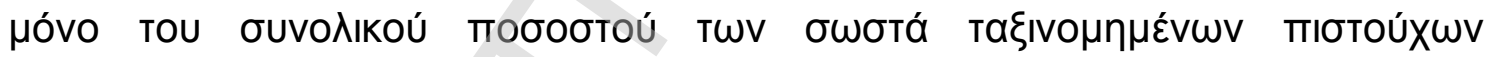

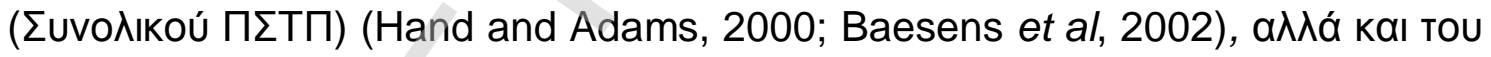

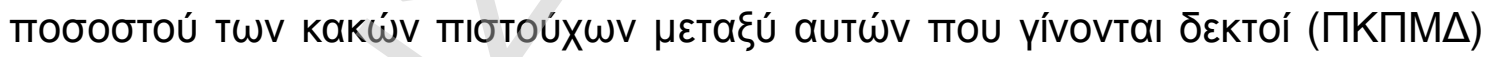

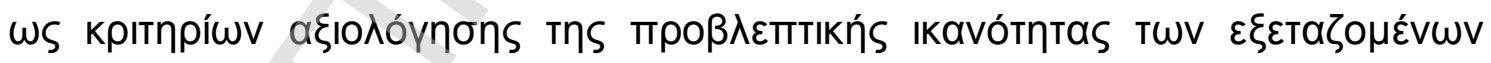

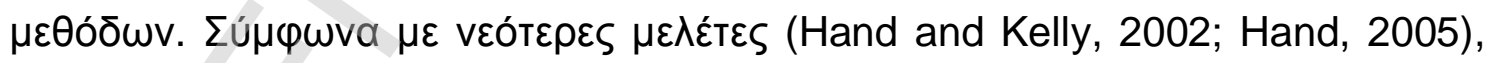

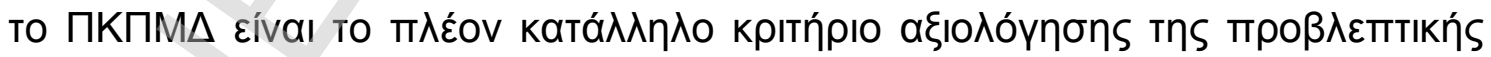

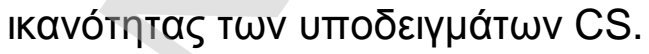

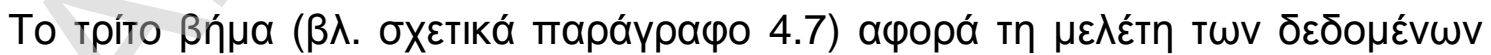

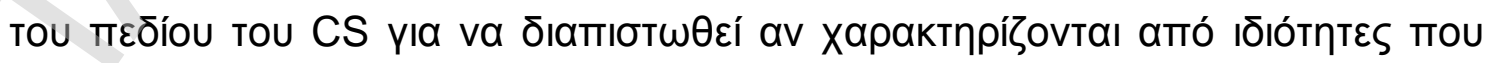

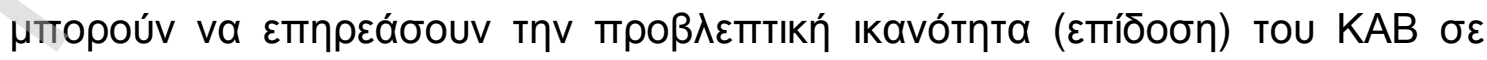

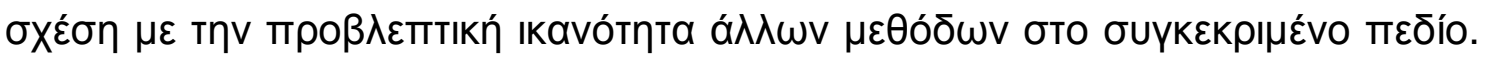




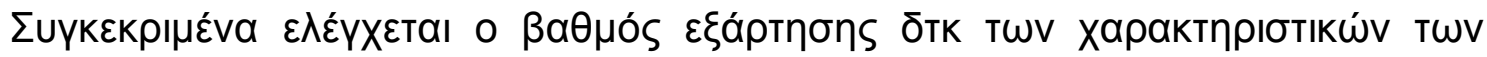

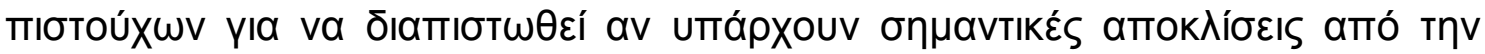

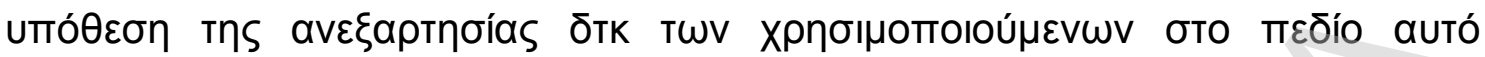

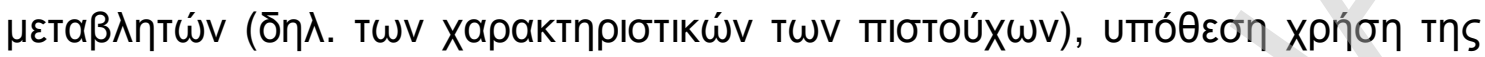

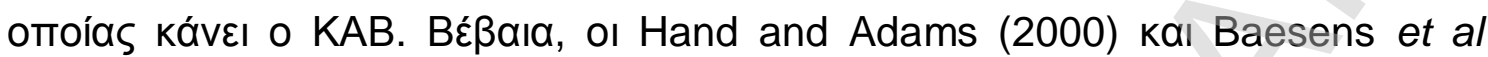

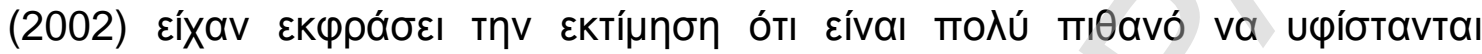

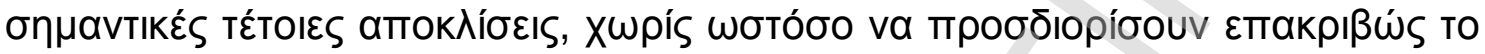

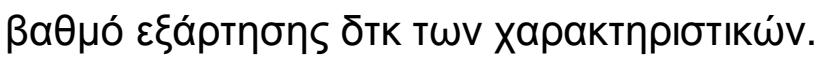

\section{$4.2 \Delta \varepsilon i ́ \gamma \mu \alpha$}

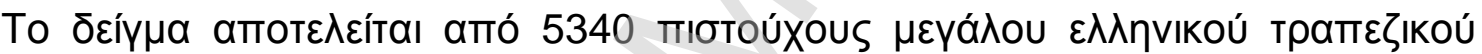

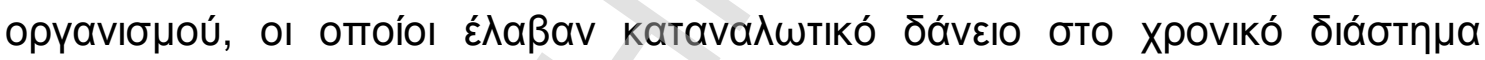

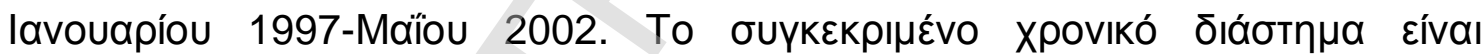

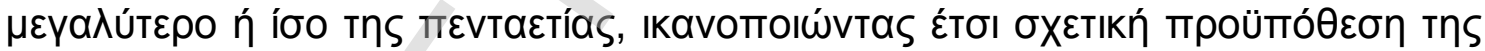

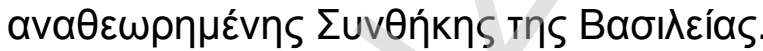

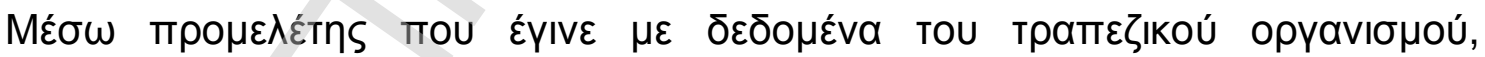

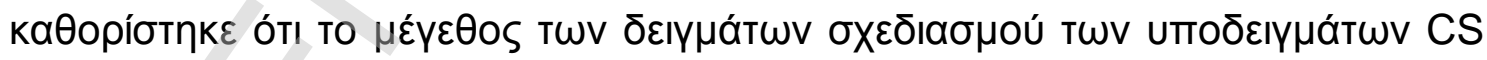

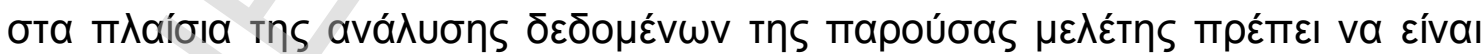

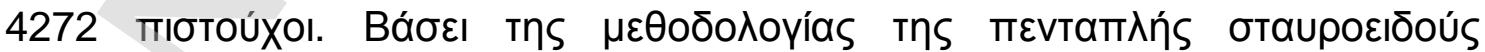

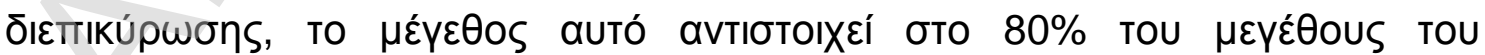

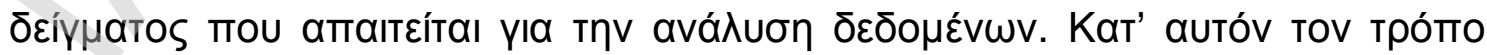

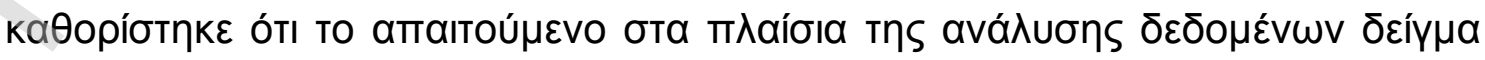




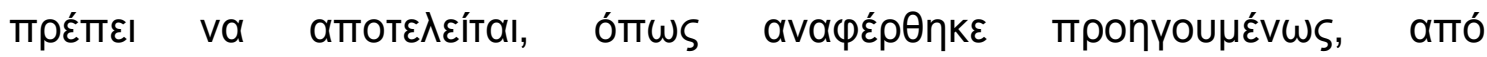
$(100 / 80)^{*} 4272=5340$ mıбтои́Xous.

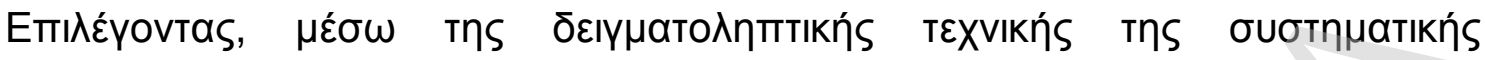

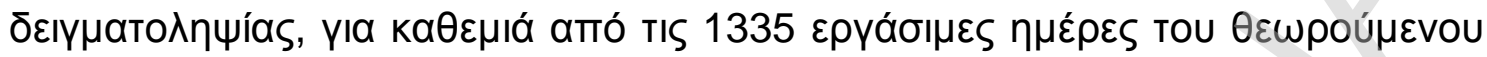

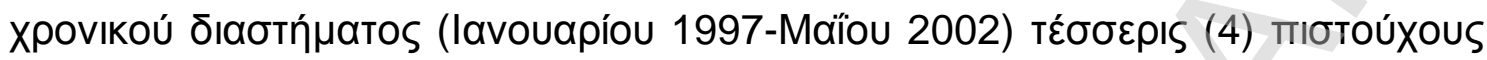

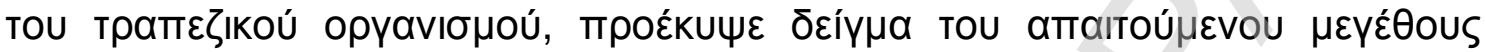

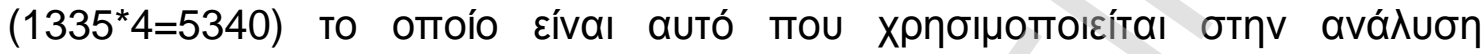
$\varepsilon \delta о \mu \varepsilon ́ v \omega v$.

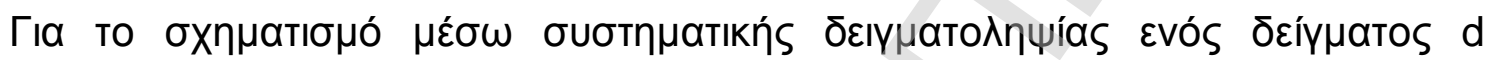

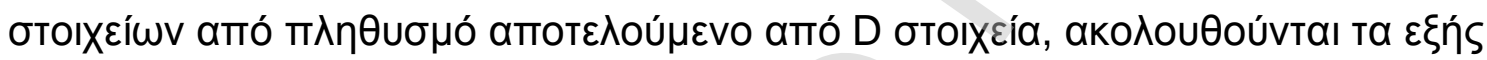
oтóסıı (Stehman and Overton, 1994; Gundersen et al, 1999; Rose et al, 2006; Zhang, 2008):

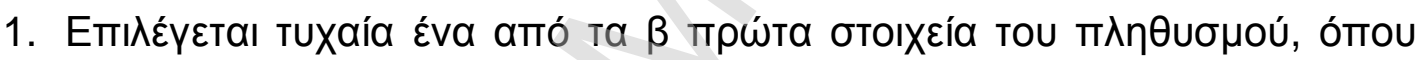

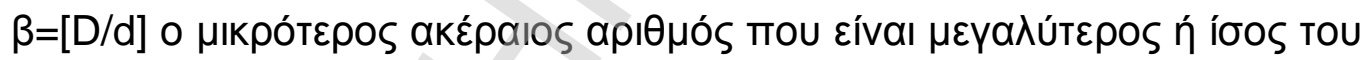
$\mathrm{D} / \mathrm{d}$.

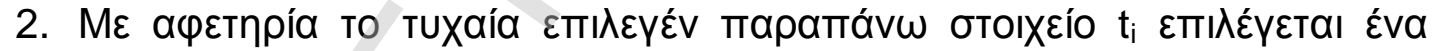

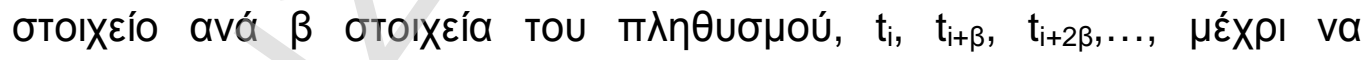

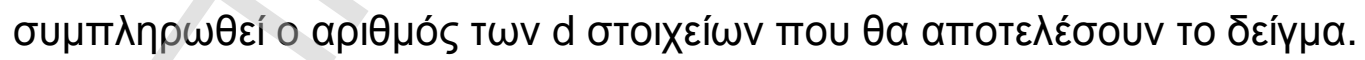

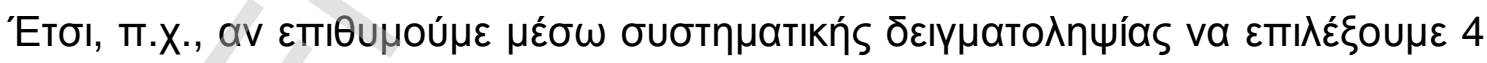

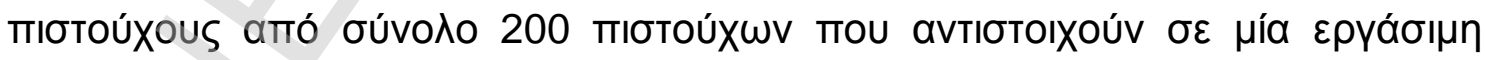
$\eta \mu \varepsilon ́ \rho \alpha:$

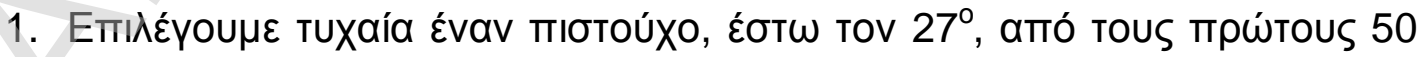

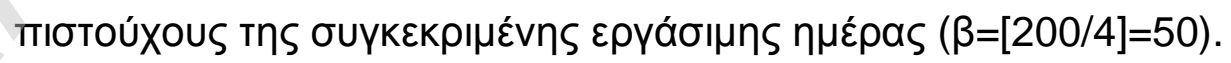

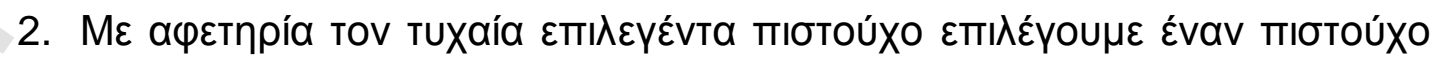

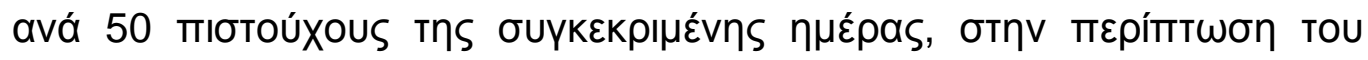




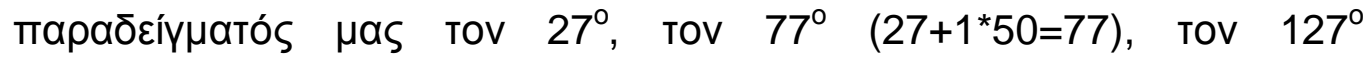
$(27+2 * 50=127)$ kaı tov $177^{\circ}\left(27+3^{\star} 50=177\right)$.

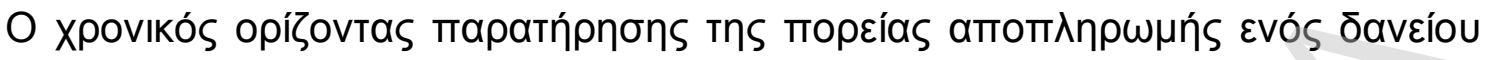

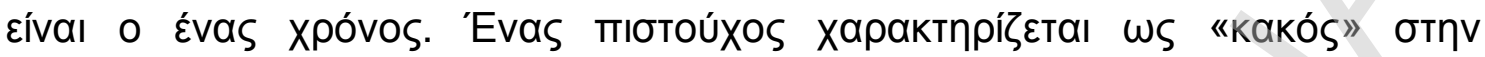

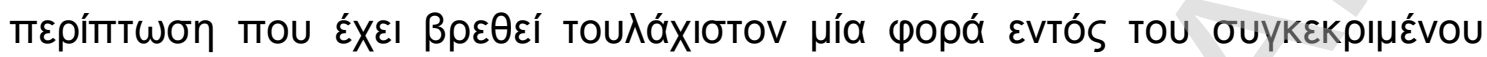

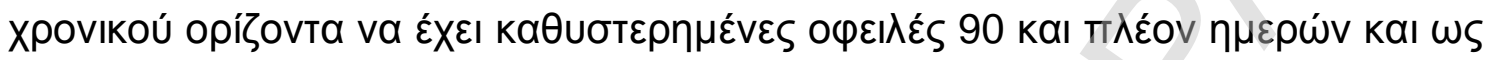

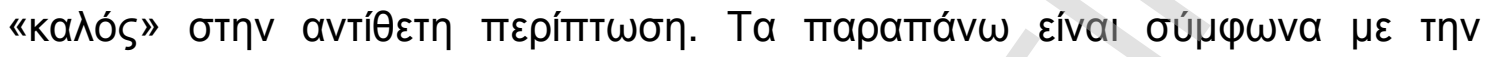

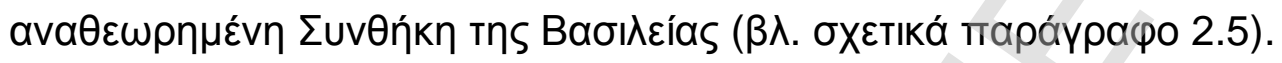

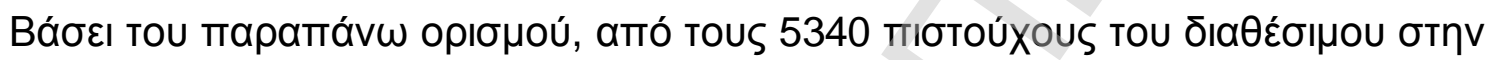

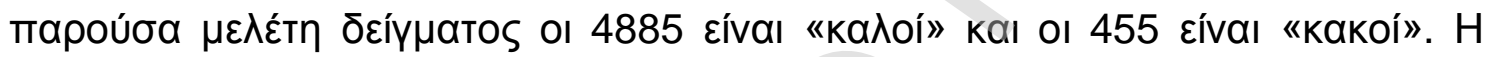

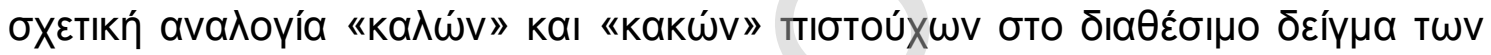

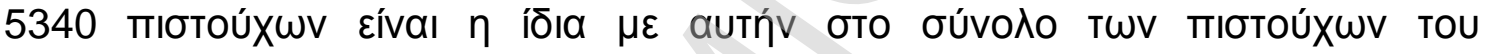

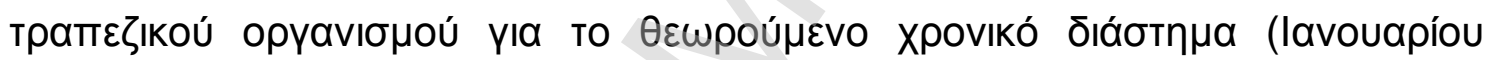
1997-Maiiou 2002).

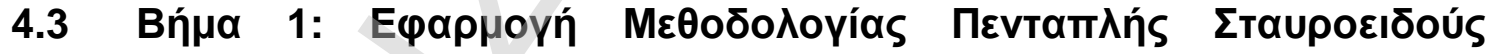

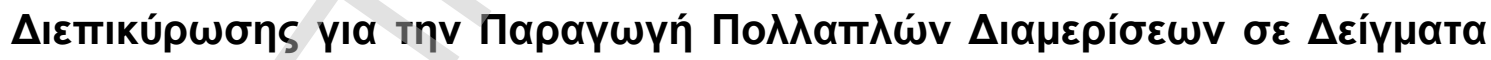

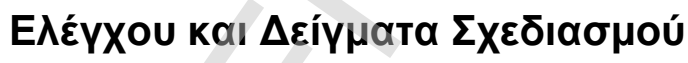

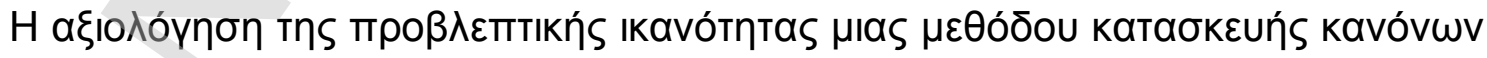

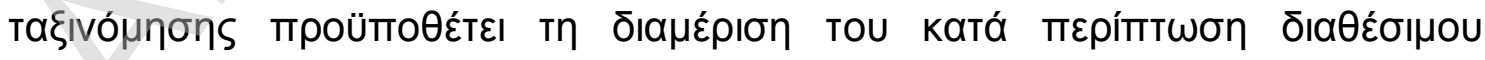

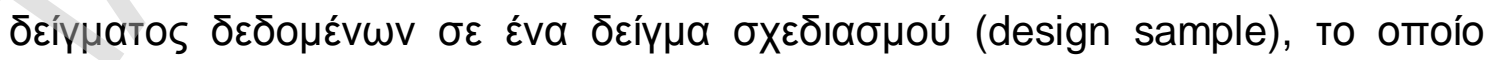

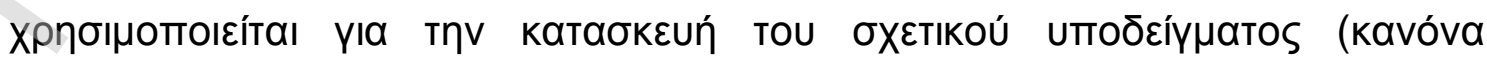

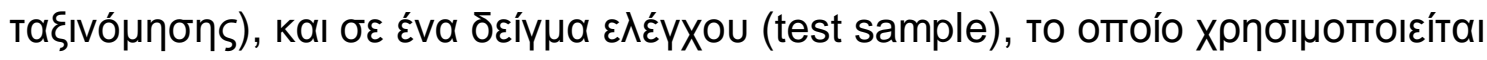




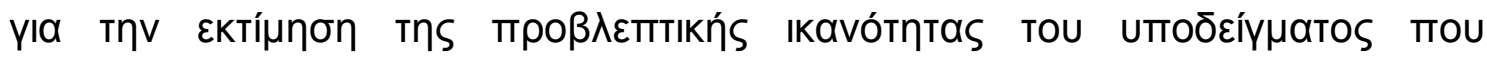

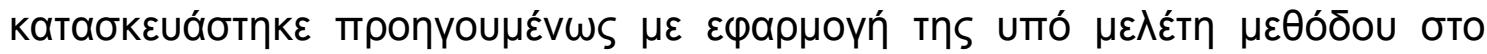

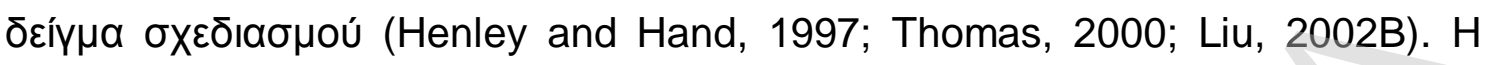

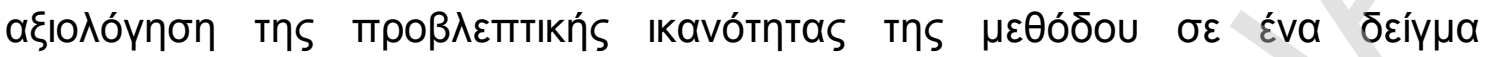

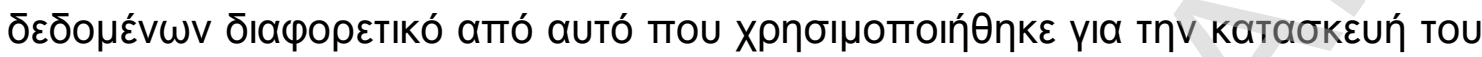

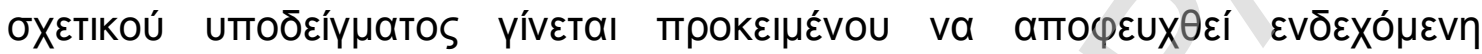

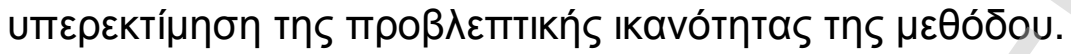

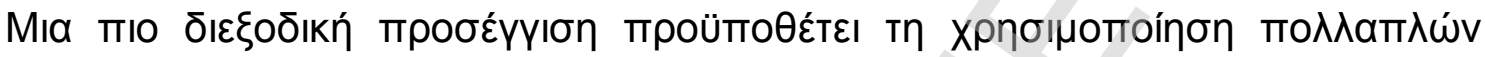

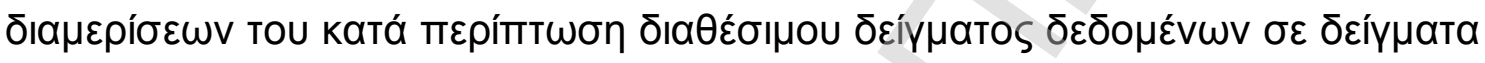

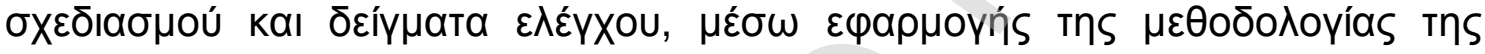

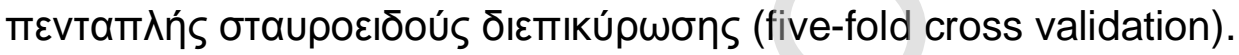

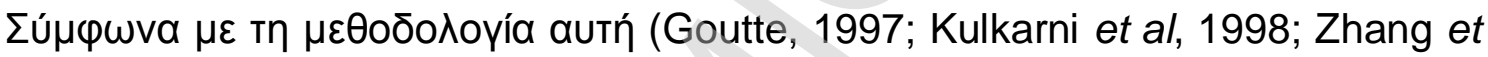

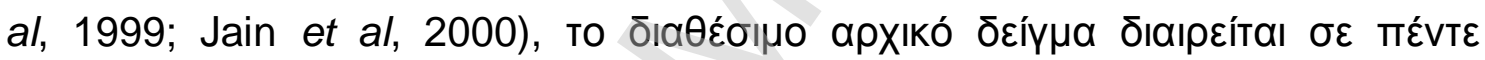

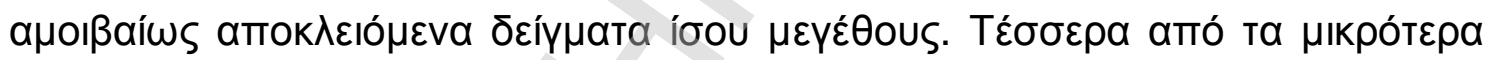

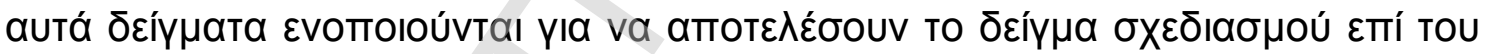

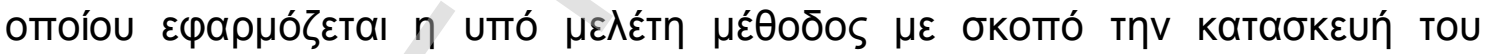

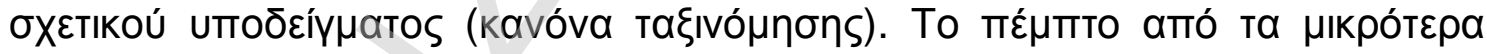

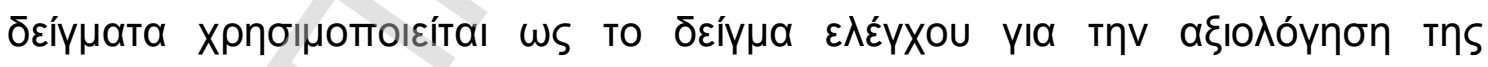

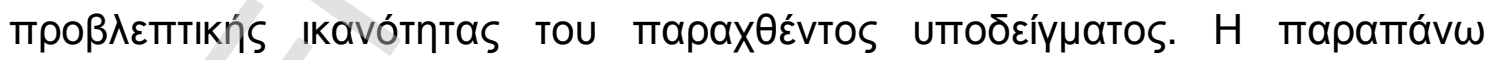

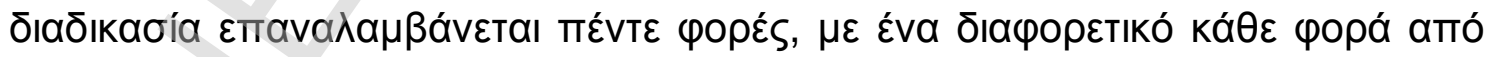

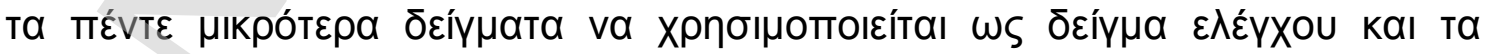

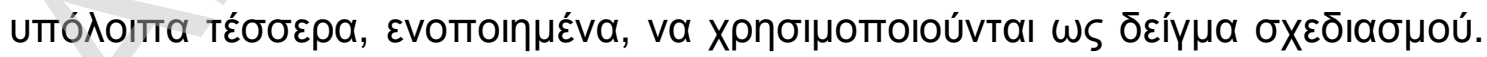

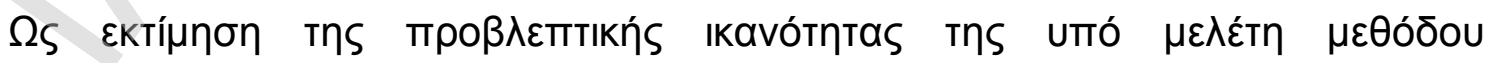

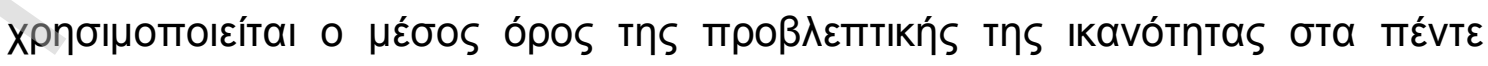

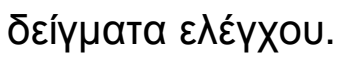




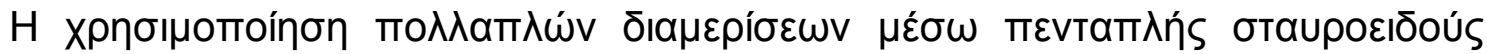

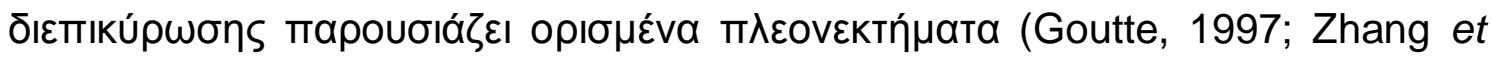

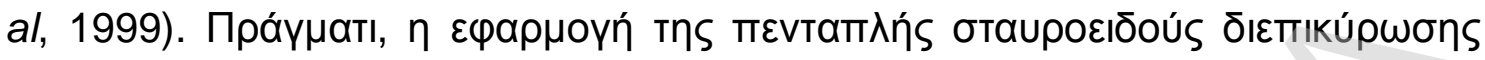

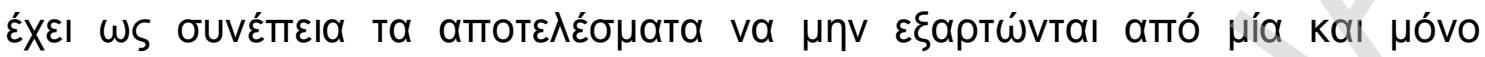

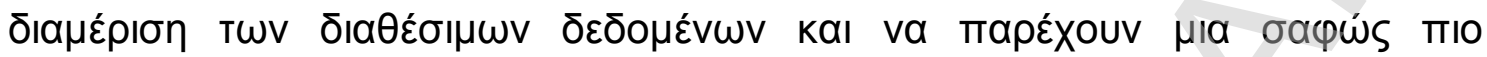

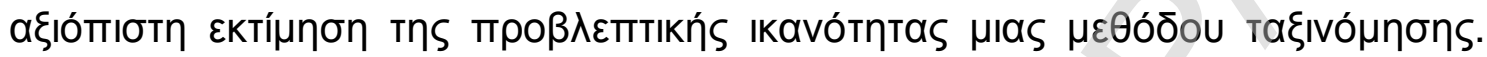

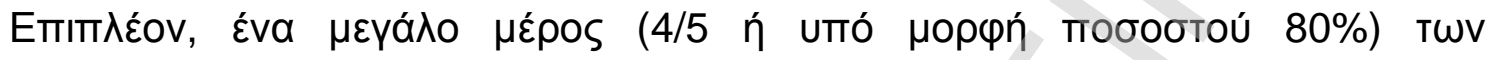

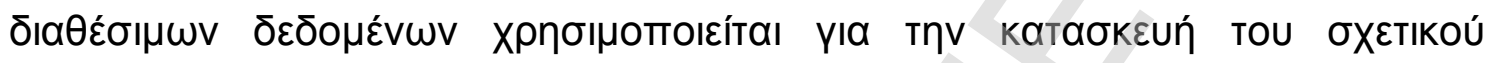

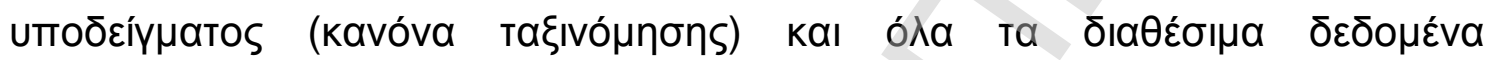

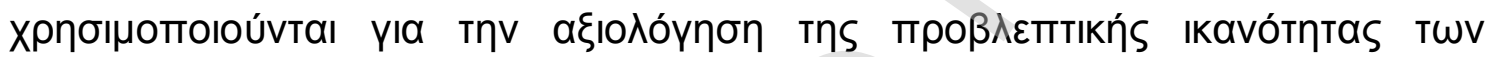

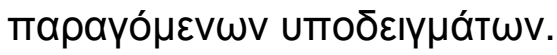

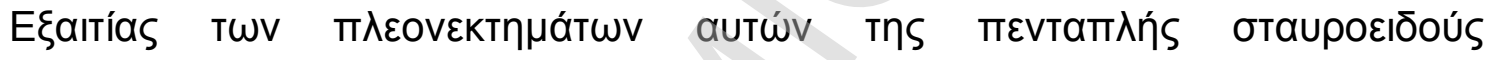

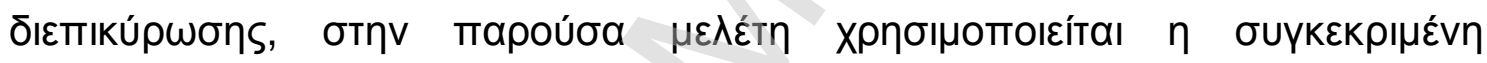

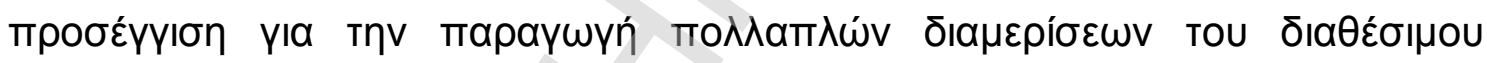

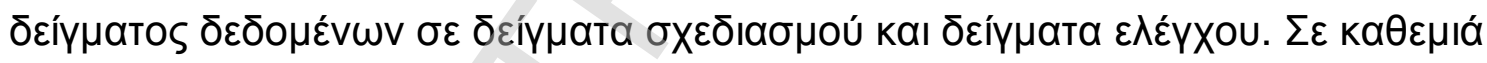

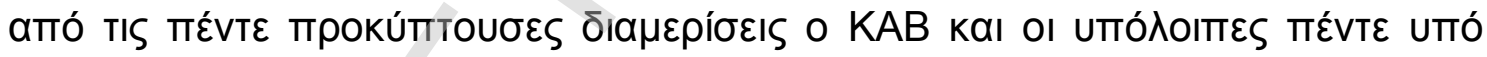

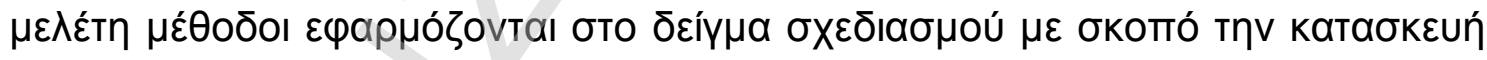

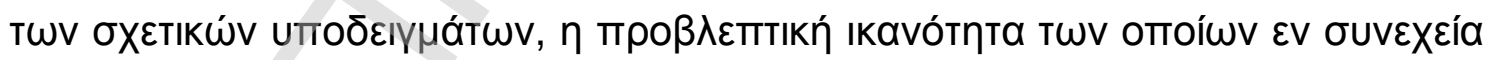

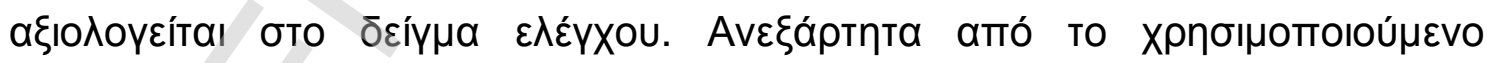

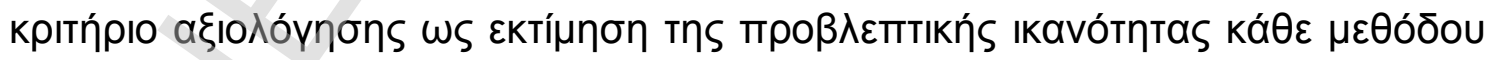

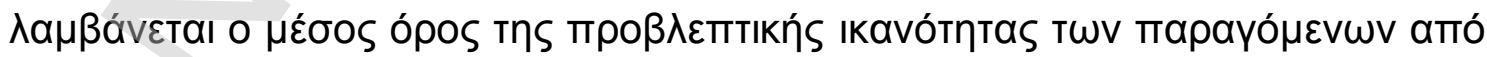

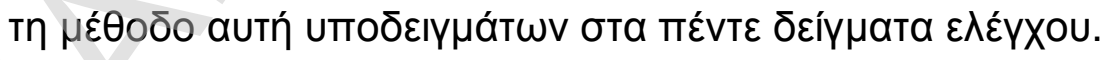

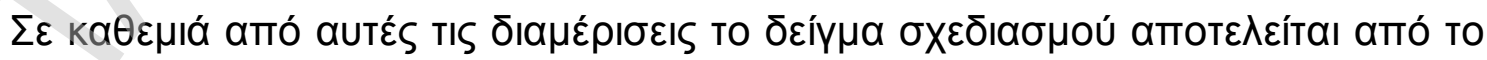

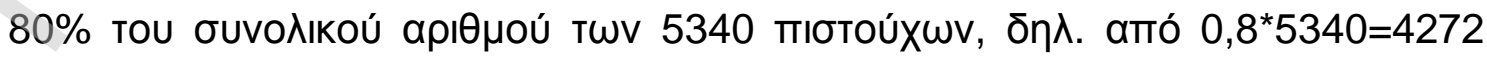

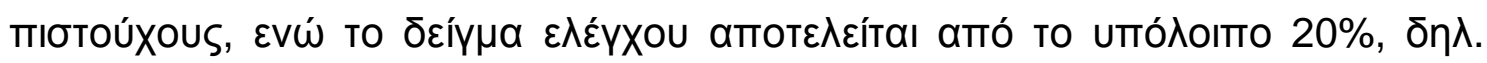




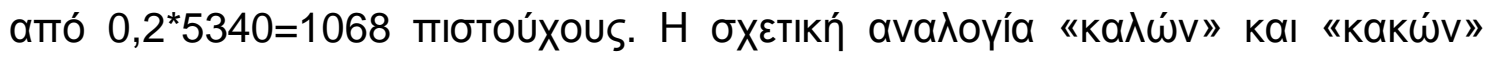

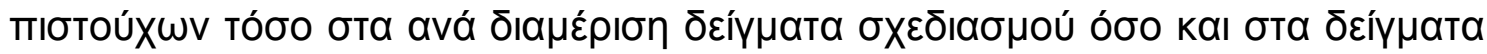

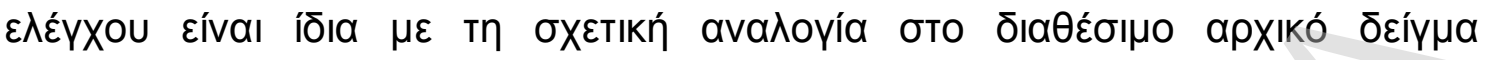

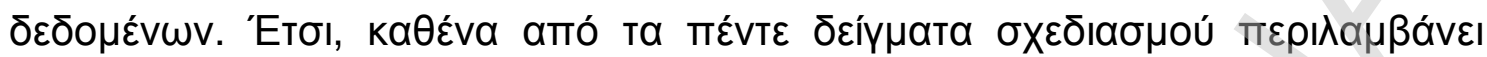

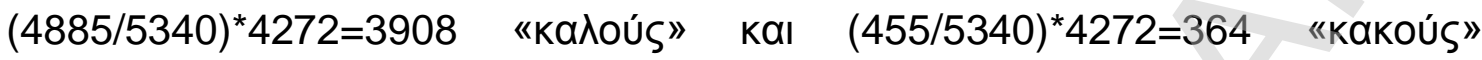

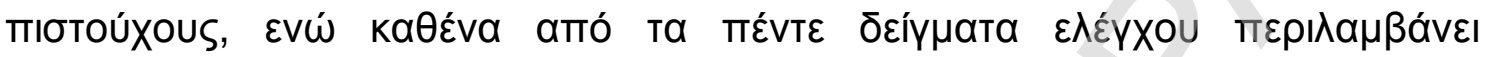

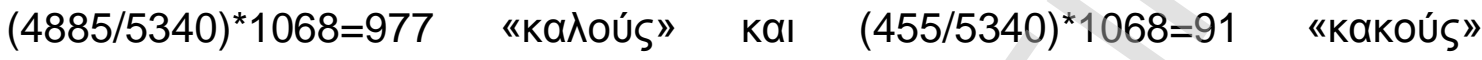
пıтTоúxous.

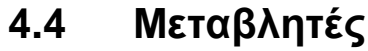

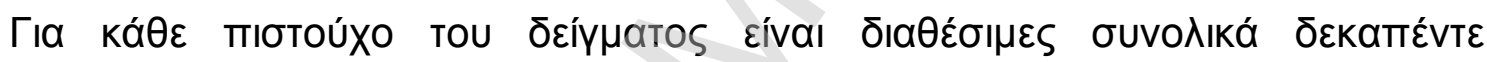

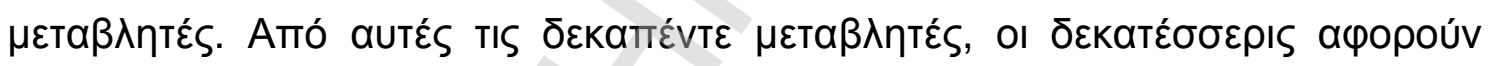

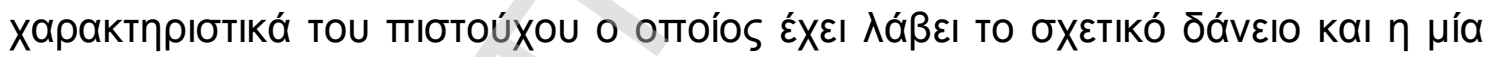

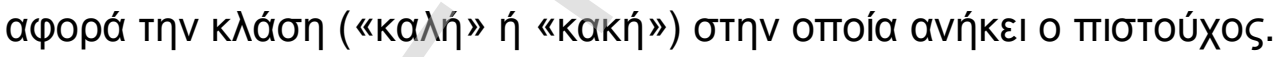

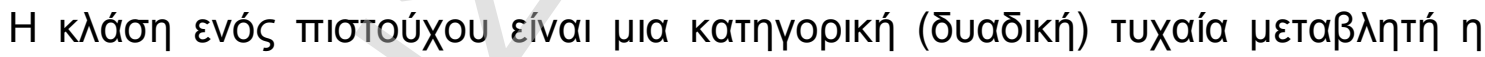

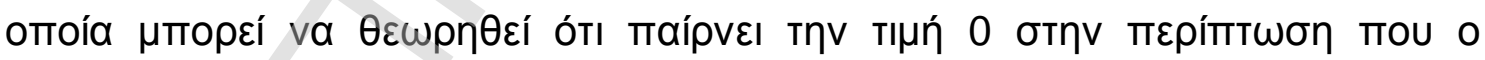

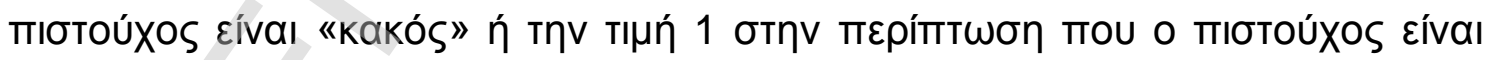

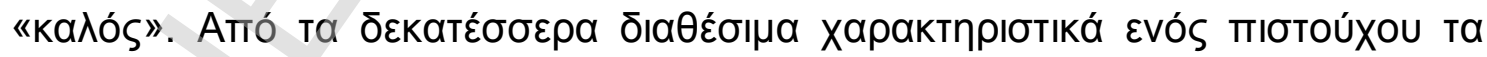

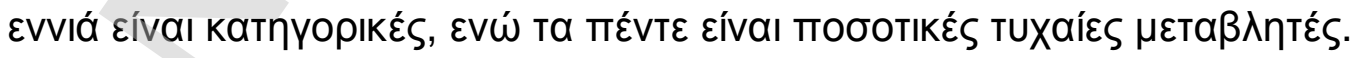

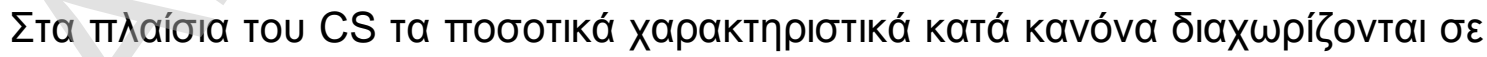

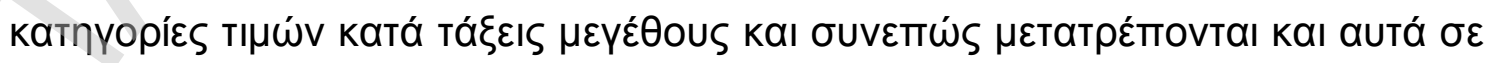
Katnyopikó (Hand and Henley, 1997; Thomas, 2000; Thomas et al, 2002). As

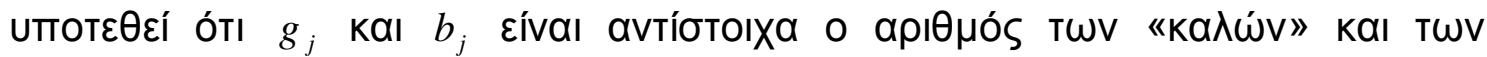




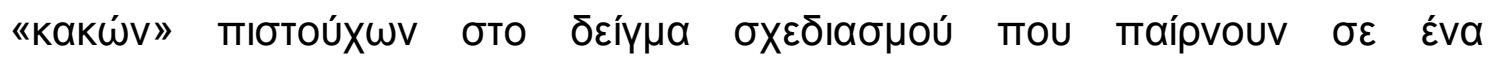

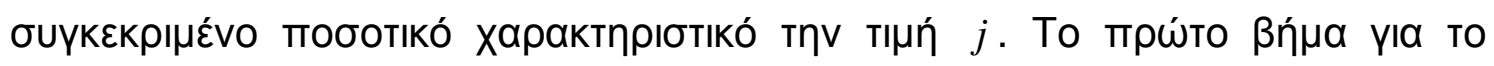

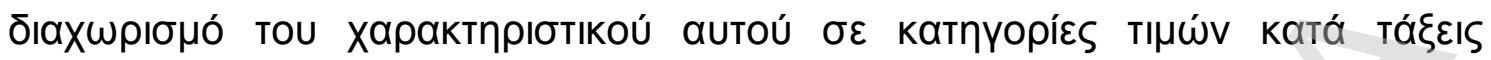

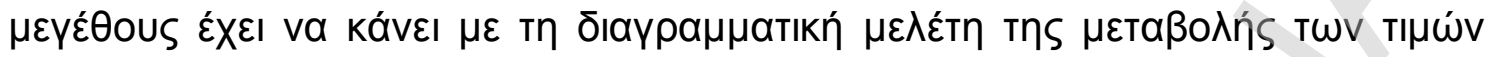

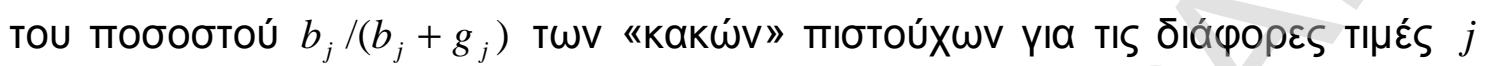
тоU характпрібтікоú (Crook et al, 1992; Yobas et al, 2000; Hamilton and Khan, 2001).

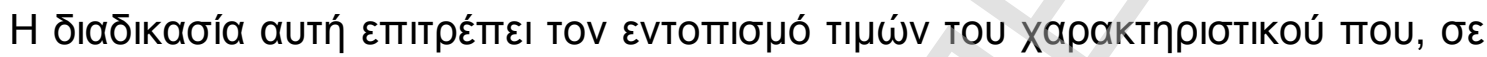

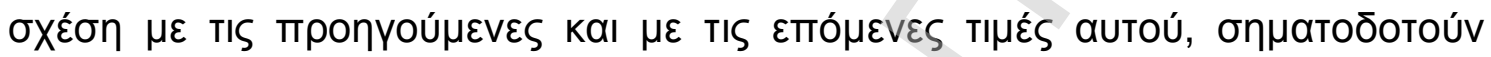

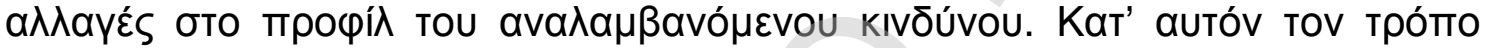

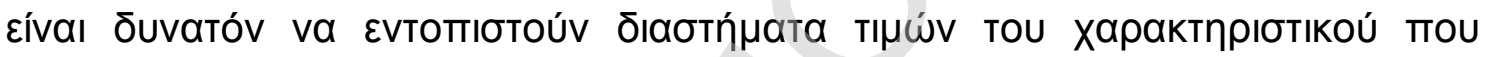

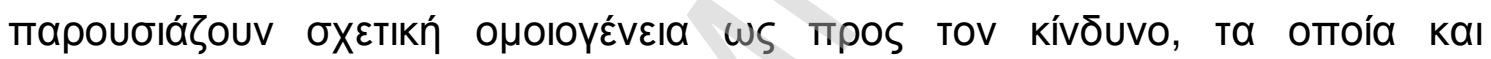

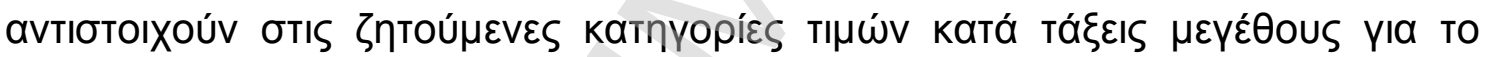

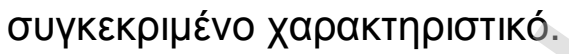

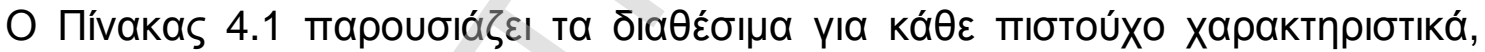

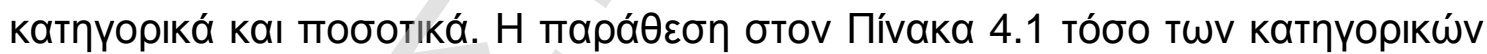

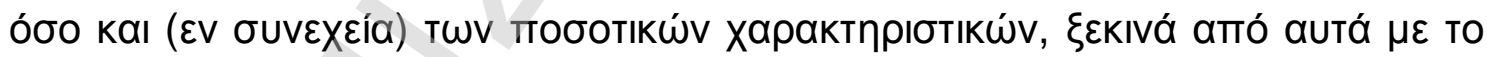

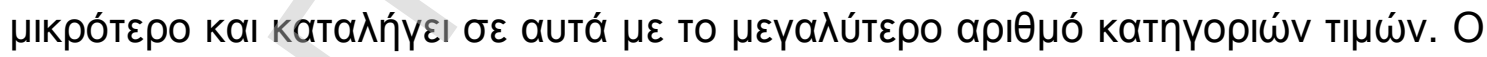

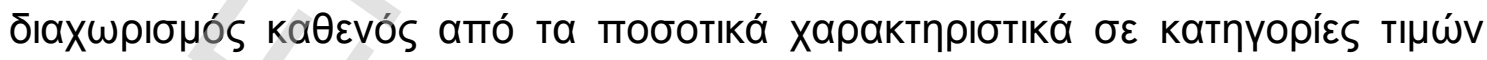

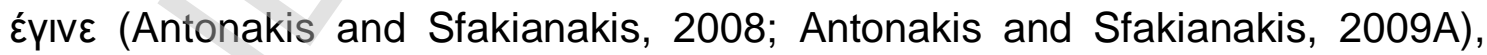

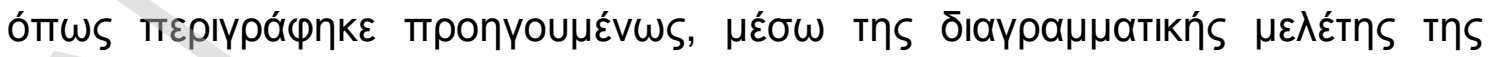

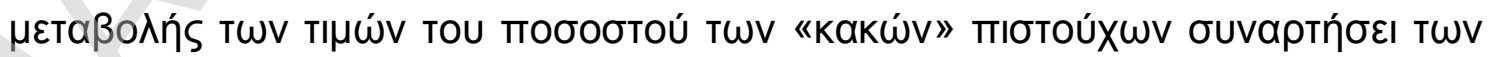

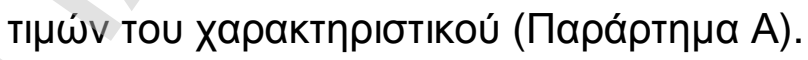




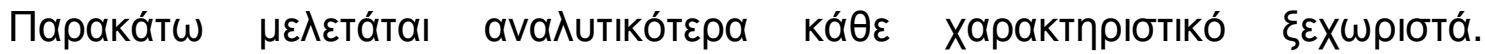

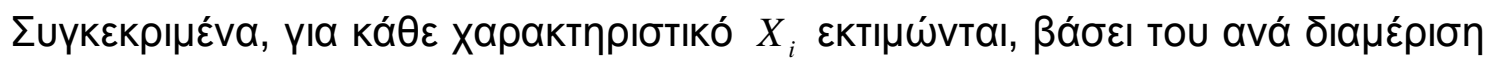

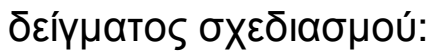

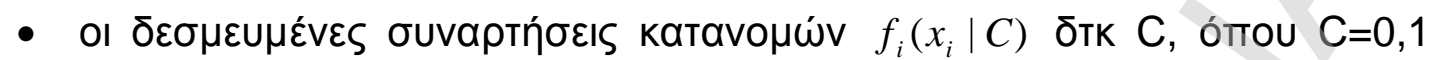

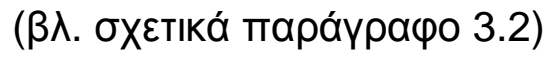

$$
f_{i}\left(x_{i} \mid C\right)=\frac{n_{C}\left(x_{i}\right)+1 / 2}{N_{i C}+1}
$$

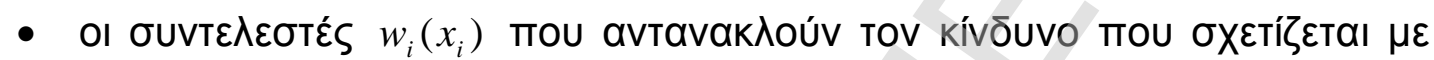

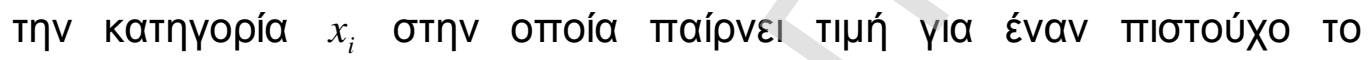

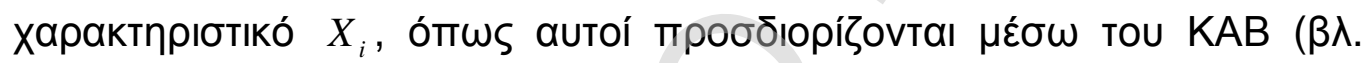

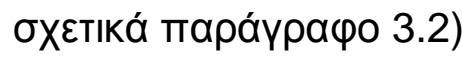

$$
w_{i}\left(x_{i}\right)=\ln \frac{f_{i}\left(x_{i} \mid 1\right)}{f_{i}\left(x_{i} \mid 0\right)}
$$

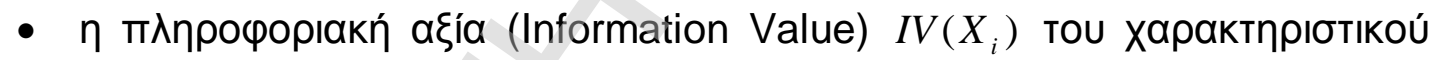
$X_{i}$

$$
I V\left(X_{i}\right)=\sum_{x_{i}}\left[f_{i}\left(x_{i} \mid 1\right)-f_{i}\left(x_{i} \mid 0\right)\right] * \ln \frac{f_{i}\left(x_{i} \mid 1\right)}{f_{i}\left(x_{i} \mid 0\right)}
$$

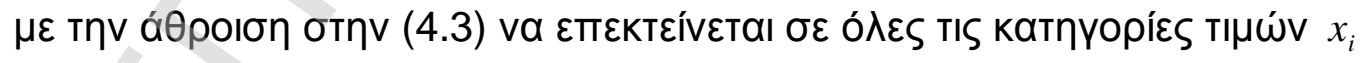
Tou характпрібтікоÚ $X_{i}$

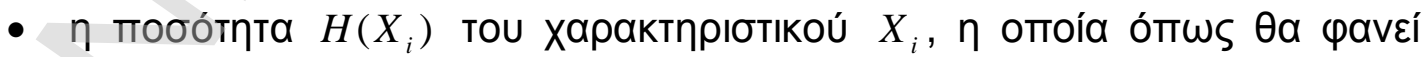

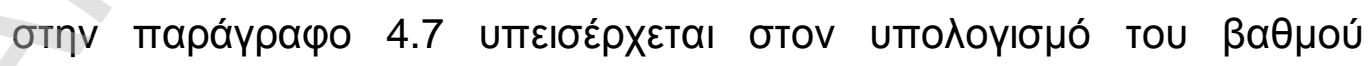

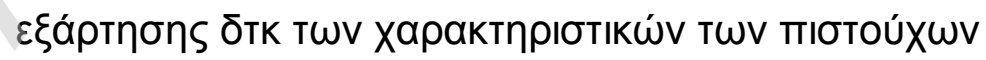

$$
H\left(X_{i}\right)=\sum_{C=0}^{1} P(C) \sum_{x_{i}}-P\left(C \cap\left\{X_{i}=x_{i}\right\}\right) \log _{2} P\left(C \cap\left\{X_{i}=x_{i}\right\}\right)
$$




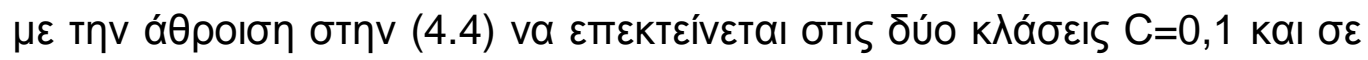

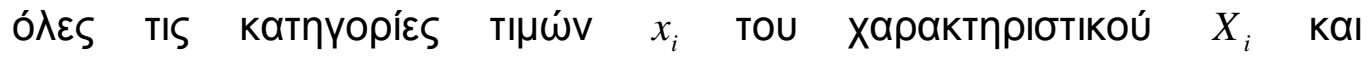

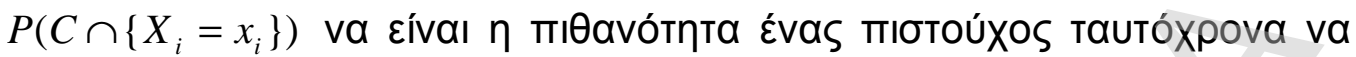

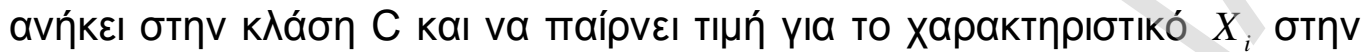
катпүopía $x_{i}$.

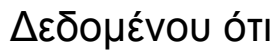

$$
P\left(C \cap\left\{X_{i}=x_{i}\right\}\right)=P(C) P\left(X_{i}=x_{i} \mid C\right)=P(C) f_{i}\left(x_{i} \mid C\right)
$$

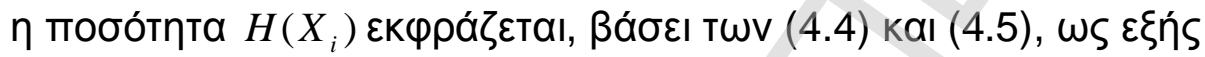

$$
H\left(X_{i}\right)=\sum_{C=0}^{1} P(C) \sum_{x_{i}}-P(C) f_{i}\left(x_{i} \mid C\right) \log _{2}\left[P(C) f_{i}\left(x_{i} \mid C\right)\right]
$$

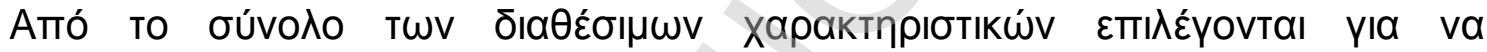

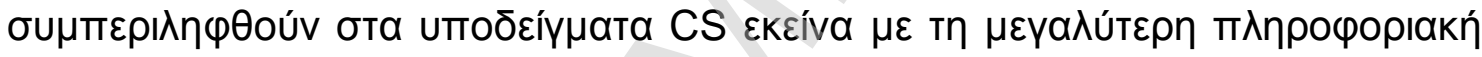
a६ía (Henley and Hand, 1996; Mester, 1997; Thomas, 2000; Fishelson-Holstine,

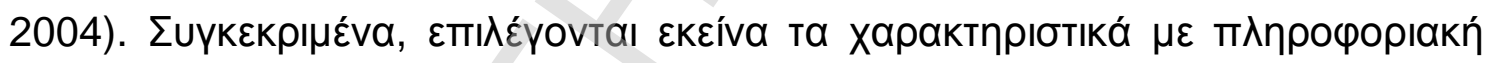

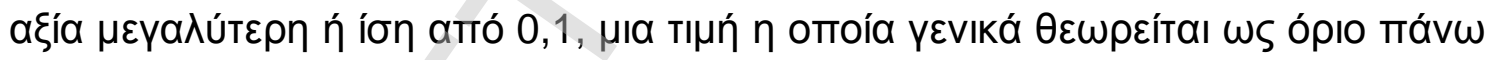

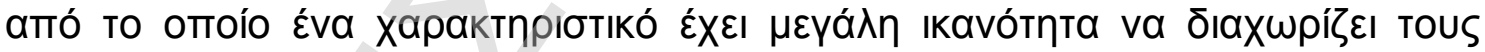

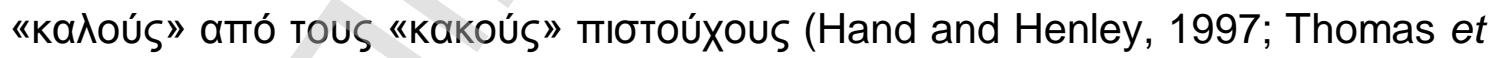
al, 2002; Antonakis and Sfakianakis, 2008; Antonakis and Sfakianakis, 2009A).

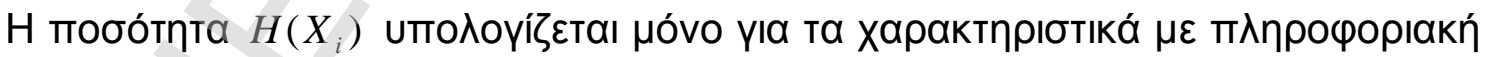

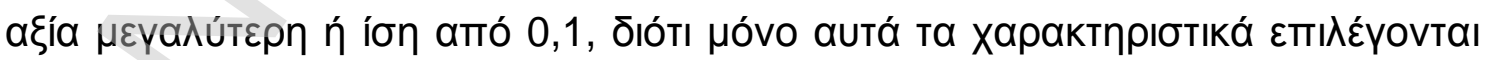

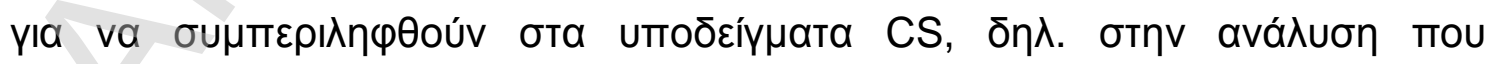

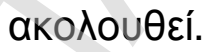


ПINAKA $\Sigma$ 4.1

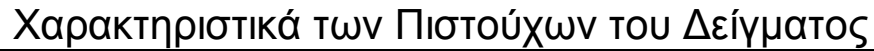

\begin{tabular}{|c|c|}
\hline \multicolumn{2}{|c|}{ 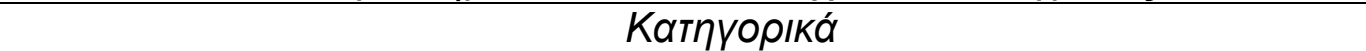 } \\
\hline 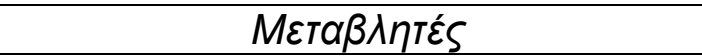 & 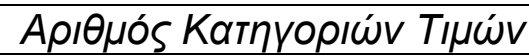 \\
\hline Катохи́ Autokivńtou & 2 \\
\hline 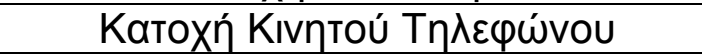 & 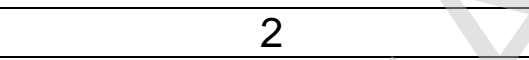 \\
\hline 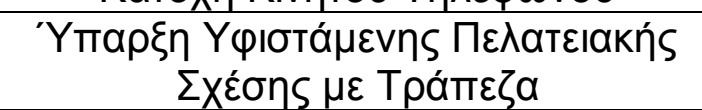 & 2 \\
\hline Фúlo & 0 \\
\hline Катохи́ Пıбтштікńs Kápтаs & 2 \\
\hline 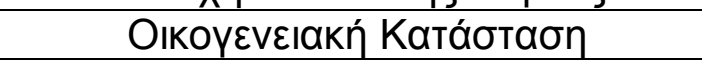 & 3 \\
\hline Túтাоs Katoıкі́as & 3 \\
\hline 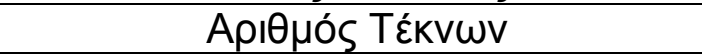 & 2 \\
\hline 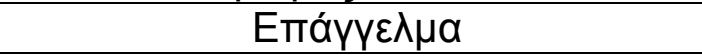 & 6 \\
\hline 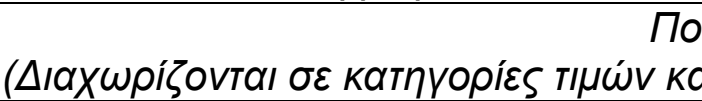 & 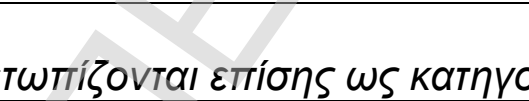 \\
\hline 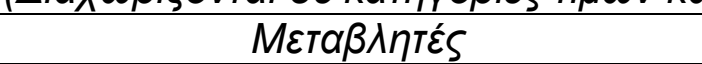 & 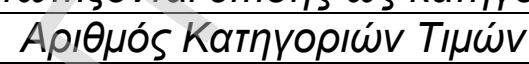 \\
\hline 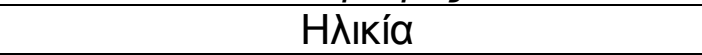 & 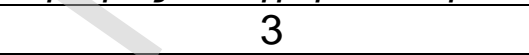 \\
\hline 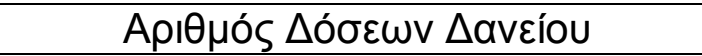 & 3 \\
\hline 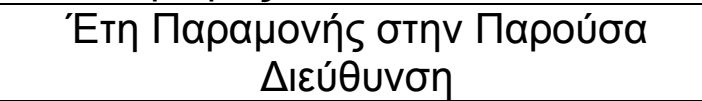 & 4 \\
\hline 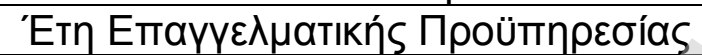 & 4 \\
\hline 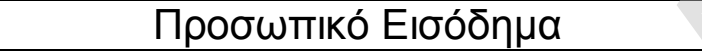 & 4 \\
\hline
\end{tabular}

\subsection{1 Катохи́ Autoкivńtou (CAR)}

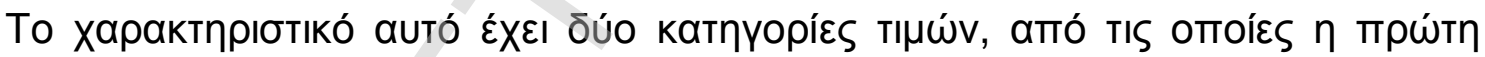

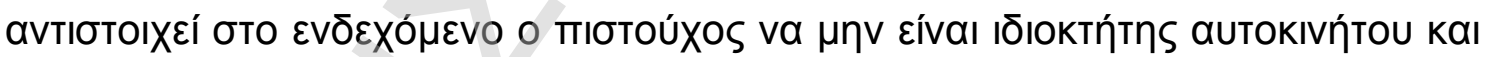

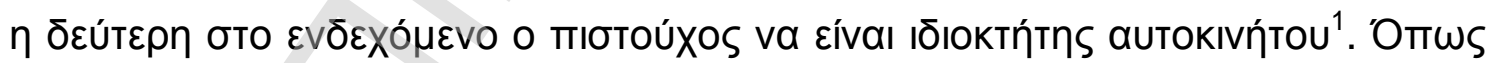

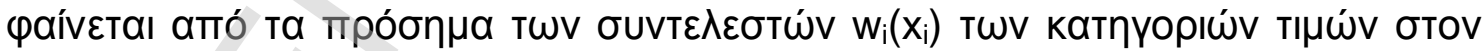

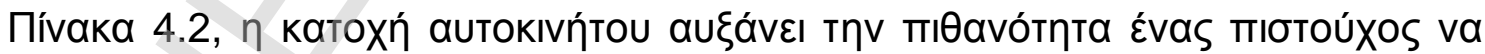

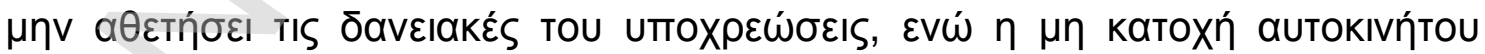

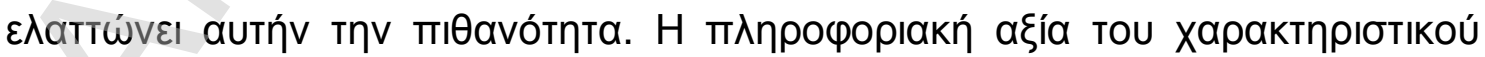

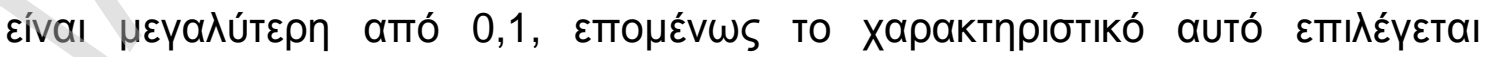

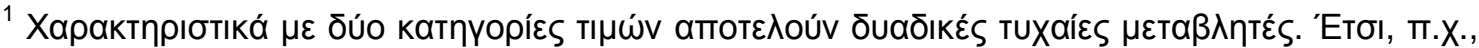

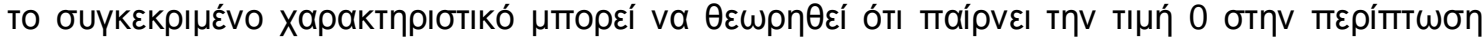

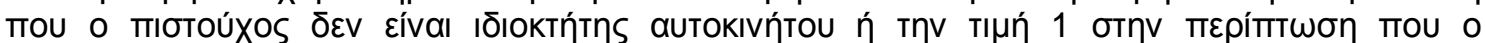

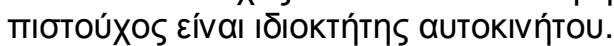




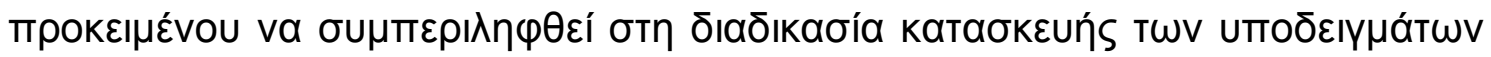
CS.

ПINAKA $\Sigma 4.2$

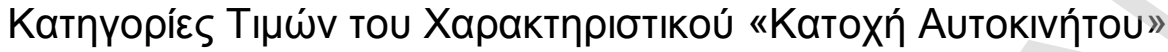

\begin{tabular}{|c|c|c|c|c|c|c|}
\hline & \multirow[b]{2}{*}{ 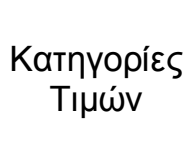 } & \multicolumn{2}{|c|}{ Пıотои́хоI } & \multirow[b]{2}{*}{$f_{i}\left(x_{i} \mid 1\right)$} & \multirow[b]{2}{*}{$f_{i}\left(x_{i} \mid 0\right)$} & \multirow{2}{*}{ 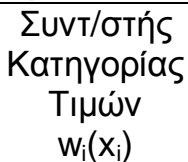 } \\
\hline & & «Какоі́» & «Ka入oí» & & & \\
\hline \multirow[t]{3}{*}{ 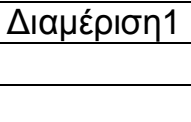 } & OXI & 200 & 997 & 0,25518 & 0,549315 & $-0,766702$ \\
\hline & Nal & 164 & 2911 & 0,74482 & 0,450685 & 0,502374 \\
\hline & Eúvoגo & 364 & 3908 & & 7 & \\
\hline \multicolumn{7}{|c|}{$I V\left(X_{i}\right)=0,373279 \mathrm{k \alpha} \mathrm{H}\left(X_{i}\right)=0,826224$} \\
\hline \multirow[t]{3}{*}{$\Delta ı \alpha \mu \varepsilon ́ p ı \sigma \eta 2$} & OXI & 194 & 1018 & 0,260553 & 0,532877 & $-0,71549$ \\
\hline & Naı & 170 & 2890 & 0,739447 & 0,467123 & 0,45931 \\
\hline & $\sum u ́ v o \lambda o$ & 364 & 3908 & - & & \\
\hline \multicolumn{7}{|c|}{$I V\left(X_{i}\right)=0,373279 \kappa \alpha ı H\left(X_{i}\right)=0,833109$} \\
\hline \multirow[t]{3}{*}{$\Delta ı \alpha \mu \varepsilon ́ p ı \sigma \eta 3$} & $\mathrm{OXI}$ & 205 & 990 & 0,25339 & 0,563014 & $-0,79838$ \\
\hline & $\mathrm{NaI}$ & 159 & 2918 & 0,74661 & 0,436986 & 0,535642 \\
\hline & $\sum u ́ v o \lambda o$ & 364 & 3908 & s & & \\
\hline \multicolumn{7}{|c|}{$I V\left(X_{i}\right)=0,413044 \mathrm{kal} H\left(X_{i}\right)=0,823866$} \\
\hline \multirow[t]{3}{*}{$\Delta ı \alpha \varepsilon_{p}$} & OXI & 196 & 1035 & 0,264902 & 0,538356 & $-0,70916$ \\
\hline & Naı & 168 & 2873 & 0,735098 & 0,461644 & 0,465211 \\
\hline & $\sum u ́ v o \lambda o$ & 364 & 3908 & & & \\
\hline \multicolumn{7}{|c|}{$I V\left(X_{i}\right)=0,321138 \mathrm{kaI} H\left(X_{i}\right)=0,838518$} \\
\hline \multirow[t]{3}{*}{$\Delta ı \alpha \mu \varepsilon ́ p ı \sigma \eta 5$} & OXI & 197 & 996 & 0,254925 & 0,541096 & $-0,75263$ \\
\hline & Nal & 167 & 2912 & 0,745075 & 0,458904 & 0,484644 \\
\hline & $\sum u ́ v o \lambda o$ & 364 & 3908 & & & \\
\hline
\end{tabular}

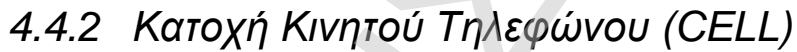

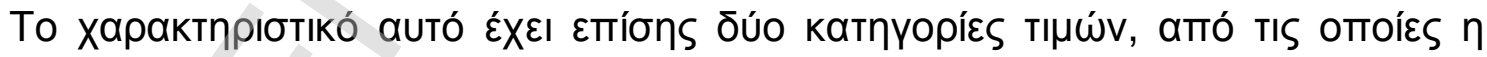

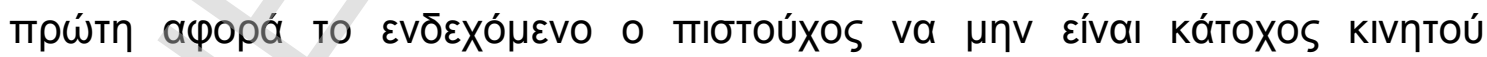

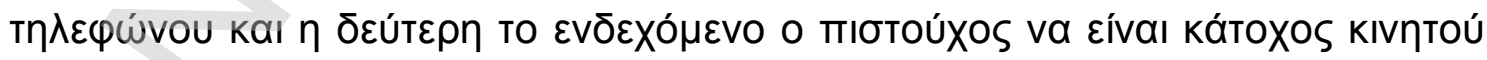

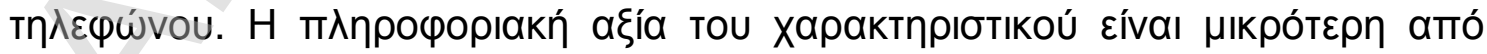

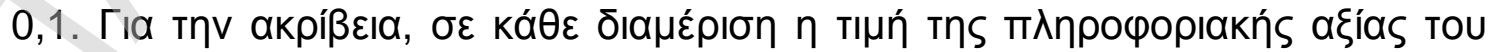

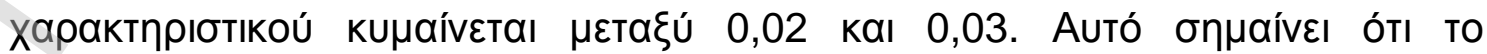




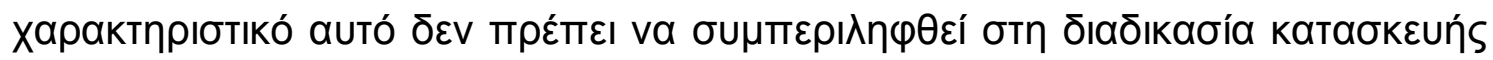

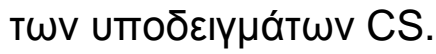

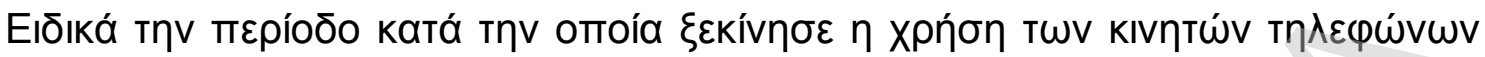

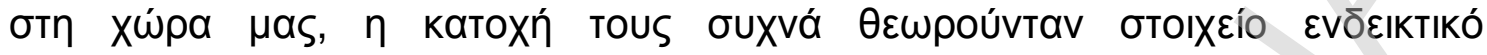

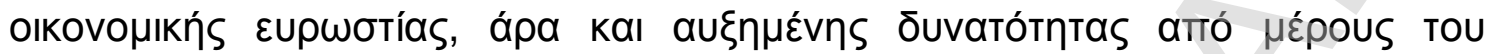

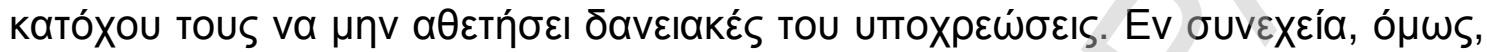

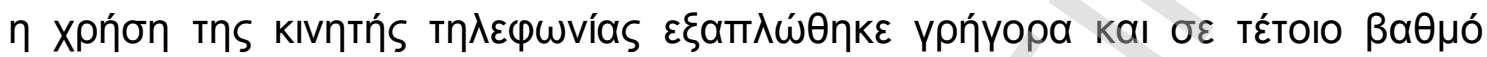

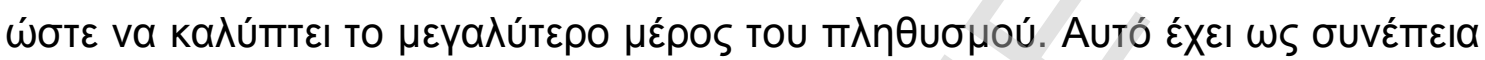

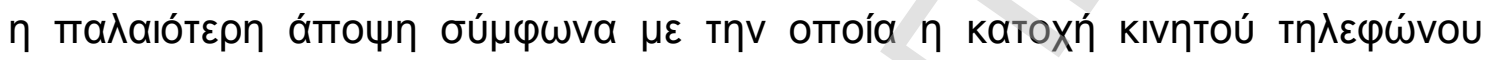

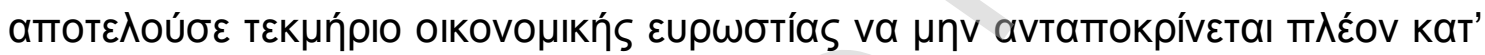

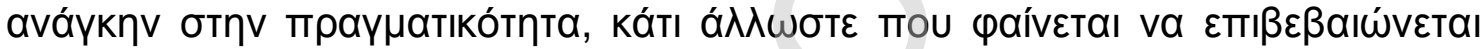

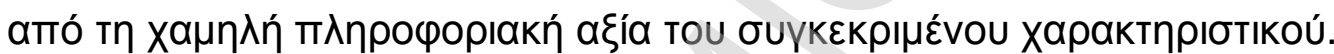

\section{ПINAKA $\Sigma 4.3$}

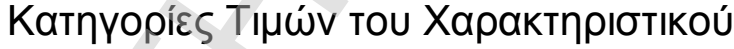

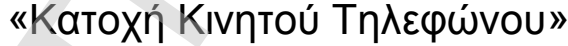

\begin{tabular}{|c|c|c|c|c|c|c|}
\hline & \multirow[b]{2}{*}{ 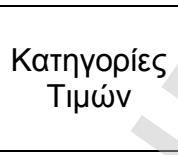 } & \multicolumn{2}{|c|}{ Пıбтои́хоІ } & \multirow[b]{2}{*}{$\mathrm{f}_{\mathrm{i}}\left(\mathrm{x}_{\mathrm{i}} \mid 1\right)$} & \multirow[b]{2}{*}{$f_{i}\left(x_{i} \mid 0\right)$} & \multirow{2}{*}{ 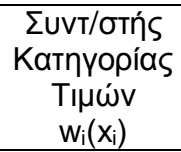 } \\
\hline & & «Какоі́» & «Ka入oí» & & & \\
\hline \multirow{3}{*}{$\Delta ı \alpha \mu \varepsilon ́ p ı \sigma \eta 1$} & OXI & 182 & 1641 & 0,419928 & 0,5 & $-0,17452$ \\
\hline & $\mathrm{Nal}$ & 182 & 2267 & 0,580072 & 0,5 & 0,148543 \\
\hline & $\sum u ́ v o \lambda o$ & 364 & 3908 & & & \\
\hline \multicolumn{7}{|c|}{$I V\left(X_{i}\right)=0,025869$} \\
\hline \multirow[t]{3}{*}{$\Delta ı \alpha \mu \varepsilon ́ p ı \sigma \eta 2$} & ${ }^{\circ} \mathrm{OXI}$ & 184 & 1658 & 0,424277 & 0,505479 & $-0,17512$ \\
\hline & $\mathrm{Nal}$ & 180 & 2250 & 0,575723 & 0,494521 & 0,152037 \\
\hline & ¿úvoגo & 364 & 3908 & & & \\
\hline \multicolumn{7}{|c|}{$I V\left(X_{i}\right)=0,026566$} \\
\hline$\Delta ı \alpha \mu \varepsilon ́ \rho ı \sigma n 3$ & OXI & 182 & 1655 & 0,42351 & 0,5 & $-0,16603$ \\
\hline 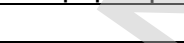 & Nal & 182 & 2253 & 0,57649 & 0,5 & 0,14235 \\
\hline 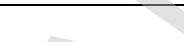 & $\sum$ ¿́voגo & 364 & 3908 & & & \\
\hline \multicolumn{7}{|c|}{$I V\left(X_{i}\right)=0,023588$} \\
\hline 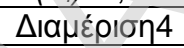 & OXI & 181 & 1641 & 0,419928 & 0,49726 & $-0,16903$ \\
\hline 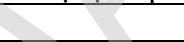 & $\mathrm{Nal}$ & 183 & 2267 & 0,580072 & 0,50274 & 0,143079 \\
\hline$\sqrt{2}$ & $\sum u ́ v o \lambda o$ & 364 & 3908 & & & \\
\hline \multicolumn{7}{|c|}{$I V\left(X_{i}\right)=0,024136$} \\
\hline 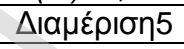 & OXI & 187 & 1697 & 0,434254 & 0,513699 & $-0,16801$ \\
\hline 8 & Nal & 177 & 2211 & 0,565746 & 0,486301 & 0,151316 \\
\hline & $\sum u ́ v o \lambda o$ & 364 & 3908 & & & \\
\hline \multicolumn{7}{|c|}{$I V\left(X_{i}\right)=0,025368$} \\
\hline
\end{tabular}




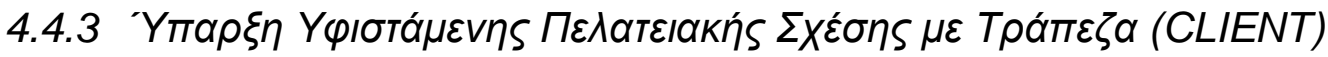

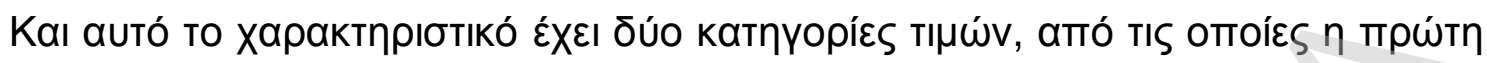

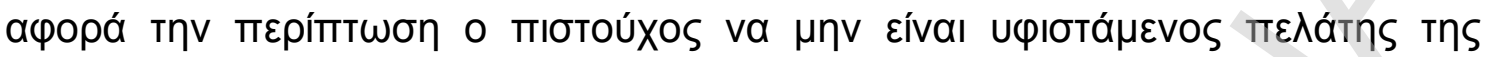

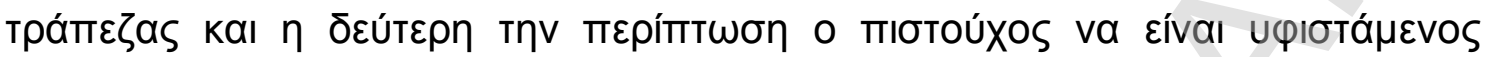

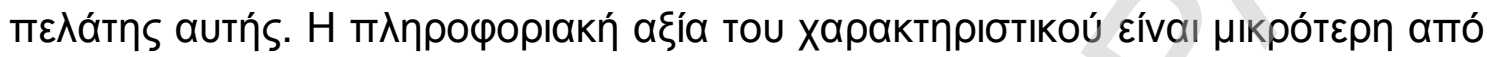

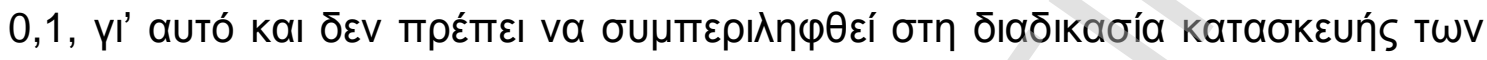

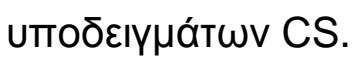

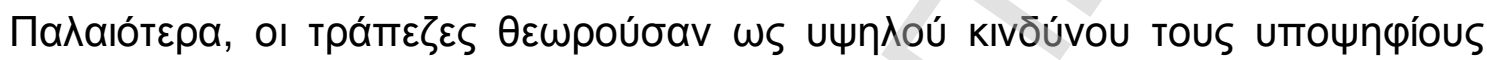

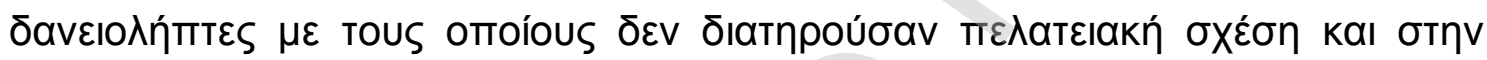

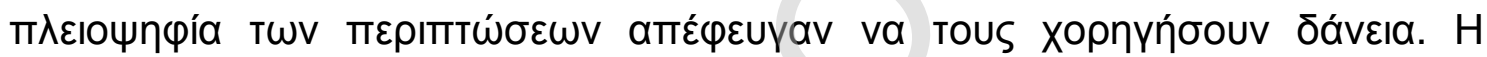

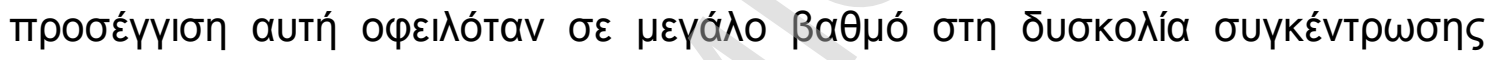

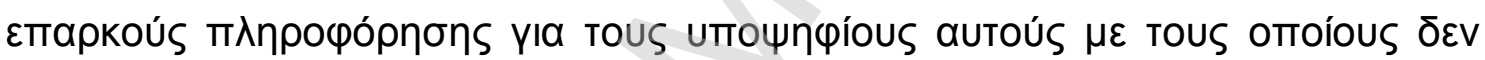

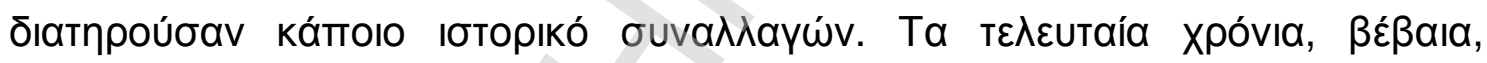

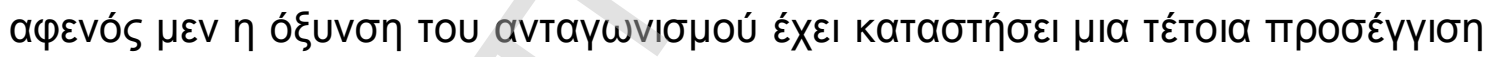

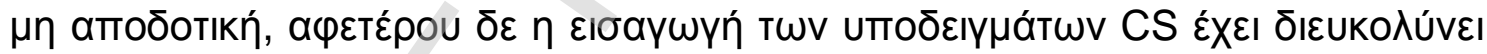

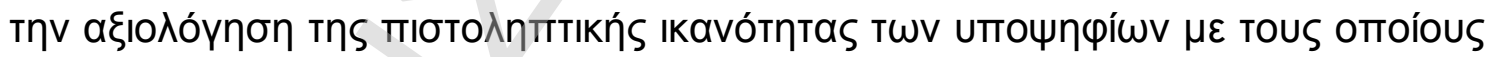

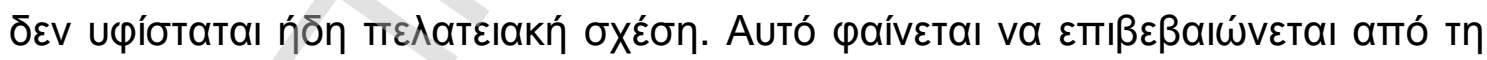

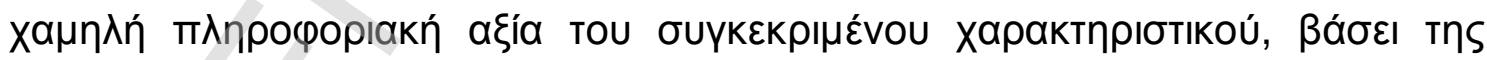

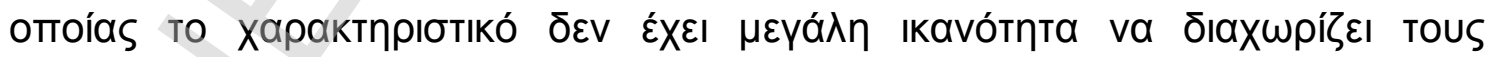

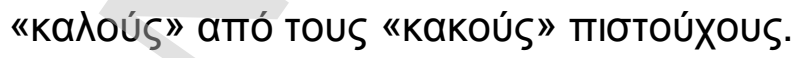


ПINAKA $\Sigma 4.4$

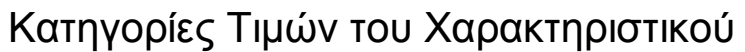

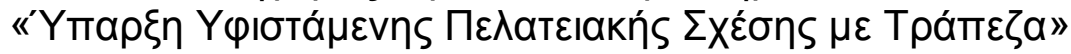

\begin{tabular}{|c|c|c|c|c|c|c|}
\hline & \multirow[b]{2}{*}{ 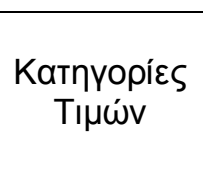 } & \multicolumn{2}{|c|}{ ПıбтоÚXOI } & \multirow[b]{2}{*}{$f_{i}\left(x_{i} \mid 1\right)$} & \multirow[b]{2}{*}{$f_{i}\left(x_{i} \mid 0\right)$} & \multirow{2}{*}{ 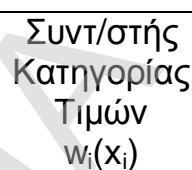 } \\
\hline & & «Какоі́» & «Ka入oí» & & & \\
\hline \multirow[t]{3}{*}{$\Delta ı \alpha \mu \varepsilon ́ p ı \sigma \eta 1$} & OXI & 309 & 2864 & 0,732796 & 0,847945 & $-0,145950$ \\
\hline & Naı & 55 & 1044 & 0,267204 & 0,152055 & 0,563771 \\
\hline & $\sum u ́ v o \lambda o$ & 364 & 3908 & & 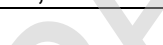 & \\
\hline \multicolumn{7}{|c|}{$I V\left(X_{i}\right)=0,081724$} \\
\hline \multirow[t]{3}{*}{$\Delta ı \alpha \mu \varepsilon ́ p ı \sigma \eta 2$} & OxI & 305 & 2882 & 0,737401 & 0,836986 & $-0,126680$ \\
\hline & $\mathrm{Nal}$ & 59 & 1026 & 0,262599 & 0,163014 & 0,476794 \\
\hline & ¿úvoגo & 364 & 3908 & 7 & 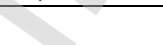 & \\
\hline \multicolumn{7}{|c|}{$I V\left(X_{i}\right)=0,060097$} \\
\hline \multirow[t]{3}{*}{$\Delta ı \alpha \mu \varepsilon ́ p ı \sigma \eta 3$} & OXI & 304 & 2858 & 0,731261 & 0,834247 & $-0,131760$ \\
\hline & $\mathrm{Nal}$ & 60 & 1050 & 0,268739 & 0,165753 & 0,483239 \\
\hline & ¿úvoגo & 364 & 3908 & 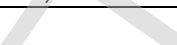 & & \\
\hline \multicolumn{7}{|c|}{$I V\left(X_{i}\right)=0,063336$} \\
\hline \multirow[t]{3}{*}{$\Delta ı \alpha \mu \varepsilon ́ p ı \sigma\rceil 4$} & OXI & 303 & 2855 & 0,730494 & 0,831507 & $-0,129520$ \\
\hline & Nal & 61 & 1053 & 0,269506 & 0,168493 & 0,469697 \\
\hline & $\sum u ́ v o \lambda o$ & 364 & 3908 & & & \\
\hline \multicolumn{7}{|c|}{$I V\left(X_{i}\right)=0,060529$} \\
\hline \multirow[t]{3}{*}{$\Delta ı \alpha \mu \varepsilon ́ p ı \sigma\rceil 5$} & OXI & 299 & 2853 & 0,729982 & 0,820548 & $-0,116950$ \\
\hline & $\mathrm{Nal}$ & 65 & 1055 & 0,270018 & 0,179452 & 0,408580 \\
\hline & ¿úvoגo & 364 & 3908 & & & \\
\hline
\end{tabular}

4.4.4 Фú^o (GENDER)

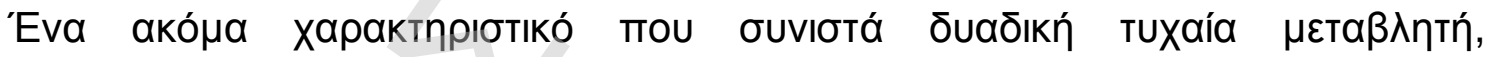

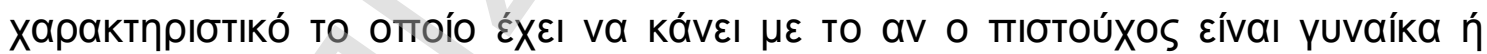

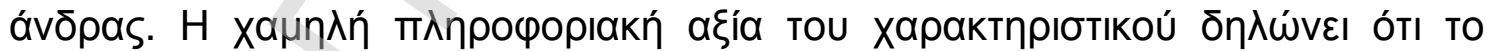

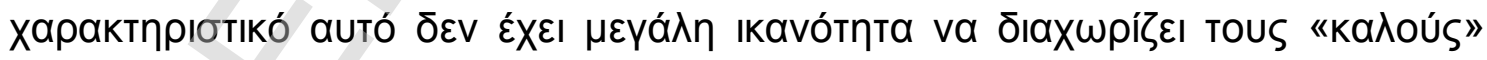

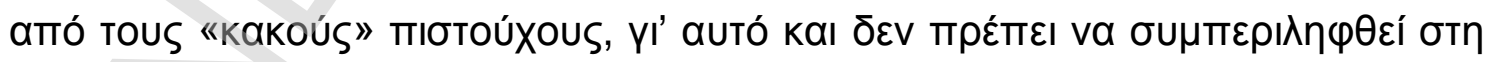

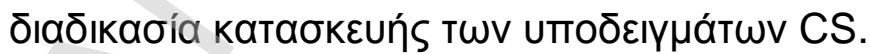


ПINAKA 4.5

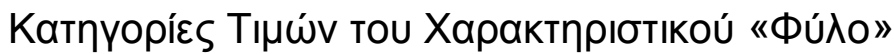

\begin{tabular}{|c|c|c|c|c|c|c|}
\hline & \multirow[b]{2}{*}{ 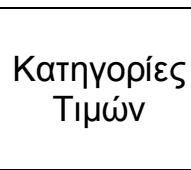 } & \multicolumn{2}{|c|}{ ПıотоúXOI } & \multirow[b]{2}{*}{$\mathrm{f}_{\mathrm{i}}\left(\mathrm{x}_{\mathrm{i}} \mid 1\right)$} & \multirow[b]{2}{*}{$\mathrm{f}_{\mathrm{i}}\left(\mathrm{x}_{\mathrm{i}} \mid 0\right)$} & \multirow{2}{*}{ 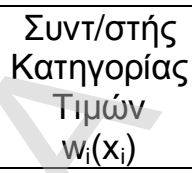 } \\
\hline & & «Какоі́» & «Ka入oí» & & & \\
\hline \multirow[t]{3}{*}{$\Delta ı \alpha \mu \varepsilon ́ p ı \sigma \eta 1$} & Гuvaíka & 127 & 1014 & 0,259529 & 0,349315 & $-0,2971050$ \\
\hline & 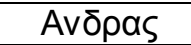 & 237 & 2894 & 0,740471 & 0,650685 & 0,1292605 \\
\hline & ¿úvoגo & 364 & 3908 & & +2 & 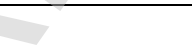 \\
\hline \multicolumn{7}{|c|}{$I V\left(X_{i}\right)=0,038282$} \\
\hline \multirow[t]{3}{*}{ 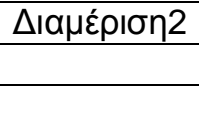 } & Гuvaíka & 132 & 1006 & 0,257483 & 0,363014 & $-0,343490$ \\
\hline & 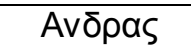 & 232 & 2902 & 0,742517 & 0,636986 & 0,153298 \\
\hline & Eúvoגo & 364 & 3908 & & & \\
\hline \multicolumn{7}{|c|}{$I V\left(X_{i}\right)=0,052426$} \\
\hline \multirow[t]{3}{*}{$\Delta ı \alpha \mu \varepsilon ́ p ı \sigma \eta 3$} & Гuvaíka & 126 & 1009 & 0,25825 & 0,346575 & $-0,294170$ \\
\hline & Avס̄pas & 238 & 2899 & 0,74175 & 0,653425 & 0,126785 \\
\hline & ¿úvoגo & 364 & 3908 & N & & \\
\hline \multicolumn{7}{|c|}{$I V\left(X_{i}\right)=0,037181$} \\
\hline \multirow[t]{3}{*}{$\Delta ı а \mu \varepsilon ́ p ı \sigma \eta 4$} & Гuvaíka & 134 & 1014 & 0,259529 & 0,368493 & $-0,350550$ \\
\hline & Avס̄pas & 230 & 2894 & 0,740471 & 0,631507 & 0,159177 \\
\hline & ¿úvoגo & 364 & 3908 & & & \\
\hline \multicolumn{7}{|c|}{$I V\left(X_{i}\right)=0,055542$} \\
\hline \multirow[t]{3}{*}{$\Delta ı \alpha \mu \varepsilon ́ p ı \sigma \eta 5$} & Гuvaíka & 127 & 1014 & 0,259529 & 0,349315 & $-0,297100$ \\
\hline & Avסраs & 237 & 2894 & 0,740471 & 0,650685 & 0,129261 \\
\hline & ¿úvoגo & 364 & 3908 & & & \\
\hline
\end{tabular}

\subsection{5 Катохи́ Пıбтштıкńs Kápтаs (CRD)}

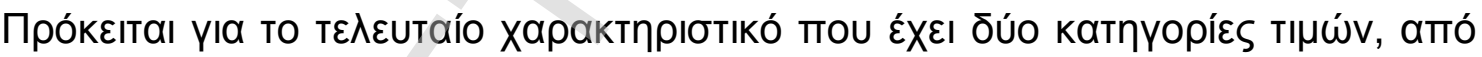

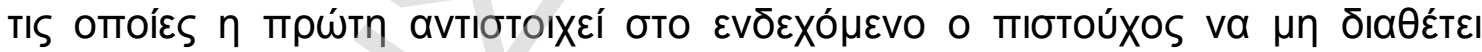

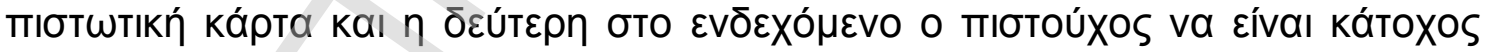

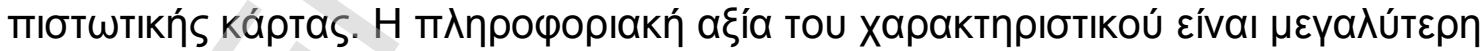

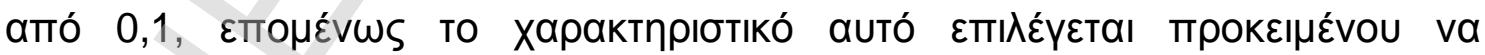

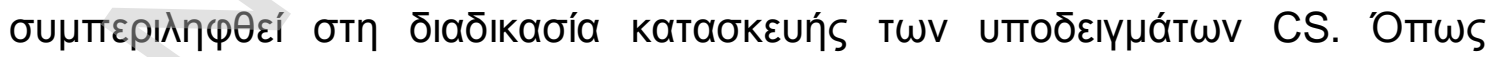

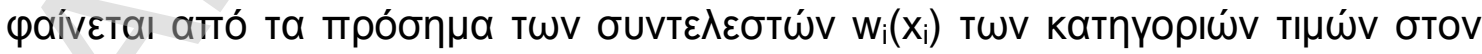

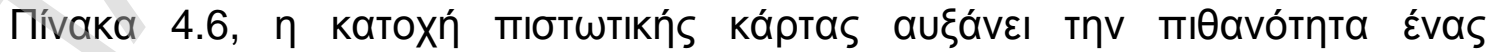

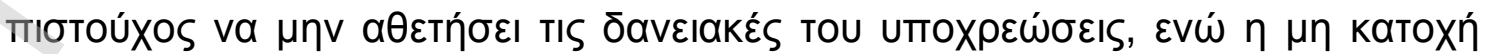

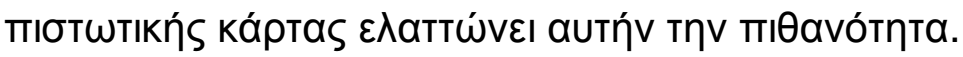




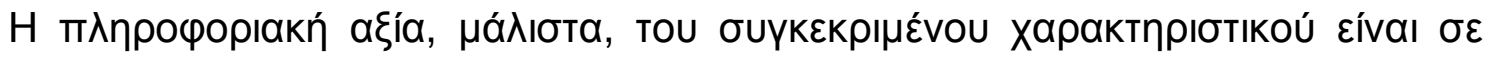

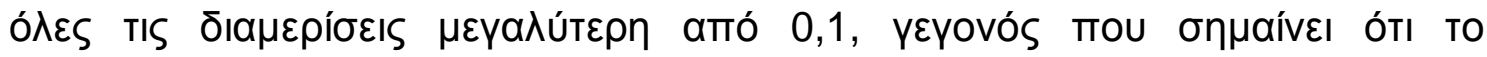

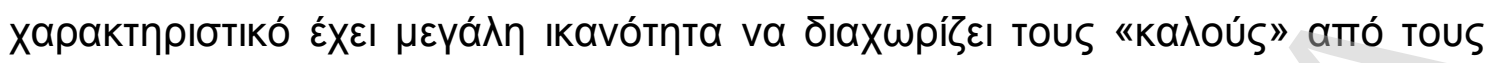

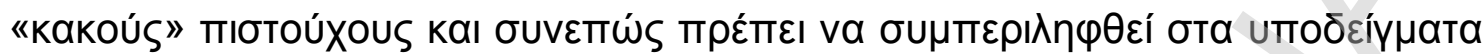

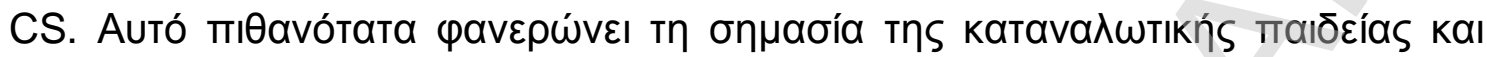

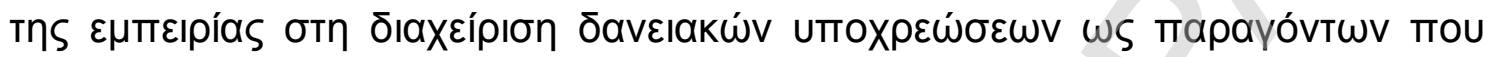

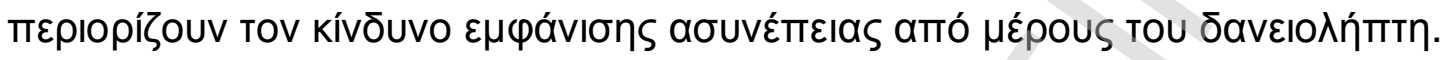

ПINAKA $\Sigma$ 4.6

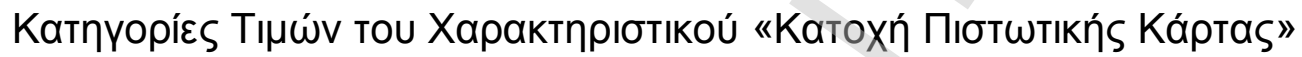

\begin{tabular}{|c|c|c|c|c|c|c|}
\hline & \multirow[b]{2}{*}{ 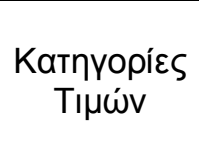 } & \multicolumn{2}{|c|}{ Пıбтои́XоІ } & \multirow[b]{2}{*}{$f_{i}\left(x_{i} \mid 1\right)$} & \multirow[b]{2}{*}{$f_{i}\left(x_{i} \mid 0\right)$} & \multirow{2}{*}{ 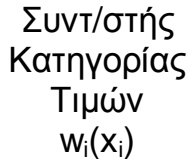 } \\
\hline & & «Какоі́» & «Ka入oí» & & & \\
\hline \multirow[t]{3}{*}{$\Delta ı \alpha \mu \varepsilon ́ p ı \sigma \eta 1$} & OXI & 309 & 2509 & 0,64198 & 0,847945 & $-0,278259$ \\
\hline & Naı & 55 & 1399 & 0,35802 & 0,152055 & 0,856348 \\
\hline & $\sum u ́ v o \lambda o$ & 364 & 3908 & & & \\
\hline \multicolumn{7}{|c|}{$I V\left(X_{i}\right)=0,233689 \mathrm{kal} H\left(X_{i}\right)=0,925275$} \\
\hline \multirow[t]{3}{*}{$\Delta ı а \mu \varepsilon ́ p ı \sigma \eta 2$} & OXI & 301 & 2537 & 0,649143 & 0,826027 & $-0,240970$ \\
\hline & Nal & 63 & 1371 & 0,350857 & 0,173973 & 0,701481 \\
\hline & $\sum u ́ v o \lambda o$ & 364 & 3908 & & & \\
\hline \multicolumn{7}{|c|}{$I V\left(X_{i}\right)=0,166706 \mathrm{kal} H\left(X_{i}\right)=0,920465$} \\
\hline \multirow[t]{3}{*}{$\Delta ı а \mu \varepsilon ́ p ı \sigma \eta 3$} & OXI & 309 & 2519 & 0,644538 & 0,847945 & $-0,274280$ \\
\hline & $\mathrm{Nal}$ & 55 & 1389 & 0,355462 & 0,152055 & 0,849177 \\
\hline & Eúvoגo & 364 & 3908 & & & \\
\hline \multicolumn{7}{|c|}{$I V\left(X_{i}\right)=0,228519 \mathrm{k \alpha I} H\left(X_{i}\right)=0,923454$} \\
\hline \multirow[t]{3}{*}{$\Delta ı \alpha \mu \varepsilon ́ p ı \sigma \eta 4$} & OXI & 305 & 2509 & 0,64198 & 0,836986 & $-0,265250$ \\
\hline & $\mathrm{Nal}$ & 59 & 1399 & 0,35802 & 0,163014 & 0,786754 \\
\hline & $\sum u ́ v o \lambda o$ & 364 & 3908 & & & \\
\hline \multicolumn{7}{|c|}{$I V\left(X_{i}\right)=0,205148 \mathrm{Kal} H\left(X_{i}\right)=0,925468$} \\
\hline \multirow[t]{3}{*}{ 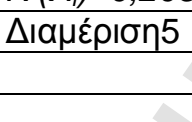 } & OXI & 312 & 2522 & 0,645306 & 0,856164 & $-0,282740$ \\
\hline & $\mathrm{NaI}$ & 52 & 1386 & 0,354694 & 0,143836 & 0,902585 \\
\hline & Eúvoגo & 364 & 3908 & & & \\
\hline
\end{tabular}




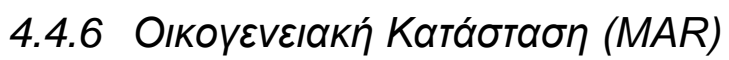

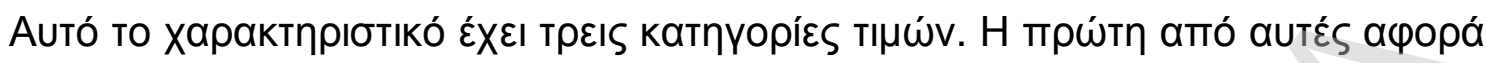

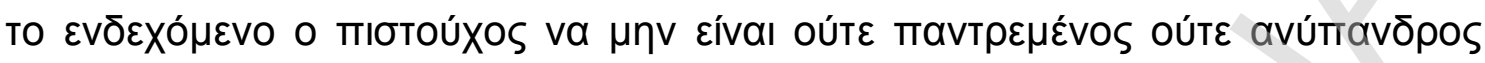
(

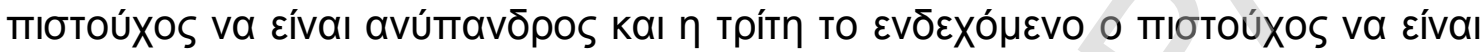

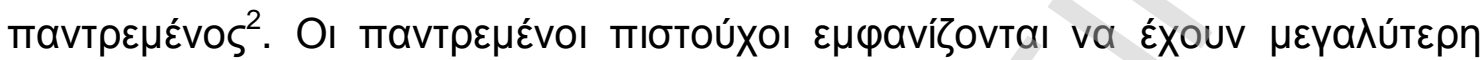

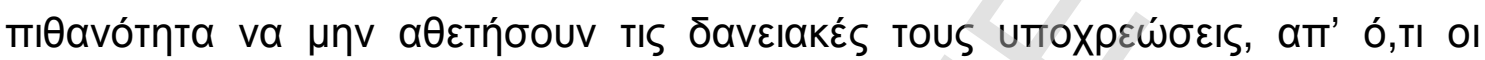

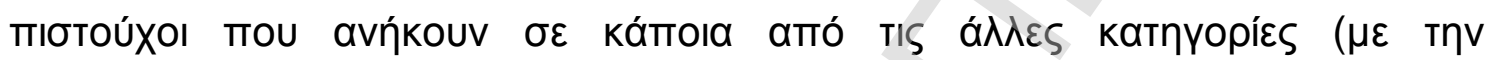

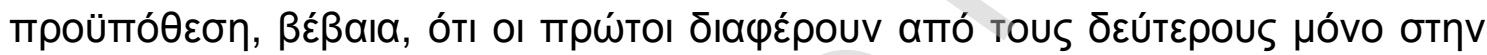

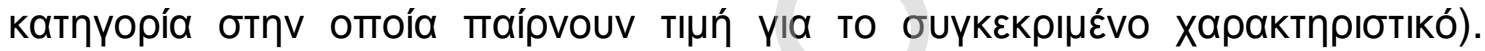

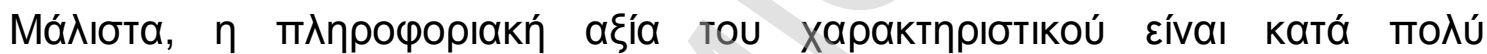

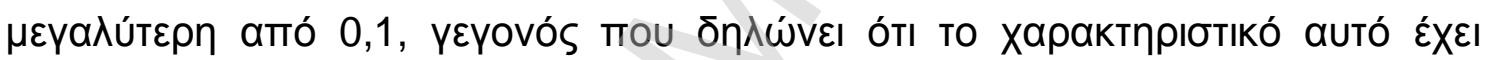

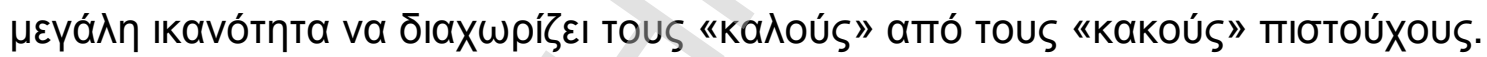

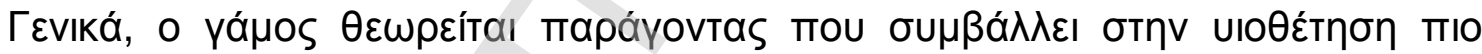

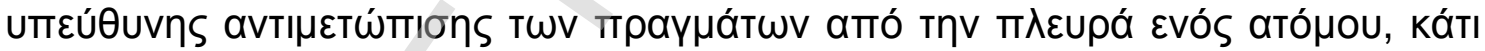

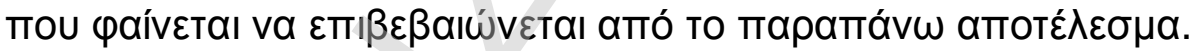

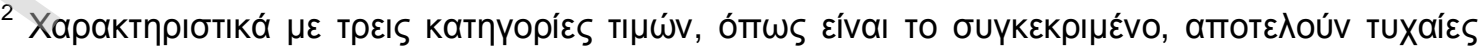

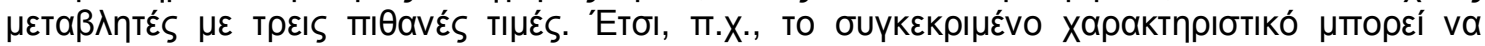

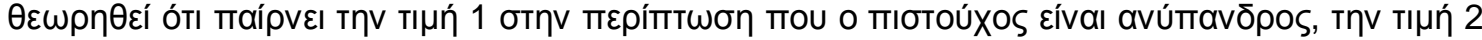

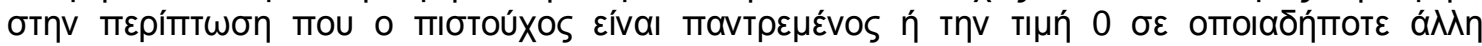

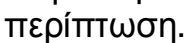


ПINAKA $\Sigma 4.7$

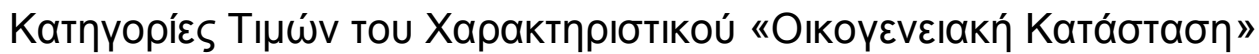

\begin{tabular}{|c|c|c|c|c|c|c|}
\hline & \multirow{2}{*}{ 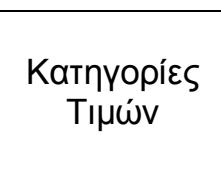 } & \multicolumn{2}{|c|}{ ПıбтоúXOI } & \multirow[b]{2}{*}{$f_{i}\left(x_{i} \mid 1\right)$} & \multirow[b]{2}{*}{$\mathrm{f}_{\mathrm{i}}\left(\mathrm{x}_{\mathrm{i}} \mid 0\right)$} & \multirow{2}{*}{ 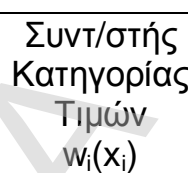 } \\
\hline & & «Какоі́» & «Ka入oí» & & & \\
\hline \multirow{4}{*}{$\Delta ı \alpha \mu \varepsilon ́ p ı \sigma \eta 1$} & $A \lambda \lambda \mathrm{O}$ & 125 & 182 & 0,046687 & 0,343836 & $-1,996695$ \\
\hline & Avúmavס̄pos & 166 & 965 & 0,246994 & 0,456164 & $-0,613489$ \\
\hline & 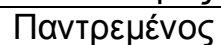 & 73 & 2761 & 0,706447 & 0,201370 & 1,255104 \\
\hline & ¿úvoגo & 364 & 3908 & & & \\
\hline \multicolumn{5}{|c|}{$I V\left(X_{i}\right)=1,355563$ кaı $H\left(X_{i}\right)=1,030451$} & & \\
\hline \multirow[t]{4}{*}{$\Delta ı \alpha \mu \varepsilon ́ p ı \sigma \eta n$} & $\mathrm{~A} \wedge \lambda \mathrm{O}$ & 127 & 192 & 0,049245 & 0,349315 & $-1,95916$ \\
\hline & Avúmavס̄рos & 167 & 984 & 0,251855 & 0,458904 & $-0,59999$ \\
\hline & 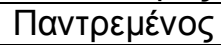 & 70 & 2732 & 0,699028 & 0,193151 & 1,28622 \\
\hline & $\sum u ́ v o \lambda o$ & 364 & 3908 & 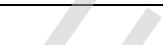 & & \\
\hline \multicolumn{5}{|c|}{$I V\left(X_{i}\right)=1,362781 \mathrm{k \alpha I} H\left(X_{i}\right)=1,044764$} & 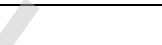 & \\
\hline \multirow[t]{4}{*}{$\Delta ı а \mu \varepsilon ́ p ı \sigma\rceil 3$} & $A \lambda \lambda \mathrm{O}$ & 118 & 174 & 0,044641 & 0,324658 & $-1,984130$ \\
\hline & Avúтாаvס̄рos & 176 & 995 & 0,254669 & 0,483562 & $-0,641220$ \\
\hline & 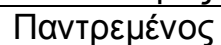 & 70 & 2739 & 0,700819 & 0,193151 & 1,288778 \\
\hline & ¿úvoגo & 364 & 3908 & 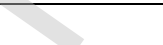 & & \\
\hline \multicolumn{7}{|c|}{ 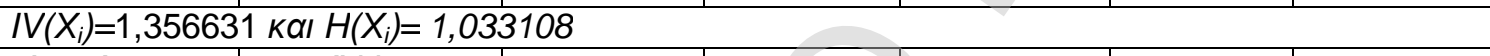 } \\
\hline \multirow[t]{4}{*}{$\Delta ı \alpha \mu \varepsilon ́ p ı \sigma \eta ~ 4$} & $A \lambda \lambda O$ & 119 & 184 & 0,047199 & 0,327397 & $-1,936810$ \\
\hline & Avúmavס̄pos & 175 & 983 & 0,251599 & 0,480822 & $-0,647660$ \\
\hline & 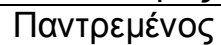 & 70 & 2741 & 0,701330 & 0,193151 & 1,289508 \\
\hline & 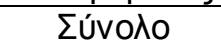 & 364 & 3908 & & & \\
\hline \multicolumn{3}{|c|}{$I V\left(X_{i}\right)=1,346451 \kappa \alpha I H\left(X_{i}\right)=1,037762$} & & & & \\
\hline \multirow[t]{4}{*}{$\Delta ı а \mu \varepsilon ́ p ı \sigma \eta ~ 5$} & $A \wedge \lambda \mathrm{O}$ & 123 & 172 & 0,044129 & 0,338356 & $-2,036980$ \\
\hline & Avúmavס̄os & 172 & 961 & 0,245971 & 0,472603 & $-0,653040$ \\
\hline & 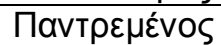 & 69 & 2775 & 0,710028 & 0,190411 & 1,316120 \\
\hline & $\sum u ́ v o \lambda o$ & 364 & 3908 & & & \\
\hline
\end{tabular}

\subsubsection{Túmos KatoIkías (HOME)}

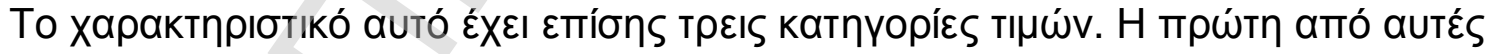

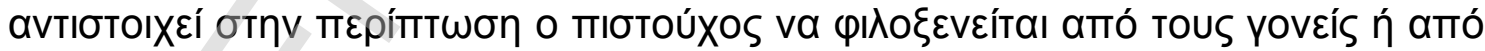

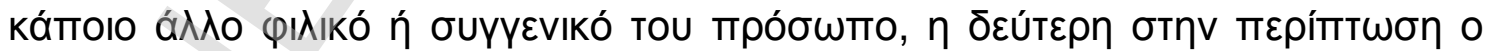

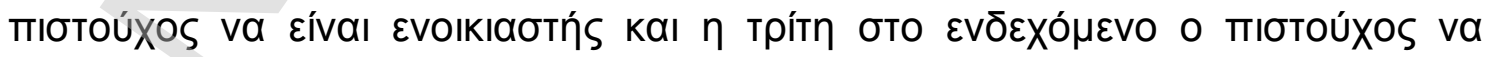

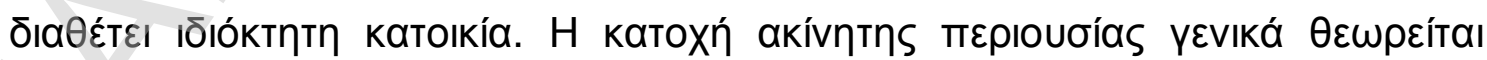

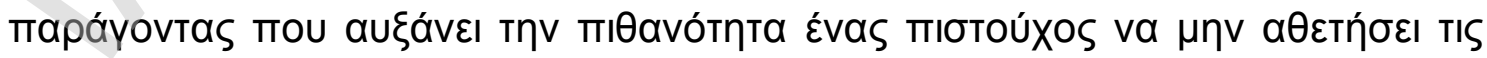

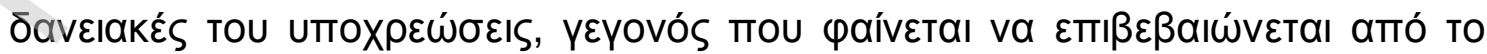

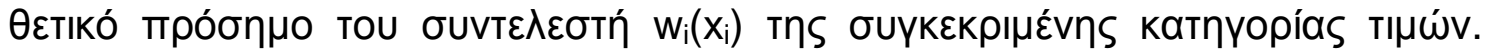




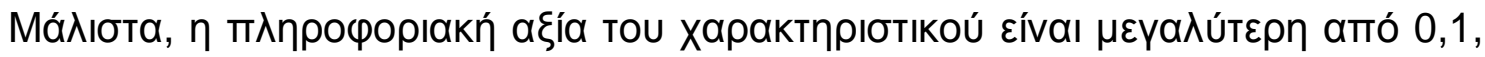

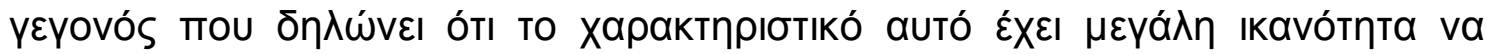

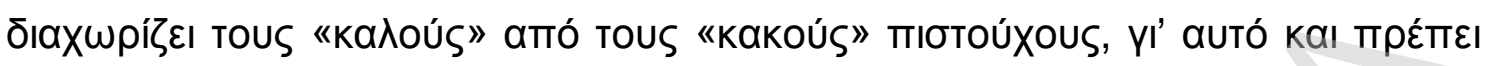

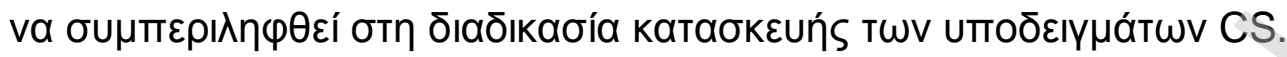

ПINAKA 5.8

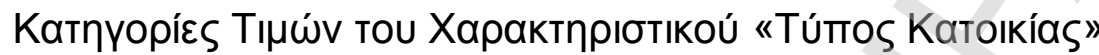

\begin{tabular}{|c|c|c|c|c|c|c|}
\hline & \multirow[b]{2}{*}{ 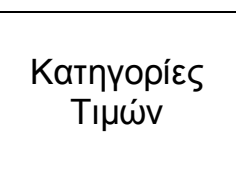 } & \multicolumn{2}{|c|}{ ПıбтоúXoı } & & \multirow{2}{*}{$\mathrm{f}_{\mathrm{i}}\left(\mathrm{x}_{\mathrm{i}} \mid 0\right)$} & \multirow{2}{*}{ 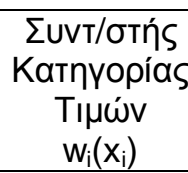 } \\
\hline & & «Какоі́» & «Ka入oí» & & & \\
\hline \multirow[t]{4}{*}{ 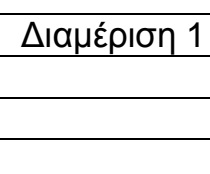 } & 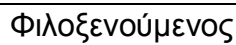 & 65 & 599 & 0,153364 & 0,179452 & $-0,157094$ \\
\hline & Evolkıaotńs & 154 & 742 & 0,189946 & 0,423288 & $-0,801311$ \\
\hline & ІঠІокти́тnS & 145 & 2567 & 0,656818 & 0,398630 & 0,499372 \\
\hline & 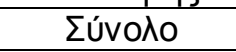 & 364 & 3908 & & & \\
\hline \multicolumn{7}{|c|}{$I V\left(X_{i}\right)=0,320009 \mathrm{k} \alpha \mathrm{I} H\left(X_{i}\right)=1,205649$} \\
\hline \multirow[t]{4}{*}{$\Delta ı \alpha \mu \varepsilon ́ p ı \sigma \eta n 2$} & 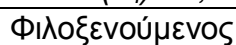 & 65 & 611 & 0,156434 & 0,179452 & $-0,137270$ \\
\hline & Evolkıaotńs & 159 & 745 & 0,190714 & 0,436986 & $-0,829130$ \\
\hline & Iঠıокти́т̄S & 140 & 2552 & 0,652980 & 0,384932 & 0,528482 \\
\hline & ¿úvoגo & 364 & 3908 & & & \\
\hline \multicolumn{7}{|c|}{$I V\left(X_{i}\right)=0,34901 \mathrm{k \alpha} \mathrm{H}\left(X_{i}\right)=1,212124$} \\
\hline \multirow[t]{4}{*}{$\Delta ı \alpha \mu \varepsilon ́ p ı \sigma \eta ~ 3$} & 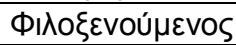 & 71 & 607 & 0,155411 & 0,19589 & $-0,231480$ \\
\hline & Evoıkıaбтńs & 162 & 732 & 0,187388 & 0,445205 & $-0,865350$ \\
\hline & ІঠІокти́тnS & 131 & 2569 & 0,657329 & 0,360274 & 0,601320 \\
\hline & ¿úvoגo & 364 & 3908 & & & \\
\hline \multicolumn{7}{|c|}{$I V\left(X_{i}\right)=0,411099 \kappa \alpha ı H\left(X_{i}\right)=1,205464$} \\
\hline \multirow[t]{4}{*}{$\Delta ı \alpha \mu \varepsilon ́ p ı \sigma \eta ~ 4$} & 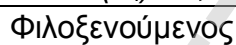 & 66 & 611 & 0,156434 & 0,182192 & $-0,152430$ \\
\hline & Evolkıaotńs & 163 & 739 & 0,189179 & 0,447945 & $-0,861980$ \\
\hline & Iঠıокти́т̄ns & 135 & 2558 & 0,654515 & 0,371233 & 0,567065 \\
\hline & ¿úvoגo & 364 & 3908 & & & \\
\hline \multicolumn{7}{|c|}{$I V\left(X_{i}\right)=0,387617 \kappa \alpha I H\left(X_{i}\right)=1,209836$} \\
\hline \multirow[t]{4}{*}{$\Delta ı \alpha \mu \varepsilon ́ p ı \sigma \eta ~ 5$} & 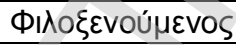 & 69 & 600 & 0,153620 & 0,190411 & $-0,214700$ \\
\hline & Evolkıaotńs & 154 & 738 & 0,188923 & 0,423288 & $-0,806710$ \\
\hline & ІঠІокти́т̄' & 141 & 2570 & 0,657585 & 0,387671 & 0,528416 \\
\hline & Eúvoגo & 364 & 3908 & & & \\
\hline
\end{tabular}




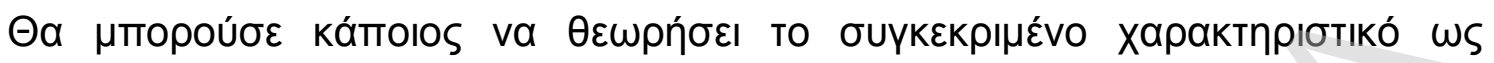

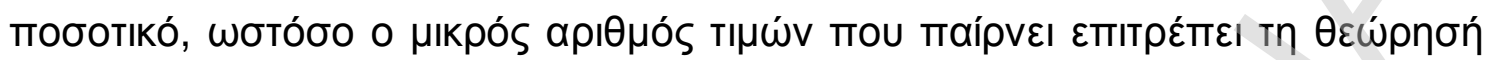

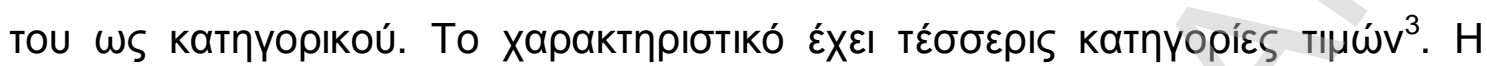

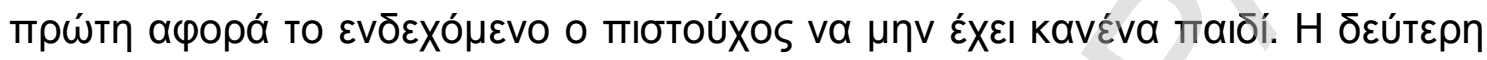

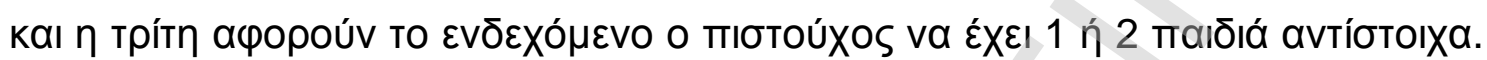

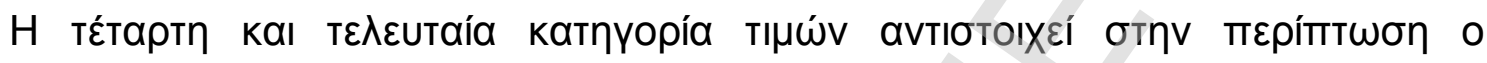

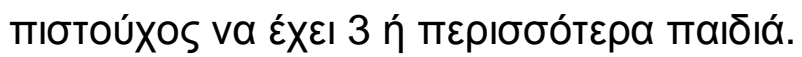

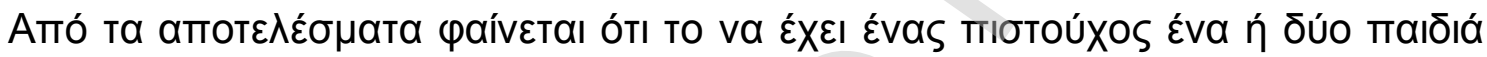

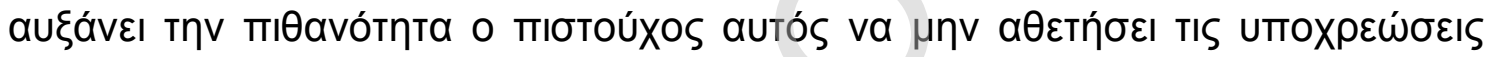

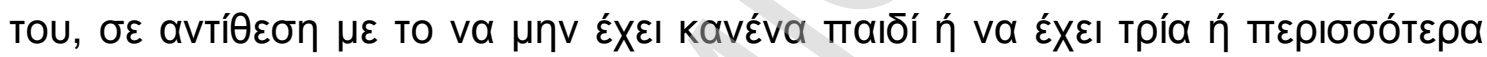

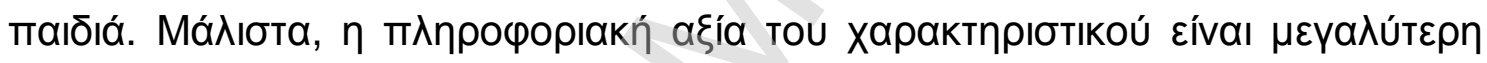

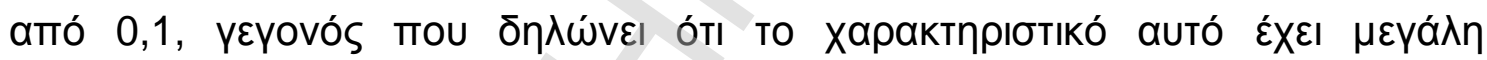

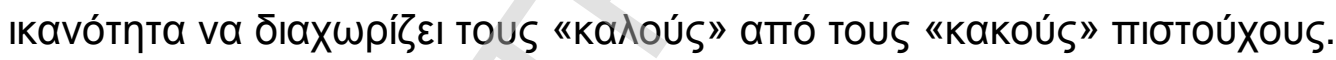

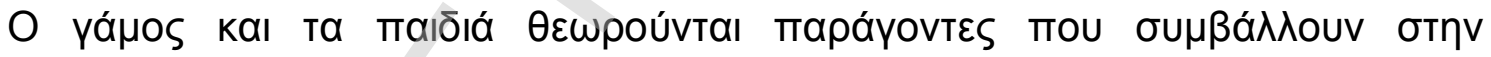

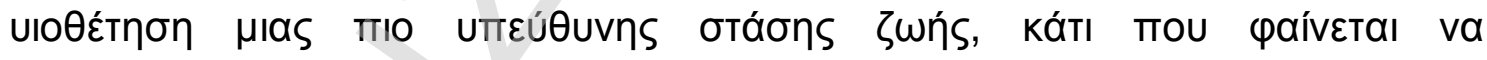

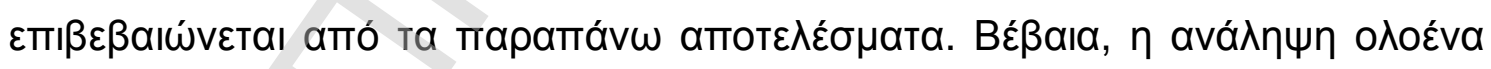

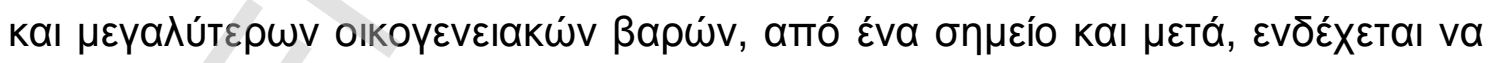

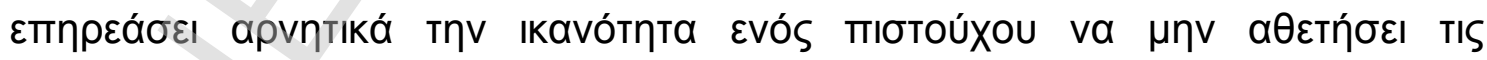

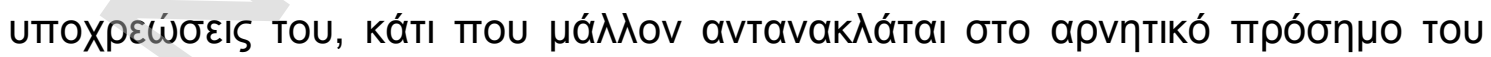

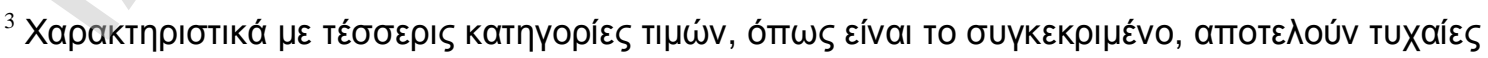

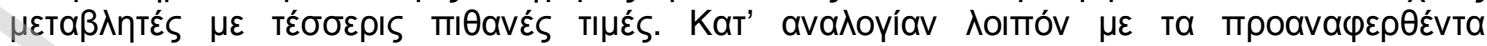

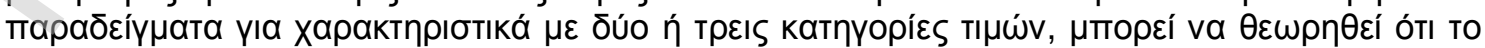

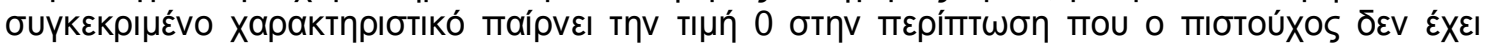

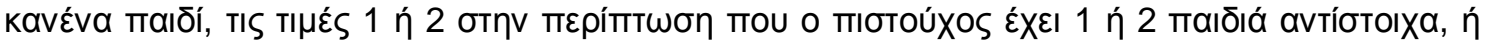

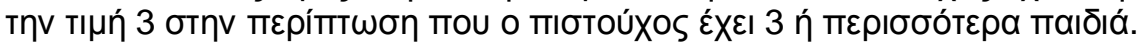




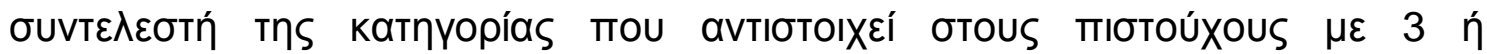

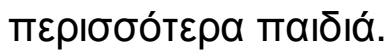

ПINAKA 4.9

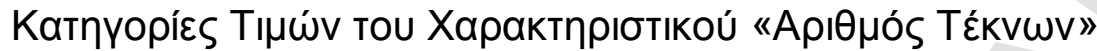

\begin{tabular}{|c|c|c|c|c|c|c|}
\hline & \multirow[b]{2}{*}{ 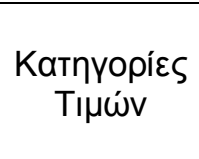 } & \multicolumn{2}{|c|}{ ПıбтоúXol } & \multirow[b]{2}{*}{$f_{i}\left(x_{i} \mid 1\right)$} & \multirow[b]{2}{*}{$f_{i}\left(x_{i} \mid 0\right)$} & \multirow{2}{*}{ 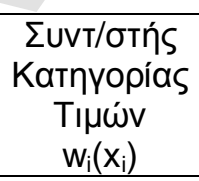 } \\
\hline & & «Какоі́» & «Ka入oí» & & & \\
\hline \multirow[t]{5}{*}{$\Delta ı а \mu \varepsilon ́ p ı \sigma \eta ~ 1$} & 0 & 198 & 1421 & 0,363648 & 0,543836 & $-0,402461$ \\
\hline & 1 & 16 & 622 & 0,159248 & 0,045205 & 1,259244 \\
\hline & 2 & 98 & 1390 & 0,355718 & 0,269863 & 0,276223 \\
\hline & $3+$ & 52 & 475 & 0,121642 & 0,143836 & $-0,167586$ \\
\hline & $\sum u ́ v o \lambda o$ & 364 & 3908 & & & \\
\hline \multicolumn{7}{|c|}{$I V\left(X_{i}\right)=0,24356$ каı $H\left(X_{i}\right)=1,69562$} \\
\hline \multirow[t]{5}{*}{$\Delta ı \alpha \mu \varepsilon ́ p ı \sigma \eta 2$} & 0 & 197 & 1440 & 0,368509 & 0,541096 & $-0,384130$ \\
\hline & 1 & 18 & 656 & 0,167946 & 0,050685 & 1,198012 \\
\hline & 2 & 97 & 1347 & 0,344717 & 0,267123 & 0,255014 \\
\hline & $3+$ & 52 & 465 & 0,119084 & 0,143836 & $-0,188840$ \\
\hline & Eúvoגo & 364 & 3908 & & & \\
\hline \multicolumn{7}{|c|}{$I V\left(X_{i}\right)=0,231238 \mathrm{kal} H\left(X_{i}\right)=1,700174$} \\
\hline \multirow[t]{5}{*}{$\Delta ı \alpha \mu \varepsilon ́ p ı \sigma \eta ~ 3$} & 0 & 207 & 1435 & 0,367229 & 0,568493 & $-0,437000$ \\
\hline & 1 & 18 & 637 & 0,163085 & 0,050685 & 1,168644 \\
\hline & 2 & 91 & 1355 & 0,346764 & 0,250685 & 0,324447 \\
\hline & $3+$ & 48 & 481 & 0,123177 & 0,132877 & $-0,075800$ \\
\hline & Eúvoגo & 364 & 3908 & & & \\
\hline \multicolumn{7}{|c|}{$I V\left(X_{i}\right)=0,251216$ каı $H\left(X_{i}\right)=1,70084$} \\
\hline \multirow[t]{5}{*}{$\Delta ı \alpha \mu \varepsilon ́ p ı \sigma \eta ~ 4$} & 0 & 201 & 1442 & 0,369020 & 0,552055 & $-0,402800$ \\
\hline & 1 & 17 & 623 & 0,159504 & 0,047945 & 1,202008 \\
\hline & 2 & 94 & 1377 & 0,352392 & 0,258904 & 0,308286 \\
\hline & $3+$ & 52 & 466 & 0,119340 & 0,143836 & $-0,186690$ \\
\hline & $\sum u ́ v o \lambda o$ & 364 & 3908 & & & \\
\hline \multicolumn{7}{|c|}{$I V\left(X_{i}\right)=0,241214$ каı $H\left(X_{i}\right)=1,692636$} \\
\hline \multirow[t]{5}{*}{$\Delta ı \alpha \mu \varepsilon ́ p ı \sigma\rceil ~ 5$} & 0 & 197 & 1426 & 0,364927 & 0,541096 & $-0,393900$ \\
\hline & 1 & 15 & 642 & 0,164364 & 0,042466 & 1,353387 \\
\hline & 2 & 96 & 1375 & 0,351880 & 0,264384 & 0,285890 \\
\hline & $3+$ & 56 & 465 & 0,119084 & 0,154795 & $-0,262270$ \\
\hline & ¿úvoגo & 364 & 3908 & & & \\
\hline
\end{tabular}




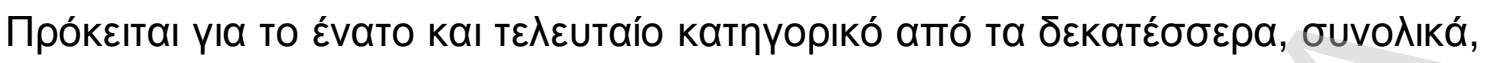

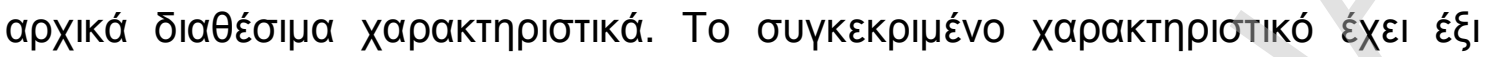

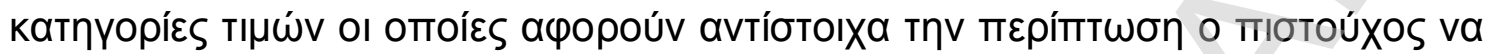
Eíval:

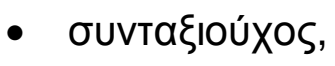

- TEXVIKóS,

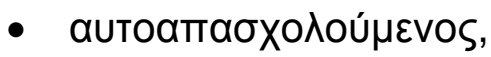

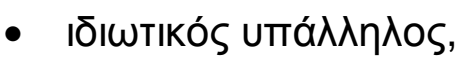

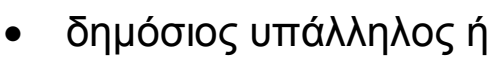

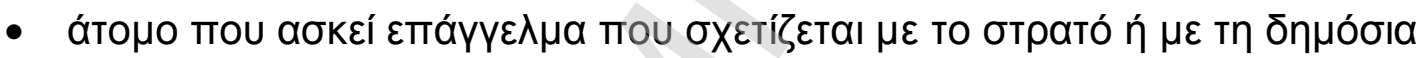

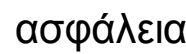

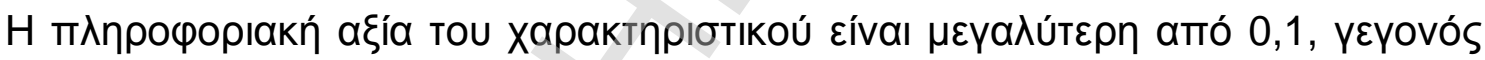

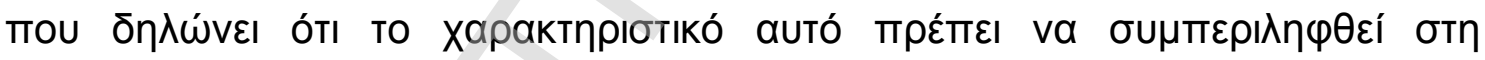

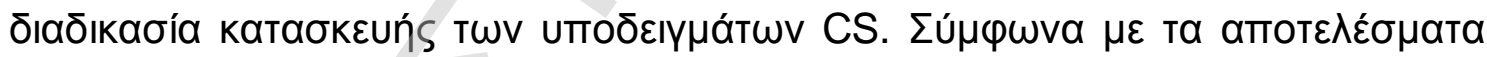

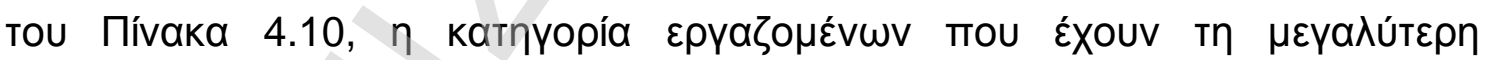

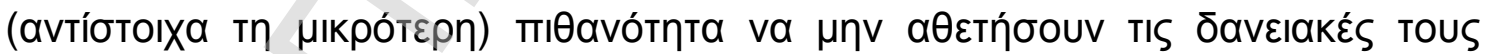

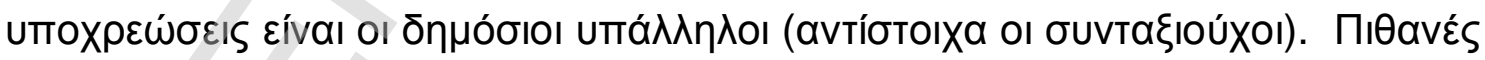

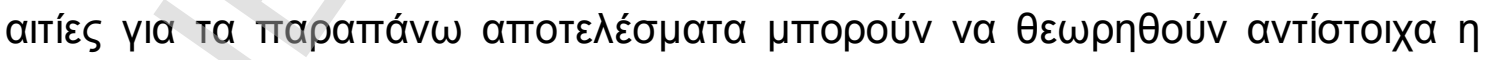

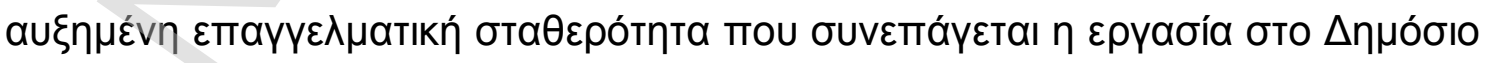

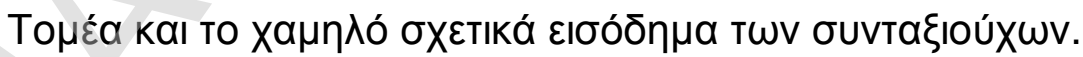


ПINAKA $\Sigma 4.10$

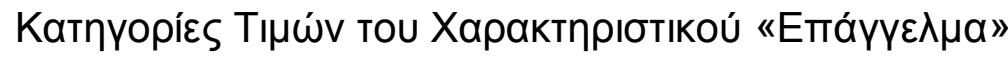

\begin{tabular}{|c|c|c|c|c|c|c|}
\hline & \multirow{2}{*}{ 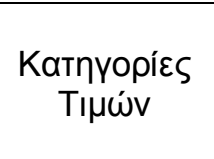 } & \multicolumn{2}{|c|}{ ПıбтоúXOI } & \multirow[b]{2}{*}{$\mathrm{f}_{\mathrm{i}}\left(\mathrm{x}_{\mathrm{i}} \mid 1\right)$} & \multirow[b]{2}{*}{$\mathrm{f}_{\mathrm{i}}\left(\mathrm{x}_{\mathrm{i}} \mid 0\right)$} & \multirow{2}{*}{ 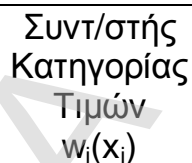 } \\
\hline & & «Какоі́» & «Ka入oí» & & & \\
\hline \multirow{7}{*}{$\Delta ı \alpha \mu \varepsilon ́ p ı \sigma \eta 1$} & EuvtakıoúXOS & 105 & 448 & 0,114735 & 0,289041 & $-0,923942$ \\
\hline & TEXVIKós & 58 & 674 & 0,172551 & 0,160274 & 0,073805 \\
\hline & 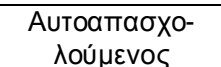 & 70 & 904 & 0,231389 & 0,193151 & 0,180630 \\
\hline & 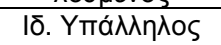 & 18 & 245 & 0,062804 & 0,050685 & 0,214387 \\
\hline & 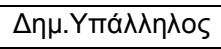 & 98 & 1418 & 0,362881 & 0,269863 & 0,296159 \\
\hline & 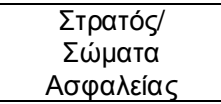 & 15 & 219 & 0,056152 & 0,042466 & 0,279373 \\
\hline & ¿úvoגo & 364 & 3908 & 7 & & \\
\hline \multicolumn{5}{|c|}{$I V\left(X_{i}\right)=0,202831 \mathrm{k \alpha I} H\left(X_{i}\right)=2,074391$} & \multicolumn{2}{|l|}{ 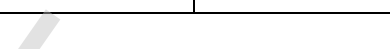 } \\
\hline \multirow[t]{7}{*}{$\Delta ı \alpha \mu \varepsilon ́ p ı \sigma \eta 2$} & EUvTakıoúxos & 100 & 468 & 0,119852 & 0,275342 & $-0,83176$ \\
\hline & TEXVIKós & 63 & 677 & 0,173318 & 0,173973 & $-0,00377$ \\
\hline & 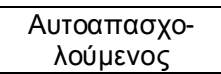 & 69 & 870 & 0,222691 & 0,190411 & 0,156601 \\
\hline & 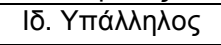 & 17 & 254 & 0,065106 & 0,047945 & 0,30596 \\
\hline & 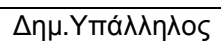 & 97 & 1426 & 0,364927 & 0,267123 & 0,311987 \\
\hline & 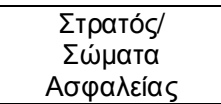 & 18 & 213 & 0,054618 & 0,050685 & 0,074727 \\
\hline & $\Sigma u ́ v o \lambda o$ & 364 & 3908 & & & \\
\hline \multicolumn{7}{|c|}{$I V\left(X_{i}\right)=0,170447 \kappa \alpha I H\left(X_{i}\right)=2,078632$} \\
\hline \multirow[t]{7}{*}{ 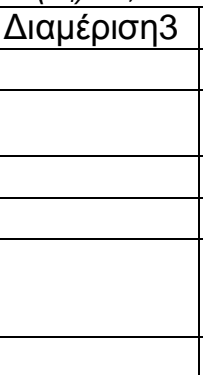 } & Euvtakııóxos & 100 & 447 & 0,114479 & 0,275342 & $-0,87762$ \\
\hline & TEXVIKós & 67 & 654 & 0,167434 & 0,184932 & $-0,0994$ \\
\hline & 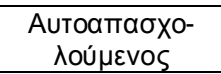 & 82 & 979 & 0,250576 & 0,226027 & 0,103104 \\
\hline & Iठ. Yாव́ं $\lambda \lambda \eta \lambda \propto S$ & 20 & 256 & 0,065618 & 0,056164 & 0,155564 \\
\hline & 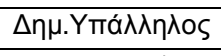 & 80 & 1351 & 0,345741 & 0,220548 & 0,449574 \\
\hline & 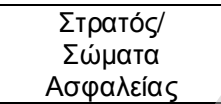 & & 221 & 0,056664 & 0,042466 & 0,288443 \\
\hline & ¿úvoגo & 364 & 3908 & & & \\
\hline \multicolumn{7}{|c|}{$I V\left(X_{i}\right)=0,207296 k \alpha I H\left(X_{i}\right)=2,085628$} \\
\hline \multirow[t]{7}{*}{ 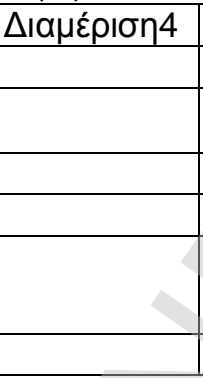 } & 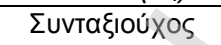 & 97 & 465 & 0,119084 & 0,267123 & $-0,80788$ \\
\hline & TEXVIKós & 64 & 764 & 0,195574 & 0,176712 & 0,101417 \\
\hline & 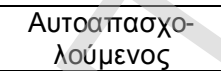 & 83 & 878 & 0,224738 & 0,228767 & $-0,01777$ \\
\hline & 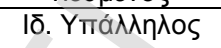 & 18 & 253 & 0,06485 & 0,050685 & 0,246454 \\
\hline & 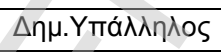 & 84 & 1327 & 0,339601 & 0,231507 & 0,383162 \\
\hline & 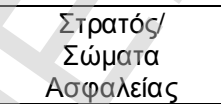 & 18 & 221 & 0,056664 & 0,050685 & 0,111512 \\
\hline & 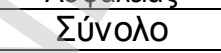 & 364 & 3908 & & & \\
\hline \multicolumn{7}{|c|}{$I V\left(X_{i}\right)=0,167158$ KaI $H\left(X_{i}\right)=2,100319$} \\
\hline \multirow[t]{2}{*}{$\Delta ı a \mu \varepsilon ́ p ı \sigma \eta 5$} & EuvtakıoúXos & 94 & 464 & 0,118828 & 0,258904 & $-0,77878$ \\
\hline & TEXVIKós & 63 & 684 & 0,175109 & 0,173973 & 0,006509 \\
\hline 8 & 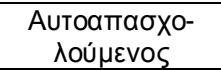 & 83 & 874 & 0,223715 & 0,228767 & $-0,02233$ \\
\hline$-\sqrt{-1}$ & Iठ. Yாव́́ $\lambda \lambda \eta \lambda \circ \varsigma$ & 19 & 240 & 0,061525 & 0,053425 & 0,141166 \\
\hline & 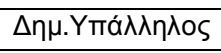 & 87 & 1420 & 0,363392 & 0,239726 & 0,415986 \\
\hline$\nabla$ & 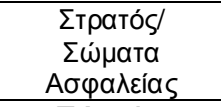 & 18 & 226 & 0,057943 & 0,050685 & 0,133835 \\
\hline & 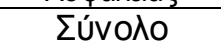 & 364 & 3908 & & & \\
\hline
\end{tabular}




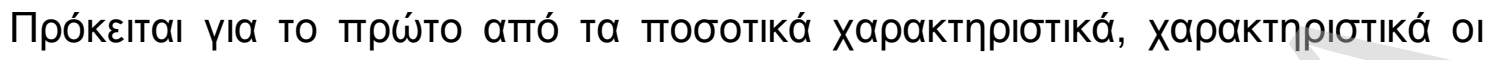

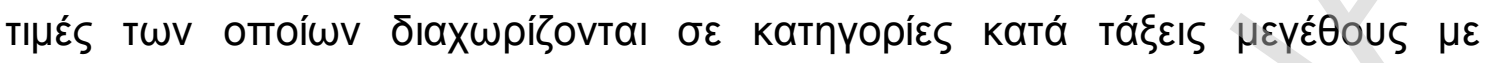

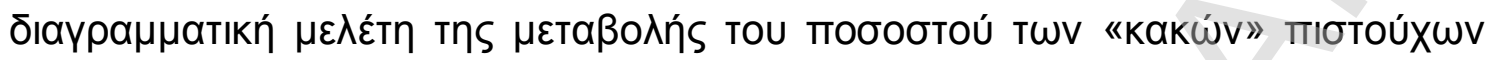

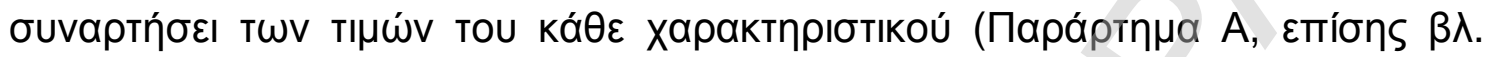

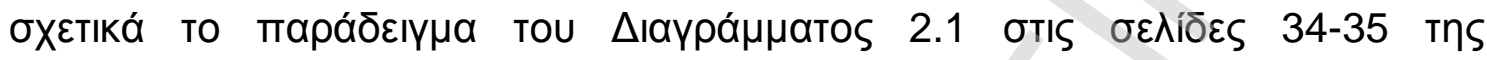

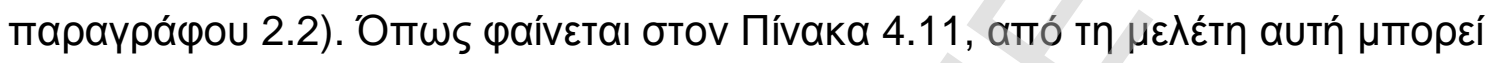

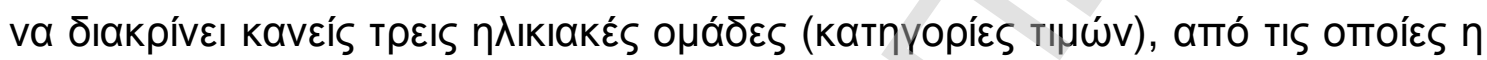

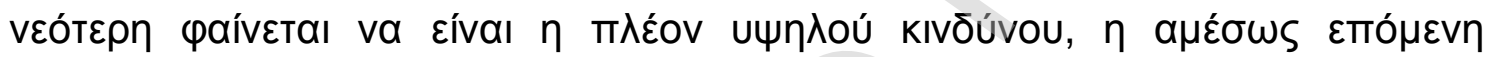

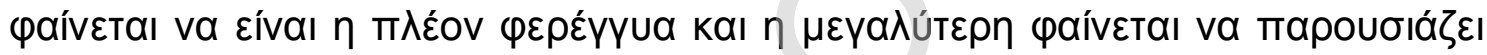

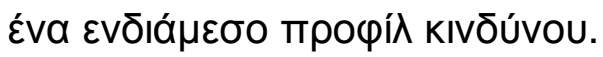

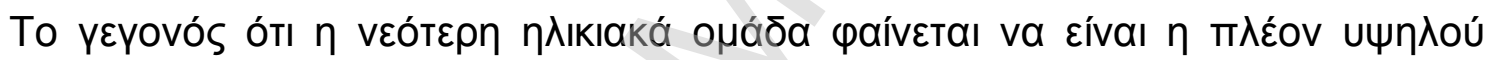

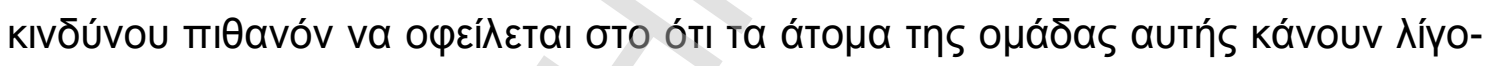

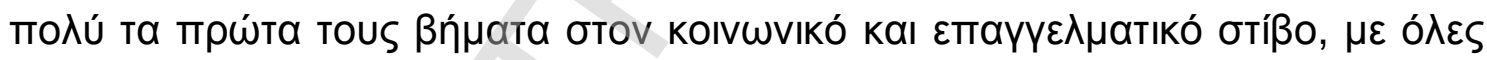

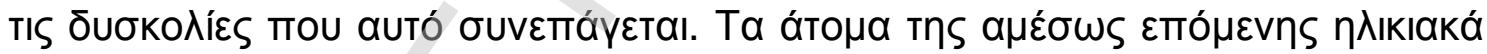

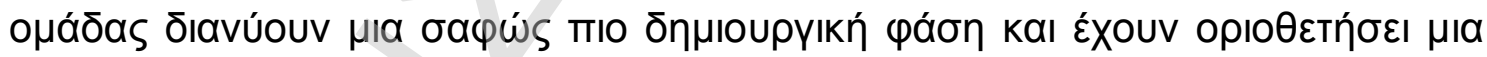

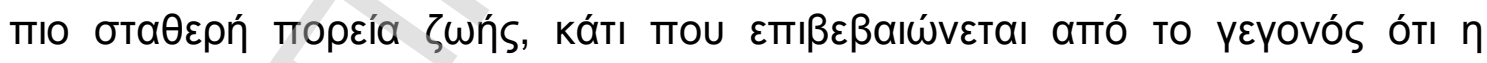

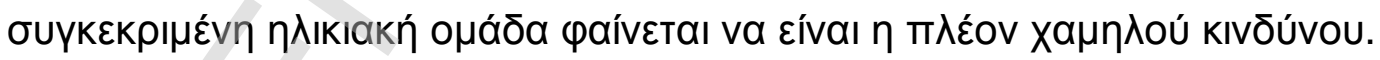

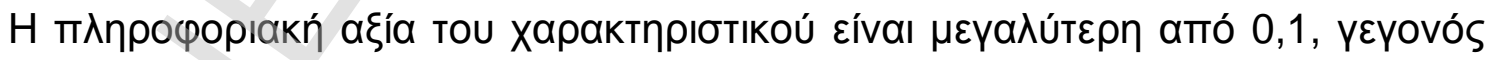

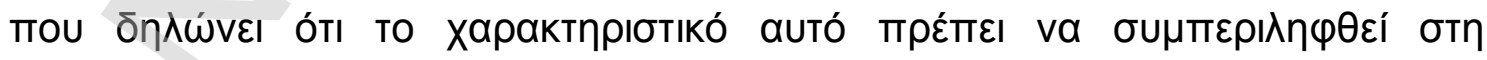

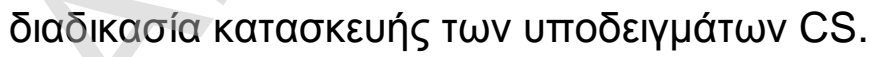


ПINAKA $\Sigma 4.11$

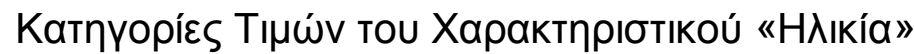

\begin{tabular}{|c|c|c|c|c|c|c|}
\hline & \multirow{2}{*}{ 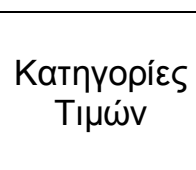 } & \multicolumn{2}{|c|}{ ПıбтоúXOI } & \multirow[b]{2}{*}{$f_{i}\left(x_{i} \mid 1\right)$} & \multirow[b]{2}{*}{$f_{i}\left(x_{i} \mid 0\right)$} & \multirow{2}{*}{$\begin{array}{c}\text { ¿uvt/otńs } \\
\text { Katnyopías } \\
\text { Tipúv } \\
w_{i}\left(x_{i}\right)\end{array}$} \\
\hline & & «Какоі́» & «Ka入oí» & & & \\
\hline \multirow[t]{4}{*}{ 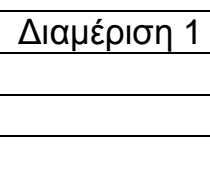 } & $20-39$ & 218 & 1533 & 0,392300 & 0,598630 & $-0,42262$ \\
\hline & $40-58$ & 69 & 1809 & 0,462906 & 0,190411 & 0,88834 \\
\hline & $59+$ & 77 & 566 & 0,144922 & 0,212329 & $-0,38194$ \\
\hline & $\sum u ́ v o \lambda o$ & 364 & 3908 & & $\overline{7}$ & \\
\hline \multicolumn{5}{|c|}{$I V\left(X_{i}\right)=0,355012 \mathrm{k \alpha I} H\left(X_{i}\right)=1,3549442$} & & \\
\hline \multirow[t]{4}{*}{$\Delta ı \alpha \mu \varepsilon ́ p ı \sigma \eta 2$} & $20-39$ & 228 & 1550 & 0,396649 & 0,626027 & $-0,45634$ \\
\hline & $40-58$ & 64 & 1797 & 0,459836 & 0,176712 & 0,95634 \\
\hline & $59+$ & 72 & 561 & 0,143643 & 0,198630 & $-0,32411$ \\
\hline & $\sum u ́ v o \lambda o$ & 364 & 3908 & 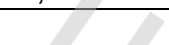 & & \\
\hline \multicolumn{5}{|c|}{$I V\left(X_{i}\right)=0,393262 \mathrm{kal} H\left(X_{i}\right)=1,353642$} & -7 & \\
\hline \multirow[t]{4}{*}{$\Delta ı \alpha \mu \varepsilon$ pı } & $20-39$ & 226 & 1560 & 0,399207 & 0,620548 & $-0,44112$ \\
\hline & $40-58$ & 65 & 1797 & 0,459836 & 0,179452 & 0,94096 \\
\hline & $59+$ & 73 & 551 & 0,141085 & 0,201370 & $-0,35578$ \\
\hline & $\sum u ́ v o \lambda o$ & 364 & 3908 & 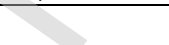 & & \\
\hline \multicolumn{7}{|c|}{$I V\left(X_{i}\right)=0,382918 \mathrm{k \alpha I} H\left(X_{i}\right)=1,350536$} \\
\hline \multirow[t]{4}{*}{$\Delta ı \alpha \mu \varepsilon ́ p ı \sigma \eta 4$} & $20-39$ & 233 & 1545 & 0,395370 & 0,639726 & $-0,48122$ \\
\hline & $40-62$ & 78 & 1993 & 0,509977 & 0,215068 & 0,86341 \\
\hline & $63+$ & 53 & 370 & 0,094781 & 0,146575 & $-0,43597$ \\
\hline & ¿úvoגo & 364 & 3908 & & & \\
\hline \multicolumn{3}{|c|}{$I V\left(X_{i}\right)=0,394796 \kappa \alpha I H\left(X_{i}\right)=1,269931$} & & & & \\
\hline \multirow[t]{4}{*}{$\Delta ı а \mu \varepsilon ́ p ı \sigma\rceil 5$} & $20-39$ & 231 & 1520 & 0,388974 & 0,634247 & $-0,48892$ \\
\hline & $40-58$ & 66 & 1809 & 0,462906 & 0,182192 & 0,93246 \\
\hline & $59+$ & 67 & 579 & 0,148248 & 0,184932 & $-0,22110$ \\
\hline & $\sum u ́ v o \lambda o$ & 364 & 3908 & & & \\
\hline
\end{tabular}

4.4.11

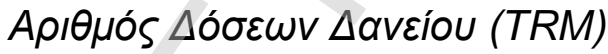

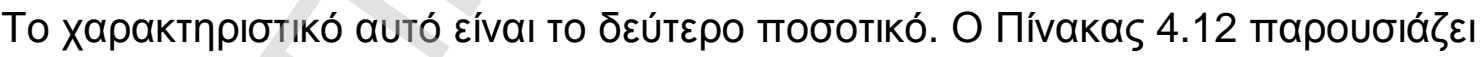

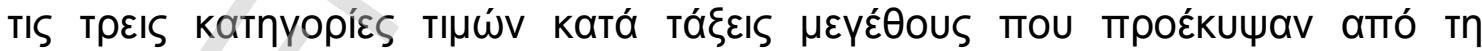

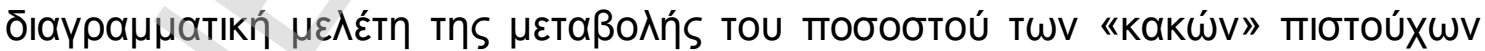

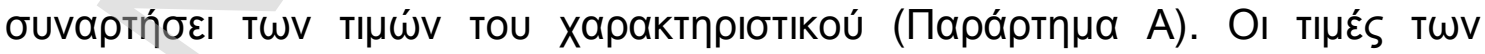

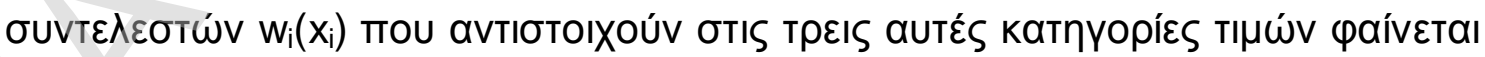

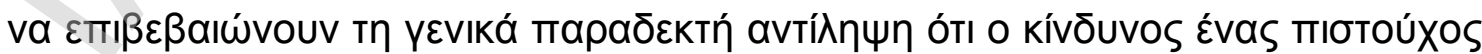

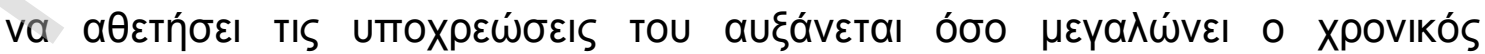

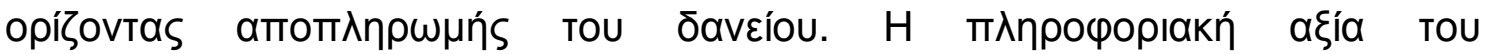




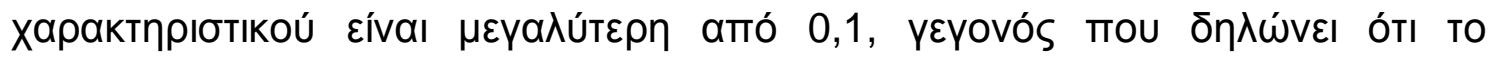

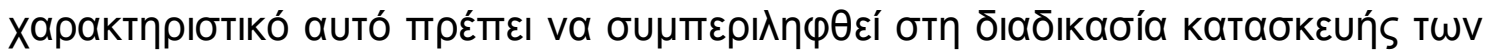

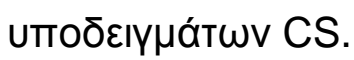

ПINAKA 4.12

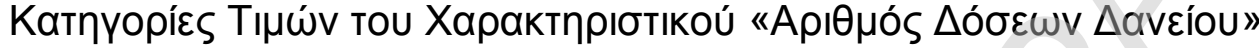

\begin{tabular}{|c|c|c|c|c|c|c|}
\hline & \multirow[b]{2}{*}{ 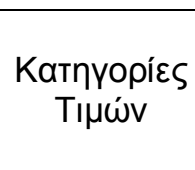 } & \multicolumn{2}{|c|}{ Пıбтои́XoI } & \multirow[b]{2}{*}{$f_{i}\left(x_{i} \mid 1\right)$} & \multirow{2}{*}{$f_{i}\left(x_{i} \mid 0\right)$} & \multirow{2}{*}{ 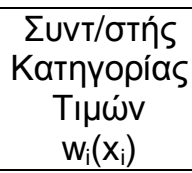 } \\
\hline & & «Какоі́» & «Ка入০ó» & & & \\
\hline \multirow[t]{4}{*}{$\Delta ı \alpha \mu \varepsilon ́ p ı \sigma \eta 1$} & $6-22$ & 37 & 973 & 0,249041 & 0,10274 & 0,885417 \\
\hline & $23-40$ & 272 & 2532 & 0,647864 & 0,746575 & $-0,141816$ \\
\hline & $41+$ & 55 & 403 & 0,103223 & 0,152055 & $-0,387346$ \\
\hline & ¿úvoגo & 364 & 3908 & 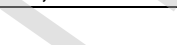 & & \\
\hline \multicolumn{7}{|c|}{$I V\left(X_{i}\right)=0,162451 \kappa \alpha ı \mathrm{H}\left(X_{i}\right)=1,181596$} \\
\hline \multirow[t]{4}{*}{ 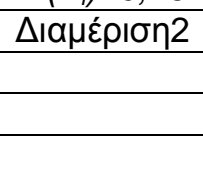 } & $6-22$ & 31 & 961 & 0,245971 & 0,086301 & 1,047368 \\
\hline & $23-41$ & 286 & 2563 & 0,655794 & 0,784932 & $-0,179750$ \\
\hline & $42+$ & 47 & 384 & 0,098363 & 0,130137 & $-0,279930$ \\
\hline & Eúvoגo & 364 & 3908 & & & \\
\hline \multicolumn{7}{|c|}{$I V\left(X_{i}\right)=0,199339 \kappa \alpha ı H\left(X_{i}\right)=1,166303$} \\
\hline \multirow[t]{4}{*}{$\Delta ı а \mu \varepsilon ́ p ı \sigma \eta 3$} & $6-21$ & 28 & 928 & 0,237529 & 0,078082 & 1,112527 \\
\hline & $22-40$ & 284 & 2604 & 0,666283 & 0,779452 & $-0,156880$ \\
\hline & $41+$ & 52 & 376 & 0,096316 & 0,143836 & $-0,401030$ \\
\hline & $\sum u ́ v o \lambda o$ & 364 & 3908 & & & \\
\hline \multicolumn{7}{|c|}{$I V\left(X_{i}\right)=0,214199$ каı $H\left(X_{i}\right)=1,151373$} \\
\hline \multirow[t]{4}{*}{$\Delta ı \alpha \mu \varepsilon ́ p ı \sigma \eta 4$} & $6-21$ & 31 & 902 & 0,230877 & 0,086301 & 0,984042 \\
\hline & $22-40$ & 284 & 2642 & 0,676004 & 0,779452 & $-0,142390$ \\
\hline & $41+$ & 49 & 364 & 0,093246 & 0,135616 & $-0,374590$ \\
\hline & ¿úvoגo & 364 & 3908 & & & \\
\hline \multicolumn{7}{|c|}{$I V\left(X_{i}\right)=0,17287 \kappa \alpha ı H\left(X_{i}\right)=1,13571$} \\
\hline \multirow[t]{4}{*}{$\Delta ı a \mu \varepsilon ́ p ı \sigma \eta 5$} & $6-21$ & 30 & 865 & 0,221412 & 0,083562 & 0,974441 \\
\hline & $22-40$ & 277 & 2666 & 0,682144 & 0,760274 & $-0,108440$ \\
\hline & $41+$ & 57 & 377 & 0,096572 & 0,157534 & $-0,489350$ \\
\hline & ¿úvoגo & 364 & 3908 & & & \\
\hline
\end{tabular}

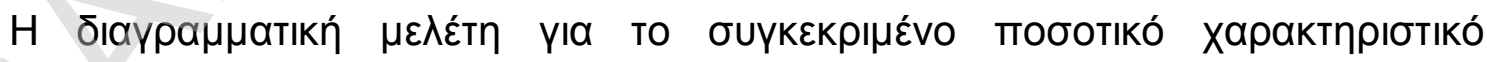

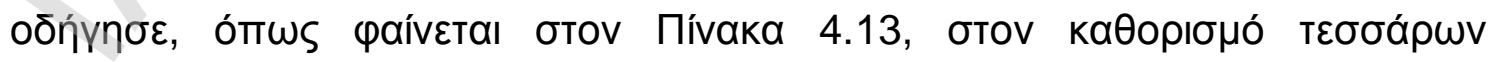

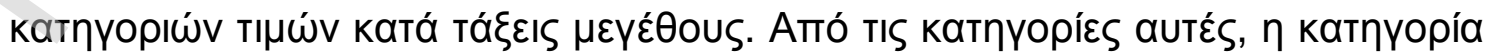

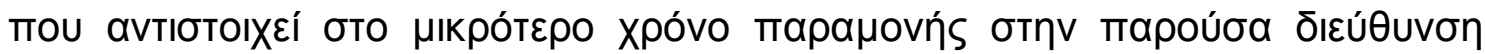




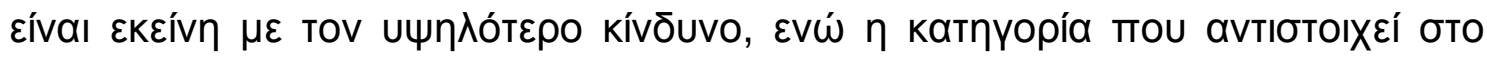

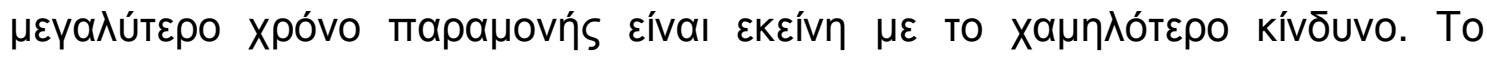

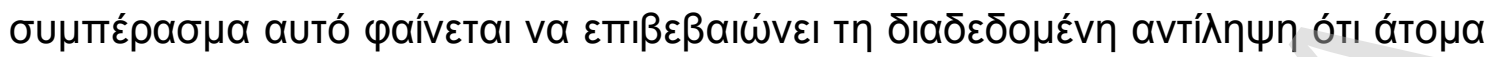

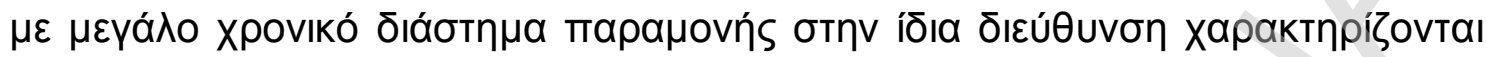

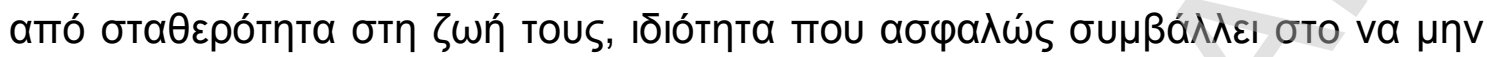

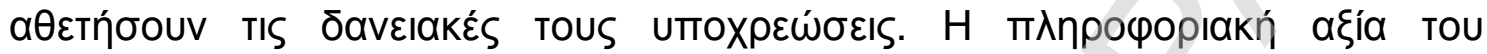

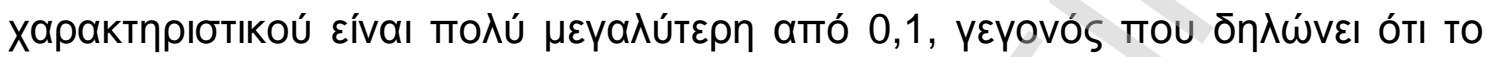

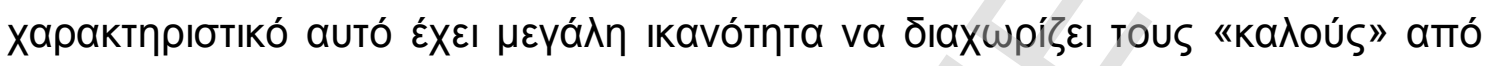
TOus «Kakoúৎ» тіఠтоúXous.

ПINAKA 4.13

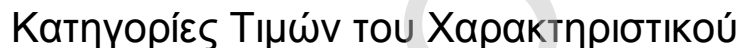

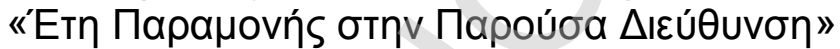

\begin{tabular}{|c|c|c|c|c|c|c|}
\hline & \multirow[b]{2}{*}{ 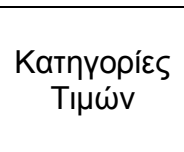 } & \multicolumn{2}{|c|}{ Пıбтои́XоІ } & \multirow[b]{2}{*}{$f_{i}\left(x_{i} \mid 1\right)$} & \multirow[b]{2}{*}{$\mathrm{f}_{\mathrm{i}}\left(\mathrm{x}_{\mathrm{i}} \mid 0\right)$} & \multirow{2}{*}{ 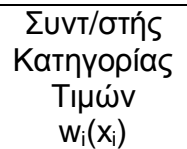 } \\
\hline & & «Какоі́»» & «Ka入oí» & & & \\
\hline \multirow[t]{5}{*}{$\Delta ı \alpha \mu \varepsilon ́ p ı \sigma \eta 1$} & $1-3$ & 198 & 798 & 0,204272 & 0,543836 & $-0,979194$ \\
\hline & $4-17$ & 81 & 1667 & 0,426580 & 0,223288 & 0,647338 \\
\hline & $18-38$ & 79 & 1194 & 0,305577 & 0,217808 & 0,338586 \\
\hline & $39+$ & 6 & 249 & 0,063827 & 0,017808 & 1,276517 \\
\hline & $\sum u ́ v o \lambda o$ & 364 & 3908 & & & \\
\hline \multicolumn{7}{|c|}{$I V\left(X_{i}\right)=0,552558 \mathrm{k \alpha} I H\left(X_{i}\right)=1,624516$} \\
\hline \multirow[t]{5}{*}{$\Delta ı а \mu \varepsilon ́ \rho ı \sigma \eta 2$} & $1-3$ & 195 & 791 & 0,202481 & 0,535616 & $-0,972770$ \\
\hline & $4-18$ & 89 & 1703 & 0,435789 & 0,245205 & 0,575062 \\
\hline & 19-38 & 75 & 1172 & 0,299949 & 0,206849 & 0,371621 \\
\hline & $39+$ & 5 & 242 & 0,062036 & 0,015068 & 1,415114 \\
\hline & $\sum u ́ v o \lambda o$ & 364 & 3908 & & & \\
\hline \multicolumn{7}{|c|}{$\left.I V\left(X_{i}\right)=0,534724 \kappa \alpha ı\right) ~ H\left(X_{i}\right)=1,616312$} \\
\hline \multirow[t]{5}{*}{$\Delta ı а \mu \varepsilon ́ p ı \sigma \eta ~ 3$} & $1-3$ & 199 & 819 & 0,209644 & 0,546575 & $-0,958260$ \\
\hline & $4-18$ & 86 & 1699 & 0,434766 & 0,236986 & 0,606805 \\
\hline & $19-37$ & 77 & 1134 & 0,290228 & 0,212329 & 0,312530 \\
\hline & $38+$ & 2 & 256 & 0,065618 & 0,006849 & 2,259698 \\
\hline & $\sum u ́ v o \lambda o$ & 364 & 3908 & & & \\
\hline \multicolumn{7}{|c|}{$I V\left(X_{i}\right)=0,600026 \mathrm{kal} H\left(X_{i}\right)=1,626166$} \\
\hline \multirow[t]{2}{*}{$\Delta ı \alpha \mu \varepsilon ́ p ı \sigma \eta ~ 4$} & $1-3$ & 191 & 819 & 0,209644 & 0,524658 & $-0,917330$ \\
\hline & $4-18$ & 85 & 1684 & 0,430929 & 0,234247 & 0,609568 \\
\hline 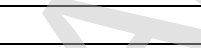 & $19-38$ & 83 & 1159 & 0,296623 & 0,228767 & 0,259758 \\
\hline 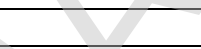 & $39+$ & 5 & 246 & 0,063060 & 0,015068 & 1,431474 \\
\hline- & ¿úvoגo & 364 & 3908 & & & \\
\hline \multicolumn{7}{|c|}{$I V\left(X_{i}\right)=0,495187 \kappa \alpha ı H\left(X_{i}\right)=1,623746$} \\
\hline \multirow[t]{5}{*}{ 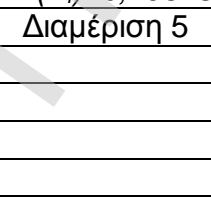 } & $1-3$ & 201 & 809 & 0,207086 & 0,552055 & $-0,980510$ \\
\hline & $4-17$ & 81 & 1640 & 0,419673 & 0,223288 & 0,631014 \\
\hline & $18-38$ & 76 & 1203 & 0,307879 & 0,209589 & 0,384559 \\
\hline & $39+$ & 6 & 256 & 0,065618 & 0,017808 & 1,304187 \\
\hline & $\sum u ́ v o \lambda o$ & 364 & 3908 & & & \\
\hline
\end{tabular}




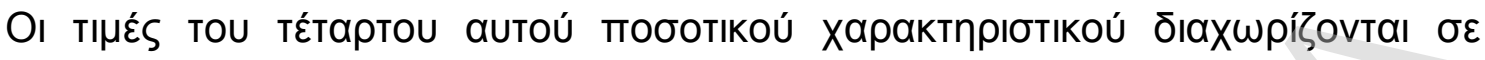

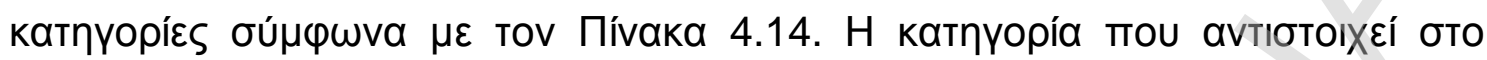

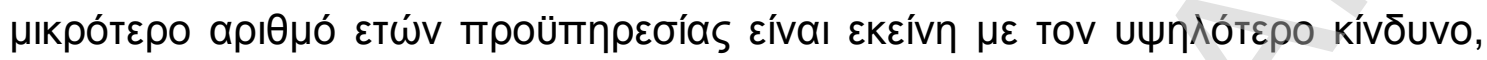

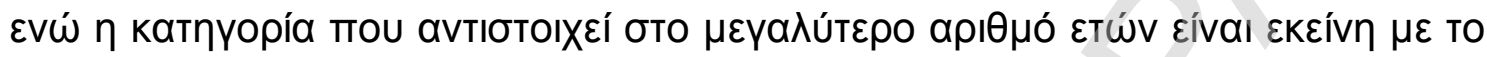

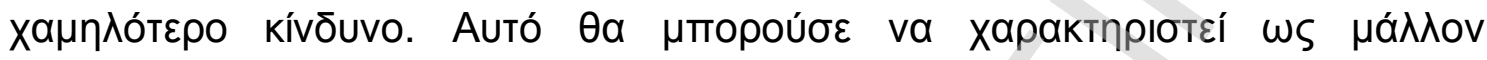

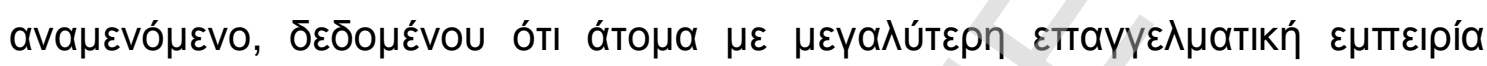

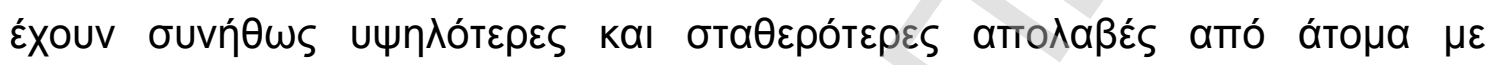

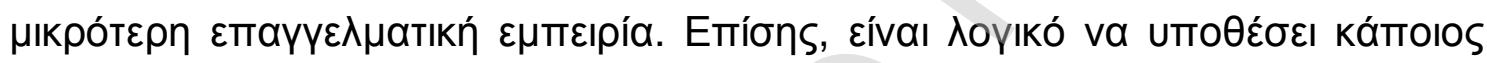

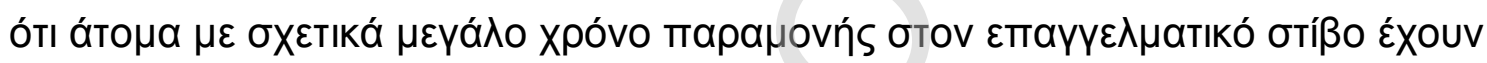

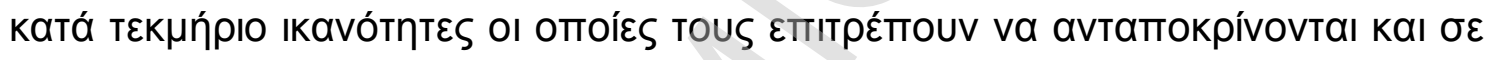

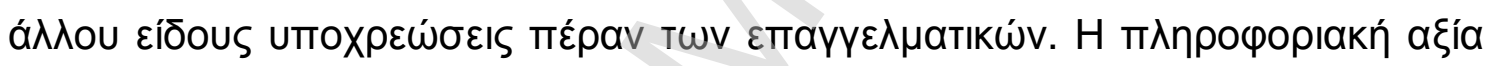

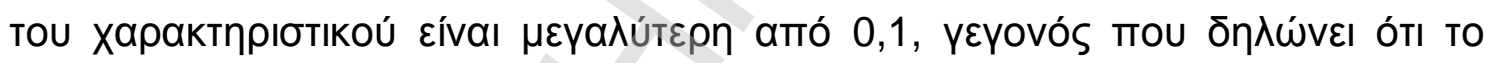

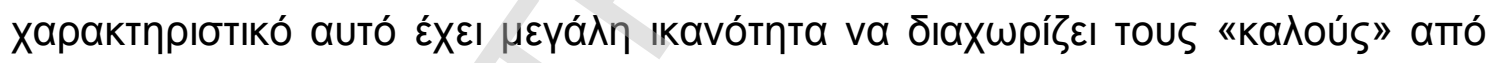
TOuৎ «Kakoúৎ» тіఠтоúXous. 
ПINAKA $\Sigma 4.14$

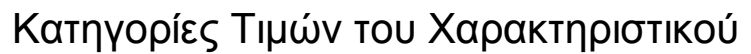

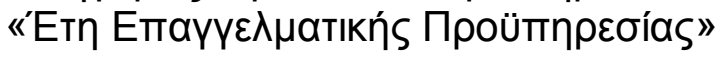

\begin{tabular}{|c|c|c|c|c|c|c|}
\hline & \multirow[b]{2}{*}{ 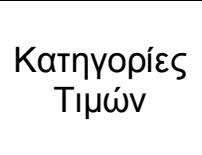 } & \multicolumn{2}{|c|}{ Пıбтои́Xol } & \multirow[b]{2}{*}{$\mathrm{f}_{\mathrm{i}}\left(\mathrm{x}_{\mathrm{i}} \mid 1\right)$} & \multirow[b]{2}{*}{$\mathrm{f}_{\mathrm{i}}\left(\mathrm{x}_{\mathrm{i}} \mid 0\right)$} & \multirow{2}{*}{ 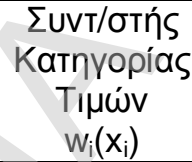 } \\
\hline & & «Какоі́» & «Ka入oí» & & & \\
\hline \multirow[t]{5}{*}{$\Delta ı \alpha \mu \varepsilon ́ p ı \sigma \eta 1$} & $1-3$ & 121 & 802 & 0,205295 & 0,332877 & $-0,483322$ \\
\hline & $4-8$ & 116 & 1008 & 0,257994 & 0,319178 & $-0,212811$ \\
\hline & $9-12$ & 56 & 651 & 0,166667 & 0,154795 & 0,073897 \\
\hline & $13+$ & 71 & 1447 & 0,370299 & 0,195890 & 0,636756 \\
\hline & Eúvoגo & 364 & 3908 & & $\overline{3}$ & \\
\hline \multicolumn{5}{|c|}{$I V\left(X_{i}\right)=0,186617 \mathrm{k \alpha I} H\left(X_{i}\right)=1,766541$} & & \\
\hline \multirow[t]{5}{*}{$\Delta ı а \mu \varepsilon ́ p ı \sigma \eta 2$} & $1-3$ & 118 & 798 & 0,204272 & 0,324658 & $-0,463320$ \\
\hline & $4-9$ & 123 & 1134 & 0,290228 & 0,338356 & $-0,153430$ \\
\hline & $10-12$ & 46 & 558 & 0,142875 & 0,127397 & 0,114663 \\
\hline & $13+$ & 77 & 1418 & 0,362881 & 0,212329 & 0,535938 \\
\hline & Eúvoגo & 364 & 3908 & & & \\
\hline \multicolumn{7}{|c|}{$I V\left(X_{i}\right)=0,145622 \mathrm{kal} H\left(X_{i}\right)=1,752199$} \\
\hline \multirow[t]{5}{*}{$\Delta ı \alpha \mu \varepsilon ́ p ı \sigma \eta ~ 3$} & $1-3$ & 128 & 814 & 0,208365 & 0,352055 & $-0,524490$ \\
\hline & $4-9$ & 108 & 1114 & 0,285111 & 0,297260 & $-0,041730$ \\
\hline & $10-12$ & 47 & 550 & 0,140829 & 0,130137 & 0,078958 \\
\hline & $13+$ & 81 & 1430 & 0,365950 & 0,223288 & 0,494037 \\
\hline & ¿úvoגo & 364 & 3908 & & & \\
\hline \multicolumn{7}{|c|}{$I V\left(X_{i}\right)=0,147196 \kappa \alpha I H\left(X_{i}\right)=1,75127$} \\
\hline \multirow{5}{*}{$\Delta ı \alpha \mu \dot{p} ı \sigma \eta n$} & $1-2$ & 82 & 511 & 0,130852 & 0,226027 & $-0,546590$ \\
\hline & $3-8$ & 141 & 1358 & 0,347531 & 0,387671 & $-0,109300$ \\
\hline & $9-12$ & 59 & 644 & 0,164876 & 0,163014 & 0,011359 \\
\hline & $13+$ & 82 & 1395 & 0,356997 & 0,226027 & 0,457070 \\
\hline & $\sum u ́ v o \lambda o$ & 364 & 3908 & & & \\
\hline \multicolumn{7}{|c|}{$I V\left(X_{i}\right)=0,116293 \kappa \alpha ı H\left(X_{i}\right)=1,714898$} \\
\hline \multirow[t]{5}{*}{$\Delta ı \alpha \mu \varepsilon ́ p ı \sigma \eta ~ 5$} & $1-3$ & 124 & 802 & 0,205295 & 0,341096 & $-0,5077130$ \\
\hline & $4-8$ & 108 & 1014 & 0,259529 & 0,297260 & $-0,1357390$ \\
\hline & $9-12$ & 55 & 642 & 0,164364 & 0,152055 & 0,0778443 \\
\hline & $13+$ & 77 & 1450 & 0,371067 & 0,212329 & 0,5582462 \\
\hline & ¿úvoגo & 364 & 3908 & & & \\
\hline
\end{tabular}

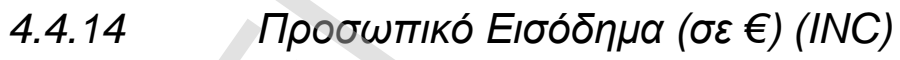

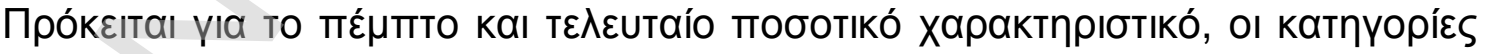

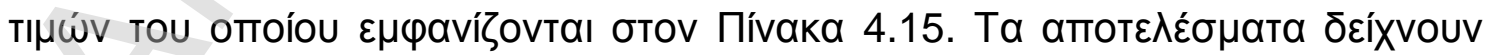

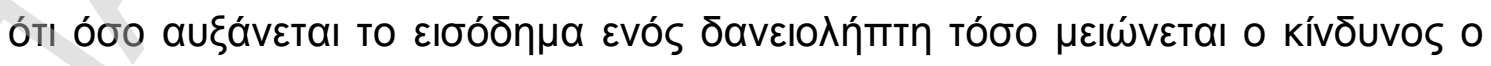

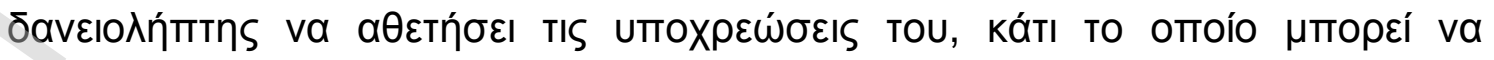

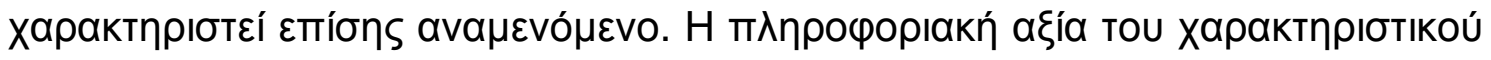

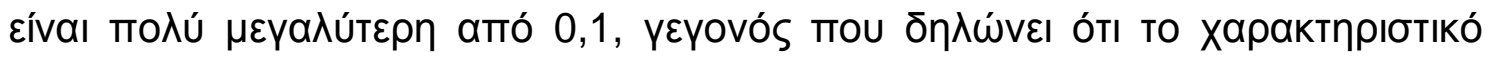




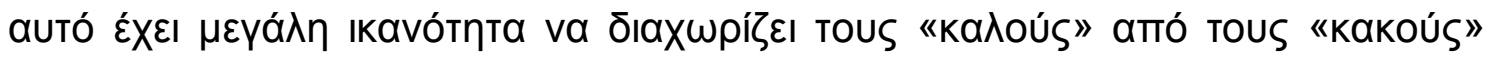

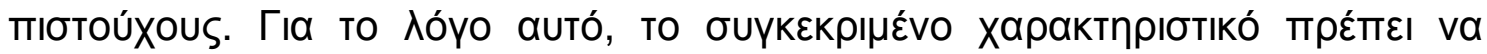

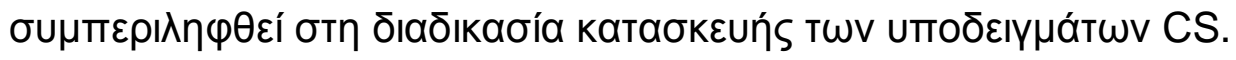

ПINAKA 24.15

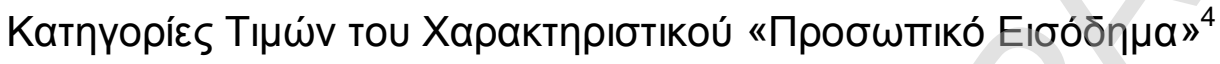

\begin{tabular}{|c|c|c|c|c|c|c|}
\hline & \multirow[b]{2}{*}{ 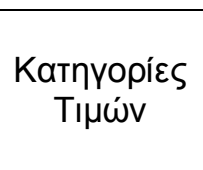 } & \multicolumn{2}{|c|}{ Пıбтои́XoI } & \multirow[b]{2}{*}{$f_{i}\left(x_{i} \mid 1\right)$} & \multirow{2}{*}{$f_{i}\left(x_{i} \mid 0\right)$} & \multirow{2}{*}{ 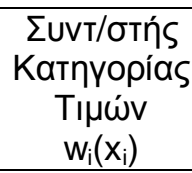 } \\
\hline & & «Какоі́» & «Ka入oí» & & & \\
\hline \multirow[t]{5}{*}{$\Delta ı \alpha \mu \varepsilon ́ p ı \sigma \eta 1$} & $\ldots-7579$ & 157 & 644 & 0,164876 & 0,431507 & $-0,962090$ \\
\hline & $7580-9459$ & 86 & 715 & 0,183039 & 0,236986 & $-0,258302$ \\
\hline & $9460-18656$ & 115 & 2020 & 0,516884 & 0,316438 & 0,490690 \\
\hline & $18657+$ & 6 & 529 & 0,135457 & 0,017808 & 2,028991 \\
\hline & $\sum u ́ v o \lambda o$ & 364 & 3908 & 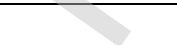 & & \\
\hline \multicolumn{7}{|c|}{$I V\left(X_{i}\right)=0,607522 \kappa \alpha ı H\left(X_{i}\right)=1,618169$} \\
\hline \multirow[t]{5}{*}{$\Delta ı \alpha \mu \varepsilon ́ p ı \sigma \eta 2$} & .7542 & 161 & 639 & 0,163597 & 0,442466 & $-0,994960$ \\
\hline & $7543-9453$ & 92 & 710 & 0,181760 & 0,253425 & $-0,332380$ \\
\hline & $9454-18486$ & 107 & 2029 & 0,519186 & 0,294521 & 0,566914 \\
\hline & $18487+$ & 4 & 530 & 0,135712 & 0,012329 & 2,398603 \\
\hline & $\sum u ́ v o \lambda o$ & 364 & 3908 & & & \\
\hline \multicolumn{7}{|c|}{$I V\left(X_{i}\right)=0,724598 \mathrm{k \alpha I} H\left(X_{i}\right)=1,615021$} \\
\hline \multirow[t]{5}{*}{ 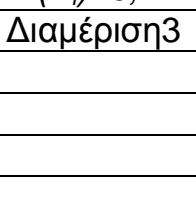 } & $\ldots-7508$ & 166 & 636 & 0,162829 & 0,456164 & $-1,030150$ \\
\hline & $7509-9430$ & 90 & 708 & 0,181248 & 0,247945 & $-0,313340$ \\
\hline & $9431-18414$ & 102 & 2036 & 0,520977 & 0,280822 & 0,617986 \\
\hline & $18415+$ & 6 & 528 & 0,135201 & 0,017808 & 2,027101 \\
\hline & Eúvoגo & 364 & 3908 & & & \\
\hline \multicolumn{7}{|c|}{$I V\left(X_{i}\right)=0,709457 \kappa \alpha I H\left(X_{i}\right)=1,612564$} \\
\hline \multirow[t]{5}{*}{$\Delta ı \alpha \mu \dot{p ı \sigma \eta 4}$} &.-7550 & 167 & 634 & 0,162318 & 0,458904 & $-1,039290$ \\
\hline & $7551-9474$ & 89 & 712 & 0,182272 & 0,245205 & $-0,296600$ \\
\hline & $9475-18532$ & 102 & 2034 & 0,520466 & 0,280822 & 0,617003 \\
\hline & $18533+$ & 6 & 528 & 0,135201 & 0,017808 & 2,027101 \\
\hline & ¿úvoגo & 364 & 3908 & & & \\
\hline \multicolumn{7}{|c|}{$I V\left(X_{i}\right)=0,712732 \kappa \alpha ı H\left(X_{i}\right)=1,613127$} \\
\hline \multirow[t]{5}{*}{ 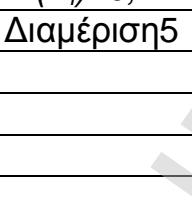 } & .7579 & 166 & 636 & 0,162829 & 0,456164 & $-1,030150$ \\
\hline & $7580-9464$ & 91 & 709 & 0,181504 & 0,250685 & $-0,322920$ \\
\hline & $9465-18748$ & 105 & 2031 & 0,519698 & 0,289041 & 0,586679 \\
\hline & $18749+$ & 2 & 532 & 0,136224 & 0,006849 & 2,990153 \\
\hline & $\Sigma u ́ v o \lambda o$ & 364 & 3908 & & & \\
\hline
\end{tabular}

${ }^{4} \wedge$

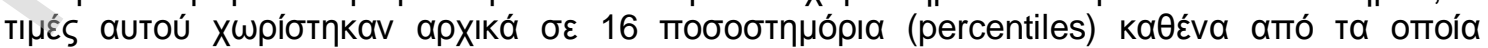

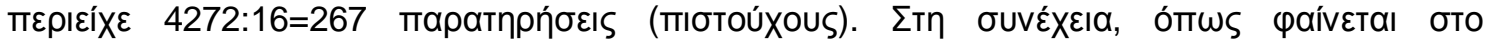

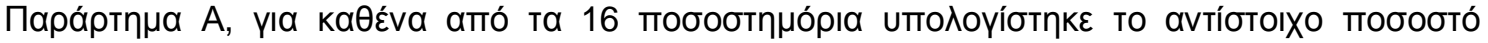

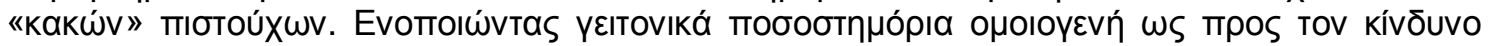

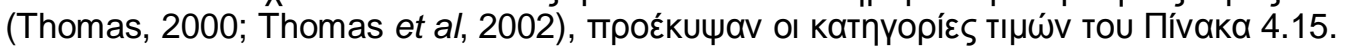




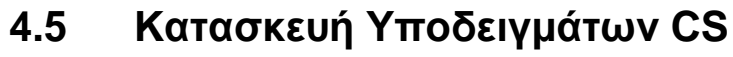

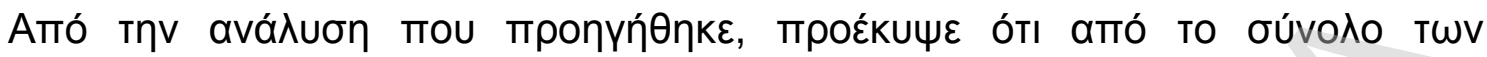

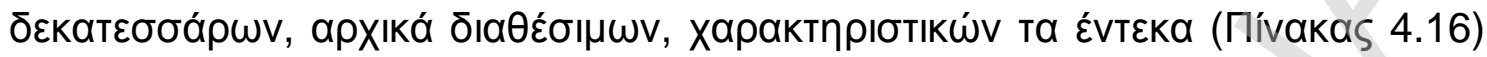

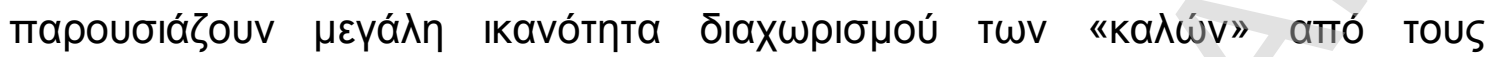

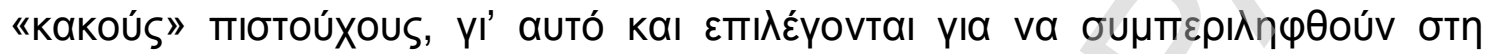

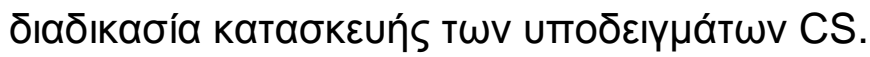

ПINAKA 4.16

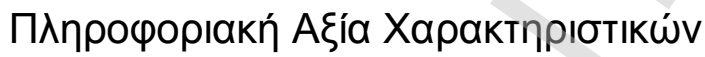

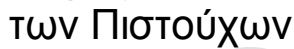

\begin{tabular}{|c|c|}
\hline Характпрıбтіко́ & 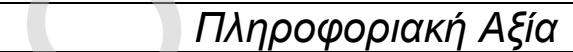 \\
\hline 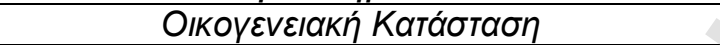 & $\mu \varepsilon T \alpha \xi u ́$ 1,35 кaı 1,43(*) \\
\hline 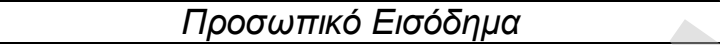 & 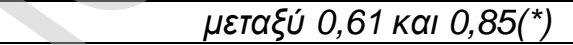 \\
\hline 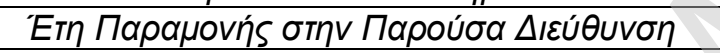 & $\mu \varepsilon T \alpha \xi u ́ ~ 0,50$ каı 0,60( $\left.{ }^{*}\right)$ \\
\hline Hגıкía & 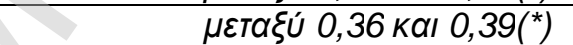 \\
\hline Катохи́ АUтокіvи́тоU & 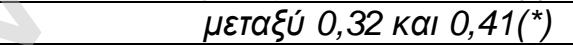 \\
\hline Túmos Katolkías & 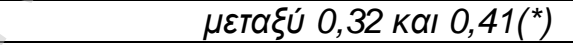 \\
\hline 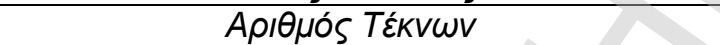 & 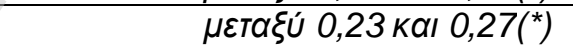 \\
\hline Kaтохи́ Пıбтштікńs Káptas & $\mu \varepsilon T \alpha \xi u ́ ~ 0,17$ каı $0,25\left(^{*}\right)$ \\
\hline 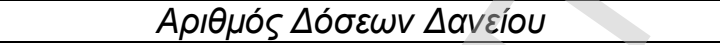 & 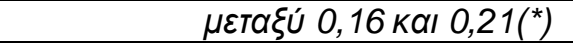 \\
\hline Eпর́์ $\gamma \varepsilon \lambda \mu \alpha$ & 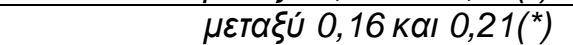 \\
\hline 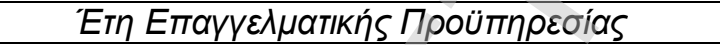 & 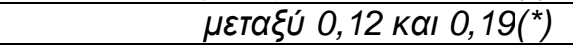 \\
\hline 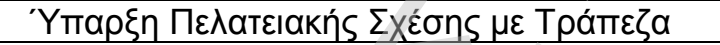 & $\mu \varepsilon т \alpha \xi u ́ ~ 0,05$ каı 0,08 \\
\hline Фúlo & $\mu \varepsilon т \alpha \xi u ́ ~ 0,04$ каı 0,06 \\
\hline 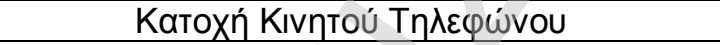 & $\mu \varepsilon т \alpha \xi u ́$ 0,02 каı 0,03 \\
\hline
\end{tabular}

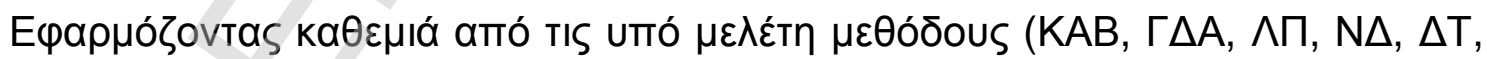

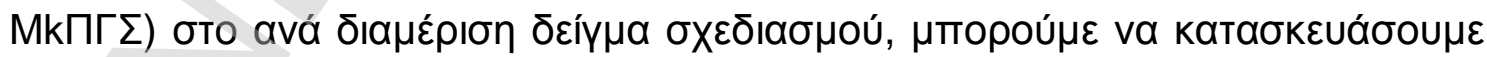

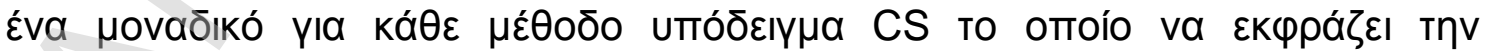

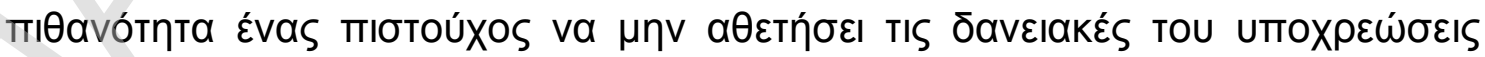

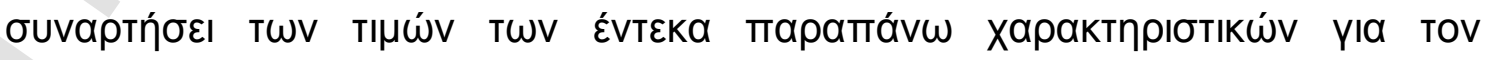

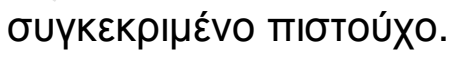




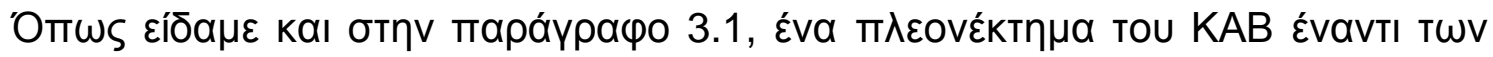

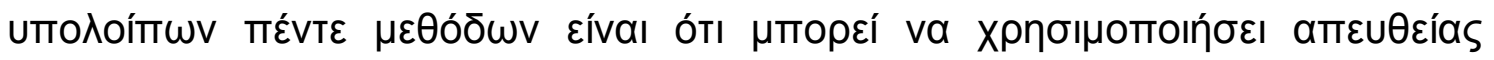

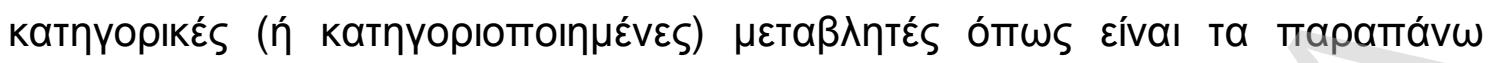

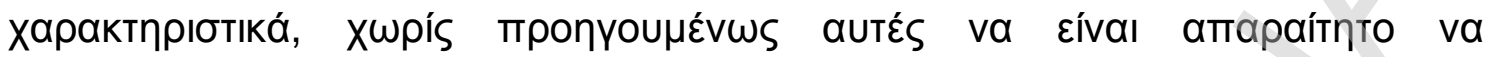

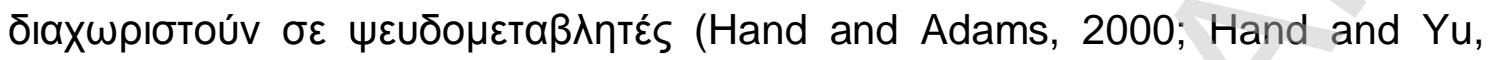
2001; Baesens et al, 2002; Antonakis and Sfakianakis, 2008; Antonakis and

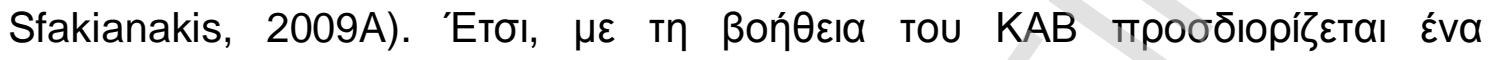

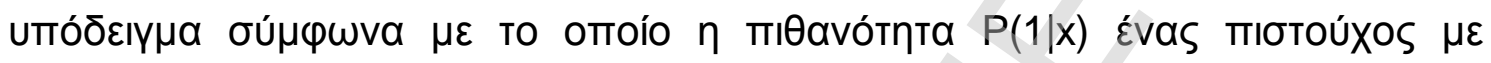

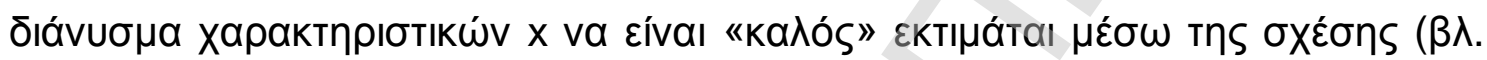

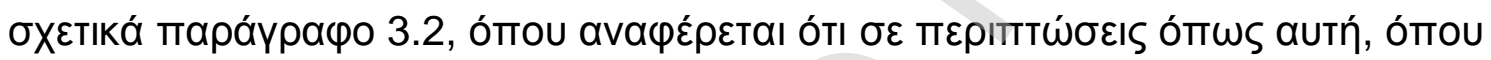

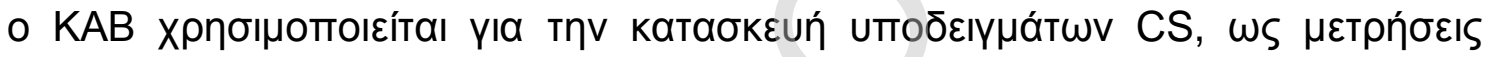

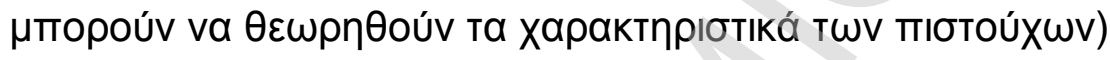

$$
\ln \frac{P(1 \mid x)}{1-P(1 \mid x)}=\Sigma w_{i}\left(x_{i}\right)+b
$$

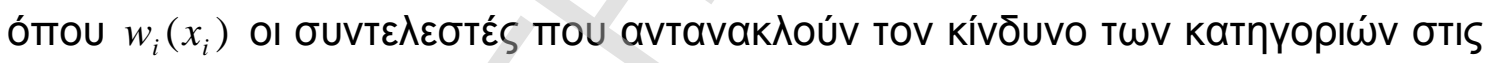

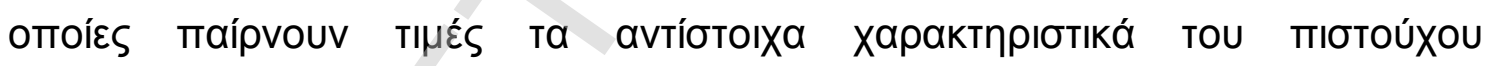

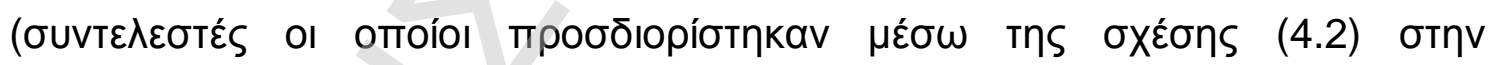

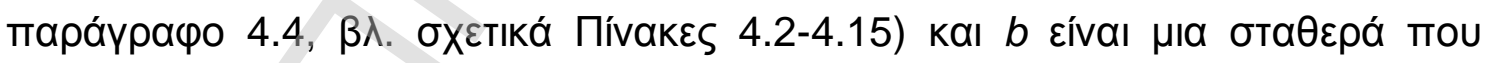

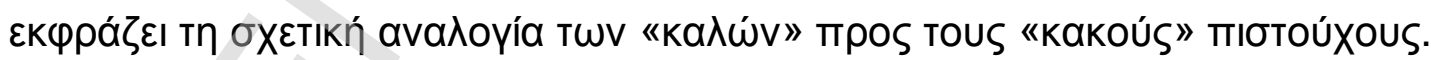

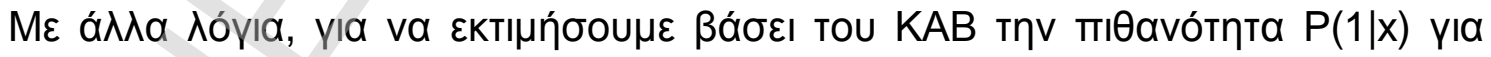

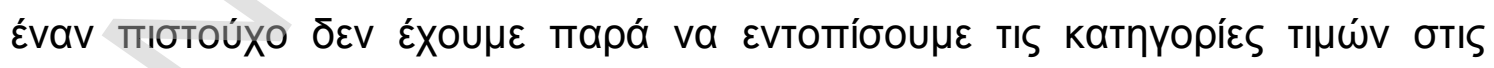

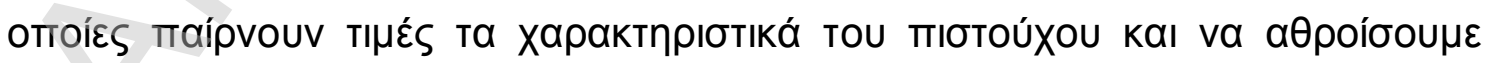

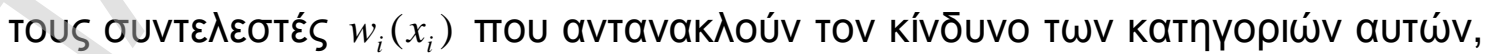

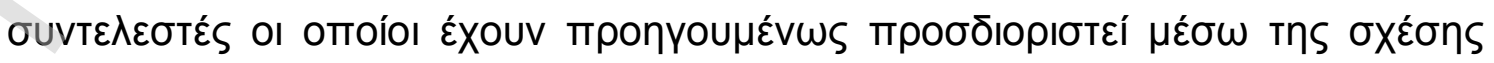




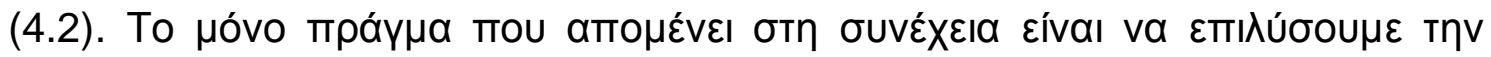

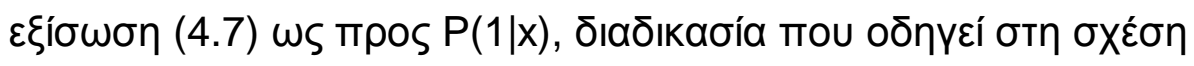

$$
P(1 \mid x)=\frac{e^{\sum w_{i}\left(x_{i}\right)+b}}{1+e^{\sum w_{i}\left(x_{i}\right)+b}}
$$

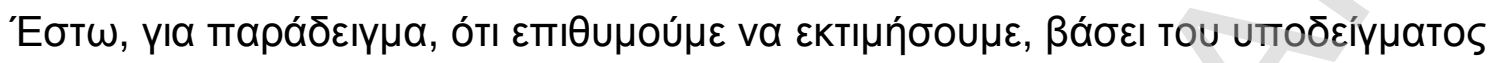

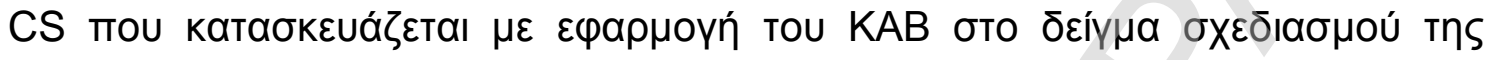

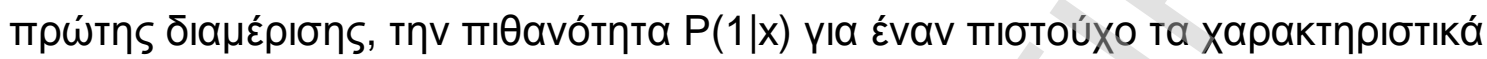

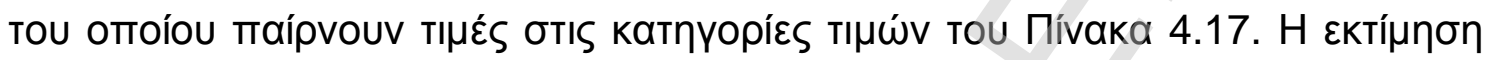

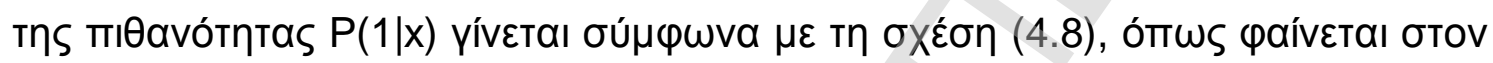
Пі́vaка 4.17.

ПINAKA $\Sigma 4.17$

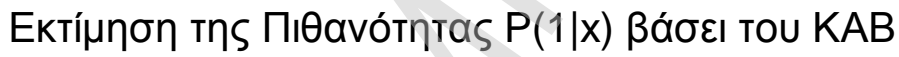

\begin{tabular}{|c|c|c|}
\hline ХарактпрІбтіко́ & 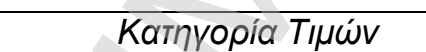 & 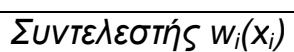 \\
\hline Катохи́ Autokivńтоu & $2^{n}(\mathrm{Na} \mathrm{I})$ & 0,502374 \\
\hline Катохи́ ПıбтштІки́s Кáртаs & $2^{n}(\mathrm{Nal})$ & 0,856348 \\
\hline 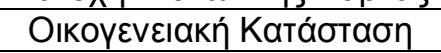 & 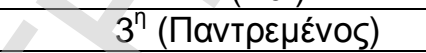 & 1,255104 \\
\hline Túmо Kaтоікі́as & $3^{n}$ (IঠıоктńтnS) & 0,499372 \\
\hline 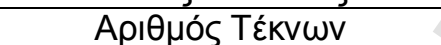 & $2^{n}(2)$ & 0,276223 \\
\hline 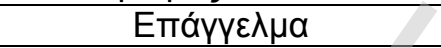 & $5^{n}(\Delta \eta \mu$. Yாá́ $\lambda \lambda \eta \lambda \circ \varsigma)$ & 0,296159 \\
\hline H入ıкía & $2^{n}(40-58)$ & 0,888340 \\
\hline 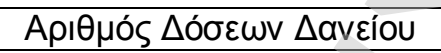 & $1^{n}(6-22)$ & 0,885417 \\
\hline 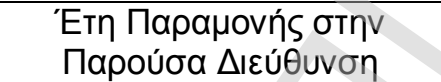 & $2^{n}(4-17)$ & 0,647338 \\
\hline 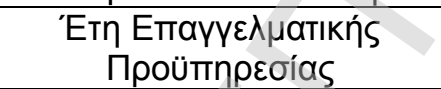 & $4^{n}(13+)$ & 0,636756 \\
\hline 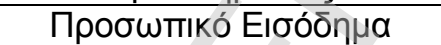 & $4^{n}(18657+)$ & 2,028991 \\
\hline \multicolumn{2}{|c|}{$\Sigma т \alpha \theta \varepsilon \rho \alpha ́$ b $=\ln (3908 / 364)$} & 2,373627 \\
\hline \multicolumn{2}{|c|}{$A \theta \rho o ı \sigma \mu \alpha \Sigma w_{i}\left(x_{i}\right)+b$} & 11,146049 \\
\hline 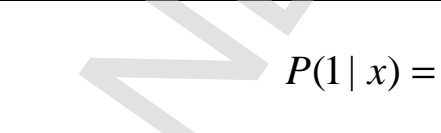 & $\frac{e^{\sum w_{i}\left(x_{i}\right)+b}}{+e^{\sum w_{i}\left(x_{i}\right)+}}$ & 0,999986 \\
\hline
\end{tabular}

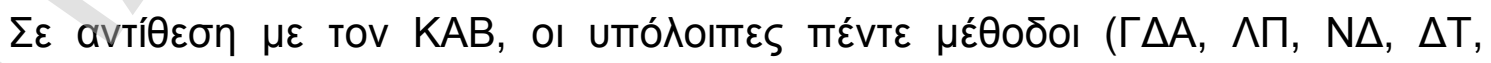

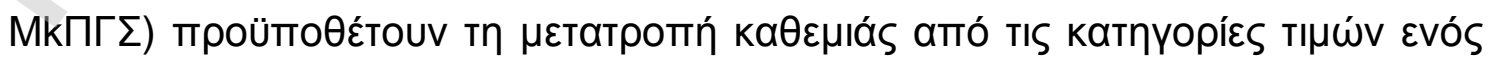

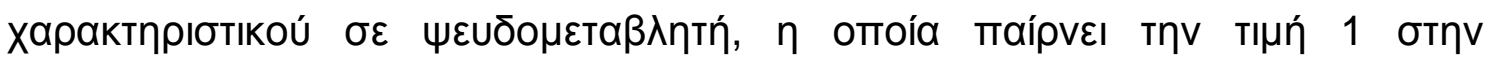




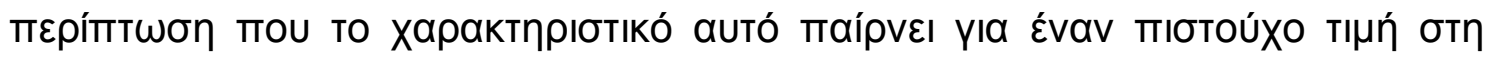

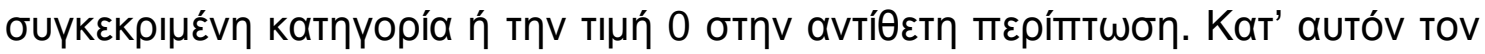

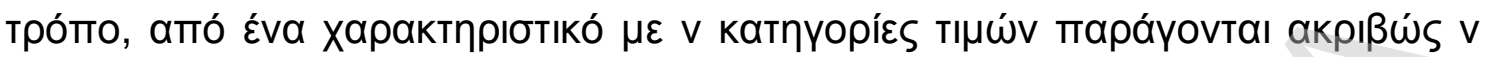

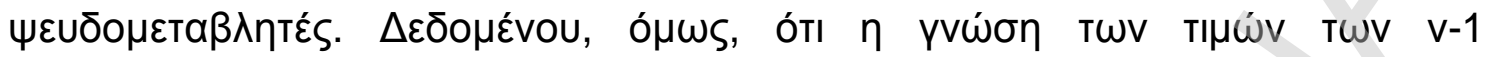

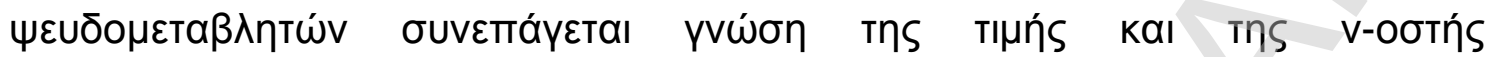

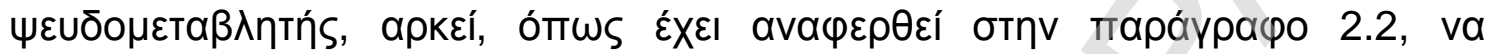

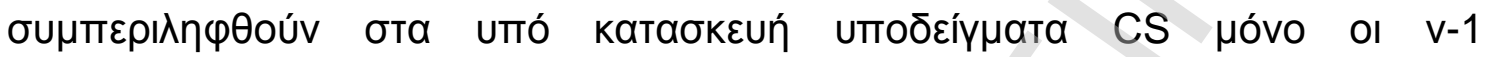

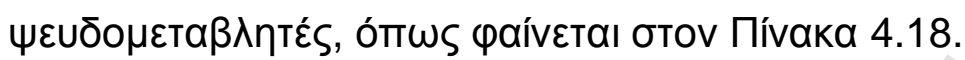

ПINAKA 4.18

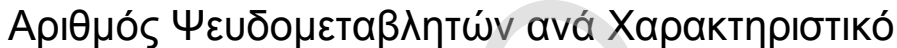

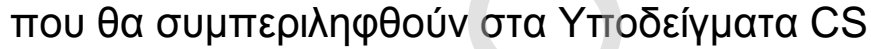

\begin{tabular}{|c|c|c|}
\hline ХарактпрІбтіко́ & 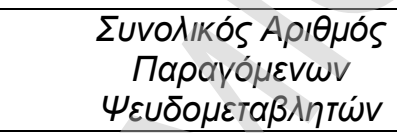 & 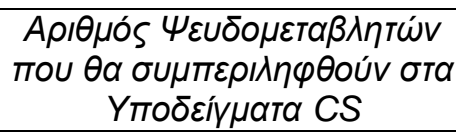 \\
\hline Катохи́ AutoKıvńтоu & 2 & $2-1=1$ \\
\hline Катохи́ Пıбтштікńs Káртаs & 2 & $2-1=1$ \\
\hline 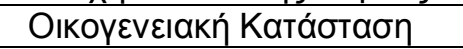 & 3 & $3-1=2$ \\
\hline Túmоs Katoıкі́as & $-{ }_{-1}$ & $3-1=2$ \\
\hline 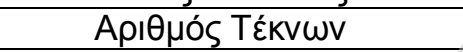 & + & $4-1=3$ \\
\hline 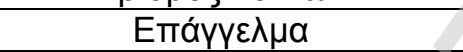 & 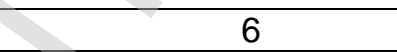 & $6-1=5$ \\
\hline H入ıќ́a & 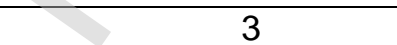 & $3-1=2$ \\
\hline 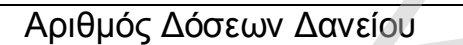 & 3 & $3-1=2$ \\
\hline 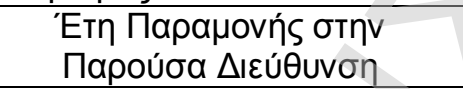 & $\overline{3}$ & $4-1=3$ \\
\hline 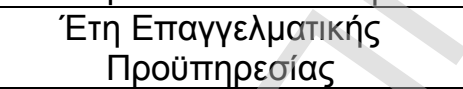 & 4 & $4-1=3$ \\
\hline 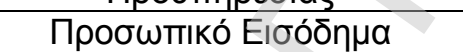 & 4 & $4-1=3$ \\
\hline ¿ÚVO\O & 38 & 27 \\
\hline
\end{tabular}

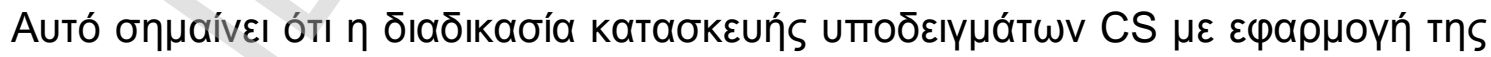

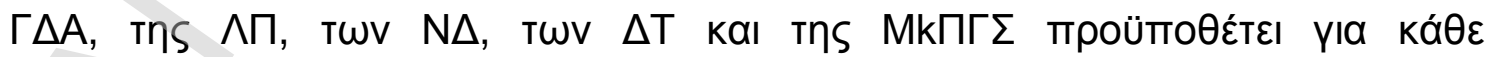

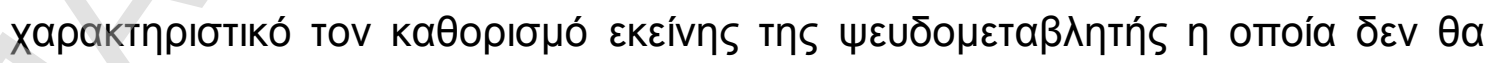

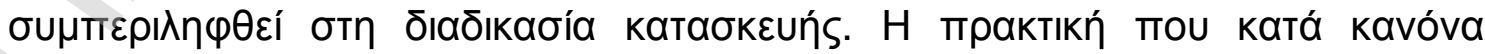

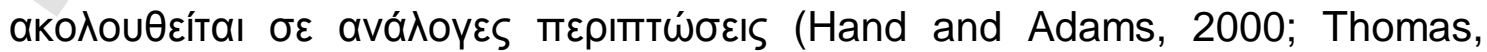
2000; Baesens et al, 2002; Antonakis and Sfakianakis, 2008; Antonakis and 


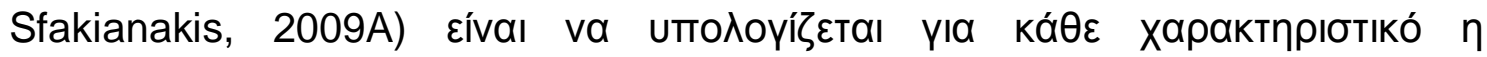

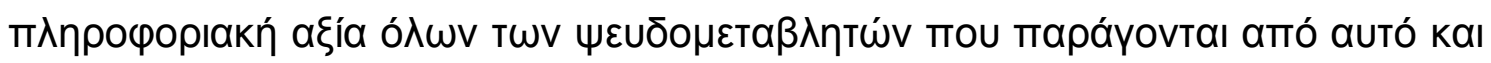

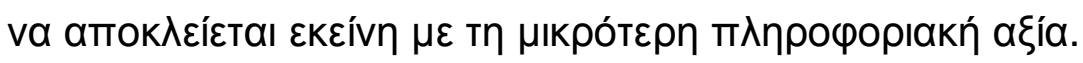

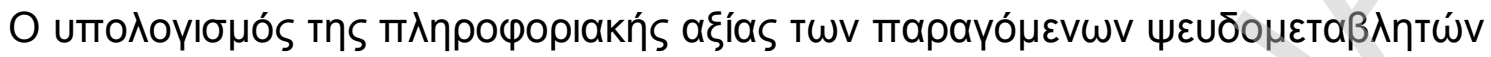

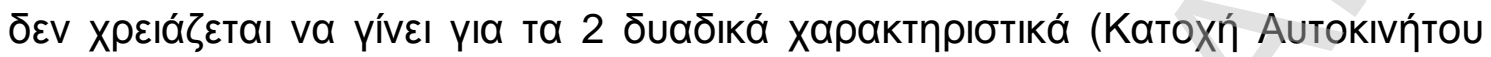

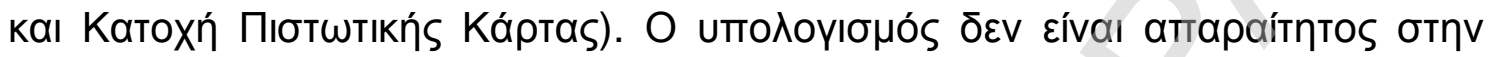

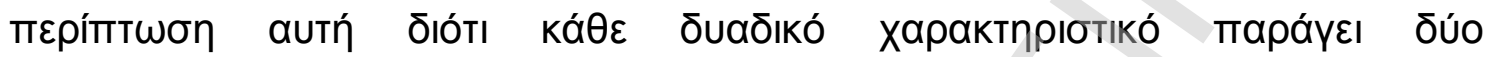

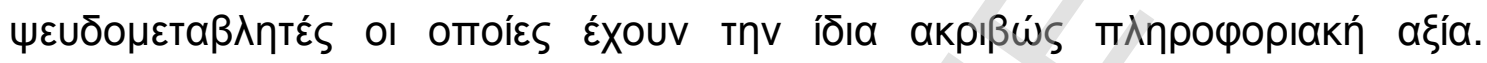

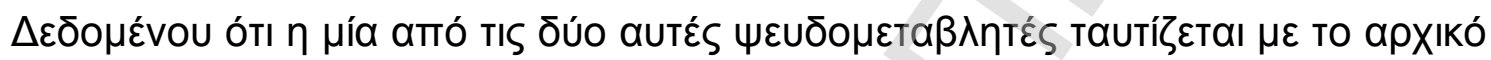

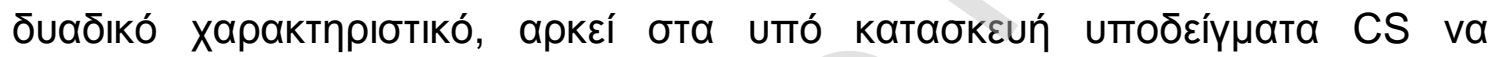

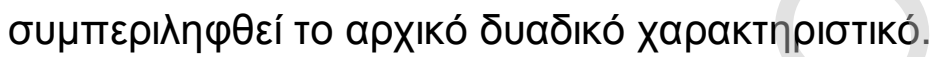

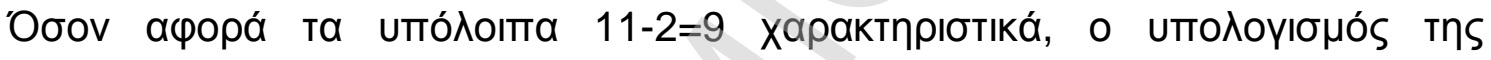

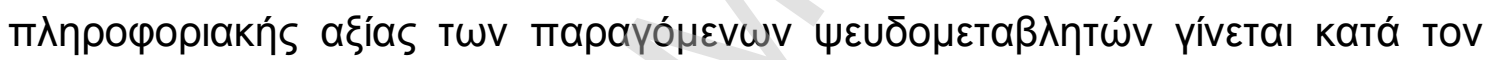

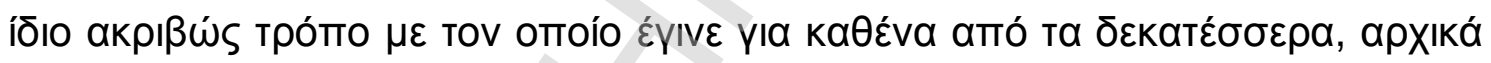

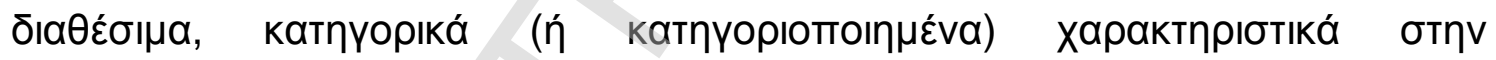

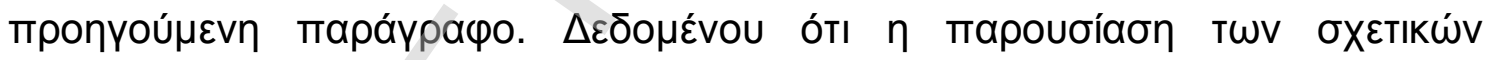

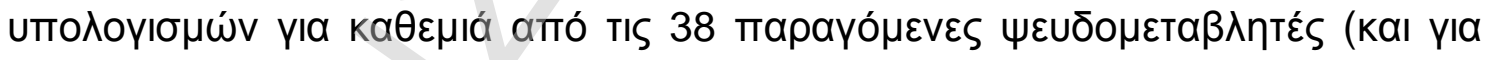

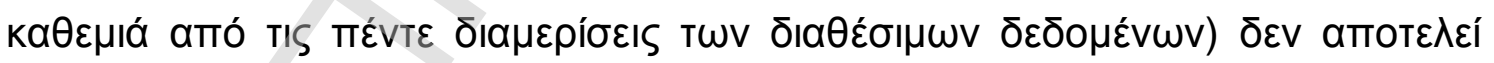

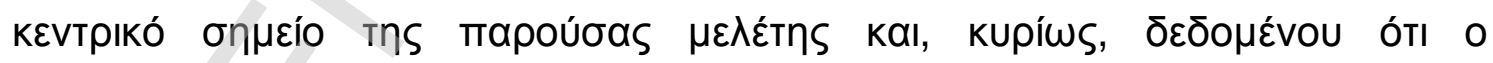

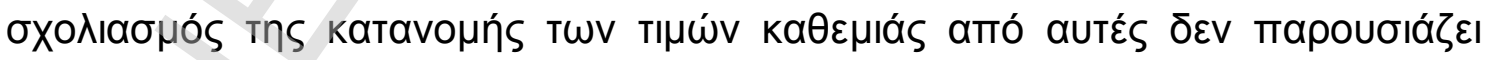

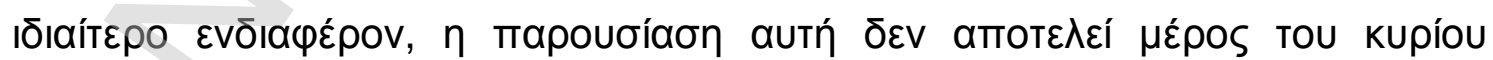

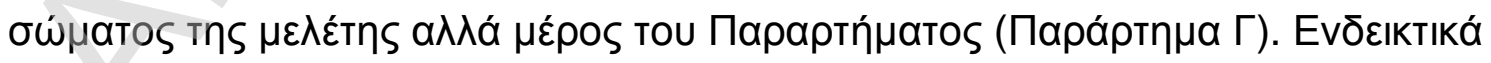

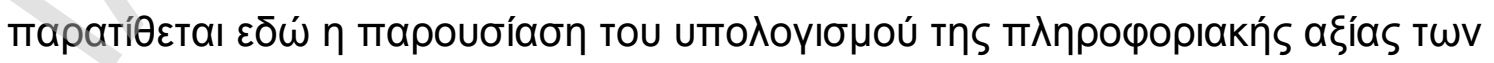

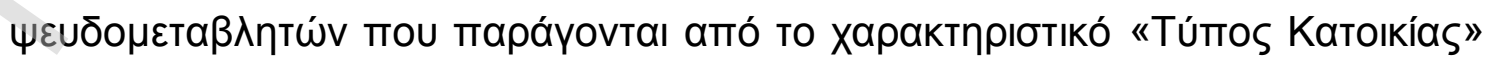

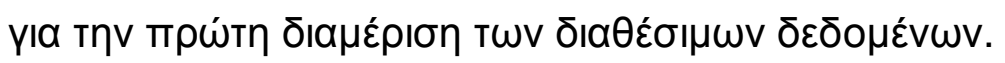


ПINAKA 4.19

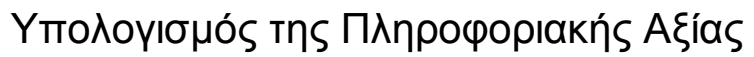

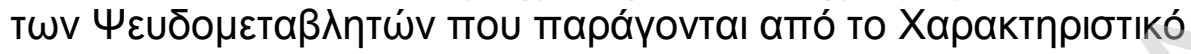
«Túmos Katoıí́as"

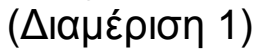

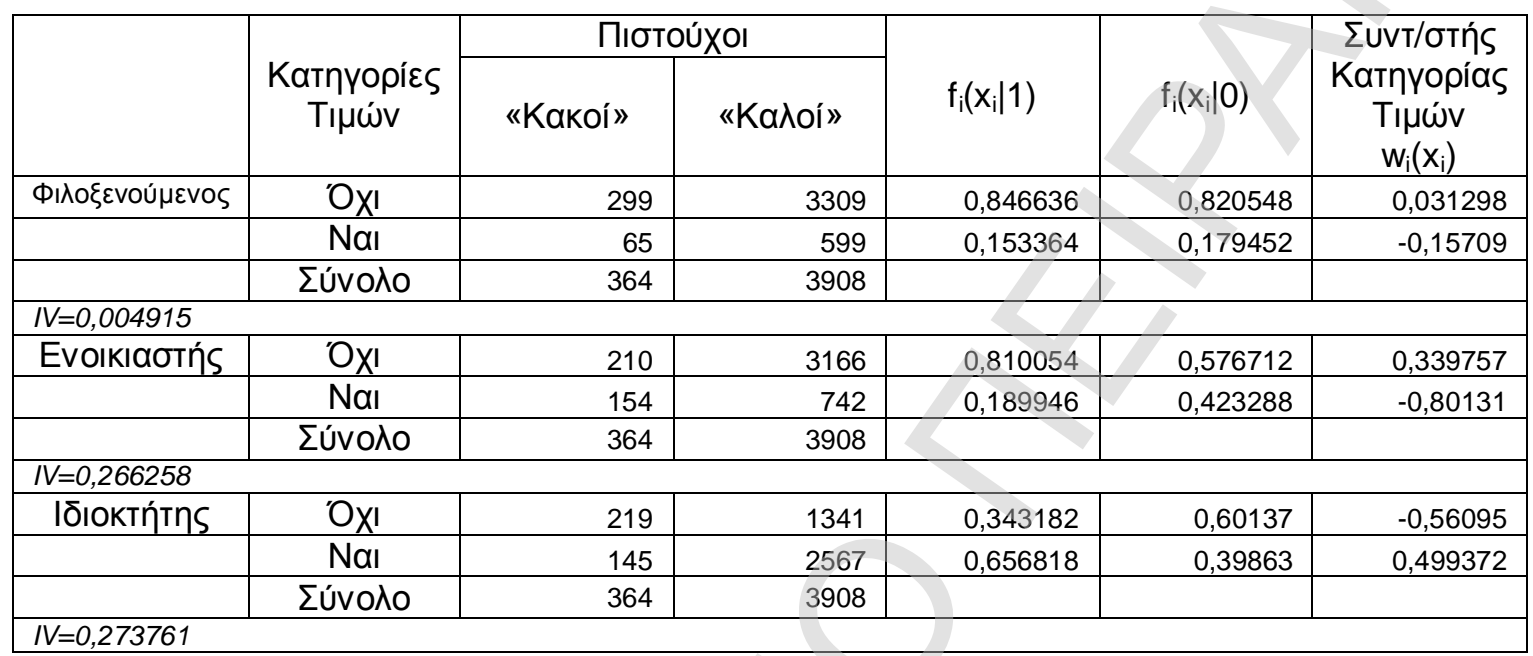

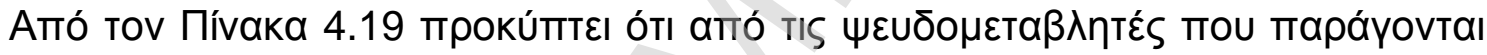

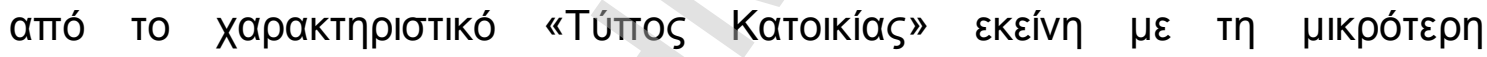

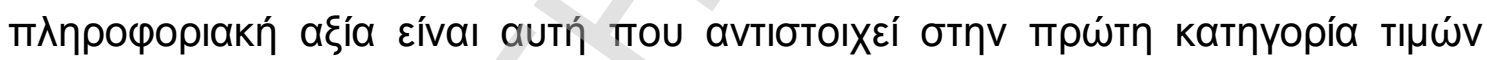

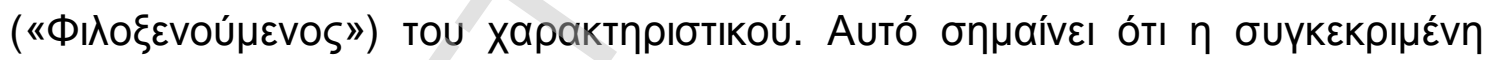

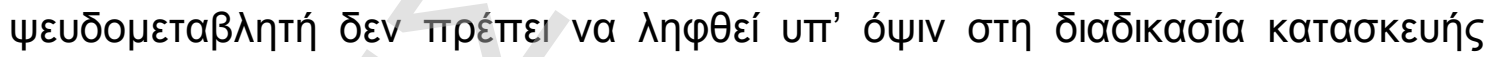

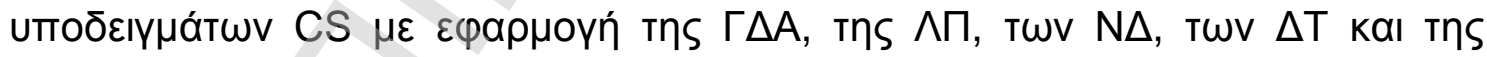

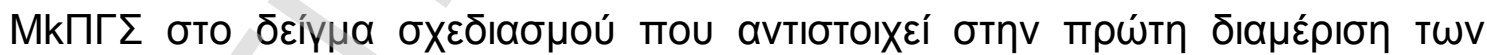

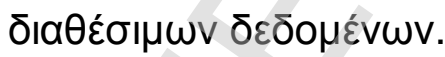

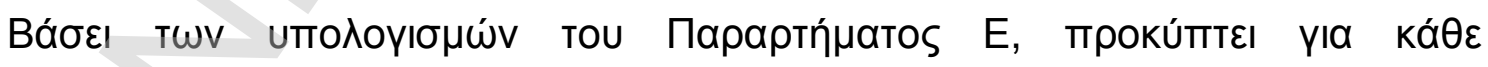

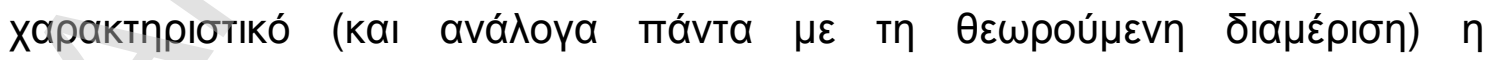

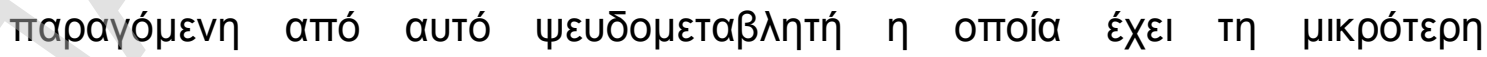

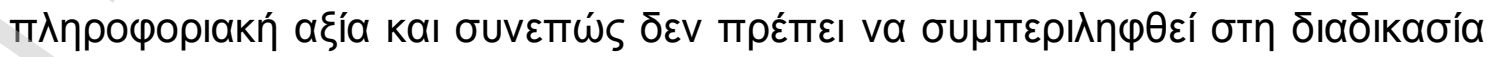

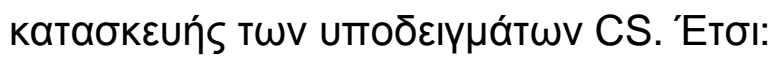




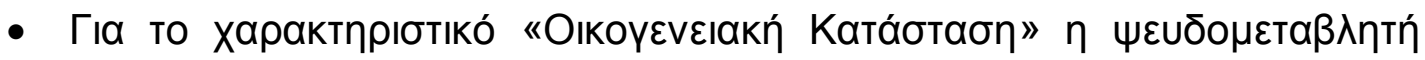

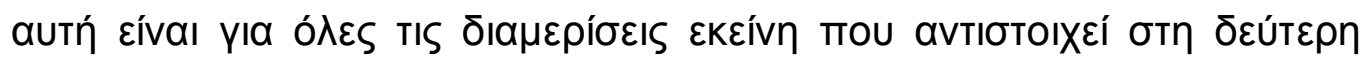

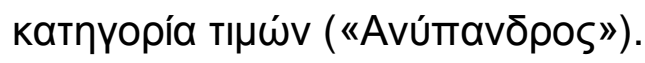

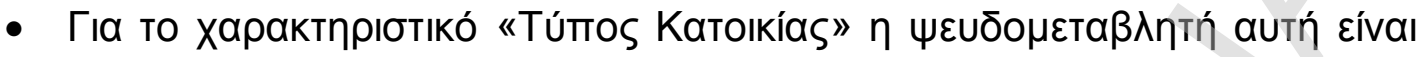

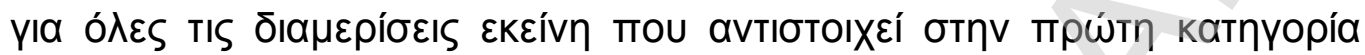

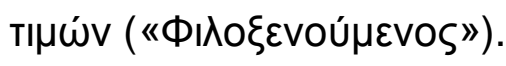

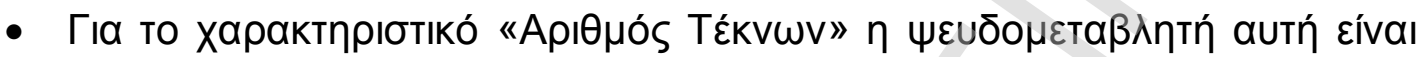

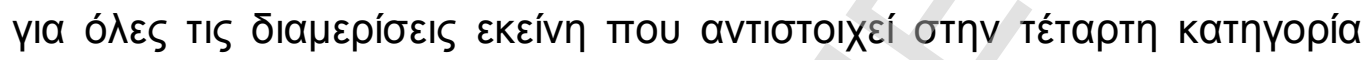

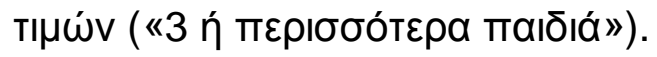

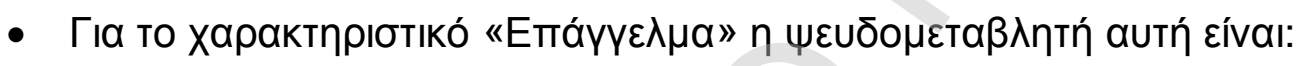

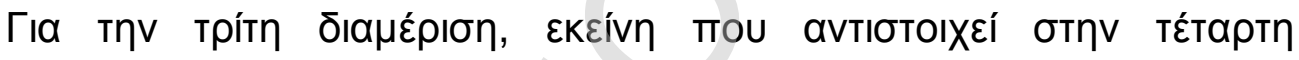

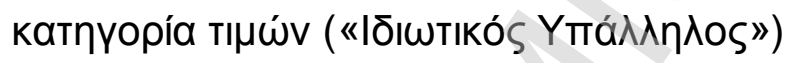

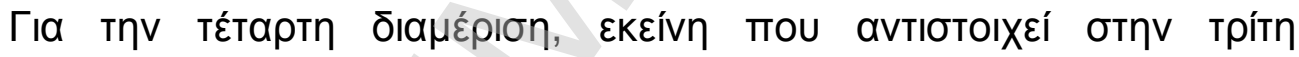

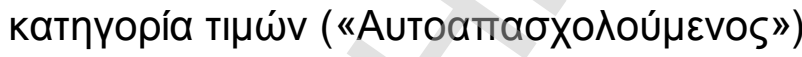

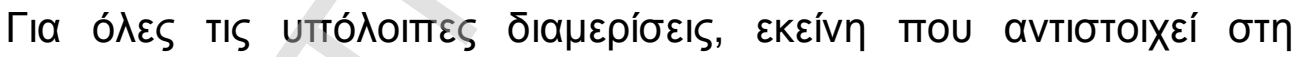

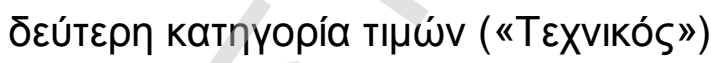

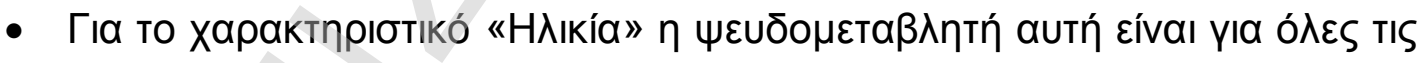

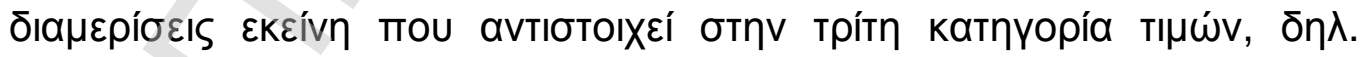

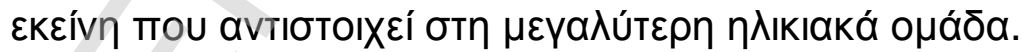

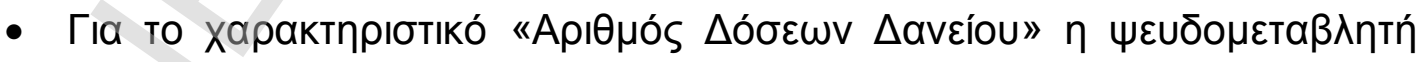
autń દívaı:

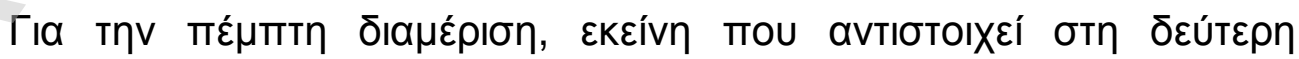

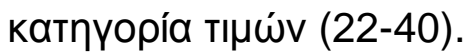




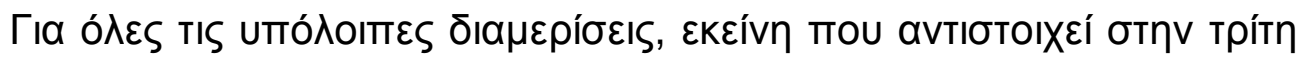

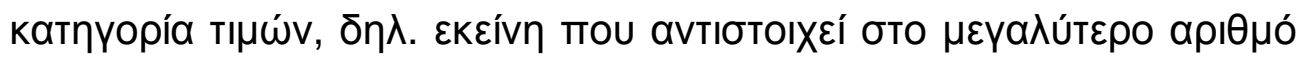

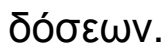

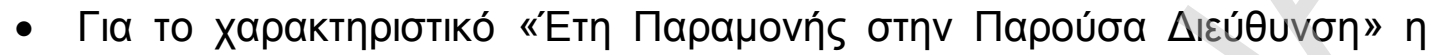

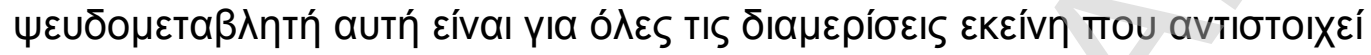

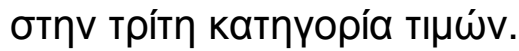

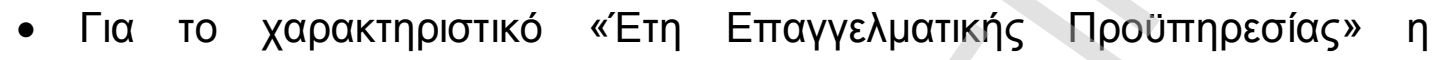

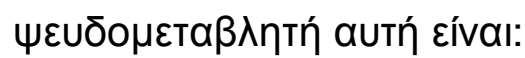

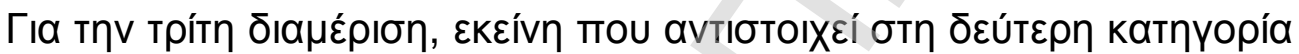
Tıน́́v.

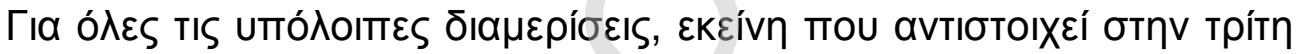

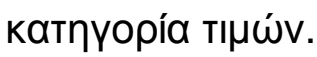

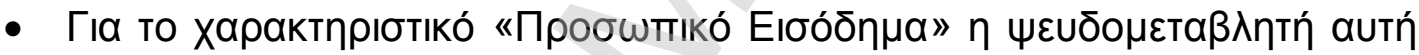

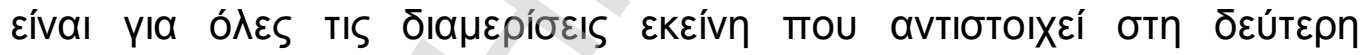

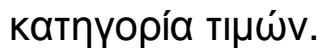

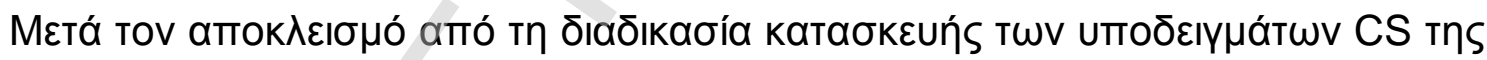

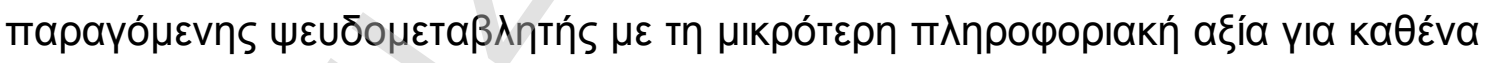

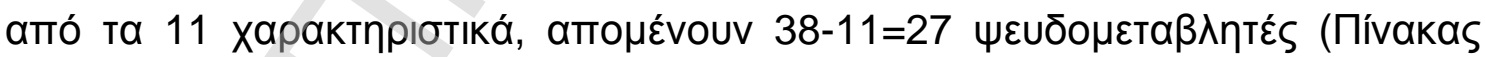

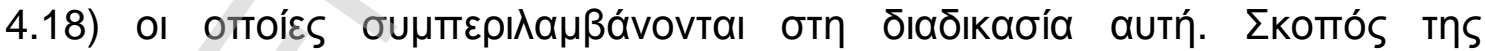

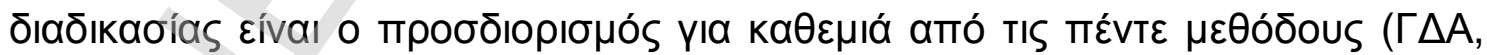

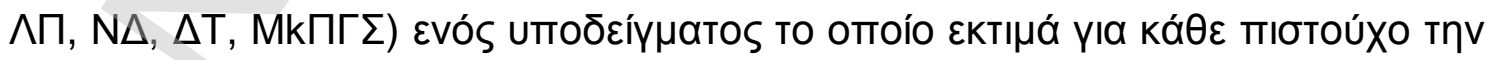

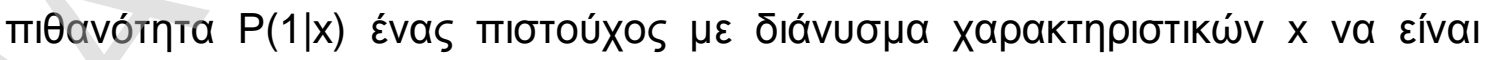

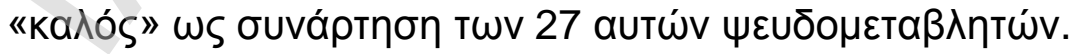

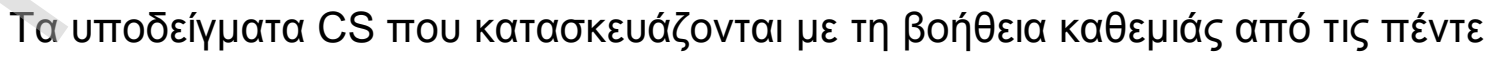

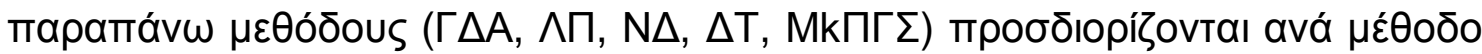




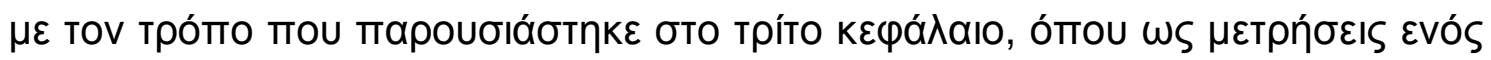

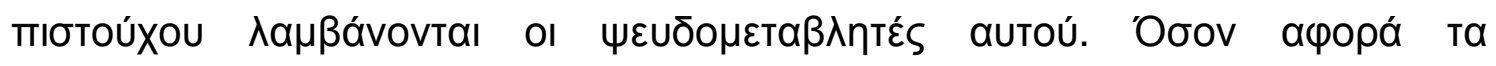

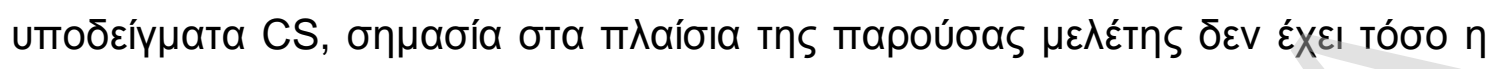

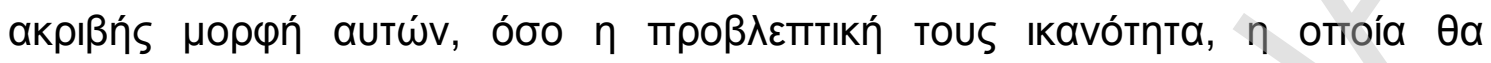

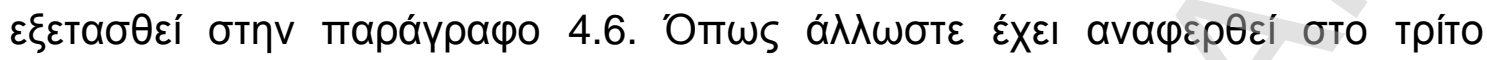

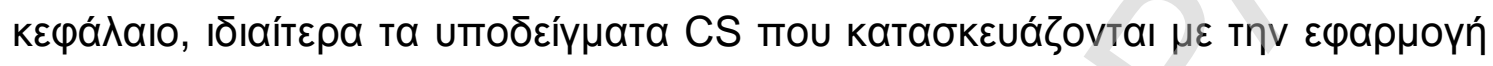

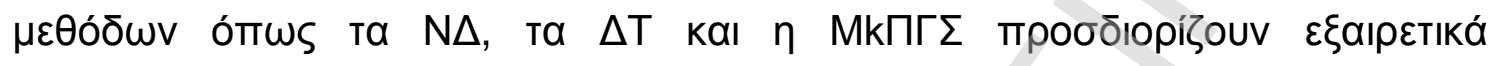

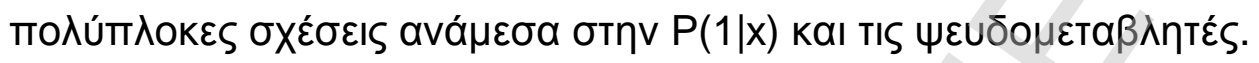

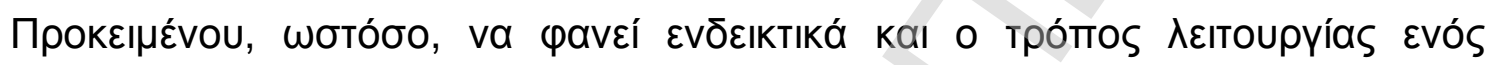

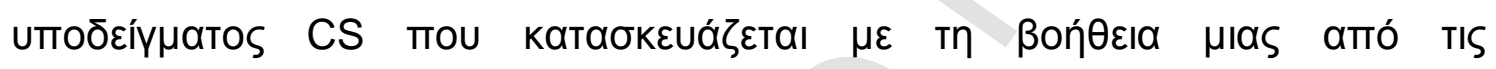

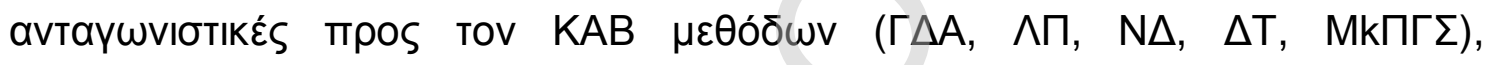

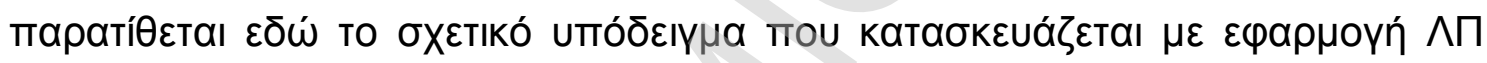

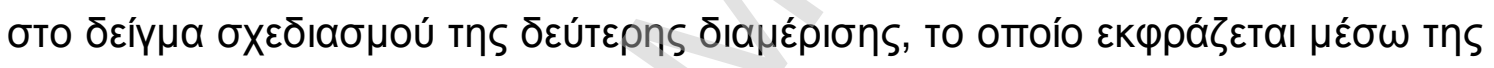

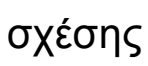

$$
\begin{aligned}
& \ln \frac{P(1 \mid x)}{1-P(1 \mid x)}=-2,4376359(J O B 1)-0,40653166(J O B 3)+0,66156745(J O B 4)- \\
& -0,00960249(J O B 5)+0,12935023(J O B 6)-0,4808135(\text { HOME2 })+ \\
& +0,35022455(\text { HOME3) }-1,76735914(\text { MAR1 })+2,29769301(\text { MAR3 })+ \\
& +0,90539736(C A R)+1,57383215(K I D 1)+2,23818636(K I D 2)+ \\
& +0,612418(K I D 3)-0,83994561(I N C 1)+0,93871403(I N C 3)+ \\
& +2,41247201(I N C 4)+1,240695(C R D)-1,18997824(A G E 1)+ \\
& +0,50103116(A G E 2)+0,25477916(J Y R 1)+0,11969619(J Y R 2)- \\
& -0,19925466(J Y R 4)-1,50437856(H Y R 1)+0,06509197(H Y R 2)+ \\
& +1,76217389(\text { HYR } 4)+2,74322462(\text { TRM } 1)+0,66159451(\text { TRM } 2)
\end{aligned}
$$




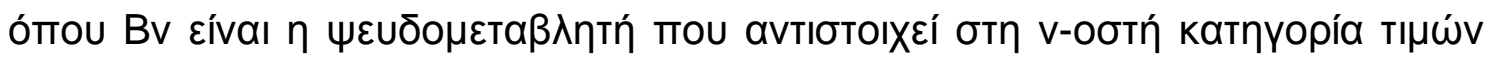

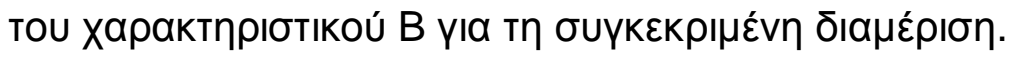

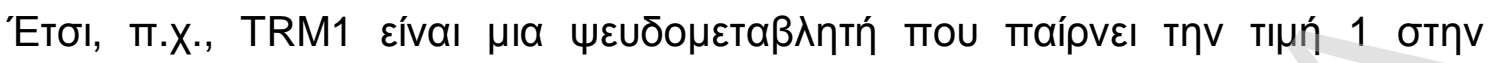

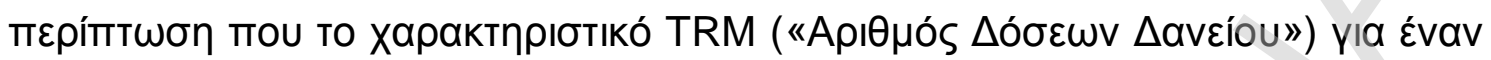

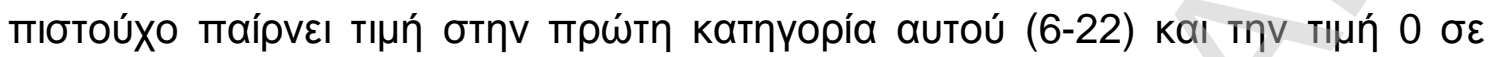

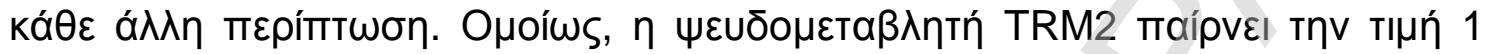

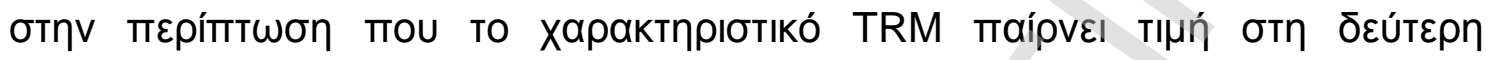

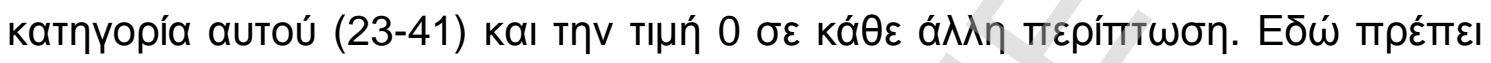

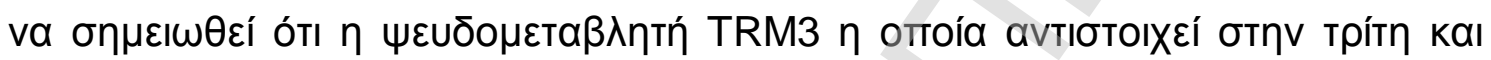

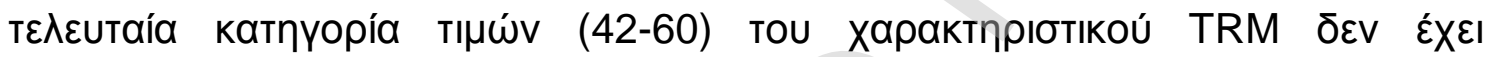

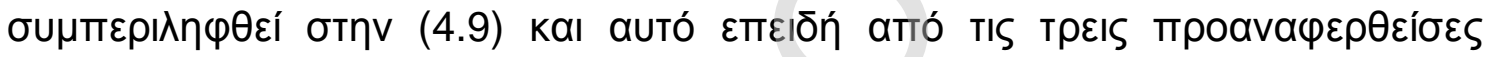

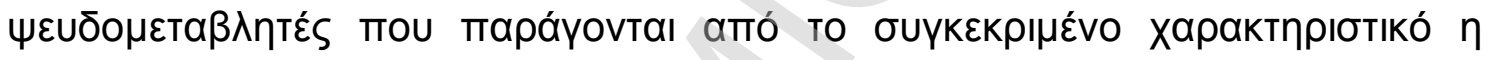

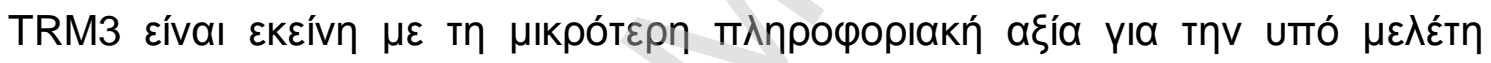

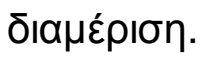

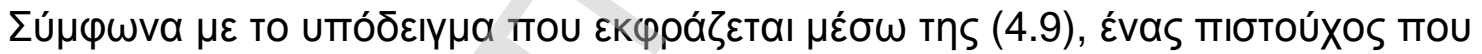

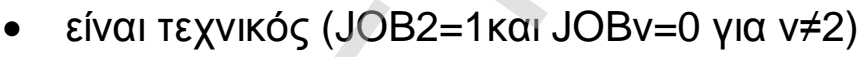

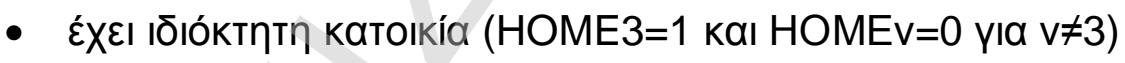

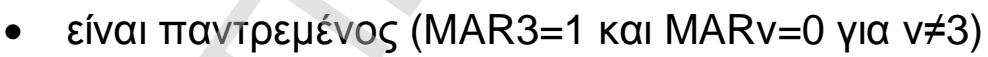

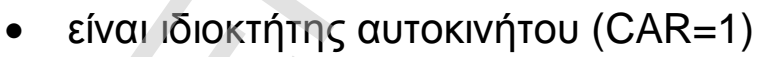

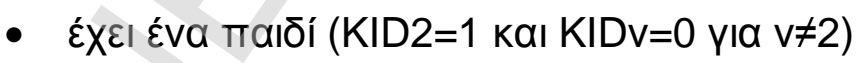

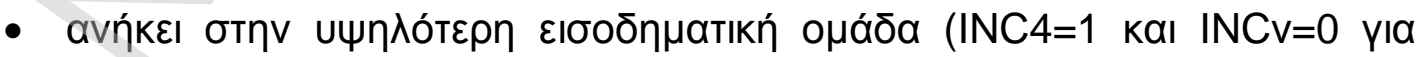
$v \neq 4)$

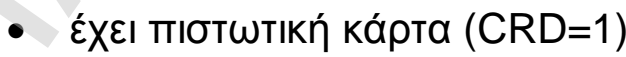

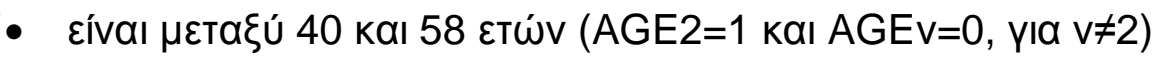




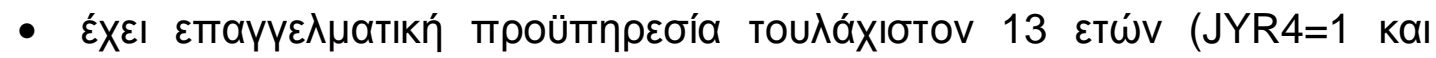
$J Y R v=0$ YIa $\vee \neq 4$ )

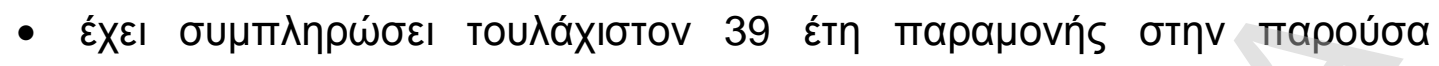

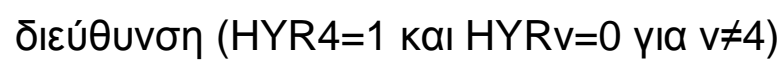

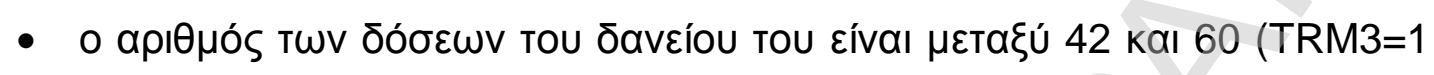
$\kappa \alpha I T R M v=0$ үı $v \neq 3$ )

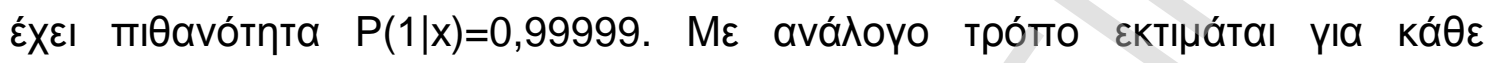

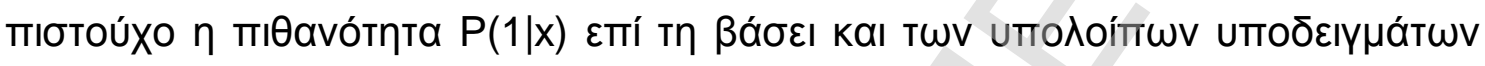

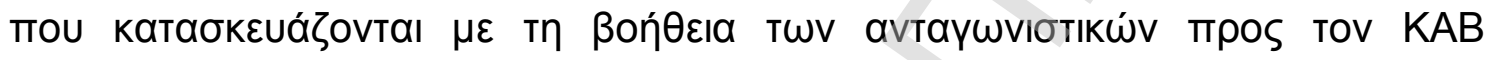
$\mu \varepsilon \theta 0 ́ \delta \omega v$.

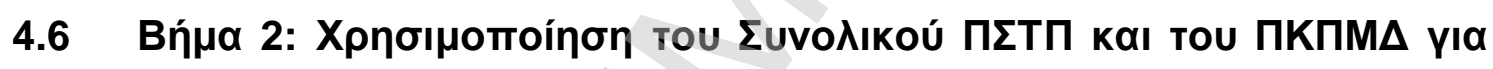

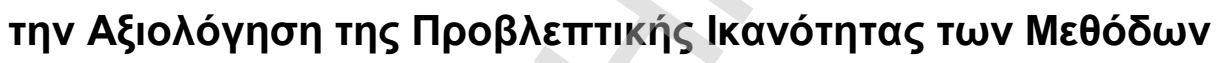

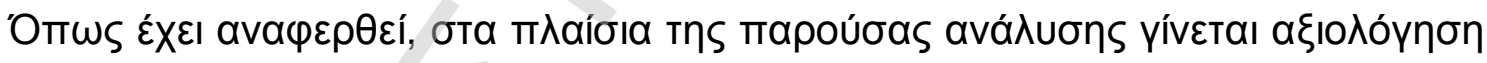

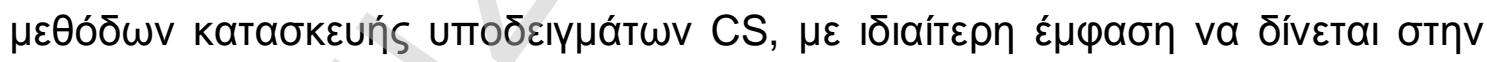

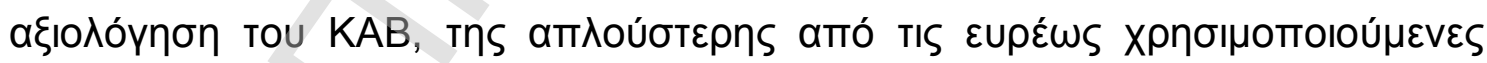

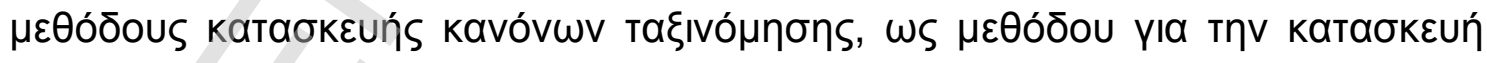

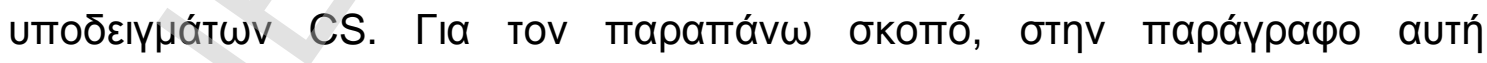

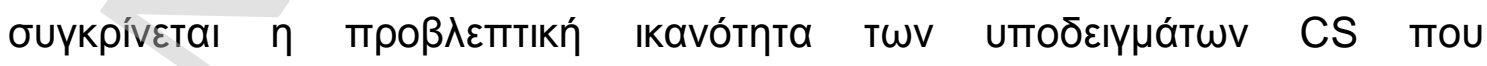

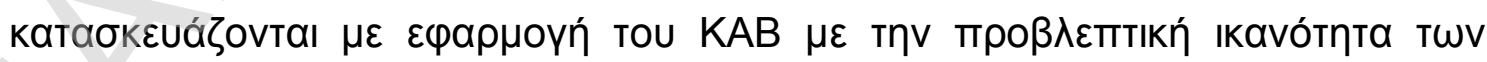

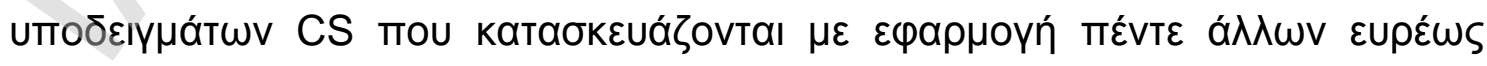

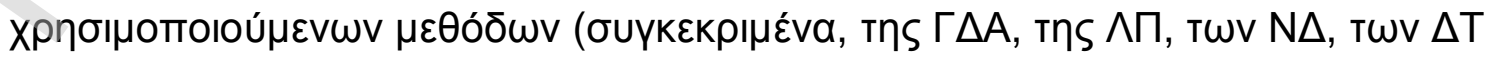

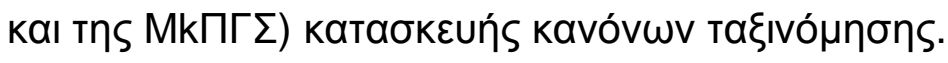




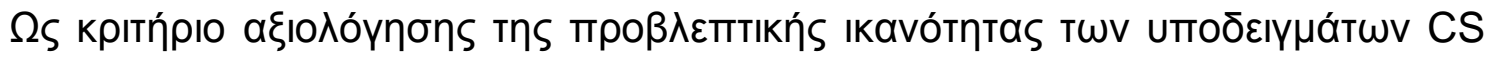

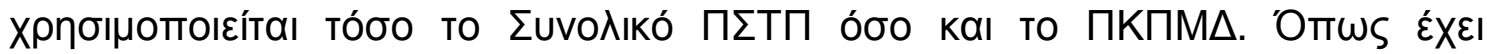

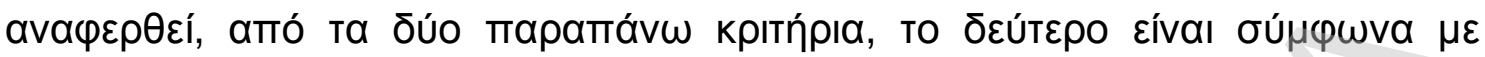

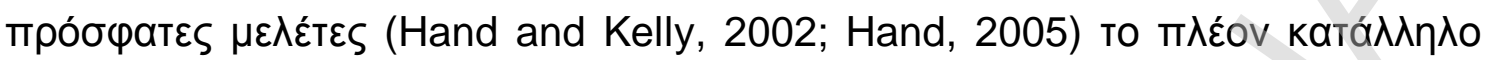

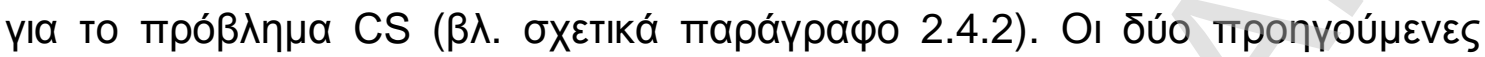

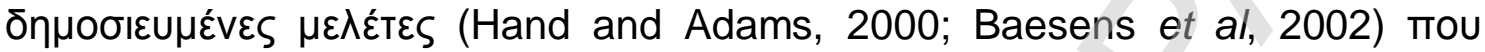

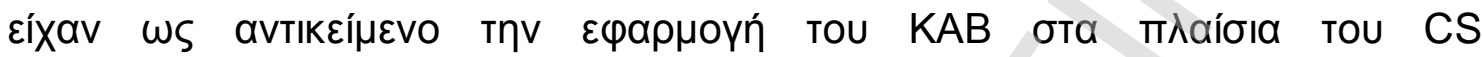

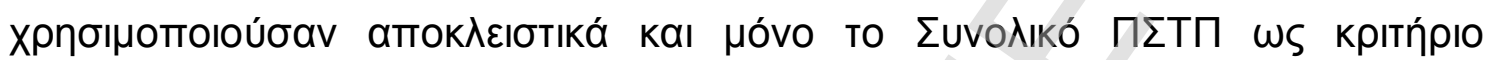

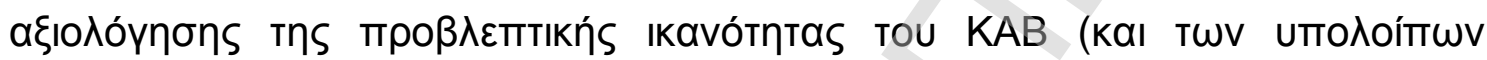

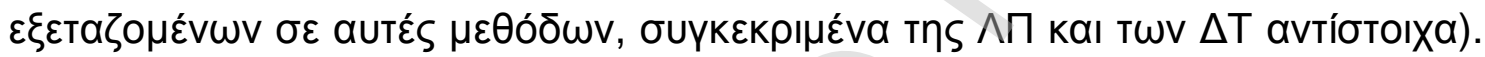

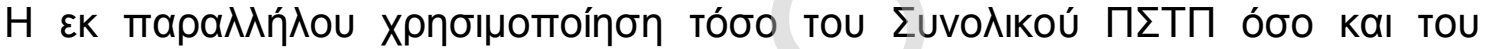

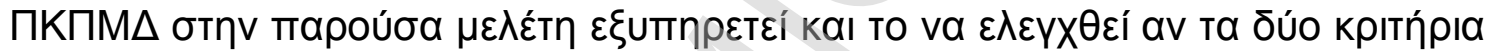

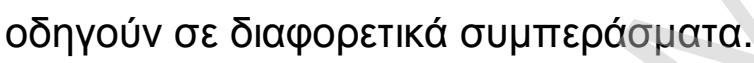

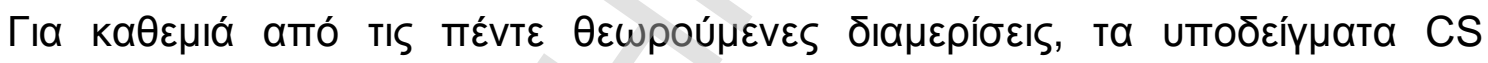

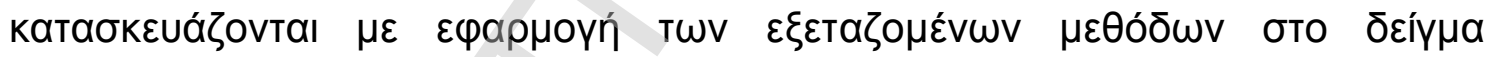

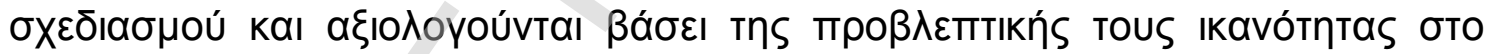

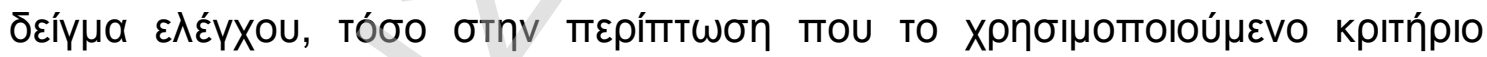

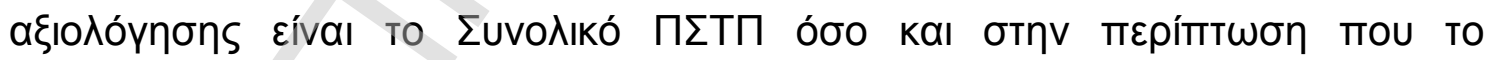

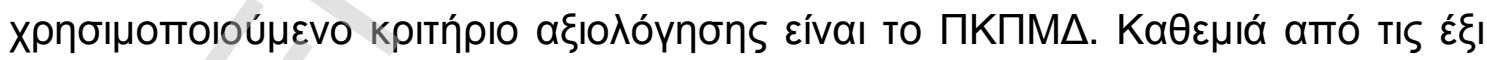

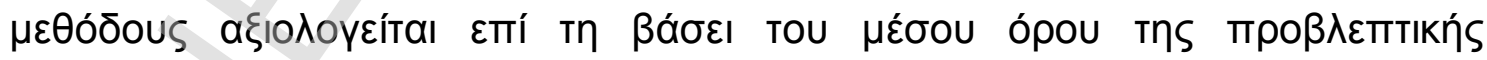

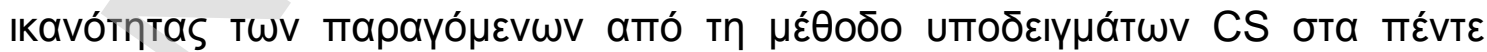

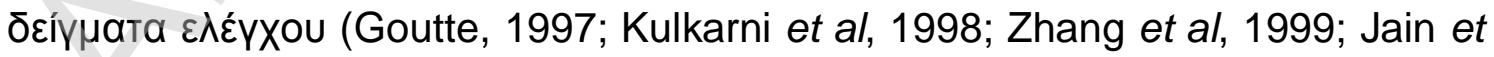
al, 2000).

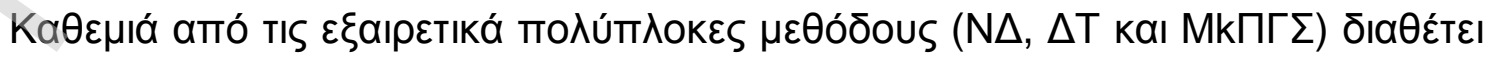

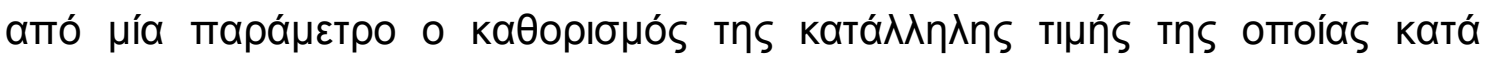




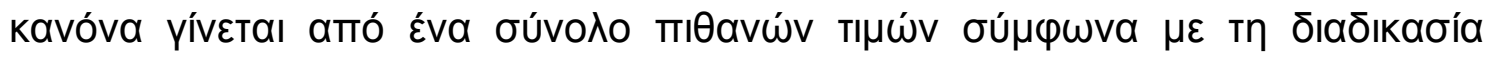

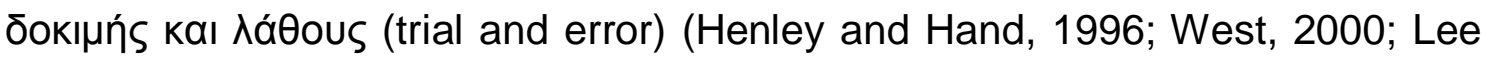

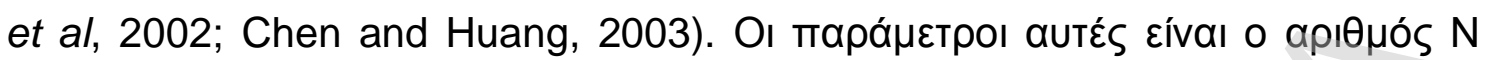

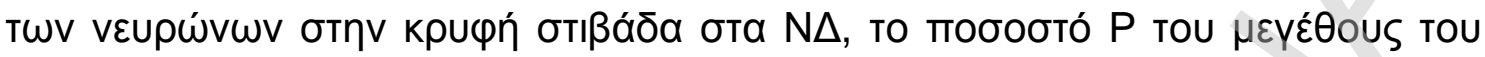

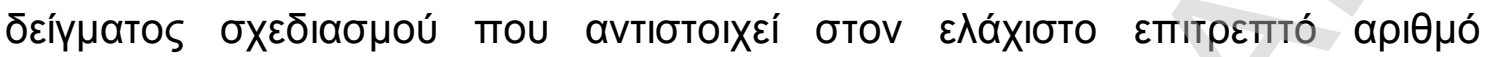

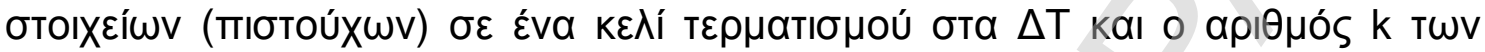

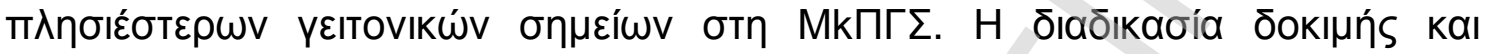

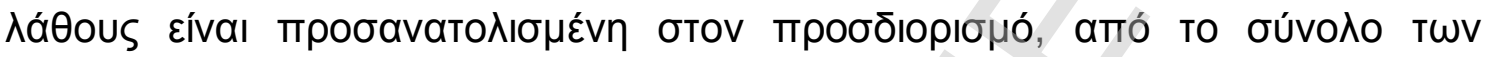

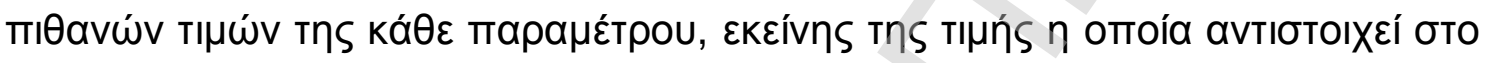

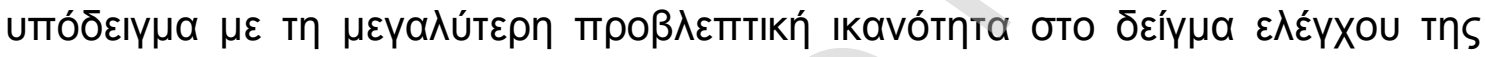

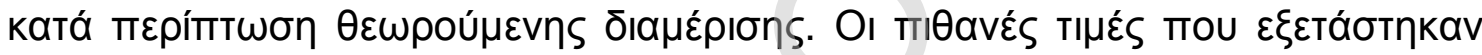

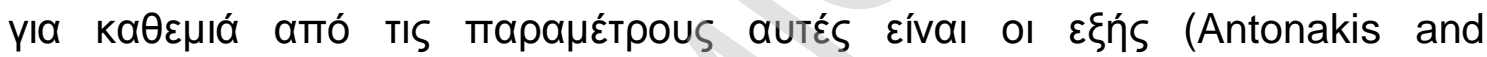
Sfakianakis, 2008; Antonakis and Sfakianakis, 2009A):

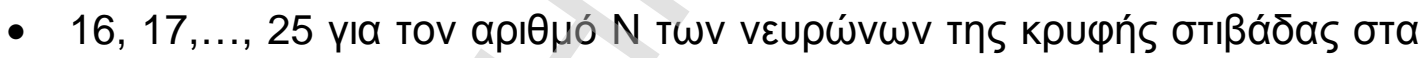
$\mathrm{N} \Delta$

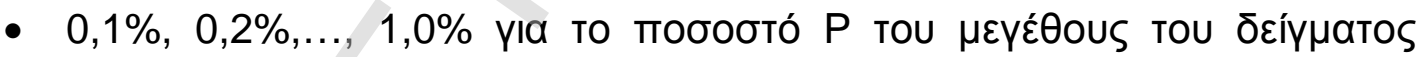

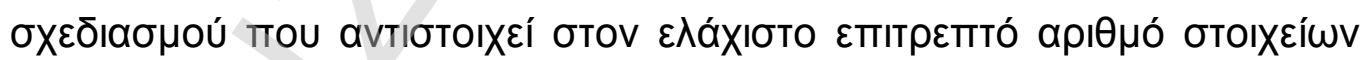

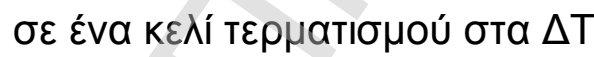

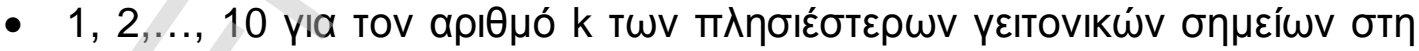
МkПГ $\Sigma$

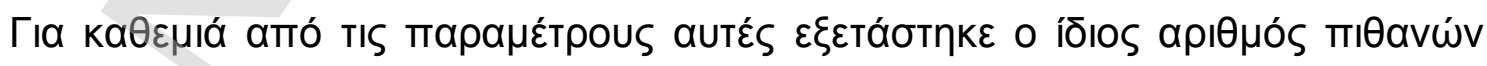

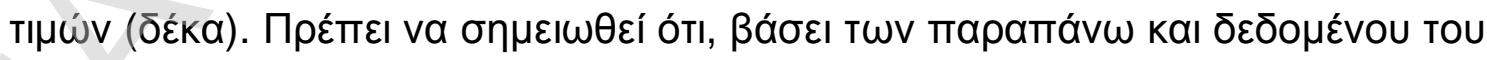

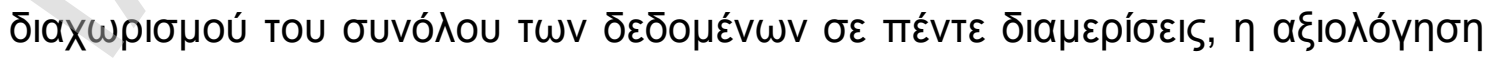

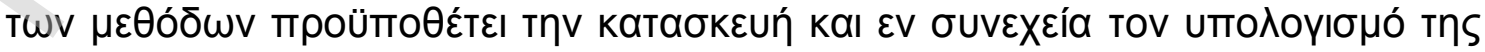




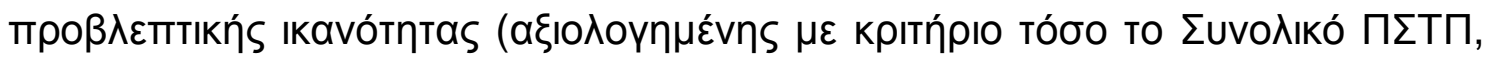

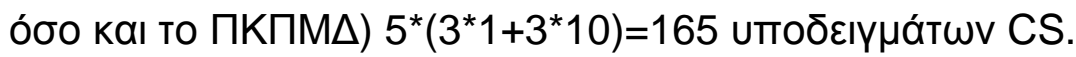

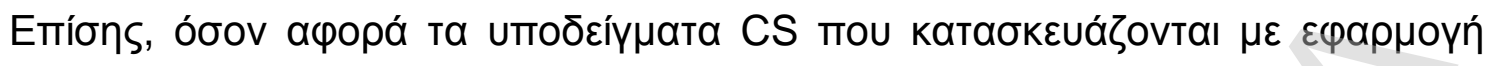

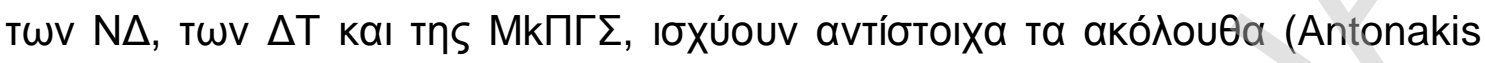
and Sfakianakis, 2008; Antonakis and Sfakianakis, 2009A):

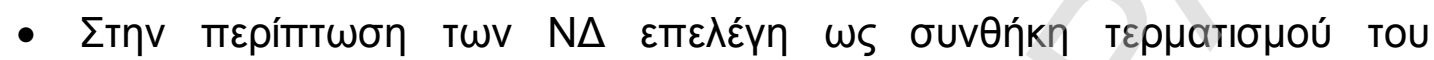

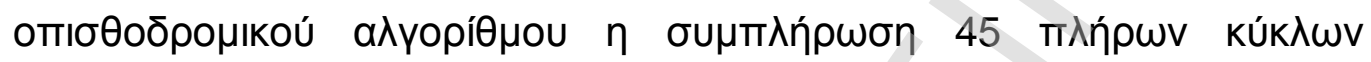

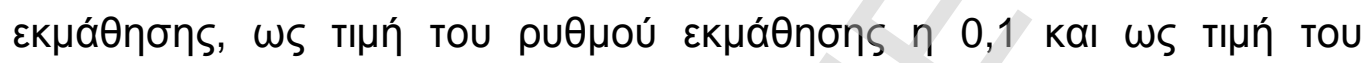

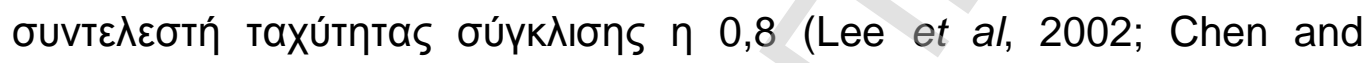
Huang, 2003; Malhotra and Malhotra, 2003).

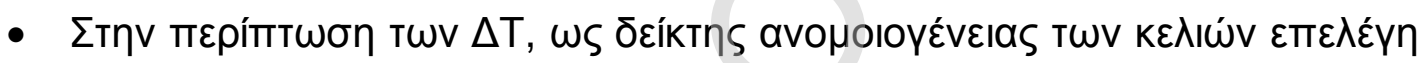
o

$$
i(v)=2 p(l) p(r) \sum_{j=0}^{1}|p(j \mid l)-p(j \mid r)|
$$

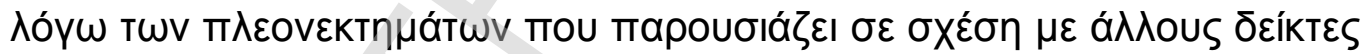

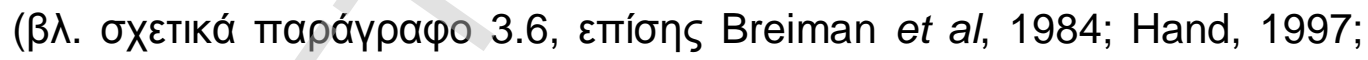
Hastie et al, 2001; Thomas et al, 2002).

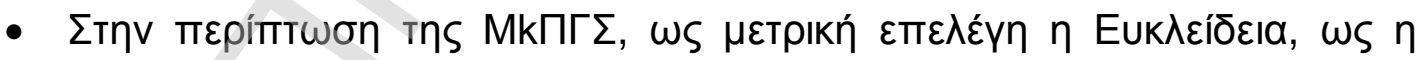

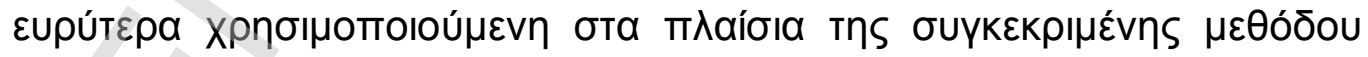

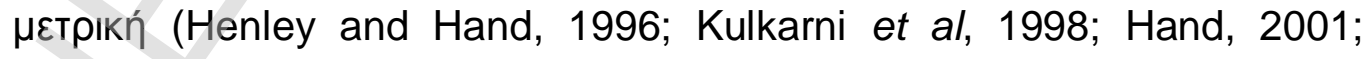
Webb, 2002).

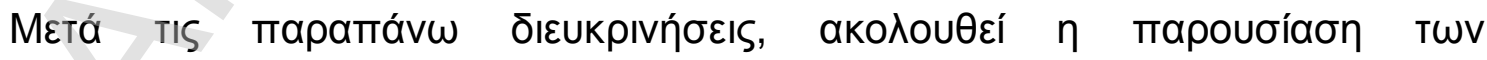

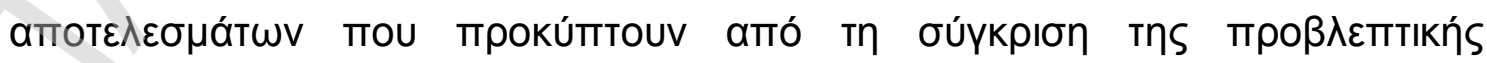

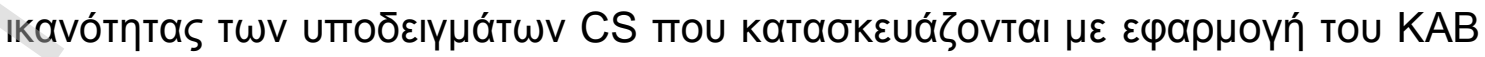




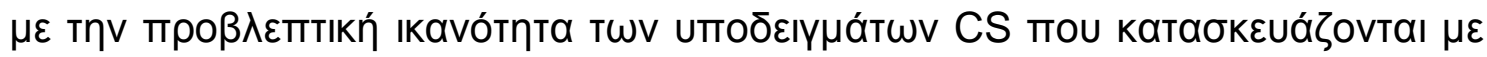

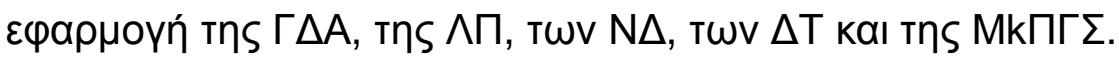

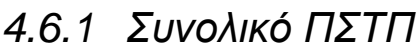

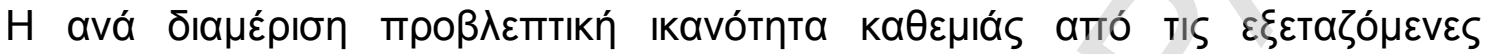

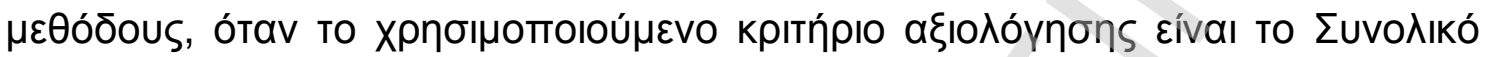

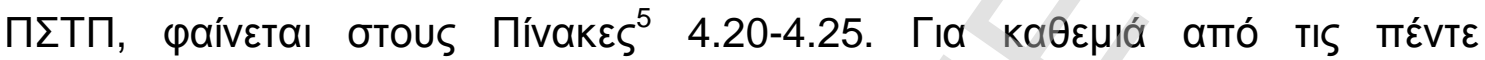

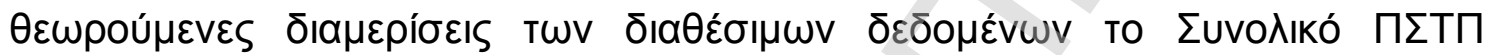

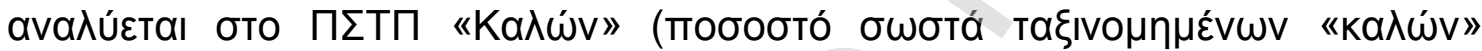

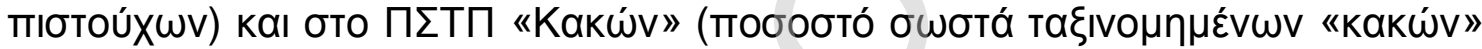

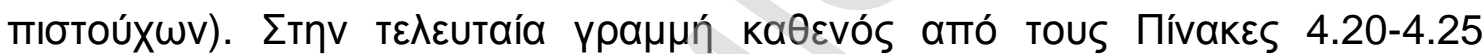

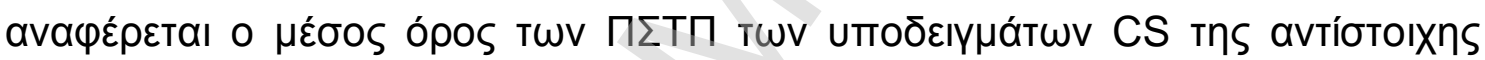

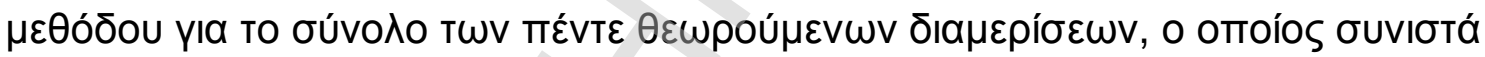

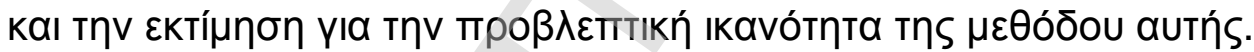

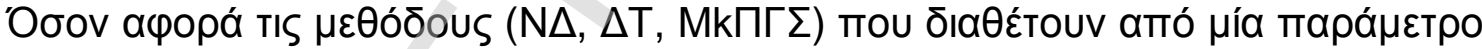

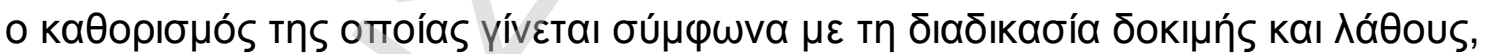

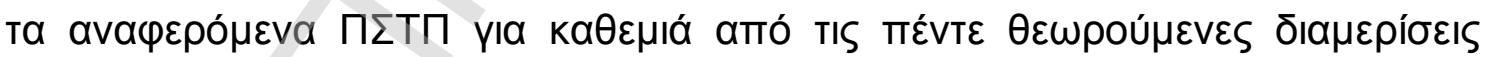

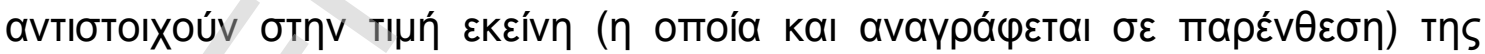

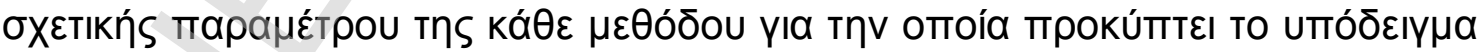

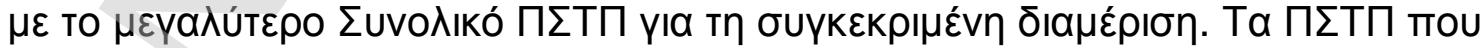

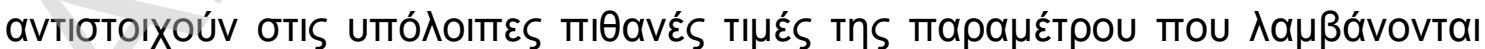

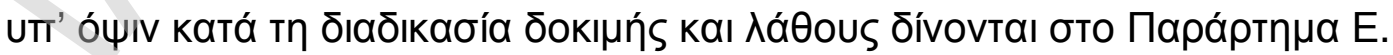

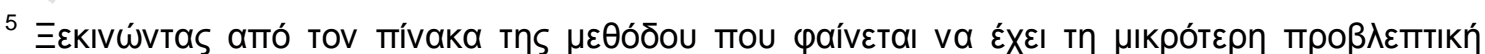

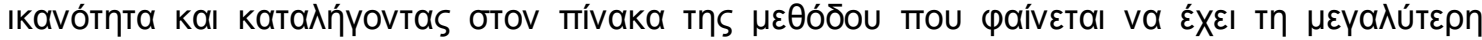

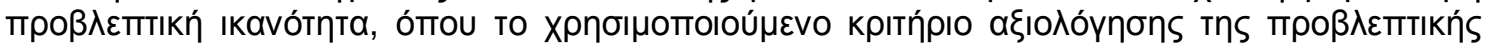

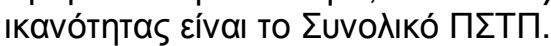


ПINAKA $\Sigma$ 4.20

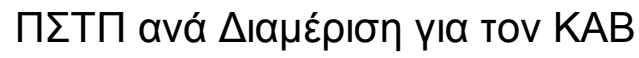

\begin{tabular}{|c|c|c|c|}
\hline$\Delta ı \mu \varepsilon ́ \rho ı \sigma \eta$ & 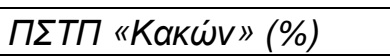 & 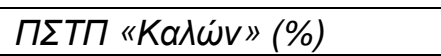 & 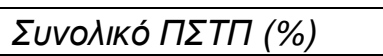 \\
\hline 1 & 57,14285714 & 94,16581372 & 91,01123596 \\
\hline 2 & 59,34065934 & 95,70112590 & 92,60299625 \\
\hline 3 & 43,95604396 & 95,08700102 & 90,73033708 \\
\hline 4 & 50,54945055 & 95,18935517 & 91,38576779 \\
\hline 5 & 50,54945055 & 93,85875128 & 90,16853933 \\
\hline 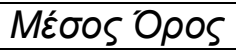 & 52,30769231 & 94,80040942 & 91,17977528 \\
\hline
\end{tabular}

ПINAKA $\Sigma$ 4.21

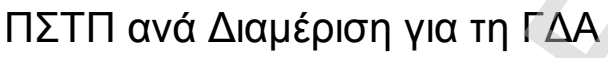

\begin{tabular}{|c|c|c|c|}
\hline$\Delta ı \mu \varepsilon \dot{\rho} \rho \sigma \eta$ & 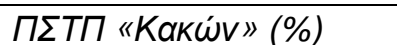 & 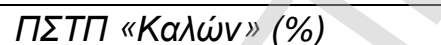 & 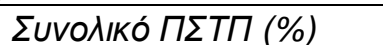 \\
\hline 1 & 49,45054945 & 96,72466735 & 92,69662921 \\
\hline 2 & 45,05494505 & 97,54350051 & 93,07116105 \\
\hline 3 & 46,15384615 & 96,72466735 & 92,41573034 \\
\hline 4 & 45,05494505 & 97,13408393 & 92,69662921 \\
\hline 5 & 50,54945055 & 95,90583419 & 92,04119850 \\
\hline MÉoOS Opos & 47,25274725 & 96,80655067 & 92,58426966 \\
\hline
\end{tabular}

ПINAKA 4.22

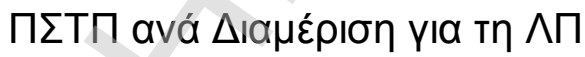

\begin{tabular}{|c|c|c|c|}
\hline$\Delta ı \alpha \mu \varepsilon \rho ı \eta$ & 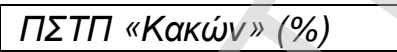 & 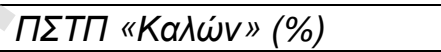 & 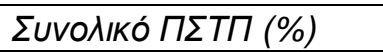 \\
\hline 1 & 49,45054945 & 97,13408393 & 93,07116105 \\
\hline 2 & 47,25274725 & 98,97645855 & 94,56928839 \\
\hline 3 & 40,65934066 & 98,56704197 & 93,63295880 \\
\hline 4 & 47,25274725 & 97,85056295 & 93,53932584 \\
\hline 5 & 42,85714286 & 97,74820880 & 93,07116105 \\
\hline 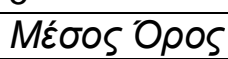 & 45,49450549 & 98,05527124 & 93,57677903 \\
\hline
\end{tabular}

ПINAKA $\Sigma$ 4.23

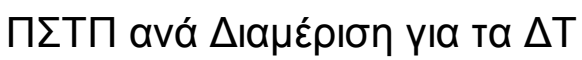

\begin{tabular}{|c|c|c|c|}
\hline$\Delta ı \alpha \mu \varepsilon ́ \rho ı \sigma \eta$ & 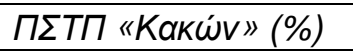 & 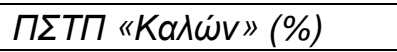 & 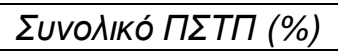 \\
\hline $1(P=0,3 \% \eta \mathrm{P}=0,4 \%)$ & 48,35164835 & 98,05527124 & 93,82022472 \\
\hline $2(P=0,5 \%)$ & 52,74725275 & 98,36233367 & 94,47565543 \\
\hline $3(P=0,2 \%)$ & 47,25274725 & 97,33879222 & 93,07116105 \\
\hline $4(P=0,5 \%)$ & 37,36263736 & 98,97645855 & 93,72659176 \\
\hline $5(P=0,4 \%)$ & 48,35164835 & 97,95291709 & 93,72659176 \\
\hline 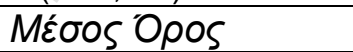 & 46,81318681 & 98,13715455 & 93,76404494 \\
\hline
\end{tabular}


ПINAKA $\Sigma 4.24$

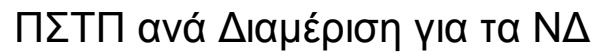

\begin{tabular}{|c|c|c|c|}
\hline$\triangle ı \mu \varepsilon \dot{\rho} \rho \sigma \eta$ & 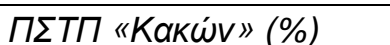 & 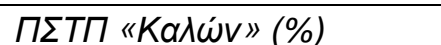 & 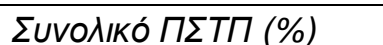 \\
\hline $1(\mathrm{~N}=22)$ & 67,03296703 & 97,03172979 & 94,47565543 \\
\hline $2(\mathrm{~N}=23)$ & 61,53846154 & 97,54350051 & 94,47565543 \\
\hline $3(\mathrm{~N}=22)$ & 60,43956044 & 96,92937564 & 93,82022472 \\
\hline $4(\mathrm{~N}=22)$ & 69,23076923 & 96,51995906 & 94,19475655 \\
\hline $5(\mathrm{~N}=17)$ & 56,04395604 & 97,03172979 & 93,53932584 \\
\hline Méoos Opos & 62,85714286 & 97,01125896 & 94,10112360 \\
\hline
\end{tabular}

ПINAKA $\Sigma$ 4.25

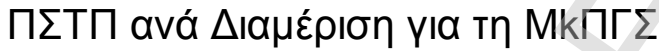

\begin{tabular}{|c|c|c|c|}
\hline 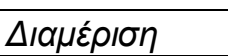 & 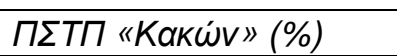 & 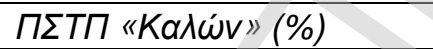 & 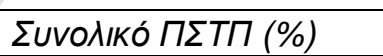 \\
\hline $1(\mathrm{k}=3)$ & 58,24175824 & 98,15762538 & 94,75655431 \\
\hline $2(k=9)$ & 46,15384615 & 98,97645855 & 94,47565543 \\
\hline $3(k=6)$ & 38,46153846 & 98,36233367 & 93,25842697 \\
\hline $4(k=7)$ & 45,05494505 & 98,56704197 & 94,00749064 \\
\hline $5(k=5)$ & 51,64835165 & 98,36233367 & 94,38202247 \\
\hline 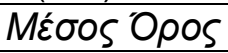 & 47,91208791 & 98,48515865 & 94,17602996 \\
\hline
\end{tabular}

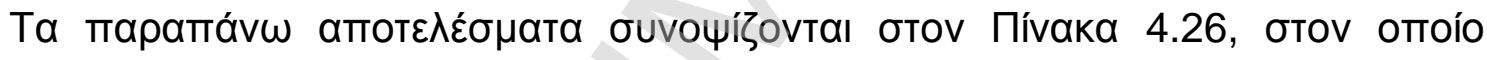

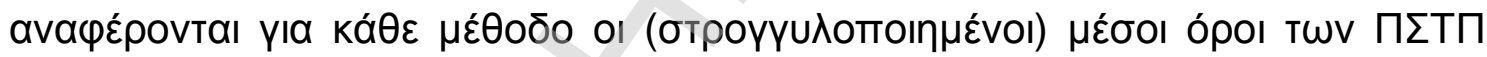

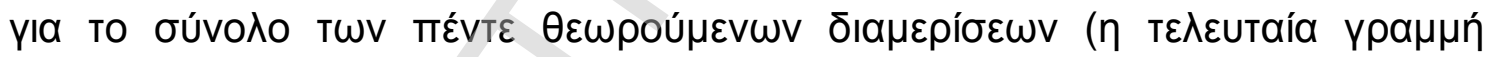

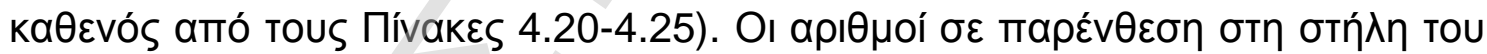

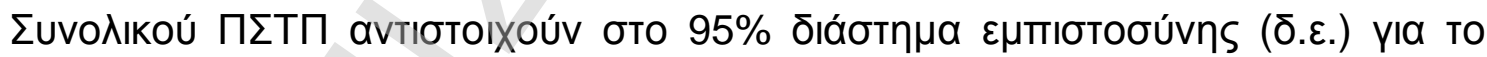

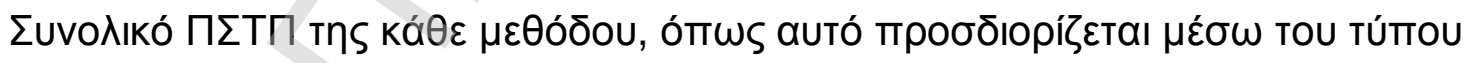

$$
p_{M} \pm z_{a / 2} \sqrt{\frac{p_{M} q_{M}}{n}}
$$

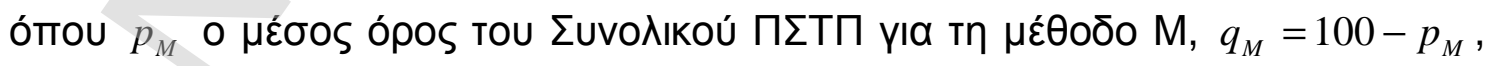
$n=1068,100 *(1-a)=95 \Rightarrow a=0,05 \mathrm{Kal} z_{a / 2}=z_{0,05 / 2}=z_{0,025}=1,96$. 


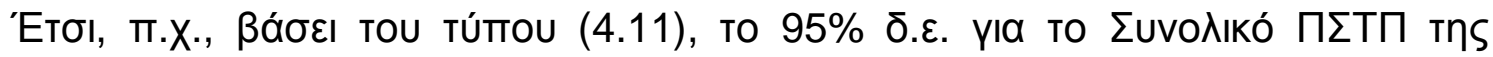

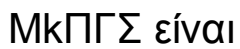

$$
94,2 \% \pm 1,96 * \sqrt{\frac{94,2 \% *(100-94,2) \%}{1068}}=(94,2 \pm 1,4) \%
$$

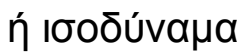

$$
[(94,2-1,4) \%,(94,2+1,4) \%]=[92,8 \%, 95,6 \%]
$$

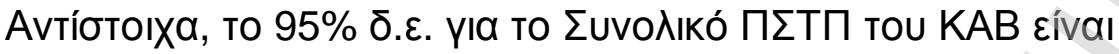

$$
91,2 \% \pm 1,96 \sqrt{\frac{91,2 \% *(100-91,2) \%}{1068}}=(91,2 \pm 1,7) \%
$$

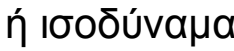

$$
[(91,2-1,7) \%,(91,2+1,7) \%]=[89,5 \%, 92,9 \%]
$$

\begin{tabular}{|c|c|c|c|}
\hline Мદ́Өoठоక & 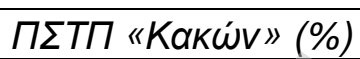 & 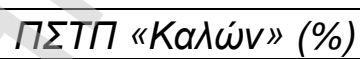 & 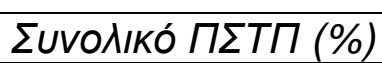 \\
\hline MkПГ $\Sigma$ & 47,9 & 98,5 & $94,2( \pm 1,4)$ \\
\hline $\mathrm{N} \Delta$ & 62,9 & 97,0 & $94,1( \pm 1,4)$ \\
\hline$\Delta \mathrm{T}$ & 46,8 & 98,1 & $93,8( \pm 1,4)$ \\
\hline$\wedge \Pi$ & 45,5 & 98,1 & $93,6( \pm 1,5)$ \\
\hline$\Gamma \Delta \mathrm{A}$ & 47,3 & 96,8 & $92,6( \pm 1,6)$ \\
\hline KAB & 52,3 & 94,8 & $91,2( \pm 1,7)$ \\
\hline
\end{tabular}

ПINAKA $\Sigma 4.26$

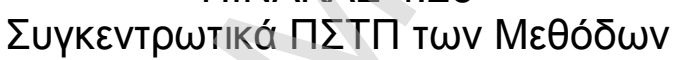

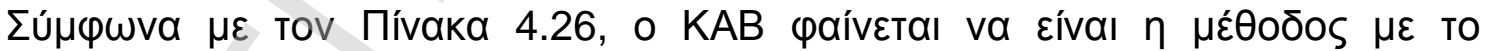

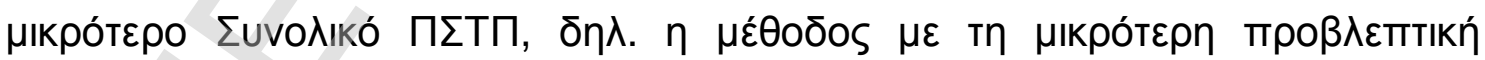

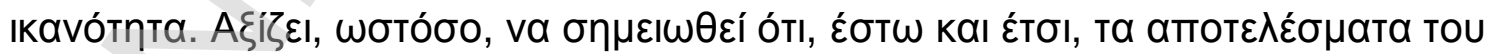

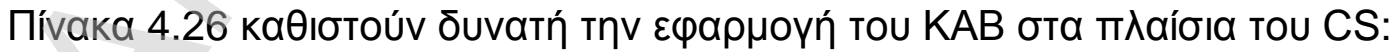

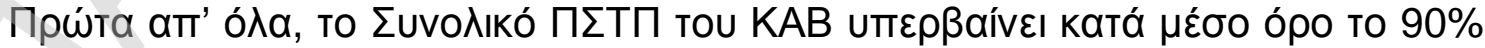

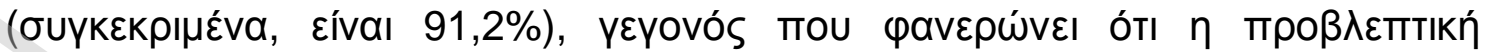

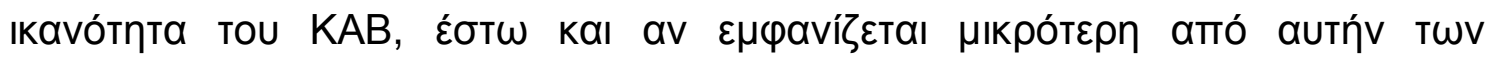

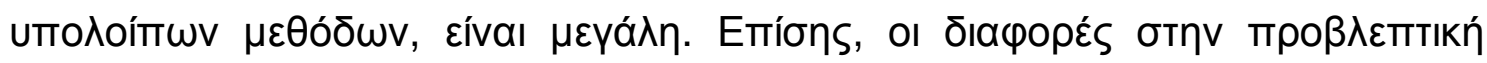




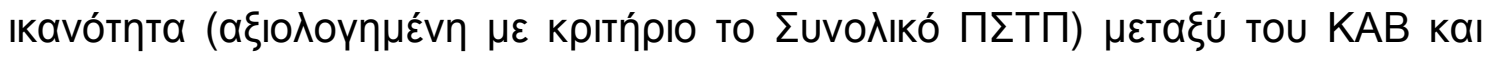

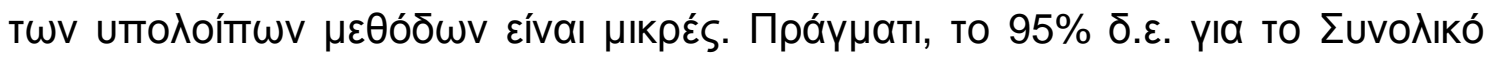

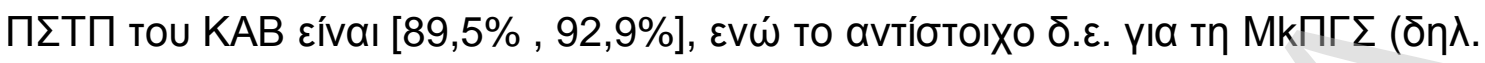

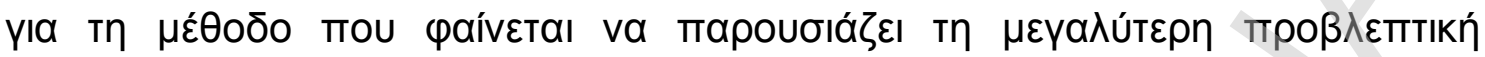

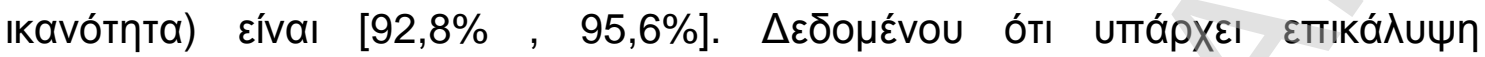

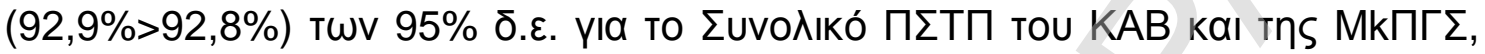

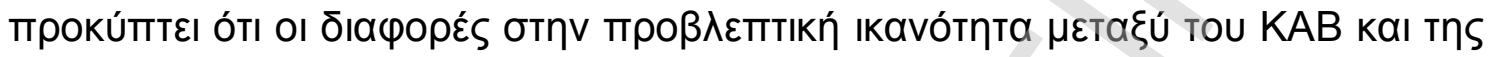

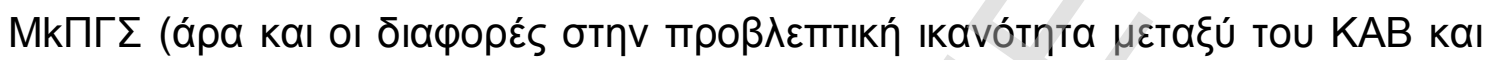

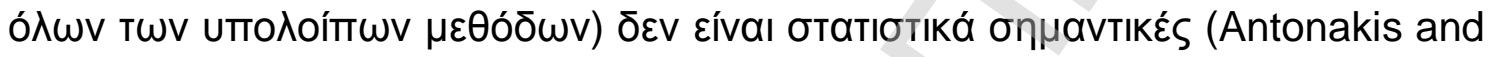
Sfakianakis, 2008; Antonakis and Sfakianakis, 2009A).

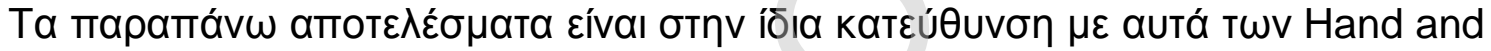

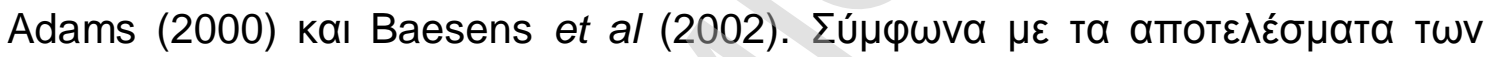

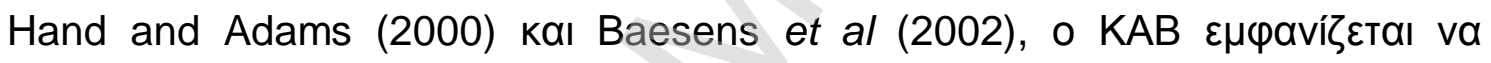

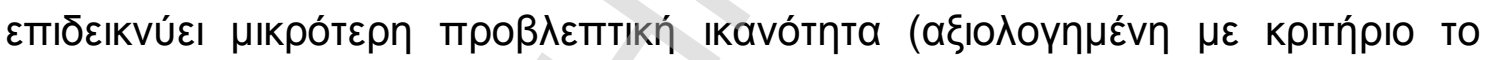

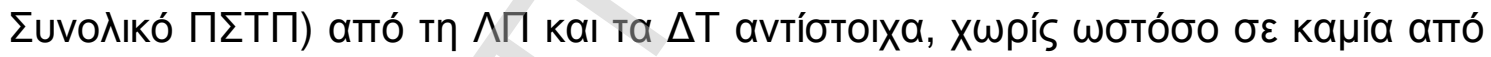

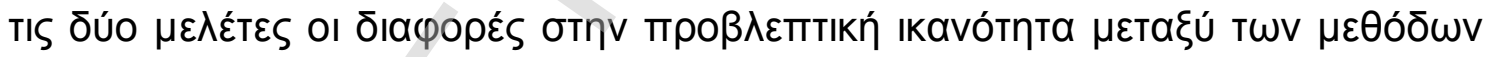

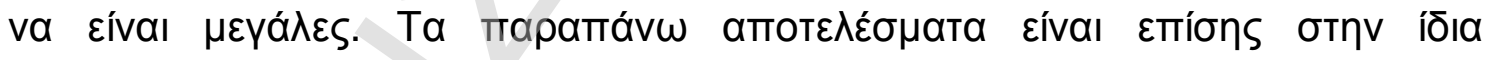

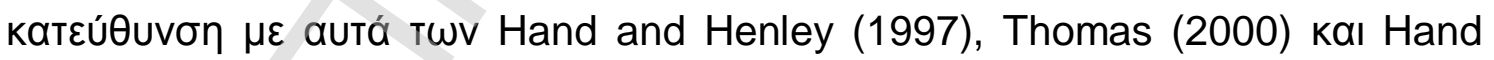

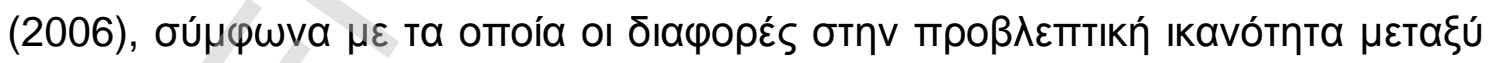

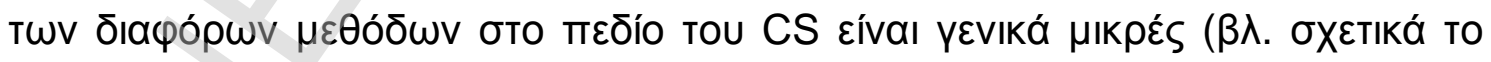

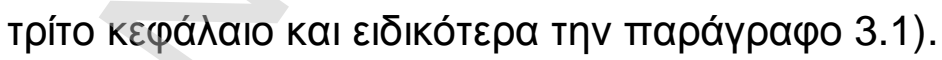

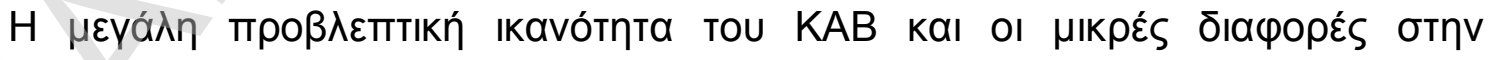

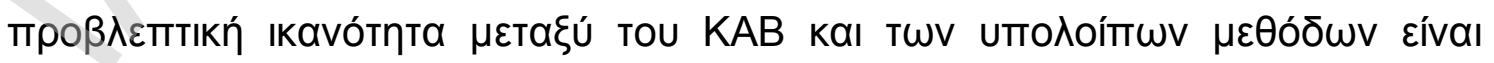

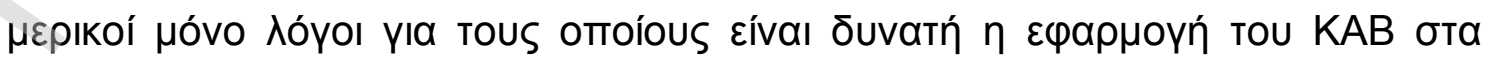

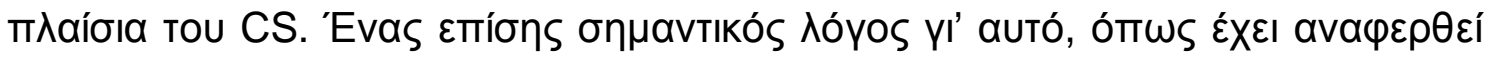




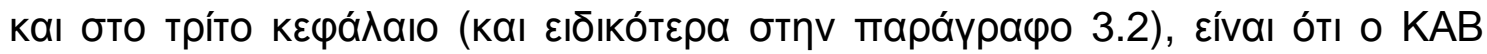

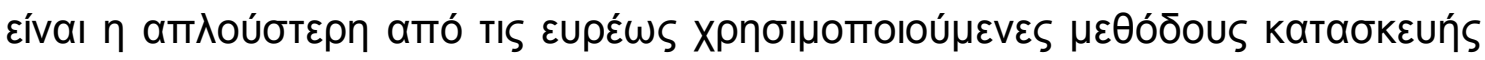

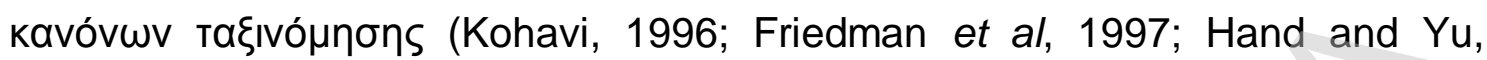

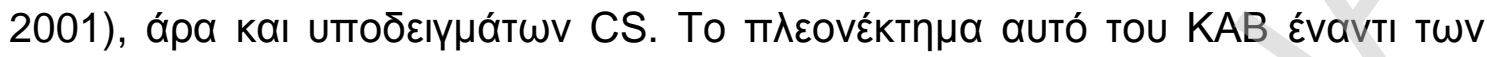

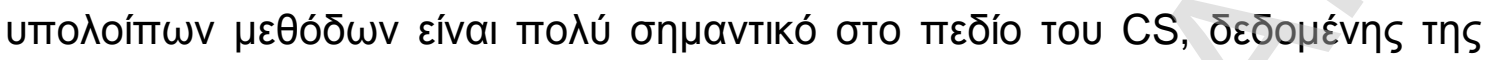

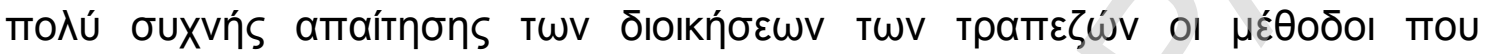

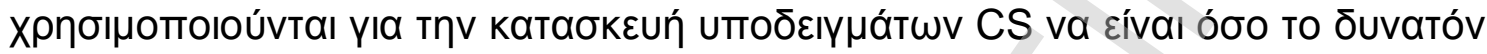

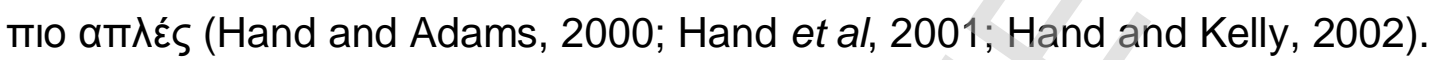

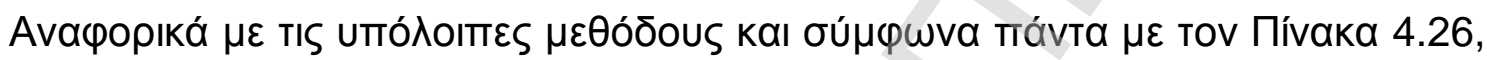

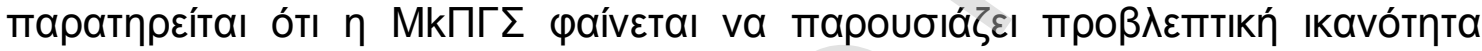

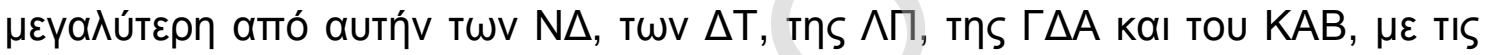

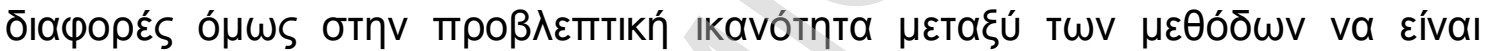

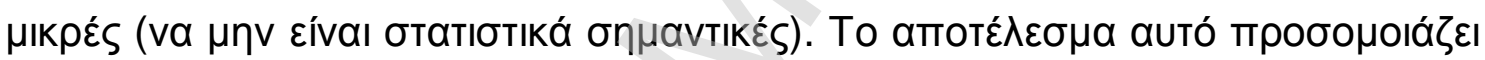

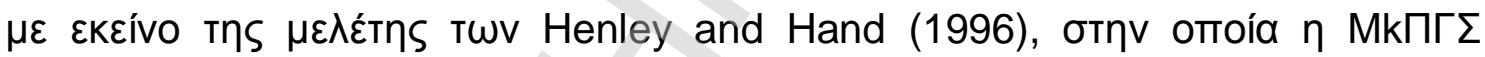

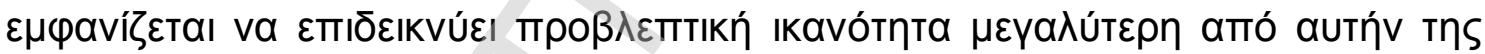

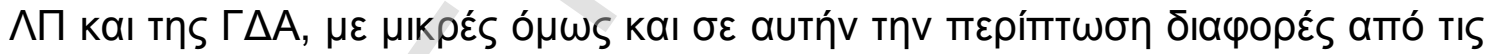

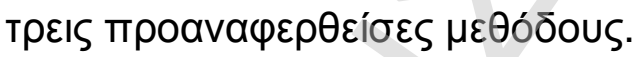

\subsection{2 ПКПM}

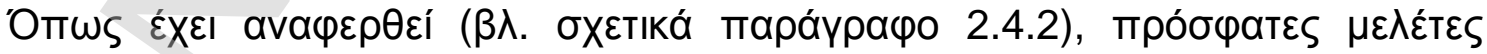

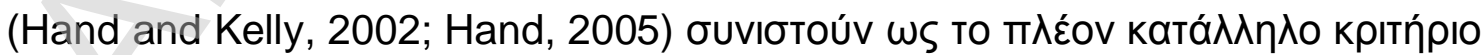

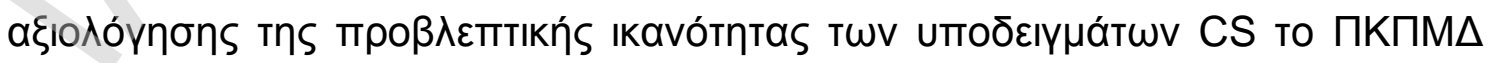

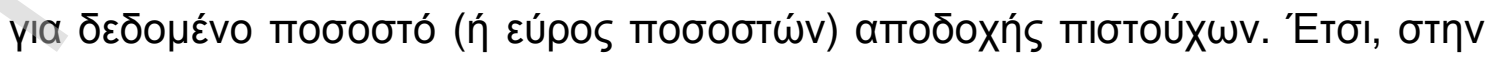

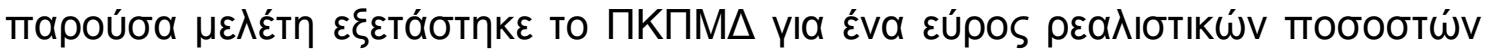




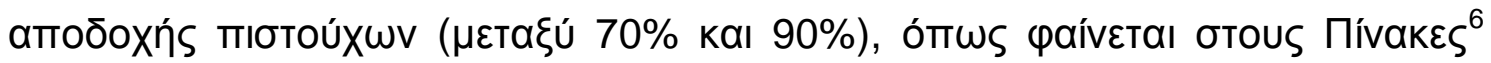

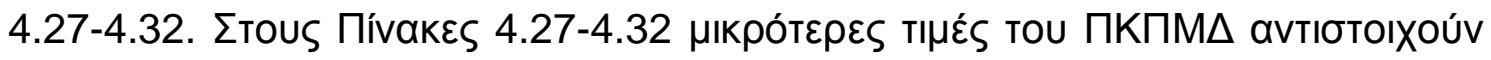

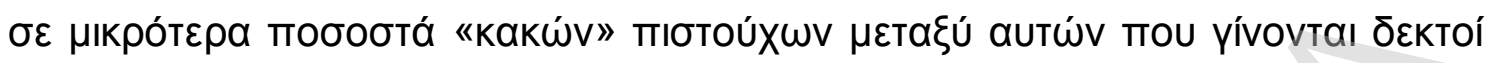

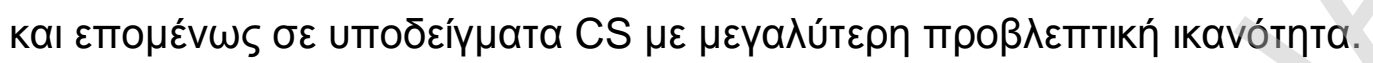

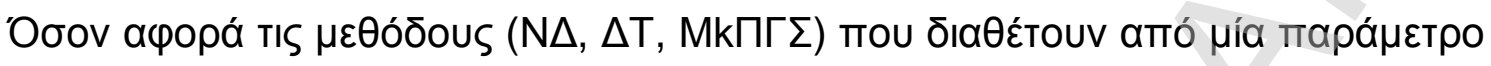

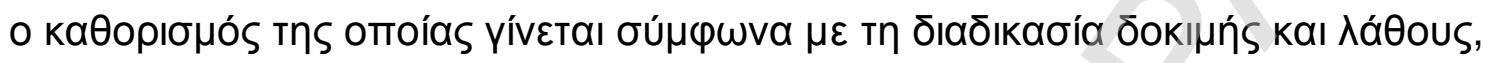

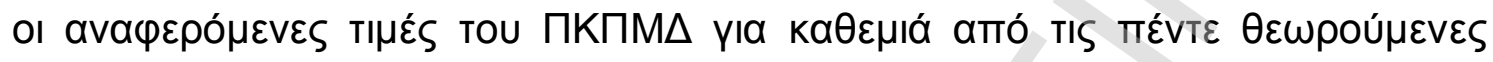

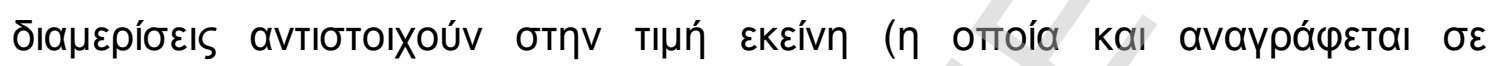

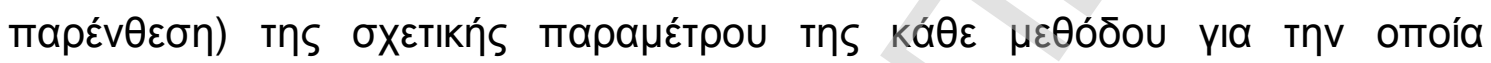

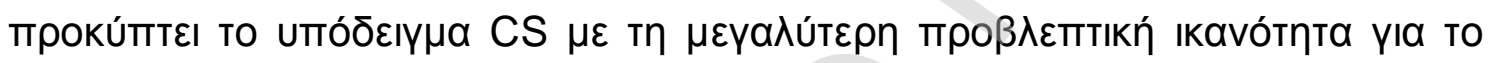

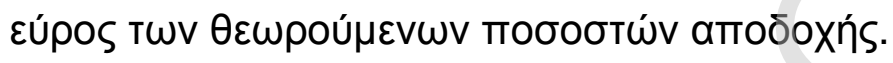

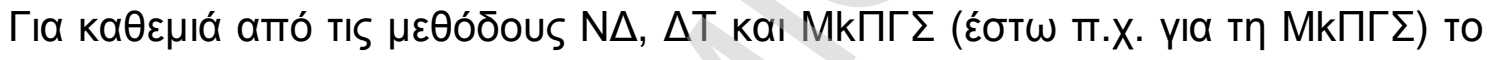

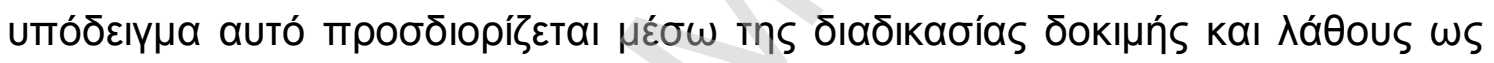

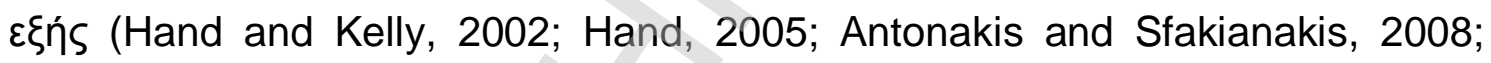
Antonakis and Sfakianakis, 2009A):

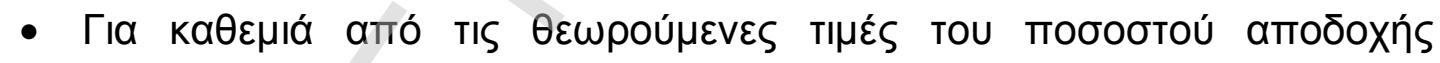

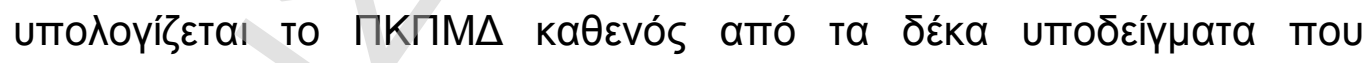

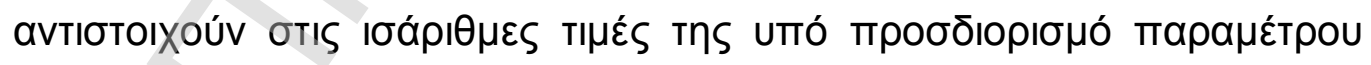

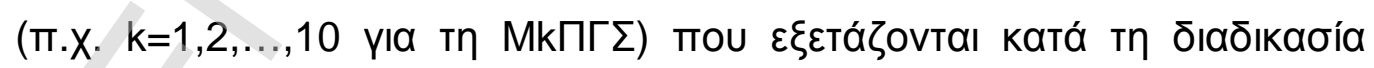

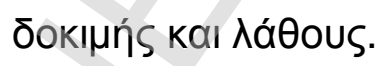

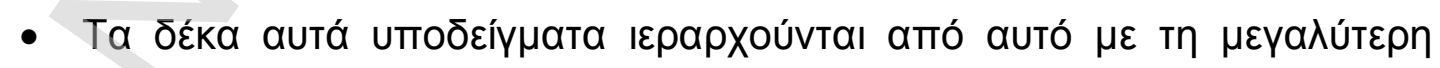

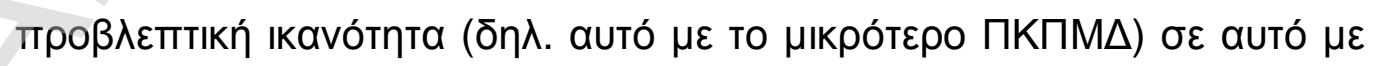

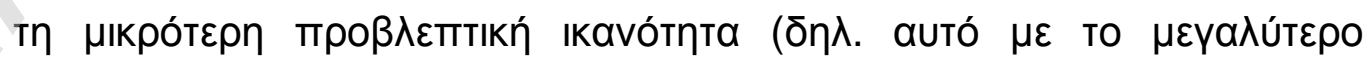

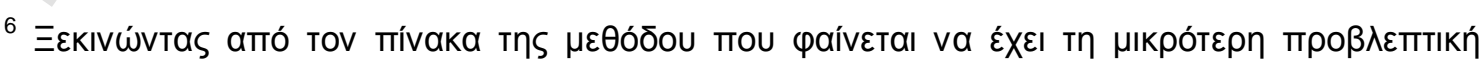

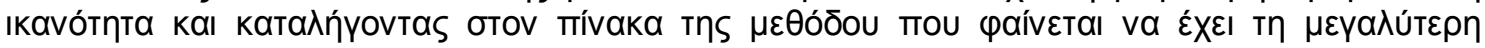

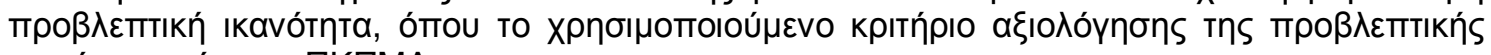

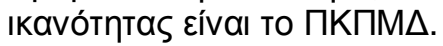




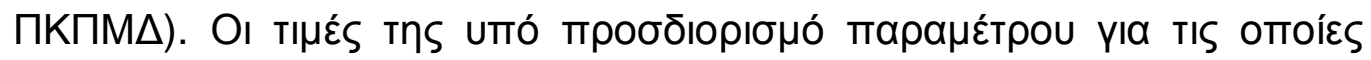

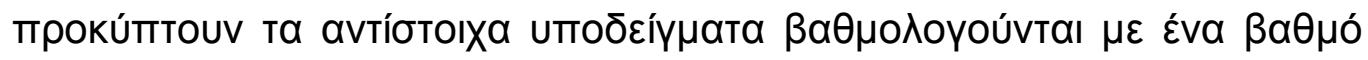

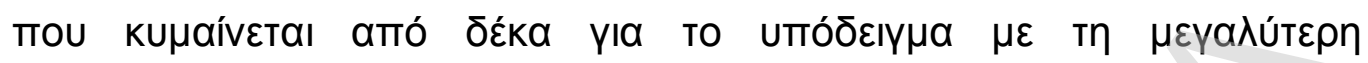

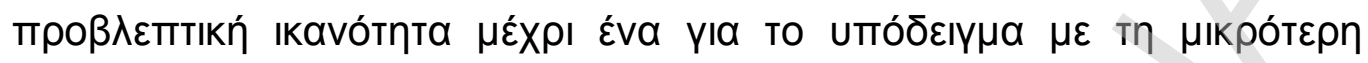

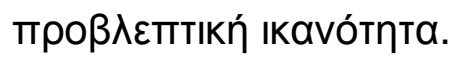

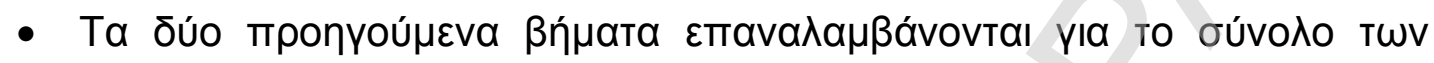

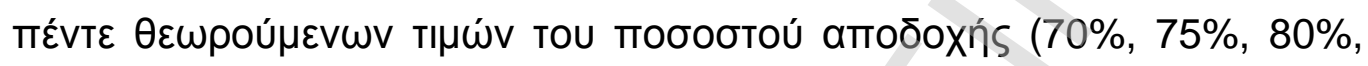

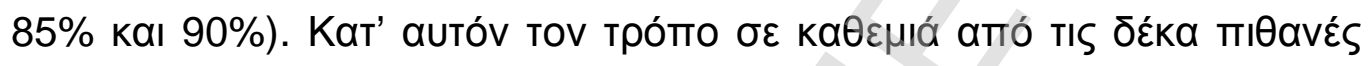

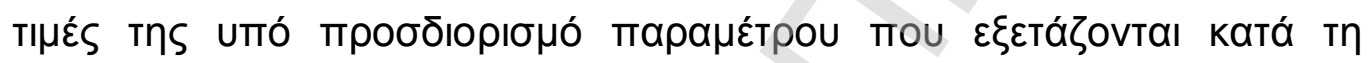

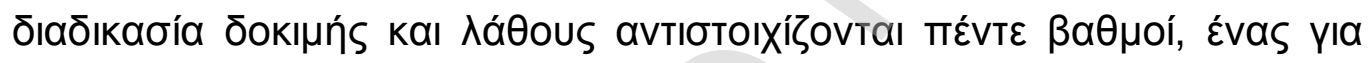

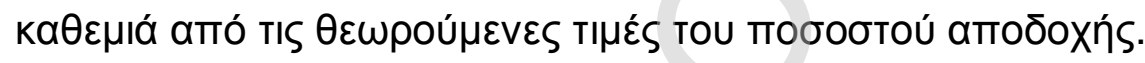

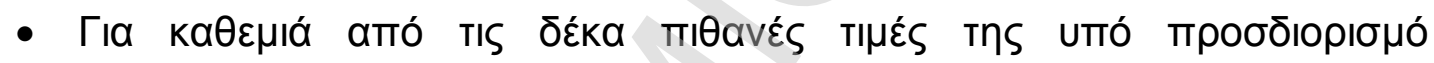

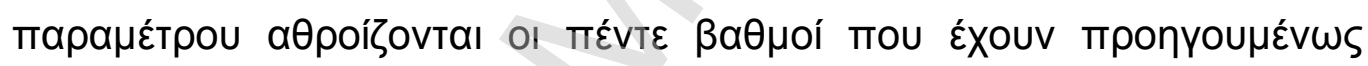

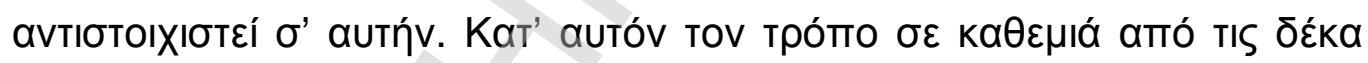

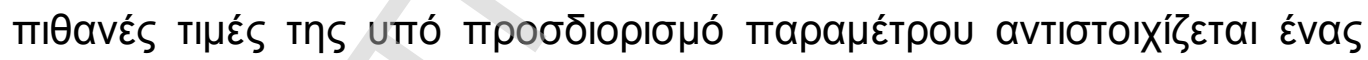

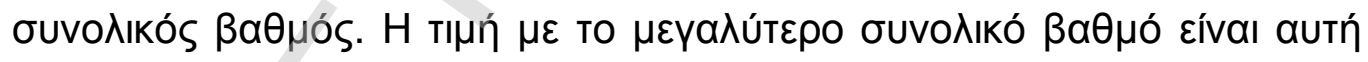

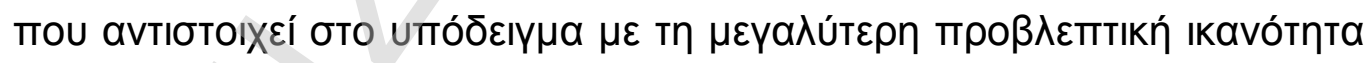

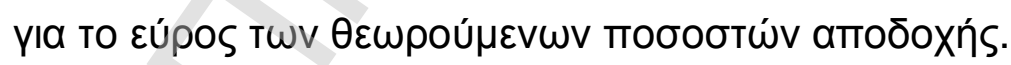

ПINAKA 4.27

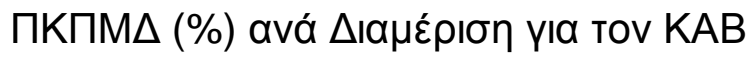

\begin{tabular}{|c|c|c|c|c|c|}
\hline \multirow{2}{*}{$\Delta ı а \varepsilon \varepsilon \rho ı \sigma \eta$} & \multicolumn{5}{|c|}{ Побобто́ Апобохи́s } \\
\hline & $70 \%$ & $75 \%$ & $80 \%$ & $85 \%$ & $90 \%$ \\
\hline 1 & 1,604278 & 2,122347 & 2,693208 & 3,193833 & 4,162331 \\
\hline $2>$ & 1,470588 & 1,872659 & 2,224824 & 2,643172 & 3,433923 \\
\hline 3 & 1,871658 & 2,496879 & 3,161593 & 3,854626 & 4,890739 \\
\hline 4 & 1,069519 & 1,747815 & 2,34192 & 2,973568 & 4,266389 \\
\hline 5 & 2,005348 & 2,496879 & 3,044496 & 3,744493 & 4,682622 \\
\hline MÉoOs Opos & 1,604278 & 2,147316 & 2,693208 & 3,281938 & 4,287201 \\
\hline
\end{tabular}


ПINAKA 4.28

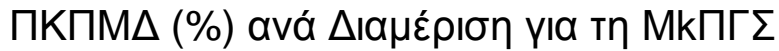

\begin{tabular}{|c|c|c|c|c|c|}
\hline \multirow{2}{*}{ 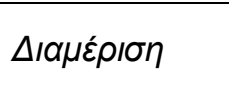 } & \multicolumn{5}{|c|}{ Побобто́ Апобохи́s } \\
\hline & $70 \%$ & $75 \%$ & $80 \%$ & $85 \%$ & $90 \%$ \\
\hline $1(k=7)$ & 0,935829 & 1,123596 & 2,107728 & 1,982379 & 2,913632 \\
\hline $2(k=6)$ & 1,604278 & 1,997503 & 1,990632 & 2,312775 & 2,809573 \\
\hline $3(k=4)$ & 1,604278 & 1,498127 & 2,107728 & 2,973568 & 3,746098 \\
\hline $4(k=9)$ & 1,069519 & 1,123596 & 2,224824 & 2,202643 & 3,433923 \\
\hline $5(\mathrm{k}=10)$ & 1,470588 & 2,122347 & 1,990632 & 3,193833 & 3,537981 \\
\hline 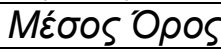 & 1,336898 & 1,573034 & 2,084309 & 2,53304 & 3,288241 \\
\hline
\end{tabular}

ПINAKA 4.29

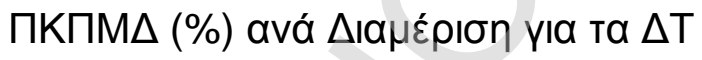

\begin{tabular}{|c|c|c|c|c|c|}
\hline \multirow{2}{*}{ 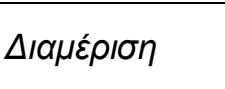 } & \multicolumn{5}{|c|}{ Побоото́ Апобохи́ऽ } \\
\hline & $70 \%$ & $75 \%$ & $80 \%$ & $85 \%$ & $90 \%$ \\
\hline $1(P=0,3 \%)$ & 1,069519 & 1,248439 & 1,873536 & 1,872247 & 3,121748 \\
\hline $2(P=0,4 \%)$ & 1,470588 & 1,747815 & 1,990632 & 2,643172 & 3,01769 \\
\hline $3(P=0,6 \%)$ & 1,069519 & 1,248439 & 1,75644 & 2,753304 & 3,537981 \\
\hline $4(P=0,7 \%)$ & 0,935829 & 0,998752 & 1,639344 & 2,202643 & 3,433923 \\
\hline $5(P=0,3 \%)$ & 1,336898 & 1,373283 & 2,107728 & 2,422907 & 3,329865 \\
\hline 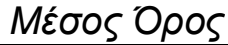 & 1,176471 & 1,323346 & 1,873536 & 2,378855 & 3,288241 \\
\hline
\end{tabular}

ПINAKA $\Sigma$ 4.30

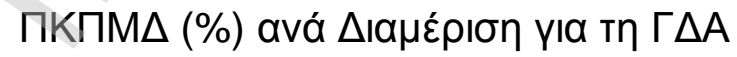

\begin{tabular}{|c|c|c|c|c|c|}
\hline \multirow{2}{*}{$\Delta ı \alpha \mu \varepsilon \rho ı \sigma \eta$} & \multicolumn{5}{|c|}{ Побобто́ Апобохи́s } \\
\hline & $70 \%$ & $75 \%$ & $80 \%$ & $85 \%$ & $90 \%$ \\
\hline- & 0,802139 & 1,248439 & 1,639344 & 2,53304 & 3,537981 \\
\hline 2 & 0,935829 & 1,373283 & 1,405152 & 1,762115 & 3,01769 \\
\hline 3 & 0,935829 & 1,622971 & 1,75644 & 2,312775 & 3,850156 \\
\hline 4 & 0,668449 & 0,998752 & 1,639344 & 2,092511 & 3,954214 \\
\hline$5 \longdiv { - 1 }$ & 1,069519 & 1,498127 & 1,75644 & 2,422907 & 3,954214 \\
\hline 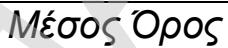 & 0,882353 & 1,348315 & 1,639344 & 2,22467 & 3,662851 \\
\hline
\end{tabular}


ПINAKA $\Sigma 4.31$

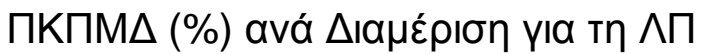

\begin{tabular}{|c|c|c|c|c|c|}
\hline \multirow{2}{*}{$\triangle ı \alpha \mu \varepsilon ́ \rho ı \sigma \eta$} & \multicolumn{5}{|c|}{ Побобто́ Аாобохи́s } \\
\hline & $70 \%$ & $75 \%$ & $80 \%$ & $85 \%$ & $90 \%$ \\
\hline 1 & 0,802139 & 1,248439 & 1,522248 & 1,982379 & 3,433923 \\
\hline 2 & 0,668449 & 0,998752 & 1,17096 & 1,651982 & 2,39334 \\
\hline 3 & 0,802139 & 0,998752 & 1,522248 & 2,53304 & 3,537981 \\
\hline 4 & 0,534759 & 1,373283 & 1,639344 & 2,092511 & 3,329865 \\
\hline 5 & 1,203209 & 1,747815 & 1,990632 & 2,753304 & 3,64204 \\
\hline 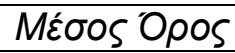 & 0,802139 & 1,273408 & 1,569087 & 2,202643 & 3,26743 \\
\hline
\end{tabular}

ПINAKA 24.32

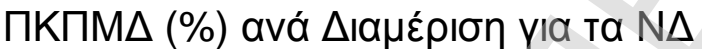

\begin{tabular}{|c|c|c|c|c|c|}
\hline \multirow{2}{*}{$\Delta ı \alpha \mu \varepsilon ́ \rho ı \sigma \eta$} & \multicolumn{5}{|c|}{ Побобто́ Атобохи́s } \\
\hline & $70 \%$ & $75 \%$ & $80 \%$ & $85 \%$ & $90 \%$ \\
\hline $1(\mathrm{~N}=20)$ & 0,668449 & 0,62422 & 0,819672 & 1,431718 & 2,809573 \\
\hline $2(\mathrm{~N}=24)$ & 0,40107 & 0,499376 & 1,405152 & 1,872247 & 2,913632 \\
\hline $3(\mathrm{~N}=22)$ & 0,802139 & 0,873908 & 1,405152 & 2,202643 & 2,913632 \\
\hline $4(\mathrm{~N}=24)$ & 0,40107 & 0,873908 & 1,17096 & 2,092511 & 3,121748 \\
\hline $5(\mathrm{~N}=21)$ & 1,203209 & 1,248439 & 1,75644 & 2,312775 & 3,225806 \\
\hline 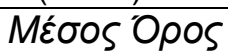 & 0,695187 & 0,82397 & 1,311475 & 1,982379 & 2,996878 \\
\hline
\end{tabular}

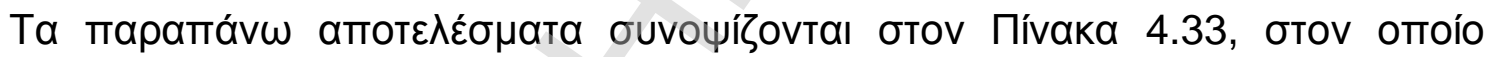

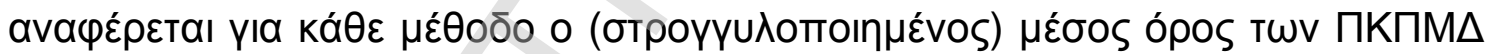

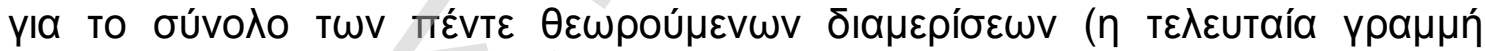

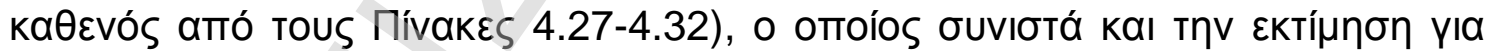

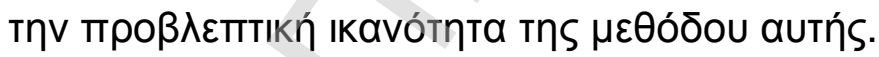

ПINAKA $\Sigma 4.33$

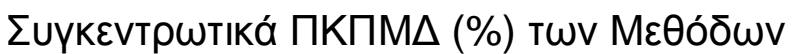

\begin{tabular}{|c|c|c|c|c|c|}
\hline \multirow{2}{*}{ 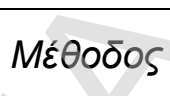 } & \multicolumn{5}{|c|}{ Побобтó Аாобохи́s } \\
\hline & $70 \%$ & $75 \%$ & $80 \%$ & $85 \%$ & $90 \%$ \\
\hline $\mathrm{N} \Delta$ & 0,7 & 0,8 & 1,3 & 2,0 & 3,0 \\
\hline$\Lambda \Pi$ & 0,8 & 1,3 & 1,6 & 2,2 & 3,3 \\
\hline$\Gamma \Delta \mathrm{A}$ & 0,9 & 1,3 & 1,6 & 2,2 & 3,7 \\
\hline$\Delta T$ & 1,2 & 1,3 & 1,9 & 2,4 & 3,3 \\
\hline MkПГ $\Sigma$ & 1,3 & 1,6 & 2,1 & 2,5 & 3,3 \\
\hline KAB & 1,6 & 2,1 & 2,7 & 3,3 & 4,3 \\
\hline
\end{tabular}




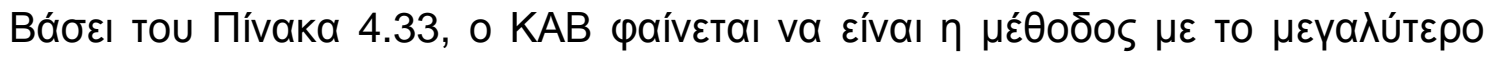

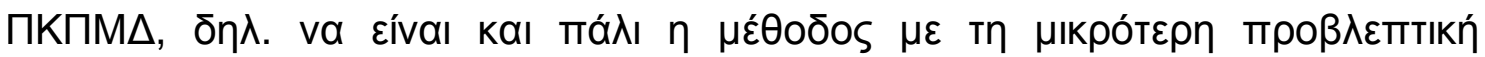

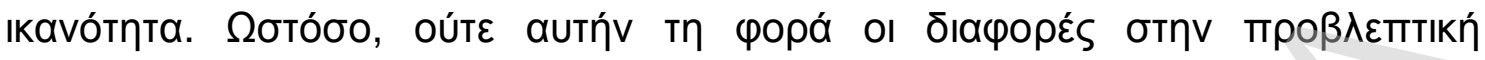

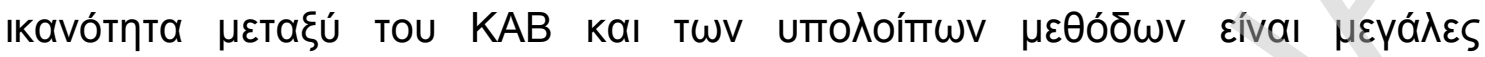
(Antonakis and Sfakianakis, 2008; Antonakis and Sfakianakis, 2009A). To

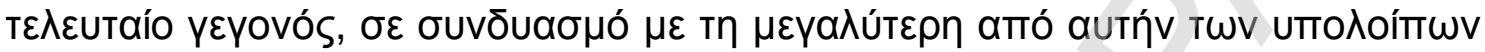

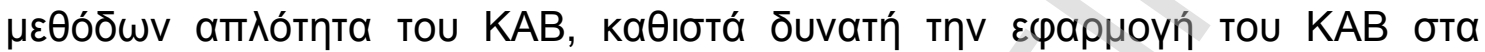

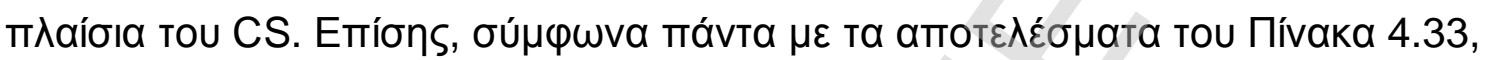

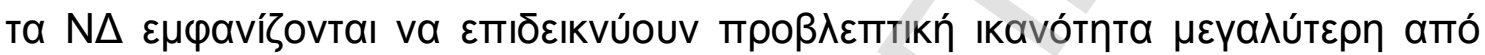

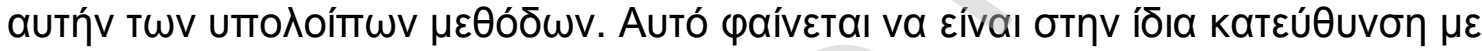

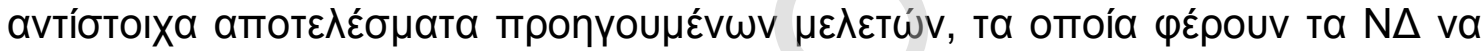

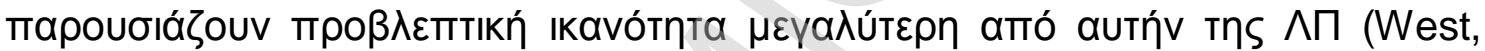
2000; Lee et al, 2002; Ong et al, 2005), tns Г $\Delta \mathrm{A}$ (West, 2000; Lee et al, 2002; Chen and Huang, 2003; Malhotra, 2003) kaı TwV $\Delta T$ (West, 2000; Chen and Huang, 2003; Ong et al, 2005).

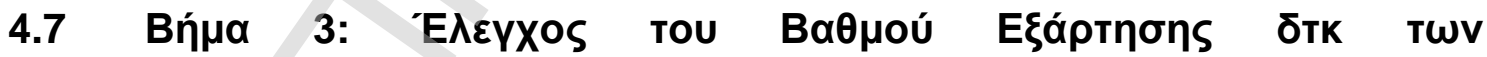

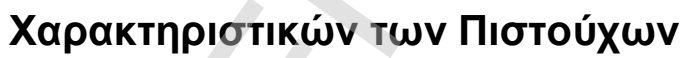

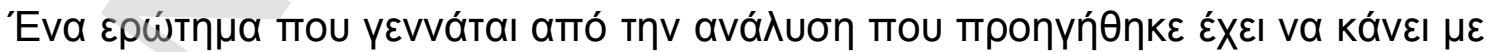

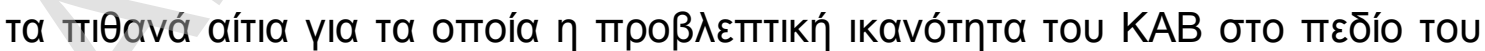

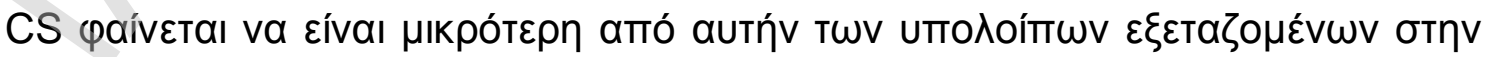

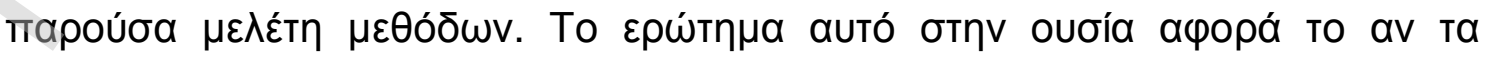

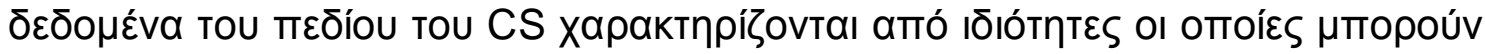




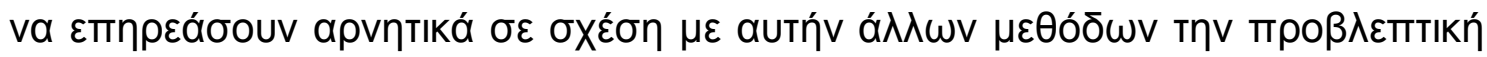

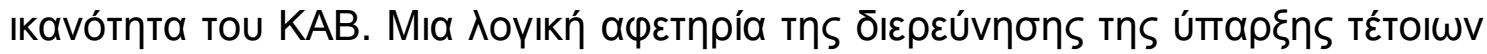

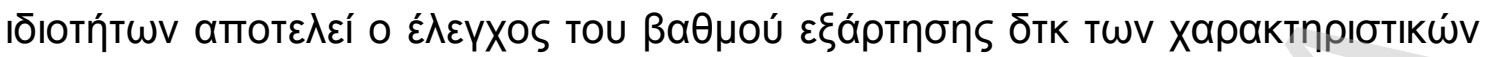

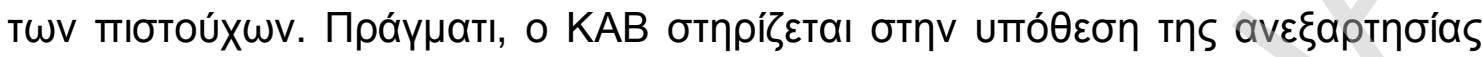

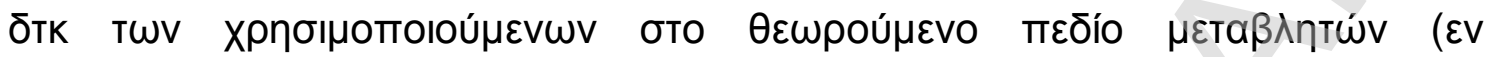

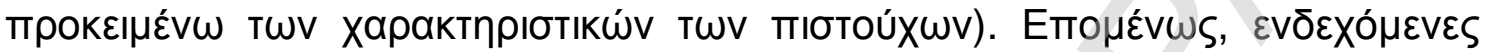

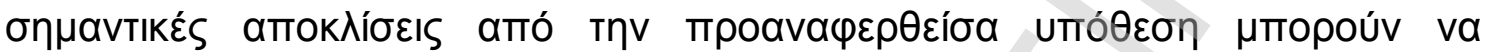

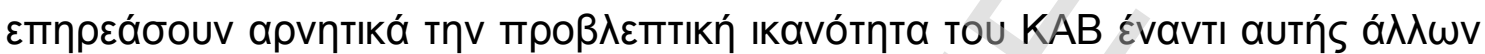

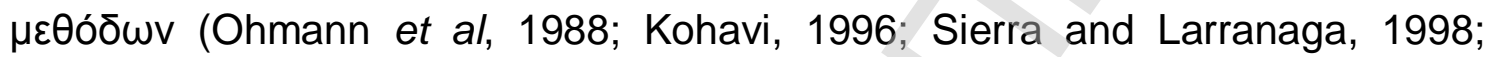

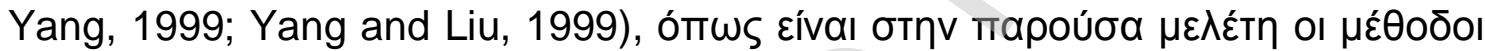

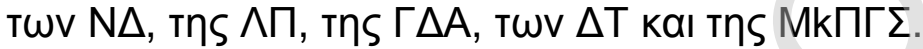

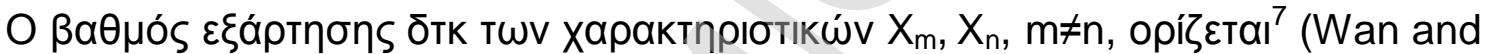
Wong, 1989; Kononenko, 1991; Antonakis and Sfakianakis, 2008; Antonakis

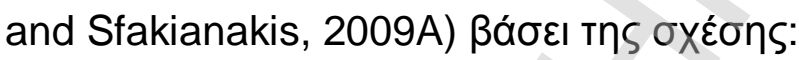

$$
D\left(X_{m}, X_{n}\right)=H\left(X_{m}\right)+H\left(X_{n}\right)-H\left(X_{m} X_{n}\right)
$$

óтоण:

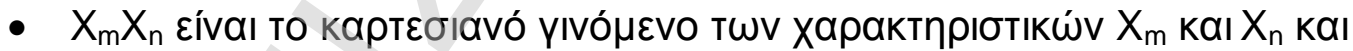

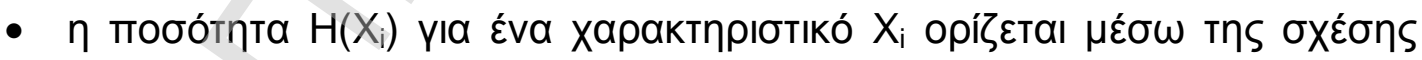

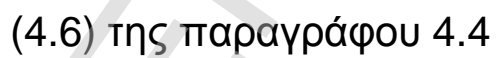

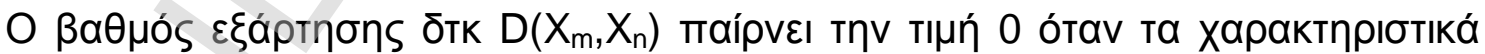

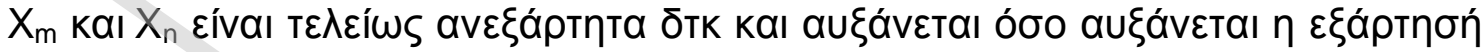

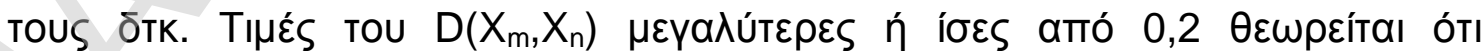

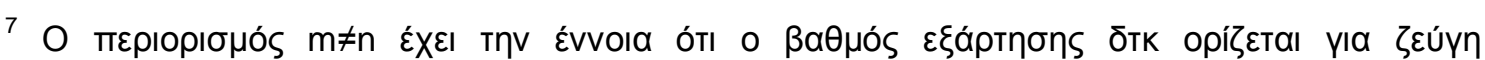

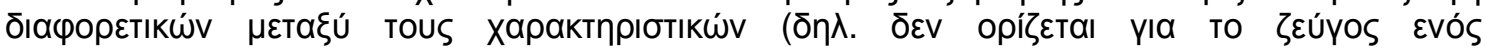

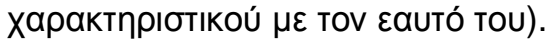




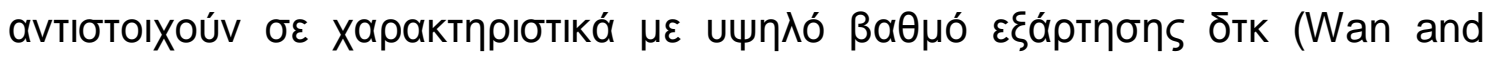
Wong, 1989; Kononenko, 1991).

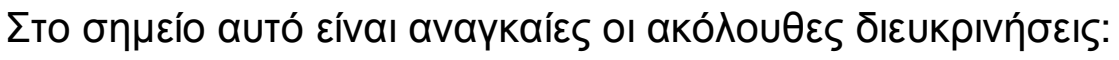

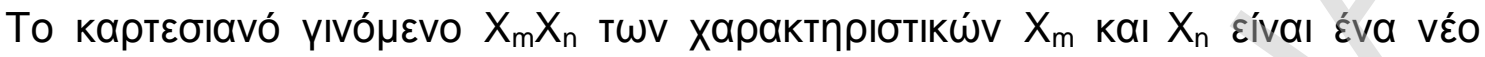

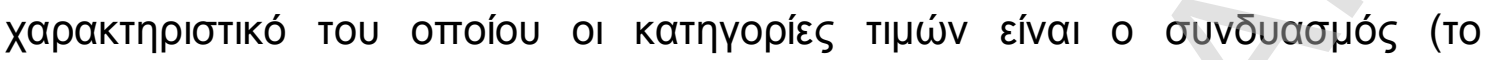

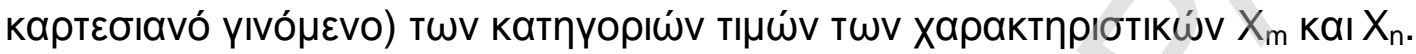

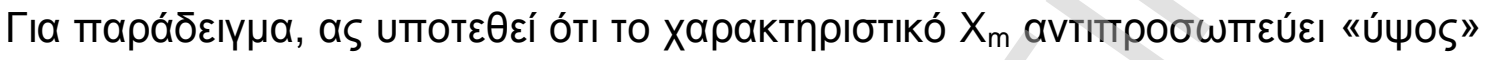

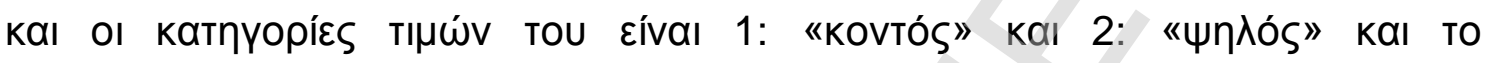

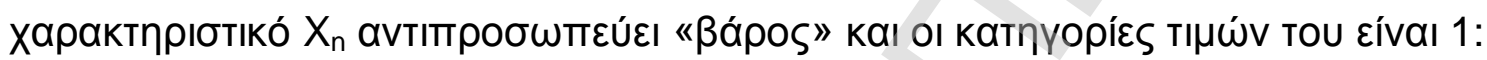

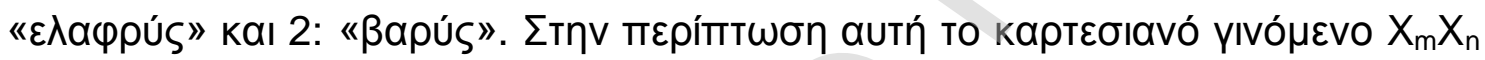

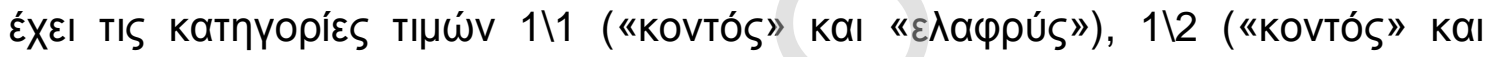

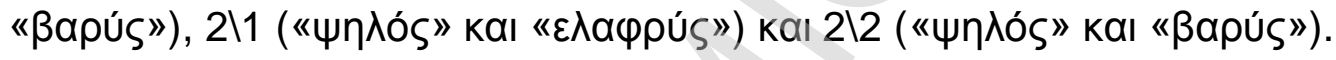

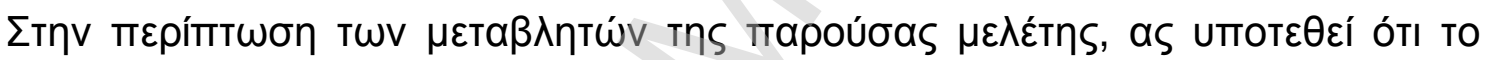

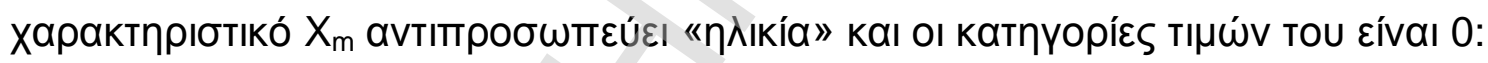

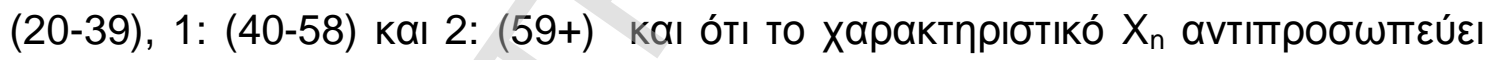

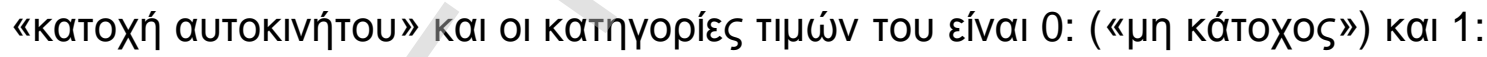

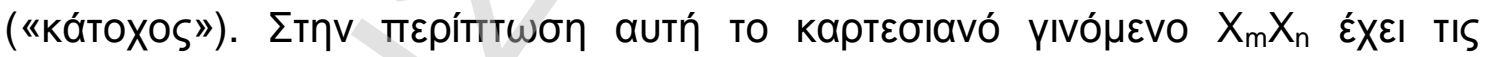

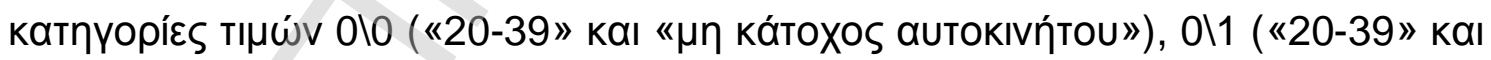

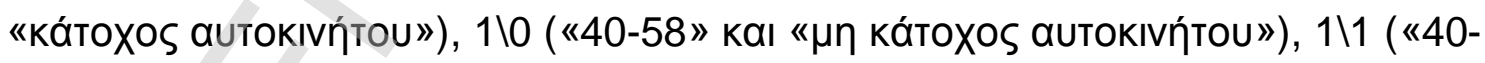

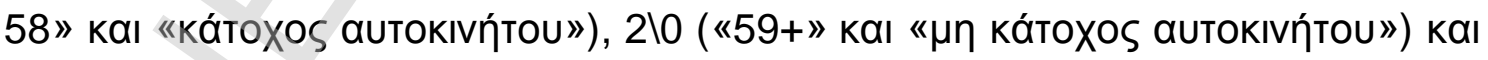

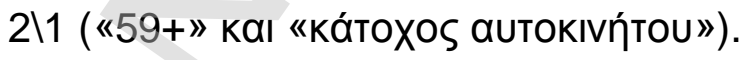

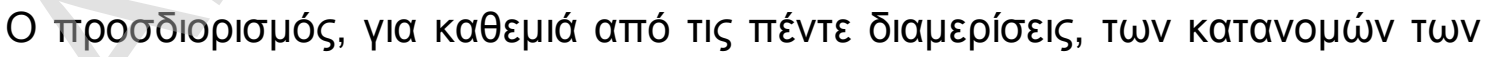

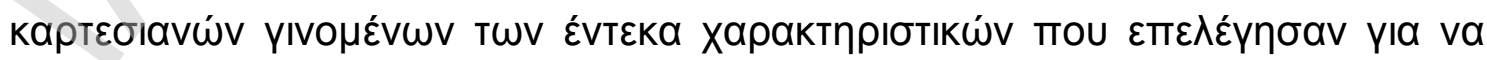

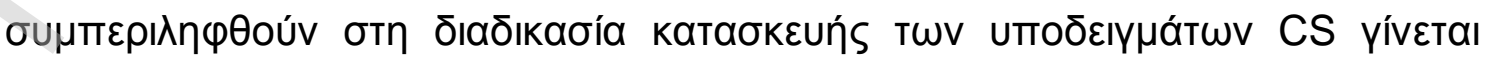

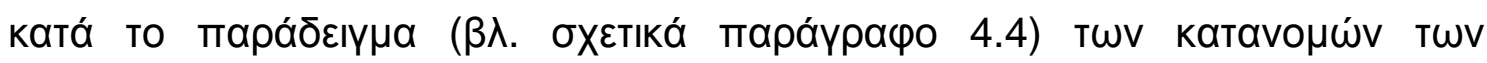




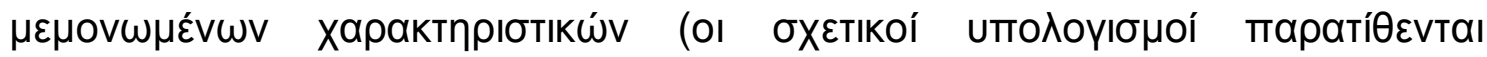

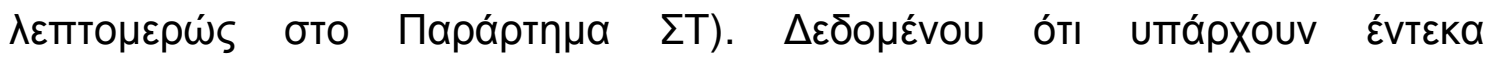

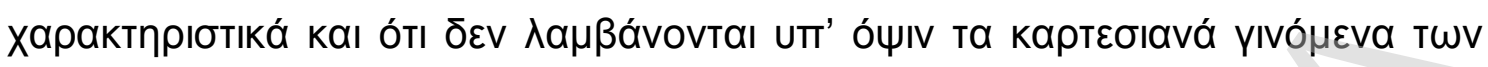

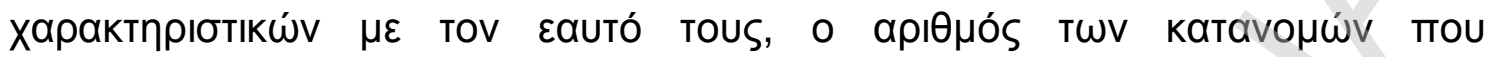

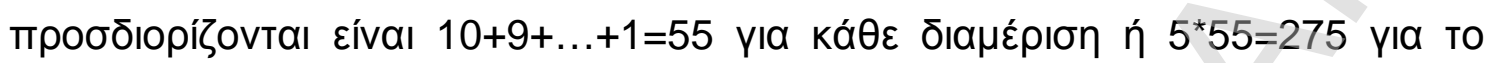

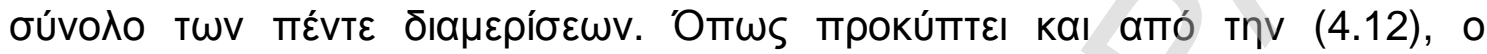

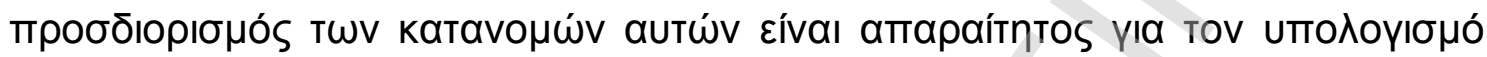

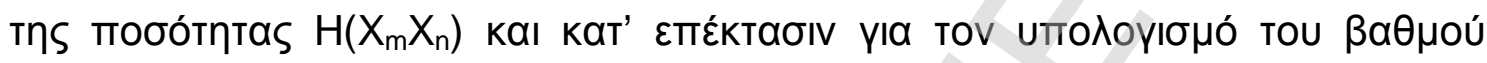

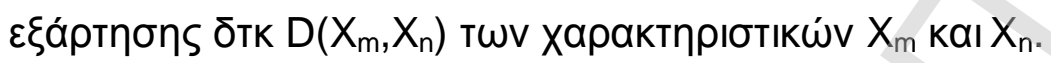

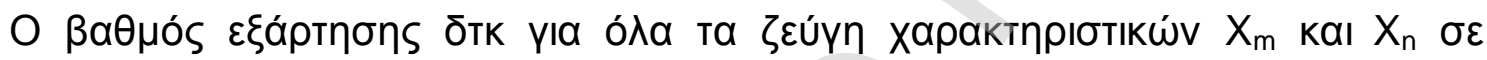

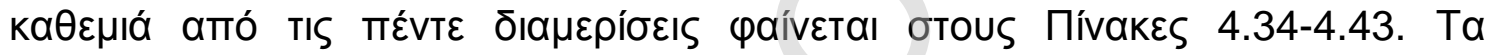

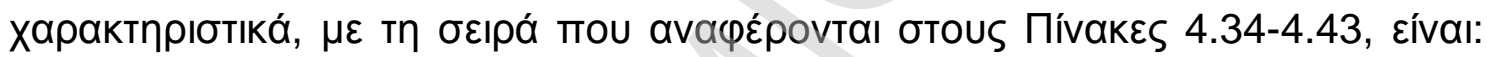

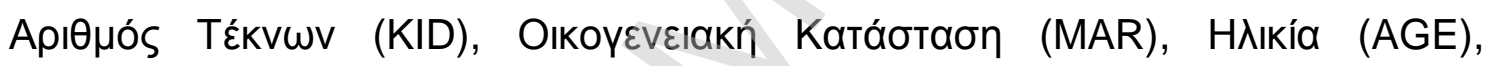

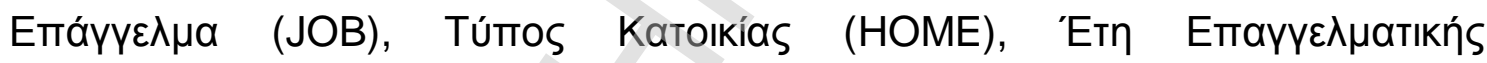

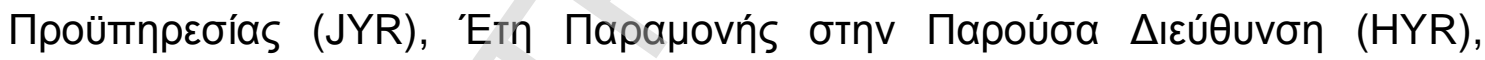

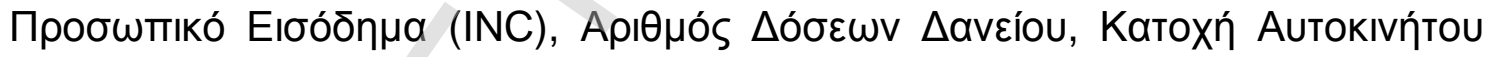

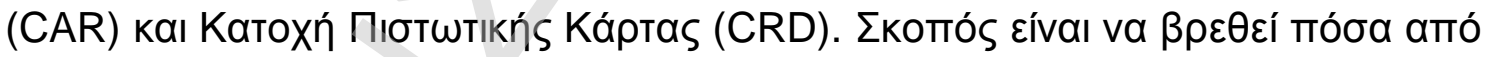

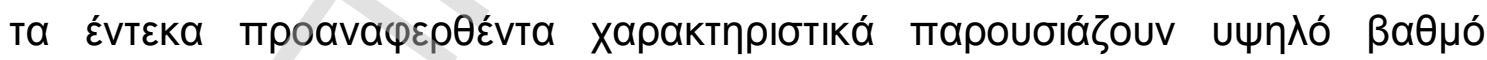

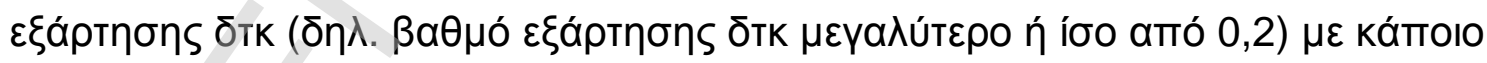

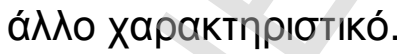


ПINAKA $\Sigma 4.34$

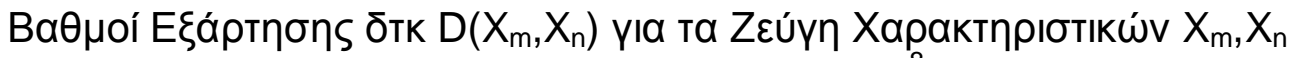

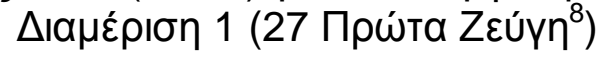

\begin{tabular}{|c|c|c|c|c|c|c|}
\hline$\alpha / \alpha$ & $\mathrm{X}_{\mathrm{m}}$ & $X_{n}$ & $\mathrm{H}\left(\mathrm{X}_{\mathrm{m}}\right)$ & $H\left(X_{n}\right)$ & $\mathrm{H}\left(\mathrm{X}_{\mathrm{m}} \mathrm{X}_{\mathrm{n}}\right)$ & $\mathrm{D}\left(\mathrm{X}_{\mathrm{m}}, \mathrm{X}_{\mathrm{n}}\right)$ \\
\hline 1 & $K I D$ & MAR & 1,69562 & 1,030451 & 2,241864 & $0,484207\left(^{*}\right)$ \\
\hline 2 & $A G E$ & $J O B$ & 1,354944 & 2,074391 & 3,117816 & $0,311519\left(^{*}\right)$ \\
\hline 3 & AGE & MAR & 1,354944 & 1,030451 & 2,095862 & $\left.0,289533^{*}\right)$ \\
\hline 4 & AGE & KID & 1,354944 & 1,69562 & 2,776811 & $0,273753\left(^{*}\right)$ \\
\hline 5 & HOME & MAR & 1,205649 & 1,030451 & 1,979822 & $0,256278\left(^{*}\right)$ \\
\hline 6 & AGE & $J Y R$ & 1,354944 & 1,766541 & 2,868479 & $0,253006\left(^{*}\right)$ \\
\hline 7 & AGE & HOME & 1,354944 & 1,205649 & 2,334095 & $0,226498\left(^{*}\right)$ \\
\hline 8 & KID & HOME & 1,69562 & 1,205649 & 2,67735 & $0,223919\left(^{*}\right)$ \\
\hline 9 & HYR & HOME & 1,624516 & 1,205649 & 2,607622 & $0,222543\left(^{*}\right)$ \\
\hline 10 & AGE & HYR & 1,354944 & 1,624516 & 2,785087 & 0,194373 \\
\hline 11 & JYR & MAR & 1,766541 & 1,030451 & 2,605441 & 0,191551 \\
\hline 12 & KID & JYR & 1,69562 & 1,766541 & 3,276577 & 0,185584 \\
\hline 13 & INC & JOB & 1,618169 & 2,074391 & 3,513939 & 0,178621 \\
\hline 14 & INC & JYR & 1,618169 & 1,766541 & 3,207693 & 0,177017 \\
\hline 15 & HOME & JYR & 1,205649 & 1,766541 & 2,799122 & 0,173068 \\
\hline 16 & JOB & MAR & 2,074391 & 1,030451 & 2,944913 & 0,159929 \\
\hline 17 & AGE & INC & 1,354944 & 1,618169 & 2,814824 & 0,158289 \\
\hline 18 & HOME & JOB & 1,205649 & 2,074391 & 3,12259 & 0,15745 \\
\hline 19 & JOB & JYR & 2,074391 & 1,766541 & 3,685408 & 0,155524 \\
\hline 20 & HYR & JOB & 1,624516 & 2,074391 & 3,543485 & 0,155422 \\
\hline 21 & INC & TRM & 1,618169 & 1,181596 & 2,648053 & 0,151712 \\
\hline 22 & INC & MAR & 1,618169 & 1,030451 & 2,499802 & 0,148818 \\
\hline 23 & HYR & MAR & 1,624516 & 1,030451 & 2,506314 & 0,148653 \\
\hline 24 & KID & HYR & 1,69562 & 1,624516 & 3,172916 & 0,14722 \\
\hline 25 & KID & INC & 1,69562 & 1,618169 & 3,168574 & 0,145215 \\
\hline 26 & CAR & INC & 0,826224 & 1,618169 & 2,299723 & 0,14467 \\
\hline 27 & CAR & JOB & 0,826224 & 2,074391 & 2,756416 & 0,144199 \\
\hline
\end{tabular}

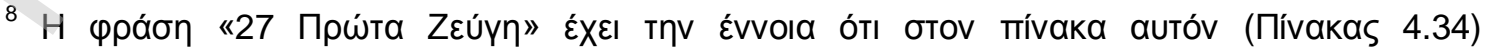

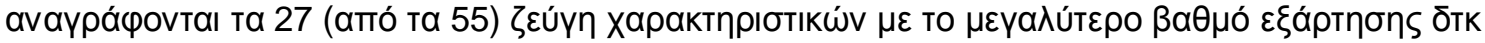

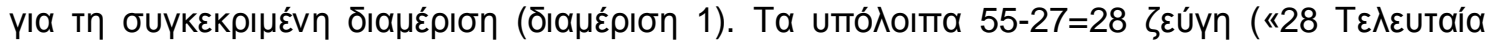

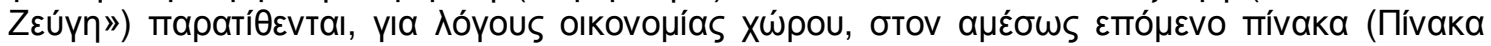
4.35). 
ПINAKA 24.35

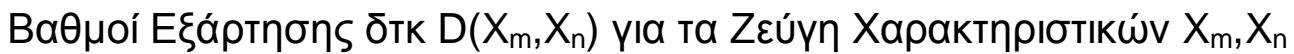

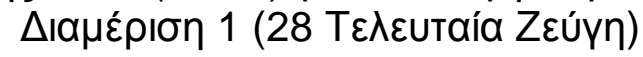

\begin{tabular}{|l|l|l|l|l|l|l|}
\hline$\alpha / \alpha$ & $\mathrm{X}_{\mathrm{m}}$ & $\mathrm{X}_{\mathrm{n}}$ & $\mathrm{H}\left(\mathrm{X}_{\mathrm{m}}\right)$ & $\mathrm{H}\left(\mathrm{X}_{n}\right)$ & $\mathrm{H}\left(\mathrm{X}_{\mathrm{m}} \mathrm{X}_{\mathrm{n}}\right)$ & $\mathrm{D}\left(\mathrm{X}_{\mathrm{m}}, \mathrm{X}_{\mathrm{n}}\right)$ \\
\hline 28 & KID & JOB & 1,69562 & 2,074391 & 3,626677 & 0,143334 \\
\hline 29 & AGE & CRD & 1,354944 & 0,925275 & 2,137662 & 0,142557 \\
\hline 30 & CAR & MAR & 0,826224 & 1,030451 & 1,714178 & 0,142497 \\
\hline 31 & CAR & KID & 0,826224 & 1,69562 & 2,379949 & 0,141895 \\
\hline 32 & HYR & JYR & 1,624516 & 1,766541 & 3,249327 & 0,14173 \\
\hline 33 & HOME & INC & 1,205649 & 1,618169 & 2,68247 & 0,141348 \\
\hline 34 & CRD & JOB & 0,925275 & 2,074391 & 2,859905 & 0,139761 \\
\hline 35 & MAR & TRM & 1,030451 & 1,181596 & 2,072639 & 0,139408 \\
\hline 36 & HOME & TRM & 1,205649 & 1,181596 & 2,249001 & 0,138244 \\
\hline 37 & AGE & TRM & 1,354944 & 1,181596 & 2,399321 & 0,137219 \\
\hline 38 & CAR & JYR & 0,826224 & 1,766541 & 2,455718 & 0,137047 \\
\hline 39 & KID & TRM & 1,69562 & 1,181596 & 2,7407 & 0,136516 \\
\hline 40 & CRD & MAR & 0,925275 & 1,030451 & 1,819912 & 0,135814 \\
\hline 41 & JYR & TRM & 1,766541 & 1,181596 & 2,812694 & 0,135443 \\
\hline 42 & CRD & KID & 0,925275 & 1,69562 & 2,485552 & 0,135343 \\
\hline 43 & CAR & HOME & 0,826224 & 1,205649 & 1,896973 & 0,1349 \\
\hline 44 & AGE & CAR & 1,354944 & 0,826224 & 2,046332 & 0,134836 \\
\hline 45 & CAR & CRD & 0,826224 & 0,925275 & 1,616986 & 0,134513 \\
\hline 46 & CAR & TRM & 0,826224 & 1,181596 & 1,87339 & 0,13443 \\
\hline 47 & JOB & TRM & 2,074391 & 1,181596 & 3,121873 & 0,134114 \\
\hline 48 & CRD & INC & 0,925275 & 1,618169 & 2,409781 & 0,133663 \\
\hline 49 & CRD & HOME & 0,925275 & 1,205649 & 1,997489 & 0,133435 \\
\hline 50 & CRD & TRM & 0,925275 & 1,181596 & 1,973443 & 0,133428 \\
\hline 51 & CRD & JYR & 0,925275 & 1,766541 & 2,55861 & 0,133206 \\
\hline 52 & CRD & HYR & 0,925275 & 1,624516 & 2,417238 & 0,132553 \\
\hline 53 & CXR & HYR & 0,826224 & 1,624516 & 2,318306 & 0,132434 \\
\hline 54 & HYR & INC & 1,624516 & 1,618169 & 3,110895 & 0,13179 \\
\hline 55 & HYR & TRM & 1,624516 & 1,181596 & 2,67547 & 0,130642 \\
\hline & & & & & & \\
\hline
\end{tabular}


ПINAKA $\Sigma 4.36$

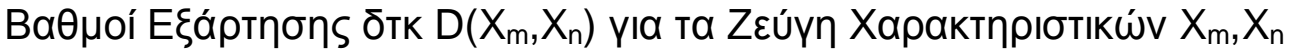

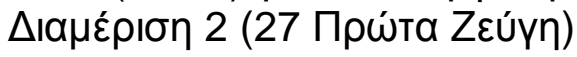

\begin{tabular}{|c|c|c|c|c|c|c|}
\hline$\alpha / \alpha$ & $\mathrm{X}_{\mathrm{m}}$ & $X_{n}$ & $\mathrm{H}\left(\mathrm{X}_{\mathrm{m}}\right)$ & $\mathrm{H}\left(\mathrm{X}_{n}\right)$ & $\mathrm{H}\left(\mathrm{X}_{\mathrm{m}} \mathrm{X}_{\mathrm{n}}\right)$ & $\mathrm{D}\left(\mathrm{X}_{\mathrm{m}}, \mathrm{X}_{\mathrm{n}}\right)$ \\
\hline 1 & $K I D$ & $M A R$ & 1,700714 & 1,044764 & 2,259167 & $0,486311\left(^{*}\right)$ \\
\hline 2 & $A G E$ & $J O B$ & 1,353642 & 2,078632 & 3,110058 & $0,322216\left(^{*}\right)$ \\
\hline 3 & AGE & MAR & 1,353642 & 1,044764 & 2,095785 & $0,302621\left(^{*}\right)$ \\
\hline 4 & AGE & KID & 1,353642 & 1,700714 & 2,782454 & $\left.0,271902^{*}\right)$ \\
\hline 5 & HOME & MAR & 1,212124 & 1,044764 & 1,987988 & $0,268900\left(^{*}\right)$ \\
\hline 6 & AGE & JYR & 1,353642 & 1,752199 & 2,850704 & $0,255137\left(^{*}\right)$ \\
\hline 7 & $H Y R$ & HOME & 1,616312 & 1,212124 & 2,598424 & $0,230012\left(^{*}\right)$ \\
\hline 8 & $\mathrm{KID}$ & HOME & 1,700714 & 1,212124 & 2,685862 & $0,226976\left(^{*}\right)$ \\
\hline 9 & AGE & HOME & 1,353642 & 1,212124 & 2,340458 & $0,225308\left(^{*}\right)$ \\
\hline 10 & AGE & HYR & 1,353642 & 1,616312 & 2,772713 & 0,197241 \\
\hline 11 & JYR & MAR & 1,752199 & 1,044764 & 2,601481 & 0,195482 \\
\hline 12 & KID & JYR & 1,700714 & 1,752199 & 3,266239 & 0,186674 \\
\hline 13 & INC & JYR & 1,615021 & 1,752199 & 3,194672 & 0,172548 \\
\hline 14 & HOME & JYR & 1,212124 & 1,752199 & 2,793248 & 0,171075 \\
\hline 15 & INC & $\mathrm{JOB}$ & 1,615021 & 2,078632 & 3,522951 & 0,170702 \\
\hline 16 & JOB & MAR & 2,078632 & 1,044764 & 2,957048 & 0,166348 \\
\hline 17 & HOME & $\mathrm{JOB}$ & 1,212124 & 2,078632 & 3,131317 & 0,159439 \\
\hline 18 & AGE & INC & 1,353642 & 1,615021 & 2,811308 & 0,157355 \\
\hline 19 & JOB & JYR & 2,078632 & 1,752199 & 3,67387 & 0,156961 \\
\hline 20 & HYR & JOB & 1,616312 & 2,078632 & 3,540859 & 0,154085 \\
\hline 21 & INC & TRM & 1,615021 & 1,166303 & 2,627355 & 0,153969 \\
\hline 22 & HYR & MAR & 1,616312 & 1,044764 & 2,50824 & 0,152836 \\
\hline 23 & INC & MAR & 1,615021 & 1,044764 & 2,509386 & 0,150399 \\
\hline 24 & $\mathrm{KID}$ & HYR & 1,700714 & 1,616312 & 3,169332 & 0,147694 \\
\hline 25 & KID & JOB & 1,700714 & 2,078632 & 3,634207 & 0,145139 \\
\hline 26 & KID & INC & 1,700714 & 1,615021 & 3,17119 & 0,144545 \\
\hline 27 & CAR & INC & 0,833109 & 1,615021 & 2,30398 & 0,14415 \\
\hline
\end{tabular}


ПINAKA $\Sigma$ 4.37

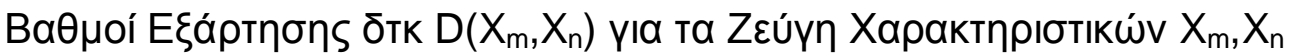

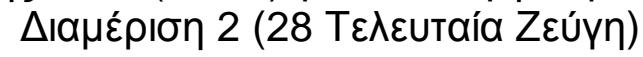

\begin{tabular}{|c|c|c|c|c|c|c|}
\hline$\alpha / \alpha$ & $X_{m}$ & $X_{n}$ & $\mathrm{H}\left(\mathrm{X}_{\mathrm{m}}\right)$ & $\mathrm{H}\left(\mathrm{X}_{n}\right)$ & $\mathrm{H}\left(\mathrm{X}_{\mathrm{m}} \mathrm{X}_{\mathrm{n}}\right)$ & $\mathrm{D}\left(\mathrm{X}_{\mathrm{m}}, \mathrm{X}_{\mathrm{n}}\right)$ \\
\hline 28 & CAR & $\mathrm{JOB}$ & 0,833109 & 2,078632 & 2,768171 & 0,14357 \\
\hline 29 & HOME & INC & 1,212124 & 1,615021 & 2,684559 & 0,142586 \\
\hline 30 & MAR & TRM & 1,044764 & 1,166303 & 2,069121 & 0,141946 \\
\hline 31 & CAR & KID & 0,833109 & 1,700714 & 2,392802 & 0,141021 \\
\hline 32 & AGE & CRD & 1,353642 & 0,920465 & 2,133634 & 0,140473 \\
\hline 33 & HOME & TRM & 1,212124 & 1,166303 & 2,238864 & 0,139563 \\
\hline 34 & CAR & MAR & 0,833109 & 1,044764 & 1,738586 & 0,139287 \\
\hline 35 & HYR & JYR & 1,616312 & 1,752199 & 3,229307 & 0,139204 \\
\hline 36 & KID & TRM & 1,700714 & 1,166303 & 2,729414 & 0,137603 \\
\hline 37 & CRD & $\mathrm{JOB}$ & 0,920465 & 2,078632 & 2,861726 & 0,137371 \\
\hline 38 & AGE & TRM & 1,353642 & 1,166303 & 2,383525 & 0,13642 \\
\hline 39 & CAR & JYR & 0,833109 & 1,752199 & 2,448941 & 0,136367 \\
\hline 40 & CRD & INC & 0,920465 & 1,615021 & 2,399601 & 0,135885 \\
\hline 41 & CRD & MAR & 0,920465 & 1,044764 & 1,829825 & 0,135404 \\
\hline 42 & CAR & TRM & 0,833109 & 1,166303 & 1,864046 & 0,135366 \\
\hline 43 & CRD & KID & 0,920465 & 1,700714 & 2,486326 & 0,134853 \\
\hline 44 & CAR & CRD & 0,833109 & 0,920465 & 1,618942 & 0,134632 \\
\hline 45 & JYR & TRM & 1,752199 & 1,166303 & 2,78398 & 0,134522 \\
\hline 46 & AGE & CAR & 1,353642 & 0,833109 & 2,05241 & 0,134341 \\
\hline 47 & CAR & HOME & 0,833109 & 1,212124 & 1,911154 & 0,134079 \\
\hline 48 & CRD & JYR & 0,920465 & 1,752199 & 2,539187 & 0,133477 \\
\hline 49 & CRD & HOME & 0,920465 & 1,212124 & 1,999193 & 0,133396 \\
\hline 50 & CRD & TRM & 0,920465 & 1,166303 & 1,953474 & 0,133294 \\
\hline 51 & CRD & HYR & 0,920465 & 1,616312 & 2,404073 & 0,132704 \\
\hline 52 & CAR & HYR & 0,833109 & 1,616312 & 2,317091 & 0,13233 \\
\hline 53 & JOB & TRM & 2,078632 & 1,166303 & 3,113209 & 0,131726 \\
\hline 54 & HYR & INC & 1,616312 & 1,615021 & 3,100057 & 0,131276 \\
\hline 55 & HYR & TRM & 1,616312 & 1,166303 & 2,65193 & 0,130685 \\
\hline
\end{tabular}


ПINAKA $\Sigma 4.38$

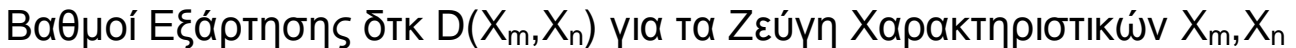

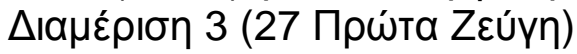

\begin{tabular}{|c|c|c|c|c|c|c|}
\hline$\alpha / \alpha$ & $X_{m}$ & $X_{n}$ & $\mathrm{H}\left(\mathrm{X}_{\mathrm{m}}\right)$ & $\mathrm{H}\left(\mathrm{X}_{n}\right)$ & $\mathrm{H}\left(\mathrm{X}_{\mathrm{m}} \mathrm{X}_{\mathrm{n}}\right)$ & $\mathrm{D}\left(\mathrm{X}_{\mathrm{m}}, \mathrm{X}_{\mathrm{n}}\right)$ \\
\hline 1 & $K I D$ & $M A R$ & 1,70084 & 1,033108 & 2,242995 & $0,490953\left(^{*}\right)$ \\
\hline 2 & $A G E$ & $J O B$ & 1,350536 & 2,085628 & 3,111418 & $0,324746\left(^{*}\right)$ \\
\hline 3 & AGE & MAR & 1,350536 & 1,033108 & 2,086335 & $0,297309\left(^{*}\right)$ \\
\hline 4 & AGE & KID & 1,350536 & 1,70084 & 2,768719 & $0,282657\left(^{*}\right)$ \\
\hline 5 & HOME & MAR & 1,205464 & 1,033108 & 1,969524 & $0,269048\left(^{*}\right)$ \\
\hline 6 & AGE & JYR & 1,350536 & 1,75127 & 2,842057 & $0,259749\left(^{*}\right)$ \\
\hline 7 & KID & HOME & 1,70084 & 1,205464 & 2,677225 & $0,229079\left(^{*}\right)$ \\
\hline 8 & AGE & HOME & 1,350536 & 1,205464 & 2,329021 & $0,226979\left(^{*}\right)$ \\
\hline 9 & $H Y R$ & HOME & 1,626166 & 1,205464 & 2,608026 & $0,223604\left(^{*}\right)$ \\
\hline 10 & JYR & MAR & 1,75127 & 1,033108 & 2,587421 & 0,196957 \\
\hline 11 & AGE & HYR & 1,350536 & 1,626166 & 2,780515 & 0,196187 \\
\hline 12 & KID & JYR & 1,70084 & 1,75127 & 3,261323 & 0,190787 \\
\hline 13 & HOME & JYR & 1,205464 & 1,75127 & 2,78033 & 0,176404 \\
\hline 14 & INC & JYR & 1,612564 & 1,75127 & 3,19043 & 0,173404 \\
\hline 15 & INC & $\mathrm{JOB}$ & 1,612564 & 2,085628 & 3,525071 & 0,173121 \\
\hline 16 & JOB & MAR & 2,085628 & 1,033108 & 2,952743 & 0,165993 \\
\hline 17 & AGE & INC & 1,350536 & 1,612564 & 2,7989 & 0,1642 \\
\hline 18 & HOME & JOB & 1,205464 & 2,085628 & 3,132637 & 0,158455 \\
\hline 19 & INC & TRM & 1,612564 & 1,151373 & 2,607297 & 0,15664 \\
\hline 20 & HYR & JOB & 1,626166 & 2,085628 & 3,556341 & 0,155453 \\
\hline 21 & JOB & JYR & 2,085628 & 1,75127 & 3,683268 & 0,15363 \\
\hline 22 & INC & MAR & 1,612564 & 1,033108 & 2,492496 & 0,153176 \\
\hline 23 & HYR & MAR & 1,626166 & 1,033108 & 2,50914 & 0,150134 \\
\hline 24 & KID & HYR & 1,70084 & 1,626166 & 3,178223 & 0,148783 \\
\hline 25 & KID & INC & 1,70084 & 1,612564 & 3,165543 & 0,147861 \\
\hline 26 & $\mathrm{KID}$ & JOB & 1,70084 & 2,085628 & 3,639737 & 0,146731 \\
\hline 27 & CAR & INC & 0,823866 & 1,612564 & 2,28984 & 0,14659 \\
\hline
\end{tabular}


ПINAKA 5.39

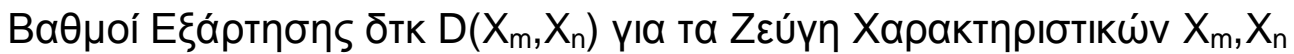

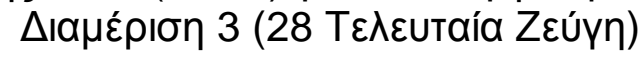

\begin{tabular}{|c|c|c|c|c|c|c|}
\hline$\alpha / \alpha$ & $X_{m}$ & $X_{n}$ & $\mathrm{H}\left(\mathrm{X}_{\mathrm{m}}\right)$ & $\mathrm{H}\left(\mathrm{X}_{n}\right)$ & $\mathrm{H}\left(\mathrm{X}_{\mathrm{m}} \mathrm{X}_{\mathrm{n}}\right)$ & $\mathrm{D}\left(\mathrm{X}_{\mathrm{m}}, \mathrm{X}_{\mathrm{n}}\right)$ \\
\hline 28 & CAR & $\mathrm{JOB}$ & 0,823866 & 2,085628 & 2,763198 & 0,146296 \\
\hline 29 & HOME & INC & 1,205464 & 1,612564 & 2,673486 & 0,144542 \\
\hline 30 & MAR & TRM & 1,033108 & 1,151373 & 2,041028 & 0,143453 \\
\hline 31 & CAR & MAR & 0,823866 & 1,033108 & 1,716058 & 0,140916 \\
\hline 32 & CAR & KID & 0,823866 & 1,70084 & 2,38458 & 0,140126 \\
\hline 33 & AGE & CRD & 1,350536 & 0,923454 & 2,133901 & 0,140089 \\
\hline 34 & HOME & TRM & 1,205464 & 1,151373 & 2,216824 & 0,140013 \\
\hline 35 & AGE & TRM & 1,350536 & 1,151373 & 2,363061 & 0,138848 \\
\hline 36 & HYR & JYR & 1,626166 & 1,75127 & 3,239264 & 0,138172 \\
\hline 37 & KID & TRM & 1,70084 & 1,151373 & 2,714045 & 0,138168 \\
\hline 38 & CRD & $\mathrm{JOB}$ & 0,923454 & 2,085628 & 2,873061 & 0,136021 \\
\hline 39 & JYR & TRM & 1,75127 & 1,151373 & 2,767181 & 0,135462 \\
\hline 40 & CAR & JYR & 0,823866 & 1,75127 & 2,439752 & 0,135384 \\
\hline 41 & AGE & CAR & 1,350536 & 0,823866 & 2,039047 & 0,135355 \\
\hline 42 & CRD & MAR & 0,923454 & 1,033108 & 1,821222 & 0,13534 \\
\hline 43 & CRD & JYR & 0,923454 & 1,75127 & 2,539831 & 0,134893 \\
\hline 44 & CRD & KID & 0,923454 & 1,70084 & 2,489601 & 0,134693 \\
\hline 45 & CAR & CRD & 0,823866 & 0,923454 & 1,612736 & 0,134584 \\
\hline 46 & CAR & TRM & 0,823866 & 1,151373 & 1,840705 & 0,134534 \\
\hline 47 & CRD & INC & 0,923454 & 1,612564 & 2,401498 & 0,13452 \\
\hline 48 & CAR & HOME & 0,823866 & 1,205464 & 1,894895 & 0,134435 \\
\hline 49 & JOB & TRM & 2,085628 & 1,151373 & 3,102672 & 0,134329 \\
\hline 50 & CRD & HOME & 0,923454 & 1,205464 & 1,995157 & 0,133761 \\
\hline 51 & CRD & TRM & 0,923454 & 1,151373 & 1,941413 & 0,133414 \\
\hline 52 & CAR & HYR & 0,823866 & 1,626166 & 2,31667 & 0,133362 \\
\hline 53 & CRD & HYR & 0,923454 & 1,626166 & 2,416696 & 0,132924 \\
\hline 54 & HYR & INC & 1,626166 & 1,612564 & 3,108203 & 0,130527 \\
\hline 55 & HYR & TRM & 1,626166 & 1,151373 & 2,647111 & 0,130428 \\
\hline
\end{tabular}


ПINAKA $\Sigma 4.40$

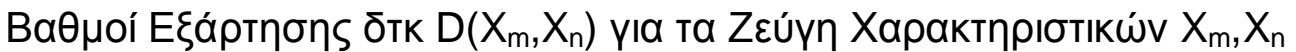

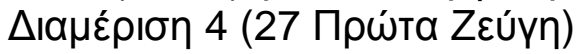

\begin{tabular}{|c|c|c|c|c|c|c|}
\hline$\alpha / \alpha$ & $X_{m}$ & $X_{n}$ & $\mathrm{H}\left(\mathrm{X}_{\mathrm{m}}\right)$ & $H\left(X_{n}\right)$ & $\mathrm{H}\left(\mathrm{X}_{\mathrm{m}} \mathrm{X}_{\mathrm{n}}\right)$ & $\mathrm{D}\left(\mathrm{X}_{\mathrm{m}}, \mathrm{X}_{\mathrm{n}}\right)$ \\
\hline 1 & $K I D$ & $M A R$ & 1,692636 & 1,037762 & 2,246326 & $0,484072\left(^{*}\right)$ \\
\hline 2 & $A G E$ & $J O B$ & 1,269931 & 2,100319 & 3,06169 & $0,308560\left(^{*}\right)$ \\
\hline 3 & AGE & MAR & 1,269931 & 1,037762 & 2,009937 & $0,297756\left(^{*}\right)$ \\
\hline 4 & AGE & KID & 1,269931 & 1,692636 & 2,687254 & $0,275313\left(^{*}\right)$ \\
\hline 5 & HOME & MAR & 1,209836 & 1,037762 & 1,97883 & $0,268768\left(^{*}\right)$ \\
\hline 6 & AGE & JYR & 1,269931 & 1,714898 & 2,730614 & $0,254215\left(^{*}\right)$ \\
\hline 7 & $H Y R$ & HOME & 1,623746 & 1,209836 & 2,603059 & $0,230523\left(^{*}\right)$ \\
\hline 8 & $\mathrm{KID}$ & HOME & 1,692636 & 1,209836 & 2,674903 & $0,227569\left(^{*}\right)$ \\
\hline 9 & AGE & HOME & 1,269931 & 1,209836 & 2,256098 & $0,223669\left(^{*}\right)$ \\
\hline 10 & JYR & MAR & 1,714898 & 1,037762 & 2,555216 & 0,197444 \\
\hline 11 & AGE & HYR & 1,269931 & 1,623746 & 2,703067 & 0,19061 \\
\hline 12 & KID & JYR & 1,692636 & 1,714898 & 3,219719 & 0,187815 \\
\hline 13 & INC & JOB & 1,613127 & 2,100319 & 3,532337 & 0,181109 \\
\hline 14 & HOME & JYR & 1,209836 & 1,714898 & 2,748939 & 0,175795 \\
\hline 15 & INC & JYR & 1,613127 & 1,714898 & 3,153948 & 0,174077 \\
\hline 16 & JOB & MAR & 2,100319 & 1,037762 & 2,973221 & 0,16486 \\
\hline 17 & $\mathrm{JOB}$ & JYR & 2,100319 & 1,714898 & 3,652843 & 0,162374 \\
\hline 18 & HOME & $\mathrm{JOB}$ & 1,209836 & 2,100319 & 3,14978 & 0,160375 \\
\hline 19 & AGE & INC & 1,269931 & 1,613127 & 2,722794 & 0,160264 \\
\hline 20 & INC & TRM & 1,613127 & 1,13571 & 2,593046 & 0,155791 \\
\hline 21 & INC & MAR & 1,613127 & 1,037762 & 2,498043 & 0,152846 \\
\hline 22 & HYR & JOB & 1,623746 & 2,100319 & 3,572234 & 0,151831 \\
\hline 23 & HYR & MAR & 1,623746 & 1,037762 & 2,512323 & 0,149185 \\
\hline 24 & $\mathrm{KID}$ & INC & 1,692636 & 1,613127 & 3,156981 & 0,148782 \\
\hline 25 & KID & HYR & 1,692636 & 1,623746 & 3,167868 & 0,148514 \\
\hline 26 & CAR & $\mathrm{JOB}$ & 0,838518 & 2,100319 & 2,792958 & 0,145879 \\
\hline 27 & KID & JOB & 1,692636 & 2,100319 & 3,647745 & 0,14521 \\
\hline
\end{tabular}


ПINAKA $\Sigma 4.41$

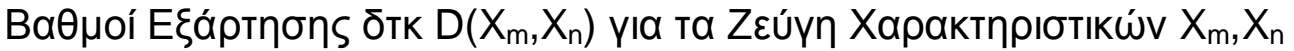

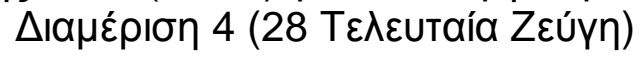

\begin{tabular}{|c|c|c|c|c|c|c|}
\hline$\alpha / \alpha$ & $X_{m}$ & $X_{n}$ & $\mathrm{H}\left(\mathrm{X}_{\mathrm{m}}\right)$ & $\mathrm{H}\left(\mathrm{X}_{\mathrm{n}}\right)$ & $\mathrm{H}\left(\mathrm{X}_{\mathrm{m}} \mathrm{X}_{\mathrm{n}}\right)$ & $\mathrm{D}\left(\mathrm{X}_{\mathrm{m}}, \mathrm{X}_{\mathrm{n}}\right)$ \\
\hline 28 & HOME & INC & 1,209836 & 1,613127 & 2,67868 & 0,144283 \\
\hline 29 & CAR & INC & 0,838518 & 1,613127 & 2,307504 & 0,144141 \\
\hline 30 & MAR & TRM & 1,037762 & 1,13571 & 2,030453 & 0,143019 \\
\hline 31 & CAR & MAR & 0,838518 & 1,037762 & 1,733503 & 0,142777 \\
\hline 32 & CAR & $\mathrm{KID}$ & 0,838518 & 1,692636 & 2,38977 & 0,141384 \\
\hline 33 & HOME & TRM & 1,209836 & 1,13571 & 2,204809 & 0,140737 \\
\hline 34 & AGE & CRD & 1,269931 & 0,925468 & 2,055588 & 0,139811 \\
\hline 35 & $\mathrm{KID}$ & TRM & 1,692636 & 1,13571 & 2,689531 & 0,138815 \\
\hline 36 & HYR & JYR & 1,623746 & 1,714898 & 3,200068 & 0,138576 \\
\hline 37 & AGE & TRM & 1,269931 & 1,13571 & 2,267118 & 0,138523 \\
\hline 38 & CRD & $\mathrm{JOB}$ & 0,925468 & 2,100319 & 2,887319 & 0,138468 \\
\hline 39 & CRD & INC & 0,925468 & 1,613127 & 2,402414 & 0,136181 \\
\hline 40 & CRD & MAR & 0,925468 & 1,037762 & 1,82722 & 0,13601 \\
\hline 41 & CAR & JYR & 0,838518 & 1,714898 & 2,417684 & 0,135732 \\
\hline 42 & JYR & TRM & 1,714898 & 1,13571 & 2,714989 & 0,135619 \\
\hline 43 & CAR & HOME & 0,838518 & 1,209836 & 1,912966 & 0,135388 \\
\hline 44 & CAR & TRM & 0,838518 & 1,13571 & 1,839265 & 0,134963 \\
\hline 45 & AGE & CAR & 1,269931 & 0,838518 & 1,973561 & 0,134888 \\
\hline 46 & CAR & CRD & 0,838518 & 0,925468 & 1,629412 & 0,134574 \\
\hline 47 & CRD & $\mathrm{KID}$ & 0,925468 & 1,692636 & 2,483686 & 0,134418 \\
\hline 48 & CRD & HOME & 0,925468 & 1,209836 & 2,001057 & 0,134247 \\
\hline 49 & CRD & TRM & 0,925468 & 1,13571 & 1,927027 & 0,134151 \\
\hline 50 & $\mathrm{JOB}$ & TRM & 2,100319 & 1,13571 & 3,102537 & 0,133492 \\
\hline 51 & CRD & JYR & 0,925468 & 1,714898 & 2,506952 & 0,133414 \\
\hline 52 & CRD & HYR & 0,925468 & 1,623746 & 2,416923 & 0,132291 \\
\hline 53 & CAR & HYR & 0,838518 & 1,623746 & 2,330004 & 0,13226 \\
\hline 54 & HYR & INC & 1,623746 & 1,613127 & 3,105694 & 0,131179 \\
\hline 55 & HYR & TRM & 1,623746 & 1,13571 & 2,629004 & 0,130452 \\
\hline
\end{tabular}


ПINAKA $\Sigma 4.42$

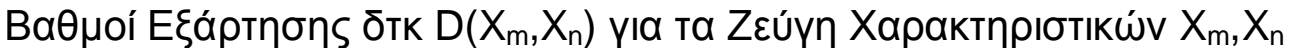

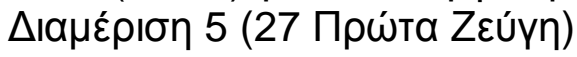

\begin{tabular}{|c|c|c|c|c|c|c|}
\hline$\alpha / \alpha$ & $X_{m}$ & $X_{n}$ & $\mathrm{H}\left(\mathrm{X}_{\mathrm{m}}\right)$ & $\mathrm{H}\left(\mathrm{X}_{n}\right)$ & $\mathrm{H}\left(\mathrm{X}_{\mathrm{m}} \mathrm{X}_{\mathrm{n}}\right)$ & $\mathrm{D}\left(\mathrm{X}_{\mathrm{m}}, \mathrm{X}_{\mathrm{n}}\right)$ \\
\hline 1 & $K I D$ & $M A R$ & 1,697113 & 1,020545 & 2,245852 & $0,471806\left(^{*}\right)$ \\
\hline 2 & $A G E$ & $J O B$ & 1,358452 & 2,079563 & 3,118547 & $0,319468\left(^{*}\right)$ \\
\hline 3 & AGE & MAR & 1,358452 & 1,020545 & 2,081146 & $0,297851\left(^{*}\right)$ \\
\hline 4 & AGE & KID & 1,358452 & 1,697113 & 2,779991 & $0,275574\left(^{*}\right)$ \\
\hline 5 & HOME & MAR & 1,204648 & 1,020545 & 1,962661 & $0,262532\left(^{*}\right)$ \\
\hline 6 & AGE & $J Y R$ & 1,358452 & 1,765009 & 2,869129 & $0,254332\left(^{*}\right)$ \\
\hline 7 & $H Y R$ & HOME & 1,631839 & 1,204648 & 2,606065 & $0,230422\left(^{*}\right)$ \\
\hline 8 & AGE & HOME & 1,358452 & 1,204648 & 2,336275 & $0,226825\left(^{*}\right)$ \\
\hline 9 & KID & HOME & 1,697113 & 1,204648 & 2,676509 & $0,225252\left(^{*}\right)$ \\
\hline 10 & AGE & HYR & 1,358452 & 1,631839 & 2,792078 & 0,198213 \\
\hline 11 & JYR & MAR & 1,765009 & 1,020545 & 2,594635 & 0,190919 \\
\hline 12 & KID & JYR & 1,697113 & 1,765009 & 3,276088 & 0,186034 \\
\hline 13 & INC & JYR & 1,614189 & 1,765009 & 3,203096 & 0,176102 \\
\hline 14 & INC & JOB & 1,614189 & 2,079563 & 3,523514 & 0,170238 \\
\hline 15 & HOME & JYR & 1,204648 & 1,765009 & 2,800373 & 0,169284 \\
\hline 16 & JOB & MAR & 2,079563 & 1,020545 & 2,935267 & 0,164841 \\
\hline 17 & HOME & JOB & 1,204648 & 2,079563 & 3,123684 & 0,160527 \\
\hline 18 & HYR & $\mathrm{JOB}$ & 1,631839 & 2,079563 & 3,551302 & 0,1601 \\
\hline 19 & AGE & INC & 1,358452 & 1,614189 & 2,816239 & 0,156402 \\
\hline 20 & $\mathrm{JOB}$ & JYR & 2,079563 & 1,765009 & 3,689743 & 0,154829 \\
\hline 21 & INC & TRM & 1,614189 & 1,131362 & 2,593856 & 0,151695 \\
\hline 22 & KID & HYR & 1,697113 & 1,631839 & 3,1794 & 0,149552 \\
\hline 23 & HYR & MAR & 1,631839 & 1,020545 & 2,50362 & 0,148764 \\
\hline 24 & INC & MAR & 1,614189 & 1,020545 & 2,486589 & 0,148145 \\
\hline 25 & CAR & $\mathrm{JOB}$ & 0,825909 & 2,079563 & 2,761168 & 0,144304 \\
\hline 26 & CAR & INC & 0,825909 & 1,614189 & 2,295989 & 0,144109 \\
\hline 27 & KID & $\mathrm{JOB}$ & 1,697113 & 2,079563 & 3,632865 & 0,143811 \\
\hline
\end{tabular}


ПINAKA $\Sigma 4.43$

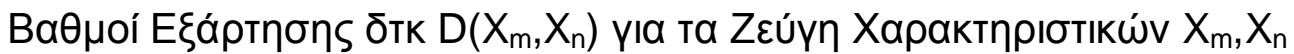

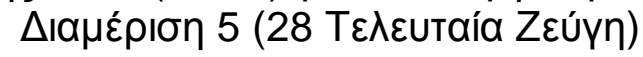

\begin{tabular}{|c|c|c|c|c|c|c|}
\hline$\alpha / \alpha$ & $X_{m}$ & $X_{n}$ & $\mathrm{H}\left(\mathrm{X}_{\mathrm{m}}\right)$ & $\mathrm{H}\left(\mathrm{X}_{n}\right)$ & $\mathrm{H}\left(\mathrm{X}_{\mathrm{m}} \mathrm{X}_{\mathrm{n}}\right)$ & $\mathrm{D}\left(\mathrm{X}_{\mathrm{m}}, \mathrm{X}_{\mathrm{n}}\right)$ \\
\hline 28 & KID & INC & 1,697113 & 1,614189 & 3,167675 & 0,143627 \\
\hline 29 & AGE & CRD & 1,358452 & 0,922751 & 2,137944 & 0,143259 \\
\hline 30 & HOME & INC & 1,204648 & 1,614189 & 2,677726 & 0,141111 \\
\hline 31 & MAR & TRM & 1,020545 & 1,131362 & 2,011171 & 0,140736 \\
\hline 32 & HYR & JYR & 1,631839 & 1,765009 & 3,256865 & 0,139983 \\
\hline 33 & CAR & KID & 0,825909 & 1,697113 & 2,383335 & 0,139687 \\
\hline 34 & HOME & TRM & 1,204648 & 1,131362 & 2,196354 & 0,139656 \\
\hline 35 & CAR & MAR & 0,825909 & 1,020545 & 1,706928 & 0,139526 \\
\hline 36 & CRD & $\mathrm{JOB}$ & 0,922751 & 2,079563 & 2,864708 & 0,137606 \\
\hline 37 & CRD & MAR & 0,922751 & 1,020545 & 1,806006 & 0,13729 \\
\hline 38 & AGE & TRM & 1,358452 & 1,131362 & 2,352793 & 0,137021 \\
\hline 39 & JYR & TRM & 1,765009 & 1,131362 & 2,759753 & 0,136618 \\
\hline 40 & KID & TRM & 1,697113 & 1,131362 & 2,692021 & 0,136454 \\
\hline 41 & CRD & KID & 0,922751 & 1,697113 & 2,483568 & 0,136296 \\
\hline 42 & CAR & JYR & 0,825909 & 1,765009 & 2,45553 & 0,135388 \\
\hline 43 & CAR & CRD & 0,825909 & 0,922751 & 1,613921 & 0,134739 \\
\hline 44 & AGE & CAR & 1,358452 & 0,825909 & 2,049738 & 0,134623 \\
\hline 45 & CAR & HOME & 0,825909 & 1,204648 & 1,896189 & 0,134368 \\
\hline 46 & CAR & TRM & 0,825909 & 1,131362 & 1,823121 & 0,13415 \\
\hline 47 & CRD & JYR & 0,922751 & 1,765009 & 2,553758 & 0,134002 \\
\hline 48 & CRD & HOME & 0,922751 & 1,204648 & 1,993422 & 0,133977 \\
\hline 49 & CRD & INC & 0,922751 & 1,614189 & 2,403064 & 0,133876 \\
\hline 50 & CRD & TRM & 0,922751 & 1,131362 & 1,920551 & 0,133562 \\
\hline 51 & JOB & TRM & 2,079563 & 1,131362 & 3,077812 & 0,133113 \\
\hline 52 & CRD & HYR & 0,922751 & 1,631839 & 2,421903 & 0,132687 \\
\hline 53 & CAR & HYR & 0,825909 & 1,631839 & 2,325263 & 0,132485 \\
\hline 54 & HYR & INC & 1,631839 & 1,614189 & 3,114653 & 0,131375 \\
\hline 55 & HYR & TRM & 1,631839 & 1,131362 & 2,632894 & 0,130307 \\
\hline
\end{tabular}




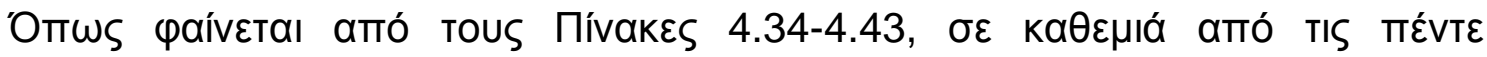

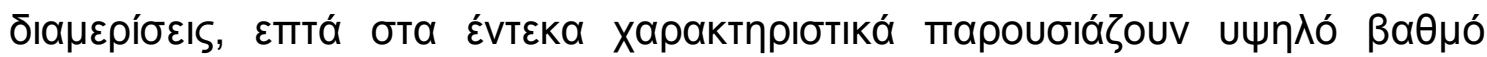

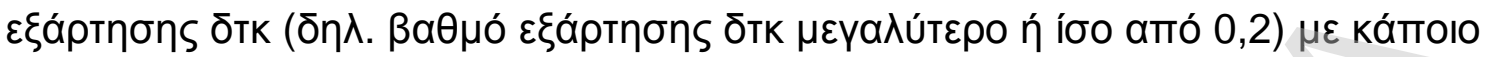

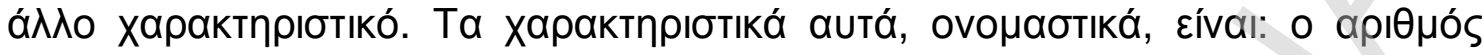

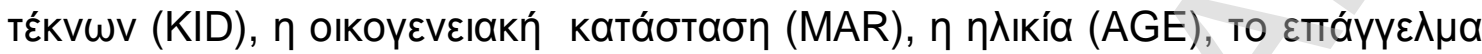

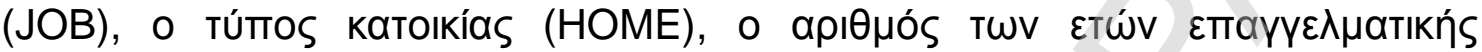

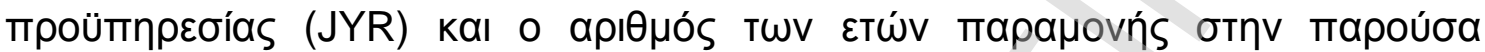

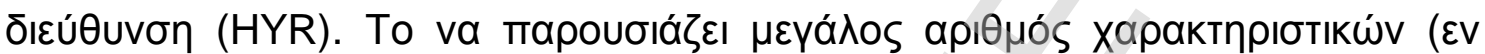

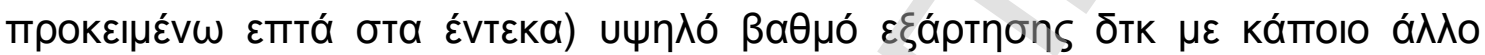

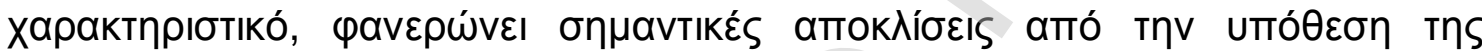

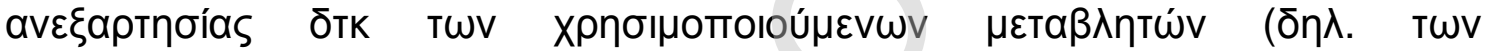

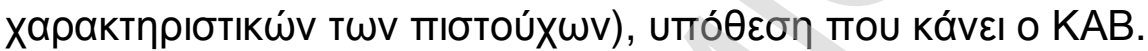

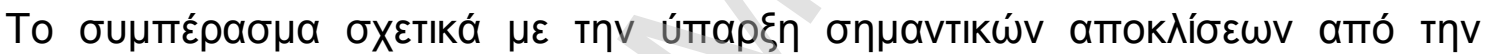

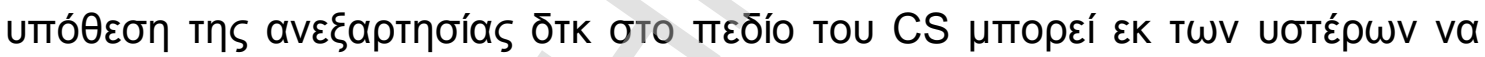

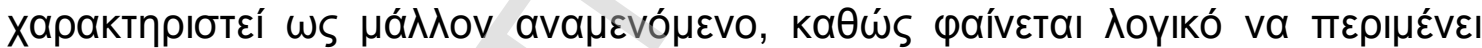

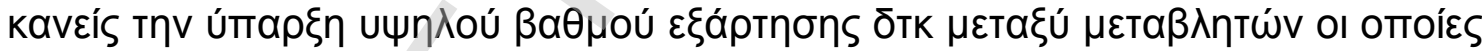

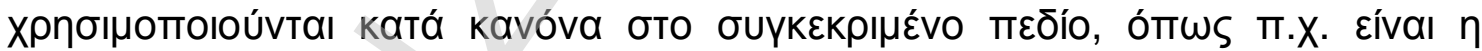

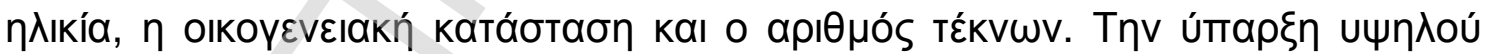

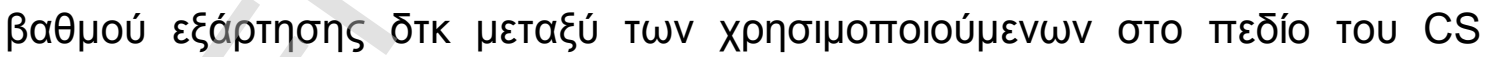

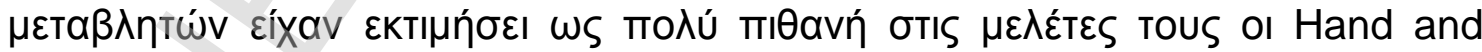

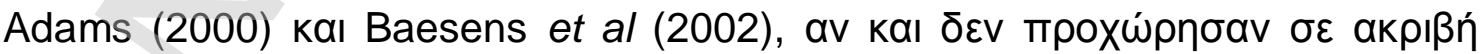

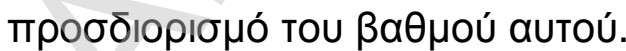

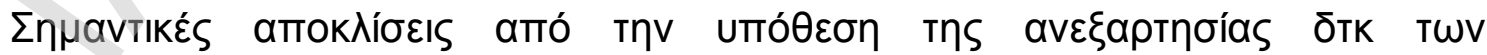

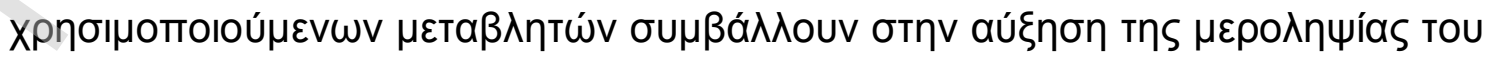

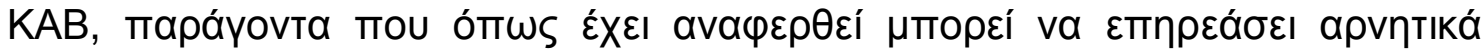




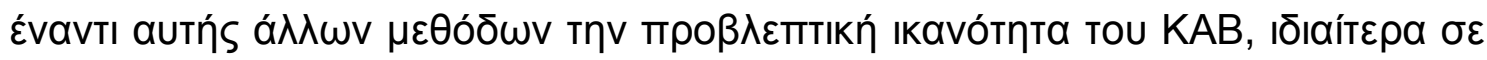

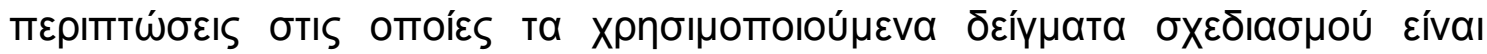

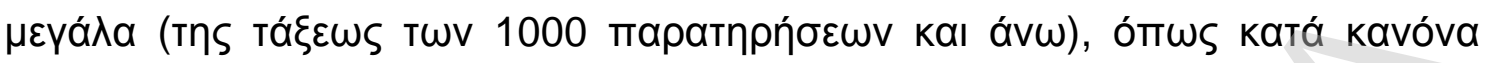

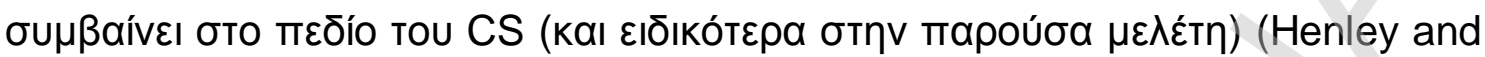

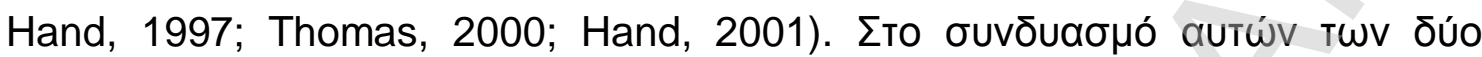

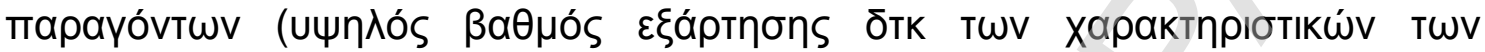

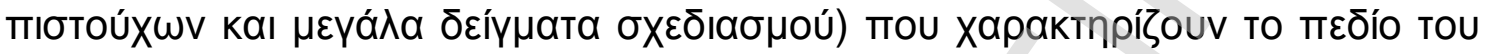

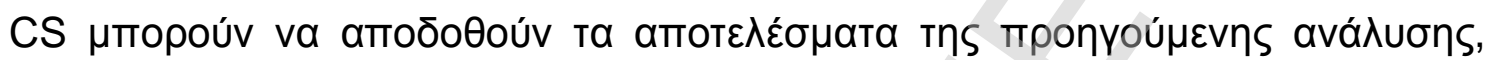

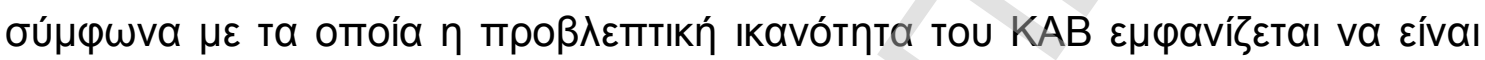

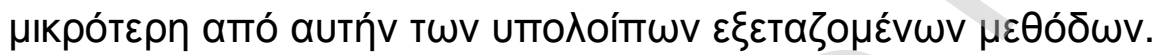

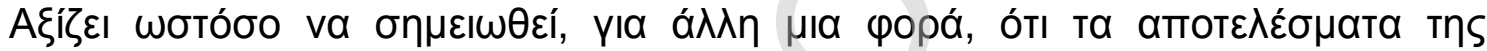

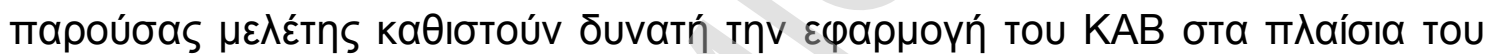

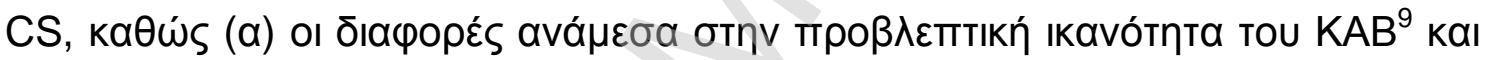

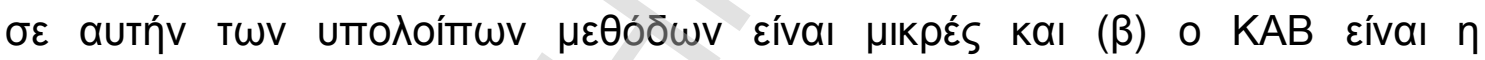

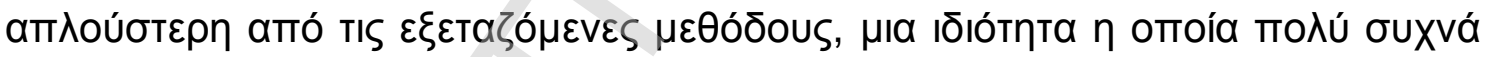

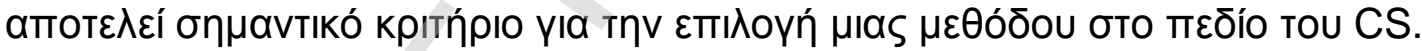

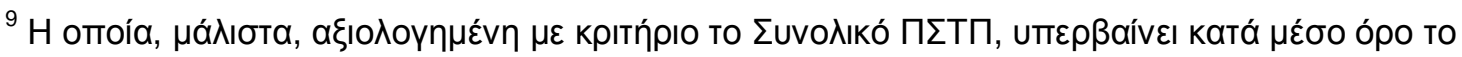

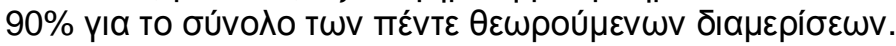




\section{KEФA^AIO 5

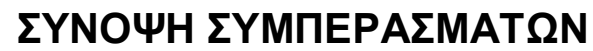

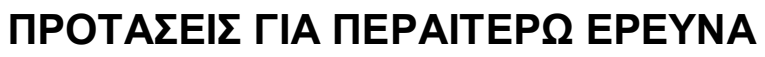

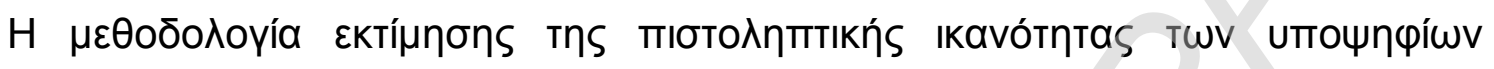

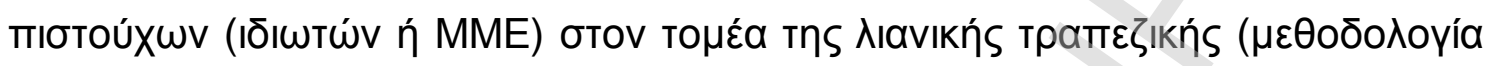

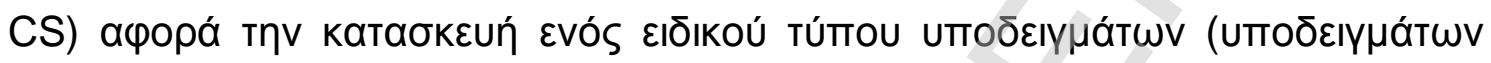

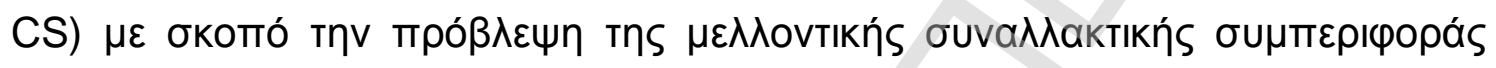

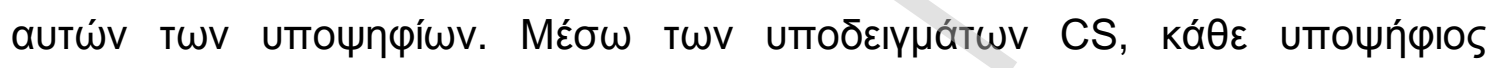

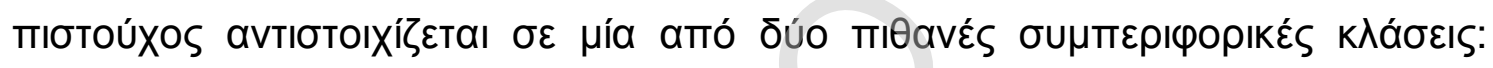

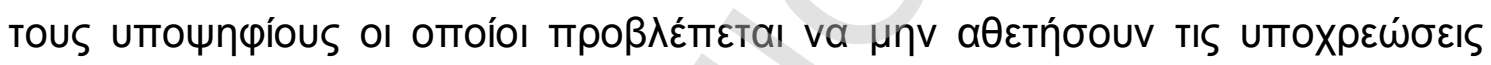

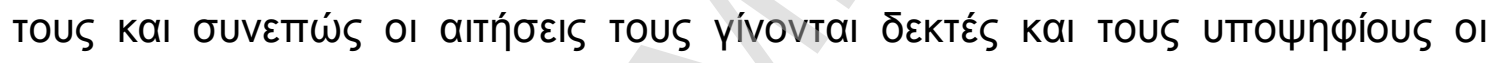

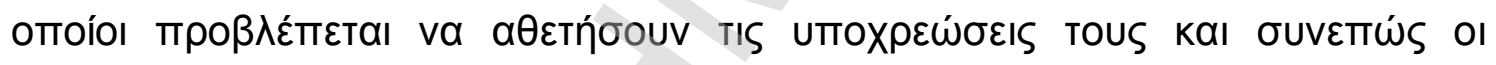

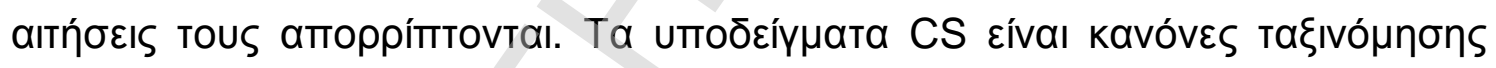

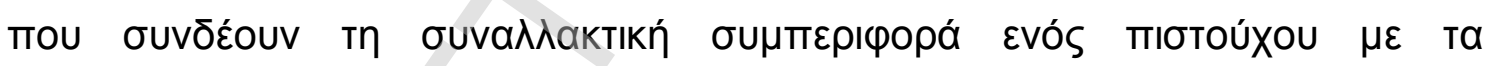

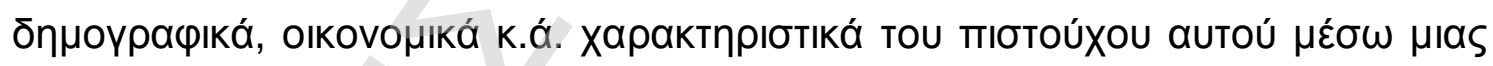

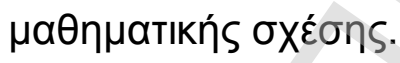

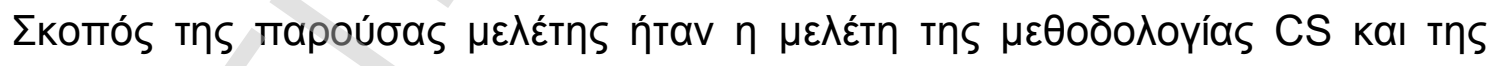

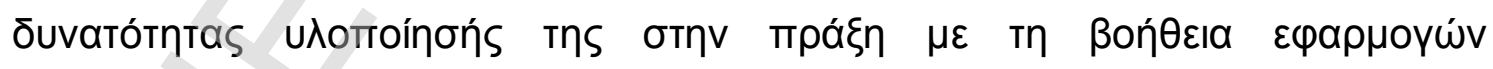

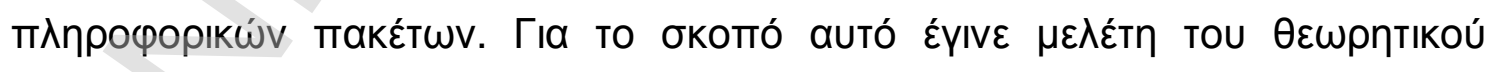

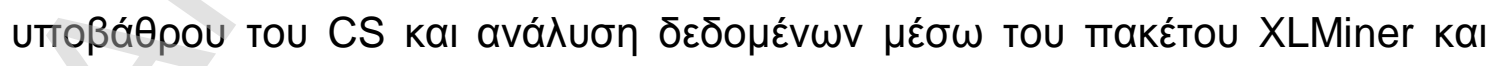

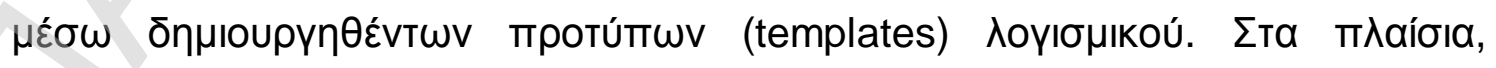

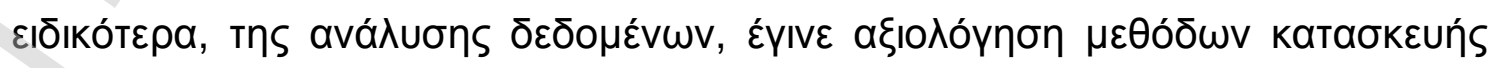

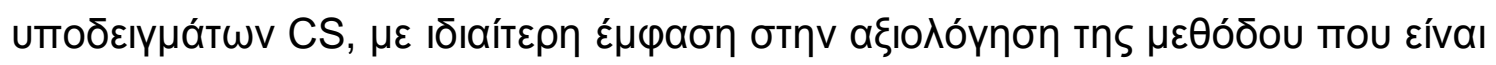

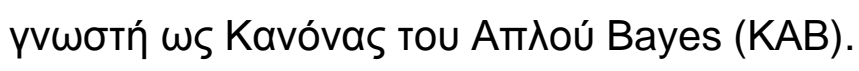




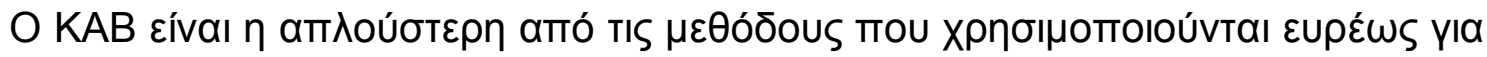

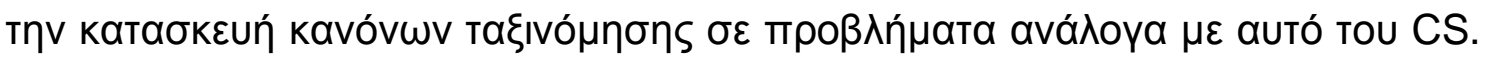

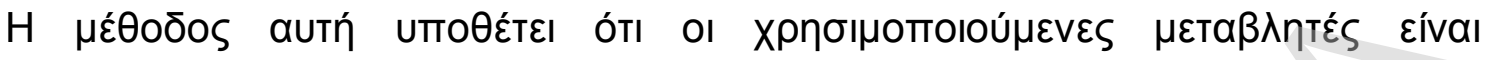

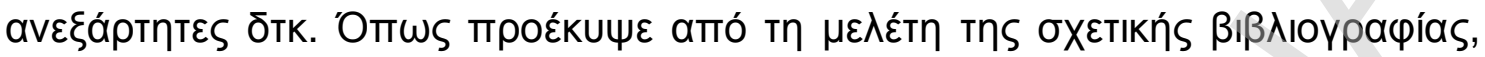

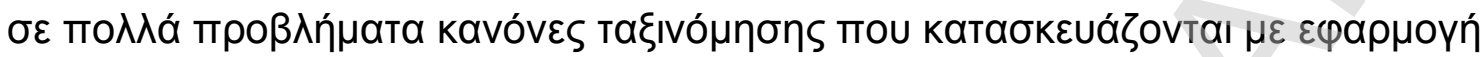

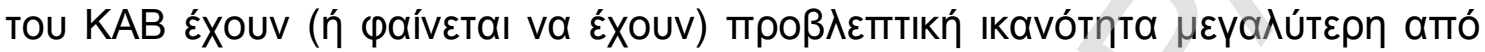

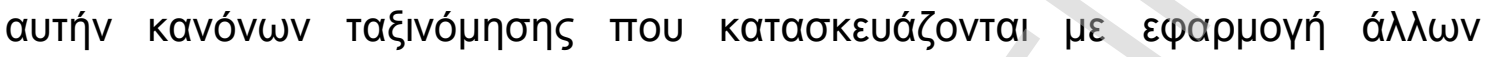

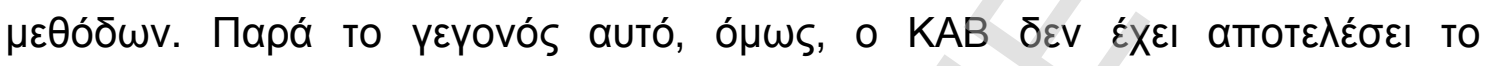

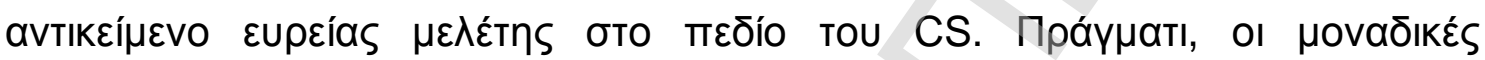

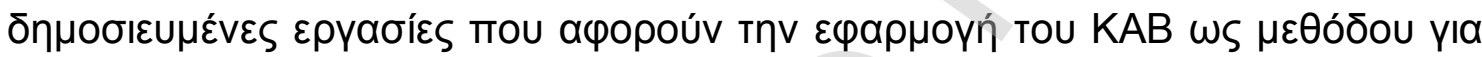

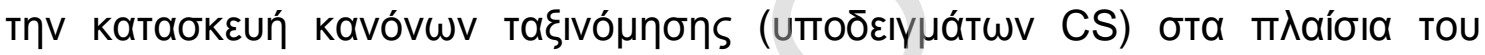

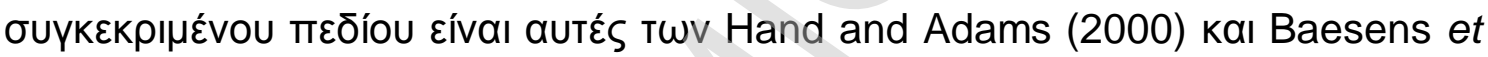

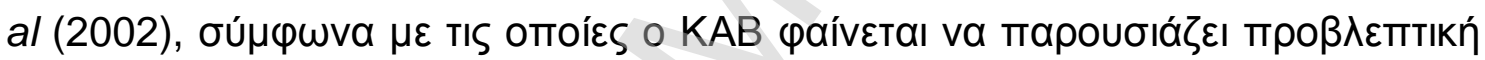

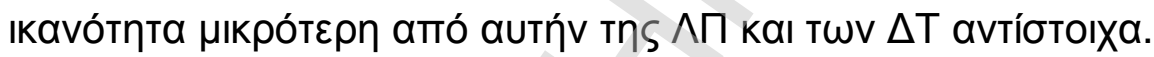

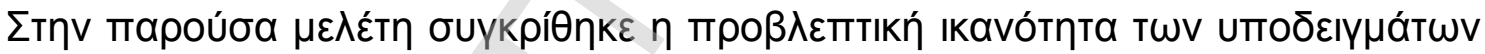

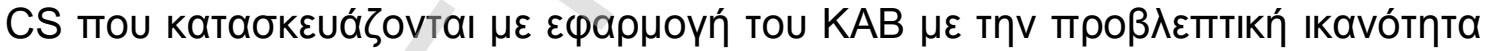

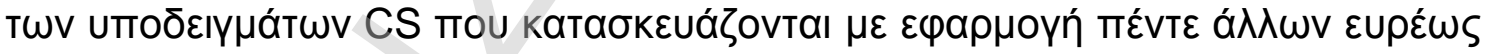

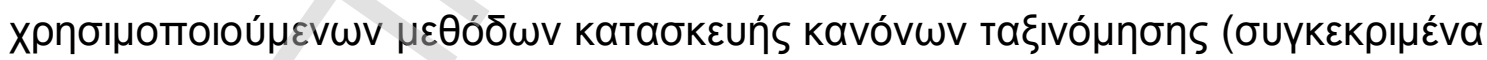

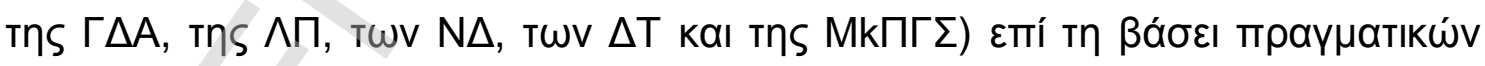

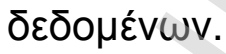

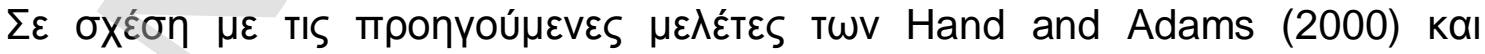

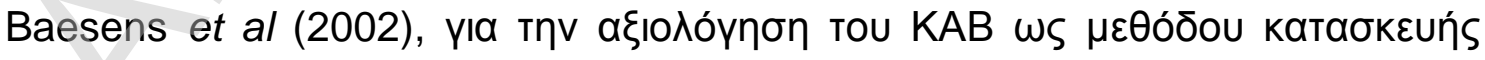

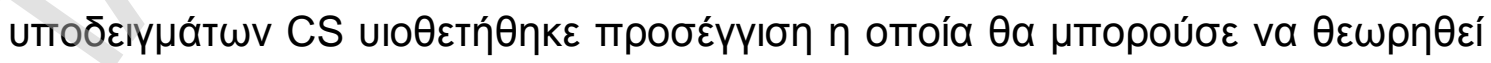

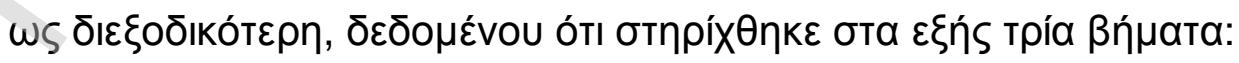




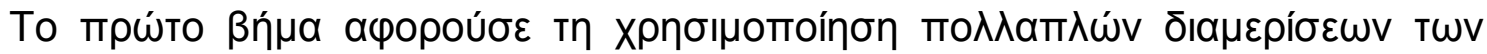

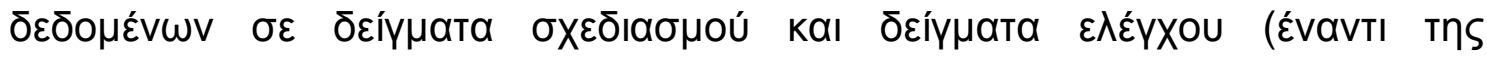

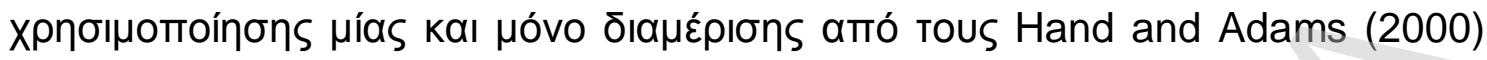

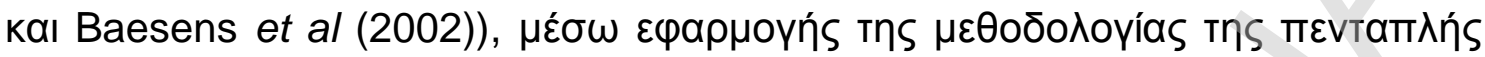

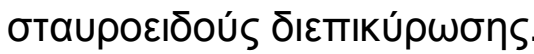

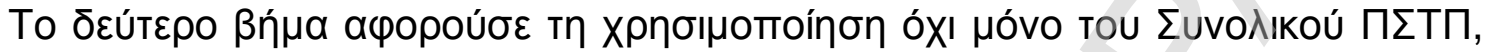

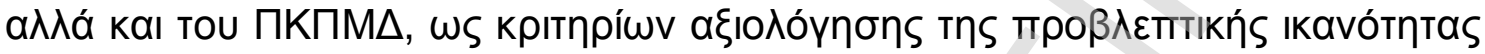

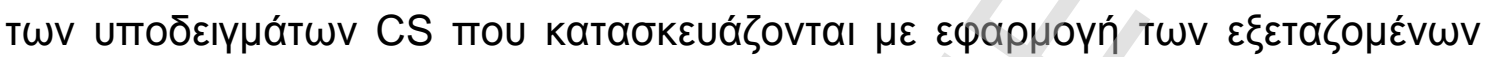

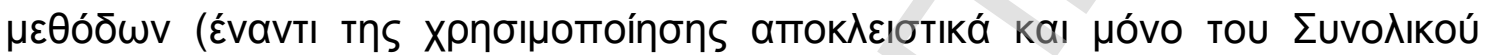

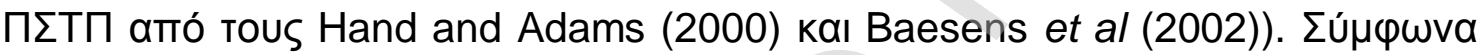

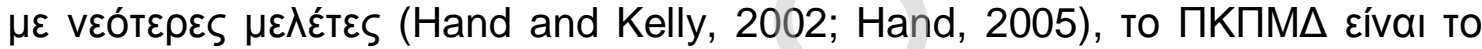

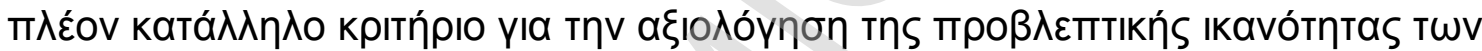

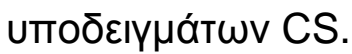

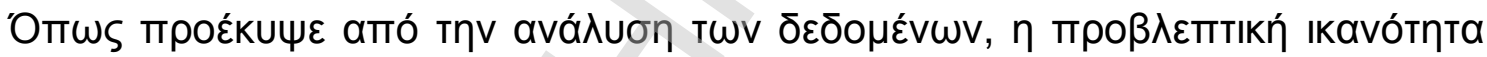

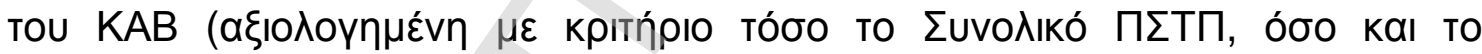

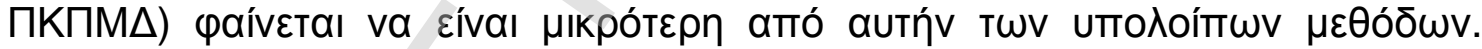

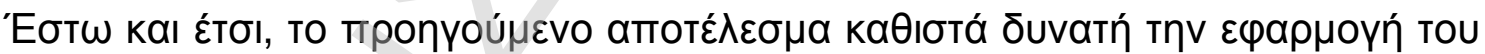

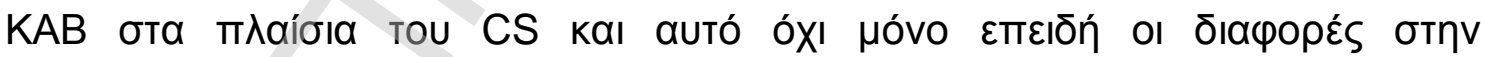

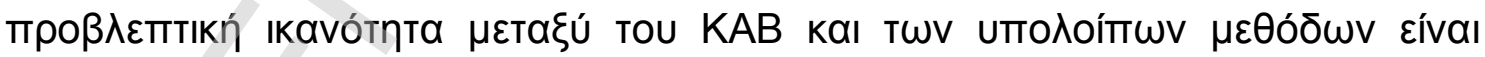

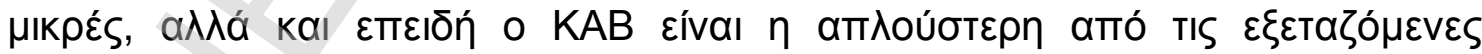

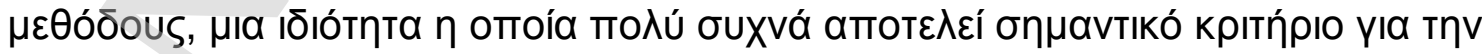

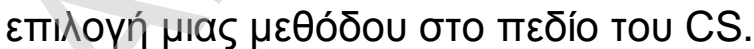

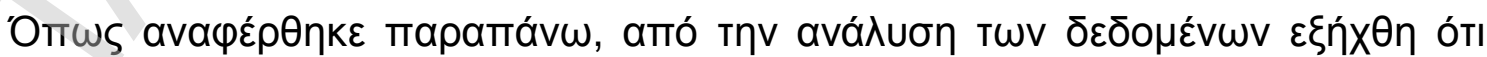

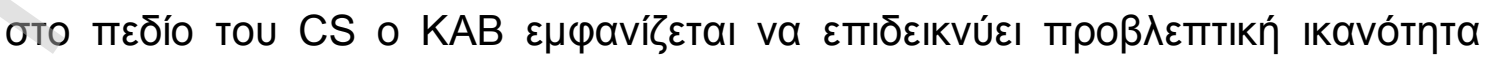

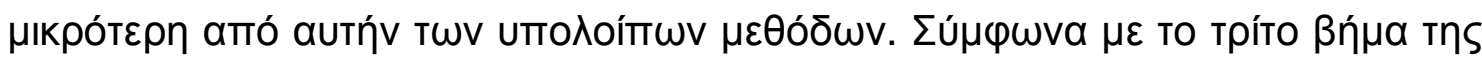




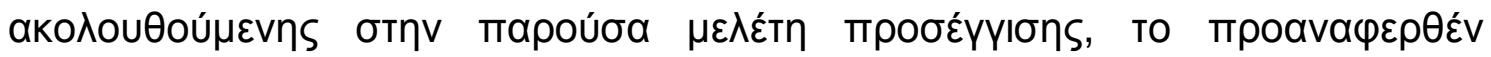

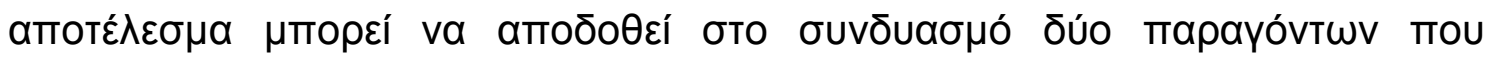

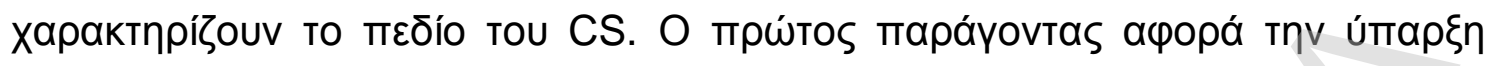

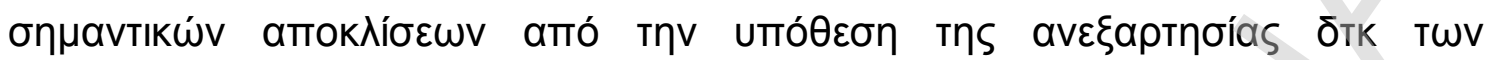

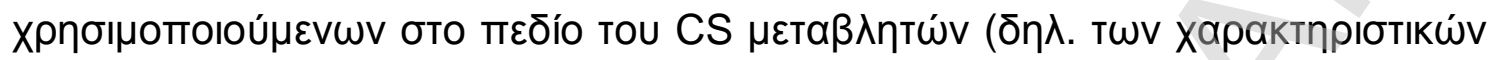

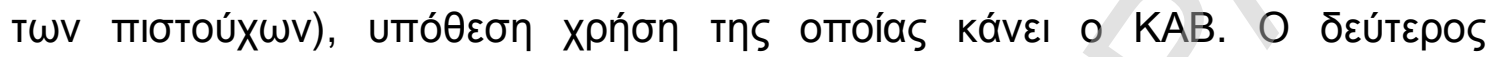

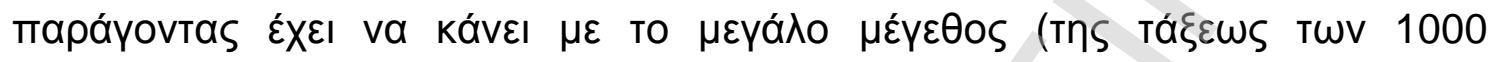

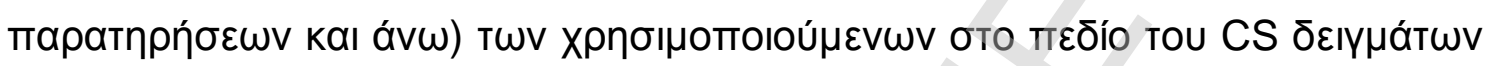

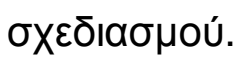

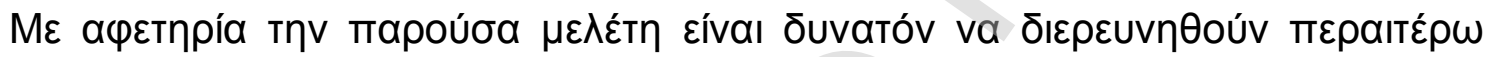

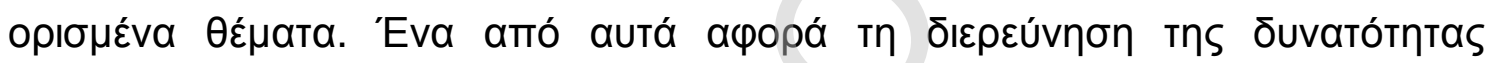

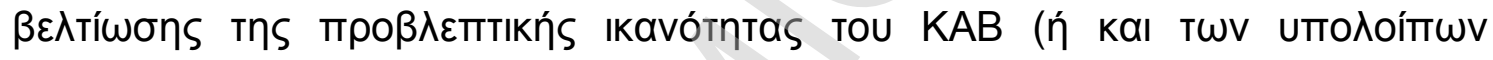

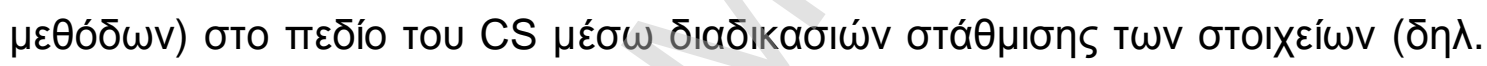

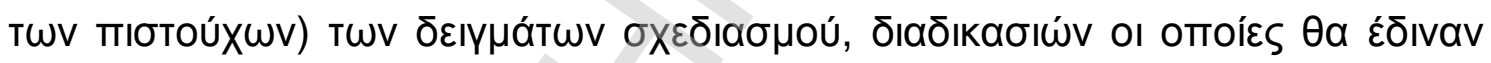

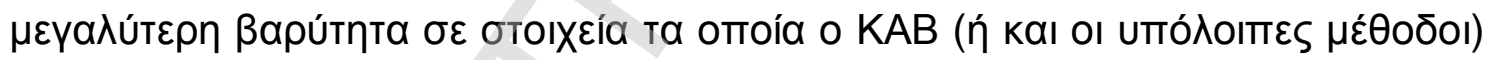

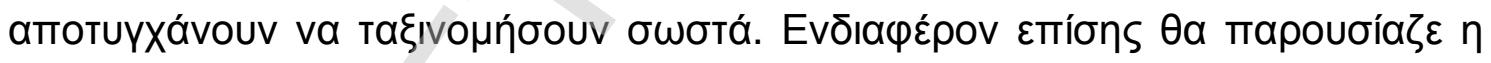

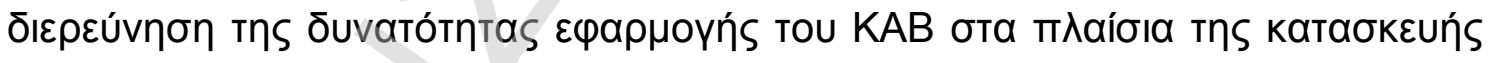

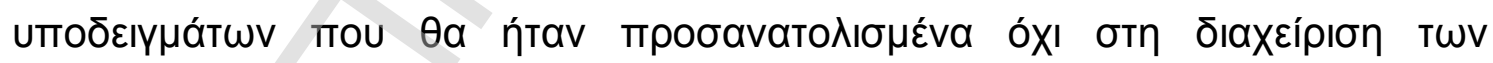

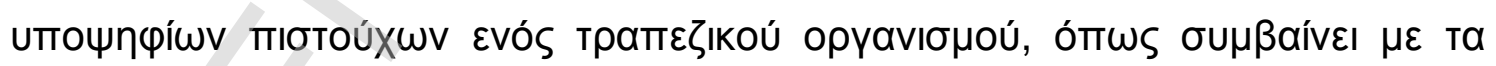

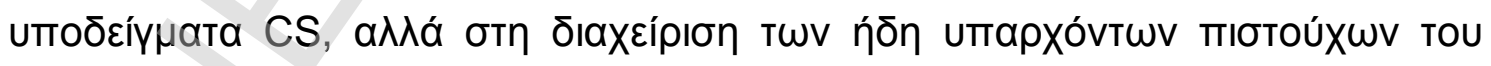
opyaviouoú autoú. 


\section{ВІВАІОГРАФІА}


Adams, N.M. and Hand, D.J. (2000). Improving the practice of classifier performance assessment. Neural Computation, 12, 305-311.

Allen, L., DeLong, G. and Saunders, A. (2004). Issues in the credit risk modeling of retail markets. Journal of Banking and Finance, 28, 727-752.

Alvarez, M., Moreno, I.M., Jos, A., Camean, A.M. and Gonzalez, A.G. (2007). Differentiation of two Andalusian DO 'fino' wines according to their metal content frpm ICP-OES by using supervised pattern recognition methods. Microchemical Journal, 87, 72-76.

Antonakis, A.C. and Sfakianakis, M.E. (2005). Knowledge management for evaluating borrower creditworthiness as a competitive advantage in the banking sector. Proceedings of the Conference on Knowledge Management and Corporate Governance Systems, 758-764. Tripoli, Greece: Hellenic Society for Systemic Studies.

Antonakis, A.C. and Sfakianakis, M.E. (2007). Knowledge management as a means of achieving organizational excellence: A retail-banking case. To appear in: Proceedings of the International Conference on Managing Global Trends and Challenges in a Turbulent Economy. Chios, Greece: Department of Business Administration, University of the Aegean.

Antonakis, A.C. and Sfakianakis, M.E. (2008). Naïve Bayes as a means of constructing application scorecards. In: Moutinho, L. and Huarng, K.H. (Eds): Advances in Doctoral Research in Management, 2, 47-62. Singapore: World Scientific Publishing Co.

Antonakis, A.C. and Sfakianakis, M.E. (2009A). Assessing Naïve Bayes as a method for screening credit applicants. Journal of Applied Statistics, 36, 537545. 
Antonakis, A.C. and Sfakianakis, M.E. (2009B). Electronic systems of trust in retail lending: new application areas. Under Revision.

Ash, D. and Vlatsa, D. (2001). Scorecard modeling with continuous vs. classed variables. In: Mays, E. (Ed.): Handbook of Credit Scoring, 71-85. New York, NY: American Management Association.

Asikainen, A., Kolehmainen, M., Ruuskanen, J. and Tuppurainen, K. (2006). Structure-based classification of active and inactive estrogenic compounds by decision tree, LVQ and kNN methods. Chemosphere, 62, 658-673.

Avery, R.B., Bostic, R.W., Calem, P.S. and Canner, G.B. (1996). Credit risk, credit scoring and the performance of home mortgages. Federal Reserve Bulletin, 82, 621-648.

Baesens, B., Egmont-Petersen, M., Castelo, R. \& Vanthienen, J. (2002). Learning Bayesian network classifiers for credit scoring. Proceedings of the $16^{\text {th }}$ International Conference on Pattern Recognition (ICPR'02), Quebec City, Quebec: IEEE Computer Society Press, 3, 49-52.

Bailey, M. (2004). Cut-off strategies. In: Bailey, M. (Ed.): Credit Scoring: The Principles and Practicalities, 69-76, Bristol: White Box Publishing, $2^{\text {nd }}$ edition.

Barfield, G. (2004). Nature of risk: deep impact. Balance Sheet, 12, 32-37.

Barron, J.M. and Staten, M. (2003). The value of comprehensive credit reports: Lessons from the US experience. In: Miller, M.J. (Ed.): Credit Reporting Systems and the International Economy, 273-310, Cambridge, MA: The MIT Press.

Baumgartner, C., Böhm, C. and Baumgartner, D. (2005). Modelling of classification rules on metabolic patterns including machine learning and expert knowledge. Journal of Biomedical Informatics, 38, 89-98. 
Behr, P. and Guettler, A. (2007). Credit risk assessment and relationship lending: an empirical analysis of German Small and Medium-sized Enterprises, Journal of Small Business Management, 45, 194-213.

Behrman, M., Linder, R., Assadi, A.H., Stacey, B.R. and Backonja, M.M. (2007). Classification of patients with pain based on neuropathic pain symptoms: comparison of an artificial neural network against an established scoring system. European Journal of Pain, 11, 370-376.

Berger, A.N., Frame, W.S. and Miller, N.H. (2005). Credit scoring and the availability, price and risk of small business credit. Journal of Money, Credit and Banking, 37, 191-222.

Bertelli, D., Plessi, M., Sabatini, A.G., Lolli, M. and Grillenzoni, F. (2007). Classification of Italian honeys by mid-infrared diffuse reflectance spectroscopy (DRIFTS). Food Chemistry, 101, 1565-1570.

Bishop, C.M. (1995). Neural Networks for Pattern Recognition. Oxford: Oxford Univeristy Press.

Bishop, M. (2002). The next chapter in small business scoring. The RMA Journal, 84, 48-52.

Blunt, G. and Hand, D.J. (2000). The UK credit card market. Technical Report, Department of Mathematics, Imperial College, London.

Boyle, M., Crook, J.N., Hamilton, R. and Thomas, L.C. (1992). Methods for credit scoring applied to slow payers. In: Thomas, L.C., Crook, J.N. and Edelman, B.D. (Eds.): Credit Scoring and Credit Control, 75-90, Oxford: Clarendon Press.

Breiman, L., Friedman, J.H., Olshen, R.A. and Stone, C.J. (1984). Classification and regression trees. Belmont, CA: Wadsworth. 
Brill, J. (1998). The importance of credit scoring models in improving cash-flows and collections. Business Credit, 1, 16-17.

Brown, D. and Edelman, D. (2001). Some views on setting scorecard cut-offs. Proceedings of the Credit Scoring and Credit Control VII Conference, Credit Research Centre, University of Edinburgh, September 2001.

Buckinx, W. and Van den Poel, D. (2005). Customer base analysis: partial defection of behaviourally loyal clients in a non-contractual FMCG retail setting. European Journal of Operational Research, 164, 252-268.

Burton, D., Knights, D., Leyshon, A., Alferoff, C. and Signoretta, P. (2004). Making a market: the UK retail financial services industry and the rise of the complex sub-prime credit market. Competition and Change, 8, 3-25.

Camdeviren, H.A., Yazici, A.C., Akkus, Z., Bugdayci, R. and Sungur, M.A. (2007). Comparison of logistic regression model and classification tree: an application to postpartum depression data. Expert Systems with Applications, 32, 987-994.

Caruana, J. (2005). Implementation of Basel II. Financial Markets, Institutions and Instruments, 14, 253-265.

Chaterjee, S., Corbae, D. and Rios-Rull, J.V. (2005). A recursive equilibrium model with credit scoring and competitive pricing of default risk. Working Paper, Research Automation Department, Macro Team, Federal Reserve Bank of Atlanta.

Chen, M.C. and Huang, S.H. (2003). Credit scoring and rejected instances reassigning through evolutionary computational techniques. Expert Systems with Applications, 24, 433-441. 
Chen, K., Kurgan, L. and Rahbari, M. (2007). Prediction of protein crystallization using collocation of amino acid pairs. Biochemical and Biophysical Research Communications, 355, 764-769.

Cheng, B. and Titterington, D.M. (1994). Neural networks: a review from a statistical perspective (with discussion). Statistical Science, 9, 2-54.

Chrástek, R., Wolf, M., Donath, K., Niemann, H., Paulus, D., Hothorn, T., Lausen, B., Lämmer, R., Mardin, C.Y. and Michelson, G. (2005). Automated segmentation of the optic nerve head for diagnosis of glaucoma. Medical Image Analysis, 9, 297-314.

Clark, P. and Niblett, T. (1989). The CN2 induction algorithm. Machine Learning, 3, 261-283.

Crook, J.N., Edelman, D.B. and Thomas, L.C. (2007). Recent developments in consumer credit risk assessment. European Journal of Operational Research, $183,1447-1465$.

Crook, J.N., Hamilton, R. and Thomas, L.C. (1992). A comparison of a credit scoring model with a credit performance model. The Service Industries Journal, $12,558-579$

Cybenko, G. (1989). Approximation by superpositions of a sigmoidal function. Mathematical Control Signal Systems, 2, 303-314.

Davis, R.H., Edelman, D.B. and Gammerman, A.J. (1992). Machine-learning algorithms for credit-card applications. IMA Journal of Mathematics Applied in Business and Industry, 4, 43-52.

Desai, V.S., Crook, J.N. and Overstreet, G.A. (1996). A comparison of neural networks and linear scoring models in the credit union environment. European Journal of Operational Research, 95, 24-37. 
Dragovic, S. and Onjia, A. (2007). Classification of soil samples according to geographic origin using gamma-ray spectrometry and pattern recognition methods. Applied Radiation and Isotopes, 65, 218-224.

Duda, R.O., Hart, P.E. and Stork, D.G. (2000). Pattern Classification. New York, NY: Wiley Interscience, $2^{\text {nd }}$ edition.

Duin, R.P.W. (1996). A note on comparing classifiers. Pattern Recognition Letters, 17, 529-536.

Durand, D. (1941). Risk Elements in Consumer Installment Financing. New York: National Bureau of Economic Research.

Edelman, D.B. (2005). Credit scoring as a strategic management tool. Proceedings of the Credit Scoring and Credit Control IX Conference, Credit Research Centre, University of Edinburgh, September 2005.

Emmons, W.R., Lskavyan, V. and Yeager, T.J. (2005). Basel II will trickle down to community bankers, consumers. The Regional Economist, April, 12-13.

Fishelson-Holstine, H. (2004). The role of credit scoring in increasing homeownership for underserved populations. Proceedings of the Building Assets, Building Credit Symposium on Improving Financial Services in LowIncome Communities, Joint Center for Housing Studies, Harvard University, November 2003.

Fisher, R.A. (1936). The use of multiple measurements in taxonomic problems. Annals of Eugenics, 7, 179-188.

Fix, E. and Hodges, J.L. (1951). Discriminatory analysis-nonparametric discrimination: consistency properties. Project 21-49-004, Rep. 4, 261-279, USAF School of Aviation Medicine, Randolf Field, TX. 
Freeman, J.A. and Skapura, D.M. (1992). Neural metworks: algorithms, application and programming techniques. MI, USA: Addison-Wesley.

Friedman, J.H. (1997). On bias, variance 0/1-loss and the curse-ofdimensionality. Data Mining and Knowledge Discovery, 1, 55-77.

Friedman, N., Geiger, D. and Goldszmidt, M. (1997). Bayesian network classifiers. Machine Learning, 29, 131-163.

Gammerman, A. and Thatcher, A.R. (1991). Bayesian diagnostic probabilities without assuming independence of symptoms. Methods of Information in Medicine, 30, 15-22.

Gardener, E., Howcroft, B. and Williams, J. (1999). The new retail banking revolution. The Service Industries Journal, 19, 83-100.

Garside, T. and Bech, J. (2003). Dealing with Basel II: the impact of the New Basel Capital Accord. Balance Sheet, 11, 26-31.

Gates, S.W., Perry, V.G. and Zorn, P.M. (2002). Automated underwriting in mortgage lending: good news for the underserved? Housing Policy Debate, 13, 369-391.

Ginoris, Y.P., Amaral, A.L., Nicolau, A., Coelho, M.A.Z. and Ferreira, E.C. (2007). Recognition of protozoa and metazoa using image analysis tools, discriminant analysis, neural networks and decision trees. Analytica Chimica Acta, 595, 160-169.

Gordy, M.B. (2003). A risk-factor model foundation for ratings-based bank capital rules. Journal of Financial Intermediation, 12, 199-232.

Goutte, C. (1997). Note on cross validation. Neural Computation, 9, 1211-1215. 
Granitto, P.M., Navone, H.D., Verdes, P.F. and Ceccatto, H.A. (2002). Weed seeds identification by machine vision. Computers and Electronics in Agriculture, 33, 91-103.

Granitto, P.M, Verdes, P.F. and Ceccatto, H.A. (2005). Large-scale investigation of weed seed identification by machine vision. Computers and Electronics in Agriculture, 47, 15-24.

Gomha, M.A., Sheir, K.Z., Showky, S., Abdel-Khalek, M., Mokhtar, A.A. and Madbouly, K. (2004). Can we improve the prediction of stone-free status after extracorporeal shock wave lithotripsy for ureteral stones? A neural network or a statistical model? The Journal of Urology, 172, 175-179.

Gundersen, H.J.G., Jensen, E.B.V., Kieu, K. and Nielsen, J. (1999). The efficiency of systematic sampling. Journal of Microscopy, 193, 199-211.

Hamilton, R. and Khan, M. (2001). Revolving credit card holders: who are they and how can they be identified? The Service Industries Journal, 21, 37-48.

Hand, D.J. (1997). Construction and Assessment of Classification Rules. Chichester: John Wiley and Sons.

Hand, D.J. (1998). Consumer credit and statistics. In: Hand, D.J. and Jacka, S.D. (Eds.): Statistics in Finance, London: Arnold, 69-81.

Hand, D.J. (2001). Modeling consumer credit risk. IMA Journal of Management Mathematics, 12, 139-155.

Hand, D.J. (2005). Good practice in retail credit scorecard assessment. Journal of the Operational Research Society, 56, 1109-1117.

Hand, D.J. (2006). Classifier technology and the illusion of progress (with discussion). Statistical Science, 21, 1-34. 
Hand, D.J. and Adams, N.M. (2000). Defining attributes for scorecard construction. Journal of Applied Statistics, 27, 527-540.

Hand, D.J., Adams, N.M. and Kelly, M.G. (2001). Multiple classifier systems based on interpretable linear classifiers. In Kittler, J. and Roli, F. (Eds.): Proceedings of the $2^{\text {nd }}$ International Workshop on Multiple Classifier Systems (MCS 2001), Cambridge, UK, July 2-4, Berlin: Springer-Verlag, 136-147.

Hand, D.J. and Henley, W.E. (1997). Statistical classification methods in consumer credit scoring: a review. Journal of the Royal Statistical Society, Series A, 160, 523-541.

Hand, D.J. and Kelly, M.G. (2002). Superscorecards. IMA Journal of Management Mathematics, 13, 273-281.

Hand, D.J. and Vinciotti, V. (2003). Local versus global models for classification problems: fitting models where it matters. The American Statistician, 57, 124131.

Hand, D.J. and Yu, K. (2001). Idiot's Bayes-Not so stupid after all. International Statistical Review, 69, 385-398.

Hastie, T., Tibshirani, R. and Friedman, J. (2001). The Elements of Statistical Learning. New York: Springer.

Haykin, S. (1999). Neural Networks: A Comprehensive Foundation. London: Prentice-Hall International.

Henley, W.E. and Hand, D.J. (1996). A k-nearest neighbour classifier for assessing consumer credit risk. The Statistician, 45, 77-95.

Hilden, J. (1984). Statistical diagnosis based on conditional independence. Computers in Biology and Medicine, 14, 429-435. 
Hornik, K., Stinchcombe, M. and White, H. (1989). Multilayer feedforward networks are universal approximators. Neural Networks, 2, 336-359.

Hunt, R.M. (2003). The development and regulation of consumer credit reporting in America. Proceedings of the European Experience and Lessons from the US Conference on the Economics of Consumer Credit, Finance and Consumption Programme, European University Institute, Economics Department, Florence, May 13-14.

Jain, A.K., Duin, R.P.W. and Mao, J. (2000). Statistical pattern recognition: a review. IEEE Transactions on Pattern Analysis and Machine Learning, 22, 4-37. Jappelli, T. and Pagano, M. (2002). Information sharing, lending and defaults: cross-country evidence. Journal of Banking \& Finance, 26, 2017-2045.

Jekova, I., Bortolan, G. and Christov, I. (2008). Assessment and comparison of different methods for heartbeat classification. Medical Engineering and Physics, 30, 248-257.

Jennings, A. (2003). Basel II: transforming the compliance challenge into business opportunity. Viewpoints, September, 4-6.

Jentzsch, N. (2001). The economics and regulation of financial privacy-a comparative analysis of the United States and Europe. Working Paper N. 128/2001, John F. Kennedy Institute of North American Studies, Berlin.

Jentzsch, N. and Riestra, A.S.J. (2003). Information sharing and its implications for consumer credit markets: United States vs. Europe. Proceedings of the European Experience and Lessons from the US Conference on the Economics of Consumer Credit, Finance and Consumption Programme, European University Institute, Economics Department, Florence, May 13-14. 
Katos, V. (2007). Network intrusion detection: evaluating cluster, discriminant, and logit analysis. Information Sciences, 177, 3060-3073.

Kelly, M.G., Hand, D.J. and Adams, N.M. (1999). Classification problems: how to be both judge and jury. In: Hand, D.J., Kok, J. and Berthold, M.R. (Eds.): Advances in Intelligent Data Analysis, Lecture Notes in Computer Science, $1642,235-244$.

Kim, H.-J., Choo, H., Cho, Y.-S., Koh, H.Y., No, K.T. and Pae, A.N. (2006). Classification of dopamine, serotonin, and dual antagonists by decision trees. Bioorganic and Medicinal Chemistry, 14, 2763-2770.

Kindred, D. and Bailey, M. (2004). The scorecard build process. In: Bailey, M. (Ed.): Credit Scoring: The Principles and Practicalities, 42-51. Bristol: White Box Publishing, $2^{\text {nd }}$ edition.

Kitsantas, P., Hollander, M. and Li, L. (2006). Using classification trees to assess low birth weight outcomes. Artificial Intelligence in Medicine, 38, 275289.

Kohavi, R. (1996). Scaling up the accuracy of naïve-Bayes classifiers: a decision- tree hybrid. Proceedings of the 2nd International Conference on Knowledge Discovery and Data Mining, 202-207. Portland, OR: AAAI Press.

Kononenko, I. (1991). Semi-naïve Bayesian classifier. Proceedings of the $6^{\text {th }}$ European Working Session on Learning, Porto, Portugal: Springer-Verlag, 206219.

Kotsiantis, S., Pierrakeas, C. and Pintelas, P. (2004). Predicting students' performance in distance learning using machine learning techniques. Applied Artificial Intelligence, 18, 411-426. 
Krall, M. (2008). Understanding Basel II: operational and strategic implications. Financial Markets, Institutions and Instruments, 17, 97-108.

Kulkarni, S.R., Lugosi, G. and Venkatesh, S.S. (1998). Learning pattern classification-a survey. IEEE Transactions on Information Theory, 44, 21782206.

Kurt, I., Ture, M. and Kurum, A.T. (2008). Comparing performances of logistic regression, classification and regression tree, and neural networks for predicting coronary artery disease. Expert Systems with Applications, 34, 366-374.

Langley, P., Iba, W. and Thompson, K. (1992). An analysis of Bayesian classifiers. Proceedings of the 10th National Conference on Artificial Intelligence, 223-228. San Jose, CA: AAAI Press.

Lee, T.S. and Chen, I.F. (2005). A two-stage hybrid credit scoring model using artificial neural networks and multivariate adaptive regression splines. Expert Systems with Applications, 28, 743-752.

Lee, T.S., Chiu, C.C., Lu, C.J. and Chen, I.F. (2002). Credit scoring using the hybrid neural discriminant technique. Expert Systems with Applications, 23, 245-254.

Lehmann, C., Koenig, T., Jelic, V., Prichep, L., John, R.E., Wahlund, L.-O., Dodge, Y. and Dierks, T. (2007). Application and comparison of classification algorithms for recognition of Alzheimer's disease in electrical brain activity (EEG). Journal of Neuroscience Methods, 161, 342-350.

Leonard, K.J. (1995). The development of credit scoring quality measures for consumer credit applications. International Journal of Quality and Reliability, 12, 79-85. 
Leonard, K.J. (1996). Information systems and benchmarking in the credit scoring industry. International Journal of Benchmarking for Quality Management and Technology, 3, 38-44.

Le Pan, N. (2008). Reminder on Basel II. Financial Markets, Institutions and Instruments, 17, 19-22.

Leyshon, A. and Thrift, N. (1999). Lists come alive: electronic systems of knowledge and the rise of credit scoring in retail banking. Economy and Society, 28, 434-466.

Leyshon, A., Thrift, N. and Pratt, J. (1998), Reading financial services: texts, consumers and financial literacy. Environment and Planning D: Society and Space, 16, 29-55.

Li, Y. and Anderson-Sprecher, R. (2006). Facies identification frpm well logs: a comparison of discriminant analysis and naïve Bayes classifier. Journal of Petroleum Science and Engineering, 53, 149-157.

Liu, Y. (2001). New issues in credit scoring application. Arbeitsbericht Nr. 16/2001, Institut fuer Wirtschaftsinformatik, Georg-August-Universitaet Goettingen.

Liu, Y. (2002A). A framework of data mining application process for credit scoring. Arbeitsbericht Nr. 01/2002, Institut fuer Wirtschaftsinformatik, GeorgAugust-Universitaet Goettingen.

Liu, Y. (2002B). The evaluation of classification models for credit scoring. Arbeitsbericht Nr. 02/2002, Institut fuer Wirtschaftsinformatik, Georg-AugustUniversitaet Goettingen.

Longenecker, J.G., Moore, C.W. and Petty, J.W. (1997). Credit scoring and the small business: a review and the need for research. Proceedings of the 1997 
United States Association for Small Business and Enterpreneurship (USASBE) Annual National Conference, San Francisco, California, 21-24 June.

Malhotra, R. and Malhotra, D.K. (2003). Evaluating consumer loans using neural networks, Omega, 31, 83-96.

Maloof, M.A., Langley, P., Binford, T.O., Nevatia, R. and Sage, S. (2003). Improved rooftop detection in aerial images with machine learning. Machine Learning, 53, 157-191.

Mani, S., Shankle, W.R., Dick, M.B. \& Pazzani, M.J. (1999). Machine learning model for guideline development. Artificial Intelligence in Medicine, 16, 51-71. Marron, D. (2007). 'Lending by numbers': credit scoring and the constitution of risk within American consumer credit. Economy and Society, 36, 103-133. Marshall, C. (2003). What lies beyond compliance? The Banker, October, 4-6. Mays, E. (2001). The basics of scorecard development and validation. In: Mays, E. (Ed.): Handbook of Credit Scoring, 89-105. New York, NY: American Management Association.

Mays, E. (2004). Scorecard policies: How to use your scorecard to your best advantage. In: Mays, E. (Ed.): Credit Scoring for Risk Managers: The Handbook for Lenders, 187-200, Mason, OH: Thomson/South-Western.

McLachlan, G.J. (1992). Discriminant Analysis and Statistical Pattern Recognition. New York: John Wiley.

McNab, H. and Wynn, A. (2003). Principles and Practice of Consumer Credit Risk Management. Canterbury, Kent: Institute of Financial Services, $2^{\text {nd }}$ edition. Mester, L.J. (1997). What's the point of credit scoring? Federal Reserve Bank of Philadelphia Business Review, September/October, 3-16. 
Michie, D., Spiegelhalter, D.J. and Taylor, C.C. (1994). Machine Learning, Neural and Statistical Classification. New York: Horwood.

Miller, M.J. (2003). Credit reporting systems around the globe: the state of the art in public credit registries and private credit reporting. In: Miller, M.J. (Ed.): Credit Reporting Systems and the International Economy, 25-80, Cambridge, MA: The MIT Press.

Myers, J.H. and Forgy, E.W. (1963). The development of numerical credit evaluation systems. Journal of the American Statistical Association, 58, 799806.

Nattkemper, T.W., Arnrich, B. , Lichte, O., Timm, W., Degenhard, A., Pointon, L., Hayes, C., Leach, M.O. and The UK MARIBS Breast Screening Study (2005). Artificial Intelligence in Medicine, 34, 129-139.

Nel, A. (2004). New accounts scorecard monitoring. In: Bailey, M. (Ed.): Consumer Credit Quality: Underwriting, Scoring, Fraud Prevention and Collections, 60-70, Bristol: White Box Publishing.

O'Farrell, M., Lewis, E., Flanagan, C., Lyons, W. and Jackman, N. (2005). Comparison of $k-\mathrm{NN}$ and neural network methods in the classification of spectral data from an optical fibre-based sensor system used for quality control in the food industry. Sensors and Actuators, 111-112, 354-362.

Ohmann, C., Moustakis, V., Yang, O. and Lang, K. (1996). Evaluation of automatic knowledge acquisition techniques in the diagnosis of acute abdominal pain. Artificial Intelligence in Medicine, 8, 23-36.

Ohmann, C., Yang, O., Kuenneke, M., Stoeltzing, H., Thon, K. and Lorenz, W. (1988). Bayes theorem and conditional dependence of symptoms: different 
models applied to data of upper gastrointestinal bleeding. Methods of Information in Medicine, 27, 73-83.

Ong, C.S., Huang, J.J. and Tzeng, G.H. (2005). Building credit scoring models using genetic programming. Expert Systems with Applications, 29, 41-47.

Ozturk, A. and Arslan, A. (2007). Classification of transcranial Doppler signals using their chaotic invariant measures. Computer Methods and Programs in Biomedicine, 86, 171-180.

Park, Y.S. and Chung, Y.J. (2006). Hazard rating of pine trees from a forest insect pest using artificial neural networks. Forest Ecology and Management, $222,222-233$.

Pazzani, M., Muramatsu, J. and Billsus, D. (1996). Identifying interesting web sites. Proceedings of the 13th National Conference on Artificial Intelligence, 5461. Portland, OR: AAAI Press.

Penny, W.D. and Frost, D.P. (1997). Modeling of the level of observation in an acute psychiatric ward. Computers and Biomedical Research, 30, 1-17.

Perli, R. and Nayda, W.I. (2004). Economic and regulatory capital allocation for revolving retail exposures. Journal of Banking and Finance, 28, 789-809.

Poulakis, V., Witzsch, U., de Vries, R., Emmerlich, V., Meves, M., Altmannsberger, H.-M. and Becht, E. (2004). Preoperative neural network using combined magnetic resonance imaging variables, prostate specific antigen and gleason score to predict prostate cancer stage. The Journal of Urology, 172, 1306-1310.

Poynton, M.R. and McDaniel, A.M. (2006). Classification of smoking cessation status with a backpropagation neural network. Journal of Biomedical Informatics, 39, 680-686. 
Petersen, M.A. and Rajan, R.G. (2002). Does distance still matter? The information revolution in small business lending. Journal of Finance, 57, 25332570.

Ren, S. and Schultz, T.W. (2002). Identifying the mechanism of aquatic toxicity of selected compounds by hydrophobicity and electrophilicity descriptors. Toxicology Letters, 129, 151-160.

Repley, B. (1994). Neural networks and related methods for classification. Journal of the Royal Statistical Society, Series B, 56, 409-456.

Riestra, A.S.J. (2002). Credit bureaus in today's credit markets. Research Report N. 4, European Credit Research Institute, Brussels.

Rose, A.M.C., Grais, R.F., Coulombier, H. and Ritter, H. (2006). Systematic sampling methods for measuring crude mortality. Bulletin of the World Health Organization, 84, 290-296.

Rosenberg, E. and Gleit, E. (1994). Quantitative methods in credit management: a survey. Operations Research, 42, 589-613.

Rowe, D., Jovic, D. and Reeves, R. (2004). Bank capital management in the light of Basel II-how to manage capital in financial institutions. Balance Sheet, $12,15-21$.

Rumelhart, D.E., Hinton, G.E. and Williams, R.J. (1986). Learning representation by back-propagating errors. Nature, 323, 533-536.

Russek, E., Kronmal, R.A. and Fisher, L.D. (1983). The effect of assuming independence in applying Bayes' theorem to risk estimation and classification in diagnosis. Computers and Biomedical Research, 16, 537-552.

Rutherford, R. (1994/1995). Securitizing small business loans: a banker's action plan. Commercial Lending Review, 10, 62-74. 
Safavian, S.R. and Landgrebe, D. (1991). A survey of decision tree classifier methodology. IEEE Transactions on Systems, Man and Cybernetics, 21, 660674.

Schiffman, R. (2001). Evaluating and monitoring your model. In: Mays, E. (Ed.): Handbook of Credit Scoring, 285-300. New York, NY: American Management Association.

Sharda, R. and Delen, D. (2006). Predicting box-office success of motion pictures with neural networks. Expert Systems with Applications, 30, 243-254.

Sierra, B. and Larranaga, P. (1998). Predicting skin melanoma. An empirical comparison between different approaches. Artificial Intelligence in Medicine, 14, 215-230.

Stehman, S.V. and Overton, W.S. (1994). Comparison of variance estimators of the Horwitz-Thompson estimator for randomized variable probability systematic sampling. Journal of the American Statistical Association, 89, 30-43.

Straka, J.W. (2000). A shift in the mortgage landscape: the 1990s move to automated credit evaluations. Journal of Housing Research, 11, 207-232.

Su C.-T., Yang, C.-H., Hsu, K.-H. and Chiu, W.-K. (2006). Data mining for the diagnosis of type II diabetes from three-dimensional body surface anthropometrical scanning data. Computers and Mathematics with Applications, $51,1075-1092$.

Taylor, P.C. and Silverman, B.W. (1993). Block diagrams and splitting criteria for classification trees. Statistics and Computing, 3, 147-161.

Thessler, S., Sesnie, S., Ramos-Bendana, Z.S., Ruokolainen, K., Tomppo, E. and Finegan, B. (2008). Using k-nn and discriminant analyses to classify rain 
forest types in a Landsat TM image over northern Costa Rica. To appear in: Remote Sensing of Environment.

Thomas, L.C. (1998). Methodologies for classifying applicants for credit. In: Hand, D.J. \& Jacka, S.D. (Eds.) Statistics in Finance, London: Arnold, 83-103. Thomas, L.C. (2000). A survey of credit and behavioural scoring: forecasting financial risk of lending to consumers. International Journal of Forecasting, 16, 149-172.

Thomas, L.C. (2003). Consumer credit modelling: context and current issues. Proceedings of the Banff Credit Risk Conference, Banff, Canada, October 1116.

Thomas, L.C., Edelman, D.B. and Crook, J.N. (2002). Credit Scoring and its Applications. Philadelphia: Society for Industrial and Applied Mathematics.

Thomas, L.C., Edelman, D.B. and Crook, J.N. (2004). Readings in Credit Scoring. New York: Oxford University Press.

Thomas, L.C., Oliver, R.W. and Hand, D.J. (2005). A survey of the issues in consumer credit modelling research. Journal of the Operational Research Society, 56, 1006-1015.

Thomas, S., Leese, M., Walsh, E., McCrone, P., Moran, P., Burns, T., Creed, F., Tyrer, P. and Fahy, T. (2005). A comparison of statistical models in predicting violence in psychotic illness. Comprehensive Psychiatry, 46, 296303.

Titterington, D.M., Murray, G.D., Murray, L.S., Spiegelhalter, D.J., Skene, A.M., Habbema, J.D.F. and Gelpke, G.J. (1981). Comparison of discrimination techniques applied to a complex data set of head injured patients. Journal of the Royal Statistical Society, Series A, 144, 145-175. 
Ture, M., Kurt, I., Kurum, A.T. and Ozdamar, K. (2005). Comparing classification techniques for predicting essential hypertension. Expert Systems with Applications, 29, 583-588.

Valenti, P., Cazamajou, E., Scarpettini, M., Aizemberg, A., Silva, W. and Kochen, S. (2006). Automatic detection of interictal spikes using data mining models. Journal of Neuroscience Methods, 150, 105-110.

Van Looy, S., Vander Cruyssen, B., Meeus, J., Wyns, B., Westhoven, R., Durez, P., Van den Bosch, F., Vastesaeger, N., Geldhof, A., Boullart, L. and De Keyser, F. (2006). Prediction of dose escalation for rheumatoid arthritis patients under infliximab treatment. Engineering Applications of Artificial Intelligence, 19, 819-828.

Wan, S.J. and Wong, S.K.M. (1989). A measure for concept dissimilarity and its applications in machine learning. Proceedings of the International Conference on Computing and Information, Toronto, Ontario: North-Holland, 267-273.

Wang, D., Lim, J.-S., Han, M.-M. and Lee, B.-W. (2005). Learning similarity for semantic images classification. Neurocomputing, 67, 363-368.

Webb, A.R. (2002). Statistical Pattern Recognition. Chichester: John Wiley and Sons, $2^{\text {nd }}$ edition.

West, D. (2000). Neural network credit scoring models. Computers \& Operations Research, 27, 1131-1152.

Westlake, M. (2003). Can Basel II be made to work? The Banker, August, 6164.

Wyatt, K. (2004). CP3: Standardized approach for credit risk and current capital requirements. Journal of Financial Services Research, 26, 105-119. 
Yamamura, S., Kawada, K., Takehira, R., Nishizawa, K., Katayama, S., Hirano, M. and Momose, Y. (2004). Artificial neural network modeling to predict the plasma concentration of aminoglycosides in burn patients. Biomedecine and Pharmacotherapy, 58, 239-244.

Yang, Y. (1999). An evaluation of statistical approaches to text categorization. Journal of Information Retrieval, 1, 67-88.

Yang, Y. and Liu, X. (1999). A re-examination of text categorization methods. Proceedings of the 22nd Annual International ACM SIGIR Conference on Research and Development in Information Retrieval, 42-49. Berkeley, CA: ACM Press.

Yobas, M.B., Crook, J.N. and Ross, P. (2000). Credit scoring using neural and evolutionary techniques. IMA Journal of Mathematics Applied in Business and Industry, 11, 111-125.

Zhang, G., Hu, M., Patuwo, B. and Indro, D. (1999). Artificial neural networks in bankruptcy prediction: general framework and cross-validation analysis. European Journal of Operations Research, 116, 16-32.

Zhang, G., Patuwo, B.E. and Hu, M.,Y. (1998). Forecasting with artificial neural networks: the state of the art. International Journal of Forecasting, 14, 35-62.

Zhang, G.P. (2000). Neural networks for classification: a survey. IEEE Transactions on Systems, Man and Cybernetics, Part C: Applications and Reviews, 30, 451-462.

Zhang, L.C. (2008). On some common practices of systematic sampling. Journal of Official Statistics, 24, 557-569. 


\section{ПАРАРТНMА A}

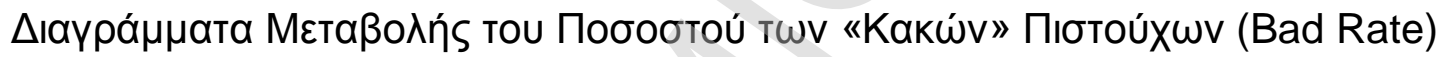

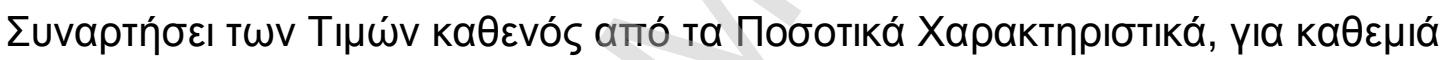

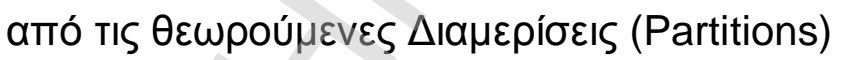



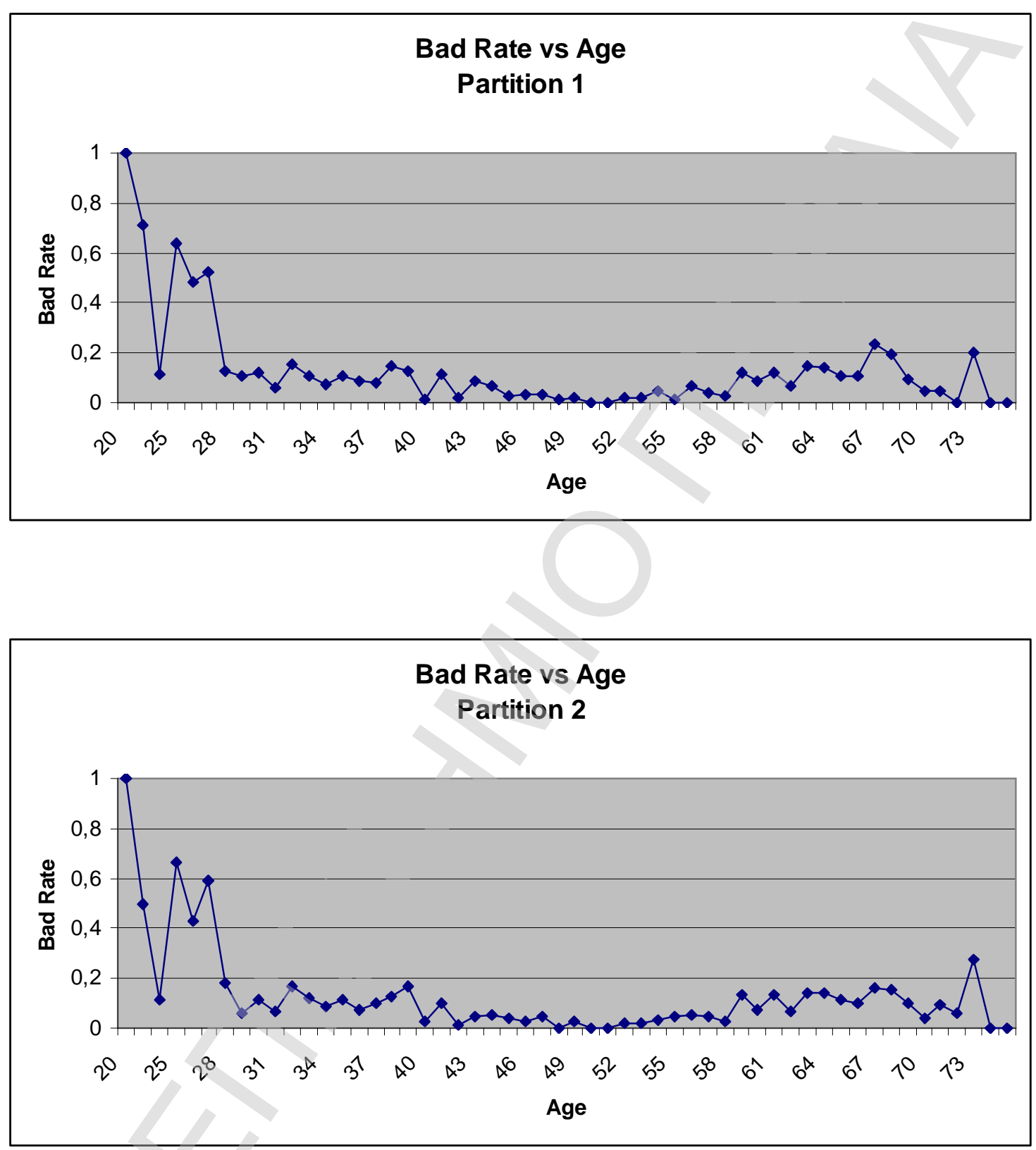

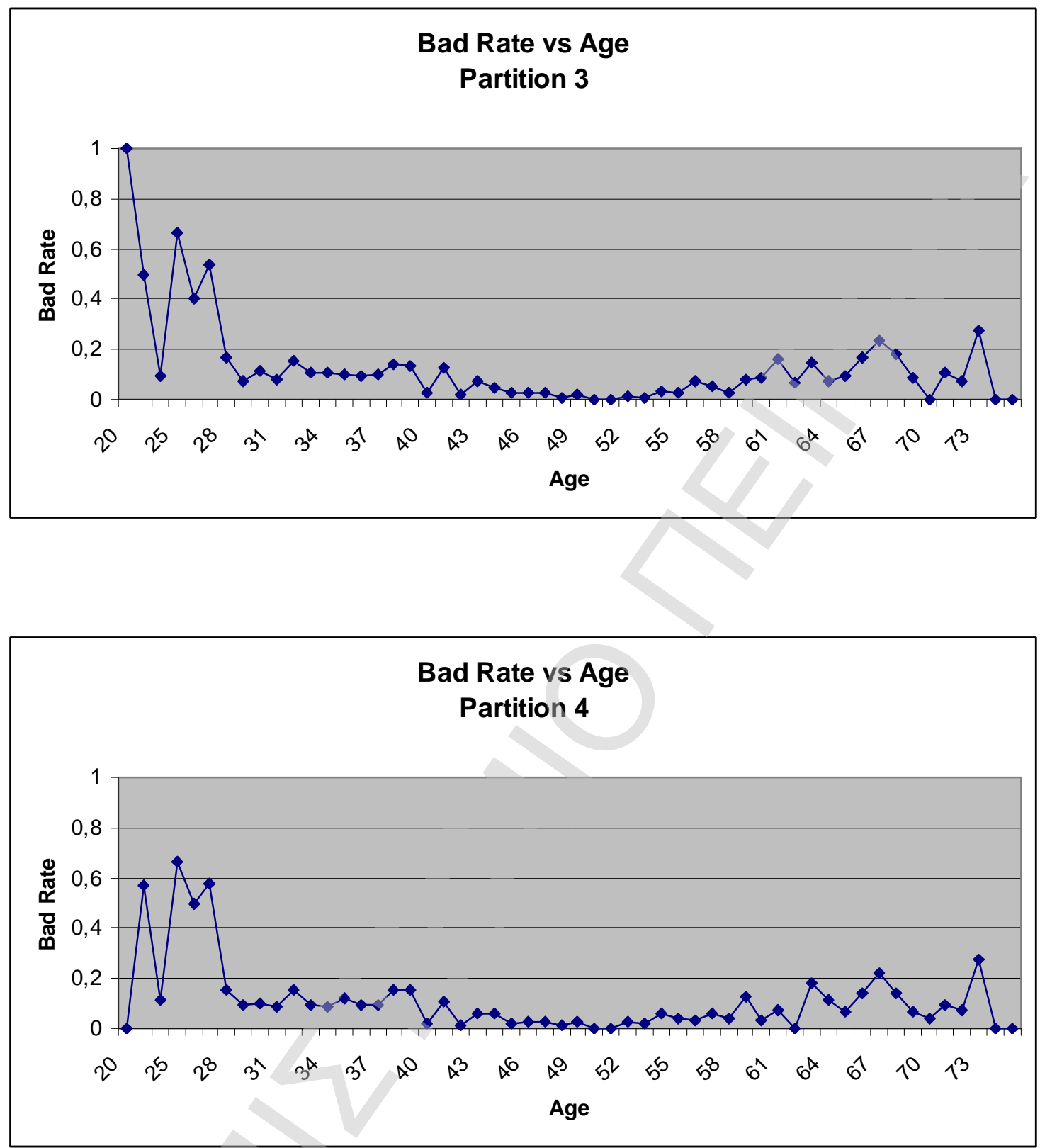


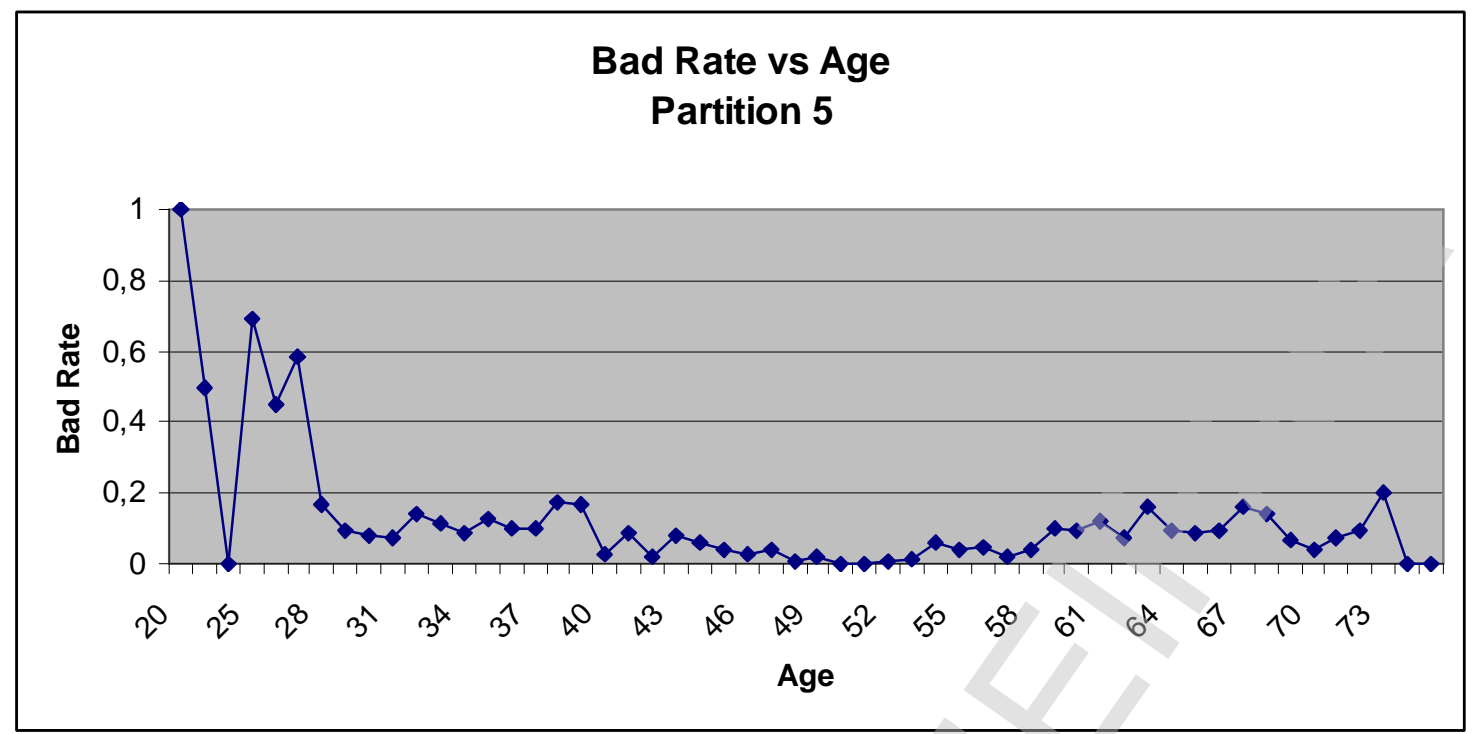




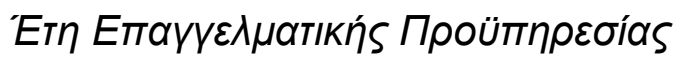
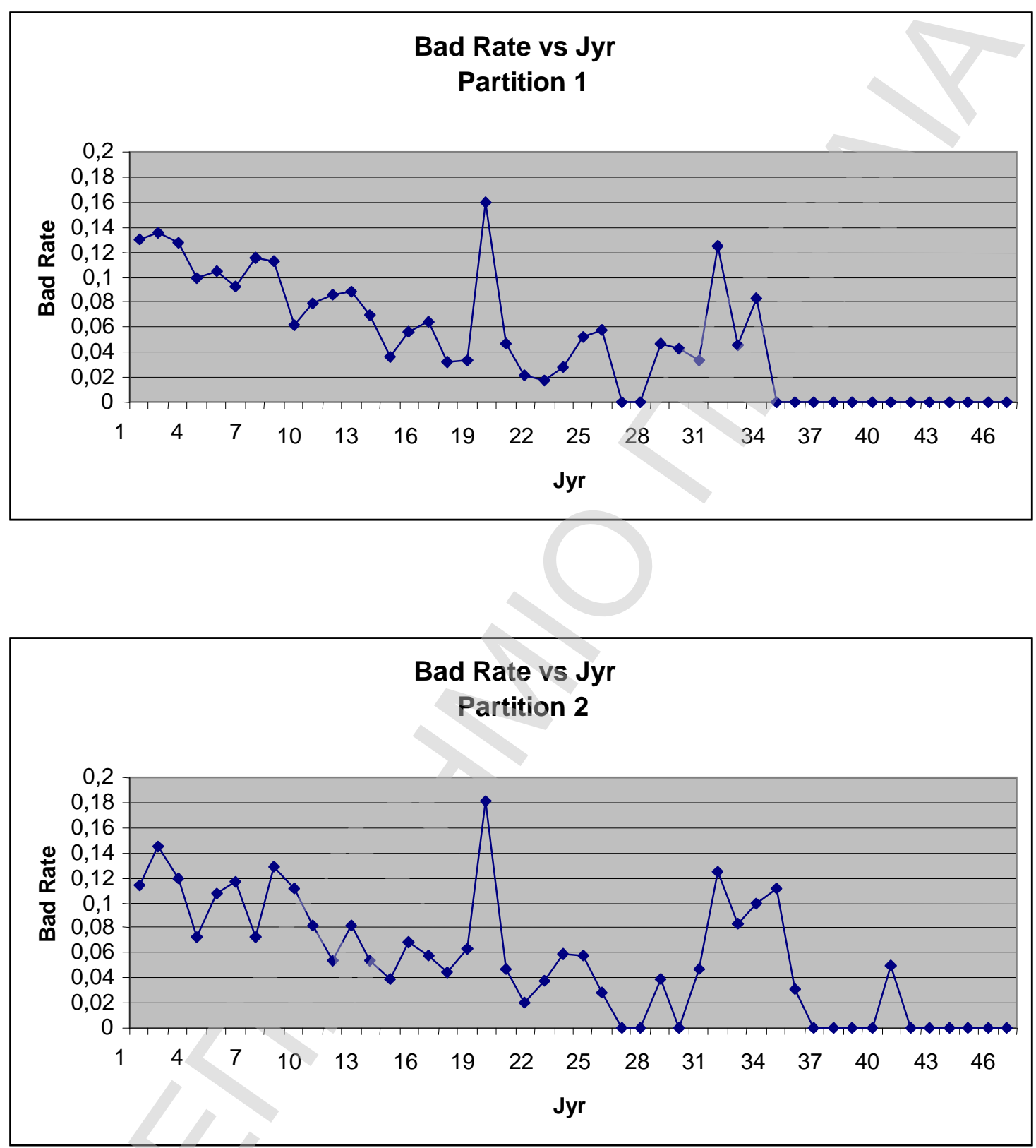

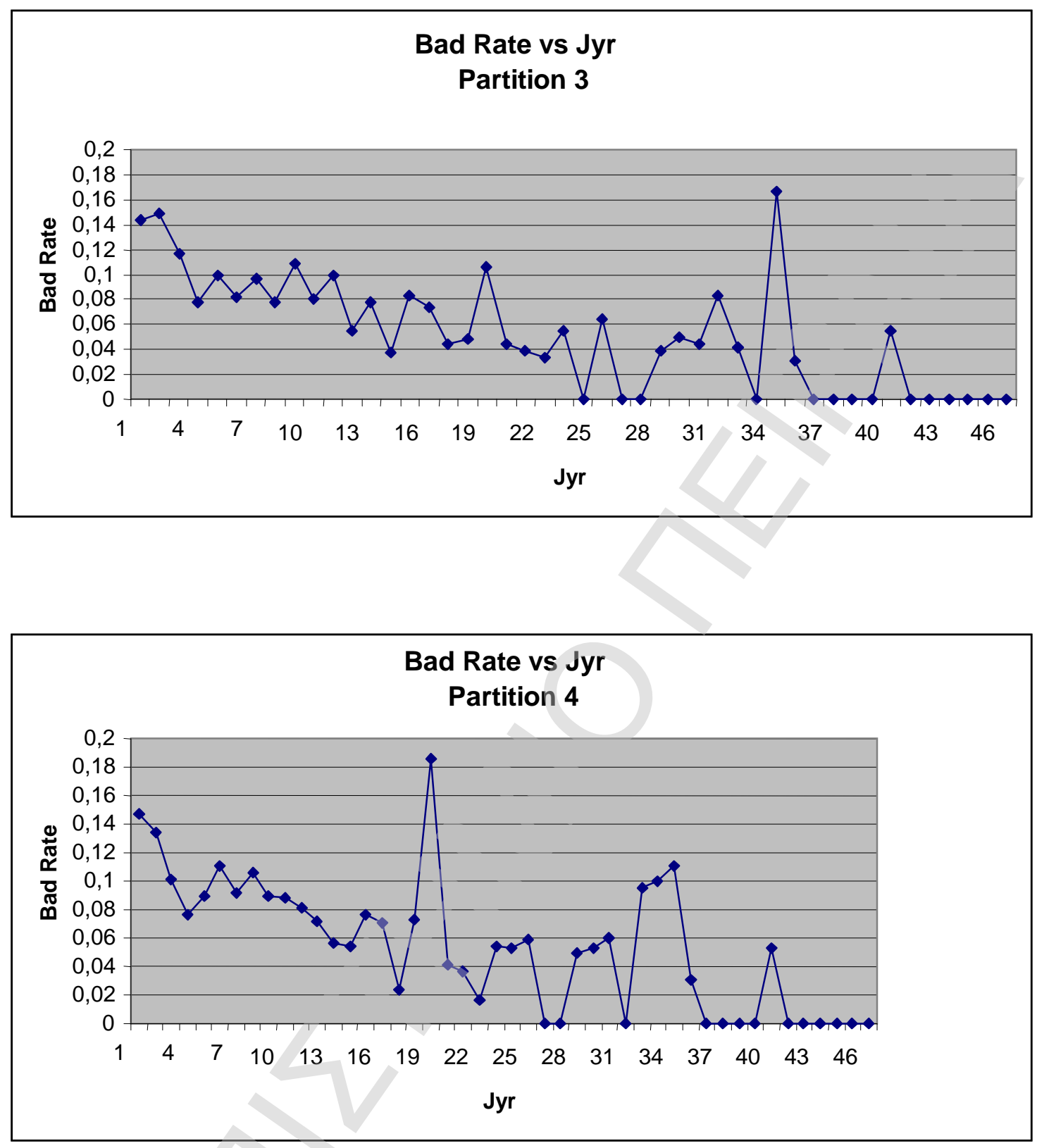


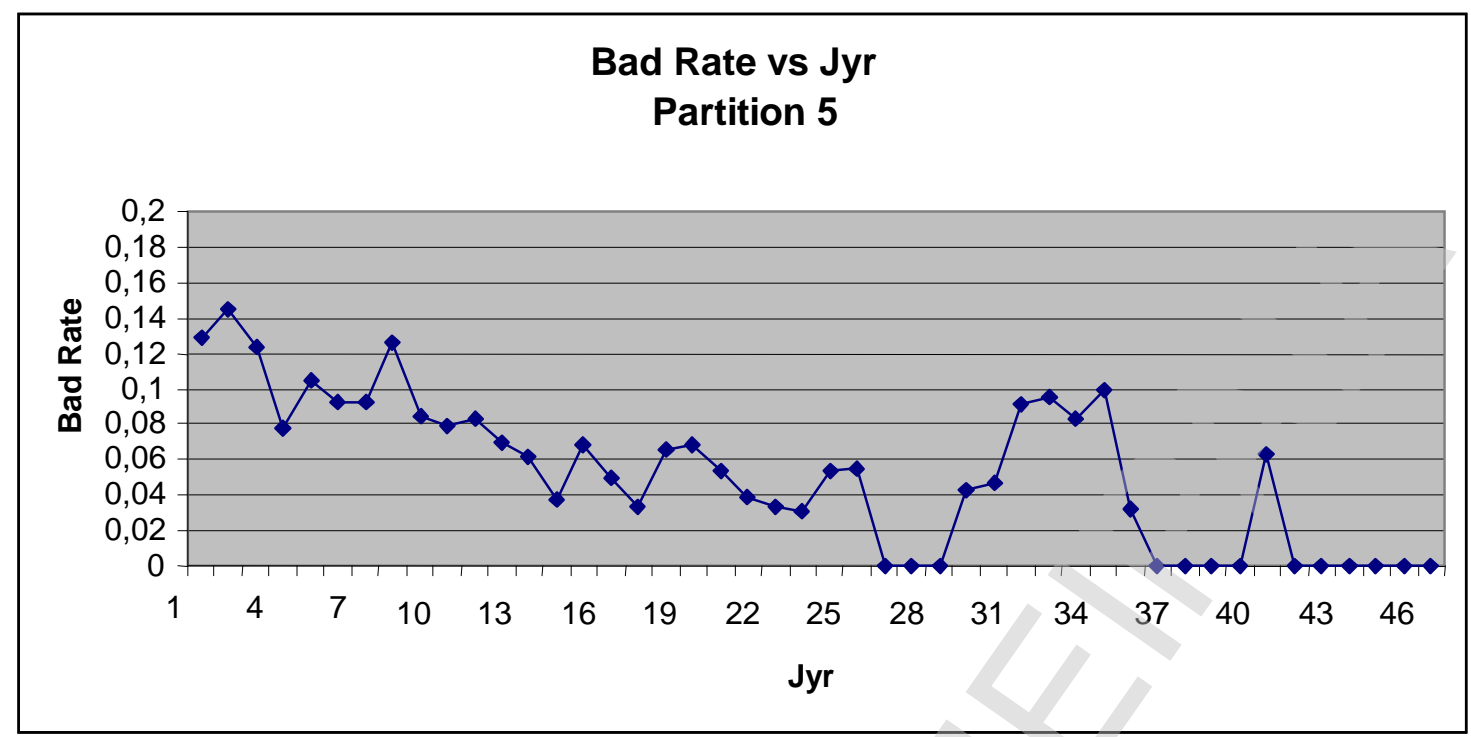




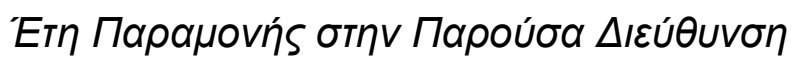
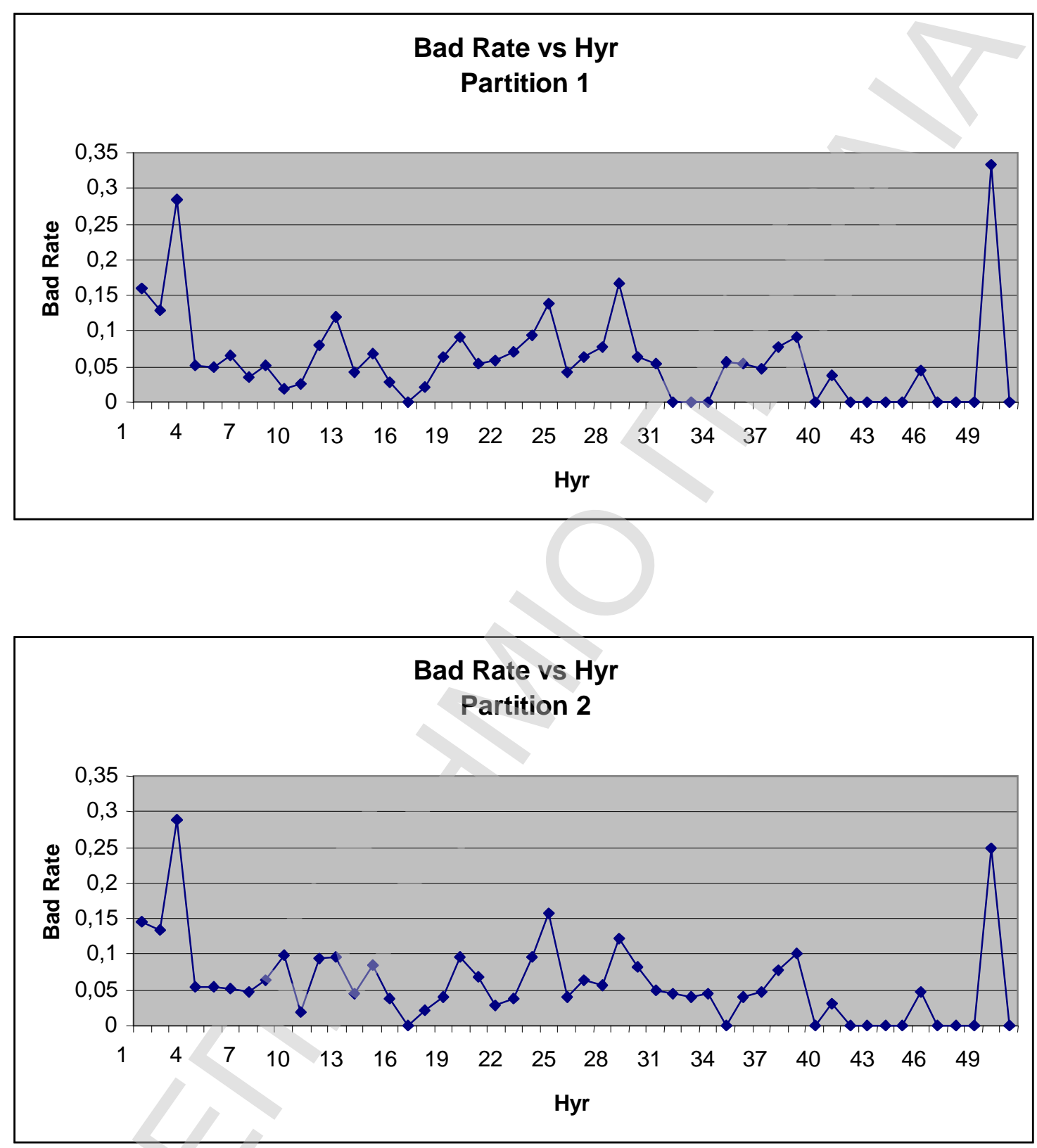

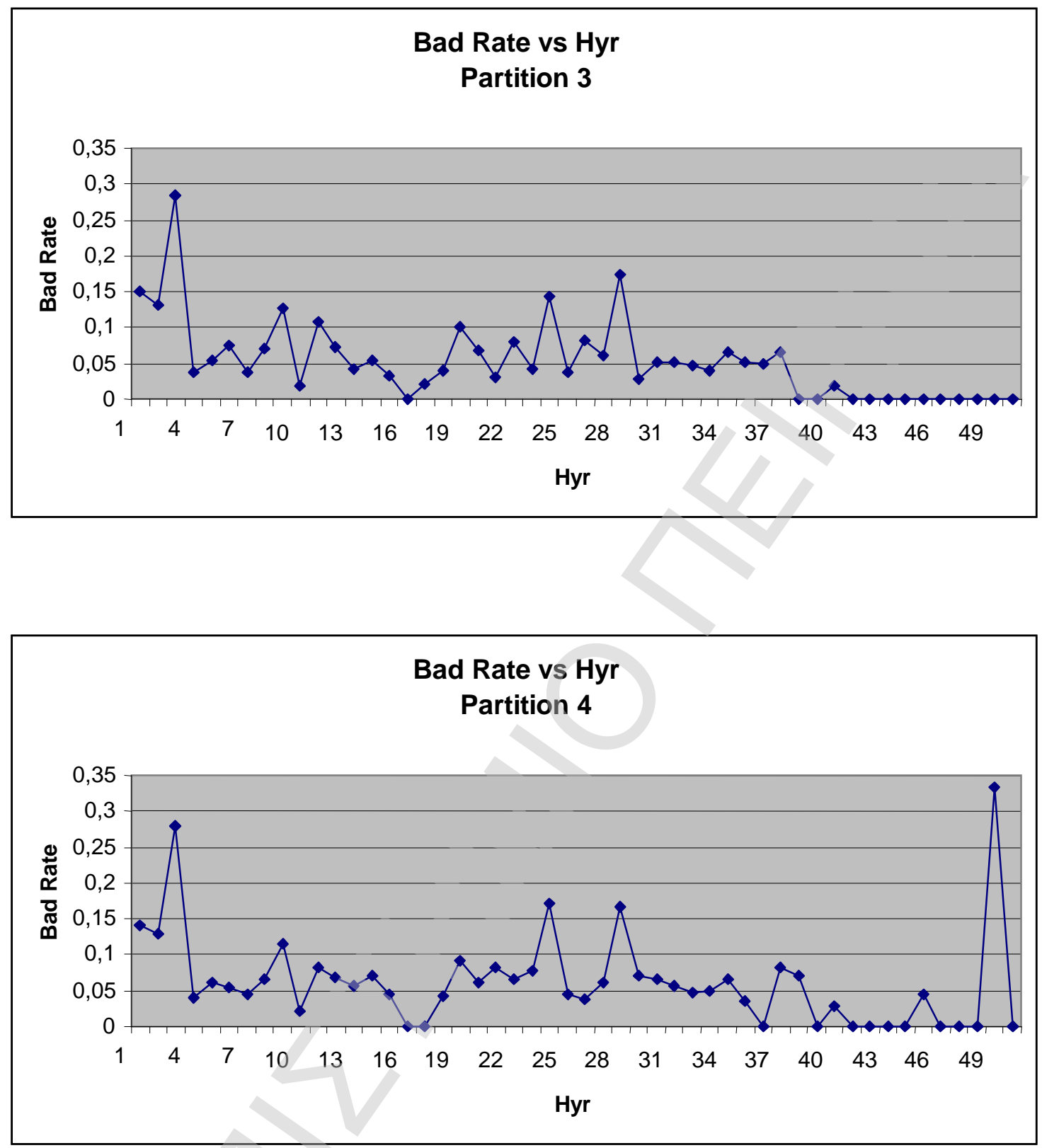


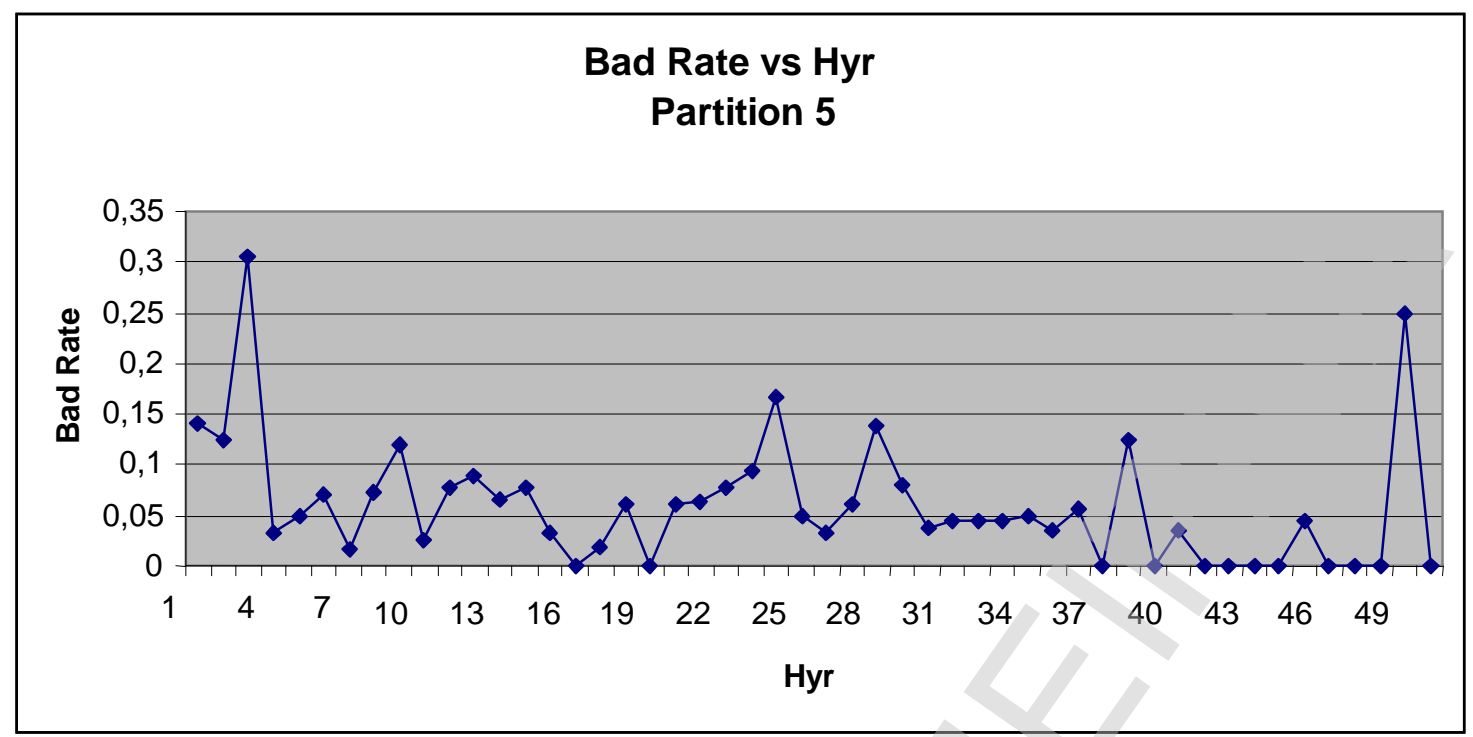




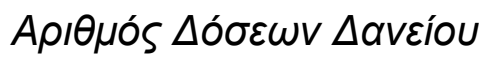
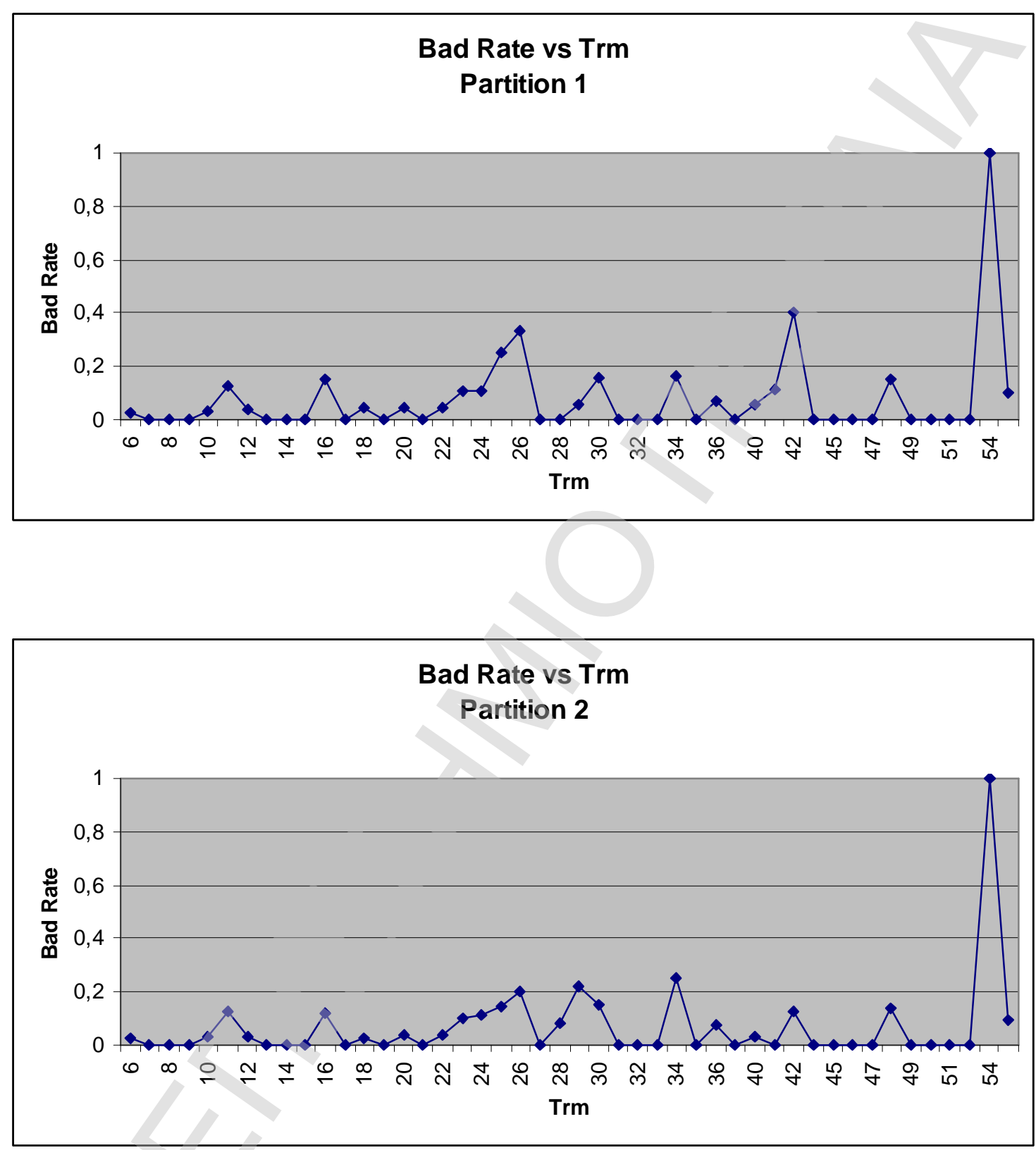

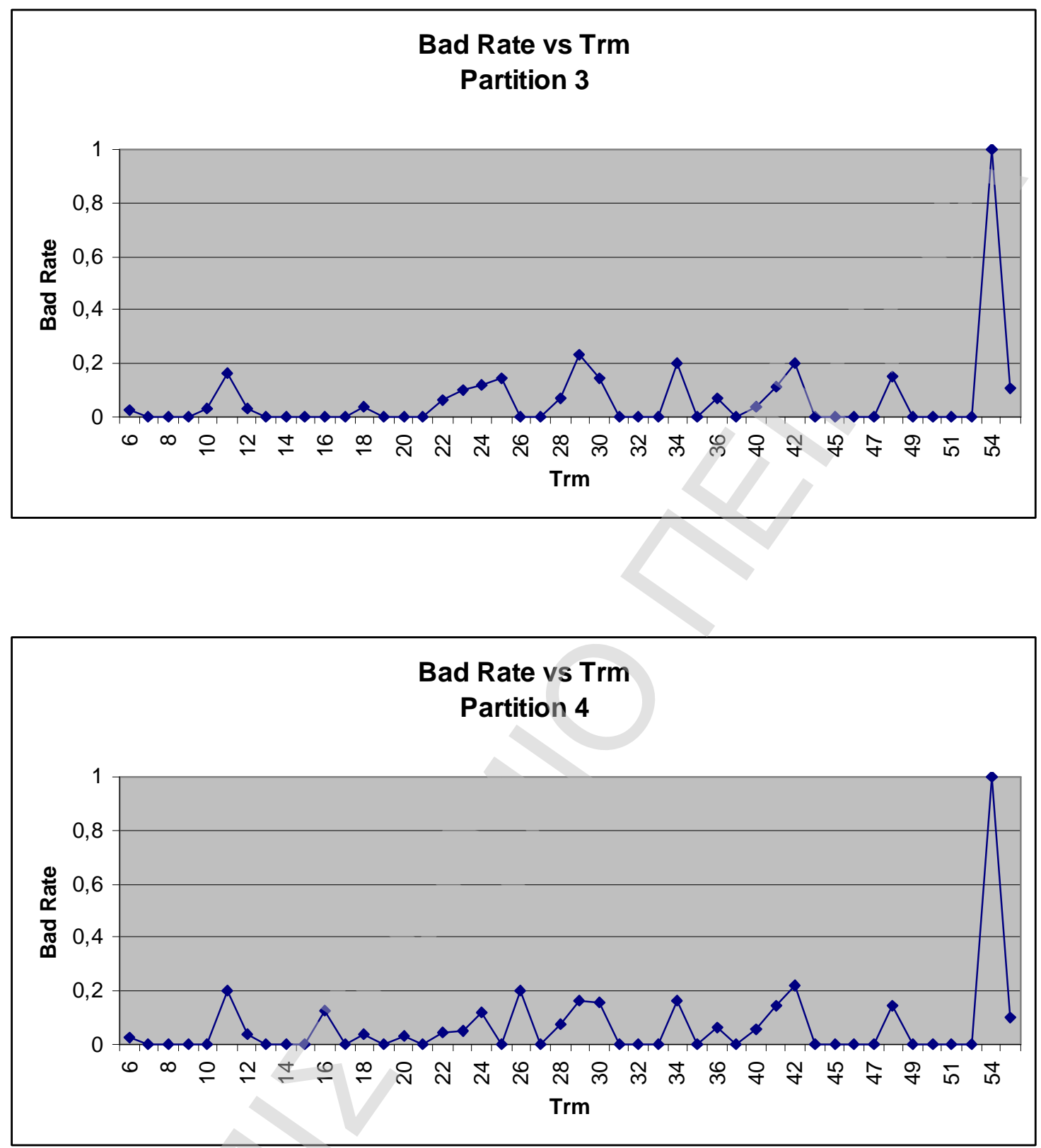


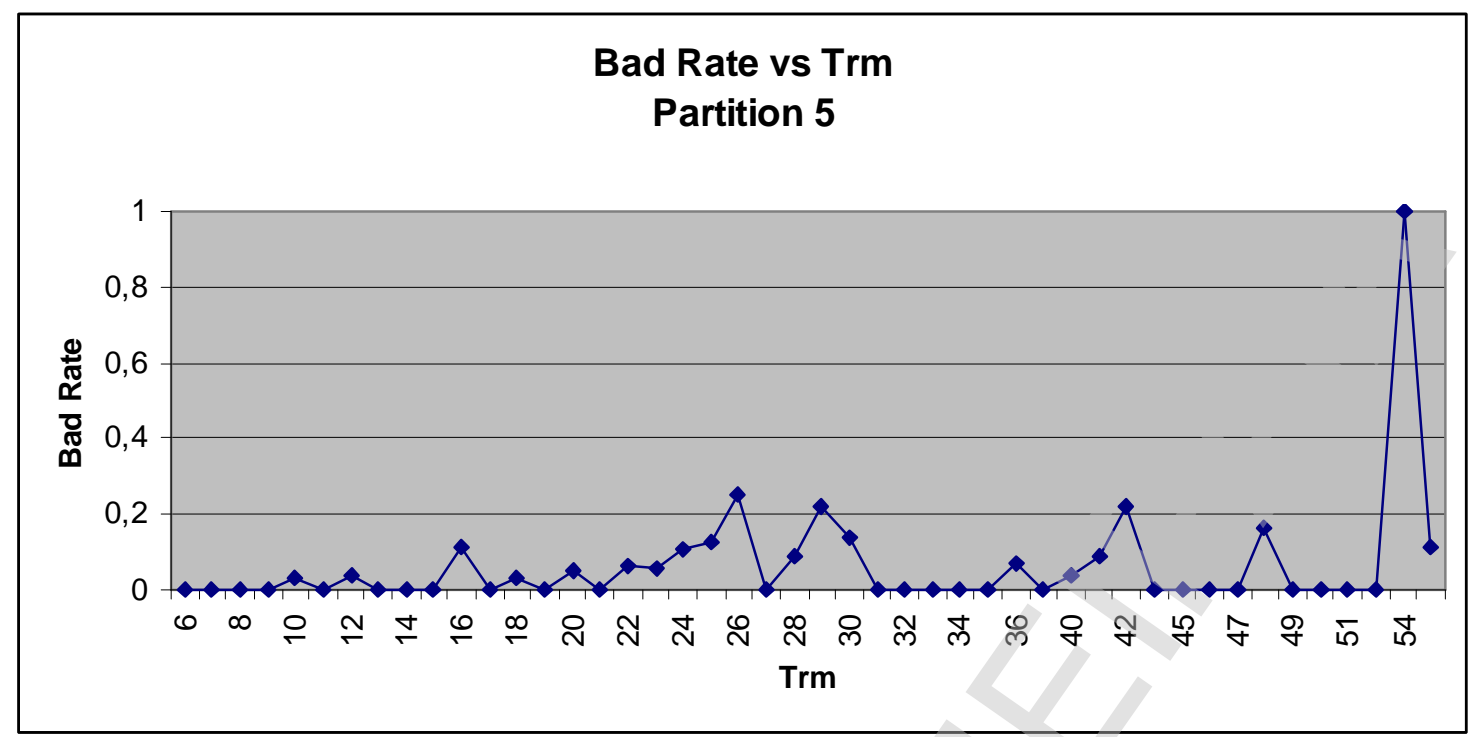




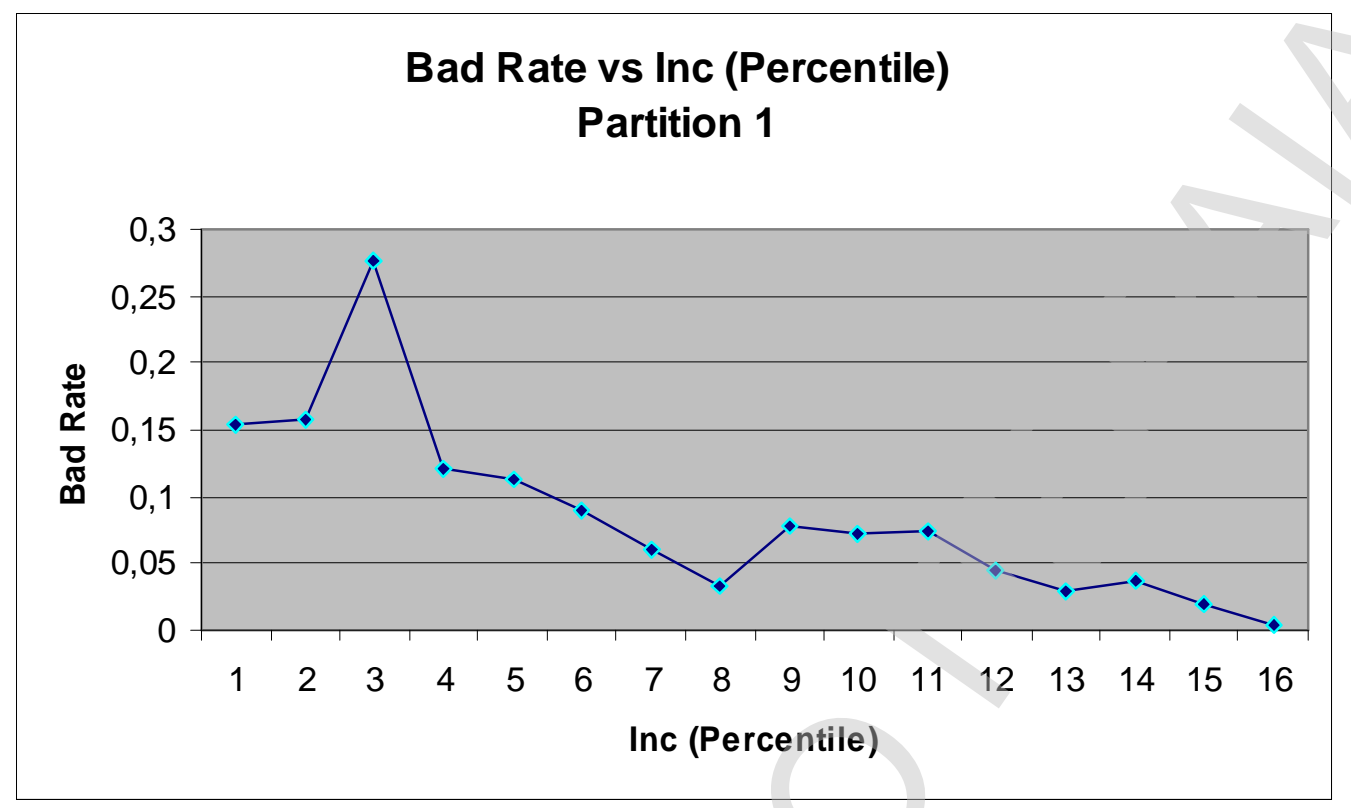

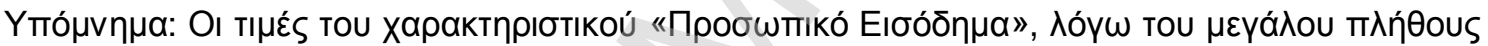

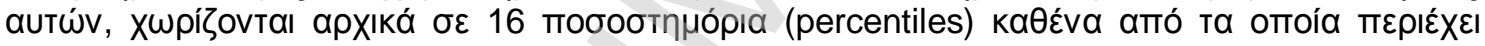

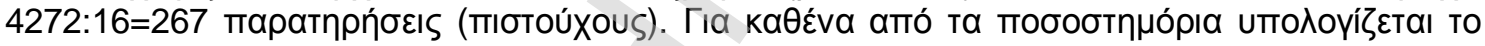

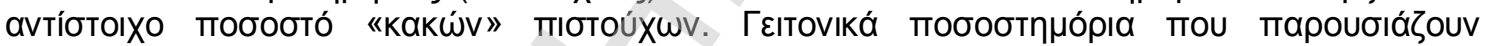

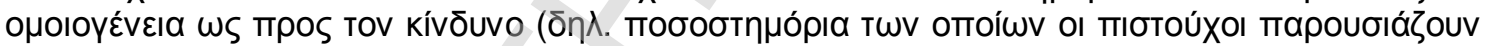

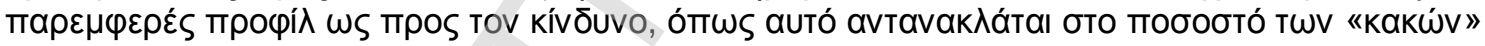

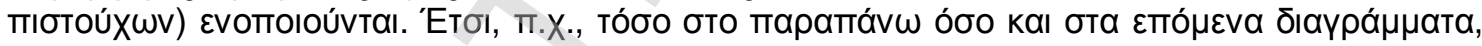

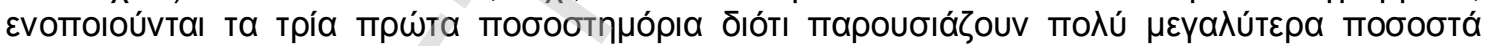

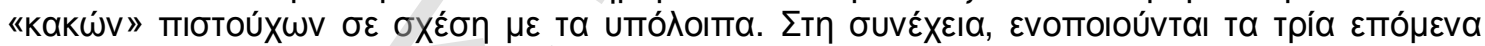

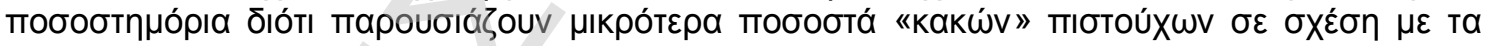

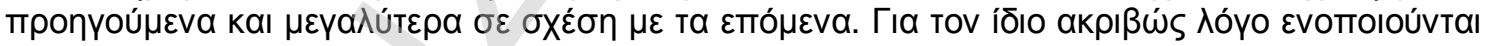

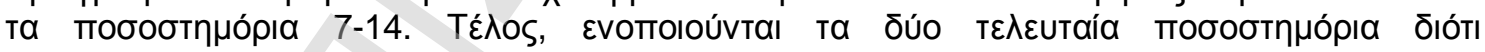

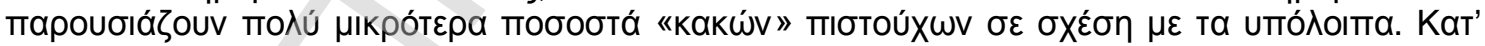

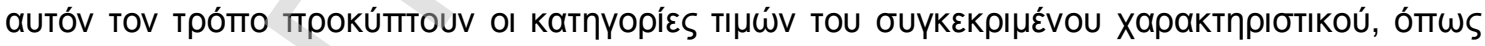

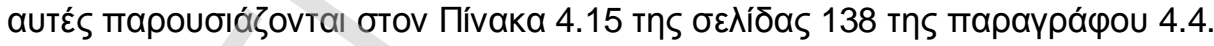



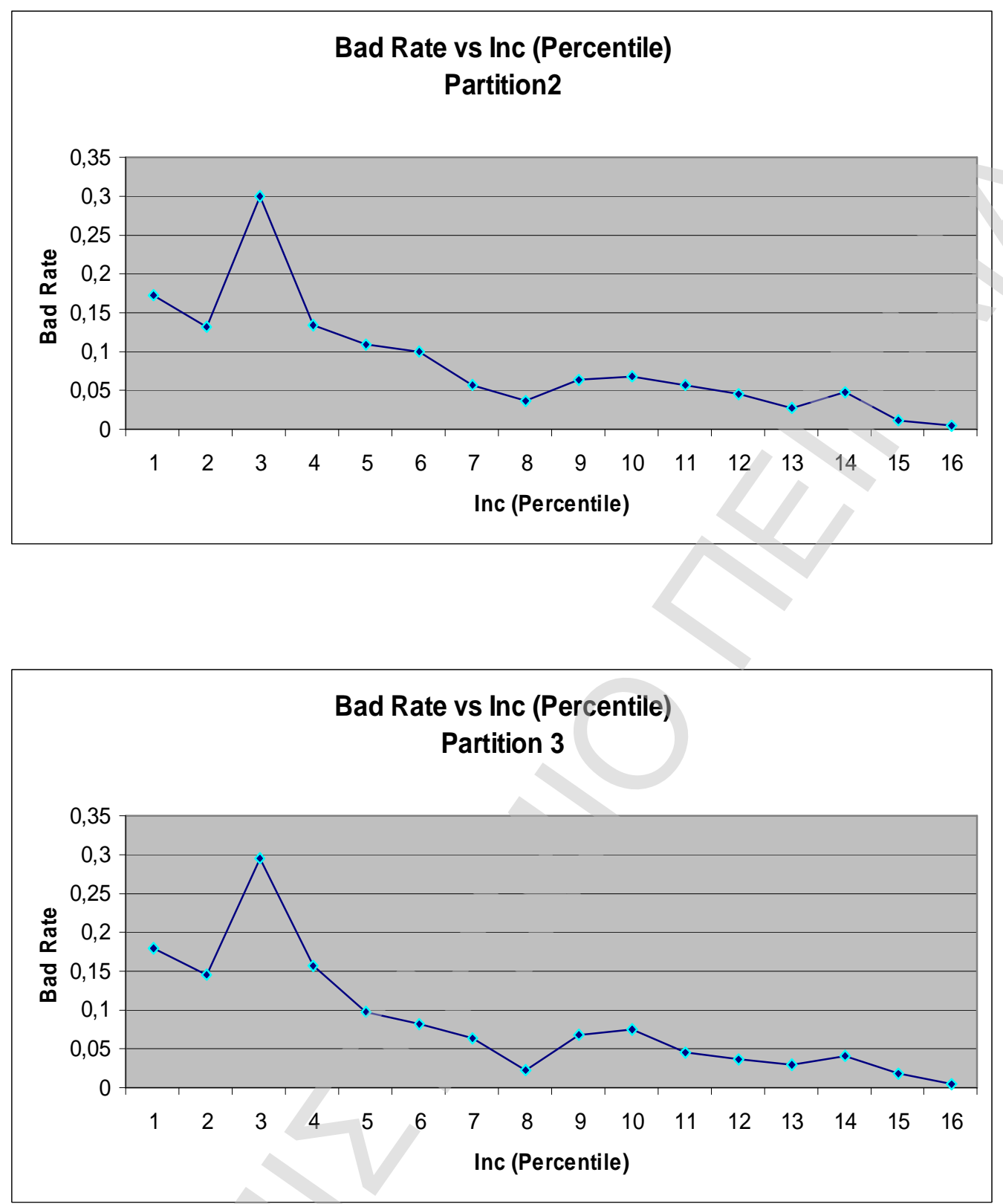

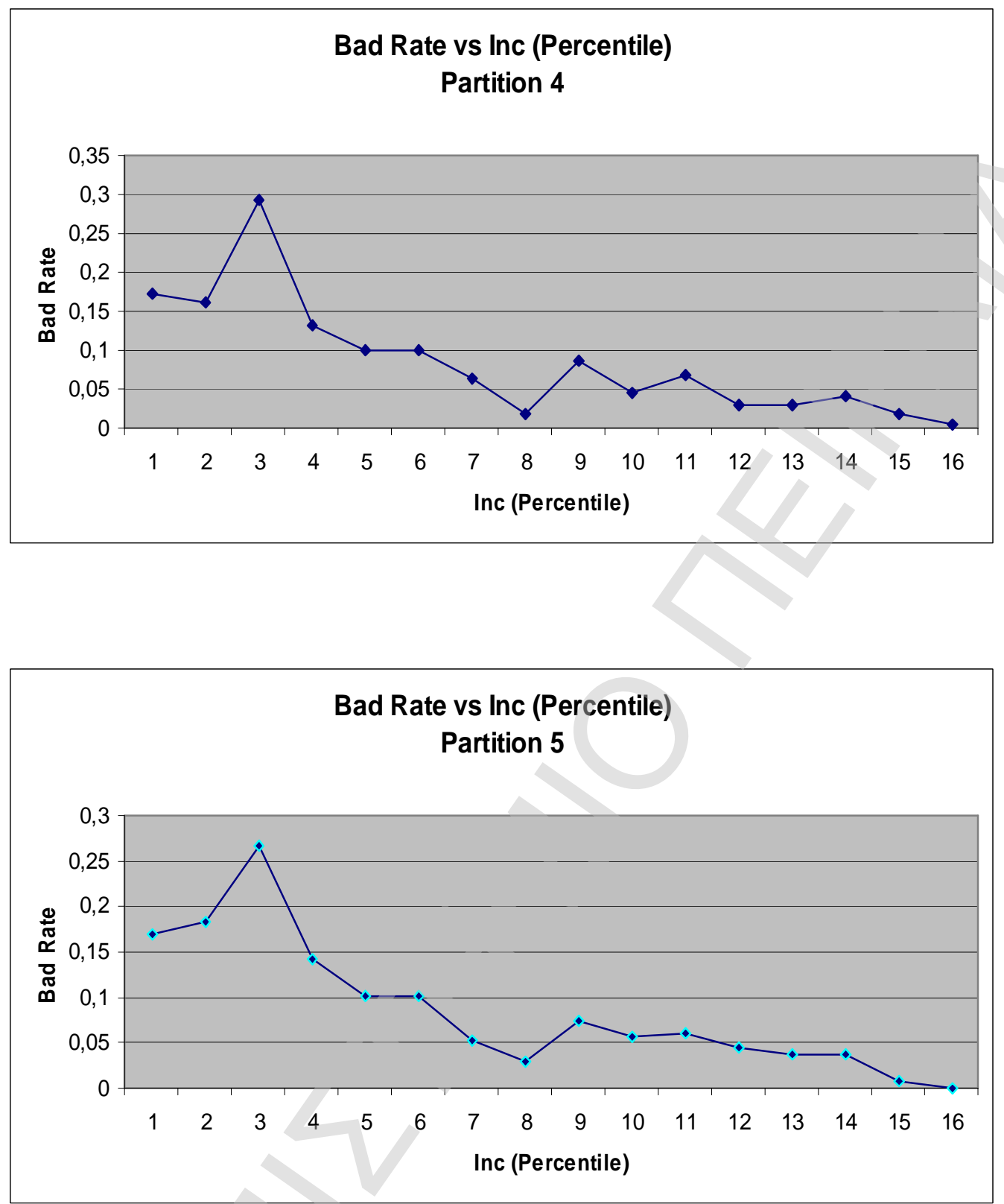
ПАРАРТНMА B

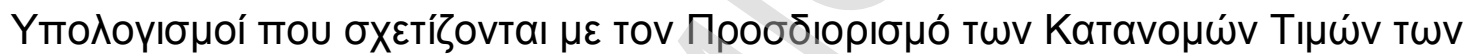

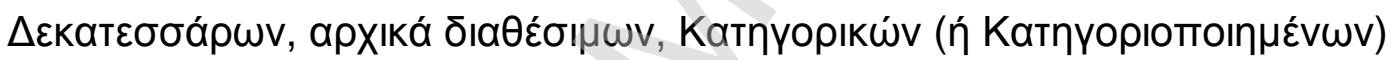
Характпрібтіки́v 
$\Delta ı \alpha \mu \dot{p}$ ¡ơ 1:

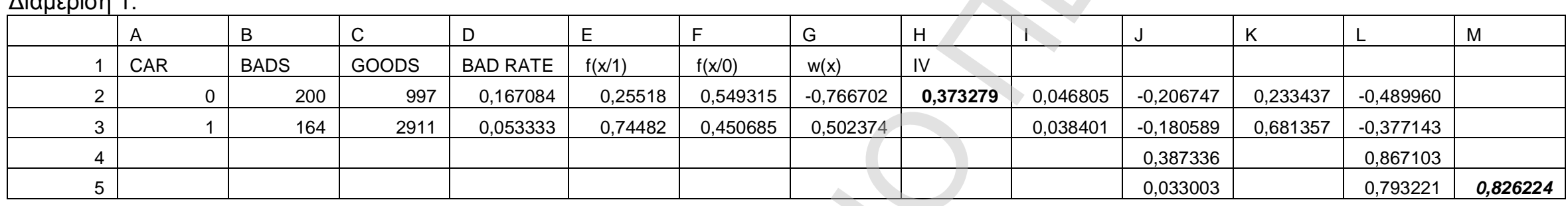

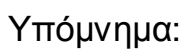

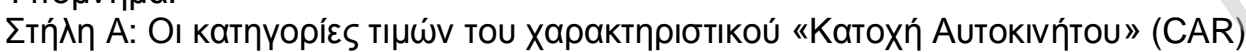

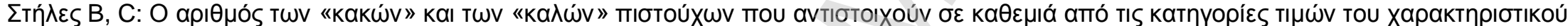

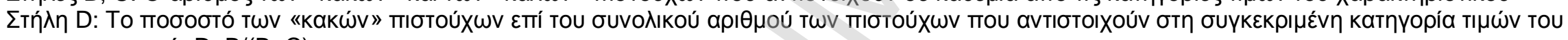

xapaktпрібтікоú: $\mathrm{D}=\mathrm{B} /(\mathrm{B}+\mathrm{C})$

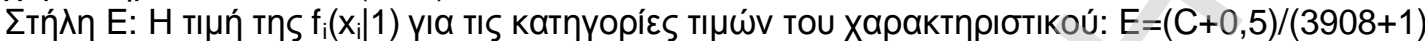

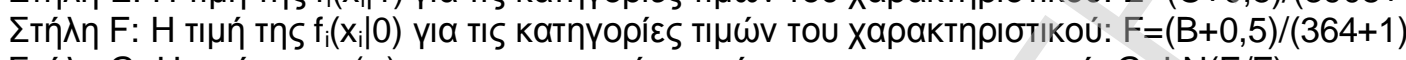

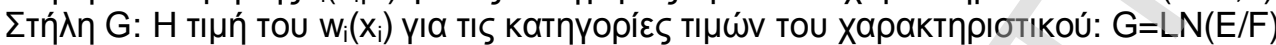

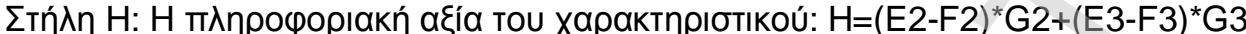

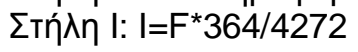

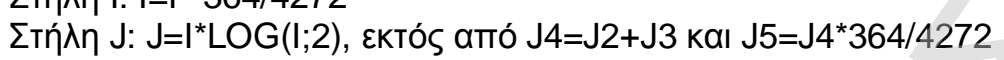

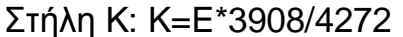

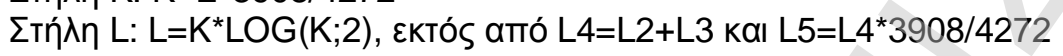

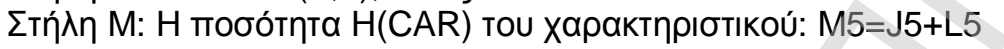




\begin{tabular}{|c|c|c|c|c|c|c|c|c|c|c|c|c|}
\hline CAR & BADS & GOODS & $\begin{array}{l}\text { BAD } \\
\text { RATE }\end{array}$ & $f(x / 1)$ & $f(x / 0)$ & $w(x)$ & IV & & & & & \\
\hline 0 & 194 & 1018 & 0,160066 & 0,260553 & 0,532877 & $-0,71549$ & 0,319925 & 0,045404 & $-0,202550$ & 0,238352 & $-0,493111$ & \\
\hline 1 & 170 & 2890 & 0,055556 & 0,739447 & 0,467123 & 0,45931 & & 0,039802 & $-0,185119$ & 0,676442 & $-0,381487$ & \\
\hline & & & & & & & & 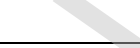 & 0,387669 & & 0,874598 & \\
\hline & & & & & & & & & 0,033032 & & 0,800077 & 9 \\
\hline
\end{tabular}

\begin{tabular}{|c|c|c|c|c|c|c|c|c|c|c|c|c|}
\hline on 3: & & & & & & & & & & & & \\
\hline CAR & BADS & GOODS & $\begin{array}{l}\text { BAD } \\
\text { RATE }\end{array}$ & $f(x / 1)$ & $f(x / 0)$ & $w(x)$ & IV & ) & & & & \\
\hline 0 & 205 & 990 & 0,171548 & 0,25339 & 0,563014 & $-0,79838$ & 0,413044 & 0.047972 & $-0,210198$ & 0,231799 & $-0,488877$ & \\
\hline 1 & 159 & 2918 & 0,051674 & 0,74661 & 0,436986 & 0,535642 & 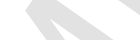 & 0.037234 & $-0,176758$ & 0,682995 & $-0,375684$ & \\
\hline & & & & & & & 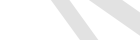 & & 0,386956 & & 0,864561 & \\
\hline & & & & & & & 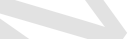 & & 0,032971 & & 0,790895 & 866 \\
\hline
\end{tabular}

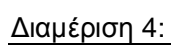

\begin{tabular}{|r|r|r|l|l|l|l|l|l|l|l|l|l|}
\hline \multicolumn{1}{|l|}{ CAR } & BADS & GOODS & $\begin{array}{l}\text { BAD } \\
\text { RATE }\end{array}$ & $\mathrm{f}(\mathrm{x} / 1)$ & $\mathrm{f}(\mathrm{x} / 0)$ & $\mathrm{w}(\mathrm{x})$ & $\mathrm{IV}$ & & & & & \\
\hline 0 & 196 & 1035 & 0,15922 & 0,264902 & 0,538356 & $-0,70916$ & 0,321138 & 0,045871 & $-0,203956$ & 0,24233 & $-0,495554$ & \\
\hline 1 & 168 & 2873 & 0,055245 & 0,735098 & 0,461644 & 0,465211 & & 0,039335 & $-0,183617$ & 0,672464 & $-0,384966$ & \\
\hline & & & & & & & & & 0,387572 & & 0,880521 & \\
\hline & & & & & & & & & 0,033023 & & 0,805495 & 0,838518 \\
\hline
\end{tabular}

\begin{tabular}{|c|c|c|c|c|c|c|c|c|c|c|c|c|}
\hline \multicolumn{13}{|l|}{ 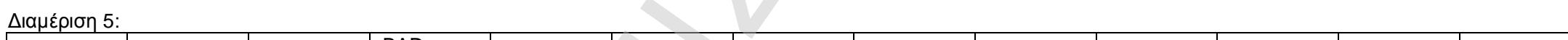 } \\
\hline CAR & BADS & GOODS & $\begin{array}{l}\text { BAD } \\
\text { RATE }\end{array}$ & $f(x / 1)$ & $f(x / 0)$ & $w(x)$ & IV & & & & & \\
\hline 0 & 197 & 996 & 0,16513 & 0,254925 & 0,541096 & $-0,75263$ & 0,354072 & 0,046105 & $-0,204656$ & 0,233203 & $-0,489806$ & \\
\hline 1 & 167 & 2912 & 0,054238 & 0,745075 & 0,458904 & 0,484644 & & 0,039101 & $-0,182863$ & 0,681591 & $-0,376935$ & \\
\hline & & & & 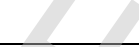 & & & & & 0,387519 & & 0,866741 & \\
\hline & & & & +8 & 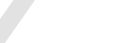 & & & & 0,033019 & & 0,79289 & 0,825909 \\
\hline
\end{tabular}




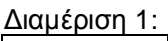

\begin{tabular}{|r|r|r|r|r|r|r|r|}
\hline \multicolumn{1}{|l|}{ CELL } & \multicolumn{1}{l|}{ BADS } & \multicolumn{1}{l|}{ GOODS } & BAD RATE & $\mathrm{f}(\mathrm{x} / 1)$ & $\mathrm{f}(\mathrm{x} / 0)$ & $\mathrm{w}(\mathrm{x})$ & \\
\hline 0 & 182 & 1641 & 0,099835 & 0,419928 & 0,5 & $-0,17452$ & 0,025869 \\
\hline 1 & 182 & 2267 & 0,074316 & 0,580072 & 0,5 & 0,148543 & \\
\hline & & & & & & & \\
\hline
\end{tabular}

\begin{tabular}{|c|c|c|c|c|c|c|c|}
\hline CELL & BADS & GOODS & $\begin{array}{l}\text { BAD } \\
\text { RATE }\end{array}$ & $f(x / 1)$ & $f(x / 0)$ & $w(x)$ & \\
\hline 0 & 184 & 1658 & 0,099891 & 0,424277 & 0,505479 & $-0,17512$ & 0,026566 \\
\hline 1 & 180 & 2250 & 0,074074 & 0,575723 & 0,494521 & 0,152037 & r \\
\hline
\end{tabular}

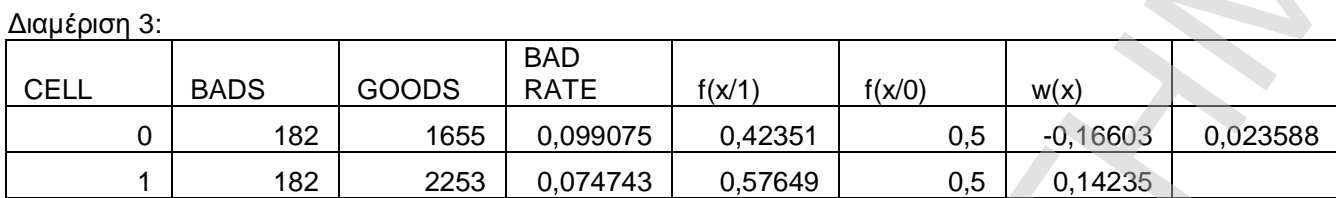

\begin{tabular}{|c|c|c|c|c|c|c|c|}
\hline CELL & BADS & GOODS & $\begin{array}{l}\text { BAD } \\
\text { RATE }\end{array}$ & $f(x / 1)$ & $f(x / 0)$ & $w(x)$ & \\
\hline 0 & 181 & 1641 & 0,099341 & 0,419928 & 0,49726 & $-0,16903$ & 0,024136 \\
\hline 1 & 183 & 2267 & 0,074694 & 0,580072 & 0,50274 & 0,143079 & \\
\hline
\end{tabular}

\begin{tabular}{|c|c|c|c|c|c|c|c|}
\hline CELL & BADS & GOODS & $\begin{array}{l}\text { BAD } \\
\text { RATE }\end{array}$ & $f(x / 1)$ & $f(x / 0)$ & $w(x)$ & \\
\hline 0 & 187 & 1697 & 0,099257 & 0,434254 & 0,513699 & $-0,16801$ & 0,025368 \\
\hline 1 & 177 & 2211 & 0,074121 & 0,565746 & 0,486301 & 0,151316 & \\
\hline
\end{tabular}




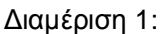

\begin{tabular}{|r|r|r|l|l|l|l|l|}
\hline \multicolumn{1}{|l|}{ CLIENT } & \multicolumn{1}{|l|}{ BADS } & GOODS & $\begin{array}{l}\text { BAD } \\
\text { RATE }\end{array}$ & $\mathrm{f}(\mathrm{x} / 1)$ & $\mathrm{f}(\mathrm{x} / 0)$ & $\mathrm{w}(\mathrm{x})$ & \\
\hline 0 & 309 & 2864 & 0,097384 & 0,732796 & 0,847945 & $-0,14595$ & 0,081724 \\
\hline 1 & 55 & 1044 & 0,050045 & 0,267204 & 0,152055 & 0,563771 & \\
\hline & & & & & & & \\
\hline
\end{tabular}

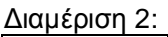

\begin{tabular}{|r|r|r|l|l|l|l|l|}
\hline \multicolumn{1}{|l|}{ CLIENT } & \multicolumn{1}{|l|}{ BADS } & GOODS & BAD RATE & $f(x / 1)$ & $f(x / 0)$ & $w(x)$ & \\
\hline 0 & 305 & 2882 & 0,0957013 & 0,737401 & 0,836986 & $-0,12668$ & 0,060097 \\
\hline 1 & 59 & 1026 & 0,0543779 & 0,262599 & 0,163014 & 0,476794 & \\
\hline
\end{tabular}

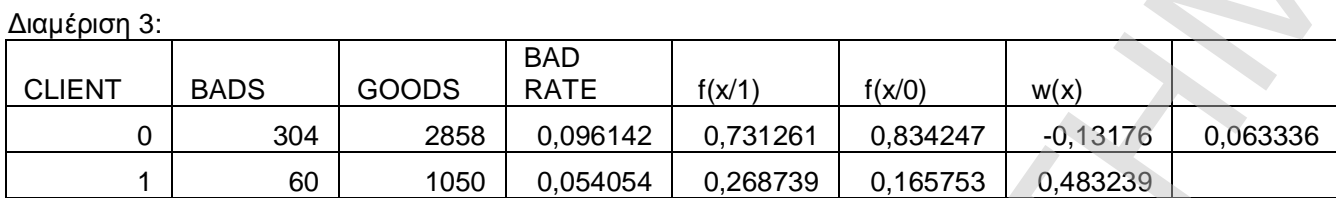

\begin{tabular}{|c|c|c|c|c|c|c|c|}
\hline \multicolumn{8}{|c|}{ 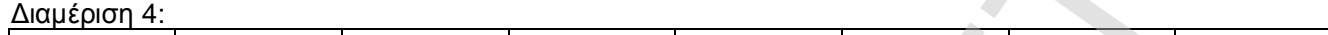 } \\
\hline CLIENT & BADS & GOODS & $\begin{array}{l}\text { BAD } \\
\text { RATE }\end{array}$ & $f(x / 1)$ & $f(x / 0)$ & $w(x)$ & \\
\hline 0 & 303 & 2855 & 0,095947 & 0,730494 & 0,831507 & $-0,12952$ & 0,060529 \\
\hline 1 & 61 & 1053 & 0,054758 & 0,269506 & 0,168493 & 0,469697 & \\
\hline
\end{tabular}

\begin{tabular}{|c|c|c|c|c|c|c|c|}
\hline CLIENT & BADS & GOODS & $\begin{array}{l}\text { BAD } \\
\text { RATE }\end{array}$ & $f(x / 1)$ & $f(x / 0)$ & $w(x)$ & \\
\hline 0 & 299 & 2853 & 0,09486 & 0,729982 & 0,820548 & $-0,11695$ & 0,047595 \\
\hline 1 & 65 & 1055 & 0,058036 & 0,270018 & 0,179452 & 0,40858 & \\
\hline
\end{tabular}




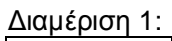

\begin{tabular}{|r|r|r|r|l|l|l|l|}
\hline \multicolumn{1}{|l|}{ GENDER } & \multicolumn{1}{l|}{ BADS } & GOODS & BAD RATE & $f(x / 1)$ & $f(x / 0)$ & $w(x)$ & \\
\hline 0 & 127 & 1014 & 0,1113059 & 0,259529 & 0,349315 & $-0,297105$ & 0,038282 \\
\hline 1 & 237 & 2894 & 0,0756947 & 0,740471 & 0,650685 & 0,1292605 & \\
\hline & & & & & & & \\
\hline
\end{tabular}

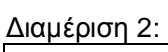

\begin{tabular}{|r|r|r|r|r|l|l|l|}
\hline \multicolumn{1}{|c|}{ GENDER } & \multicolumn{1}{l|}{ BADS } & \multicolumn{1}{l|}{ GOODS } & BAD RATE & $\mathrm{f}(\mathrm{x} / 1)$ & $\mathrm{f}(\mathrm{x} / 0)$ & $\mathrm{w}(\mathrm{x})$ & \\
\hline 0 & 132 & 1006 & 0,115993 & 0,257483 & 0,363014 & $-0,34349$ & 0,052426 \\
\hline 1 & 232 & 2902 & 0,0740268 & 0,742517 & 0,636986 & 0,153298 & \\
\hline
\end{tabular}

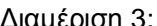

\begin{tabular}{|r|r|r|r|l|l|l|l|}
\hline \multicolumn{1}{|c|}{ GENDER } & \multicolumn{1}{|c|}{ BADS } & GOODS & BAD RATE & $\mathrm{f}(\mathrm{x} / 1)$ & $\mathrm{f}(\mathrm{x} / 0)$ & $\mathrm{w}(\mathrm{x})$ & \\
\hline 0 & 126 & 1009 & 0,1110132 & 0,25825 & 0,346575 & $-0,29417$ & 0,037181 \\
\hline 1 & 238 & 2899 & 0,0758687 & 0,74175 & 0,653425 & 0,126785 & \\
\hline
\end{tabular}

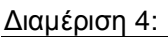

\begin{tabular}{|r|r|r|r|l|l|l|l|}
\hline \multicolumn{1}{|l|}{ GENDER } & \multicolumn{1}{l|}{ BADS } & \multicolumn{1}{l|}{ GOODS } & BAD RATE & $\mathrm{f}(\mathrm{x} / 1)$ & $\mathrm{f}(\mathrm{x} / 0)$ & $\mathrm{w}(\mathrm{x})$ & \\
\hline 0 & 134 & 1014 & 0,1167247 & 0,259529 & 0,368493 & $-0,35055$ & 0,055542 \\
\hline 1 & 230 & 2894 & 0,0736236 & 0,740471 & 0,631507 & 0,159177 & \\
\hline
\end{tabular}

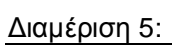

\begin{tabular}{|r|r|r|r|l|l|r|r|}
\hline \multicolumn{1}{|l|}{ GENDER } & \multicolumn{1}{l|}{ BADS } & \multicolumn{1}{l|}{ GOODS } & BAD RATE & $\mathrm{f}(\mathrm{x} / 1)$ & $\mathrm{f}(\mathrm{x} / 0)$ & $\mathrm{w}(\mathrm{x})$ & \\
\hline 0 & 127 & 1014 & 0,1113059 & 0,259529 & 0,349315 & $-0,2971$ & 0,038282 \\
\hline 1 & 237 & 2894 & 0,0756947 & 0,740471 & 0,650685 & 0,129261 & \\
\hline
\end{tabular}




\begin{tabular}{|c|c|c|c|c|c|c|c|c|c|c|c|c|}
\hline \multicolumn{13}{|c|}{ 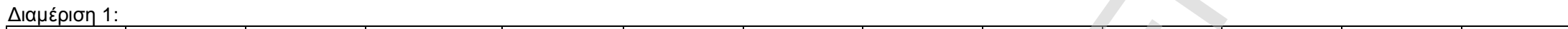 } \\
\hline CRD & BADS & GOODS & BAD RATE & $f(x / 1)$ & $f(x / 0)$ & $w(x)$ & & & 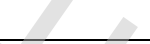 & & & \\
\hline 0 & 309 & 2509 & 0,109652 & 0,64198 & 0,847945 & $-0,278259$ & 0,057312 & 0,07225 & $-0,273890$ & 0,587279 & $-0,450961$ & \\
\hline \multirow[t]{3}{*}{1} & 55 & 1399 & 0,037827 & 0,35802 & 0,152055 & 0,856348 & 0,176378 & 0,012956 & $-0,081237$ & 0,327515 & $-0,527419$ & \\
\hline & & & & & & & 0,233689 & 8 & 0,355127 & & 0,978380 & \\
\hline & & & & & & & & & 0,030259 & & 0,895016 & 0,925275 \\
\hline
\end{tabular}

\begin{tabular}{|c|c|c|c|c|c|c|c|c|c|c|c|c|}
\hline \multicolumn{13}{|l|}{$\Delta ı \alpha \mu \varepsilon ́ p ı \sigma \eta ~ 2:$} \\
\hline CRD & BADS & GOODS & BAD RATE & $f(x / 1)$ & $f(x / 0)$ & $w(x)$ & 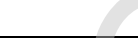 & D & & & & \\
\hline 0 & 301 & 2537 & 0,106061 & 0,649143 & 0,826027 & $-0,24097$ & 0,042625 & 0,070382 & $-0,269469$ & 0,593832 & $-0,446486$ & \\
\hline 1 & 63 & 1371 & 0,043933 & 0,350857 & 0,173973 & 0,701481 & 0,124081 & 0,014824 & $-0,090067$ & 0,320962 & $-0,526225$ & \\
\hline & & & & & & & 0,166706 & & 0,359536 & & 0,972712 & \\
\hline & & & & & & & se & 7 & 0,030635 & & 0,889831 & 0,920465 \\
\hline
\end{tabular}

\begin{tabular}{|c|c|c|c|c|c|c|c|c|c|c|c|c|}
\hline \multicolumn{13}{|l|}{ 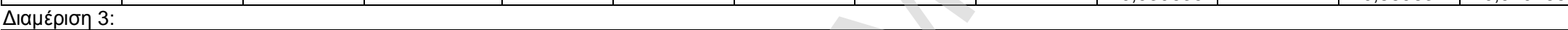 } \\
\hline CRD & BADS & GOODS & BAD RATE & $f(x / 1)$ & $f(x / 0)$ & $w(x)$ & + & & & & & \\
\hline 0 & 309 & 2519 & 0,109264 & 0,644538 & 0,847945 & $-0,27428$ & 0,055791 & 0,07225 & $-0,273890$ & 0,58962 & $-0,449375$ & \\
\hline \multirow[t]{3}{*}{1} & 55 & 1389 & 0,038089 & 0,355462 & 0,152055 & 0,849177 & 0,172728 & 0,012956 & $-0,081237$ & 0,325174 & $-0,527015$ & \\
\hline & & & & & & 2 & 0,228519 & & 0,355127 & & 0,976390 & \\
\hline & & & & & & K & & & 0,030259 & & 0,893195 & 0,923454 \\
\hline
\end{tabular}

\begin{tabular}{|c|c|c|c|c|c|c|c|c|c|c|c|c|}
\hline \multicolumn{13}{|c|}{ 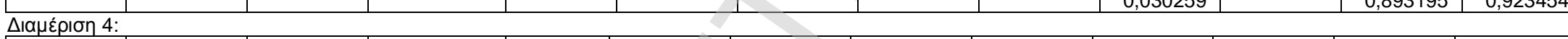 } \\
\hline CRD & BADS & GOODS & BAD RATE & $f(x / 1)$ & $f(x / 0)$ & $w(x)$ & & & & & & \\
\hline 0 & 305 & 2509 & 0,108387 & 0,64198 & 0,836986 & $-0,26525$ & 0,051726 & 0,071316 & $-0,271688$ & 0,587279 & $-0,450961$ & \\
\hline \multirow[t]{3}{*}{1} & 59 & 1399 & 0,040466 & 0,35802 & 0,163014 & 0,786754 & 0,153422 & 0,01389 & $-0,085697$ & 0,327515 & $-0,527419$ & \\
\hline & & & & & & & 0,205148 & & 0,357386 & & 0,978380 & \\
\hline & & & & & & & & & 0,030451 & & 0,895016 & 0,925468 \\
\hline
\end{tabular}

\begin{tabular}{|c|c|c|c|c|c|c|c|c|c|c|c|c|}
\hline \multicolumn{13}{|l|}{$\Delta ı \mu \varepsilon \varepsilon_{\rho} \mid \sigma \eta$ 5: } \\
\hline CRD & BADS & GOODS & BAD RATE & $f(x / 1)$ & $f(x / 0)$ & $w(x)$ & & & & & & \\
\hline 0 & 312 & 2522 & 0,110092 & 0,645306 & 0,856164 & $-0,28274$ & 0,059618 & 0,07295 & $-0,275529$ & 0,590322 & $-0,448896$ & \\
\hline \multirow[t]{3}{*}{1} & 52 & 1386 & 0,036161 & 0,354694 & 0,143836 & 0,902585 & 0,190318 & 0,012256 & $-0,077828$ & 0,324472 & $-0,526889$ & \\
\hline & & & & 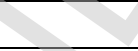 & & & 0,249936 & & 0,353358 & & 0,975785 & \\
\hline & & & & $x_{0}$ & & & & & 0,030108 & & 0.892642 & 0.922751 \\
\hline
\end{tabular}




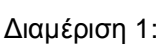

\begin{tabular}{|c|c|c|c|c|c|c|c|c|c|c|c|c|}
\hline MAR & BADS & GOODS & BAD RATE & $f(x / 1)$ & $f(x / 0)$ & $w(x)$ & & & 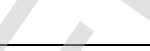 & & & \\
\hline 0 & 125 & 182 & 0,407166 & 0,046687 & 0,343836 & $-1,996695$ & 0,593315 & 0,029297 & $-0,149212$ & 0,042709 & $-0,194297$ & \\
\hline 1 & 166 & 965 & 0,146773 & 0,246994 & 0,456164 & $-0,613489$ & 0,128324 & 0,038868 & $-0,182107$ & 0,225949 & $-0,484871$ & \\
\hline \multirow[t]{3}{*}{2} & 73 & 2761 & 0,025759 & 0,706447 & 0,20137 & 1,255104 & 0,633924 & 0,017158 & $-0,100631$ & 0,646253 & $-0,407029$ & \\
\hline & & & & & & & 1,355563 & 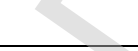 & 0,431950 & & 1,086197 & \\
\hline & & & & & & & & & 0,036805 & & 0,993646 & 1,030451 \\
\hline
\end{tabular}

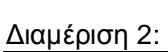

\begin{tabular}{|c|c|c|c|c|c|c|c|c|c|c|c|c|}
\hline MAR & BADS & GOODS & BAD RATE & $f(x / 1)$ & $f(x / 0)$ & $w(x)$ & & & & & & \\
\hline 0 & 127 & 192 & 0,398119 & 0,049245 & 0,349315 & $-1,95916$ & 0,587885 & 0,029764 & $-0,150911$ & 0,045049 & $-0,201476$ & \\
\hline 1 & 167 & 984 & 0,145091 & 0,251855 & 0,458904 & $-0,59999$ & 0,124227 & 0,039101 & $-0,182863$ & 0,230395 & $-0,487935$ & \\
\hline 2 & 70 & 2732 & 0,024982 & 0,699028 & 0,193151 & 1,28622 & 0,650669 & 0,016458 & $-0,097513$ & 0,639467 & $-0,412494$ & \\
\hline & & & & & & & 1,362781 & & 0,431287 & & 1,101905 & \\
\hline & & & & & & & 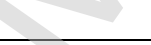 & & 0,036748 & & 1,008016 & 1,044764 \\
\hline
\end{tabular}

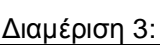

\begin{tabular}{|c|c|c|c|c|c|c|c|c|c|c|c|c|}
\hline MAR & BADS & GOODS & BAD RATE & $f(x / 1)$ & $f(x / 0)$ & $w(x)$ & & & & & & \\
\hline 0 & 118 & 174 & 0,40411 & 0,044641 & 0,324658 & $-1,98413$ & 0,555589 & 0,027663 & $-0,143180$ & 0,040837 & $-0,188421$ & \\
\hline 1 & 176 & 995 & 0,150299 & 0,254669 & 0,483562 & $-0,64122$ & 0,14677 & 0,041202 & $-0,189577$ & 0,232969 & $-0,489652$ & \\
\hline 2 & 70 & 2739 & 0,02492 & 0,700819 & 0,193151 & 1,288778 & 0,654272 & 0,016458 & $-0,097513$ & 0,641105 & $-0,411184$ & \\
\hline & & & & & & & 1,356631 & & 0,430270 & & 1,089257 & \\
\hline & & & & & & & & & 0,036662 & & 0,996446 & 1,033108 \\
\hline
\end{tabular}

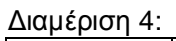

\begin{tabular}{|c|c|c|c|c|c|c|c|c|c|c|c|c|}
\hline MAR & BADS & GOODS & BAD RATE & $f(x / 1)$ & $f(x / 0)$ & $w(x)$ & & & & & & \\
\hline 0 & 119 & 184 & 0,392739 & 0,047199 & 0,327397 & $-1,93681$ & 0,54269 & 0,027896 & $-0,144050$ & 0,043177 & $-0,195747$ & \\
\hline 1 & 175 & 983 & 0,151123 & 0,251599 & 0,480822 & $-0,64766$ & 0,148459 & 0,040969 & $-0,188839$ & 0,230161 & $-0,487777$ & \\
\hline 2 & 70 & 2741 & 0,024902 & 0,70133 & 0,193151 & 1,289508 & 0,655302 & 0,016458 & $-0,097513$ & 0,641573 & $-0,410809$ & \\
\hline & & & & & & & 1,346451 & & 0,430402 & & 1,094333 & \\
\hline & & & & 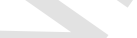 & & & & & 0,036673 & & 1,001089 & 1,037762 \\
\hline
\end{tabular}




\begin{tabular}{|c|c|c|c|c|c|c|c|c|c|c|c|c|}
\hline MAR & BADS & GOODS & BAD RATE & $f(x / 1)$ & $f(x / 0)$ & $w(x)$ & & & 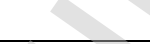 & 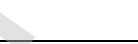 & & \\
\hline 0 & 123 & 172 & 0,416949 & 0,044129 & 0,338356 & $-2,03698$ & 0,599336 & 0,028830 & $-0,147502$ & 0,040369 & $-0,186933$ & \\
\hline 1 & 172 & 961 & 0,151809 & 0,245971 & 0,472603 & $-0,65304$ & 0,148 & 0,040269 & $-0,186613$ & 0,225013 & $-0,484210$ & \\
\hline \multirow[t]{3}{*}{2} & 69 & 2775 & 0,024262 & 0,710028 & 0,190411 & 1,31612 & 0,683879 & 0,016224 & $-0,096464$ & 0,649529 & $-0,404354$ & \\
\hline & & & & & & & 1,431215 & 0 & 0,430579 & & 1,075496 & \\
\hline & & & & & & & & 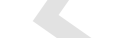 & 0,036688 & & 0,983857 & 1,020545 \\
\hline
\end{tabular}

7) Túmos Katoıкі́as (HOME)

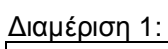

\begin{tabular}{|c|c|c|c|c|c|c|c|c|c|c|c|c|}
\hline HOME & BADS & GOODS & BAD RATE & $f(x / 1)$ & $f(x / 0)$ & $w(x)$ & 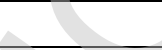 & & & & & \\
\hline 0 & 65 & 599 & 0,097892 & 0,153364 & 0,179452 & $-0,157094$ & 0,004098 & 0,015290 & $-0,092220$ & 0,140296 & $-0,397523$ & \\
\hline 1 & 154 & 742 & 0,171875 & 0,189946 & 0,423288 & $-0,801311$ & 0,186979 & 0,036067 & $-0,172874$ & 0,173762 & $-0,438717$ & \\
\hline \multirow[t]{3}{*}{2} & 145 & 2567 & 0,053466 & 0,656818 & 0,39863 & 0,499372 & 0,128932 & 0,033966 & $-0,165745$ & 0,600853 & $-0,441577$ & \\
\hline & & & & & & 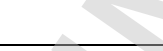 & 0,320009 & & 0,430839 & & 1,277816 & \\
\hline & & & & & & & 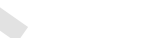 & & 0,03671 & & 1,168939 & 1,205649 \\
\hline
\end{tabular}

$\Delta$ ıане́pıơ 2:

\begin{tabular}{|c|c|c|c|c|c|c|c|c|c|c|c|c|}
\hline HOME & BADS & GOODS & $\begin{array}{l}\text { BAD } \\
\text { RATE }\end{array}$ & $f(x / 1)$ & $f(x / 0)$ & $w(x)$ & & & & & & \\
\hline 0 & 65 & 611 & 0,096154 & 0,156434 & 0,179452 & $-0,13727$ & 0,00316 & 0,015290 & $-0,092220$ & 0,143105 & $-0,401388$ & \\
\hline 1 & 159 & 745 & 0,175885 & 0,190714 & 0,436986 & $-0,82913$ & 0,204192 & 0,037234 & $-0,176758$ & 0,174464 & $-0,439474$ & \\
\hline 2 & 140 & 2552 & 0,052006 & 0,65298 & 0,384932 & 0,528482 & 0,141659 & 0,032798 & $-0,161704$ & 0,597342 & $-0,444046$ & \\
\hline & & & & & $\Delta$ & 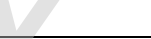 & 0,34901 & & 0,430682 & & 1,284909 & \\
\hline & & & & & & & & & 0,036697 & & 1,175427 & 1,212124 \\
\hline
\end{tabular}

\begin{tabular}{|c|c|c|c|c|c|c|c|c|c|c|c|c|}
\hline HOME & BADS & GOODS & $\begin{array}{l}\text { BAD } \\
\text { RATE }\end{array}$ & $f(x / 1)$ & $f(x / 0)$ & $w(x)$ & & & & & & \\
\hline 0 & 71 & 607 & 0,10472 & 0,155411 & 0,19589 & $-0,23148$ & 0,00937 & 0,016691 & $-0,098557$ & 0,142169 & $-0,400109$ & \\
\hline 1 & 162 & 732 & 0,181208 & 0,187388 & 0,445205 & $-0,86535$ & 0,223103 & 0,037934 & $-0,179063$ & 0,171421 & $-0,436161$ & \\
\hline 2 & 131 & 2569 & 0,048519 & 0,657329 & 0,360274 & 0,60132 & 0,178625 & 0,030698 & $-0,154278$ & 0,601321 & $-0,441245$ & \\
\hline & & & 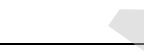 & $\vec{P}$ & & & 0,411099 & & 0,431897 & & 1,277515 & \\
\hline & & & & 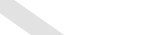 & & & & & 0,0368 & & 1,168663 & 1,205464 \\
\hline
\end{tabular}


$\Delta ı \mu \varepsilon \dot{p} ı \sigma n ~ 4:$

\begin{tabular}{|c|c|c|c|c|c|c|c|c|c|c|c|c|}
\hline HOME & BADS & GOODS & BAD RATE & $f(x / 1)$ & $f(x / 0)$ & $w(x)$ & & & 2 & 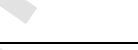 & & \\
\hline 0 & 66 & 611 & 0,0974889 & 0,156434 & 0,182192 & $-0,15243$ & 0,003926 & 0,015524 & $-0,093288$ & 0,143105 & $-0,401388$ & \\
\hline 1 & 163 & 739 & 0,1807095 & 0,189179 & 0,447945 & $-0,86198$ & 0,223051 & 0,038168 & $-0,179827$ & 0,173060 & $-0,437955$ & \\
\hline \multirow[t]{3}{*}{2} & 135 & 2558 & 0,05013 & 0,654515 & 0,371233 & 0,567065 & 0,16064 & 0,031631 & $-0,157603$ & 0,598747 & $-0,443062$ & \\
\hline & & & & & & & 0,387617 & 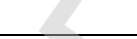 & 0,430718 & & 1,282405 & \\
\hline & & & & & & & & & 0,0367 & & 1,173137 & 1,20 \\
\hline
\end{tabular}

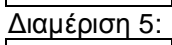

\begin{tabular}{|c|c|c|c|c|c|c|c|c|c|c|c|c|}
\hline HOME & BADS & GOODS & BAD RATE & $f(x / 1)$ & $f(x / 0)$ & $w(x)$ & & 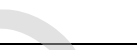 & & & & \\
\hline 0 & 69 & 600 & 0,103139 & 0,15362 & 0,190411 & $-0,2147$ & 0,007899 & 0,016224 & $-0,096464$ & 0,140531 & $-0,397848$ & \\
\hline 1 & 154 & 738 & 0,1726457 & 0,188923 & 0,423288 & $-0,80671$ & 0,189065 & 0,036067 & $-0,172874$ & 0,172826 & $-0,437700$ & \\
\hline \multirow[t]{3}{*}{2} & 141 & 2570 & 0,0520103 & 0,657585 & 0,387671 & 0,528416 & 0,142627 & 0,033032 & $-0,162517$ & 0,601555 & $-0,441079$ & \\
\hline & & & & & & & 0,339591 & & 0,431855 & & 1,276627 & \\
\hline & & & & & & & & & 0,036797 & & 1,167851 & 1,204648 \\
\hline
\end{tabular}

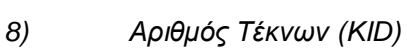

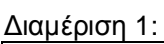

\begin{tabular}{|c|c|c|c|c|c|c|c|c|c|c|c|c|}
\hline KID & BADS & GOODS & BAD RATE & $f(x / 1)$ & $f(x / 0)$ & $w(x)$ & & & & & & \\
\hline 0 & 198 & 1421 & 0,122298 & 0,363648 & 0,543836 & $-0,402461$ & 0,072518 & 0,046338 & $-0,205354$ & 0,332663 & $-0,528224$ & \\
\hline 1 & 16 & 622 & 0,025078 & 0,159248 & 0,045205 & 1,259244 & 0,143607 & 0,003852 & $-0,030892$ & 0,145679 & $-0,404862$ & \\
\hline 2 & 98 & 1390 & 0,06586 & 0,355718 & 0,269863 & 0,276223 & 0,023715 & 0,022994 & $-0,125147$ & 0,325408 & $-0,527056$ & \\
\hline $3+$ & 52 & 475 & 0,098672 & 0,121642 & 0,143836 & $-0,167586$ & 0,003719 & 0,012256 & $-0,077828$ & 0,111278 & $-0,352501$ & \\
\hline & & & & 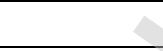 & 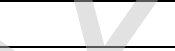 & & 0,24356 & & 0,439222 & & 1,812644 & \\
\hline & & & & & 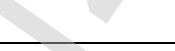 & & & & 0,037424 & & 1,658196 & 1,69562 \\
\hline
\end{tabular}

\begin{tabular}{|c|c|c|c|c|c|c|c|c|c|c|c|c|}
\hline \multicolumn{13}{|l|}{ 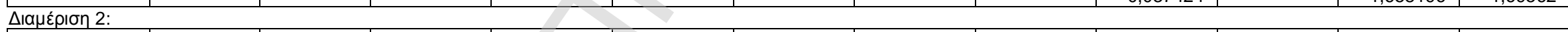 } \\
\hline KID & BADS & GOODS & BAD RATE & $f(x / 1)$ & $f(x / 0)$ & $w(x)$ & & & & & & \\
\hline 0 & 197 & 1440 & 0,120342 & 0,368509 & 0,541096 & $-0,38413$ & 0,066296 & 0,046105 & $-0,204656$ & 0,337109 & $-0,528827$ & \\
\hline 1 & 18 & 656 & 0,026706 & 0,167946 & 0,050685 & 1,198012 & 0,14048 & 0,004319 & $-0,033924$ & 0,153636 & $-0,415187$ & \\
\hline 2 & 97 & 1347 & 0,067175 & 0,344717 & 0,267123 & 0,255014 & 0,019788 & 0,022761 & $-0,124211$ & 0,315345 & $-0,525049$ & \\
\hline $3+$ & 52 & 465 & 0,10058 & 0,119084 & 0,143836 & $-0,18884$ & 0,004674 & 0,012256 & $-0,077828$ & 0,108937 & $-0,348429$ & \\
\hline & & & & 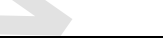 & & & 0,231238 & & 0,440620 & & 1,817492 & \\
\hline & & & & & & & & & 0,037543 & & 1,662631 & 1,700174 \\
\hline
\end{tabular}




\begin{tabular}{|c|c|c|c|c|c|c|c|c|c|c|c|c|}
\hline KID & BADS & GOODS & BAD RATE & $f(x / 1)$ & $f(x / 0)$ & $w(x)$ & & 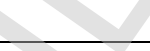 & & & & \\
\hline 0 & 207 & 1435 & 0,126066 & 0,367229 & 0,568493 & $-0,437$ & 0,087953 & 0,048439 & $-0,211566$ & 0,335939 & $-0,528677$ & \\
\hline 1 & 18 & 637 & 0,027481 & 0,163085 & 0,050685 & 1,168644 & 0,131356 & 0,004319 & $-0,033924$ & 0,149189 & $-0,409492$ & \\
\hline 2 & 91 & 1355 & 0,062932 & 0,346764 & 0,250685 & 0,324447 & 0,031173 & 0,02136 & $-0,118525$ & 0,317218 & $-0,525457$ & \\
\hline $3+$ & 48 & 481 & 0,090737 & 0,123177 & 0,132877 & $-0,0758$ & 0,000735 & 0,011322 & $-0,073193$ & 0,112682 & $-0,354911$ & \\
\hline & & & & & & & 0,251216 & & 0,437208 & & 1,818537 & \\
\hline & & & & & & & 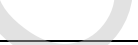 & & 0,037253 & & 1,663587 & 1,70084 \\
\hline
\end{tabular}

\begin{tabular}{|c|c|c|c|c|c|c|c|c|c|c|c|c|}
\hline KID & BADS & GOODS & BAD RATE & $f(x / 1)$ & $f(x / 0)$ & $w(x)$ & & & & & & \\
\hline 0 & 201 & 1442 & 0,122337 & 0,36902 & 0,552055 & $-0,4028$ & 0,073726 & 0,047038 & $-0,207440$ & 0,337577 & $-0,528886$ & \\
\hline 1 & 17 & 623 & 0,026563 & 0,159504 & 0,047945 & 1,202008 & 0,134094 & 0,004085 & $-0,032418$ & 0,145913 & $-0,405174$ & \\
\hline 2 & 94 & 1377 & 0,063902 & 0,352392 & 0,258904 & 0,308286 & 0,028821 & 0,02206 & $-0,121384$ & 0,322366 & $-0,526497$ & \\
\hline \multirow[t]{3}{*}{$3+$} & 52 & 466 & 0,100386 & 0,11934 & 0,143836 & $-0,18669$ & 0,004573 & 0,012256 & $-0,077828$ & 0,109172 & $-0,348839$ & \\
\hline & & & & & 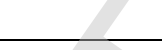 & - & 0,241214 & & 0,439070 & & 1,809396 & \\
\hline & & & & & 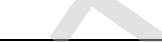 & & & & 0,037411 & & 1,655225 & 1,692636 \\
\hline
\end{tabular}

\begin{tabular}{|c|c|c|c|c|c|c|c|c|c|c|c|c|}
\hline \multicolumn{13}{|l|}{ 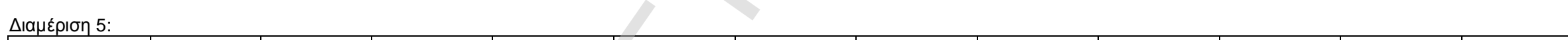 } \\
\hline KID & BADS & GOODS & BAD RATE & $f(x / 1)$ & $f(x / 0)$ & $w(x)$ & & & & & & \\
\hline 0 & 197 & 1426 & 0,12138 & 0,364927 & 0,541096 & $-0,3939$ & 0,069393 & 0,046105 & $-0,204656$ & 0,333833 & $-0,528391$ & \\
\hline 1 & 15 & 642 & 0,022831 & 0,164364 & 0,042466 & 1,353387 & 0,164976 & 0,003618 & $-0,029346$ & 0,150359 & $-0,411009$ & \\
\hline 2 & 96 & 1375 & 0,065262 & 0,35188 & 0,264384 & 0,28589 & 0,025014 & 0,022527 & $-0,123273$ & 0,321898 & $-0,526408$ & \\
\hline $3+$ & 56 & 465 & 0,107486 & 0,119084 & 0,154795 & $-0,26227$ & 0,009366 & 0,013189 & $-0,082361$ & 0,108937 & $-0,348429$ & \\
\hline & & & & 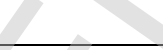 & & & 0,268749 & & 0,439636 & & 1,814237 & \\
\hline & & & & 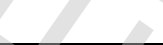 & & & & & 0,03746 & & 1,659653 & 1,697113 \\
\hline
\end{tabular}




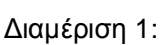

\begin{tabular}{|c|c|c|c|c|c|c|c|c|c|c|c|c|}
\hline $\mathrm{JOB}$ & BADS & GOODS & BAD RATE & $f(x / 1)$ & $f(x / 0)$ & $w(x)$ & & & 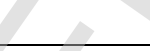 & & & \\
\hline 0 & 105 & 448 & 0,1898734 & 0,114735 & 0,289041 & $-0,923942$ & 0,161048 & 0,024628 & $-0,131601$ & 0,104959 & $-0,341338$ & \\
\hline 2 & 58 & 674 & 0,079235 & 0,172551 & 0,160274 & 0,073805 & 0,000906 & 0,013656 & $-0,084591$ & 0,157848 & $-0,420411$ & \\
\hline 3 & 70 & 904 & 0,0718686 & 0,231389 & 0,193151 & 0,180630 & 0,006907 & 0,016458 & $-0,097513$ & 0,211673 & $-0,474167$ & \\
\hline 4 & 18 & 245 & 0,0684411 & 0,062804 & 0,050685 & 0,214387 & 0,002598 & 0,004319 & $-0,033924$ & 0,057453 & $-0,236790$ & \\
\hline 5 & 98 & 1418 & 0,0646438 & 0,362881 & 0,269863 & 0,296159 & 0,027548 & 0,022994 & $-0,125147$ & 0,331961 & $-0,528122$ & \\
\hline \multirow[t]{3}{*}{6} & 15 & 219 & 0,0641026 & 0,056152 & 0,042466 & 0,279373 & 0,003824 & 0,003618 & $-0,029346$ & 0,051368 & $-0,220008$ & \\
\hline & & & & & & & 0,202831 & 1 & 0,502122 & & 2,220836 & \\
\hline & & & & & & & & . & 0,042784 & & 2,031607 & 2,074391 \\
\hline
\end{tabular}

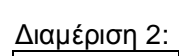

\begin{tabular}{|c|c|c|c|c|c|c|c|c|c|c|c|c|}
\hline $\mathrm{JOB}$ & BADS & GOODS & BAD RATE & $f(x / 1)$ & $f(x / 0)$ & $w(x)$ & & 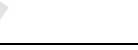 & & & & \\
\hline 0 & 100 & 468 & 0,176056 & 0,119852 & 0,275342 & $-0,83176$ & 0,129331 & 0,023461 & $-0,127008$ & 0,109640 & $-0,349658$ & \\
\hline 2 & 63 & 677 & 0,085135 & 0,173318 & 0,173973 & $-0,00377$ & $2,47 \mathrm{E}-06$ & 0,014824 & $-0,090067$ & 0,158550 & $-0,421266$ & \\
\hline 3 & 69 & 870 & 0,073482 & 0,222691 & 0,190411 & 0,156601 & 0,005055 & 0,016224 & $-0,096464$ & 0,203717 & $-0,467604$ & \\
\hline 4 & 17 & 254 & 0,062731 & 0,065106 & 0,047945 & 0,30596 & 0,005251 & 0,004085 & $-0,032418$ & 0,059559 & $-0,242377$ & \\
\hline 5 & 97 & 1426 & 0,06369 & 0,364927 & 0,267123 & 0,311987 & 0,030514 & 0,022761 & $-0,124211$ & 0,333833 & $-0,528391$ & \\
\hline 6 & 18 & 213 & 0,077922 & 0,054618 & 0,050685 & 0,074727 & 0,000294 & 0,004319 & $-0,033924$ & 0,049964 & $-0,215992$ & \\
\hline & & & & & 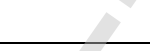 & $>$ & 0,170447 & & 0,504092 & & 2,225288 & \\
\hline & & & & & & & & & 0,042952 & & 2,03568 & 2,078632 \\
\hline
\end{tabular}

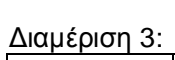

\begin{tabular}{|c|c|c|c|c|c|c|c|c|c|c|c|c|}
\hline $\mathrm{JOB}$ & BADS & GOODS & BAD RATE & $f(x / 1)$ & $f(x / 0)$ & $w(x)$ & & & & & & \\
\hline 0 & 100 & 447 & 0,182815 & 0,114479 & 0,275342 & $-0,87762$ & 0,141177 & 0,023461 & $-0,127008$ & 0,104725 & $-0,340914$ & \\
\hline 2 & 67 & 654 & 0,092926 & 0,167434 & 0,184932 & $-0,0994$ & 0,001739 & 0,015757 & $-0,094352$ & 0,153168 & $-0,414597$ & \\
\hline 3 & 82 & 979 & 0,077286 & 0,250576 & 0,226027 & 0,103104 & 0,002531 & 0,019259 & $-0,109744$ & 0,229225 & $-0,487141$ & \\
\hline 4 & 20 & 256 & 0,072464 & 0,065618 & 0,056164 & 0,155564 & 0,001471 & 0,004786 & $-0,036883$ & 0,060027 & $-0,243604$ & \\
\hline 5 & 80 & 1351 & 0,055905 & 0,345741 & 0,220548 & 0,449574 & 0,056283 & 0,018792 & $-0,107748$ & 0,316281 & $-0,525255$ & \\
\hline \multirow[t]{3}{*}{6} & 15 & 221 & 0,063559 & 0,056664 & 0,042466 & 0,288443 & 0,004095 & 0,003618 & $-0,029346$ & 0,051836 & $-0,221335$ & \\
\hline & & & & & & & 0,207296 & & 0,505081 & & 2,232844 & \\
\hline & & & & & & & & & 0,043036 & & 2,042592 & 2,085628 \\
\hline
\end{tabular}




\begin{tabular}{|c|c|c|c|c|c|c|c|c|c|c|c|c|}
\hline $\mathrm{JOB}$ & BADS & GOODS & BAD RATE & $f(x / 1)$ & $f(x / 0)$ & $w(x)$ & & & 2 & 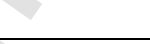 & & \\
\hline 0 & 97 & 465 & 0,172598 & 0,119084 & 0,267123 & $-0,80788$ & 0,119598 & 0,022761 & $-0,124211$ & 0,108937 & $-0,348429$ & \\
\hline 2 & 64 & 764 & 0,077295 & 0,195574 & 0,176712 & 0,101417 & 0,001913 & 0,015057 & $-0,091146$ & 0,178910 & $-0,444179$ & \\
\hline 3 & 83 & 878 & 0,086368 & 0,224738 & 0,228767 & $-0,01777$ & $7,16 \mathrm{E}-05$ & 0,019492 & $-0,110735$ & 0,205589 & $-0,469188$ & \\
\hline 4 & 18 & 253 & 0,066421 & 0,06485 & 0,050685 & 0,246454 & 0,003491 & 0,004319 & $-0,033924$ & 0,059325 & $-0,241761$ & \\
\hline 5 & 84 & 1327 & 0,059532 & 0,339601 & 0,231507 & 0,383162 & 0,041418 & 0,019726 & $-0,111722$ & 0,310665 & $-0,523958$ & \\
\hline \multirow[t]{3}{*}{6} & 18 & 221 & 0,075314 & 0,056664 & 0,050685 & 0,111512 & 0,000667 & 0,004319 & $-0,033924$ & 0,051836 & $-0,221335$ & \\
\hline & & & & & & & 0,167158 & - & 0,505663 & & 2,248849 & \\
\hline & & & & & & & & & 0,043085 & & 2,057234 & 2,100319 \\
\hline
\end{tabular}

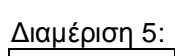

\begin{tabular}{|c|c|c|c|c|c|c|c|c|c|c|c|c|}
\hline $\mathrm{JOB}$ & BADS & GOODS & BAD RATE & $f(x / 1)$ & $f(x / 0)$ & $w(x)$ & & & & & & \\
\hline 0 & 94 & 464 & 0,168459 & 0,118828 & 0,258904 & $-0,77878$ & 0,109088 & 0,022060 & $-0,121384$ & 0,108703 & $-0,348017$ & \\
\hline 2 & 63 & 684 & 0,084337 & 0,175109 & 0,173973 & 0,006509 & $7,4 \mathrm{E}-06$ & 0,014824 & $-0,090067$ & 0,160188 & $-0,423243$ & \\
\hline 3 & 83 & 874 & 0,086729 & 0,223715 & 0,228767 & $-0,02233$ & 0,000113 & 0,019492 & $-0,110735$ & 0,204653 & $-0,468399$ & \\
\hline 4 & 19 & 240 & 0,073359 & 0,061525 & 0,053425 & 0,141166 & 0,001143 & 0,004552 & $-0,035412$ & 0,056282 & $-0,233638$ & \\
\hline 5 & 87 & 1420 & 0,057731 & 0,363392 & 0,239726 & 0,415986 & 0,051443 & 0,020426 & $-0,114661$ & 0,332429 & $-0,528190$ & \\
\hline \multirow[t]{3}{*}{6} & 18 & 226 & 0,07377 & 0,057943 & 0,050685 & 0,133835 & 0,000971 & 0,004319 & $-0,033924$ & 0,053006 & $-0,224624$ & \\
\hline & & & & & 4 & 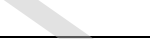 & 0,162766 & & 0,506183 & & 2,226112 & \\
\hline & & & & & 8 & $\theta$ & & & 0,04313 & & 2,036434 & 2,079563 \\
\hline
\end{tabular}

10) HAikía (AGE)

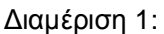

\begin{tabular}{|c|c|c|c|c|c|c|c|c|c|c|c|c|}
\hline AGE & BADS & GOODS & BAD RATE & $f(x / 1)$ & $f(x / 0)$ & $w(x)$ & IV & & & & & \\
\hline & & & & & & & 0,3550124 & & & & & \\
\hline $20-39$ & 218 & 1533 & 0,124500286 & 0,3922998 & 0,5986301 & $-0,422618$ & & 0,0510069 & $-0,218981$ & 0,3588735 & $-0,530577$ & \\
\hline $40-58$ & 69 & 1809 & 0,036741214 & 0,4629061 & 0,190411 & 0,888340 & & 0,0162242 & $-0,096464$ & 0,4234637 & $-0,524964$ & \\
\hline \multirow[t]{3}{*}{$59+$} & 77 & 566 & 0,119751166 & 0,144922 & 0,2123288 & $-0,381940$ & & 0,0180917 & $-0,104724$ & 0,1325738 & $-0,386470$ & \\
\hline & 364 & 3908 & & 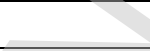 & & & & & 0,4201693 & & 1,4420112 & \\
\hline & & & & & & & & & 0,0358009 & & 1,3191432 & 1,3549442 \\
\hline
\end{tabular}




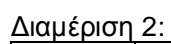

\begin{tabular}{|c|c|c|c|c|c|c|c|c|c|c|c|c|}
\hline AGE & BADS & GOODS & BAD RATE & $f(x / 1)$ & $f(x / 0)$ & $w(x)$ & & & 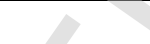 & 8 & & \\
\hline & & & & & & & 0,393262 & & 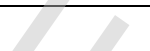 & 7 & & \\
\hline $20-39$ & 228 & 1550 & 0,128234 & 0,396649 & 0,626027 & $-0,45634$ & & 0,053341 & $-0,225559$ & 0,362852 & $-0,530688$ & \\
\hline $40-58$ & 64 & 1797 & 0,034390 & 0,459836 & 0,176712 & 0,956347 & & 0,015057 & $-0,091146$ & 0,420655 & $-0,525520$ & \\
\hline \multirow[t]{3}{*}{$59+$} & 72 & 561 & 0,113744 & 0,143643 & 0,19863 & $-0,32411$ & & 0,016924 & $-0,099596$ & 0,131404 & $-0,384740$ & \\
\hline & 364 & 3908 & & & & & & 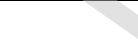 & 0,416302 & & 1,440948 & \\
\hline & & & & & & & & & 0,035471 & & 1,318171 & 1,353642 \\
\hline
\end{tabular}

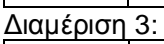

\begin{tabular}{|c|c|c|c|c|c|c|c|c|c|c|c|c|}
\hline AGE & BADS & GOODS & BAD RATE & $f(x / 1)$ & $f(x / 0)$ & $w(x)$ & & & & & & \\
\hline & & & & & & & 0,382918 & 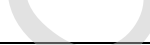 & & & & \\
\hline $20-39$ & 226 & 1560 & 0,126540 & 0,399207 & 0,620548 & $-0,44112$ & & 0,052874 & $-0,224256$ & 0,365192 & $-0,530724$ & \\
\hline $40-58$ & 65 & 1797 & 0,034909 & 0,459836 & 0,179452 & 0,940962 & 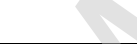 & 0,01529 & $-0,092220$ & 0,420655 & $-0,525520$ & \\
\hline \multirow[t]{3}{*}{$59+$} & 73 & 551 & 0,116987 & 0,141085 & 0,20137 & $-0,35578$ & 8 & 0,017158 & $-0,100631$ & 0,129063 & $-0,381234$ & \\
\hline & 364 & 3908 & & & & & 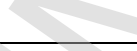 & 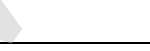 & 0,417106 & & 1,437478 & \\
\hline & & & & & & & & & 0,03554 & & 1,314996 & 1,350536 \\
\hline
\end{tabular}

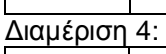

\begin{tabular}{|c|c|c|c|c|c|c|c|c|c|c|c|c|}
\hline AGE & BADS & GOODS & BAD RATE & $f(x / 1)$ & $f(x / 0)$ & $w(x)$ & 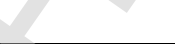 & & & & & \\
\hline & & & & & & 8 & 0,394796 & & & & & \\
\hline 20-39 & 233 & 1545 & 0,131046 & 0,39537 & 0,639726 & $-0,48122$ & & 0,054508 & $-0,228793$ & 0,361682 & $-0,530662$ & \\
\hline $40-62$ & 78 & 1993 & 0,037663 & 0,509977 & 0,215068 & 0,863409 & $\nabla$ & 0,018325 & $-0,105737$ & 0,466524 & $-0,513166$ & \\
\hline \multirow[t]{3}{*}{$59+$} & 53 & 370 & 0,125296 & 0,094781 & 0,146575 & $-0,43597$ & & 0,012489 & $-0,078971$ & 0,086705 & $-0,305873$ & \\
\hline & 364 & 3908 & & & $\Delta$ & 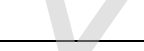 & & & 0,413500 & & 1,349701 & \\
\hline & & & & & & 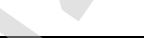 & & & 0,035233 & & 1,234699 & 1,269931 \\
\hline
\end{tabular}

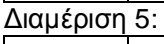

\begin{tabular}{|c|c|c|c|c|c|c|c|c|c|c|c|c|}
\hline AGE & BADS & GOODS & BAD RATE & $f(x / 1)$ & $f(x / 0)$ & $w(x)$ & & & & & & \\
\hline & & & & & 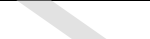 & & 0,389787 & & & & & \\
\hline $20-39$ & 231 & 1520 & 0,131925 & 0,388974 & 0,634247 & $-0,48892$ & & 0,054042 & $-0,227504$ & 0,355831 & $-0,530450$ & \\
\hline $40-58$ & 66 & 1809 & 0,035200 & 0,462906 & 0,182192 & 0,932464 & & 0,015524 & $-0,093288$ & 0,423464 & $-0,524964$ & \\
\hline $59+$ & 67 & 579 & 0,103715 & 0,148248 & 0,184932 & $-0,2211$ & & 0,015757 & $-0,094352$ & 0,135616 & $-0,390900$ & \\
\hline & 364 & 3908 & & & 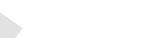 & & & & 0,415144 & & 1,446313 & \\
\hline & & & & 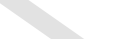 & & & & & 0,035373 & & 1,323079 & 1,358452 \\
\hline
\end{tabular}


$\Delta ı \mu \varepsilon \dot{p} ı \sigma n ~ 1:$

\begin{tabular}{|c|c|c|c|c|c|c|c|c|c|c|c|c|}
\hline TRM & BADS & GOODS & BAD RATE & $f(x / 1)$ & $f(x / 0)$ & $w(x)$ & & 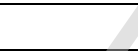 & $x+y$ & & & \\
\hline & & & & & & & 0,162451 & + & $\nabla$ & & & \\
\hline 6-22 & 37 & 973 & 0,036634 & 0,249041 & 0,10274 & 0,885417 & & 0,008754 & $-0,059841$ & 0,227821 & $-0,486176$ & \\
\hline $23-40$ & 272 & 2532 & 0,097004 & 0,647864 & 0,746575 & $-0,141816$ & & 0,063613 & $-0,252831$ & 0,592662 & $-0,447293$ & \\
\hline \multirow[t]{3}{*}{$41+$} & 55 & 403 & 0,120087 & 0,103223 & 0,152055 & $-0,387346$ & & 0,012956 & $-0,081237$ & 0,094428 & $-0,321494$ & \\
\hline & 364 & 3908 & & & & & & & 0,393910 & & 1,254963 & \\
\hline & & & & & & & & 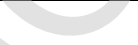 & 0,033563 & & 1,148032 & 1,181596 \\
\hline
\end{tabular}

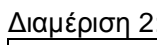

\begin{tabular}{|c|c|c|c|c|c|c|c|c|c|c|c|c|}
\hline TRM & BADS & GOODS & BAD RATE & $f(x / 1)$ & $f(x / 0)$ & $w(x)$ & 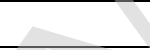 & 8 & & & & \\
\hline & & & & & & & 0,199339 & & & & & \\
\hline $6-22$ & 31 & 961 & 0,03125 & 0,245971 & 0,08630 & 1,047367 & $x$ & 0,007353 & $-0,052116$ & 0,225013 & $-0,484210$ & \\
\hline $23-40$ & 286 & 2563 & 0,100386 & 0,655794 & 0,78493 & $-0,179749$ & 3 & 0,066881 & $-0,260987$ & 0,599917 & $-0,442238$ & \\
\hline \multirow[t]{3}{*}{$41+$} & 47 & 384 & 0,109049 & 0,098363 & 0,13014 & $-0,279925$ & 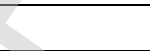 & 0,011088 & $-0,072017$ & 0,089982 & $-0,312617$ & \\
\hline & 364 & 3908 & & & & 8 & 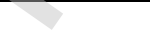 & & 0,385120 & & 1,239064 & \\
\hline & & & & & & 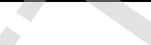 & & & 0,03281455 & & 1,133488 & 1,166303 \\
\hline
\end{tabular}

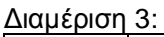

\begin{tabular}{|c|c|c|c|c|c|c|c|c|c|c|c|c|}
\hline TRM & BADS & GOODS & BAD RATE & $f(x / 1)$ & $f(x / 0)$ & $w(x)$ & & & & & & \\
\hline & & & & & 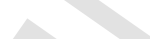 & 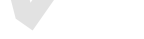 & 0,214199 & & & & & \\
\hline $6-21$ & 28 & 928 & 0,029289 & 0,237529 & 0,078082 & 1,112527 & & 0,006653 & $-0,048113$ & 0,217290 & $-0,478539$ & \\
\hline $22-40$ & 284 & 2604 & 0,098338 & 0,666283 & 0,779452 & $-0,15688$ & & 0,066414 & $-0,259836$ & 0,609512 & $-0,435359$ & \\
\hline $41+$ & 52 & 376 & 0,121495 & 0,096316 & 0,143836 & $-0,40103$ & & 0,012256 & $-0,077828$ & 0,088109 & $-0,308785$ & \\
\hline & 364 & 3908 & & 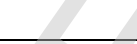 & & & & & 0,385778 & & 1,222682 & \\
\hline & & & & & & & & & 0,032871 & & 1,118503 & 1,151373 \\
\hline
\end{tabular}




\begin{tabular}{|c|c|c|c|c|c|c|c|c|c|c|c|c|}
\hline TRM & BADS & GOODS & BAD RATE & $f(x / 1)$ & $f(x / 0)$ & $w(x)$ & & 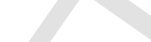 & & & & \\
\hline & & & & & & & 0,17287 & 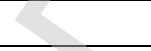 & 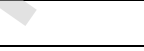 & & & \\
\hline $6-21$ & 31 & 902 & 0,0332262 & 0,230877 & 0,086301 & 0,984042 & & 0,007353 & $-0,052116$ & 0,211205 & $-0,473793$ & \\
\hline $22-40$ & 284 & 2642 & 0,0970608 & 0,676004 & 0,779452 & $-0,14239$ & & 0,066414 & $-0,259836$ & 0,618404 & $-0,428788$ & \\
\hline $41+$ & 49 & 364 & 0,1186441 & 0,093246 & 0,135616 & $-0,37459$ & & 0,011555 & $-0,074362$ & 0,085301 & $-0,302929$ & \\
\hline & 364 & 3908 & & & & & & & 0,386314 & & 1,205510 & \\
\hline & & & & & & & & & 0,032916 & & 1,102793 & 1,13571 \\
\hline
\end{tabular}

\begin{tabular}{|c|c|c|c|c|c|c|c|c|c|c|c|c|}
\hline TRM & BADS & GOODS & BAD RATE & $f(x / 1)$ & $f(x / 0)$ & $w(x)$ & +8 & & & & & \\
\hline & & & & & & 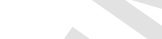 & 0,172632 & & & & & \\
\hline $6-22$ & 30 & 865 & 0,03352 & 0,221412 & 0,083562 & 0,974441 & 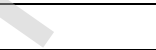 & 0,007120 & $-0,050793$ & 0,202546 & $-0,466601$ & \\
\hline $22-40$ & 277 & 2666 & 0,094122 & 0,682144 & 0,760274 & $-0,10844$ & 8 & 0,064780 & $-0,255771$ & 0,624021 & $-0,424542$ & \\
\hline \multirow[t]{3}{*}{$41+$} & 57 & 377 & 0,131336 & 0,096572 & 0,157534 & $-0,48935$ & & 0,013423 & $-0,083479$ & 0,088343 & $-0,309267$ & \\
\hline & 364 & 3908 & & & 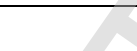 & $\nabla$ & & & 0,390043 & & 1,200411 & \\
\hline & & & & & & - & & & 0,033234 & & 1,098128 & 1,131362 \\
\hline
\end{tabular}

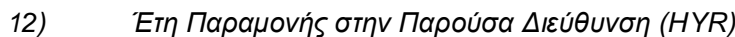

\begin{tabular}{|c|c|c|c|c|c|c|c|c|c|c|c|c|}
\hline HYR & BADS & GOODS & BAD RATE & $f(x / 1)$ & $f(x / 0)$ & $w(x)$ & & & & & & \\
\hline $1-3$ & 198 & 798 & 0,19880 & 0,204272 & 0,543836 & $-0,979194$ & 0,332498 & 0,046338 & $-0,205354$ & 0,186867 & $-0,452202$ & \\
\hline $4-17$ & 81 & 1667 & 0,04634 & 0,42658 & 0,223288 & 0,647338 & 0,131599 & 0,019025 & $-0,108748$ & 0,390233 & $-0,529777$ & \\
\hline $18-38$ & 79 & 1194 & 0,06206 & 0,305577 & 0,217808 & 0,338586 & 0,029717 & 0,018559 & $-0,106745$ & 0,27954 & $-0,514039$ & \\
\hline \multirow[t]{3}{*}{$39+$} & 6 & 249 & 0,02353 & 0,063827 & 0,017808 & 1,276517 & 0,058744 & 0,001517 & $-0,014209$ & 0,058389 & $-0,239286$ & \\
\hline & 364 & 3908 & & 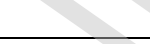 & 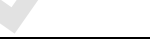 & & 0,552558 & & 0,435056 & & 1,735305 & \\
\hline & & & & & & & & & 0,037069 & & 1,587446 & 1,624516 \\
\hline
\end{tabular}


$\Delta ı \mu \varepsilon \dot{p} ı \sigma n ~ 2:$

\begin{tabular}{|c|c|c|c|c|c|c|c|c|c|c|c|c|}
\hline HYR & BADS & GOODS & BAD RATE & $f(x / 1)$ & $f(x / 0)$ & $w(x)$ & & & 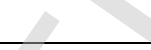 & o & & \\
\hline $1-3$ & 195 & 791 & 0,19777 & 0,202481 & 0,535616 & $-0,97277$ & 0,324064 & 0,045638 & $-0,203254$ & 0,185229 & $-0,450591$ & \\
\hline $4-18$ & 89 & 1703 & 0,04967 & 0,435789 & 0,245205 & 0,575062 & 0,109597 & 0,020893 & $-0,116600$ & 0,398657 & $-0,528930$ & \\
\hline $19-38$ & 75 & 1172 & 0,06014 & 0,299949 & 0,206849 & 0,371621 & 0,034598 & 0,017625 & $-0,102686$ & 0,274391 & $-0,511930$ & \\
\hline \multirow[t]{3}{*}{$39+$} & 5 & 242 & 0,02024 & 0,062036 & 0,015068 & 1,415114 & 0,066465 & 0,001284 & $-0,012332$ & 0,05675 & $-0,234903$ & \\
\hline & 364 & 3908 & & & & & 0,534724 & 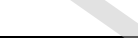 & 0,434873 & & 1,726354 & \\
\hline & & & & & & & & & 0,037054 & & 1,579259 & 1,616312 \\
\hline
\end{tabular}

\begin{tabular}{|c|c|c|c|c|c|c|c|c|c|c|c|c|}
\hline \multicolumn{13}{|c|}{$\Delta ı \mu \mu \dot{\rho} \rho \mid \emptyset\rceil$ 3: } \\
\hline HYR & BADS & GOODS & BAD RATE & $f(x / 1)$ & $f(x / 0)$ & $w(x)$ & & & & & & \\
\hline $1-3$ & 199 & 819 & 0,19548 & 0,209644 & 0,546575 & $-0,95826$ & 0,322867 & 0,046571 & $-0,206051$ & 0,191781 & $-0,456913$ & \\
\hline $4-18$ & 86 & 1699 & 0,04818 & 0,434766 & 0,236986 & 0,606805 & 0,120014 & 0,020193 & $-0,113685$ & 0,397721 & $-0,529037$ & \\
\hline $19-37$ & 77 & 1134 & 0,06358 & 0,290228 & 0,212329 & 0,31253 & 0,024346 & 0,018092 & $-0,104724$ & 0,265499 & $-0,507958$ & \\
\hline \multirow[t]{3}{*}{$38+$} & 2 & 256 & 0,00775 & 0,065618 & 0,006849 & 2,259698 & 0,132799 & 0,000584 & $-0,006269$ & 0,060027 & $-0,243604$ & \\
\hline & 364 & 3908 & & & & & 0,600026 & & 0,430730 & & 1,737512 & \\
\hline & & & & & & & ( & & 0,036701 & & 1,589465 & 1,626166 \\
\hline
\end{tabular}

\begin{tabular}{|c|c|c|c|c|c|c|c|c|c|c|c|c|}
\hline \multicolumn{13}{|c|}{ 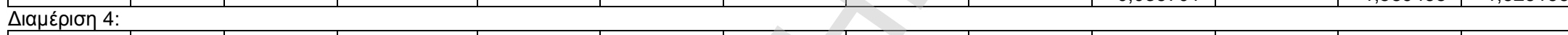 } \\
\hline HYR & BADS & GOODS & BAD RATE & $f(x / 1)$ & $f(x / 0)$ & $w(x)$ & & & & & & \\
\hline $1-3$ & 191 & 819 & 0,18911 & 0,209644 & 0,524658 & $-0,91733$ & 0,288972 & 0,044704 & $-0,200428$ & 0,191781 & $-0,456913$ & \\
\hline $4-18$ & 85 & 1684 & 0,04805 & 0,430929 & 0,234247 & 0,609568 & 0,119891 & 0,019959 & $-0,112706$ & 0,394211 & $-0,529410$ & \\
\hline $19-38$ & 83 & 1159 & 0,06683 & 0,296623 & 0,228767 & 0,259758 & 0,017626 & 0,019492 & $-0,110735$ & 0,271349 & $-0,510619$ & \\
\hline \multirow[t]{3}{*}{$39+$} & 5 & 246 & 0,01992 & 0,06306 & 0,015068 & 1,431474 & 0,068698 & 0,001284 & $-0,012332$ & 0,057687 & $-0,237416$ & \\
\hline & 364 & 3908 & & & 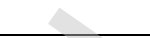 & 2 & 0,495187 & & 0,436201 & & 1,734357 & \\
\hline & & & & & & 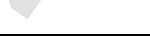 & & & 0,037167 & & 1,586579 & 1,623746 \\
\hline
\end{tabular}

\begin{tabular}{|c|c|c|c|c|c|c|c|c|c|c|c|c|}
\hline \multicolumn{13}{|c|}{ 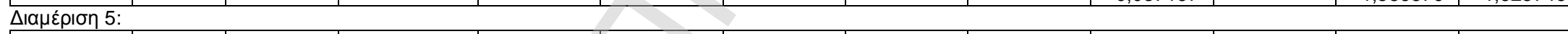 } \\
\hline HYR & BADS & GOODS & BAD RATE & $f(x / 1)$ & $f(x / 0)$ & $w(x)$ & & & & & & \\
\hline $1-3$ & 201 & 809 & 0,19901 & 0,207086 & 0,552055 & $-0,98051$ & 0,338246 & 0,047038 & $-0,207440$ & 0,189441 & $-0,454693$ & \\
\hline 4-17 & 81 & 1640 & 0,04707 & 0,419673 & 0,223288 & 0,631014 & 0,123922 & 0,019025 & $-0,108748$ & 0,383914 & $-0,530241$ & \\
\hline 19-38 & 76 & 1203 & 0,05942 & 0,307879 & 0,209589 & 0,384559 & 0,037798 & 0,017858 & $-0,103708$ & 0,281646 & $-0,514862$ & \\
\hline $39+$ & 6 & 256 & 0,02290 & 0,065618 & 0,017808 & 1,304187 & 0,062353 & 0,001517 & $-0,014209$ & 0,060027 & $-0,243604$ & \\
\hline & 364 & 3908 & & & & & 0,562318 & & 0,434105 & & 1,743399 & \\
\hline & & & & 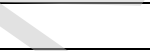 & & & & & 0,036988 & & 1,594851 & 1,631839 \\
\hline
\end{tabular}




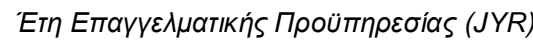

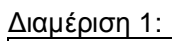

\begin{tabular}{|c|c|c|c|c|c|c|c|c|c|c|c|c|}
\hline JYR & BADS & GOODS & BAD RATE & $f(x / 1)$ & $f(x / 0)$ & $w(x)$ & & 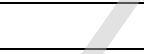 & 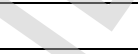 & & & \\
\hline $1-3$ & 121 & 802 & 0,13109 & 0,205295 & 0,332877 & $-0,483322$ & 0,061663 & 0,028363 & $-0,145782$ & 0,187803 & $-0,453114$ & \\
\hline $4-8$ & 116 & 1008 & 0,10320 & 0,257994 & 0,319178 & $-0,212811$ & 0,013021 & 0,027196 & $-0,141431$ & 0,236012 & $-0,491629$ & \\
\hline $9-12$ & 56 & 651 & 0,07921 & 0,166667 & 0,154795 & 0,073897 & 0,000877 & 0,013189 & $-0,082361$ & 0,152466 & $-0,413707$ & \\
\hline \multirow[t]{3}{*}{$13+$} & 71 & 1447 & 0,04677 & 0,370299 & 0,19589 & 0,636756 & 0,111056 & 0,016691 & $-0,098557$ & 0,338748 & $-0,529028$ & \\
\hline & 364 & 3908 & & & & & 0,186617 & & 0,468131 & & 1,887478 & \\
\hline & & & & & & & & 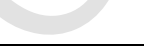 & 0,039888 & & 1,726653 & 1,766541 \\
\hline
\end{tabular}

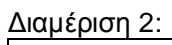

\begin{tabular}{|c|c|c|c|c|c|c|c|c|c|c|c|c|}
\hline JYR & BADS & GOODS & BAD RATE & $f(x / 1)$ & $f(x / 0)$ & $w(x)$ & 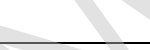 & & & & & \\
\hline $1-3$ & 118 & 798 & 0,12882 & 0,204272 & 0,324658 & $-0,46332$ & 0,055777 & 0,027663 & $-0,143180$ & 0,186867 & $-0,452202$ & \\
\hline $4-9$ & 123 & 1134 & 0,09785 & 0,290228 & 0,338356 & $-0,15343$ & 0,007385 & 0,028830 & $-0,147502$ & 0,265499 & $-0,507958$ & \\
\hline $10-12$ & 46 & 558 & 0,07616 & 0,142875 & 0,127397 & 0,114663 & 0,001775 & 0,010855 & $-0,070834$ & 0,130702 & $-0,383694$ & \\
\hline \multirow[t]{3}{*}{$13+$} & 77 & 1418 & 0,05151 & 0,362881 & 0,212329 & 0,535938 & 0,080686 & 0,018092 & $-0,104724$ & 0,331961 & $-0,528122$ & \\
\hline & 364 & 3908 & & & & 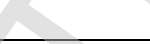 & 0,145622 & & 0,466241 & & 1,871976 & \\
\hline & & & & & & & & & 0,039727 & & 1,712473 & 1,752199 \\
\hline
\end{tabular}

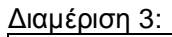

\begin{tabular}{|c|c|c|c|c|c|c|c|c|c|c|c|c|}
\hline JYR & BADS & GOODS & BAD RATE & $f(x / 1)$ & $f(x / 0)$ & $w(x)$ & & & & & & \\
\hline $1-3$ & 128 & 814 & 0,13588 & 0,208365 & 0,352055 & $-0,52449$ & 0,075364 & 0,029997 & $-0,151757$ & 0,190611 & $-0,455808$ & \\
\hline 4-9 & 108 & 1114 & 0,08838 & 0,285111 & 0,29726 & $-0,04173$ & 0,000507 & 0,025328 & $-0,134319$ & 0,260818 & $-0,505696$ & \\
\hline $10-12$ & 47 & 550 & 0,07873 & 0,140829 & 0,130137 & 0,078958 & 0,000844 & 0,011088 & $-0,072017$ & 0,128829 & $-0,380880$ & \\
\hline \multirow[t]{3}{*}{$13+$} & 81 & 1430 & 0,05361 & 0,36595 & 0,223288 & 0,494037 & 0,070481 & 0,019025 & $-0,108748$ & 0,334769 & $-0,528521$ & \\
\hline & 364 & 3908 & & 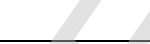 & & & 0,147196 & & 0,466841 & & 1,870904 & \\
\hline & & & & 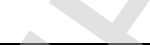 & 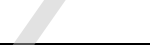 & & & & 0,039778 & & 1,711492 & 1,75127 \\
\hline
\end{tabular}




\begin{tabular}{|c|c|c|c|c|c|c|c|c|c|c|c|c|}
\hline JYR & BADS & GOODS & BAD RATE & $f(x / 1)$ & $f(x / 0)$ & $w(x)$ & & & 2 & & & \\
\hline $1-2$ & 82 & 511 & 0,13828 & 0,130852 & 0,226027 & $-0,54659$ & 0,052022 & 0,019259 & $-0,109744$ & 0,119703 & $-0,366586$ & \\
\hline $3-8$ & 141 & 1358 & 0,09406 & 0,347531 & 0,387671 & $-0,1093$ & 0,004387 & 0,033032 & $-0,162517$ & 0,317920 & $-0,525606$ & \\
\hline $9-12$ & 59 & 644 & 0,08393 & 0,164876 & 0,163014 & 0,011359 & 2,12E-05 & 0,013890 & $-0,085697$ & 0,150828 & $-0,411613$ & \\
\hline \multirow[t]{3}{*}{$13+$} & 82 & 1395 & 0,05552 & 0,356997 & 0,226027 & 0,45707 & 0,059862 & 0,019259 & $-0,109744$ & 0,326578 & $-0,527260$ & \\
\hline & 364 & 3908 & & & & & 0,116293 & & 0,467701 & & 1,831065 & \\
\hline & & & & & & & & - & 0,039851 & & 1,675047 & 1,714898 \\
\hline
\end{tabular}

\begin{tabular}{|c|c|c|c|c|c|c|c|c|c|c|c|c|}
\hline \begin{tabular}{|l|} 
JYR \\
\end{tabular} & RADS & gnons & 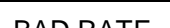 & $f(x / 1)$ & $f(x / 0)$ & wor & & & & & & \\
\hline $1-3$ & 124 & 802 & 0,13391 & 0,205295 & 0,341096 & $-0,507713$ & 0,068948 & 0,029063 & $-0,148359$ & 0,187803 & $-0,453114$ & \\
\hline $4-8$ & 108 & 1014 & 0,09626 & 0,259529 & 0,29726 & $-0,135739$ & 0,005122 & 0,025328 & $-0,134319$ & 0,237416 & $-0,492522$ & \\
\hline $9-12$ & 55 & 642 & 0,07891 & 0,164364 & 0,152055 & 0,0778443 & 0,000958 & 0,012956 & $-0,081237$ & 0,150359 & $-0,411009$ & \\
\hline \multirow[t]{3}{*}{$13+$} & 77 & 1450 & 0,05043 & 0,371067 & 0,212329 & 0,5582462 & 0,088615 & 0,018092 & $-0,104724$ & 0,339450 & $-0,529111$ & \\
\hline & 364 & 3908 & & & & 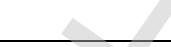 & 0,163642 & & 0,468639 & & 1,885756 & \\
\hline & & & & & & & & & 0,039931 & & 1,725078 & 1,765009 \\
\hline
\end{tabular}

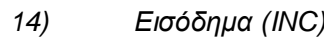

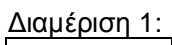

\begin{tabular}{|c|c|c|c|c|c|c|c|c|c|c|c|c|}
\hline INC & BADS & GOODS & BAD RATE & $f(x / 1)$ & $f(x / 0)$ & $w(x)$ & & & & & & \\
\hline$\ldots-7579$ & 157 & 644 & 0,196005 & 0,164876 & 0,431507 & $-0,962090$ & 0,256523 & 0,036767 & $-0,175211$ & 0,150828 & $-0,411613$ & \\
\hline $\begin{array}{r}7580- \\
9459 \\
\end{array}$ & 86 & 715 & 0,1073658 & 0,183039 & 0,236986 & $-0,258302$ & 0,013935 & 0,020193 & $-0,113685$ & 0,167443 & $-0,431711$ & \\
\hline $\begin{array}{r}9460- \\
18656 \\
\end{array}$ & 115 & 2020 & 0,0538642 & 0,516884 & 0,316438 & 0,490690 & 0,098357 & 0,026962 & $-0,140553$ & 0,472842 & $-0,510939$ & \\
\hline \multirow[t]{3}{*}{$18657+$} & 6 & 529 & 0,011215 & 0,135457 & 0,017808 & 2,028991 & 0,238708 & 0,001517 & $-0,014209$ & 0,123915 & $-0,373303$ & \\
\hline & 364 & 3908 & & + & 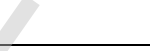 & & 0,607522 & & 0,443658 & & 1,727566 & \\
\hline & & & & & & & & & 0,037802 & & 1,580367 & 1,618169 \\
\hline
\end{tabular}




\begin{tabular}{|c|c|c|c|c|c|c|c|c|c|c|c|c|}
\hline INC & BADS & GOODS & BAD RATE & $f(x / 1)$ & $f(x / 0)$ & $w(x)$ & & & 2 & & & \\
\hline$\ldots-7542$ & 161 & 639 & 0,20125 & 0,163597 & 0,442466 & $-0,99496$ & 0,277463 & 0,037701 & $-0,178297$ & 0,149657 & $-0,410101$ & \\
\hline $\begin{array}{r}7543- \\
9453 \\
\end{array}$ & 92 & 710 & 0,11471 & 0,18176 & 0,253425 & $-0,33238$ & 0,02382 & 0,021593 & $-0,119482$ & 0,166273 & $-0,430377$ & \\
\hline $\begin{array}{r}9454- \\
18486 \\
\end{array}$ & 107 & 2029 & 0,05009 & 0,519186 & 0,294521 & 0,566914 & 0,127366 & 0,025095 & $-0,133416$ & 0,474949 & $-0,510169$ & \\
\hline \multirow[t]{3}{*}{$18487+$} & 4 & 530 & 0,00749 & 0,135712 & 0,012329 & 2,398603 & 0,295948 & 0,001050 & $-0,010394$ & 0,124149 & $-0,373670$ & \\
\hline & 364 & 3908 & & & & & 0,724598 & 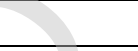 & 0,441589 & & 1,724317 & \\
\hline & & & & & & & & & 0,037626 & & 1,577395 & 1,615021 \\
\hline
\end{tabular}

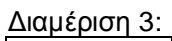

\begin{tabular}{|c|c|c|c|c|c|c|c|c|c|c|c|c|}
\hline INC & BADS & GOODS & BAD RATE & $f(x / 1)$ & $f(x / 0)$ & $w(x)$ & 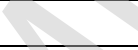 & & & & & \\
\hline$\ldots-7508$ & 166 & 636 & 0,20698 & 0,162829 & 0,456164 & $-1,03015$ & 0,302179 & 0,038868 & $-0,182107$ & 0,148955 & $-0,409187$ & \\
\hline $\begin{array}{r}7509- \\
9430 \\
\end{array}$ & 90 & 708 & 0,11278 & 0,181248 & 0,247945 & $-0,31334$ & 0,020899 & 0,021126 & $-0,117564$ & 0,165805 & $-0,429840$ & \\
\hline $\begin{array}{c}9431- \\
18414\end{array}$ & 102 & 2036 & 0,04771 & 0,520977 & 0,280822 & 0,617986 & 0,148413 & 0,023928 & $-0,128855$ & 0,476587 & $-0,509561$ & \\
\hline \multirow[t]{3}{*}{$18415+$} & 6 & 528 & 0,01124 & 0,135201 & 0,017808 & 2,027101 & 0,237967 & 0,001517 & $-0,014209$ & 0,123681 & $-0,372936$ & \\
\hline & 364 & 3908 & & & & ( & 0,709457 & & 0,442735 & & 1,721524 & \\
\hline & & & & & 7 & 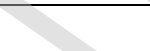 & & & 0,037724 & & 1,574840 & 1,612564 \\
\hline
\end{tabular}

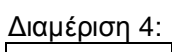

\begin{tabular}{|c|c|c|c|c|c|c|c|c|c|c|c|c|}
\hline INC & BADS & GOODS & BAD RATE & $f(x / 1)$ & $f(x / 0)$ & $w(x)$ & & & & & & \\
\hline$\ldots-7550$ & 167 & 634 & 0,20849 & 0,162318 & 0,458904 & $-1,03929$ & 0,308238 & 0,039101 & $-0,182863$ & 0,148487 & $-0,408576$ & \\
\hline $\begin{array}{r}7551- \\
9474 \\
\end{array}$ & 89 & 712 & 0,11111 & 0,182272 & 0,245205 & $-0,2966$ & 0,018666 & 0,020893 & $-0,116600$ & 0,166741 & $-0,430912$ & \\
\hline $\begin{array}{r}9475- \\
18532 \\
\end{array}$ & 102 & 2034 & 0,04775 & 0,520466 & 0,280822 & 0,617003 & 0,147861 & 0,023928 & $-0,128855$ & 0,476119 & $-0,509736$ & \\
\hline \multirow[t]{3}{*}{$18533+$} & 6 & 528 & 0,01124 & 0,135201 & 0,017808 & 2,027101 & 0,237967 & 0,001517 & $-0,014209$ & 0,123681 & $-0,372936$ & \\
\hline & 364 & 3908 & & 2 & 2 & & 0,712732 & & 0,442527 & & 1,722160 & \\
\hline & & & & & & & & & 0,037706 & & 1,575421 & 1,613127 \\
\hline
\end{tabular}




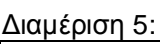

\begin{tabular}{|c|c|c|c|c|c|c|c|c|c|c|c|c|}
\hline INC & BADS & GOODS & BAD RATE & $f(x / 1)$ & $f(x / 0)$ & $w(x)$ & & & 8 & & & \\
\hline$\ldots-7579$ & 166 & 636 & 0,20698 & 0,162829 & 0,456164 & $-1,03015$ & 0,302179 & 0,038868 & $-0,182107$ & 0,148955 & $-0,409187$ & \\
\hline $\begin{array}{r}7580- \\
9464 \\
\end{array}$ & 91 & 709 & 0,11375 & 0,181504 & 0,250685 & $-0,32292$ & 0,02234 & 0,021360 & $-0,118525$ & 0,166039 & $-0,430108$ & \\
\hline $\begin{array}{r}9465- \\
18748 \\
\end{array}$ & 105 & 2031 & 0,04916 & 0,519698 & 0,289041 & 0,586679 & 0,135322 & 0,024628 & $-0,131601$ & 0,475417 & $-0,509996$ & \\
\hline \multirow[t]{3}{*}{$18749+$} & 2 & 532 & 0,00375 & 0,136224 & 0,006849 & 2,990153 & 0,38685 & 0,000584 & $-0,006269$ & 0,124617 & $-0,374403$ & \\
\hline & 364 & 3908 & & & & & 0,846691 & & 0,438503 & & 1,723695 & \\
\hline & & & & & & & & & 0,037363 & & 1,576826 & 1,614189 \\
\hline
\end{tabular}




\section{ПАРАРТНMA $\Delta$}

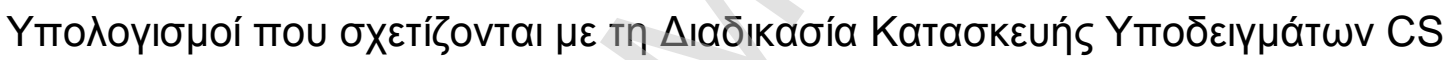




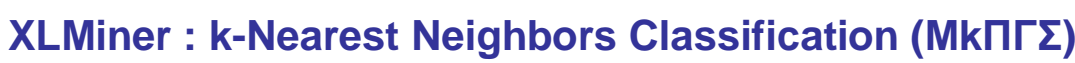

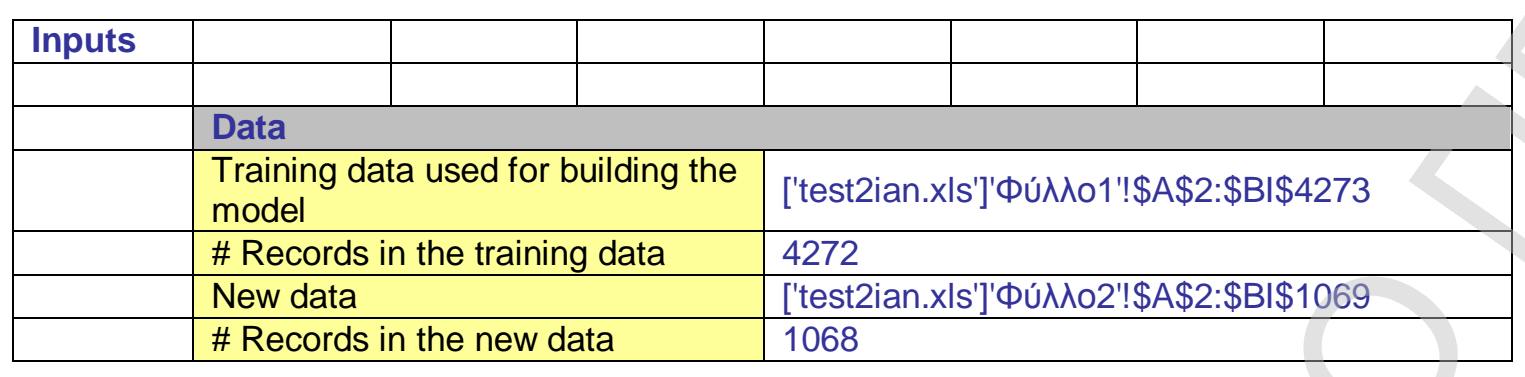

Yто́ $\mu v \eta \mu \alpha:$

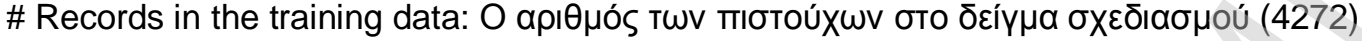

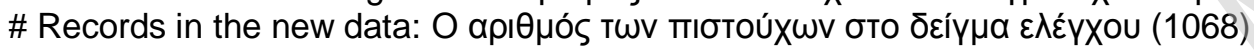

\begin{tabular}{|c|c|c|c|c|c|c|c|c|c|c|c|c|c|c|c|c|c|c|c|c|c|c|c|c|c|c|c|}
\hline & & & & & & & & & & & & 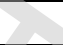 & 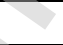 & & & & & & & & & & & & & & \\
\hline \multicolumn{28}{|c|}{ Variables } \\
\hline $\begin{array}{l}\text { \# Input } \\
\text { Variabl } \\
\text { es }\end{array}$ & \multicolumn{9}{|l|}{27} & & & & & & & & & & & & & & & & & & \\
\hline $\begin{array}{l}\text { Input } \\
\text { variable } \\
\text { s }\end{array}$ & $\begin{array}{l}\text { JO } \\
\text { B1 }\end{array}$ & $\begin{array}{l}\text { JO } \\
\text { B3 }\end{array}$ & $\begin{array}{l}\text { JO } \\
\text { B4 }\end{array}$ & $\begin{array}{l}\text { JO } \\
\text { B5 }\end{array}$ & $\begin{array}{l}\mathrm{JO} \\
\mathrm{B} 6\end{array}$ & $\begin{array}{l}\mathrm{HO} \\
\text { ME2 }\end{array}$ & $\begin{array}{l}\mathrm{HO} \\
\mathrm{ME} 3\end{array}$ & $\begin{array}{l}\text { MA } \\
\text { R1 }\end{array}$ & $\begin{array}{l}\text { MA } \\
\text { R3 }\end{array}$ & $\begin{array}{l}\text { C } \\
A \\
R\end{array}$ & $\begin{array}{l}\mathrm{KI} \\
\mathrm{D} 1\end{array}$ & $\begin{array}{l}\mathrm{KI} \\
\mathrm{D} 2\end{array}$ & $\begin{array}{l}\mathrm{KI} \\
\mathrm{D} 3\end{array}$ & $\begin{array}{l}\text { IN } \\
\text { C1 }\end{array}$ & $\begin{array}{l}\text { IN } \\
\text { C3 }\end{array}$ & $\begin{array}{l}\text { IN } \\
\text { C4 }\end{array}$ & $\begin{array}{l}C \\
R \\
D\end{array}$ & $\begin{array}{l}\text { AG } \\
\text { E1 }\end{array}$ & $\begin{array}{l}\text { AG } \\
\text { E2 }\end{array}$ & $\begin{array}{l}\text { JY } \\
\text { R1 }\end{array}$ & $\begin{array}{l}\text { JY } \\
\text { R2 }\end{array}$ & $\begin{array}{l}\text { JY } \\
\text { R4 }\end{array}$ & $\begin{array}{l}\text { HY } \\
\text { R1 }\end{array}$ & $\begin{array}{l}\text { HY } \\
\text { R2 }\end{array}$ & $\begin{array}{l}\text { HY } \\
\text { R4 }\end{array}$ & $\begin{array}{l}\text { TR } \\
\text { M1 }\end{array}$ & $\begin{array}{l}\text { TR } \\
\text { M2 }\end{array}$ \\
\hline $\begin{array}{l}\text { Output } \\
\text { variable }\end{array}$ & \multicolumn{10}{|c|}{ STATUS } & & & & & & & & & & & & & & & & & \\
\hline
\end{tabular}

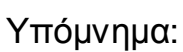

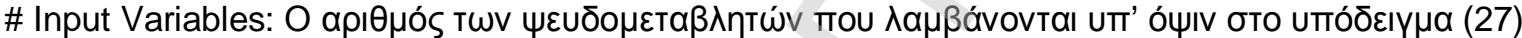

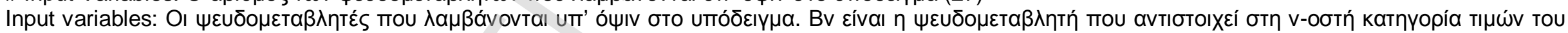

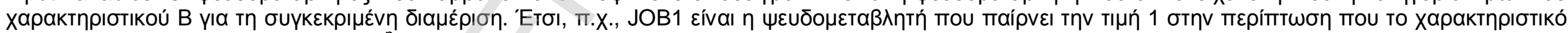

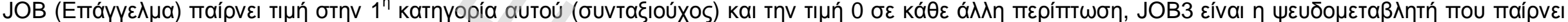

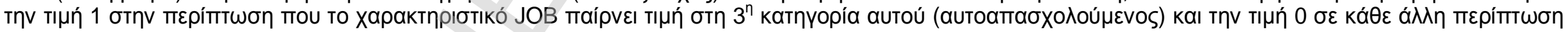
KOK.

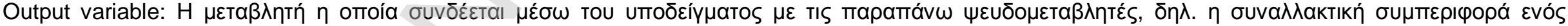

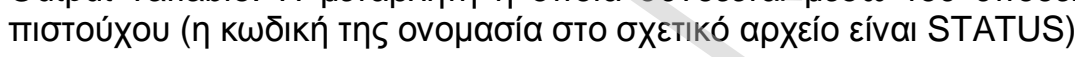




\begin{tabular}{|l|l|l|l|l|l|}
\hline & & & & & \\
\hline Parameters/Options & 9 & & \\
\hline \# Nearest neighbors & 9 & \\
\hline
\end{tabular}

Yтó $\mu v \eta \mu \alpha$ :

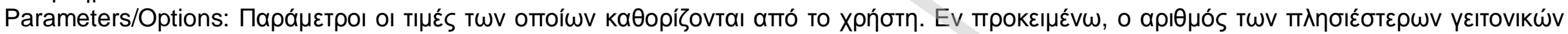
on $\mu \varepsilon^{i} \omega \mathrm{v}$.

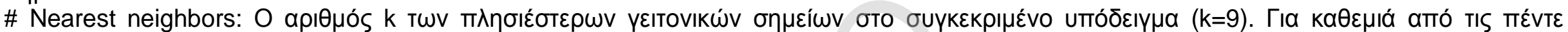

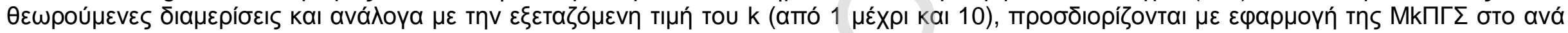

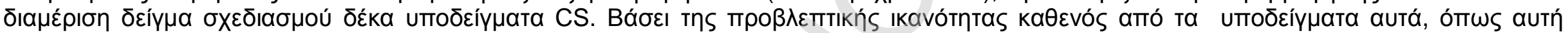

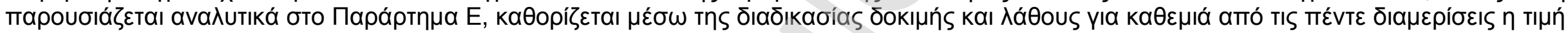

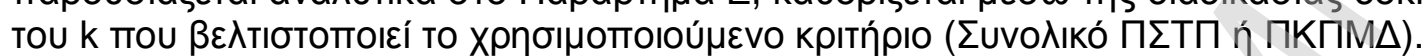




\section{XLMiner : k-Nearest Neighbors - Classification of Test Data}

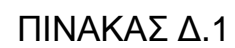

\begin{tabular}{|c|c|c|c|c|c|c|c|c|c|c|c|c|c|c|c|c|c|c|c|c|c|c|c|c|c|c|c|c|}
\hline $\begin{array}{r}\text { Ro } \\
\text { w } \\
\text { Id. } \\
\end{array}$ & $\begin{array}{r}\text { Prob. } \\
\text { for } 1 \\
\text { (succ } \\
\text { ess) } \\
\end{array}$ & $\begin{array}{l}\text { JO } \\
\text { B1 }\end{array}$ & $\begin{array}{l}\text { JO } \\
\text { B3 }\end{array}$ & $\begin{array}{l}\text { JO } \\
\text { B4 }\end{array}$ & $\begin{array}{l}\text { JO } \\
\text { B5 }\end{array}$ & $\begin{array}{l}\text { JO } \\
\text { B6 }\end{array}$ & $\begin{array}{r}\text { HO } \\
\text { ME2 }\end{array}$ & $\begin{array}{r}\text { HO } \\
\text { ME3 }\end{array}$ & $\begin{array}{r}\text { MA } \\
\text { R1 }\end{array}$ & $\begin{array}{r}\text { MA } \\
\text { R3 }\end{array}$ & $\begin{array}{l}\mathrm{C} \\
\mathrm{A} \\
\mathrm{R} \\
\end{array}$ & $\begin{array}{c}\text { KI } \\
\text { D1 }\end{array}$ & $\begin{array}{l}\text { KI } \\
\text { D2 }\end{array}$ & $\begin{array}{c}\text { KI } \\
\text { D3 }\end{array}$ & $\begin{array}{l}\text { IN } \\
\text { C1 }\end{array}$ & $\begin{array}{l}\text { IN } \\
\text { C3 }\end{array}$ & $\begin{array}{l}\text { IN } \\
\text { C4 }\end{array}$ & $\begin{array}{l}C \\
R \\
D\end{array}$ & $\begin{array}{c}A G \\
\text { E1 }\end{array}$ & $\begin{array}{l}\text { AG } \\
\text { E2 }\end{array}$ & $\begin{array}{l}\text { JY } \\
\text { R1 }\end{array}$ & $\begin{array}{l}\text { JY } \\
\text { R2 }\end{array}$ & $\begin{array}{l}\text { JY } \\
\text { R4 }\end{array}$ & $\begin{array}{l}\text { HY } \\
\text { R1 }\end{array}$ & $\begin{array}{l}\text { HY } \\
\text { R2 }\end{array}$ & $\begin{array}{l}\text { HY } \\
\text { R4 }\end{array}$ & $\begin{array}{l}\text { TR } \\
\text { M1 }\end{array}$ & $\begin{array}{l}\text { TR } \\
\text { M2 }\end{array}$ \\
\hline 1 & $\begin{array}{r}0,888 \\
889 \\
\end{array}$ & 0 & 0 & 0 & 1 & 0 & 0 & 0 & 0 & 0 & 0 & 1 & 0 & 0 & 1 & 0 & 0 & 0 & 1 & 0 & 1 & 0 & 0 & 1 & 0 & 0 & 1 & 0 \\
\hline 2 & $\begin{array}{r}0,888 \\
889 \\
\end{array}$ & 0 & 0 & 0 & 1 & 0 & 0 & 0 & 0 & 0 & 0 & 1 & 0 & 0 & 1 & 0 & 0 & 0 & 1 & 0 & 1 & 0 & 0 & 1 & 0 & 0 & 1 & 0 \\
\hline 3 & $\begin{array}{r}0,777 \\
778 \\
\end{array}$ & 0 & 0 & 1 & 0 & 0 & 1 & 0 & 0 & 0 & 0 & 1 & 0 & 0 & 1 & 0 & 0 & 0 & 1 & 0 & 1 & 0 & 0 & 1 & 0 & 0 & 1 & 0 \\
\hline 4 & 1 & 0 & 0 & 0 & 0 & 0 & 0 & 1 & 0 & 1 & 1 & 1 & 0 & 0 & 1 & 0 & 0 & 0 & 1 & 0 & 1 & 0 & 0 & 1 & 0 & 0 & 1 & 0 \\
\hline 5 & $\begin{array}{r}0,888 \\
889 \\
\end{array}$ & 0 & 0 & 1 & 0 & 0 & 1 & 0 & 0 & 1 & 0 & 0 & 0 & 1 & 1 & 0 & 0 & 1 & 1 & 0 & 1 & 0 & 0 & 1 & 0 & 0 & 1 & 0 \\
\hline 6 & 1 & 0 & 0 & 0 & 1 & 0 & 0 & 0 & 0 & 0 & 0 & 1 & 0 & 0 & 0 & 0 & 0 & 0 & 1 & 0 & 1 & 0 & 0 & 1 & 0 & 0 & 1 & 0 \\
\hline 7 & $\begin{array}{r}0,666 \\
667 \\
\end{array}$ & 0 & 0 & 0 & 0 & 0 & 1 & 0 & 0 & 0 & 0 & 1 & 0 & 0 & 0 & 0 & 0 & 0 & 1 & 0 & 1 & 0 & 0 & 1 & 0 & 0 & 1 & 0 \\
\hline 8 & $\begin{array}{r}0,666 \\
667 \\
\end{array}$ & 0 & 1 & 0 & 0 & 0 & 1 & 0 & 0 & 0 & 0 & 1 & 0 & 0 & 0 & 0 & 0 & 0 & 1 & 0 & 1 & 0 & 0 & 1 & 0 & 0 & 1 & 0 \\
\hline$\ldots$ & $\ldots$ & $\ldots$ & $\ldots$ & $\ldots$ & $\ldots$ & $\ldots$ & $\ldots$ & $\ldots$ & $\ldots$ & $\ldots$ & $\ldots$ & $\ldots$ & $\ldots$ & $\ldots$ & $\ldots$ & $\ldots$ & $\ldots$ & $\ldots$ & $\ldots$ & $\ldots$ & $\ldots$ & $\ldots$ & $\ldots$ & $\ldots$ & $\ldots$ & $\ldots$ & $\ldots$ & $\ldots$ \\
\hline $\begin{array}{l}10 \\
61 \\
\end{array}$ & 1 & 0 & 0 & 0 & 1 & 0 & 0 & 1 & 0 & 0 & 1 & 0 & 0 & 1 & 0 & 1 & 0 & 0 & 0 & 0 & 0 & 0 & 1 & 0 & 0 & 0 & 0 & 0 \\
\hline $\begin{array}{l}10 \\
62 \\
\end{array}$ & 1 & 0 & 1 & 0 & 0 & 0 & 0 & 1 & 0 & 1 & 1 & 0 & 1 & 0 & 0 & 1 & 0 & 0 & 0 & 1 & 0 & 1 & 0 & 0 & 0 & 1 & 0 & 0 \\
\hline $\begin{array}{l}10 \\
63 \\
\end{array}$ & 1 & 0 & 1 & 0 & 0 & 0 & 0 & 1 & 0 & 1 & 1 & 0 & 0 & 1 & 0 & 0 & 1 & 1 & 0 & 1 & 0 & 1 & 0 & 0 & 0 & 1 & 0 & 0 \\
\hline $\begin{array}{l}10 \\
64 \\
\end{array}$ & 1 & 0 & 1 & 0 & 0 & 0 & 0 & 1 & 0 & 1 & 1 & 0 & 0 & 0 & 1 & 0 & 0 & 0 & 0 & 1 & 0 & 0 & 1 & 0 & 0 & 1 & 0 & 0 \\
\hline $\begin{array}{l}10 \\
65 \\
\end{array}$ & 1 & 0 & 1 & 0 & 0 & 0 & 0 & 1 & 1 & 0 & 1 & 0 & 0 & 1 & 0 & 0 & 0 & 0 & 0 & 1 & 0 & 0 & 1 & 0 & 0 & 1 & 0 & 0 \\
\hline $\begin{array}{l}10 \\
66 \\
\end{array}$ & 1 & 0 & 0 & 0 & 1 & 0 & 0 & 1 & 0 & 1 & 1 & 0 & 1 & 0 & 0 & 1 & 0 & 1 & 0 & 1 & 0 & 0 & 1 & 0 & 0 & 1 & 0 & 0 \\
\hline $\begin{array}{l}10 \\
67 \\
\end{array}$ & 1 & 0 & 0 & 0 & 0 & 0 & 0 & 1 & 0 & 1 & 1 & 0 & 1 & 0 & 0 & 0 & 1 & 1 & 0 & 1 & 0 & 0 & 1 & 0 & 0 & 1 & 0 & 0 \\
\hline $\begin{array}{l}10 \\
68 \\
\end{array}$ & 1 & 1 & 0 & 0 & 0 & 0 & 0 & 1 & 0 & 1 & 0 & 0 & 0 & 1 & 0 & 1 & 0 & 0 & 0 & 0 & 0 & 0 & 1 & 0 & 0 & 1 & 0 & 0 \\
\hline
\end{tabular}

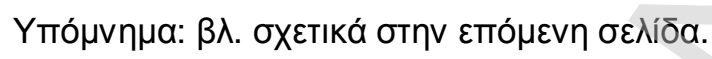




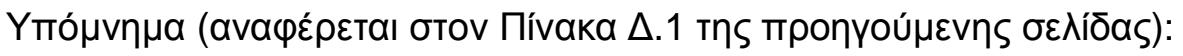

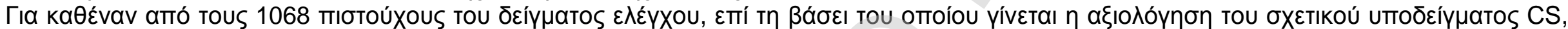

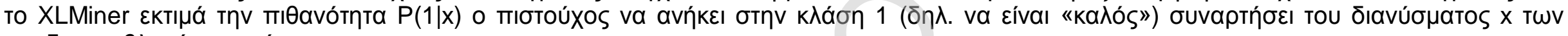

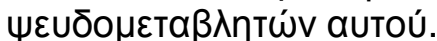

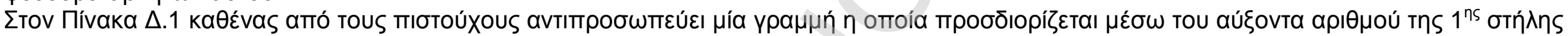

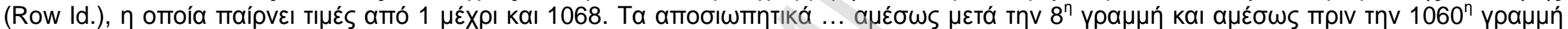

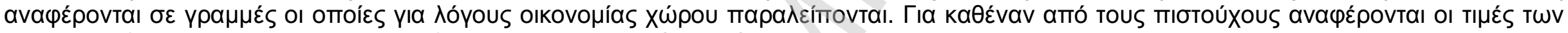

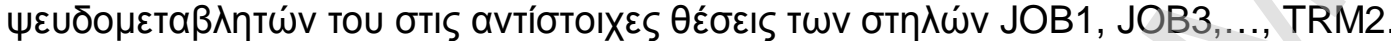

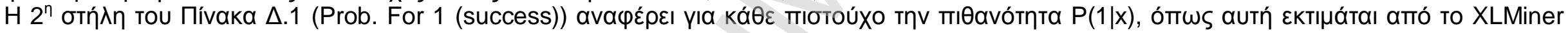

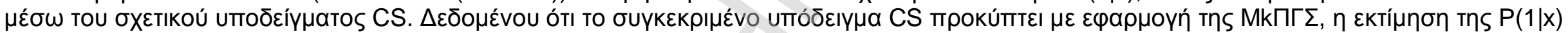

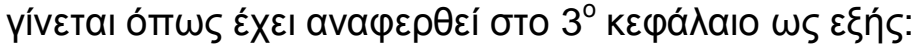

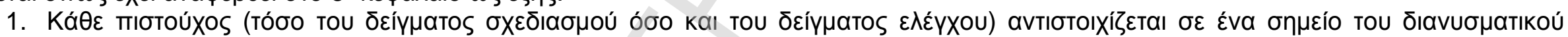

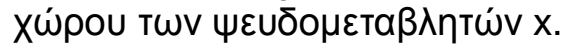

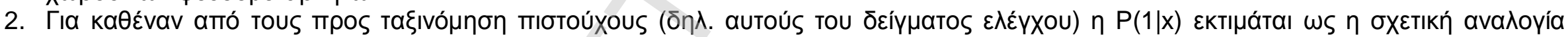

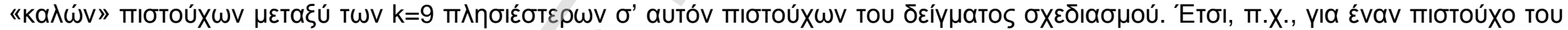

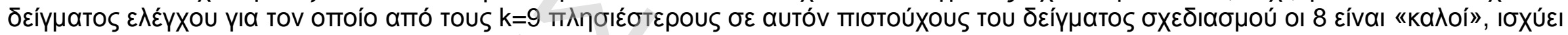

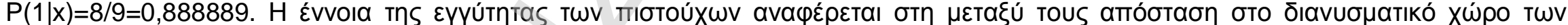

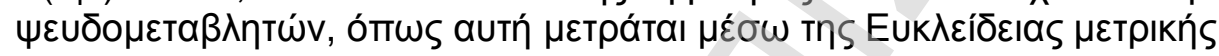




\section{XLMiner : Neural Network Classification (N $\Delta$ )}

\section{Inputs}

\begin{tabular}{|c|c|c|}
\hline & \multicolumn{2}{|l|}{ Data } \\
\hline & Training data used for building the model & ['test2ian.xls']'Data_Partition1'!\$C\$19:\$BJ $\$ 4290$ \\
\hline & \# Records in the training data & 4272 \\
\hline & Validation data & ['test2ian.xls']'Data_Partition1!! \$C \$4291:\$BJ\$5358 \\
\hline & \# Records in the va & 1068 \\
\hline
\end{tabular}

\begin{tabular}{|c|c|c|c|c|c|c|c|c|c|c|c|c|c|c|c|c|c|c|c|c|c|c|c|c|c|c|c|}
\hline \multicolumn{28}{|c|}{ Variables } \\
\hline \begin{tabular}{|l|l}
$\#$ \\
Input \\
Varia \\
bles \\
\end{tabular} & 27 & & & & & & & & & & & & & & & & & & & & & & & & & & \\
\hline $\begin{array}{l}\text { Input } \\
\text { varia } \\
\text { bles }\end{array}$ & $\begin{array}{l}\text { JO } \\
\text { B1 }\end{array}$ & $\begin{array}{l}\mathrm{JO} \\
\mathrm{B} 3\end{array}$ & $\begin{array}{l}\mathrm{JO} \\
\mathrm{B} 4\end{array}$ & $\begin{array}{l}\text { JO } \\
\text { B5 }\end{array}$ & $\begin{array}{l}\mathrm{JO} \\
\mathrm{B} 6\end{array}$ & $\begin{array}{l}\text { HO } \\
\text { ME2 }\end{array}$ & $\begin{array}{l}\mathrm{HO} \\
\text { ME3 }\end{array}$ & $\begin{array}{l}\text { MA } \\
\text { R1 }\end{array}$ & \begin{tabular}{|l} 
MA \\
R3
\end{tabular} & \begin{tabular}{|l|}
$C$ \\
$A$ \\
R
\end{tabular} & $\begin{array}{l}\mathrm{KI} \\
\mathrm{D} 1\end{array}$ & \begin{tabular}{|l}
$\mathrm{KI}$ \\
$\mathrm{D} 2$
\end{tabular} & $\begin{array}{l}\mathrm{KI} \\
\mathrm{D} 3\end{array}$ & $\begin{array}{l}\text { IN } \\
\text { C1 }\end{array}$ & $\begin{array}{l}\text { IN } \\
\text { C3 }\end{array}$ & $\begin{array}{l}\text { IN } \\
\text { C4 }\end{array}$ & $\begin{array}{l}\text { C } \\
R \\
D\end{array}$ & \begin{tabular}{|l} 
AG \\
$\mathrm{E} 1$
\end{tabular} & $\begin{array}{l}\mathrm{AG} \\
\mathrm{E} 2\end{array}$ & \begin{tabular}{|l} 
JY \\
R1
\end{tabular} & \begin{tabular}{|l|} 
JY \\
R2
\end{tabular} & $\begin{array}{l}\text { JY } \\
\text { R4 }\end{array}$ & \begin{tabular}{|l} 
HY \\
R1
\end{tabular} & \begin{tabular}{|l} 
HY \\
R2
\end{tabular} & \begin{tabular}{|l} 
HY \\
R4
\end{tabular} & \begin{tabular}{|l} 
TR \\
M1
\end{tabular} & \begin{tabular}{|l} 
TR \\
M2
\end{tabular} \\
\hline \begin{tabular}{|l} 
Outp \\
ut \\
varia \\
ble
\end{tabular} & STA & & & & & & & & & & & & & & & & & & & & & & & & & & \\
\hline
\end{tabular}

Yто́ $\mu v \eta \mu \alpha:$

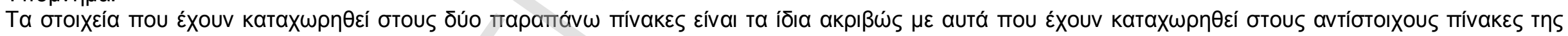
MkПГг. 


\begin{tabular}{|l|l|l|l|l|}
\hline & & Parameters/Options & \\
\hline & & \# Hidden layers & 1 & \\
\hline & & \# Nodes in HiddenLayer & 23 & \\
\hline & & Hidden layer sigmoid & Standard & \\
\hline & & Output layer sigmoid & Standard & \\
\hline & & \# Epochs & 45 & \\
\hline & & Step size for gradient descent & 0,1 & \\
\hline & & Weight change momentum & 0,8 & \\
\hline
\end{tabular}

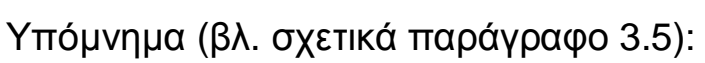

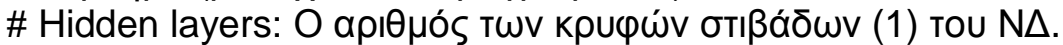

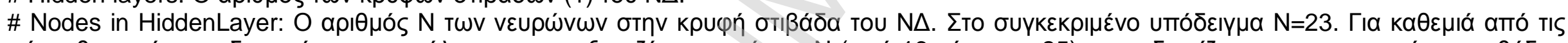

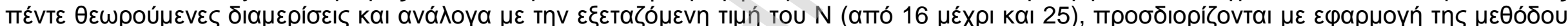

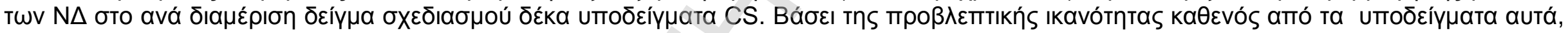

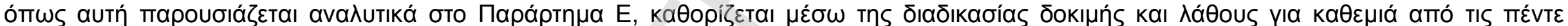

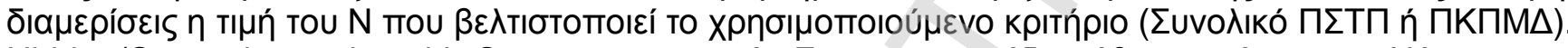

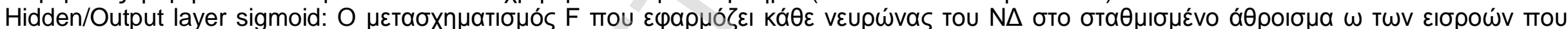
$\lambda \alpha \mu \beta a ́ v \varepsilon ı$ a

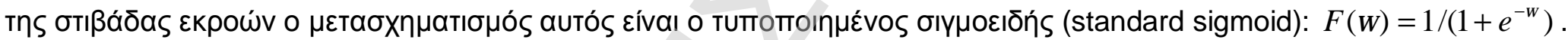

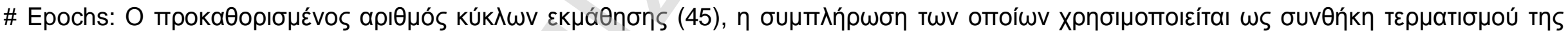

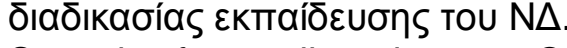

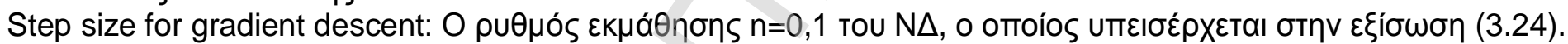

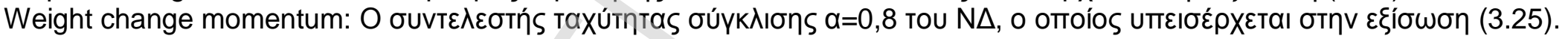


The Neural Network Model / Inter-layer connection weights

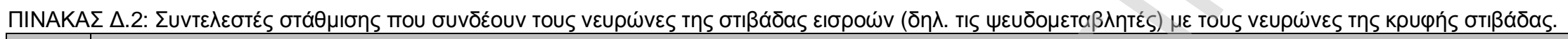

\begin{tabular}{|c|c|c|c|c|c|c|c|c|c|c|c|c|c|c|c|c|c|c|c|}
\hline & \multicolumn{19}{|c|}{ Input Layer } \\
\hline $\begin{array}{l}\text { Hidde } \\
\mathrm{n} \\
\text { Layer }\end{array}$ & JOB1 & JOB3 & JOB4 & JOB5 & JOB6 & $\begin{array}{r}\text { HOM } \\
\text { E2 }\end{array}$ & $\begin{array}{r}\text { HOM } \\
\text { E3 }\end{array}$ & IAR1 & MAR3 & CAR & KID1 & KID2 & KID3 & INC1 & INC3 & INC4 & CRD & GE1 & GE2 \\
\hline $\begin{array}{l}\text { Node } \\
\# 1\end{array}$ & $\begin{array}{r}1,197 \\
5 \\
\end{array}$ & $\begin{array}{r}1,116 \\
68 \\
\end{array}$ & $\begin{array}{r}0,2706 \\
36 \\
\end{array}$ & $\begin{array}{r}1,027 \\
02 \\
\end{array}$ & $\begin{array}{r}0,171 \\
988 \\
\end{array}$ & $\begin{array}{r}1,767 \\
39 \\
\end{array}$ & $\begin{array}{r}0,159 \\
23\end{array}$ & $\begin{array}{r}1,172 \\
37\end{array}$ & $\begin{array}{r}1,271 \\
62\end{array}$ & $\begin{array}{r}1,814 \\
19\end{array}$ & $\begin{array}{r}1,3539 \\
1\end{array}$ & $\begin{array}{r}2,0507 \\
6 \\
\end{array}$ & $-0,839$ & $\begin{array}{r}0,895 \\
287 \\
\end{array}$ & $\begin{array}{r}0939 \\
773\end{array}$ & $\begin{array}{l}, 787 \\
958\end{array}$ & $\begin{array}{r}0,357 \\
357 \\
\end{array}$ & $\begin{array}{r}0,463 \\
074 \\
\end{array}$ & $\begin{array}{r}0,1774 \\
31 \\
\end{array}$ \\
\hline $\begin{array}{l}\text { Node } \\
\# 2\end{array}$ & $\begin{array}{r}4,711 \\
35\end{array}$ & $\begin{array}{r}1,237 \\
5\end{array}$ & $\begin{array}{r}0,6146 \\
16\end{array}$ & $\begin{array}{r}0,928 \\
816\end{array}$ & $\begin{array}{r}0,683 \\
299\end{array}$ & $\begin{array}{r}0,126 \\
717 \\
\end{array}$ & $\begin{array}{r}1,722 \\
78\end{array}$ & $\begin{array}{r}0,488 \\
459 \\
\end{array}$ & $\begin{array}{r}0,325 \\
153 \\
\end{array}$ & $\begin{array}{r}1,567 \\
17\end{array}$ & $\begin{array}{r}2,6057 \\
6 \\
\end{array}$ & $\begin{array}{r}0,5140 \\
03\end{array}$ & $\begin{array}{r}0,939 \\
916\end{array}$ & $\begin{array}{r}0,225 \\
48 \\
\end{array}$ & $\begin{array}{r}0,4188 \\
53 \\
\end{array}$ & $\begin{array}{r}0,360 \\
007\end{array}$ & $\begin{array}{r}0,107 \\
427\end{array}$ & $\begin{array}{r}0,212 \\
597 \\
\end{array}$ & $\begin{array}{r}0,4281 \\
51 \\
\end{array}$ \\
\hline $\begin{array}{l}\text { Node } \\
\text { \# } 3\end{array}$ & $\begin{array}{r}0,380 \\
273\end{array}$ & $-1,515$ & $\begin{array}{r}0,2467 \\
37\end{array}$ & $\begin{array}{r}0,145 \\
784\end{array}$ & $\begin{array}{r}0,192 \\
808\end{array}$ & $\begin{array}{r}1,494 \\
89 \\
\end{array}$ & $\begin{array}{r}0,717 \\
296\end{array}$ & $\begin{array}{r}0,796 \\
051 \\
\end{array}$ & $\begin{array}{r}1,081 \\
79\end{array}$ & $\begin{array}{r}0,149 \\
651\end{array}$ & $\begin{array}{r}0,2180 \\
57\end{array}$ & $\begin{array}{r}0,0881 \\
805 \\
\end{array}$ & $\begin{array}{r}0,210 \\
684\end{array}$ & $\begin{array}{r}1,910 \\
73 \\
\end{array}$ & $\begin{array}{r}0,7513 \\
47\end{array}$ & $\begin{array}{r}0,324 \\
431\end{array}$ & $\begin{array}{r}0,781 \\
238\end{array}$ & $\begin{array}{r}1,130 \\
49 \\
\end{array}$ & $\begin{array}{r}0,8991 \\
74\end{array}$ \\
\hline $\begin{array}{l}\text { Node } \\
\# 4\end{array}$ & $\begin{array}{r}3,643 \\
39 \\
\end{array}$ & $\begin{array}{r}1,209 \\
69 \\
\end{array}$ & $\begin{array}{r}0,9012 \\
4 \\
\end{array}$ & $\begin{array}{r}0,713 \\
162\end{array}$ & $\begin{array}{r}0,428 \\
63 \\
\end{array}$ & $\begin{array}{r}2,351 \\
53\end{array}$ & $\begin{array}{r}2,570 \\
06\end{array}$ & $\begin{array}{r}2,625 \\
14\end{array}$ & $\begin{array}{r}1,292 \\
65\end{array}$ & $\begin{array}{r}0,287 \\
764\end{array}$ & $\begin{array}{r}2,0904 \\
8\end{array}$ & $\begin{array}{r}1,5072 \\
9\end{array}$ & $\begin{array}{r}0,209 \\
532\end{array}$ & $\begin{array}{r}0,016 \\
7754\end{array}$ & $\begin{array}{r}0,9206 \\
1\end{array}$ & $\begin{array}{r}1,460 \\
22\end{array}$ & $\begin{array}{r}1,852 \\
87\end{array}$ & $\begin{array}{r}- \\
2,411 \\
39 \\
\end{array}$ & $\begin{array}{r}0,6827 \\
34 \\
\end{array}$ \\
\hline $\begin{array}{l}\text { Node } \\
\# 5\end{array}$ & $\begin{array}{r}2,025 \\
85 \\
\end{array}$ & $\begin{array}{r}1,609 \\
67 \\
\end{array}$ & $\begin{array}{r}0,1850 \\
47 \\
\end{array}$ & $\begin{array}{r}0,851 \\
177 \\
\end{array}$ & $\begin{array}{r}1,670 \\
46\end{array}$ & $\begin{array}{r}1,480 \\
28\end{array}$ & $\begin{array}{r}1,925 \\
16\end{array}$ & $\begin{array}{r}1,585 \\
04\end{array}$ & $\begin{array}{r}1,776 \\
4 \\
\end{array}$ & $\begin{array}{r}1,838 \\
36\end{array}$ & $\begin{array}{r}3,5619 \\
2\end{array}$ & $\begin{array}{r}3,0775 \\
4\end{array}$ & $\begin{array}{r}- \\
1,819 \\
38 \\
\end{array}$ & $\begin{array}{r}2,251 \\
38 \\
\end{array}$ & $\begin{array}{r}1,1461 \\
2\end{array}$ & $\begin{array}{r}2,246 \\
19\end{array}$ & $\begin{array}{r}0,977 \\
616\end{array}$ & $\begin{array}{r}1,634 \\
68\end{array}$ & $\begin{array}{r}0,7451 \\
62 \\
\end{array}$ \\
\hline $\begin{array}{l}\text { Node } \\
\# 6\end{array}$ & $\begin{array}{r}1,998 \\
25 \\
\end{array}$ & $\begin{array}{r}0,699 \\
017 \\
\end{array}$ & $\begin{array}{r}1,3968 \\
3 \\
\end{array}$ & $\begin{array}{r}2,553 \\
72 \\
\end{array}$ & $\begin{array}{r}- \\
0,999 \\
06\end{array}$ & $\begin{array}{r}0,645 \\
379 \\
\end{array}$ & $\begin{array}{r}1,124 \\
83 \\
\end{array}$ & $\begin{array}{r}1,631 \\
94\end{array}$ & $\begin{array}{r}2,479 \\
02 \\
\end{array}$ & $\begin{array}{r}- \\
1,820 \\
13\end{array}$ & $\begin{array}{r}0,7402 \\
66 \\
\end{array}$ & $\begin{array}{r}2,0171 \\
6 \\
\end{array}$ & $\begin{array}{r}2,341 \\
21 \\
\end{array}$ & $\begin{array}{r}4,153 \\
3 \\
\end{array}$ & $\begin{array}{r}2,1568 \\
3 \\
\end{array}$ & $\begin{array}{r}0,879 \\
553 \\
\end{array}$ & $\begin{array}{r}2,876 \\
69 \\
\end{array}$ & $\begin{array}{r}1,391 \\
81\end{array}$ & $\begin{array}{r}2,4157 \\
7 \\
\end{array}$ \\
\hline $\begin{array}{l}\text { Node } \\
\# 7\end{array}$ & $\begin{array}{r}2,333 \\
39 \\
\end{array}$ & $\begin{array}{r}0,730 \\
117 \\
\end{array}$ & $\begin{array}{r}0,3718 \\
36 \\
\end{array}$ & $\begin{array}{r}2,751 \\
57\end{array}$ & $\begin{array}{r}0,678 \\
904 \\
\end{array}$ & $\begin{array}{r}0,523 \\
789 \\
\end{array}$ & $\begin{array}{r}0,166 \\
142 \\
\end{array}$ & $\begin{array}{r}0,901 \\
086\end{array}$ & $\begin{array}{r}0,495 \\
885\end{array}$ & $\begin{array}{r}0,625 \\
862\end{array}$ & $\begin{array}{r}0,1238 \\
16 \\
\end{array}$ & $\begin{array}{r}1,3158 \\
4\end{array}$ & $\begin{array}{r}560 \\
32\end{array}$ & $\begin{array}{r}0,441 \\
848 \\
\end{array}$ & $\begin{array}{r}1,4816 \\
8 \\
\end{array}$ & $\begin{array}{r}, 802 \\
521\end{array}$ & $\begin{array}{r}1,868 \\
45\end{array}$ & $\begin{array}{r}3,599 \\
19 \\
\end{array}$ & $\begin{array}{r}0,6817 \\
09 \\
\end{array}$ \\
\hline $\begin{array}{l}\text { Node } \\
\# 8\end{array}$ & $\begin{array}{r}1,736 \\
09\end{array}$ & $\begin{array}{r}0,508 \\
629 \\
\end{array}$ & $\begin{array}{r}0,2758 \\
69\end{array}$ & $\begin{array}{r}0,041 \\
608 \\
\end{array}$ & $\begin{array}{r}0,917 \\
176 \\
\end{array}$ & $\begin{array}{r}0,105 \\
565 \\
\end{array}$ & $\begin{array}{r}- \\
0,048 \\
801\end{array}$ & $\begin{array}{r}3,292 \\
37 \\
\end{array}$ & $\begin{array}{r}1,576 \\
05\end{array}$ & $\begin{array}{r}1,010 \\
04 \\
\end{array}$ & 1,0063 & $\begin{array}{r}0,7603 \\
42 \\
\end{array}$ & $\begin{array}{r}0,934 \\
971 \\
\end{array}$ & $\begin{array}{r}0,625 \\
291 \\
\end{array}$ & $\begin{array}{r}1,0418 \\
6\end{array}$ & $\begin{array}{r}0,298 \\
346\end{array}$ & $\begin{array}{r}1,433 \\
21\end{array}$ & $\begin{array}{r}2,037 \\
02 \\
\end{array}$ & $\begin{array}{r}0,4438 \\
83\end{array}$ \\
\hline $\begin{array}{l}\text { Node } \\
\# 9\end{array}$ & $\begin{array}{r}0,765 \\
538 \\
\end{array}$ & $\begin{array}{r}0,579 \\
419\end{array}$ & $\begin{array}{r}0,1697 \\
21 \\
\end{array}$ & $\begin{array}{r}0,503 \\
795\end{array}$ & $\begin{array}{r}0,301 \\
816 \\
\end{array}$ & $\begin{array}{r}0,908 \\
311 \\
\end{array}$ & $\begin{array}{r}0,698 \\
046\end{array}$ & $\begin{array}{r}0,701 \\
596 \\
\end{array}$ & $\begin{array}{r}1,038 \\
65\end{array}$ & $\begin{array}{r}2,333 \\
16\end{array}$ & $\begin{array}{r}0,6435 \\
17 \\
\end{array}$ & $\begin{array}{r}1,3254 \\
6\end{array}$ & $\begin{array}{r}0,431 \\
134\end{array}$ & $\begin{array}{r}1,197 \\
09 \\
\end{array}$ & $\begin{array}{r}- \\
1,4809 \\
6 \\
\end{array}$ & $\begin{array}{r}0,632 \\
702\end{array}$ & $\begin{array}{r}0,716 \\
421 \\
\end{array}$ & $\begin{array}{r}0,640 \\
171 \\
\end{array}$ & $\begin{array}{r}0,1744 \\
93\end{array}$ \\
\hline $\begin{array}{l}\text { Node } \\
\# 10\end{array}$ & $\begin{array}{r}0,978 \\
349 \\
\end{array}$ & $\begin{array}{l}0,011 \\
2889\end{array}$ & $\begin{array}{r}0,2137 \\
56\end{array}$ & $\begin{array}{r}0,062 \\
6568\end{array}$ & $\begin{array}{r}0,011 \\
0198\end{array}$ & $\begin{array}{r}0,394 \\
325 \\
\end{array}$ & $\begin{array}{r}0,107 \\
319 \\
\end{array}$ & $\begin{array}{r}0,276 \\
104 \\
\end{array}$ & $\begin{array}{r}2,012 \\
77\end{array}$ & $\begin{array}{c}0,079 \\
3924\end{array}$ & $\begin{array}{r}0,9990 \\
15 \\
\end{array}$ & $\begin{array}{r}0,4941 \\
38\end{array}$ & $\begin{array}{r}0,923 \\
401\end{array}$ & $\begin{array}{r}1,844 \\
57 \\
\end{array}$ & $\begin{array}{r}0,9846 \\
38\end{array}$ & $\begin{array}{r}0,510 \\
362\end{array}$ & $\begin{array}{r}0,046 \\
4992\end{array}$ & $\begin{array}{r}2,517 \\
86 \\
\end{array}$ & $\begin{array}{r}0,1442 \\
75 \\
\end{array}$ \\
\hline $\begin{array}{l}\text { Node } \\
\# 11\end{array}$ & $\begin{array}{r}3,717 \\
8 \\
\end{array}$ & $\begin{array}{r}1,133 \\
56 \\
\end{array}$ & $\begin{array}{r}0,0170 \\
723\end{array}$ & $\begin{array}{r}1,029 \\
95\end{array}$ & $\begin{array}{r}0,823 \\
902\end{array}$ & $\begin{array}{r}0,593 \\
967\end{array}$ & $\begin{array}{r}0,485 \\
276\end{array}$ & $\begin{array}{r}1,393 \\
39\end{array}$ & $\begin{array}{r}0,857 \\
492 \\
\end{array}$ & $\begin{array}{r}2,150 \\
24\end{array}$ & $\begin{array}{r}0,2692 \\
5 \\
\end{array}$ & $\begin{array}{r}0,9389 \\
85\end{array}$ & 1,944 & $\begin{array}{r}0,207 \\
148 \\
\end{array}$ & 1,44 & $\begin{array}{r}1,410 \\
64\end{array}$ & $\begin{array}{r}0,821 \\
291 \\
\end{array}$ & $\begin{array}{r}1,272 \\
03 \\
\end{array}$ & $\begin{array}{r}0,0486 \\
124 \\
\end{array}$ \\
\hline $\begin{array}{l}\text { Node } \\
\# 12\end{array}$ & $\begin{array}{r}1,903 \\
5 \\
\end{array}$ & $\begin{array}{r}1,315 \\
36 \\
\end{array}$ & $\begin{array}{r}1,1861 \\
5\end{array}$ & $\begin{array}{r}2,138 \\
59 \\
\end{array}$ & $\begin{array}{r}0,856 \\
893 \\
\end{array}$ & $\begin{array}{r}0,070 \\
915\end{array}$ & $\begin{array}{r}0,179 \\
326 \\
\end{array}$ & $\begin{array}{r}0,660 \\
931 \\
\end{array}$ & $\begin{array}{r}0,332 \\
207 \\
\end{array}$ & $\begin{array}{r}1,825 \\
62\end{array}$ & $\begin{array}{r}2,2325 \\
1\end{array}$ & $\begin{array}{r}1,3611 \\
6\end{array}$ & $\begin{array}{r}0,278 \\
175\end{array}$ & $\begin{array}{r}1,566 \\
42 \\
\end{array}$ & $\begin{array}{r}0,0439 \\
535 \\
\end{array}$ & $\begin{array}{r}0,763 \\
499\end{array}$ & $\begin{array}{r}0,284 \\
226 \\
\end{array}$ & $\begin{array}{r}0,726 \\
547\end{array}$ & $\begin{array}{r}0,9179 \\
28 \\
\end{array}$ \\
\hline
\end{tabular}




\begin{tabular}{|c|c|c|c|c|c|c|c|c|c|c|c|c|c|c|c|c|c|c|c|}
\hline $\begin{array}{l}\text { Node } \\
\# 13\end{array}$ & $\begin{array}{r}0,674 \\
833 \\
\end{array}$ & $\begin{array}{r}1,427 \\
56 \\
\end{array}$ & $\begin{array}{r}0,0417 \\
813 \\
\end{array}$ & $\begin{array}{r}1,681 \\
17\end{array}$ & $\begin{array}{r}0,510 \\
153\end{array}$ & $\begin{array}{r}3,717 \\
08 \\
\end{array}$ & $\begin{array}{r}1,402 \\
41\end{array}$ & $\begin{array}{r}2,065 \\
03 \\
\end{array}$ & $\begin{array}{r}0,280 \\
385\end{array}$ & $\begin{array}{r}0,363 \\
294\end{array}$ & $\begin{array}{r}1,2178 \\
8\end{array}$ & 2,6298 & $\begin{array}{r}1,598 \\
11 \\
\end{array}$ & $\begin{array}{r}3,547 \\
57\end{array}$ & $\begin{array}{r}1,1796 \\
4\end{array}$ & $\begin{array}{r}2,163 \\
98\end{array}$ & $\begin{array}{r}1,593 \\
82\end{array}$ & $\begin{array}{r}1,704 \\
17\end{array}$ & $\begin{array}{r}0,9538 \\
67 \\
\end{array}$ \\
\hline $\begin{array}{l}\text { Node } \\
\# 14\end{array}$ & $\begin{array}{r}1,333 \\
04 \\
\end{array}$ & $\begin{array}{r}0,412 \\
673\end{array}$ & $\begin{array}{r}0,3692 \\
19\end{array}$ & $\begin{array}{r}0,149 \\
496\end{array}$ & $\begin{array}{r}0,495 \\
537\end{array}$ & $\begin{array}{r}1,366 \\
9 \\
\end{array}$ & $\begin{array}{r}0,221 \\
393\end{array}$ & $\begin{array}{r}0,935 \\
896 \\
\end{array}$ & $\begin{array}{r}1,156 \\
77\end{array}$ & $\begin{array}{r}1,591 \\
33\end{array}$ & $\begin{array}{r}2,1338 \\
7\end{array}$ & $\begin{array}{r}0,7094 \\
37\end{array}$ & $\begin{array}{r}1,432 \\
67 \\
\end{array}$ & $\begin{array}{r}1,259 \\
44 \\
\end{array}$ & $\begin{array}{r}2,1252 \\
5\end{array}$ & $\begin{array}{r}2,053 \\
86\end{array}$ & $\begin{array}{r}1,217 \\
59\end{array}$ & $\begin{array}{r}0,339 \\
334\end{array}$ & $\begin{array}{r}0,1156 \\
21\end{array}$ \\
\hline $\begin{array}{l}\text { Node } \\
\# 15\end{array}$ & $\begin{array}{r}2,788 \\
36\end{array}$ & $\begin{array}{r}0,896 \\
428\end{array}$ & $\begin{array}{r}0,0675 \\
539\end{array}$ & 1,493 & $\begin{array}{r}0,610 \\
156\end{array}$ & $\begin{array}{r}2,564 \\
65 \\
\end{array}$ & $\begin{array}{r}1,698 \\
24\end{array}$ & $\begin{array}{r}0,448 \\
999\end{array}$ & $\begin{array}{r}0,708 \\
449 \\
\end{array}$ & $\begin{array}{r}3,326 \\
23\end{array}$ & $\begin{array}{r}0,0566 \\
223 \\
\end{array}$ & $\begin{array}{r}0,1792 \\
9 \\
\end{array}$ & $\begin{array}{r}0,126 \\
467\end{array}$ & $\begin{array}{r}1,468 \\
28 \\
\end{array}$ & $\begin{array}{r}1,0106 \\
7\end{array}$ & $\begin{array}{r}0,680 \\
348\end{array}$ & $\begin{array}{r}3,374 \\
29\end{array}$ & $\begin{array}{r}0,739 \\
391\end{array}$ & $\begin{array}{r}0,5859 \\
38 \\
\end{array}$ \\
\hline $\begin{array}{l}\text { Node } \\
\# 16\end{array}$ & $\begin{array}{r}1,235 \\
15\end{array}$ & $\begin{array}{r}0,136 \\
095\end{array}$ & $\begin{array}{r}1,1191 \\
6\end{array}$ & $\begin{array}{r}2,203 \\
97 \\
\end{array}$ & $\begin{array}{r}2,544 \\
87 \\
\end{array}$ & 1,21 & $\begin{array}{r}2,505 \\
57\end{array}$ & $\begin{array}{r}2,839 \\
75 \\
\end{array}$ & $\begin{array}{r}1,619 \\
46\end{array}$ & $\begin{array}{r}0,506 \\
139 \\
\end{array}$ & $\begin{array}{r}0,7410 \\
87\end{array}$ & $\begin{array}{r}0,4795 \\
02\end{array}$ & $\begin{array}{r}1,071 \\
36 \\
\end{array}$ & $\begin{array}{r}2,088 \\
67\end{array}$ & $\begin{array}{r}1,0230 \\
9\end{array}$ & $\begin{array}{r}0,750 \\
104\end{array}$ & $\begin{array}{r}0,459 \\
607 \\
\end{array}$ & $\begin{array}{r}0,156 \\
223\end{array}$ & $\begin{array}{r}0,3195 \\
93\end{array}$ \\
\hline $\begin{array}{l}\text { Node } \\
\# 17\end{array}$ & $\begin{array}{r}0,548 \\
091\end{array}$ & $\begin{array}{r}0,931 \\
261 \\
\end{array}$ & $\begin{array}{r}0,7137 \\
19 \\
\end{array}$ & $\begin{array}{r}0,641 \\
559\end{array}$ & $\begin{array}{r}1,084 \\
4 \\
\end{array}$ & $\begin{array}{r}1,284 \\
58 \\
\end{array}$ & $\begin{array}{r}0,104 \\
218\end{array}$ & $\begin{array}{r}2,075 \\
7 \\
\end{array}$ & $\begin{array}{r}0,562 \\
078 \\
\end{array}$ & $\begin{array}{r}3,220 \\
21\end{array}$ & $\begin{array}{r}0,7487 \\
03\end{array}$ & $\begin{array}{r}0,6201 \\
44\end{array}$ & $\begin{array}{r}0,059 \\
4169\end{array}$ & $\begin{array}{r}1,107 \\
94 \\
\end{array}$ & $\begin{array}{r}1,2088 \\
9 \\
\end{array}$ & $\begin{array}{r}1,245 \\
11\end{array}$ & $\begin{array}{r}2,456 \\
02\end{array}$ & $\begin{array}{r}0,115 \\
174 \\
\end{array}$ & $\begin{array}{r}0,5774 \\
2\end{array}$ \\
\hline $\begin{array}{l}\text { Node } \\
\# 18\end{array}$ & $\begin{array}{r}2,163 \\
48 \\
\end{array}$ & $\begin{array}{r}0,753 \\
162\end{array}$ & $\begin{array}{r}0,5235 \\
85\end{array}$ & $\begin{array}{r}1,803 \\
77\end{array}$ & $\begin{array}{r}0,460 \\
909 \\
\end{array}$ & $\begin{array}{r}0,521 \\
613\end{array}$ & $\begin{array}{r}0,427 \\
771 \\
\end{array}$ & $\begin{array}{r}0,465 \\
935\end{array}$ & $\begin{array}{r}2,345 \\
6\end{array}$ & $\begin{array}{r}0,130 \\
692 \\
\end{array}$ & $\begin{array}{r}1,4758 \\
4 \\
\end{array}$ & $\begin{array}{r}0,7558 \\
66\end{array}$ & $\begin{array}{r}1,474 \\
07\end{array}$ & $\begin{array}{r}3,067 \\
74 \\
\end{array}$ & 1,3971 & $\begin{array}{r}0,336 \\
704\end{array}$ & $\begin{array}{r}0,465 \\
847\end{array}$ & $\begin{array}{r}2,924 \\
91 \\
\end{array}$ & $\begin{array}{r}0,4250 \\
56\end{array}$ \\
\hline $\begin{array}{l}\text { Node } \\
\# 19\end{array}$ & $\begin{array}{r}0,032 \\
2545\end{array}$ & $\begin{array}{r}0,119 \\
49\end{array}$ & $\begin{array}{r}2,2765 \\
7\end{array}$ & $\begin{array}{r}0,820 \\
723 \\
\end{array}$ & $\begin{array}{r}0,979 \\
201 \\
\end{array}$ & $\begin{array}{r}1,232 \\
46 \\
\end{array}$ & $\begin{array}{r}4,299 \\
44\end{array}$ & $\begin{array}{r}1,316 \\
67 \\
\end{array}$ & $\begin{array}{r}2,452 \\
09 \\
\end{array}$ & $\begin{array}{r}0,690 \\
815\end{array}$ & $\begin{array}{r}0,2894 \\
95\end{array}$ & $\begin{array}{r}0,0972 \\
59\end{array}$ & $\begin{array}{r}0,958 \\
108\end{array}$ & $\begin{array}{r}1,387 \\
78\end{array}$ & $\begin{array}{r}0,3534 \\
11 \\
\end{array}$ & $\begin{array}{r}1,171 \\
76\end{array}$ & $\begin{array}{r}1,317 \\
12 \\
\end{array}$ & $\begin{array}{r}0,139 \\
521\end{array}$ & $\begin{array}{r}1,0470 \\
5\end{array}$ \\
\hline $\begin{array}{l}\text { Node } \\
\# 20\end{array}$ & $\begin{array}{r}1,577 \\
85\end{array}$ & $\begin{array}{r}1,655 \\
1\end{array}$ & $\begin{array}{r}0,5002 \\
31\end{array}$ & $\begin{array}{r}2,881 \\
73 \\
\end{array}$ & $\begin{array}{r}1,279 \\
28 \\
\end{array}$ & $\begin{array}{r}0,115 \\
131\end{array}$ & $\begin{array}{r}0,740 \\
729 \\
\end{array}$ & $\begin{array}{r}1,857 \\
23 \\
\end{array}$ & $\begin{array}{r}2,125 \\
95 \\
\end{array}$ & $\begin{array}{r}1,767 \\
37\end{array}$ & $\begin{array}{r}0,0190 \\
698\end{array}$ & $\begin{array}{r}0,4467 \\
43\end{array}$ & $\begin{array}{r}0,344 \\
986 \\
\end{array}$ & $\begin{array}{r}1,005 \\
39 \\
\end{array}$ & $\begin{array}{r}1,3012 \\
6 \\
\end{array}$ & $\begin{array}{r}0,370 \\
587\end{array}$ & $\begin{array}{r}1,370 \\
24\end{array}$ & $\begin{array}{r}0,742 \\
662 \\
\end{array}$ & $\begin{array}{r}0,4531 \\
93\end{array}$ \\
\hline $\begin{array}{l}\text { Node } \\
\# 21\end{array}$ & $\begin{array}{r}2,079 \\
19\end{array}$ & $\begin{array}{r}0,148 \\
374 \\
\end{array}$ & $\begin{array}{r}0,1310 \\
43 \\
\end{array}$ & $\begin{array}{r}0,848 \\
118 \\
\end{array}$ & $\begin{array}{r}0,544 \\
852\end{array}$ & $\begin{array}{r}0,145 \\
541\end{array}$ & $\begin{array}{r}0,438 \\
234 \\
\end{array}$ & $\begin{array}{r}2,696 \\
7\end{array}$ & $\begin{array}{r}1,624 \\
27\end{array}$ & $\begin{array}{r}0,407 \\
867\end{array}$ & $\begin{array}{r}0,7833 \\
03\end{array}$ & $\begin{array}{r}0,0497 \\
877 \\
\end{array}$ & $\begin{array}{r}0,526 \\
523 \\
\end{array}$ & $\begin{array}{r}0,426 \\
468 \\
\end{array}$ & $\begin{array}{r}2,0929 \\
8\end{array}$ & $\begin{array}{r}0,349 \\
287\end{array}$ & $\begin{array}{r}1,330 \\
7\end{array}$ & $\begin{array}{r}0,730 \\
747 \\
\end{array}$ & $\begin{array}{r}0,1737 \\
43 \\
\end{array}$ \\
\hline $\begin{array}{l}\text { Node } \\
\# 22\end{array}$ & $\begin{array}{r}0,923 \\
307 \\
\end{array}$ & $\begin{array}{r}0,011 \\
8508\end{array}$ & $\begin{array}{r}2,2051 \\
8\end{array}$ & $\begin{array}{r}1,498 \\
51 \\
\end{array}$ & $\begin{array}{r}0,681 \\
098\end{array}$ & $\begin{array}{r}0,542 \\
576 \\
\end{array}$ & $\begin{array}{r}1,143 \\
83 \\
\end{array}$ & $\begin{array}{r}0,706 \\
778 \\
\end{array}$ & $\begin{array}{r}0,936 \\
625\end{array}$ & $\begin{array}{r}1,856 \\
91\end{array}$ & $\begin{array}{r}0,1775 \\
36 \\
\end{array}$ & $\begin{array}{r}0,8831 \\
66\end{array}$ & $\begin{array}{r}0,724 \\
914\end{array}$ & $\begin{array}{r}3,229 \\
26 \\
\end{array}$ & $\begin{array}{r}0,9492 \\
94\end{array}$ & $\begin{array}{r}1,513 \\
38\end{array}$ & $\begin{array}{r}1,056 \\
35\end{array}$ & $\begin{array}{r}0,606 \\
046\end{array}$ & $\begin{array}{r}0,5085 \\
79 \\
\end{array}$ \\
\hline $\begin{array}{l}\text { Node } \\
\# 23\end{array}$ & $\begin{array}{r}1,591 \\
59\end{array}$ & $\begin{array}{r}0,245 \\
971\end{array}$ & $\begin{array}{r}0,6119 \\
72 \\
\end{array}$ & $\begin{array}{r}0,358 \\
68\end{array}$ & $\begin{array}{r}1,515 \\
46\end{array}$ & $\begin{array}{r}2,213 \\
46\end{array}$ & $\begin{array}{r}0,248 \\
32\end{array}$ & $\begin{array}{r}0,679 \\
851\end{array}$ & $\begin{array}{r}1,803 \\
64\end{array}$ & $\begin{array}{r}2,199 \\
71\end{array}$ & $\begin{array}{r}1,6783 \\
5\end{array}$ & $\begin{array}{r}1,2314 \\
5\end{array}$ & $\begin{array}{r}0,189 \\
064\end{array}$ & $\begin{array}{r}0,075 \\
4731\end{array}$ & $\begin{array}{r}2,4172 \\
9\end{array}$ & $\begin{array}{r}1,360 \\
68\end{array}$ & $\begin{array}{r}0,666 \\
287\end{array}$ & $\begin{array}{r}0,113 \\
319\end{array}$ & $\begin{array}{r}0,1243 \\
79\end{array}$ \\
\hline
\end{tabular}

Yтó $\mu v \eta \mu \alpha:$

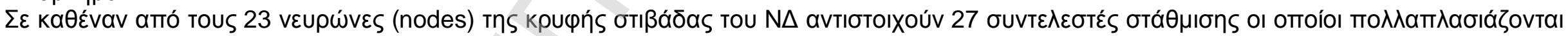

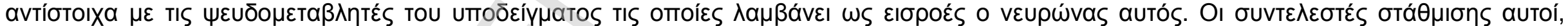

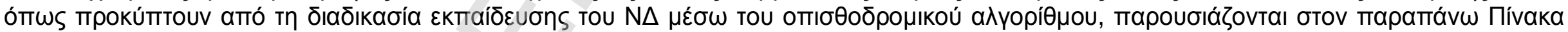

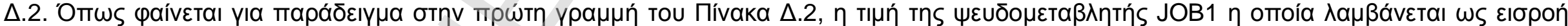

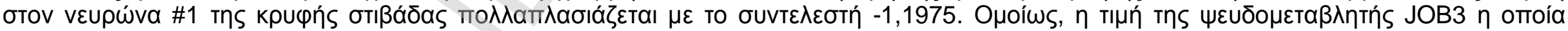

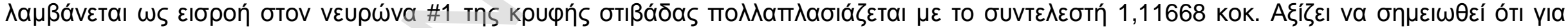

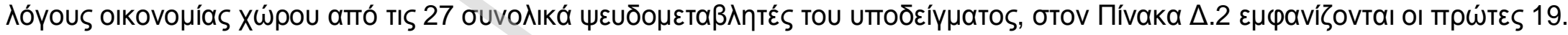




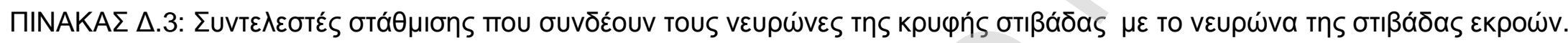

\begin{tabular}{|c|c|c|c|c|c|c|c|c|c|c|c|c|c|c|c|c|c|c|c|c|c|c|c|}
\hline & Hidde & Layer & & & & & & & & & & & & & & & & & & & & & \\
\hline $\begin{array}{l}\text { Out } \\
\text { put } \\
\text { Lay } \\
\text { er }\end{array}$ & $\begin{array}{r}\text { Nod } \\
\text { e \# } 1\end{array}$ & $\begin{array}{r}\text { Node } \\
\text { \# } 2\end{array}$ & $\begin{array}{l}\text { Nod } \\
\text { e \# } 3\end{array}$ & $\begin{array}{r}\text { Nod } \\
\text { e \# } 4\end{array}$ & $\begin{array}{r}\text { Nod } \\
\text { e \# } 5\end{array}$ & $\begin{array}{r}\text { Nod } \\
\text { e\# } 6\end{array}$ & $\begin{array}{r}\text { Nod } \\
\text { e \# } 7\end{array}$ & $\begin{array}{r}\text { Nod } \\
\text { e\# } 8\end{array}$ & $\begin{array}{l}\text { Nod } \\
\text { e \# } 9\end{array}$ & $\begin{array}{r}\text { Nod } \\
\text { e \# } \\
10\end{array}$ & $\begin{array}{r}\text { Nod } \\
\text { e\# } \\
11\end{array}$ & $\begin{array}{r}\text { Nod } \\
\text { e\# } \\
12\end{array}$ & $\begin{array}{r}\text { Nod } \\
\text { e\# } \\
13\end{array}$ & $\begin{array}{r}\text { Nod } \\
\text { e\# } \\
14\end{array}$ & $\begin{array}{r}\text { Node } \\
\# 15\end{array}$ & $\begin{array}{r}\text { Nod } \\
\text { e \# } \\
16\end{array}$ & $\begin{array}{r}\text { Nod } \\
\text { e \# } \\
17\end{array}$ & $\begin{array}{r}\text { Nod } \\
\text { e\# } \\
18\end{array}$ & $\begin{array}{r}\text { Nod } \\
\text { e \# } \\
19\end{array}$ & $\begin{array}{r}\text { Nod } \\
\text { e \# } \\
20\end{array}$ & $\begin{array}{r}\text { Nod } \\
\text { e \# } \\
21\end{array}$ & $\begin{array}{r}\text { Nod } \\
\text { e \# } \\
22\end{array}$ & $\begin{array}{r}\text { Nod } \\
\text { e \# } \\
23\end{array}$ \\
\hline 1 & $\begin{array}{r}2,80 \\
701\end{array}$ & $\begin{array}{r}5,032 \\
82 \\
\end{array}$ & $\begin{array}{r}2,14 \\
093\end{array}$ & $\begin{array}{r}2,97 \\
828\end{array}$ & $\begin{array}{r}2,76 \\
941\end{array}$ & $\begin{array}{r}3,32 \\
681\end{array}$ & $\begin{array}{r}3,70 \\
858\end{array}$ & $\begin{array}{r}3,65 \\
567\end{array}$ & $\begin{array}{r}1,85 \\
998\end{array}$ & $\begin{array}{r}2,54 \\
121\end{array}$ & $\begin{array}{r}3,44 \\
69\end{array}$ & $\begin{array}{r}1,99 \\
52\end{array}$ & $\begin{array}{r}3,54 \\
414\end{array}$ & $\begin{array}{r}1,51 \\
102\end{array}$ & $\begin{array}{r}3,602 \\
21\end{array}$ & $\begin{array}{r}3,59 \\
367\end{array}$ & $\begin{array}{r}2,29 \\
089\end{array}$ & $\begin{array}{r}2,90 \\
134\end{array}$ & $\begin{array}{r}3,06 \\
003\end{array}$ & $\begin{array}{r}2,97 \\
174\end{array}$ & $\begin{array}{r}2,68 \\
645\end{array}$ & $\begin{array}{r}1,43 \\
665\end{array}$ & $\begin{array}{r}2,35 \\
768\end{array}$ \\
\hline
\end{tabular}

Yто́́

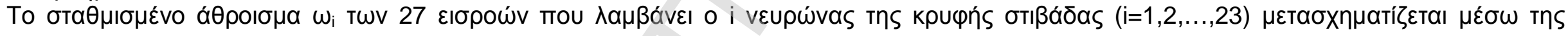

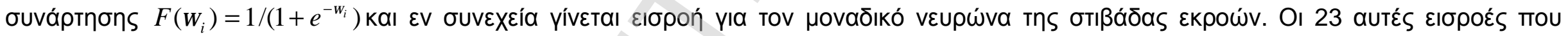

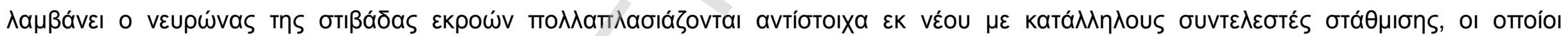

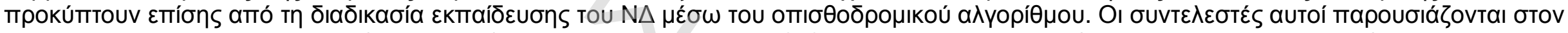

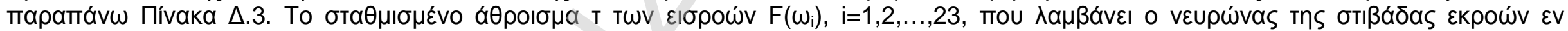

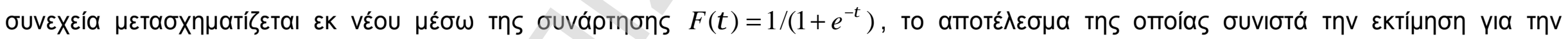
піӨ аvótпта $\mathrm{P}(1 \mid \mathrm{x})$. 


\section{XLMiner : Neural Network - Classification of Test Data}

\begin{tabular}{|c|c|c|c|c|c|c|c|c|c|c|c|c|c|c|c|c|c|c|c|c|c|c|c|c|c|c|c|c|}
\hline \begin{tabular}{|r|}
$R$ \\
0 \\
w \\
Id. \\
\end{tabular} & $\begin{array}{r}\text { Prob. } \\
\text { for } 1 \\
\text { (succe } \\
\text { ss) } \\
\end{array}$ & $\begin{array}{l}\text { JO } \\
\text { B1 }\end{array}$ & $\begin{array}{l}\text { JO } \\
\text { B3 }\end{array}$ & $\begin{array}{l}\text { JO } \\
\text { B4 }\end{array}$ & $\begin{array}{l}\text { JO } \\
\text { B5 }\end{array}$ & $\begin{array}{l}\text { JO } \\
\text { B6 }\end{array}$ & $\begin{array}{r}\text { HO } \\
\text { ME2 }\end{array}$ & $\begin{array}{r}\text { HO } \\
\text { ME3 }\end{array}$ & $\begin{array}{r}\text { MA } \\
\text { R1 }\end{array}$ & $\begin{array}{r}\text { MA } \\
\text { R3 }\end{array}$ & $\begin{array}{l}\mathbf{C} \\
\mathbf{A} \\
\mathbf{R}\end{array}$ & $\begin{array}{l}\mathrm{KI} \\
\mathrm{D} 1\end{array}$ & $\begin{array}{l}\mathrm{KI} \\
\mathrm{D} 2\end{array}$ & $\begin{array}{l}\mathrm{KI} \\
\mathrm{D} 3\end{array}$ & $\begin{array}{l}\text { IN } \\
\text { C1 }\end{array}$ & $\begin{array}{l}\text { IN } \\
\text { C3 }\end{array}$ & $\begin{array}{l}\text { IN } \\
\text { C4 }\end{array}$ & $\begin{array}{l}C \\
R \\
D\end{array}$ & $\begin{array}{l}\mathrm{AG} \\
\mathrm{E} 1\end{array}$ & $\begin{array}{l}\mathrm{AG} \\
\mathrm{E} 2\end{array}$ & $\begin{array}{l}\text { JY } \\
\text { R1 }\end{array}$ & $\begin{array}{l}\text { JY } \\
\text { R2 }\end{array}$ & $\begin{array}{l}\text { JY } \\
\text { R4 }\end{array}$ & $\begin{array}{l}\text { HY } \\
\text { R1 }\end{array}$ & $\begin{array}{l}\text { HY } \\
\text { R2 }\end{array}$ & $\begin{array}{l}\text { HY } \\
\text { R4 }\end{array}$ & $\begin{array}{l}\text { TR } \\
\text { M1 }\end{array}$ & $\begin{array}{l}\text { TR } \\
\text { M2 }\end{array}$ \\
\hline 1 & $\begin{array}{r}0,90127 \\
0224 \\
\end{array}$ & 0 & 0 & 0 & 1 & 0 & 0 & 0 & 0 & 0 & 0 & 1 & 0 & 0 & 1 & 0 & 0 & 0 & 1 & 0 & 1 & 0 & 0 & 1 & 0 & 0 & 1 & 0 \\
\hline 2 & $\begin{array}{r}0,90127 \\
0224 \\
\end{array}$ & 0 & 0 & 0 & 1 & 0 & 0 & 0 & 0 & 0 & 0 & 1 & 0 & 0 & 1 & 0 & 0 & 0 & 1 & 0 & 1 & 0 & 0 & 1 & 0 & 0 & 1 & 0 \\
\hline 3 & $\begin{array}{r}0,95813 \\
4967 \\
\end{array}$ & 0 & 0 & 1 & 0 & 0 & 1 & 0 & 0 & 0 & 0 & 1 & 0 & 0 & 1 & 0 & 0 & 0 & 1 & 0 & 1 & 0 & 0 & 1 & 0 & 0 & 1 & 0 \\
\hline 4 & $\begin{array}{r}0,99999 \\
9993 \\
\end{array}$ & 0 & 0 & 0 & 0 & 0 & 0 & 1 & 0 & 1 & 1 & 1 & 0 & 0 & 1 & 0 & 0 & 0 & 1 & 0 & 1 & 0 & 0 & 1 & 0 & 0 & 1 & 0 \\
\hline 5 & $\begin{array}{r}0,99996 \\
8258 \\
\end{array}$ & 0 & 0 & 1 & 0 & 0 & 1 & 0 & 0 & 1 & 0 & 0 & 0 & 1 & 1 & 0 & 0 & 1 & 1 & 0 & 1 & 0 & 0 & 1 & 0 & 0 & 1 & 0 \\
\hline 6 & $\begin{array}{r}0,99882 \\
2126 \\
\end{array}$ & 0 & 0 & 0 & 1 & 0 & 0 & 0 & 0 & 0 & 0 & 1 & 0 & 0 & 0 & 0 & 0 & 0 & 1 & 0 & 1 & 0 & 0 & 1 & 0 & 0 & 1 & 0 \\
\hline 7 & $\begin{array}{r}0,83387 \\
7994 \\
\end{array}$ & 0 & 0 & 0 & 0 & 0 & 1 & 0 & 0 & 0 & 0 & 1 & 0 & 0 & 0 & 0 & 0 & 0 & 1 & 0 & 1 & 0 & 0 & 1 & 0 & 0 & 1 & 0 \\
\hline 8 & $\begin{array}{r}0,98392 \\
2743 \\
\end{array}$ & 0 & 1 & 0 & 0 & 0 & 1 & 0 & 0 & 0 & 0 & 1 & 0 & 0 & 0 & 0 & 0 & 0 & 1 & 0 & 1 & 0 & 0 & 1 & 0 & 0 & 1 & 0 \\
\hline$\ldots$ & $\ldots$ & $\ldots$ & $\ldots$ & $\ldots$ & $\ldots$ & $\ldots$ & $\ldots$ & $\ldots$ & $\ldots$ & $\ldots$ & $\ldots$ & $\ldots$ & $\ldots$ & $\ldots$ & $\ldots$ & $\ldots$ & $\ldots$ & $\ldots$ & $\ldots$ & $\ldots$ & $\ldots$ & $\ldots$ & $\ldots$ & $\ldots$ & $\ldots$ & $\ldots$ & $\ldots$ & $\ldots$ \\
\hline $\begin{array}{l}10 \\
61 \\
\end{array}$ & $\begin{array}{r}0,99529 \\
8281 \\
\end{array}$ & 0 & 0 & 0 & 1 & 0 & 0 & 1 & 0 & 0 & 1 & 0 & 0 & 1 & 0 & 1 & 0 & 0 & 0 & 0 & 0 & 0 & 1 & 0 & 0 & 0 & 0 & 0 \\
\hline $\begin{array}{l}10 \\
62 \\
\end{array}$ & 1 & 0 & 1 & 0 & 0 & 0 & 0 & 1 & 0 & 1 & 1 & 0 & 1 & 0 & 0 & 1 & 0 & 0 & 0 & 1 & 0 & 1 & 0 & 0 & 0 & 1 & 0 & 0 \\
\hline $\begin{array}{l}10 \\
63 \\
\end{array}$ & 1 & 0 & 1 & 0 & 0 & 0 & 0 & 1 & 0 & 1 & 1 & 0 & 0 & 1 & 0 & 0 & 1 & 1 & 0 & 1 & 0 & 1 & 0 & 0 & 0 & 1 & 0 & 0 \\
\hline $\begin{array}{l}10 \\
64 \\
\end{array}$ & $\begin{array}{r}0,99999 \\
8758 \\
\end{array}$ & 0 & 1 & 0 & 0 & 0 & 0 & 1 & 0 & 1 & 1 & 0 & 0 & 0 & 1 & 0 & 0 & 0 & 0 & 1 & 0 & 0 & 1 & 0 & 0 & 1 & 0 & 0 \\
\hline $\begin{array}{l}10 \\
65 \\
\end{array}$ & $\begin{array}{r}0,98438 \\
8196 \\
\end{array}$ & 0 & 1 & 0 & 0 & 0 & 0 & 1 & 1 & 0 & 1 & 0 & 0 & 1 & 0 & 0 & 0 & 0 & 0 & 1 & 0 & 0 & 1 & 0 & 0 & 1 & 0 & 0 \\
\hline $\begin{array}{l}10 \\
66 \\
\end{array}$ & 1 & 0 & 0 & 0 & 1 & 0 & 0 & 1 & 0 & 1 & 1 & 0 & 1 & 0 & 0 & 1 & 0 & 1 & 0 & 1 & 0 & 0 & 1 & 0 & 0 & 1 & 0 & 0 \\
\hline $\begin{array}{l}10 \\
67 \\
\end{array}$ & 1 & 0 & 0 & 0 & 0 & 0 & 0 & 1 & 0 & 1 & 1 & 0 & 1 & 0 & 0 & 0 & 1 & 1 & 0 & 1 & 0 & 0 & 1 & 0 & 0 & 1 & 0 & 0 \\
\hline $\begin{array}{l}10 \\
68 \\
\end{array}$ & $\begin{array}{r}0,99993 \\
4332 \\
\end{array}$ & 1 & 0 & 0 & 0 & 0 & 0 & & 0 & 1 & 0 & 0 & 0 & 1 & 0 & 1 & 0 & 0 & 0 & 0 & 0 & 0 & 1 & 0 & 0 & 1 & 0 & 0 \\
\hline
\end{tabular}

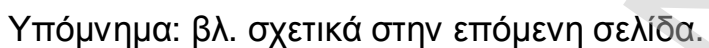




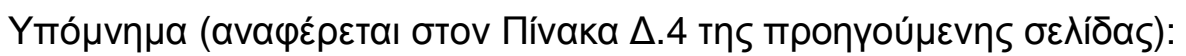

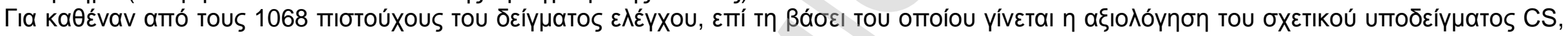

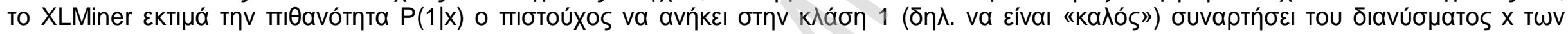

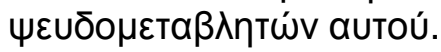

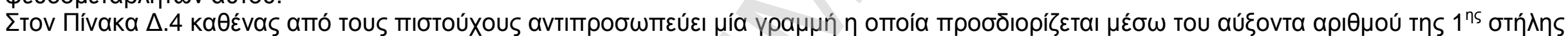

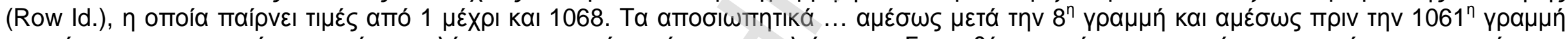

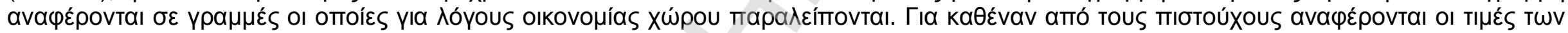

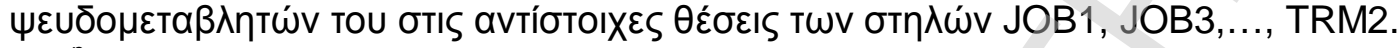

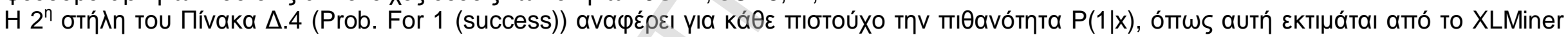

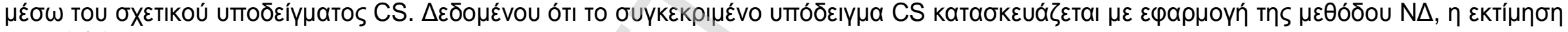

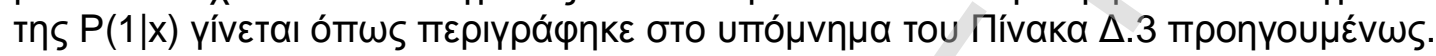


XLMiner : Classification Tree $(\Delta \mathrm{T})$

Inputs

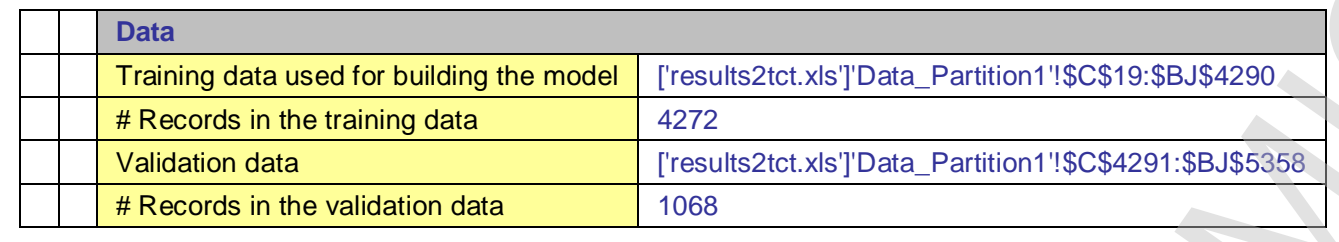

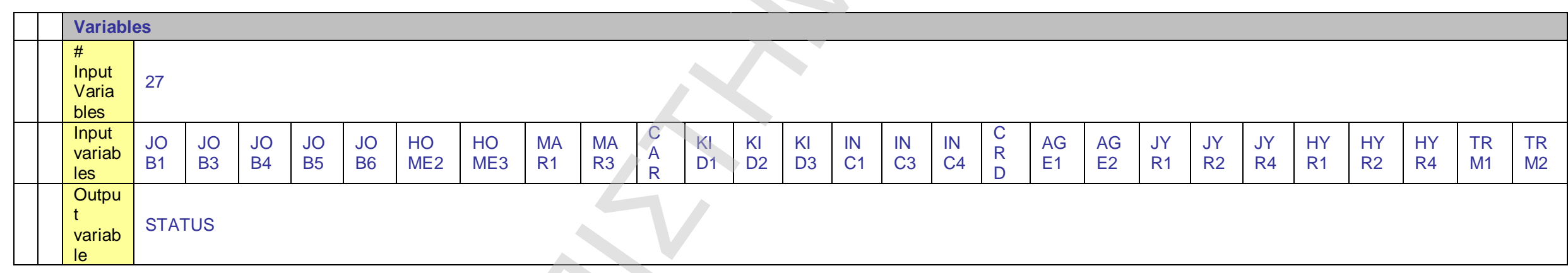

Yто́

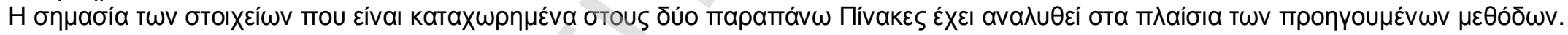




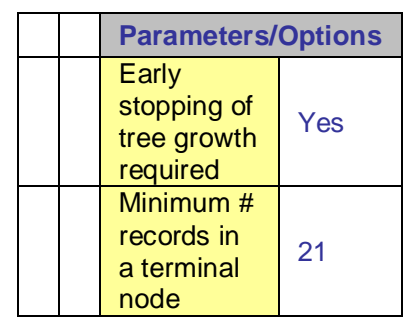

Yтó

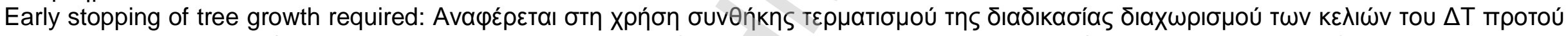

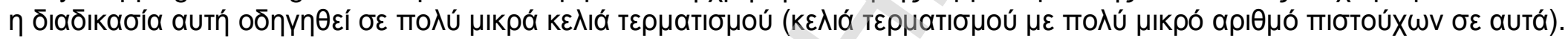

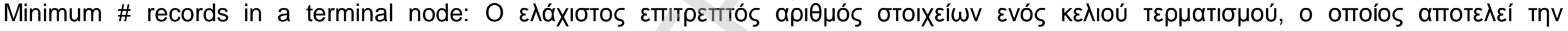

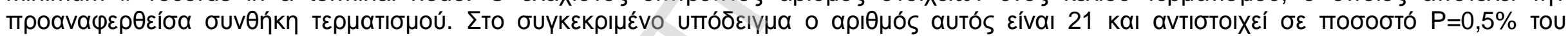

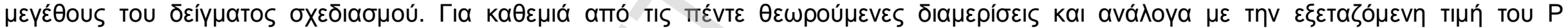

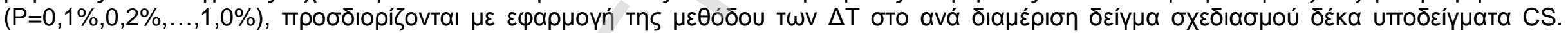

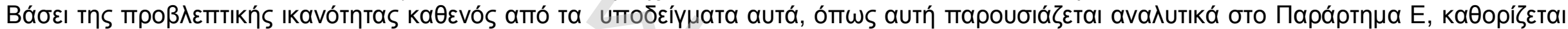

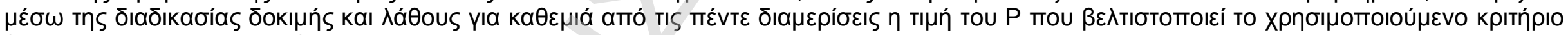

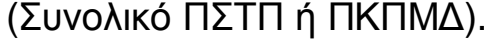


The Classification Tree Model

\section{ПINAKA $\Sigma \Delta .5$}

\begin{tabular}{|c|c|c|c|c|c|c|c|c|c|c|}
\hline Level & NodelD & ParentID & SplitVar & SplitValue & Cases & LeftChild & RightChild & $\begin{array}{l}\text { Node } \\
\text { Type }\end{array}$ & Operator & $\begin{array}{l}\text { Prob. } \\
\text { For } 1\end{array}$ \\
\hline 0 & 0 & $\mathrm{~N} / \mathrm{A}$ & MAR3 & 0,5 & 4272 & 1 & 2 & Decision & $<=$ & 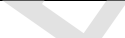 \\
\hline 1 & 1 & 0 & MAR1 & 0,5 & 1470 & 3 & 4 & Decision & $<=$ & 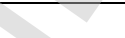 \\
\hline 1 & 2 & 0 & HYR1 & 0,5 & 2802 & 5 & 6 & Decision & $<=$ & $F$ \\
\hline 2 & 3 & 1 & HYR1 & 0,5 & 1151 & 7 & 8 & Decision & $<=$ & \\
\hline 2 & 4 & 1 & JOB1 & 0,5 & 319 & 9 & 10 & Decision & $<=$ & \\
\hline 2 & 5 & 2 & AGE1 & 0,5 & 2247 & 11 & 12 & Decision & $<=$ & \\
\hline 2 & 6 & 2 & INC1 & 0,5 & 555 & 13 & 14 & Decision & $<=$ & \\
\hline 3 & 7 & 3 & CAR & 0,5 & 795 & 15 & 16 & Decision & $<=$ & \\
\hline 3 & 8 & 3 & TRM1 & 0,5 & 356 & 17 & 18 & Decision & $<=$ & \\
\hline 3 & 9 & 4 & AGE1 & 0,5 & 215 & 19 & 20 & Decision & $<=$ & \\
\hline 3 & 10 & 4 & CAR & 0,5 & 104 & 21 & 22 & Decision & $<=$ & \\
\hline 3 & 11 & 5 & JOB5 & 0,5 & 1765 & 23 & 24 & Decision & $<=$ & \\
\hline 3 & 12 & 5 & JYR4 & 0,5 & 482 & 25 & 26 & Decision & $<=$ & \\
\hline 3 & 13 & 6 & JYR2 & 0,5 & 441 & 27 & 28 & Decision & $<=$ & \\
\hline 3 & 14 & 6 & KID3 & 0,5 & 114 & 29 & 30 & Decision & $<=$ & \\
\hline 4 & 15 & 7 & CRD & 0,5 & 279 & 31 & 32 & Decision & $<=$ & \\
\hline 4 & 16 & 7 & TRM2 & 0,5 & 516 & 33 & 34 & Decision & $<=$ & \\
\hline 4 & 17 & 8 & INC1 & 0,5 & 266 & 35 & 36 & Decision & $<=$ & \\
\hline 4 & 18 & 8 & CAR & 0,5 & 90 & 37 & 38 & Decision & $<=$ & \\
\hline 4 & 19 & 9 & KID2 & 0,5 & 143 & 39 & 40 & Decision & $<=$ & \\
\hline 4 & 20 & 9 & KID3 & 0,5 & 72 & 41 & 42 & Decision & $<=$ & \\
\hline 4 & 21 & 10 & INC3 & 0,5 & 64 & 43 & 44 & Decision & $<=$ & \\
\hline 4 & 22 & 10 & $\mathrm{~N} / \mathrm{A}$ & $\mathrm{N} / \mathrm{A}$ & 40 & $\mathrm{~N} / \mathrm{A}$ & $N / A$ & Terminal & & 0,35 \\
\hline 4 & 23 & 11 & $\mathrm{~N} / \mathrm{A}$ & $\mathrm{N} / \mathrm{A}$ & 1162 & $\mathrm{~N} / \mathrm{A}$ & $\mathrm{N} / \mathrm{A}$ & Terminal & & 1 \\
\hline 4 & 24 & 11 & JYR4 & 0,5 & 603 & 45 & 46 & Decision & $<=$ & \\
\hline 4 & 25 & 12 & HOME2 & 0,5 & 381 & 47 & 48 & Decision & $<=$ & \\
\hline 4 & 26 & 12 & INC3 & 0,5 & 101 & 49 & 50 & Decision & $<=$ & \\
\hline 4 & 27 & 13 & JYR4 & 0,5 & 303 & 51 & 52 & Decision & $<=$ & \\
\hline 4 & 28 & 13 & JOB5 & 0,5 & 138 & 53 & 54 & Decision & $<=$ & \\
\hline 4 & 29 & 14 & KID2 & 0,5 & 73 & 55 & 56 & Decision & $<=$ & \\
\hline 4 & 30 & 14 & $N / A$ & $\mathrm{~N} / \mathrm{A}$ & 41 & $\mathrm{~N} / \mathrm{A}$ & $\mathrm{N} / \mathrm{A}$ & Terminal & & 0,585366 \\
\hline
\end{tabular}




\begin{tabular}{|c|c|c|c|c|c|c|c|c|c|c|c|}
\hline & 5 & 31 & 15 & HOME3 & 0,5 & 186 & 57 & 58 & Decision & $<=$ & \\
\hline & 5 & 32 & 15 & N/A & $N / A$ & 93 & $N / A$ & $\mathrm{~N} / \mathrm{A}$ & Terminal & & 1 \\
\hline & 5 & 33 & 16 & JOB5 & 0,5 & 189 & 59 & 60 & Decision & $<=$ & 2 \\
\hline & 5 & 34 & 16 & CRD & 0,5 & 327 & 61 & 62 & Decision & $<=$ & 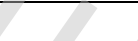 \\
\hline & 5 & 35 & 17 & CRD & 0,5 & 187 & 63 & 64 & Decision & $<=$ & 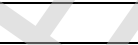 \\
\hline & 5 & 36 & 17 & HOME2 & 0,5 & 79 & 65 & 66 & Decision & $<=$ & 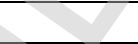 \\
\hline & 5 & 37 & 18 & $\mathrm{~N} / \mathrm{A}$ & $\mathrm{N} / \mathrm{A}$ & 41 & $\mathrm{~N} / \mathrm{A}$ & $\mathrm{N} / \mathrm{A}$ & Terminal & 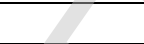 & 0,829268 \\
\hline & 5 & 38 & 18 & $\mathrm{~N} / \mathrm{A}$ & $\mathrm{N} / \mathrm{A}$ & 49 & $N / A$ & $\mathrm{~N} / \mathrm{A}$ & Terminal & 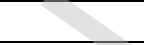 & 1 \\
\hline & 5 & 39 & 19 & KID3 & 0,5 & 98 & 67 & 68 & Decision & $<=$ & \\
\hline & 5 & 40 & 19 & $\mathrm{~N} / \mathrm{A}$ & $N / A$ & 45 & $\mathrm{~N} / \mathrm{A}$ & $\mathrm{N} / \mathrm{A}$ & Terminal & & 1 \\
\hline & 5 & 41 & 20 & $\mathrm{~N} / \mathrm{A}$ & $\mathrm{N} / \mathrm{A}$ & 42 & $\mathrm{~N} / \mathrm{A}$ & $\mathrm{N} / \mathrm{A}$ & Terminal & 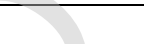 & 0,642857 \\
\hline & 5 & 42 & 20 & $\mathrm{~N} / \mathrm{A}$ & $\mathrm{N} / \mathrm{A}$ & 30 & $\mathrm{~N} / \mathrm{A}$ & $\mathrm{N} / \mathrm{A}$ & Terminal & & 0,366667 \\
\hline & 5 & 43 & 21 & $\mathrm{~N} / \mathrm{A}$ & $\mathrm{N} / \mathrm{A}$ & 38 & $\mathrm{~N} / \mathrm{A}$ & $\mathrm{N} / \mathrm{A}$ & Terminal & $F$ & 0,105263 \\
\hline & 5 & 44 & 21 & $\mathrm{~N} / \mathrm{A}$ & $\mathrm{N} / \mathrm{A}$ & 26 & $\mathrm{~N} / \mathrm{A}$ & $\mathrm{N} / \mathrm{A}$ & Terminal & & 0,115385 \\
\hline & 5 & 45 & 24 & INC3 & 0,5 & 187 & 69 & 70 & Decision & $<=$ & \\
\hline & 5 & 46 & 24 & $\mathrm{~N} / \mathrm{A}$ & $\mathrm{N} / \mathrm{A}$ & 416 & $\mathrm{~N} / \mathrm{A}$ & $\mathrm{N} / \mathrm{A}$ & Terminal & & 1 \\
\hline & 5 & 47 & 25 & HYR2 & 0,5 & 319 & 71 & 72 & Decision & $<=$ & \\
\hline & 5 & 48 & 25 & JOB5 & 0,5 & 62 & 73 & 74 & Decision & $<=$ & \\
\hline & 5 & 49 & 26 & JOB3 & 0,5 & 44 & 75 & 76 & Decision & $<=$ & \\
\hline & 5 & 50 & 26 & JOB5 & 0,5 & 57 & 77 & 78 & Decision & $<=$ & \\
\hline & 5 & 51 & 27 & $\mathrm{~N} / \mathrm{A}$ & $\mathrm{N} / \mathrm{A}$ & 136 & $\mathrm{~N} / \mathrm{A}$ & $\mathrm{N} / \mathrm{A}$ & Terminal & & 1 \\
\hline & 5 & 52 & 27 & AGE1 & 0,5 & 167 & 79 & 80 & Decision & $<=$ & \\
\hline & 5 & 53 & 28 & HOME3 & 0,5 & 93 & 81 & 82 & Decision & $<=$ & \\
\hline & 5 & 54 & 28 & CRD & 0,5 & 45 & 83 & 84 & Decision & $<=$ & \\
\hline & 5 & 55 & 29 & $\mathrm{~N} / \mathrm{A}$ & $\mathrm{N} / \mathrm{A}$ & 44 & $N / A$ & $\mathrm{~N} / \mathrm{A}$ & Terminal & & 0,795455 \\
\hline & 5 & 56 & 29 & $\mathrm{~N} / \mathrm{A}$ & $\mathrm{N} / \mathrm{A}$ & 29 & $\mathrm{~N} / \mathrm{A}$ & $\mathrm{N} / \mathrm{A}$ & Terminal & & 1 \\
\hline & 6 & 57 & 31 & TRM2 & 0,5 & 124 & 85 & 86 & Decision & $<=$ & \\
\hline & 6 & 58 & 31 & JOB5 & 0,5 & 62 & 87 & 88 & Decision & $<=$ & \\
\hline & 6 & 59 & 33 & $\mathrm{~N} / \mathrm{A}$ & $\mathrm{N} / \mathrm{A}$ & 115 & $\mathrm{~N} / \mathrm{A}$ & $\mathrm{N} / \mathrm{A}$ & Terminal & & 1 \\
\hline & 6 & 60 & 33 & JYR2 & 0,5 & 74 & 89 & 90 & Decision & $<=$ & \\
\hline & 6 & 61 & 34 & JOB4 & 0,5 & 197 & 91 & 92 & Decision & $<=$ & \\
\hline & 6 & 62 & 34 & JOB5 & 0,5 & 130 & 93 & 94 & Decision & $<=$ & \\
\hline & 6 & 63 & 35 & CAR & 0,5 & 118 & 95 & 96 & Decision & $<=$ & \\
\hline & 6 & 64 & 35 & JYR2 & 0,5 & 69 & 97 & 98 & Decision & $<=$ & \\
\hline & 6 & 65 & 36 & $\mathrm{~N} / \mathrm{A}$ & $\mathrm{N} / \mathrm{A}$ & 38 & $N / A$ & $\mathrm{~N} / \mathrm{A}$ & Terminal & & 0,421053 \\
\hline & 6 & 66 & 36 & $\mathrm{~N} / \mathrm{A}$ & N/A & 41 & $\mathrm{~N} / \mathrm{A}$ & $\mathrm{N} / \mathrm{A}$ & Terminal & & 0,219512 \\
\hline
\end{tabular}




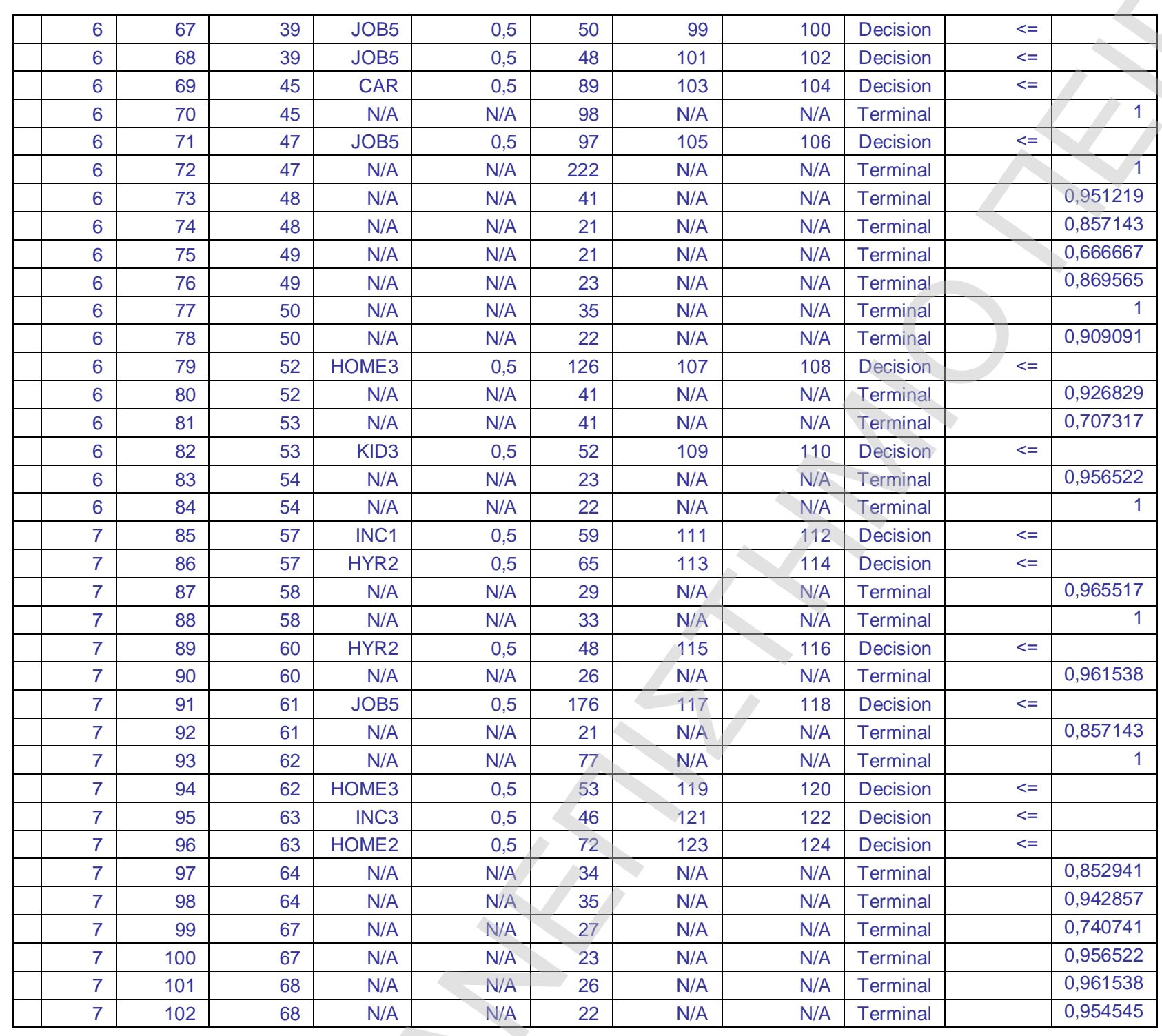




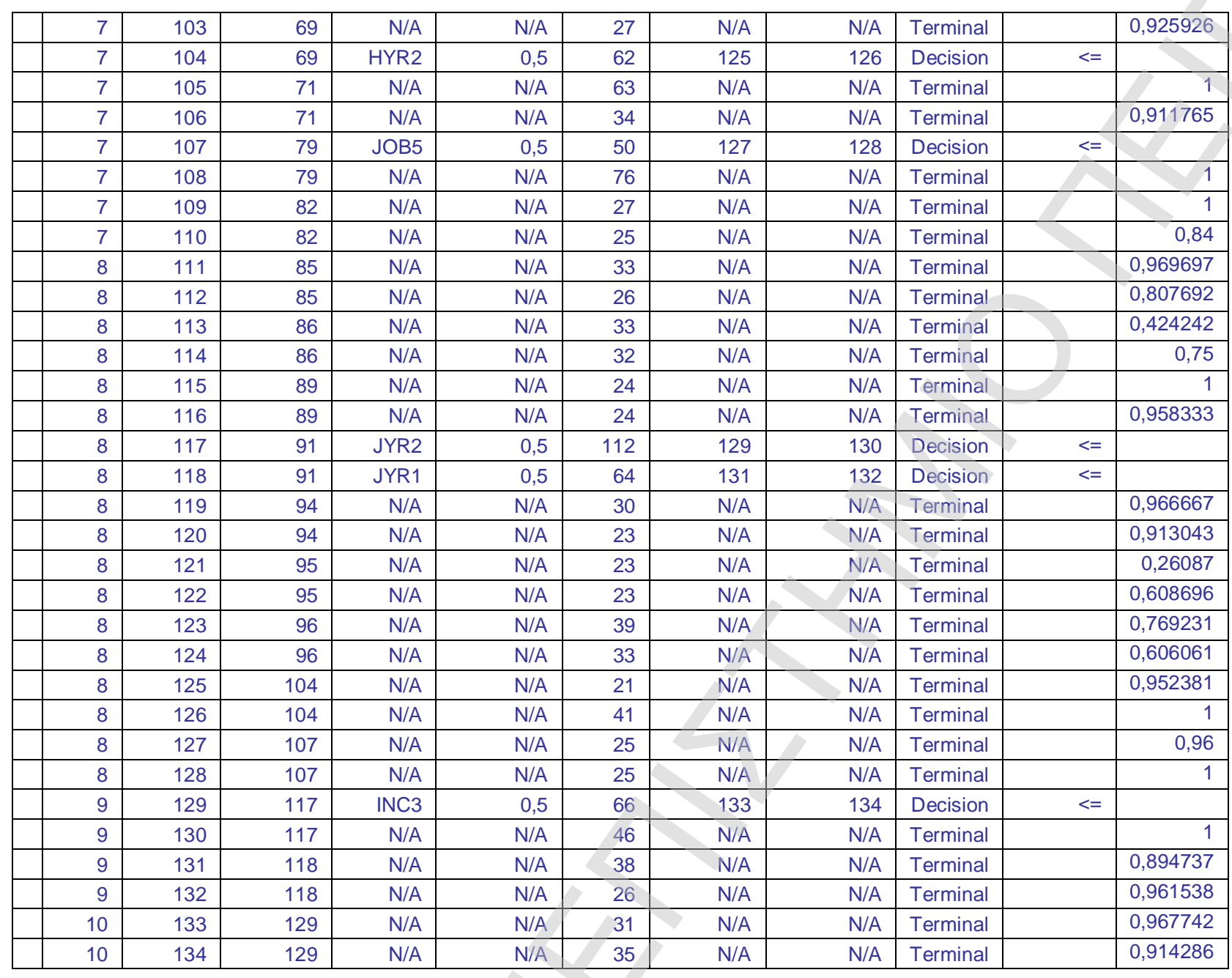

Yтó 


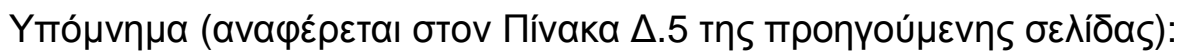

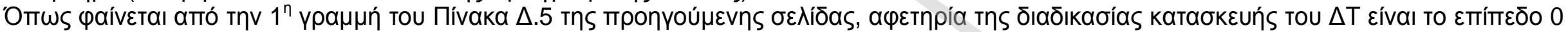

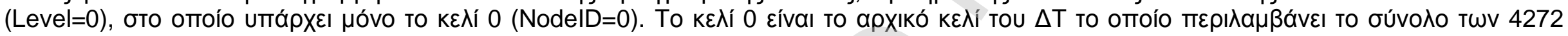

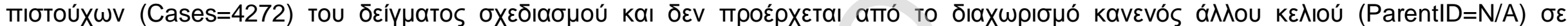

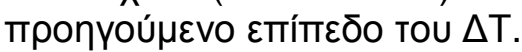

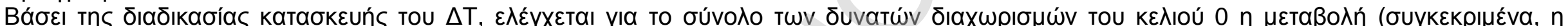

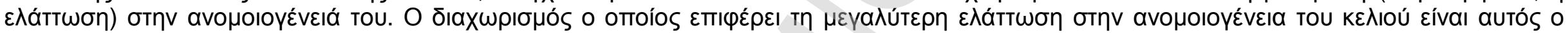

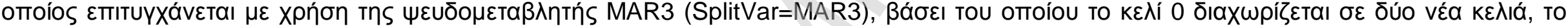

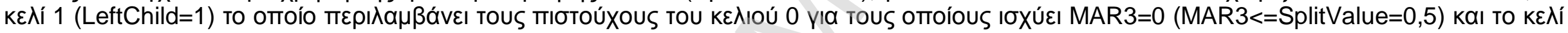

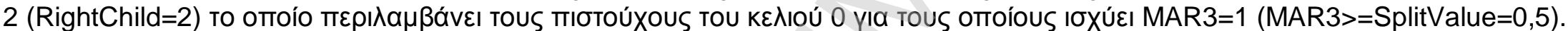

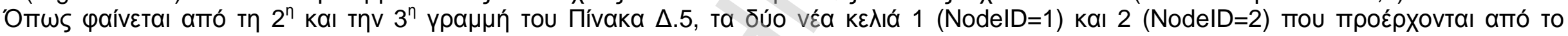

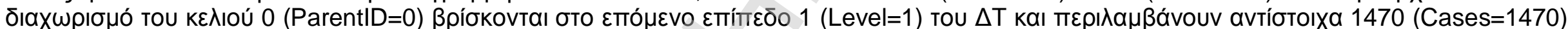

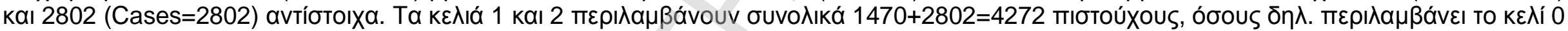

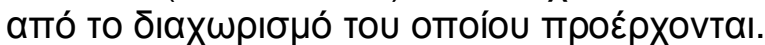

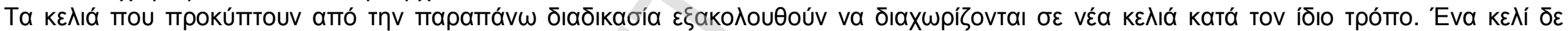

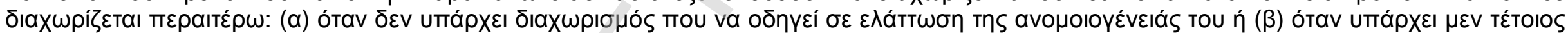

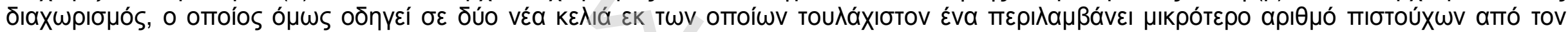

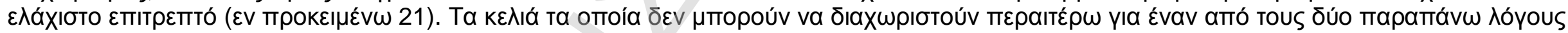

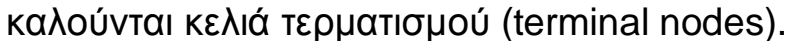

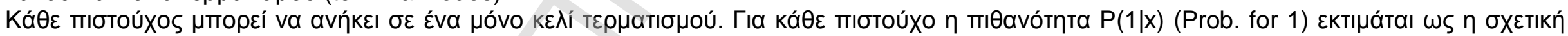

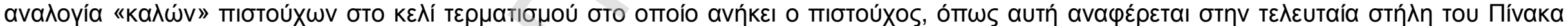
$\Delta .5$. 


\section{XLMiner : Classification Tree - Classification of Test Data}

\begin{tabular}{|c|c|c|c|c|c|c|c|c|c|c|c|c|c|c|c|c|c|c|c|c|c|c|c|c|c|c|c|c|}
\hline $\begin{array}{r}\text { Ro } \\
\text { w } \\
\text { Id. }\end{array}$ & $\begin{array}{r}\text { Prob. } \\
\text { for } 1 \\
\text { (succe } \\
\text { ss) } \\
\end{array}$ & $\begin{array}{l}\text { JO } \\
\text { B1 }\end{array}$ & $\begin{array}{l}\text { JO } \\
\text { B3 }\end{array}$ & $\begin{array}{l}\text { JO } \\
\text { B4 }\end{array}$ & $\begin{array}{l}\text { JO } \\
\text { B5 }\end{array}$ & $\begin{array}{l}\text { JO } \\
\text { B6 }\end{array}$ & $\begin{array}{r}\text { HO } \\
\text { ME2 }\end{array}$ & $\begin{array}{r}\text { HO } \\
\text { ME3 }\end{array}$ & $\begin{array}{r}\text { MA } \\
\text { R1 }\end{array}$ & $\begin{array}{r}\text { MA } \\
\text { R3 }\end{array}$ & $\begin{array}{l}\mathbf{C} \\
\mathbf{A} \\
\mathbf{R}\end{array}$ & $\begin{array}{l}\mathrm{KI} \\
\mathrm{D} 1\end{array}$ & $\begin{array}{l}\mathrm{KI} \\
\mathrm{D} 2\end{array}$ & $\begin{array}{l}\text { KI } \\
\text { D3 }\end{array}$ & $\begin{array}{l}\text { IN } \\
\text { C1 }\end{array}$ & $\begin{array}{l}\text { IN } \\
\text { C3 }\end{array}$ & $\begin{array}{l}\text { IN } \\
\text { C4 }\end{array}$ & $\begin{array}{l}\mathrm{C} \\
\mathrm{R} \\
\mathrm{D}\end{array}$ & $\begin{array}{l}\mathrm{AG} \\
\mathrm{E} 1\end{array}$ & $\begin{array}{l}\text { AG } \\
\text { E2 }\end{array}$ & $\begin{array}{l}\text { JY } \\
\text { R1 }\end{array}$ & $\begin{array}{l}\text { JY } \\
\text { R2 }\end{array}$ & $\begin{array}{l}\text { JY } \\
\text { R4 }\end{array}$ & $\begin{array}{l}\text { HY } \\
\text { R1 }\end{array}$ & $\begin{array}{l}\text { HY } \\
\text { R2 }\end{array}$ & $\begin{array}{l}\text { HY } \\
\text { R4 }\end{array}$ & $\begin{array}{l}\text { TR } \\
\text { M1 }\end{array}$ & $\begin{array}{l}\text { TR } \\
\text { M2 }\end{array}$ \\
\hline 1 & $\begin{array}{r}0,82926 \\
7979 \\
\end{array}$ & 0 & 0 & 0 & 1 & 0 & 0 & 0 & 0 & 0 & 0 & 1 & 0 & 0 & 1 & 0 & 0 & 0 & 1 & 0 & 1 & 0 & 0 & 1 & 0 & 0 & 1 & 0 \\
\hline 2 & $\begin{array}{r}0,82926 \\
7979 \\
\end{array}$ & 0 & 0 & 0 & 1 & 0 & 0 & 0 & 0 & 0 & 0 & 1 & 0 & 0 & 1 & 0 & 0 & 0 & 1 & 0 & 1 & 0 & 0 & 1 & 0 & 0 & 1 & 0 \\
\hline 3 & $\begin{array}{r}0,82926 \\
7979 \\
\end{array}$ & 0 & 0 & 1 & 0 & 0 & 1 & 0 & 0 & 0 & 0 & 1 & 0 & 0 & 1 & 0 & 0 & 0 & 1 & 0 & 1 & 0 & 0 & 1 & 0 & 0 & 1 & 0 \\
\hline 4 & $\begin{array}{r}0,79545 \\
4979 \\
\end{array}$ & 0 & 0 & 0 & 0 & 0 & 0 & 1 & 0 & 1 & 1 & 1 & 0 & 0 & 1 & 0 & 0 & 0 & 1 & 0 & 1 & 0 & 0 & 1 & 0 & 0 & 1 & 0 \\
\hline 5 & $\begin{array}{r}0,58536 \\
6011 \\
\end{array}$ & 0 & 0 & 1 & 0 & 0 & 1 & 0 & 0 & 1 & 0 & 0 & 0 & 1 & 1 & 0 & 0 & 1 & 1 & 0 & 1 & 0 & 0 & 1 & 0 & 0 & 1 & 0 \\
\hline 6 & $\begin{array}{r}0,82926 \\
7979 \\
\end{array}$ & 0 & 0 & 0 & 1 & 0 & 0 & 0 & 0 & 0 & 0 & 1 & 0 & 0 & 0 & 0 & 0 & 0 & 1 & 0 & 1 & 0 & 0 & 1 & 0 & 0 & 1 & 0 \\
\hline 7 & $\begin{array}{r}0,82926 \\
7979 \\
\end{array}$ & 0 & 0 & 0 & 0 & 0 & 1 & 0 & 0 & 0 & 0 & 1 & 0 & 0 & 0 & 0 & 0 & 0 & 1 & 0 & 1 & 0 & 0 & 1 & 0 & 0 & 1 & 0 \\
\hline 8 & $\begin{array}{r}0,82926 \\
7979 \\
\end{array}$ & 0 & 1 & 0 & 0 & 0 & 1 & 0 & 0 & 0 & 0 & 1 & 0 & 0 & 0 & 0 & 0 & 0 & 1 & 0 & 1 & 0 & 0 & 1 & 0 & 0 & 1 & 0 \\
\hline$\ldots$ & $\ldots$ & $\ldots$ & $\ldots$ & $\ldots$ & $\ldots$ & $\ldots$ & $\ldots$ & $\ldots$ & $\ldots$ & $\ldots$ & $\ldots$ & $\ldots$ & $\ldots$ & $\ldots$ & $\ldots$ & $\ldots$ & $\ldots$ & $\ldots$ & $\ldots$ & $\ldots$ & $\ldots$ & $\ldots$ & $\ldots$ & $\ldots$ & $\ldots$ & $\ldots$ & $\ldots$ & $\ldots$ \\
\hline $\begin{array}{l}10 \\
61 \\
\end{array}$ & 1 & 0 & 0 & 0 & 1 & 0 & 0 & 1 & 0 & 0 & 1 & 0 & 0 & 1 & 0 & 1 & 0 & 0 & 0 & 0 & 0 & 0 & 1 & 0 & 0 & 0 & 0 & 0 \\
\hline $\begin{array}{l}10 \\
62 \\
\end{array}$ & 1 & 0 & 1 & 0 & 0 & 0 & 0 & 1 & 0 & 1 & 1 & 0 & 1 & 0 & 0 & 1 & 0 & 0 & 0 & 1 & 0 & 1 & 0 & 0 & 0 & 1 & 0 & 0 \\
\hline $\begin{array}{l}10 \\
63 \\
\end{array}$ & 1 & 0 & 1 & 0 & 0 & 0 & 0 & 1 & 0 & 1 & 1 & 0 & 0 & 1 & 0 & 0 & 1 & 1 & 0 & 1 & 0 & 1 & 0 & 0 & 0 & 1 & 0 & 0 \\
\hline $\begin{array}{l}10 \\
64 \\
\end{array}$ & 1 & 0 & 1 & 0 & 0 & 0 & 0 & 1 & 0 & 1 & 1 & 0 & 0 & 0 & 1 & 0 & 0 & 0 & 0 & 1 & 0 & 0 & 1 & 0 & 0 & 1 & 0 & 0 \\
\hline $\begin{array}{l}10 \\
65 \\
\end{array}$ & $\begin{array}{r}0,96153 \\
8017 \\
\end{array}$ & 0 & 1 & 0 & 0 & 0 & 0 & 1 & 1 & 0 & 1 & 0 & 0 & 1 & 0 & 0 & 0 & 0 & 0 & 1 & 0 & 0 & 1 & 0 & 0 & 1 & 0 & 0 \\
\hline $\begin{array}{l}10 \\
66 \\
\end{array}$ & 1 & 0 & 0 & 0 & 1 & 0 & 0 & 1 & 0 & 1 & 1 & 0 & 1 & 0 & 0 & 1 & 0 & 1 & 0 & 1 & 0 & 0 & 1 & 0 & 0 & 1 & 0 & 0 \\
\hline $\begin{array}{l}10 \\
67 \\
\end{array}$ & 1 & 0 & 0 & 0 & 0 & 0 & 0 & 1 & 0 & 1 & 1 & 0 & 1 & 0 & 0 & 0 & 1 & 1 & 0 & 1 & 0 & 0 & 1 & 0 & 0 & 1 & 0 & 0 \\
\hline $\begin{array}{l}10 \\
68 \\
\end{array}$ & 1 & 1 & 0 & 0 & 0 & 0 & 0 & 1 & 0 & 1 & 0 & 0 & 0 & 1 & 0 & 1 & 0 & 0 & 0 & 0 & 0 & 0 & 1 & 0 & 0 & 1 & 0 & 0 \\
\hline
\end{tabular}

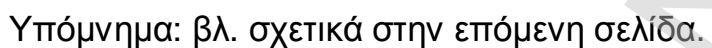




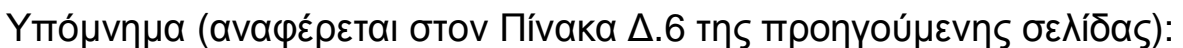

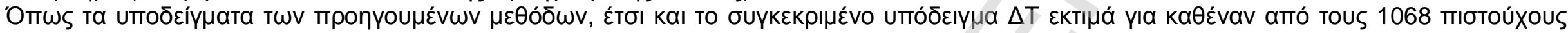

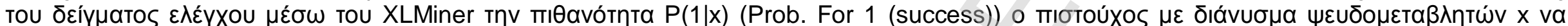

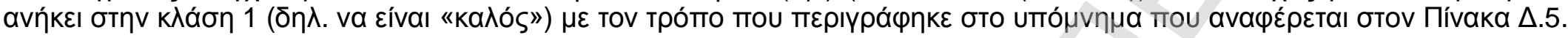

\section{XLMiner : Logistic Regression (^П)}

\section{Inputs}

\begin{tabular}{|c|c|}
\hline \multicolumn{2}{|c|}{ Data } \\
\hline Training data used for building the model & 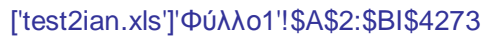 \\
\hline \# Records in the training data & 4272 \\
\hline New data & 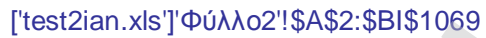 \\
\hline \# Records in the new data & 1068 \\
\hline
\end{tabular}

\begin{tabular}{|c|c|c|c|c|c|c|c|c|c|c|c|c|c|c|c|c|c|c|c|c|c|c|c|c|c|c|c|}
\hline \multicolumn{28}{|c|}{ Variables } \\
\hline $\begin{array}{l}\# \\
\text { Input } \\
\text { Varia } \\
\text { bles }\end{array}$ & \multicolumn{27}{|l|}{27} \\
\hline $\begin{array}{l}\text { Input } \\
\text { variab } \\
\text { les }\end{array}$ & $\begin{array}{l}\mathrm{JO} \\
\mathrm{B} 1\end{array}$ & $\begin{array}{l}\mathrm{JO} \\
\mathrm{B} 3\end{array}$ & $\begin{array}{l}\mathrm{JO} \\
\mathrm{B} 4\end{array}$ & $\begin{array}{l}\mathrm{JO} \\
\mathrm{B} 5\end{array}$ & $\begin{array}{l}\mathrm{JO} \\
\mathrm{B} 6\end{array}$ & $\begin{array}{l}\mathrm{HO} \\
\mathrm{ME} 2\end{array}$ & $\begin{array}{l}\mathrm{HO} \\
\text { ME3 }\end{array}$ & $\begin{array}{l}\text { MA } \\
\text { R1 }\end{array}$ & $\begin{array}{l}\text { MA } \\
\text { R3 }\end{array}$ & $\begin{array}{l}C \\
A \\
R\end{array}$ & $\begin{array}{l}\mathrm{KI} \\
\mathrm{D} 1\end{array}$ & $\begin{array}{l}\mathrm{KI} \\
\mathrm{D} 2\end{array}$ & $\begin{array}{l}\mathrm{KI} \\
\mathrm{D} 3\end{array}$ & $\begin{array}{l}\text { IN } \\
\text { C1 }\end{array}$ & $\begin{array}{l}\text { IN } \\
\text { C3 }\end{array}$ & $\begin{array}{l}\text { IN } \\
\text { C4 }\end{array}$ & $\begin{array}{l}C \\
R \\
D\end{array}$ & $\begin{array}{l}\text { AG } \\
\text { E1 }\end{array}$ & $\begin{array}{l}\text { AG } \\
\text { E2 }\end{array}$ & $\begin{array}{l}\text { JY } \\
\text { R1 }\end{array}$ & $\begin{array}{l}\text { JY } \\
\text { R2 }\end{array}$ & $\begin{array}{l}\text { JY } \\
\text { R4 }\end{array}$ & $\begin{array}{l}\mathrm{HY} \\
\mathrm{R} 1\end{array}$ & $\begin{array}{l}\text { HY } \\
\text { R2 }\end{array}$ & $\begin{array}{l}\text { HY } \\
\text { R4 }\end{array}$ & $\begin{array}{l}\text { TR } \\
\text { M1 }\end{array}$ & $\begin{array}{l}\text { TR } \\
\text { M2 }\end{array}$ \\
\hline $\begin{array}{l}\text { Outpu } \\
\mathrm{t} \\
\text { variab } \\
\text { le }\end{array}$ & \multicolumn{27}{|c|}{ STATUS } \\
\hline
\end{tabular}

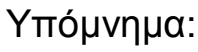

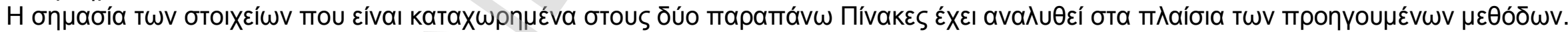


The Logistic Regression Model

ПINAKA $\Delta .7$
\begin{tabular}{|l|l|r|}
\hline $\begin{array}{l}\text { Input } \\
\text { Variables }\end{array}$ & Coefficient \\
\hline & JOB1 & $-2,4376359$ \\
\hline & JOB3 & $-0,4065317$ \\
\hline & JOB4 & 0,66156745 \\
\hline & JOB5 & $-0,0096025$ \\
\hline & JOB6 & 0,12935023 \\
\hline & HOME2 & $-0,4808135$ \\
\hline & HOME3 & 0,35022455 \\
\hline & MAR1 & $-1,7673591$ \\
\hline & MAR3 & 2,29769301 \\
\hline & CAR & 0,90539736 \\
\hline & KID1 & 1,57383215 \\
\hline & KID2 & 2,23818636 \\
\hline & KID3 & 0,612418 \\
\hline & INC1 & $-0,8399456$ \\
\hline & INC3 & 0,93871403 \\
\hline & INC4 & 2,41247201 \\
\hline & CRD & 1,240695 \\
\hline & AGE1 & $-1,1899782$ \\
\hline AGE2 & 0,50103116 \\
\hline JYR1 & 0,25477916 \\
\hline JYR2 & 0,11969619 \\
\hline JYR4 & $-0,1992547$ \\
\hline HYR1 & $-1,5043786$ \\
\hline HYR2 & 0,06509197 \\
\hline HYR4 & 1,76217389 \\
\hline & TRM1 & 2,74322462 \\
\hline TRM2 & 0,66159451 \\
\hline
\end{tabular}

Yтó $\mu v \eta \mu \alpha:$

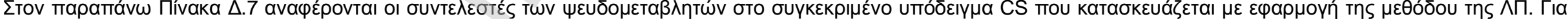

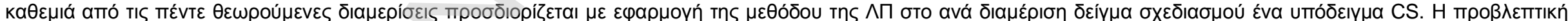

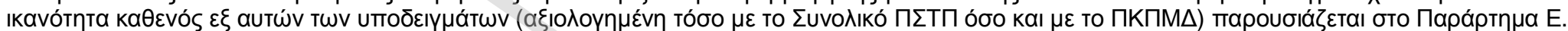




\section{XLMiner: Logistic Regression - Classification of Test Data}

ПINAKA $\Sigma$ $\Delta .8$

\begin{tabular}{|c|c|c|c|c|c|c|c|c|c|c|c|c|c|c|c|c|c|c|c|c|c|c|c|c|c|c|c|c|}
\hline $\begin{array}{l}\text { Ro } \\
\text { w } \\
\text { Id. }\end{array}$ & $\begin{array}{r}\text { Prob. } \\
\text { for } 1 \\
\text { (succe } \\
\text { ss) } \\
\end{array}$ & $\begin{array}{l}\text { JO } \\
\text { B1 }\end{array}$ & $\begin{array}{l}\text { JO } \\
\text { B3 }\end{array}$ & $\begin{array}{l}\text { JO } \\
\text { B4 }\end{array}$ & $\begin{array}{l}\text { JO } \\
\text { B5 }\end{array}$ & $\begin{array}{l}\text { JO } \\
\text { B6 }\end{array}$ & $\begin{array}{r}\text { HO } \\
\text { ME2 }\end{array}$ & $\begin{array}{r}\text { HO } \\
\text { ME3 }\end{array}$ & $\begin{array}{l}\text { MA } \\
\text { R1 }\end{array}$ & $\begin{array}{l}\text { MA } \\
\text { R3 }\end{array}$ & $\begin{array}{l}\mathrm{C} \\
\mathrm{A} \\
\mathrm{R}\end{array}$ & $\begin{array}{l}\mathrm{KI} \\
\mathrm{D} 1\end{array}$ & $\begin{array}{r}\mathrm{KI} \\
\mathrm{D} 2\end{array}$ & $\begin{array}{l}\mathrm{KI} \\
\mathrm{D} 3\end{array}$ & $\begin{array}{l}\text { IN } \\
\text { C1 }\end{array}$ & $\begin{array}{l}\text { IN } \\
\text { C3 }\end{array}$ & $\begin{array}{l}\text { IN } \\
\text { C4 }\end{array}$ & $\begin{array}{l}\text { C } \\
R \\
D\end{array}$ & $\begin{array}{l}\mathrm{AG} \\
\mathrm{E} 1\end{array}$ & $\begin{array}{l}\text { AG } \\
\text { E2 }\end{array}$ & $\begin{array}{l}\text { JY } \\
\text { R1 }\end{array}$ & $\begin{array}{l}\text { JY } \\
\text { R2 }\end{array}$ & $\begin{array}{l}\text { JY } \\
\text { R4 }\end{array}$ & $\begin{array}{l}\text { HY } \\
\text { R1 }\end{array}$ & $\begin{array}{l}\text { HY } \\
\text { R2 }\end{array}$ & $\begin{array}{l}\text { HY } \\
\text { R4 }\end{array}$ & $\begin{array}{l}\text { TR } \\
\text { M1 }\end{array}$ & $\begin{array}{l}\text { TR } \\
\text { M2 }\end{array}$ \\
\hline 1 & $\begin{array}{r}0,73651 \\
4586 \\
\end{array}$ & 0 & 0 & 0 & 1 & 0 & 0 & 0 & 0 & 0 & 0 & 1 & 0 & 0 & 1 & 0 & 0 & 0 & 1 & 0 & 1 & 0 & 0 & 1 & 0 & 0 & 1 & 0 \\
\hline 2 & $\begin{array}{r}0,73651 \\
4586 \\
\end{array}$ & 0 & 0 & 0 & 1 & 0 & 0 & 0 & 0 & 0 & 0 & 1 & 0 & 0 & 1 & 0 & 0 & 0 & 1 & 0 & 1 & 0 & 0 & 1 & 0 & 0 & 1 & 0 \\
\hline 3 & $\begin{array}{r}0,77176 \\
2036 \\
\end{array}$ & 0 & 0 & 1 & 0 & 0 & 1 & 0 & 0 & 0 & 0 & 1 & 0 & 0 & 1 & 0 & 0 & 0 & 1 & 0 & 1 & 0 & 0 & 1 & 0 & 0 & 1 & 0 \\
\hline 4 & $\begin{array}{r}0,98995 \\
7624 \\
\end{array}$ & 0 & 0 & 0 & 0 & 0 & 0 & 1 & 0 & 1 & 1 & 1 & 0 & 0 & 1 & 0 & 0 & 0 & 1 & 0 & 1 & 0 & 0 & 1 & 0 & 0 & 1 & 0 \\
\hline 5 & $\begin{array}{r}0,97801 \\
708 \\
\end{array}$ & 0 & 0 & 1 & 0 & 0 & 1 & 0 & 0 & 1 & 0 & 0 & 0 & 1 & 1 & 0 & 0 & 1 & 1 & 0 & 1 & 0 & 0 & 1 & 0 & 0 & 1 & 0 \\
\hline 6 & $\begin{array}{r}0,86621 \\
2396 \\
\end{array}$ & 0 & 0 & 0 & 1 & 0 & 0 & 0 & 0 & 0 & 0 & 1 & 0 & 0 & 0 & 0 & 0 & 0 & 1 & 0 & 1 & 0 & 0 & 1 & 0 & 0 & 1 & 0 \\
\hline 7 & $\begin{array}{r}0,80165 \\
4241 \\
\end{array}$ & 0 & 0 & 0 & 0 & 0 & 1 & 0 & 0 & 0 & 0 & 1 & 0 & 0 & 0 & 0 & 0 & 0 & 1 & 0 & 1 & 0 & 0 & 1 & 0 & 0 & 1 & 0 \\
\hline 8 & $\begin{array}{r}0,72911 \\
4383 \\
\end{array}$ & 0 & 1 & 0 & 0 & 0 & 1 & 0 & 0 & 0 & 0 & 1 & 0 & 0 & 0 & 0 & 0 & 0 & 1 & 0 & 1 & 0 & 0 & 1 & 0 & 0 & 1 & 0 \\
\hline$\ldots$ & & $\cdots$ & $\ldots$ & & $\ldots$ & $\ldots$ & & & $\ldots$ & $\ldots$ & $\ldots$ & $\ldots$ & & $\ldots$ & $\ldots$ & $\ldots$ & & $\ldots$ & $\ldots$ & $\ldots$ & $\ldots$ & $\ldots$ & $\ldots$ & $\ldots$ & $\ldots$ & $\ldots$ & $\ldots$ & $\ldots$ \\
\hline $\begin{array}{l}10 \\
61 \\
\end{array}$ & $\begin{array}{r}0,93072 \\
6098 \\
\end{array}$ & 0 & 0 & 0 & 1 & 0 & 0 & 1 & 0 & 0 & 1 & 0 & 0 & 1 & 0 & 1 & 0 & 0 & 0 & 0 & 0 & 0 & 1 & 0 & 0 & 0 & 0 & 0 \\
\hline $\begin{array}{l}10 \\
62 \\
\end{array}$ & $\begin{array}{r}0,99983 \\
4535 \\
\end{array}$ & 0 & 1 & 0 & 0 & 0 & 0 & 1 & 0 & 1 & 1 & 0 & 1 & 0 & 0 & 1 & 0 & 0 & 0 & 1 & 0 & 1 & 0 & 0 & 0 & 1 & 0 & 0 \\
\hline $\begin{array}{l}10 \\
63 \\
\end{array}$ & $\begin{array}{r}0,99994 \\
4289 \\
\end{array}$ & 0 & 1 & 0 & 0 & 0 & 0 & 1 & 0 & 1 & 1 & 0 & 0 & 1 & 0 & 0 & 1 & 1 & 0 & 1 & 0 & 1 & 0 & 0 & 0 & 1 & 0 & 0 \\
\hline $\begin{array}{l}10 \\
64 \\
\end{array}$ & $\begin{array}{r}0,98751 \\
6532 \\
\end{array}$ & 0 & 1 & 0 & 0 & 0 & 0 & 1 & 0 & 1 & 1 & 0 & 0 & 0 & 1 & 0 & 0 & 0 & 0 & 1 & 0 & 0 & 1 & 0 & 0 & 1 & 0 & 0 \\
\hline $\begin{array}{l}10 \\
65 \\
\end{array}$ & $\begin{array}{r}0,85297 \\
1476 \\
\end{array}$ & 0 & 1 & 0 & 0 & 0 & 0 & 1 & 1 & 0 & 1 & 0 & 0 & 1 & 0 & 0 & 0 & 0 & 0 & 1 & 0 & 0 & 1 & 0 & 0 & 1 & 0 & 0 \\
\hline $\begin{array}{l}10 \\
66 \\
\end{array}$ & $\begin{array}{r}0,99995 \\
5734 \\
\end{array}$ & 0 & 0 & 0 & 1 & 0 & 0 & 1 & 0 & 1 & 1 & 0 & 1 & 0 & 0 & 1 & 0 & 1 & 0 & 1 & 0 & 0 & 1 & 0 & 0 & 1 & 0 & 0 \\
\hline $\begin{array}{l}10 \\
67 \\
\end{array}$ & $\begin{array}{r}0,99998 \\
9957 \\
\end{array}$ & 0 & 0 & 0 & 0 & 0 & 0 & 1 & 0 & 1 & 1 & 0 & 1 & 0 & 0 & 0 & 1 & 1 & 0 & 1 & 0 & 0 & 1 & 0 & 0 & 1 & 0 & 0 \\
\hline $\begin{array}{l}10 \\
68 \\
\end{array}$ & $\begin{array}{r}0,96525 \\
4205 \\
\end{array}$ & 1 & 0 & 0 & 0 & 0 & 0 & & 0 & 1 & 0 & 0 & 0 & 1 & 0 & 1 & 0 & 0 & 0 & 0 & 0 & 0 & 1 & 0 & 0 & 1 & 0 & 0 \\
\hline
\end{tabular}

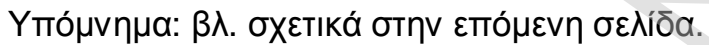




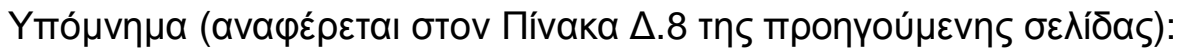

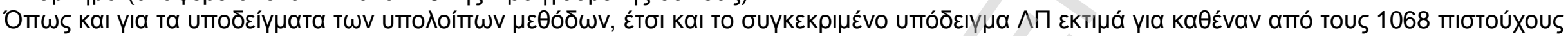

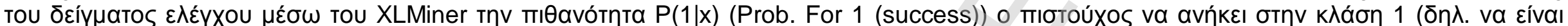

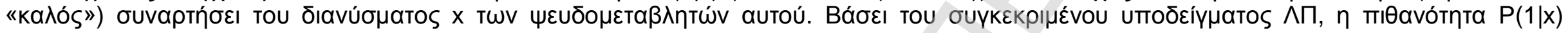

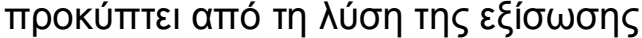

$$
\ln \frac{P(1 \mid x)}{1-P(1 \mid x)}=\sum \omega_{k} x_{k}
$$

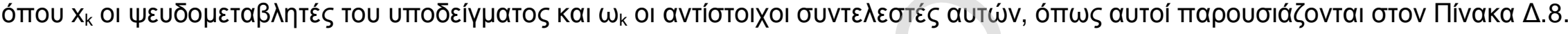

\section{XLMiner : Linear Discriminant Analysis ( $\Gamma \Delta \mathrm{A})$}

Inputs

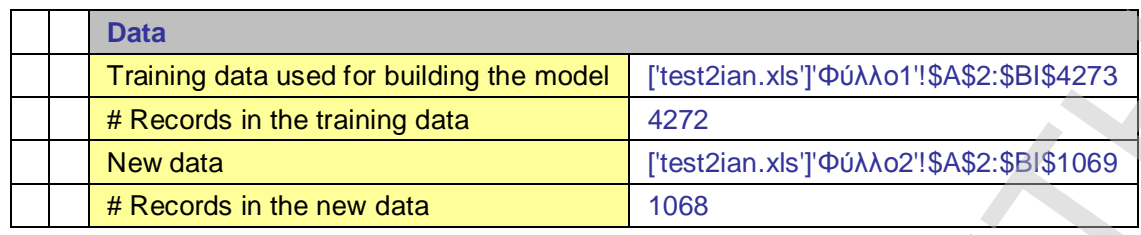

\begin{tabular}{|c|c|c|c|c|c|c|c|c|c|c|c|c|c|c|c|c|c|c|c|c|c|c|c|c|c|c|c|}
\hline \multicolumn{28}{|c|}{ Variables } \\
\hline $\begin{array}{l}\# \\
\text { Input } \\
\text { Inaria } \\
\text { bles } \\
\end{array}$ & 27 & & & & & & & & & & 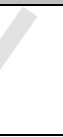 & & & & & & & & & & & & & & & & \\
\hline $\begin{array}{l}\text { Input } \\
\text { variab } \\
\text { les }\end{array}$ & $\begin{array}{l}\text { JO } \\
\text { B1 }\end{array}$ & $\begin{array}{l}\text { JO } \\
\text { B3 }\end{array}$ & \begin{tabular}{|l}
$\mathrm{JO}$ \\
$\mathrm{B} 4$
\end{tabular} & $\begin{array}{l}\text { JO } \\
\text { B5 }\end{array}$ & $\begin{array}{l}\mathrm{JO} \\
\mathrm{B} 6\end{array}$ & $\begin{array}{l}\mathrm{HO} \\
\mathrm{ME} 2\end{array}$ & $\begin{array}{l}\mathrm{HO} \\
\mathrm{ME} 3\end{array}$ & $\begin{array}{l}\text { MA } \\
\text { R1 }\end{array}$ & $\begin{array}{l}\text { MA } \\
\text { R3 }\end{array}$ & $\begin{array}{l}\mathrm{C} \\
\mathrm{A} \\
\mathrm{R}\end{array}$ & $\begin{array}{l}\mathrm{KI} \\
\mathrm{D} 1\end{array}$ & \begin{tabular}{|l} 
KI \\
D2
\end{tabular} & $\begin{array}{l}\mathrm{Kl} \\
\mathrm{D} 3\end{array}$ & $\begin{array}{l}\text { IN } \\
\text { C1 }\end{array}$ & $\begin{array}{l}\text { IN } \\
\text { C3 }\end{array}$ & $\begin{array}{l}\text { IN } \\
\text { C4 }\end{array}$ & $\begin{array}{l}C \mathrm{C} \\
\mathrm{R} \\
\mathrm{D}\end{array}$ & $\begin{array}{l}\text { AG } \\
\mathrm{E} 1\end{array}$ & $\begin{array}{l}\text { AG } \\
\text { E2 }\end{array}$ & $\begin{array}{l}\text { JY } \\
\text { R1 }\end{array}$ & $\begin{array}{l}\text { JY } \\
\text { R2 }\end{array}$ & $\begin{array}{l}\text { JY } \\
\text { R4 }\end{array}$ & $\begin{array}{l}\text { HY } \\
\text { R1 }\end{array}$ & $\begin{array}{l}\text { HY } \\
\text { R2 }\end{array}$ & $\begin{array}{l}\text { HY } \\
\text { R4 }\end{array}$ & \begin{tabular}{|l} 
TR \\
M1
\end{tabular} & \begin{tabular}{|l} 
TR \\
M2
\end{tabular} \\
\hline $\begin{array}{l}\text { Outpu } \\
\text { t } \\
\text { variab } \\
\text { le }\end{array}$ & STA & & & & & & & & & & & & & & & & & & & & & & & & & & \\
\hline
\end{tabular}

Yтópvnua:

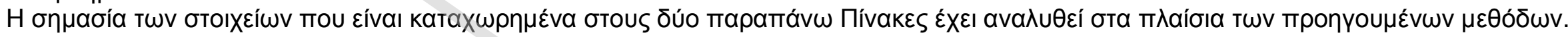


XLMiner : Linear Discriminant Analysis - Classification of Test Data

ПINAKA $\Sigma \Delta .9$

\begin{tabular}{|c|c|c|c|c|c|c|c|c|c|c|c|c|c|c|c|c|c|c|c|c|c|c|c|c|c|c|c|c|}
\hline $\begin{array}{r}\text { Ro } \\
\text { w } \\
\text { Id. }\end{array}$ & $\begin{array}{r}\text { Prob. } \\
\text { for } 1 \\
\text { (succe } \\
\text { ss) } \\
\end{array}$ & $\begin{array}{l}\text { JO } \\
\text { B1 }\end{array}$ & $\begin{array}{l}\text { JO } \\
\text { B3 }\end{array}$ & $\begin{array}{l}\text { JO } \\
\text { B4 }\end{array}$ & $\begin{array}{l}\text { JO } \\
\text { B5 }\end{array}$ & $\begin{array}{l}\text { JO } \\
\text { B6 }\end{array}$ & $\begin{array}{r}\text { HO } \\
\text { ME2 }\end{array}$ & $\begin{array}{r}\text { HO } \\
\text { ME3 }\end{array}$ & $\begin{array}{r}\text { MA } \\
\text { R1 }\end{array}$ & $\begin{array}{r}\text { MA } \\
\text { R3 }\end{array}$ & $\begin{array}{l}\mathbf{C} \\
\mathbf{A} \\
\mathrm{R}\end{array}$ & $\begin{array}{l}\mathrm{KI} \\
\mathrm{D} 1\end{array}$ & $\begin{array}{l}\mathrm{KI} \\
\mathrm{D} 2\end{array}$ & $\begin{array}{l}\text { KI } \\
\text { D3 }\end{array}$ & $\begin{array}{l}\text { IN } \\
\text { C1 }\end{array}$ & $\begin{array}{l}\text { IN } \\
\text { C3 }\end{array}$ & $\begin{array}{l}\text { IN } \\
\text { C4 }\end{array}$ & $\begin{array}{l}C \\
R \\
D\end{array}$ & $\begin{array}{l}\mathrm{AG} \\
\mathrm{E} 1\end{array}$ & $\begin{array}{c}\text { AG } \\
\text { E2 }\end{array}$ & $\begin{array}{l}\text { JY } \\
\text { R1 }\end{array}$ & $\begin{array}{l}\text { JY } \\
\text { R2 }\end{array}$ & $\begin{array}{l}\text { JY } \\
\text { R4 }\end{array}$ & $\begin{array}{l}\text { HY } \\
\text { R1 }\end{array}$ & $\begin{array}{l}\text { HY } \\
\text { R2 }\end{array}$ & $\begin{array}{l}\text { HY } \\
\text { R4 }\end{array}$ & $\begin{array}{l}\text { TR } \\
\text { M1 }\end{array}$ & $\begin{array}{l}\text { TR } \\
\text { M2 }\end{array}$ \\
\hline 1 & $\begin{array}{r}0,75497 \\
4488\end{array}$ & 0 & 0 & 0 & 1 & 0 & 0 & 0 & 0 & 0 & 0 & 1 & 0 & 0 & 1 & 0 & 0 & 0 & 1 & 0 & 1 & 0 & 0 & 1 & 0 & 0 & 1 & 0 \\
\hline 2 & $\begin{array}{r}0,75497 \\
4488 \\
\end{array}$ & 0 & 0 & 0 & 1 & 0 & 0 & 0 & 0 & 0 & 0 & 1 & 0 & 0 & 1 & 0 & 0 & 0 & 1 & 0 & 1 & 0 & 0 & 1 & 0 & 0 & 1 & 0 \\
\hline 3 & $\begin{array}{r}0,70271 \\
373\end{array}$ & 0 & 0 & 1 & 0 & 0 & 1 & 0 & 0 & 0 & 0 & 1 & 0 & 0 & 1 & 0 & 0 & 0 & 1 & 0 & 1 & 0 & 0 & 1 & 0 & 0 & 1 & 0 \\
\hline 4 & $\begin{array}{r}0,97857 \\
9927 \\
\end{array}$ & 0 & 0 & 0 & 0 & 0 & 0 & 1 & 0 & 1 & 1 & 1 & 0 & 0 & 1 & 0 & 0 & 0 & 1 & 0 & 1 & 0 & 0 & 1 & 0 & 0 & 1 & 0 \\
\hline 5 & $\begin{array}{r}0,94608 \\
4318 \\
\end{array}$ & 0 & 0 & 1 & 0 & 0 & 1 & 0 & 0 & 1 & 0 & 0 & 0 & 1 & 1 & 0 & 0 & 1 & 1 & 0 & 1 & 0 & 0 & 1 & 0 & 0 & 1 & 0 \\
\hline 6 & $\begin{array}{r}0,92145 \\
2149 \\
\end{array}$ & 0 & 0 & 0 & 1 & 0 & 0 & 0 & 0 & 0 & 0 & 1 & 0 & 0 & 0 & 0 & 0 & 0 & 1 & 0 & 1 & 0 & 0 & 1 & 0 & 0 & 1 & 0 \\
\hline 7 & $\begin{array}{r}0,80457 \\
088 \\
\end{array}$ & 0 & 0 & 0 & 0 & 0 & 1 & 0 & 0 & 0 & 0 & 1 & 0 & 0 & 0 & 0 & 0 & 0 & 1 & 0 & 1 & 0 & 0 & 1 & 0 & 0 & 1 & 0 \\
\hline 8 & $\begin{array}{r}0,76022 \\
7487 \\
\end{array}$ & 0 & 1 & 0 & 0 & 0 & 1 & 0 & 0 & 0 & 0 & 1 & 0 & 0 & 0 & 0 & 0 & 0 & 1 & 0 & 1 & 0 & 0 & 1 & 0 & 0 & 1 & 0 \\
\hline & & $\ldots$ & $\ldots$ & $\ldots$ & $\ldots$ & $\ldots$ & $\ldots$ & $\ldots$ & $\ldots$ & $\ldots$ & $\ldots$ & $\ldots$ & $\ldots$ & $\ldots$ & $\ldots$ & $\ldots$ & $\ldots$ & $\ldots$ & $\ldots$ & $\ldots$ & $\ldots$ & $\ldots$ & $\ldots$ & $\ldots$ & $\ldots$ & $\ldots$ & $\ldots$ & $\ldots$ \\
\hline $\begin{array}{l}10 \\
61 \\
\end{array}$ & $\begin{array}{r}0,97546 \\
8852 \\
\end{array}$ & 0 & 0 & 0 & 1 & 0 & 0 & 1 & 0 & 0 & 1 & 0 & 0 & 1 & 0 & 1 & 0 & 0 & 0 & 0 & 0 & 0 & 1 & 0 & 0 & 0 & 0 & 0 \\
\hline $\begin{array}{l}10 \\
62 \\
\end{array}$ & $\begin{array}{r}0,99945 \\
5465 \\
\end{array}$ & 0 & 1 & 0 & 0 & 0 & 0 & 1 & 0 & 1 & 1 & 0 & 1 & 0 & 0 & 1 & 0 & 0 & 0 & 1 & 0 & 1 & 0 & 0 & 0 & 1 & 0 & 0 \\
\hline $\begin{array}{l}10 \\
63 \\
\end{array}$ & $\begin{array}{r}0,99947 \\
0565 \\
\end{array}$ & 0 & 1 & 0 & 0 & 0 & 0 & 1 & 0 & 1 & 1 & 0 & 0 & 1 & 0 & 0 & 1 & 1 & 0 & 1 & 0 & 1 & 0 & 0 & 0 & 1 & 0 & 0 \\
\hline $\begin{array}{l}10 \\
64 \\
\end{array}$ & $\begin{array}{r}0,96850 \\
238 \\
\end{array}$ & 0 & 1 & 0 & 0 & 0 & 0 & 1 & 0 & 1 & 1 & 0 & 0 & 0 & 1 & 0 & 0 & 0 & 0 & 1 & 0 & 0 & 1 & 0 & 0 & 1 & 0 & 0 \\
\hline $\begin{array}{l}10 \\
65 \\
\end{array}$ & $\begin{array}{r}0,28497 \\
7604 \\
\end{array}$ & 0 & 1 & 0 & 0 & 0 & 0 & 1 & 1 & 0 & 1 & 0 & 0 & 1 & 0 & 0 & 0 & 0 & 0 & 1 & 0 & 0 & 1 & 0 & 0 & 1 & 0 & 0 \\
\hline $\begin{array}{l}10 \\
66 \\
\end{array}$ & $\begin{array}{r}0,99969 \\
8616 \\
\end{array}$ & 0 & 0 & 0 & 1 & 0 & 0 & 1 & 0 & 1 & 1 & 0 & 1 & 0 & 0 & 1 & 0 & 1 & 0 & 1 & 0 & 0 & 1 & 0 & 0 & 1 & 0 & 0 \\
\hline $\begin{array}{l}10 \\
67 \\
\end{array}$ & $\begin{array}{r}0,99976 \\
1215 \\
\end{array}$ & 0 & 0 & 0 & 0 & 0 & 0 & 1 & 0 & 1 & 1 & 0 & 1 & 0 & 0 & 0 & 1 & 1 & 0 & 1 & 0 & 0 & 1 & 0 & 0 & 1 & 0 & 0 \\
\hline $\begin{array}{l}10 \\
68 \\
\end{array}$ & $\begin{array}{r}0,94948 \\
7877 \\
\end{array}$ & 1 & 0 & 0 & 0 & 0 & 0 & & 0 & 1 & 0 & 0 & 0 & 1 & 0 & 1 & 0 & 0 & 0 & 0 & 0 & 0 & 1 & 0 & 0 & 1 & 0 & 0 \\
\hline
\end{tabular}

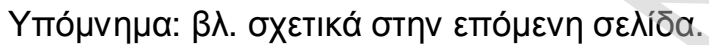




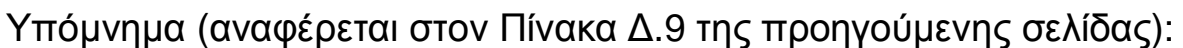

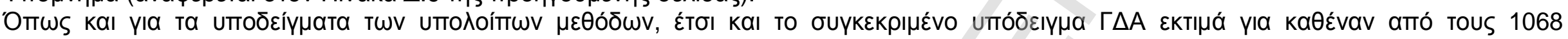

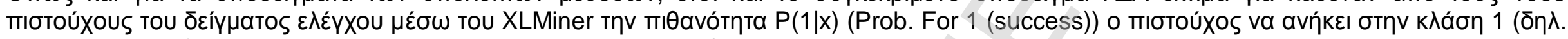

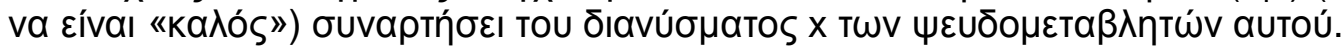

\section{Simple Bayes Rule Classification (KAB)}

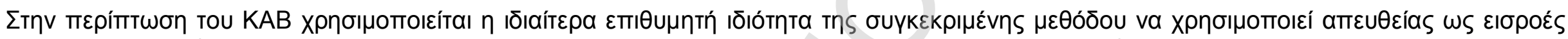

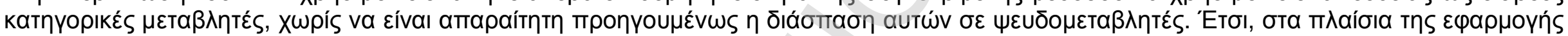

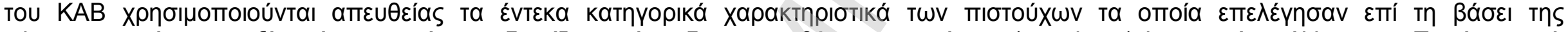

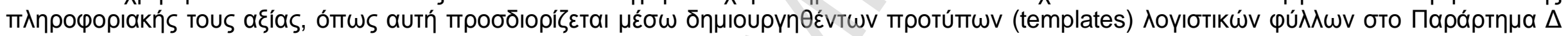

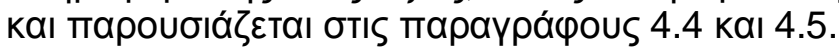

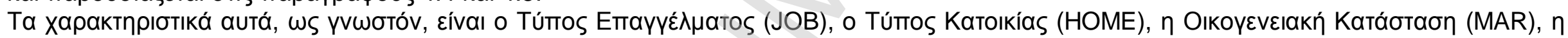

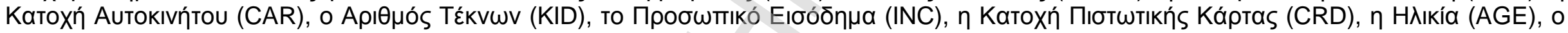

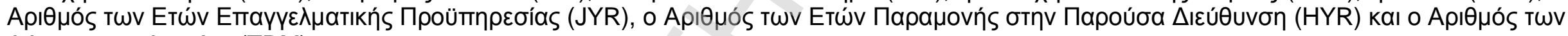

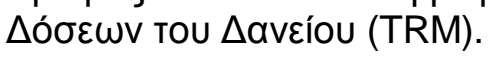

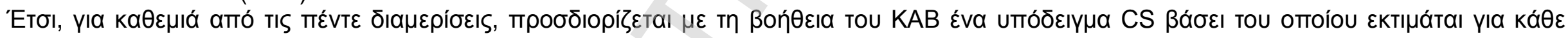

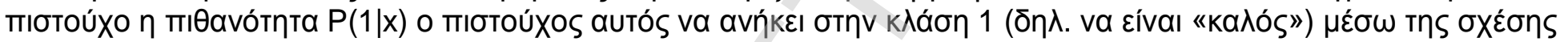

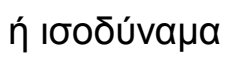

$$
\ln \frac{P(1 \mid x)}{1-P(1 \mid x)}=\Sigma w_{i}\left(x_{i}\right)+b
$$

$$
P(1 \mid x)=\frac{e^{\sum w_{i}\left(x_{i}\right)+b}}{1+e^{\sum w_{i}\left(x_{i}\right)+b}},
$$

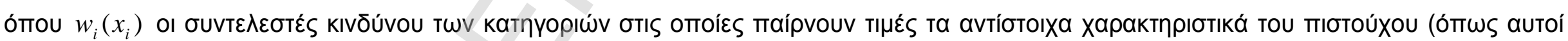

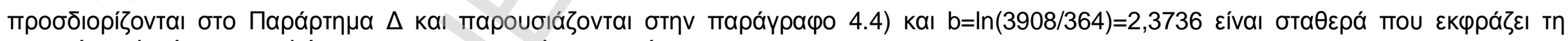

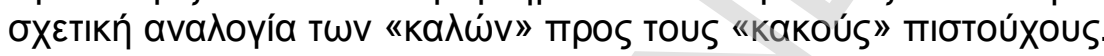




\section{Simple Bayes Rule - Classification of Test Data}

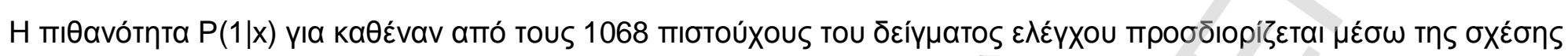

Uтто入оүí̧ovтаs

$$
P(1 \mid x)=\frac{e^{\sum w_{i}\left(x_{i}\right)+b}}{1+e^{\sum w_{i}\left(x_{i}\right)+b}}
$$

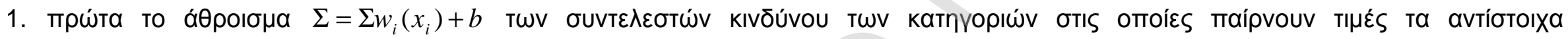

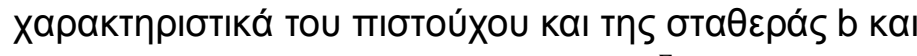

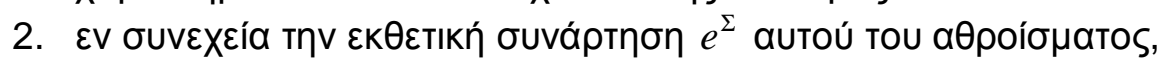

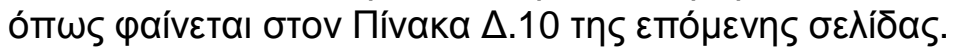

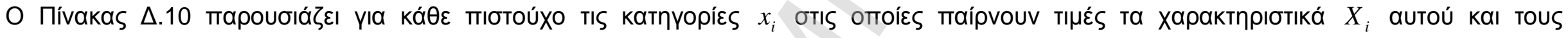

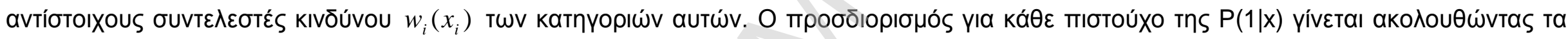

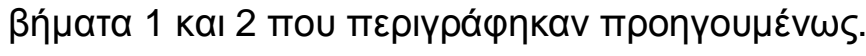




\begin{tabular}{|c|c|c|c|c|c|c|c|c|c|c|c|c|c|c|c|c|c|c|c|c|c|c|c|c|c|c|}
\hline $\begin{array}{r}\text { Row } \\
\text { Id. }\end{array}$ & $\begin{array}{l}\mathrm{J} \\
\mathrm{O} \\
\mathrm{B} \\
\end{array}$ & $\begin{array}{l}\mathrm{HO} \\
\mathrm{ME}\end{array}$ & $\begin{array}{r}M \\
\mathrm{AR} \\
\end{array}$ & $\begin{array}{l}\text { C } \\
A \\
R\end{array}$ & $\begin{array}{c}\mathrm{KI} \\
\mathrm{D} \\
\end{array}$ & $\begin{array}{r}\text { IN } \\
\text { C }\end{array}$ & $\begin{array}{l}\text { C } \\
R \\
D \\
\end{array}$ & $\begin{array}{l}\mathrm{A} \\
\mathrm{G} \\
\mathrm{E}\end{array}$ & $\begin{array}{r}J Y \\
R \\
\end{array}$ & $\begin{array}{l}\mathrm{H} \\
\mathrm{Y} \\
\mathrm{R} \\
\end{array}$ & $\begin{array}{r}\text { TR } \\
\mathrm{M} \\
\end{array}$ & $\begin{array}{r}W \\
\text { JOB }\end{array}$ & $\begin{array}{r}\mathrm{W} \\
\mathrm{HOM} \\
\mathrm{E} \\
\end{array}$ & $\begin{array}{r}W \\
\text { MAR } \\
\end{array}$ & $\begin{array}{r}W \\
C A R \\
\end{array}$ & $\begin{array}{r}W \\
\text { KID } \\
\end{array}$ & $\begin{array}{r}\text { W } \\
\text { INC }\end{array}$ & $\begin{array}{r}W \\
\text { CRD } \\
\end{array}$ & $\begin{array}{r}W \\
A G E\end{array}$ & $\begin{array}{r}W \\
\text { JYR }\end{array}$ & $\begin{array}{r}W \\
\text { HYR } \\
\end{array}$ & $\begin{array}{r}W \\
\text { TRM } \\
\end{array}$ & b & $\Sigma$ & $e^{\Sigma}$ & $P(1 \mid x)$ \\
\hline 1 & 2 & 2 & 2 & 1 & 1 & 3 & 1 & 1 & 3 & 3 & 2 & $\begin{array}{r}0,00 \\
38\end{array}$ & $\begin{array}{r}0,528 \\
5\end{array}$ & $\begin{array}{r}1,28 \\
62\end{array}$ & $\begin{array}{r}0,45 \\
93\end{array}$ & $\begin{array}{r}1,19 \\
80\end{array}$ & $\begin{array}{r}2,39 \\
86\end{array}$ & $\begin{array}{r}0,70 \\
15\end{array}$ & $\begin{array}{r}0,95 \\
63\end{array}$ & $\begin{array}{r}0,53 \\
59\end{array}$ & $\begin{array}{r}1,41 \\
51\end{array}$ & $\begin{array}{r}0,27 \\
99\end{array}$ & $\begin{array}{r}2,37 \\
36\end{array}$ & $\begin{array}{r}11,5 \\
694\end{array}$ & $\begin{array}{r}1058 \\
13,6\end{array}$ & $\begin{array}{r}0,999 \\
991\end{array}$ \\
\hline 2 & 5 & 2 & 2 & 1 & 2 & 3 & 1 & 1 & 3 & 1 & 0 & $\begin{array}{r}0,31 \\
20\end{array}$ & $\begin{array}{r}0,528 \\
5\end{array}$ & $\begin{array}{r}1,28 \\
62 \\
\end{array}$ & $\begin{array}{r}0,45 \\
93\end{array}$ & $\begin{array}{r}0,25 \\
50\end{array}$ & $\begin{array}{r}2,39 \\
86\end{array}$ & $\begin{array}{r}0,70 \\
15 \\
\end{array}$ & $\begin{array}{r}0,95 \\
63\end{array}$ & $\begin{array}{r}0,53 \\
59\end{array}$ & $\begin{array}{r}0,57 \\
51\end{array}$ & $\begin{array}{r}1,04 \\
74\end{array}$ & $\begin{array}{r}2,37 \\
36\end{array}$ & \begin{tabular}{r|}
11,4 \\
294 \\
\end{tabular} & $\begin{array}{r}9199 \\
0,37\end{array}$ & $\begin{array}{r}0,999 \\
989 \\
\end{array}$ \\
\hline 3 & 3 & 2 & 2 & 1 & 2 & 3 & 1 & 1 & 3 & 1 & 0 & $\begin{array}{r}0,15 \\
66 \\
\end{array}$ & $\begin{array}{r}0,528 \\
5 \\
\end{array}$ & $\begin{array}{r}1,28 \\
62 \\
\end{array}$ & $\begin{array}{r}0,45 \\
93 \\
\end{array}$ & $\begin{array}{r}0,25 \\
50 \\
\end{array}$ & $\begin{array}{r}2,39 \\
86 \\
\end{array}$ & $\begin{array}{r}0,70 \\
15 \\
\end{array}$ & $\begin{array}{r}0,95 \\
63 \\
\end{array}$ & $\begin{array}{r}0,53 \\
59 \\
\end{array}$ & $\begin{array}{r}0,57 \\
51 \\
\end{array}$ & $\begin{array}{r}1,04 \\
74 \\
\end{array}$ & $\begin{array}{r}2,37 \\
36 \\
\end{array}$ & \begin{tabular}{r|}
11,2 \\
741 \\
\end{tabular} & $\begin{array}{r}7875 \\
1,54 \\
\end{array}$ & $\begin{array}{r}0,999 \\
987 \\
\end{array}$ \\
\hline 4 & 5 & 2 & 2 & 1 & 1 & 3 & 0 & 1 & 3 & 2 & 0 & $\begin{array}{r}0,31 \\
20 \\
\end{array}$ & $\begin{array}{r}0,528 \\
5 \\
\end{array}$ & $\begin{array}{r}1,28 \\
62 \\
\end{array}$ & $\begin{array}{r}0,45 \\
93\end{array}$ & $\begin{array}{r}1,19 \\
80\end{array}$ & $\begin{array}{r}2,39 \\
86\end{array}$ & $\begin{array}{r}0,24 \\
10 \\
\end{array}$ & $\begin{array}{r}0,95 \\
63\end{array}$ & $\begin{array}{r}0,53 \\
59\end{array}$ & $\begin{array}{r}0,37 \\
16\end{array}$ & $\begin{array}{r}1,04 \\
74\end{array}$ & $\begin{array}{r}2,37 \\
36\end{array}$ & $\begin{array}{r}11,2 \\
265\end{array}$ & $\begin{array}{r}7509 \\
7,7\end{array}$ & $\begin{array}{r}0,999 \\
987\end{array}$ \\
\hline 5 & 3 & 2 & 2 & 1 & 2 & 3 & 1 & 1 & 3 & 2 & 0 & $\begin{array}{r}0,15 \\
66 \\
\end{array}$ & $\begin{array}{r}0,528 \\
5 \\
\end{array}$ & $\begin{array}{r}1,28 \\
62 \\
\end{array}$ & $\begin{array}{r}0,45 \\
93 \\
\end{array}$ & $\begin{array}{r}0,25 \\
50 \\
\end{array}$ & $\begin{array}{r}2,39 \\
86 \\
\end{array}$ & $\begin{array}{r}0,70 \\
15 \\
\end{array}$ & $\begin{array}{r}0,95 \\
63 \\
\end{array}$ & $\begin{array}{r}0,53 \\
59 \\
\end{array}$ & $\begin{array}{r}0,37 \\
16 \\
\end{array}$ & $\begin{array}{r}1,04 \\
74 \\
\end{array}$ & $\begin{array}{r}2,37 \\
36 \\
\end{array}$ & $\begin{array}{l}11,0 \\
706 \\
\end{array}$ & $\begin{array}{r}6425 \\
4,83 \\
\end{array}$ & $\begin{array}{r}0,999 \\
984 \\
\end{array}$ \\
\hline 6 & 3 & 2 & 2 & 1 & 2 & 3 & 1 & 1 & 3 & 2 & 0 & $\begin{array}{r}0,15 \\
66 \\
\end{array}$ & $\begin{array}{r}0,528 \\
5 \\
\end{array}$ & $\begin{array}{r}1,28 \\
62 \\
\end{array}$ & $\begin{array}{r}0,45 \\
93 \\
\end{array}$ & $\begin{array}{r}0,25 \\
50 \\
\end{array}$ & $\begin{array}{r}2,39 \\
86 \\
\end{array}$ & $\begin{array}{r}0,70 \\
15 \\
\end{array}$ & $\begin{array}{r}0,95 \\
63 \\
\end{array}$ & $\begin{array}{r}0,53 \\
59 \\
\end{array}$ & $\begin{array}{r}0,37 \\
16 \\
\end{array}$ & $\begin{array}{r}1,04 \\
74 \\
\end{array}$ & $\begin{array}{r}2,37 \\
36 \\
\end{array}$ & $\begin{array}{l}11,0 \\
706 \\
\end{array}$ & $\begin{array}{r}6425 \\
4,83 \\
\end{array}$ & $\begin{array}{r}0,999 \\
984 \\
\end{array}$ \\
\hline 7 & 5 & 2 & 2 & 1 & 2 & 3 & 1 & 1 & 3 & 3 & 1 & $\begin{array}{r}0,31 \\
20 \\
\end{array}$ & $\begin{array}{r}0,528 \\
5 \\
\end{array}$ & $\begin{array}{r}1,28 \\
62 \\
\end{array}$ & $\begin{array}{r}0,45 \\
93 \\
\end{array}$ & $\begin{array}{r}0,25 \\
50 \\
\end{array}$ & $\begin{array}{r}2,39 \\
86 \\
\end{array}$ & $\begin{array}{r}0,70 \\
15 \\
\end{array}$ & $\begin{array}{r}0,95 \\
63 \\
\end{array}$ & $\begin{array}{r}0,53 \\
59 \\
\end{array}$ & $\begin{array}{r}1,41 \\
51 \\
\end{array}$ & $\begin{array}{r}0,17 \\
98 \\
\end{array}$ & $\begin{array}{r}2,37 \\
36 \\
\end{array}$ & $\begin{array}{r}11,0 \\
424 \\
\end{array}$ & $\begin{array}{r}6246 \\
5,72 \\
\end{array}$ & $\begin{array}{r}0,999 \\
984 \\
\end{array}$ \\
\hline & & 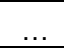 & & $\ldots$ & & & & & & & & .. & $\ldots$ & $\ldots$ & & $\ldots$ & & $\ldots$ & $\ldots$ & $\ldots$ &.. & $\ldots$ & & $\ldots$ & & \\
\hline $\begin{array}{l}106 \\
2\end{array}$ & 5 & 1 & 1 & 0 & 0 & 0 & 0 & 0 & 1 & 0 & 1 & $\begin{array}{r}0,31 \\
20\end{array}$ & $\begin{array}{r}- \\
0,829 \\
1\end{array}$ & $\begin{array}{r}0,60 \\
00 \\
\end{array}$ & $\begin{array}{r}- \\
0,71 \\
55\end{array}$ & $\begin{array}{r}0,38 \\
41\end{array}$ & $\begin{array}{r}0,99 \\
50\end{array}$ & $\begin{array}{r}- \\
0,24 \\
10\end{array}$ & $\begin{array}{r}0,45 \\
63\end{array}$ & $\begin{array}{r}- \\
0,15 \\
34 \\
\end{array}$ & $\begin{array}{r}- \\
0,97 \\
28\end{array}$ & $\begin{array}{r}0,17 \\
98\end{array}$ & $\begin{array}{r}2,37 \\
36\end{array}$ & $\begin{array}{r}2,84 \\
13\end{array}$ & $\begin{array}{r}0,058 \\
347\end{array}$ & $\begin{array}{r}0,055 \\
13 \\
\end{array}$ \\
\hline $\begin{array}{l}106 \\
3 \\
\end{array}$ & 4 & 1 & 1 & 0 & 0 & 0 & 0 & 0 & 1 & 0 & 1 & $\begin{array}{r}0,30 \\
60 \\
\end{array}$ & $\begin{array}{r}0,829 \\
1 \\
\end{array}$ & $\begin{array}{r}0,60 \\
00 \\
\end{array}$ & $\begin{array}{r}0,71 \\
55 \\
\end{array}$ & $\begin{array}{r}0,38 \\
41 \\
\end{array}$ & $\begin{array}{r}0,99 \\
50 \\
\end{array}$ & $\begin{array}{r}0,24 \\
10 \\
\end{array}$ & $\begin{array}{r}0,45 \\
63 \\
\end{array}$ & $\begin{array}{r}0,15 \\
34 \\
\end{array}$ & $\begin{array}{r}0,97 \\
28 \\
\end{array}$ & $\begin{array}{r}0,17 \\
98 \\
\end{array}$ & $\begin{array}{r}2,37 \\
36 \\
\end{array}$ & $\begin{array}{r}2,84 \\
74 \\
\end{array}$ & $\begin{array}{r}0,057 \\
996 \\
\end{array}$ & $\begin{array}{r}0,054 \\
817 \\
\end{array}$ \\
\hline $\begin{array}{l}106 \\
4\end{array}$ & 5 & 1 & 1 & 0 & 0 & 0 & 0 & 0 & 0 & 0 & 1 & $\begin{array}{r}0,31 \\
20 \\
\end{array}$ & $\begin{array}{r}- \\
0,829 \\
1\end{array}$ & $\begin{array}{r}0,60 \\
00 \\
\end{array}$ & $\begin{array}{r}- \\
0,71 \\
55 \\
\end{array}$ & $\begin{array}{r}0,38 \\
41 \\
\end{array}$ & $\begin{array}{r}0,99 \\
50\end{array}$ & $\begin{array}{r}0,24 \\
10 \\
\end{array}$ & $\begin{array}{r}0,45 \\
63 \\
\end{array}$ & $\begin{array}{r}0,46 \\
33 \\
\end{array}$ & $\begin{array}{r}- \\
0,97 \\
28\end{array}$ & $\begin{array}{r}- \\
0,17 \\
98 \\
\end{array}$ & $\begin{array}{r}2,37 \\
36 \\
\end{array}$ & $\begin{array}{r}3,15 \\
12 \\
\end{array}$ & $\begin{array}{r}0,042 \\
799 \\
\end{array}$ & $\begin{array}{r}0,041 \\
043 \\
\end{array}$ \\
\hline $\begin{array}{l}106 \\
5 \\
\end{array}$ & 3 & 1 & 1 & 0 & 0 & 0 & 0 & 0 & 0 & 0 & 1 & $\begin{array}{r}0,15 \\
66 \\
\end{array}$ & $\begin{array}{r}0,829 \\
1 \\
\end{array}$ & $\begin{array}{r}0,60 \\
00 \\
\end{array}$ & $\begin{array}{r}0,71 \\
55 \\
\end{array}$ & $\begin{array}{r}0,38 \\
41 \\
\end{array}$ & $\begin{array}{r}0,99 \\
50 \\
\end{array}$ & $\begin{array}{r}0,24 \\
10 \\
\end{array}$ & $\begin{array}{r}0,45 \\
63 \\
\end{array}$ & $\begin{array}{r}0,46 \\
33 \\
\end{array}$ & $\begin{array}{r}0,97 \\
28 \\
\end{array}$ & $\begin{array}{r}0,17 \\
98 \\
\end{array}$ & $\begin{array}{r}2,37 \\
36 \\
\end{array}$ & $\begin{array}{r}3,30 \\
66 \\
\end{array}$ & $\begin{array}{r}0,036 \\
64 \\
\end{array}$ & $\begin{array}{r}0,035 \\
345 \\
\end{array}$ \\
\hline $\begin{array}{l}106 \\
6\end{array}$ & 0 & 1 & 0 & 0 & 0 & 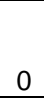 & 0 & 2 & 0 & 2 & 1 & $\begin{array}{r}0,83 \\
18 \\
\end{array}$ & $\begin{array}{r}0,829 \\
1 \\
\end{array}$ & $\begin{array}{r}1,95 \\
92 \\
\end{array}$ & $\begin{array}{r}0,71 \\
55 \\
\end{array}$ & $\begin{array}{r}0,38 \\
41 \\
\end{array}$ & $\begin{array}{r}0,99 \\
50 \\
\end{array}$ & $\begin{array}{r}0,24 \\
10 \\
\end{array}$ & $\begin{array}{r}0,32 \\
41 \\
\end{array}$ & $\begin{array}{r}0,46 \\
33 \\
\end{array}$ & $\begin{array}{r}0,37 \\
16 \\
\end{array}$ & $\begin{array}{r}0,17 \\
98 \\
\end{array}$ & $\begin{array}{r}2,37 \\
36 \\
\end{array}$ & $\begin{array}{r}4,17 \\
75 \\
\end{array}$ & $\begin{array}{r}0,015 \\
336 \\
\end{array}$ & $\begin{array}{r}0,015 \\
105 \\
\end{array}$ \\
\hline $\begin{array}{l}106 \\
7 \\
\end{array}$ & 0 & 2 & 0 & 0 & 0 & 0 & 0 & 2 & 0 & 0 & 2 & $\begin{array}{r}0,83 \\
18 \\
\end{array}$ & $\begin{array}{r}0,528 \\
5 \\
\end{array}$ & $\begin{array}{r}1,95 \\
92 \\
\end{array}$ & $\begin{array}{r}0,71 \\
55 \\
\end{array}$ & $\begin{array}{r}0,38 \\
41 \\
\end{array}$ & $\begin{array}{r}0,99 \\
50 \\
\end{array}$ & $\begin{array}{r}0,24 \\
10 \\
\end{array}$ & $\begin{array}{r}0,32 \\
41 \\
\end{array}$ & $\begin{array}{r}0,46 \\
33 \\
\end{array}$ & $\begin{array}{r}0,97 \\
28\end{array}$ & $\begin{array}{r}0,27 \\
99\end{array}$ & $\begin{array}{r}2,37 \\
36\end{array}$ & $\begin{array}{r}4,26 \\
45\end{array}$ & $\begin{array}{r}0,014 \\
059\end{array}$ & $\begin{array}{r}0,013 \\
864\end{array}$ \\
\hline $\begin{array}{l}106 \\
8\end{array}$ & 0 & 1 & 0 & 0 & 3 & 0 & 0 & 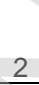 & 2 & 0 & 1 & $\begin{array}{r}0,83 \\
18\end{array}$ & $\begin{array}{r}0,829 \\
1\end{array}$ & $\begin{array}{r}1,95 \\
92 \\
\end{array}$ & $\begin{array}{r}0,71 \\
55\end{array}$ & $\begin{array}{r}0,18 \\
88 \\
\end{array}$ & $\begin{array}{r}0,99 \\
50\end{array}$ & $\begin{array}{r}0,24 \\
10 \\
\end{array}$ & $\begin{array}{r}0,32 \\
41\end{array}$ & $\begin{array}{r}0,11 \\
47 \\
\end{array}$ & $\begin{array}{r}0,97 \\
28\end{array}$ & $\begin{array}{r}0,17 \\
98\end{array}$ & $\begin{array}{r}2,37 \\
36 \\
\end{array}$ & $\begin{array}{r}4,74 \\
86 \\
\end{array}$ & $\begin{array}{r}0,008 \\
663 \\
\end{array}$ & $\begin{array}{r}0,008 \\
589 \\
\end{array}$ \\
\hline
\end{tabular}




\section{ПАРАРТНMА Г}

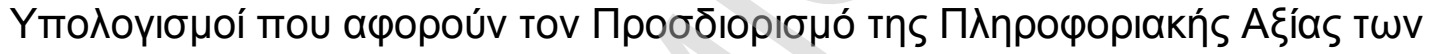

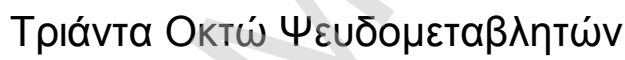




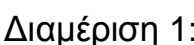

\begin{tabular}{|r|lr|r|r|r|r|r|r|}
\hline & A & B & C & D & E & F & G \\
\hline 1 & MAR1 & & & & & & \\
\hline 2 & & 0 & 239 & 3726 & 0,953313 & 0,656164 & 0,373532 & 0,110994 \\
\hline 3 & 1 & 125 & 182 & 0,046687 & 0,343836 & $-1,9967$ & 0,593315 \\
\hline 4 & & & & & & & & 0,704309 \\
\hline 5 & MAR2 & & & & & & \\
\hline 6 & & 0 & 198 & 2943 & 0,753006 & 0,543836 & 0,325426 & 0,068069 \\
\hline 7 & & 1 & 166 & 965 & 0,246994 & 0,456164 & $-0,61349$ & 0,128324 \\
\hline 8 & & & & & & & & 0,196393 \\
\hline 9 & MAR3 & & & & & & \\
\hline 10 & & 0 & 291 & 1147 & 0,293553 & 0,79863 & $-1,00084$ & 0,5055 \\
\hline 11 & & 1 & 73 & 2761 & 0,706447 & 0,20137 & 1,255104 & 0,633924 \\
\hline 12 & & & & & & & & 1,139424 \\
\hline
\end{tabular}

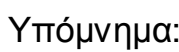

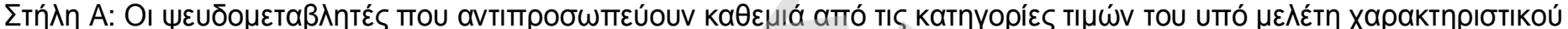

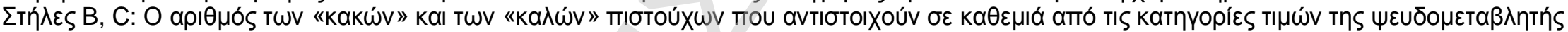

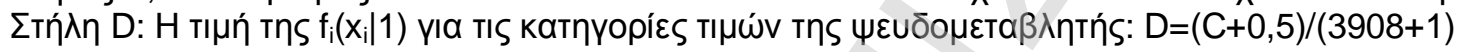

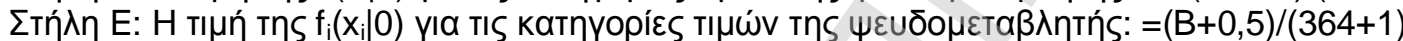

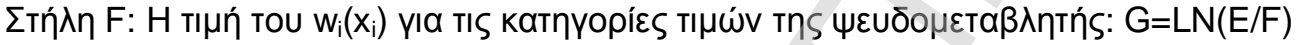

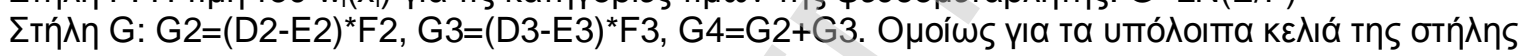

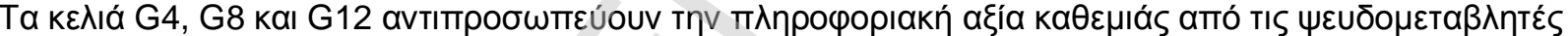




\begin{tabular}{|c|c|c|c|c|c|c|}
\hline \multicolumn{7}{|l|}{ MAR1 } \\
\hline 0 & 237 & 3716 & 0,950755 & 0,650685 & 0,379231 & 0,113796 \\
\hline \multirow[t]{2}{*}{1} & 127 & 192 & 0,049245 & 0,349315 & $-1,95916$ & 0,587885 \\
\hline & & & & & & 0,70168 \\
\hline \multicolumn{7}{|l|}{ MAR2 } \\
\hline 0 & 197 & 2924 & 0,748145 & 0,541096 & 0,324001 & 0,067084 \\
\hline \multirow[t]{2}{*}{1} & 167 & 984 & 0,251855 & 0,458904 & $-0,59999$ & 0,124227 \\
\hline & & & & & & 0,191312 \\
\hline \multicolumn{7}{|l|}{ MAR3 } \\
\hline 0 & 294 & 1176 & 0,300972 & 0,806849 & $-0,98612$ & 0,498855 \\
\hline \multirow[t]{2}{*}{1} & 70 & 2732 & 0,699028 & 0,193151 & 1,28622 & 0,650669 \\
\hline & & & & & & 1,149525 \\
\hline
\end{tabular}

\begin{tabular}{|c|c|c|c|c|c|c|}
\hline MAR1 & & & & & 1 & 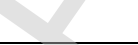 \\
\hline 0 & 246 & 3734 & 0,955359 & 0,675342 & 0,346868 & 0,097129 \\
\hline \multirow[t]{2}{*}{1} & 118 & 174 & 0,044641 & 0,324658 & $-1,98413$ & 0,555589 \\
\hline & & & & & 2 & 0,652718 \\
\hline \multicolumn{7}{|l|}{ MAR2 } \\
\hline 0 & 188 & 2913 & 0,745331 & 0,516438 & 0,366873 & 0,083975 \\
\hline \multirow[t]{2}{*}{1} & 176 & 995 & 0,254669 & 0,483562 & $-0,64122$ & 0,14677 \\
\hline & & & & 2 & . & 0,230744 \\
\hline MAR3 & & & & $<$ & 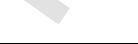 & \\
\hline 0 & 294 & 1169 & 0,299181 & 0,806849 & $-0,99209$ & 0,503651 \\
\hline \multirow[t]{2}{*}{1} & 70 & 2739 & 0,700819 & 0,193151 & 1,288778 & 0,654272 \\
\hline & & & 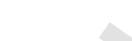 & 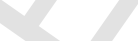 & & 1,157922 \\
\hline
\end{tabular}




\begin{tabular}{|c|c|c|c|c|c|c|}
\hline MAR1 & & & & & & \\
\hline 0 & 245 & 3724 & 0,952801 & 0,672603 & 0,348251 & 0,09758 \\
\hline \multirow[t]{2}{*}{1} & 119 & 184 & 0,047199 & 0,327397 & $-1,93681$ & 0,54269 \\
\hline & & & & & & 0,64027 \\
\hline \multicolumn{7}{|l|}{ MAR2 } \\
\hline 0 & 189 & 2925 & 0,748401 & 0,519178 & 0,365692 & 0,083825 \\
\hline \multirow[t]{2}{*}{1} & 175 & 983 & 0,251599 & 0,480822 & $-0,64766$ & 0,148459 \\
\hline & & & & & & 0,232284 \\
\hline \multicolumn{7}{|l|}{ MAR3 } \\
\hline 0 & 294 & 1167 & 0,29867 & 0,806849 & $-0,9938$ & 0,505028 \\
\hline \multirow[t]{2}{*}{1} & 70 & 2741 & 0,70133 & 0,193151 & 1,289508 & 0,655302 \\
\hline & & & & & & 1,16033 \\
\hline
\end{tabular}

\begin{tabular}{|c|c|c|c|c|c|c|}
\hline MAR1 & & & & & & + \\
\hline 0 & 241 & 3736 & 0,955871 & 0,661644 & 0,367896 & 0,108245 \\
\hline \multirow[t]{2}{*}{1} & 123 & 172 & 0,044129 & 0,338356 & $-2,03698$ & 0,599336 \\
\hline & & & & & 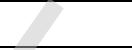 & 0,707581 \\
\hline \multicolumn{7}{|l|}{ MAR2 } \\
\hline 0 & 192 & 2947 & 0,754029 & 0,527397 & 0,357477 & 0,081016 \\
\hline \multirow[t]{2}{*}{1} & 172 & 961 & 0,245971 & 0,472603 & $-0,65304$ & 0,148 \\
\hline & & & & 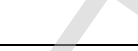 & 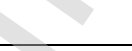 & 0,229016 \\
\hline \multicolumn{7}{|l|}{ MAR3 } \\
\hline 0 & 295 & 1133 & 0,289972 & 0,809589 & $-1,02674$ & 0,533513 \\
\hline \multirow[t]{2}{*}{1} & 69 & 2775 & 0,710028 & 0,190411 & 1,31612 & 0,683879 \\
\hline & & & 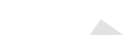 & 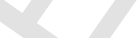 & & 1,217392 \\
\hline
\end{tabular}




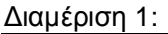

\begin{tabular}{|r|r|r|r|r|r|r|}
\hline \multicolumn{1}{|l|}{ HOME1 } & & & & & & \\
\hline 0 & 299 & 3309 & 0,846636 & 0,820548 & 0,031298 & 0,000817 \\
\hline 1 & 65 & 599 & 0,153364 & 0,179452 & $-0,15709$ & 0,004098 \\
\hline & & & & & & 0,004915 \\
\hline HOME2 & & & & & & \\
\hline 0 & 210 & 3166 & 0,810054 & 0,576712 & 0,339757 & 0,079279 \\
\hline 1 & 154 & 742 & 0,189946 & 0,423288 & $-0,80131$ & 0,186979 \\
\hline & & & & & & 0,266258 \\
\hline HOME3 & & & & & & \\
\hline 0 & 219 & 1341 & 0,343182 & 0,60137 & $-0,56095$ & 0,14483 \\
\hline 1 & 145 & 2567 & 0,656818 & 0,39863 & 0,499372 & 0,128932 \\
\hline & & & & & & 0,273761 \\
\hline
\end{tabular}

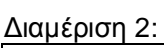

\begin{tabular}{|c|c|c|c|c|c|c|}
\hline HOME1 & & & & & 7 & 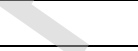 \\
\hline 0 & 299 & 3297 & 0,843566 & 0,820548 & 0,027666 & 0,000637 \\
\hline \multirow[t]{2}{*}{1} & 65 & 611 & 0,156434 & 0,179452 & $-0,13727$ & 0,00316 \\
\hline & & & & & 0 & 0,003797 \\
\hline \multicolumn{7}{|l|}{ HOME2 } \\
\hline 0 & 205 & 3163 & 0,809286 & 0,563014 & 0,362849 & 0,08936 \\
\hline \multirow[t]{2}{*}{1} & 159 & 745 & 0,190714 & 0,436986 & $-0,82913$ & 0,204192 \\
\hline & & & & & 8 & 0,293551 \\
\hline \multicolumn{7}{|l|}{ HOME3 } \\
\hline 0 & 224 & 1356 & 0,34702 & 0,615068 & $-0,57235$ & 0,153418 \\
\hline \multirow[t]{2}{*}{1} & 140 & 2552 & 0,65298 & 0,384932 & 0,528482 & 0,141659 \\
\hline & & & & & & 0,295077 \\
\hline
\end{tabular}




\begin{tabular}{|c|c|c|c|c|c|c|}
\hline HOME1 & & & & & & \\
\hline 0 & 293 & 3301 & 0,844589 & 0,80411 & 0,049115 & 0,001988 \\
\hline \multirow[t]{2}{*}{1} & 71 & 607 & 0,155411 & 0,19589 & $-0,23148$ & 0,00937 \\
\hline & & & & & & 0,011359 \\
\hline \multicolumn{7}{|l|}{ HOME2 } \\
\hline 0 & 202 & 3176 & 0,812612 & 0,554795 & 0,381656 & 0,098398 \\
\hline \multirow[t]{2}{*}{1} & 162 & 732 & 0,187388 & 0,445205 & $-0,86535$ & 0,223103 \\
\hline & & & & & & 0,321501 \\
\hline \multicolumn{7}{|l|}{ HOME3 } \\
\hline 0 & 233 & 1339 & 0,342671 & 0,639726 & $-0,62427$ & 0,185443 \\
\hline \multirow[t]{2}{*}{1} & 131 & 2569 & 0,657329 & 0,360274 & 0,60132 & 0,178625 \\
\hline & & & & & & 0,364068 \\
\hline
\end{tabular}

\begin{tabular}{|c|c|c|c|c|c|c|}
\hline \multicolumn{7}{|l|}{ HOME1 } \\
\hline 0 & 298 & 3297 & 0,843566 & 0,817808 & 0,03101 & 0,000799 \\
\hline \multirow[t]{2}{*}{1} & 66 & 611 & 0,156434 & 0,182192 & $-0,15243$ & 0,003926 \\
\hline & & & & & 2 & 0,004725 \\
\hline HOME2 & & & & & 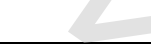 & 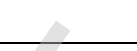 \\
\hline 0 & 201 & 3169 & 0,810821 & 0,552055 & 0,3844 & 0,09947 \\
\hline \multirow[t]{2}{*}{1} & 163 & 739 & 0,189179 & 0,447945 & $-0,86198$ & 0,223051 \\
\hline & & & & & & 0,322521 \\
\hline \multicolumn{7}{|l|}{ HOME3 } \\
\hline 0 & 229 & 1350 & 0,345485 & 0,628767 & $-0,59881$ & 0,169633 \\
\hline \multirow[t]{2}{*}{1} & 135 & 2558 & 0,654515 & 0,371233 & 0,567065 & 0,16064 \\
\hline & & & & $\sqrt{ }$ & 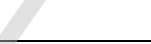 & 0,330273 \\
\hline
\end{tabular}




\begin{tabular}{|c|c|c|c|c|c|c|}
\hline \multicolumn{7}{|l|}{ HOME1 } \\
\hline 0 & 295 & 3308 & 0,84638 & 0,809589 & 0.044442 & 0,001635 \\
\hline \multirow{2}{*}{1} & 69 & 600 & 0,15362 & 0,190411 & $-0,2147$ & 0,007899 \\
\hline & & & & & & 0,009534 \\
\hline \multicolumn{7}{|l|}{ HOME2 } \\
\hline 0 & 210 & 3170 & 0,811077 & 0,576712 & 0,341019 & 0,079923 \\
\hline \multirow[t]{2}{*}{1} & 154 & 738 & 0,188923 & 0,423288 & $-0,80671$ & 0,189065 \\
\hline & & & & & & 0,268988 \\
\hline \multicolumn{7}{|l|}{ HOME3 } \\
\hline 0 & 223 & 1338 & 0,342415 & 0,612329 & $-0,58125$ & 0,156886 \\
\hline 1 & 141 & 2570 & 0,657585 & 0,387671 & 0,528416 & 0,142627 \\
\hline & & & & & & 0,299513 \\
\hline
\end{tabular}

3) $\quad A \rho ı \theta \mu o ́ s ~ T E ́ k v \omega v$ (KID)

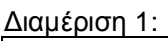

\begin{tabular}{|c|c|c|c|c|c|c|}
\hline \multicolumn{2}{|l|}{ KID1 } & \multirow[b]{2}{*}{2487} & \multirow[b]{2}{*}{0,636352} & \multirow[b]{2}{*}{0,456164} & \multirow[b]{2}{*}{0,332899} & \multirow{2}{*}{0,059984} \\
\hline 0 & 166 & & & & & \\
\hline 1 & 198 & 1421 & 0,363648 & 0,543836 & $-0,40246$ & 0,072518 \\
\hline & & & & & 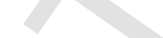 & 0,132503 \\
\hline \multicolumn{2}{|l|}{ KID2 } & & & & 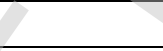 & \\
\hline 0 & 348 & 3286 & 0,840752 & 0,954795 & $-0,1272$ & 0,014506 \\
\hline \multirow[t]{2}{*}{1} & 16 & 622 & 0,159248 & 0,045205 & 1,259244 & 0,143607 \\
\hline & & & & $2=$ & - & 0,158113 \\
\hline \multicolumn{2}{|l|}{ KID3 } & & & 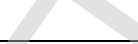 & $\gamma$ & \\
\hline 0 & 266 & 2518 & 0,644282 & 0,730137 & $-0,12509$ & 0,01074 \\
\hline \multirow[t]{2}{*}{1} & 98 & 1390 & 0,355718 & 0,269863 & 0,276223 & 0,023715 \\
\hline & & & 7 & 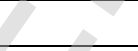 & & 0,034455 \\
\hline \multicolumn{2}{|l|}{ KID4 } & & & 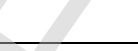 & & \\
\hline 0 & 312 & 3433 & 0,878358 & 0,856164 & 0,025591 & 0,000568 \\
\hline \multirow[t]{2}{*}{1} & 52 & 475 & 0,121642 & 0,143836 & $-0,16759$ & 0,003719 \\
\hline & & & & & & 0,004287 \\
\hline
\end{tabular}




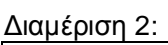

\begin{tabular}{|c|c|c|c|c|c|c|}
\hline \multicolumn{7}{|l|}{ KID1 } \\
\hline 0 & 167 & 2468 & 0,631491 & 0,458904 & 0,319243 & 0,055097 \\
\hline \multirow[t]{2}{*}{1} & 197 & 1440 & 0,368509 & 0,541096 & $-0,38413$ & 0,066296 \\
\hline & & & & & & 0,121394 \\
\hline \multicolumn{2}{|l|}{ KID2 } & & & & & +2 \\
\hline 0 & 346 & 3252 & 0,832054 & 0,949315 & $-0,13184$ & 0,01546 \\
\hline \multirow[t]{2}{*}{1} & 18 & 656 & 0,167946 & 0,050685 & 1,198012 & 0,14048 \\
\hline & & & & & & 0,15594 \\
\hline \multicolumn{2}{|l|}{ KID3 } & & & & $\Delta$ & 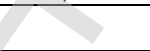 \\
\hline 0 & 267 & 2561 & 0,655283 & 0,732877 & $-0,11191$ & 0,008684 \\
\hline \multirow[t]{2}{*}{1} & 97 & 1347 & 0,344717 & 0,267123 & 0,255014 & 0,019788 \\
\hline & & & & & & 0,028471 \\
\hline \multicolumn{2}{|l|}{ KID4 } & & & & & \\
\hline 0 & 312 & 3443 & 0,880916 & 0,856164 & 0,0285 & 0,000705 \\
\hline \multirow[t]{2}{*}{1} & 52 & 465 & 0,119084 & 0,143836 & $-0,18884$ & 0,004674 \\
\hline & & & & 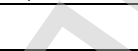 & 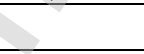 & 0,005379 \\
\hline
\end{tabular}




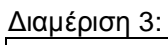

\begin{tabular}{|c|c|c|c|c|c|c|}
\hline \multicolumn{7}{|l|}{ KID1 } \\
\hline 0 & 157 & 2473 & 0,632771 & 0,431507 & 0,382824 & 0,077049 \\
\hline \multirow[t]{2}{*}{1} & 207 & 1435 & 0,367229 & 0,568493 & $-0,437$ & 0,087953 \\
\hline & & & & & & 0,165001 \\
\hline \multicolumn{7}{|l|}{ KID2 } \\
\hline 0 & 346 & 3271 & 0,836915 & 0,949315 & $-0,12602$ & 0,014165 \\
\hline \multirow[t]{2}{*}{1} & 18 & 637 & 0,163085 & 0,050685 & 1,168644 & 0,131356 \\
\hline & & & & & & 0,14552 \\
\hline \multicolumn{7}{|l|}{ KID3 } \\
\hline 0 & 273 & 2553 & 0,653236 & 0,749315 & $-0,13722$ & 0,013184 \\
\hline \multirow[t]{2}{*}{1} & 91 & 1355 & 0,346764 & 0,250685 & 0,324447 & 0,031173 \\
\hline & & & & & $\Delta$ & 0,044357 \\
\hline \multicolumn{2}{|l|}{ KID4 } & & & & 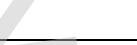 & \\
\hline 0 & 316 & 3427 & 0,876823 & 0,867123 & 0,011124 & 0,000108 \\
\hline \multirow[t]{2}{*}{1} & 48 & 481 & 0,123177 & 0,132877 & $-0,0758$ & 0,000735 \\
\hline & & & & 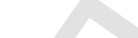 & 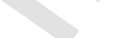 & 0,000843 \\
\hline
\end{tabular}




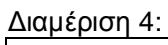

\begin{tabular}{|c|c|c|c|c|c|c|}
\hline \multicolumn{7}{|l|}{ KID1 } \\
\hline 0 & 163 & 2466 & 0,63098 & 0,447945 & 0,342603 & 0,062708 \\
\hline \multirow[t]{2}{*}{1} & 201 & 1442 & 0,36902 & 0,552055 & $-0,4028$ & 0,073726 \\
\hline & & & & & & 0,136434 \\
\hline \multicolumn{7}{|l|}{ KID2 } \\
\hline 0 & 347 & 3285 & 0,840496 & 0,952055 & $-0,12463$ & 0,013904 \\
\hline \multirow[t]{2}{*}{1} & 17 & 623 & 0,159504 & 0,047945 & 1,202008 & 0,134094 \\
\hline & & & & & & 0,147998 \\
\hline \multicolumn{7}{|l|}{ KID3 } \\
\hline 0 & 270 & 2531 & 0,647608 & 0,741096 & $-0,13484$ & 0,012606 \\
\hline \multirow[t]{2}{*}{1} & 94 & 1377 & 0,352392 & 0,258904 & 0,308286 & 0,028821 \\
\hline & & & & & 2 & 0,041427 \\
\hline \multicolumn{2}{|l|}{ KID4 } & & & & 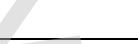 & \\
\hline 0 & 312 & 3442 & 0,88066 & 0,856164 & 0,028209 & 0,000691 \\
\hline \multirow[t]{2}{*}{1} & 52 & 466 & 0,11934 & 0,143836 & $-0,18669$ & 0,004573 \\
\hline & & & & 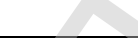 & 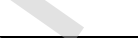 & 0,005264 \\
\hline
\end{tabular}




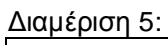

\begin{tabular}{|c|c|c|c|c|c|c|}
\hline \multicolumn{7}{|l|}{ KID1 } \\
\hline 0 & 167 & 2482 & 0,635073 & 0,458904 & 0,324899 & 0,057237 \\
\hline \multirow[t]{2}{*}{1} & 197 & 1426 & 0,364927 & 0,541096 & $-0,3939$ & 0,069393 \\
\hline & & & & & & 0,12663 \\
\hline \multicolumn{7}{|l|}{ KID2 } \\
\hline 0 & 349 & 3266 & 0,835636 & 0,957534 & $-0,13617$ & 0,016599 \\
\hline \multirow[t]{2}{*}{1} & 15 & 642 & 0,164364 & 0,042466 & 1,353387 & 0,164976 \\
\hline & & & & & & 0,181575 \\
\hline \multicolumn{7}{|l|}{ KID3 } \\
\hline 0 & 268 & 2533 & 0,64812 & 0,735616 & $-0,12663$ & 0,01108 \\
\hline \multirow[t]{2}{*}{1} & 96 & 1375 & 0,35188 & 0,264384 & 0,28589 & 0,025014 \\
\hline & & & & & $\Delta$ & 0,036094 \\
\hline \multicolumn{2}{|l|}{ KID4 } & & & & 2 & \\
\hline 0 & 308 & 3443 & 0,880916 & 0,845205 & 0,041382 & 0,001478 \\
\hline \multirow[t]{2}{*}{1} & 56 & 465 & 0,119084 & 0,154795 & $-0,26227$ & 0,009366 \\
\hline & & & & 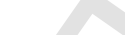 & 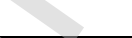 & 0,010843 \\
\hline
\end{tabular}




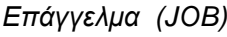

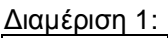

\begin{tabular}{|c|c|c|c|c|c|c|}
\hline \multicolumn{7}{|l|}{ JOB1 } \\
\hline 0 & 259 & 3460 & 0,885265 & 0,710959 & 0,219272 & 0,03822 \\
\hline \multirow[t]{2}{*}{1} & 105 & 448 & 0,114735 & 0,289041 & $-0,92394$ & 0,161048 \\
\hline & & & & & & 0,199269 \\
\hline \multicolumn{7}{|l|}{ JOB2 } \\
\hline 0 & 306 & 3234 & 0,827449 & 0,839726 & $-0,01473$ & 0,000181 \\
\hline \multirow[t]{2}{*}{1} & 58 & 674 & 0,172551 & 0,160274 & 0,073805 & 0,000906 \\
\hline & & & & & & 0,001087 \\
\hline \multicolumn{7}{|l|}{ JOB3 } \\
\hline 0 & 294 & 3004 & 0,768611 & 0,806849 & $-0,04855$ & 0,001857 \\
\hline \multirow[t]{2}{*}{1} & 70 & 904 & 0,231389 & 0,193151 & 0,18063 & 0,006907 \\
\hline & & & & & & 0,008764 \\
\hline \multicolumn{7}{|l|}{ JOB4 } \\
\hline 0 & 346 & 3663 & 0,937196 & 0,949315 & $-0,01285$ & 0,000156 \\
\hline \multirow[t]{2}{*}{1} & 18 & 245 & 0,062804 & 0,050685 & 0,214387 & 0,002598 \\
\hline & & & & & 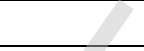 & 0,002754 \\
\hline \multicolumn{7}{|l|}{ JOB5 } \\
\hline 0 & 266 & 2490 & 0,637119 & 0,730137 & $-0,13627$ & 0,012676 \\
\hline \multirow[t]{2}{*}{1} & 98 & 1418 & 0,362881 & 0,269863 & 0,296159 & 0,027548 \\
\hline & & & & & + & 0,040224 \\
\hline \multicolumn{7}{|l|}{ JOB6 } \\
\hline 0 & 349 & 3689 & 0,943848 & 0,957534 & $-0,0144$ & 0,000197 \\
\hline \multirow[t]{2}{*}{1} & 15 & 219 & 0,056152 & 0,042466 & 0,279373 & 0,003824 \\
\hline & & & & ( & 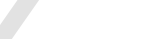 & 0,004021 \\
\hline
\end{tabular}




\begin{tabular}{|c|c|c|c|c|c|c|}
\hline JOB1 & & & & & & \\
\hline 0 & 264 & 3440 & 0,880148 & 0,724658 & 0,194391 & 0,030226 \\
\hline \multirow[t]{2}{*}{1} & 100 & 468 & 0,119852 & 0,275342 & $-0,83176$ & 0,129331 \\
\hline & & & & & & 0,159557 \\
\hline \multicolumn{7}{|l|}{ JOB2 } \\
\hline 0 & 301 & 3231 & 0,826682 & 0,826027 & 0,000792 & $5,19 \mathrm{E}-07$ \\
\hline \multirow[t]{2}{*}{1} & 63 & 677 & 0,173318 & 0,173973 & $-0,00377$ & 2,47E-06 \\
\hline & & & & & & 2,99E-06 \\
\hline \multicolumn{7}{|l|}{ JOB3 } \\
\hline 0 & 295 & 3038 & 0,777309 & 0,809589 & $-0,04069$ & 0,001313 \\
\hline \multirow[t]{2}{*}{1} & 69 & 870 & 0,222691 & 0,190411 & 0,156601 & 0,005055 \\
\hline & & & & & & 0,006369 \\
\hline \multicolumn{7}{|l|}{ JOB4 } \\
\hline 0 & 347 & 3654 & 0,934894 & 0,952055 & $-0,01819$ & 0,000312 \\
\hline \multirow[t]{2}{*}{1} & 17 & 254 & 0,065106 & 0,047945 & 0,30596 & 0,005251 \\
\hline & & & & & -2 & 0,005563 \\
\hline \multicolumn{7}{|l|}{ JOB5 } \\
\hline 0 & 267 & 2482 & 0,635073 & 0,732877 & $-0,14324$ & 0,014009 \\
\hline \multirow[t]{2}{*}{1} & 97 & 1426 & 0,364927 & 0,267123 & 0,311987 & 0,030514 \\
\hline & & & & & & 0,044523 \\
\hline \multicolumn{7}{|l|}{ JOB6 } \\
\hline 0 & 346 & 3695 & 0,945382 & 0,949315 & $-0,00415$ & 1,63E-05 \\
\hline \multirow[t]{2}{*}{1} & 18 & 213 & 0,054618 & 0,050685 & 0,074727 & 0,000294 \\
\hline & & & & 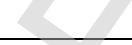 & s. & 0,00031 \\
\hline
\end{tabular}




\begin{tabular}{|c|c|c|c|c|c|c|}
\hline \multicolumn{7}{|l|}{ JOB1 } \\
\hline 0 & 264 & 3461 & 0,885521 & 0,724658 & 0,200477 & 0,032249 \\
\hline \multirow[t]{2}{*}{1} & 100 & 447 & 0,114479 & 0,275342 & $-0,87762$ & 0,141177 \\
\hline & & & & & & 0,173426 \\
\hline \multicolumn{7}{|l|}{ JOB2 } \\
\hline 0 & 297 & 3254 & 0,832566 & 0,815068 & 0,02124 & 0,000372 \\
\hline \multirow[t]{2}{*}{1} & 67 & 654 & 0,167434 & 0,184932 & $-0,0994$ & 0,001739 \\
\hline & & & & & & 0,002111 \\
\hline \multicolumn{7}{|l|}{ JOB3 } \\
\hline 0 & 282 & 2929 & 0,749424 & 0,773973 & $-0,03223$ & 0,000791 \\
\hline \multirow[t]{2}{*}{1} & 82 & 979 & 0,250576 & 0,226027 & 0,103104 & 0,002531 \\
\hline & & & & & & 0,003322 \\
\hline \multicolumn{7}{|l|}{\begin{tabular}{|l|} 
JOB4 \\
\end{tabular}} \\
\hline 0 & 344 & 3652 & 0,934382 & 0,943836 & $-0,01007$ & $9,52 \mathrm{E}-05$ \\
\hline \multirow[t]{2}{*}{1} & 20 & 256 & 0,065618 & 0,056164 & 0,155564 & 0,001471 \\
\hline & & & & & 3 & 0,001566 \\
\hline \multicolumn{7}{|l|}{ JOB5 } \\
\hline 0 & 284 & 2557 & 0,654259 & 0,779452 & $-0,17509$ & 0,02192 \\
\hline \multirow[t]{2}{*}{1} & 80 & 1351 & 0,345741 & 0,220548 & 0,449574 & 0,056283 \\
\hline & & & & & & 0,078203 \\
\hline \multicolumn{7}{|l|}{ JOB6 } \\
\hline 0 & 349 & 3687 & 0,943336 & 0,957534 & $-0,01494$ & 0,000212 \\
\hline \multirow[t]{2}{*}{1} & 15 & 221 & 0,056664 & 0,042466 & 0,288443 & 0,004095 \\
\hline & & & & & & 0,004308 \\
\hline
\end{tabular}




\begin{tabular}{|c|c|c|c|c|c|c|}
\hline JOB1 & & & & & & \\
\hline 0 & 267 & 3443 & 0,880916 & 0,732877 & 0,183985 & 0,027237 \\
\hline \multirow[t]{2}{*}{1} & 97 & 465 & 0,119084 & 0,267123 & $-0,80788$ & 0,119598 \\
\hline & & & & & & 0,146835 \\
\hline \multicolumn{7}{|l|}{ JOB2 } \\
\hline 0 & 300 & 3144 & 0,804426 & 0,823288 & $-0,02318$ & 0,000437 \\
\hline \multirow[t]{2}{*}{1} & 64 & 764 & 0,195574 & 0,176712 & 0,101417 & 0,001913 \\
\hline & & & & & & 0,00235 \\
\hline \multicolumn{7}{|l|}{ JOB3 } \\
\hline 0 & 281 & 3030 & 0,775262 & 0,771233 & 0,005211 & $2,1 \mathrm{E}-05$ \\
\hline \multirow[t]{2}{*}{1} & 83 & 878 & 0,224738 & 0,228767 & $-0,01777$ & 7,16E-05 \\
\hline & & & & & & 9,26E-05 \\
\hline \multicolumn{7}{|l|}{ JOB4 } \\
\hline 0 & 346 & 3655 & 0,93515 & 0,949315 & $-0,01503$ & 0,000213 \\
\hline \multirow[t]{2}{*}{1} & 18 & 253 & 0,06485 & 0,050685 & 0,246454 & 0,003491 \\
\hline & & & & & & 0,003704 \\
\hline \multicolumn{7}{|l|}{ JOB5 } \\
\hline 0 & 280 & 2581 & 0,660399 & 0,768493 & $-0,15159$ & 0,016386 \\
\hline \multirow[t]{2}{*}{1} & 84 & 1327 & 0,339601 & 0,231507 & 0,383162 & 0,041418 \\
\hline & & & & & & 0,057803 \\
\hline \multicolumn{7}{|l|}{ JOB6 } \\
\hline 0 & 346 & 3687 & 0,943336 & 0,949315 & $-0,00632$ & 3,78E-05 \\
\hline \multirow[t]{2}{*}{1} & 18 & 221 & 0,056664 & 0,050685 & 0,111512 & 0,000667 \\
\hline & & & & 2 & 2 & 0,000705 \\
\hline
\end{tabular}




\begin{tabular}{|c|c|c|c|c|c|c|}
\hline JOB1 & & & & & & \\
\hline 0 & 270 & 3444 & 0,881172 & 0,741096 & 0,173122 & 0,02425 \\
\hline \multirow[t]{2}{*}{1} & 94 & 464 & 0,118828 & 0,258904 & $-0,77878$ & 0,109088 \\
\hline & & & & & & 0,133338 \\
\hline \multicolumn{7}{|l|}{ JOB2 } \\
\hline 0 & 301 & 3224 & 0,824891 & 0,826027 & $-0,00138$ & $1,56 \mathrm{E}-06$ \\
\hline \multirow[t]{2}{*}{1} & 63 & 684 & 0,175109 & 0,173973 & 0,006509 & $7,4 \mathrm{E}-06$ \\
\hline & & & & & & 8,96E-06 \\
\hline \multicolumn{7}{|l|}{ JOB3 } \\
\hline 0 & 281 & 3034 & 0,776285 & 0,771233 & 0,00653 & 3,3E-05 \\
\hline \multirow[t]{2}{*}{1} & 83 & 874 & 0,223715 & 0,228767 & $-0,02233$ & 0,000113 \\
\hline & & & & & & 0,000146 \\
\hline \multicolumn{7}{|l|}{ JOB4 } \\
\hline 0 & 345 & 3668 & 0,938475 & 0,946575 & $-0,00859$ & $6,96 \mathrm{E}-05$ \\
\hline \multirow[t]{2}{*}{1} & 19 & 240 & 0,061525 & 0,053425 & 0,141166 & 0,001143 \\
\hline & & & & & & 0,001213 \\
\hline \multicolumn{7}{|l|}{ JOB5 } \\
\hline 0 & 277 & 2488 & 0,636608 & 0,760274 & $-0,17753$ & 0,021954 \\
\hline \multirow[t]{2}{*}{1} & 87 & 1420 & 0,363392 & 0,239726 & 0,415986 & 0,051443 \\
\hline & & & & & & 0,073397 \\
\hline \multicolumn{7}{|l|}{ JOB6 } \\
\hline 0 & 346 & 3682 & 0,942057 & 0,949315 & $-0,00768$ & 5,57E-05 \\
\hline \multirow[t]{2}{*}{1} & 18 & 226 & 0,057943 & 0,050685 & 0,133835 & 0,000971 \\
\hline & & & & 24 & 2 & 0,001027 \\
\hline
\end{tabular}




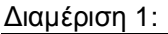

\begin{tabular}{|c|c|c|c|c|c|c|}
\hline \multicolumn{7}{|l|}{ AGE1 } \\
\hline 0 & 146 & 2375 & 0,6077 & 0,40137 & 0,414798 & 0,085585 \\
\hline \multirow[t]{2}{*}{1} & 218 & 1533 & 0,3923 & 0,59863 & $-0,42262$ & 0,087199 \\
\hline & & & & & & 0,172784 \\
\hline \multicolumn{7}{|l|}{ AGE2 } \\
\hline 0 & 295 & 2099 & 0,537094 & 0,809589 & $-0,41035$ & 0,111819 \\
\hline \multirow[t]{2}{*}{1} & 69 & 1809 & 0,462906 & 0,190411 & 0,88834 & 0,242068 \\
\hline & & & & & & 0,353888 \\
\hline \multicolumn{7}{|l|}{ AGE3 } \\
\hline 0 & 287 & 3342 & 0,855078 & 0,787671 & 0,082112 & 0,005535 \\
\hline \multirow[t]{2}{*}{1} & 77 & 566 & 0,144922 & 0,212329 & $-0,38194$ & 0,025745 \\
\hline & & & & & & 0,03128 \\
\hline
\end{tabular}

\begin{tabular}{|c|c|c|c|c|c|c|}
\hline AGE1 & & & & & & 8 \\
\hline 0 & 136 & 2358 & 0,603351 & 0,373973 & 0,478317 & 0,109716 \\
\hline \multirow[t]{2}{*}{1} & 228 & 1550 & 0,396649 & 0,626027 & $-0,45634$ & 0,104675 \\
\hline & & & & & & 0,214391 \\
\hline \multicolumn{7}{|l|}{ AGE2 } \\
\hline 0 & 300 & 2111 & 0,540164 & 0,823288 & $-0,42143$ & 0,119318 \\
\hline \multirow[t]{2}{*}{1} & 64 & 1797 & 0,459836 & 0,176712 & 0,956347 & 0,270765 \\
\hline & & & & & $\nabla$ & 0,390083 \\
\hline \multicolumn{7}{|l|}{ AGE3 } \\
\hline 0 & 292 & 3347 & 0,856357 & 0,80137 & 0,066365 & 0,003649 \\
\hline \multirow[t]{2}{*}{1} & 72 & 561 & 0,143643 & 0,19863 & $-0,32411$ & 0,017822 \\
\hline & & & & & & 0,021471 \\
\hline
\end{tabular}




\begin{tabular}{|c|c|c|c|c|c|c|}
\hline AGE1 & & & & & & \\
\hline 0 & 138 & 2348 & 0,600793 & 0,379452 & 0,459522 & 0,101711 \\
\hline \multirow[t]{2}{*}{1} & 226 & 1560 & 0,399207 & 0,620548 & $-0,44112$ & 0,097639 \\
\hline & & & & & & 0,19935 \\
\hline \multicolumn{7}{|l|}{ AGE2 } \\
\hline 0 & 299 & 2111 & 0,540164 & 0,820548 & $-0,4181$ & 0,117229 \\
\hline \multirow[t]{2}{*}{1} & 65 & 1797 & 0,459836 & 0,179452 & 0,940962 & 0,263831 \\
\hline & & & & & & 0,38106 \\
\hline \multicolumn{7}{|l|}{ AGE3 } \\
\hline 0 & 291 & 3357 & 0,858915 & 0,79863 & 0,072772 & 0,004387 \\
\hline \multirow[t]{2}{*}{1} & 73 & 551 & 0,141085 & 0,20137 & $-0,35578$ & 0,021448 \\
\hline & & & & & & 0,025836 \\
\hline
\end{tabular}

\begin{tabular}{|c|c|c|c|c|c|c|}
\hline AGE1 & & & & & & + \\
\hline 0 & 131 & 2363 & 0,60463 & 0,360274 & 0,517752 & 0,126516 \\
\hline \multirow[t]{2}{*}{1} & 233 & 1545 & 0,39537 & 0,639726 & $-0,48122$ & 0,117589 \\
\hline & & & & & 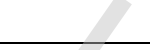 & 0,244105 \\
\hline AGE2 & & & & & + & $\Delta$ \\
\hline 0 & 286 & 1915 & 0,490023 & 0,784932 & $-0,47114$ & 0,138944 \\
\hline \multirow[t]{2}{*}{1} & 78 & 1993 & 0,509977 & 0,215068 & 0,863409 & 0,254627 \\
\hline & & & & & 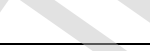 & 0,393571 \\
\hline \multicolumn{7}{|l|}{ AGE3 } \\
\hline 0 & 311 & 3538 & 0,905219 & 0,853425 & 0,058919 & 0,003052 \\
\hline \multirow[t]{2}{*}{1} & 53 & 370 & 0,094781 & 0,146575 & $-0,43597$ & 0,022581 \\
\hline & & & & - & $t$ & 0,025632 \\
\hline
\end{tabular}




\begin{tabular}{|c|c|c|c|c|c|c|}
\hline \multicolumn{7}{|l|}{ AGE1 } \\
\hline 0 & 133 & 2388 & 0,611026 & 0,365753 & 0,51318 & 0,125869 \\
\hline \multirow[t]{2}{*}{1} & 231 & 1520 & 0,388974 & 0,634247 & $-0,48892$ & 0,11992 \\
\hline & & & & & & 0,245789 \\
\hline \multicolumn{7}{|l|}{ AGE2 } \\
\hline 0 & 298 & 2099 & 0,537094 & 0,817808 & $-0,42045$ & 0,118028 \\
\hline \multirow[t]{2}{*}{1} & 66 & 1809 & 0,462906 & 0,182192 & 0,932464 & 0,261756 \\
\hline & & & & & & 0,379784 \\
\hline \multicolumn{7}{|l|}{ AGE3 } \\
\hline 0 & 297 & 3329 & 0,851752 & 0,815068 & 0,044024 & 0,001615 \\
\hline \multirow[t]{2}{*}{1} & 67 & 579 & 0,148248 & 0,184932 & $-0,2211$ & 0,008111 \\
\hline & & & & & & 0,009726 \\
\hline
\end{tabular}

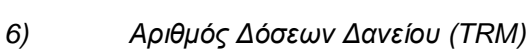

\begin{tabular}{|c|c|c|c|c|c|c|}
\hline TRM1 & & & & & & 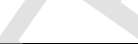 \\
\hline 0 & 327 & 2935 & 0,750959 & 0,89726 & $-0,17799$ & 0,026041 \\
\hline \multirow[t]{2}{*}{1} & 37 & 973 & 0,249041 & 0,10274 & 0,885417 & 0,129537 \\
\hline & & & & & & 0,155578 \\
\hline \multicolumn{7}{|l|}{ TRM2 } \\
\hline 0 & 92 & 1376 & 0,352136 & 0,253425 & 0,328951 & 0,032471 \\
\hline \multirow[t]{2}{*}{1} & 272 & 2532 & 0,647864 & 0,746575 & $-0,14182$ & 0,013999 \\
\hline & & & & & $\nabla$ & 0,04647 \\
\hline \multicolumn{7}{|l|}{ TRM3 } \\
\hline 0 & 309 & 3505 & 0,896777 & 0,847945 & 0,055991 & 0,002734 \\
\hline \multirow[t]{2}{*}{1} & 55 & 403 & 0,103223 & 0,152055 & $-0,38735$ & 0,018915 \\
\hline & & & & & & 0,021649 \\
\hline
\end{tabular}




\begin{tabular}{|c|c|c|c|c|c|c|}
\hline \multicolumn{7}{|l|}{ TRM1 } \\
\hline 0 & 333 & 2947 & 0,754029 & 0,913699 & $-0,19207$ & 0,030668 \\
\hline \multirow[t]{2}{*}{1} & 31 & 961 & 0,245971 & 0,086301 & 1,047368 & 0,167233 \\
\hline & & & & & & 0,1979 \\
\hline \multicolumn{7}{|l|}{ TRM2 } \\
\hline 0 & 78 & 1345 & 0,344206 & 0,215068 & 0,470283 & 0,060731 \\
\hline \multirow[t]{2}{*}{1} & 286 & 2563 & 0,655794 & 0,784932 & $-0,17975$ & 0,023212 \\
\hline & & & & & & 0,083943 \\
\hline \multicolumn{7}{|l|}{ TRM3 } \\
\hline 0 & 317 & 3524 & 0,901637 & 0,869863 & 0,035877 & 0,00114 \\
\hline \multirow[t]{2}{*}{1} & 47 & 384 & 0,098363 & 0,130137 & $-0,27993$ & 0,008894 \\
\hline & & & & & & 0,010034 \\
\hline
\end{tabular}

\begin{tabular}{|c|c|c|c|c|c|c|}
\hline TRM1 & & & & & & +2 \\
\hline 0 & 336 & 2980 & 0,762471 & 0,921918 & $-0,18989$ & 0,030278 \\
\hline \multirow[t]{2}{*}{1} & 28 & 928 & 0,237529 & 0,078082 & 1,112527 & 0,177389 \\
\hline & & & & & 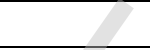 & 0,207666 \\
\hline TRM2 & & & & & $\square$ & 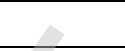 \\
\hline 0 & 80 & 1304 & 0,333717 & 0,220548 & 0,414178 & 0,046872 \\
\hline \multirow[t]{2}{*}{1} & 284 & 2604 & 0,666283 & 0,779452 & $-0,15688$ & 0,017754 \\
\hline & & & & & & 0,064626 \\
\hline \multicolumn{7}{|l|}{ TRM3 } \\
\hline 0 & 312 & 3532 & 0,903684 & 0,856164 & 0,054017 & 0,002567 \\
\hline \multirow[t]{2}{*}{1} & 52 & 376 & 0,096316 & 0,143836 & $-0,40103$ & 0,019057 \\
\hline & & & & r & 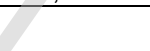 & 0,021624 \\
\hline
\end{tabular}




\begin{tabular}{|c|c|c|c|c|c|c|}
\hline TRM1 & & & & & & \\
\hline 0 & 333 & 3006 & 0,769123 & 0,913699 & $-0,17225$ & 0,024903 \\
\hline \multirow[t]{2}{*}{1} & 31 & 902 & 0,230877 & 0,086301 & 0,984042 & 0,142269 \\
\hline & & & & & & 0,167172 \\
\hline \multicolumn{7}{|l|}{ TRM2 } \\
\hline 0 & 80 & 1266 & 0,323996 & 0,220548 & 0,384616 & 0,039788 \\
\hline \multirow[t]{2}{*}{1} & 284 & 2642 & 0,676004 & 0,779452 & $-0,14239$ & 0,01473 \\
\hline & & & & & & 0,054518 \\
\hline \multicolumn{7}{|l|}{ TRM3 } \\
\hline 0 & 315 & 3544 & 0,906754 & 0,864384 & 0,047854 & 0,002028 \\
\hline \multirow[t]{2}{*}{1} & 49 & 364 & 0,093246 & 0,135616 & $-0,37459$ & 0,015871 \\
\hline & & & & & & 0,017899 \\
\hline
\end{tabular}

\begin{tabular}{|c|c|c|c|c|c|c|}
\hline TRM1 & & & & & & 5 \\
\hline 0 & 334 & 3043 & 0,778588 & 0,916438 & $-0,16301$ & 0,022471 \\
\hline \multirow[t]{2}{*}{1} & 30 & 865 & 0,221412 & 0,083562 & 0,974441 & 0,134327 \\
\hline & & & & & 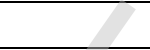 & 0,156799 \\
\hline TRM2 & & & & & 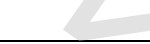 & $\Delta$ \\
\hline 0 & 87 & 1242 & 0,317856 & 0,239726 & 0,282102 & 0,022041 \\
\hline \multirow[t]{2}{*}{1} & 277 & 2666 & 0,682144 & 0,760274 & $-0,10844$ & 0,008472 \\
\hline & & & & & & 0,030513 \\
\hline \multicolumn{7}{|l|}{ TRM3 } \\
\hline 0 & 307 & 3531 & 0,903428 & 0,842466 & 0,069863 & 0,004259 \\
\hline \multirow[t]{2}{*}{1} & 57 & 377 & 0,096572 & 0,157534 & $-0,48935$ & 0,029832 \\
\hline & & & & 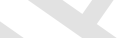 & 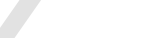 & 0,034091 \\
\hline
\end{tabular}




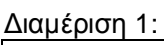

\begin{tabular}{|c|c|c|c|c|c|c|}
\hline \multicolumn{7}{|c|}{ HYR1 } \\
\hline 0 & 166 & 3110 & 0,795728 & 0,456164 & 0,556404 & 0,188934 \\
\hline \multirow[t]{2}{*}{1} & 198 & 798 & 0,204272 & 0,543836 & $-0,97919$ & 0,332498 \\
\hline & & & & & & 0,521433 \\
\hline \multicolumn{7}{|l|}{ HYR2 } \\
\hline 0 & 283 & 2241 & 0,57342 & 0,776712 & $-0,30345$ & 0,061689 \\
\hline \multirow[t]{2}{*}{1} & 81 & 1667 & 0,42658 & 0,223288 & 0,647338 & 0,131599 \\
\hline & & & & & & 0,193288 \\
\hline \multicolumn{7}{|c|}{ HYR3 } \\
\hline 0 & 285 & 2714 & 0,694423 & 0,782192 & $-0,11902$ & 0,010446 \\
\hline \multirow[t]{2}{*}{1} & 79 & 1194 & 0,305577 & 0,217808 & 0,338586 & 0,029717 \\
\hline & & & & & 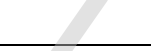 & 0,040163 \\
\hline \multicolumn{2}{|l|}{ HYR4 } & & & & 8 & 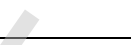 \\
\hline 0 & 358 & 3659 & 0,936173 & 0,982192 & $-0,04799$ & 0,002208 \\
\hline \multirow[t]{2}{*}{1} & 6 & 249 & 0,063827 & 0,017808 & 1,276517 & 0,058744 \\
\hline & & & & 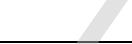 & 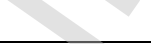 & 0,060952 \\
\hline
\end{tabular}




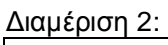

\begin{tabular}{|c|c|c|c|c|c|c|}
\hline \multicolumn{7}{|c|}{ HYR1 } \\
\hline 0 & 169 & 3117 & 0,797519 & 0,464384 & 0,540794 & 0,180157 \\
\hline \multirow[t]{2}{*}{1} & 195 & 791 & 0,202481 & 0,535616 & $-0,97277$ & 0,324064 \\
\hline & & & & & & 0,504221 \\
\hline \multicolumn{7}{|c|}{ HYR2 } \\
\hline 0 & 275 & 2205 & 0,564211 & 0,754795 & $-0,29102$ & 0,055463 \\
\hline \multirow[t]{2}{*}{1} & 89 & 1703 & 0,435789 & 0,245205 & 0,575062 & 0,109597 \\
\hline & & & & & & 0,165061 \\
\hline \multicolumn{2}{|c|}{ HYR3 } & & & & & -1 \\
\hline 0 & 289 & 2736 & 0,700051 & 0,793151 & $-0,12486$ & 0,011624 \\
\hline \multirow[t]{2}{*}{1} & 75 & 1172 & 0,299949 & 0,206849 & 0,371621 & 0,034598 \\
\hline & & & & & $\Delta$ & 0,046222 \\
\hline \multicolumn{7}{|l|}{ HYR4 } \\
\hline 0 & 359 & 3666 & 0,937964 & 0,984932 & $-0,04886$ & 0,002295 \\
\hline \multirow[t]{2}{*}{1} & 5 & 242 & 0,062036 & 0,015068 & 1,415114 & 0,066465 \\
\hline & & & & & & 0,06876 \\
\hline
\end{tabular}




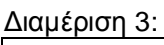

\begin{tabular}{|c|c|c|c|c|c|c|}
\hline \multicolumn{7}{|l|}{ HYR1 } \\
\hline 0 & 165 & 3089 & 0,790356 & 0,453425 & 0,555654 & 0,187217 \\
\hline \multirow[t]{2}{*}{1} & 199 & 819 & 0,209644 & 0,546575 & $-0,95826$ & 0,322867 \\
\hline & & & & & & 0,510084 \\
\hline \multicolumn{7}{|l|}{ HYR2 } \\
\hline 0 & 278 & 2209 & 0,565234 & 0,763014 & $-0,30004$ & 0,059341 \\
\hline \multirow[t]{2}{*}{1} & 86 & 1699 & 0,434766 & 0,236986 & 0,606805 & 0,120014 \\
\hline & & & & & & 0,179355 \\
\hline \multicolumn{7}{|l|}{ HYR3 } \\
\hline 0 & 287 & 2774 & 0,709772 & 0,787671 & $-0,10414$ & 0,008112 \\
\hline \multirow[t]{2}{*}{1} & 77 & 1134 & 0,290228 & 0,212329 & 0,31253 & 0,024346 \\
\hline & & & & & 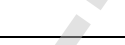 & 0,032458 \\
\hline \multicolumn{7}{|l|}{ HYR4 } \\
\hline 0 & 362 & 3652 & 0,934382 & 0,993151 & $-0,061$ & 0,003585 \\
\hline \multirow[t]{2}{*}{1} & 2 & 256 & 0,065618 & 0,006849 & 2,259698 & 0,132799 \\
\hline & & & & & & 0,136384 \\
\hline
\end{tabular}




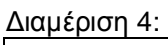

\begin{tabular}{|c|c|c|c|c|c|c|}
\hline \multicolumn{7}{|l|}{ HYR1 } \\
\hline 0 & 173 & 3089 & 0,790356 & 0,475342 & 0,508447 & 0,160168 \\
\hline \multirow[t]{2}{*}{1} & 191 & 819 & 0,209644 & 0,524658 & $-0,91733$ & 0,288972 \\
\hline & & & & & & 0,44914 \\
\hline \multicolumn{7}{|l|}{ HYR2 } \\
\hline 0 & 279 & 2224 & 0,569071 & 0,765753 & $-0,29685$ & 0,058386 \\
\hline \multirow[t]{2}{*}{1} & 85 & 1684 & 0,430929 & 0,234247 & 0,609568 & 0,119891 \\
\hline & & & & & & 0,178277 \\
\hline \multicolumn{7}{|l|}{ HYR3 } \\
\hline 0 & 281 & 2749 & 0,703377 & 0,771233 & $-0,0921$ & 0,006249 \\
\hline \multirow[t]{2}{*}{1} & 83 & 1159 & 0,296623 & 0,228767 & 0,259758 & 0,017626 \\
\hline & & & & & $\Delta$ & 0,023876 \\
\hline \multicolumn{7}{|l|}{ HYR4 } \\
\hline 0 & 359 & 3662 & 0,93694 & 0,984932 & $-0,04995$ & 0,002397 \\
\hline \multirow[t]{2}{*}{1} & 5 & 246 & 0,06306 & 0,015068 & 1,431474 & 0,068698 \\
\hline & & & & & & 0,071095 \\
\hline
\end{tabular}




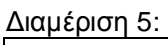

\begin{tabular}{|c|c|c|c|c|c|c|}
\hline \multicolumn{7}{|c|}{ HYR1 } \\
\hline 0 & 163 & 3099 & 0,792914 & 0,447945 & 0,571044 & 0,196992 \\
\hline \multirow[t]{2}{*}{1} & 201 & 809 & 0,207086 & 0,552055 & $-0,98051$ & 0,338246 \\
\hline & & & & & & 0,535238 \\
\hline \multicolumn{7}{|c|}{ HYR2 } \\
\hline 0 & 283 & 2268 & 0,580327 & 0,776712 & $-0,29148$ & 0,057242 \\
\hline \multirow[t]{2}{*}{1} & 81 & 1640 & 0,419673 & 0,223288 & 0,631014 & 0,123922 \\
\hline & & & & & & 0,181163 \\
\hline \multicolumn{7}{|c|}{ HYR3 } \\
\hline 0 & 288 & 2705 & 0,692121 & 0,790411 & $-0,13279$ & 0,013052 \\
\hline \multirow[t]{2}{*}{1} & 76 & 1203 & 0,307879 & 0,209589 & 0,384559 & 0,037798 \\
\hline & & & & & $\Delta$ & 0,050851 \\
\hline \multicolumn{7}{|c|}{ HYR4 } \\
\hline 0 & 358 & 3652 & 0,934382 & 0,982192 & $-0,0499$ & 0,002386 \\
\hline \multirow[t]{2}{*}{1} & 6 & 256 & 0,065618 & 0,017808 & 1,304187 & 0,062353 \\
\hline & & & & & 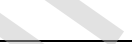 & 0,064738 \\
\hline
\end{tabular}




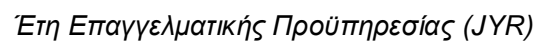

\begin{tabular}{|c|c|c|c|c|c|c|}
\hline JYR1 & & & & & & \\
\hline 0 & 243 & 3106 & 0,794705 & 0,667123 & 0,174996 & 0,022326 \\
\hline \multirow[t]{2}{*}{1} & 121 & 802 & 0,205295 & 0,332877 & $-0,48332$ & 0,061663 \\
\hline & & & & & & 0,083989 \\
\hline \multicolumn{7}{|l|}{ JYR2 } \\
\hline 0 & 248 & 2900 & 0,742006 & 0,680822 & 0,086056 & 0,005265 \\
\hline \multirow[t]{2}{*}{1} & 116 & 1008 & 0,257994 & 0,319178 & $-0,21281$ & 0,013021 \\
\hline & & & & & & 0,018286 \\
\hline JYR3 & & & & & & 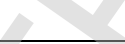 \\
\hline 0 & 308 & 3257 & 0,833333 & 0,845205 & $-0,01415$ & 0,000168 \\
\hline \multirow[t]{2}{*}{1} & 56 & 651 & 0,166667 & 0,154795 & 0,073897 & 0,000877 \\
\hline & & & & & 2 & 0,001045 \\
\hline JYR4 & & & & & 2 & 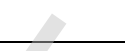 \\
\hline 0 & 293 & 2461 & 0,629701 & 0,80411 & $-0,24449$ & 0,042641 \\
\hline \multirow[t]{2}{*}{1} & 71 & 1447 & 0,370299 & 0,19589 & 0,636756 & 0,111056 \\
\hline & & & & & 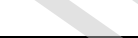 & 0,153697 \\
\hline
\end{tabular}




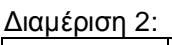

\begin{tabular}{|c|c|c|c|c|c|c|}
\hline JYR1 & & & & & & \\
\hline 0 & 246 & 3110 & 0,795728 & 0,675342 & 0,164037 & 0,019748 \\
\hline \multirow[t]{2}{*}{1} & 118 & 798 & 0,204272 & 0,324658 & $-0,46332$ & 0,055777 \\
\hline & & & & & & 0,075524 \\
\hline \multicolumn{7}{|l|}{ JYR2 } \\
\hline 0 & 241 & 2774 & 0,709772 & 0,661644 & 0,070217 & 0,003379 \\
\hline \multirow[t]{2}{*}{1} & 123 & 1134 & 0,290228 & 0,338356 & $-0,15343$ & 0,007385 \\
\hline & & & & & & 0,010764 \\
\hline \multicolumn{7}{|l|}{ JYR3 } \\
\hline 0 & 318 & 3350 & 0,857125 & 0,872603 & $-0,0179$ & 0,000277 \\
\hline \multirow[t]{2}{*}{1} & 46 & 558 & 0,142875 & 0,127397 & 0,114663 & 0,001775 \\
\hline & & & & & $\Delta$ & 0,002052 \\
\hline JYR4 & & & & & 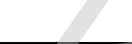 & \\
\hline 0 & 287 & 2490 & 0,637119 & 0,787671 & $-0,21212$ & 0,031936 \\
\hline \multirow[t]{2}{*}{1} & 77 & 1418 & 0,362881 & 0,212329 & 0,535938 & 0,080686 \\
\hline & & & & & & 0,112622 \\
\hline
\end{tabular}




\begin{tabular}{|c|c|c|c|c|c|c|}
\hline JYR1 & & & & & & \\
\hline 0 & 236 & 3094 & 0,791635 & 0,647945 & 0,200294 & 0,02878 \\
\hline \multirow[t]{2}{*}{1} & 128 & 814 & 0,208365 & 0,352055 & $-0,52449$ & 0,075364 \\
\hline & & & & & & 0,104144 \\
\hline \multicolumn{7}{|l|}{ JYR2 } \\
\hline 0 & 256 & 2794 & 0,714889 & 0,70274 & 0,01714 & 0,000208 \\
\hline \multirow[t]{2}{*}{1} & 108 & 1114 & 0,285111 & 0,29726 & $-0,04173$ & 0,000507 \\
\hline & & & & & & 0,000715 \\
\hline JYR3 & & & & & & $\Delta+$ \\
\hline 0 & 317 & 3358 & 0,859171 & 0,869863 & $-0,01237$ & 0,000132 \\
\hline \multirow[t]{2}{*}{1} & 47 & 550 & 0,140829 & 0,130137 & 0,078958 & 0,000844 \\
\hline & & & & & $\Delta$ & 0,000976 \\
\hline \multicolumn{7}{|l|}{ JYR4 } \\
\hline 0 & 283 & 2478 & 0,63405 & 0,776712 & $-0,20294$ & 0,028952 \\
\hline \multirow[t]{2}{*}{1} & 81 & 1430 & 0,36595 & 0,223288 & 0,494037 & 0,070481 \\
\hline & & & & & & 0,099433 \\
\hline
\end{tabular}




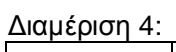

\begin{tabular}{|c|c|c|c|c|c|c|}
\hline JYR1 & & & & & & \\
\hline 0 & 282 & 3397 & 0,869148 & 0,773973 & 0,115977 & 0,011038 \\
\hline \multirow[t]{2}{*}{1} & 82 & 511 & 0,130852 & 0,226027 & $-0,54659$ & 0,052022 \\
\hline & & & & & & 0,06306 \\
\hline \multicolumn{7}{|l|}{ JYR2 } \\
\hline 0 & 223 & 2550 & 0,652469 & 0,612329 & 0,063494 & 0,002549 \\
\hline \multirow[t]{2}{*}{1} & 141 & 1358 & 0,347531 & 0,387671 & $-0,1093$ & 0,004387 \\
\hline & & & & & & 0,006936 \\
\hline \multicolumn{7}{|l|}{ JYR3 } \\
\hline 0 & 305 & 3264 & 0,835124 & 0,836986 & $-0,00223$ & $4,15 \mathrm{E}-06$ \\
\hline \multirow[t]{2}{*}{1} & 59 & 644 & 0,164876 & 0,163014 & 0,011359 & $2,12 \mathrm{E}-05$ \\
\hline & & & & & $\Delta$ & 2,53E-05 \\
\hline JYR4 & & & & & 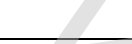 & \\
\hline 0 & 282 & 2513 & 0,643003 & 0,773973 & $-0,18539$ & 0,02428 \\
\hline \multirow[t]{2}{*}{1} & 82 & 1395 & 0,356997 & 0,226027 & 0,45707 & 0,059862 \\
\hline & & & & & & 0,084142 \\
\hline
\end{tabular}




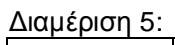

\begin{tabular}{|c|c|c|c|c|c|c|}
\hline JYR1 & & & & & & \\
\hline 0 & 240 & 3106 & 0,794705 & 0,658904 & 0,187392 & 0,025448 \\
\hline \multirow[t]{2}{*}{1} & 124 & 802 & 0,205295 & 0,341096 & $-0,50771$ & 0,068948 \\
\hline & & & & & & 0,094396 \\
\hline \multicolumn{7}{|l|}{ JYR2 } \\
\hline 0 & 256 & 2894 & 0,740471 & 0,70274 & 0,052299 & 0,001973 \\
\hline \multirow[t]{2}{*}{1} & 108 & 1014 & 0,259529 & 0,29726 & $-0,13574$ & 0,005122 \\
\hline & & & & & & 0,007095 \\
\hline \multicolumn{7}{|l|}{ JYR3 } \\
\hline 0 & 309 & 3266 & 0,835636 & 0,847945 & $-0,01462$ & 0,00018 \\
\hline \multirow[t]{2}{*}{1} & 55 & 642 & 0,164364 & 0,152055 & 0,077844 & 0,000958 \\
\hline & & & & & $\Delta$ & 0,001138 \\
\hline \multicolumn{7}{|l|}{ JYR4 } \\
\hline 0 & 287 & 2458 & 0,628933 & 0,787671 & $-0,22506$ & 0,035725 \\
\hline \multirow[t]{2}{*}{1} & 77 & 1450 & 0,371067 & 0,212329 & 0,558246 & 0,088615 \\
\hline & & & & & & 0,12434 \\
\hline
\end{tabular}




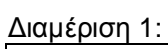

\begin{tabular}{|c|c|c|c|c|c|c|}
\hline INC1 & & & & & & \\
\hline 0 & 207 & 3264 & 0,835124 & 0,568493 & 0,384591 & 0,102544 \\
\hline \multirow[t]{2}{*}{1} & 157 & 644 & 0,164876 & 0,431507 & $-0,96209$ & 0,256523 \\
\hline & & & & & & 0,359067 \\
\hline \multicolumn{7}{|l|}{ INC2 } \\
\hline 0 & 278 & 3193 & 0,816961 & 0,763014 & 0,068315 & 0,003685 \\
\hline \multirow[t]{2}{*}{1} & 86 & 715 & 0,183039 & 0,236986 & $-0,2583$ & 0,013935 \\
\hline & & & & & & 0,01762 \\
\hline INC3 & & & & & & $2+2$ \\
\hline 0 & 249 & 1888 & 0,483116 & 0,683562 & $-0,34706$ & 0,069567 \\
\hline \multirow[t]{2}{*}{1} & 115 & 2020 & 0,516884 & 0,316438 & 0,49069 & 0,098357 \\
\hline & & & & & 2 & 0,167924 \\
\hline INC4 & & & & & 2 & 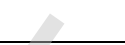 \\
\hline 0 & 358 & 3379 & 0,864543 & 0,982192 & $-0,12759$ & 0,01501 \\
\hline \multirow[t]{2}{*}{1} & 6 & 529 & 0,135457 & 0,017808 & 2,028991 & 0,238708 \\
\hline & & & & & & 0,253718 \\
\hline
\end{tabular}




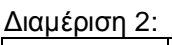

\begin{tabular}{|c|c|c|c|c|c|c|}
\hline INC1 & & & & & & \\
\hline 0 & 203 & 3269 & 0,836403 & 0,557534 & 0,405587 & 0,113106 \\
\hline \multirow[t]{2}{*}{1} & 161 & 639 & 0,163597 & 0,442466 & $-0,99496$ & 0,277463 \\
\hline & & & & & & 0,390568 \\
\hline \multicolumn{7}{|l|}{ INC2 } \\
\hline 0 & 272 & 3198 & 0,81824 & 0,746575 & 0,091659 & 0,006569 \\
\hline \multirow[t]{2}{*}{1} & 92 & 710 & 0,18176 & 0,253425 & $-0,33238$ & 0,02382 \\
\hline & & & & & & 0,030389 \\
\hline \multicolumn{7}{|l|}{ INC3 } \\
\hline 0 & 257 & 1879 & 0,480814 & 0,705479 & $-0,3834$ & 0,086137 \\
\hline \multirow[t]{2}{*}{1} & 107 & 2029 & 0,519186 & 0,294521 & 0,566914 & 0,127366 \\
\hline & & & & & $\Delta$ & 0,213503 \\
\hline \multicolumn{7}{|l|}{ INC4 } \\
\hline 0 & 360 & 3378 & 0,864288 & 0,987671 & $-0,13344$ & 0,016465 \\
\hline \multirow[t]{2}{*}{1} & 4 & 530 & 0,135712 & 0,012329 & 2,398603 & 0,295948 \\
\hline & & & & & ( & 0,312413 \\
\hline
\end{tabular}




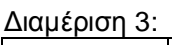

\begin{tabular}{|c|c|c|c|c|c|c|}
\hline \multicolumn{7}{|l|}{ INC1 } \\
\hline 0 & 198 & 3272 & 0,837171 & 0,543836 & 0,431381 & 0,126539 \\
\hline \multirow[t]{2}{*}{1} & 166 & 636 & 0,162829 & 0,456164 & $-1,03015$ & 0,302179 \\
\hline & & & & & & 0,428718 \\
\hline \multicolumn{7}{|l|}{ INC2 } \\
\hline 0 & 274 & 3200 & 0,818752 & 0,752055 & 0,084972 & 0,005667 \\
\hline \multirow[t]{2}{*}{1} & 90 & 708 & 0,181248 & 0,247945 & $-0,31334$ & 0,020899 \\
\hline & & & & & & 0,026566 \\
\hline \multicolumn{7}{|l|}{ INC3 } \\
\hline 0 & 262 & 1872 & 0,479023 & 0,719178 & $-0,40636$ & 0,09759 \\
\hline \multirow[t]{2}{*}{1} & 102 & 2036 & 0,520977 & 0,280822 & 0,617986 & 0,148413 \\
\hline & & & & & $\Delta$ & 0,246002 \\
\hline \multicolumn{7}{|l|}{ INC4 } \\
\hline 0 & 358 & 3380 & 0,864799 & 0,982192 & $-0,12729$ & 0,014943 \\
\hline \multirow[t]{2}{*}{1} & 6 & 528 & 0,135201 & 0,017808 & 2,027101 & 0,237967 \\
\hline & & & & & 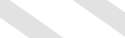 & 0,252909 \\
\hline
\end{tabular}




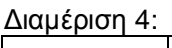

\begin{tabular}{|c|c|c|c|c|c|c|}
\hline \multicolumn{7}{|l|}{ INC1 } \\
\hline 0 & 197 & 3274 & 0,837682 & 0,541096 & 0,437042 & 0,129621 \\
\hline \multirow[t]{2}{*}{1} & 167 & 634 & 0,162318 & 0,458904 & $-1,03929$ & 0,308238 \\
\hline & & & & & & 0,437859 \\
\hline \multicolumn{7}{|l|}{ INC2 } \\
\hline 0 & 275 & 3196 & 0,817728 & 0,754795 & 0,080085 & 0,00504 \\
\hline \multirow[t]{2}{*}{1} & 89 & 712 & 0,182272 & 0,245205 & $-0,2966$ & 0,018666 \\
\hline & & & & & & 0,023706 \\
\hline \multicolumn{7}{|l|}{ INC3 } \\
\hline 0 & 262 & 1874 & 0,479534 & 0,719178 & $-0,40529$ & 0,097126 \\
\hline \multirow[t]{2}{*}{1} & 102 & 2034 & 0,520466 & 0,280822 & 0,617003 & 0,147861 \\
\hline & & & & & $\Delta$ & 0,244987 \\
\hline \multicolumn{7}{|l|}{ INC4 } \\
\hline 0 & 358 & 3380 & 0,864799 & 0,982192 & $-0,12729$ & 0,014943 \\
\hline \multirow[t]{2}{*}{1} & 6 & 528 & 0,135201 & 0,017808 & 2,027101 & 0,237967 \\
\hline & & & & & ( & 0,252909 \\
\hline
\end{tabular}




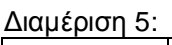

\begin{tabular}{|c|c|c|c|c|c|c|}
\hline \multicolumn{7}{|l|}{ INC1 } \\
\hline 0 & 198 & 3272 & 0,837171 & 0,543836 & 0,431381 & 0,126539 \\
\hline \multirow[t]{2}{*}{1} & 166 & 636 & 0,162829 & 0,456164 & $-1,03015$ & 0,302179 \\
\hline & & & & & & 0,428718 \\
\hline \multicolumn{7}{|l|}{ INC2 } \\
\hline 0 & 273 & 3199 & 0,818496 & 0,749315 & 0,088309 & 0,006109 \\
\hline \multirow[t]{2}{*}{1} & 91 & 709 & 0,181504 & 0,250685 & $-0,32292$ & 0,02234 \\
\hline & & & & & & 0,028449 \\
\hline \multicolumn{7}{|l|}{ INC3 } \\
\hline 0 & 259 & 1877 & 0,480302 & 0,710959 & $-0,3922$ & 0,090464 \\
\hline \multirow[t]{2}{*}{1} & 105 & 2031 & 0,519698 & 0,289041 & 0,586679 & 0,135322 \\
\hline & & & & & $\Delta$ & 0,225785 \\
\hline \multicolumn{7}{|l|}{ INC4 } \\
\hline 0 & 362 & 3376 & 0,863776 & 0,993151 & $-0,13957$ & 0,018057 \\
\hline \multirow[t]{2}{*}{1} & 2 & 532 & 0,136224 & 0,006849 & 2,990153 & 0,38685 \\
\hline & & & & & ( & 0,404907 \\
\hline
\end{tabular}




\section{ПAPAPTHMA E}

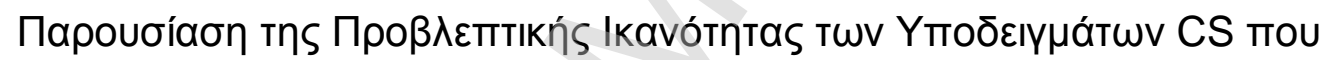

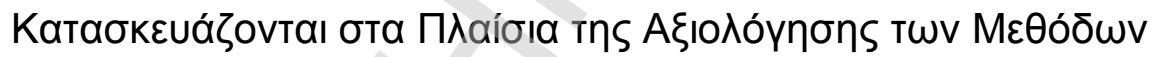




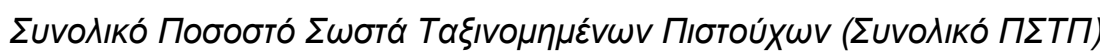
(Percentage of Correctly Classified Cases-PCC)

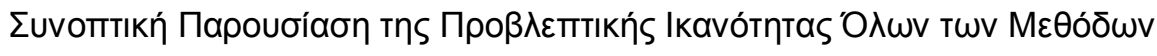

1) $\quad M k \Pi \Gamma \Sigma$

\begin{tabular}{|rrrrrrr|}
\hline \multicolumn{1}{l|}{ k-NN } & \multicolumn{1}{l|}{} & & & \\
\hline Partition & BA & PCC Bads(\%) & GR & PCC Goods(\%) & TM & PCC Total(\%) \\
\hline 1 & 38 & 58,24175824 & 18 & 98,15762538 & 56 & 94,75655431 \\
\hline 2 & 49 & 46,15384615 & 10 & 98,97645855 & 59 & 94,47565543 \\
\hline 3 & 56 & 38,46153846 & 16 & 98,36233367 & 72 & 93,25842697 \\
\hline 4 & 50 & 45,05494505 & 14 & 98,56704197 & 64 & 94,00749064 \\
\hline 5 & 44 & 51,64835165 & 16 & 98,36233367 & 60 & 94,38202247 \\
\hline Average & & 47,91208791 & & 98,48515865 & & 94,17602996 \\
\hline
\end{tabular}

Yтó

Partition: $\triangle$ ıа

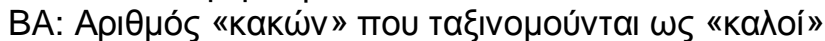

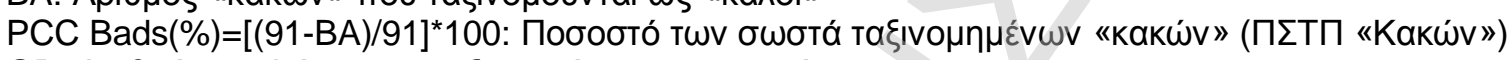

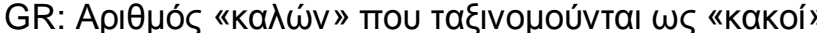

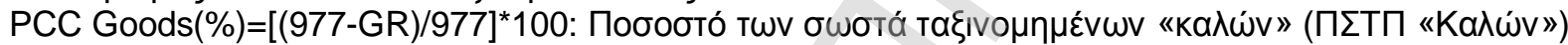

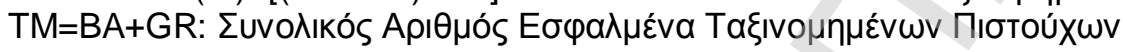

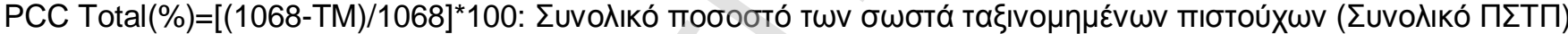

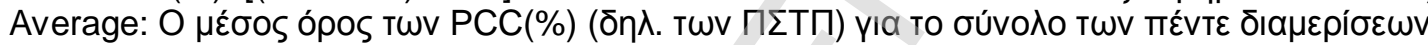


NN

\begin{tabular}{|r|r|r|r|r|r|r|}
\hline Partition & BA & PCC Bads(\%) & GR & PCC Goods(\%) & TM & PCC Total(\%) \\
\hline 1 & 30 & 67,03296703 & 29 & 97,03172979 & 59 & 94,47565543 \\
\hline 2 & 35 & 61,53846154 & 24 & 97,54350051 & 59 & 94,47565543 \\
\hline 3 & 36 & 60,43956044 & 30 & 96,92937564 & 66 & 93,82022472 \\
\hline 4 & 28 & 69,23076923 & 34 & 96,51995906 & 62 & 94,19475655 \\
\hline 5 & 40 & 56,04395604 & 29 & 97,03172979 & 69 & 93,53932584 \\
\hline Average & & 62,85714286 & & 97,01125896 & & 94,1011236 \\
\hline
\end{tabular}

3)

$\Delta T$

\section{CT}

\begin{tabular}{|r|r|r|r|r|r|r|}
\hline \multicolumn{1}{|l}{ Partition } & BA & PCC Bads(\%) & GR & PCC Goods(\%) & TM & PCC Total(\%) \\
\hline 1 & 47 & 48,35164835 & 19 & 98,05527124 & 66 & 93,82022472 \\
\hline 2 & 43 & 52,74725275 & 16 & 98,36233367 & 59 & 94,47565543 \\
\hline 3 & 48 & 47,25274725 & 26 & 97,33879222 & 74 & 93,07116105 \\
\hline 4 & 57 & 37,36263736 & 10 & 98,97645855 & 67 & 93,72659176 \\
\hline 5 & 47 & 48,35164835 & 20 & 97,95291709 & 67 & 93,72659176 \\
\hline Average & & 46,81318681 & & 98,13715455 & & 93,76404494 \\
\hline
\end{tabular}

4)

$\wedge \Pi$

LRA

\begin{tabular}{|r|r|r|r|r|r|r|}
\hline Partition & BA & PCC Bads(\%) & GR & PCC Goods(\%) & TM & PCC Total(\%) \\
\hline 1 & 46 & 49,45054945 & 28 & 97,13408393 & 74 & 93,07116105 \\
\hline 2 & 48 & 47,25274725 & 10 & 98,97645855 & 58 & 94,56928839 \\
\hline 3 & 54 & 40,65934066 & 14 & 98,56704197 & 68 & 93,6329588 \\
\hline 4 & 48 & 47,25274725 & 21 & 97,85056295 & 69 & 93,53932584 \\
\hline 5 & 52 & 42,85714286 & 22 & 97,7482088 & 74 & 93,07116105 \\
\hline Average & & 45,49450549 & & 98,05527124 & & 93,57677903 \\
\hline
\end{tabular}




\section{LDA}

\begin{tabular}{|r|r|r|r|r|r|r|}
\hline Partition & BA & PCC Bads(\%) & GR & PCC Goods(\%) & TM & PCC Total(\%) \\
\hline 1 & 46 & 49,45054945 & 32 & 96,72466735 & 78 & 92,69662921 \\
\hline 2 & 50 & 45,05494505 & 24 & 97,54350051 & 74 & 93,07116105 \\
\hline 3 & 49 & 46,15384615 & 32 & 96,72466735 & 81 & 92,41573034 \\
\hline 4 & 50 & 45,05494505 & 28 & 97,13408393 & 78 & 92,69662921 \\
\hline 5 & 45 & 50,54945055 & 40 & 95,90583419 & 85 & 92,0411985 \\
\hline Average & & 47,25274725 & & 96,80655067 & & 92,58426966 \\
\hline
\end{tabular}

6)

$K A B$

\begin{tabular}{|r|r|c|r|r|r|r|}
\hline \multicolumn{1}{|l|}{ NBR } \\
\hline Partition & BA & PCC Bads(\%) & GR & PCC Goods(\%) & TM & PCC Total $(\%)$ \\
\hline 1 & 39 & 57,14285714 & 57 & 94,16581372 & 96 & 91,01123596 \\
\hline 2 & 37 & 59,34065934 & 42 & 95,7011259 & 79 & 92,60299625 \\
\hline 3 & 51 & 43,95604396 & 48 & 95,08700102 & 99 & 90,73033708 \\
\hline 4 & 45 & 50,54945055 & 47 & 95,18935517 & 92 & 91,38576779 \\
\hline 5 & 45 & 50,54945055 & 60 & 93,85875128 & 105 & 90,16853933 \\
\hline Average & & 52,30769231 & & 94,80040942 & & 91,17977528 \\
\hline
\end{tabular}




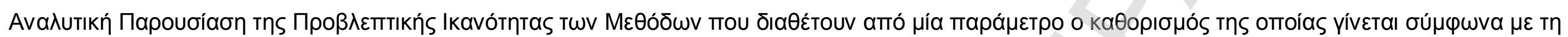

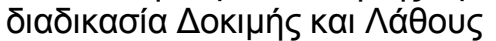

\section{1) $\quad M k \Pi \Gamma \Sigma$}

$\Delta$ ıане́pıơ 1:

\begin{tabular}{|c|c|c|c|c|c|c|c|c|c|c|}
\hline 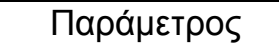 & \multicolumn{10}{|c|}{$\mathrm{K}$} \\
\hline тро К KaӨopıбно́ & $\mathrm{k}=1$ & $\mathrm{k}=2$ & $k=3$ & $\mathrm{k}=4$ & $\mathrm{k}=5$ & $\mathrm{k}=6$ & $\mathrm{k}=7$ & $\mathrm{k}=8$ & $\mathrm{k}=9$ & $\mathrm{~K}=10$ \\
\hline BA & 41 & 53 & 38 & 50 & 44 & 53 & 47 & 49 & 46 & 52 \\
\hline GR & 36 & 8 & 18 & 13 & 22 & 16 & 22 & 15 & 19 & 14 \\
\hline TM & 77 & 61 & 56 & 63 & 66 & 69 & 69 & 64 & 65 & 66 \\
\hline
\end{tabular}

$\triangle$ ıане́pıơ 2:

\begin{tabular}{|c|c|c|c|c|c|c|c|c|c|c|}
\hline \multirow{2}{*}{ 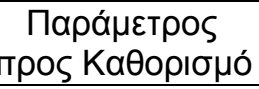 } & \multicolumn{10}{|c|}{$\bar{K}$} \\
\hline & $\mathrm{k}=1$ & $k=2$ & $k=3$ & $\mathrm{k}=4$ & $\mathrm{k}=5$ & $\mathrm{k}=6$ & $\mathrm{k}=7$ & $\mathrm{k}=8$ & $k=9$ & $K=10$ \\
\hline BA & 46 & 54 & 44 & 55 & 46 & 55 & 46 & 51 & 49 & 54 \\
\hline GR & 44 & 14 & 16 & 1 & 16 & 6 & 15 & 11 & 10 & 5 \\
\hline TM & 90 & 68 & 60 & 66 & 62 & 61 & 61 & 62 & 59 & 59 \\
\hline
\end{tabular}

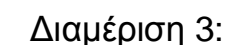

\begin{tabular}{|c|c|c|c|c|c|c|c|c|c|c|}
\hline \multirow{2}{*}{ 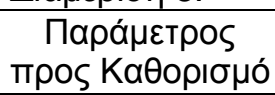 } & \multicolumn{10}{|c|}{$\mathrm{K}$} \\
\hline & $k=1$ & $k=2$ & $\mathrm{k}=3$ & $\mathrm{k}=4$ & $k=5$ & $k=6$ & $k=7$ & $\mathrm{k}=8$ & $k=9$ & $\mathrm{~K}=10$ \\
\hline BA & 52 & 59 & 52 & 62 & 53 & 56 & 55 & 60 & 57 & 57 \\
\hline GR & 38 & 19 & 25 & 10 & 20 & 16 & 21 & 17 & 18 & 15 \\
\hline TM & 90 & 78 & 77 & 72 & 73 & 72 & 76 & 77 & 75 & 72 \\
\hline
\end{tabular}

$\Delta$ ıан́́pıơ 4:

\begin{tabular}{|c|c|c|c|c|c|c|c|c|c|c|}
\hline \multirow{2}{*}{ 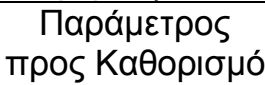 } & \multicolumn{10}{|c|}{$\mathrm{K}$} \\
\hline & $\mathrm{k}=1$ & $k=2$ & $\mathrm{k}=3$ & $\mathrm{k}=4$ & $\mathrm{k}=5$ & $\mathrm{k}=6$ & $k=7$ & $\mathrm{~K}=8$ & $\mathrm{k}=9$ & $\mathrm{k}=10$ \\
\hline BA & 47 & 61 & 47 & 53 & 51 & 54 & 50 & 55 & 53 & 56 \\
\hline GR & 39 & 15 & 20 & 15 & 18 & 10 & 14 & 11 & 16 & 11 \\
\hline$\overline{\mathrm{TM}}$ & 86 & 76 & 67 & 68 & 69 & 64 & 64 & 66 & 69 & 67 \\
\hline
\end{tabular}




\begin{tabular}{|c|c|c|c|c|c|c|c|c|c|c|}
\hline \multirow{2}{*}{ 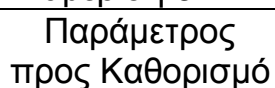 } & \multicolumn{10}{|c|}{$\mathrm{K}$} \\
\hline & $\mathrm{k}=1$ & $\mathrm{k}=2$ & $\mathrm{k}=3$ & $\mathrm{k}=4$ & $k=5$ & $\mathrm{k}=6$ & $\mathrm{k}=7$ & $\mathrm{~K}=8$ & $\mathrm{k}=9$ & $\mathrm{k}=10$ \\
\hline BA & 45 & 52 & 46 & 50 & 44 & 47 & 46 & 49 & 47 & 50 \\
\hline GR & 39 & 17 & 26 & 13 & 16 & 13 & 19 & 16 & 18 & 14 \\
\hline TM & 84 & 69 & 72 & 63 & 60 & 60 & 65 & 65 & 65 & 64 \\
\hline
\end{tabular}

2) $\quad N \Delta$

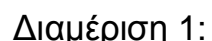

\begin{tabular}{|c|c|c|c|c|c|c|c|c|c|c|}
\hline Пара́ & \multicolumn{10}{|c|}{$\mathrm{N}$} \\
\hline трољ KaӨорıбно́ & $\mathrm{N}=16$ & $\mathrm{~N}=17$ & $\mathrm{~N}=18$ & $\mathrm{~N}=19$ & $\mathrm{~N}=20$ & $\mathrm{~N}=21$ & $\mathrm{~N}=22$ & $\mathrm{~N}=23$ & $N=24$ & $\mathrm{~N}=25$ \\
\hline BA & 30 & 37 & 27 & 26 & 27 & 32 & 30 & 26 & 32 & 36 \\
\hline GR & 32 & 35 & 36 & 39 & 34 & 29 & 29 & 41 & 29 & 31 \\
\hline TM & 62 & 72 & 63 & 65 & 61 & 61 & 59 & 67 & 61 & 67 \\
\hline
\end{tabular}

\begin{tabular}{|c|c|c|c|c|c|c|c|c|c|c|}
\hline \multirow{2}{*}{ 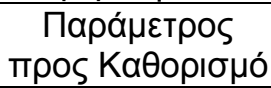 } & \multicolumn{10}{|c|}{$\mathrm{N}$} \\
\hline & $\mathrm{N}=16$ & $\mathrm{~N}=17$ & $\mathrm{~N}=18$ & $\mathrm{~N}=19$ & $\mathrm{~N}=20$ & $\mathrm{~N}=21$ & $\mathrm{~N}=22$ & $\mathrm{~N}=23$ & $\mathrm{~N}=24$ & $\mathrm{~N}=25$ \\
\hline BA & 38 & 41 & 38 & 39 & 38 & 36 & 39 & 35 & 40 & 41 \\
\hline GR & 25 & 28 & 26 & 31 & 28 & 27 & 29 & 24 & 22 & 23 \\
\hline TM & 63 & 69 & 64 & 70 & 66 & 63 & 68 & 59 & 62 & 64 \\
\hline
\end{tabular}

\begin{tabular}{|c|c|c|c|c|c|c|c|c|c|c|}
\hline \multirow{2}{*}{ 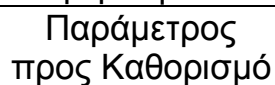 } & \multicolumn{10}{|c|}{$\bar{N}$} \\
\hline & $\mathrm{N}=16$ & $\mathrm{~N}=17$ & $\mathrm{~N}=18$ & $\mathrm{~N}=19$ & $\mathrm{~N}=20$ & $\mathrm{~N}=21$ & $\mathrm{~N}=22$ & $\mathrm{~N}=23$ & $\mathrm{~N}=24$ & $\mathrm{~N}=25$ \\
\hline BA & 44 & 41 & 46 & 42 & 41 & 44 & 36 & 43 & 45 & 39 \\
\hline GR & 25 & 33 & 25 & 28 & 25 & 29 & 30 & 25 & 23 & 34 \\
\hline TM & 69 & 74 & 71 & 70 & 66 & 73 & 66 & 68 & 68 & 73 \\
\hline
\end{tabular}




\begin{tabular}{|c|c|c|c|c|c|c|c|c|c|c|}
\hline \multirow{2}{*}{ 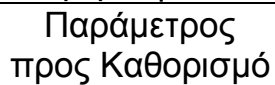 } & \multicolumn{10}{|c|}{$\mathrm{N}$} \\
\hline & $\mathrm{N}=16$ & $\mathrm{~N}=17$ & $\mathrm{~N}=18$ & $\mathrm{~N}=19$ & $\mathrm{~N}=20$ & $\mathrm{~N}=21$ & $\mathrm{~N}=22$ & $\mathrm{~N}=23$ & $\mathrm{~N}=24$ & $\mathrm{~N}=25$ \\
\hline $\mathrm{BA}$ & 37 & 41 & 38 & 36 & 45 & 41 & 28 & 38 & 41 & 43 \\
\hline GR & 33 & 37 & 35 & 29 & 32 & 31 & 34 & 37 & 32 & 30 \\
\hline TM & 70 & 78 & 73 & 65 & 77 & 72 & 62 & 75 & 73 & 73 \\
\hline
\end{tabular}

\begin{tabular}{|c|c|c|c|c|c|c|c|c|c|c|}
\hline \multirow{2}{*}{ 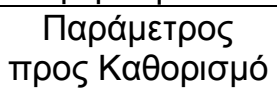 } & \multicolumn{10}{|c|}{$\mathrm{N}$} \\
\hline & $\mathrm{N}=16$ & $\mathrm{~N}=17$ & $\mathrm{~N}=18$ & $\mathrm{~N}=19$ & $\mathrm{~N}=20$ & $\mathrm{~N}=21$ & $\mathrm{~N}=22$ & $\mathrm{~N}=23$ & $\mathrm{~N}=24$ & $N=25$ \\
\hline BA & 39 & 40 & 38 & 41 & 41 & 39 & 39 & 39 & 35 & 40 \\
\hline GR & 31 & 29 & 35 & 32 & 35 & 33 & 36 & 33 & 36 & 36 \\
\hline TM & 70 & 69 & 73 & 73 & 76 & 72 & 75 & 72 & 71 & 76 \\
\hline
\end{tabular}

3)

$\Delta T$

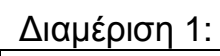

\begin{tabular}{|c|c|c|c|c|c|c|c|c|c|c|}
\hline \multirow{2}{*}{ 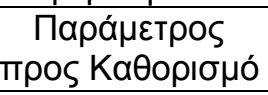 } & \multicolumn{10}{|c|}{$P$} \\
\hline & $P=0,1 \%$ & $P=0,2 \%$ & $P=0,3 \%$ & $P=0,4 \%$ & $P=0,5 \%$ & $P=0,6 \%$ & $P=0,7 \%$ & $P=0,8 \%$ & $P=0,9 \%$ & $P=1,0 \%$ \\
\hline $\mathrm{BA}$ & 38 & 37 & 47 & 47 & 44 & 49 & 47 & 54 & 54 & 54 \\
\hline GR & 36 & 30 & 19 & 19 & 25 & 24 & 32 & 31 & 31 & 31 \\
\hline TM & 74 & 67 & 66 & 66 & 69 & 73 & 79 & 85 & 85 & 85 \\
\hline
\end{tabular}

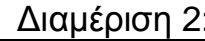

\begin{tabular}{|c|c|c|c|c|c|c|c|c|c|c|}
\hline \multirow{2}{*}{ 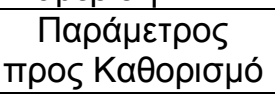 } & \multicolumn{10}{|c|}{$P$} \\
\hline & $P=0,1 \%$ & $P=0,2 \%$ & $P=0,3 \%$ & $P=0,4 \%$ & $P=0,5 \%$ & $P=0,6 \%$ & $P=0,7 \%$ & $P=0,8 \%$ & $\mathrm{P}=0,9 \%$ & $P=1,0 \%$ \\
\hline BA & 40 & 44 & 49 & 44 & 43 & 41 & 41 & 49 & 52 & 52 \\
\hline GR & 21 & 23 & 18 & 17 & 16 & 19 & 19 & 16 & 15 & 15 \\
\hline TM & 61 & 67 & 67 & 61 & 59 & 60 & 60 & 65 & 67 & 67 \\
\hline
\end{tabular}




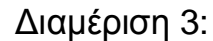

\begin{tabular}{|c|c|c|c|c|c|c|c|c|c|c|}
\hline Пара́ & \multicolumn{10}{|c|}{$P$} \\
\hline трољ КаӨорıбно́ & $P=0,1 \%$ & $P=0,2 \%$ & $P=0,3 \%$ & $\mathrm{P}=0,4 \%$ & $P=0,5 \%$ & $\mathrm{P}=0,6 \%$ & $\mathrm{P}=0,7 \%$ & $P=0,8 \%$ & $P=0,9 \%$ & $P=1,0 \%$ \\
\hline BA & 51 & 48 & 51 & 55 & 52 & 56 & 67 & 67 & 67 & 68 \\
\hline GR & 31 & 26 & 32 & 25 & 27 & 27 & 21 & 21 & 21 & 17 \\
\hline TM & 82 & 74 & 83 & 80 & 79 & 83 & 88 & 88 & 88 & 85 \\
\hline
\end{tabular}

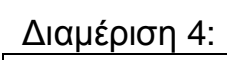

\begin{tabular}{|c|c|c|c|c|c|c|c|c|c|c|}
\hline 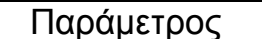 & \multicolumn{10}{|c|}{$\mathrm{P}$} \\
\hline тро К KaӨорıбно́ & $P=0,1 \%$ & $P=0,2 \%$ & $P=0,3 \%$ & $P=0,4 \%$ & $P=0,5 \%$ & $P=0,6 \%$ & $P=0,7 \%$ & $P=0,8 \%$ & $P=0,9 \%$ & $P=1,0 \%$ \\
\hline $\mathrm{BA}$ & 49 & 53 & 51 & 56 & 57 & 51 & 57 & 57 & 57 & 57 \\
\hline GR & 38 & 18 & 18 & 13 & 10 & 19 & 12 & 12 & 12 & 12 \\
\hline TM & 87 & 71 & 69 & 69 & 67 & 70 & 69 & 69 & 69 & 69 \\
\hline
\end{tabular}

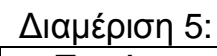

\begin{tabular}{|c|c|c|c|c|c|c|c|c|c|c|}
\hline \multirow{2}{*}{ 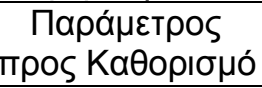 } & \multicolumn{10}{|c|}{$P$} \\
\hline & $P=0,1 \%$ & $P=0,2 \%$ & $P=0,3 \%$ & $P=0,4 \%$ & $P=0,5 \%$ & $P=0,6 \%$ & $P=0,7 \%$ & $P=0,8 \%$ & $P=0,9 \%$ & $P=1,0 \%$ \\
\hline $\mathrm{BA}$ & 42 & 48 & 48 & 47 & 44 & 47 & 45 & 43 & 47 & 51 \\
\hline GR & 32 & 24 & 23 & 20 & 31 & 31 & 28 & 35 & 28 & 20 \\
\hline TM & 74 & 72 & 71 & 67 & 75 & 78 & 73 & 78 & 75 & 71 \\
\hline
\end{tabular}




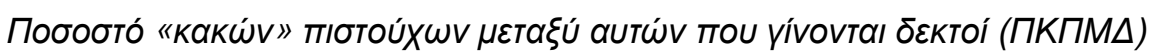
(Bad Rate amongst Accepts-BRA)

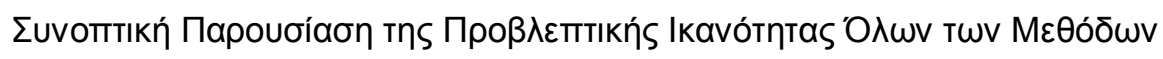

1) $\quad N \Delta$

\begin{tabular}{|c|c|c|c|c|c|c|c|c|c|c|}
\hline \multicolumn{11}{|l|}{$\mathrm{NN}$} \\
\hline & \multicolumn{10}{|c|}{ Accept Rate } \\
\hline & \multicolumn{2}{|c|}{$70 \%(748)$} & \multicolumn{2}{|c|}{$75 \%(801)$} & \multicolumn{2}{|c|}{$80 \%(854)$} & \multicolumn{2}{|c|}{$85 \%(908)$} & \multicolumn{2}{|c|}{$90 \%(961)$} \\
\hline Partition & $\mathrm{BA}$ & BRA (\%) & $\mathrm{BA}$ & BRA (\%) & $\mathrm{BA}$ & BRA (\%) & $\mathrm{BA}$ & BRA (\%) & $\mathrm{BA}$ & BRA (\%) \\
\hline 1 & 5 & 0,668449 & 5 & 0,62422 & 7 & 0,819672 & 13 & 1,431718 & 27 & 2,809573 \\
\hline 2 & 3 & 0,40107 & 4 & 0,499376 & 12 & 1,405152 & 17 & 1,872247 & 28 & 2,913632 \\
\hline 3 & 6 & 0,802139 & 7 & 0,873908 & 12 & 1,405152 & 20 & 2,202643 & 28 & 2,913632 \\
\hline 4 & 3 & 0,40107 & 7 & 0,873908 & 10 & 1,17096 & 19 & 2,092511 & 30 & 3,121748 \\
\hline 5 & 9 & 1,203209 & 10 & 1,248439 & 15 & 1,75644 & 21 & 2,312775 & 31 & 3,225806 \\
\hline Average & & 0,695187 & & 0,82397 & & 1,311475 & & 1,982379 & & 2,996878 \\
\hline
\end{tabular}

Yтópuvnu:

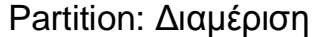

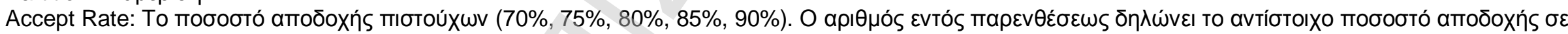

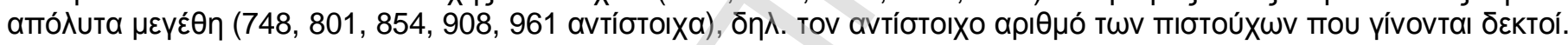

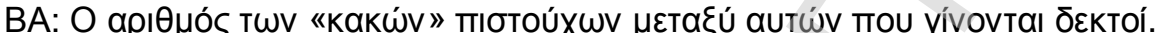

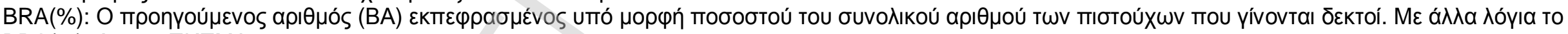
BRA(\%) gíval To ПKПM $\Delta$.

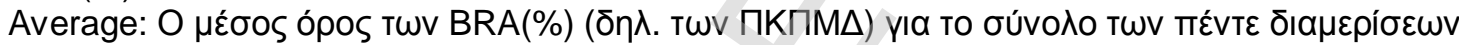




\begin{tabular}{|c|c|c|c|c|c|c|c|c|c|c|}
\hline \multicolumn{11}{|l|}{ LRA } \\
\hline & \multicolumn{10}{|c|}{ Accept Rate } \\
\hline Partition & \multicolumn{2}{|c|}{$70 \%(748)$} & \multicolumn{2}{|c|}{$75 \%(801)$} & \multicolumn{2}{|c|}{$80 \%(854)$} & \multicolumn{2}{|c|}{$85 \%(908)$} & \multicolumn{2}{|c|}{$90 \%(961)$} \\
\hline 1 & 6 & 0,802139 & 10 & 1,248439 & 13 & 1,522248 & 18 & 1,982379 & 33 & 3,433923 \\
\hline 2 & 5 & 0,668449 & 8 & 0,998752 & 10 & 1,17096 & 15 & 1,651982 & 23 & 2,39334 \\
\hline 3 & 6 & 0,802139 & 8 & 0,998752 & 13 & 1,522248 & 23 & 2,53304 & 34 & 3,537981 \\
\hline 4 & 4 & 0,534759 & 11 & 1,373283 & 14 & 1,639344 & 19 & 2,092511 & 32 & 3,329865 \\
\hline 5 & 9 & 1,203209 & 14 & 1,747815 & 17 & 1,990632 & 25 & 2,753304 & 35 & 3,64204 \\
\hline Average & & 0,802139 & & 1,273408 & & 1,569087 & & 2,202643 & & 3,26743 \\
\hline
\end{tabular}

3)

\section{$\Gamma \Delta A$}

\begin{tabular}{|c|c|c|c|c|c|c|c|c|c|c|}
\hline \multicolumn{11}{|l|}{ LDA } \\
\hline & \multicolumn{10}{|c|}{ Accept Rate } \\
\hline Partition & \multicolumn{2}{|c|}{$70 \%(748)$} & \multicolumn{2}{|c|}{$75 \%(801)$} & \multicolumn{2}{|c|}{$80 \%(854)$} & \multicolumn{2}{|c|}{$85 \%(908)$} & \multicolumn{2}{|c|}{$90 \%(961)$} \\
\hline 1 & 6 & 0,802139 & 10 & 1,248439 & 14 & 1,639344 & 23 & 2,53304 & 34 & 3,537981 \\
\hline 2 & 7 & 0,935829 & 11 & 1,373283 & 12 & 1,405152 & 16 & 1,762115 & 29 & 3,01769 \\
\hline 3 & 7 & 0,935829 & 13 & 1,622971 & 15 & 1,75644 & 21 & 2,312775 & 37 & 3,850156 \\
\hline 4 & 5 & 0,668449 & 8 & 0,998752 & 14 & 1,639344 & 19 & 2,092511 & 38 & 3,954214 \\
\hline 5 & 8 & 1,069519 & 12 & 1,498127 & 15 & 1,75644 & 22 & 2,422907 & 38 & 3,954214 \\
\hline Average & & 0,882353 & & 1,348315 & $\nabla$ & 1,639344 & & 2,22467 & & 3,662851 \\
\hline
\end{tabular}




\begin{tabular}{|c|c|c|c|c|c|c|c|c|c|c|}
\hline \multicolumn{11}{|l|}{ CT } \\
\hline & \multicolumn{10}{|c|}{ Accept Rate } \\
\hline Partition & \multicolumn{2}{|c|}{$70 \%(748)$} & \multicolumn{2}{|c|}{$75 \%(801)$} & \multicolumn{2}{|c|}{$80 \%(854)$} & \multicolumn{2}{|c|}{$85 \%(908)$} & \multicolumn{2}{|c|}{$90 \%(961)$} \\
\hline 1 & 8 & 1,069519 & 10 & 1,248439 & 16 & 1,873536 & 17 & 1,872247 & 30 & 3,121748 \\
\hline 2 & 11 & 1,470588 & 14 & 1,747815 & 17 & 1,990632 & 24 & 2,643172 & 29 & 3,01769 \\
\hline 3 & 8 & 1,069519 & 10 & 1,248439 & 15 & 1,75644 & 25 & 2,753304 & 34 & 3,537981 \\
\hline 4 & 7 & 0,935829 & 8 & 0,998752 & 14 & 1,639344 & 20 & 2,202643 & 33 & 3,433923 \\
\hline 5 & 10 & 1,336898 & 11 & 1,373283 & 18 & 2,107728 & 22 & 2,422907 & 32 & 3,329865 \\
\hline Average & & 1176471 & & 1.323346 & & 1873536 & & 2378855 & & 3288241 \\
\hline
\end{tabular}

5) $М k \Pi \Gamma \Sigma$

\begin{tabular}{|c|c|c|c|c|c|c|c|c|c|c|}
\hline \multicolumn{11}{|l|}{ k-NN } \\
\hline & \multicolumn{10}{|c|}{ Accept Rate } \\
\hline Partition & \multicolumn{2}{|c|}{$70 \%(748)$} & \multicolumn{2}{|c|}{$75 \%(801)$} & \multicolumn{2}{|c|}{$80 \%(854)$} & \multicolumn{2}{|c|}{$85 \%(908)$} & \multicolumn{2}{|c|}{$90 \%(961)$} \\
\hline 1 & 7 & 0,935829 & 9 & 1,123596 & 18 & 2,107728 & 18 & 1,982379 & 28 & 2,913632 \\
\hline 2 & 12 & 1,604278 & 16 & 1,997503 & 17 & 1,990632 & 21 & 2,312775 & 27 & 2,809573 \\
\hline 3 & 12 & 1,604278 & 12 & 1,498127 & 18 & 2,107728 & 27 & 2,973568 & 36 & 3,746098 \\
\hline 4 & 8 & 1,069519 & 9 & 1,123596 & 19 & 2,224824 & 20 & 2,202643 & 33 & 3,433923 \\
\hline 5 & 11 & 1,470588 & 17 & 2,122347 & 17 & 1,990632 & 29 & 3,193833 & 34 & 3,537981 \\
\hline Average & & 1,336898 & & 1,573034 & $\nabla$ & 2,084309 & & 2,53304 & & 3,288241 \\
\hline
\end{tabular}


6) $K A B$

\begin{tabular}{|c|c|c|c|c|c|c|c|c|c|c|}
\hline \multicolumn{11}{|l|}{ NBR } \\
\hline & \multicolumn{10}{|c|}{ Accept Rate } \\
\hline Partition & \multicolumn{2}{|c|}{$70 \%(748)$} & \multicolumn{2}{|c|}{$75 \%(801)$} & \multicolumn{2}{|c|}{$80 \%(854)$} & \multicolumn{2}{|c|}{$85 \%(908)$} & \multicolumn{2}{|c|}{$90 \%(961)$} \\
\hline 1 & 12 & 1,604278 & 17 & 2,122347 & 23 & 2,693208 & 29 & 3,193833 & 40 & 4,162331 \\
\hline 2 & 11 & 1,470588 & 15 & 1,872659 & 19 & 2,224824 & 24 & 2,643172 & 33 & 3,433923 \\
\hline 3 & 14 & 1,871658 & 20 & 2,496879 & 27 & 3,161593 & 35 & 3,854626 & 47 & 4,890739 \\
\hline 4 & 8 & 1,069519 & 14 & 1,747815 & 20 & 2,34192 & 27 & 2,973568 & 41 & 4,266389 \\
\hline 5 & 15 & 2,005348 & 20 & 2,496879 & 26 & 3,044496 & 34 & 3,744493 & 45 & 4,682622 \\
\hline Average & & 1,604278 & & 2,147316 & & 2,693208 & 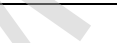 & 3,281938 & & 4,287201 \\
\hline
\end{tabular}




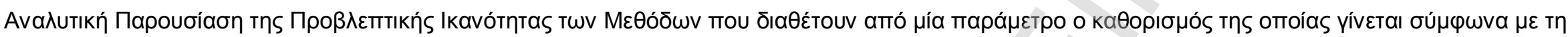

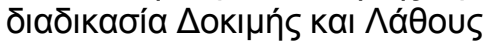

1) $N \Delta$

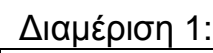

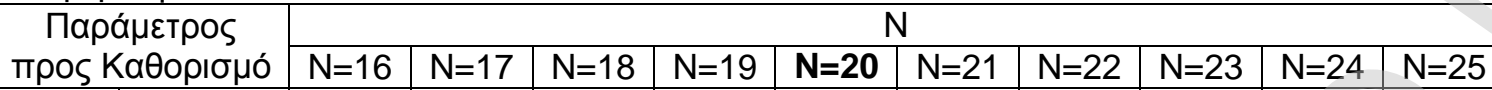

\begin{tabular}{|l|r|r|r|r|r|r|r|r|r|r|r|r|}
\hline \multirow{2}{*}{ 은 } & $90 \%$ & 24 & 25 & 25 & 26 & $\mathbf{2 7}$ & 23 & 24 & 26 & 24 & 26 \\
\cline { 2 - 13 } & $85 \%$ & 18 & 17 & 16 & 18 & $\mathbf{1 3}$ & 17 & 16 & 17 & 17 & 14 \\
\cline { 2 - 11 }
\end{tabular}

\begin{tabular}{|r|r|r|r|r|r|r|r|r|r|r|r|}
\multirow{2}{*}{ 은 운 } & $80 \%$ & 12 & 11 & 11 & 11 & $\mathbf{7}$ & 13 & 12 & 8 & 12 & 10 \\
\cline { 2 - 12 } & $75 \%$ & 8 & 7 & 8 & 4 & $\mathbf{5}$ & 12 & 5 & 7 & 8 & 5 \\
\cline { 2 - 11 } & $70 \%$ & 5 & 5 & 3 & 2 & $\mathbf{5}$ & 8 & 5 & 5 & 6 & 5 \\
\hline
\end{tabular}

\begin{tabular}{|c|c|c|c|c|c|c|c|c|c|c|c|}
\hline \multirow{2}{*}{\multicolumn{2}{|c|}{ 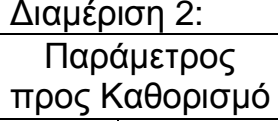 }} & \multicolumn{10}{|c|}{$\mathrm{N}$} \\
\hline & & $\mathrm{N}=16$ & $\mathrm{~N}=17$ & $\mathrm{~N}=18$ & $\mathrm{~N}=19$ & $\mathrm{~N}=20$ & $\mathrm{~N}=21$ & $\mathrm{~N}=22$ & $\mathrm{~N}=23$ & $\mathrm{~N}=24$ & $\mathrm{~N}=25$ \\
\hline \multirow{5}{*}{ 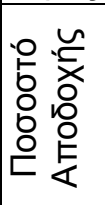 } & $90 \%$ & 28 & 31 & 28 & 26 & 32 & 28 & 29 & 25 & 28 & 27 \\
\hline & $85 \%$ & 19 & 15 & 18 & 20 & 22 & 17 & 18 & 18 & 17 & 18 \\
\hline & $80 \%$ & 13 & 12 & 13 & 14 & 14 & 10 & 11 & 12 & 12 & 10 \\
\hline & $75 \%$ & 10 & 8 & 9 & 9 & 11 & 5 & 9 & 9 & 4 & 8 \\
\hline & $70 \%$ & 9 & 6 & 5 & 4 & 7 & 5 & 6 & 6 & 3 & 6 \\
\hline
\end{tabular}

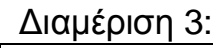

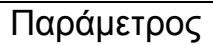

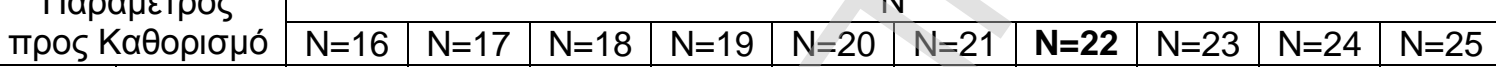

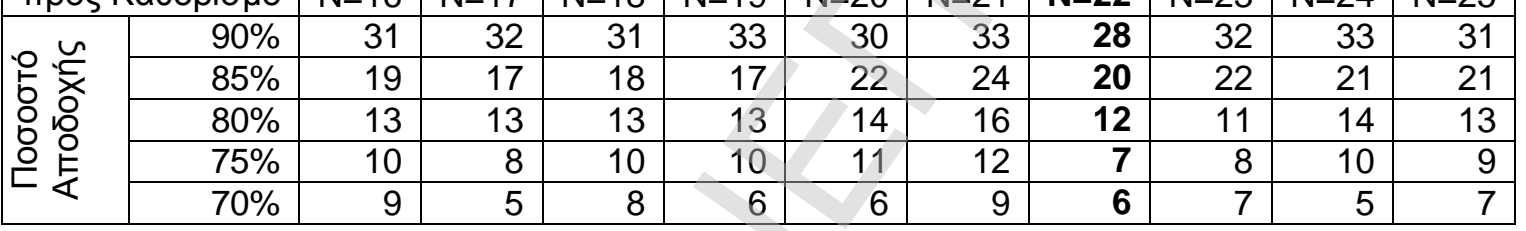




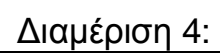

\begin{tabular}{|c|c|c|c|c|c|c|c|c|c|c|}
\hline \multirow{2}{*}{ 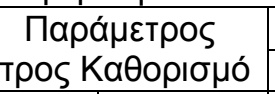 } & \multicolumn{10}{|c|}{$\mathrm{N}$} \\
\hline & $\mathrm{N}=16$ & $\mathrm{~N}=17$ & $\mathrm{~N}=18$ & $\mathrm{~N}=19$ & $\mathrm{~N}=20$ & $\mathrm{~N}=21$ & $\mathrm{~N}=22$ & $\mathrm{~N}=23$ & $\mathrm{~N}=24$ & $\mathrm{~N}=25$ \\
\hline $90 \%$ & 29 & 30 & 29 & 28 & 28 & 32 & 25 & 32 & 30 & 32 \\
\hline $85 \%$ & 18 & 22 & 18 & 19 & 19 & 17 & 21 & 19 & 19 & 21 \\
\hline $80 \%$ & 13 & 13 & 14 & 14 & 13 & 12 & 16 & 14 & 10 & 14 \\
\hline $75 \%$ & 8 & 9 & 9 & $\frac{8}{-1}$ & 8 & 7 & 8 & 7 & 7 & 10 \\
\hline $70 \%$ & 3 & 5 & 3 & 5 & 3 & 4 & 3 & 5 & 3 & \\
\hline
\end{tabular}

\begin{tabular}{|c|c|c|c|c|c|c|c|c|c|c|c|}
\hline \multirow{2}{*}{\multicolumn{2}{|c|}{ 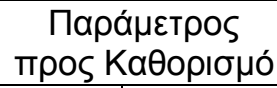 }} & \multicolumn{10}{|c|}{$\mathrm{N}$} \\
\hline & & $\mathrm{N}=16$ & $\mathrm{~N}=17$ & $\mathrm{~N}=18$ & $\mathrm{~N}=19$ & $\mathrm{~N}=20$ & $\mathrm{~N}=21$ & $\mathrm{~N}=22$ & $\mathrm{~N}=23$ & $\mathrm{~N}=24$ & $\mathrm{~N}=25$ \\
\hline \multirow{5}{*}{ 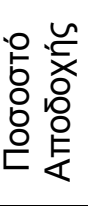 } & $90 \%$ & 33 & 33 & 34 & 33 & 33 & 31 & 31 & 34 & 32 & 33 \\
\hline & $85 \%$ & 22 & 19 & 24 & 25 & 26 & 21 & 19 & 19 & 23 & 24 \\
\hline & $80 \%$ & 13 & 14 & 14 & 18 & 17 & 15 & 14 & 13 & 19 & 14 \\
\hline & $75 \%$ & 11 & 13 & 10 & 12 & 10 & 10 & 12 & 11 & 12 & 11 \\
\hline & $70 \%$ & 9 & 8 & 9 & 9 & 10 & 9 & 10 & 8 & 10 & 7 \\
\hline
\end{tabular}

2)

$\Delta T$

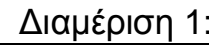

\begin{tabular}{|c|c|c|c|c|c|c|c|c|c|c|}
\hline \multirow{2}{*}{ 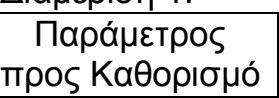 } & \multicolumn{10}{|c|}{$\mathrm{P}$} \\
\hline & $P=0,1 \%$ & $P=0,2 \%$ & $P=0,3 \%$ & $P=0,4 \%$ & $P=0,5 \%$ & $\mathrm{P}=0,6 \%$ & $P=0,7 \%$ & $\mathrm{P}=0,8 \%$ & $\mathrm{P}=0,9 \%$ & $P=1,0 \%$ \\
\hline $90 \%$ & 35 & 30 & 30 & 32 & 31 & 31 & 31 & 37 & 35 & 38 \\
\hline $85 \%$ & 23 & 22 & 17 & 20 & 21 & 19 & 19 & 22 & 24 & 23 \\
\hline $80 \%$ & 14 & 17 & 16 & 16 & 14 & 15 & 17 & 16 & 16 & 16 \\
\hline $75 \%$ & 14 & 12 & 10 & 11 & 12 & 11 & 11 & 14 & 14 & 12 \\
\hline $70 \%$ & 14 & 12 & 8 & 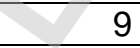 & 9 & 7 & 9 & 10 & 11 & 9 \\
\hline
\end{tabular}




\begin{tabular}{|c|c|c|c|c|c|c|c|c|c|c|c|}
\hline \multirow{2}{*}{\multicolumn{2}{|c|}{ 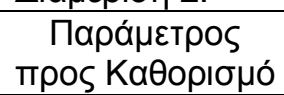 }} & \multicolumn{10}{|c|}{$\mathrm{P}$} \\
\hline & & $\mathrm{P}=0,1 \%$ & $\mathrm{P}=0,2 \%$ & $\mathrm{P}=0,3 \%$ & $P=0,4 \%$ & $P=0,5 \%$ & $\mathrm{P}=0,6 \%$ & $P=0,7 \%$ & $P=0,8 \%$ & $P=0,9 \%$ & $P=1,0 \%$ \\
\hline \multirow{5}{*}{ 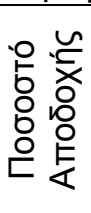 } & $90 \%$ & 27 & 31 & 34 & 29 & 33 & 33 & 33 & 34 & 34 & 33 \\
\hline & $85 \%$ & 22 & 22 & 20 & 24 & 26 & 22 & 22 & 23 & 25 & 27 \\
\hline & $80 \%$ & 22 & 16 & 17 & 17 & 16 & 19 & 20 & 18 & 17 & 19 \\
\hline & $75 \%$ & 22 & 16 & 15 & 14 & 12 & 16 & 16 & 14 & 14 & 16 \\
\hline & $70 \%$ & 22 & 16 & 15 & 11 & 12 & 11 & 13 & 11 & 12 & 13 \\
\hline
\end{tabular}

\begin{tabular}{|c|c|c|c|c|c|c|c|c|c|c|c|}
\hline \multirow{2}{*}{\multicolumn{2}{|c|}{ 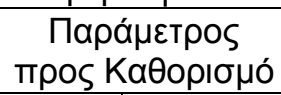 }} & \multicolumn{10}{|c|}{$\mathrm{P}$} \\
\hline & & $P=0,1 \%$ & $\mathrm{P}=0,2 \%$ & $\mathrm{P}=0,3 \%$ & $\mathrm{P}=0,4 \%$ & $\mathrm{P}=0,5 \%$ & $P=0,6 \%$ & $P=0,7 \%$ & $\mathrm{P}=0,8 \%$ & $\mathrm{P}=0,9 \%$ & $P=1,0 \%$ \\
\hline \multirow{5}{*}{ 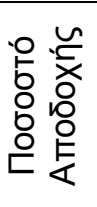 } & $90 \%$ & 39 & 35 & 34 & 35 & 37 & 34 & 34 & 34 & 33 & 36 \\
\hline & $85 \%$ & 25 & 21 & 21 & 21 & 23 & 25 & 25 & 25 & 25 & 28 \\
\hline & $80 \%$ & 21 & 18 & 17 & 19 & 17 & -2 & 21 & 22 & 22 & 22 \\
\hline & $75 \%$ & 21 & 11 & 14 & 15 & 13 & 10 & 15 & 15 & 16 & 15 \\
\hline & $70 \%$ & 21 & 11 & 12 & 12 & 8 & 8 & 10 & 9 & 11 & 10 \\
\hline
\end{tabular}

\begin{tabular}{|c|c|c|c|c|c|c|c|c|c|c|c|}
\hline \multicolumn{12}{|c|}{ Плمáuยтonc } \\
\hline \multicolumn{2}{|c|}{ 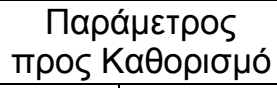 } & $P=0,1 \%$ & $P=0,2 \%$ & $P=0,3 \%$ & $P=0,4 \%$ & $P=0,5 \%$ & $P=0,6 \%$ & $P=0,7 \%$ & $P=0,8 \%$ & $P=0,9 \%$ & $P=1,0 \%$ \\
\hline \multirow{5}{*}{ 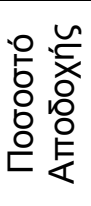 } & $90 \%$ & 37 & 35 & 32 & 34 & 34 & 34 & 33 & 31 & 31 & 38 \\
\hline & $85 \%$ & 26 & 28 & 28 & 25 & 22 & 20 & 20 & 23 & 23 & 23 \\
\hline & $80 \%$ & 25 & 21 & 17 & 14 & 17 & 17 & 14 & 15 & 16 & 17 \\
\hline & $75 \%$ & 25 & 13 & 13 & 12 & 14 & 11 & 8 & 11 & 11 & 13 \\
\hline & $70 \%$ & 25 & 12 & 11 & 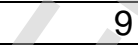 & 10 & 9 & 7 & 9 & 9 & 9 \\
\hline
\end{tabular}




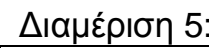

\begin{tabular}{|c|c|c|c|c|c|c|c|c|c|c|c|}
\hline \multirow{2}{*}{\multicolumn{2}{|c|}{ 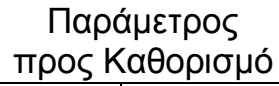 }} & \multicolumn{10}{|c|}{$P$} \\
\hline & & $P=0,1 \%$ & $P=0,2 \%$ & $P=0,3 \%$ & $P=0,4 \%$ & $P=0,5 \%$ & $P=0,6 \%$ & $P=0,7 \%$ & $P=0,8 \%$ & $P=0,9 \%$ & $P=1,0 \%$ \\
\hline \multirow{5}{*}{ 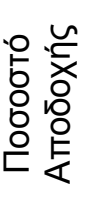 } & $90 \%$ & 33 & 33 & 32 & 37 & 39 & 35 & 35 & 35 & 37 & 40 \\
\hline & $85 \%$ & 23 & 19 & 22 & 22 & 23 & 25 & 27 & 27 & 27 & 31 \\
\hline & $80 \%$ & 17 & 18 & 18 & 17 & 20 & 15 & 18 & 20 & 20 & 23 \\
\hline & $75 \%$ & 17 & 12 & 11 & 13 & 15 & 14 & 18 & 15 & 15 & 14 \\
\hline & $70 \%$ & 17 & 12 & 10 & 11 & 11 & 11 & 12 & 9 & 9 & 9 \\
\hline
\end{tabular}

3) $\quad M k \Pi \Gamma \Sigma$

\begin{tabular}{|c|c|c|c|c|c|c|c|c|c|c|c|}
\hline \multirow{2}{*}{\multicolumn{2}{|c|}{ 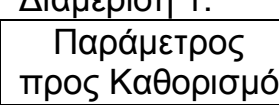 }} & \multicolumn{10}{|c|}{ K } \\
\hline & & $\mathrm{k}=1$ & $\mathrm{~K}=2$ & $\mathrm{k}=3$ & $\mathrm{k}=4$ & $\mathrm{k}=5$ & $\mathrm{k}=6$ & $\mathrm{k}=7$ & $\mathrm{k}=8$ & $\mathrm{k}=9$ & $k=10$ \\
\hline \multirow{5}{*}{$\begin{array}{l}0 \\
0 \\
0 \\
0 \\
0 \\
0 \\
0 \\
\\
\end{array}$} & $90 \%$ & 37 & 53 & 35 & 29 & 28 & 32 & 28 & 37 & 33 & 31 \\
\hline & $85 \%$ & 34 & 23 & 32 & 29 & 22 & 19 & 18 & 16 & 24 & 20 \\
\hline & $80 \%$ & 3 & 23 & 19 & 16 & 22 & 19 & 18 & 15 & 15 & 14 \\
\hline & $75 \%$ & 34 & 23 & 19 & 15 & 10 & 8 & 9 & 15 & 15 & 14 \\
\hline & $70 \%$ & 34 & 23 & 19 & 15 & 10 & 7 & 7 & 7 & 5 & \\
\hline
\end{tabular}

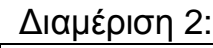

\begin{tabular}{|c|c|c|c|c|c|c|c|c|c|c|}
\hline \multirow{2}{*}{ 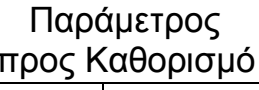 } & \multicolumn{10}{|c|}{$\mathrm{n}$} \\
\hline & $\mathrm{k}=1$ & $\mathrm{~K}=2$ & $\mathrm{k}=3$ & $\mathrm{k}=4$ & $\mathrm{k}=5$ & $\mathrm{k}=6$ & $\mathrm{k}=7$ & $\mathrm{k}=8$ & $\mathrm{k}=9$ & $\mathrm{k}=10$ \\
\hline $90 \%$ & 42 & 47 & 41 & 38 & 32 & 27 & 35 & 33 & 30 & 28 \\
\hline $85 \%$ & 42 & 26 & 26 & 28 & 32 & 21 & 25 & 24 & 23 & 23 \\
\hline $80 \%$ & 42 & 26 & 25 & 23 & 20 & 17 & 25 & 24 & 23 & 22 \\
\hline $75 \%$ & 42 & 26 & 25 & 23 & 20 & 16 & 16 & 16 & 17 & 14 \\
\hline $70 \%$ & 42 & 26 & 25 & 23 & 20 & 12 & 16 & 16 & 16 & 13 \\
\hline
\end{tabular}




\begin{tabular}{|c|c|c|c|c|c|c|c|c|c|c|c|}
\hline \multirow{2}{*}{\multicolumn{2}{|c|}{ 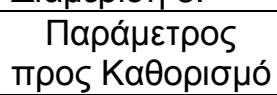 }} & \multicolumn{10}{|c|}{$\mathrm{K}$} \\
\hline & & $\mathrm{k}=1$ & $\mathrm{~K}=2$ & $\mathrm{k}=3$ & $\mathrm{k}=4$ & $\mathrm{k}=5$ & $\mathrm{k}=6$ & $\mathrm{k}=7$ & $\mathrm{k}=8$ & $\mathrm{k}=9$ & $\mathrm{k}=10$ \\
\hline \multirow{5}{*}{ 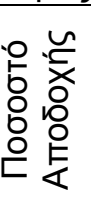 } & $90 \%$ & 49 & 59 & 50 & 36 & 37 & 41 & 40 & 35 & 37 & 41 \\
\hline & $85 \%$ & 49 & 31 & 30 & 27 & 35 & 29 & 23 & 22 & 30 & 27 \\
\hline & $80 \%$ & 49 & 31 & 24 & 18 & 18 & 17 & 23 & 22 & 18 & 17 \\
\hline & $75 \%$ & 49 & 31 & 24 & 12 & 16 & 13 & 12 & 13 & 13 & 17 \\
\hline & $70 \%$ & 49 & 31 & 24 & 12 & 16 & 13 & 12 & 12 & 12 & 17 \\
\hline
\end{tabular}

\begin{tabular}{|c|c|c|c|c|c|c|c|c|c|c|c|}
\hline \multirow{2}{*}{\multicolumn{2}{|c|}{ 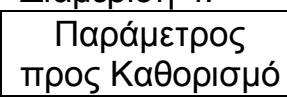 }} & \multicolumn{10}{|c|}{$\mathrm{K}$} \\
\hline & & $\mathrm{K}=1$ & $\mathrm{k}=2$ & $\mathrm{k}=3$ & $\mathrm{k}=4$ & $\mathrm{k}=5$ & $\mathrm{k}=6$ & $\mathrm{k}=7$ & $\mathrm{k}=8$ & $k=9$ & $k=10$ \\
\hline \multirow{5}{*}{ 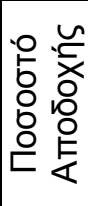 } & $90 \%$ & 40 & 39 & 46 & 38 & 35 & 42 & 43 & 36 & 33 & 35 \\
\hline & $85 \%$ & 40 & 29 & 23 & 38 & 32 & 31 & 25 & 20 & 20 & 26 \\
\hline & $80 \%$ & 40 & 29 & 19 & 14 & 12 & 12 & 25 & 20 & 19 & 16 \\
\hline & $75 \%$ & 40 & 29 & 19 & 14 & 11 & 10 & 8 & 8 & 9 & 11 \\
\hline & $70 \%$ & 40 & 29 & 19 & 14 & 11 & 10 & 8 & 8 & 8 & 7 \\
\hline
\end{tabular}

\begin{tabular}{|c|c|c|c|c|c|c|c|c|c|c|c|}
\hline \multicolumn{12}{|c|}{ 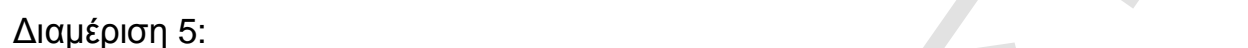 } \\
\hline \multirow{2}{*}{\multicolumn{2}{|c|}{ 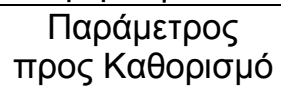 }} & \multicolumn{8}{|c|}{$\mathrm{K}$} & \multicolumn{2}{|l|}{ 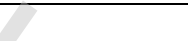 } \\
\hline & & $\mathrm{K}=1$ & $\mathrm{k}=2$ & $\mathrm{k}=3$ & $\mathrm{k}=4$ & $\mathrm{k}=5$ & $\mathrm{k}=6$ & $\mathrm{k}=7$ & $\mathrm{k}=8$ & $\mathrm{k}=9$ & $k=10$ \\
\hline \multirow{5}{*}{ 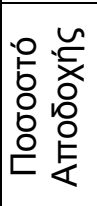 } & $90 \%$ & 41 & 52 & 44 & 37 & 39 & 40 & 38 & 36 & 35 & 34 \\
\hline & $85 \%$ & 41 & 31 & 29 & 37 & 32 & 27 & 26 & 26 & 27 & 29 \\
\hline & $80 \%$ & 41 & 31 & 24 & 22 & 32 & 27 & 26 & 23 & 20 & 17 \\
\hline & $75 \%$ & 41 & 31 & 24 & 22 & 20 & 17 & 20 & 23 & 20 & 17 \\
\hline & $70 \%$ & 41 & 31 & 24 & 22 & 20 & 17 & 15 & 23 & 10 & 11 \\
\hline
\end{tabular}




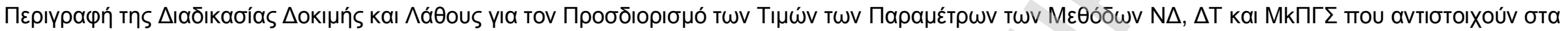

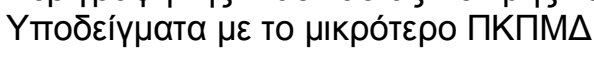

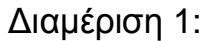

\begin{tabular}{|c|c|c|c|c|}
\hline \multicolumn{5}{|c|}{ 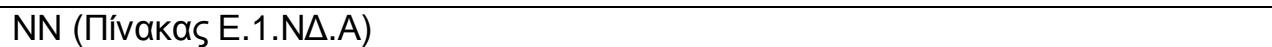 } \\
\hline \multicolumn{5}{|c|}{ Accept Rate } \\
\hline $70 \%$ & $75 \%$ & $80 \%$ & $85 \%$ & $90 \%$ \\
\hline $\mathrm{N}=19(02): 10$ & $\mathrm{~N}=19(04): 10$ & $\mathrm{~N}=20(07): 10$ & $\mathrm{~N}=20(13): 10$ & $\mathrm{~N}=21(23): 10$ \\
\hline $\mathrm{N}=18(03): 9$ & $\mathrm{~N}=20(05): 8$ & $\mathrm{~N}=23(08): 9$ & $\mathrm{~N}=25(14): 9$ & $\mathrm{~N}=16(24): 8$ \\
\hline $\mathrm{N}=16(05): 5,5$ & $\mathrm{~N}=22(05): 8$ & $\mathrm{~N}=25(10): 8$ & $\mathrm{~N}=18(16): \quad 7,5$ & $\mathrm{~N}=22(24): 8$ \\
\hline $\mathrm{N}=17(05): 5,5$ & $\mathrm{~N}=25(05): 8$ & $\mathrm{~N}=17(11): 6$ & $\mathrm{~N}=22(16): 7,5$ & $\mathrm{~N}=24(24): 8$ \\
\hline $\mathrm{N}=20(05): 5,5$ & $\mathrm{~N}=17(07): 5,5$ & $\mathrm{~N}=18(11): 6$ & $\mathrm{~N}=17(17): 4,5$ & $\mathrm{~N}=17(25): 5,5$ \\
\hline $\mathrm{N}=22(05): 5,5$ & $\mathrm{~N}=23(07): 5,5$ & $\mathrm{~N}=19(11): 6$ & $\mathrm{~N}=21(17): 4,5$ & $\mathrm{~N}=18(25): 5,5$ \\
\hline $\mathrm{N}=23(05): 5,5$ & $\mathrm{~N}=16(08): 3$ & $\mathrm{~N}=16(12): 3$ & $\mathrm{~N}=23(17): 4,5$ & $\mathrm{~N}=19(26): 3$ \\
\hline $\mathrm{N}=25(05): 5,5$ & $\mathrm{~N}=18(08): 3$ & $\mathrm{~N}=22(12): 3$ & $\mathrm{~N}=24(17): 4,5$ & $\mathrm{~N}=23(26): 3$ \\
\hline $\mathrm{N}=24(06): 2$ & $\mathrm{~N}=24(08): 3$ & $\mathrm{~N}=24(12): 3$ & $\mathrm{~N}=16(18): \quad 1,5$ & $\mathrm{~N}=25(26): 3$ \\
\hline $\mathrm{N}=21(08): 1$ & $\mathrm{~N}=21(12): 1$ & $\mathrm{~N}=21(13): 1$ & $\mathrm{~N}=19(18): \quad 1,5$ & $\mathrm{~N}=20(27): 1$ \\
\hline 55 & 55 & 55 & 55 & 55 \\
\hline
\end{tabular}

Yто́

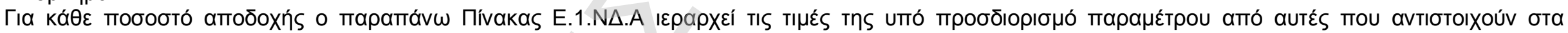

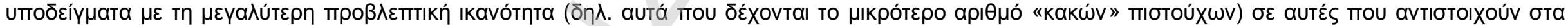

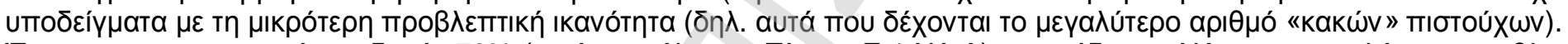

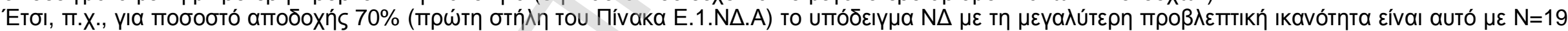

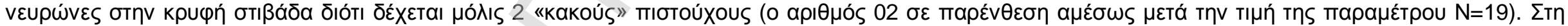

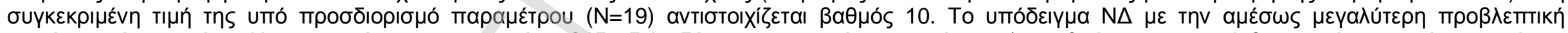

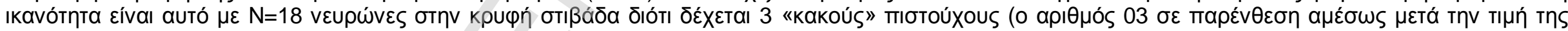

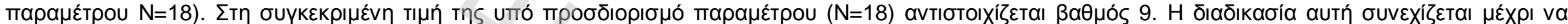

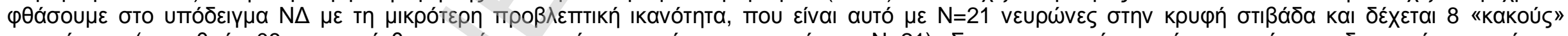

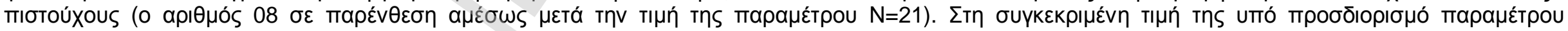

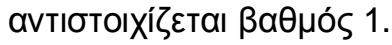




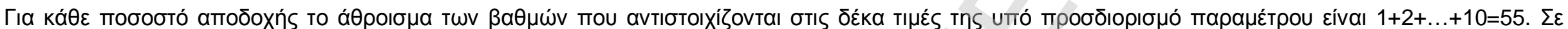

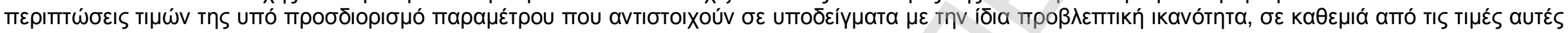

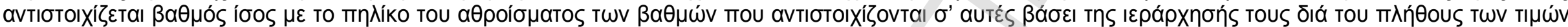

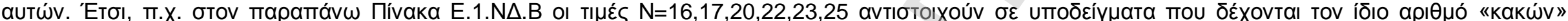

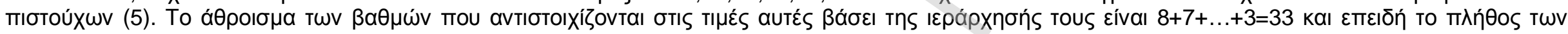

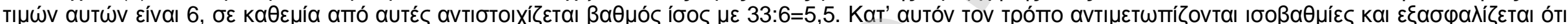

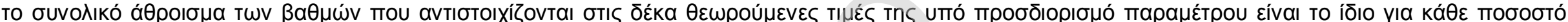
атообохи́s (55).

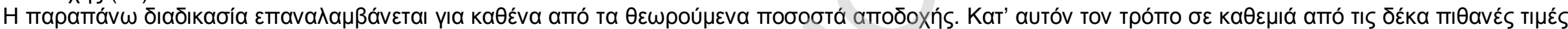

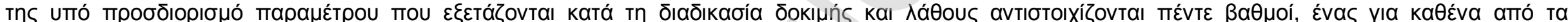

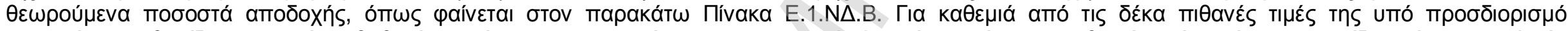

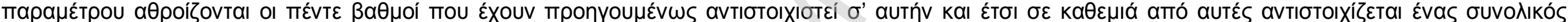

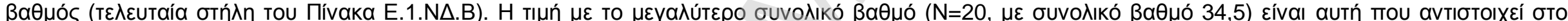

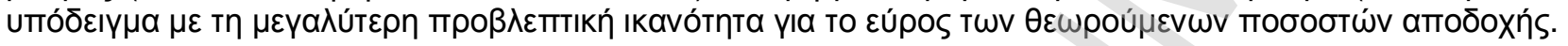

\begin{tabular}{|c|c|c|c|c|c|c|}
\hline \multicolumn{7}{|c|}{$\mathrm{NN}$ (Пívakas E.1.N $\Delta$. B) } \\
\hline & $70 \%$ & $75 \%$ & $80 \%$ & $85 \%$ & $90 \%$ & \\
\hline $\mathrm{N}=16$ & 5,5 & 3 & 3 & 1,5 & 8 & 21 \\
\hline $\mathrm{N}=17$ & 5,5 & 5,5 & 6 & 4,5 & 5,5 & 27 \\
\hline $\mathrm{N}=18$ & 9 & 3 & 6 & 7,5 & 5,5 & 31 \\
\hline $\mathrm{N}=19$ & 10 & 10 & 6 & 1,5 & 3 & 30,5 \\
\hline $\mathrm{N}=20$ & 5,5 & 8 & 10 & 10 & 1 & 34,5 \\
\hline $\mathrm{N}=21$ & 1 & 1 & 1 & 4,5 & 10 & 17,5 \\
\hline $\mathrm{N}=22$ & 5,5 & 8 & 3 & 7,5 & 8 & 32 \\
\hline $\mathrm{N}=23$ & 5,5 & 5,5 & 9 & 4,5 & 3 & 27,5 \\
\hline $\mathrm{N}=24$ & 2 & 3 & 3 & 4,5 & 8 & 20,5 \\
\hline $\mathrm{N}=25$ & 5,5 & 8 & 8 & 9 & 3 & 33,5 \\
\hline
\end{tabular}




\begin{tabular}{|c|c|c|c|c|}
\hline \multicolumn{5}{|c|}{ 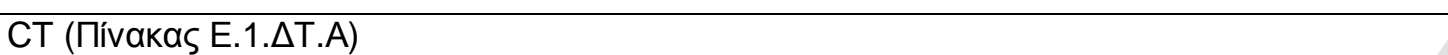 } \\
\hline \multicolumn{5}{|c|}{ Accept Rate } \\
\hline $70 \%$ & $75 \%$ & $80 \%$ & $85 \%$ & $90 \%$ \\
\hline $\mathrm{P}=0,6 \%(07): 10$ & $P=0,3 \%(10): 10$ & $P=0,1 \%(14): 9,5$ & $P=0,3 \%(17): 10$ & $P=0,2 \%(30): 9,5$ \\
\hline $\mathrm{P}=0,3 \%(08): 9$ & $\mathrm{P}=0,4 \%(11): 8$ & $\mathrm{P}=0,5 \%(14): 9,5$ & $P=0,6 \%(19): 8,5$ & $\mathrm{P}=0,3 \%(30): 9,5$ \\
\hline$P=0,4 \%(09): \quad 6,5$ & $P=0,6 \%(11): 8$ & $P=0,6 \%(15): 8$ & $P=0,7 \%(19): \quad 8,5$ & $P=0,5 \%(31): 7$ \\
\hline $\mathrm{P}=0,5 \%(09): \quad 6,5$ & $\mathrm{P}=0,7 \%(11): 8$ & $\mathrm{P}=0,3 \%(16): 5$ & $\mathrm{P}=0,4 \%(20): 7$ & $P=0,6 \%(31): 7$ \\
\hline$P=0,7 \%(09): 6,5$ & $P=0,2 \%(12): 5$ & $P=0,4 \%(16): 5$ & $P=0,5 \%(21): \quad 6$ & $\mathrm{P}=0,7 \%(31): 7$ \\
\hline$P=1,0 \%(09): \quad 6,5$ & $P=0,5 \%(12): 5$ & $P=0,8 \%(16): 5$ & $P=0,2 \%(22): 4,5$ & $\mathrm{P}=0,4 \%(32): 5$ \\
\hline$P=0,8 \%(10): 4$ & $P=1,0 \%(12): 5$ & $\mathrm{P}=0,9 \%(16): 5$ & $P=0,8 \%(22): 4,5$ & $\mathrm{P}=0,1 \%(35): 3,5$ \\
\hline $\mathrm{P}=0,9 \%(11): 3$ & $\mathrm{P}=0,1 \%(14): 2$ & $P=1,0 \%(16): 5$ & $P=0,1 \%(23): 2,5$ & $\mathrm{P}=0,9 \%(35): 3,5$ \\
\hline$P=0,2 \%(12): 2$ & $P=0,8 \%(14): 2$ & $\mathrm{P}=0,2 \%(17): 1,5$ & $P=1,0 \%(23): 2,5$ & $P=0,8 \%(37): 2$ \\
\hline $\mathrm{P}=0,1 \%(14): \quad 1$ & $P=0,9 \%(14): 2$ & $\mathrm{P}=0,7 \%(17): 1,5$ & $\mathrm{P}=0,9 \%(24): 1$ & $P=1,0 \%(38): 1$ \\
\hline
\end{tabular}

\begin{tabular}{|c|c|c|c|c|c|c|}
\hline \multicolumn{7}{|c|}{ 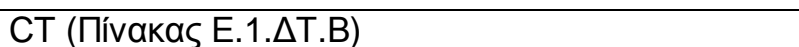 } \\
\hline \multicolumn{7}{|c|}{ Accept Rate } \\
\hline & $70 \%$ & $75 \%$ & $80 \%$ & $85 \%$ & $90 \%$ & \\
\hline$P=0,1 \%$ & 1 & 2 & 9,5 & 2,5 & 3,5 & 18,5 \\
\hline $\mathrm{P}=0,2 \%$ & 2 & 5 & 1,5 & 4,5 & 9,5 & 22,5 \\
\hline$P=0,3 \%$ & 9 & 10 & 5 & 10 & 9,5 & 43,5 \\
\hline$P=0,4 \%$ & 6,5 & 8 & 5 & 7 & 5 & 31,5 \\
\hline$P=0,5 \%$ & 6,5 & 5 & 9,5 & 6 & 7 & 34 \\
\hline$P=0,6 \%$ & 10 & 8 & 8 & 8,5 & 7 & 41,5 \\
\hline$P=0,7 \%$ & 6,5 & 8 & 1,5 & 8,5 & 7 & 31,5 \\
\hline$P=0,8 \%$ & 4 & 2 & 5 & 4,5 & 2 & 17,5 \\
\hline$P=0,9 \%$ & 3 & 2 & 5 & 1 & 3,5 & 14,5 \\
\hline$P=1,0 \%$ & 6,5 & 5 & 5 & 2,5 & 1 & 20 \\
\hline
\end{tabular}




\begin{tabular}{|c|c|c|c|c|}
\hline \multicolumn{5}{|c|}{ 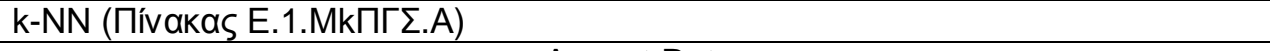 } \\
\hline \multicolumn{5}{|c|}{ Accept Rate } \\
\hline $70 \%$ & $75 \%$ & $80 \%$ & $85 \%$ & $90 \%$ \\
\hline$K=9(05): 10$ & $\mathrm{~K}=6(08): 10$ & $\mathrm{~K}=10(14): 10$ & $\mathrm{~K}=8(16): 10$ & $K=5(28): 9,5$ \\
\hline $\mathrm{K}=6(07): 7,5$ & $K=7(09): 9$ & $\mathrm{~K}=8(15): 8,5$ & $K=7(18): 9$ & $K=7(28): 9,5$ \\
\hline$K=7(07): 7,5$ & $K=5(10): 8$ & $K=9(15): 8,5$ & $K=6(19): 8$ & $K=4(29): 8$ \\
\hline $\mathrm{K}=8(07): \quad 7,5$ & $\mathrm{~K}=10(14): 7$ & $K=4(16): 7$ & $\mathrm{~K}=5(22): 6,5$ & $\mathrm{~K}=10(31): 7$ \\
\hline $\mathrm{K}=10(07): \quad 7,5$ & $K=4(15): 5$ & $K=7(18): 6$ & $\mathrm{~K}=10(22): 6,5$ & $\mathrm{~K}=6(32): 6$ \\
\hline$K=5(10): 5$ & $K=8(15): 5$ & $K=3(19): 4,5$ & $K=2(23): 5$ & $K=9(33): 5$ \\
\hline$K=4(15): 4$ & $K=9(15): 5$ & $\mathrm{~K}=6(19): 4,5$ & $K=9(24): 4$ & $K=3(35): 4$ \\
\hline$K=3(19): 3$ & $K=3(19): 3$ & $K=5(22): 3$ & $K=4(29): 3$ & $K=1(37): 2,5$ \\
\hline$K=2(23): 2$ & $K=2(23): 2$ & $K=2(23): 2$ & $\mathrm{~K}=3(32): 2$ & $K=8(37): 2,5$ \\
\hline $\mathrm{K}=1(34): 1$ & $K=1(34): 1$ & $K=1(34): 1$ & $K=1(34): 1$ & $K=2(53): 1$ \\
\hline
\end{tabular}

\begin{tabular}{|l|c|c|c|c|l|l|}
\hline \multicolumn{7}{|c|}{ k-NN (Пívakas E.1.MkПГट.B) } \\
\hline \multicolumn{7}{|c|}{ Accept Rate } \\
\hline $\mathrm{K}=1$ & 1 & 1 & 1 & 1 & 2,5 & 6,5 \\
\hline $\mathrm{K}=2$ & 2 & 2 & 2 & 5 & 1 & 12 \\
\hline $\mathrm{K}=3$ & 3 & 3 & 4,5 & 2 & 4 & 16,5 \\
\hline $\mathrm{K}=4$ & 4 & 5 & 7 & 3 & 8 & 27 \\
\hline $\mathrm{K}=5$ & 5 & 8 & 3 & 6,5 & 9,5 & 32 \\
\hline $\mathrm{K}=6$ & 7,5 & 10 & 4,5 & 8 & 6 & 36 \\
\hline $\mathrm{K}=7$ & 7,5 & 9 & 6 & 9 & 9,5 & $\mathbf{4 1}$ \\
\hline $\mathrm{K}=8$ & 7,5 & 5 & 8,5 & 10 & 2,5 & 33,5 \\
\hline $\mathrm{K}=9$ & 10 & 5 & 8,5 & 4 & 5 & 32,5 \\
\hline $\mathrm{K}=10$ & 7,5 & 7 & 10 & 6,5 & 7 & 38 \\
\hline
\end{tabular}




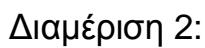

1) $N \Delta$

\begin{tabular}{|c|c|c|c|c|}
\hline \multicolumn{5}{|c|}{ NN (Пívakas E.2.Nঃ.A) } \\
\hline \multicolumn{5}{|c|}{ Accept Rate } \\
\hline $70 \%$ & $75 \%$ & $80 \%$ & $85 \%$ & $90 \%$ \\
\hline$N=24(03): 10$ & $\mathrm{~N}=24(04): 10$ & $\mathrm{~N}=21(10): 9,5$ & $N=17(15): 10$ & $N=23(25): 10$ \\
\hline $\mathrm{N}=19(04): 9$ & $\mathrm{~N}=21(05): 9$ & $\mathrm{~N}=25(10): 9,5$ & $\mathrm{~N}=21(17): 8,5$ & $\mathrm{~N}=19(26): 9$ \\
\hline $\mathrm{N}=18(05): \quad 7,5$ & $\mathrm{~N}=17(08): \quad 7,5$ & $\mathrm{~N}=22(11): 8$ & $\mathrm{~N}=24(17): 8,5$ & $\mathrm{~N}=25(27): 8$ \\
\hline $\mathrm{N}=21(05): 7,5$ & $\mathrm{~N}=25(08): 7,5$ & $\mathrm{~N}=23(12): 6$ & $\mathrm{~N}=18(18): 5,5$ & $\mathrm{~N}=16(28)$ \\
\hline $\mathrm{N}=17(06): 4,5$ & $\mathrm{~N}=18(09): 4,5$ & $\mathrm{~N}=24(12): 6$ & $\mathrm{~N}=22(18): 5,5$ & $\mathrm{~N}=18(28): 5,5$ \\
\hline $\mathrm{N}=22(06): 4,5$ & $\mathrm{~N}=19(09): 4,5$ & $\mathrm{~N}=17(12): 6$ & $\mathrm{~N}=23(18): \quad 5,5$ & $\mathrm{~N}=21(28): 5,5$ \\
\hline $\mathrm{N}=23(06): 4,5$ & $\mathrm{~N}=22(09): 4,5$ & $\mathrm{~N}=16(13): 3,5$ & $\mathrm{~N}=25(18): \quad 5,5$ & $\mathrm{~N}=24(28)$ \\
\hline $\mathrm{N}=25(06): 4,5$ & $\mathrm{~N}=23(09): 4,5$ & $\mathrm{~N}=18(13): 3,5$ & $\mathrm{~N}=16(19): 3$ & $\mathrm{~N}=22(29): 3$ \\
\hline $\mathrm{N}=20(07): 2$ & $\mathrm{~N}=16(10): 2$ & $\mathrm{~N}=19(14): 1,5$ & $\mathrm{~N}=19(20): 2$ & $\mathrm{~N}=17(31): 2$ \\
\hline $\mathrm{N}=16(07): 1$ & $\mathrm{~N}=20(11): 1$ & $\mathrm{~N}=20(14): 1,5$ & $\mathrm{~N}=20(22): 1$ & $\mathrm{~N}=20(32): 1$ \\
\hline
\end{tabular}

\begin{tabular}{|l|c|c|l|c|c|c|}
\hline \multicolumn{7}{|c|}{ NN (Пívakas E.2.N $\Delta$. B) } \\
\hline \multicolumn{7}{|c|}{ Accept Rate } \\
\hline & $70 \%$ & $75 \%$ & $80 \%$ & $85 \%$ & $90 \%$ & \\
\hline $\mathrm{N}=16$ & 1 & 2 & 3,5 & 3 & 5,5 & 15 \\
\hline $\mathrm{N}=17$ & 4,5 & 7,5 & 6 & 10 & 2 & 30 \\
\hline $\mathrm{N}=18$ & 7,5 & 4,5 & 3,5 & 5,5 & 5,5 & 26,5 \\
\hline $\mathrm{N}=19$ & 9 & 4,5 & 1,5 & 2 & 9 & 26 \\
\hline $\mathrm{N}=20$ & 2 & 1 & 1,5 & 1 & 1 & 6,5 \\
\hline $\mathrm{N}=21$ & 7,5 & 9 & 9,5 & 8,5 & 5,5 & 40 \\
\hline $\mathrm{N}=22$ & 4,5 & 4,5 & 8 & 5,5 & 3 & 25,5 \\
\hline $\mathrm{N}=23$ & 4,5 & 4,5 & 6 & 5,5 & 10 & 30,5 \\
\hline $\mathrm{N}=\mathbf{2 4}$ & 10 & 10 & 6 & 8,5 & 5,5 & $\mathbf{4 0}$ \\
\hline $\mathrm{N}=25$ & 4,5 & 7,5 & 9,5 & 5,5 & 8 & 35 \\
\hline
\end{tabular}




\begin{tabular}{|c|c|c|c|c|}
\hline \multicolumn{5}{|c|}{ 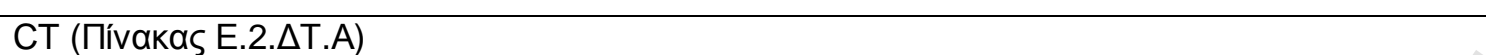 } \\
\hline \multicolumn{5}{|c|}{ Accept Rate } \\
\hline $70 \%$ & $75 \%$ & $80 \%$ & $85 \%$ & $90 \%$ \\
\hline $\mathrm{P}=0,4 \%(11): 9$ & $P=0,5 \%(12): 10$ & $\mathrm{P}=0,2 \%(16): 9,5$ & 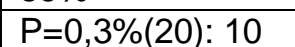 & $P=0,1 \%(27): 10$ \\
\hline $\mathrm{P}=0,6 \%(11): 9$ & $\mathrm{P}=0,4 \%(14): 8$ & $\mathrm{P}=0,5 \%(16): 9,5$ & $\mathrm{P}=0,1 \%(22): \quad 7,5$ & $P=0,4 \%(29): 9$ \\
\hline$P=0,8 \%(11): 9$ & $\mathrm{P}=0,8 \%(14): 8$ & $\mathrm{P}=0,3 \%(17): 7$ & $P=0,2 \%(22): \quad 7,5$ & $P=0,2 \%(31): 8$ \\
\hline $\mathrm{P}=0,5 \%(12): 6,5$ & $\mathrm{P}=0,9 \%(14): 8$ & $\mathrm{P}=0,4 \%(17): 7$ & $\mathrm{P}=0,6 \%(22): \quad 7,5$ & $\mathrm{P}=0,5 \%(33): 5,5$ \\
\hline $\mathrm{P}=0,9 \%(12): 6,5$ & $P=0,3 \%(15): 6$ & $\mathrm{P}=0,9 \%(17): 7$ & $P=0,7 \%(22): \quad 7,5$ & $\mathrm{P}=0,6 \%(33): 5,5$ \\
\hline $\mathrm{P}=0,7 \%(13): 4,5$ & $P=0,2 \%(16): \quad 3,5$ & $\mathrm{P}=0,8 \%(18): 5$ & $P=0,8 \%(23): 5$ & $\mathrm{P}=0,7 \%(33): \quad 5,5$ \\
\hline$P=1,0 \%(13): 4,5$ & $P=0,6 \%(16): \quad 3,5$ & $\mathrm{P}=0,6 \%(19): 3,5$ & $P=0,4 \%(24): 4$ & $\mathrm{P}=1,0 \%(33): 5,5$ \\
\hline$P=0,3 \%(15): 3$ & $\mathrm{P}=0,7 \%(16): \quad 3,5$ & $\mathrm{P}=1,0 \%(19): 3,5$ & \begin{tabular}{|l}
$P=0,9 \%(25): 3$ \\
\end{tabular} & $P=0,3 \%(34): 2$ \\
\hline$P=0,2 \%(16): 2$ & $P=1,0 \%(16): \quad 3,5$ & $\mathrm{P}=0,7 \%(20): 2$ & $P=0,5 \%(26): 2$ & $P=0,8 \%(34): 2$ \\
\hline $\mathrm{P}=0,1 \%(22): 1$ & $\mathrm{P}=0,1 \%(22): 1$ & $\mathrm{P}=0,1 \%(22): 1$ & \begin{tabular}{|l}
$P=1,0 \%(27): 1$ \\
\end{tabular} & $\mathrm{P}=0,9 \%(34): 2$ \\
\hline
\end{tabular}

\begin{tabular}{|l|l|l|l|r|l|l|}
\hline \multicolumn{7}{|c|}{ CT (Пívakas E.2. $\mathrm{T}$ T.B) } \\
\hline & $70 \%$ & $75 \%$ & $80 \%$ & $85 \%$ & $90 \%$ & \\
\hline $\mathrm{P}=0,1 \%$ & 1 & 1 & 1 & 7,5 & 10 & 20,5 \\
\hline $\mathrm{P}=0,2 \%$ & 2 & 3,5 & 9,5 & 7,5 & 8 & 30,5 \\
\hline $\mathrm{P}=0,3 \%$ & 3 & 6 & 7 & 10 & 2 & 28 \\
\hline $\mathrm{P}=\mathbf{0 , 4 \%}$ & 9 & 8 & 7 & 4 & 9 & $\mathbf{3 7}$ \\
\hline $\mathrm{P}=0,5 \%$ & 6,5 & 10 & 9,5 & 2 & 5,5 & 33,5 \\
\hline $\mathrm{P}=0,6 \%$ & 9 & 3,5 & 3,5 & 7,5 & 5,5 & 29 \\
\hline $\mathrm{P}=0,7 \%$ & 4,5 & 3,5 & 2 & 7,5 & 5,5 & 23 \\
\hline $\mathrm{P}=0,8 \%$ & 9 & 8 & 5 & 5 & 2 & 29 \\
\hline $\mathrm{P}=0,9 \%$ & 6,5 & 8 & 7 & 3 & 2 & 26,5 \\
\hline $\mathrm{P}=1,0 \%$ & 4,5 & 3,5 & 3,5 & 1 & 5,5 & 18 \\
\hline
\end{tabular}




\begin{tabular}{|c|c|c|c|c|}
\hline \multicolumn{5}{|c|}{ k-NN (Пívakas Е.2.MkПГГ.А) } \\
\hline \multicolumn{5}{|c|}{ Accept Rate } \\
\hline $70 \%$ & $75 \%$ & $80 \%$ & $85 \%$ & $90 \%$ \\
\hline $\mathrm{K}=6(12): 10$ & $\mathrm{~K}=10(14): 10$ & $\mathrm{~K}=6(17): 10$ & $\mathrm{~K}=6(21): 10$ & $\mathrm{~K}=6(27): 10$ \\
\hline $\mathrm{K}=10(13): 9$ & $\mathrm{~K}=6(16): 8$ & $K=5(20): 9$ & $K=9(23): 8,5$ & $\mathrm{~K}=10(28): 9$ \\
\hline$K=7(16): 7$ & $K=7(16): 8$ & $K=10(22): 8$ & $\mathrm{~K}=10(23): 8,5$ & $\mathrm{~K}=9(30): 8$ \\
\hline $\mathrm{K}=8(16): 7$ & $\mathrm{~K}=8(16): 8$ & $\mathrm{~K}=4(23): 6,5$ & $\mathrm{~K}=8(24): 7$ & $\mathrm{~K}=5(32):$ \\
\hline$K=9(16): 7$ & $K=9(17): 6$ & $\mathrm{~K}=9(23): 6,5$ & $\mathrm{~K}=7(25): 6$ & $\mathrm{~K}=8(33): 6$ \\
\hline$K=5(20): 5$ & $K=5(20): 5$ & $K=8(24): 5$ & $\mathrm{~K}=2(26): 4,5$ & $K=7(35): 5$ \\
\hline $\mathrm{K}=4(23): 4$ & $\mathrm{~K}=4(23): 4$ & $\mathrm{~K}=3(25): 3,5$ & $\mathrm{~K}=3(26): 4,5$ & $\mathrm{~K}=4(38): 4$ \\
\hline $\mathrm{K}=3(25): 3$ & $\mathrm{~K}=3(25): 3$ & $\mathrm{~K}=7(25): 3,5$ & $\mathrm{~K}=4(28): 3$ & $\mathrm{~K}=3(41): 3$ \\
\hline$K=2(26): 2$ & $K=2(26): 2$ & $K=2(26): 2$ & $\mathrm{~K}=5(32): 2$ & $K=1(42): 2$ \\
\hline $\mathrm{K}=1(42): 1$ & $\mathrm{~K}=1(42): 1$ & $K=1(42): 1$ & $\mathrm{~K}=1(42): 1$ & $\mathrm{~K}=2(47):$ \\
\hline
\end{tabular}

\begin{tabular}{|c|c|c|c|c|c|c|}
\hline \multicolumn{7}{|c|}{ k-NN (Пі́vakas Е.2.MkПГц.В) } \\
\hline \multicolumn{7}{|c|}{ Accept Rate } \\
\hline & $70 \%$ & $75 \%$ & $80 \%$ & $85 \%$ & $90 \%$ & \\
\hline$K=1$ & 1 & 1 & 1 & 1 & 2 & 6 \\
\hline $\mathrm{K}=2$ & 2 & 2 & 2 & 4,5 & 1 & 11,5 \\
\hline $\mathrm{K}=3$ & 3 & 3 & 3,5 & 4,5 & 3 & 17 \\
\hline$K=4$ & 4 & 4 & 6,5 & 3 & 4 & 21,5 \\
\hline$K=5$ & 5 & 5 & 9 & 2 & 7 & 28 \\
\hline$K=6$ & 10 & 8 & 10 & 10 & 10 & 48 \\
\hline$K=7$ & 7 & 8 & 3,5 & 6 & 5 & 29 \\
\hline$K=8$ & 7 & 8 & 5 & 7 & 6 & 33 \\
\hline$K=9$ & 7 & 6 & 6,5 & 8,5 & 8 & 36 \\
\hline$K=10$ & 9 & 10 & 8 & 8,5 & 9 & 44,5 \\
\hline
\end{tabular}




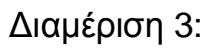

1) $N \Delta$

\begin{tabular}{|c|c|c|c|c|}
\hline \multicolumn{5}{|c|}{ 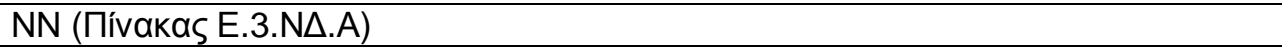 } \\
\hline \multicolumn{5}{|c|}{ Accept Rate } \\
\hline $70 \%$ & $75 \%$ & $80 \%$ & $85 \%$ & $90 \%$ \\
\hline $\mathrm{N}=17(05): 9,5$ & $\mathrm{~N}=22(07): 10$ & $\mathrm{~N}=23(11): 10$ & $\mathrm{~N}=17(17): 9,5$ & $\mathrm{~N}=22(28): 10$ \\
\hline $\mathrm{N}=24(05): 9,5$ & $\mathrm{~N}=17(08): 8,5$ & $\mathrm{~N}=22(12): 9$ & $\mathrm{~N}=19(17): 9,5$ & $\mathrm{~N}=20(30): 9$ \\
\hline $\mathrm{N}=19(06): 7$ & $\mathrm{~N}=23(08): 8,5$ & $\mathrm{~N}=16(13): 6$ & $\mathrm{~N}=18(18): 8$ & $\mathrm{~N}=16(31): 7$ \\
\hline $\mathrm{N}=20(06): 7$ & $\mathrm{~N}=25(09): 7$ & $\mathrm{~N}=17(13): 6$ & $\mathrm{~N}=16(19): 7$ & $\mathrm{~N}=18(31): 7$ \\
\hline $\mathrm{N}=22(06): 7$ & $\mathrm{~N}=16(10): \quad 4,5$ & $\mathrm{~N}=18(13): 6$ & $\mathrm{~N}=22(20): 6$ & $\mathrm{~N}=25(31): 7$ \\
\hline $\mathrm{N}=23(07): 4,5$ & $\mathrm{~N}=18(10): 4,5$ & $\mathrm{~N}=19(13): 6$ & $\mathrm{~N}=24(21): 4,5$ & $\mathrm{~N}=17(32): 4,5$ \\
\hline $\mathrm{N}=25(07): 4,5$ & $\mathrm{~N}=19(10): 4,5$ & $\mathrm{~N}=25(13): 6$ & $\mathrm{~N}=25(21): 4,5$ & $\mathrm{~N}=23(32): 4,5$ \\
\hline $\mathrm{N}=18(08): 3$ & $\mathrm{~N}=24(10)$ & $\mathrm{N}=20(14): 2,5$ & $\mathrm{~N}=20(22): 2,5$ & $N=19(33): 2$ \\
\hline $\mathrm{N}=16(09): 1,5$ & $\mathrm{~N}=20(11): 2$ & $\mathrm{~N}=24(14): 2,5$ & $\mathrm{~N}=23(22): 2,5$ & $\mathrm{~N}=21(33): 2$ \\
\hline $\mathrm{N}=21(09): 1,5$ & $\mathrm{~N}=21(12): 1$ & $\mathrm{~N}=21(16): 1$ & $\mathrm{~N}=21(24): 1$ & $\mathrm{~N}=24(33): 2$ \\
\hline
\end{tabular}

\begin{tabular}{|l|l|r|c|l|l|l|}
\hline \multicolumn{7}{|c|}{ NN (Пivakas E.3.N $\Delta$. B) } \\
\hline \multicolumn{7}{|c|}{ Accept Rate } \\
\hline & $70 \%$ & $75 \%$ & $80 \%$ & $85 \%$ & $90 \%$ & \\
\hline $\mathrm{N}=16$ & 1,5 & 4,5 & 6 & 7 & 7 & 26 \\
\hline $\mathrm{N}=17$ & 9,5 & 8,5 & 6 & 9,5 & 4,5 & 38 \\
\hline $\mathrm{N}=18$ & 3 & 4,5 & 6 & 8 & 7 & 28,5 \\
\hline $\mathrm{N}=19$ & 7 & 4,5 & 6 & 9,5 & 2 & 29 \\
\hline $\mathrm{N}=20$ & 7 & 2 & 2,5 & 2,5 & 9 & 23 \\
\hline $\mathrm{N}=21$ & 1,5 & 1 & 1 & 1 & 2 & 6,5 \\
\hline $\mathrm{N}=22$ & 7 & 10 & 9 & 6 & 10 & $\mathbf{4 2}$ \\
\hline $\mathrm{N}=23$ & 4,5 & 8,5 & 10 & 2,5 & 4,5 & 30 \\
\hline $\mathrm{N}=24$ & 9,5 & 4,5 & 2,5 & 4,5 & 2 & 23 \\
\hline $\mathrm{N}=25$ & 4,5 & 7 & 6 & 4,5 & 7 & 29 \\
\hline
\end{tabular}




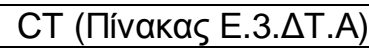

\begin{tabular}{|l|l|l|l|l|}
\hline \multicolumn{7}{|c|}{ Accept Rate } \\
\hline $70 \%$ & $75 \%$ & $80 \%$ & $85 \%$ & $90 \%$ \\
\hline$P=0,5 \%(08): 9,5$ & $P=0,6 \%(10): 10$ & $P=0,6 \%(15): 10$ & $P=0,2 \%(21): 9$ & $P=0,9 \%(33): 10$ \\
\hline$P=0,6 \%(08): 9,5$ & $P=0,2 \%(11): 9$ & $P=0,3 \%(17): 8,5$ & $P=0,3 \%(21): 9$ & $P=0,3 \%(34): 7,5$ \\
\hline$P=0,8 \%(09): 8$ & $P=0,5 \%(13): 8$ & $P=0,5 \%(17): 8,5$ & $P=0,4 \%(21): 9$ & $P=0,6 \%(34): 7,5$ \\
\hline$P=0,7 \%(10): 6,5$ & $P=0,3 \%(14): 7$ & $P=0,2 \%(18): 7$ & $P=0,5 \%(23): 7$ & $P=0,7 \%(34): 7,5$ \\
\hline$P=1,0 \%(10): 6,5$ & $P=0,4 \%(15): 4,5$ & $P=0,4 \%(19): 6$ & $P=0,1 \%(25): 4$ & $P=0,8 \%(34): 7,5$ \\
\hline$P=0,2 \%(11): 4,5$ & $P=0,7 \%(15): 4,5$ & $P=0,1 \%(21): 4,5$ & $P=0,6 \%(25): 4$ & $P=0,2 \%(35): 4,5$ \\
\hline$P=0,9 \%(11): 4,5$ & $P=0,8 \%(15): 4,5$ & $P=0,7 \%(21): 4,5$ & $P=0,7 \%(25): 4$ & $P=0,4 \%(35): 4,5$ \\
\hline$P=0,3 \%(12): 2,5$ & $P=1,0 \%(15): 4,5$ & $P=0,8 \%(22): 2$ & $P=0,8 \%(25): 4$ & $P=1,0 \%(36): 3$ \\
\hline$P=0,4 \%(12): 2,5$ & $P=0,9 \%(16): 2$ & $P=0,9 \%(22): 2$ & $P=0,9 \%(25): 4$ & $P=0,5 \%(37): 2$ \\
\hline$P=0,1 \%(21): 1$ & $P=0,1 \%(21): 1$ & $P=1,0 \%(22): 2$ & $P=1,0 \%(28): 1$ & $P=0,1 \%(39): 1$ \\
\hline
\end{tabular}

\begin{tabular}{|c|c|c|c|c|c|c|}
\hline \multicolumn{7}{|c|}{ 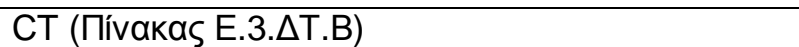 } \\
\hline \multicolumn{7}{|c|}{ Accept Rate } \\
\hline & $70 \%$ & $75 \%$ & $80 \%$ & $85 \%$ & $90 \%$ & \\
\hline $\mathrm{P}=0,1 \%$ & 1 & 1 & 4,5 & 4 & 1 & 11,5 \\
\hline $\mathrm{P}=0,2 \%$ & 4,5 & 9 & 7 & 9 & 4,5 & 34 \\
\hline $\mathrm{P}=0,3 \%$ & 2,5 & 7 & 8,5 & 9 & 7,5 & 34,5 \\
\hline$P=0,4 \%$ & 2,5 & 4,5 & 6 & 9 & 4,5 & 26,5 \\
\hline $\mathrm{P}=0,5 \%$ & 9,5 & 8 & 8,5 & 7 & 2 & 35 \\
\hline$P=0,6 \%$ & 9,5 & 10 & 10 & 4 & 7,5 & 41 \\
\hline$P=0,7 \%$ & 6,5 & 4,5 & 4,5 & 4 & 7,5 & 27 \\
\hline $\mathrm{P}=0,8 \%$ & 8 & 4,5 & 2 & 4 & 7,5 & 26 \\
\hline $\mathrm{P}=0,9 \%$ & 4,5 & 2 & 2 & 4 & 10 & 22,5 \\
\hline$P=1,0 \%$ & 6,5 & 4,5 & 2 & 1 & 3 & 17 \\
\hline
\end{tabular}




\begin{tabular}{|c|c|c|c|c|}
\hline \multicolumn{5}{|c|}{ 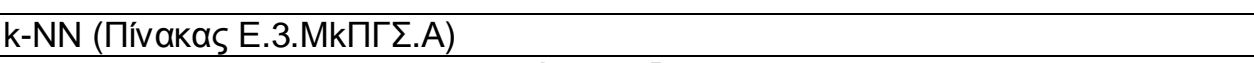 } \\
\hline \multicolumn{5}{|c|}{ Accept Rate } \\
\hline $70 \%$ & $75 \%$ & $80 \%$ & $85 \%$ & $90 \%$ \\
\hline $\mathrm{K}=4(12): 8,5$ & $\mathrm{~K}=4(12): 9,5$ & $\mathrm{~K}=6(17): 9,5$ & $\mathrm{~K}=8(22): 10$ & $\mathrm{~K}=8(35): 10$ \\
\hline $\mathrm{K}=7(12): 8,5$ & $\mathrm{~K}=7(12): 9,5$ & $\mathrm{~K}=10(17): 9,5$ & $\mathrm{~K}=7(23): 9$ & $\mathrm{~K}=4(36): 9$ \\
\hline$K=8(12): 8,5$ & $K=6(13): 7$ & $\mathrm{~K}=4(18): 7$ & $\mathrm{~K}=4(27): \quad 7,5$ & $\mathrm{~K}=5(37): \quad 7,5$ \\
\hline $\mathrm{K}=9(12): 8,5$ & $\mathrm{~K}=8(13): 7$ & $\mathrm{~K}=5(18): 7$ & $\mathrm{~K}=10(27): \quad 7,5$ & $\mathrm{~K}=9(37): \quad 7,5$ \\
\hline $\mathrm{K}=6(13): 6$ & $\mathrm{~K}=9(13): 7$ & $\mathrm{~K}=9(18): 7$ & $\mathrm{~K}=6(29): 6$ & $\mathrm{~K}=7(40): 6$ \\
\hline $\mathrm{K}=5(16): 5$ & $K=5(16): 5$ & $K=8(22): 5$ & $\mathrm{~K}=3(30): 4,5$ & $\mathrm{~K}=6(41): 4,5$ \\
\hline $\mathrm{K}=10(17): 4$ & $\mathrm{~K}=10(17): 4$ & $K=7(23): 4$ & $\mathrm{~K}=9(30): 4,5$ & $\mathrm{~K}=10(41): \quad 4,5$ \\
\hline $\mathrm{K}=3(24): 3$ & $\mathrm{~K}=3(24): 3$ & $K=3(24): 3$ & $\mathrm{~K}=2(31): 3$ & $\mathrm{~K}=1(49): 3$ \\
\hline $\mathrm{K}=2(31): 2$ & $K=2(31): 2$ & $K=2(31): 2$ & $\mathrm{~K}=5(35): 2$ & $\mathrm{~K}=3(50): 2$ \\
\hline $\mathrm{K}=1(49): 1$ & $\mathrm{~K}=1(49): 1$ & $\mathrm{~K}=1(49): 1$ & $\mathrm{~K}=1(49): \quad 1$ & $\mathrm{~K}=2(59): 1$ \\
\hline
\end{tabular}

\begin{tabular}{|c|c|c|c|c|c|c|}
\hline \multicolumn{7}{|c|}{ k-NN (Пívakas E.3.MkПГГ.B) } \\
\hline \multicolumn{7}{|c|}{ Accept Rate } \\
\hline & $70 \%$ & $75 \%$ & $80 \%$ & $85 \%$ & $90 \%$ & \\
\hline$K=1$ & 1 & 1 & 1 & 1 & 3 & 7 \\
\hline$K=2$ & 2 & 2 & 2 & 3 & 1 & 10 \\
\hline $\mathrm{K}=3$ & 3 & 3 & 3 & 4,5 & 2 & 15,5 \\
\hline$K=4$ & 8,5 & 9,5 & 7 & 7,5 & 9 & 41,5 \\
\hline$K=5$ & 5 & 5 & 7 & 2 & 7,5 & 26,5 \\
\hline$K=6$ & 6 & 7 & 9,5 & 6 & 4,5 & 33 \\
\hline$K=7$ & 8.5 & 9,5 & 4 & 9 & 6 & 37 \\
\hline$K=8$ & 8,5 & 7 & 5 & 10 & 10 & 40,5 \\
\hline$K=9$ & 8,5 & 7 & 7 & 4,5 & 7,5 & 34,5 \\
\hline $\mathrm{K}=10$ & 4 & 4 & 9,5 & 7,5 & 4,5 & 29,5 \\
\hline
\end{tabular}




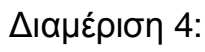

1) $N \Delta$

\begin{tabular}{|c|c|c|c|c|}
\hline \multicolumn{5}{|c|}{ NN (Пívakas E.4.NA.A) } \\
\hline \multicolumn{5}{|c|}{ Accept Rate } \\
\hline $70 \%$ & $75 \%$ & $80 \%$ & $85 \%$ & $90 \%$ \\
\hline$N=16(03): 8$ & $\mathrm{~N}=21(07): 9$ & $N=24(10): 10$ & $N=21(17): 10$ & $N=22(25): 10$ \\
\hline $\mathrm{N}=18(03): 8$ & $\mathrm{~N}=23(07): 9$ & $\mathrm{~N}=21(12): 9$ & $\mathrm{~N}=16(18): 8,5$ & $\mathrm{~N}=19(28): 8,5$ \\
\hline $\mathrm{N}=20(03): 8$ & $\mathrm{~N}=24(07): 9$ & $\mathrm{~N}=16(13): 7$ & $\mathrm{~N}=18(18): 8,5$ & $\mathrm{~N}=20(28): \quad 8,5$ \\
\hline $\mathrm{N}=22(03): 8$ & $\mathrm{~N}=16(08): 5,5$ & $\mathrm{~N}=17(13): 7$ & $\mathrm{~N}=19(19): 5,5$ & $\mathrm{~N}=16(29): \quad 6,5$ \\
\hline $\mathrm{N}=24(03): 8$ & $\mathrm{~N}=19(08): 5,5$ & $\mathrm{~N}=20(13): 7$ & $\mathrm{~N}=20(19): 5,5$ & $\mathrm{~N}=18(29): \quad 6,5$ \\
\hline $\mathrm{N}=21(04): 5$ & $\mathrm{~N}=20(08): 5,5$ & $\mathrm{~N}=18(14): \quad 3,5$ & $\mathrm{~N}=23(19): 5,5$ & $\mathrm{~N}=17(30): 4,5$ \\
\hline $\mathrm{N}=17(05): 2,5$ & $\mathrm{~N}=22(08): 5,5$ & $\mathrm{~N}=19(14): \quad 3,5$ & $\mathrm{~N}=24(19): 5,5$ & $\mathrm{~N}=24(30): 4,5$ \\
\hline $\mathrm{N}=19(05): 2,5$ & $\mathrm{~N}=17(09): 2,5$ & $\mathrm{~N}=23(14): \quad 3,5$ & $\mathrm{~N}=22(21): 2,5$ & $\mathrm{~N}=21(32): 2$ \\
\hline $\mathrm{N}=23(05): 2,5$ & $\mathrm{~N}=18(09): 2,5$ & $\mathrm{~N}=25(14): \quad 3,5$ & $\mathrm{~N}=25(21): 2,5$ & $\mathrm{~N}=23(32): 2$ \\
\hline $\mathrm{N}=25(05): 2,5$ & $\mathrm{~N}=25(10): 1$ & $\mathrm{~N}=22(16): 1$ & $\mathrm{~N}=17(22): 1$ & $\mathrm{~N}=25(32): 2$ \\
\hline
\end{tabular}

\begin{tabular}{|l|l|l|r|r|r|r|}
\hline \multicolumn{7}{|c|}{$\mathrm{NN}$ (Пivakas E.4.N $\Delta . B$ ) } \\
\hline \multicolumn{7}{|c|}{ Accept Rate } \\
\hline & $70 \%$ & $75 \%$ & $80 \%$ & $85 \%$ & $90 \%$ & \\
\hline $\mathrm{N}=16$ & 8 & 5,5 & 7 & 8,5 & 6,5 & 35,5 \\
\hline $\mathrm{N}=17$ & 2,5 & 2,5 & 7 & 1 & 4,5 & 17,5 \\
\hline $\mathrm{N}=18$ & 8 & 2,5 & 3,5 & 8,5 & 6,5 & 29 \\
\hline $\mathrm{N}=19$ & 2,5 & 5,5 & 3,5 & 5,5 & 8,5 & 25,5 \\
\hline $\mathrm{N}=20$ & 8 & 5,5 & 7 & 5,5 & 8,5 & 34,5 \\
\hline $\mathrm{N}=21$ & 5 & 9 & 9 & 10 & 2 & 35 \\
\hline $\mathrm{N}=22$ & 8 & 5,5 & 1 & 2,5 & 10 & 27 \\
\hline $\mathrm{N}=23$ & 2,5 & 9 & 3,5 & 5,5 & 2 & 22,5 \\
\hline $\mathrm{N}=\mathbf{2 4}$ & 8 & 9 & 10 & 5,5 & 4,5 & $\mathbf{3 7}$ \\
\hline $\mathrm{N}=25$ & 2,5 & 1 & 3,5 & 2,5 & 2 & 11,5 \\
\hline
\end{tabular}




\begin{tabular}{|l|l|l|l|l|}
\hline \multicolumn{7}{|l|}{ CT (Пívakas E.4.4T.A) } \\
\hline \multicolumn{7}{|l|}{ Accept Rate } \\
\hline $70 \%$ & $75 \%$ & $80 \%$ & $85 \%$ & $90 \%$ \\
\hline $\mathrm{P}=0,7 \%(07): 10$ & $\mathrm{P}=0,7 \%(08): 10$ & $\mathrm{P}=0,4 \%(14): 9,5$ & $\mathrm{P}=0,6 \%(20): 9,5$ & $\mathrm{P}=0,8 \%(31): 9,5$ \\
\hline $\mathrm{P}=0,4 \%(09): 7$ & $\mathrm{P}=0,6 \%(11): 8$ & $\mathrm{P}=0,7 \%(14): 9,5$ & $\mathrm{P}=0,7 \%(20): 9,5$ & $\mathrm{P}=0,9 \%(31): 9,5$ \\
\hline $\mathrm{P}=0,6 \%(09): 7$ & $\mathrm{P}=0,8 \%(11): 8$ & $\mathrm{P}=0,8 \%(15): 8$ & $\mathrm{P}=0,5 \%(22): 8$ & $\mathrm{P}=0,3 \%(32): 8$ \\
\hline $\mathrm{P}=0,8 \%(09): 7$ & $\mathrm{P}=0,9 \%(11): 8$ & $\mathrm{P}=0,9 \%(16): 7$ & $\mathrm{P}=0,8 \%(23): 6$ & $\mathrm{P}=0,7 \%(33): 7$ \\
\hline $\mathrm{P}=0,9 \%(09): 7$ & $\mathrm{P}=0,4 \%(12): 6$ & $\mathrm{P}=0,3 \%(17): 4,5$ & $\mathrm{P}=0,9 \%(23): 6$ & $\mathrm{P}=0,4 \%(34): 5$ \\
\hline $\mathrm{P}=1,0 \%(09): 7$ & $\mathrm{P}=0,2 \%(13): 4$ & $\mathrm{P}=0,5 \%(17): 4,5$ & $\mathrm{P}=1,0 \%(23): 6$ & $\mathrm{P}=0,5 \%(34): 5$ \\
\hline $\mathrm{P}=0,5 \%(10): 4$ & $\mathrm{P}=0,3 \%(13): 4$ & $\mathrm{P}=0,6 \%(17): 4,5$ & $\mathrm{P}=0,4 \%(25): 4$ & $\mathrm{P}=0,6 \%(34): 5$ \\
\hline $\mathrm{P}=0,3 \%(11): 3$ & $\mathrm{P}=1,0 \%(13): 4$ & $\mathrm{P}=1,0 \%(17): 4,5$ & $\mathrm{P}=0,1 \%(26): 3$ & $\mathrm{P}=0,2 \%(35): 3$ \\
\hline $\mathrm{P}=0,2 \%(12): 2$ & $\mathrm{P}=0,5 \%(14): 2$ & $\mathrm{P}=0,2 \%(21): 2$ & $\mathrm{P}=0,2 \%(28): 1,5$ & $\mathrm{P}=0,1 \%(37): 2$ \\
\hline $\mathrm{P}=0,1 \%(25): 1$ & $\mathrm{P}=0,1 \%(25): 1$ & $\mathrm{P}=0,1 \%(25): 1$ & $\mathrm{P}=0,3 \%(28): 1,5$ & $\mathrm{P}=1,0 \%(38): 1$ \\
\hline
\end{tabular}

\begin{tabular}{|c|c|c|c|c|c|c|}
\hline \multicolumn{7}{|c|}{ 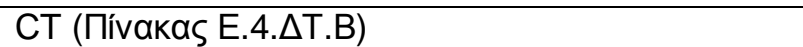 } \\
\hline \multicolumn{7}{|c|}{ Accept Rate } \\
\hline & $70 \%$ & $75 \%$ & $80 \%$ & $85 \%$ & $90 \%$ & \\
\hline$P=0,1 \%$ & 1 & 1 & 1 & 3 & 2 & 8 \\
\hline$P=0,2 \%$ & 2 & 4 & 2 & 1,5 & 3 & 12,5 \\
\hline$P=0,3 \%$ & 3 & 4 & 4,5 & 1,5 & 8 & 21 \\
\hline$P=0,4 \%$ & 7 & 6 & 9,5 & 4 & 5 & 31,5 \\
\hline$P=0,5 \%$ & 4 & 2 & 4,5 & 8 & 5 & 23,5 \\
\hline$P=0,6 \%$ & 7 & 8 & 4,5 & 9,5 & 5 & 34 \\
\hline$P=0,7 \%$ & 10 & 10 & 9,5 & 9,5 & 7 & 46 \\
\hline $\mathrm{P}=0,8 \%$ & 7 & 8 & 8 & 6 & 9,5 & 38,5 \\
\hline$P=0,9 \%$ & 7 & 8 & 7 & 6 & 9,5 & 37,5 \\
\hline$P=1,0 \%$ & 7 & 4 & 4,5 & 6 & 1 & 22,5 \\
\hline
\end{tabular}




\begin{tabular}{|c|c|c|c|c|}
\hline \multicolumn{5}{|c|}{ 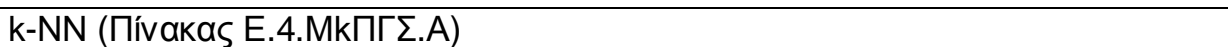 } \\
\hline \multicolumn{5}{|c|}{ Accept Rate } \\
\hline $70 \%$ & $75 \%$ & $80 \%$ & $85 \%$ & $90 \%$ \\
\hline $\mathrm{K}=10(07): 10$ & $K=7(08): 9,5$ & $K=5(12): 9,5$ & $K=8(20): 9,5$ & $\mathrm{~K}=9(33): 10$ \\
\hline$K=7(08): 8$ & $K=8(08): 9,5$ & $K=6(12): 9,5$ & $K=9(20): 9,5$ & $\mathrm{~K}=5(35): 8,5$ \\
\hline$K=8(08): 8$ & $K=9(09): 8$ & $K=4(14): 8$ & $K=3(23): 8$ & $\mathrm{~K}=10(35): 8,5$ \\
\hline$K=9(08): 8$ & $K=6(10): 7$ & $K=10(16): 7$ & $K=7(25): 7$ & $\mathrm{~K}=8(36): 7$ \\
\hline$K=6(10): 6$ & $K=5(11): 5,5$ & $K=3(19): 5,5$ & $\mathrm{~K}=10(26): 6$ & $K=4(38): 6$ \\
\hline$K=5(11): 5$ & $K=10(11): 5,5$ & $K=9(19): 5,5$ & $K=2(29): 5$ & $K=2(39): 5$ \\
\hline$K=4(14): 4$ & $\mathrm{~K}=4(14): 4$ & $\mathrm{~K}=8(20): 4$ & $\mathrm{~K}=6(31): 4$ & $\mathrm{~K}=1(40): 4$ \\
\hline $\mathrm{K}=3(19): 3$ & $\mathrm{~K}=3(19): 3$ & $\mathrm{~K}=7(25): 3$ & $\mathrm{~K}=5(32): 3$ & $K=6(42): 3$ \\
\hline$K=2(29): 2$ & $K=2(29): 2$ & $\mathrm{~K}=2(29): 2$ & $\mathrm{~K}=4(38): 2$ & $K=7(43): 2$ \\
\hline$K=1(40): 1$ & $K=1(40): 1$ & $K=1(40): 1$ & $K=1(40): 1$ & $\mathrm{~K}=3(46): 1$ \\
\hline
\end{tabular}

\begin{tabular}{|l|c|l|l|l|c|c|}
\hline \multicolumn{7}{|c|}{ k-NN (Пívakas E.4.MkПГ Accept Rate } \\
\hline & $70 \%$ & $75 \%$ & $80 \%$ & $85 \%$ & $90 \%$ & \\
\hline $\mathrm{K}=1$ & 1 & 1 & 1 & 1 & 4 & 8 \\
\hline $\mathrm{K}=2$ & 2 & 2 & 2 & 5 & 5 & 16 \\
\hline $\mathrm{K}=3$ & 3 & 3 & 5,5 & 8 & 1 & 20,5 \\
\hline $\mathrm{K}=4$ & 4 & 4 & 8 & 2 & 6 & 24 \\
\hline $\mathrm{K}=5$ & 5 & 5,5 & 9,5 & 3 & 8,5 & 31,5 \\
\hline $\mathrm{K}=6$ & 6 & 7 & 9,5 & 4 & 3 & 29,5 \\
\hline $\mathrm{K}=7$ & 8 & 9,5 & 3 & 7 & 2 & 29,5 \\
\hline $\mathrm{K}=8$ & 8 & 9,5 & 4 & 9,5 & 7 & 38 \\
\hline $\mathrm{K}=9$ & 8 & 8 & 5,5 & 9,5 & 10 & 41 \\
\hline $\mathrm{K}=10$ & 10 & 5,5 & 7 & 6 & 8,5 & 37 \\
\hline
\end{tabular}




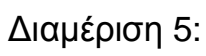

1) $N \Delta$

\begin{tabular}{|c|c|c|c|c|}
\hline \multicolumn{5}{|c|}{ NN (Пívakas E.5.N $\Delta . A)$} \\
\hline \multicolumn{5}{|c|}{ Accept Rate } \\
\hline $70 \%$ & $75 \%$ & $80 \%$ & $85 \%$ & $90 \%$ \\
\hline$N=25(07): 10$ & $\mathrm{~N}=18(10): 9$ & $N=16(13): 9,5$ & $\mathrm{~N}=17(19): 9$ & $\mathrm{~N}=21(31): 9,5$ \\
\hline $\mathrm{N}=17(08): 8,5$ & $\mathrm{~N}=20(10): 9$ & $\mathrm{~N}=23(13): 9,5$ & $\mathrm{~N}=22(19): 9$ & $\mathrm{~N}=22(31): 9,5$ \\
\hline $\mathrm{N}=23(08): 8,5$ & $\mathrm{~N}=21(10): 9$ & $\mathrm{~N}=17(14): 6,5$ & $\mathrm{~N}=23(19): 9$ & $\mathrm{~N}=24(32): 8$ \\
\hline $\mathrm{N}=16(09): 5,5$ & $\mathrm{~N}=16(11): 6$ & $\mathrm{~N}=18(14): 6,5$ & $\mathrm{~N}=21(21): 7$ & $\mathrm{~N}=16(33): 5$ \\
\hline $\mathrm{N}=18(09): 5,5$ & $\mathrm{~N}=23(11): 6$ & $\mathrm{~N}=22(14): 6.5$ & $\mathrm{~N}=16(22): 6$ & $\mathrm{~N}=17(33): 5$ \\
\hline $\mathrm{N}=19(09): 5,5$ & $\mathrm{~N}=25(11): 6$ & $\mathrm{~N}=25(14): 6,5$ & $\mathrm{~N}=24(23): 5$ & $\mathrm{~N}=19(33): 5$ \\
\hline $\mathrm{N}=21(09): \quad 5,5$ & $\mathrm{~N}=19(12): 3$ & $\mathrm{~N}=21(15): 4$ & $\mathrm{~N}=18(24): 3,5$ & $\mathrm{~N}=20(33): 5$ \\
\hline $\mathrm{N}=20(10): 2$ & $\mathrm{~N}=22(12): 3$ & $\mathrm{~N}=20(17): 3$ & $\mathrm{~N}=25(24): 3,5$ & $\mathrm{~N}=25(33): 5$ \\
\hline $\mathrm{N}=22(10): 2$ & $\mathrm{~N}=24(12): 3$ & $\mathrm{~N}=19(18): 2$ & $\mathrm{~N}=19(25): 2$ & $\mathrm{~N}=18(34): 1,5$ \\
\hline $\mathrm{N}=24(10): 2$ & $\mathrm{~N}=17(13): 1$ & $\mathrm{~N}=24(19): 1$ & $\mathrm{~N}=20(26): 1$ & $\mathrm{~N}=23(34): 1,5$ \\
\hline
\end{tabular}

\begin{tabular}{|l|r|l|l|l|l|l|}
\hline \multicolumn{7}{|c|}{ NN (Пivakas E.5.N $\Delta$. B) } \\
\hline \multicolumn{7}{|c|}{ Accept Rate } \\
\hline & $70 \%$ & $75 \%$ & $80 \%$ & $85 \%$ & $90 \%$ & \\
\hline $\mathrm{N}=16$ & 5,5 & 6 & 9,5 & 6 & 5 & 32 \\
\hline $\mathrm{N}=17$ & 8,5 & 1 & 6,5 & 9 & 5 & 30 \\
\hline $\mathrm{N}=18$ & 5,5 & 9 & 6,5 & 3,5 & 1,5 & 26 \\
\hline $\mathrm{N}=19$ & 5,5 & 3 & 2 & 2 & 5 & 17,5 \\
\hline $\mathrm{N}=20$ & 2 & 9 & 3 & 1 & 5 & 20 \\
\hline $\mathrm{N}=\mathbf{2 1}$ & 5,5 & 9 & 4 & 7 & 9,5 & $\mathbf{3 5}$ \\
\hline $\mathrm{N}=22$ & 2 & 3 & 6,5 & 9 & 9,5 & 30 \\
\hline $\mathrm{N}=23$ & 8,5 & 6 & 9,5 & 9 & 1,5 & 34,5 \\
\hline $\mathrm{N}=24$ & 2 & 3 & 1 & 5 & 8 & 19 \\
\hline $\mathrm{N}=25$ & 10 & 6 & 6,5 & 3,5 & 5 & 31 \\
\hline
\end{tabular}




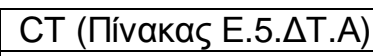

\begin{tabular}{|l|l|l|l|l|}
\hline \multicolumn{7}{|c|}{ Accept Rate } \\
\hline $70 \%$ & $75 \%$ & $80 \%$ & $85 \%$ & $90 \%$ \\
\hline$P=0,9 \%(09): 9,5$ & $P=0,3 \%(11): 10$ & $P=0,6 \%(15): 10$ & $P=0,2 \%(19): 10$ & $P=0,3 \%(32): 10$ \\
\hline$P=1,0 \%(09): 9,5$ & $P=0,2 \%(12): 9$ & $P=0,1 \%(17): 8$ & $P=0,3 \%(22): 8,5$ & $P=0,1 \%(33): 8,5$ \\
\hline$P=0,3 \%(10): 8$ & $P=0,4 \%(13): 8$ & $P=0,4 \%(17): 8$ & $P=0,4 \%(22): 8,5$ & $P=0,2 \%(33): 8,5$ \\
\hline$P=0,4 \%(11): 5,5$ & $P=0,6 \%(14): 6,5$ & $P=0,8 \%(17): 8$ & $P=0,1 \%(23): 6,5$ & $P=0,6 \%(35): 6$ \\
\hline$P=0,5 \%(11): 5,5$ & $P=1,0 \%(14): 6,5$ & $P=0,2 \%(18): 5$ & $P=0,5 \%(23): 6,5$ & $P=0,7 \%(35): 6$ \\
\hline$P=0,6 \%(11): 5,5$ & $P=0,5 \%(15): 4,5$ & $P=0,3 \%(18): 5$ & $P=0,6 \%(25): 5$ & $P=0,8 \%(35): 6$ \\
\hline$P=0,8 \%(11): 5,5$ & $P=0,9 \%(15): 4,5$ & $P=0,7 \%(18): 5$ & $P=0,7 \%(27): 3$ & $P=0,4 \%(37): 3,5$ \\
\hline$P=0,2 \%(12): 2,5$ & $P=0,8 \%(16): 3$ & $P=0,5 \%(20): 2$ & $P=0,8 \%(27): 3$ & $P=0,9 \%(37): 3,5$ \\
\hline$P=0,7 \%(12): 2,5$ & $P=0,1 \%(17): 2$ & $P=0,9 \%(20): 2$ & $P=0,9 \%(27): 3$ & $P=0,5 \%(39): 2$ \\
\hline$P=0,1 \%(17): 1$ & $P=0,7 \%(18): 1$ & $P=1,0 \%(20): 2$ & $P=1,0 \%(31): 1$ & $P=1,0 \%(40): 1$ \\
\hline
\end{tabular}

\begin{tabular}{|c|c|c|c|c|c|c|}
\hline \multicolumn{7}{|c|}{ 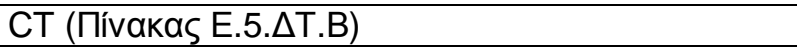 } \\
\hline \multicolumn{7}{|c|}{ Accept Rate } \\
\hline & $70 \%$ & $75 \%$ & $80 \%$ & $85 \%$ & $90 \%$ & \\
\hline $\mathrm{P}=0,1 \%$ & 1 & 2 & 8 & 6,5 & 8,5 & 26 \\
\hline $\mathrm{P}=0,2 \%$ & 2,5 & 9 & 5 & 10 & 8,5 & 35 \\
\hline$P=0,3 \%$ & 8 & 10 & 5 & 8,5 & 10 & 41,5 \\
\hline $\mathrm{P}=0,4 \%$ & 5,5 & 8 & 8 & 8,5 & 3,5 & 33,5 \\
\hline$P=0,5 \%$ & 5,5 & 4,5 & 2 & 6,5 & 2 & 20,5 \\
\hline $\mathrm{P}=0,6 \%$ & 5,5 & 6,5 & 10 & 5 & 6 & 33 \\
\hline $\mathrm{P}=0,7 \%$ & 2,5 & 1 & 5 & 3 & 6 & 17,5 \\
\hline $\mathrm{P}=0,8 \%$ & 5,5 & 3 & 8 & 3 & 6 & 25,5 \\
\hline $\mathrm{P}=0,9 \%$ & 9,5 & 4,5 & 2 & 3 & 3,5 & 22,5 \\
\hline $\mathrm{P}=1,0 \%$ & 9,5 & 6,5 & 2 & 1 & 1 & 20 \\
\hline
\end{tabular}




\begin{tabular}{|c|c|c|c|c|}
\hline \multicolumn{5}{|c|}{ 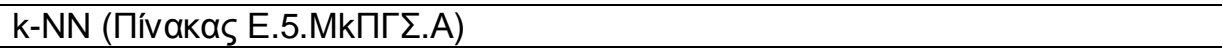 } \\
\hline \multicolumn{5}{|c|}{ Accept Rate } \\
\hline $70 \%$ & $75 \%$ & $80 \%$ & $85 \%$ & $90 \%$ \\
\hline $\mathrm{K}=9(10): 10$ & $\mathrm{~K}=6(17): 9,5$ & $\mathrm{~K}=10(17): 10$ & $\mathrm{~K}=7(26): 9,5$ & $\mathrm{~K}=10(34): 10$ \\
\hline $\mathrm{K}=8(11): 8,5$ & $\mathrm{~K}=10(17): 9,5$ & $\mathrm{~K}=9(20): 9$ & $\mathrm{~K}=8(26): 9,5$ & $\mathrm{~K}=9(35): 9$ \\
\hline $\mathrm{K}=10(11): 8,5$ & $\mathrm{~K}=5(20): 7$ & $\mathrm{~K}=4(22): 8$ & $\mathrm{~K}=6(27): 7,5$ & $\mathrm{~K}=8(36): 8$ \\
\hline $\mathrm{K}=7(15): 7$ & $K=7(20): 7$ & $\mathrm{~K}=8(23): 7$ & $\mathrm{~K}=9(27): 7,5$ & $\mathrm{~K}=4(37): 7$ \\
\hline$K=6(17): 6$ & $K=9(20): 7$ & $\mathrm{~K}=3(24): 6$ & $\mathrm{~K}=3(29): 5,5$ & $K=7(38): 6$ \\
\hline $\mathrm{K}=5(20): 5$ & $K=4(22): 5$ & $\mathrm{~K}=7(26): 5$ & $\mathrm{~K}=10(29): 5,5$ & $\mathrm{~K}=5(39): 5$ \\
\hline $\mathrm{K}=4(22): 4$ & $\mathrm{~K}=8(23): 4$ & $\mathrm{~K}=6(27): 4$ & $\mathrm{~K}=2(31): 4$ & $\mathrm{~K}=6(40): 4$ \\
\hline $\mathrm{K}=3(24): 3$ & $K=3(24): 3$ & $\mathrm{~K}=2(31): 3$ & $K=5(32): 3$ & $\mathrm{~K}=1(41): 3$ \\
\hline$K=2(31): 2$ & $K=2(31): 2$ & $K=5(32): 2$ & $K=4(37): 2$ & $\mathrm{~K}=3(44): 2$ \\
\hline $\mathrm{K}=1(41): 1$ & $\mathrm{~K}=1(41): 1$ & $\mathrm{~K}=1(41): 1$ & $\mathrm{~K}=1(41): 1$ & $\mathrm{~K}=2(52): 1$ \\
\hline
\end{tabular}

\begin{tabular}{|l|c|l|c|l|c|c|}
\hline \multicolumn{7}{|c|}{ k-NN (Пívakas E.5.MkПГ Accept Rate } \\
\hline & $70 \%$ & $75 \%$ & $80 \%$ & $85 \%$ & $90 \%$ & \\
\hline $\mathrm{K}=1$ & 1 & 1 & 1 & 1 & 3 & 7 \\
\hline $\mathrm{K}=2$ & 2 & 2 & 3 & 4 & 1 & 12 \\
\hline $\mathrm{K}=3$ & 3 & 3 & 6 & 5,5 & 2 & 19,5 \\
\hline $\mathrm{K}=4$ & 4 & 5 & 8 & 2 & 7 & 26 \\
\hline $\mathrm{K}=5$ & 5 & 7 & 2 & 3 & 5 & 22 \\
\hline $\mathrm{K}=6$ & 6 & 9,5 & 4 & 7,5 & 4 & 31 \\
\hline $\mathrm{K}=7$ & 7 & 7 & 5 & 9,5 & 6 & 34,5 \\
\hline $\mathrm{K}=8$ & 8,5 & 4 & 7 & 9,5 & 8 & 37 \\
\hline $\mathrm{K}=9$ & 10 & 7 & 9 & 7,5 & 9 & 42,5 \\
\hline $\mathrm{K}=10$ & 8,5 & 9,5 & 10 & 5,5 & 10 & 43,5 \\
\hline
\end{tabular}




\section{ПАРАРТНMА $\Sigma T$}

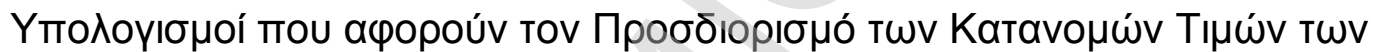

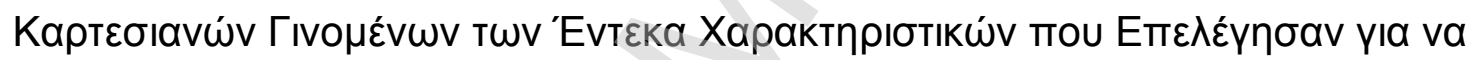

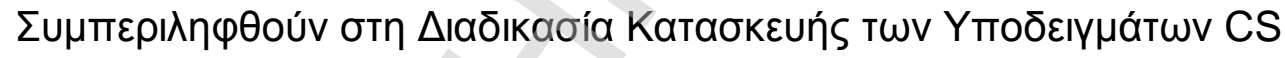




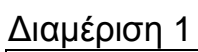

\begin{tabular}{|c|c|c|c|c|c|c|c|c|c|c|c|}
\hline & A & $\mathrm{B}$ & C & $D$ & $E$ & $\mathrm{~F}$ & $\mathrm{G}$ & $\mathrm{H}$ & 1 & $\mathrm{~J}$ & $\mathrm{~K}$ \\
\hline 1 & AGE\CAR & BADS & GOODS & $f(x / 1)$ & $f(x / 0)$ & $w(x)$ & & & & & \\
\hline 2 & $0 \backslash 0$ & 111 & 432 & 0,110642 & 0,305479 & $-1,01558$ & 0,026029 & 0,137009 & 0,101215 & 0,334465 & \\
\hline 3 & $0 \backslash 1$ & 107 & 1101 & 0,281786 & 0,294521 & $-0,0442$ & 0,025095 & 0,133416 & 0,257776 & 0,504161 & \\
\hline 4 & $1 \backslash 0$ & 38 & 410 & 0,105014 & 0,105479 & $-0,00442$ & 0,008987 & $0,061096^{-}$ & 0,096066 & 0,324687 & \\
\hline 5 & $1 \backslash 1$ & 31 & 1399 & 0,35802 & 0,086301 & 1,422743 & 0,007353 & $0,052116^{-}$ & 0,327515 & $0,527419^{-}$ & \\
\hline 6 & 210 & 51 & 155 & 0,03978 & 0,141096 & $-1,26608$ & 0,012022 & $0,076680^{-}$ & 0,036391 & $0,173957^{-}$ & \\
\hline 7 & $2 \backslash 1$ & 26 & 411 & 0,10527 & 0,072603 & 0,371525 & 0,006186 & 0,045386 & 0,0963 & $0,325140^{-}$ & \\
\hline 8 & & 364 & 3908 & & & 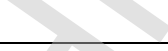 & & 0,505703 & & 2,18983 & \\
\hline 9 & & & & & & $y$ & & 0,043089 & & 2,003243 & 2,046332 \\
\hline
\end{tabular}

Yтó

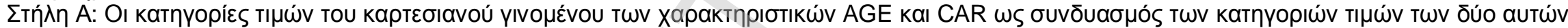
характпрібтіки́v

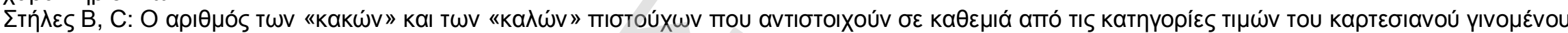

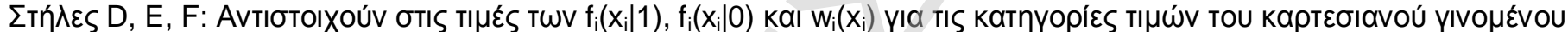

$\Sigma T \eta ́ \lambda \eta \mathrm{G}: \mathrm{G}=\mathrm{E}^{\star} 364 / 4272$

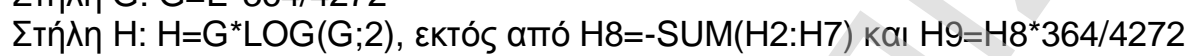

$\sum т n ́ \lambda \eta$ I: I=D*3908/4272

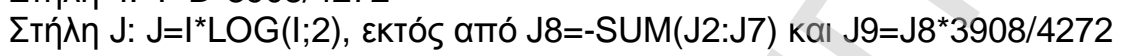

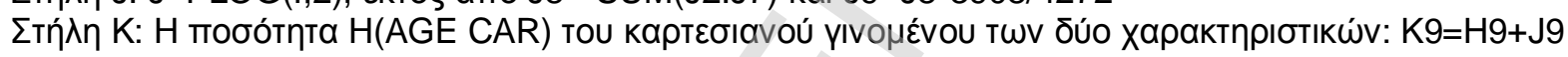




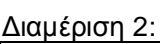

\begin{tabular}{|c|c|c|c|c|c|c|c|c|c|c|}
\hline AGE\CAR & BADS & GOODS & $f(x / 1)$ & $f(x / 0)$ & $w(x)$ & & & & 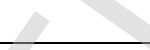 & \\
\hline $0 \backslash 0$ & 112 & 437 & 0,111921 & 0,308219 & $-1,01302$ & 0,026262 & $-0,137899$ & 0,102385 & $-0,336634$ & \\
\hline $0 \backslash 1$ & 116 & 1113 & 0,284855 & 0,319178 & $-0,11377$ & 0,027196 & $-0,141431$ & 0,260584 & $-0,505580$ & \\
\hline 110 & 37 & 422 & 0,108084 & 0,10274 & 0,050709 & 0,008754 & $-0,059841$ & 0,098875 & $-0,330069$ & \\
\hline $1 \backslash 1$ & 27 & 1375 & 0,35188 & 0,075342 & 1,541247 & 0,00642 & $-0,046756$ & 0,321898 & $-0,526408$ & \\
\hline 210 & 45 & 159 & 0,040803 & 0,124658 & $-1,11681$ & 0,010622 & $-0,069644$ & 0,037327 & $-0,177064$ & \\
\hline \multirow[t]{3}{*}{$2 \backslash 1$} & 27 & 402 & 0,102968 & 0,075342 & 0,31237 & 0,00642 & $-0,046756$ & 0,094194 & $-0,321034$ & \\
\hline & 364 & 3908 & & & & & 0,502328 & 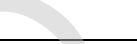 & 2,196788 & \\
\hline & & & & & & & 0,042801 & & 2,009609 & 2,05241 \\
\hline
\end{tabular}

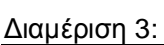

\begin{tabular}{|l|r|r|r|r|r|r|r|r|r|l|}
\hline AGEICAR & \multicolumn{1}{|c|}{ BADS } & \multicolumn{1}{l|}{ GOODS } & $\mathrm{f}(\mathrm{x} / 1)$ & $\mathrm{f}(\mathrm{x} / 0)$ & $\mathrm{w}(\mathrm{x})$ & & & & & \\
\hline 010 & 119 & 440 & 0,112689 & 0,327397 & $-1,06655$ & 0,027896 & $-0,144050$ & 0,103087 & $-0,337926$ & \\
\hline 011 & 107 & 1120 & 0,286646 & 0,294521 & $-0,0271$ & 0,025095 & $-0,133416$ & 0,262222 & $-0,506387$ & \\
\hline 110 & 37 & 398 & 0,101944 & 0,10274 & $-0,00777$ & 0,008754 & $-0,059841$ & 0,093258 & $-0,319187$ & \\
\hline $1 \backslash 1$ & 28 & 1399 & 0,35802 & 0,078082 & 1,522827 & 0,006653 & $-0,048113$ & 0,327515 & $-0,527419$ & \\
\hline 210 & 49 & 152 & 0,039013 & 0,135616 & $-1,24595$ & 0,011555 & $-0,074362$ & 0,035688 & $-0,171604$ & \\
\hline 211 & 24 & 399 & 0,1022 & 0,067123 & 0,420401 & 0,005719 & $-0,042609$ & 0,093492 & $-0,319650$ & \\
\hline & 364 & 3908 & & & & & 0,502391 & & 2,182175 & \\
\hline & & & & & & & 0,042807 & & 1,99624 & 2,039047 \\
\hline
\end{tabular}

$\triangle$ ıанદ́pıơ 4:

\begin{tabular}{|l|r|r|l|r|r|r|r|r|r|l|}
\hline AGEICAR & \multicolumn{1}{l|}{ BADS } & \multicolumn{1}{l}{ GOODS } & $\mathrm{f}(\mathrm{x} / 1)$ & $\mathrm{f}(\mathrm{x} / 0)$ & $\mathrm{w}(\mathrm{x})$ & & & & & \\
\hline $0 \backslash 0$ & 113 & 450 & 0,115247 & 0,310959 & $-0,99258$ & 0,026496 & $-0,138787$ & 0,105427 & $-0,342183$ & \\
\hline $0 \backslash 1$ & 120 & 1095 & 0,280251 & 0,330137 & $-0,16382$ & 0,02813 & $-0,144917$ & 0,256372 & $-0,503435$ & \\
\hline 110 & 45 & 475 & 0,121642 & 0,124658 & $-0,02448$ & 0,010622 & $-0,069644$ & 0,111278 & $-0,352501$ & \\
\hline $1 \backslash 1$ & 33 & 1518 & 0,388463 & 0,091781 & 1,442793 & 0,00782 & $-0,054731$ & 0,355363 & $-0,530427$ & \\
\hline 210 & 38 & 110 & 0,028268 & 0,105479 & $-1,31678$ & 0,008987 & $-0,061096$ & 0,025859 & $-0,136361$ & \\
\hline $2 \backslash 1$ & 15 & 260 & 0,066641 & 0,042466 & 0,450623 & 0,003618 & $-0,029346$ & 0,060963 & $-0,246042$ & \\
\hline & 364 & 3908 & & & & & 0,498521 & & 2,110949 & \\
\hline & & & & & & & 0,042477 & & 1,931084 & 1,973561 \\
\hline
\end{tabular}




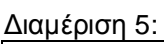

\begin{tabular}{|c|c|c|c|c|c|c|c|c|c|c|}
\hline AGE\CAR & BADS & GOODS & $f(x / 1)$ & $f(x / 0)$ & $w(x)$ & & & & 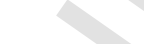 & 1 \\
\hline 010 & 117 & 425 & 0,108851 & 0,321918 & $-1,08431$ & 0,027429 & $-0,142307$ & 0,099577 & $-0,331396$ & \\
\hline $0 \backslash 1$ & 114 & 1095 & 0,280251 & 0,313699 & $-0,11275$ & 0,026729 & $-0,139671$ & 0,256372 & $-0,503435$ & \\
\hline 110 & 35 & 412 & 0,105526 & 0,09726 & 0,081564 & 0,008287 & $-0,057305$ & 0,096534 & $-0,325592$ & \\
\hline $1 \backslash 1$ & 31 & 1397 & 0,357508 & 0,086301 & 1,421313 & 0,007353 & $-0,052116$ & 0,327046 & $-0,527340$ & \\
\hline 210 & 45 & 159 & 0,040803 & 0,124658 & $-1,11681$ & 0,010622 & $-0,069644$ & 0,037327 & $-0,177064$ & \\
\hline \multirow[t]{3}{*}{$2 \backslash 1$} & 22 & 420 & 0,107572 & 0,061644 & 0,55679 & 0,005252 & $-0,039776$ & 0,098406 & $-0,329180$ & \\
\hline & 364 & 3908 & & & & & 0,500819 & & 2,194007 & \\
\hline & & & & & & & 0,042673 & 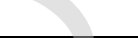 & 2,007065 & 2,049738 \\
\hline
\end{tabular}

2) $\quad H(A G E C R D)$

$\triangle$ ıан́́pıon 1:

\begin{tabular}{|l|r|r|l|l|l|l|l|l|l|l|}
\hline AGEICRD & \multicolumn{1}{|c|}{ BADS } & GOODS & $f(x / 1)$ & $f(x / 0)$ & $w(x)$ & & & & \\
\hline 010 & 182 & 902 & 0,230877 & 0,500000 & $-0,772721$ & 0,042603 & $-0,193967$ & 0,211205 & $-0,473793$ & \\
\hline $0 \backslash 1$ & 36 & 631 & 0,161550 & 0,100000 & 0,479646 & 0,008521 & $-0,058578$ & 0,147785 & $-0,407655$ & \\
\hline 110 & 59 & 1169 & 0,299181 & 0,163014 & 0,607216 & 0,013890 & $-0,085697$ & 0,273689 & $-0,511632$ & \\
\hline $1 \backslash 1$ & 10 & 640 & 0,163853 & 0,028767 & 1,739734 & 0,002451 & $-0,021257$ & 0,149891 & $-0,410404$ & \\
\hline 210 & 68 & 438 & 0,112177 & 0,187671 & $-0,514613$ & 0,015991 & $-0,095411$ & 0,102619 & $-0,337065$ & \\
\hline 211 & 9 & 128 & 0,032873 & 0,026027 & 0,233498 & 0,002218 & $-0,019553$ & 0,030072 & $-0,152027$ & \\
\hline & 364 & 3908 & & & & & 0,474463 & & 2,292576 & \\
\hline & & & & & & & 0,040427 & & 2,097234 & 2,137662 \\
\hline
\end{tabular}

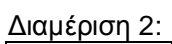

\begin{tabular}{|l|r|r|l|l|l|l|l|l|l|l|}
\hline AGEICRD & \multicolumn{1}{l|}{ BADS } & GOODS & $\mathrm{f}(\mathrm{x} / 1)$ & $\mathrm{f}(\mathrm{x} / 0)$ & $\mathrm{w}(\mathrm{x})$ & & & & & \\
\hline 010 & 188 & 935 & 0,239320 & 0,516438 & $-0,769156$ & 0,044004 & $-0,198291$ & 0,218928 & $-0,479775$ & \\
\hline 011 & 40 & 615 & 0,157457 & 0,110959 & 0,349993 & 0,009454 & $-0,063579$ & 0,144041 & $-0,402659$ & \\
\hline 110 & 52 & 1171 & 0,299693 & 0,143836 & 0,734088 & 0,012256 & $-0,077828$ & 0,274157 & $-0,511831$ & \\
\hline 111 & 12 & 626 & 0,160271 & 0,034247 & 1,543281 & 0,002918 & $-0,024572$ & 0,146615 & $-0,406108$ & \\
\hline 210 & 61 & 431 & 0,110386 & 0,168493 & $-0,422909$ & 0,014357 & $-0,087893$ & 0,100981 & $-0,334029$ & \\
\hline 211 & 11 & 130 & 0,033384 & 0,031507 & 0,057887 & 0,002685 & $-0,022929$ & 0,030540 & $-0,153712$ & \\
\hline & 364 & 3908 & & & & & 0,475092 & & 2,288114 & \\
\hline & & & & & & & 0,040481 & & 2,093153 & 2,133634 \\
\hline
\end{tabular}




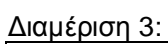

\begin{tabular}{|l|r|r|l|l|l|l|l|l|l|l|}
\hline AGEICRD & \multicolumn{1}{l|}{ BADS } & GOODS & $f(x / 1)$ & $f(x / 0)$ & $w(x)$ & & & & \\
\hline 010 & 191 & 933 & 0,238808 & 0,524658 & $-0,787086$ & 0,044704 & $-0,200428$ & 0,218460 & $-0,479423$ & \\
\hline 011 & 35 & 627 & 0,160527 & 0,097260 & 0,501071 & 0,008287 & $-0,057305$ & 0,146849 & $-0,406419$ & \\
\hline 110 & 57 & 1167 & 0,298670 & 0,157534 & 0,639696 & 0,013423 & $-0,083479$ & 0,273221 & $-0,511432$ & \\
\hline $1 \backslash 1$ & 8 & 630 & 0,161294 & 0,023288 & 1,935307 & 0,001984 & $-0,017813$ & 0,147551 & $-0,407346$ & \\
\hline 210 & 61 & 419 & 0,107316 & 0,168493 & $-0,451113$ & 0,014357 & $-0,087893$ & 0,098172 & $-0,328734$ & \\
\hline 211 & 12 & 132 & 0,033896 & 0,034247 & $-0,010286$ & 0,002918 & $-0,024572$ & 0,031008 & $-0,155388$ & \\
\hline & 364 & 3908 & & & & & 0,471490 & & 2,288742 & \\
\hline & & & & & & & 0,040174 & & 2,093727 & 2,133901 \\
\hline
\end{tabular}

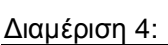

\begin{tabular}{|l|r|r|l|l|l|l|l|l|l|l|}
\hline AGEICRD & \multicolumn{1}{|c|}{ BADS } & GOODS & $\mathrm{f}(\mathrm{x} / 1)$ & $\mathrm{f}(\mathrm{x} / 0)$ & $\mathrm{w}(\mathrm{x})$ & & & & & \\
\hline 010 & 195 & 920 & 0,235482 & 0,535616 & $-0,821783$ & 0,045638 & $-0,203254$ & 0,215418 & $-0,477105$ & \\
\hline 011 & 38 & 625 & 0,160015 & 0,105479 & 0,416754 & 0,008987 & $-0,061096$ & 0,146381 & $-0,405797$ & \\
\hline 110 & 66 & 1301 & 0,332950 & 0,182192 & 0,602931 & 0,015524 & $-0,093288$ & 0,304580 & $-0,522387$ & \\
\hline 111 & 12 & 692 & 0,177155 & 0,034247 & 1,643440 & 0,002918 & $-0,024572$ & 0,162061 & $-0,425473$ & \\
\hline 210 & 44 & 288 & 0,073804 & 0,121918 & $-0,501934$ & 0,010388 & $-0,068447$ & 0,067515 & $-0,262543$ & \\
\hline 211 & 9 & 82 & 0,021105 & 0,026027 & $-0,209633$ & 0,002218 & $-0,019553$ & 0,019307 & $-0,109948$ & \\
\hline & 364 & 3908 & & & & & 0,470209 & & 2,203254 & \\
\hline & & & & & & & 0,040065 & & 2,015524 & 2,055588 \\
\hline
\end{tabular}

$\triangle$ ıанદ́pıơ 5:

\begin{tabular}{|l|r|r|l|l|l|l|l|l|l|l|}
\hline AGEICRD & \multicolumn{1}{|c|}{ BADS } & GOODS & $\mathrm{f}(\mathrm{x} / 1)$ & $\mathrm{f}(\mathrm{x} / 0)$ & $\mathrm{w}(\mathrm{x})$ & & & & \\
\hline 010 & 196 & 898 & 0,229854 & 0,538356 & $-0,851075$ & 0,045871 & $-0,203956$ & 0,210269 & $-0,473041$ & \\
\hline 011 & 35 & 622 & 0,159248 & 0,097260 & 0,493071 & 0,008287 & $-0,057305$ & 0,145679 & $-0,404862$ & \\
\hline 110 & 55 & 1172 & 0,299949 & 0,152055 & 0,679371 & 0,012956 & $-0,081237$ & 0,274391 & $-0,511930$ & \\
\hline 111 & 11 & 637 & 0,163085 & 0,031507 & 1,644068 & 0,002685 & $-0,022929$ & 0,149189 & $-0,409492$ & \\
\hline 210 & 61 & 452 & 0,115759 & 0,168493 & $-0,375389$ & 0,014357 & $-0,087893$ & 0,105895 & $-0,343025$ & \\
\hline 211 & 6 & 127 & 0,032617 & 0,017808 & 0,605175 & 0,001517 & $-0,014209$ & 0,029838 & $-0,151180$ & \\
\hline & 364 & 3908 & & & & & 0,467529 & & 2,293530 & \\
\hline & & & & & & & 0,039836 & & 2,098108 & 2,137944 \\
\hline
\end{tabular}




\begin{tabular}{|c|c|c|c|c|c|c|c|c|c|c|c|}
\hline $0 \backslash 1$ & 0 & 249 & 0,063827 & 0,00137 & 3,841467 & 0,239927 & 0,000117 & $-0,001525$ & 0,058389 & $-0,239286$ & \\
\hline $0 \backslash 3$ & 23 & 67 & 0,017268 & 0,064384 & $-1,316012$ & 0,062005 & 0,005486 & $-0,041199$ & 0,015797 & $-0,094530$ & \\
\hline $1 \backslash 0$ & 26 & 319 & 0,081734 & 0,072603 & 0,118473 & 0,001082 & 0,006186 & $-0,045386$ & 0,07477 & $-0,279745$ & \\
\hline $1 \backslash 1$ & 3 & 289 & 0,07406 & 0,009589 & 2,044253 & 0,131795 & 0,000817 & $-0,008381$ & 0,06775 & $-0,263115$ & \\
\hline 113 & 20 & 310 & 0,079432 & 0,056164 & 0,346620 & 0,008065 & 0,004786 & $-0,036883$ & 0,072664 & $-0,274860$ & \\
\hline 210 & 24 & 137 & 0,035175 & 0,067123 & $-0,646189$ & 0,020644 & 0,005719 & $-0,042609$ & 0,032178 & $-0,159532$ & \\
\hline $2 \backslash 1$ & 13 & 84 & 0,021617 & 0,036986 & $-0,537078$ & 0,008255 & 0,003151 & $-0,026188$ & 0,019775 & $-0,111930$ & \\
\hline $2 \backslash 2$ & 31 & 247 & 0,063315 & 0,086301 & $-0,309716$ & 0,007119 & 0,007353 & $-0,052116$ & 0,057921 & $-0,238041$ & \\
\hline $2 \backslash 3$ & 9 & 98 & 0,025198 & 0,026027 & $-0,032375$ & $2,68 \mathrm{E}-05$ & 0,002218 & $-0,019553$ & 0,023051 & $-0,125376$ & \\
\hline
\end{tabular}




\begin{tabular}{|c|c|c|c|c|c|c|c|c|c|c|c|}
\hline $0 \backslash 1$ & 0 & 243 & 0,062292 & 0,00137 & 3,817125 & 0,232548 & 0,000117 & $-0,001525$ & 0,056984 & $-0,235533$ & \\
\hline 013 & 26 & 73 & 0,018803 & 0,072603 & $-1,350999$ & 0,072684 & 0,006186 & $-0,045386$ & 0,017201 & $-0,100820$ & \\
\hline 110 & 24 & 313 & 0,0802 & 0,067123 & 0,177987 & 0,002327 & 0,005719 & $-0,042609$ & 0,073366 & $-0,276498$ & \\
\hline 113 & 19 & 302 & 0,077386 & 0,053425 & 0,370527 & 0,008878 & 0,004552 & $-0,035412$ & 0,070792 & $-0,270444$ & \\
\hline 210 & 20 & 143 & 0,03671 & 0,056164 & $-0,425229$ & 0,008273 & 0,004786 & $-0,036883$ & 0,033582 & $-0,164424$ & \\
\hline $2 \backslash 1$ & 14 & 95 & 0,024431 & 0,039726 & $-0,486162$ & 0,007436 & 0,003385 & $-0,027779$ & 0,022349 & $-0,122555$ & \\
\hline 212 & 31 & 233 & 0,059734 & 0,086301 & $-0,367945$ & 0,009775 & 0,007353 & $-0,052116$ & 0,054644 & $-0,229166$ & \\
\hline 213 & 7 & 90 & 0,023152 & 0,020548 & 0,119307 & 0,000311 & 0,001751 & $-0,016033$ & 0,021179 & $-0,117781$ & \\
\hline 010 & 156 & 990 & 0,25339 & 0,428767 & $-0,525986$ & 0,092246 & 0,036534 & $-0,174434$ & 0,231799 & $-0,488877$ & \\
\hline $0 \backslash 1$ & 0 & 261 & 0,066897 & 0,00137 & 3,888442 & 0,254798 & 0,000117 & $-0,001525$ & 0,061197 & $-0,246648$ & \\
\hline 012 & 47 & 245 & 0,062804 & 0,130137 & $-0,728572$ & 0,049057 & 0,011088 & $-0,072017$ & 0,057453 & $-0,236790$ & \\
\hline 013 & 23 & 64 & 0,0165 & 0,064384 & $-1,361475$ & 0,065192 & 0,005486 & $-0,041199$ & 0,015094 & $-0,091319$ & \\
\hline 110 & 26 & 305 & 0,078153 & 0,072603 & 0,073666 & 0,000409 & 0,006186 & $-0,045386$ & 0,071494 & $-0,272108$ & \\
\hline $1 \backslash 1$ & 5 & 294 & 0,075339 & 0,015068 & 1,609391 & 0,096999 & 0,001284 & $-0,012332$ & 0,06892 & $-0,265957$ & \\
\hline 112 & 18 & 884 & 0,226273 & 0,050685 & 1,496112 & 0,262699 & 0,004319 & $-0,033924$ & 0,206993 & $-0,470360$ & \\
\hline 113 & 16 & 314 & 0,080455 & 0,045205 & 0,576484 & 0,020321 & 0,003852 & $-0,030892$ & 0,0736 & $-0,277042$ & \\
\hline 210 & 25 & 140 & 0,035943 & 0,069863 & $-0,664610$ & 0,022544 & 0,005953 & $-0,044004$ & 0,03288 & $-0,161989$ & \\
\hline $2 \backslash 1$ & 13 & 82 & 0,021105 & 0,036986 & $-0,561031$ & 0,00891 & 0,003151 & $-0,026188$ & 0,019307 & $-0,109948$ & \\
\hline
\end{tabular}




\begin{tabular}{|c|c|c|c|c|c|c|c|c|c|c|c|}
\hline $0 \backslash 1$ & 0 & 241 & 0,061781 & 0,00137 & 3,808877 & 0,230097 & 0,000117 & $-0,001525$ & 0,056516 & $-0,234271$ & \\
\hline 013 & 27 & 59 & 0,015221 & 0,075342 & $-1,599349$ & 0,096155 & 0,00642 & $-0,046756$ & 0,013924 & $-0,085861$ & \\
\hline 110 & 28 & 359 & 0,091967 & 0,078082 & 0,163671 & 0,002273 & 0,006653 & $-0,048113$ & 0,084131 & $-0,300450$ & \\
\hline 113 & 20 & 354 & 0,090688 & 0,056164 & 0,479144 & 0,016542 & 0,004786 & $-0,036883$ & 0,082961 & $-0,297948$ & \\
\hline 210 & 14 & 99 & 0,025454 & 0,039726 & $-0,445131$ & 0,006353 & 0,003385 & $-0,027779$ & 0,023285 & $-0,126309$ & \\
\hline $2 \backslash 1$ & 10 & 57 & 0,01471 & 0,028767 & $-0,670730$ & 0,009429 & 0,002451 & $-0,021257$ & 0,013456 & $-0,083639$ & \\
\hline 212 & 24 & 161 & 0,041315 & 0,067123 & $-0,485307$ & 0,012525 & 0,005719 & $-0,042609$ & 0,037795 & $-0,178605$ & \\
\hline 213 & 5 & 53 & 0,013686 & 0,015068 & $-0,096206$ & 0,000133 & 0,001284 & $-0,012332$ & 0,01252 & $-0,079123$ & \\
\hline 010 & 152 & 961 & 0,245971 & 0,417808 & $-0,529810$ & 0,091041 & 0,0356 & $-0,171306$ & 0,225013 & $-0,484210$ & \\
\hline $0 \backslash 1$ & 0 & 250 & 0,064083 & 0,00137 & 3,845467 & 0,241161 & 0,000117 & $-0,001525$ & 0,058623 & $-0,239907$ & \\
\hline 012 & 50 & 252 & 0,064595 & 0,138356 & $-0,761702$ & 0,056184 & 0,011789 & $-0,075524$ & 0,059091 & $-0,241145$ & \\
\hline 013 & 29 & 57 & 0,01471 & 0,080822 & $-1,703745$ & 0,112638 & 0,006887 & $-0,049459$ & 0,013456 & $-0,083639$ & \\
\hline 110 & 25 & 312 & 0,079944 & 0,069863 & 0,134787 & 0,001359 & 0,005953 & $-0,044004$ & 0,073132 & $-0,275953$ & \\
\hline $1 \backslash 1$ & 4 & 314 & 0,080455 & 0,012329 & 1,875767 & 0,12779 & 0,00105 & $-0,010394$ & 0,0736 & $-0,277042$ & \\
\hline 112 & 19 & 883 & 0,226017 & 0,053425 & 1,442337 & 0,248936 & 0,004552 & $-0,035412$ & 0,206759 & $-0,470165$ & \\
\hline 113 & 18 & 300 & 0,076874 & 0,050685 & 0,416538 & 0,010909 & 0,004319 & $-0,033924$ & 0,070324 & $-0,269329$ & \\
\hline 210 & 20 & 153 & 0,039268 & 0,056164 & $-0,357864$ & 0,006046 & 0,004786 & $-0,036883$ & 0,035922 & $-0,172391$ & \\
\hline $2 \backslash 1$ & 11 & 78 & 0,020082 & 0,031507 & $-0,450388$ & 0,005146 & 0,002685 & $-0,022929$ & 0,018371 & $-0,105934$ & \\
\hline
\end{tabular}




\begin{tabular}{|c|c|c|c|c|c|c|c|c|c|c|}
\hline AGE\HYR & BADS & GOODS & $f(x / 1)$ & $f(x / 0)$ & $w(x)$ & & & & & \\
\hline 313 & 29 & 263 & 0,067409 & 0,080822 & $-0,18148$ & 0,006887 & $-0,049459$ & 0,061665 & $-0,247856$ & \\
\hline 113 & 42 & 460 & 0,117805 & 0,116438 & 0,011669 & 0,009921 & $-0,066028$ & 0,107767 & $-0,346365$ & \\
\hline 213 & 8 & 471 & 0,120619 & 0,023288 & 1,644713 & 0,001984 & $-0,017813$ & 0,110342 & $-0,350881$ & \\
\hline $3 \backslash 1$ & 25 & 50 & 0,012919 & 0,069863 & $-1,68784$ & 0,005953 & $-0,044004$ & 0,011818 & $-0,075670$ & \\
\hline $1 \backslash 1$ & 128 & 449 & 0,114991 & 0,352055 & $-1,11893$ & 0,029997 & $-0,151757$ & 0,105193 & $-0,341761$ & \\
\hline $2 \backslash 1$ & 45 & 299 & 0,076618 & 0,124658 & $-0,48674$ & 0,010622 & $-0,069644$ & 0,07009 & $-0,268770$ & \\
\hline 312 & 19 & 189 & 0,048478 & 0,053425 & $-0,09716$ & 0,004552 & $-0,035412$ & 0,044347 & $-0,199341$ & \\
\hline 112 & 47 & 622 & 0,159248 & 0,130137 & 0,201874 & 0,011088 & $-0,072017$ & 0,145679 & $-0,404862$ & \\
\hline 212 & 15 & 856 & 0,21911 & 0,042466 & 1,640875 & 0,003618 & $-0,029346$ & 0,20044 & $-0,464772$ & \\
\hline 314 & 4 & 64 & 0,0165 & 0,012329 & 0,291448 & 0,00105 & $-0,010394$ & 0,015094 & $-0,091319$ & \\
\hline $1 \backslash 4$ & 1 & 2 & 0,00064 & 0,00411 & $-1,86031$ & 0,00035 & $-0,004020$ & 0,000585 & $-0,006283$ & \\
\hline \multirow[t]{3}{*}{$2 \backslash 4$} & 1 & 183 & 0,046943 & 0,00411 & 2,43561 & 0,00035 & $-0,004020$ & 0,042943 & $-0,195023$ & \\
\hline & 364 & 3908 & & $\Delta$ & 8 & & 0,553915 & & 2,992903 & \\
\hline & & & & 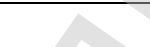 & 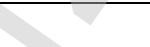 & & 0,047197 & & 2,737890 & 2,785087 \\
\hline
\end{tabular}




\begin{tabular}{|c|c|c|c|c|c|c|c|c|c|c|}
\hline AGEHYR & BADS & GOODS & $f(x / 1)$ & $f(x / 0)$ & $w(x)$ & & & & 8 & \\
\hline 010 & 135 & 459 & 0,117549 & 0,371233 & $-1,14997$ & 0,031631 & $-0,157603$ & 0,107533 & $-0,345950$ & \\
\hline $0 \backslash 1$ & 52 & 619 & 0,15848 & 0,143836 & 0,09696 & 0,012256 & $-0,077828$ & 0,144977 & $-0,403921$ & \\
\hline 012 & 40 & 470 & 0,120363 & 0,110959 & 0,081354 & 0,009454 & $-0,063579$ & 0,110108 & $-0,350474$ & \\
\hline 013 & 1 & 2 & 0,00064 & 0,00411 & $-1,86031$ & 0,00035 & $-0,004020$ & 0,000585 & $-0,006283$ & \\
\hline 110 & 38 & 287 & 0,073548 & 0,105479 & $-0,36057$ & 0,008987 & $-0,061096$ & 0,067281 & $-0,261970$ & \\
\hline $1 \backslash 1$ & 17 & 887 & 0,22704 & 0,047945 & 1,555068 & 0,004085 & $-0,032418$ & 0,207695 & $-0,470940$ & \\
\hline 112 & 8 & 449 & 0,114991 & 0,023288 & 1,59693 & 0,001984 & $-0,017813$ & 0,105193 & $-0,341761$ & \\
\hline 113 & 1 & 174 & 0,044641 & 0,00411 & 2,38532 & 0,00035 & $-0,004020$ & 0,040837 & $-0,188421$ & \\
\hline 210 & 22 & 45 & 0,01164 & 0,061644 & $-1,66694$ & 0,005252 & $-0,039776$ & 0,010648 & $-0,069779$ & \\
\hline $2 \backslash 1$ & 20 & 197 & 0,050524 & 0,056164 & $-0,10583$ & 0,004786 & $-0,036883$ & 0,046219 & $-0,205000$ & \\
\hline 212 & 27 & 253 & 0,06485 & 0,075342 & $-0,14996$ & 0,00642 & $-0,046756$ & 0,059325 & $-0,241761$ & \\
\hline \multirow[t]{3}{*}{213} & 3 & 66 & 0,017012 & 0,009589 & 0,573299 & 0,000817 & $-0,008381$ & 0,015562 & $-0,093465$ & \\
\hline & 364 & 3908 & & & & - & 0,550171 & & 2,979726 & \\
\hline & & & & & & + & 0,046878 & & 2,725835 & 2,772713 \\
\hline \multicolumn{11}{|c|}{ 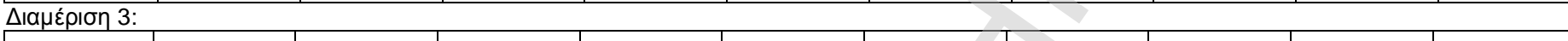 } \\
\hline AGEHYR & BADS & GOODS & $f(x / 1)$ & $f(x / 0)$ & $w(x)$ & $-\quad$ & 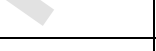 & & & \\
\hline 010 & 134 & 466 & 0,11934 & 0,368493 & $-1,12745$ & 0,031398 & $-0,156775$ & 0,109172 & $-0,348839$ & \\
\hline $0 \backslash 1$ & 53 & 635 & 0,162574 & 0,146575 & 0,103591 & 0,012489 & $-0,078971$ & 0,148721 & $-0,408882$ & \\
\hline 012 & 39 & 455 & 0,116526 & 0,108219 & 0,073956 & 0,009221 & $-0,062341$ & 0,106597 & $-0,344283$ & \\
\hline 013 & 0 & 4 & 0,001151 & 0,00137 & $-0,17391$ & 0,000117 & $-0,001525$ & 0,001053 & $-0,010416$ & \\
\hline 110 & 41 & 302 & 0,077386 & 0,113699 & $-0,38475$ & 0,009688 & $-0,064808$ & 0,070792 & $-0,270444$ & \\
\hline $1 \backslash 1$ & 15 & 883 & 0,226017 & 0,042466 & 1,671912 & 0,003618 & $-0,029346$ & 0,206759 & $-0,470165$ & \\
\hline 112 & 8 & 432 & 0,110642 & 0,023288 & 1,558377 & 0,001984 & $-0,017813$ & 0,101215 & $-0,334465$ & \\
\hline 113 & 1 & 180 & 0,046175 & 0,00411 & 2,419126 & 0,00035 & $-0,004020$ & 0,042241 & $-0,192839$ & \\
\hline 210 & 24 & 51 & 0,013175 & 0,067123 & $-1,62823$ & 0,005719 & $-0,042609$ & 0,012052 & $-0,076827$ & \\
\hline $2 \backslash 1$ & 18 & 181 & 0,046431 & 0,050685 & $-0,08765$ & 0,004319 & $-0,033924$ & 0,042475 & $-0,193569$ & \\
\hline 212 & 30 & 247 & 0,063315 & 0,083562 & $-0,27746$ & 0,00712 & $-0,050793$ & 0,057921 & $-0,238041$ & \\
\hline \multirow[t]{3}{*}{213} & 1 & 72 & 0,018547 & 0,00411 & 1,506982 & 0,00035 & $-0,004020$ & 0,016967 & $-0,099783$ & \\
\hline & 364 & 3908 & & 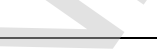 & & & 0,546945 & & 2,988555 & \\
\hline & & & + & & & & 0,046603 & & 2,733912 & 2,780515 \\
\hline
\end{tabular}




\begin{tabular}{|c|c|c|c|c|c|c|c|c|c|c|}
\hline AGEHYR & BADS & GOODS & $f(x / 1)$ & $f(x / 0)$ & $w(x)$ & & & & 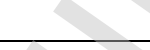 & \\
\hline 010 & 134 & 463 & 0,118573 & 0,368493 & $-1,1339$ & 0,031398 & $-0,156775$ & 0,108469 & $-0,347605$ & \\
\hline $0 \backslash 1$ & 53 & 624 & 0,15976 & 0,146575 & 0,08613 & 0,012489 & $-0,078971$ & 0,146147 & $-0,405486$ & \\
\hline 012 & 45 & 455 & 0,116526 & 0,124658 & $-0,06746$ & 0,010622 & $-0,069644$ & 0,106597 & $-0,344283$ & \\
\hline 013 & 1 & 3 & 0,000895 & 0,00411 & $-1,52384$ & 0,00035 & $-0,004020$ & 0,000819 & $-0,008399$ & \\
\hline $1 \backslash 0$ & 44 & 327 & 0,083781 & 0,121918 & $-0,37514$ & 0,010388 & $-0,068447$ & 0,076642 & $-0,284015$ & \\
\hline $1 \backslash 1$ & 18 & 941 & 0,240854 & 0,050685 & 1,558564 & 0,004319 & $-0,033924$ & 0,220332 & $-0,480819$ & \\
\hline 112 & 15 & 533 & 0,13648 & 0,042466 & 1,16748 & 0,003618 & $-0,029346$ & 0,124851 & $-0,374768$ & \\
\hline 113 & 1 & 192 & 0,049245 & 0,00411 & 2,483492 & 0,00035 & $-0,004020$ & 0,045049 & $-0,201476$ & \\
\hline 210 & 13 & 29 & 0,007547 & 0,036986 & $-1,58944$ & 0,003151 & $-0,026188$ & 0,006904 & $-0,049557$ & \\
\hline $2 \backslash 1$ & 14 & 119 & 0,03057 & 0,039726 & $-0,26197$ & 0,003385 & $-0,027779$ & 0,027966 & $-0,144309$ & \\
\hline 212 & 23 & 171 & 0,043873 & 0,064384 & $-0,38356$ & 0,005486 & $-0,041199$ & 0,040135 & $-0,186186$ & \\
\hline \multirow[t]{3}{*}{213} & 3 & 51 & 0,013175 & 0,009589 & 0,317679 & 0,000817 & $-0,008381$ & 0,012052 & $-0,076827$ & \\
\hline & 364 & 3908 & & & & 4 & 0,548693 & & 2,903731 & \\
\hline & & & & & & $\Delta$ & 0,046752 & & 2,656315 & 2,703067 \\
\hline \multicolumn{11}{|l|}{ 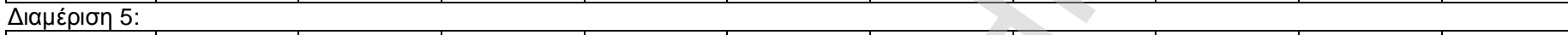 } \\
\hline AGEHYR & BADS & GOODS & $f(x / 1)$ & $f(x / 0)$ & $w(x)$ & 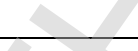 & $\nabla$ & & & \\
\hline 010 & 137 & 467 & 0,119596 & 0,376712 & $-1,14736$ & 0,032098 & $-0,159251$ & 0,109406 & $-0,349249$ & \\
\hline $0 \backslash 1$ & 49 & 597 & 0,152852 & 0,135616 & 0,119642 & 0,011555 & $-0,074362$ & 0,139828 & $-0,396871$ & \\
\hline $0 \backslash 2$ & 44 & 454 & 0,11627 & 0,121918 & $-0,04743$ & 0,010388 & $-0,068447$ & 0,106363 & $-0,343865$ & \\
\hline $0 \backslash 3$ & 1 & 2 & 0,00064 & 0,00411 & $-1,86031$ & 0,00035 & $-0,004020$ & 0,000585 & $-0,006283$ & \\
\hline 110 & 43 & 289 & 0,07406 & 0,119178 & $-0,47575$ & 0,010155 & $-0,067241$ & 0,06775 & $-0,263115$ & \\
\hline $1 \backslash 1$ & 16 & 855 & 0,218854 & 0,045205 & 1,577186 & 0,003852 & $-0,030892$ & 0,200206 & $-0,464567$ & \\
\hline $1 \backslash 2$ & 6 & 483 & 0,123689 & 0,017808 & 1,93811 & 0,001517 & $-0,014209$ & 0,11315 & $-0,355708$ & \\
\hline 113 & 1 & 182 & 0,046687 & 0,00411 & 2,430146 & 0,00035 & $-0,004020$ & 0,042709 & $-0,194297$ & \\
\hline 210 & 21 & 53 & 0,013686 & 0,058904 & $-1,45951$ & 0,005019 & $-0,038337$ & 0,01252 & $-0,079123$ & \\
\hline $2 \backslash 1$ & 16 & 188 & 0,048222 & 0,045205 & 0,064598 & 0,003852 & $-0,030892$ & 0,044113 & $-0,198626$ & \\
\hline 212 & 26 & 266 & 0,068176 & 0,072603 & $-0,06291$ & 0,006186 & $-0,045386$ & 0,062367 & $-0,249660$ & \\
\hline \multirow[t]{3}{*}{213} & 4 & 72 & 0,018547 & 0,012329 & 0,40837 & 0,00105 & $-0,010394$ & 0,016967 & $-0,099783$ & \\
\hline & 364 & 3908 & & 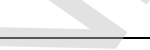 & & & 0,547451 & & 3,001147 & \\
\hline & & & 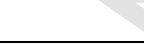 & & & & 0,046646 & & 2,745431 & 2,792078 \\
\hline
\end{tabular}




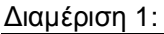

\begin{tabular}{|c|c|c|c|c|c|c|c|c|c|c|}
\hline AGE\HOME & BADS & GOODS & $f(x / 1)$ & $f(x / 0)$ & $w(x)$ & & & 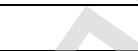 & 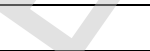 & \\
\hline 310 & 6 & 16 & 0,004221 & 0,017808 & $-1,43958$ & 0,001517 & $-0,014209$ & 0,003861 & $-0,030955$ & \\
\hline 110 & 56 & 476 & 0,121898 & 0,154795 & $-0,23891$ & 0,013189 & $-0,082361$ & 0,111512 & $-0,352905$ & \\
\hline 210 & 3 & 107 & 0,027501 & 0,009589 & 1,053588 & 0,000817 & $-0,008381$ & 0,025157 & $-0,133658$ & \\
\hline $3 \backslash 1$ & 25 & 73 & 0,018803 & 0,069863 & $-1,31253$ & 0,005953 & $-0,044004$ & 0,017201 & $-0,100820$ & \\
\hline $1 \backslash 1$ & 95 & 361 & 0,092479 & 0,261644 & $-1,04$ & 0,022294 & $-0,122330$ & 0,084599 & $-0,301445$ & \\
\hline $2 \backslash 1$ & 34 & 308 & 0,07892 & 0,094521 & $-0,18038$ & 0,008054 & $-0,056023$ & 0,072196 & $-0,273763$ & \\
\hline 312 & 46 & 477 & 0,122154 & 0,127397 & $-0,04203$ & 0,010855 & $-0,070834$ & 0,111746 & $-0,353307$ & \\
\hline 112 & 67 & 696 & 0,178179 & 0,184932 & $-0,0372$ & 0,015757 & $-0,094352$ & 0,162997 & $-0,426576$ & \\
\hline \multirow[t]{3}{*}{212} & 32 & 1394 & 0,356741 & 0,089041 & 1,387912 & 0,007587 & $-0,053429$ & 0,326344 & $-0,527220$ & \\
\hline & 364 & 3908 & & & & 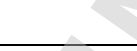 & 0,545922 & & 2,500649 & \\
\hline & & & & & & & 0,046516 & & 2,287579 & 2,334095 \\
\hline
\end{tabular}

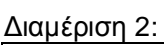

\begin{tabular}{|c|c|c|c|c|c|c|c|c|c|c|}
\hline AGEHOME & BADS & GOODS & $f(x / 1)$ & $f(x / 0)$ & $w(x)$ & 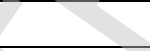 & & & & \\
\hline 010 & 55 & 481 & 0,123177 & 0,152055 & $-0,21062$ & 0,012956 & $-0,081237$ & 0,112682 & $-0,354911$ & \\
\hline $0 \backslash 1$ & 108 & 368 & 0,09427 & 0,29726 & $-1,14845$ & 0,025328 & $-0,134319$ & 0,086237 & $-0,304896$ & \\
\hline 012 & 65 & 701 & 0,179458 & 0,179452 & $3,12 \mathrm{E}-05$ & 0,01529 & $-0,092220$ & 0,164167 & $-0,427944$ & \\
\hline $1 \backslash 0$ & 4 & 113 & 0,029036 & 0,012329 & 0,856586 & 0,00105 & $-0,010394$ & 0,026562 & $-0,139037$ & \\
\hline $1 \backslash 1$ & 31 & 312 & 0,079944 & 0,086301 & $-0,07652$ & 0,007353 & $-0,052116$ & 0,073132 & $-0,275953$ & \\
\hline 112 & 29 & 1372 & 0,351113 & 0,080822 & 1,468859 & 0,006887 & $-0,049459$ & 0,321196 & $-0,526271$ & \\
\hline 210 & 6 & 17 & 0,004477 & 0,017808 & $-1,38074$ & 0,001517 & $-0,014209$ & 0,004095 & $-0,032484$ & \\
\hline $2 \backslash 1$ & 20 & 65 & 0,016756 & 0,056164 & $-1,20951$ & 0,004786 & $-0,036883$ & 0,015328 & $-0,092395$ & \\
\hline \multirow[t]{3}{*}{212} & 46 & 479 & 0,122666 & 0,127397 & $-0,03785$ & 0,010855 & $-0,070834$ & 0,112214 & $-0,354111$ & \\
\hline & 364 & 3908 & & 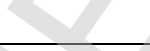 & & & 0,541671 & & 2,508001 & \\
\hline & & & & & & & 0,046154 & & 2,294305 & 2,340458 \\
\hline
\end{tabular}




\begin{tabular}{|c|c|c|c|c|c|c|c|c|c|c|}
\hline AGEHOME & BADS & GOODS & $f(x / 1)$ & $f(x / 0)$ & $w(x)$ & & & & + & \\
\hline 010 & 61 & 482 & 0,123433 & 0,168493 & $-0,3112$ & 0,014357 & $-0,087893$ & 0,112916 & $-0,355310$ & \\
\hline $0 \backslash 1$ & 106 & 366 & 0,093758 & 0,291781 & $-1,13529$ & 0,024861 & $-0,132510$ & 0,085769 & $-0,303914$ & \\
\hline 012 & 59 & 712 & 0,182272 & 0,163014 & 0,111664 & 0,01389 & $-0,085697$ & 0,166741 & $-0,430912$ & \\
\hline 110 & 5 & 113 & 0,029036 & 0,015068 & 0,655915 & 0,001284 & $-0,012332$ & 0,026562 & $-0,139037$ & \\
\hline $1 \backslash 1$ & 33 & 298 & 0,076362 & 0,091781 & $-0,18392$ & 0,00782 & $-0,054731$ & 0,069856 & $-0,268209$ & \\
\hline 112 & 27 & 1386 & 0,354694 & 0,075342 & 1,549212 & 0,00642 & $-0,046756$ & 0,324472 & $-0,526889$ & \\
\hline 210 & 5 & 12 & 0,003198 & 0,015068 & $-1,55016$ & 0,001284 & $-0,012332$ & 0,002925 & $-0,024623$ & \\
\hline 211 & 23 & 68 & 0,017524 & 0,064384 & $-1,30131$ & 0,005486 & $-0,041199$ & 0,016031 & $-0,095591$ & \\
\hline \multirow[t]{3}{*}{212} & 45 & 471 & 0,120619 & 0,124658 & $-0,03293$ & 0,010622 & $-0,069644$ & 0,110342 & $-0,350881$ & \\
\hline & 364 & 3908 & & & & & 0,543096 & & 2,495366 & \\
\hline & & & & & & & 0,046275 & & 2,282746 & 2,329021 \\
\hline
\end{tabular}

\begin{tabular}{|c|c|c|c|c|c|c|c|c|c|c|}
\hline AGEHOME & BADS & GOODS & $f(x / 1)$ & $f(x / 0)$ & $w(x)$ & 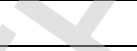 & & & & \\
\hline $0 \backslash 0$ & 57 & 482 & 0,123433 & 0,157534 & $-0,24394$ & 0,013423 & $-0,083479$ & 0,112916 & $-0,355310$ & \\
\hline $0 \backslash 1$ & 108 & 360 & 0,092223 & 0,29726 & $-1,1704$ & 0,025328 & $-0,134319$ & 0,084365 & $-0,300948$ & \\
\hline 012 & 68 & 703 & 0,179969 & 0,187671 & $-0,04191$ & 0,015991 & $-0,095411$ & 0,164635 & $-0,428488$ & \\
\hline 110 & 3 & 117 & 0,030059 & 0,009589 & 1,142536 & 0,000817 & $-0,008381$ & 0,027498 & $-0,142563$ & \\
\hline $1 \backslash 1$ & 37 & 338 & 0,086595 & 0,10274 & $-0,17096$ & 0,008754 & $-0,059841$ & 0,079217 & $-0,289779$ & \\
\hline 112 & 38 & 1538 & 0,393579 & 0,105479 & 1,316765 & 0,008987 & $-0,061096$ & 0,360044 & $-0,530617$ & \\
\hline 210 & 6 & 12 & 0,003198 & 0,017808 & $-1,71721$ & 0,001517 & $-0,014209$ & 0,002925 & $-0,024623$ & \\
\hline $2 \backslash 1$ & 18 & 41 & 0,010617 & 0,050685 & $-1,56322$ & 0,004319 & $-0,033924$ & 0,009712 & $-0,064934$ & \\
\hline \multirow[t]{3}{*}{212} & 29 & 317 & 0,081223 & 0,080822 & 0,004948 & 0,006887 & $-0,049459$ & 0,074302 & $-0,278667$ & \\
\hline & 364 & 3908 & & & & & 0,540118 & & 2,415928 & \\
\hline & & & & & & & 0,046021 & & 2,210076 & 2,256098 \\
\hline
\end{tabular}


$\Delta ı \alpha \mu \dot{p ı \mid \sigma \eta ~ 5: ~}$

\begin{tabular}{|c|c|c|c|c|c|c|c|c|c|c|}
\hline AGEHOME & BADS & GOODS & $f(x / 1)$ & $f(x / 0)$ & $w(x)$ & & & & 8 & \\
\hline 010 & 59 & 471 & 0,120619 & 0,163014 & $-0,3012$ & 0,01389 & $-0,085697$ & 0,110342 & $-0,350881$ & \\
\hline $0 \backslash 1$ & 103 & 361 & 0,092479 & 0,283562 & $-1,12045$ & 0,024161 & $-0,129774$ & 0,084599 & $-0,301445$ & \\
\hline 012 & 69 & 688 & 0,176132 & 0,190411 & $-0,07795$ & 0,016224 & $-0,096464$ & 0,161125 & $-0,424362$ & \\
\hline 110 & 5 & 116 & 0,029803 & 0,015068 & 0,682004 & 0,001284 & $-0,012332$ & 0,027264 & $-0,141686$ & \\
\hline $1 \backslash 1$ & 32 & 306 & 0,078409 & 0,089041 & $-0,12716$ & 0,007587 & $-0,053429$ & 0,071728 & $-0,272661$ & \\
\hline 112 & 29 & 1387 & 0,35495 & 0,080822 & 1,479729 & 0,006887 & $-0,049459$ & 0,324706 & $-0,526931$ & \\
\hline 210 & 5 & 13 & 0,003454 & 0,015068 & $-1,4732$ & 0,001284 & $-0,012332$ & 0,003159 & $-0,026242$ & \\
\hline $2 \backslash 1$ & 19 & 71 & 0,018291 & 0,053425 & $-1,07186$ & 0,004552 & $-0,035412$ & 0,016733 & $-0,098742$ & \\
\hline \multirow[t]{3}{*}{212} & 43 & 495 & 0,126759 & 0,119178 & 0,061667 & 0,010155 & $-0,067241$ & 0,115958 & $-0,360435$ & \\
\hline & 364 & 3908 & & & & & 0,542141 & 3 & 2,503385 & \\
\hline & & & & & & & 0,046194 & & 2,290082 & 2,336275 \\
\hline
\end{tabular}

6) $\quad H(A G E I N C)$

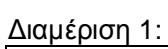

\begin{tabular}{|c|c|c|c|c|c|c|c|c|c|c|}
\hline AGEIINC & BADS & GOODS & $f(x / 1)$ & $f(x / 0)$ & $w(x)$ & 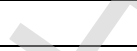 & 8 & & & \\
\hline $1 \backslash 1$ & 16 & 82 & 0,021105 & 0,045205 & $-0,7617$ & 0,003852 & $-0,030892$ & 0,019307 & $-0,109948$ & \\
\hline $2 \backslash 1$ & 55 & 355 & 0,090944 & 0,152055 & $-0,514$ & 0,012956 & $-0,081237$ & 0,083195 & $-0,298450$ & \\
\hline $3 \backslash 1$ & 15 & 278 & 0,071246 & 0,042466 & 0,517439 & 0,003618 & $-0,029346$ & 0,065175 & $-0,256760$ & \\
\hline 112 & 23 & 66 & 0,017012 & 0,064384 & $-1,33094$ & 0,005486 & $-0,041199$ & 0,015562 & $-0,093465$ & \\
\hline 212 & 104 & 346 & 0,088642 & 0,286301 & $-1,17244$ & 0,024395 & $-0,130689$ & 0,081089 & $-0,293894$ & \\
\hline 312 & 30 & 232 & 0,059478 & 0,083562 & $-0,33998$ & 0,00712 & $-0,050793$ & 0,05441 & $-0,228522$ & \\
\hline 113 & 34 & 313 & 0,0802 & 0,094521 & $-0,1643$ & 0,008054 & $-0,056023$ & 0,073366 & $-0,276498$ & \\
\hline 213 & 58 & 722 & 0,18483 & 0,160274 & 0,142551 & 0,013656 & $-0,084591$ & 0,169081 & $-0,433560$ & \\
\hline 313 & 23 & 985 & 0,252111 & 0,064384 & 1,365009 & 0,005486 & $-0,041199$ & 0,230629 & $-0,488093$ & \\
\hline $1 \backslash 4$ & 4 & 105 & 0,026989 & 0,012329 & 0,783494 & 0,00105 & $-0,010394$ & 0,024689 & $-0,131840$ & \\
\hline $2 \backslash 4$ & 1 & 110 & 0,028268 & 0,00411 & 1,928411 & 0,00035 & $-0,004020$ & 0,025859 & $-0,136361$ & \\
\hline \multirow[t]{3}{*}{$3 \backslash 4$} & 1 & 314 & 0,080455 & 0,00411 & 2,974379 & 0,00035 & $-0,004020$ & 0,0736 & $-0,277042$ & \\
\hline & 364 & 3908 & & + & & & 0,564404 & & 3,024433 & \\
\hline & & & . & & & & 0,048091 & & 2,766733 & 2,814824 \\
\hline
\end{tabular}




\begin{tabular}{|c|c|c|c|c|c|c|c|c|c|c|}
\hline AGEINC & BADS & GOODS & $f(x / 1)$ & $f(x / 0)$ & $w(x)$ & & & & 8 & \\
\hline 010 & 113 & 351 & 0,089921 & 0,310959 & $-1,24073$ & 0,026496 & $-0,138787$ & 0,082259 & $-0,296435$ & \\
\hline $0 \backslash 1$ & 63 & 352 & 0,090177 & 0,173973 & $-0,65713$ & 0,014824 & $-0,090067$ & 0,082493 & $-0,296940$ & \\
\hline 012 & 51 & 728 & 0,186365 & 0,141096 & 0,278266 & 0,012022 & $-0,076680$ & 0,170485 & $-0,435126$ & \\
\hline 013 & 1 & 119 & 0,03057 & 0,00411 & 2,006712 & 0,00035 & $-0,004020$ & 0,027966 & $-0,144309$ & \\
\hline 110 & 30 & 225 & 0,057687 & 0,083562 & $-0,37055$ & 0,00712 & $-0,050793$ & 0,052772 & $-0,223969$ & \\
\hline 111 & 11 & 278 & 0,071246 & 0,031507 & 0,815932 & 0,002685 & $-0,022929$ & 0,065175 & $-0,256760$ & \\
\hline 112 & 22 & 989 & 0,253134 & 0,061644 & 1,412545 & 0,005252 & $-0,039776$ & 0,231565 & $-0,488721$ & \\
\hline 113 & 1 & 305 & 0,078153 & 0,00411 & 2,945345 & 0,00035 & $-0,004020$ & 0,071494 & $-0,272108$ & \\
\hline 210 & 18 & 63 & 0,016245 & 0,050685 & $-1,13787$ & 0,004319 & $-0,033924$ & 0,01486 & $-0,090238$ & \\
\hline $2 \backslash 1$ & 18 & 80 & 0,020594 & 0,050685 & $-0,90065$ & 0,004319 & $-0,033924$ & 0,018839 & $-0,107949$ & \\
\hline 212 & 34 & 312 & 0,079944 & 0,094521 & $-0,16749$ & 0,008054 & $-0,056023$ & 0,073132 & $-0,275953$ & \\
\hline \multirow[t]{3}{*}{213} & 2 & 106 & 0,027245 & 0,006849 & 1,380715 & 0,000584 & $-0,006269$ & 0,024923 & $-0,132751$ & \\
\hline & 364 & 3908 & & & & & 0,557211 & & 3,021260 & \\
\hline & & & & & & $\Delta$ & 0,047478 & & 2,763830 & 2,811308 \\
\hline \multicolumn{11}{|c|}{ 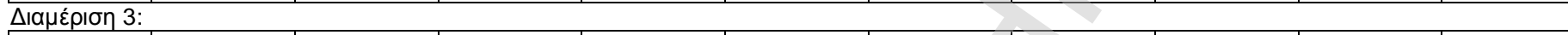 } \\
\hline AGEINC & BADS & GOODS & $f(x / 1)$ & $f(x / 0)$ & $w(x)$ & 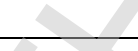 & 8 & & & \\
\hline 010 & 115 & 350 & 0,089665 & 0,316438 & $-1,26105$ & 0,026962 & $-0,140553$ & 0,082025 & $-0,295929$ & \\
\hline $0 \backslash 1$ & 61 & 369 & 0,094525 & 0,168493 & $-0,57803$ & 0,014357 & $-0,087893$ & 0,086471 & $-0,305385$ & \\
\hline 012 & 49 & 740 & 0,189435 & 0,135616 & 0,334213 & 0,011555 & $-0,074362$ & 0,173294 & $-0,438209$ & \\
\hline 013 & 1 & 101 & 0,025966 & 0,00411 & 1,843454 & 0,00035 & $-0,004020$ & 0,023753 & $-0,128166$ & \\
\hline 110 & 29 & 216 & 0,055385 & 0,080822 & $-0,37794$ & 0,006887 & $-0,049459$ & 0,050666 & $-0,218007$ & \\
\hline 111 & 13 & 264 & 0,067664 & 0,036986 & 0,604012 & 0,003151 & $-0,026188$ & 0,061899 & $-0,248459$ & \\
\hline 112 & 22 & 994 & 0,254413 & 0,061644 & 1,417585 & 0,005252 & $-0,039776$ & 0,232735 & $-0,489498$ & \\
\hline 113 & 1 & 323 & 0,082758 & 0,00411 & 3,002594 & 0,00035 & $-0,004020$ & 0,075706 & $-0,281888$ & \\
\hline 210 & 22 & 70 & 0,018035 & 0,061644 & $-1,22904$ & 0,005252 & $-0,039776$ & 0,016499 & $-0,097697$ & \\
\hline 211 & 16 & 75 & 0,019314 & 0,045205 & $-0,85037$ & 0,003852 & $-0,030892$ & 0,017669 & $-0,102879$ & \\
\hline 212 & 31 & 302 & 0,077386 & 0,086301 & $-0,10905$ & 0,007353 & $-0,052116$ & 0,070792 & $-0,270444$ & \\
\hline \multirow[t]{3}{*}{213} & 4 & 104 & 0,026733 & 0,012329 & 0,77397 & 0,00105 & $-0,010394$ & 0,024455 & $-0,130927$ & \\
\hline & 364 & 3908 & & - & & & 0,559448 & & 3,007488 & \\
\hline & & & & & & & 0,047668 & & 2,751232 & 2,798900 \\
\hline
\end{tabular}




\begin{tabular}{|c|c|c|c|c|c|c|c|c|c|c|}
\hline AGEINC & BADS & GOODS & $f(x / 1)$ & $f(x / 0)$ & $w(x)$ & & & & 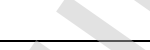 & \\
\hline $0 \backslash 0$ & 120 & 345 & 0,088386 & 0,330137 & $-1,3178$ & 0,02813 & $-0,144917$ & 0,080855 & $-0,293383$ & \\
\hline $0 \backslash 1$ & 59 & 369 & 0,094525 & 0,163014 & $-0,54497$ & 0,01389 & $-0,085697$ & 0,086471 & $-0,305385$ & \\
\hline 012 & 53 & 716 & 0,183295 & 0,146575 & 0,223557 & 0,012489 & $-0,078971$ & 0,167677 & $-0,431977$ & \\
\hline $0 \backslash 3$ & 1 & 115 & 0,029547 & 0,00411 & 1,972666 & 0,00035 & $-0,004020$ & 0,02703 & $-0,140806$ & \\
\hline 110 & 34 & 244 & 0,062548 & 0,094521 & $-0,41288$ & 0,008054 & $-0,056023$ & 0,057219 & $-0,236162$ & \\
\hline $1 \backslash 1$ & 15 & 295 & 0,075595 & 0,042466 & 0,576689 & 0,003618 & $-0,029346$ & 0,069154 & $-0,266522$ & \\
\hline 112 & 26 & 1102 & 0,282041 & 0,072603 & 1,357051 & 0,006186 & $-0,045386$ & 0,25801 & $-0,504281$ & \\
\hline $1 \backslash 3$ & 3 & 352 & 0,090177 & 0,009589 & 2,241148 & 0,000817 & $-0,008381$ & 0,082493 & $-0,296940$ & \\
\hline 210 & 13 & 45 & 0,01164 & 0,036986 & $-1,15612$ & 0,003151 & $-0,026188$ & 0,010648 & $-0,069779$ & \\
\hline $2 \backslash 1$ & 15 & 48 & 0,012407 & 0,042466 & $-1,23042$ & 0,003618 & $-0,029346$ & 0,01135 & $-0,073335$ & \\
\hline 212 & 23 & 216 & 0,055385 & 0,064384 & $-0,15055$ & 0,005486 & $-0,041199$ & 0,050666 & $-0,218007$ & \\
\hline \multirow[t]{3}{*}{213} & 2 & 61 & 0,015733 & 0,006849 & 0,831607 & 0,000584 & $-0,006269$ & 0,014392 & $-0,088061$ & \\
\hline & 364 & 3908 & & & & & 0,555744 & & 2,924638 & \\
\hline & & & & & & $\Delta$ & 0,047353 & & 2,675441 & 2,722794 \\
\hline \multicolumn{11}{|c|}{ 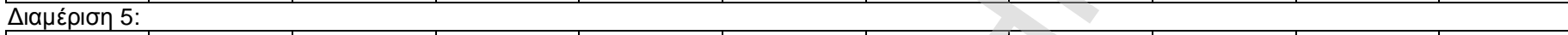 } \\
\hline AGEINC & BADS & GOODS & $f(x / 1)$ & $f(x / 0)$ & $w(x)$ & 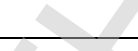 & $\nabla$ & & & \\
\hline 010 & 115 & 345 & 0,088386 & 0,316438 & $-1,27542$ & 0,026962 & $-0,140553$ & 0,080855 & $-0,293383$ & \\
\hline $0 \backslash 1$ & 63 & 344 & 0,08813 & 0,173973 & $-0,68009$ & 0,014824 & $-0,090067$ & 0,080621 & $-0,292871$ & \\
\hline 012 & 53 & 713 & 0,182528 & 0,146575 & 0,219361 & 0,012489 & $-0,078971$ & 0,166975 & $-0,431179$ & \\
\hline 013 & 0 & 118 & 0,030315 & 0,00137 & 3,096921 & 0,000117 & $-0,001525$ & 0,027732 & $-0,143437$ & \\
\hline 110 & 31 & 221 & 0,056664 & 0,086301 & $-0,4207$ & 0,007353 & $-0,052116$ & 0,051836 & $-0,221335$ & \\
\hline $1 \backslash 1$ & 12 & 280 & 0,071757 & 0,034247 & 0,739706 & 0,002918 & $-0,024572$ & 0,065643 & $-0,257926$ & \\
\hline 112 & 23 & 996 & 0,254925 & 0,064384 & 1,376109 & 0,005486 & $-0,041199$ & 0,233203 & $-0,489806$ & \\
\hline 113 & 0 & 312 & 0,079944 & 0,00137 & 4,066612 & 0,000117 & $-0,001525$ & 0,073132 & $-0,275953$ & \\
\hline 210 & 20 & 70 & 0,018035 & 0,056164 & $-1,13595$ & 0,004786 & $-0,036883$ & 0,016499 & $-0,097697$ & \\
\hline $2 \backslash 1$ & 16 & 85 & 0,021873 & 0,045205 & $-0,72598$ & 0,003852 & $-0,030892$ & 0,020009 & $-0,112915$ & \\
\hline 212 & 29 & 322 & 0,082502 & 0,080822 & 0,020573 & 0,006887 & $-0,049459$ & 0,075472 & $-0,281354$ & \\
\hline \multirow[t]{3}{*}{213} & 2 & 102 & 0,026222 & 0,006849 & 1,342433 & 0,000584 & $-0,006269$ & 0,023987 & $-0,129090$ & \\
\hline & 364 & 3908 & & . & & & 0,554031 & & 3,026946 & \\
\hline & & & & & & & 0,047207 & & 2,769032 & 2,816239 \\
\hline
\end{tabular}




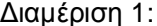

\begin{tabular}{|c|c|c|c|c|c|c|c|c|c|c|}
\hline AGE।JOB & BADS & GOODS & $f(x / 1)$ & $f(x / 0)$ & $w(x)$ & & & 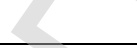 & $\nabla$ & \\
\hline $1 \backslash 1$ & 0 & 56 & 0,014454 & 0,00137 & 2,356248 & 0,000117 & $-0,001525$ & 0,013222 & $-0,082519$ & \\
\hline $2 \backslash 1$ & 47 & 287 & 0,073548 & 0,130137 & $-0,57065$ & 0,011088 & $-0,072017$ & 0,067281 & $-0,261970$ & \\
\hline $3 \backslash 1$ & 11 & 331 & 0,084804 & 0,031507 & 0,990141 & 0,002685 & $-0,022929$ & 0,077578 & $-0,286125$ & \\
\hline 112 & 77 & 323 & 0,082758 & 0,212329 & $-0,94222$ & 0,018092 & $-0,104724$ & 0,075706 & $-0,281888$ & \\
\hline 212 & 4 & 8 & 0,002174 & 0,012329 & $-1,73515$ & 0,00105 & $-0,010394$ & 0,001989 & $-0,017850$ & \\
\hline 312 & 24 & 117 & 0,030059 & 0,067123 & $-0,80337$ & 0,005719 & $-0,042609$ & 0,027498 & $-0,142563$ & \\
\hline 113 & 0 & 88 & 0,02264 & 0,00137 & 2,80501 & 0,000117 & $-0,001525$ & 0,020711 & $-0,115846$ & \\
\hline 213 & 57 & 356 & 0,0912 & 0,157534 & $-0,54659$ & 0,013423 & $-0,083479$ & 0,083429 & $-0,298952$ & \\
\hline 313 & 13 & 460 & 0,117805 & 0,036986 & 1,158484 & 0,003151 & $-0,026188$ & 0,107767 & $-0,346365$ & \\
\hline $1 \backslash 4$ & 0 & 7 & 0,001919 & 0,00137 & 0,336911 & 0,000117 & $-0,001525$ & 0,001755 & $-0,016067$ & \\
\hline $2 \backslash 4$ & 14 & 161 & 0,041315 & 0,039726 & 0,039217 & 0,003385 & $-0,027779$ & 0,037795 & $-0,178605$ & \\
\hline $3 \backslash 4$ & 4 & 77 & 0,019826 & 0,012329 & 0,475061 & 0,00105 & $-0,010394$ & 0,018137 & $-0,104920$ & \\
\hline 115 & 0 & 91 & 0,023408 & 0,00137 & 2,838347 & 0,000117 & $-0,001525$ & 0,021413 & $-0,118743$ & \\
\hline 215 & 85 & 576 & 0,14748 & 0,234247 & $-0,46268$ & 0,019959 & $-0,112706$ & 0,134914 & $-0,389886$ & \\
\hline 315 & 13 & 751 & 0,192249 & 0,036986 & 1,648242 & 0,003151 & $-0,026188$ & 0,175868 & $-0,440978$ & \\
\hline 116 & 0 & 1 & 0,000384 & 0,00137 & $-1,27253$ & 0,000117 & $-0,001525$ & 0,000351 & $-0,004028$ & \\
\hline 216 & 11 & 145 & 0,037222 & 0,031507 & 0,16669 & 0,002685 & $-0,022929$ & 0,03405 & $-0,166036$ & \\
\hline \multirow[t]{4}{*}{316} & 4 & 73 & 0,018803 & 0,012329 & 0,422068 & 0,00105 & $-0,010394$ & 0,017201 & $-0,100820$ & \\
\hline & 364 & 3908 & & & 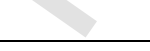 & & $-0,580355$ & & $-3,354161$ & \\
\hline & & & & 2 & & & 0,580355 & & 3,354161 & \\
\hline & & & & 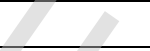 & 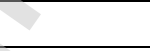 & & 0,04945 & & 3,068367 & 3,117816 \\
\hline
\end{tabular}




\begin{tabular}{|c|c|c|c|c|c|c|c|c|c|c|}
\hline AGEJOB & BADS & GOODS & $f(x / 1)$ & $f(x / 0)$ & $w(x)$ & & & 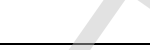 & + & \\
\hline 010 & 3 & 8 & 0,002174 & 0,009589 & $-1,48384$ & 0,000817 & $-0,008381$ & 0,001989 & $-0,017850$ & \\
\hline 012 & 53 & 299 & 0,076618 & 0,146575 & $-0,64871$ & 0,012489 & $-0,078971$ & 0,07009 & $-0,268770$ & \\
\hline 013 & 57 & 350 & 0,089665 & 0,157534 & $-0,56356$ & 0,013423 & $-0,083479$ & 0,082025 & $-0,295929$ & \\
\hline $0 \backslash 4$ & 14 & 169 & 0,043361 & 0,039726 & 0,087565 & 0,003385 & $-0,027779$ & 0,039667 & $-0,184686$ & \\
\hline 015 & 87 & 579 & 0,148248 & 0,239726 & $-0,48061$ & 0,020426 & $-0,114661$ & 0,135616 & $-0,390900$ & \\
\hline 016 & 14 & 145 & 0,037222 & 0,039726 & $-0,06511$ & 0,003385 & $-0,027779$ & 0,03405 & $-0,166036$ & \\
\hline 110 & 25 & 123 & 0,031594 & 0,069863 & $-0,79358$ & 0,005953 & $-0,044004$ & 0,028902 & $-0,147766$ & \\
\hline 112 & 10 & 332 & 0,08506 & 0,028767 & 1,084125 & 0,002451 & $-0,021257$ & 0,077812 & $-0,286650$ & \\
\hline 113 & 12 & 443 & 0,113456 & 0,034247 & 1,19783 & 0,002918 & $-0,024572$ & 0,103789 & $-0,339211$ & \\
\hline 114 & 3 & 78 & 0,020082 & 0,009589 & 0,739196 & 0,000817 & $-0,008381$ & 0,018371 & $-0,105934$ & \\
\hline 115 & 10 & 754 & 0,193016 & 0,028767 & 1,903541 & 0,002451 & $-0,021257$ & 0,17657 & $-0,441723$ & \\
\hline 116 & 4 & 67 & 0,017268 & 0,012329 & 0,336911 & 0,00105 & $-0,010394$ & 0,015797 & $-0,094530$ & \\
\hline 210 & 72 & 337 & 0,086339 & 0,19863 & $-0,83316$ & 0,016924 & $-0,099596$ & 0,078983 & $-0,289260$ & \\
\hline 212 & 0 & 46 & 0,011896 & 0,00137 & 2,16146 & 0,000117 & $-0,001525$ & 0,010882 & $-0,070972$ & \\
\hline 213 & 0 & 77 & 0,019826 & 0,00137 & 2,672286 & 0,000117 & $-0,001525$ & 0,018137 & $-0,104920$ & \\
\hline 214 & 0 & 7 & 0,001919 & 0,00137 & 0,336911 & 0,000117 & $-0,001525$ & 0,001755 & $-0,016067$ & \\
\hline 215 & 0 & 93 & 0,023919 & 0,00137 & 2,859969 & 0,000117 & $-0,001525$ & 0,021881 & $-0,120656$ & \\
\hline \multirow[t]{4}{*}{216} & 0 & 1 & 0,000384 & 0,00137 & $-1,27253$ & 0,000117 & $-0,001525$ & 0,000351 & $-0,004028$ & \\
\hline & 364 & 3908 & & & & & $-0,578135$ & & $-3,345887$ & \\
\hline & & & & 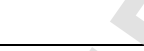 & & & 0,578135 & & 3,345887 & \\
\hline & & & & 8 & 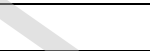 & & 0,049261 & & 3,060798 & 3,110058 \\
\hline
\end{tabular}




\begin{tabular}{|c|c|c|c|c|c|c|c|c|c|c|}
\hline AGEJOB & BADS & GOODS & $f(x / 1)$ & $f(x / 0)$ & $w(x)$ & & & 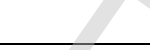 & . & \\
\hline 010 & 5 & 5 & 0,001407 & 0,015068 & $-2,37114$ & 0,001284 & $-0,012332$ & 0,001287 & $-0,012358$ & \\
\hline 012 & 54 & 280 & 0,071757 & 0,149315 & $-0,73277$ & 0,012723 & $-0,080107$ & 0,065643 & $-0,257926$ & \\
\hline 013 & 68 & 401 & 0,102712 & 0,187671 & $-0,60277$ & 0,015991 & $-0,095411$ & 0,09396 & $-0,320574$ & \\
\hline 014 & 16 & 175 & 0,044896 & 0,045205 & $-0,00686$ & 0,003852 & $-0,030892$ & 0,041071 & $-0,189162$ & \\
\hline 015 & 70 & 545 & 0,13955 & 0,193151 & $-0,32505$ & 0,016458 & $-0,097513$ & 0,127659 & $-0,379101$ & \\
\hline 016 & 13 & 154 & 0,039524 & 0,036986 & 0,066365 & 0,003151 & $-0,026188$ & 0,036156 & $-0,173175$ & \\
\hline 110 & 22 & 117 & 0,030059 & 0,061644 & $-0,71822$ & 0,005252 & $-0,039776$ & 0,027498 & $-0,142563$ & \\
\hline 112 & 13 & 324 & 0,083014 & 0,036986 & 0,808456 & 0,003151 & $-0,026188$ & 0,07594 & $-0,282421$ & \\
\hline 113 & 14 & 487 & 0,124712 & 0,039726 & 1,144002 & 0,003385 & $-0,027779$ & 0,114086 & $-0,357295$ & \\
\hline 114 & 4 & 74 & 0,019059 & 0,012329 & 0,435582 & 0,00105 & $-0,010394$ & 0,017435 & $-0,101852$ & \\
\hline 115 & 10 & 728 & 0,186365 & 0,028767 & 1,868473 & 0,002451 & $-0,021257$ & 0,170485 & $-0,435126$ & \\
\hline 116 & 2 & 67 & 0,017268 & 0,006849 & 0,924697 & 0,000584 & $-0,006269$ & 0,015797 & $-0,094530$ & \\
\hline 210 & 73 & 325 & 0,083269 & 0,20137 & $-0,88306$ & 0,017158 & $-0,100631$ & 0,076174 & $-0,282953$ & \\
\hline 212 & 0 & 50 & 0,012919 & 0,00137 & 2,243981 & 0,000117 & $-0,001525$ & 0,011818 & $-0,075670$ & \\
\hline 213 & 0 & 91 & 0,023408 & 0,00137 & 2,838347 & 0,000117 & $-0,001525$ & 0,021413 & $-0,118743$ & \\
\hline 214 & 0 & 7 & 0,001919 & 0,00137 & 0,336911 & 0,000117 & $-0,001525$ & 0,001755 & $-0,016067$ & \\
\hline 215 & 0 & 78 & 0,020082 & 0,00137 & 2,685106 & 0,000117 & $-0,001525$ & 0,018371 & $-0,105934$ & \\
\hline \multirow[t]{4}{*}{216} & 0 & 0 & 0,000128 & 0,00137 & $-2,37114$ & 0,000117 & $-0,001525$ & 0,000117 & $-0,001528$ & \\
\hline & 364 & 3908 & & & & & $-0,582361$ & & $-3,346980$ & \\
\hline & & & & $>$ & & & 0,582361 & & 3,346980 & \\
\hline & & & & $\bar{P}$ & 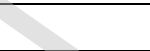 & & 0,049621 & & 3,061797 & 3,111418 \\
\hline
\end{tabular}




\begin{tabular}{|c|c|c|c|c|c|c|c|c|c|c|}
\hline AGEJOB & BADS & GOODS & $f(x / 1)$ & $f(x / 0)$ & $w(x)$ & & & - & 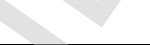 & \\
\hline 010 & 4 & 6 & 0,001663 & 0,012329 & $-2,00341$ & 0,00105 & $-0,010394$ & 0,001521 & $-0,014239$ & \\
\hline 012 & 49 & 330 & 0,084548 & 0,135616 & $-0,47251$ & 0,011555 & $-0,074362$ & 0,077344 & $-0,285599$ & \\
\hline 013 & 72 & 361 & 0,092479 & 0,19863 & $-0,76446$ & 0,016924 & $-0,099596$ & 0,084599 & $-0,301445$ & \\
\hline 014 & 16 & 173 & 0,044385 & 0,045205 & $-0,01832$ & 0,003852 & $-0,030892$ & 0,040603 & $-0,187678$ & \\
\hline 015 & 77 & 529 & 0,135457 & 0,212329 & $-0,44948$ & 0,018092 & $-0,104724$ & 0,123915 & $-0,373303$ & \\
\hline 016 & 15 & 146 & 0,037478 & 0,042466 & $-0,12495$ & 0,003618 & $-0,029346$ & 0,034284 & $-0,166838$ & \\
\hline 110 & 40 & 199 & 0,051036 & 0,110959 & $-0,77663$ & 0,009454 & $-0,063579$ & 0,046687 & $-0,206397$ & \\
\hline 112 & 15 & 407 & 0,104247 & 0,042466 & 0,898061 & 0,003618 & $-0,029346$ & 0,095364 & $-0,323324$ & \\
\hline 113 & 11 & 474 & 0,121387 & 0,031507 & 1,348775 & 0,002685 & $-0,022929$ & 0,111044 & $-0,352097$ & \\
\hline 114 & 2 & 75 & 0,019314 & 0,006849 & 1,036702 & 0,000584 & $-0,006269$ & 0,017669 & $-0,102879$ & \\
\hline 115 & 7 & 764 & 0,195574 & 0,020548 & 2,253179 & 0,001751 & $-0,016033$ & 0,17891 & $-0,444179$ & \\
\hline 116 & 3 & 74 & 0,019059 & 0,009589 & 0,686897 & 0,000817 & $-0,008381$ & 0,017435 & $-0,101852$ & \\
\hline 210 & 53 & 260 & 0,066641 & 0,146575 & $-0,78822$ & 0,012489 & $-0,078971$ & 0,060963 & $-0,246042$ & \\
\hline 212 & 0 & 27 & 0,007035 & 0,00137 & 1,636194 & 0,000117 & $-0,001525$ & 0,006436 & $-0,046849$ & \\
\hline 213 & 0 & 43 & 0,011128 & 0,00137 & 2,094769 & 0,000117 & $-0,001525$ & 0,01018 & $-0,067372$ & \\
\hline 214 & 0 & 5 & 0,001407 & 0,00137 & 0,026756 & 0,000117 & $-0,001525$ & 0,001287 & $-0,012358$ & \\
\hline 215 & 0 & 34 & 0,008826 & 0,00137 & 1,862967 & 0,000117 & $-0,001525$ & 0,008074 & $-0,056133$ & \\
\hline \multirow[t]{4}{*}{216} & 0 & 1 & 0,000384 & 0,00137 & $-1,27253$ & 0,000117 & $-0,001525$ & 0,000351 & $-0,004028$ & \\
\hline & 364 & 3908 & & & & & $-0,582449$ & & $-3,292613$ & \\
\hline & & & & 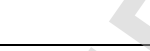 & & & 0,582449 & & 3,292613 & \\
\hline & & & & 8 & 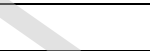 & & 0,049628 & & 3,012062 & 3,06169 \\
\hline
\end{tabular}




\begin{tabular}{|c|c|c|c|c|c|c|c|c|c|c|}
\hline AGEJOB & BADS & GOODS & $f(x / 1)$ & $f(x / 0)$ & $w(x)$ & & & 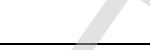 & 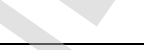 & \\
\hline 010 & 4 & 5 & 0,001407 & 0,012329 & $-2,17047$ & 0,00105 & $-0,010394$ & 0,001287 & $-0,012358$ & \\
\hline 012 & 51 & 292 & 0,074827 & 0,141096 & $-0,63426$ & 0,012022 & $-0,076680$ & 0,068452 & $-0,264824$ & \\
\hline 013 & 68 & 343 & 0,087874 & 0,187671 & $-0,75879$ & 0,015991 & $-0,095411$ & 0,080387 & $-0,292358$ & \\
\hline 014 & 16 & 166 & 0,042594 & 0,045205 & $-0,0595$ & 0,003852 & $-0,030892$ & 0,038965 & $-0,182421$ & \\
\hline 015 & 77 & 564 & 0,14441 & 0,212329 & $-0,38548$ & 0,018092 & $-0,104724$ & 0,132106 & $-0,385780$ & \\
\hline 016 & 15 & 150 & 0,038501 & 0,042466 & $-0,09802$ & 0,003618 & $-0,029346$ & 0,03522 & $-0,170025$ & \\
\hline 110 & 23 & 126 & 0,032361 & 0,064384 & $-0,6879$ & 0,005486 & $-0,041199$ & 0,029604 & $-0,150330$ & \\
\hline 112 & 12 & 335 & 0,085828 & 0,034247 & 0,918754 & 0,002918 & $-0,024572$ & 0,078515 & $-0,288219$ & \\
\hline 113 & 15 & 446 & 0,114224 & 0,042466 & 0,98946 & 0,003618 & $-0,029346$ & 0,104491 & $-0,340489$ & \\
\hline 114 & 3 & 66 & 0,017012 & 0,009589 & 0,573299 & 0,000817 & $-0,008381$ & 0,015562 & $-0,093465$ & \\
\hline 115 & 10 & 761 & 0,194807 & 0,028767 & 1,912775 & 0,002451 & $-0,021257$ & 0,178208 & $-0,443447$ & \\
\hline 116 & 3 & 75 & 0,019314 & 0,009589 & 0,70023 & 0,000817 & $-0,008381$ & 0,017669 & $-0,102879$ & \\
\hline 210 & 67 & 333 & 0,085316 & 0,184932 & $-0,77362$ & 0,015757 & $-0,094352$ & 0,078047 & $-0,287174$ & \\
\hline 212 & 0 & 57 & 0,01471 & 0,00137 & 2,373793 & 0,000117 & $-0,001525$ & 0,013456 & $-0,083639$ & \\
\hline 213 & 0 & 85 & 0,021873 & 0,00137 & 2,770524 & 0,000117 & $-0,001525$ & 0,020009 & $-0,112915$ & \\
\hline 214 & 0 & 8 & 0,002174 & 0,00137 & 0,462074 & 0,000117 & $-0,001525$ & 0,001989 & $-0,017850$ & \\
\hline 215 & 0 & 95 & 0,024431 & 0,00137 & 2,881134 & 0,000117 & $-0,001525$ & 0,022349 & $-0,122555$ & \\
\hline \multirow[t]{4}{*}{216} & 0 & 1 & 0,000384 & 0,00137 & $-1,27253$ & 0,000117 & $-0,001525$ & 0,000351 & $-0,004028$ & \\
\hline & 364 & 3908 & & & & & $-0,582560$ & & $-3,354755$ & \\
\hline & & & & 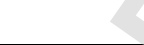 & & & 0,582560 & & 3,354755 & \\
\hline & & & & 2 & & & 0,049638 & & 3,06891 & 3,118547 \\
\hline
\end{tabular}




\begin{tabular}{|c|c|c|c|c|c|c|c|c|c|c|}
\hline AGE\JYR & BADS & GOODS & $f(x / 1)$ & $f(x / 0)$ & $w(x)$ & & & & & \\
\hline $1 \backslash 1$ & 15 & 72 & 0,018547 & 0,042466 & $-0,82839$ & 0,003618 & $-0,029346$ & 0,016967 & $-0,099783$ & \\
\hline $2 \backslash 1$ & 30 & 299 & 0,076618 & 0,083562 & $-0,08675$ & 0,00712 & $-0,050793$ & 0,07009 & $-0,268770$ & \\
\hline $3 \backslash 1$ & 11 & 280 & 0,071757 & 0,031507 & 0,823087 & 0,002685 & $-0,022929$ & 0,065643 & $-0,257926$ & \\
\hline 112 & 24 & 110 & 0,028268 & 0,067123 & $-0,8648$ & 0,005719 & $-0,042609$ & 0,025859 & $-0,136361$ & \\
\hline $2 \backslash 2$ & 75 & 462 & 0,118317 & 0,206849 & $-0,55863$ & 0,017625 & $-0,102686$ & 0,108235 & $-0,347193$ & \\
\hline 312 & 22 & 230 & 0,058966 & 0,061644 & $-0,0444$ & 0,005252 & $-0,039776$ & 0,053942 & $-0,227228$ & \\
\hline 113 & 28 & 116 & 0,029803 & 0,078082 & $-0,96315$ & 0,006653 & $-0,048113$ & 0,027264 & $-0,141686$ & \\
\hline 213 & 67 & 588 & 0,15055 & 0,184932 & $-0,20569$ & 0,015757 & $-0,094352$ & 0,137722 & $-0,393909$ & \\
\hline 313 & 21 & 304 & 0,077897 & 0,058904 & 0,279479 & 0,005019 & $-0,038337$ & 0,07126 & $-0,271555$ & \\
\hline $1 \backslash 4$ & 10 & 268 & 0,068688 & 0,028767 & 0,870336 & 0,002451 & $-0,021257$ & 0,062835 & $-0,250856$ & \\
\hline $2 \backslash 4$ & 46 & 184 & 0,047199 & 0,127397 & $-0,99294$ & 0,010855 & $-0,070834$ & 0,043177 & $-0,195747$ & \\
\hline \multirow[t]{4}{*}{$3 \backslash 4$} & 15 & 995 & 0,254669 & 0,042466 & 1,791266 & 0,003618 & $-0,029346$ & 0,232969 & $-0,489652$ & \\
\hline & 364 & 3908 & & & $\triangle$ & & $-0,590379$ & & $-3,080666$ & \\
\hline & & & & & & & 0,590379 & & 3,080666 & \\
\hline & & & & 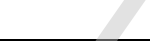 & ser & & 0,050304 & & 2,818175 & 2,868479 \\
\hline
\end{tabular}




\begin{tabular}{|c|c|c|c|c|c|c|c|c|c|c|}
\hline \multicolumn{11}{|c|}{ 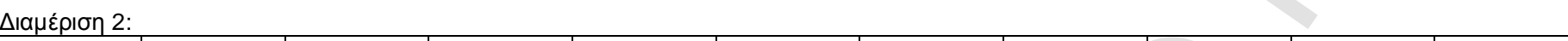 } \\
\hline AGEJYR & BADS & GOODS & $f(x / 1)$ & $f(x / 0)$ & $w(x)$ & & & 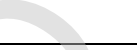 & & \\
\hline $0 \backslash 0$ & 78 & 459 & 0,117549 & 0,215068 & $-0,6041$ & 0,018325 & $-0,105737$ & 0,107533 & $-0,345950$ & \\
\hline $0 \backslash 1$ & 74 & 672 & 0,172039 & 0,20411 & $-0,17094$ & 0,017391 & $-0,101661$ & 0,15738 & $-0,419839$ & \\
\hline 012 & 27 & 235 & 0,060246 & 0,075342 & $-0,22361$ & 0,00642 & $-0,046756$ & 0,055112 & $-0,230451$ & \\
\hline 013 & 49 & 184 & 0,047199 & 0,135616 & $-1,05546$ & 0,011555 & $-0,074362$ & 0,043177 & $-0,195747$ & \\
\hline 110 & 19 & 230 & 0,058966 & 0,053425 & 0,098697 & 0,004552 & $-0,035412$ & 0,053942 & $-0,227228$ & \\
\hline $1 \backslash 1$ & 23 & 323 & 0,082758 & 0,064384 & 0,251059 & 0,005486 & $-0,041199$ & 0,075706 & $-0,281888$ & \\
\hline 112 & 6 & 253 & 0,06485 & 0,017808 & 1,292422 & 0,001517 & $-0,014209$ & 0,059325 & $-0,241761$ & \\
\hline $1 \backslash 3$ & 16 & 991 & 0,253645 & 0,045205 & 1,724719 & 0,003852 & $-0,030892$ & 0,232033 & $-0,489033$ & \\
\hline $2 \backslash 0$ & 21 & 109 & 0,028012 & 0,058904 & $-0,74327$ & 0,005019 & $-0,038337$ & 0,025625 & $-0,135463$ & \\
\hline $2 \backslash 1$ & 26 & 139 & 0,035687 & 0,072603 & $-0,71022$ & 0,006186 & $-0,045386$ & 0,032646 & $-0,161172$ & \\
\hline 212 & 13 & 70 & 0,018035 & 0,036986 & $-0,71822$ & 0,003151 & $-0,026188$ & 0,016499 & $-0,097697$ & \\
\hline \multirow[t]{4}{*}{213} & 12 & 243 & 0,062292 & 0,034247 & 0,598249 & 0,002918 & $-0,024572$ & 0,056984 & $-0,235533$ & \\
\hline & 364 & 3908 & & & & $P$ & $-0,584711$ & & $-3,061763$ & \\
\hline & & & & & & 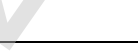 & 0,584711 & & 3,061763 & \\
\hline & & & & & 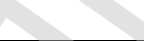 & & 0,049821 & & 2,800883 & 2,850704 \\
\hline
\end{tabular}




\begin{tabular}{|c|c|c|c|c|c|c|c|c|c|c|}
\hline AGFIYR & אחקת & תחת & $f(x / 1)$ & $f(x / 0) \quad x$ & 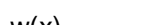 & & & & & \\
\hline $0 \backslash 0$ & 83 & 466 & 0,11934 & 0,228767 & $-0,65073$ & 0,019492 & $-0,110735$ & 0,109172 & -0348839 & \\
\hline $0 \backslash 1$ & 68 & 677 & 0,173318 & 0,187671 & $-0,07956$ & 0,015991 & $-0,095411$ & 0,15855 & $-0,421266$ & \\
\hline $0 \backslash 2$ & 25 & 237 & 0,060757 & 0,069863 & $-0,13965$ & 0,005953 & $-0,044004$ & 0,05558 & $-0,231730$ & \\
\hline 013 & 50 & 180 & 0,046175 & 0,138356 & $-1,09738$ & 0,011789 & $-0,075524$ & 0,042241 & $-0,192839$ & \\
\hline 110 & 21 & 239 & 0,061269 & 0,058904 & 0,039361 & 0,005019 & $-0,038337$ & 0,056048 & $-0,233003$ & \\
\hline $1 \backslash 1$ & 20 & 323 & 0,082758 & 0,056164 & 0,387635 & 0,004786 & $-0,036883$ & 0,075706 & $-0,281888$ & \\
\hline 112 & 7 & 243 & 0,062292 & 0,020548 & 1,109074 & 0,001751 & $-0,016033$ & 0,056984 & $-0,235533$ & \\
\hline 113 & 17 & 992 & 0,253901 & 0,047945 & 1,666887 & 0,004085 & $-0,032418$ & 0,232267 & $-0,489188$ & \\
\hline 210 & 24 & 109 & 0,028012 & 0,067123 & $-0,87389$ & 0,005719 & $-0,042609$ & 0,025625 & $-0,135463$ & \\
\hline $2 \backslash 1$ & 20 & 114 & 0,029291 & 0,056164 & $-0,65099$ & 0,004786 & $-0,036883$ & 0,026796 & $-0,139923$ & \\
\hline 212 & 15 & 70 & 0,018035 & 0,042466 & $-0,85637$ & 0,003618 & $-0,029346$ & 0,016499 & $-0,097697$ & \\
\hline 213 & 14 & 258 & 0,066129 & 0,039726 & 0,509608 & 0,003385 & $-0,027779$ & 0,060495 & $-0,244825$ & \\
\hline & 364 & 3908 & & & & 2 & $-0,585961$ & & $-3,052195$ & \\
\hline & & & & & & 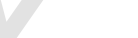 & 0,585961 & & 3,052195 & \\
\hline & & & & & 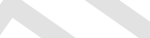 & & 0,049927 & & 2,79213 & 2,842057 \\
\hline
\end{tabular}




\begin{tabular}{|c|c|c|c|c|c|c|c|c|c|c|}
\hline$\pi$ & & & & & & & & & & \\
\hline AGEJYR & BADS & GOODS & $f(x / 1)$ & $f(x / 0)$ & $w(x)$ & & & 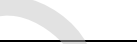 & & \\
\hline $0 \backslash 0$ & 55 & 266 & 0,068176 & 0,152055 & $-0,80215$ & 0,012956 & $-0,081237$ & 0,062367 & $-0,249660$ & \\
\hline $0 \backslash 1$ & 87 & 809 & 0,207086 & 0,239726 & $-0,14636$ & 0,020426 & $-0,114661$ & 0,189441 & $-0,454693$ & \\
\hline 012 & 35 & 296 & 0,075851 & 0,09726 & $-0,24862$ & 0,008287 & $-0,057305$ & 0,069388 & $-0,267085$ & \\
\hline $0 \backslash 3$ & 56 & 174 & 0,044641 & 0,154795 & $-1,24346$ & 0,013189 & $-0,082361$ & 0,040837 & $-0,188421$ & \\
\hline 110 & 18 & 182 & 0,046687 & 0,050685 & $-0,08216$ & 0,004319 & $-0,033924$ & 0,042709 & $-0,194297$ & \\
\hline $1 \backslash 1$ & 30 & 442 & 0,1132 & 0,083562 & 0,303574 & 0,00712 & $-0,050793$ & 0,103555 & $-0,338783$ & \\
\hline 112 & 13 & 292 & 0,074827 & 0,036986 & 0,704635 & 0,003151 & $-0,026188$ & 0,068452 & $-0,264824$ & \\
\hline $1 \backslash 3$ & 17 & 1077 & 0,275646 & 0,047945 & 1,749058 & 0,004085 & $-0,032418$ & 0,252159 & $-0,501190$ & \\
\hline $2 \backslash 0$ & 9 & 63 & 0,016245 & 0,026027 & $-0,47139$ & 0,002218 & $-0,019553$ & 0,01486 & $-0,090238$ & \\
\hline $2 \backslash 1$ & 24 & 107 & 0,027501 & 0,067123 & $-0,89232$ & 0,005719 & $-0,042609$ & 0,025157 & $-0,133658$ & \\
\hline 212 & 11 & 56 & 0,014454 & 0,031507 & $-0,77925$ & 0,002685 & $-0,022929$ & 0,013222 & $-0,082519$ & \\
\hline 213 & 9 & 144 & 0,036966 & 0,026027 & 0,350848 & 0,002218 & $-0,019553$ & 0,033816 & $-0,165231$ & \\
\hline & 364 & 3908 & & & & $P$ & $-0,583530$ & & $-2,930598$ & \\
\hline & & & & & & 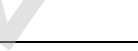 & 0,583530 & & 2,930598 & \\
\hline & & & & & 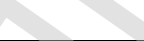 & & 0,04972 & & 2,680894 & 2,730614 \\
\hline
\end{tabular}




\begin{tabular}{|c|c|c|c|c|c|c|c|c|c|c|}
\hline AGEJYR & BADS & GOODS & $f(x / 1)$ & $f(x / 0)$ & $w(x)$ & & & & 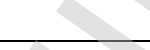 & \\
\hline $0 \backslash 0$ & 77 & 456 & 0,116782 & 0,212329 & $-0,59783$ & 0,018092 & $-0,104724$ & 0,106831 & $-0,344701$ & \\
\hline $0 \backslash 1$ & 68 & 594 & 0,152085 & 0,187671 & $-0,21025$ & 0,015991 & $-0,095411$ & 0,139126 & $-0,395889$ & \\
\hline 012 & 35 & 288 & 0,073804 & 0,09726 & $-0,27598$ & 0,008287 & $-0,057305$ & 0,067515 & $-0,262543$ & \\
\hline $0 \backslash 3$ & 51 & 182 & 0,046687 & 0,141096 & $-1,10597$ & 0,012022 & $-0,076680$ & 0,042709 & $-0,194297$ & \\
\hline $1 \backslash 0$ & 25 & 231 & 0,059222 & 0,069863 & $-0,16524$ & 0,005953 & $-0,044004$ & 0,054176 & $-0,227876$ & \\
\hline $1 \backslash 1$ & 16 & 295 & 0,075595 & 0,045205 & 0,514169 & 0,003852 & $-0,030892$ & 0,069154 & $-0,266522$ & \\
\hline 112 & 8 & 278 & 0,071246 & 0,023288 & 1,118212 & 0,001984 & $-0,017813$ & 0,065175 & $-0,256760$ & \\
\hline 113 & 17 & 1005 & 0,257227 & 0,047945 & 1,6799 & 0,004085 & $-0,032418$ & 0,23531 & $-0,491178$ & \\
\hline $2 \backslash 0$ & 22 & 115 & 0,029547 & 0,061644 & $-0,73538$ & 0,005252 & $-0,039776$ & 0,02703 & $-0,140806$ & \\
\hline $2 \backslash 1$ & 24 & 125 & 0,032105 & 0,067123 & $-0,73751$ & 0,005719 & $-0,042609$ & 0,02937 & $-0,149478$ & \\
\hline 212 & 12 & 76 & 0,01957 & 0,034247 & $-0,55958$ & 0,002918 & $-0,024572$ & 0,017903 & $-0,103902$ & \\
\hline \multirow[t]{4}{*}{$2 \backslash 3$} & 9 & 263 & 0,067409 & 0,026027 & 0,951622 & 0,002218 & $-0,019553$ & 0,061665 & $-0,247856$ & \\
\hline & 364 & 3908 & & & & 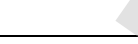 & $-0,585755$ & & $-3,081807$ & \\
\hline & & & & & & 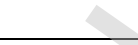 & 0,585755 & & 3,081807 & \\
\hline & & & & & & & 0,04991 & & 2,819219 & 2,869129 \\
\hline
\end{tabular}

9) $\quad H(A G E M A R)$

\begin{tabular}{|c|c|c|c|c|c|c|c|c|c|c|}
\hline AGEIMAR & BADS & GOODS & $f(x / 1)$ & $f(x / 0)$ & $w(x)$ & & & & & \\
\hline $1 \backslash 1$ & 3 & 25 & 0,006523 & 0,009589 & $-0,38522$ & 0,000817 & $-0,008381$ & 0,005968 & $-0,044092$ & \\
\hline 211 & 141 & 791 & 0,202481 & 0,387671 & $-0,64951$ & 0,033032 & $-0,162517$ & 0,185229 & $-0,450591$ & \\
\hline $3 \backslash 1$ & 22 & 149 & 0,038245 & 0,061644 & $-0,47736$ & 0,005252 & $-0,039776$ & 0,034986 & $-0,169231$ & \\
\hline 112 & 61 & 30 & 0,007803 & 0,168493 & $-3,07245$ & 0,014357 & $-0,087893$ & 0,007138 & $-0,050894$ & \\
\hline 212 & 34 & 35 & 0,009082 & 0,094521 & $-2,34257$ & 0,008054 & $-0,056023$ & 0,008308 & $-0,057418$ & \\
\hline 312 & 30 & 117 & 0,030059 & 0,083562 & $-1,02243$ & 0,00712 & $-0,050793$ & 0,027498 & $-0,142563$ & \\
\hline 113 & 13 & 511 & 0,130852 & 0,036986 & 1,263518 & 0,003151 & $-0,026188$ & 0,119703 & $-0,366586$ & \\
\hline 213 & 43 & 707 & 0,180993 & 0,119178 & 0,417837 & 0,010155 & $-0,067241$ & 0,165571 & $-0,429570$ & \\
\hline \multirow[t]{4}{*}{$3 \backslash 3$} & 17 & 1543 & 0,394858 & 0,047945 & 2,108467 & 0,004085 & $-0,032418$ & 0,361214 & $-0,530650$ & \\
\hline & 364 & 3908 & & 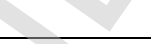 & & & $-0,531229$ & & $-2,241596$ & \\
\hline & & & & 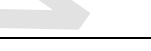 & & & 0,531229 & & 2,241596 & \\
\hline & & & & & & & 0,045264 & & 2,050598 & 2,095862 \\
\hline
\end{tabular}




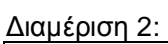

\begin{tabular}{|c|c|c|c|c|c|c|c|c|c|c|}
\hline AGEMAR & BADS & GOODS & $f(x / 1)$ & $f(x / 0)$ & $w(x)$ & & & & 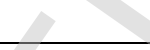 & \\
\hline $0 \backslash 0$ & 37 & 38 & 0,009849 & 0,10274 & $-2,34482$ & 0,008754 & $-0,059841$ & 0,00901 & $-0,061216$ & 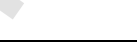 \\
\hline $0 \backslash 1$ & 145 & 821 & 0,210156 & 0,39863 & $-0,64018$ & 0,033966 & $-0,165745$ & 0,192249 & $-0,457352$ & \\
\hline 012 & 46 & 691 & 0,176899 & 0,127397 & 0,328271 & 0,010855 & $-0,070834$ & 0,161827 & $-0,425196$ & \\
\hline $1 \backslash 0$ & 30 & 122 & 0,031338 & 0,083562 & $-0,98076$ & 0,00712 & $-0,050793$ & 0,028668 & $-0,146906$ & \\
\hline $1 \backslash 1$ & 20 & 143 & 0,03671 & 0,056164 & $-0,42523$ & 0,004786 & $-0,036883$ & 0,033582 & $-0,164424$ & \\
\hline 112 & 14 & 1532 & 0,392044 & 0,039726 & 2,289368 & 0,003385 & $-0,027779$ & 0,35864 & $-0,530569$ & \\
\hline $2 \backslash 0$ & 60 & 32 & 0,008314 & 0,165753 & $-2,99254$ & 0,014123 & $-0,086798$ & 0,007606 & $-0,053534$ & \\
\hline $2 \backslash 1$ & 2 & 20 & 0,005244 & 0,006849 & $-0,26701$ & 0,000584 & $-0,006269$ & 0,004797 & $-0,036957$ & \\
\hline \multirow[t]{4}{*}{212} & 10 & 509 & 0,13034 & 0,028767 & 1,510915 & 0,002451 & $-0,021257$ & 0,119234 & $-0,365826$ & \\
\hline & 364 & 3908 & & & & & $-0,526200$ & & $-2,241980$ & \\
\hline & & & & & & & 0,526200 & $\nabla$ & 2,241980 & \\
\hline & & & & & & & 0,044835 & & 2,05095 & 2,095785 \\
\hline
\end{tabular}

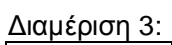

\begin{tabular}{|l|r|r|l|l|l|l|l|l|l|l|}
\hline AGEMAR & \multicolumn{1}{l|}{ BADS } & \multicolumn{1}{l}{ GOODS } & $\mathrm{f}(\mathrm{x} / \mathrm{1})$ & $\mathrm{f}(\mathrm{x} / 0)$ & $\mathrm{w}(\mathrm{x})$ & & & & & \\
\hline 010 & 32 & 36 & 0,009337 & 0,089041 & $-2,25507$ & 0,007587 & $-0,053429$ & 0,008542 & $-0,058693$ & \\
\hline 011 & 150 & 823 & 0,210668 & 0,412329 & $-0,67154$ & 0,035133 & $-0,169728$ & 0,192718 & $-0,457789$ & \\
\hline 012 & 44 & 701 & 0,179458 & 0,121918 & 0,386592 & 0,010388 & $-0,068447$ & 0,164167 & $-0,427944$ & \\
\hline 110 & 28 & 115 & 0,029547 & 0,078082 & $-0,97177$ & 0,006653 & $-0,048113$ & 0,02703 & $-0,140806$ & \\
\hline 111 & 23 & 150 & 0,038501 & 0,064384 & $-0,51418$ & 0,005486 & $-0,041199$ & 0,03522 & $-0,170025$ & \\
\hline 112 & 14 & 1532 & 0,392044 & 0,039726 & 2,289368 & 0,003385 & $-0,027779$ & 0,35864 & $-0,530569$ & \\
\hline 210 & 58 & 23 & 0,006012 & 0,160274 & $-3,28317$ & 0,013656 & $-0,084591$ & 0,0055 & $-0,041282$ & \\
\hline 211 & 3 & 22 & 0,005756 & 0,009589 & $-0,51039$ & 0,000817 & $-0,008381$ & 0,005266 & $-0,039856$ & \\
\hline 212 & 12 & 506 & 0,129573 & 0,034247 & 1,330656 & 0,002918 & $-0,024572$ & 0,118532 & $-0,364682$ & \\
\hline & 364 & 3908 & & & & & $-0,526238$ & & $-2,231646$ & \\
\hline & & & & & & & 0,526238 & & 2,231646 & \\
\hline & & & & & & & 0,044839 & & 2,041496 & 2,086335 \\
\hline
\end{tabular}




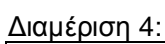

\begin{tabular}{|c|c|c|c|c|c|c|c|c|c|c|}
\hline AGEMAR & BADS & GOODS & $f(x / 1)$ & $f(x / 0)$ & $w(x)$ & & & & 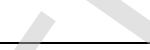 & \\
\hline $0 \backslash 0$ & 35 & 32 & 0,008314 & 0,09726 & $-2,45943$ & 0,008287 & $-0,057305$ & 0,007606 & $-0,053534$ & 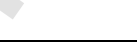 \\
\hline $0 \backslash 1$ & 152 & 814 & 0,208365 & 0,417808 & $-0,69573$ & 0,0356 & $-0,171306$ & 0,190611 & $-0,455808$ & \\
\hline $0 \backslash 2$ & 46 & 699 & 0,178946 & 0,127397 & 0,339774 & 0,010855 & $-0,070834$ & 0,163699 & $-0,427399$ & \\
\hline $1 \backslash 0$ & 39 & 125 & 0,032105 & 0,108219 & $-1,21513$ & 0,009221 & $-0,062341$ & 0,02937 & $-0,149478$ & \\
\hline $1 \backslash 1$ & 21 & 156 & 0,040036 & 0,058904 & $-0,38614$ & 0,005019 & $-0,038337$ & 0,036625 & $-0,174737$ & \\
\hline $1 \backslash 2$ & 18 & 1712 & 0,438092 & 0,050685 & 2,156799 & 0,004319 & $-0,033924$ & 0,400764 & $-0,528678$ & \\
\hline $2 \backslash 0$ & 45 & 27 & 0,007035 & 0,124658 & $-2,87467$ & 0,010622 & $-0,069644$ & 0,006436 & $-0,046849$ & \\
\hline $2 \backslash 1$ & 2 & 13 & 0,003454 & 0,006849 & $-0,68474$ & 0,000584 & $-0,006269$ & 0,003159 & $-0,026242$ & \\
\hline \multirow[t]{4}{*}{212} & 6 & 330 & 0,084548 & 0,017808 & 1,557665 & 0,001517 & $-0,014209$ & 0,077344 & $-0,285599$ & \\
\hline & 364 & 3908 & & & & & $-0,524170$ & & $-2,148324$ & \\
\hline & & & & & & & 0,524170 & $\nabla$ & 2,148324 & \\
\hline & & & & & & & 0,044662 & & 1,965274 & 2,009937 \\
\hline
\end{tabular}

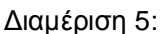

\begin{tabular}{|l|r|r|r|r|r|r|r|r|l|l|}
\hline AGEMAR & \multicolumn{1}{|l|}{ BADS } & \multicolumn{1}{l}{ GOODS } & $\mathrm{f}(\mathrm{x} / \mathrm{1})$ & $\mathrm{f}(\mathrm{x} / 0)$ & $\mathrm{w}(\mathrm{x})$ & & & & & \\
\hline 010 & 38 & 31 & 0,008058 & 0,105479 & $-2,57181$ & 0,008987 & $-0,061096$ & 0,007372 & $-0,052220$ & \\
\hline 011 & 148 & 795 & 0,203505 & 0,406849 & $-0,69275$ & 0,034666 & $-0,168142$ & 0,186165 & $-0,451514$ & \\
\hline 012 & 45 & 694 & 0,177667 & 0,124658 & 0,35434 & 0,010622 & $-0,069644$ & 0,162529 & $-0,426026$ & \\
\hline 110 & 30 & 111 & 0,028524 & 0,083562 & $-1,07484$ & 0,00712 & $-0,050793$ & 0,026094 & $-0,137256$ & \\
\hline $1 \backslash 1$ & 22 & 145 & 0,037222 & 0,061644 & $-0,50448$ & 0,005252 & $-0,039776$ & 0,03405 & $-0,166036$ & \\
\hline 112 & 14 & 1553 & 0,397416 & 0,039726 & 2,302978 & 0,003385 & $-0,027779$ & 0,363554 & $-0,530701$ & \\
\hline 210 & 55 & 30 & 0,007803 & 0,152055 & $-2,9698$ & 0,012956 & $-0,081237$ & 0,007138 & $-0,050894$ & \\
\hline 211 & 2 & 21 & 0,0055 & 0,006849 & $-0,21938$ & 0,000584 & $-0,006269$ & 0,005031 & $-0,038414$ & \\
\hline 212 & 10 & 528 & 0,135201 & 0,028767 & 1,547528 & 0,002451 & $-0,021257$ & 0,123681 & $-0,372936$ & \\
\hline & 364 & 3908 & & & & & $-0,525993$ & & $-2,225997$ & \\
\hline & & & & & & & 0,525993 & & 2,225997 & \\
\hline & & & & & & & 0,044818 & & 2,036328 & 2,081146 \\
\hline
\end{tabular}




\begin{tabular}{|c|c|c|c|c|c|c|c|c|c|c|}
\hline AGEITRM & BADS & GOODS & $f(x / 1)$ & $f(x / 0)$ & $w(x)$ & & & 2 & 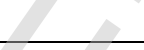 & \\
\hline $1 \backslash 1$ & 15 & 51 & 0,013175 & 0,042466 & $-1,1704$ & 0,003618 & $-0,029346$ & 0,012052 & $-0,076827$ & \\
\hline $2 \backslash 1$ & 32 & 138 & 0,035431 & 0,089041 & $-0,92151$ & 0,007587 & $-0,053429$ & 0,032412 & $-0,160353$ & \\
\hline $3 \backslash 1$ & 8 & 214 & 0,054873 & 0,023288 & 0,857104 & 0,001984 & $-0,017813$ & 0,050198 & $-0,216665$ & \\
\hline 112 & 13 & 128 & 0,032873 & 0,036986 & $-0,1179$ & 0,003151 & $-0,026188$ & 0,030072 & $-0,152027$ & \\
\hline 212 & 17 & 458 & 0,117293 & 0,047945 & 0,89462 & 0,004085 & $-0,032418$ & 0,107299 & $-0,345535$ & \\
\hline 312 & 7 & 387 & 0,09913 & 0,020548 & 1,573673 & 0,001751 & $-0,016033$ & 0,090684 & $-0,314039$ & \\
\hline 113 & 49 & 387 & 0,09913 & 0,135616 & $-0,3134$ & 0,011555 & $-0,074362$ & 0,090684 & $-0,314039$ & \\
\hline 213 & 169 & 937 & 0,239831 & 0,464384 & $-0,66078$ & 0,039568 & $-0,184369$ & 0,219396 & $-0,480124$ & \\
\hline \multirow[t]{4}{*}{313} & 54 & 1208 & 0,309158 & 0,149315 & 0,727795 & 0,012723 & $-0,080107$ & 0,282816 & $-0,515309$ & \\
\hline & 364 & 3908 & & & & & $-0,514065$ & & $-2,574918$ & \\
\hline & & & & & & 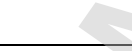 & 0,514065 & & 2,574918 & \\
\hline & & & & & & $\Delta$ & 0,043801 & & 2,35552 & 2,399321 \\
\hline
\end{tabular}

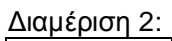

\begin{tabular}{|l|r|r|r|r|r|r|r|r|r|r|}
\hline AGETRM & \multicolumn{1}{l|}{ BADS } & GOODS & $\mathrm{f}(\mathrm{x} / 1)$ & $\mathrm{f}(\mathrm{x} / 0)$ & $\mathrm{w}(\mathrm{x})$ & & & & \\
\hline 010 & 15 & 452 & 0,115759 & 0,042466 & 1,002808 & 0,003618 & $-0,029346$ & 0,105895 & $-0,343025$ & \\
\hline $0 \backslash 1$ & 183 & 965 & 0,246994 & 0,50274 & $-0,71071$ & 0,042836 & $-0,194692$ & 0,225949 & $-0,484871$ & \\
\hline 012 & 30 & 133 & 0,034152 & 0,083562 & $-0,89476$ & 0,00712 & $-0,050793$ & 0,031242 & $-0,156222$ & \\
\hline 110 & 6 & 383 & 0,098107 & 0,017808 & 1,706398 & 0,001517 & $-0,014209$ & 0,089748 & $-0,312141$ & \\
\hline $1 \backslash 1$ & 53 & 1212 & 0,310182 & 0,146575 & 0,749618 & 0,012489 & $-0,078971$ & 0,283752 & $-0,515662$ & \\
\hline 112 & 5 & 202 & 0,051804 & 0,015068 & 1,234852 & 0,001284 & $-0,012332$ & 0,04739 & $-0,208480$ & \\
\hline 210 & 10 & 126 & 0,032361 & 0,028767 & 0,117728 & 0,002451 & $-0,021257$ & 0,029604 & $-0,150330$ & \\
\hline $2 \backslash 1$ & 50 & 386 & 0,098874 & 0,138356 & $-0,33598$ & 0,011789 & $-0,075524$ & 0,09045 & $-0,313566$ & \\
\hline 212 & 12 & 49 & 0,012663 & 0,034247 & $-0,9949$ & 0,002918 & $-0,024572$ & 0,011584 & $-0,074506$ & \\
\hline & 364 & 3908 & & & & & $-0,501697$ & & $-2,558802$ & \\
\hline & & & & & & & 0,501697 & & 2,558802 & \\
\hline & & & & & & & 0,042748 & & 2,340777 & 2,383525 \\
\hline
\end{tabular}




\begin{tabular}{|c|c|c|c|c|c|c|c|c|c|c|}
\hline AGETRM & BADS & GOODS & $f(x / 1)$ & $f(x / 0)$ & $w(x)$ & & & & 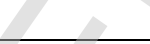 & 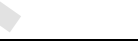 \\
\hline $0 \backslash 0$ & 17 & 449 & 0,114991 & 0,047945 & 0,874795 & 0,004085 & $-0,032418$ & 0,105193 & $-0,341761$ & \\
\hline $0 \backslash 1$ & 177 & 992 & 0,253901 & 0,486301 & $-0,64988$ & 0,041436 & $-0,190314$ & 0,232267 & $-0,489188$ & \\
\hline 012 & 32 & 119 & 0,03057 & 0,089041 & $-1,06906$ & 0,007587 & $-0,053429$ & 0,027966 & $-0,144309$ & \\
\hline 110 & 4 & 358 & 0,091711 & 0,012329 & 2,006712 & 0,00105 & $-0,010394$ & 0,083897 & $-0,299952$ & \\
\hline $1 \backslash 1$ & 55 & 1226 & 0,313763 & 0,152055 & 0,724397 & 0,012956 & $-0,081237$ & 0,287029 & $-0,516862$ & \\
\hline 112 & 6 & 213 & 0,054618 & 0,017808 & 1,120695 & 0,001517 & $-0,014209$ & 0,049964 & $-0,215992$ & \\
\hline 210 & 7 & 121 & 0,031082 & 0,020548 & 0,413872 & 0,001751 & $-0,016033$ & 0,028434 & $-0,146043$ & \\
\hline $2 \backslash 1$ & 52 & 386 & 0,098874 & 0,143836 & $-0,37482$ & 0,012256 & $-0,077828$ & 0,09045 & $-0,313566$ & \\
\hline \multirow[t]{4}{*}{212} & 14 & 44 & 0,011384 & 0,039726 & $-1,2498$ & 0,003385 & $-0,027779$ & 0,010414 & $-0,068580$ & \\
\hline & 364 & 3908 & & & & & $-0,503641$ & 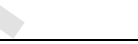 & $-2,536251$ & \\
\hline & & & & & & & 0,503641 & & 2,536251 & \\
\hline & & & & & & & 0,042913 & & 2,320147 & 2,363061 \\
\hline
\end{tabular}

\begin{tabular}{|c|c|c|c|c|c|c|c|c|c|c|}
\hline AGETRM & BADS & GOODS & $f(x / 1)$ & $f(x / 0)$ & $w(x)$ & 8 & & & & \\
\hline $0 \backslash 0$ & 17 & 437 & 0,111921 & 0,047945 & 0,847736 & 0,004085 & $-0,032418$ & 0,102385 & $-0,336634$ & \\
\hline $0 \backslash 1$ & 187 & 979 & 0,250576 & 0,513699 & $-0,71788$ & 0,04377 & $-0,197575$ & 0,229225 & $-0,487141$ & \\
\hline $0 \backslash 2$ & 29 & 129 & 0,033129 & 0,080822 & $-0,89185$ & 0,006887 & $-0,049459$ & 0,030306 & $-0,152871$ & \\
\hline 110 & 6 & 375 & 0,09606 & 0,017808 & 1,685317 & 0,001517 & $-0,014209$ & 0,087875 & $-0,308302$ & \\
\hline $1 \backslash 1$ & 60 & 1409 & 0,360578 & 0,165753 & 0,777207 & 0,014123 & $-0,086798$ & 0,329855 & $-0,527800$ & \\
\hline 112 & 12 & 209 & 0,053594 & 0,034247 & 0,447856 & 0,002918 & $-0,024572$ & 0,049028 & $-0,213283$ & \\
\hline 210 & 8 & 90 & 0,023152 & 0,023288 & $-0,00586$ & 0,001984 & $-0,017813$ & 0,021179 & $-0,117781$ & \\
\hline $2 \backslash 1$ & 37 & 254 & 0,065106 & 0,10274 & $-0,45618$ & 0,008754 & $-0,059841$ & 0,059559 & $-0,242377$ & \\
\hline \multirow[t]{4}{*}{$2 \backslash 2$} & 8 & 26 & 0,006779 & 0,023288 & $-1,23406$ & 0,001984 & $-0,017813$ & 0,006202 & $-0,045477$ & \\
\hline & 364 & 3908 & & 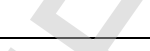 & 2 & & $-0,500498$ & & $-2,431665$ & \\
\hline & & & & & & & 0,500498 & & 2,431665 & \\
\hline & & & & & & & 0,042645 & & 2,224473 & 2,267118 \\
\hline
\end{tabular}




\begin{tabular}{|c|c|c|c|c|c|c|c|c|c|c|}
\hline AGETRM & BADS & GOODS & $f(x / 1)$ & $f(x / 0)$ & $w(x)$ & & & & 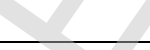 & \\
\hline 010 & 18 & 407 & 0,104247 & 0,050685 & 0,721131 & 0,004319 & $-0,033924$ & 0,095364 & $-0,323324$ & \\
\hline $0 \backslash 1$ & 176 & 981 & 0,251087 & 0,483562 & $-0,65538$ & 0,041202 & $-0,189577$ & 0,229693 & $-0,487459$ & \\
\hline 012 & 37 & 132 & 0,033896 & 0,10274 & $-1,1089$ & 0,008754 & $-0,059841$ & 0,031008 & $-0,155388$ & \\
\hline 110 & 4 & 338 & 0,086595 & 0,012329 & 1,949307 & 0,00105 & $-0,010394$ & 0,079217 & $-0,289779$ & \\
\hline $1 \backslash 1$ & 56 & 1271 & 0,325275 & 0,154795 & 0,742572 & 0,013189 & $-0,082361$ & 0,29756 & $-0,520357$ & \\
\hline 112 & 6 & 200 & 0,051292 & 0,017808 & 1,057873 & 0,001517 & $-0,014209$ & 0,046922 & $-0,207093$ & \\
\hline 210 & 8 & 120 & 0,030826 & 0,023288 & 0,280444 & 0,001984 & $-0,017813$ & 0,0282 & $-0,145177$ & \\
\hline 211 & 45 & 414 & 0,106037 & 0,124658 & $-0,16178$ & 0,010622 & $-0,069644$ & 0,097002 & $-0,326494$ & \\
\hline \multirow[t]{4}{*}{212} & 14 & 45 & 0,01164 & 0,039726 & $-1,22758$ & 0,003385 & $-0,027779$ & 0,010648 & $-0,069779$ & \\
\hline & 364 & 3908 & & & & & $-0,505543$ & & $-2,524850$ & \\
\hline & & & & & & - & 0,505543 & & 2,524850 & \\
\hline & & & & & & & 0,043075 & & 2,309718 & 2,352793 \\
\hline
\end{tabular}

11) $\quad H(C A R C R D)$

\begin{tabular}{|c|c|c|c|c|c|c|c|c|c|c|}
\hline CARICRD & BADS & GOODS & $f(x / 1)$ & $f(x / 0)$ & $w(x)$ & & & & & \\
\hline $1 \backslash 0$ & 127 & 1831 & 0,468534 & 0,349315 & 0,293635 & 0,029764 & $-0,150911$ & 0,428612 & $-0,523873$ & \\
\hline $2 \backslash 0$ & 182 & 678 & 0,173574 & 0,5 & $-1,05801$ & 0,042603 & $-0,193967$ & 0,158784 & $-0,421550$ & \\
\hline $1 \backslash 1$ & 37 & 1080 & 0,276413 & 0,10274 & 0,989699 & 0,008754 & $-0,059841$ & 0,252861 & $-0,501571$ & \\
\hline \multirow[t]{4}{*}{211} & 18 & 319 & 0,081734 & 0,050685 & 0,477847 & 0,004319 & $-0,033924$ & 0,07477 & $-0,279745$ & \\
\hline & 364 & 3908 & & 2 & & & $-0,438643$ & & $-1,726739$ & \\
\hline & & & & 12 & F & & 0,438643 & & 1,726739 & \\
\hline & & & & 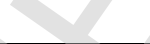 & 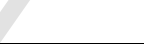 & & 0,037375 & & 1,579611 & 1,616986 \\
\hline
\end{tabular}




\begin{tabular}{|c|c|c|c|c|c|c|c|c|c|c|}
\hline CARCRD & BADS & GOODS & $f(x / 1)$ & $f(x / 0)$ & $w(x)$ & & & & & \\
\hline $0 \backslash 0$ & 172 & 701 & 0.179458 & 0,472603 & $-0,96832$ & 0.040269 & -0.186613 & 0.164167 & $-0,427944$ & \\
\hline $0 \backslash 1$ & 22 & 317 & 0,081223 & 0,061644 & 0,275823 & 0.005252 & -0.039776 & 0.074302 & $-0,278667$ & \\
\hline 110 & 129 & 1836 & 0,469813 & 0,354795 & 0,280796 & 0,030231 & $-0,152600$ & 0,429782 & $-0,523613$ & \\
\hline $1 \backslash 1$ & 41 & 1054 & 0,269762 & 0,113699 & 0,863989 & 0,009688 & $-0,064808$ & 0,246777 & $-0,498174$ & \\
\hline & 364 & 3908 & & & & & $-0,443795$ & & $-1,728398$ & \\
\hline & & & & & & & 0,443795 & & 1,728398 & \\
\hline & & & & & & & 0,037814 & & 1,581128 & 1,618942 \\
\hline
\end{tabular}

\begin{tabular}{|c|c|c|c|c|c|c|c|c|c|c|}
\hline CARCRD & BADS & GOODS & $f(x / 1)$ & $f(x / 0)$ & $w(x)$ & & 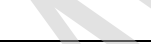 & 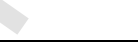 & & \\
\hline 010 & 186 & 677 & 0,173318 & 0,510959 & $-1,08116$ & 0,043537 & $-0,196857$ & 0,15855 & $-0,421266$ & \\
\hline $0 \backslash 1$ & 19 & 313 & 0,0802 & 0,053425 & 0,406245 & 0,004552 & $-0,035412$ & 0,073366 & $-0,276498$ & \\
\hline 110 & 123 & 1842 & 0,471348 & 0,338356 & 0,331498 & 0,02883 & $-0,147502$ & 0,431186 & $-0,523295$ & \\
\hline \multirow[t]{4}{*}{$1 \backslash 1$} & 36 & 1076 & 0,27539 & 0,1 & 1,013019 & 0,008521 & $-0,058578$ & 0,251925 & $-0,501062$ & \\
\hline & 364 & 3908 & & & & 2 & $-0,438349$ & & $-1,722121$ & \\
\hline & & & & & & & 0,438349 & & 1,722121 & \\
\hline & & & & & & & 0,03735 & & 1,575386 & 1,612736 \\
\hline
\end{tabular}

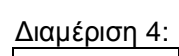

\begin{tabular}{|l|r|r|l|l|l|l|l|l|l|l|}
\hline CARCRD & \multicolumn{1}{|c|}{ BADS } & GOODS & $\mathrm{f}(\mathrm{x} / 1)$ & $\mathrm{f}(\mathrm{x} / 0)$ & $\mathrm{w}(\mathrm{x})$ & & & & \\
\hline 010 & 176 & 704 & 0,180225 & 0,483562 & $-0,98697$ & 0,041202 & $-0,189577$ & 0,164869 & $-0,428759$ & \\
\hline $0 \backslash 1$ & 20 & 331 & 0,084804 & 0,056164 & 0,412063 & 0,004786 & $-0,036883$ & 0,077578 & $-0,286125$ & \\
\hline 110 & 129 & 1805 & 0,461883 & 0,354795 & 0,263772 & 0,030231 & $-0,152600$ & 0,422528 & $-0,525152$ & \\
\hline 111 & 39 & 1068 & 0,273344 & 0,108219 & 0,926571 & 0,009221 & $-0,062341$ & 0,250053 & $-0,500030$ & \\
\hline & 364 & 3908 & & & & & $-0,441401$ & & $-1,740066$ & \\
\hline & & & & & & & 0,441401 & & 1,740066 & \\
\hline & & & & & & & 0,03761 & & 1,591802 & 1,629412 \\
\hline
\end{tabular}




\begin{tabular}{|c|c|c|c|c|c|c|c|c|c|c|}
\hline CARCRD & BADS & GOODS & $f(x / 1)$ & $f(x / 0)$ & $w(x)$ & & & ( & 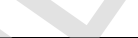 & \\
\hline $0 \backslash 0$ & 180 & 684 & 0,175109 & 0,494521 & $-1,03818$ & 0,042136 & $-0,192511$ & 0,160188 & $-0,423243$ & \\
\hline $0 \backslash 1$ & 17 & 312 & 0,079944 & 0,047945 & 0,511264 & 0,004085 & $-0,032418$ & 0,073132 & $-0,275953$ & \\
\hline 110 & 132 & 1838 & 0,470325 & 0,363014 & 0,258983 & 0,030931 & $-0,155113$ & 0,43025 & $-0,523508$ & \\
\hline \multirow[t]{4}{*}{$1 \backslash 1$} & 35 & 1074 & 0,274878 & 0,09726 & 1,038939 & 0,008287 & $-0,057305$ & 0,251457 & $-0,500806$ & \\
\hline & 364 & 3908 & & & & & $-0,437347$ & . & $-1,723510$ & \\
\hline & & & & & & & 0,437347 & ( & 1,723510 & \\
\hline & & & & & & & 0,037265 & & 1,576657 & 1,613921 \\
\hline
\end{tabular}

12) $\quad H(C A R K I D)$

\begin{tabular}{|c|c|c|c|c|c|c|c|c|c|c|}
\hline CAR\KID & BADS & GOODS & $f(x / 1)$ & $f(x / 0)$ & $w(x)$ & +2 & 8 & & & \\
\hline $1 \backslash 1$ & 28 & 366 & 0,093758 & 0,078082 & 0,182955 & 0,006653 & $-0,048113$ & 0,085769 & $-0,303914$ & \\
\hline $2 \backslash 1$ & 24 & 109 & 0,028012 & 0,067123 & $-0,87389$ & 0,005719 & $-0,042609$ & 0,025625 & $-0,135463$ & \\
\hline 112 & 78 & 954 & 0,24418 & 0,215068 & 0,12695 & 0,018325 & $-0,105737$ & 0,223374 & $-0,483039$ & \\
\hline 212 & 120 & 467 & 0,119596 & 0,330137 & $-1,01539$ & 0,02813 & $-0,144917$ & 0,109406 & $-0,349249$ & \\
\hline $1 \backslash 3$ & 4 & 486 & 0,124456 & 0,012329 & 2,31202 & 0,00105 & $-0,010394$ & 0,113852 & $-0,356900$ & \\
\hline 213 & 12 & 136 & 0,034919 & 0,034247 & 0,019456 & 0,002918 & $-0,024572$ & 0,031944 & $-0,158708$ & \\
\hline $1 \backslash 4$ & 54 & 1105 & 0,282809 & 0,149315 & 0,638713 & 0,012723 & $-0,080107$ & 0,258712 & $-0,504639$ & \\
\hline \multirow[t]{4}{*}{$2 \backslash 4$} & 44 & 285 & 0,073037 & 0,121918 & $-0,51239$ & 0,010388 & $-0,068447$ & 0,066813 & $-0,260821$ & \\
\hline & 364 & 3908 & & $\Delta$ & & & $-0,524896$ & & $-2,552733$ & \\
\hline & & & & 8 & 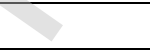 & & 0,524896 & & 2,552733 & \\
\hline & & & & 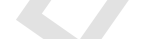 & 0 & & 0,044724 & & 2,335225 & 2,379949 \\
\hline
\end{tabular}




\begin{tabular}{|c|c|c|c|c|c|c|c|c|c|c|}
\hline CARKID & BADS & GOODS & $f(x / 1)$ & $f(x / 0)$ & $w(x)$ & & & & 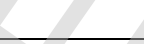 & \\
\hline $0 \backslash 0$ & 114 & 472 & 0,120875 & 0,313699 & $-0,95368$ & 0,026729 & $-0,139671$ & 0,110576 & $-0,351287$ & \\
\hline $0 \backslash 1$ & 13 & 144 & 0,036966 & 0,036986 & $-0,00055$ & 0,003151 & $-0,026188$ & 0,033816 & $-0,165231$ & \\
\hline $0 \backslash 2$ & 45 & 288 & 0,073804 & 0,124658 & $-0,52416$ & 0,010622 & $-0,069644$ & 0,067515 & $-0,262543$ & \\
\hline $0 \backslash 3$ & 22 & 114 & 0,029291 & 0,061644 & $-0,74408$ & 0,005252 & $-0,039776$ & 0,026796 & $-0,139923$ & \\
\hline 110 & 83 & 968 & 0,247762 & 0,228767 & 0,079762 & 0,019492 & $-0,110735$ & 0,226651 & $-0,485363$ & \\
\hline $1 \backslash 1$ & 5 & 512 & 0,131108 & 0,015068 & 2,163413 & 0,001284 & $-0,012332$ & 0,119937 & $-0,366965$ & \\
\hline 112 & 52 & 1059 & 0,271041 & 0,143836 & 0,6336 & 0,012256 & $-0,077828$ & 0,247947 & $-0,498844$ & \\
\hline \multirow[t]{4}{*}{113} & 30 & 351 & 0,089921 & 0,083562 & 0,073344 & 0,00712 & $-0,050793$ & 0,082259 & $-0,296435$ & \\
\hline & 364 & 3908 & & & & & $-0,526968$ & & $-2,566590$ & \\
\hline & & & & & & & 0,526968 & & 2,566590 & \\
\hline & & & & & & & 0,044901 & & 2,347902 & 2,392802 \\
\hline
\end{tabular}

\begin{tabular}{|c|c|c|c|c|c|c|c|c|c|c|}
\hline CARKID & BADS & GOODS & $f(x / 1)$ & $f(x / 0)$ & $w(x)$ & 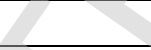 & & & & \\
\hline 010 & 126 & 458 & 0,117293 & 0,346575 & $-1,08342$ & 0,02953 & $-0,150063$ & 0,107299 & $-0,345535$ & \\
\hline $0 \backslash 1$ & 13 & 139 & 0,035687 & 0,036986 & $-0,03576$ & 0,003151 & $-0,026188$ & 0,032646 & $-0,161172$ & \\
\hline 012 & 44 & 281 & 0,072013 & 0,121918 & $-0,5265$ & 0,010388 & $-0,068447$ & 0,065877 & $-0,258508$ & \\
\hline 013 & 22 & 112 & 0,02878 & 0,061644 & $-0,7617$ & 0,005252 & $-0,039776$ & 0,026328 & $-0,138148$ & \\
\hline 110 & 81 & 977 & 0,250064 & 0,223288 & 0,113256 & 0,019025 & $-0,108748$ & 0,228757 & $-0,486821$ & \\
\hline $1 \backslash 1$ & 5 & 498 & 0,127526 & 0,015068 & 2,135716 & 0,001284 & $-0,012332$ & 0,11666 & $-0,361602$ & \\
\hline 112 & 47 & 1074 & 0,274878 & 0,130137 & 0,747741 & 0,011088 & $-0,072017$ & 0,251457 & $-0,500806$ & \\
\hline \multirow[t]{4}{*}{113} & 26 & 369 & 0,094525 & 0,072603 & 0,263866 & 0,006186 & $-0,045386$ & 0,086471 & $-0,305385$ & \\
\hline & 364 & 3908 & & 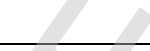 & 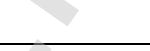 & & $-0,522957$ & & $-2,557976$ & \\
\hline & & & & + & 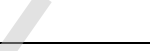 & & 0,522957 & & 2,557976 & \\
\hline & & & & & & & 0,044559 & & 2,340021 & 2,38458 \\
\hline
\end{tabular}




\begin{tabular}{|c|c|c|c|c|c|c|c|c|c|c|}
\hline CARKID & BADS & GOODS & $f(x / 1)$ & $f(x / 0)$ & $w(x)$ & & & & 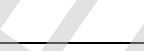 & \\
\hline 010 & 120 & 483 & 0,123689 & 0,330137 & $-0,98174$ & 0,02813 & $-0,144917$ & 0,11315 & $-0,355708$ & \\
\hline $0 \backslash 1$ & 12 & 140 & 0,035943 & 0,034247 & 0,048339 & 0,002918 & $-0,024572$ & 0,03288 & $-0,161989$ & \\
\hline 012 & 40 & 293 & 0,075083 & 0,110959 & $-0,39056$ & 0,009454 & $-0,063579$ & 0,068686 & $-0,265391$ & \\
\hline 013 & 24 & 119 & 0,03057 & 0,067123 & $-0,7865$ & 0,005719 & $-0,042609$ & 0,027966 & $-0,144309$ & \\
\hline 110 & 81 & 959 & 0,245459 & 0,223288 & 0,09467 & 0,019025 & $-0,108748$ & 0,224545 & $-0,483877$ & \\
\hline $1 \backslash 1$ & 5 & 483 & 0,123689 & 0,015068 & 2,105164 & 0,001284 & $-0,012332$ & 0,11315 & $-0,355708$ & \\
\hline 112 & 54 & 1084 & 0,277437 & 0,149315 & 0,619534 & 0,012723 & $-0,080107$ & 0,253797 & $-0,502075$ & \\
\hline \multirow[t]{4}{*}{113} & 28 & 347 & 0,088897 & 0,078082 & 0,129721 & 0,006653 & $-0,048113$ & 0,081323 & $-0,294405$ & \\
\hline & 364 & 3908 & & & & & $-0,524978$ & & $-2,563461$ & \\
\hline & & & & & & & 0,524978 & & 2,563461 & \\
\hline & & & & & & & 0,044731 & & 2,345039 & 2,38977 \\
\hline
\end{tabular}

\begin{tabular}{|c|c|c|c|c|c|c|c|c|c|c|}
\hline CARKID & BADS & GOODS & $f(x / 1)$ & $f(x / 0)$ & $w(x)$ & 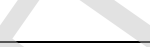 & & & & \\
\hline 010 & 120 & 456 & 0,116782 & 0,330137 & $-1,0392$ & 0,02813 & $-0,144917$ & 0,106831 & $-0,344701$ & \\
\hline $0 \backslash 1$ & 10 & 145 & 0,037222 & 0,028767 & 0,257661 & 0,002451 & $-0,021257$ & 0,03405 & $-0,166036$ & \\
\hline 012 & 43 & 293 & 0,075083 & 0,119178 & $-0,46202$ & 0,010155 & $-0,067241$ & 0,068686 & $-0,265391$ & \\
\hline 013 & 24 & 102 & 0,026222 & 0,067123 & $-0,93995$ & 0,005719 & $-0,042609$ & 0,023987 & $-0,129090$ & \\
\hline 110 & 77 & 970 & 0,248273 & 0,212329 & 0,156394 & 0,018092 & $-0,104724$ & 0,227119 & $-0,485689$ & \\
\hline $1 \backslash 1$ & 5 & 497 & 0,12727 & 0,015068 & 2,133708 & 0,001284 & $-0,012332$ & 0,116426 & $-0,361214$ & \\
\hline 112 & 53 & 1082 & 0,276925 & 0,146575 & 0,636207 & 0,012489 & $-0,078971$ & 0,253329 & $-0,501824$ & \\
\hline \multirow[t]{4}{*}{113} & 32 & 363 & 0,092991 & 0,089041 & 0,0434 & 0,007587 & $-0,053429$ & 0,085067 & $-0,302435$ & \\
\hline & 364 & 3908 & & 2 & $\nabla$ & & $-0,525480$ & & $-2,556379$ & \\
\hline & & & & 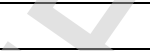 & 2 & & 0,525480 & & 2,556379 & \\
\hline & & & & & & & 0,044774 & & 2,33856 & 2,383335 \\
\hline
\end{tabular}




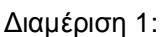

\begin{tabular}{|c|c|c|c|c|c|c|c|c|c|c|}
\hline CAR\HYR & BADS & GOODS & $f(x / 1)$ & $f(x / 0)$ & $w(x)$ & & & & 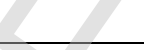 & \\
\hline $1 \backslash 1$ & 28 & 895 & 0,229087 & 0,078082 & 1,076339 & 0,006653 & $-0,048113$ & 0,209567 & $-0,472472$ & \\
\hline $2 \backslash 1$ & 51 & 299 & 0,076618 & 0,141096 & $-0,61061$ & 0,012022 & $-0,076680$ & 0,07009 & $-0,268770$ & \\
\hline 112 & 95 & 578 & 0,147992 & 0,261644 & $-0,56983$ & 0,022294 & $-0,122330$ & 0,135382 & $-0,390563$ & \\
\hline 212 & 103 & 220 & 0,056408 & 0,283562 & $-1,61481$ & 0,024161 & $-0,129774$ & 0,051602 & $-0,220672$ & \\
\hline 113 & 40 & 1257 & 0,321694 & 0,110959 & 1,064439 & 0,009454 & $-0,063579$ & 0,294283 & $-0,519328$ & \\
\hline 213 & 41 & 410 & 0,105014 & 0,113699 & $-0,07946$ & 0,009688 & $-0,064808$ & 0,096066 & $-0,324687$ & \\
\hline $1 \backslash 4$ & 1 & 181 & 0,046431 & 0,00411 & 2,424651 & 0,00035 & $-0,004020$ & 0,042475 & $-0,193569$ & \\
\hline \multirow[t]{4}{*}{$2 \backslash 4$} & 5 & 68 & 0,017524 & 0,015068 & 0,150946 & 0,001284 & $-0,012332$ & 0,016031 & $-0,095591$ & \\
\hline & 364 & 3908 & & & & & $-0,521635$ & & $-2,485652$ & \\
\hline & & & & & & 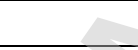 & 0,521635 & & 2,485652 & \\
\hline & & & & & & & 0,044446 & & 2,27386 & 2,318306 \\
\hline
\end{tabular}

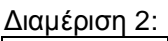

\begin{tabular}{|c|c|c|c|c|c|c|c|c|c|c|}
\hline CARHYR & BADS & GOODS & $f(x / 1)$ & $f(x / 0)$ & $w(x)$ & ser & & & & \\
\hline $0 \backslash 0$ & 99 & 222 & 0,05692 & 0,272603 & $-1,56637$ & 0,023227 & $-0,126079$ & 0,05207 & $-0,221995$ & \\
\hline $0 \backslash 1$ & 44 & 434 & 0,111154 & 0,121918 & $-0,09243$ & 0,010388 & $-0,068447$ & 0,101683 & $-0,335335$ & \\
\hline 012 & 47 & 296 & 0,075851 & 0,130137 & $-0,53982$ & 0,011088 & $-0,072017$ & 0,069388 & $-0,267085$ & \\
\hline 013 & 4 & 66 & 0,017012 & 0,012329 & 0,321985 & 0,00105 & $-0,010394$ & 0,015562 & $-0,093465$ & \\
\hline 110 & 96 & 569 & 0,145689 & 0,264384 & $-0,59592$ & 0,022527 & $-0,123273$ & 0,133276 & $-0,387501$ & \\
\hline $1 \backslash 1$ & 45 & 1269 & 0,324763 & 0,124658 & 0,957527 & 0,010622 & $-0,069644$ & 0,297092 & $-0,520213$ & \\
\hline 112 & 28 & 876 & 0,224226 & 0,078082 & 1,054893 & 0,006653 & $-0,048113$ & 0,205121 & $-0,468794$ & \\
\hline \multirow[t]{4}{*}{113} & 1 & 176 & 0,045152 & 0,00411 & 2,396716 & 0,00035 & $-0,004020$ & 0,041305 & $-0,189901$ & \\
\hline & 364 & 3908 & & -4 & 2 & & $-0,521987$ & & $-2,484291$ & \\
\hline & & & & & & & 0,521987 & & 2,484291 & \\
\hline & & & & & & & 0,044476 & & 2,272614 & 2,317091 \\
\hline
\end{tabular}




\begin{tabular}{|c|c|c|c|c|c|c|c|c|c|c|}
\hline CARHYR & BADS & GOODS & $f(x / 1)$ & $f(x / 0)$ & $w(x)$ & & & & 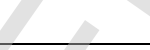 & 8 \\
\hline $0 \backslash 0$ & 99 & 236 & 0,060501 & 0,272603 & $-1,50535$ & 0,023227 & $-0,126079$ & 0,055346 & $-0,231091$ & \\
\hline $0 \backslash 1$ & 49 & 411 & 0,10527 & 0,135616 & $-0,2533$ & 0,011555 & $-0,074362$ & 0,0963 & $-0,325140$ & \\
\hline $0 \backslash 2$ & 55 & 271 & 0,069455 & 0,152055 & $-0,78356$ & 0,012956 & $-0,081237$ & 0,063537 & $-0,252640$ & \\
\hline $0 \backslash 3$ & 2 & 72 & 0,018547 & 0,006849 & 0,996156 & 0,000584 & $-0,006269$ & 0,016967 & $-0,099783$ & \\
\hline $1 \backslash 0$ & 100 & 583 & 0,149271 & 0,275342 & $-0,61225$ & 0,023461 & $-0,127008$ & 0,136552 & $-0,392243$ & \\
\hline $1 \backslash 1$ & 37 & 1288 & 0,329624 & 0,10274 & 1,165754 & 0,008754 & $-0,059841$ & 0,301538 & $-0,521537$ & \\
\hline 112 & 22 & 863 & 0,2209 & 0,061644 & 1,276339 & 0,005252 & $-0,039776$ & 0,202078 & $-0,466198$ & \\
\hline \multirow[t]{4}{*}{113} & 0 & 184 & 0,047199 & 0,00137 & 3,539657 & 0,000117 & $-0,001525$ & 0,043177 & $-0,195747$ & \\
\hline & 364 & 3908 & & & & & $-0,516097$ & & $-2,484379$ & \\
\hline & & & & & & & 0,516097 & 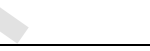 & 2,484379 & \\
\hline & & & & & & & 0,043975 & & 2,272695 & 2,31667 \\
\hline
\end{tabular}

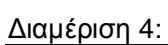

\begin{tabular}{|l|r|r|l|l|l|l|l|l|l|l|}
\hline CARHYR & \multicolumn{1}{|l|}{ BADS } & GOODS & $\mathrm{f}(\mathrm{x} / 1)$ & $\mathrm{f}(\mathrm{x} / 0)$ & $\mathrm{w}(\mathrm{x})$ & & & & \\
\hline 010 & 98 & 230 & 0,058966 & 0,269863 & $-1,52095$ & 0,022994 & $-0,125147$ & 0,053942 & $-0,227228$ & \\
\hline $0 \backslash 1$ & 40 & 431 & 0,110386 & 0,110959 & $-0,00517$ & 0,009454 & $-0,063579$ & 0,100981 & $-0,334029$ & \\
\hline 012 & 54 & 309 & 0,079176 & 0,149315 & $-0,63438$ & 0,012723 & $-0,080107$ & 0,07243 & $-0,274312$ & \\
\hline 013 & 4 & 65 & 0,016756 & 0,012329 & 0,306833 & 0,00105 & $-0,010394$ & 0,015328 & $-0,092395$ & \\
\hline 110 & 93 & 589 & 0,150806 & 0,256164 & $-0,52983$ & 0,021827 & $-0,120435$ & 0,137956 & $-0,394240$ & \\
\hline 111 & 45 & 1253 & 0,32067 & 0,124658 & 0,944843 & 0,010622 & $-0,069644$ & 0,293347 & $-0,519025$ & \\
\hline 112 & 29 & 850 & 0,217575 & 0,080822 & 0,990295 & 0,006887 & $-0,049459$ & 0,199036 & $-0,463535$ & \\
\hline 113 & 1 & 181 & 0,046431 & 0,00411 & 2,424651 & 0,00035 & $-0,004020$ & 0,042475 & $-0,193569$ & \\
\hline & 364 & 3908 & & & & & $-0,522785$ & & $-2,498332$ & \\
\hline & & & & & & & 0,522785 & & 2,498332 & \\
\hline & & & & & & & 0,044544 & & 2,285459 & 2,330004 \\
\hline
\end{tabular}




\begin{tabular}{|c|c|c|c|c|c|c|c|c|c|c|}
\hline \multicolumn{11}{|c|}{$\triangle$} \\
\hline CARHYR & BADS & GOODS & $f(x / 1)$ & $f(x / 0)$ & $w(x)$ & & & & 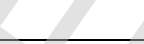 & \\
\hline $0 \backslash 0$ & 101 & 224 & 0,057432 & 0,278082 & $-1,57732$ & 0,023694 & $-0,127933$ & 0,052538 & $-0,223313$ & \\
\hline $0 \backslash 1$ & 38 & 411 & 0,10527 & 0,105479 & $-0,00199$ & 0,008987 & $-0,061096$ & 0,0963 & $-0,325140$ & \\
\hline 012 & 53 & 295 & 0,075595 & 0,146575 & $-0,66215$ & 0,012489 & $-0,078971$ & 0,069154 & $-0,266522$ & \\
\hline 013 & 5 & 66 & 0,017012 & 0,015068 & 0,121314 & 0,001284 & $-0,012332$ & 0,015562 & $-0,093465$ & \\
\hline 110 & 100 & 585 & 0,149783 & 0,275342 & $-0,60883$ & 0,023461 & $-0,127008$ & 0,13702 & $-0,392911$ & \\
\hline $1 \backslash 1$ & 43 & 1229 & 0,314531 & 0,119178 & 0,970462 & 0,010155 & $-0,067241$ & 0,287731 & $-0,517112$ & \\
\hline 112 & 23 & 908 & 0,232412 & 0,064384 & 1,283655 & 0,005486 & $-0,041199$ & 0,212609 & $-0,474910$ & \\
\hline \multirow[t]{4}{*}{$1 \backslash 3$} & 1 & 190 & 0,048734 & 0,00411 & 2,473048 & 0,00035 & $-0,004020$ & 0,044581 & $-0,200055$ & \\
\hline & 364 & 3908 & & & & & $-0,519800$ & & $-2,493428$ & \\
\hline & & & & & & & 0,519800 & & 2,493428 & \\
\hline & & & & & & & 0,04429 & & 2,280973 & 2,325263 \\
\hline
\end{tabular}

\section{4) H(CAR HOME)}

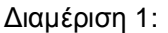

\begin{tabular}{|c|c|c|c|c|c|c|c|c|c|c|}
\hline CAR\HOME & BADS & GOODS & $f(x / 1)$ & $f(x / 0)$ & $w(x)$ & 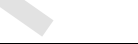 & & & & \\
\hline $1 \backslash 1$ & 25 & 420 & 0,107572 & 0,069863 & 0,431627 & 0,005953 & $-0,044004$ & 0,098406 & $-0,329180$ & \\
\hline $2 \backslash 1$ & 40 & 179 & 0,04592 & 0,110959 & $-0,88227$ & 0,009454 & $-0,063579$ & 0,042007 & $-0,192108$ & \\
\hline 112 & 60 & 529 & 0,135457 & 0,165753 & $-0,20185$ & 0,014123 & $-0,086798$ & 0,123915 & $-0,373303$ & \\
\hline 212 & 94 & 213 & 0,054618 & 0,258904 & $-1,5561$ & 0,02206 & $-0,121384$ & 0,049964 & $-0,215992$ & \\
\hline 113 & 79 & 1962 & 0,502047 & 0,217808 & 0,835078 & 0,018559 & $-0,106745$ & 0,459269 & $-0,515570$ & \\
\hline \multirow[t]{4}{*}{213} & 66 & 605 & 0,154899 & 0,182192 & $-0,16229$ & 0,015524 & $-0,093288$ & 0,141701 & $-0,399466$ & \\
\hline & 364 & 3908 & & 2 & & & $-0,515798$ & & $-2,025619$ & \\
\hline & & & & 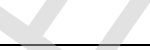 & & & 0,515798 & & 2,025619 & \\
\hline & & & & 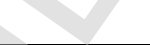 & & & 0,043949 & & 1,853024 & 1,896973 \\
\hline
\end{tabular}




\begin{tabular}{|c|c|c|c|c|c|c|c|c|c|c|}
\hline CARHOME & BADS & GOODS & $f(x / 1)$ & $f(x / 0)$ & $w(x)$ & & & & + & \\
\hline 010 & 40 & 175 & 0,044896 & 0,110959 & $-0,9048$ & 0,009454 & $-0,063579$ & 0,041071 & $-0,189162$ & \\
\hline $0 \backslash 1$ & 91 & 219 & 0,056152 & 0,250685 & $-1,49613$ & 0,02136 & $-0,118525$ & 0,051368 & $-0,220008$ & \\
\hline 012 & 63 & 624 & 0,15976 & 0,173973 & $-0,08523$ & 0,014824 & $-0,090067$ & 0,146147 & $-0,405486$ & \\
\hline 110 & 25 & 436 & 0,111665 & 0,069863 & 0,46897 & 0,005953 & $-0,044004$ & 0,102151 & $-0,336202$ & \\
\hline $1 \backslash 1$ & 68 & 526 & 0,134689 & 0,187671 & $-0,33172$ & 0,015991 & $-0,095411$ & 0,123213 & $-0,372198$ & \\
\hline \multirow[t]{4}{*}{112} & 77 & 1928 & 0,493349 & 0,212329 & 0,84308 & 0,018092 & $-0,104724$ & 0,451312 & $-0,518017$ & \\
\hline & 364 & 3908 & & & & & $-0,516310$ & 2 & $-2,041073$ & \\
\hline & & & & & & & 0,516310 & & 2,041073 & \\
\hline & & & & & & & 0,043993 & & 1,867162 & 1,911154 \\
\hline
\end{tabular}

\begin{tabular}{|c|c|c|c|c|c|c|c|c|c|c|}
\hline САРНML & & & & & & & & & & \\
\hline UARAUIVIE & BADS & GUUDS & $1(x / 1)$ & $x(x, 0)$ & & & & & & \\
\hline 010 & 50 & 172 & 0,044129 & 0,138356 & $-1,14272$ & 0,011789 & $-0,075524$ & 0,040369 & $-0,186933$ & \\
\hline $0 \backslash 1$ & 89 & 212 & 0,054362 & 0,245205 & $-1,50644$ & 0,020893 & $-0,116600$ & 0,04973 & $-0,215317$ & \\
\hline 012 & 66 & 606 & 0,155155 & 0,182192 & $-0,16064$ & 0,015524 & $-0,093288$ & 0,141935 & $-0,399788$ & \\
\hline 110 & 21 & 435 & 0,11141 & 0,058904 & 0,637302 & 0,005019 & $-0,038337$ & 0,101917 & $-0,335769$ & \\
\hline $1 \backslash 1$ & 73 & 520 & 0,133154 & 0,20137 & $-0,41364$ & 0,017158 & $-0,100631$ & 0,121809 & $-0,369971$ & \\
\hline \multirow[t]{4}{*}{112} & 65 & 1963 & 0,502302 & 0,179452 & 1,029294 & 0,01529 & $-0,092220$ & 0,459503 & $-0,515495$ & \\
\hline & 364 & 3908 & & 8 & 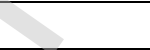 & & $-0,516601$ & & $-2,023272$ & \\
\hline & & & & & 2 & & 0,516601 & & 2,023272 & \\
\hline & & & & & & & 0,044017 & & 1,850877 & 1,894895 \\
\hline
\end{tabular}




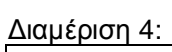

\begin{tabular}{|c|c|c|c|c|c|c|c|c|c|c|}
\hline CARHOME & BADS & GOODS & $f(x / 1)$ & $f(x / 0)$ & $w(x)$ & & & & - & \\
\hline 010 & 40 & 187 & 0,047966 & 0,110959 & $-0,83866$ & 0,009454 & $-0,063579$ & 0,043879 & $-0,197909$ & \\
\hline $0 \backslash 1$ & 94 & 227 & 0,058199 & 0,258904 & $-1,49259$ & 0,02206 & $-0,121384$ & 0,05324 & $-0,225277$ & \\
\hline 012 & 62 & 621 & 0,158992 & 0,171233 & $-0,07417$ & 0,01459 & $-0,088983$ & 0,145445 & $-0,404549$ & \\
\hline 110 & 26 & 424 & 0,108596 & 0,072603 & 0,402628 & 0,006186 & $-0,045386$ & 0,099343 & $-0,330954$ & \\
\hline $1 \backslash 1$ & 69 & 512 & 0,131108 & 0,190411 & $-0,37317$ & 0,016224 & $-0,096464$ & 0,119937 & $-0,366965$ & \\
\hline \multirow[t]{4}{*}{112} & 73 & 1937 & 0,495651 & 0,20137 & 0,900729 & 0,017158 & $-0,100631$ & 0,453419 & $-0,517389$ & \\
\hline & 364 & 3908 & & & & & $-0,516427$ & 2 & $-2,043043$ & \\
\hline & & & & & & & 0,516427 & & 2,043043 & \\
\hline & & & & & & & 0,044003 & & 1,868963 & 1,912966 \\
\hline
\end{tabular}

\begin{tabular}{|c|c|c|c|c|c|c|c|c|c|c|}
\hline САРНML & & & & & & & & & & \\
\hline UARAUIVIE & BADS & GUUDS & & 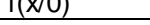 & & & & & & \\
\hline $0 \backslash 0$ & 46 & 167 & 0,04285 & 0,127397 & $-1,08961$ & 0,010855 & $-0,070834$ & 0,039199 & $-0,183178$ & \\
\hline $0 \backslash 1$ & 88 & 217 & 0,055641 & 0,242466 & $-1,47194$ & 0,02066 & $-0,115632$ & 0,0509 & $-0,218676$ & \\
\hline 012 & 63 & 612 & 0,15669 & 0,173973 & $-0,10463$ & 0,014824 & $-0,090067$ & 0,143339 & $-0,401707$ & \\
\hline 110 & 23 & 433 & 0,110898 & 0,064384 & 0,543752 & 0,005486 & $-0,041199$ & 0,101449 & $-0,334900$ & \\
\hline $1 \backslash 1$ & 66 & 521 & 0,13341 & 0,182192 & $-0,31163$ & 0,015524 & $-0,093288$ & 0,122043 & $-0,370344$ & \\
\hline \multirow[t]{4}{*}{112} & 78 & 1958 & 0,501023 & 0,215068 & 0,845696 & 0,018325 & $-0,105737$ & 0,458333 & $-0,515868$ & \\
\hline & 364 & 3908 & & 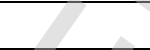 & 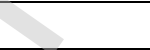 & & $-0,516758$ & & $-2,024673$ & \\
\hline & & & & & 2 & & 0,516758 & & 2,024673 & \\
\hline & & & & & & & 0,044031 & & 1,852159 & 1,896189 \\
\hline
\end{tabular}




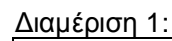

\begin{tabular}{|c|c|c|c|c|c|c|c|c|c|c|}
\hline CARIINC & BADS & GOODS & $f(x / 1)$ & $f(x / 0)$ & $w(x)$ & & & & 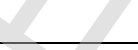 & \\
\hline $1 \backslash 1$ & 29 & 495 & 0,126759 & 0,080822 & 0,450038 & 0,006887 & $-0,049459$ & 0,115958 & $-0,360435$ & \\
\hline $2 \backslash 1$ & 57 & 220 & 0,056408 & 0,157534 & $-1,02703$ & 0,013423 & $-0,083479$ & 0,051602 & $-0,220672$ & \\
\hline 112 & 66 & 415 & 0,106293 & 0,182192 & $-0,53886$ & 0,015524 & $-0,093288$ & 0,097236 & $-0,326944$ & \\
\hline 212 & 91 & 229 & 0,058711 & 0,250685 & $-1,45158$ & 0,02136 & $-0,118525$ & 0,053708 & $-0,226579$ & \\
\hline 113 & 68 & 1552 & 0,39716 & 0,187671 & 0,749649 & 0,015991 & $-0,095411$ & 0,36332 & $-0,530697$ & \\
\hline 213 & 47 & 468 & 0,119852 & 0,130137 & $-0,08233$ & 0,011088 & $-0,072017$ & 0,10964 & $-0,349658$ & \\
\hline $1 \backslash 4$ & 1 & 449 & 0,114991 & 0,00411 & 3,331531 & 0,00035 & $-0,004020$ & 0,105193 & $-0,341761$ & \\
\hline \multirow[t]{4}{*}{$2 \backslash 4$} & 5 & 80 & 0,020594 & 0,015068 & 0,31237 & 0,001284 & $-0,012332$ & 0,018839 & $-0,107949$ & \\
\hline & 364 & 3908 & & & & & $-0,528531$ & & $-2,464696$ & \\
\hline & & & & & & 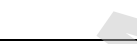 & 0,528531 & & 2,464696 & \\
\hline & & & & & & & 0,045034 & & 2,254689 & 2,299723 \\
\hline
\end{tabular}

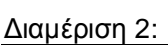

\begin{tabular}{|l|r|r|l|l|l|l|l|l|l|l|}
\hline \multicolumn{1}{|c|}{ CARINC } & \multicolumn{1}{l|}{ BADS } & GOODS & $\mathrm{f}(\mathrm{x} / 1)$ & $\mathrm{f}(\mathrm{x} / 0)$ & $\mathrm{w}(\mathrm{x})$ & & & & \\
\hline 010 & 88 & 233 & 0,059734 & 0,242466 & $-1,40096$ & 0,02066 & $-0,115632$ & 0,054644 & $-0,229166$ & \\
\hline 011 & 59 & 211 & 0,054106 & 0,163014 & $-1,10289$ & 0,01389 & $-0,085697$ & 0,049496 & $-0,214641$ & \\
\hline 012 & 44 & 494 & 0,126503 & 0,121918 & 0,036918 & 0,010388 & $-0,068447$ & 0,115724 & $-0,360045$ & \\
\hline 013 & 3 & 80 & 0,020594 & 0,009589 & 0,764355 & 0,000817 & $-0,008381$ & 0,018839 & $-0,107949$ & \\
\hline 110 & 73 & 406 & 0,103991 & 0,20137 & $-0,66084$ & 0,017158 & $-0,100631$ & 0,09513 & $-0,322867$ & \\
\hline 111 & 33 & 499 & 0,127782 & 0,091781 & 0,330923 & 0,00782 & $-0,054731$ & 0,116894 & $-0,361989$ & \\
\hline 112 & 63 & 1535 & 0,392811 & 0,173973 & 0,814432 & 0,014824 & $-0,090067$ & 0,359342 & $-0,530594$ & \\
\hline 113 & 1 & 450 & 0,115247 & 0,00411 & 3,333753 & 0,00035 & $-0,004020$ & 0,105427 & $-0,342183$ & \\
\hline & 364 & 3908 & & & & & $-0,527606$ & & $-2,469435$ & \\
\hline & & & & & & & 0,527606 & & 2,469435 & \\
\hline & & & & & & & 0,044955 & & 2,259024 & 2,30398 \\
\hline
\end{tabular}




\begin{tabular}{|c|c|c|c|c|c|c|c|c|c|c|}
\hline CARINC & BADS & GOODS & $f(x / 1)$ & $f(x / 0)$ & $w(x)$ & & & & 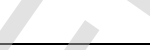 & 8 \\
\hline $0 \backslash 0$ & 100 & 225 & 0,057687 & 0,275342 & $-1,56298$ & 0,023461 & $-0,127008$ & 0,052772 & $-0,223969$ & \\
\hline $0 \backslash 1$ & 57 & 223 & 0,057176 & 0,157534 & $-1,01351$ & 0,013423 & $-0,083479$ & 0,052304 & $-0,222655$ & \\
\hline $0 \backslash 2$ & 43 & 470 & 0,120363 & 0,119178 & 0,009896 & 0,010155 & $-0,067241$ & 0,110108 & $-0,350474$ & \\
\hline $0 \backslash 3$ & 5 & 72 & 0,018547 & 0,015068 & 0,207699 & 0,001284 & $-0,012332$ & 0,016967 & $-0,099783$ & \\
\hline 110 & 66 & 411 & 0,10527 & 0,182192 & $-0,54853$ & 0,015524 & $-0,093288$ & 0,0963 & $-0,325140$ & \\
\hline 111 & 33 & 485 & 0,124201 & 0,091781 & 0,302494 & 0,00782 & $-0,054731$ & 0,113618 & $-0,356503$ & \\
\hline $1 \backslash 2$ & 59 & 1566 & 0,400742 & 0,163014 & 0,899483 & 0,01389 & $-0,085697$ & 0,366596 & $-0,530735$ & \\
\hline \multirow[t]{4}{*}{113} & 1 & 456 & 0,116782 & 0,00411 & 3,346984 & 0,00035 & $-0,004020$ & 0,106831 & $-0,344701$ & \\
\hline & 364 & 3908 & & & & & $-0,527797$ & & $-2,453960$ & \\
\hline & & & & & & & 0,527797 & 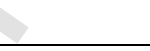 & 2,453960 & \\
\hline & & & & & & & 0,044971 & & 2,244868 & 2,28984 \\
\hline
\end{tabular}

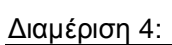

\begin{tabular}{|c|c|c|c|c|c|c|c|c|c|c|}
\hline CARINC & BADS & GOODS & $f(x / 1)$ & $f(x / 0)$ & $w(x)$ & & 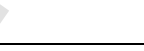 & & & \\
\hline 010 & 94 & 227 & 0,058199 & 0,258904 & $-1,49259$ & 0,02206 & $-0,121384$ & 0,05324 & $-0,225277$ & \\
\hline $0 \backslash 1$ & 59 & 229 & 0,058711 & 0,163014 & $-1,02121$ & 0,01389 & $-0,085697$ & 0,053708 & $-0,226579$ & \\
\hline 012 & 38 & 496 & 0,127015 & 0,105479 & 0,185786 & 0,008987 & $-0,061096$ & 0,116192 & $-0,360825$ & \\
\hline 013 & 5 & 83 & 0,021361 & 0,015068 & 0,348959 & 0,001284 & $-0,012332$ & 0,019541 & $-0,110941$ & \\
\hline 110 & 73 & 407 & 0,104247 & 0,20137 & $-0,65838$ & 0,017158 & $-0,100631$ & 0,095364 & $-0,323324$ & \\
\hline $1 \backslash 1$ & 30 & 483 & 0,123689 & 0,083562 & 0,392185 & 0,00712 & $-0,050793$ & 0,11315 & $-0,355708$ & \\
\hline 112 & 64 & 1538 & 0,393579 & 0,176712 & 0,800758 & 0,015057 & $-0,091146$ & 0,360044 & $-0,530617$ & \\
\hline \multirow[t]{4}{*}{113} & 1 & 445 & 0,113968 & 0,00411 & 3,322593 & 0,00035 & $-0,004020$ & 0,104257 & $-0,340064$ & \\
\hline & 364 & 3908 & & & 2 & & $-0,527100$ & & $-2,473335$ & \\
\hline & & & & 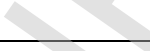 & & & 0,527100 & & 2,473335 & \\
\hline & & & & 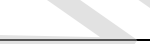 & & & 0,044912 & & 2,262592 & 2,307504 \\
\hline
\end{tabular}




\begin{tabular}{|c|c|c|c|c|c|c|c|c|c|c|}
\hline CARINC & BADS & GOODS & $f(x / 1)$ & $f(x / 0)$ & $w(x)$ & & & & 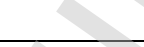 & 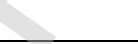 \\
\hline 010 & 96 & 224 & 0,057432 & 0,264384 & $-1,52681$ & 0,022527 & $-0,123273$ & 0,052538 & $-0,223313$ & \\
\hline $0 \backslash 1$ & 56 & 220 & 0,056408 & 0,154795 & $-1,00948$ & 0,013189 & $-0,082361$ & 0,051602 & $-0,220672$ & \\
\hline 012 & 43 & 470 & 0,120363 & 0,119178 & 0,009896 & 0,010155 & $-0,067241$ & 0,110108 & $-0,350474$ & \\
\hline 013 & 2 & 82 & 0,021105 & 0,006849 & 1,125368 & 0,000584 & $-0,006269$ & 0,019307 & $-0,109948$ & \\
\hline 110 & 70 & 412 & 0,105526 & 0,193151 & $-0,60452$ & 0,016458 & $-0,097513$ & 0,096534 & $-0,325592$ & \\
\hline 111 & 35 & 489 & 0,125224 & 0,09726 & 0,252712 & 0,008287 & $-0,057305$ & 0,114554 & $-0,358084$ & \\
\hline 112 & 62 & 1561 & 0,399463 & 0,171233 & 0,847096 & 0,01459 & $-0,088983$ & 0,365426 & $-0,530726$ & \\
\hline \multirow[t]{4}{*}{113} & 0 & 450 & 0,115247 & 0,00137 & 4,432366 & 0,000117 & $-0,001525$ & 0,105427 & $-0,342183$ & \\
\hline & 364 & 3908 & & & & & $-0,524470$ & & $-2,460992$ & \\
\hline & & & & & & & 0,524470 & 2 & 2,460992 & \\
\hline & & & & & & & 0,044688 & & 2,251301 & 2,295989 \\
\hline
\end{tabular}

16) $\quad H(C A R J O B)$

\begin{tabular}{|c|c|c|c|c|c|c|c|c|c|c|}
\hline CAR\JOB & BADS & GOODS & $f(x / 1)$ & $f(x / 0)$ & $w(x)$ & 8 & 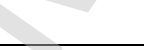 & & & \\
\hline $1 \backslash 1$ & 16 & 503 & 0,128805 & 0,045205 & 1,047084 & 0,003852 & $-0,030892$ & 0,11783 & $-0,363532$ & \\
\hline $2 \backslash 1$ & 42 & 171 & 0,043873 & 0,116438 & $-0,97606$ & 0,009921 & $-0,066028$ & 0,040135 & $-0,186186$ & \\
\hline 112 & 35 & 311 & 0,079688 & 0,09726 & $-0,19927$ & 0,008287 & $-0,057305$ & 0,072898 & $-0,275407$ & \\
\hline 212 & 70 & 137 & 0,035175 & 0,193151 & $-1,70313$ & 0,016458 & $-0,097513$ & 0,032178 & $-0,159532$ & \\
\hline 113 & 42 & 749 & 0,191737 & 0,116438 & 0,498763 & 0,009921 & $-0,066028$ & 0,1754 & $-0,440478$ & \\
\hline 213 & 28 & 155 & 0,03978 & 0,078082 & $-0,6744$ & 0,006653 & $-0,048113$ & 0,036391 & $-0,173957$ & \\
\hline $1 \backslash 4$ & 10 & 152 & 0,039013 & 0,028767 & 0,30465 & 0,002451 & $-0,021257$ & 0,035688 & $-0,171604$ & \\
\hline $2 \backslash 4$ & 8 & 93 & 0,023919 & 0,023288 & 0,026756 & 0,001984 & $-0,017813$ & 0,021881 & $-0,120656$ & \\
\hline 115 & 49 & 1008 & 0,257994 & 0,135616 & 0,643107 & 0,011555 & $-0,074362$ & 0,236012 & $-0,491629$ & \\
\hline 215 & 49 & 410 & 0,105014 & 0,135616 & $-0,25574$ & 0,011555 & $-0,074362$ & 0,096066 & $-0,324687$ & \\
\hline 116 & 12 & 188 & 0,048222 & 0,034247 & 0,34223 & 0,002918 & $-0,024572$ & 0,044113 & $-0,198626$ & \\
\hline \multirow[t]{4}{*}{216} & 3 & 31 & 0,008058 & 0,009589 & $-0,17391$ & 0,000817 & $-0,008381$ & 0,007372 & $-0,052220$ & \\
\hline & 364 & 3908 & & 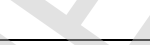 & & & $-0,586627$ & & $-2,958515$ & \\
\hline & & & & 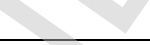 & & & 0,586627 & & 2,958515 & \\
\hline & & & & ${ }_{2}$ & & & 0,049984 & & 2,706431 & 2,756416 \\
\hline
\end{tabular}




\begin{tabular}{|c|c|c|c|c|c|c|c|c|c|c|}
\hline \multicolumn{11}{|c|}{ 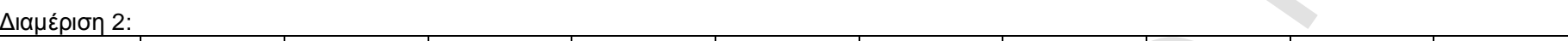 } \\
\hline CARJOB & BADS & GOODS & $f(x / 1)$ & $f(x / 0)$ & $w(x)$ & & & 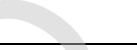 & & \\
\hline $0 \backslash 0$ & 65 & 156 & 0,040036 & 0,179452 & $-1,50013$ & 0,01529 & $-0,092220$ & 0,036625 & $-0,174737$ & \\
\hline $0 \backslash 2$ & 46 & 170 & 0,043617 & 0,127397 & $-1,07186$ & 0,010855 & $-0,070834$ & 0,039901 & $-0,185437$ & \\
\hline $0 \backslash 3$ & 26 & 153 & 0,039268 & 0,072603 & $-0,61458$ & 0,006186 & $-0,045386$ & 0,035922 & $-0,172391$ & \\
\hline $0 \backslash 4$ & 5 & 93 & 0,023919 & 0,015068 & 0,462074 & 0,001284 & $-0,012332$ & 0,021881 & $-0,120656$ & \\
\hline $0 \backslash 5$ & 46 & 413 & 0,105782 & 0,127397 & $-0,18593$ & 0,010855 & $-0,070834$ & 0,096768 & $-0,326044$ & \\
\hline $0 \backslash 6$ & 6 & 33 & 0,00857 & 0,017808 & $-0,7314$ & 0,001517 & $-0,014209$ & 0,00784 & $-0,054839$ & \\
\hline $1 \backslash 0$ & 35 & 312 & 0,079944 & 0,09726 & $-0,19607$ & 0,008287 & $-0,057305$ & 0,073132 & $-0,275953$ & \\
\hline 112 & 17 & 507 & 0,129829 & 0,047945 & 0,996156 & 0,004085 & $-0,032418$ & 0,118766 & $-0,365064$ & \\
\hline $1 \backslash 3$ & 43 & 717 & 0,183551 & 0,119178 & 0,431872 & 0,010155 & $-0,067241$ & 0,167911 & $-0,432242$ & \\
\hline $1 \backslash 4$ & 12 & 161 & 0,041315 & 0,034247 & 0,187637 & 0,002918 & $-0,024572$ & 0,037795 & $-0,178605$ & \\
\hline 115 & 51 & 1013 & 0,259273 & 0,141096 & 0,608444 & 0,012022 & $-0,076680$ & 0,237182 & $-0,492374$ & \\
\hline \multirow[t]{4}{*}{116} & 12 & 180 & 0,046175 & 0,034247 & 0,298863 & 0,002918 & $-0,024572$ & 0,042241 & $-0,192839$ & \\
\hline & 364 & 3908 & & & & $P$ & $-0,588604$ & & $-2,971181$ & \\
\hline & & & & & & 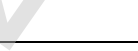 & 0,588604 & & 2,971181 & \\
\hline & & & & & 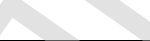 & & 0,050153 & & 2,718019 & 2,768171 \\
\hline
\end{tabular}




\begin{tabular}{|c|c|c|c|c|c|c|c|c|c|c|}
\hline CARIOB & RARS & אחת & $f(x / 1)$ & $f(x / 0)$ & (v) & & & & & \\
\hline $0 \backslash 0$ & 68 & 136 & 0,034919 & 0,187671 & -1.68165 & 0.015991 & $-0,095411$ & 0,031944 & -0.158708 & \\
\hline $0 \backslash 2$ & 52 & 154 & 0,039524 & 0,143836 & $-1,29176$ & 0,012256 & $-0,077828$ & 0.036156 & -0.173175 & \\
\hline 013 & 31 & 163 & 0,041827 & 0,086301 & $-0,72431$ & 0,007353 & $-0,052116$ & 0,038263 & $-0,180138$ & \\
\hline $0 \backslash 4$ & 8 & 102 & 0,026222 & 0,023288 & 0,118657 & 0,001984 & $-0,017813$ & 0,023987 & $-0,129090$ & \\
\hline $0 \backslash 5$ & 42 & 399 & 0,1022 & 0,116438 & $-0,13043$ & 0,009921 & $-0,066028$ & 0,093492 & $-0,319650$ & \\
\hline 016 & 4 & 36 & 0,009337 & 0,012329 & $-0,2779$ & 0,00105 & $-0,010394$ & 0,008542 & $-0,058693$ & \\
\hline 110 & 32 & 311 & 0,079688 & 0,089041 & $-0,11098$ & 0,007587 & $-0,053429$ & 0,072898 & $-0,275407$ & \\
\hline 112 & 15 & 500 & 0,128038 & 0,042466 & 1,103628 & 0,003618 & $-0,029346$ & 0,117128 & $-0,362376$ & \\
\hline 113 & 51 & 816 & 0,208877 & 0,141096 & 0,392306 & 0,012022 & $-0,076680$ & 0,191079 & $-0,456251$ & \\
\hline $1 \backslash 4$ & 12 & 154 & 0,039524 & 0,034247 & 0,143326 & 0,002918 & $-0,024572$ & 0,036156 & $-0,173175$ & \\
\hline 115 & 38 & 952 & 0,243668 & 0,105479 & 0,837292 & 0,008987 & $-0,061096$ & 0,222906 & $-0,482702$ & \\
\hline 116 & 11 & 185 & 0,047455 & 0,031507 & 0,409568 & 0,002685 & $-0,022929$ & 0,043411 & $-0,196470$ & \\
\hline & 364 & 3908 & & & & 2 & $-0,587642$ & & $-2,965834$ & \\
\hline & & & & & & 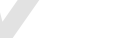 & 0,587642 & & 2,965834 & \\
\hline & & & & & & & 0,050071 & & 2,713127 & 2,763198 \\
\hline
\end{tabular}




\begin{tabular}{|c|c|c|c|c|c|c|c|c|c|c|}
\hline 0 & & & & & & & & & & \\
\hline CARJOB & BADS & GOODS & $f(x / 1)$ & $f(x / 0)$ & $w(x)$ & & & 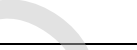 & & \\
\hline $0 \backslash 0$ & 67 & 144 & 0,036966 & 0,184932 & $-1,60999$ & 0,015757 & $-0,094352$ & 0,033816 & $-0,165231$ & \\
\hline $0 \backslash 2$ & 47 & 196 & 0,050269 & 0,130137 & $-0,95121$ & 0,011088 & $-0,072017$ & 0,045985 & $-0,204299$ & \\
\hline $0 \backslash 3$ & 30 & 152 & 0,039013 & 0,083562 & $-0,7617$ & 0,00712 & $-0,050793$ & 0,035688 & $-0,171604$ & \\
\hline $0 \backslash 4$ & 7 & 101 & 0,025966 & 0,020548 & 0,234016 & 0,001751 & $-0,016033$ & 0,023753 & $-0,128166$ & \\
\hline $0 \backslash 5$ & 39 & 408 & 0,104502 & 0,108219 & $-0,03495$ & 0,009221 & $-0,062341$ & 0,095598 & $-0,323779$ & \\
\hline $0 \backslash 6$ & 6 & 34 & 0,008826 & 0,017808 & $-0,70198$ & 0,001517 & $-0,014209$ & 0,008074 & $-0,056133$ & \\
\hline $1 \backslash 0$ & 30 & 321 & 0,082246 & 0,083562 & $-0,01587$ & 0,00712 & $-0,050793$ & 0,075238 & $-0,280818$ & \\
\hline 112 & 17 & 568 & 0,145434 & 0,047945 & 1,109661 & 0,004085 & $-0,032418$ & 0,133042 & $-0,387158$ & \\
\hline $1 \backslash 3$ & 53 & 726 & 0,185853 & 0,146575 & 0,237417 & 0,012489 & $-0,078971$ & 0,170017 & $-0,434606$ & \\
\hline $1 \backslash 4$ & 11 & 152 & 0,039013 & 0,031507 & 0,213678 & 0,002685 & $-0,022929$ & 0,035688 & $-0,171604$ & \\
\hline 115 & 45 & 919 & 0,235226 & 0,124658 & 0,634978 & 0,010622 & $-0,069644$ & 0,215184 & $-0,476924$ & \\
\hline 116 & 12 & 187 & 0,047966 & 0,034247 & 0,336911 & 0,002918 & $-0,024572$ & 0,043879 & $-0,197909$ & \\
\hline & 364 & 3908 & & & & $P$ & $-0,589073$ & & $-2,998233$ & \\
\hline & & & & & & 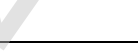 & 0,589073 & & 2,998233 & \\
\hline & & & & & 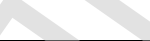 & & 0,050193 & & 2,742765 & 2,792958 \\
\hline
\end{tabular}




\begin{tabular}{|c|c|c|c|c|c|c|c|c|c|c|}
\hline CARJOB & BADS & GOODS & $f(x / 1)$ & $f(x / 0)$ & $w(x)$ & & & & 8 & \\
\hline $0 \backslash 0$ & 62 & 143 & 0,03671 & 0,171233 & $-1,53997$ & 0,01459 & $-0,088983$ & 0,033582 & $-0,164424$ & \\
\hline $0 \backslash 2$ & 46 & 173 & 0,044385 & 0,127397 & $-1,05441$ & 0,010855 & $-0,070834$ & 0,040603 & $-0,187678$ & \\
\hline 013 & 30 & 146 & 0,037478 & 0,083562 & $-0,80184$ & 0,00712 & $-0,050793$ & 0,034284 & $-0,166838$ & \\
\hline $0 \backslash 4$ & 8 & 91 & 0,023408 & 0,023288 & 0,005133 & 0,001984 & $-0,017813$ & 0,021413 & $-0,118743$ & \\
\hline $0 \backslash 5$ & 46 & 409 & 0,104758 & 0,127397 & $-0,19565$ & 0,010855 & $-0,070834$ & 0,095832 & $-0,324233$ & \\
\hline $0 \backslash 6$ & 5 & 34 & 0,008826 & 0,015068 & $-0,53493$ & 0,001284 & $-0,012332$ & 0,008074 & $-0,056133$ & \\
\hline 110 & 32 & 321 & 0,082246 & 0,089041 & $-0,07938$ & 0,007587 & $-0,053429$ & 0,075238 & $-0,280818$ & \\
\hline $1 \backslash 2$ & 17 & 511 & 0,130852 & 0,047945 & 1,004007 & 0,004085 & $-0,032418$ & 0,119703 & $-0,366586$ & \\
\hline 113 & 53 & 728 & 0,186365 & 0,146575 & 0,240166 & 0,012489 & $-0,078971$ & 0,170485 & $-0,435126$ & \\
\hline $1 \backslash 4$ & 11 & 149 & 0,038245 & 0,031507 & 0,19381 & 0,002685 & $-0,022929$ & 0,034986 & $-0,169231$ & \\
\hline $1 \backslash 5$ & 41 & 1011 & 0,258762 & 0,113699 & 0,822357 & 0,009688 & $-0,064808$ & 0,236714 & $-0,492077$ & \\
\hline \multirow[t]{4}{*}{116} & 13 & 192 & 0,049245 & 0,036986 & 0,286267 & 0,003151 & $-0,026188$ & 0,045049 & $-0,201476$ & \\
\hline & 364 & 3908 & & & & & $-0,590332$ & & $-2,963365$ & \\
\hline & & & & & & $\Delta$ & 0,590332 & & 2,963365 & \\
\hline & & & & & & & 0,0503 & & 2,710869 & 2,761168 \\
\hline
\end{tabular}

17) $\quad H(C A R J Y R)$

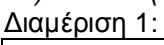

\begin{tabular}{|c|c|c|c|c|c|c|c|c|c|c|}
\hline CAR\JYR & BADS & GOODS & $f(x / 1)$ & $f(x / 0)$ & $w(x)$ & - & & & & \\
\hline $1 \backslash 1$ & 28 & 489 & 0,125224 & 0,078082 & 0,472341 & 0,006653 & $-0,048113$ & 0,114554 & $-0,358084$ & \\
\hline $2 \backslash 1$ & 28 & 162 & 0,041571 & 0,078082 & $-0,63037$ & 0,006653 & $-0,048113$ & 0,038029 & $-0,179372$ & \\
\hline 112 & 40 & 554 & 0,141852 & 0,110959 & 0,245625 & 0,009454 & $-0,063579$ & 0,129765 & $-0,382292$ & \\
\hline 212 & 81 & 248 & 0,063571 & 0,223288 & $-1,2563$ & 0,019025 & $-0,108748$ & 0,058155 & $-0,238664$ & \\
\hline 113 & 49 & 726 & 0,185853 & 0,135616 & 0,315126 & 0,011555 & $-0,074362$ & 0,170017 & $-0,434606$ & \\
\hline 213 & 67 & 282 & 0,072269 & 0,184932 & $-0,93959$ & 0,015757 & $-0,094352$ & 0,066111 & $-0,259088$ & \\
\hline $1 \backslash 4$ & 47 & 1142 & 0,292274 & 0,130137 & 0,809105 & 0,011088 & $-0,072017$ & 0,267371 & $-0,508830$ & \\
\hline \multirow[t]{4}{*}{214} & 24 & 305 & 0,078153 & 0,067123 & 0,152137 & 0,005719 & $-0,042609$ & 0,071494 & $-0,272108$ & \\
\hline & 364 & 3908 & & 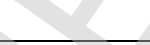 & & & $-0,551893$ & & $-2,633045$ & \\
\hline & & & & & & & 0,551893 & & 2,633045 & \\
\hline & & & & $x_{2}$ & & & 0,047025 & & 2,408694 & 2,455718 \\
\hline
\end{tabular}




\begin{tabular}{|c|c|c|c|c|c|c|c|c|c|c|}
\hline CARJYR & BADS & GOODS & $f(x / 1)$ & $f(x / 0)$ & $w(x)$ & & & & 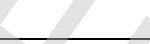 & \\
\hline $0 \backslash 0$ & 78 & 252 & 0,064595 & 0,215068 & $-1,20283$ & 0,018325 & $-0,105737$ & 0,059091 & $-0,241145$ & \\
\hline $0 \backslash 1$ & 66 & 314 & 0,080455 & 0,182192 & $-0,81736$ & 0,015524 & $-0,093288$ & 0,0736 & $-0,277042$ & \\
\hline $0 \backslash 2$ & 24 & 140 & 0,035943 & 0,067123 & $-0,62461$ & 0,005719 & $-0,042609$ & 0,03288 & $-0,161989$ & \\
\hline 013 & 26 & 312 & 0,079944 & 0,072603 & 0,09632 & 0,006186 & $-0,045386$ & 0,073132 & $-0,275953$ & \\
\hline $1 \backslash 0$ & 40 & 546 & 0,139806 & 0,110959 & 0,231093 & 0,009454 & $-0,063579$ & 0,127893 & $-0,379458$ & \\
\hline $1 \backslash 1$ & 57 & 820 & 0,2099 & 0,157534 & 0,286989 & 0,013423 & $-0,083479$ & 0,192015 & $-0,457132$ & \\
\hline 112 & 22 & 418 & 0,107061 & 0,061644 & 0,552022 & 0,005252 & $-0,039776$ & 0,097938 & $-0,328288$ & \\
\hline \multirow[t]{4}{*}{113} & 51 & 1106 & 0,283065 & 0,141096 & 0,696236 & 0,012022 & $-0,076680$ & 0,258946 & $-0,504757$ & \\
\hline & 364 & 3908 & & & & & $-0,550533$ & & $-2,625763$ & \\
\hline & & & & & & & 0,550533 & & 2,625763 & \\
\hline & & & & & & & 0,046909 & & 2,402032 & 2,448941 \\
\hline
\end{tabular}

$\Delta$ IIa ÉpıII 3:
\begin{tabular}{|l|r|r|r|r|r|r|r|r|r|r|}
\hline CARJYR & BADS & GOODS & $\mathrm{f}(\mathrm{x} / 1)$ & $\mathrm{f}(\mathrm{x} / 0)$ & $\mathrm{w}(\mathrm{x})$ & & & & & \\
\hline 010 & 88 & 245 & 0,062804 & 0,242466 & $-1,35085$ & 0,02066 & $-0,115632$ & 0,057453 & $-0,236790$ & \\
\hline 011 & 64 & 297 & 0,076106 & 0,176712 & $-0,84239$ & 0,015057 & $-0,091146$ & 0,069622 & $-0,267648$ & \\
\hline 012 & 24 & 135 & 0,034664 & 0,067123 & $-0,66084$ & 0,005719 & $-0,042609$ & 0,03171 & $-0,157882$ & \\
\hline 013 & 29 & 313 & 0,0802 & 0,080822 & $-0,00773$ & 0,006887 & $-0,049459$ & 0,073366 & $-0,276498$ & \\
\hline 110 & 40 & 569 & 0,145689 & 0,110959 & 0,272317 & 0,009454 & $-0,063579$ & 0,133276 & $-0,387501$ & \\
\hline 111 & 44 & 817 & 0,209133 & 0,121918 & 0,539622 & 0,010388 & $-0,068447$ & 0,191313 & $-0,456472$ & \\
\hline 112 & 23 & 415 & 0,106293 & 0,064384 & 0,501343 & 0,005486 & $-0,041199$ & 0,097236 & $-0,326944$ & \\
\hline 113 & 52 & 1117 & 0,285879 & 0,143836 & 0,686897 & 0,012256 & $-0,077828$ & 0,26152 & $-0,506043$ & \\
\hline & 364 & 3908 & & & & & $-0,549899$ & & $-2,615777$ & \\
\hline & & & & & & & 0,549899 & & 2,615777 & \\
\hline & & & & & & & 0,046855 & & 2,392897 & 2,439752 \\
\hline
\end{tabular}




\begin{tabular}{|c|c|c|c|c|c|c|c|c|c|c|}
\hline \multicolumn{11}{|c|}{ 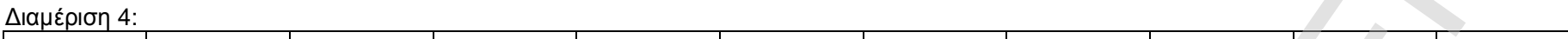 } \\
\hline CARJYR & BADS & GOODS & $f(x / 1)$ & $f(x / 0)$ & $w(x)$ & & & & 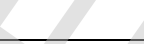 & \\
\hline $0 \backslash 0$ & 55 & 151 & 0,038757 & 0,152055 & $-1,36694$ & 0,012956 & $-0,081237$ & 0,035454 & $-0,170815$ & \\
\hline $0 \backslash 1$ & 85 & 407 & 0,104247 & 0,234247 & $-0,80961$ & 0,019959 & $-0,112706$ & 0,095364 & $-0,323324$ & \\
\hline 012 & 30 & 160 & 0,041059 & 0,083562 & $-0,71057$ & 0,00712 & $-0,050793$ & 0,037561 & $-0,177836$ & \\
\hline 013 & 26 & 317 & 0,081223 & 0,072603 & 0,112194 & 0,006186 & $-0,045386$ & 0,074302 & $-0,278667$ & \\
\hline 110 & 27 & 360 & 0,092223 & 0,075342 & 0,202166 & 0,00642 & $-0,046756$ & 0,084365 & $-0,300948$ & \\
\hline $1 \backslash 1$ & 56 & 951 & 0,243413 & 0,154795 & 0,45266 & 0,013189 & $-0,082361$ & 0,222672 & $-0,482532$ & \\
\hline 112 & 29 & 484 & 0,123945 & 0,080822 & 0,427588 & 0,006887 & $-0,049459$ & 0,113384 & $-0,356106$ & \\
\hline \multirow[t]{4}{*}{$1 \backslash 3$} & 56 & 1078 & 0,275902 & 0,154795 & 0,577946 & 0,013189 & $-0,082361$ & 0,252393 & $-0,501317$ & \\
\hline & 364 & 3908 & & & & & $-0,551059$ & & $-2,591545$ & \\
\hline & & & & & & & 0,551059 & & 2,591545 & \\
\hline & & & & & & & 0,046954 & & 2,37073 & 2,417684 \\
\hline
\end{tabular}

\begin{tabular}{|c|c|c|c|c|c|c|c|c|c|c|}
\hline CARJYR & BADS & GOODS & $f(x / 1)$ & $f(x / 0)$ & $w(x)$ & 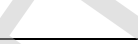 & & & & \\
\hline 010 & 85 & 237 & 0,060757 & 0,234247 & $-1,34949$ & 0,019959 & $-0,112706$ & 0,05558 & $-0,231730$ & \\
\hline $0 \backslash 1$ & 61 & 284 & 0,072781 & 0,168493 & $-0,83944$ & 0,014357 & $-0,087893$ & 0,066579 & $-0,260244$ & \\
\hline 012 & 24 & 154 & 0,039524 & 0,067123 & $-0,52962$ & 0,005719 & $-0,042609$ & 0,036156 & $-0,173175$ & \\
\hline 013 & 27 & 321 & 0,082246 & 0,075342 & 0,087672 & 0,00642 & $-0,046756$ & 0,075238 & $-0,280818$ & \\
\hline 110 & 39 & 565 & 0,144666 & 0,108219 & 0,29027 & 0,009221 & $-0,062341$ & 0,13234 & $-0,386125$ & \\
\hline $1 \backslash 1$ & 47 & 730 & 0,186876 & 0,130137 & 0,36186 & 0,011088 & $-0,072017$ & 0,170953 & $-0,435645$ & \\
\hline 112 & 31 & 488 & 0,124968 & 0,086301 & 0,370212 & 0,007353 & $-0,052116$ & 0,11432 & $-0,357690$ & \\
\hline \multirow[t]{4}{*}{113} & 50 & 1129 & 0,288949 & 0,138356 & 0,736417 & 0,011789 & $-0,075524$ & 0,264328 & $-0,507404$ & \\
\hline & 364 & 3908 & & 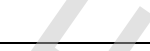 & 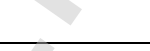 & & $-0,551963$ & & $-2,632832$ & \\
\hline & & & & + & 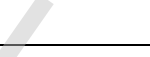 & & 0,551963 & & 2,632832 & \\
\hline & & & & & & & 0,047031 & & 2,408499 & 2,45553 \\
\hline
\end{tabular}




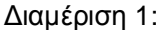

\begin{tabular}{|c|c|c|c|c|c|c|c|c|c|c|}
\hline CARIMAR & BADS & GOODS & $f(x / 1)$ & $f(x / 0)$ & $w(x)$ & & & 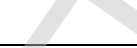 & $\nabla$ & \\
\hline $1 \backslash 1$ & 67 & 637 & 0,163085 & 0,184932 & $-0,12571$ & 0,015757 & $-0,094352$ & 0,149189 & $-0,409492$ & \\
\hline $2 \backslash 1$ & 99 & 328 & 0,084037 & 0,272603 & $-1,17676$ & 0,023227 & $-0,126079$ & 0,076876 & $-0,284544$ & \\
\hline 112 & 53 & 116 & 0,029803 & 0,146575 & $-1,59293$ & 0,012489 & $-0,078971$ & 0,027264 & $-0,141686$ & \\
\hline $2 \backslash 2$ & 72 & 66 & 0,017012 & 0,19863 & $-2,45752$ & 0,016924 & $-0,099596$ & 0,015562 & $-0,093465$ & \\
\hline 113 & 44 & 2158 & 0,552187 & 0,121918 & 1,51054 & 0,010388 & $-0,068447$ & 0,505138 & $-0,497688$ & \\
\hline \multirow[t]{4}{*}{213} & 29 & 603 & 0,154387 & 0,080822 & 0,647216 & 0,006887 & $-0,049459$ & 0,141233 & $-0,398820$ & \\
\hline & 364 & 3908 & & & & & $-0,516904$ & & $-1,825695$ & \\
\hline & & & & & & & 0,516904 & & 1,825695 & \\
\hline & & & & & & & 0,044043 & & 1,670135 & 1,714178 \\
\hline
\end{tabular}

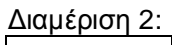

\begin{tabular}{|l|r|r|l|l|l|l|l|l|l|l|}
\hline CARMAR & \multicolumn{1}{l|}{ BADS } & GOODS & $f(x / 1)$ & $f(x / 0)$ & $w(x)$ & & & & & \\
\hline 010 & 69 & 63 & 0,016245 & 0,190411 & $-2,46143$ & 0,016224 & $-0,096464$ & 0,01486 & $-0,090238$ & \\
\hline 011 & 98 & 328 & 0,084037 & 0,269863 & $-1,16666$ & 0,022994 & $-0,125147$ & 0,076876 & $-0,284544$ & \\
\hline 012 & 27 & 627 & 0,160527 & 0,075342 & 0,756418 & 0,00642 & $-0,046756$ & 0,146849 & $-0,406419$ & \\
\hline 110 & 58 & 129 & 0,033129 & 0,160274 & $-1,57649$ & 0,013656 & $-0,084591$ & 0,030306 & $-0,152871$ & \\
\hline $1 \backslash 1$ & 69 & 656 & 0,167946 & 0,190411 & $-0,12554$ & 0,016224 & $-0,096464$ & 0,153636 & $-0,415187$ & \\
\hline 112 & 43 & 2105 & 0,538629 & 0,119178 & 1,508408 & 0,010155 & $-0,067241$ & 0,492734 & $-0,503140$ & \\
\hline & 364 & 3908 & & & & & $-0,516664$ & & $-1,852399$ & \\
\hline & & & & & & & 0,516664 & & 1,852399 & \\
\hline & & & & & & & 0,044023 & & 1,694563 & 1,738586 \\
\hline
\end{tabular}




\begin{tabular}{|c|c|c|c|c|c|c|c|c|c|c|}
\hline CARMAR & BADS & GOODS & $f(x / 1)$ & $f(x / 0)$ & $w(x)$ & & & 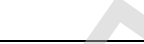 & + & \\
\hline $0 \backslash 0$ & 74 & 61 & 0,015733 & 0,20411 & $-2,5629$ & 0,017391 & $-0,101661$ & 0,014392 & $-0,088061$ & \\
\hline $0 \backslash 1$ & 105 & 328 & 0,084037 & 0,289041 & $-1,23531$ & 0,024628 & $-0,131601$ & 0,076876 & $-0,284544$ & \\
\hline $0 \backslash 2$ & 26 & 601 & 0,153876 & 0,072603 & 0,751142 & 0,006186 & $-0,045386$ & 0,140765 & $-0,398173$ & \\
\hline 110 & 44 & 113 & 0,029036 & 0,121918 & $-1,43483$ & 0,010388 & $-0,068447$ & 0,026562 & $-0,139037$ & \\
\hline $1 \backslash 1$ & 71 & 667 & 0,17076 & 0,19589 & $-0,1373$ & 0,016691 & $-0,098557$ & 0,15621 & $-0,418399$ & \\
\hline \multirow[t]{4}{*}{112} & 44 & 2138 & 0,547071 & 0,121918 & 1,501231 & 0,010388 & $-0,068447$ & 0,500457 & $-0,499797$ & \\
\hline & 364 & 3908 & & & & & $-0,514099$ & & $-1,828011$ & \\
\hline & & & & & & & 0,514099 & 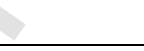 & 1,828011 & \\
\hline & & & & & & & 0,043804 & & 1,672253 & 1,716058 \\
\hline
\end{tabular}

\begin{tabular}{|c|c|c|c|c|c|c|c|c|c|c|}
\hline CARMAR & BADS & GOODS & $f(x / 1)$ & $f(x / 0)$ & $w(x)$ & 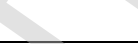 & & & & \\
\hline 010 & 68 & 70 & 0,018035 & 0,187671 & $-2,34236$ & 0,015991 & $-0,095411$ & 0,016499 & $-0,097697$ & \\
\hline $0 \backslash 1$ & 103 & 343 & 0,087874 & 0,283562 & $-1,17152$ & 0,024161 & $-0,129774$ & 0,080387 & $-0,292358$ & \\
\hline 012 & 25 & 622 & 0,159248 & 0,069863 & 0,823926 & 0,005953 & $-0,044004$ & 0,145679 & $-0,404862$ & \\
\hline 110 & 51 & 114 & 0,029291 & 0,141096 & $-1,57215$ & 0,012022 & $-0,076680$ & 0,026796 & $-0,139923$ & \\
\hline $1 \backslash 1$ & 72 & 640 & 0,163853 & 0,19863 & $-0,19248$ & 0,016924 & $-0,099596$ & 0,149891 & $-0,410404$ & \\
\hline \multirow[t]{4}{*}{112} & 45 & 2119 & 0,54221 & 0,124658 & 1,470084 & 0,010622 & $-0,069644$ & 0,496011 & $-0,501743$ & \\
\hline & 364 & 3908 & & $\Delta$ & & & $-0,515108$ & & $-1,846987$ & \\
\hline & & & & 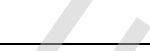 & $\nabla$ & & 0,515108 & & 1,846987 & \\
\hline & & & & & 2 & & 0,04389 & & 1,689612 & 1,733503 \\
\hline
\end{tabular}




\begin{tabular}{|c|c|c|c|c|c|c|c|c|c|c|}
\hline CARMAR & BADS & GOODS & $f(x / 1)$ & $f(x / 0)$ & $w(x)$ & & & 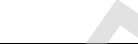 & $y$ & \\
\hline 010 & 69 & 60 & 0,015477 & 0,190411 & $-2,50982$ & 0,016224 & $-0,096464$ & 0,014158 & $-0,086964$ & \\
\hline $0 \backslash 1$ & 103 & 313 & 0,0802 & 0,283562 & $-1,26291$ & 0,024161 & $-0,129774$ & 0,073366 & $-0,276498$ & \\
\hline 012 & 25 & 623 & 0,159504 & 0,069863 & 0,825531 & 0,005953 & $-0,044004$ & 0,145913 & $-0,405174$ & \\
\hline 110 & 54 & 112 & 0,02878 & 0,149315 & $-1,64639$ & 0,012723 & $-0,080107$ & 0,026328 & $-0,138148$ & \\
\hline $1 \backslash 1$ & 69 & 648 & 0,165899 & 0,190411 & $-0,1378$ & 0,016224 & $-0,096464$ & 0,151764 & $-0,412813$ & \\
\hline \multirow[t]{4}{*}{112} & 44 & 2152 & 0,550652 & 0,121918 & 1,507757 & 0,010388 & $-0,068447$ & 0,503733 & $-0,498327$ & \\
\hline & 364 & 3908 & & & & & $-0,515260$ & & $-1,817923$ & \\
\hline & & & & & & & 0,515260 & 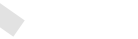 & 1,817923 & \\
\hline & & & & & & & 0,043903 & & 1,663025 & 1,706928 \\
\hline
\end{tabular}

\section{9) $\quad H(C A R$ TRM}

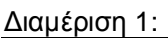

\begin{tabular}{|c|c|c|c|c|c|c|c|c|c|c|}
\hline CARITRM & BADS & GOODS & $f(x / 1)$ & $f(x / 0)$ & $w(x)$ & & & & & \\
\hline $1 \backslash 1$ & 38 & 295 & 0,075595 & 0,105479 & $-0,33313$ & 0,008987 & $-0,061096$ & 0,069154 & $-0,266522$ & \\
\hline $2 \backslash 1$ & 17 & 108 & 0,027756 & 0,047945 & $-0,54659$ & 0,004085 & $-0,032418$ & 0,025391 & $-0,134562$ & \\
\hline 112 & 3 & 691 & 0,176899 & 0,009589 & 2,914961 & 0,000817 & $-0,008381$ & 0,161827 & $-0,425196$ & \\
\hline 212 & 34 & 282 & 0,072269 & 0,094521 & $-0,26842$ & 0,008054 & $-0,056023$ & 0,066111 & $-0,259088$ & \\
\hline 113 & 123 & 1925 & 0,492581 & 0,338356 & 0,37556 & 0,02883 & $-0,147502$ & 0,45061 & $-0,518223$ & \\
\hline \multirow[t]{4}{*}{213} & 149 & 607 & 0,155411 & 0,409589 & $-0,96908$ & 0,034899 & $-0,168936$ & 0,142169 & $-0,400109$ & \\
\hline & 364 & 3908 & & $\Delta$ & & & $-0,474355$ & & $-2,003700$ & \\
\hline & & & & 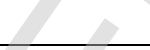 & $\nabla$ & & 0,474355 & & 2,003700 & \\
\hline & & & & 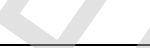 & & & 0,040418 & & 1,832973 & 1,87339 \\
\hline
\end{tabular}




\begin{tabular}{|c|c|c|c|c|c|c|c|c|c|c|}
\hline CARTRM & BADS & GOODS & $f(x / 1)$ & $f(x / 0)$ & $w(x)$ & & & 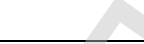 & + & \\
\hline $0 \backslash 0$ & 27 & 298 & 0,076362 & 0,075342 & 0,013444 & 0,00642 & $-0,046756$ & 0,069856 & $-0,268209$ & \\
\hline $0 \backslash 1$ & 151 & 618 & 0,158225 & 0,415068 & $-0,96443$ & 0,035366 & $-0,170518$ & 0,144743 & $-0,403606$ & \\
\hline $0 \backslash 2$ & 16 & 102 & 0,026222 & 0,045205 & $-0,54464$ & 0,003852 & $-0,030892$ & 0,023987 & $-0,129090$ & \\
\hline $1 \backslash 0$ & 4 & 663 & 0,169737 & 0,012329 & 2,622312 & 0,00105 & $-0,010394$ & 0,155274 & $-0,417239$ & \\
\hline $1 \backslash 1$ & 135 & 1945 & 0,497698 & 0,371233 & 0,293163 & 0,031631 & $-0,157603$ & 0,455291 & $-0,516819$ & \\
\hline \multirow[t]{4}{*}{112} & 31 & 282 & 0,072269 & 0,086301 & $-0,17745$ & 0,007353 & $-0,052116$ & 0,066111 & $-0,259088$ & \\
\hline & 364 & 3908 & & & & & $-0,468280$ & & $-1,994050$ & \\
\hline & & & & & & & 0,468280 & 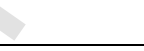 & 1,994050 & \\
\hline & & & & & & & 0,0399 & & 1,824145 & 1,864046 \\
\hline
\end{tabular}

\begin{tabular}{|c|c|c|c|c|c|c|c|c|c|c|}
\hline CARTRM & BADS & GOODS & $f(x / 1)$ & $f(x / 0)$ & $w(x)$ & 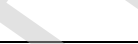 & & & & \\
\hline 010 & 27 & 260 & 0,066641 & 0,075342 & $-0,12272$ & 0,00642 & $-0,046756$ & 0,060963 & $-0,246042$ & \\
\hline $0 \backslash 1$ & 159 & 618 & 0,158225 & 0,436986 & $-1,01589$ & 0,037234 & $-0,176758$ & 0,144743 & $-0,403606$ & \\
\hline 012 & 19 & 112 & 0,02878 & 0,053425 & $-0,6186$ & 0,004552 & $-0,035412$ & 0,026328 & $-0,138148$ & \\
\hline 110 & 1 & 668 & 0,171016 & 0,00411 & 3,728432 & 0,00035 & $-0,004020$ & 0,156444 & $-0,418688$ & \\
\hline $1 \backslash 1$ & 125 & 1986 & 0,508186 & 0,343836 & 0,390684 & 0,029297 & $-0,149212$ & 0,464886 & $-0,513723$ & \\
\hline \multirow[t]{4}{*}{112} & 33 & 264 & 0,067664 & 0,091781 & $-0,30484$ & 0,00782 & $-0,054731$ & 0,061899 & $-0,248459$ & \\
\hline & 364 & 3908 & & $\Delta$ & & & $-0,466889$ & & $-1,968666$ & \\
\hline & & & & 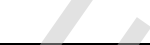 & $\nabla$ & & 0,466889 & & 1,968666 & \\
\hline & & & & & 2 & & 0,039782 & & 1,800924 & 1,840705 \\
\hline
\end{tabular}




\begin{tabular}{|c|c|c|c|c|c|c|c|c|c|c|}
\hline CARTRM & BADS & GOODS & $f(x / 1)$ & $f(x / 0)$ & $w(x)$ & & & 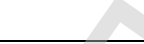 & + & \\
\hline $0 \backslash 0$ & 28 & 277 & 0,07099 & 0,078082 & $-0,09522$ & 0,006653 & $-0,048113$ & 0,064941 & $-0,256175$ & \\
\hline $0 \backslash 1$ & 155 & 653 & 0,167178 & 0,426027 & $-0,93544$ & 0,0363 & $-0,173655$ & 0,152934 & $-0,414301$ & \\
\hline $0 \backslash 2$ & 13 & 105 & 0,026989 & 0,036986 & $-0,31512$ & 0,003151 & $-0,026188$ & 0,024689 & $-0,131840$ & \\
\hline 110 & 3 & 625 & 0,160015 & 0,009589 & 2,814649 & 0,000817 & $-0,008381$ & 0,146381 & $-0,405797$ & \\
\hline $1 \backslash 1$ & 129 & 1989 & 0,508954 & 0,354795 & 0,360818 & 0,030231 & $-0,152600$ & 0,465588 & $-0,513485$ & \\
\hline \multirow[t]{4}{*}{112} & 36 & 259 & 0,066385 & 0,1 & $-0,4097$ & 0,008521 & $-0,058578$ & 0,060729 & $-0,245434$ & \\
\hline & 364 & 3908 & & & & & $-0,467514$ & & $-1,967033$ & \\
\hline & & & & & & & 0,467514 & 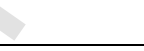 & 1,967033 & \\
\hline & & & & & & & 0,039835 & & 1,79943 & 1,839265 \\
\hline
\end{tabular}

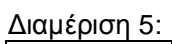

\begin{tabular}{|c|c|c|c|c|c|c|c|c|c|c|}
\hline CARTRM & BADS & GOODS & $f(x / 1)$ & $f(x / 0)$ & $w(x)$ & 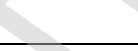 & & & & \\
\hline $0 \backslash 0$ & 28 & 253 & 0,06485 & 0,078082 & $-0,18568$ & 0,006653 & $-0,048113$ & 0,059325 & $-0,241761$ & \\
\hline $0 \backslash 1$ & 150 & 647 & 0,165643 & 0,412329 & $-0,91198$ & 0,035133 & $-0,169728$ & 0,15153 & $-0,412513$ & \\
\hline 012 & 19 & 96 & 0,024687 & 0,053425 & $-0,77201$ & 0,004552 & $-0,035412$ & 0,022583 & $-0,123499$ & \\
\hline $1 \backslash 0$ & 2 & 612 & 0,15669 & 0,006849 & 3,130119 & 0,000584 & $-0,006269$ & 0,143339 & $-0,401707$ & \\
\hline $1 \backslash 1$ & 127 & 2019 & 0,516628 & 0,349315 & 0,391349 & 0,029764 & $-0,150911$ & 0,472608 & $-0,511023$ & \\
\hline \multirow[t]{4}{*}{112} & 38 & 281 & 0,072013 & 0,105479 & $-0,38167$ & 0,008987 & $-0,061096$ & 0,065877 & $-0,258508$ & \\
\hline & 364 & 3908 & & 2 & & & $-0,471530$ & & $-1,949011$ & \\
\hline & & & & 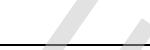 & 8 & & 0,471530 & & 1,949011 & \\
\hline & & & & +2 & 8 & & 0,040177 & & 1,782944 & 1,823121 \\
\hline
\end{tabular}




\begin{tabular}{|c|c|c|c|c|c|c|c|c|c|c|}
\hline CRD $\backslash K I D$ & BADS & GOODS & $f(x / 1)$ & $f(x / 0)$ & $w(x)$ & & & P & + & \\
\hline $0 \backslash 1$ & 36 & 321 & 0,082246 & 0,1 & $-0,19545$ & 0,008521 & $-0,058578$ & 0,075238 & $-0,280818$ & \\
\hline $1 \backslash 1$ & 16 & 154 & 0,039524 & 0,045205 & $-0,13431$ & 0,003852 & $-0,030892$ & 0,036156 & $-0,173175$ & \\
\hline 012 & 169 & 855 & 0,218854 & 0,464384 & $-0,75231$ & 0,039568 & $-0,184369$ & 0,200206 & $-0,464567$ & \\
\hline 112 & 29 & 566 & 0,144922 & 0,080822 & 0,583947 & 0,006887 & $-0,049459$ & 0,132574 & $-0,386470$ & \\
\hline 013 & 15 & 391 & 0,100153 & 0,042466 & 0,858006 & 0,003618 & $-0,029346$ & 0,09162 & $-0,315923$ & \\
\hline 113 & 1 & 231 & 0,059222 & 0,00411 & 2,667975 & 0,00035 & $-0,004020$ & 0,054176 & $-0,227876$ & \\
\hline $0 \backslash 4$ & 89 & 942 & 0,24111 & 0,245205 & $-0,01684$ & 0,020893 & $-0,116600$ & 0,220566 & $-0,480992$ & \\
\hline \multirow[t]{4}{*}{$1 \backslash 4$} & 9 & 448 & 0,114735 & 0,026027 & 1,483477 & 0,002218 & $-0,019553$ & 0,104959 & $-0,341338$ & \\
\hline & 364 & 3908 & & & & & $-0,492817$ & & $-2,671159$ & \\
\hline & & & & & & & 0,492817 & & 2,671159 & \\
\hline & & & & & & & 0,041991 & & 2,443561 & 2,485552 \\
\hline
\end{tabular}

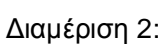

\begin{tabular}{|l|r|r|l|l|l|l|l|l|l|l|}
\hline CRDKID & \multicolumn{1}{|c|}{ BADS } & GOODS & $\mathrm{f}(\mathrm{x} / 1)$ & $\mathrm{f}(\mathrm{x} / 0)$ & $\mathrm{w}(\mathrm{x})$ & & & & \\
\hline 010 & 166 & 891 & 0,228063 & 0,456164 & $-0,69323$ & 0,038868 & $-0,182107$ & 0,208631 & $-0,471709$ & \\
\hline 011 & 15 & 417 & 0,106805 & 0,042466 & 0,922305 & 0,003618 & $-0,029346$ & 0,097704 & $-0,327841$ & \\
\hline 012 & 86 & 906 & 0,231901 & 0,236986 & $-0,02169$ & 0,020193 & $-0,113685$ & 0,212141 & $-0,474540$ & \\
\hline 013 & 34 & 323 & 0,082758 & 0,094521 & $-0,1329$ & 0,008054 & $-0,056023$ & 0,075706 & $-0,281888$ & \\
\hline 110 & 31 & 549 & 0,140573 & 0,086301 & 0,487882 & 0,007353 & $-0,052116$ & 0,128595 & $-0,380525$ & \\
\hline 111 & 3 & 239 & 0,061269 & 0,009589 & 1,854651 & 0,000817 & $-0,008381$ & 0,056048 & $-0,233003$ & \\
\hline 112 & 11 & 441 & 0,112944 & 0,031507 & 1,276691 & 0,002685 & $-0,022929$ & 0,103321 & $-0,338355$ & \\
\hline 113 & 18 & 142 & 0,036454 & 0,050685 & $-0,32957$ & 0,004319 & $-0,033924$ & 0,033348 & $-0,163615$ & \\
\hline & & & & & & & $-0,498511$ & & $-2,671476$ & \\
\hline & & & & & & & 0,498511 & & 2,671476 & \\
\hline & & & & & & & 0,042476 & & 2,44385 & 2,486326 \\
\hline
\end{tabular}




\begin{tabular}{|c|c|c|c|c|c|c|c|c|c|c|}
\hline CRDKID & BADS & GOODS & $f(x / 1)$ & $f(x / 0)$ & $w(x)$ & & & & 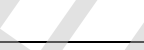 & \\
\hline $0 \backslash 0$ & 178 & 876 & 0,224226 & 0,489041 & $-0,77979$ & 0,041669 & $-0,191048$ & 0,205121 & $-0,468794$ & \\
\hline $0 \backslash 1$ & 15 & 403 & 0,103223 & 0,042466 & 0,888197 & 0,003618 & $-0,029346$ & 0,094428 & $-0,321494$ & \\
\hline $0 \backslash 2$ & 81 & 904 & 0,231389 & 0,223288 & 0,03564 & 0,019025 & $-0,108748$ & 0,211673 & $-0,474167$ & \\
\hline $0 \backslash 3$ & 35 & 336 & 0,086083 & 0,09726 & $-0,12207$ & 0,008287 & $-0,057305$ & 0,078749 & $-0,288740$ & \\
\hline 110 & 29 & 559 & 0,143131 & 0,080822 & 0,571514 & 0,006887 & $-0,049459$ & 0,130936 & $-0,384043$ & \\
\hline $1 \backslash 1$ & 3 & 234 & 0,05999 & 0,009589 & 1,833553 & 0,000817 & $-0,008381$ & 0,054878 & $-0,229809$ & \\
\hline 112 & 10 & 451 & 0,115503 & 0,028767 & 1,390061 & 0,002451 & $-0,021257$ & 0,105661 & $-0,342605$ & \\
\hline \multirow[t]{4}{*}{113} & 13 & 145 & 0,037222 & 0,036986 & 0,006347 & 0,003151 & $-0,026188$ & 0,03405 & $-0,166036$ & \\
\hline & 364 & 3908 & & & & & $-0,491732$ & & $-2,675687$ & \\
\hline & & & & & & & 0,491732 & & 2,675687 & \\
\hline & & & & & & & 0,041899 & & 2,447703 & 2,489601 \\
\hline
\end{tabular}

\begin{tabular}{|c|c|c|c|c|c|c|c|c|c|c|}
\hline CRDKID & BADS & GOODS & $f(x / 1)$ & $f(x / 0)$ & $w(x)$ & 1 & & & & \\
\hline 010 & 172 & 880 & 0,225249 & 0,472603 & $-0,74105$ & 0,040269 & $-0,186613$ & 0,206057 & $-0,469580$ & \\
\hline $0 \backslash 1$ & 14 & 392 & 0,100409 & 0,039726 & 0,927248 & 0,003385 & $-0,027779$ & 0,091854 & $-0,316392$ & \\
\hline 012 & 81 & 913 & 0,233691 & 0,223288 & 0,045541 & 0,019025 & $-0,108748$ & 0,21378 & $-0,475831$ & \\
\hline 013 & 38 & 324 & 0,083014 & 0,105479 & $-0,23951$ & 0,008987 & $-0,061096$ & 0,07594 & $-0,282421$ & \\
\hline 110 & 29 & 562 & 0,143899 & 0,080822 & 0,576861 & 0,006887 & $-0,049459$ & 0,131638 & $-0,385087$ & \\
\hline $1 \backslash 1$ & 3 & 231 & 0,059222 & 0,009589 & 1,820677 & 0,000817 & $-0,008381$ & 0,054176 & $-0,227876$ & \\
\hline 112 & 13 & 464 & 0,118828 & 0,036986 & 1,167132 & 0,003151 & $-0,026188$ & 0,108703 & $-0,348017$ & \\
\hline \multirow[t]{4}{*}{113} & 14 & 142 & 0,036454 & 0,039726 & $-0,08595$ & 0,003385 & $-0,027779$ & 0,033348 & $-0,163615$ & \\
\hline & 364 & 3908 & & 2 & $\nabla$ & & $-0,496041$ & & $-2,668819$ & \\
\hline & & & & +3 & 3 & & 0,496041 & & 2,668819 & \\
\hline & & & & & & & 0,042266 & & 2,44142 & 2,483686 \\
\hline
\end{tabular}




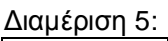

\begin{tabular}{|l|r|r|l|l|l|l|l|l|l|l|}
\hline CRDKID & \multicolumn{1}{|l|}{ BADS } & GOODS & $\mathrm{f}(\mathrm{x} / 1)$ & $\mathrm{f}(\mathrm{x} / 0)$ & $\mathrm{w}(\mathrm{x})$ & & & & \\
\hline 010 & 175 & 854 & 0,218598 & 0,480822 & $-0,78826$ & 0,040969 & $-0,188839$ & 0,199972 & $-0,464361$ & \\
\hline 011 & 13 & 413 & 0,105782 & 0,036986 & 1,050828 & 0,003151 & $-0,026188$ & 0,096768 & $-0,326044$ & \\
\hline 012 & 83 & 923 & 0,23625 & 0,228767 & 0,032185 & 0,019492 & $-0,110735$ & 0,21612 & $-0,477646$ & \\
\hline 013 & 41 & 332 & 0,08506 & 0,113699 & $-0,29019$ & 0,009688 & $-0,064808$ & 0,077812 & $-0,286650$ & \\
\hline 110 & 22 & 572 & 0,146457 & 0,061644 & 0,865358 & 0,005252 & $-0,039776$ & 0,133978 & $-0,388527$ & \\
\hline 111 & 2 & 229 & 0,058711 & 0,006849 & 2,148473 & 0,000584 & $-0,006269$ & 0,053708 & $-0,226579$ & \\
\hline 112 & 13 & 452 & 0,115759 & 0,036986 & 1,140959 & 0,003151 & $-0,026188$ & 0,105895 & $-0,343025$ & \\
\hline 113 & 15 & 133 & 0,034152 & 0,042466 & $-0,21788$ & 0,003618 & $-0,029346$ & 0,031242 & $-0,156222$ & \\
\hline & 364 & 3908 & & & & & $-0,492149$ & & $-2,669054$ & \\
\hline & & & & & & & 0,492149 & & 2,669054 & \\
\hline & & & & & & & 0,041934 & & 2,441634 & 2,483568 \\
\hline
\end{tabular}

21) H(CRD HYR)

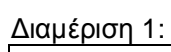

\begin{tabular}{|c|c|c|c|c|c|c|c|c|c|c|}
\hline CRDIHYR & BADS & GOODS & $f(x / 1)$ & $f(x / 0)$ & $w(x)$ & & & & & \\
\hline $0 \backslash 1$ & 70 & 787 & 0,201458 & 0,193151 & 0,042111 & 0,016458 & $-0,097513$ & 0,184293 & $-0,449661$ & \\
\hline $1 \backslash 1$ & 9 & 407 & 0,104247 & 0,026027 & 1,38761 & 0,002218 & $-0,019553$ & 0,095364 & $-0,323324$ & \\
\hline 012 & 167 & 492 & 0,125991 & 0,458904 & $-1,29263$ & 0,039101 & $-0,182863$ & 0,115256 & $-0,359263$ & \\
\hline 112 & 31 & 306 & 0,078409 & 0,086301 & $-0,09591$ & 0,007353 & $-0,052116$ & 0,071728 & $-0,272661$ & \\
\hline 013 & 66 & 1068 & 0,273344 & 0,182192 & 0,40567 & 0,015524 & $-0,093288$ & 0,250053 & $-0,500030$ & \\
\hline 113 & 15 & 599 & 0,153364 & 0,042466 & 1,284116 & 0,003618 & $-0,029346$ & 0,140296 & $-0,397523$ & \\
\hline $0 \backslash 4$ & 6 & 162 & 0,041571 & 0,017808 & 0,847736 & 0,001517 & $-0,014209$ & 0,038029 & $-0,179372$ & \\
\hline \multirow[t]{4}{*}{$1 \backslash 4$} & 0 & 87 & 0,022384 & 0,00137 & 2,793646 & 0,000117 & $-0,001525$ & 0,020477 & $-0,114873$ & \\
\hline & 364 & 3908 & & 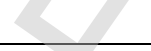 & 2 & & $-0,490414$ & & $-2,596706$ & \\
\hline & & & & & & & 0,490414 & & 2,596706 & \\
\hline & & & & & & & 0,041786 & & 2,375451 & 2,417238 \\
\hline
\end{tabular}




\begin{tabular}{|c|c|c|c|c|c|c|c|c|c|c|}
\hline \multicolumn{11}{|l|}{ 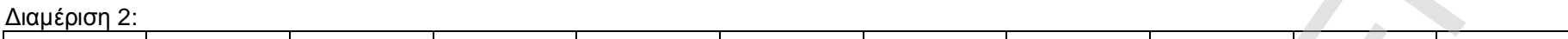 } \\
\hline CRDHYR & BADS & GOODS & $f(x / 1)$ & $f(x / 0)$ & $w(x)$ & & & & 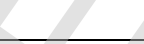 & \\
\hline $0 \backslash 0$ & 161 & 488 & 0,124968 & 0,442466 & $-1,26431$ & 0,037701 & $-0,178297$ & 0,11432 & $-0,357690$ & \\
\hline $0 \backslash 1$ & 70 & 1124 & 0,287669 & 0,193151 & 0,398342 & 0,016458 & $-0,097513$ & 0,263158 & $-0,506842$ & \\
\hline 012 & 65 & 770 & 0,197109 & 0,179452 & 0,09385 & 0,01529 & $-0,092220$ & 0,180314 & $-0,445631$ & \\
\hline 013 & 5 & 155 & 0,03978 & 0,015068 & 0,970758 & 0,001284 & $-0,012332$ & 0,036391 & $-0,173957$ & \\
\hline 110 & 34 & 303 & 0,077641 & 0,094521 & $-0,19672$ & 0,008054 & $-0,056023$ & 0,071026 & $-0,271000$ & \\
\hline $1 \backslash 1$ & 19 & 579 & 0,148248 & 0,053425 & 1,020612 & 0,004552 & $-0,035412$ & 0,135616 & $-0,390900$ & \\
\hline 112 & 10 & 402 & 0,102968 & 0,028767 & 1,27518 & 0,002451 & $-0,021257$ & 0,094194 & $-0,321034$ & \\
\hline \multirow[t]{4}{*}{$1 \backslash 3$} & 0 & 87 & 0,022384 & 0,00137 & 2,793646 & 0,000117 & $-0,001525$ & 0,020477 & $-0,114873$ & \\
\hline & 364 & 3908 & & & & & $-0,494579$ & & $-2,581928$ & \\
\hline & & & & & & & 0,494579 & & 2,581928 & \\
\hline & & & & & & & 0,042141 & & 2,361932 & 2,404073 \\
\hline
\end{tabular}

\begin{tabular}{|c|c|c|c|c|c|c|c|c|c|c|}
\hline CRDHYR & BADS & GOODS & $f(x / 1)$ & $f(x / 0)$ & $w(x)$ & 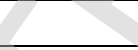 & & & & \\
\hline 010 & 169 & 497 & 0,12727 & 0,464384 & $-1,2944$ & 0,039568 & $-0,184369$ & 0,116426 & $-0,361214$ & \\
\hline $0 \backslash 1$ & 71 & 1111 & 0,284344 & 0,19589 & 0,372629 & 0,016691 & $-0,098557$ & 0,260116 & $-0,505346$ & \\
\hline 012 & 67 & 745 & 0,190714 & 0,184932 & 0,030788 & 0,015757 & $-0,094352$ & 0,174464 & $-0,439474$ & \\
\hline 013 & 2 & 166 & 0,042594 & 0,006849 & 1,827565 & 0,000584 & $-0,006269$ & 0,038965 & $-0,182421$ & \\
\hline 110 & 30 & 322 & 0,082502 & 0,083562 & $-0,01276$ & 0,00712 & $-0,050793$ & 0,075472 & $-0,281354$ & \\
\hline $1 \backslash 1$ & 15 & 588 & 0,15055 & 0,042466 & 1,265597 & 0,003618 & $-0,029346$ & 0,137722 & $-0,393909$ & \\
\hline 112 & 10 & 389 & 0,099642 & 0,028767 & 1,242349 & 0,002451 & $-0,021257$ & 0,091152 & $-0,314983$ & \\
\hline \multirow[t]{4}{*}{113} & 0 & 90 & 0,023152 & 0,00137 & 2,827358 & 0,000117 & $-0,001525$ & 0,021179 & $-0,117781$ & \\
\hline & 364 & 3908 & & 2 & 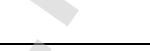 & & $-0,486469$ & & $-2,596482$ & \\
\hline & & & & + & 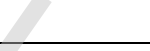 & & 0,486469 & & 2,596482 & \\
\hline & & & & & & & 0,04145 & & 2,375246 & 2,416696 \\
\hline
\end{tabular}




\begin{tabular}{|c|c|c|c|c|c|c|c|c|c|c|}
\hline CRDHYR & BADS & GOODS & $f(x / 1)$ & $f(x / 0)$ & $w(x)$ & & & & & \\
\hline 010 & 161 & 511 & 0,130852 & 0,442466 & $-1,2183$ & 0,037701 & $-0,178297$ & 0,119703 & $-0,366586$ & \\
\hline $0 \backslash 1$ & 68 & 1078 & 0,275902 & 0,187671 & 0,385353 & 0,015991 & $-0,095411$ & 0,252393 & $-0,501317$ & \\
\hline 012 & 71 & 760 & 0,194551 & 0,19589 & $-0,00686$ & 0,016691 & $-0,098557$ & 0,177974 & $-0,443202$ & \\
\hline 013 & 5 & 160 & 0,041059 & 0,015068 & 1,002406 & 0,001284 & $-0,012332$ & 0,037561 & $-0,177836$ & \\
\hline 110 & 30 & 308 & 0,07892 & 0,083562 & $-0,05714$ & 0,00712 & $-0,050793$ & 0,072196 & $-0,273763$ & \\
\hline $1 \backslash 1$ & 17 & 606 & 0,155155 & 0,047945 & 1,174364 & 0,004085 & $-0,032418$ & 0,141935 & $-0,399788$ & \\
\hline 112 & 12 & 399 & 0,1022 & 0,034247 & 1,093346 & 0,002918 & $-0,024572$ & 0,093492 & $-0,319650$ & \\
\hline \multirow[t]{4}{*}{113} & 0 & 86 & 0,022128 & 0,00137 & 2,782152 & 0,000117 & $-0,001525$ & 0,020243 & $-0,113896$ & \\
\hline & 364 & 3908 & & & & & $-0,493904$ & & $-2,596037$ & \\
\hline & & & & & & & 0,493904 & & 2,596037 & \\
\hline & & & & & & & 0,042084 & & 2,374839 & 2,416923 \\
\hline
\end{tabular}

\begin{tabular}{|c|c|c|c|c|c|c|c|c|c|c|}
\hline CRDHYR & BADS & GOODS & $f(x / 1)$ & $f(x / 0)$ & $w(x)$ & & & & & \\
\hline 010 & 174 & 496 & 0,127015 & 0,478082 & $-1,32548$ & 0,040735 & $-0,188099$ & 0,116192 & $-0,360825$ & \\
\hline $0 \backslash 1$ & 65 & 1069 & 0,273599 & 0,179452 & 0,421757 & 0,01529 & $-0,092220$ & 0,250287 & $-0,500160$ & \\
\hline 012 & 67 & 789 & 0,20197 & 0,184932 & 0,088133 & 0,015757 & $-0,094352$ & 0,184761 & $-0,450127$ & \\
\hline 013 & 6 & 168 & 0,043106 & 0,017808 & 0,883994 & 0,001517 & $-0,014209$ & 0,039433 & $-0,183933$ & \\
\hline 110 & 27 & 313 & 0,0802 & 0,075342 & 0,062474 & 0,00642 & $-0,046756$ & 0,073366 & $-0,276498$ & \\
\hline $1 \backslash 1$ & 16 & 571 & 0,146201 & 0,045205 & 1,173765 & 0,003852 & $-0,030892$ & 0,133744 & $-0,388186$ & \\
\hline 112 & 9 & 414 & 0,106037 & 0,026027 & 1,404642 & 0,002218 & $-0,019553$ & 0,097002 & $-0,326494$ & \\
\hline \multirow[t]{4}{*}{113} & 0 & 88 & 0,02264 & 0,00137 & 2,80501 & 0,000117 & $-0,001525$ & 0,020711 & $-0,115846$ & \\
\hline & 364 & 3908 & & & & & $-0,487606$ & & $-2,602068$ & \\
\hline & & & & & & & 0,487606 & & 2,602068 & \\
\hline & & & & & & & 0,041547 & & 2,380356 & 2,421903 \\
\hline
\end{tabular}




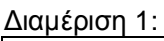

\begin{tabular}{|c|c|c|c|c|c|c|c|c|c|c|}
\hline CRD & BADS & GOODS & $f(x / 1)$ & $f(x / 0)$ & $w(x)$ & & & 2 & 8 & \\
\hline $0 \backslash 1$ & 59 & 372 & 0,095293 & 0,163014 & $-0,53688$ & 0,01389 & $-0,085697$ & 0,087173 & $-0,306848$ & \\
\hline $1 \backslash 1$ & 6 & 227 & 0,058199 & 0,017808 & 1,184209 & 0,001517 & $-0,014209$ & 0,05324 & $-0,225277$ & \\
\hline 012 & 138 & 457 & 0,117038 & 0,379452 & $-1,17623$ & 0,032332 & $-0,160071$ & 0,107065 & $-0,345118$ & \\
\hline 112 & 16 & 285 & 0,073037 & 0,045205 & 0,479742 & 0,003852 & $-0,030892$ & 0,066813 & $-0,260821$ & \\
\hline 013 & 112 & 1680 & 0,429905 & 0,308219 & 0,332754 & 0,026262 & $-0,137899$ & 0,393275 & $-0,529501$ & \\
\hline \multirow[t]{4}{*}{113} & 33 & 887 & 0,22704 & 0,091781 & 0,905724 & 0,00782 & $-0,054731$ & 0,207695 & $-0,470940$ & \\
\hline & 364 & 3908 & & & & & $-0,483499$ & & $-2,138506$ & \\
\hline & & & & & & & 0,483499 & & 2,138506 & \\
\hline & & & & & & & 0,041197 & & 1,956292 & 1,997489 \\
\hline
\end{tabular}

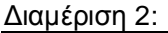

\begin{tabular}{|c|c|c|c|c|c|c|c|c|c|c|}
\hline CRDHOME & BADS & GOODS & $f(x / 1)$ & $f(x / 0)$ & $w(x)$ & 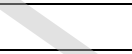 & & & & \\
\hline 010 & 60 & 389 & 0,099642 & 0,165753 & $-0,50892$ & 0,014123 & $-0,086798$ & 0,091152 & $-0,314983$ & \\
\hline $0 \backslash 1$ & 137 & 462 & 0,118317 & 0,376712 & $-1,15812$ & 0,032098 & $-0,159251$ & 0,108235 & $-0,347193$ & \\
\hline $0 \backslash 2$ & 104 & 1686 & 0,43144 & 0,286301 & 0,410084 & 0,024395 & $-0,130689$ & 0,394679 & $-0,529363$ & \\
\hline 110 & 5 & 222 & 0,05692 & 0,015068 & 1,329039 & 0,001284 & $-0,012332$ & 0,05207 & $-0,221995$ & \\
\hline $1 \backslash 1$ & 22 & 283 & 0,072525 & 0,061644 & 0,162557 & 0,005252 & $-0,039776$ & 0,066345 & $-0,259667$ & \\
\hline \multirow[t]{4}{*}{$1 \backslash 2$} & 36 & 866 & 0,221668 & 0,1 & 0,79601 & 0,008521 & $-0,058578$ & 0,202781 & $-0,466803$ & \\
\hline & 364 & 3908 & & 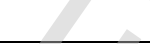 & 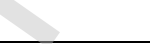 & & $-0,487423$ & & $-2,140003$ & \\
\hline & & & & 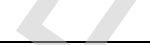 & 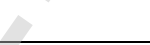 & & 0,487423 & & 2,140003 & \\
\hline & & & & & & & 0,041531 & & 1,957662 & 1,999193 \\
\hline
\end{tabular}




\begin{tabular}{|c|c|c|c|c|c|c|c|c|c|c|}
\hline CRDHOME & BADS & GOODS & $f(x / 1)$ & $f(x / 0)$ & $w(x)$ & & & 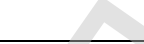 & + & \\
\hline 010 & 66 & 373 & 0,095549 & 0,182192 & $-0,64542$ & 0,015524 & $-0,093288$ & 0,087407 & $-0,307333$ & \\
\hline $0 \backslash 1$ & 142 & 452 & 0,115759 & 0,390411 & $-1,21569$ & 0,033265 & $-0,163327$ & 0,105895 & $-0,343025$ & \\
\hline 012 & 101 & 1694 & 0,433487 & 0,278082 & 0,443945 & 0,023694 & $-0,127933$ & 0,396551 & $-0,529166$ & \\
\hline $1 \backslash 0$ & 5 & 234 & 0,05999 & 0,015068 & 1,381568 & 0,001284 & $-0,012332$ & 0,054878 & $-0,229809$ & \\
\hline $1 \backslash 1$ & 20 & 280 & 0,071757 & 0,056164 & 0,245009 & 0,004786 & $-0,036883$ & 0,065643 & $-0,257926$ & \\
\hline \multirow[t]{4}{*}{112} & 30 & 875 & 0,22397 & 0,083562 & 0,985929 & 0,00712 & $-0,050793$ & 0,204887 & $-0,468597$ & \\
\hline & 364 & 3908 & & & & & $-0,484557$ & & $-2,135857$ & \\
\hline & & & & & & & 0,484557 & & 2,135857 & \\
\hline & & & & & & & 0,041287 & & 1,953869 & 1,995157 \\
\hline
\end{tabular}

\begin{tabular}{|c|c|c|c|c|c|c|c|c|c|c|}
\hline CRDHOME & BADS & GOODS & $f(x / 1)$ & $f(x / 0)$ & $w(x)$ & & & & & \\
\hline 010 & 60 & 391 & 0,100153 & 0,165753 & $-0,5038$ & 0,014123 & $-0,086798$ & 0,09162 & $-0,315923$ & \\
\hline $0 \backslash 1$ & 144 & 438 & 0,112177 & 0,39589 & $-1,26106$ & 0,033732 & $-0,164941$ & 0,102619 & $-0,337065$ & \\
\hline 012 & 101 & 1680 & 0,429905 & 0,278082 & 0,435648 & 0,023694 & $-0,127933$ & 0,393275 & $-0,529501$ & \\
\hline 110 & 6 & 220 & 0,056408 & 0,017808 & 1,152956 & 0,001517 & $-0,014209$ & 0,051602 & $-0,220672$ & \\
\hline $1 \backslash 1$ & 19 & 301 & 0,07713 & 0,053425 & 0,367216 & 0,004552 & $-0,035412$ & 0,070558 & $-0,269887$ & \\
\hline \multirow[t]{4}{*}{112} & 34 & 878 & 0,224738 & 0,094521 & 0,866117 & 0,008054 & $-0,056023$ & 0,205589 & $-0,469188$ & \\
\hline & 364 & 3908 & & 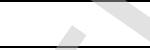 & & & $-0,485316$ & & $-2,142237$ & \\
\hline & & & & 2 & + & & 0,485316 & & 2,142237 & \\
\hline & & & & $2-\sqrt{2}$ & 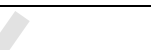 & & 0,041352 & & 1,959705 & 2,001057 \\
\hline
\end{tabular}




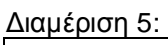

\begin{tabular}{|l|r|r|l|l|l|l|l|l|l|l|}
\hline CRDHOME & \multicolumn{1}{|l|}{ BADS } & GOODS & $\mathrm{f}(\mathrm{x} / 1)$ & $\mathrm{f}(\mathrm{x} / 0)$ & $\mathrm{w}(\mathrm{x})$ & & & & \\
\hline 010 & 63 & 371 & 0,095037 & 0,173973 & $-0,60463$ & 0,014824 & $-0,090067$ & 0,086939 & $-0,306361$ & \\
\hline 011 & 139 & 451 & 0,115503 & 0,382192 & $-1,19663$ & 0,032565 & $-0,160889$ & 0,105661 & $-0,342605$ & \\
\hline 012 & 110 & 1700 & 0,435022 & 0,30274 & 0,362523 & 0,025795 & $-0,136115$ & 0,397955 & $-0,529011$ & \\
\hline 110 & 6 & 229 & 0,058711 & 0,017808 & 1,192961 & 0,001517 & $-0,014209$ & 0,053708 & $-0,226579$ & \\
\hline 111 & 15 & 287 & 0,073548 & 0,042466 & 0,549243 & 0,003618 & $-0,029346$ & 0,067281 & $-0,261970$ & \\
\hline 112 & 31 & 870 & 0,222691 & 0,086301 & 0,947941 & 0,007353 & $-0,052116$ & 0,203717 & $-0,467604$ & \\
\hline & 364 & 3908 & & & & & $-0,482742$ & & $-2,134130$ & \\
\hline & & & & & & & 0,482742 & & 2,134130 & \\
\hline & & & & & & & 0,041133 & & 1,952289 & 1,993422 \\
\hline
\end{tabular}

23)

$H(C R D I N C)$

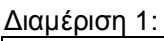

\begin{tabular}{|c|c|c|c|c|c|c|c|c|c|c|}
\hline CRDIINC & BADS & GOODS & $f(x / 1)$ & $f(x / 0)$ & $w(x)$ & & & & & \\
\hline $0 \backslash 1$ & 77 & 473 & 0,121131 & 0,212329 & $-0,56127$ & 0,018092 & $-0,104724$ & 0,11081 & $-0,351693$ & \\
\hline $1 \backslash 1$ & 9 & 242 & 0,062036 & 0,026027 & 0,86857 & 0,002218 & $-0,019553$ & 0,05675 & $-0,234903$ & \\
\hline 012 & 133 & 435 & 0,11141 & 0,365753 & $-1,18875$ & 0,031164 & $-0,155945$ & 0,101917 & $-0,335769$ & \\
\hline 112 & 24 & 209 & 0,053594 & 0,067123 & $-0,22509$ & 0,005719 & $-0,042609$ & 0,049028 & $-0,213283$ & \\
\hline 013 & 95 & 1289 & 0,32988 & 0,261644 & 0,231744 & 0,022294 & $-0,122330$ & 0,301772 & $-0,521604$ & \\
\hline 113 & 20 & 731 & 0,187132 & 0,056164 & 1,203533 & 0,004786 & $-0,036883$ & 0,171187 & $-0,435903$ & \\
\hline $0 \backslash 4$ & 4 & 312 & 0,079944 & 0,012329 & 1,869388 & 0,00105 & $-0,010394$ & 0,073132 & $-0,275953$ & \\
\hline \multirow[t]{4}{*}{$1 \backslash 4$} & 2 & 217 & 0,055641 & 0,006849 & 2,094769 & 0,000584 & $-0,006269$ & 0,0509 & $-0,218676$ & \\
\hline & 364 & 3908 & & & & & $-0,498707$ & & $-2,587783$ & \\
\hline & & & & & & & 0,498707 & & 2,587783 & \\
\hline & & & & & & & 0,042493 & & 2,367288 & 2,409781 \\
\hline
\end{tabular}




\begin{tabular}{|c|c|c|c|c|c|c|c|c|c|c|}
\hline CRDINC & BADS & GOODS & $f(x / 1)$ & $f(x / 0)$ & $w(x)$ & & & & 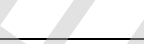 & \\
\hline $0 \backslash 0$ & 133 & 440 & 0,112689 & 0,365753 & $-1,17733$ & 0,031164 & $-0,155945$ & 0,103087 & $-0,337926$ & \\
\hline $0 \backslash 1$ & 80 & 462 & 0,118317 & 0,220548 & $-0,62275$ & 0,018792 & $-0,107748$ & 0,108235 & $-0,347193$ & \\
\hline $0 \backslash 2$ & 86 & 1332 & 0,34088 & 0,236986 & 0,363528 & 0,020193 & $-0,113685$ & 0,311835 & $-0,524240$ & \\
\hline 013 & 2 & 303 & 0,077641 & 0,006849 & 2,427951 & 0,000584 & $-0,006269$ & 0,071026 & $-0,271000$ & \\
\hline 110 & 28 & 199 & 0,051036 & 0,078082 & $-0,42523$ & 0,006653 & $-0,048113$ & 0,046687 & $-0,206397$ & \\
\hline $1 \backslash 1$ & 12 & 248 & 0,063571 & 0,034247 & 0,618575 & 0,002918 & $-0,024572$ & 0,058155 & $-0,238664$ & \\
\hline 112 & 21 & 693 & 0,177411 & 0,058904 & 1,102559 & 0,005019 & $-0,038337$ & 0,162295 & $-0,425750$ & \\
\hline \multirow[t]{4}{*}{113} & 2 & 227 & 0,058199 & 0,006849 & 2,13972 & 0,000584 & $-0,006269$ & 0,05324 & $-0,225277$ & \\
\hline & & & & & & & $-0,500940$ & & $-2,576446$ & \\
\hline & & & & & & & 0,500940 & & 2,576446 & \\
\hline & & & & & & & 0,042683 & & 2,356918 & 2,399601 \\
\hline
\end{tabular}

\begin{tabular}{|c|c|c|c|c|c|c|c|c|c|c|}
\hline CRDINC & BADS & GOODS & $f(x / 1)$ & $f(x / 0)$ & $w(x)$ & 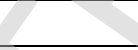 & & & & \\
\hline 010 & 148 & 440 & 0,112689 & 0,406849 & $-1,28381$ & 0,034666 & $-0,168142$ & 0,103087 & $-0,337926$ & \\
\hline $0 \backslash 1$ & 77 & 459 & 0,117549 & 0,212329 & $-0,59128$ & 0,018092 & $-0,104724$ & 0,107533 & $-0,345950$ & \\
\hline 012 & 80 & 1314 & 0,336275 & 0,220548 & 0,421815 & 0,018792 & $-0,107748$ & 0,307623 & $-0,523194$ & \\
\hline 013 & 4 & 306 & 0,078409 & 0,012329 & 1,850001 & 0,00105 & $-0,010394$ & 0,071728 & $-0,272661$ & \\
\hline 110 & 18 & 196 & 0,050269 & 0,050685 & $-0,00825$ & 0,004319 & $-0,033924$ & 0,045985 & $-0,204299$ & \\
\hline $1 \backslash 1$ & 13 & 249 & 0,063827 & 0,036986 & 0,54563 & 0,003151 & $-0,026188$ & 0,058389 & $-0,239286$ & \\
\hline 112 & 22 & 722 & 0,18483 & 0,061644 & 1,098063 & 0,005252 & $-0,039776$ & 0,169081 & $-0,433560$ & \\
\hline \multirow[t]{4}{*}{113} & 2 & 222 & 0,05692 & 0,006849 & 2,117497 & 0,000584 & $-0,006269$ & 0,05207 & $-0,221995$ & \\
\hline & 364 & 3908 & & 2 & $\nabla$ & & $-0,497165$ & & $-2,578872$ & \\
\hline & & & & +3 & 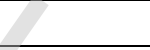 & & 0,497165 & & 2,578872 & \\
\hline & & & & & & & 0,042361 & & 2,359136 & 2,401498 \\
\hline
\end{tabular}




\begin{tabular}{|c|c|c|c|c|c|c|c|c|c|c|}
\hline CRDINC & BADS & GOODS & $f(x / 1)$ & $f(x / 0)$ & $w(x)$ & & & & $\sqrt{2}$ & \\
\hline $0 \backslash 0$ & 145 & 449 & 0,114991 & 0,39863 & $-1,24318$ & 0,033966 & $-0,165745$ & 0,105193 & $-0,341761$ & \\
\hline $0 \backslash 1$ & 76 & 463 & 0,118573 & 0,209589 & $-0,56962$ & 0,017858 & $-0,103708$ & 0,108469 & $-0,347605$ & \\
\hline 012 & 80 & 1300 & 0,332694 & 0,220548 & 0,411107 & 0,018792 & $-0,107748$ & 0,304346 & $-0,522323$ & \\
\hline 013 & 4 & 297 & 0,076106 & 0,012329 & 1,820197 & 0,00105 & $-0,010394$ & 0,069622 & $-0,267648$ & \\
\hline 110 & 22 & 185 & 0,047455 & 0,061644 & $-0,2616$ & 0,005252 & $-0,039776$ & 0,043411 & $-0,196470$ & \\
\hline $1 \backslash 1$ & 13 & 249 & 0,063827 & 0,036986 & 0,54563 & 0,003151 & $-0,026188$ & 0,058389 & $-0,239286$ & \\
\hline 112 & 22 & 734 & 0,1879 & 0,061644 & 1,114535 & 0,005252 & $-0,039776$ & 0,17189 & $-0,436676$ & \\
\hline \multirow[t]{4}{*}{113} & 2 & 231 & 0,059222 & 0,006849 & 2,15715 & 0,000584 & $-0,006269$ & 0,054176 & $-0,227876$ & \\
\hline & 364 & 3908 & & & & & $-0,499604$ & & $-2,579646$ & \\
\hline & & & & & & & 0,499604 & & 2,579646 & \\
\hline & & & & & & & 0,042569 & & 2,359845 & 2,402414 \\
\hline
\end{tabular}

\begin{tabular}{|c|c|c|c|c|c|c|c|c|c|c|}
\hline CRDINC & BADS & GOODS & $f(x / 1)$ & $f(x / 0)$ & $w(x)$ & 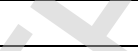 & & & & \\
\hline $0 \backslash 0$ & 146 & 438 & 0,112177 & 0,40137 & $-1,27481$ & 0,034199 & $-0,166546$ & 0,102619 & $-0,337065$ & \\
\hline $0 \backslash 1$ & 78 & 461 & 0,118061 & 0,215068 & $-0,59976$ & 0,018325 & $-0,105737$ & 0,108001 & $-0,346779$ & \\
\hline $0 \backslash 2$ & 86 & 1309 & 0,334996 & 0,236986 & 0,346117 & 0,020193 & $-0,113685$ & 0,306452 & $-0,522889$ & \\
\hline 013 & 2 & 314 & 0,080455 & 0,006849 & 2,463554 & 0,000584 & $-0,006269$ & 0,0736 & $-0,277042$ & \\
\hline $1 \backslash 0$ & 20 & 198 & 0,05078 & 0,056164 & $-0,10078$ & 0,004786 & $-0,036883$ & 0,046453 & $-0,205699$ & \\
\hline $1 \backslash 1$ & 13 & 248 & 0,063571 & 0,036986 & 0,541614 & 0,003151 & $-0,026188$ & 0,058155 & $-0,238664$ & \\
\hline 112 & 19 & 722 & 0,18483 & 0,053425 & 1,241163 & 0,004552 & $-0,035412$ & 0,169081 & $-0,433560$ & \\
\hline \multirow[t]{4}{*}{$1 \backslash 3$} & 0 & 218 & 0,055897 & 0,00137 & 3,708794 & 0,000117 & $-0,001525$ & 0,051134 & $-0,219343$ & \\
\hline & 364 & 3908 & & 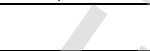 & 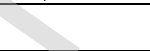 & & $-0,492245$ & & $-2,581042$ & \\
\hline & & & & & & & 0,492245 & & 2,581042 & \\
\hline & & & & & 3 & & 0,041942 & & 2,361121 & 2,403064 \\
\hline
\end{tabular}




\begin{tabular}{|c|c|c|c|c|c|c|c|c|c|c|}
\hline CRD \JOB & BADS & GOODS & $f(x / 1)$ & $f(x / 0)$ & $w(x)$ & & & & & \\
\hline $0 \backslash 1$ & 48 & 480 & 0,122921 & 0,132877 & $-0,07788$ & 0,011322 & $-0,073193$ & 0,112448 & $-0,354511$ & \\
\hline $1 \backslash 1$ & 10 & 194 & 0,049757 & 0,028767 & 0,547917 & 0,002451 & $-0,021257$ & 0,045517 & $-0,202891$ & \\
\hline 012 & 89 & 337 & 0,086339 & 0,245205 & $-1,04381$ & 0,020893 & $-0,116600$ & 0,078983 & $-0,289260$ & \\
\hline 112 & 16 & 111 & 0,028524 & 0,045205 & $-0,46048$ & 0,003852 & $-0,030892$ & 0,026094 & $-0,137256$ & \\
\hline 013 & 64 & 570 & 0,145945 & 0,176712 & $-0,19129$ & 0,015057 & $-0,091146$ & 0,13351 & $-0,387844$ & \\
\hline 113 & 6 & 334 & 0,085572 & 0,017808 & 1,569695 & 0,001517 & $-0,014209$ & 0,078281 & $-0,287697$ & \\
\hline $0 \backslash 4$ & 15 & 140 & 0,035943 & 0,042466 & $-0,16677$ & 0,003618 & $-0,029346$ & 0,03288 & $-0,161989$ & \\
\hline 114 & 3 & 105 & 0,026989 & 0,009589 & 1,034808 & 0,000817 & $-0,008381$ & 0,024689 & $-0,131840$ & \\
\hline 015 & 82 & 848 & 0,217063 & 0,226027 & $-0,04047$ & 0,019259 & $-0,109744$ & 0,198568 & $-0,463119$ & \\
\hline 115 & 16 & 570 & 0,145945 & 0,045205 & 1,172013 & 0,003852 & $-0,030892$ & 0,13351 & $-0,387844$ & \\
\hline 016 & 11 & 134 & 0,034408 & 0,031507 & 0,088078 & 0,002685 & $-0,022929$ & 0,031476 & $-0,157053$ & \\
\hline \multirow[t]{4}{*}{116} & 4 & 85 & 0,021873 & 0,012329 & 0,573299 & 0,00105 & $-0,010394$ & 0,020009 & $-0,112915$ & \\
\hline & 364 & 3908 & & & & & $-0,558984$ & & $-3,074218$ & \\
\hline & & & & & 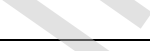 & & 0,558984 & & 3,074218 & \\
\hline & & & & & $\theta$ & & 0,047629 & & 2,812276 & 2,859905 \\
\hline
\end{tabular}




\begin{tabular}{|c|c|c|c|c|c|c|c|c|c|c|}
\hline CRDJOB & RADS & cons & $f(x / 1)$ & $f(x / 0)$ & 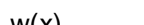 & & & & & \\
\hline 010 & 81 & 344 & 0,08813 & 0,223288 & -0.92965 & 0,019025 & -0.108748 & 0,080621 & -0292871 & \\
\hline 012 & 54 & 485 & 0.124201 & 0.149315 & -0.18416 & 0.012723 & -0.080107 & 0.113618 & -0.356503 & \\
\hline 013 & 59 & 549 & 0,140573 & 0,163014 & $-0,14811$ & 0,01389 & $-0,085697$ & 0,128595 & $-0,380525$ & \\
\hline $0 \backslash 4$ & 13 & 151 & 0,038757 & 0,036986 & 0,046756 & 0,003151 & $-0,026188$ & 0,035454 & $-0,170815$ & \\
\hline $0 \backslash 5$ & 81 & 873 & 0,223459 & 0,223288 & 0,000766 & 0,019025 & $-0,108748$ & 0,204419 & $-0,468201$ & \\
\hline 016 & 13 & 135 & 0,034664 & 0,036986 & $-0,06486$ & 0,003151 & $-0,026188$ & 0,03171 & $-0,157882$ & \\
\hline 110 & 19 & 124 & 0,03185 & 0,053425 & $-0,51725$ & 0,004552 & $-0,035412$ & 0,029136 & $-0,148624$ & \\
\hline 112 & 9 & 192 & 0,049245 & 0,026027 & 0,637665 & 0,002218 & $-0,019553$ & 0,045049 & $-0,201476$ & \\
\hline 113 & 10 & 321 & 0,082246 & 0,028767 & 1,050483 & 0,002451 & $-0,021257$ & 0,075238 & $-0,280818$ & \\
\hline 114 & 4 & 103 & 0,026477 & 0,012329 & 0,764355 & 0,00105 & $-0,010394$ & 0,024221 & $-0,130010$ & \\
\hline 115 & 16 & 553 & 0,141596 & 0,045205 & 1,141762 & 0,003852 & $-0,030892$ & 0,129531 & $-0,381940$ & \\
\hline 116 & 5 & 78 & 0,020082 & 0,015068 & 0,287211 & 0,001284 & $-0,012332$ & 0,018371 & $-0,105934$ & \\
\hline & 364 & 3908 & & & & 2 & $-0,565517$ & & $-3,075600$ & \\
\hline & & & & & 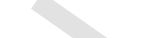 & 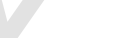 & 0,565517 & & 3,075600 & \\
\hline & & & & & N & & 0,048185 & & 2,81354 & 2,861726 \\
\hline
\end{tabular}




\begin{tabular}{|c|c|c|c|c|c|c|c|c|c|c|}
\hline CRDIOR & בחת & (1) & & & $x(x)+x$ & & & & & \\
\hline $0 \backslash 0$ & 82 & 324 & 0083014 & 0226027 & -100165 & 0019259 & -0109744 & 007594 & ח 282421 & \\
\hline 012 & 58 & 461 & 0,118061 & 0,160274 & $-0,30568$ & 0.013656 & -0.084591 & 0.108001 & -0.346779 & \\
\hline 013 & 73 & 615 & 0,157457 & 0,20137 & $-0,24599$ & 0,017158 & $-0,100631$ & 0,144041 & $-0,402659$ & \\
\hline $0 \backslash 4$ & 17 & 155 & 0,03978 & 0,047945 & $-0,18669$ & 0,004085 & $-0,032418$ & 0,036391 & $-0,173957$ & \\
\hline 015 & 69 & 823 & 0,210668 & 0,190411 & 0,101097 & 0,016224 & $-0,096464$ & 0,192718 & $-0,457789$ & \\
\hline 016 & 10 & 141 & 0,036199 & 0,028767 & 0,229785 & 0,002451 & $-0,021257$ & 0,033114 & $-0,162803$ & \\
\hline 110 & 18 & 123 & 0,031594 & 0,050685 & $-0,47267$ & 0,004319 & $-0,033924$ & 0,028902 & $-0,147766$ & \\
\hline 112 & 9 & 193 & 0,049501 & 0,026027 & 0,642846 & 0,002218 & $-0,019553$ & 0,045283 & $-0,202185$ & \\
\hline 113 & 9 & 364 & 0,093246 & 0,026027 & 1,276095 & 0,002218 & $-0,019553$ & 0,085301 & $-0,302929$ & \\
\hline 114 & 3 & 101 & 0,025966 & 0,009589 & 0,996156 & 0,000817 & $-0,008381$ & 0,023753 & $-0,128166$ & \\
\hline 115 & 11 & 528 & 0,135201 & 0,031507 & 1,456556 & 0,002685 & $-0,022929$ & 0,123681 & $-0,372936$ & \\
\hline 116 & 5 & 80 & 0,020594 & 0,015068 & 0,31237 & 0,001284 & $-0,012332$ & 0,018839 & $-0,107949$ & \\
\hline & 364 & 3908 & & & & 2 & $-0,561776$ & & $-3,088340$ & \\
\hline & & & & & ( & 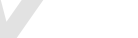 & 0,561776 & & 3,088340 & \\
\hline & & & & & N & & 0,047867 & & 2,825195 & 2,873061 \\
\hline
\end{tabular}




\begin{tabular}{|c|c|c|c|c|c|c|c|c|c|c|}
\hline 1 & & & & & & & & & & \\
\hline CRDJOB & BADS & GOODS & $f(x / 1)$ & $f(x / 0)$ & $w(x)$ & & & 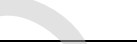 & & \\
\hline $0 \backslash 0$ & 77 & 347 & 0,088897 & 0,212329 & $-0,87065$ & 0,018092 & $-0,104724$ & 0,081323 & $-0,294405$ & \\
\hline $0 \backslash 2$ & 57 & 530 & 0,135712 & 0,157534 & $-0,1491$ & 0,013423 & $-0,083479$ & 0,124149 & $-0,373670$ & \\
\hline $0 \backslash 3$ & 72 & 559 & 0,143131 & 0,19863 & $-0,32768$ & 0,016924 & $-0,099596$ & 0,130936 & $-0,384043$ & \\
\hline $0 \backslash 4$ & 16 & 148 & 0,037989 & 0,045205 & $-0,17391$ & 0,003852 & $-0,030892$ & 0,034752 & $-0,168436$ & \\
\hline $0 \backslash 5$ & 71 & 788 & 0,201714 & 0,19589 & 0,029295 & 0,016691 & $-0,098557$ & 0,184527 & $-0,449894$ & \\
\hline $0 \backslash 6$ & 12 & 137 & 0,035175 & 0,034247 & 0,026756 & 0,002918 & $-0,024572$ & 0,032178 & $-0,159532$ & \\
\hline $1 \backslash 0$ & 20 & 118 & 0,030315 & 0,056164 & $-0,61665$ & 0,004786 & $-0,036883$ & 0,027732 & $-0,143437$ & \\
\hline 112 & 7 & 234 & 0,05999 & 0,020548 & 1,071413 & 0,001751 & $-0,016033$ & 0,054878 & $-0,229809$ & \\
\hline $1 \backslash 3$ & 11 & 319 & 0,081734 & 0,031507 & 0,953271 & 0,002685 & $-0,022929$ & 0,07477 & $-0,279745$ & \\
\hline $1 \backslash 4$ & 2 & 105 & 0,026989 & 0,006849 & 1,371281 & 0,000584 & $-0,006269$ & 0,024689 & $-0,131840$ & \\
\hline 115 & 13 & 539 & 0,138015 & 0,036986 & 1,316814 & 0,003151 & $-0,026188$ & 0,126255 & $-0,376946$ & \\
\hline 116 & 6 & 84 & 0,021617 & 0,017808 & 0,19381 & 0,001517 & $-0,014209$ & 0,019775 & $-0,111930$ & \\
\hline & 364 & 3908 & & & & $P$ & $-0,564332$ & & $-3,103687$ & \\
\hline & & & & & & 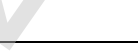 & 0,564332 & & 3,103687 & \\
\hline & & & & & 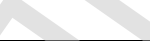 & & 0,048084 & & 2,839234 & 2,887319 \\
\hline
\end{tabular}




\begin{tabular}{|c|c|c|c|c|c|c|c|c|c|c|}
\hline CRDJOB & BADS & GOODS & $f(x / 1)$ & $f(x / 0)$ & $w(x)$ & & & & 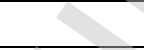 & ( \\
\hline 010 & 79 & 348 & 0,089153 & 0,217808 & $-0,89326$ & 0,018559 & $-0,106745$ & 0,081557 & $-0,294914$ & \\
\hline 012 & 54 & 474 & 0,121387 & 0,149315 & $-0,20708$ & 0,012723 & $-0,080107$ & 0,111044 & $-0,352097$ & \\
\hline 013 & 75 & 561 & 0,143643 & 0,206849 & $-0,36466$ & 0,017625 & $-0,102686$ & 0,131404 & $-0,384740$ & \\
\hline 014 & 15 & 146 & 0,037478 & 0,042466 & $-0,12495$ & 0,003618 & $-0,029346$ & 0,034284 & $-0,166838$ & \\
\hline 015 & 75 & 856 & 0,21911 & 0,206849 & 0,057582 & 0,017625 & $-0,102686$ & 0,20044 & $-0,464772$ & \\
\hline 016 & 14 & 137 & 0,035175 & 0,039726 & $-0,12166$ & 0,003385 & $-0,027779$ & 0,032178 & $-0,159532$ & \\
\hline 110 & 15 & 116 & 0,029803 & 0,042466 & $-0,35409$ & 0,003618 & $-0,029346$ & 0,027264 & $-0,141686$ & \\
\hline 112 & 9 & 210 & 0,05385 & 0,026027 & 0,727054 & 0,002218 & $-0,019553$ & 0,049262 & $-0,213963$ & \\
\hline 113 & 8 & 313 & 0,0802 & 0,023288 & 1,236594 & 0,001984 & $-0,017813$ & 0,073366 & $-0,276498$ & \\
\hline 114 & 4 & 94 & 0,024175 & 0,012329 & 0,673383 & 0,00105 & $-0,010394$ & 0,022115 & $-0,121607$ & \\
\hline 115 & 12 & 564 & 0,14441 & 0,034247 & 1,439072 & 0,002918 & $-0,024572$ & 0,132106 & $-0,385780$ & \\
\hline \multirow[t]{4}{*}{116} & 4 & 89 & 0,022896 & 0,012329 & 0,619022 & 0,00105 & $-0,010394$ & 0,020945 & $-0,116816$ & \\
\hline & 364 & 3908 & & & & & $-0,561422$ & & $-3,079242$ & \\
\hline & & & & & & $\Delta$ & 0,561422 & & 3,079242 & \\
\hline & & & & & & & 0,047837 & & 2,816872 & 2,864708 \\
\hline
\end{tabular}

25) $H(C R D J Y R)$

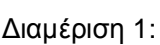

\begin{tabular}{|c|c|c|c|c|c|c|c|c|c|c|}
\hline CRDIJYR & BADS & GOODS & $f(x / 1)$ & $f(x / 0)$ & $w(x)$ & $>$ & & & & \\
\hline $0 \backslash 1$ & 47 & 406 & 0,103991 & 0,130137 & $-0,22429$ & 0,011088 & $-0,072017$ & 0,09513 & $-0,322867$ & \\
\hline $1 \backslash 1$ & 9 & 245 & 0,062804 & 0,026027 & 0,880866 & 0,002218 & $-0,019553$ & 0,057453 & $-0,236790$ & \\
\hline 012 & 106 & 520 & 0,133154 & 0,291781 & $-0,78449$ & 0,024861 & $-0,132510$ & 0,121809 & $-0,369971$ & \\
\hline 112 & 15 & 282 & 0,072269 & 0,042466 & 0,531699 & 0,003618 & $-0,029346$ & 0,066111 & $-0,259088$ & \\
\hline 013 & 97 & 621 & 0,158992 & 0,267123 & $-0,51886$ & 0,022761 & $-0,124211$ & 0,145445 & $-0,404549$ & \\
\hline 113 & 19 & 387 & 0,09913 & 0,053425 & 0,618162 & 0,004552 & $-0,035412$ & 0,090684 & $-0,314039$ & \\
\hline $0 \backslash 4$ & 59 & 962 & 0,246227 & 0,163014 & 0,412418 & 0,01389 & $-0,085697$ & 0,225247 & $-0,484375$ & \\
\hline \multirow[t]{4}{*}{$1 \backslash 4$} & 12 & 485 & 0,124201 & 0,034247 & 1,288311 & 0,002918 & $-0,024572$ & 0,113618 & $-0,356503$ & \\
\hline & 364 & 3908 & & 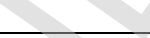 & & & $-0,523319$ & & $-2,748182$ & \\
\hline & & & & 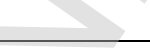 & & & 0,523319 & & 2,748182 & \\
\hline & & & & & & & 0,04459 & & 2,51402 & 2,55861 \\
\hline
\end{tabular}




\begin{tabular}{|c|c|c|c|c|c|c|c|c|c|c|}
\hline CRDJYR & BADS & GOODS & $f(x / 1)$ & $f(x / 0)$ & $w(x)$ & & & & 2 & \\
\hline $0 \backslash 0$ & 102 & 508 & 0,130084 & 0,280822 & $-0,76954$ & 0,023928 & $-0,128855$ & 0,119 & $-0,365446$ & \\
\hline $0 \backslash 1$ & 100 & 711 & 0,182016 & 0,275342 & $-0,41392$ & 0,023461 & $-0,127008$ & 0,166507 & $-0,430645$ & \\
\hline 012 & 36 & 355 & 0,090944 & 0,1 & $-0,09493$ & 0,008521 & $-0,058578$ & 0,083195 & $-0,298450$ & \\
\hline 013 & 63 & 963 & 0,246482 & 0,173973 & 0,348393 & 0,014824 & $-0,090067$ & 0,225481 & $-0,484541$ & \\
\hline $1 \backslash 0$ & 16 & 290 & 0,074316 & 0,045205 & 0,497104 & 0,003852 & $-0,030892$ & 0,067984 & $-0,263686$ & \\
\hline $1 \backslash 1$ & 23 & 423 & 0,10834 & 0,064384 & 0,520414 & 0,005486 & $-0,041199$ & 0,099109 & $-0,330512$ & \\
\hline 112 & 10 & 203 & 0,052059 & 0,028767 & 0,593151 & 0,002451 & $-0,021257$ & 0,047624 & $-0,209171$ & \\
\hline \multirow[t]{4}{*}{113} & 14 & 455 & 0,116526 & 0,039726 & 1,076108 & 0,003385 & $-0,027779$ & 0,106597 & $-0,344283$ & \\
\hline & 364 & 3908 & & & & & $-0,525635$ & & $-2,726734$ & \\
\hline & & & & & & & 0,525635 & & 2,726734 & \\
\hline & & & & & & & 0,044787 & & 2,4944 & 2,539187 \\
\hline
\end{tabular}

\begin{tabular}{|c|c|c|c|c|c|c|c|c|c|c|}
\hline CRDJYR & BADS & GOODS & $f(x / 1)$ & $f(x / 0)$ & $w(x)$ & 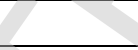 & & & & \\
\hline 010 & 113 & 516 & 0,132131 & 0,310959 & $-0,85587$ & 0,026496 & $-0,138787$ & 0,120873 & $-0,368473$ & \\
\hline $0 \backslash 1$ & 92 & 670 & 0,171527 & 0,253425 & $-0,39032$ & 0,021593 & $-0,119482$ & 0,156912 & $-0,419265$ & \\
\hline 012 & 38 & 358 & 0,091711 & 0,105479 & $-0,13987$ & 0,008987 & $-0,061096$ & 0,083897 & $-0,299952$ & \\
\hline 013 & 66 & 975 & 0,249552 & 0,182192 & 0,314609 & 0,015524 & $-0,093288$ & 0,228289 & $-0,486499$ & \\
\hline 110 & 15 & 298 & 0,076362 & 0,042466 & 0,58679 & 0,003618 & $-0,029346$ & 0,069856 & $-0,268209$ & \\
\hline $1 \backslash 1$ & 16 & 444 & 0,113712 & 0,045205 & 0,92245 & 0,003852 & $-0,030892$ & 0,104023 & $-0,339638$ & \\
\hline 112 & 9 & 192 & 0,049245 & 0,026027 & 0,637665 & 0,002218 & $-0,019553$ & 0,045049 & $-0,201476$ & \\
\hline \multirow[t]{4}{*}{113} & 15 & 455 & 0,116526 & 0,042466 & 1,009416 & 0,003618 & $-0,029346$ & 0,106597 & $-0,344283$ & \\
\hline & 364 & 3908 & & 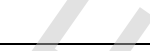 & 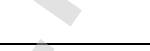 & & $-0,521790$ & & $-2,727796$ & \\
\hline & & & & + & 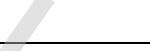 & & 0,521790 & & 2,727796 & \\
\hline & & & & & & & 0,04446 & & 2,495371 & 2,539831 \\
\hline
\end{tabular}




\begin{tabular}{|c|c|c|c|c|c|c|c|c|c|c|}
\hline CRDJYR & BADS & GOODS & $f(x / 1)$ & $f(x / 0)$ & $w(x)$ & & & & 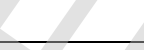 & \\
\hline $0 \backslash 0$ & 74 & 338 & 0,086595 & 0,20411 & $-0,85741$ & 0,017391 & $-0,101661$ & 0,079217 & $-0,289779$ & \\
\hline $0 \backslash 1$ & 115 & 835 & 0,213738 & 0,316438 & $-0,39238$ & 0,026962 & $-0,140553$ & 0,195526 & $-0,460379$ & \\
\hline $0 \backslash 2$ & 49 & 408 & 0,104502 & 0,135616 & $-0,26062$ & 0,011555 & $-0,074362$ & 0,095598 & $-0,323779$ & \\
\hline 013 & 67 & 928 & 0,237529 & 0,184932 & 0,250303 & 0,015757 & $-0,094352$ & 0,21729 & $-0,478539$ & \\
\hline 110 & 8 & 173 & 0,044385 & 0,023288 & 0,644972 & 0,001984 & $-0,017813$ & 0,040603 & $-0,187678$ & \\
\hline $1 \backslash 1$ & 26 & 523 & 0,133922 & 0,072603 & 0,612253 & 0,006186 & $-0,045386$ & 0,122511 & $-0,371088$ & \\
\hline 112 & 10 & 236 & 0,060501 & 0,028767 & 0,743433 & 0,002451 & $-0,021257$ & 0,055346 & $-0,231091$ & \\
\hline \multirow[t]{4}{*}{113} & 15 & 467 & 0,119596 & 0,042466 & 1,03542 & 0,003618 & $-0,029346$ & 0,109406 & $-0,349249$ & \\
\hline & 364 & 3908 & & & & & $-0,524730$ & & $-2,691581$ & \\
\hline & & & & & & & 0,524730 & & 2,691581 & \\
\hline & & & & & & & 0,04471 & & 2,462242 & 2,506952 \\
\hline
\end{tabular}

\begin{tabular}{|c|c|c|c|c|c|c|c|c|c|c|}
\hline CRDJYR & BADS & GOODS & $f(x / 1)$ & $f(x / 0)$ & $w(x)$ & 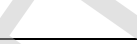 & & & & \\
\hline $0 \backslash 0$ & 111 & 510 & 0,130596 & 0,305479 & $-0,84977$ & 0,026029 & $-0,137009$ & 0,119468 & $-0,366207$ & \\
\hline $0 \backslash 1$ & 89 & 622 & 0,159248 & 0,245205 & $-0,43163$ & 0,020893 & $-0,116600$ & 0,145679 & $-0,404862$ & \\
\hline 012 & 47 & 406 & 0,103991 & 0,130137 & $-0,22429$ & 0,011088 & $-0,072017$ & 0,09513 & $-0,322867$ & \\
\hline 013 & 65 & 984 & 0,251855 & 0,179452 & 0,338944 & 0,01529 & $-0,092220$ & 0,230395 & $-0,487935$ & \\
\hline 110 & 13 & 292 & 0,074827 & 0,036986 & 0,704635 & 0,003151 & $-0,026188$ & 0,068452 & $-0,264824$ & \\
\hline $1 \backslash 1$ & 19 & 392 & 0,100409 & 0,053425 & 0,630983 & 0,004552 & $-0,035412$ & 0,091854 & $-0,316392$ & \\
\hline 112 & 8 & 236 & 0,060501 & 0,023288 & 0,954743 & 0,001984 & $-0,017813$ & 0,055346 & $-0,231091$ & \\
\hline \multirow[t]{4}{*}{113} & 12 & 466 & 0,11934 & 0,034247 & 1,24839 & 0,002918 & $-0,024572$ & 0,109172 & $-0,348839$ & \\
\hline & 364 & 3908 & & 2 & 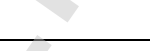 & & $-0,521831$ & & $-2,743017$ & \\
\hline & & & & + & 2 & & 0,521831 & & 2,743017 & \\
\hline & & & & & & & 0,044463 & & 2,509295 & 2,553758 \\
\hline
\end{tabular}




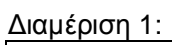

\begin{tabular}{|c|c|c|c|c|c|c|c|c|c|c|}
\hline CRDIMAR & BADS & GOODS & $f(x / 1)$ & $f(x / 0)$ & $w(x)$ & & & 8 & 8 & \\
\hline $0 \backslash 1$ & 142 & 564 & 0,14441 & 0,390411 & $-0,99454$ & 0,033265 & $-0,163327$ & 0,132106 & $-0,385780$ & \\
\hline $1 \backslash 1$ & 24 & 401 & 0,102712 & 0,067123 & 0,425395 & 0,005719 & $-0,042609$ & 0,09396 & $-0,320574$ & \\
\hline 012 & 102 & 108 & 0,027756 & 0,280822 & $-2,31425$ & 0,023928 & $-0,128855$ & 0,025391 & $-0,134562$ & \\
\hline 112 & 23 & 74 & 0,019059 & 0,064384 & $-1,21734$ & 0,005486 & $-0,041199$ & 0,017435 & $-0,101852$ & \\
\hline 013 & 65 & 1837 & 0,470069 & 0,179452 & 0,962972 & 0,01529 & $-0,092220$ & 0,430016 & $-0,523561$ & \\
\hline \multirow[t]{4}{*}{113} & 8 & 924 & 0,236506 & 0,023288 & 2,318047 & 0,001984 & $-0,017813$ & 0,216354 & $-0,477825$ & \\
\hline & 364 & 3908 & & & & & $-0,486023$ & & $-1,944153$ & \\
\hline & & & & & & & 0,486023 & & 1,944153 & \\
\hline & & & & & & 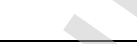 & 0,041412 & & 1,7785 & 1,819912 \\
\hline
\end{tabular}

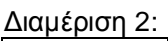

\begin{tabular}{|l|r|r|l|l|l|l|r|r|r|r|}
\hline CRDMAR & \multicolumn{1}{|c|}{ BADS } & GOODS & $\mathrm{f}(\mathrm{x} / 1)$ & $\mathrm{f}(\mathrm{x} / 0)$ & $\mathrm{w}(\mathrm{x})$ & & & & \\
\hline 010 & 96 & 118 & 0,030315 & 0,264384 & $-2,16577$ & 0,022527 & $-0,123273$ & 0,027732 & $-0,143437$ & \\
\hline 011 & 143 & 586 & 0,150038 & 0,393151 & $-0,9633$ & 0,033499 & $-0,164136$ & 0,137254 & $-0,393244$ & \\
\hline 012 & 62 & 1833 & 0,469046 & 0,171233 & 1,007676 & 0,01459 & $-0,088983$ & 0,42908 & $-0,523770$ & \\
\hline 110 & 31 & 74 & 0,019059 & 0,086301 & $-1,51033$ & 0,007353 & $-0,052116$ & 0,017435 & $-0,101852$ & \\
\hline 111 & 24 & 398 & 0,101944 & 0,067123 & 0,417895 & 0,005719 & $-0,042609$ & 0,093258 & $-0,319187$ & \\
\hline 112 & 8 & 899 & 0,23011 & 0,023288 & 2,290633 & 0,001984 & $-0,017813$ & 0,210503 & $-0,473229$ & \\
\hline & 364 & 3908 & & & & & $-0,488929$ & & $-1,954719$ & \\
\hline & & & & & & & 0,488929 & & 1,954719 & \\
\hline & & & & & & & 0,04166 & & 1,788166 & 1,829825 \\
\hline
\end{tabular}




\begin{tabular}{|c|c|c|c|c|c|c|c|c|c|c|}
\hline CRDMAR & BADS & GOODS & $f(x / 1)$ & $f(x / 0)$ & $w(x)$ & & & $\hat{x}$ & + & \\
\hline 010 & 93 & 112 & 0,02878 & 0,256164 & $-2,18615$ & 0,021827 & $-0,120435$ & 0,026328 & $-0,138148$ & \\
\hline $0 \backslash 1$ & 153 & 585 & 0,149783 & 0,420548 & $-1,03237$ & 0,035833 & $-0,172091$ & 0,13702 & $-0,392911$ & \\
\hline 012 & 63 & 1822 & 0,466232 & 0,173973 & 0,985785 & 0,014824 & $-0,090067$ & 0,426506 & $-0,524330$ & \\
\hline 110 & 25 & 62 & 0,015989 & 0,069863 & $-1,47465$ & 0,005953 & $-0,044004$ & 0,014626 & $-0,089152$ & \\
\hline $1 \backslash 1$ & 23 & 410 & 0,105014 & 0,064384 & 0,489236 & 0,005486 & $-0,041199$ & 0,096066 & $-0,324687$ & \\
\hline \multirow[t]{4}{*}{112} & 7 & 917 & 0,234715 & 0,020548 & 2,43561 & 0,001751 & $-0,016033$ & 0,214716 & $-0,476562$ & \\
\hline & 364 & 3908 & & & & & $-0,483830$ & & $-1,945790$ & \\
\hline & & & & & & & 0,483830 & 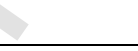 & 1,945790 & \\
\hline & & & & & & & 0,041225 & & 1,779997 & 1,821222 \\
\hline
\end{tabular}

\begin{tabular}{|c|c|c|c|c|c|c|c|c|c|c|}
\hline CRDMAR & BADS & GOODS & $f(x / 1)$ & $f(x / 0)$ & $w(x)$ & 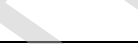 & & & & \\
\hline $0 \backslash 0$ & 89 & 110 & 0,028268 & 0,245205 & $-2,16036$ & 0,020893 & $-0,116600$ & 0,025859 & $-0,136361$ & \\
\hline $0 \backslash 1$ & 152 & 574 & 0,146969 & 0,417808 & $-1,0448$ & 0,0356 & $-0,171306$ & 0,134446 & $-0,389208$ & \\
\hline 012 & 64 & 1825 & 0,466999 & 0,176712 & 0,971804 & 0,015057 & $-0,091146$ & 0,427208 & $-0,524180$ & \\
\hline $1 \backslash 0$ & 30 & 74 & 0,019059 & 0,083562 & $-1,47807$ & 0,00712 & $-0,050793$ & 0,017435 & $-0,101852$ & \\
\hline $1 \backslash 1$ & 23 & 409 & 0,104758 & 0,064384 & 0,486797 & 0,005486 & $-0,041199$ & 0,095832 & $-0,324233$ & \\
\hline \multirow[t]{4}{*}{112} & 6 & 916 & 0,234459 & 0,017808 & 2,57762 & 0,001517 & $-0,014209$ & 0,214482 & $-0,476380$ & \\
\hline & 364 & 3908 & & $\Delta$ & & & $-0,485253$ & & $-1,952213$ & \\
\hline & & & & 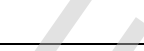 & 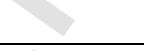 & & 0,485253 & & 1,952213 & \\
\hline & & & & 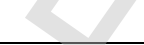 & 2 & & 0,041346 & & 1,785873 & 1,82722 \\
\hline
\end{tabular}




\begin{tabular}{|c|c|c|c|c|c|c|c|c|c|c|}
\hline CRDMAR & BADS & GOODS & $f(x / 1)$ & $f(x / 0)$ & $w(x)$ & & & 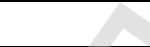 & 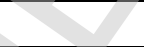 & \\
\hline $0 \backslash 0$ & 96 & 100 & 0,02571 & 0,264384 & $-2,33052$ & 0,022527 & $-0,123273$ & 0,023519 & $-0,127240$ & \\
\hline $0 \backslash 1$ & 154 & 555 & 0,142108 & 0,423288 & $-1,09146$ & 0,036067 & $-0,172874$ & 0,13 & $-0,382643$ & \\
\hline $0 \backslash 2$ & 62 & 1867 & 0,477744 & 0,171233 & 1,02605 & 0,01459 & $-0,088983$ & 0,437037 & $-0,521898$ & \\
\hline $1 \backslash 0$ & 27 & 72 & 0,018547 & 0,075342 & $-1,40174$ & 0,00642 & $-0,046756$ & 0,016967 & $-0,099783$ & \\
\hline $1 \backslash 1$ & 18 & 406 & 0,103991 & 0,050685 & 0,718674 & 0,004319 & $-0,033924$ & 0,09513 & $-0,322867$ & \\
\hline \multirow[t]{4}{*}{112} & 7 & 908 & 0,232412 & 0,020548 & 2,425752 & 0,001751 & $-0,016033$ & 0,212609 & $-0,474910$ & \\
\hline & 364 & 3908 & & & & & $-0,481843$ & & $-1,929342$ & \\
\hline & & & & & & & 0,481843 & 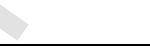 & 1,929342 & \\
\hline & & & & & & & 0,041056 & & 1,76495 & 1,806006 \\
\hline
\end{tabular}

27)

\section{H(CRD TRM)}

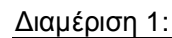

\begin{tabular}{|c|c|c|c|c|c|c|c|c|c|c|}
\hline CRDITRM & BADS & GOODS & $f(x / 1)$ & $f(x / 0)$ & $w(x)$ & & & & & \\
\hline $0 \backslash 1$ & 37 & 242 & 0,062036 & 0,10274 & $-0,50448$ & 0,008754 & $-0,059841$ & 0,05675 & $-0,234903$ & \\
\hline $1 \backslash 1$ & 18 & 161 & 0,041315 & 0,050685 & $-0,20441$ & 0,004319 & $-0,033924$ & 0,037795 & $-0,178605$ & \\
\hline 012 & 37 & 642 & 0,164364 & 0,10274 & 0,469886 & 0,008754 & $-0,059841$ & 0,150359 & $-0,411009$ & \\
\hline 112 & 0 & 331 & 0,084804 & 0,00137 & 4,125635 & 0,000117 & $-0,001525$ & 0,077578 & $-0,286125$ & \\
\hline 013 & 235 & 1625 & 0,415835 & 0,645205 & $-0,43928$ & 0,054975 & $-0,230076$ & 0,380404 & $-0,530434$ & \\
\hline \multirow[t]{4}{*}{113} & 37 & 907 & 0,232157 & 0,10274 & 0,815213 & 0,008754 & $-0,059841$ & 0,212375 & $-0,474725$ & \\
\hline & 364 & 3908 & & $\Delta$ & & & $-0,445048$ & & $-2,115801$ & \\
\hline & & & & 2 & P & & 0,445048 & & 2,115801 & \\
\hline & & & & & 8 & & 0,037921 & & 1,935522 & 1,973443 \\
\hline
\end{tabular}




\begin{tabular}{|c|c|c|c|c|c|c|c|c|c|c|}
\hline CRDTRM & BADS & GOODS & $f(x / 1)$ & $f(x / 0)$ & $w(x)$ & & & $\hat{x}$ & + & \\
\hline 010 & 30 & 643 & 0,16462 & 0,083562 & 0,678056 & 0,00712 & $-0,050793$ & 0,150593 & $-0,411311$ & \\
\hline $0 \backslash 1$ & 241 & 1659 & 0,424533 & 0,661644 & $-0,44374$ & 0,056376 & $-0,233891$ & 0,38836 & $-0,529930$ & \\
\hline 012 & 30 & 235 & 0,060246 & 0,083562 & $-0,32716$ & 0,00712 & $-0,050793$ & 0,055112 & $-0,230451$ & \\
\hline 110 & 1 & 318 & 0,081479 & 0,00411 & 2,987018 & 0,00035 & $-0,004020$ & 0,074536 & $-0,279206$ & \\
\hline $1 \backslash 1$ & 45 & 904 & 0,231389 & 0,124658 & 0,61853 & 0,010622 & $-0,069644$ & 0,211673 & $-0,474167$ & \\
\hline \multirow[t]{4}{*}{112} & 17 & 149 & 0,038245 & 0,047945 & $-0,22604$ & 0,004085 & $-0,032418$ & 0,034986 & $-0,169231$ & \\
\hline & 364 & 3908 & & & & & $-0,441559$ & & $-2,094297$ & \\
\hline & & & & & & & 0,441559 & 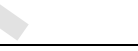 & 2,094297 & \\
\hline & & & & & & & 0,037623 & & 1,91585 & 1,953474 \\
\hline
\end{tabular}

\begin{tabular}{|c|c|c|c|c|c|c|c|c|c|c|}
\hline CRDTRM & BADS & GOODS & $f(x / 1)$ & $f(x / 0)$ & $w(x)$ & 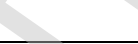 & & & & \\
\hline $0 \backslash 0$ & 28 & 610 & 0,156178 & 0,078082 & 0,693235 & 0,006653 & $-0,048113$ & 0,142871 & $-0,401069$ & \\
\hline $0 \backslash 1$ & 244 & 1686 & 0,43144 & 0,669863 & $-0,43994$ & 0,057076 & $-0,235780$ & 0,394679 & $-0,529363$ & \\
\hline 012 & 37 & 223 & 0,057176 & 0,10274 & $-0,58607$ & 0,008754 & $-0,059841$ & 0,052304 & $-0,222655$ & \\
\hline $1 \backslash 0$ & 0 & 318 & 0,081479 & 0,00137 & 4,08563 & 0,000117 & $-0,001525$ & 0,074536 & $-0,279206$ & \\
\hline $1 \backslash 1$ & 40 & 918 & 0,234971 & 0,110959 & 0,7503 & 0,009454 & $-0,063579$ & 0,21495 & $-0,476743$ & \\
\hline \multirow[t]{4}{*}{112} & 15 & 153 & 0,039268 & 0,042466 & $-0,07828$ & 0,003618 & $-0,029346$ & 0,035922 & $-0,172391$ & \\
\hline & 364 & 3908 & & $\Delta$ & & & $-0,438185$ & & $-2,081427$ & \\
\hline & & & & 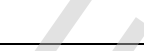 & 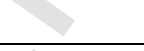 & & 0,438185 & & 2,081427 & \\
\hline & & & & 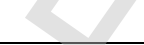 & 2 & & 0,037336 & & 1,904077 & 1,941413 \\
\hline
\end{tabular}




\begin{tabular}{|c|c|c|c|c|c|c|c|c|c|c|}
\hline CRDTRM & BADS & GOODS & $f(x / 1)$ & $f(x / 0)$ & $w(x)$ & & & $\hat{x}$ & + & \\
\hline 010 & 31 & 595 & 0,152341 & 0,086301 & 0,568274 & 0,007353 & $-0,052116$ & 0,13936 & $-0,396217$ & \\
\hline $0 \backslash 1$ & 243 & 1706 & 0,436557 & 0,667123 & $-0,42406$ & 0,056843 & $-0,235152$ & 0,399359 & $-0,528848$ & \\
\hline 012 & 31 & 208 & 0,053338 & 0,086301 & $-0,48119$ & 0,007353 & $-0,052116$ & 0,048794 & $-0,212602$ & \\
\hline 110 & 0 & 307 & 0,078665 & 0,00137 & 4,050483 & 0,000117 & $-0,001525$ & 0,071962 & $-0,273212$ & \\
\hline $1 \backslash 1$ & 41 & 936 & 0,239575 & 0,113699 & 0,745317 & 0,009688 & $-0,064808$ & 0,219162 & $-0,479950$ & \\
\hline \multirow[t]{4}{*}{112} & 18 & 156 & 0,040036 & 0,050685 & $-0,23585$ & 0,004319 & $-0,033924$ & 0,036625 & $-0,174737$ & \\
\hline & 364 & 3908 & & & & & $-0,439641$ & & $-2,065566$ & \\
\hline & & & & & & & 0,439641 & 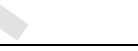 & 2,065566 & \\
\hline & & & & & & & 0,03746 & & 1,889567 & 1,927027 \\
\hline
\end{tabular}

\begin{tabular}{|c|c|c|c|c|c|c|c|c|c|c|}
\hline CRDTRM & BADS & GOODS & $f(x / 1)$ & $f(x / 0)$ & $w(x)$ & 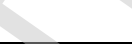 & & & & \\
\hline $0 \backslash 0$ & 30 & 569 & 0,145689 & 0,083562 & 0,555893 & 0,00712 & $-0,050793$ & 0,133276 & $-0,387501$ & \\
\hline $0 \backslash 1$ & 241 & 1731 & 0,442952 & 0,661644 & $-0,40127$ & 0,056376 & $-0,233891$ & 0,40521 & $-0,528093$ & \\
\hline 012 & 41 & 222 & 0,05692 & 0,113699 & $-0,69191$ & 0,009688 & $-0,064808$ & 0,05207 & $-0,221995$ & \\
\hline 110 & 0 & 296 & 0,075851 & 0,00137 & 4,014055 & 0,000117 & $-0,001525$ & 0,069388 & $-0,267085$ & \\
\hline $1 \backslash 1$ & 36 & 935 & 0,23932 & 0,1 & 0,872629 & 0,008521 & $-0,058578$ & 0,218928 & $-0,479775$ & \\
\hline \multirow[t]{4}{*}{112} & 16 & 155 & 0,03978 & 0,045205 & $-0,12785$ & 0,003852 & $-0,030892$ & 0,036391 & $-0,173957$ & \\
\hline & 364 & 3908 & & $\Delta$ & & & $-0,440487$ & & $-2,058407$ & \\
\hline & & & & 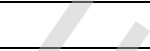 & 8 & & 0,440487 & & 2,058407 & \\
\hline & & & & $4=$ & 2 & & 0,037532 & & 1,883019 & 1,920551 \\
\hline
\end{tabular}




\begin{tabular}{|c|c|c|c|c|c|c|c|c|c|c|}
\hline KID\HOME & BADS & GOODS & $f(x / 1)$ & $f(x / 0)$ & $w(x)$ & & $\triangle$ & 2 & & \\
\hline $1 \backslash 1$ & 1 & 19 & 0,004988 & 0,00411 & 0,19381 & 0,00035 & $-0,004020$ & 0,004563 & $-0,035484$ & \\
\hline $2 \backslash 1$ & 54 & 454 & 0,11627 & 0,149315 & $-0,25014$ & 0,012723 & $-0,080107$ & 0,106363 & $-0,343865$ & \\
\hline $3 \backslash 1$ & 0 & 59 & 0,015221 & 0,00137 & 2,407984 & 0,000117 & $-0,001525$ & 0,013924 & $-0,085861$ & \\
\hline $4 \backslash 1$ & 10 & 67 & 0,017268 & 0,028767 & $-0,51039$ & 0,002451 & $-0,021257$ & 0,015797 & $-0,094530$ & \\
\hline 112 & 21 & 76 & 0,01957 & 0,058904 & $-1,1019$ & 0,005019 & $-0,038337$ & 0,017903 & $-0,103902$ & \\
\hline 212 & 95 & 337 & 0,086339 & 0,261644 & $-1,1087$ & 0,022294 & $-0,122330$ & 0,078983 & $-0,289260$ & \\
\hline 312 & 5 & 121 & 0,031082 & 0,015068 & 0,724027 & 0,001284 & $-0,012332$ & 0,028434 & $-0,146043$ & \\
\hline 412 & 33 & 208 & 0,053338 & 0,091781 & $-0,54275$ & 0,00782 & $-0,054731$ & 0,048794 & $-0,212602$ & \\
\hline 113 & 30 & 380 & 0,097339 & 0,083562 & 0,15262 & 0,00712 & $-0,050793$ & 0,089046 & $-0,310708$ & \\
\hline 213 & 49 & 630 & 0,161294 & 0,135616 & 0,173401 & 0,011555 & $-0,074362$ & 0,147551 & $-0,407346$ & \\
\hline 313 & 11 & 442 & 0,1132 & 0,031507 & 1,278954 & 0,002685 & $-0,022929$ & 0,103555 & $-0,338783$ & \\
\hline \multirow[t]{4}{*}{413} & 55 & 1115 & 0,285367 & 0,152055 & 0,629535 & 0,012956 & $-0,081237$ & 0,261052 & $-0,505812$ & \\
\hline & 364 & 3908 & & 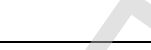 & $>$ & & $-0,563960$ & & $-2,874195$ & \\
\hline & & & & 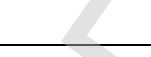 & 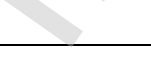 & & 0,563960 & & 2,874195 & \\
\hline & & & & $\Delta$ & & & 0,048053 & & 2,629297 & 2,67735 \\
\hline
\end{tabular}




\begin{tabular}{|c|c|c|c|c|c|c|c|c|c|c|}
\hline KIDHOME & BADS & GOODS & $f(x / 1)$ & $f(x / 0)$ & $w(x)$ & & & - & & \\
\hline 010 & 54 & 469 & 0,120107 & 0,149315 & $-0,21767$ & 0,012723 & $-0,080107$ & 0,109874 & $-0,350066$ & \\
\hline $0 \backslash 1$ & 98 & 338 & 0,086595 & 0,269863 & $-1,13667$ & 0,022994 & $-0,125147$ & 0,079217 & $-0,289779$ & \\
\hline 012 & 45 & 633 & 0,162062 & 0,124658 & 0,262408 & 0,010622 & $-0,069644$ & 0,148253 & $-0,408269$ & \\
\hline 110 & 0 & 57 & 0,01471 & 0,00137 & 2,373793 & 0,000117 & $-0,001525$ & 0,013456 & $-0,083639$ & \\
\hline $1 \backslash 1$ & 5 & 132 & 0,033896 & 0,015068 & 0,810695 & 0,001284 & $-0,012332$ & 0,031008 & $-0,155388$ & \\
\hline 112 & 13 & 467 & 0,119596 & 0,036986 & 1,17357 & 0,003151 & $-0,026188$ & 0,109406 & $-0,349249$ & \\
\hline 210 & 10 & 63 & 0,016245 & 0,028767 & $-0,57147$ & 0,002451 & $-0,021257$ & 0,01486 & $-0,090238$ & \\
\hline $2 \backslash 1$ & 37 & 201 & 0,051548 & 0,10274 & $-0,68969$ & 0,008754 & $-0,059841$ & 0,047156 & $-0,207788$ & \\
\hline 212 & 50 & 1083 & 0,277181 & 0,138356 & 0,694839 & 0,011789 & $-0,075524$ & 0,253563 & $-0,501949$ & \\
\hline 310 & 1 & 22 & 0,005756 & 0,00411 & 0,336911 & 0,00035 & $-0,004020$ & 0,005266 & $-0,039856$ & \\
\hline $3 \backslash 1$ & 19 & 74 & 0,019059 & 0,053425 & $-1,03075$ & 0,004552 & $-0,035412$ & 0,017435 & $-0,101852$ & \\
\hline \multirow[t]{4}{*}{312} & 32 & 369 & 0,094525 & 0,089041 & 0,059771 & 0,007587 & $-0,053429$ & 0,086471 & $-0,305385$ & \\
\hline & 364 & 3908 & & & & 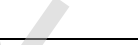 & $-0,564426$ & & $-2,883457$ & \\
\hline & & & & & 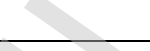 & 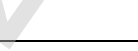 & 0,564426 & & 2,883457 & \\
\hline & & & & & 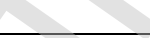 & & 0,048092 & & 2,637769 & 2,685862 \\
\hline
\end{tabular}




\begin{tabular}{|c|c|c|c|c|c|c|c|c|c|c|}
\hline KIDHOME & BADS & GOSOS & $f(x / 1)$ & $f(x / 0)$ & $w(x)+2$ & & & & & \\
\hline $0 \backslash 0$ & 60 & 467 & 0,119596 & 0,165753 & $-0,32638$ & 0,014123 & $-0,086798$ & 0,109406 & $-0,349249$ & \\
\hline $0 \backslash 1$ & 103 & 342 & 0,087618 & 0,283562 & $-1,17444$ & 0,024161 & $-0,129774$ & 0,080153 & $-0,291844$ & \\
\hline 012 & 44 & 626 & 0,160271 & 0,121918 & 0,27352 & 0,010388 & $-0,068447$ & 0,146615 & $-0,406108$ & \\
\hline 110 & 0 & 54 & 0,013942 & 0,00137 & 2,320208 & 0,000117 & $-0,001525$ & 0,012754 & $-0,080261$ & \\
\hline $1 \backslash 1$ & 4 & 118 & 0,030315 & 0,012329 & 0,899696 & 0,00105 & $-0,010394$ & 0,027732 & $-0,143437$ & \\
\hline 112 & 14 & 465 & 0,119084 & 0,039726 & 1,097824 & 0,003385 & $-0,027779$ & 0,108937 & $-0,348429$ & \\
\hline 210 & 9 & 66 & 0,017012 & 0,026027 & $-0,42523$ & 0,002218 & $-0,019553$ & 0,015562 & $-0,093465$ & \\
\hline $2 \backslash 1$ & 34 & 196 & 0,050269 & 0,094521 & $-0,63144$ & 0,008054 & $-0,056023$ & 0,045985 & $-0,204299$ & \\
\hline 212 & 48 & 1093 & 0,279739 & 0,132877 & 0,744436 & 0,011322 & $-0,073193$ & 0,255904 & $-0,503190$ & \\
\hline 310 & 2 & 20 & 0,005244 & 0,006849 & $-0,26701$ & 0,000584 & $-0,006269$ & 0,004797 & $-0,036957$ & \\
\hline $3 \backslash 1$ & 21 & 76 & 0,01957 & 0,058904 & $-1,1019$ & 0,005019 & $-0,038337$ & 0,017903 & $-0,103902$ & \\
\hline \multirow[t]{4}{*}{312} & 25 & 385 & 0,098619 & 0,069863 & 0,344723 & 0,005953 & $-0,044004$ & 0,090216 & $-0,313092$ & \\
\hline & 364 & 3908 & & & & 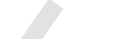 & $-0,562095$ & & $-2,874233$ & \\
\hline & & & & & & 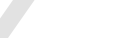 & 0,562095 & & 2,874233 & \\
\hline & & & & & & & 0,047894 & & 2,629331 & 2,677225 \\
\hline
\end{tabular}




\begin{tabular}{|c|c|c|c|c|c|c|c|c|c|c|}
\hline KIDHOME & $\mathrm{B} \triangle \mathrm{C}$ & Sחת & $f(x / 1)$ & $f(x / 0)$ & 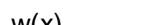 & & & & & \\
\hline 010 & 57 & 472 & 0120875 & 0157534 & -026489 & 0013423 & -0083479 & 0110576 & -0351287 & \\
\hline $0 \backslash 1$ & 103 & 338 & 0.086595 & 0.283562 & -1.18619 & 0.024161 & -0.129774 & 0.079217 & -0.289779 & \\
\hline $0 \backslash 2$ & 41 & 632 & 0,161806 & 0,113699 & 0,352847 & 0,009688 & $-0,064808$ & 0,148019 & $-0,407962$ & \\
\hline 110 & 0 & 51 & 0,013175 & 0,00137 & 2,263589 & 0,000117 & $-0,001525$ & 0,012052 & $-0,076827$ & \\
\hline $1 \backslash 1$ & 3 & 118 & 0,030315 & 0,009589 & 1,15101 & 0,000817 & $-0,008381$ & 0,027732 & $-0,143437$ & \\
\hline 112 & 14 & 454 & 0,11627 & 0,039726 & 1,07391 & 0,003385 & $-0,027779$ & 0,106363 & $-0,343865$ & \\
\hline 210 & 7 & 66 & 0,017012 & 0,020548 & $-0,18884$ & 0,001751 & $-0,016033$ & 0,015562 & $-0,093465$ & \\
\hline $2 \backslash 1$ & 35 & 211 & 0,054106 & 0,09726 & $-0,58645$ & 0,008287 & $-0,057305$ & 0,049496 & $-0,214641$ & \\
\hline 212 & 52 & 1100 & 0,28153 & 0,143836 & 0,671567 & 0,012256 & $-0,077828$ & 0,257542 & $-0,504041$ & \\
\hline 310 & 2 & 22 & 0,005756 & 0,006849 & $-0,17391$ & 0,000584 & $-0,006269$ & 0,005266 & $-0,039856$ & \\
\hline $3 \backslash 1$ & 22 & 72 & 0,018547 & 0,061644 & $-1,20107$ & 0,005252 & $-0,039776$ & 0,016967 & $-0,099783$ & \\
\hline \multirow[t]{4}{*}{312} & 28 & 372 & 0,095293 & 0,078082 & 0,199193 & 0,006653 & $-0,048113$ & 0,087173 & $-0,306848$ & \\
\hline & 364 & 3908 & & & & 2 & $-0,561070$ & & $-2,871790$ & \\
\hline & & & & & 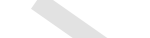 & 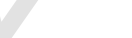 & 0,561070 & & 2,871790 & \\
\hline & & & & & N & & 0,047807 & & 2,627097 & 2,674903 \\
\hline
\end{tabular}




\begin{tabular}{|c|c|c|c|c|c|c|c|c|c|c|}
\hline \multicolumn{11}{|l|}{ 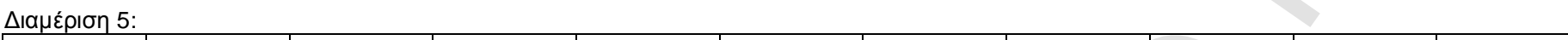 } \\
\hline KIDHOME & BADS & GOODS & $f(x / 1)$ & $f(x / 0)$ & $w(x)$ & & & 工 & & \\
\hline $0 \backslash 0$ & 59 & 462 & 0,118317 & 0,163014 & $-0,32047$ & 0,01389 & $-0,085697$ & 0,108235 & $-0,347193$ & \\
\hline $0 \backslash 1$ & 93 & 325 & 0,083269 & 0,256164 & $-1,12374$ & 0,021827 & $-0,120435$ & 0,076174 & $-0,282953$ & \\
\hline 012 & 45 & 639 & 0,163597 & 0,124658 & 0,271835 & 0,010622 & $-0,069644$ & 0,149657 & $-0,410101$ & \\
\hline 110 & 0 & 55 & 0,014198 & 0,00137 & 2,338391 & 0,000117 & $-0,001525$ & 0,012988 & $-0,081393$ & \\
\hline $1 \backslash 1$ & 3 & 131 & 0,03364 & 0,009589 & 1,255104 & 0,000817 & $-0,008381$ & 0,030774 & $-0,154551$ & \\
\hline $1 \backslash 2$ & 12 & 456 & 0,116782 & 0,034247 & 1,226721 & 0,002918 & $-0,024572$ & 0,106831 & $-0,344701$ & \\
\hline 210 & 8 & 62 & 0,015989 & 0,023288 & $-0,37604$ & 0,001984 & $-0,017813$ & 0,014626 & $-0,089152$ & \\
\hline 211 & 37 & 204 & 0,052315 & 0,10274 & $-0,67491$ & 0,008754 & $-0,059841$ & 0,047858 & $-0,209861$ & \\
\hline $2 \backslash 2$ & 51 & 1109 & 0,283832 & 0,141096 & 0,698943 & 0,012022 & $-0,076680$ & 0,259648 & $-0,505112$ & \\
\hline 310 & 2 & 21 & 0,0055 & 0,006849 & $-0,21938$ & 0,000584 & $-0,006269$ & 0,005031 & $-0,038414$ & \\
\hline $3 \backslash 1$ & 21 & 78 & 0,020082 & 0,058904 & $-1,07609$ & 0,005019 & $-0,038337$ & 0,018371 & $-0,105934$ & \\
\hline $3 \backslash 2$ & 33 & 366 & 0,093758 & 0,091781 & 0,021314 & 0,00782 & $-0,054731$ & 0,085769 & $-0,303914$ & \\
\hline & 364 & 3908 & & & & 2 & $-0,563925$ & & $-2,873280$ & \\
\hline & & & & & & 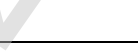 & 0,563925 & & 2,873280 & \\
\hline & & & & & 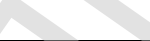 & & 0,04805 & & 2,628459 & 2,676509 \\
\hline
\end{tabular}




\begin{tabular}{|c|c|c|c|c|c|c|c|c|c|c|}
\hline KID $\backslash H Y R$ & BADS & GOODS & $f(x / 1)$ & $f(x / 0)$ & $w(x)$ & & & 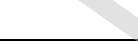 & & \\
\hline $1 \backslash 1$ & 5 & 159 & 0,040803 & 0,015068 & 0,996156 & 0,001284 & $-0,012332$ & 0,037327 & $-0,177064$ & \\
\hline $2 \backslash 1$ & 43 & 507 & 0,129829 & 0,119178 & 0,085596 & 0,010155 & $-0,067241$ & 0,118766 & $-0,365064$ & \\
\hline $3 \backslash 1$ & 9 & 141 & 0,036199 & 0,026027 & 0,329868 & 0,002218 & $-0,019553$ & 0,033114 & $-0,162803$ & \\
\hline $4 \backslash 1$ & 22 & 387 & 0,09913 & 0,061644 & 0,475061 & 0,005252 & $-0,039776$ & 0,090684 & $-0,314039$ & \\
\hline $1 \backslash 2$ & 25 & 85 & 0,021873 & 0,069863 & $-1,1613$ & 0,005953 & $-0,044004$ & 0,020009 & $-0,112915$ & \\
\hline 212 & 125 & 347 & 0,088897 & 0,343836 & $-1,35268$ & 0,029297 & $-0,149212$ & 0,081323 & $-0,294405$ & \\
\hline 312 & 6 & 148 & 0,037989 & 0,017808 & 0,757643 & 0,001517 & $-0,014209$ & 0,034752 & $-0,168436$ & \\
\hline 412 & 42 & 218 & 0,055897 & 0,116438 & $-0,73386$ & 0,009921 & $-0,066028$ & 0,051134 & $-0,219343$ & \\
\hline 113 & 20 & 192 & 0,049245 & 0,056164 & $-0,13147$ & 0,004786 & $-0,036883$ & 0,045049 & $-0,201476$ & \\
\hline 213 & 26 & 513 & 0,131364 & 0,072603 & 0,592966 & 0,006186 & $-0,045386$ & 0,120171 & $-0,367343$ & \\
\hline $3 \backslash 3$ & 1 & 297 & 0,076106 & 0,00411 & 2,91881 & 0,00035 & $-0,004020$ & 0,069622 & $-0,267648$ & \\
\hline $4 \backslash 3$ & 34 & 665 & 0,170248 & 0,094521 & 0,58844 & 0,008054 & $-0,056023$ & 0,155742 & $-0,417820$ & \\
\hline $1 \backslash 4$ & 2 & 39 & 0,010105 & 0,006849 & 0,38887 & 0,000584 & $-0,006269$ & 0,009244 & $-0,062464$ & \\
\hline $2 \backslash 4$ & 4 & 54 & 0,013942 & 0,012329 & 0,122984 & 0,00105 & $-0,010394$ & 0,012754 & $-0,080261$ & \\
\hline $3 \backslash 4$ & 0 & 36 & 0,009337 & 0,00137 & $\begin{array}{r}1,91932 \\
\end{array}$ & 0,000117 & $-0,001525$ & 0,008542 & $-0,058693$ & \\
\hline \multirow[t]{4}{*}{$4 \backslash 4$} & 0 & 120 & 0,030826 & 0,00137 & 3,113657 & 0,000117 & $-0,001525$ & 0,0282 & $-0,145177$ & \\
\hline & 364 & 3908 & & 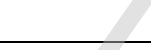 & 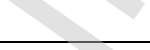 & & $-0,574381$ & & $-3,414950$ & \\
\hline & & & & 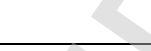 & & & 0,574381 & & 3,414950 & \\
\hline & & & & 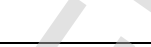 & & & 0,048941 & & 3,123976 & 3,172916 \\
\hline
\end{tabular}




\begin{tabular}{|c|c|c|c|c|c|c|c|c|c|c|}
\hline KIDHYR & & (1) & $f(x / 1)$ & $f(x / 0)$ & w(y) & & & & & \\
\hline $0 \backslash 0$ & 122 & 351 & 0.089921 & 0335616 & -131704 & 0,028597 & -0.146643 & 0082259 & -0296435 & \\
\hline $0 \backslash 1$ & 33 & 526 & 0,134689 & 0,091781 & 0,383566 & 0,00782 & $-0,054731$ & 0,123213 & $-0,372198$ & \\
\hline 012 & 39 & 509 & 0,13034 & 0,108219 & 0,18599 & 0,009221 & $-0,062341$ & 0,119234 & $-0,365826$ & \\
\hline 013 & 3 & 54 & 0,013942 & 0,009589 & 0,374298 & 0,000817 & $-0,008381$ & 0,012754 & $-0,080261$ & \\
\hline $1 \backslash 0$ & 5 & 152 & 0,039013 & 0,015068 & 0,951277 & 0,001284 & $-0,012332$ & 0,035688 & $-0,171604$ & \\
\hline $1 \backslash 1$ & 2 & 321 & 0,082246 & 0,006849 & 2,485567 & 0,000584 & $-0,006269$ & 0,075238 & $-0,280818$ & \\
\hline 112 & 11 & 142 & 0,036454 & 0,031507 & 0,145855 & 0,002685 & $-0,022929$ & 0,033348 & $-0,163615$ & \\
\hline 113 & 0 & 41 & 0,010617 & 0,00137 & 2,047701 & 0,000117 & $-0,001525$ & 0,009712 & $-0,064934$ & \\
\hline 210 & 45 & 211 & 0,054106 & 0,124658 & $-0,83463$ & 0,010622 & $-0,069644$ & 0,049496 & $-0,214641$ & \\
\hline $2 \backslash 1$ & 33 & 652 & 0,166922 & 0,091781 & 0,598126 & 0,00782 & $-0,054731$ & 0,1527 & $-0,414004$ & \\
\hline 212 & 19 & 373 & 0,095549 & 0,053425 & 0,581364 & 0,004552 & $-0,035412$ & 0,087407 & $-0,307333$ & \\
\hline 213 & 0 & 111 & 0,028524 & 0,00137 & 3,036032 & 0,000117 & $-0,001525$ & 0,026094 & $-0,137256$ & \\
\hline 310 & 23 & 77 & 0,019826 & 0,064384 & $-1,17786$ & 0,005486 & $-0,041199$ & 0,018137 & $-0,104920$ & \\
\hline $3 \backslash 1$ & 21 & 204 & 0,052315 & 0,058904 & $-0,11862$ & 0,005019 & $-0,038337$ & 0,047858 & $-0,209861$ & \\
\hline 312 & 6 & 148 & 0,037989 & 0,017808 & 0,757643 & 0,001517 & $-0,014209$ & 0,034752 & $-0,168436$ & \\
\hline 313 & 2 & 36 & 0,009337 & 0,006849 & 0,309882 & 0,000584 & $-0,006269$ & 0,008542 & $-0,058693$ & \\
\hline & 364 & 3908 & & & 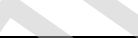 & & $-0,576479$ & & $-3,410836$ & \\
\hline & & & & & & & 0,576479 & & 3,410836 & \\
\hline & & & & & & & 0,049119 & & 3,120212 & 3,169332 \\
\hline
\end{tabular}




\begin{tabular}{|c|c|c|c|c|c|c|c|c|c|c|}
\hline \multicolumn{11}{|c|}{ 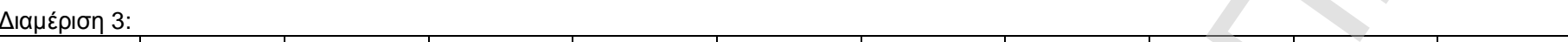 } \\
\hline KIDHYR & BADS & GOODS & $f(x / 1)$ & $f(x / 0)$ & $w(x)$ & & & 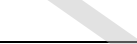 & & \\
\hline $0 \backslash 0$ & 128 & 363 & 0,092991 & 0,352055 & $-1,33129$ & 0,029997 & $-0,151757$ & 0,085067 & $-0,302435$ & \\
\hline $0 \backslash 1$ & 33 & 513 & 0,131364 & 0,091781 & 0,358565 & 0,00782 & $-0,054731$ & 0,120171 & $-0,367343$ & \\
\hline 012 & 45 & 500 & 0,128038 & 0,124658 & 0,026756 & 0,010622 & $-0,069644$ & 0,117128 & $-0,362376$ & \\
\hline $0 \backslash 3$ & 1 & 59 & 0,015221 & 0,00411 & 1,309372 & 0,00035 & $-0,004020$ & 0,013924 & $-0,085861$ & \\
\hline 110 & 5 & 147 & 0,037733 & 0,015068 & 0,917941 & 0,001284 & $-0,012332$ & 0,034518 & $-0,167638$ & \\
\hline 111 & 2 & 312 & 0,079944 & 0,006849 & 2,457174 & 0,000584 & $-0,006269$ & 0,073132 & $-0,275953$ & \\
\hline $1 \backslash 2$ & 11 & 127 & 0,032617 & 0,031507 & 0,03463 & 0,002685 & $-0,022929$ & 0,029838 & $-0,151180$ & \\
\hline 113 & 0 & 51 & 0,013175 & 0,00137 & 2,263589 & 0,000117 & $-0,001525$ & 0,012052 & $-0,076827$ & \\
\hline 210 & 42 & 229 & 0,058711 & 0,116438 & $-0,68474$ & 0,009921 & $-0,066028$ & 0,053708 & $-0,226579$ & \\
\hline 211 & 30 & 667 & 0,17076 & 0,083562 & 0,714673 & 0,00712 & $-0,050793$ & 0,15621 & $-0,418399$ & \\
\hline 212 & 19 & 350 & 0,089665 & 0,053425 & 0,517807 & 0,004552 & $-0,035412$ & 0,082025 & $-0,295929$ & \\
\hline 213 & 0 & 109 & 0,028012 & 0,00137 & 3,017932 & 0,000117 & $-0,001525$ & 0,025625 & $-0,135463$ & \\
\hline 310 & 24 & 80 & 0,020594 & 0,067123 & $-1,18156$ & 0,005719 & $-0,042609$ & 0,018839 & $-0,107949$ & \\
\hline $3 \backslash 1$ & 21 & 207 & 0,053083 & 0,058904 & $-0,10406$ & 0,005019 & $-0,038337$ & 0,04856 & $-0,211919$ & \\
\hline 312 & 2 & 157 & 0,040292 & 0,006849 & 1,771995 & 0,000584 & $-0,006269$ & 0,036859 & $-0,175515$ & \\
\hline \multirow[t]{4}{*}{313} & 1 & 37 & 0,009593 & 0,00411 & 0,847736 & 0,00035 & $-0,004020$ & 0,008776 & $-0,059959$ & \\
\hline & 364 & 3908 & & & & & $-0,568200$ & & $-3,421326$ & \\
\hline & & & & & & & 0,568200 & & 3,421326 & \\
\hline & & & & & & & 0,048414 & & 3,129809 & 3,178223 \\
\hline
\end{tabular}




\begin{tabular}{|c|c|c|c|c|c|c|c|c|c|c|}
\hline KIDHYR & BADS & GOOS & $f(x / 1)$ & $f(x / 0)$ & $w(x)$ & & & & & \\
\hline 010 & 125 & 363 & 0.092991 & 0,343836 & $-1,30767$ & 0.029297 & $-0,149212$ & 0.085067 & $-0,302435$ & \\
\hline $0 \backslash 1$ & 29 & 518 & 0,132643 & 0,080822 & 0,49541 & 0,006887 & -0.049459 & 0,121341 & $-0,369223$ & \\
\hline 012 & 43 & 507 & 0,129829 & 0,119178 & 0,085596 & 0,010155 & $-0,067241$ & 0,118766 & $-0,365064$ & \\
\hline 013 & 4 & 54 & 0,013942 & 0,012329 & 0,122984 & 0,00105 & $-0,010394$ & 0,012754 & $-0,080261$ & \\
\hline $1 \backslash 0$ & 4 & 152 & 0,039013 & 0,012329 & 1,151948 & 0,00105 & $-0,010394$ & 0,035688 & $-0,171604$ & \\
\hline $1 \backslash 1$ & 2 & 301 & 0,07713 & 0,006849 & 2,42134 & 0,000584 & $-0,006269$ & 0,070558 & $-0,269887$ & \\
\hline 112 & 11 & 133 & 0,034152 & 0,031507 & 0,080615 & 0,002685 & $-0,022929$ & 0,031242 & $-0,156222$ & \\
\hline 113 & 0 & 37 & 0,009593 & 0,00137 & 1,946349 & 0,000117 & $-0,001525$ & 0,008776 & $-0,059959$ & \\
\hline 210 & 39 & 228 & 0,058455 & 0,108219 & $-0,6159$ & 0,009221 & $-0,062341$ & 0,053474 & $-0,225929$ & \\
\hline $2 \backslash 1$ & 32 & 664 & 0,169992 & 0,089041 & 0,646655 & 0,007587 & $-0,053429$ & 0,155508 & $-0,417529$ & \\
\hline 212 & 23 & 369 & 0,094525 & 0,064384 & 0,384011 & 0,005486 & $-0,041199$ & 0,086471 & $-0,305385$ & \\
\hline 213 & 0 & 116 & 0,029803 & 0,00137 & 3,079899 & 0,000117 & $-0,001525$ & 0,027264 & $-0,141686$ & \\
\hline 310 & 23 & 76 & 0,01957 & 0,064384 & $-1,19085$ & 0,005486 & $-0,041199$ & 0,017903 & $-0,103902$ & \\
\hline $3 \backslash 1$ & 22 & 201 & 0,051548 & 0,061644 & $-0,17887$ & 0,005252 & $-0,039776$ & 0,047156 & $-0,207788$ & \\
\hline 312 & 6 & 150 & 0,038501 & 0,017808 & 0,771021 & 0,001517 & $-0,014209$ & 0,03522 & $-0,170025$ & \\
\hline 313 & 1 & 39 & 0,010105 & 0,00411 & 0,899696 & 0,00035 & $-0,004020$ & 0,009244 & $-0,062464$ & \\
\hline & 364 & 3908 & & & 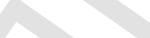 & & $-0,575122$ & & $-3,409362$ & \\
\hline & & & & & . & & 0,575122 & & 3,409362 & \\
\hline & & & & & & & 0,049004 & & 3,118864 & 3,167868 \\
\hline
\end{tabular}




\begin{tabular}{|c|c|c|c|c|c|c|c|c|c|c|}
\hline \multicolumn{11}{|c|}{ 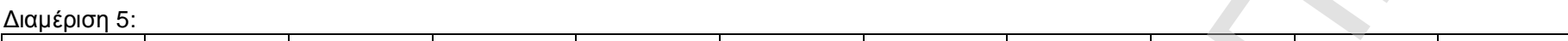 } \\
\hline KIDHYR & BADS & GOODS & $f(x / 1)$ & $f(x / 0)$ & $w(x)$ & & & 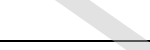 & & \\
\hline $0 \backslash 0$ & 124 & 360 & 0,092223 & 0,341096 & $-1,30795$ & 0,029063 & $-0,148359$ & 0,084365 & $-0,300948$ & \\
\hline $0 \backslash 1$ & 27 & 490 & 0,12548 & 0,075342 & 0,5101 & 0,00642 & $-0,046756$ & 0,114788 & $-0,358478$ & \\
\hline 012 & 42 & 518 & 0,132643 & 0,116438 & 0,130296 & 0,009921 & $-0,066028$ & 0,121341 & $-0,369223$ & \\
\hline 013 & 4 & 58 & 0,014965 & 0,012329 & 0,19381 & 0,00105 & $-0,010394$ & 0,01369 & $-0,084753$ & \\
\hline 110 & 4 & 157 & 0,040292 & 0,012329 & 1,184209 & 0,00105 & $-0,010394$ & 0,036859 & $-0,175515$ & \\
\hline $1 \backslash 1$ & 1 & 304 & 0,077897 & 0,00411 & 2,942066 & 0,00035 & $-0,004020$ & 0,07126 & $-0,271555$ & \\
\hline $1 \backslash 2$ & 10 & 138 & 0,035431 & 0,028767 & 0,208356 & 0,002451 & $-0,021257$ & 0,032412 & $-0,160353$ & \\
\hline 113 & 0 & 43 & 0,011128 & 0,00137 & 2,094769 & 0,000117 & $-0,001525$ & 0,01018 & $-0,067372$ & \\
\hline 210 & 48 & 218 & 0,055897 & 0,132877 & $-0,86592$ & 0,011322 & $-0,073193$ & 0,051134 & $-0,219343$ & \\
\hline $2 \backslash 1$ & 29 & 647 & 0,165643 & 0,080822 & 0,717589 & 0,006887 & $-0,049459$ & 0,15153 & $-0,412513$ & \\
\hline 212 & 19 & 393 & 0,100665 & 0,053425 & 0,633527 & 0,004552 & $-0,035412$ & 0,092088 & $-0,316860$ & \\
\hline 213 & 0 & 117 & 0,030059 & 0,00137 & 3,088446 & 0,000117 & $-0,001525$ & 0,027498 & $-0,142563$ & \\
\hline 310 & 25 & 74 & 0,019059 & 0,069863 & $-1,29902$ & 0,005953 & $-0,044004$ & 0,017435 & $-0,101852$ & \\
\hline $3 \backslash 1$ & 24 & 199 & 0,051036 & 0,067123 & $-0,274$ & 0,005719 & $-0,042609$ & 0,046687 & $-0,206397$ & \\
\hline 312 & 5 & 154 & 0,039524 & 0,015068 & 0,964306 & 0,001284 & $-0,012332$ & 0,036156 & $-0,173175$ & \\
\hline \multirow[t]{4}{*}{313} & 2 & 38 & 0,009849 & 0,006849 & 0,363228 & 0,000584 & $-0,006269$ & 0,00901 & $-0,061216$ & \\
\hline & 364 & 3908 & & & & & $-0,573537$ & & $-3,422116$ & \\
\hline & & & & & - & & 0,573537 & & 3,422116 & \\
\hline & & & & & & & 0,048869 & & 3,130531 & 3,1794 \\
\hline
\end{tabular}




\begin{tabular}{|c|c|c|c|c|c|c|c|c|c|c|}
\hline KIDVINC & BADS & GOODS & $f(x / 1)$ & $f(x / 0)$ & $w(x)$ & & & & 7 & \\
\hline $1 \backslash 1$ & 13 & 77 & 0,019826 & 0,036986 & $-0,62355$ & 0,003151 & $-0,026188$ & 0,018137 & $-0,104920$ & \\
\hline $2 \backslash 1$ & 52 & 309 & 0,079176 & 0,143836 & $-0,59699$ & 0,012256 & $-0,077828$ & 0,07243 & $-0,274312$ & \\
\hline $3 \backslash 1$ & 4 & 115 & 0,029547 & 0,012329 & 0,874054 & 0,00105 & $-0,010394$ & 0,02703 & $-0,140806$ & \\
\hline $4 \backslash 1$ & 17 & 214 & 0,054873 & 0,047945 & 0,134969 & 0,004085 & $-0,032418$ & 0,050198 & $-0,216665$ & \\
\hline 112 & 22 & 67 & 0,017268 & 0,061644 & $-1,27253$ & 0,005252 & $-0,039776$ & 0,015797 & $-0,094530$ & \\
\hline 212 & 91 & 320 & 0,08199 & 0,250685 & $-1,1176$ & 0,02136 & $-0,118525$ & 0,075004 & $-0,280282$ & \\
\hline 312 & 3 & 73 & 0,018803 & 0,009589 & 0,673383 & 0,000817 & $-0,008381$ & 0,017201 & $-0,100820$ & \\
\hline 412 & 41 & 184 & 0,047199 & 0,113699 & $-0,87918$ & 0,009688 & $-0,064808$ & 0,043177 & $-0,195747$ & \\
\hline 113 & 17 & 272 & 0,069711 & 0,047945 & 0,374298 & 0,004085 & $-0,032418$ & 0,063771 & $-0,253232$ & \\
\hline 213 & 53 & 647 & 0,165643 & 0,146575 & 0,122298 & 0,012489 & $-0,078971$ & 0,15153 & $-0,412513$ & \\
\hline 313 & 7 & 348 & 0,089153 & 0,020548 & 1,467596 & 0,001751 & $-0,016033$ & 0,081557 & $-0,294914$ & \\
\hline 413 & 38 & 753 & 0,19276 & 0,105479 & 0,602931 & 0,008987 & $-0,061096$ & 0,176336 & $-0,441475$ & \\
\hline 114 & 0 & 59 & 0,015221 & 0,00137 & 2,407984 & 0,000117 & $-0,001525$ & 0,013924 & $-0,085861$ & \\
\hline 214 & 2 & 145 & 0,037222 & 0,006849 & 1,692746 & 0,000584 & $-0,006269$ & 0,03405 & $-0,166036$ & \\
\hline 314 & 2 & 86 & 0,022128 & 0,006849 & 1,172714 & 0,000584 & $-0,006269$ & 0,020243 & $-0,113896$ & \\
\hline \multirow[t]{4}{*}{$4 \backslash 4$} & 2 & 239 & 0,061269 & 0,006849 & 2,191123 & 0,000584 & $-0,006269$ & 0,056048 & $-0,233003$ & \\
\hline & 364 & 3908 & & 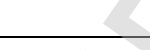 & $\nabla$ & & $-0,587168$ & & $-3,409012$ & \\
\hline & & & & 2 & & & 0,587168 & & 3,409012 & \\
\hline & & & & 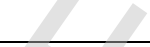 & & & 0,05003 & & 3,118544 & 4 \\
\hline
\end{tabular}




\begin{tabular}{|c|c|c|c|c|c|c|c|c|c|c|}
\hline \multicolumn{11}{|c|}{ 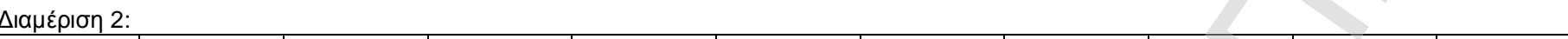 } \\
\hline KIDINC & BADS & GOODS & $f(x / 1)$ & $f(x / 0)$ & $w(x)$ & & & & & \\
\hline $0 \backslash 0$ & 93 & 323 & 0,082758 & 0,256164 & $-1,1299$ & 0,021827 & $-0,120435$ & 0,075706 & $-0,281888$ & \\
\hline $0 \backslash 1$ & 53 & 303 & 0,077641 & 0,146575 & $-0,63544$ & 0,012489 & $-0,078971$ & 0,071026 & $-0,271000$ & \\
\hline 012 & 50 & 668 & 0,171016 & 0,138356 & 0,211924 & 0,011789 & $-0,075524$ & 0,156444 & $-0,418688$ & \\
\hline 013 & 1 & 146 & 0,037478 & 0,00411 & 2,210421 & 0,00035 & $-0,004020$ & 0,034284 & $-0,166838$ & \\
\hline 110 & 3 & 84 & 0,021617 & 0,009589 & 0,812849 & 0,000817 & $-0,008381$ & 0,019775 & $-0,111930$ & \\
\hline $1 \backslash 1$ & 6 & 106 & 0,027245 & 0,017808 & 0,425203 & 0,001517 & $-0,014209$ & 0,024923 & $-0,132751$ & \\
\hline 112 & 7 & 365 & 0,093502 & 0,020548 & 1,515224 & 0,001751 & $-0,016033$ & 0,085535 & $-0,303422$ & \\
\hline 113 & 2 & 101 & 0,025966 & 0,006849 & 1,332629 & 0,000584 & $-0,006269$ & 0,023753 & $-0,128166$ & \\
\hline 210 & 43 & 166 & 0,042594 & 0,119178 & $-1,02891$ & 0,010155 & $-0,067241$ & 0,038965 & $-0,182421$ & \\
\hline $2 \backslash 1$ & 20 & 214 & 0,054873 & 0,056164 & $-0,02325$ & 0,004786 & $-0,036883$ & 0,050198 & $-0,216665$ & \\
\hline 212 & 33 & 742 & 0,189946 & 0,091781 & 0,727338 & 0,00782 & $-0,054731$ & 0,173762 & $-0,438717$ & \\
\hline 213 & 1 & 225 & 0,057687 & 0,00411 & 2,641716 & 0,00035 & $-0,004020$ & 0,052772 & $-0,223969$ & \\
\hline 310 & 22 & 66 & 0,017012 & 0,061644 & $-1,28745$ & 0,005252 & $-0,039776$ & 0,015562 & $-0,093465$ & \\
\hline $3 \backslash 1$ & 13 & 87 & 0,022384 & 0,036986 & $-0,50219$ & 0,003151 & $-0,026188$ & 0,020477 & $-0,114873$ & \\
\hline 312 & 17 & 254 & 0,065106 & 0,047945 & 0,30596 & 0,004085 & $-0,032418$ & 0,059559 & $-0,242377$ & \\
\hline \multirow[t]{4}{*}{313} & 0 & 58 & 0,014965 & 0,00137 & 2,391034 & 0,000117 & $-0,001525$ & 0,01369 & $-0,084753$ & \\
\hline & 364 & 3908 & & & & & $-0,586623$ & & $-3,411922$ & \\
\hline & & & & & 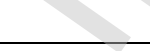 & & 0,586623 & & 3,411922 & \\
\hline & & & & & & & 0,049984 & & 3,121206 & 3,17119 \\
\hline
\end{tabular}




\begin{tabular}{|c|c|c|c|c|c|c|c|c|c|c|}
\hline KIDINC & RADS & $c_{1}$ & $f(x / 1)$ & $f(x / 0)$ & 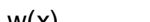 & & & & & \\
\hline 010 & 103 & 323 & 0,082758 & 0,283562 & $-1,23151$ & 0,024161 & $-0,129774$ & 0.075706 & $-0,281888$ & \\
\hline $0 \backslash 1$ & 56 & 323 & 0,082758 & 0,154795 & $-0,62618$ & 0,013189 & $-0,082361$ & 0,075706 & $-0,281888$ & \\
\hline 012 & 46 & 650 & 0,166411 & 0,127397 & 0,267149 & 0,010855 & $-0,070834$ & 0,152232 & $-0,413409$ & \\
\hline 013 & 2 & 139 & 0,035687 & 0,006849 & 1,650634 & 0,000584 & $-0,006269$ & 0,032646 & $-0,161172$ & \\
\hline 110 & 4 & 82 & 0,021105 & 0,012329 & 0,537581 & 0,00105 & $-0,010394$ & 0,019307 & $-0,109948$ & \\
\hline $1 \backslash 1$ & 5 & 104 & 0,026733 & 0,015068 & 0,573299 & 0,001284 & $-0,012332$ & 0,024455 & $-0,130927$ & \\
\hline 112 & 7 & 357 & 0,091456 & 0,020548 & 1,493093 & 0,001751 & $-0,016033$ & 0,083663 & $-0,299452$ & \\
\hline 113 & 2 & 94 & 0,024175 & 0,006849 & 1,26117 & 0,000584 & $-0,006269$ & 0,022115 & $-0,121607$ & \\
\hline 210 & 36 & 171 & 0,043873 & 0,1 & $-0,82387$ & 0,008521 & $-0,058578$ & 0,040135 & $-0,186186$ & \\
\hline $2 \backslash 1$ & 20 & 199 & 0,051036 & 0,056164 & $-0,09575$ & 0,004786 & $-0,036883$ & 0,046687 & $-0,206397$ & \\
\hline 212 & 33 & 758 & 0,194039 & 0,091781 & 0,748658 & 0,00782 & $-0,054731$ & 0,177506 & $-0,442711$ & \\
\hline 213 & 2 & 227 & 0,058199 & 0,006849 & 2,13972 & 0,000584 & $-0,006269$ & 0,05324 & $-0,225277$ & \\
\hline 310 & 23 & 60 & 0,015477 & 0,064384 & $-1,4255$ & 0,005486 & $-0,041199$ & 0,014158 & $-0,086964$ & \\
\hline $3 \backslash 1$ & 9 & 82 & 0,021105 & 0,026027 & $-0,20963$ & 0,002218 & $-0,019553$ & 0,019307 & $-0,109948$ & \\
\hline 312 & 16 & 271 & 0,069455 & 0,045205 & 0,429462 & 0,003852 & $-0,030892$ & 0,063537 & $-0,252640$ & \\
\hline 313 & 0 & 68 & 0,017524 & 0,00137 & 2,548841 & 0,000117 & $-0,001525$ & 0,016031 & $-0,095591$ & \\
\hline & 364 & 3908 & & & 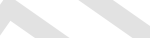 & & $-0,583898$ & & $-3,406004$ & \\
\hline & & & & & 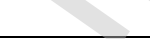 & & 0,583898 & & 3,406004 & \\
\hline & & & & & & & 0,049752 & & 3,115792 & 3,165543 \\
\hline
\end{tabular}




\begin{tabular}{|c|c|c|c|c|c|c|c|c|c|c|}
\hline KIDINC & & (1) & $f(y / 1)$ & $f(x / 0)$ & (y) & & & & & \\
\hline $0 \backslash 0$ & 100 & 317 & 0081223 & 0275342 & -122082 & 0023461 & -0.127008 & 0074302 & -0278667 & \\
\hline $0 \backslash 1$ & 55 & 335 & 0,085828 & 0,152055 & $-0,5719$ & 0,012956 & $-0,081237$ & 0,078515 & $-0,288219$ & \\
\hline 012 & 44 & 649 & 0,166155 & 0,121918 & 0,309574 & 0,010388 & $-0,068447$ & 0,151998 & $-0,413111$ & \\
\hline 013 & 2 & 141 & 0,036199 & 0,006849 & 1,664869 & 0,000584 & $-0,006269$ & 0,033114 & $-0,162803$ & \\
\hline $1 \backslash 0$ & 2 & 78 & 0,020082 & 0,006849 & 1,075668 & 0,000584 & $-0,006269$ & 0,018371 & $-0,105934$ & \\
\hline $1 \backslash 1$ & 4 & 95 & 0,024431 & 0,012329 & 0,683909 & 0,00105 & $-0,010394$ & 0,022349 & $-0,122555$ & \\
\hline 112 & 9 & 355 & 0,090944 & 0,026027 & 1,251094 & 0,002218 & $-0,019553$ & 0,083195 & $-0,298450$ & \\
\hline 113 & 2 & 95 & 0,024431 & 0,006849 & 1,271696 & 0,000584 & $-0,006269$ & 0,022349 & $-0,122555$ & \\
\hline 210 & 41 & 183 & 0,046943 & 0,113699 & $-0,88462$ & 0,009688 & $-0,064808$ & 0,042943 & $-0,195023$ & \\
\hline $2 \backslash 1$ & 18 & 200 & 0,051292 & 0,050685 & 0,011904 & 0,004319 & $-0,033924$ & 0,046922 & $-0,207093$ & \\
\hline 212 & 33 & 759 & 0,194295 & 0,091781 & 0,749975 & 0,00782 & $-0,054731$ & 0,17774 & $-0,442957$ & \\
\hline 213 & 2 & 235 & 0,060246 & 0,006849 & 2,174281 & 0,000584 & $-0,006269$ & 0,055112 & $-0,230451$ & \\
\hline 310 & 24 & 56 & 0,014454 & 0,067123 & $-1,53557$ & 0,005719 & $-0,042609$ & 0,013222 & $-0,082519$ & \\
\hline $3 \backslash 1$ & 12 & 82 & 0,021105 & 0,034247 & $-0,48407$ & 0,002918 & $-0,024572$ & 0,019307 & $-0,109948$ & \\
\hline 312 & 16 & 271 & 0,069455 & 0,045205 & 0,429462 & 0,003852 & $-0,030892$ & 0,063537 & $-0,252640$ & \\
\hline 313 & 0 & 57 & 0,01471 & 0,00137 & 2,373793 & 0,000117 & $-0,001525$ & 0,013456 & $-0,083639$ & \\
\hline & 364 & 3908 & & & 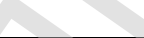 & & $-0,584776$ & & $-3,396562$ & \\
\hline & & & & & & & 0,584776 & & 3,396562 & \\
\hline & & & & & & & 0,049826 & & 3,107154 & 3,156981 \\
\hline
\end{tabular}




\begin{tabular}{|c|c|c|c|c|c|c|c|c|c|c|}
\hline KIDINC & BADS & בחת & $f(x / 1)$ & $f(x / 0)$ & $w(x)$ & & & 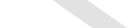 & & \\
\hline 010 & 97 & 311 & 0,079688 & 0,267123 & $-1,20959$ & 0,022761 & $-0,124211$ & 0,072898 & $-0,275407$ & \\
\hline $0 \backslash 1$ & 56 & 313 & 0,0802 & 0,154795 & $-0,65758$ & 0,013189 & $-0,082361$ & 0,073366 & $-0,276498$ & \\
\hline 012 & 43 & 655 & 0,16769 & 0,119178 & 0,341498 & 0,010155 & $-0,067241$ & 0,153402 & $-0,414892$ & \\
\hline 013 & 1 & 147 & 0,037733 & 0,00411 & 2,217224 & 0,00035 & $-0,004020$ & 0,034518 & $-0,167638$ & \\
\hline $1 \backslash 0$ & 4 & 84 & 0,021617 & 0,012329 & 0,561535 & 0,00105 & $-0,010394$ & 0,019775 & $-0,111930$ & \\
\hline $1 \backslash 1$ & 5 & 110 & 0,028268 & 0,015068 & 0,629128 & 0,001284 & $-0,012332$ & 0,025859 & $-0,136361$ & \\
\hline 112 & 6 & 356 & 0,0912 & 0,017808 & 1,633393 & 0,001517 & $-0,014209$ & 0,083429 & $-0,298952$ & \\
\hline 113 & 0 & 92 & 0,023663 & 0,00137 & 2,849216 & 0,000117 & $-0,001525$ & 0,021647 & $-0,119702$ & \\
\hline 210 & 40 & 181 & 0,046431 & 0,110959 & $-0,87119$ & 0,009454 & $-0,063579$ & 0,042475 & $-0,193569$ & \\
\hline $2 \backslash 1$ & 17 & 208 & 0,053338 & 0,047945 & 0,106599 & 0,004085 & $-0,032418$ & 0,048794 & $-0,212602$ & \\
\hline 212 & 38 & 754 & 0,193016 & 0,105479 & 0,604258 & 0,008987 & $-0,061096$ & 0,17657 & $-0,441723$ & \\
\hline 213 & 1 & 232 & 0,059478 & 0,00411 & 2,672286 & 0,00035 & $-0,004020$ & 0,05441 & $-0,228522$ & \\
\hline 310 & 25 & 60 & 0,015477 & 0,069863 & $-1,50717$ & 0,005953 & $-0,044004$ & 0,014158 & $-0,086964$ & \\
\hline $3 \backslash 1$ & 13 & 78 & 0,020082 & 0,036986 & $-0,61073$ & 0,003151 & $-0,026188$ & 0,018371 & $-0,105934$ & \\
\hline 312 & 18 & 266 & 0,068176 & 0,050685 & 0,296464 & 0,004319 & $-0,033924$ & 0,062367 & $-0,249660$ & \\
\hline \multirow[t]{4}{*}{313} & 0 & 61 & 0,015733 & 0,00137 & 2,441045 & 0,000117 & $-0,001525$ & 0,014392 & $-0,088061$ & \\
\hline & 364 & 3908 & & & 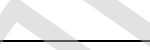 & & $-0,583047$ & & $-3,408413$ & \\
\hline & & & & & 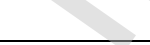 & & 0,583047 & & 3,408413 & \\
\hline & & & & & & & 0,049679 & & 3,117996 & 3,167675 \\
\hline
\end{tabular}




\begin{tabular}{|c|c|c|c|c|c|c|c|c|c|c|}
\hline & & & & & & & & & & \\
\hline KIDIJOB & BADS & GOODS & $f(x / 1)$ & $f(x / 0)$ & $w(x)$ & & & & 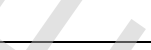 & \\
\hline $1 \backslash 1$ & 9 & 103 & 0,026477 & 0,026027 & 0,01714 & 0,002218 & $-0,019553$ & 0,024221 & $-0,130010$ & \\
\hline $2 \backslash 1$ & 41 & 226 & 0,057943 & 0,113699 & $-0,67409$ & 0,009688 & $-0,064808$ & 0,053006 & $-0,224624$ & \\
\hline $3 \backslash 1$ & 0 & 110 & 0,028268 & 0,00137 & 3,027023 & 0,000117 & $-0,001525$ & 0,025859 & $-0,136361$ & \\
\hline $4 \backslash 1$ & 8 & 235 & 0,060246 & 0,023288 & 0,950505 & 0,001984 & $-0,017813$ & 0,055112 & $-0,230451$ & \\
\hline 112 & 14 & 79 & 0,020338 & 0,039726 & $-0,66953$ & 0,003385 & $-0,027779$ & 0,018605 & $-0,106944$ & \\
\hline 212 & 29 & 103 & 0,026477 & 0,080822 & $-1,11596$ & 0,006887 & $-0,049459$ & 0,024221 & $-0,130010$ & \\
\hline 312 & 16 & 67 & 0,017268 & 0,045205 & $-0,96237$ & 0,003852 & $-0,030892$ & 0,015797 & $-0,094530$ & \\
\hline 412 & 46 & 199 & 0,051036 & 0,127397 & $-0,91478$ & 0,010855 & $-0,070834$ & 0,046687 & $-0,206397$ & \\
\hline 113 & 9 & 107 & 0,027501 & 0,026027 & 0,05506 & 0,002218 & $-0,019553$ & 0,025157 & $-0,133658$ & \\
\hline 213 & 41 & 319 & 0,081734 & 0,113699 & $-0,33008$ & 0,009688 & $-0,064808$ & 0,07477 & $-0,279745$ & \\
\hline 313 & 0 & 136 & 0,034919 & 0,00137 & 3,238332 & 0,000117 & $-0,001525$ & 0,031944 & $-0,158708$ & \\
\hline 413 & 20 & 342 & 0,087618 & 0,056164 & 0,444707 & 0,004786 & $-0,036883$ & 0,080153 & $-0,291844$ & \\
\hline $1 \backslash 4$ & 2 & 14 & 0,003709 & 0,006849 & $-0,61328$ & 0,000584 & $-0,006269$ & 0,003393 & $-0,027836$ & \\
\hline $2 \backslash 4$ & 13 & 138 & 0,035431 & 0,036986 & $-0,04296$ & 0,003151 & $-0,026188$ & 0,032412 & $-0,160353$ & \\
\hline 314 & 0 & 27 & 0,007035 & 0,00137 & 1,636194 & 0,000117 & $-0,001525$ & 0,006436 & $-0,046849$ & \\
\hline $4 \backslash 4$ & 3 & 66 & 0,017012 & 0,009589 & 0,573299 & 0,000817 & $-0,008381$ & 0,015562 & $-0,093465$ & \\
\hline 115 & 14 & 161 & 0,041315 & 0,039726 & 0,039217 & 0,003385 & $-0,027779$ & 0,037795 & $-0,178605$ & \\
\hline 215 & 64 & 534 & 0,136736 & 0,176712 & $-0,25647$ & 0,015057 & $-0,091146$ & 0,125085 & $-0,375132$ & \\
\hline 315 & 0 & 241 & 0,061781 & 0,00137 & 3,808877 & 0,000117 & $-0,001525$ & 0,056516 & $-0,234271$ & \\
\hline 415 & 20 & 482 & 0,123433 & 0,056164 & 0,787417 & 0,004786 & $-0,036883$ & 0,112916 & $-0,355310$ & \\
\hline 116 & 4 & 11 & 0,002942 & 0,012329 & $-1,43287$ & 0,00105 & $-0,010394$ & 0,002691 & $-0,022977$ & \\
\hline 216 & 10 & 101 & 0,025966 & 0,028767 & $-0,10246$ & 0,002451 & $-0,021257$ & 0,023753 & $-0,128166$ & \\
\hline 316 & 0 & 41 & 0,010617 & 0,00137 & 2,047701 & 0,000117 & $-0,001525$ & 0,009712 & $-0,064934$ & \\
\hline 416 & 1 & 66 & 0,017012 & 0,00411 & 1,420597 & 0,00035 & $-0,004020$ & 0,015562 & $-0,093465$ & \\
\hline & 364 & 3908 & & . & & & $-0,642322$ & & $-3,904646$ & \\
\hline & & & & 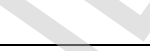 & & & 0,642322 & & 3,904646 & \\
\hline & & & & 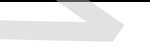 & & & 0,054730 & & 3,571947 & 3,626677 \\
\hline
\end{tabular}




\begin{tabular}{|c|c|c|c|c|c|c|c|c|c|c|}
\hline KIDJOB & BADS & GOODS & $f(x / 1)$ & $f(x / 0)$ & $w(x)$ & & & & 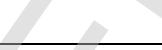 & $\theta$ \\
\hline 010 & 24 & 104 & 0,026733 & 0,067123 & $-0,92063$ & 0,005719 & $-0,042609$ & 0,024455 & $-0,130927$ & \\
\hline 012 & 47 & 243 & 0,062292 & 0,130137 & $-0,73675$ & 0,011088 & $-0,072017$ & 0,056984 & $-0,235533$ & \\
\hline 013 & 38 & 303 & 0,077641 & 0,105479 & $-0,30642$ & 0,008987 & $-0,061096$ & 0,071026 & $-0,271000$ & \\
\hline 014 & 13 & 143 & 0,03671 & 0,036986 & $-0,00749$ & 0,003151 & $-0,026188$ & 0,033582 & $-0,164424$ & \\
\hline 015 & 63 & 541 & 0,138526 & 0,173973 & $-0,22784$ & 0,014824 & $-0,090067$ & 0,126723 & $-0,377666$ & \\
\hline 016 & 12 & 106 & 0,027245 & 0,034247 & $-0,22872$ & 0,002918 & $-0,024572$ & 0,024923 & $-0,132751$ & \\
\hline 110 & 18 & 79 & 0,020338 & 0,050685 & $-0,91315$ & 0,004319 & $-0,033924$ & 0,018605 & $-0,106944$ & \\
\hline 112 & 0 & 106 & 0,027245 & 0,00137 & 2,990153 & 0,000117 & $-0,001525$ & 0,024923 & $-0,132751$ & \\
\hline 113 & 0 & 150 & 0,038501 & 0,00137 & 3,335971 & 0,000117 & $-0,001525$ & 0,03522 & $-0,170025$ & \\
\hline 114 & 0 & 33 & 0,00857 & 0,00137 & 1,833553 & 0,000117 & $-0,001525$ & 0,00784 & $-0,054839$ & \\
\hline 115 & 0 & 255 & 0,065362 & 0,00137 & 3,86523 & 0,000117 & $-0,001525$ & 0,059793 & $-0,242991$ & \\
\hline 116 & 0 & 33 & 0,00857 & 0,00137 & 1,833553 & 0,000117 & $-0,001525$ & 0,00784 & $-0,054839$ & \\
\hline 210 & 44 & 200 & 0,051292 & 0,121918 & $-0,86581$ & 0,010388 & $-0,068447$ & 0,046922 & $-0,207093$ & \\
\hline 212 & 6 & 230 & 0,058966 & 0,017808 & 1,197309 & 0,001517 & $-0,014209$ & 0,053942 & $-0,227228$ & \\
\hline 213 & 21 & 316 & 0,080967 & 0,058904 & 0,318131 & 0,005019 & $-0,038337$ & 0,074068 & $-0,278126$ & \\
\hline 214 & 3 & 66 & 0,017012 & 0,009589 & 0,573299 & 0,000817 & $-0,008381$ & 0,015562 & $-0,093465$ & \\
\hline 215 & 21 & 474 & 0,121387 & 0,058904 & 0,723069 & 0,005019 & $-0,038337$ & 0,111044 & $-0,352097$ & \\
\hline 216 & 2 & 61 & 0,015733 & 0,006849 & 0,831607 & 0,000584 & $-0,006269$ & 0,014392 & $-0,088061$ & \\
\hline 310 & 14 & 85 & 0,021873 & 0,039726 & $-0,59677$ & 0,003385 & $-0,027779$ & 0,020009 & $-0,112915$ & \\
\hline 312 & 10 & 98 & 0,025198 & 0,028767 & $-0,13246$ & 0,002451 & $-0,021257$ & 0,023051 & $-0,125376$ & \\
\hline 313 & 10 & 101 & 0,025966 & 0,028767 & $-0,10246$ & 0,002451 & $-0,021257$ & 0,023753 & $-0,128166$ & \\
\hline 314 & 1 & 12 & 0,003198 & 0,00411 & $-0,25088$ & 0,00035 & $-0,004020$ & 0,002925 & $-0,024623$ & \\
\hline 315 & 13 & 156 & 0,040036 & 0,036986 & 0,079227 & 0,003151 & $-0,026188$ & 0,036625 & $-0,174737$ & \\
\hline \multirow[t]{4}{*}{316} & 4 & 13 & 0,003454 & 0,012329 & $-1,27253$ & 0,00105 & $-0,010394$ & 0,003159 & $-0,026242$ & \\
\hline & 364 & 3908 & & & 2 & & $-0,642971$ & & $-3,912818$ & \\
\hline & & & & & 8 & & 0,642971 & & 3,912818 & \\
\hline & & & & 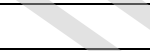 & & & 0,054785 & & 3,579422 & 3,634207 \\
\hline
\end{tabular}




\begin{tabular}{|c|c|c|c|c|c|c|c|c|c|c|}
\hline KIDJOB & BADS & GOODS & $f(x / 1)$ & $f(x / 0)$ & $w(x)$ & & & & 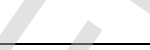 & $\theta$ \\
\hline 010 & 29 & 101 & 0,025966 & 0,080822 & $-1,13547$ & 0,006887 & $-0,049459$ & 0,023753 & $-0,128166$ & \\
\hline 012 & 50 & 224 & 0,057432 & 0,138356 & $-0,87924$ & 0,011789 & $-0,075524$ & 0,052538 & $-0,223313$ & \\
\hline 013 & 47 & 349 & 0,089409 & 0,130137 & $-0,37537$ & 0,011088 & $-0,072017$ & 0,081791 & $-0,295422$ & \\
\hline 014 & 16 & 147 & 0,037733 & 0,045205 & $-0,18067$ & 0,003852 & $-0,030892$ & 0,034518 & $-0,167638$ & \\
\hline 015 & 55 & 503 & 0,128805 & 0,152055 & $-0,16594$ & 0,012956 & $-0,081237$ & 0,11783 & $-0,363532$ & \\
\hline 016 & 10 & 111 & 0,028524 & 0,028767 & $-0,00849$ & 0,002451 & $-0,021257$ & 0,026094 & $-0,137256$ & \\
\hline 110 & 18 & 64 & 0,0165 & 0,050685 & $-1,12225$ & 0,004319 & $-0,033924$ & 0,015094 & $-0,091319$ & \\
\hline 112 & 0 & 104 & 0,026733 & 0,00137 & 2,971195 & 0,000117 & $-0,001525$ & 0,024455 & $-0,130927$ & \\
\hline 113 & 0 & 160 & 0,041059 & 0,00137 & 3,400302 & 0,000117 & $-0,001525$ & 0,037561 & $-0,177836$ & \\
\hline 114 & 0 & 38 & 0,009849 & 0,00137 & 1,972666 & 0,000117 & $-0,001525$ & 0,00901 & $-0,061216$ & \\
\hline 115 & 0 & 235 & 0,060246 & 0,00137 & 3,783719 & 0,000117 & $-0,001525$ & 0,055112 & $-0,230451$ & \\
\hline 116 & 0 & 36 & 0,009337 & 0,00137 & 1,91932 & 0,000117 & $-0,001525$ & 0,008542 & $-0,058693$ & \\
\hline 210 & 40 & 193 & 0,049501 & 0,110959 & $-0,80716$ & 0,009454 & $-0,063579$ & 0,045283 & $-0,202185$ & \\
\hline 212 & 9 & 226 & 0,057943 & 0,026027 & 0,800314 & 0,002218 & $-0,019553$ & 0,053006 & $-0,224624$ & \\
\hline 213 & 20 & 355 & 0,090944 & 0,056164 & 0,481961 & 0,004786 & $-0,036883$ & 0,083195 & $-0,298450$ & \\
\hline 214 & 2 & 60 & 0,015477 & 0,006849 & 0,815213 & 0,000584 & $-0,006269$ & 0,014158 & $-0,086964$ & \\
\hline 215 & 17 & 460 & 0,117805 & 0,047945 & 0,898972 & 0,004085 & $-0,032418$ & 0,107767 & $-0,346365$ & \\
\hline 216 & 3 & 61 & 0,015733 & 0,009589 & 0,495135 & 0,000817 & $-0,008381$ & 0,014392 & $-0,088061$ & \\
\hline 310 & 13 & 89 & 0,022896 & 0,036986 & $-0,47959$ & 0,003151 & $-0,026188$ & 0,020945 & $-0,116816$ & \\
\hline 312 & 8 & 100 & 0,02571 & 0,023288 & 0,098952 & 0,001984 & $-0,017813$ & 0,023519 & $-0,127240$ & \\
\hline 313 & 15 & 115 & 0,029547 & 0,042466 & $-0,36271$ & 0,003618 & $-0,029346$ & 0,02703 & $-0,140806$ & \\
\hline 314 & 2 & 11 & 0,002942 & 0,006849 & $-0,84508$ & 0,000584 & $-0,006269$ & 0,002691 & $-0,022977$ & \\
\hline 315 & 8 & 153 & 0,039268 & 0,023288 & 0,522495 & 0,001984 & $-0,017813$ & 0,035922 & $-0,172391$ & \\
\hline \multirow[t]{4}{*}{316} & 2 & 13 & 0,003454 & 0,006849 & $-0,68474$ & 0,000584 & $-0,006269$ & 0,003159 & $-0,026242$ & \\
\hline & 364 & 3908 & & & 2 & & $-0,642716$ & & $-3,918886$ & \\
\hline & & & & & 8 & & 0,642716 & & 3,918886 & \\
\hline & & & & -1 & & & 0,054763 & & 3,584974 & 3,639737 \\
\hline
\end{tabular}




\begin{tabular}{|c|c|c|c|c|c|c|c|c|c|c|}
\hline KIDJOB & BADS & GOODS & $f(x / 1)$ & $f(x / 0)$ & $w(x)$ & & & & 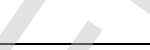 & $\theta$ \\
\hline 010 & 24 & 106 & 0,027245 & 0,067123 & $-0,90167$ & 0,005719 & $-0,042609$ & 0,024923 & $-0,132751$ & \\
\hline 012 & 49 & 260 & 0,066641 & 0,135616 & $-0,71051$ & 0,011555 & $-0,074362$ & 0,060963 & $-0,246042$ & \\
\hline 013 & 46 & 323 & 0,082758 & 0,127397 & $-0,43139$ & 0,010855 & $-0,070834$ & 0,075706 & $-0,281888$ & \\
\hline $0 \backslash 4$ & 15 & 150 & 0,038501 & 0,042466 & $-0,09802$ & 0,003618 & $-0,029346$ & 0,03522 & $-0,170025$ & \\
\hline 015 & 55 & 499 & 0,127782 & 0,152055 & $-0,17391$ & 0,012956 & $-0,081237$ & 0,116894 & $-0,361989$ & \\
\hline 016 & 12 & 104 & 0,026733 & 0,034247 & $-0,24768$ & 0,002918 & $-0,024572$ & 0,024455 & $-0,130927$ & \\
\hline 110 & 17 & 72 & 0,018547 & 0,047945 & $-0,94975$ & 0,004085 & $-0,032418$ & 0,016967 & $-0,099783$ & \\
\hline 112 & 0 & 121 & 0,031082 & 0,00137 & 3,121922 & 0,000117 & $-0,001525$ & 0,028434 & $-0,146043$ & \\
\hline 113 & 0 & 133 & 0,034152 & 0,00137 & 3,216109 & 0,000117 & $-0,001525$ & 0,031242 & $-0,156222$ & \\
\hline 114 & 0 & 32 & 0,008314 & 0,00137 & 1,803248 & 0,000117 & $-0,001525$ & 0,007606 & $-0,053534$ & \\
\hline 115 & 0 & 229 & 0,058711 & 0,00137 & 3,757911 & 0,000117 & $-0,001525$ & 0,053708 & $-0,226579$ & \\
\hline 116 & 0 & 36 & 0,009337 & 0,00137 & 1,91932 & 0,000117 & $-0,001525$ & 0,008542 & $-0,058693$ & \\
\hline 210 & 44 & 208 & 0,053338 & 0,121918 & $-0,82669$ & 0,010388 & $-0,068447$ & 0,048794 & $-0,212602$ & \\
\hline 212 & 6 & 271 & 0,069455 & 0,017808 & 1,36102 & 0,001517 & $-0,014209$ & 0,063537 & $-0,252640$ & \\
\hline 213 & 22 & 327 & 0,083781 & 0,061644 & 0,306833 & 0,005252 & $-0,039776$ & 0,076642 & $-0,284015$ & \\
\hline 214 & 2 & 58 & 0,014965 & 0,006849 & 0,781597 & 0,000584 & $-0,006269$ & 0,01369 & $-0,084753$ & \\
\hline 215 & 17 & 446 & 0,114224 & 0,047945 & 0,868099 & 0,004085 & $-0,032418$ & 0,104491 & $-0,340489$ & \\
\hline 216 & 3 & 67 & 0,017268 & 0,009589 & 0,588225 & 0,000817 & $-0,008381$ & 0,015797 & $-0,094530$ & \\
\hline 310 & 12 & 79 & 0,020338 & 0,034247 & $-0,52111$ & 0,002918 & $-0,024572$ & 0,018605 & $-0,106944$ & \\
\hline 312 & 9 & 112 & 0,02878 & 0,026027 & 0,100522 & 0,002218 & $-0,019553$ & 0,026328 & $-0,138148$ & \\
\hline 313 & 15 & 95 & 0,024431 & 0,042466 & $-0,55285$ & 0,003618 & $-0,029346$ & 0,022349 & $-0,122555$ & \\
\hline 314 & 1 & 13 & 0,003454 & 0,00411 & $-0,17391$ & 0,00035 & $-0,004020$ & 0,003159 & $-0,026242$ & \\
\hline 315 & 12 & 153 & 0,039268 & 0,034247 & 0,136832 & 0,002918 & $-0,024572$ & 0,035922 & $-0,172391$ & \\
\hline \multirow[t]{4}{*}{316} & 3 & 14 & 0,003709 & 0,009589 & $-0,94975$ & 0,000817 & $-0,008381$ & 0,003393 & $-0,027836$ & \\
\hline & 364 & 3908 & & & 2 & & $-0,642945$ & & $-3,927619$ & \\
\hline & & & & & 8 & & 0,642945 & & 3,927619 & \\
\hline & & & & 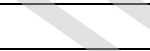 & & & 0,054783 & & 3,592962 & 3,647745 \\
\hline
\end{tabular}




\begin{tabular}{|c|c|c|c|c|c|c|c|c|c|c|}
\hline KIDJOB & BADS & GOODS & $f(x / 1)$ & $f(x / 0)$ & $w(x)$ & & & & 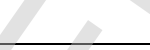 & $\theta$ \\
\hline 010 & 22 & 114 & 0,029291 & 0,061644 & $-0,74408$ & 0,005252 & $-0,039776$ & 0,026796 & $-0,139923$ & \\
\hline 012 & 48 & 235 & 0,060246 & 0,132877 & $-0,79099$ & 0,011322 & $-0,073193$ & 0,055112 & $-0,230451$ & \\
\hline 013 & 43 & 307 & 0,078665 & 0,119178 & $-0,41543$ & 0,010155 & $-0,067241$ & 0,071962 & $-0,273212$ & \\
\hline 014 & 15 & 138 & 0,035431 & 0,042466 & $-0,18111$ & 0,003618 & $-0,029346$ & 0,032412 & $-0,160353$ & \\
\hline 015 & 57 & 526 & 0,134689 & 0,157534 & $-0,15667$ & 0,013423 & $-0,083479$ & 0,123213 & $-0,372198$ & \\
\hline 016 & 12 & 106 & 0,027245 & 0,034247 & $-0,22872$ & 0,002918 & $-0,024572$ & 0,024923 & $-0,132751$ & \\
\hline 110 & 15 & 66 & 0,017012 & 0,042466 & $-0,91478$ & 0,003618 & $-0,029346$ & 0,015562 & $-0,093465$ & \\
\hline 112 & 0 & 112 & 0,02878 & 0,00137 & 3,044961 & 0,000117 & $-0,001525$ & 0,026328 & $-0,138148$ & \\
\hline 113 & 0 & 147 & 0,037733 & 0,00137 & 3,315836 & 0,000117 & $-0,001525$ & 0,034518 & $-0,167638$ & \\
\hline 114 & 0 & 30 & 0,007803 & 0,00137 & 1,739734 & 0,000117 & $-0,001525$ & 0,007138 & $-0,050894$ & \\
\hline 115 & 0 & 249 & 0,063827 & 0,00137 & 3,841467 & 0,000117 & $-0,001525$ & 0,058389 & $-0,239286$ & \\
\hline 116 & 0 & 38 & 0,009849 & 0,00137 & 1,972666 & 0,000117 & $-0,001525$ & 0,00901 & $-0,061216$ & \\
\hline 210 & 42 & 192 & 0,049245 & 0,116438 & $-0,86055$ & 0,009921 & $-0,066028$ & 0,045049 & $-0,201476$ & \\
\hline 212 & 7 & 235 & 0,060246 & 0,020548 & 1,075668 & 0,001751 & $-0,016033$ & 0,055112 & $-0,230451$ & \\
\hline 213 & 25 & 330 & 0,084548 & 0,069863 & 0,190789 & 0,005953 & $-0,044004$ & 0,077344 & $-0,285599$ & \\
\hline 214 & 2 & 58 & 0,014965 & 0,006849 & 0,781597 & 0,000584 & $-0,006269$ & 0,01369 & $-0,084753$ & \\
\hline 215 & 17 & 491 & 0,125735 & 0,047945 & 0,964122 & 0,004085 & $-0,032418$ & 0,115022 & $-0,358871$ & \\
\hline 216 & 3 & 69 & 0,017779 & 0,009589 & 0,617424 & 0,000817 & $-0,008381$ & 0,016265 & $-0,096646$ & \\
\hline 310 & 15 & 92 & 0,023663 & 0,042466 & $-0,58477$ & 0,003618 & $-0,029346$ & 0,021647 & $-0,119702$ & \\
\hline 312 & 8 & 102 & 0,026222 & 0,023288 & 0,118657 & 0,001984 & $-0,017813$ & 0,023987 & $-0,129090$ & \\
\hline 313 & 15 & 90 & 0,023152 & 0,042466 & $-0,60663$ & 0,003618 & $-0,029346$ & 0,021179 & $-0,117781$ & \\
\hline 314 & 2 & 14 & 0,003709 & 0,006849 & $-0,61328$ & 0,000584 & $-0,006269$ & 0,003393 & $-0,027836$ & \\
\hline 315 & 13 & 154 & 0,039524 & 0,036986 & 0,066365 & 0,003151 & $-0,026188$ & 0,036156 & $-0,173175$ & \\
\hline \multirow[t]{4}{*}{316} & 3 & 13 & 0,003454 & 0,009589 & $-1,02121$ & 0,000817 & $-0,008381$ & 0,003159 & $-0,026242$ & \\
\hline & 364 & 3908 & & & 2 & & $-0,645056$ & & $-3,911157$ & \\
\hline & & & & & 8 & & 0,645056 & & 3,911157 & \\
\hline & & & & -1 & & & 0,054963 & & 3,577903 & 3,632865 \\
\hline
\end{tabular}




\begin{tabular}{|c|c|c|c|c|c|c|c|c|c|}
\hline KID\JYR & BADS & GOODS & $f(x / 1)$ & $f(x / 0)$ & $w(x)$ & & & & \\
\hline $1 \backslash 1$ & 8 & 72 & 0,018547 & 0,023288 & $-0,22762$ & 0,001984 & $-0,017813$ & 0,016967 & $-0,099783$ \\
\hline 211 & 30 & 223 & 0,057176 & 0,083562 & $-0,37945$ & 0,00712 & $-0,050793$ & 0,052304 & $-0,222655$ \\
\hline $3 \backslash 1$ & 0 & 122 & 0,031338 & 0,00137 & 3,130119 & 0,000117 & $-0,001525$ & 0,028668 & $-0,146906$ \\
\hline $4 \backslash 1$ & 18 & 234 & 0,05999 & 0,050685 & 0,168545 & 0,004319 & $-0,033924$ & 0,054878 & $-0,229809$ \\
\hline 112 & 12 & 66 & 0,017012 & 0,034247 & $-0,69967$ & 0,002918 & $-0,024572$ & 0,015562 & $-0,093465$ \\
\hline 212 & 79 & 431 & 0,110386 & 0,217808 & $-0,67963$ & 0,018559 & $-0,106745$ & 0,100981 & $-0,334029$ \\
\hline 312 & 6 & 106 & 0,027245 & 0,017808 & 0,425203 & 0,001517 & $-0,014209$ & 0,024923 & $-0,132751$ \\
\hline 412 & 24 & 199 & 0,051036 & 0,067123 & $-0,274$ & 0,005719 & $-0,042609$ & 0,046687 & $-0,206397$ \\
\hline 113 & 15 & 92 & 0,023663 & 0,042466 & $-0,58477$ & 0,003618 & $-0,029346$ & 0,021647 & $-0,119702$ \\
\hline 213 & 64 & 483 & 0,123689 & 0,176712 & $-0,35675$ & 0,015057 & $-0,091146$ & 0,11315 & $-0,355708$ \\
\hline 313 & 7 & 146 & 0,037478 & 0,020548 & 0,600983 & 0,001751 & $-0,016033$ & 0,034284 & $-0,166838$ \\
\hline 413 & 30 & 287 & 0,073548 & 0,083562 & $-0,12764$ & 0,00712 & $-0,050793$ & 0,067281 & $-0,261970$ \\
\hline $1 \backslash 4$ & 17 & 245 & 0,062804 & 0,047945 & 0,269957 & 0,004085 & $-0,032418$ & 0,057453 & $-0,236790$ \\
\hline $2 \backslash 4$ & 25 & 284 & 0,072781 & 0,069863 & 0,040915 & 0,005953 & $-0,044004$ & 0,066579 & $-0,260244$ \\
\hline $3 \backslash 4$ & 3 & 248 & 0,063571 & 0,009589 & 1,89154 & 0,000817 & $-0,008381$ & 0,058155 & $-0,238664$ \\
\hline \multirow[t]{4}{*}{$4 \backslash 4$} & 26 & 670 & 0,171527 & 0,072603 & 0,859739 & 0,006186 & $-0,045386$ & 0,156912 & $-0,419265$ \\
\hline & 364 & 3908 & & & 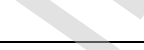 & & $-0,609697$ & & $-3,524977$ \\
\hline & & & & 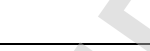 & & & 0,609697 & & 3,524977 \\
\hline & & & & 0 & & & 0,05195 & & 3,224627 \\
\hline
\end{tabular}




\begin{tabular}{|c|c|c|c|c|c|c|c|c|c|c|}
\hline KIDJYR & BADS & תחת & $f(x / 1)$ & $f(x / 0)$ & $w(x)$ & & & & & \\
\hline 010 & 77 & 442 & 0,1132 & 0,212329 & $-0,62898$ & 0,018092 & $-0,104724$ & 0,103555 & $-0,338783$ & \\
\hline $0 \backslash 1$ & 65 & 535 & 0,136992 & 0,179452 & $-0,26999$ & 0,01529 & $-0,092220$ & 0,125319 & $-0,375496$ & \\
\hline 012 & 28 & 179 & 0,04592 & 0,078082 & $-0,53087$ & 0,006653 & $-0,048113$ & 0,042007 & $-0,192108$ & \\
\hline 013 & 27 & 284 & 0,072781 & 0,075342 & $-0,03459$ & 0,00642 & $-0,046756$ & 0,066579 & $-0,260244$ & \\
\hline 110 & 4 & 108 & 0,027756 & 0,012329 & 0,811533 & 0,00105 & $-0,010394$ & 0,025391 & $-0,134562$ & \\
\hline $1 \backslash 1$ & 7 & 181 & 0,046431 & 0,020548 & 0,815213 & 0,001751 & $-0,016033$ & 0,042475 & $-0,193569$ & \\
\hline 112 & 1 & 102 & 0,026222 & 0,00411 & 1,853258 & 0,00035 & $-0,004020$ & 0,023987 & $-0,129090$ & \\
\hline 113 & 6 & 265 & 0,06792 & 0,017808 & 1,338673 & 0,001517 & $-0,014209$ & 0,062133 & $-0,249060$ & \\
\hline 210 & 23 & 182 & 0,046687 & 0,064384 & $-0,32139$ & 0,005486 & $-0,041199$ & 0,042709 & $-0,194297$ & \\
\hline $2 \backslash 1$ & 37 & 315 & 0,080711 & 0,10274 & $-0,24132$ & 0,008754 & $-0,059841$ & 0,073834 & $-0,277584$ & \\
\hline 212 & 12 & 211 & 0,054106 & 0,034247 & 0,457357 & 0,002918 & $-0,024572$ & 0,049496 & $-0,214641$ & \\
\hline 213 & 25 & 639 & 0,163597 & 0,069863 & 0,850869 & 0,005953 & $-0,044004$ & 0,149657 & $-0,410101$ & \\
\hline 310 & 14 & 66 & 0,017012 & 0,039726 & $-0,84809$ & 0,003385 & $-0,027779$ & 0,015562 & $-0,093465$ & \\
\hline $3 \backslash 1$ & 14 & 103 & 0,026477 & 0,039726 & $-0,40572$ & 0,003385 & $-0,027779$ & 0,024221 & $-0,130010$ & \\
\hline 312 & 5 & 66 & 0,017012 & 0,015068 & 0,121314 & 0,001284 & $-0,012332$ & 0,015562 & $-0,093465$ & \\
\hline \multirow[t]{4}{*}{313} & 19 & 230 & 0,058966 & 0,053425 & 0,098697 & 0,004552 & $-0,035412$ & 0,053942 & $-0,227228$ & \\
\hline & 364 & 3908 & & & 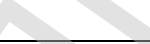 & & $-0,609388$ & & $-3,513704$ & \\
\hline & & & & & & & 0,609388 & & 3,513704 & \\
\hline & & & & & & & 0,051924 & & 3,214315 & 3,266239 \\
\hline
\end{tabular}




\begin{tabular}{|c|c|c|c|c|c|c|c|c|c|c|}
\hline & & & & & & & & & & \\
\hline KIDJYR & BADS & GOODS & $f(x / 1)$ & $f(x / 0)$ & $w(x)$ & & & 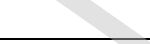 & & \\
\hline 010 & 88 & 435 & 0,11141 & 0,242466 & $-0,77765$ & 0,02066 & $-0,115632$ & 0,101917 & $-0,335769$ & \\
\hline $0 \backslash 1$ & 65 & 543 & 0,139038 & 0,179452 & $-0,25516$ & 0,01529 & $-0,092220$ & 0,127191 & $-0,378385$ & \\
\hline 012 & 27 & 188 & 0,048222 & 0,075342 & $-0,44623$ & 0,00642 & $-0,046756$ & 0,044113 & $-0,198626$ & \\
\hline 013 & 27 & 269 & 0,068943 & 0,075342 & $-0,08876$ & 0,00642 & $-0,046756$ & 0,063069 & $-0,251452$ & \\
\hline 110 & 6 & 109 & 0,028012 & 0,017808 & 0,452983 & 0,001517 & $-0,014209$ & 0,025625 & $-0,135463$ & \\
\hline $1 \backslash 1$ & 5 & 176 & 0,045152 & 0,015068 & 1,097433 & 0,001284 & $-0,012332$ & 0,041305 & $-0,189901$ & \\
\hline 112 & 1 & 95 & 0,024431 & 0,00411 & 1,782522 & 0,00035 & $-0,004020$ & 0,022349 & $-0,122555$ & \\
\hline 113 & 6 & 257 & 0,065874 & 0,017808 & 1,308078 & 0,001517 & $-0,014209$ & 0,060261 & $-0,244215$ & \\
\hline 210 & 23 & 202 & 0,051804 & 0,064384 & $-0,2174$ & 0,005486 & $-0,041199$ & 0,04739 & $-0,208480$ & \\
\hline $2 \backslash 1$ & 26 & 297 & 0,076106 & 0,072603 & 0,04713 & 0,006186 & $-0,045386$ & 0,069622 & $-0,267648$ & \\
\hline 212 & 14 & 204 & 0,052315 & 0,039726 & 0,27528 & 0,003385 & $-0,027779$ & 0,047858 & $-0,209861$ & \\
\hline 213 & 28 & 652 & 0,166922 & 0,078082 & 0,759768 & 0,006653 & $-0,048113$ & 0,1527 & $-0,414004$ & \\
\hline 310 & 11 & 68 & 0,017524 & 0,031507 & $-0,58665$ & 0,002685 & $-0,022929$ & 0,016031 & $-0,095591$ & \\
\hline $3 \backslash 1$ & 12 & 98 & 0,025198 & 0,034247 & $-0,30681$ & 0,002918 & $-0,024572$ & 0,023051 & $-0,125376$ & \\
\hline 312 & 5 & 63 & 0,016245 & 0,015068 & 0,075152 & 0,001284 & $-0,012332$ & 0,01486 & $-0,090238$ & \\
\hline 313 & 20 & 252 & 0,064595 & 0,056164 & 0,139847 & 0,004786 & $-0,036883$ & 0,059091 & $-0,241145$ & \\
\hline & 364 & 3908 & & & 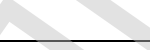 & & $-0,605328$ & & $-3,508708$ & \\
\hline & & & & & & & 0,605328 & & 3,508708 & \\
\hline & & & & & & & 0,051578 & & 3,209745 & 3,261323 \\
\hline
\end{tabular}




\begin{tabular}{|c|c|c|c|c|c|c|c|c|c|c|}
\hline & & & & & & & & & & \\
\hline KIDJYR & BADS & GOODS & $f(x / 1)$ & $f(x / 0)$ & $w(x)$ & & & 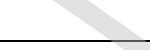 & & \\
\hline 010 & 60 & 259 & 0,066385 & 0,165753 & $-0,91503$ & 0,014123 & $-0,086798$ & 0,060729 & $-0,245434$ & \\
\hline $0 \backslash 1$ & 83 & 691 & 0,176899 & 0,228767 & $-0,25712$ & 0,019492 & $-0,110735$ & 0,161827 & $-0,425196$ & \\
\hline 012 & 33 & 226 & 0,057943 & 0,091781 & $-0,45994$ & 0,00782 & $-0,054731$ & 0,053006 & $-0,224624$ & \\
\hline 013 & 25 & 266 & 0,068176 & 0,069863 & $-0,02444$ & 0,005953 & $-0,044004$ & 0,062367 & $-0,249660$ & \\
\hline 110 & 2 & 69 & 0,017779 & 0,006849 & 0,953897 & 0,000584 & $-0,006269$ & 0,016265 & $-0,096646$ & \\
\hline $1 \backslash 1$ & 8 & 189 & 0,048478 & 0,023288 & 0,733183 & 0,001984 & $-0,017813$ & 0,044347 & $-0,199341$ & \\
\hline 112 & 1 & 117 & 0,030059 & 0,00411 & 1,989834 & 0,00035 & $-0,004020$ & 0,027498 & $-0,142563$ & \\
\hline 113 & 6 & 248 & 0,063571 & 0,017808 & 1,272501 & 0,001517 & $-0,014209$ & 0,058155 & $-0,238664$ & \\
\hline 210 & 15 & 144 & 0,036966 & 0,042466 & $-0,1387$ & 0,003618 & $-0,029346$ & 0,033816 & $-0,165231$ & \\
\hline $2 \backslash 1$ & 34 & 369 & 0,094525 & 0,094521 & 5,19E-05 & 0,008054 & $-0,056023$ & 0,086471 & $-0,305385$ & \\
\hline 212 & 16 & 232 & 0,059478 & 0,045205 & 0,27439 & 0,003852 & $-0,030892$ & 0,05441 & $-0,228522$ & \\
\hline 213 & 29 & 632 & 0,161806 & 0,080822 & 0,69415 & 0,006887 & $-0,049459$ & 0,148019 & $-0,407962$ & \\
\hline 310 & 5 & 39 & 0,010105 & 0,015068 & $-0,39959$ & 0,001284 & $-0,012332$ & 0,009244 & $-0,062464$ & \\
\hline $3 \backslash 1$ & 16 & 109 & 0,028012 & 0,045205 & $-0,47858$ & 0,003852 & $-0,030892$ & 0,025625 & $-0,135463$ & \\
\hline 312 & 9 & 69 & 0,017779 & 0,026027 & $-0,3811$ & 0,002218 & $-0,019553$ & 0,016265 & $-0,096646$ & \\
\hline 313 & 22 & 249 & 0,063827 & 0,061644 & 0,034804 & 0,005252 & $-0,039776$ & 0,058389 & $-0,239286$ & \\
\hline & 364 & 3908 & & & 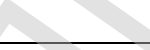 & & $-0,606852$ & & $-3,463088$ & \\
\hline & & & & & & & 0,606852 & & 3,463088 & \\
\hline & & & & & & & 0,051707 & & 3,168012 & 3,219719 \\
\hline
\end{tabular}




\begin{tabular}{|c|c|c|c|c|c|c|c|c|c|c|}
\hline & & & & & & & & & & \\
\hline KIDJYR & BADS & GOODS & $f(x / 1)$ & $f(x / 0)$ & $w(x)$ & & & 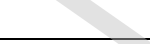 & & \\
\hline 010 & 81 & 429 & 0,109875 & 0,223288 & $-0,70912$ & 0,019025 & $-0,108748$ & 0,100513 & $-0,333154$ & \\
\hline $0 \backslash 1$ & 62 & 492 & 0,125991 & 0,171233 & $-0,30681$ & 0,01459 & $-0,088983$ & 0,115256 & $-0,359263$ & \\
\hline 012 & 30 & 220 & 0,056408 & 0,083562 & $-0,39297$ & 0,00712 & $-0,050793$ & 0,051602 & $-0,220672$ & \\
\hline 013 & 24 & 285 & 0,073037 & 0,067123 & 0,084429 & 0,005719 & $-0,042609$ & 0,066813 & $-0,260821$ & \\
\hline 110 & 5 & 109 & 0,028012 & 0,015068 & 0,620037 & 0,001284 & $-0,012332$ & 0,025625 & $-0,135463$ & \\
\hline $1 \backslash 1$ & 6 & 155 & 0,03978 & 0,017808 & 0,803704 & 0,001517 & $-0,014209$ & 0,036391 & $-0,173957$ & \\
\hline 112 & 1 & 116 & 0,029803 & 0,00411 & 1,981287 & 0,00035 & $-0,004020$ & 0,027264 & $-0,141686$ & \\
\hline 113 & 3 & 262 & 0,067153 & 0,009589 & 1,946349 & 0,000817 & $-0,008381$ & 0,061431 & $-0,247253$ & \\
\hline 210 & 25 & 195 & 0,050013 & 0,069863 & $-0,33426$ & 0,005953 & $-0,044004$ & 0,045751 & $-0,203596$ & \\
\hline $2 \backslash 1$ & 25 & 277 & 0,07099 & 0,069863 & 0,016003 & 0,005953 & $-0,044004$ & 0,064941 & $-0,256175$ & \\
\hline 212 & 18 & 240 & 0,061525 & 0,050685 & 0,19381 & 0,004319 & $-0,033924$ & 0,056282 & $-0,233638$ & \\
\hline 213 & 28 & 663 & 0,169737 & 0,078082 & 0,776485 & 0,006653 & $-0,048113$ & 0,155274 & $-0,417239$ & \\
\hline 310 & 13 & 69 & 0,017779 & 0,036986 & $-0,7325$ & 0,003151 & $-0,026188$ & 0,016265 & $-0,096646$ & \\
\hline $3 \backslash 1$ & 15 & 90 & 0,023152 & 0,042466 & $-0,60663$ & 0,003618 & $-0,029346$ & 0,021179 & $-0,117781$ & \\
\hline 312 & 6 & 66 & 0,017012 & 0,017808 & $-0,04574$ & 0,001517 & $-0,014209$ & 0,015562 & $-0,093465$ & \\
\hline 313 & 22 & 240 & 0,061525 & 0,061644 & $-0,00193$ & 0,005252 & $-0,039776$ & 0,056282 & $-0,233638$ & \\
\hline & 364 & 3908 & & & 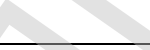 & & $-0,609639$ & & $-3,524447$ & \\
\hline & & & & & & & 0,609639 & & 3,524447 & \\
\hline & & & & & & & 0,051945 & & 3,224143 & 3,276088 \\
\hline
\end{tabular}




\begin{tabular}{|c|c|c|c|c|c|c|c|c|c|c|}
\hline KID $\backslash M A R$ & BADS & GOODS & $f(x / 1)$ & $f(x / 0)$ & $w(x)$ & & & & & \\
\hline $1 \backslash 1$ & 1 & 3 & 0,000895 & 0,00411 & $-1,52384$ & 0,00035 & $-0,004020$ & 0,000819 & $-0,008399$ & \\
\hline $2 \backslash 1$ & 165 & 937 & 0,239831 & 0,453425 & $-0,63689$ & 0,038634 & $-0,181349$ & 0,219396 & $-0,480124$ & \\
\hline $3 \backslash 1$ & 0 & 13 & 0,003454 & 0,00137 & 0,924697 & 0,000117 & $-0,001525$ & 0,003159 & $-0,026242$ & \\
\hline $4 \backslash 1$ & 0 & 12 & 0,003198 & 0,00137 & 0,847736 & 0,000117 & $-0,001525$ & 0,002925 & $-0,024623$ & \\
\hline 112 & 28 & 19 & 0,004988 & 0,078082 & $-2,75063$ & 0,006653 & $-0,048113$ & 0,004563 & $-0,035484$ & \\
\hline 212 & 27 & 48 & 0,012407 & 0,075342 & $-1,80376$ & 0,00642 & $-0,046756$ & 0,01135 & $-0,073335$ & \\
\hline 312 & 15 & 54 & 0,013942 & 0,042466 & $-1,11378$ & 0,003618 & $-0,029346$ & 0,012754 & $-0,080261$ & \\
\hline 412 & 55 & 61 & 0,015733 & 0,152055 & $-2,26849$ & 0,012956 & $-0,081237$ & 0,014392 & $-0,088061$ & \\
\hline 113 & 23 & 453 & 0,116014 & 0,064384 & 0,588855 & 0,005486 & $-0,041199$ & 0,106129 & $-0,343445$ & \\
\hline 213 & 6 & 436 & 0,111665 & 0,017808 & 1,835847 & 0,001517 & $-0,014209$ & 0,102151 & $-0,336202$ & \\
\hline 313 & 1 & 555 & 0,142108 & 0,00411 & 3,543264 & 0,00035 & $-0,004020$ & 0,13 & $-0,382643$ & \\
\hline \multirow[t]{4}{*}{$4 \backslash 3$} & 43 & 1317 & 0,337043 & 0,119178 & 1,039591 & 0,010155 & $-0,067241$ & 0,308325 & $-0,523374$ & \\
\hline & 364 & 3908 & & & 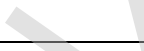 & & $-0,520541$ & & $-2,402192$ & \\
\hline & & & & & & & 0,520541 & & 2,402192 & \\
\hline & & & & 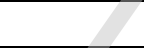 & $+x$ & & 0,044353 & & 2,197511 & 2,241864 \\
\hline
\end{tabular}




\begin{tabular}{|c|c|c|c|c|c|c|c|c|c|c|}
\hline \multicolumn{11}{|c|}{ 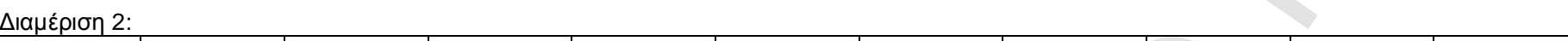 } \\
\hline KIDMAR & BADS & GOODS & $f(x / 1)$ & $f(x / 0)$ & $w(x)$ & & & 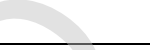 & & \\
\hline $0 \backslash 0$ & 26 & 47 & 0,012151 & 0,072603 & $-1,78755$ & 0,006186 & $-0,045386$ & 0,011116 & $-0,072157$ & \\
\hline $0 \backslash 1$ & 166 & 954 & 0,24418 & 0,456164 & $-0,62495$ & 0,038868 & $-0,182107$ & 0,223374 & $-0,483039$ & \\
\hline 012 & 5 & 439 & 0,112433 & 0,015068 & 2,00975 & 0,001284 & $-0,012332$ & 0,102853 & $-0,337496$ & \\
\hline 110 & 17 & 63 & 0,016245 & 0,047945 & $-1,0823$ & 0,004085 & $-0,032418$ & 0,01486 & $-0,090238$ & \\
\hline $1 \backslash 1$ & 0 & 15 & 0,003965 & 0,00137 & 1,062848 & 0,000117 & $-0,001525$ & 0,003627 & $-0,029406$ & \\
\hline 112 & 1 & 578 & 0,147992 & 0,00411 & 3,583834 & 0,00035 & $-0,004020$ & 0,135382 & $-0,390563$ & \\
\hline $2 \backslash 0$ & 54 & 64 & 0,0165 & 0,149315 & $-2,20267$ & 0,012723 & $-0,080107$ & 0,015094 & $-0,091319$ & \\
\hline $2 \backslash 1$ & 1 & 10 & 0,002686 & 0,00411 & $-0,42523$ & 0,00035 & $-0,004020$ & 0,002457 & $-0,021301$ & \\
\hline $2 \backslash 2$ & 42 & 1273 & 0,325787 & 0,116438 & 1,028881 & 0,009921 & $-0,066028$ & 0,298028 & $-0,520500$ & \\
\hline $3 \backslash 0$ & 30 & 18 & 0,004733 & 0,083562 & $-2,8711$ & 0,00712 & $-0,050793$ & 0,004329 & $-0,033993$ & \\
\hline $3 \backslash 1$ & 0 & 5 & 0,001407 & 0,00137 & 0,026756 & 0,000117 & $-0,001525$ & 0,001287 & $-0,012358$ & \\
\hline \multirow[t]{4}{*}{312} & 22 & 442 & 0,1132 & 0,061644 & 0,607786 & 0,005252 & $-0,039776$ & 0,103555 & $-0,338783$ & \\
\hline & 364 & 3908 & & & & $P$ & $-0,520037$ & & $-2,421154$ & \\
\hline & & & & & & 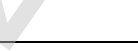 & 0,520037 & & 2,421154 & \\
\hline & & & & & 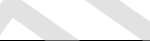 & & 0,04431 & & 2,214857 & 2,259167 \\
\hline
\end{tabular}




\begin{tabular}{|c|c|c|c|c|c|c|c|c|c|c|}
\hline \multicolumn{11}{|c|}{ 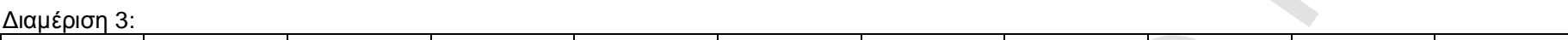 } \\
\hline KIDMAR & BADS & GOODS & $f(x / 1)$ & $f(x / 0)$ & $w(x)$ & & & 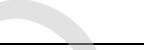 & & \\
\hline 010 & 28 & 40 & 0,010361 & 0,078082 & $-2,01974$ & 0,006653 & $-0,048113$ & 0,009478 & $-0,063703$ & \\
\hline $0 \backslash 1$ & 174 & 964 & 0,246738 & 0,478082 & $-0,66145$ & 0,040735 & $-0,188099$ & 0,225715 & $-0,484706$ & \\
\hline 012 & 5 & 431 & 0,110386 & 0,015068 & 1,99138 & 0,001284 & $-0,012332$ & 0,100981 & $-0,334029$ & \\
\hline 110 & 17 & 57 & 0,01471 & 0,047945 & $-1,18156$ & 0,004085 & $-0,032418$ & 0,013456 & $-0,083639$ & \\
\hline $1 \backslash 1$ & 0 & 15 & 0,003965 & 0,00137 & 1,062848 & 0,000117 & $-0,001525$ & 0,003627 & $-0,029406$ & \\
\hline 112 & 1 & 565 & 0,144666 & 0,00411 & 3,561106 & 0,00035 & $-0,004020$ & 0,13234 & $-0,386125$ & \\
\hline 210 & 50 & 55 & 0,014198 & 0,138356 & $-2,27673$ & 0,011789 & $-0,075524$ & 0,012988 & $-0,081393$ & \\
\hline $2\lfloor 1$ & 1 & 12 & 0,003198 & 0,00411 & $-0,25088$ & 0,00035 & $-0,004020$ & 0,002925 & $-0,024623$ & \\
\hline 212 & 40 & 1288 & 0,329624 & 0,110959 & 1,088793 & 0,009454 & $-0,063579$ & 0,301538 & $-0,521537$ & \\
\hline 310 & 23 & 22 & 0,005756 & 0,064384 & $-2,41462$ & 0,005486 & $-0,041199$ & 0,005266 & $-0,039856$ & \\
\hline $3 \backslash 1$ & 1 & 4 & 0,001151 & 0,00411 & $-1,27253$ & 0,00035 & $-0,004020$ & 0,001053 & $-0,010416$ & \\
\hline \multirow[t]{4}{*}{312} & 24 & 455 & 0,116526 & 0,067123 & 0,551583 & 0,005719 & $-0,042609$ & 0,106597 & $-0,344283$ & \\
\hline & 364 & 3908 & & & & 2 & $-0,517457$ & & $-2,403716$ & \\
\hline & & & & & 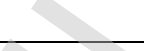 & 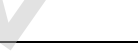 & 0,517457 & & 2,403716 & \\
\hline & & & & & 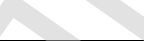 & & 0,04409 & & 2,198905 & 2,242995 \\
\hline
\end{tabular}




\begin{tabular}{|c|c|c|c|c|c|c|c|c|c|c|}
\hline KIDMAR & בחמק & אחתמת & & & (x) & & & & & \\
\hline $0 \backslash 0$ & 24 & 45 & 001164 & 0067123 & -17521 & 0005719 & -0042609 & 0010648 & 0069779 & \\
\hline $0 \backslash 1$ & 173 & 953 & 0,243924 & 0,475342 & $-0,66718$ & 0.040502 & -0.187357 & 0.22314 & $-0,482871$ & \\
\hline $0 \backslash 2$ & 4 & 444 & 0,113712 & 0,012329 & 2,221733 & 0,00105 & $-0,010394$ & 0,104023 & $-0,339638$ & \\
\hline 110 & 16 & 57 & 0,01471 & 0,045205 & $-1,12271$ & 0,003852 & $-0,030892$ & 0,013456 & $-0,083639$ & \\
\hline $1 \backslash 1$ & 0 & 14 & 0,003709 & 0,00137 & 0,996156 & 0,000117 & $-0,001525$ & 0,003393 & $-0,027836$ & \\
\hline 112 & 1 & 552 & 0,14134 & 0,00411 & 3,537849 & 0,00035 & $-0,004020$ & 0,129297 & $-0,381587$ & \\
\hline 210 & 54 & 65 & 0,016756 & 0,149315 & $-2,18729$ & 0,012723 & $-0,080107$ & 0,015328 & $-0,092395$ & \\
\hline $2 \backslash 1$ & 1 & 10 & 0,002686 & 0,00411 & $-0,42523$ & 0,00035 & $-0,004020$ & 0,002457 & $-0,021301$ & \\
\hline 212 & 39 & 1302 & 0,333205 & 0,108219 & 1,124601 & 0,009221 & $-0,062341$ & 0,304814 & $-0,522451$ & \\
\hline 310 & 25 & 17 & 0,004477 & 0,069863 & $-2,74762$ & 0,005953 & $-0,044004$ & 0,004095 & $-0,032484$ & \\
\hline $3 \backslash 1$ & 1 & 6 & 0,001663 & 0,00411 & $-0,9048$ & 0,00035 & $-0,004020$ & 0,001521 & $-0,014239$ & \\
\hline 312 & 26 & 443 & 0,113456 & 0,072603 & 0,446414 & 0,006186 & $-0,045386$ & 0,103789 & $-0,339211$ & \\
\hline & 364 & 3908 & & & & 2 & $-0,516675$ & & $-2,407429$ & \\
\hline & & & & & & 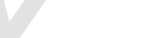 & 0,516675 & & 2,407429 & \\
\hline & & & & & 1 & & 0,044024 & & 2,202302 & 2,246326 \\
\hline
\end{tabular}




\begin{tabular}{|c|c|c|c|c|c|c|c|c|c|c|}
\hline \multicolumn{11}{|c|}{ 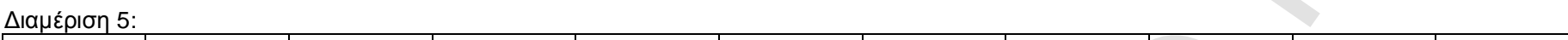 } \\
\hline KIDMAR & BADS & GOODS & $f(x / 1)$ & $f(x / 0)$ & $w(x)$ & & & 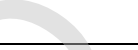 & & \\
\hline $0 \backslash 0$ & 23 & 36 & 0,009337 & 0,064384 & $-1,93083$ & 0,005486 & $-0,041199$ & 0,008542 & $-0,058693$ & \\
\hline $0 \backslash 1$ & 170 & 928 & 0,237529 & 0,467123 & $-0,6763$ & 0,039802 & $-0,185119$ & 0,21729 & $-0,478539$ & \\
\hline $0 \backslash 2$ & 4 & 462 & 0,118317 & 0,012329 & 2,26143 & 0,00105 & $-0,010394$ & 0,108235 & $-0,347193$ & \\
\hline 110 & 15 & 57 & 0,01471 & 0,042466 & $-1,06019$ & 0,003618 & $-0,029346$ & 0,013456 & $-0,083639$ & \\
\hline $1 \backslash 1$ & 0 & 15 & 0,003965 & 0,00137 & 1,062848 & 0,000117 & $-0,001525$ & 0,003627 & $-0,029406$ & \\
\hline 112 & 0 & 570 & 0,145945 & 0,00137 & 4,668521 & 0,000117 & $-0,001525$ & 0,13351 & $-0,387844$ & \\
\hline 210 & 55 & 63 & 0,016245 & 0,152055 & $-2,23648$ & 0,012956 & $-0,081237$ & 0,01486 & $-0,090238$ & \\
\hline 211 & 1 & 12 & 0,003198 & 0,00411 & $-0,25088$ & 0,00035 & $-0,004020$ & 0,002925 & $-0,024623$ & \\
\hline $2 \backslash 2$ & 40 & 1300 & 0,332694 & 0,110959 & 1,098063 & 0,009454 & $-0,063579$ & 0,304346 & $-0,522323$ & \\
\hline 310 & 30 & 16 & 0,004221 & 0,083562 & $-2,98551$ & 0,00712 & $-0,050793$ & 0,003861 & $-0,030955$ & \\
\hline $3 \backslash 1$ & 1 & 6 & 0,001663 & 0,00411 & $-0,9048$ & 0,00035 & $-0,004020$ & 0,001521 & $-0,014239$ & \\
\hline \multirow[t]{4}{*}{312} & 25 & 443 & 0,113456 & 0,069863 & 0,48488 & 0,005953 & $-0,044004$ & 0,103789 & $-0,339211$ & \\
\hline & 364 & 3908 & & & & 2 & $-0,516761$ & & $-2,406903$ & \\
\hline & & & & & & 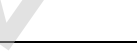 & 0,516761 & & 2,406903 & \\
\hline & & & & & 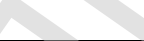 & & 0,044031 & & 2,20182 & 2,245852 \\
\hline
\end{tabular}




\begin{tabular}{|c|c|c|c|c|c|c|c|c|c|c|}
\hline KID $\backslash T R M$ & BADS & GOODS & $f(x / 1)$ & $f(x / 0)$ & $w(x)$ & & & & & \\
\hline $1 \backslash 1$ & 12 & 47 & 0,012151 & 0,034247 & $-1,03614$ & 0,002918 & $-0,024572$ & 0,011116 & $-0,072157$ & \\
\hline $2 \backslash 1$ & 21 & 127 & 0,032617 & 0,058904 & $-0,59108$ & 0,005019 & $-0,038337$ & 0,029838 & $-0,151180$ & \\
\hline $3 \backslash 1$ & 1 & 83 & 0,021361 & 0,00411 & 1,648242 & 0,00035 & $-0,004020$ & 0,019541 & $-0,110941$ & \\
\hline $4 \backslash 1$ & 21 & 146 & 0,037478 & 0,058904 & $-0,45217$ & 0,005019 & $-0,038337$ & 0,034284 & $-0,166838$ & \\
\hline 112 & 4 & 100 & 0,02571 & 0,012329 & 0,734941 & 0,00105 & $-0,010394$ & 0,023519 & $-0,127240$ & \\
\hline 212 & 16 & 431 & 0,110386 & 0,045205 & 0,892768 & 0,003852 & $-0,030892$ & 0,100981 & $-0,334029$ & \\
\hline $3 \backslash 2$ & 4 & 139 & 0,035687 & 0,012329 & 1,062848 & 0,00105 & $-0,010394$ & 0,032646 & $-0,161172$ & \\
\hline 412 & 13 & 303 & 0,077641 & 0,036986 & 0,741552 & 0,003151 & $-0,026188$ & 0,071026 & $-0,271000$ & \\
\hline 113 & 36 & 328 & 0,084037 & 0,1 & $-0,17391$ & 0,008521 & $-0,058578$ & 0,076876 & $-0,284544$ & \\
\hline 213 & 161 & 863 & 0,2209 & 0,442466 & $-0,69465$ & 0,037701 & $-0,178297$ & 0,202078 & $-0,466198$ & \\
\hline 313 & 11 & 400 & 0,102456 & 0,031507 & 1,179227 & 0,002685 & $-0,022929$ & 0,093726 & $-0,320112$ & \\
\hline \multirow[t]{4}{*}{413} & 64 & 941 & 0,240854 & 0,176712 & 0,30967 & 0,015057 & $-0,091146$ & 0,220332 & $-0,480819$ & \\
\hline & 364 & 3908 & & & 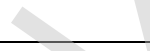 & 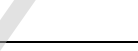 & $-0,534084$ & & $-2,946229$ & \\
\hline & & & & & & & 0,534084 & & 2,946229 & \\
\hline & & & & & 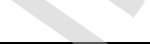 & & 0,045507 & & 2,695193 & 2,740700 \\
\hline
\end{tabular}




\begin{tabular}{|c|c|c|c|c|c|c|c|c|c|c|}
\hline & & & & & & & & & & \\
\hline KIDTRM & BADS & GOODS & $f(x / 1)$ & $f(x / 0)$ & $w(x)$ & & & 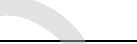 & & \\
\hline $0 \backslash 0$ & 15 & 435 & 0,11141 & 0,042466 & 0,964515 & 0,003618 & $-0,029346$ & 0,101917 & $-0,335769$ & \\
\hline $0 \backslash 1$ & 165 & 885 & 0,226529 & 0,453425 & $-0,69396$ & 0,038634 & $-0,181349$ & 0,207227 & $-0,470554$ & \\
\hline 012 & 17 & 120 & 0,030826 & 0,047945 & $-0,44169$ & 0,004085 & $-0,032418$ & 0,0282 & $-0,145177$ & \\
\hline 110 & 4 & 143 & 0,03671 & 0,012329 & 1,091118 & 0,00105 & $-0,010394$ & 0,033582 & $-0,164424$ & \\
\hline $1 \backslash 1$ & 13 & 431 & 0,110386 & 0,036986 & 1,093438 & 0,003151 & $-0,026188$ & 0,100981 & $-0,334029$ & \\
\hline 112 & 1 & 82 & 0,021105 & 0,00411 & 1,636194 & 0,00035 & $-0,004020$ & 0,019307 & $-0,109948$ & \\
\hline $2 \backslash 0$ & 10 & 291 & 0,074572 & 0,028767 & 0,952525 & 0,002451 & $-0,021257$ & 0,068218 & $-0,264255$ & \\
\hline $2 \backslash 1$ & 68 & 917 & 0,234715 & 0,187671 & 0,223679 & 0,015991 & $-0,095411$ & 0,214716 & $-0,476562$ & \\
\hline $2 \backslash 2$ & 19 & 139 & 0,035687 & 0,053425 & $-0,40349$ & 0,004552 & $-0,035412$ & 0,032646 & $-0,161172$ & \\
\hline $3 \backslash 0$ & 2 & 92 & 0,023663 & 0,006849 & 1,239778 & 0,000584 & $-0,006269$ & 0,021647 & $-0,119702$ & \\
\hline $3 \backslash 1$ & 40 & 330 & 0,084548 & 0,110959 & $-0,27183$ & 0,009454 & $-0,063579$ & 0,077344 & $-0,285599$ & \\
\hline 312 & 10 & 43 & 0,011128 & 0,028767 & $-0,94975$ & 0,002451 & $-0,021257$ & 0,01018 & $-0,067372$ & \\
\hline & 364 & 3908 & & & & $P$ & $-0,526900$ & & $-2,934561$ & \\
\hline & & & & & & 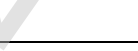 & 0,526900 & & 2,934561 & \\
\hline & & & & & 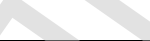 & & 0,044895 & & 2,684519 & 2,729414 \\
\hline
\end{tabular}




\begin{tabular}{|c|c|c|c|c|c|c|c|c|c|c|}
\hline KIDTRM & & & & & & & & & & \\
\hline $0 \backslash 0$ & 14 & & 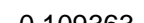 & & 1012666 & & ר? 0777 & & & \\
\hline & & & & & & & & & & \\
\hline 017 & 173 & 901 & 0,230622 & 0,475342 & $-0,72326$ & 0,040502 & $-0,187357$ & 0,210971 & $-0,473606$ & \\
\hline $0 \backslash 2$ & 20 & 107 & 0,027501 & 0,056164 & $-0,71407$ & 0,004786 & $-0,036883$ & 0,025157 & $-0,133658$ & \\
\hline 110 & 2 & 134 & 0,034408 & 0,006849 & 1,614134 & 0,000584 & $-0,006269$ & 0,031476 & $-0,157053$ & \\
\hline $1 \backslash 1$ & 14 & 427 & 0,109363 & 0,039726 & 1,012666 & 0,003385 & $-0,027779$ & 0,100045 & $-0,332277$ & \\
\hline $1 \backslash 2$ & 2 & 76 & 0,01957 & 0,006849 & 1,04986 & 0,000584 & $-0,006269$ & 0,017903 & $-0,103902$ & \\
\hline $2 \backslash 0$ & 8 & 273 & 0,069967 & 0,023288 & 1,100096 & 0,001984 & $-0,017813$ & 0,064005 & $-0,253823$ & \\
\hline $2 \backslash 1$ & 64 & 936 & 0,239575 & 0,176712 & 0,304345 & 0,015057 & $-0,091146$ & 0,219162 & $-0,479950$ & \\
\hline 212 & 19 & 146 & 0,037478 & 0,053425 & $-0,35453$ & 0,004552 & $-0,035412$ & 0,034284 & $-0,166838$ & \\
\hline $3 \backslash 0$ & 4 & 94 & 0,024175 & 0,012329 & 0,673383 & 0,00105 & $-0,010394$ & 0,022115 & $-0,121607$ & \\
\hline $3 \backslash 1$ & 33 & 340 & 0,087107 & 0,091781 & $-0,05227$ & 0,00782 & $-0,054731$ & 0,079685 & $-0,290813$ & \\
\hline 312 & 11 & 47 & 0,012151 & 0,031507 & $-0,95276$ & 0,002685 & $-0,022929$ & 0,011116 & $-0,072157$ & \\
\hline & 364 & 3908 & & & & 2 & $-0,524761$ & & $-2,917960$ & \\
\hline & & & & & & 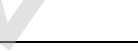 & 0,524761 & & 2,917960 & \\
\hline & & & & & & & 0,044713 & & 2,669332 & 2,714045 \\
\hline
\end{tabular}




\begin{tabular}{|c|c|c|c|c|c|c|c|c|c|c|}
\hline 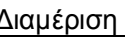 & & & & & & & & & & \\
\hline KIDTRM & BADS & GOODS & $f(x / 1)$ & $f(x / 0)$ & $w(x)$ & & & - & & \\
\hline 010 & 16 & 421 & 0,107828 & 0,045205 & 0,86932 & 0,003852 & $-0,030892$ & 0,09864 & $-0,329625$ & \\
\hline $0 \backslash 1$ & 167 & 909 & 0,232668 & 0,458904 & $-0,67923$ & 0,039101 & $-0,182863$ & 0,212843 & $-0,475095$ & \\
\hline 012 & 18 & 112 & 0,02878 & 0,050685 & $-0,56596$ & 0,004319 & $-0,033924$ & 0,026328 & $-0,138148$ & \\
\hline 110 & 3 & 131 & 0,03364 & 0,009589 & 1,255104 & 0,000817 & $-0,008381$ & 0,030774 & $-0,154551$ & \\
\hline $1 \backslash 1$ & 12 & 414 & 0,106037 & 0,034247 & 1,130205 & 0,002918 & $-0,024572$ & 0,097002 & $-0,326494$ & \\
\hline $1 \backslash 2$ & 2 & 78 & 0,020082 & 0,006849 & 1,075668 & 0,000584 & $-0,006269$ & 0,018371 & $-0,105934$ & \\
\hline 210 & 8 & 267 & 0,068432 & 0,023288 & 1,077914 & 0,001984 & $-0,017813$ & 0,062601 & $-0,250258$ & \\
\hline $2 \backslash 1$ & 68 & 981 & 0,251087 & 0,187671 & 0,291109 & 0,015991 & $-0,095411$ & 0,229693 & $-0,487459$ & \\
\hline 212 & 18 & 129 & 0,033129 & 0,050685 & $-0,42523$ & 0,004319 & $-0,033924$ & 0,030306 & $-0,152871$ & \\
\hline 310 & 4 & 83 & 0,021361 & 0,012329 & 0,54963 & 0,00105 & $-0,010394$ & 0,019541 & $-0,110941$ & \\
\hline $3 \backslash 1$ & 37 & 338 & 0,086595 & 0,10274 & $-0,17096$ & 0,008754 & $-0,059841$ & 0,079217 & $-0,289779$ & \\
\hline 312 & 11 & 45 & 0,01164 & 0,031507 & $-0,99577$ & 0,002685 & $-0,022929$ & 0,010648 & $-0,069779$ & \\
\hline & 364 & 3908 & & & & 2 & $-0,527213$ & & $-2,890934$ & \\
\hline & & & & & & 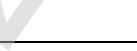 & 0,527213 & & 2,890934 & \\
\hline & & & & & 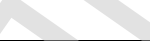 & & 0,044922 & & 2,64461 & 2,689531 \\
\hline
\end{tabular}




\begin{tabular}{|c|c|c|c|c|c|c|c|c|c|c|}
\hline KIDTRM & RARS & Sחת & $f(x / 1)$ & $f(x / 0)$ & (x) & & & & & \\
\hline $0 \backslash 0$ & 17 & 390 & 0,099898 & 0.047945 & 0,734088 & 0,004085 & $-0,032418$ & 0,091386 & -0.315453 & \\
\hline $0 \backslash 1$ & 160 & 916 & 0.234459 & 0.439726 & -0.62887 & 0,037467 & -0.177528 & 0.214482 & $-0,476380$ & \\
\hline $0 \backslash 2$ & 20 & 120 & 0,030826 & 0,056164 & $-0,59991$ & 0,004786 & $-0,036883$ & 0,0282 & $-0,145177$ & \\
\hline 110 & 3 & 129 & 0,033129 & 0,009589 & 1,239778 & 0,000817 & $-0,008381$ & 0,030306 & $-0,152871$ & \\
\hline $1 \backslash 1$ & 10 & 434 & 0,111154 & 0,028767 & 1,351681 & 0,002451 & $-0,021257$ & 0,101683 & $-0,335335$ & \\
\hline 112 & 2 & 79 & 0,020338 & 0,006849 & 1,088327 & 0,000584 & $-0,006269$ & 0,018605 & $-0,106944$ & \\
\hline 210 & 7 & 257 & 0,065874 & 0,020548 & 1,164977 & 0,001751 & $-0,016033$ & 0,060261 & $-0,244215$ & \\
\hline $2 \backslash 1$ & 66 & 989 & 0,253134 & 0,182192 & 0,328858 & 0,015524 & $-0,093288$ & 0,231565 & $-0,488721$ & \\
\hline 212 & 23 & 129 & 0,033129 & 0,064384 & $-0,66446$ & 0,005486 & $-0,041199$ & 0,030306 & $-0,152871$ & \\
\hline 310 & 3 & 89 & 0,022896 & 0,009589 & 0,870336 & 0,000817 & $-0,008381$ & 0,020945 & $-0,116816$ & \\
\hline $3 \backslash 1$ & 41 & 327 & 0,083781 & 0,113699 & $-0,30534$ & 0,009688 & $-0,064808$ & 0,076642 & $-0,284015$ & \\
\hline 312 & 12 & 49 & 0,012663 & 0,034247 & $-0,9949$ & 0,002918 & $-0,024572$ & 0,011584 & $-0,074506$ & \\
\hline & 364 & 3908 & & & & 2 & $-0,531018$ & & $-2,893302$ & \\
\hline & & & & & 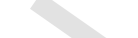 & 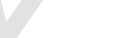 & 0,531018 & & 2,893302 & \\
\hline & & & & & +2 & & 0,045246 & & 2,646775 & 2,692021 \\
\hline
\end{tabular}




\begin{tabular}{|c|c|c|c|c|c|c|c|c|c|c|}
\hline HYR\HOME & BADS & GOODS & $f(x / 1)$ & $f(x / 0)$ & $w(x)$ & & & & & \\
\hline 111 & 21 & 284 & 0,072781 & 0,058904 & 0,211541 & 0,005019 & $-0,038337$ & 0,066579 & $-0,260244$ & \\
\hline 211 & 29 & 100 & 0,02571 & 0,080822 & $-1,14537$ & 0,006887 & $-0,049459$ & 0,023519 & $-0,127240$ & \\
\hline $3 \backslash 1$ & 14 & 197 & 0,050524 & 0,039726 & 0,24045 & 0,003385 & $-0,027779$ & 0,046219 & $-0,205000$ & \\
\hline $4 \backslash 1$ & 1 & 18 & 0,004733 & 0,00411 & 0,141166 & 0,00035 & $-0,004020$ & 0,004329 & $-0,033993$ & \\
\hline 112 & 14 & 56 & 0,014454 & 0,039726 & $-1,01105$ & 0,003385 & $-0,027779$ & 0,013222 & $-0,082519$ & \\
\hline $2 \backslash 2$ & 115 & 333 & 0,085316 & 0,316438 & $-1,31077$ & 0,026962 & $-0,140553$ & 0,078047 & $-0,287174$ & \\
\hline 312 & 25 & 352 & 0,090177 & 0,069863 & 0,255233 & 0,005953 & $-0,044004$ & 0,082493 & $-0,296940$ & \\
\hline 412 & 0 & 1 & 0,000384 & 0,00137 & $-1,27253$ & 0,000117 & $-0,001525$ & 0,000351 & $-0,004028$ & \\
\hline 113 & 44 & 854 & 0,218598 & 0,121918 & 0,583888 & 0,010388 & $-0,068447$ & 0,199972 & $-0,464361$ & \\
\hline 213 & 54 & 365 & 0,093502 & 0,149315 & $-0,46807$ & 0,012723 & $-0,080107$ & 0,085535 & $-0,303422$ & \\
\hline 313 & 42 & 1118 & 0,286135 & 0,116438 & 0,8991 & 0,009921 & $-0,066028$ & 0,261754 & $-0,506158$ & \\
\hline \multirow[t]{4}{*}{413} & 5 & 230 & 0,058966 & 0,015068 & 1,364363 & 0,001284 & $-0,012332$ & 0,053942 & $-0,227228$ & \\
\hline & 364 & 3908 & & - & & & $-0,560369$ & & $-2,798308$ & \\
\hline & & & & & & & 0,560369 & & 2,798308 & \\
\hline & & & & 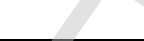 & & & 0,047747 & & 2,559875 & 2,60 \\
\hline
\end{tabular}




\begin{tabular}{|c|c|c|c|c|c|c|c|c|c|c|}
\hline HYRHOME & BADS & GOODS & $f(x / 1)$ & $f(x / 0)$ & $w(x)$ & & 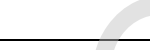 & 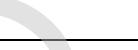 & & \\
\hline 010 & 27 & 96 & 0,024687 & 0,075342 & $-1,11578$ & 0,00642 & $-0,046756$ & 0,022583 & $-0,123499$ & \\
\hline $0 \backslash 1$ & 118 & 341 & 0,087362 & 0,324658 & $-1,3127$ & 0,027663 & $-0,143180$ & 0,079919 & $-0,291329$ & \\
\hline 012 & 50 & 354 & 0,090688 & 0,138356 & $-0,4224$ & 0,011789 & $-0,075524$ & 0,082961 & $-0,297948$ & \\
\hline $1 \backslash 0$ & 15 & 211 & 0,054106 & 0,042466 & 0,242245 & 0,003618 & $-0,029346$ & 0,049496 & $-0,214641$ & \\
\hline $1 \backslash 1$ & 27 & 358 & 0,091711 & 0,075342 & 0,196603 & 0,00642 & $-0,046756$ & 0,083897 & $-0,299952$ & \\
\hline 112 & 47 & 1134 & 0,290228 & 0,130137 & 0,802078 & 0,011088 & $-0,072017$ & 0,265499 & $-0,507958$ & \\
\hline 210 & 22 & 287 & 0,073548 & 0,061644 & 0,176568 & 0,005252 & $-0,039776$ & 0,067281 & $-0,261970$ & \\
\hline $2 \backslash 1$ & 14 & 45 & 0,01164 & 0,039726 & $-1,22758$ & 0,003385 & $-0,027779$ & 0,010648 & $-0,069779$ & \\
\hline $2 \backslash 2$ & 39 & 840 & 0,215017 & 0,108219 & 0,686557 & 0,009221 & $-0,062341$ & 0,196696 & $-0,461441$ & \\
\hline $3 \backslash 0$ & 1 & 17 & 0,004477 & 0,00411 & 0,085596 & 0,00035 & $-0,004020$ & 0,004095 & $-0,032484$ & \\
\hline $3 \backslash 1$ & 0 & 1 & 0,000384 & 0,00137 & $-1,27253$ & 0,000117 & $-0,001525$ & 0,000351 & $-0,004028$ & \\
\hline \multirow[t]{4}{*}{312} & 4 & 224 & 0,057432 & 0,012329 & 1,538659 & 0,00105 & $-0,010394$ & 0,052538 & $-0,223313$ & \\
\hline & 364 & 3908 & & & & 2 & $-0,559415$ & & $-2,788342$ & \\
\hline & & & & & & & 0,559415 & & 2,788342 & \\
\hline & & & & & & & 0,047665 & & 2,550759 & \\
\hline
\end{tabular}




\begin{tabular}{|c|c|c|c|c|c|c|c|c|c|c|}
\hline \multicolumn{11}{|l|}{ 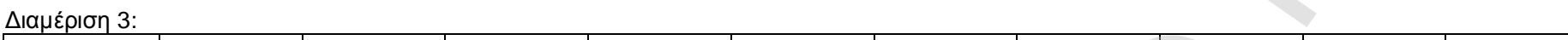 } \\
\hline HYRHOME & BADS & GOODS & $f(x / 1)$ & $f(x / 0)$ & $w(x)$ & & 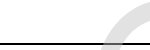 & 8 & & \\
\hline 010 & 32 & 106 & 0,027245 & 0,089041 & $-1,18423$ & 0,007587 & $-0,053429$ & 0,024923 & $-0,132751$ & \\
\hline $0 \backslash 1$ & 120 & 345 & 0,088386 & 0,330137 & $-1,3178$ & 0,02813 & $-0,144917$ & 0,080855 & $-0,293383$ & \\
\hline 012 & 47 & 368 & 0,09427 & 0,130137 & $-0,32243$ & 0,011088 & $-0,072017$ & 0,086237 & $-0,304896$ & \\
\hline 110 & 14 & 211 & 0,054106 & 0,039726 & 0,308937 & 0,003385 & $-0,027779$ & 0,049496 & $-0,214641$ & \\
\hline $1 \backslash 1$ & 32 & 337 & 0,086339 & 0,089041 & $-0,03081$ & 0,007587 & $-0,053429$ & 0,078983 & $-0,289260$ & \\
\hline 112 & 40 & 1151 & 0,294577 & 0,110959 & 0,976379 & 0,009454 & $-0,063579$ & 0,269477 & $-0,509787$ & \\
\hline 210 & 25 & 269 & 0,068943 & 0,069863 & $-0,01325$ & 0,005953 & $-0,044004$ & 0,063069 & $-0,251452$ & \\
\hline $2 \backslash 1$ & 10 & 49 & 0,012663 & 0,028767 & $-0,82054$ & 0,002451 & $-0,021257$ & 0,011584 & $-0,074506$ & \\
\hline 212 & 42 & 816 & 0,208877 & 0,116438 & 0,584383 & 0,009921 & $-0,066028$ & 0,191079 & $-0,456251$ & \\
\hline 310 & 0 & 21 & 0,0055 & 0,00137 & 1,390061 & 0,000117 & $-0,001525$ & 0,005031 & $-0,038414$ & \\
\hline $3 \backslash 1$ & 0 & 1 & 0,000384 & 0,00137 & $-1,27253$ & 0,000117 & $-0,001525$ & 0,000351 & $-0,004028$ & \\
\hline \multirow[t]{4}{*}{312} & 2 & 234 & 0,05999 & 0,006849 & 2,170025 & 0,000584 & $-0,006269$ & 0,054878 & $-0,229809$ & \\
\hline & 364 & 3908 & & & & 2 & $-0,555758$ & & $-2,799178$ & \\
\hline & & & & & & & 0,555758 & & 2,799178 & \\
\hline & & & & & 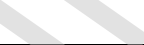 & & 0,047354 & & 2,560672 & 2,608026 \\
\hline
\end{tabular}




\begin{tabular}{|c|c|c|c|c|c|c|c|c|c|c|}
\hline HYRHOME & BADS & GOODS & $f(x / 1)$ & $f(x / 0)$ & $w(x)$ & & 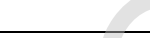 & 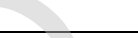 & & \\
\hline 010 & 28 & 105 & 0,026989 & 0,078082 & $-1,06233$ & 0,006653 & $-0,048113$ & 0,024689 & $-0,131840$ & \\
\hline $0 \backslash 1$ & 119 & 349 & 0,089409 & 0,327397 & $-1,29795$ & 0,027896 & $-0,144050$ & 0,081791 & $-0,295422$ & \\
\hline 012 & 44 & 365 & 0,093502 & 0,121918 & $-0,26536$ & 0,010388 & $-0,068447$ & 0,085535 & $-0,303422$ & \\
\hline 110 & 14 & 206 & 0,052827 & 0,039726 & 0,285012 & 0,003385 & $-0,027779$ & 0,048326 & $-0,211235$ & \\
\hline $1 \backslash 1$ & 28 & 343 & 0,087874 & 0,078082 & 0,118144 & 0,006653 & $-0,048113$ & 0,080387 & $-0,292358$ & \\
\hline 112 & 43 & 1135 & 0,290483 & 0,119178 & 0,890928 & 0,010155 & $-0,067241$ & 0,265733 & $-0,508068$ & \\
\hline 210 & 23 & 284 & 0,072781 & 0,064384 & 0,122593 & 0,005486 & $-0,041199$ & 0,066579 & $-0,260244$ & \\
\hline $2 \backslash 1$ & 16 & 47 & 0,012151 & 0,045205 & $-1,31377$ & 0,003852 & $-0,030892$ & 0,011116 & $-0,072157$ & \\
\hline 212 & 44 & 828 & 0,211947 & 0,121918 & 0,552988 & 0,010388 & $-0,068447$ & 0,193888 & $-0,458875$ & \\
\hline 310 & 1 & 16 & 0,004221 & 0,00411 & 0,026756 & 0,00035 & $-0,004020$ & 0,003861 & $-0,030955$ & \\
\hline $3 \backslash 1$ & 0 & 0 & 0,000128 & 0,00137 & $-2,37114$ & 0,000117 & $-0,001525$ & 0,000117 & $-0,001528$ & \\
\hline 312 & 4 & 230 & 0,058966 & 0,012329 & 1,565034 & 0,00105 & $-0,010394$ & 0,053942 & $-0,227228$ & \\
\hline & 364 & 3908 & & & & 2 & $-0,560221$ & & $-2,793334$ & \\
\hline & & & & & 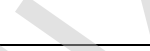 & & 0,560221 & & 2,793334 & \\
\hline & & & & & $x^{2}$ & & 0,047734 & & 2,555325 & 2,603059 \\
\hline
\end{tabular}




\begin{tabular}{|c|c|c|c|c|c|c|c|c|c|c|}
\hline \multicolumn{11}{|l|}{ 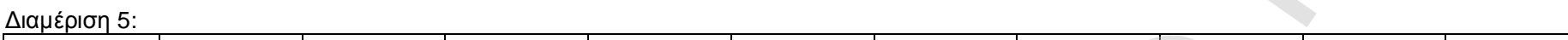 } \\
\hline HYRHOME & BADS & GOODS & $f(x / 1)$ & $f(x / 0)$ & $w(x)$ & & 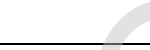 & 8 & & \\
\hline 010 & 28 & 105 & 0,026989 & 0,078082 & $-1,06233$ & 0,006653 & $-0,048113$ & 0,024689 & $-0,131840$ & \\
\hline $0 \backslash 1$ & 116 & 348 & 0,089153 & 0,319178 & $-1,27539$ & 0,027196 & $-0,141431$ & 0,081557 & $-0,294914$ & \\
\hline 012 & 57 & 356 & 0,0912 & 0,157534 & $-0,54659$ & 0,013423 & $-0,083479$ & 0,083429 & $-0,298952$ & \\
\hline 110 & 15 & 192 & 0,049245 & 0,042466 & 0,148117 & 0,003618 & $-0,029346$ & 0,045049 & $-0,201476$ & \\
\hline $1 \backslash 1$ & 24 & 338 & 0,086595 & 0,067123 & 0,254711 & 0,005719 & $-0,042609$ & 0,079217 & $-0,289779$ & \\
\hline 112 & 42 & 1110 & 0,284088 & 0,116438 & 0,891922 & 0,009921 & $-0,066028$ & 0,259882 & $-0,505229$ & \\
\hline 210 & 25 & 285 & 0,073037 & 0,069863 & 0,044424 & 0,005953 & $-0,044004$ & 0,066813 & $-0,260821$ & \\
\hline $2 \backslash 1$ & 14 & 51 & 0,013175 & 0,039726 & $-1,10371$ & 0,003385 & $-0,027779$ & 0,012052 & $-0,076827$ & \\
\hline 212 & 37 & 867 & 0,221924 & 0,10274 & 0,770135 & 0,008754 & $-0,059841$ & 0,203015 & $-0,467003$ & \\
\hline 310 & 1 & 18 & 0,004733 & 0,00411 & 0,141166 & 0,00035 & $-0,004020$ & 0,004329 & $-0,033993$ & \\
\hline $3 \backslash 1$ & 0 & 1 & 0,000384 & 0,00137 & $-1,27253$ & 0,000117 & $-0,001525$ & 0,000351 & $-0,004028$ & \\
\hline \multirow[t]{4}{*}{312} & 5 & 237 & 0,060757 & 0,015068 & 1,39428 & 0,001284 & $-0,012332$ & 0,05558 & $-0,231730$ & \\
\hline & 364 & 3908 & & & & 2 & $-0,560508$ & & $-2,796593$ & \\
\hline & & & & & & & 0,560508 & & 2,796593 & \\
\hline & & & & & $x_{1}$ & & 0,047759 & & 2,558307 & 2,606065 \\
\hline
\end{tabular}




\begin{tabular}{|c|c|c|c|c|c|c|c|c|c|c|}
\hline HYRIINC & BADS & GOODS & $f(x / 1)$ & $f(x / 0)$ & $w(x)$ & & & & & \\
\hline $1 \backslash 1$ & 17 & 235 & 0,060246 & 0,047945 & 0,228371 & 0,004085 & $-0,032418$ & 0,055112 & $-0,230451$ & \\
\hline $2 \backslash 1$ & 49 & 154 & 0,039524 & 0,135616 & $-1,23292$ & 0,011555 & $-0,074362$ & 0,036156 & $-0,173175$ & \\
\hline $3 \backslash 1$ & 17 & 272 & 0,069711 & 0,047945 & 0,374298 & 0,004085 & $-0,032418$ & 0,063771 & $-0,253232$ & \\
\hline $4 \backslash 1$ & 3 & 54 & 0,013942 & 0,009589 & 0,374298 & 0,000817 & $-0,008381$ & 0,012754 & $-0,080261$ & \\
\hline 112 & 34 & 191 & 0,04899 & 0,094521 & $-0,65721$ & 0,008054 & $-0,056023$ & 0,044815 & $-0,200767$ & \\
\hline 212 & 90 & 152 & 0,039013 & 0,247945 & $-1,84932$ & 0,021126 & $-0,117564$ & 0,035688 & $-0,171604$ & \\
\hline 312 & 31 & 264 & 0,067664 & 0,086301 & $-0,24329$ & 0,007353 & $-0,052116$ & 0,061899 & $-0,248459$ & \\
\hline 412 & 2 & 37 & 0,009593 & 0,006849 & 0,336911 & 0,000584 & $-0,006269$ & 0,008776 & $-0,059959$ & \\
\hline 113 & 26 & 602 & 0,154131 & 0,072603 & 0,752803 & 0,006186 & $-0,045386$ & 0,140999 & $-0,398497$ & \\
\hline 213 & 58 & 413 & 0,105782 & 0,160274 & $-0,41551$ & 0,013656 & $-0,084591$ & 0,096768 & $-0,326044$ & \\
\hline 313 & 30 & 879 & 0,224994 & 0,083562 & 0,990487 & 0,00712 & $-0,050793$ & 0,205823 & $-0,469384$ & \\
\hline 413 & 1 & 126 & 0,032361 & 0,00411 & 2,063638 & 0,00035 & $-0,004020$ & 0,029604 & $-0,150330$ & \\
\hline $1 \backslash 4$ & 2 & 166 & 0,042594 & 0,006849 & 1,827565 & 0,000584 & $-0,006269$ & 0,038965 & $-0,182421$ & \\
\hline 214 & 1 & 79 & 0,020338 & 0,00411 & 1,599152 & 0,00035 & $-0,004020$ & 0,018605 & $-0,106944$ & \\
\hline $3 \backslash 4$ & 3 & 252 & 0,064595 & 0,009589 & 1,907509 & 0,000817 & $-0,008381$ & 0,059091 & $-0,241145$ & \\
\hline \multirow[t]{4}{*}{$4 \backslash 4$} & 0 & 32 & 0,008314 & 0,00137 & 1,803248 & 0,000117 & $-0,001525$ & 0,007606 & $-0,053534$ & \\
\hline & 364 & 3908 & & 2 & 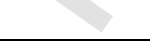 & & $-0,584536$ & & $-3,346206$ & \\
\hline & & & & 2 & & & 0,584536 & & 3,346206 & \\
\hline & & & & 8 & 8 & & 0,049806 & & 3,061089 & 3,110895 \\
\hline
\end{tabular}




\begin{tabular}{|c|c|c|c|c|c|c|c|c|c|c|}
\hline HYRINC & RADS & CORS & $f(x / 1)$ & $f(x / 0)$ & $w(y)$ & & & & & \\
\hline 010 & 95 & 157 & 0,040292 & 0,261644 & $-1,87084$ & 0,022294 & $-0,122330$ & 0,036859 & $-0,175515$ & \\
\hline $0 \backslash 1$ & 49 & 157 & 0,040292 & 0,135616 & $-1,21369$ & 0,011555 & $-0,074362$ & 0,036859 & $-0,175515$ & \\
\hline 012 & 50 & 392 & 0,100409 & 0,138356 & $-0,32058$ & 0,011789 & $-0,075524$ & 0,091854 & $-0,316392$ & \\
\hline 013 & 1 & 85 & 0,021873 & 0,00411 & 1,671912 & 0,00035 & $-0,004020$ & 0,020009 & $-0,112915$ & \\
\hline 110 & 37 & 267 & 0,068432 & 0,10274 & $-0,40636$ & 0,008754 & $-0,059841$ & 0,062601 & $-0,250258$ & \\
\hline $1 \backslash 1$ & 20 & 282 & 0,072269 & 0,056164 & 0,252114 & 0,004786 & $-0,036883$ & 0,066111 & $-0,259088$ & \\
\hline 112 & 30 & 899 & 0,23011 & 0,083562 & 1,012973 & 0,00712 & $-0,050793$ & 0,210503 & $-0,473229$ & \\
\hline 113 & 2 & 255 & 0,065362 & 0,006849 & 2,255792 & 0,000584 & $-0,006269$ & 0,059793 & $-0,242991$ & \\
\hline $2 \backslash 0$ & 27 & 178 & 0,045664 & 0,075342 & $-0,50074$ & 0,00642 & $-0,046756$ & 0,041773 & $-0,191374$ & \\
\hline $2 \backslash 1$ & 21 & 227 & 0,058199 & 0,058904 & $-0,01204$ & 0,005019 & $-0,038337$ & 0,05324 & $-0,225277$ & \\
\hline 212 & 26 & 609 & 0,155922 & 0,072603 & 0,764355 & 0,006186 & $-0,045386$ & 0,142637 & $-0,400750$ & \\
\hline $2 \backslash 3$ & 1 & 158 & 0,040547 & 0,00411 & 2,28915 & 0,00035 & $-0,004020$ & 0,037093 & $-0,176291$ & \\
\hline $3 \backslash 0$ & 2 & 37 & 0,009593 & 0,006849 & 0,336911 & 0,000584 & $-0,006269$ & 0,008776 & $-0,059959$ & \\
\hline $3 \backslash 1$ & 2 & 44 & 0,011384 & 0,006849 & 0,508059 & 0,000584 & $-0,006269$ & 0,010414 & $-0,068580$ & \\
\hline 312 & 1 & 129 & 0,033129 & 0,00411 & 2,087076 & 0,00035 & $-0,004020$ & 0,030306 & $-0,152871$ & \\
\hline 313 & 0 & 32 & 0,008314 & 0,00137 & 1,803248 & 0,000117 & $-0,001525$ & 0,007606 & $-0,053534$ & \\
\hline & 364 & 3908 & & & - & & $-0,582605$ & & $-3,334538$ & \\
\hline & & & & & & & 0,582605 & & 3,334538 & \\
\hline & & & & & & & 0,049641 & & 3,050416 & 3,100057 \\
\hline
\end{tabular}




\begin{tabular}{|c|c|c|c|c|c|c|c|c|c|c|}
\hline HYRINC & BADS & GOODS & $f(x / 1)$ & $f(x / 0)$ & $w(x)$ & & & ( & & \\
\hline $0 \backslash 0$ & 99 & 150 & 0,038501 & 0,272603 & $-1,95733$ & 0,023227 & $-0,126079$ & 0,03522 & $-0,170025$ & \\
\hline $0 \backslash 1$ & 46 & 153 & 0,039268 & 0,127397 & $-1,17689$ & 0,010855 & $-0,070834$ & 0,035922 & $-0,172391$ & \\
\hline 012 & 53 & 434 & 0,111154 & 0,146575 & $-0,27663$ & 0,012489 & $-0,078971$ & 0,101683 & $-0,335335$ & \\
\hline 013 & 1 & 82 & 0,021105 & 0,00411 & 1,636194 & 0,00035 & $-0,004020$ & 0,019307 & $-0,109948$ & \\
\hline 110 & 35 & 269 & 0,068943 & 0,09726 & $-0,3441$ & 0,008287 & $-0,057305$ & 0,063069 & $-0,251452$ & \\
\hline $1 \backslash 1$ & 22 & 297 & 0,076106 & 0,061644 & 0,210759 & 0,005252 & $-0,039776$ & 0,069622 & $-0,267648$ & \\
\hline 112 & 26 & 882 & 0,225761 & 0,072603 & 1,134475 & 0,006186 & $-0,045386$ & 0,206525 & $-0,469971$ & \\
\hline 113 & 3 & 251 & 0,064339 & 0,009589 & 1,903541 & 0,000817 & $-0,008381$ & 0,058857 & $-0,240527$ & \\
\hline 210 & 31 & 176 & 0,045152 & 0,086301 & $-0,64781$ & 0,007353 & $-0,052116$ & 0,041305 & $-0,189901$ & \\
\hline $2 \backslash 1$ & 21 & 213 & 0,054618 & 0,058904 & $-0,07556$ & 0,005019 & $-0,038337$ & 0,049964 & $-0,215992$ & \\
\hline 212 & 23 & 592 & 0,151573 & 0,064384 & 0,856211 & 0,005486 & $-0,041199$ & 0,138658 & $-0,395231$ & \\
\hline 213 & 2 & 153 & 0,039268 & 0,006849 & 1,74627 & 0,000584 & $-0,006269$ & 0,035922 & $-0,172391$ & \\
\hline 310 & 1 & 41 & 0,010617 & 0,00411 & 0,949089 & 0,00035 & $-0,004020$ & 0,009712 & $-0,064934$ & \\
\hline $3 \backslash 1$ & 1 & 45 & 0,01164 & 0,00411 & 1,041108 & 0,00035 & $-0,004020$ & 0,010648 & $-0,069779$ & \\
\hline 312 & 0 & 128 & 0,032873 & 0,00137 & 3,177937 & 0,000117 & $-0,001525$ & 0,030072 & $-0,152027$ & \\
\hline \multirow[t]{4}{*}{313} & 0 & 42 & 0,010872 & 0,00137 & 2,071512 & 0,000117 & $-0,001525$ & 0,009946 & $-0,066157$ & \\
\hline & 364 & 3908 & & & ( & & $-0,579763$ & & $-3,343707$ & \\
\hline & & & & & & & 0,579763 & & 3,343707 & \\
\hline & & & & & & & 0,049399 & & 3,058803 & 3,108203 \\
\hline
\end{tabular}




\begin{tabular}{|c|c|c|c|c|c|c|c|c|c|c|}
\hline HYRINC & BADS & GOOS & $f(x / 1)$ & $f(x / 0)$ & $w(x)$ & & & & & \\
\hline 010 & 98 & 155 & 0,03978 & 0,269863 & $-1,91455$ & 0,022994 & $-0,125147$ & 0.036391 & $-0,173957$ & \\
\hline $0 \backslash 1$ & 46 & 159 & 0,040803 & 0,127397 & $-1,13855$ & 0,010855 & $-0,070834$ & 0,037327 & $-0,177064$ & \\
\hline 012 & 46 & 419 & 0,107316 & 0,127397 & $-0,17153$ & 0,010855 & $-0,070834$ & 0,098172 & $-0,328734$ & \\
\hline 013 & 1 & 86 & 0,022128 & 0,00411 & 1,68354 & 0,00035 & $-0,004020$ & 0,020243 & $-0,113896$ & \\
\hline $1 \backslash 0$ & 36 & 272 & 0,069711 & 0,1 & $-0,36081$ & 0,008521 & $-0,058578$ & 0,063771 & $-0,253232$ & \\
\hline $1 \backslash 1$ & 19 & 278 & 0,071246 & 0,053425 & 0,287864 & 0,004552 & $-0,035412$ & 0,065175 & $-0,256760$ & \\
\hline 112 & 27 & 889 & 0,227552 & 0,075342 & 1,105334 & 0,00642 & $-0,046756$ & 0,208163 & $-0,471326$ & \\
\hline 113 & 3 & 245 & 0,062804 & 0,009589 & 1,879394 & 0,000817 & $-0,008381$ & 0,057453 & $-0,236790$ & \\
\hline 210 & 32 & 175 & 0,044896 & 0,089041 & $-0,68474$ & 0,007587 & $-0,053429$ & 0,041071 & $-0,189162$ & \\
\hline $2 \backslash 1$ & 21 & 228 & 0,058455 & 0,058904 & $-0,00766$ & 0,005019 & $-0,038337$ & 0,053474 & $-0,225929$ & \\
\hline 212 & 28 & 592 & 0,151573 & 0,078082 & 0,663307 & 0,006653 & $-0,048113$ & 0,138658 & $-0,395231$ & \\
\hline 213 & 2 & 164 & 0,042082 & 0,006849 & 1,81548 & 0,000584 & $-0,006269$ & 0,038497 & $-0,180901$ & \\
\hline 310 & 1 & 32 & 0,008314 & 0,00411 & 0,704635 & 0,00035 & $-0,004020$ & 0,007606 & $-0,053534$ & \\
\hline $3 \backslash 1$ & 3 & 47 & 0,012151 & 0,009589 & 0,236827 & 0,000817 & $-0,008381$ & 0,011116 & $-0,072157$ & \\
\hline 312 & 1 & 134 & 0,034408 & 0,00411 & 2,12496 & 0,00035 & $-0,004020$ & 0,031476 & $-0,157053$ & \\
\hline \multirow[t]{4}{*}{313} & 0 & 33 & 0,00857 & 0,00137 & 1,833553 & 0,000117 & $-0,001525$ & 0,00784 & $-0,054839$ & \\
\hline & 364 & 3908 & & & 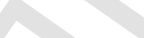 & & $-0,584055$ & & $-3,340565$ & \\
\hline & & & & & & & 0,584055 & & 3,340565 & \\
\hline & & & & & & & 0,049765 & & 3,055929 & 3,105694 \\
\hline
\end{tabular}




\begin{tabular}{|c|c|c|c|c|c|c|c|c|c|c|}
\hline \multicolumn{11}{|c|}{ 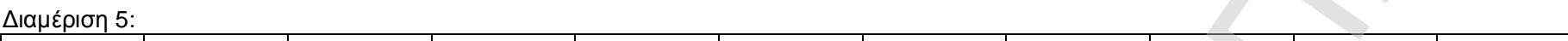 } \\
\hline HYRINC & BADS & GOODS & $f(x / 1)$ & $f(x / 0)$ & $w(x)$ & & & 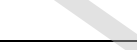 & & \\
\hline 010 & 100 & 165 & 0,042338 & 0,275342 & $-1,87233$ & 0,023461 & $-0,127008$ & 0,038731 & $-0,181662$ & \\
\hline $0 \backslash 1$ & 48 & 149 & 0,038245 & 0,132877 & $-1,24541$ & 0,011322 & $-0,073193$ & 0,034986 & $-0,169231$ & \\
\hline 012 & 53 & 405 & 0,103735 & 0,146575 & $-0,3457$ & 0,012489 & $-0,078971$ & 0,094896 & $-0,322410$ & \\
\hline 013 & 0 & 90 & 0,023152 & 0,00137 & 2,827358 & 0,000117 & $-0,001525$ & 0,021179 & $-0,117781$ & \\
\hline 110 & 31 & 237 & 0,060757 & 0,086301 & $-0,35096$ & 0,007353 & $-0,052116$ & 0,05558 & $-0,231730$ & \\
\hline $1 \backslash 1$ & 20 & 281 & 0,072013 & 0,056164 & 0,248568 & 0,004786 & $-0,036883$ & 0,065877 & $-0,258508$ & \\
\hline 112 & 29 & 881 & 0,225505 & 0,080822 & 1,026095 & 0,006887 & $-0,049459$ & 0,206291 & $-0,469775$ & \\
\hline 113 & 1 & 241 & 0,061781 & 0,00411 & 2,710265 & 0,00035 & $-0,004020$ & 0,056516 & $-0,234271$ & \\
\hline 210 & 33 & 189 & 0,048478 & 0,091781 & $-0,6383$ & 0,00782 & $-0,054731$ & 0,044347 & $-0,199341$ & \\
\hline 211 & 20 & 232 & 0,059478 & 0,056164 & 0,057326 & 0,004786 & $-0,036883$ & 0,05441 & $-0,228522$ & \\
\hline 212 & 22 & 615 & 0,157457 & 0,061644 & 0,93778 & 0,005252 & $-0,039776$ & 0,144041 & $-0,402659$ & \\
\hline 213 & 1 & 167 & 0,04285 & 0,00411 & 2,344379 & 0,00035 & $-0,004020$ & 0,039199 & $-0,183178$ & \\
\hline 310 & 2 & 45 & 0,01164 & 0,006849 & 0,530282 & 0,000584 & $-0,006269$ & 0,010648 & $-0,069779$ & \\
\hline $3 \backslash 1$ & 3 & 47 & 0,012151 & 0,009589 & 0,236827 & 0,000817 & $-0,008381$ & 0,011116 & $-0,072157$ & \\
\hline 312 & 1 & 130 & 0,033384 & 0,00411 & 2,094769 & 0,00035 & $-0,004020$ & 0,03054 & $-0,153712$ & \\
\hline \multirow[t]{4}{*}{313} & 0 & 34 & 0,008826 & 0,00137 & 1,862967 & 0,000117 & $-0,001525$ & 0,008074 & $-0,056133$ & \\
\hline & 364 & 3908 & & & & & $-0,578778$ & & $-3,350851$ & \\
\hline & & & & & & & 0,578778 & & 3,350851 & \\
\hline & & & & & & & 0,049315 & & 3,065338 & 3,114653 \\
\hline
\end{tabular}




\begin{tabular}{|c|c|c|c|c|c|c|c|c|c|c|}
\hline HYRIJOB & BADS & GOODS & $f(x / 1)$ & $f(x / 0)$ & $w(x)$ & & & & $P$ & \\
\hline $1 \backslash 1$ & 9 & 227 & 0,058199 & 0,026027 & 0,804719 & 0,002218 & $-0,019553$ & 0,05324 & $-0,225277$ & \\
\hline $2 \backslash 1$ & 41 & 138 & 0,035431 & 0,113699 & $-1,16596$ & 0,009688 & $-0,064808$ & 0,032412 & $-0,160353$ & \\
\hline $3 \backslash 1$ & 8 & 262 & 0,067153 & 0,023288 & 1,059045 & 0,001984 & $-0,017813$ & 0,061431 & $-0,247253$ & \\
\hline $4 \backslash 1$ & 0 & 47 & 0,012151 & 0,00137 & 2,182737 & 0,000117 & $-0,001525$ & 0,011116 & $-0,072157$ & \\
\hline 112 & 36 & 202 & 0,051804 & 0,1 & $-0,65771$ & 0,008521 & $-0,058578$ & 0,04739 & $-0,208480$ & \\
\hline 212 & 37 & 36 & 0,009337 & 0,10274 & $-2,39817$ & 0,008754 & $-0,059841$ & 0,008542 & $-0,058693$ & \\
\hline 312 & 27 & 157 & 0,040292 & 0,075342 & $-0,6259$ & 0,00642 & $-0,046756$ & 0,036859 & $-0,175515$ & \\
\hline 412 & 5 & 53 & 0,013686 & 0,015068 & $-0,09621$ & 0,001284 & $-0,012332$ & 0,01252 & $-0,079123$ & \\
\hline 113 & 14 & 280 & 0,071757 & 0,039726 & 0,591286 & 0,003385 & $-0,027779$ & 0,065643 & $-0,257926$ & \\
\hline 213 & 41 & 175 & 0,044896 & 0,113699 & $-0,92919$ & 0,009688 & $-0,064808$ & 0,041071 & $-0,189162$ & \\
\hline 313 & 14 & 377 & 0,096572 & 0,039726 & 0,888282 & 0,003385 & $-0,027779$ & 0,088343 & $-0,309267$ & \\
\hline 413 & 1 & 72 & 0,018547 & 0,00411 & 1,506982 & 0,00035 & $-0,004020$ & 0,016967 & $-0,099783$ & \\
\hline $1 \backslash 4$ & 1 & 72 & 0,018547 & 0,00411 & 1,506982 & 0,00035 & $-0,004020$ & 0,016967 & $-0,099783$ & \\
\hline $2 \backslash 4$ & 16 & 53 & 0,013686 & 0,045205 & $-1,19482$ & 0,003852 & $-0,030892$ & 0,01252 & $-0,079123$ & \\
\hline $3 \backslash 4$ & 1 & 113 & 0,029036 & 0,00411 & 1,955198 & 0,00035 & $-0,004020$ & 0,026562 & $-0,139037$ & \\
\hline $4 \backslash 4$ & 0 & 7 & 0,001919 & 0,00137 & 0,336911 & 0,000117 & $-0,001525$ & 0,001755 & $-0,016067$ & \\
\hline 115 & 19 & 378 & 0,096828 & 0,053425 & 0,594662 & 0,004552 & $-0,035412$ & 0,088578 & $-0,309748$ & \\
\hline $2 \backslash 5$ & 51 & 302 & 0,077386 & 0,141096 & $-0,60064$ & 0,012022 & $-0,076680$ & 0,070792 & $-0,270444$ & \\
\hline 315 & 28 & 671 & 0,171783 & 0,078082 & 0,78847 & 0,006653 & $-0,048113$ & 0,157146 & $-0,419552$ & \\
\hline 415 & 0 & 67 & 0,017268 & 0,00137 & 2,534135 & 0,000117 & $-0,001525$ & 0,015797 & $-0,094530$ & \\
\hline 116 & 0 & 35 & 0,009082 & 0,00137 & 1,89154 & 0,000117 & $-0,001525$ & 0,008308 & $-0,057418$ & \\
\hline 216 & 12 & 94 & 0,024175 & 0,034247 & $-0,34827$ & 0,002918 & $-0,024572$ & 0,022115 & $-0,121607$ & \\
\hline 316 & 3 & 87 & 0,022384 & 0,009589 & 0,847736 & 0,000817 & $-0,008381$ & 0,020477 & $-0,114873$ & \\
\hline \multirow[t]{4}{*}{416} & 0 & 3 & 0,000895 & 0,00137 & $-0,42523$ & 0,000117 & $-0,001525$ & 0,000819 & $-0,008399$ & \\
\hline & 364 & 3908 & & 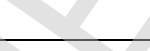 & & & $-0,643779$ & & $-3,813571$ & \\
\hline & & & & 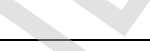 & & & 0,643779 & & 3,813571 & \\
\hline & & & & 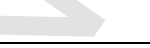 & & & 0,054854 & & 3,488632 & 3,543485 \\
\hline
\end{tabular}




\begin{tabular}{|c|c|c|c|c|c|c|c|c|c|c|}
\hline HYRJOB & BADS & GOODS & $f(x / 1)$ & $f(x / 0)$ & $w(x)$ & & & & 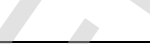 & 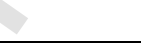 \\
\hline 010 & 34 & 36 & 0,009337 & 0,094521 & $-2,31479$ & 0,008054 & $-0,056023$ & 0,008542 & $-0,058693$ & \\
\hline 012 & 44 & 138 & 0,035431 & 0,121918 & $-1,23576$ & 0,010388 & $-0,068447$ & 0,032412 & $-0,160353$ & \\
\hline $0 \backslash 3$ & 43 & 161 & 0,041315 & 0,119178 & $-1,0594$ & 0,010155 & $-0,067241$ & 0,037795 & $-0,178605$ & \\
\hline $0 \backslash 4$ & 13 & 56 & 0,014454 & 0,036986 & $-0,93959$ & 0,003151 & $-0,026188$ & 0,013222 & $-0,082519$ & \\
\hline $0 \backslash 5$ & 47 & 312 & 0,079944 & 0,130137 & $-0,48726$ & 0,011088 & $-0,072017$ & 0,073132 & $-0,275953$ & \\
\hline 016 & 14 & 88 & 0,02264 & 0,039726 & $-0,56229$ & 0,003385 & $-0,027779$ & 0,020711 & $-0,115846$ & \\
\hline $1 \backslash 0$ & 29 & 177 & 0,045408 & 0,080822 & $-0,57656$ & 0,006887 & $-0,049459$ & 0,041539 & $-0,190639$ & \\
\hline $1 \backslash 2$ & 11 & 274 & 0,070223 & 0,031507 & 0,801465 & 0,002685 & $-0,022929$ & 0,064239 & $-0,254413$ & \\
\hline $1 \backslash 3$ & 15 & 367 & 0,094014 & 0,042466 & 0,794744 & 0,003618 & $-0,029346$ & 0,086003 & $-0,304406$ & \\
\hline $1 \backslash 4$ & 3 & 117 & 0,030059 & 0,009589 & 1,142536 & 0,000817 & $-0,008381$ & 0,027498 & $-0,142563$ & \\
\hline $1 \backslash 5$ & 28 & 685 & 0,175365 & 0,078082 & 0,809105 & 0,006653 & $-0,048113$ & 0,160422 & $-0,423524$ & \\
\hline 116 & 3 & 83 & 0,021361 & 0,009589 & 0,800944 & 0,000817 & $-0,008381$ & 0,019541 & $-0,110941$ & \\
\hline 210 & 33 & 193 & 0,049501 & 0,091781 & $-0,61741$ & 0,00782 & $-0,054731$ & 0,045283 & $-0,202185$ & \\
\hline 212 & 8 & 226 & 0,057943 & 0,023288 & 0,911539 & 0,001984 & $-0,017813$ & 0,053006 & $-0,224624$ & \\
\hline 213 & 10 & 275 & 0,070478 & 0,028767 & 0,896073 & 0,002451 & $-0,021257$ & 0,064473 & $-0,255002$ & \\
\hline $2 \backslash 4$ & 1 & 72 & 0,018547 & 0,00411 & 1,506982 & 0,00035 & $-0,004020$ & 0,016967 & $-0,099783$ & \\
\hline $2 \backslash 5$ & 22 & 369 & 0,094525 & 0,061644 & 0,427496 & 0,005252 & $-0,039776$ & 0,086471 & $-0,305385$ & \\
\hline 216 & 1 & 37 & 0,009593 & 0,00411 & 0,847736 & 0,00035 & $-0,004020$ & 0,008776 & $-0,059959$ & \\
\hline 310 & 4 & 62 & 0,015989 & 0,012329 & 0,25995 & 0,00105 & $-0,010394$ & 0,014626 & $-0,089152$ & \\
\hline 312 & 0 & 39 & 0,010105 & 0,00137 & 1,998308 & 0,000117 & $-0,001525$ & 0,009244 & $-0,062464$ & \\
\hline 313 & 1 & 67 & 0,017268 & 0,00411 & 1,435523 & 0,00035 & $-0,004020$ & 0,015797 & $-0,094530$ & \\
\hline $3 \backslash 4$ & 0 & 9 & 0,00243 & 0,00137 & 0,573299 & 0,000117 & $-0,001525$ & 0,002223 & $-0,019593$ & \\
\hline 315 & 0 & 60 & 0,015477 & 0,00137 & 2,424651 & 0,000117 & $-0,001525$ & 0,014158 & $-0,086964$ & \\
\hline \multirow[t]{4}{*}{316} & 0 & 5 & 0,001407 & 0,00137 & 0,026756 & 0,000117 & $-0,001525$ & 0,001287 & $-0,012358$ & \\
\hline & 364 & 3908 & & 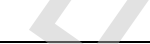 & 8 & & $-0,646433$ & & $-3,810452$ & \\
\hline & & & & + & 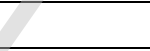 & & 0,646433 & & 3,810452 & \\
\hline & & & & & & & 0,05508 & & 3,485779 & 3,540859 \\
\hline
\end{tabular}




\begin{tabular}{|c|c|c|c|c|c|c|c|c|c|c|}
\hline HYRJOB & BADS & GOODS & $f(x / 1)$ & $f(x / 0)$ & $w(x)$ & & & & + & 8 \\
\hline 010 & 35 & 43 & 0,011128 & 0,09726 & $-2,16791$ & 0,008287 & $-0,057305$ & 0,01018 & $-0,067372$ & \\
\hline 012 & 48 & 130 & 0,033384 & 0,132877 & $-1,38133$ & 0,011322 & $-0,073193$ & 0,03054 & $-0,153712$ & \\
\hline 013 & 51 & 192 & 0,049245 & 0,141096 & $-1,05263$ & 0,012022 & $-0,076680$ & 0,045049 & $-0,201476$ & \\
\hline 014 & 16 & 61 & 0,015733 & 0,045205 & $-1,05546$ & 0,003852 & $-0,030892$ & 0,014392 & $-0,088061$ & \\
\hline 015 & 37 & 298 & 0,076362 & 0,10274 & $-0,29671$ & 0,008754 & $-0,059841$ & 0,069856 & $-0,268209$ & \\
\hline 016 & 12 & 95 & 0,024431 & 0,034247 & $-0,33774$ & 0,002918 & $-0,024572$ & 0,022349 & $-0,122555$ & \\
\hline 110 & 26 & 147 & 0,037733 & 0,072603 & $-0,65446$ & 0,006186 & $-0,045386$ & 0,034518 & $-0,167638$ & \\
\hline 112 & 10 & 285 & 0,073037 & 0,028767 & 0,931727 & 0,002451 & $-0,021257$ & 0,066813 & $-0,260821$ & \\
\hline 113 & 18 & 419 & 0,107316 & 0,050685 & 0,750153 & 0,004319 & $-0,033924$ & 0,098172 & $-0,328734$ & \\
\hline $1 \backslash 4$ & 2 & 117 & 0,030059 & 0,006849 & 1,479008 & 0,000584 & $-0,006269$ & 0,027498 & $-0,142563$ & \\
\hline 115 & 28 & 647 & 0,165643 & 0,078082 & 0,752075 & 0,006653 & $-0,048113$ & 0,15153 & $-0,412513$ & \\
\hline 116 & 2 & 84 & 0,021617 & 0,006849 & 1,149321 & 0,000584 & $-0,006269$ & 0,019775 & $-0,111930$ & \\
\hline 210 & 37 & 191 & 0,04899 & 0,10274 & $-0,74059$ & 0,008754 & $-0,059841$ & 0,044815 & $-0,200767$ & \\
\hline 212 & 9 & 194 & 0,049757 & 0,026027 & 0,648001 & 0,002218 & $-0,019553$ & 0,045517 & $-0,202891$ & \\
\hline 213 & 13 & 295 & 0,075595 & 0,036986 & 0,71484 & 0,003151 & $-0,026188$ & 0,069154 & $-0,266522$ & \\
\hline 214 & 2 & 71 & 0,018291 & 0,006849 & 0,982267 & 0,000584 & $-0,006269$ & 0,016733 & $-0,098742$ & \\
\hline 215 & 15 & 345 & 0,088386 & 0,042466 & 0,733013 & 0,003618 & $-0,029346$ & 0,080855 & $-0,293383$ & \\
\hline 216 & 1 & 38 & 0,009849 & 0,00411 & 0,874054 & 0,00035 & $-0,004020$ & 0,00901 & $-0,061216$ & \\
\hline 310 & 2 & 66 & 0,017012 & 0,006849 & 0,909772 & 0,000584 & $-0,006269$ & 0,015562 & $-0,093465$ & \\
\hline 312 & 0 & 45 & 0,01164 & 0,00137 & 2,13972 & 0,000117 & $-0,001525$ & 0,010648 & $-0,069779$ & \\
\hline 313 & 0 & 73 & 0,018803 & 0,00137 & 2,619293 & 0,000117 & $-0,001525$ & 0,017201 & $-0,100820$ & \\
\hline $3 \backslash 4$ & 0 & 7 & 0,001919 & 0,00137 & 0,336911 & 0,000117 & $-0,001525$ & 0,001755 & $-0,016067$ & \\
\hline 315 & 0 & 61 & 0,015733 & 0,00137 & 2,441045 & 0,000117 & $-0,001525$ & 0,014392 & $-0,088061$ & \\
\hline \multirow[t]{4}{*}{$3 \backslash 6$} & 0 & 4 & 0,001151 & 0,00137 & $-0,17391$ & 0,000117 & $-0,001525$ & 0,001053 & $-0,010416$ & \\
\hline & 364 & 3908 & & 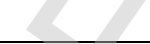 & 8 & & $-0,642814$ & & $-3,827713$ & \\
\hline & & & & - & 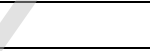 & & 0,642814 & & 3,827713 & \\
\hline & & & & & & & 0,054772 & & 3,501569 & 3,556341 \\
\hline
\end{tabular}




\begin{tabular}{|c|c|c|c|c|c|c|c|c|c|c|}
\hline HYRJOB & BADS & GOODS & $f(x / 1)$ & $f(x / 0)$ & $w(x)$ & & & & 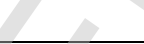 & 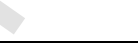 \\
\hline 010 & 28 & 39 & 0,010105 & 0,078082 & $-2,04474$ & 0,006653 & $-0,048113$ & 0,009244 & $-0,062464$ & \\
\hline 012 & 43 & 160 & 0,041059 & 0,119178 & $-1,06561$ & 0,010155 & $-0,067241$ & 0,037561 & $-0,177836$ & \\
\hline 013 & 51 & 165 & 0,042338 & 0,141096 & $-1,20375$ & 0,012022 & $-0,076680$ & 0,038731 & $-0,181662$ & \\
\hline $0 \backslash 4$ & 13 & 61 & 0,015733 & 0,036986 & $-0,85479$ & 0,003151 & $-0,026188$ & 0,014392 & $-0,088061$ & \\
\hline 015 & 43 & 304 & 0,077897 & 0,119178 & $-0,42523$ & 0,010155 & $-0,067241$ & 0,07126 & $-0,271555$ & \\
\hline 016 & 13 & 90 & 0,023152 & 0,036986 & $-0,46848$ & 0,003151 & $-0,026188$ & 0,021179 & $-0,117781$ & \\
\hline 110 & 27 & 170 & 0,043617 & 0,075342 & $-0,54659$ & 0,00642 & $-0,046756$ & 0,039901 & $-0,185437$ & \\
\hline 112 & 10 & 314 & 0,080455 & 0,028767 & 1,028469 & 0,002451 & $-0,021257$ & 0,0736 & $-0,277042$ & \\
\hline 113 & 19 & 381 & 0,097595 & 0,053425 & 0,602557 & 0,004552 & $-0,035412$ & 0,08928 & $-0,311186$ & \\
\hline 114 & 3 & 109 & 0,028012 & 0,009589 & 1,072022 & 0,000817 & $-0,008381$ & 0,025625 & $-0,135463$ & \\
\hline 115 & 22 & 625 & 0,160015 & 0,061644 & 0,953897 & 0,005252 & $-0,039776$ & 0,146381 & $-0,405797$ & \\
\hline 116 & 4 & 85 & 0,021873 & 0,012329 & 0,573299 & 0,00105 & $-0,010394$ & 0,020009 & $-0,112915$ & \\
\hline 210 & 38 & 197 & 0,050524 & 0,105479 & $-0,73606$ & 0,008987 & $-0,061096$ & 0,046219 & $-0,205000$ & \\
\hline 212 & 11 & 239 & 0,061269 & 0,031507 & 0,665067 & 0,002685 & $-0,022929$ & 0,056048 & $-0,233003$ & \\
\hline 213 & 12 & 272 & 0,069711 & 0,034247 & 0,71077 & 0,002918 & $-0,024572$ & 0,063771 & $-0,253232$ & \\
\hline 214 & 2 & 75 & 0,019314 & 0,006849 & 1,036702 & 0,000584 & $-0,006269$ & 0,017669 & $-0,102879$ & \\
\hline 215 & 19 & 335 & 0,085828 & 0,053425 & 0,474068 & 0,004552 & $-0,035412$ & 0,078515 & $-0,288219$ & \\
\hline 216 & 1 & 41 & 0,010617 & 0,00411 & 0,949089 & 0,00035 & $-0,004020$ & 0,009712 & $-0,064934$ & \\
\hline 310 & 4 & 59 & 0,015221 & 0,012329 & 0,210759 & 0,00105 & $-0,010394$ & 0,013924 & $-0,085861$ & \\
\hline 312 & 0 & 51 & 0,013175 & 0,00137 & 2,263589 & 0,000117 & $-0,001525$ & 0,012052 & $-0,076827$ & \\
\hline 313 & 1 & 60 & 0,015477 & 0,00411 & 1,326039 & 0,00035 & $-0,004020$ & 0,014158 & $-0,086964$ & \\
\hline 314 & 0 & 8 & 0,002174 & 0,00137 & 0,462074 & 0,000117 & $-0,001525$ & 0,001989 & $-0,017850$ & \\
\hline 315 & 0 & 63 & 0,016245 & 0,00137 & 2,473048 & 0,000117 & $-0,001525$ & 0,01486 & $-0,090238$ & \\
\hline \multirow[t]{4}{*}{316} & 0 & 5 & 0,001407 & 0,00137 & 0,026756 & 0,000117 & $-0,001525$ & 0,001287 & $-0,012358$ & \\
\hline & 364 & 3908 & & 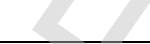 & 8 & & $-0,648439$ & & $-3,844563$ & \\
\hline & & & & 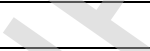 & -1 & & 0,648439 & & 3,844563 & \\
\hline & & & & 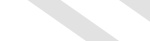 & & & 0,055251 & & 3,516984 & 3,572234 \\
\hline
\end{tabular}




\begin{tabular}{|c|c|c|c|c|c|c|c|c|c|c|}
\hline HYRJOB & BADS & GOODS & $f(x / 1)$ & $f(x / 0)$ & $w(x)$ & & & & 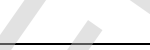 & $\theta$ \\
\hline 010 & 34 & 38 & 0,009849 & 0,094521 & $-2,26144$ & 0,008054 & $-0,056023$ & 0,00901 & $-0,061216$ & \\
\hline 012 & 42 & 130 & 0,033384 & 0,116438 & $-1,24927$ & 0,009921 & $-0,066028$ & 0,03054 & $-0,153712$ & \\
\hline 013 & 52 & 167 & 0,04285 & 0,143836 & $-1,21097$ & 0,012256 & $-0,077828$ & 0,039199 & $-0,183178$ & \\
\hline 014 & 14 & 53 & 0,013686 & 0,039726 & $-1,06561$ & 0,003385 & $-0,027779$ & 0,01252 & $-0,079123$ & \\
\hline 015 & 46 & 320 & 0,08199 & 0,127397 & $-0,44071$ & 0,010855 & $-0,070834$ & 0,075004 & $-0,280282$ & \\
\hline 016 & 13 & 101 & 0,025966 & 0,036986 & $-0,35377$ & 0,003151 & $-0,026188$ & 0,023753 & $-0,128166$ & \\
\hline 110 & 23 & 148 & 0,037989 & 0,064384 & $-0,52755$ & 0,005486 & $-0,041199$ & 0,034752 & $-0,168436$ & \\
\hline 112 & 9 & 281 & 0,072013 & 0,026027 & 1,017701 & 0,002218 & $-0,019553$ & 0,065877 & $-0,258508$ & \\
\hline 113 & 16 & 360 & 0,092223 & 0,045205 & 0,712992 & 0,003852 & $-0,030892$ & 0,084365 & $-0,300948$ & \\
\hline 114 & 3 & 114 & 0,029291 & 0,009589 & 1,116672 & 0,000817 & $-0,008381$ & 0,026796 & $-0,139923$ & \\
\hline 115 & 26 & 654 & 0,167434 & 0,072603 & 0,835587 & 0,006186 & $-0,045386$ & 0,153168 & $-0,414597$ & \\
\hline 116 & 4 & 83 & 0,021361 & 0,012329 & 0,54963 & 0,00105 & $-0,010394$ & 0,019541 & $-0,110941$ & \\
\hline 210 & 32 & 216 & 0,055385 & 0,089041 & $-0,47479$ & 0,007587 & $-0,053429$ & 0,050666 & $-0,218007$ & \\
\hline 212 & 12 & 226 & 0,057943 & 0,034247 & 0,525877 & 0,002918 & $-0,024572$ & 0,053006 & $-0,224624$ & \\
\hline 213 & 14 & 276 & 0,070734 & 0,039726 & 0,576923 & 0,003385 & $-0,027779$ & 0,064707 & $-0,255589$ & \\
\hline 214 & 2 & 67 & 0,017268 & 0,006849 & 0,924697 & 0,000584 & $-0,006269$ & 0,015797 & $-0,094530$ & \\
\hline 215 & 15 & 379 & 0,097084 & 0,042466 & 0,826875 & 0,003618 & $-0,029346$ & 0,088812 & $-0,310228$ & \\
\hline 216 & 1 & 39 & 0,010105 & 0,00411 & 0,899696 & 0,00035 & $-0,004020$ & 0,009244 & $-0,062464$ & \\
\hline 310 & 5 & 62 & 0,015989 & 0,015068 & 0,059279 & 0,001284 & $-0,012332$ & 0,014626 & $-0,089152$ & \\
\hline 312 & 0 & 47 & 0,012151 & 0,00137 & 2,182737 & 0,000117 & $-0,001525$ & 0,011116 & $-0,072157$ & \\
\hline 313 & 1 & 71 & 0,018291 & 0,00411 & 1,493093 & 0,00035 & $-0,004020$ & 0,016733 & $-0,098742$ & \\
\hline 314 & 0 & 6 & 0,001663 & 0,00137 & 0,19381 & 0,000117 & $-0,001525$ & 0,001521 & $-0,014239$ & \\
\hline 315 & 0 & 67 & 0,017268 & 0,00137 & 2,534135 & 0,000117 & $-0,001525$ & 0,015797 & $-0,094530$ & \\
\hline \multirow[t]{4}{*}{316} & 0 & 3 & 0,000895 & 0,00137 & $-0,42523$ & 0,000117 & $-0,001525$ & 0,000819 & $-0,008399$ & \\
\hline & 364 & 3908 & & & 2 & & $-0,648353$ & & $-3,821690$ & \\
\hline & & & & & & & 0,648353 & & 3,821690 & \\
\hline & & & & 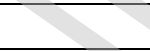 & & & 0,055244 & & 3,496059 & 3,551302 \\
\hline
\end{tabular}




\begin{tabular}{|c|c|c|c|c|c|c|c|c|c|c|}
\hline HYR\JYR & BADS & GOODS & $f(x / 1)$ & $f(x / 0)$ & $w(x)$ & & & & & \\
\hline $1 \backslash 1$ & 14 & 176 & 0,045152 & 0,039726 & 0,128033 & 0,003385 & $-0,027779$ & 0,041305 & $-0,189901$ & \\
\hline $2 \ 1$ & 28 & 141 & 0,036199 & 0,078082 & $-0,76874$ & 0,006653 & $-0,048113$ & 0,033114 & $-0,162803$ & \\
\hline $3 \backslash 1$ & 13 & 296 & 0,075851 & 0,036986 & 0,718218 & 0,003151 & $-0,026188$ & 0,069388 & $-0,267085$ & \\
\hline $4 \backslash 1$ & 1 & 38 & 0,009849 & 0,00411 & 0,874054 & 0,00035 & $-0,004020$ & 0,00901 & $-0,061216$ & \\
\hline 112 & 22 & 266 & 0,068176 & 0,061644 & 0,100719 & 0,005252 & $-0,039776$ & 0,062367 & $-0,249660$ & \\
\hline 212 & 67 & 201 & 0,051548 & 0,184932 & $-1,27748$ & 0,015757 & $-0,094352$ & 0,047156 & $-0,207788$ & \\
\hline 312 & 32 & 311 & 0,079688 & 0,089041 & $-0,11098$ & 0,007587 & $-0,053429$ & 0,072898 & $-0,275407$ & \\
\hline 412 & 0 & 24 & 0,006268 & 0,00137 & 1,520681 & 0,000117 & $-0,001525$ & 0,005734 & $-0,042694$ & \\
\hline 113 & 26 & 308 & 0,07892 & 0,072603 & 0,083438 & 0,006186 & $-0,045386$ & 0,072196 & $-0,273763$ & \\
\hline 213 & 71 & 236 & 0,060501 & 0,19589 & $-1,17489$ & 0,016691 & $-0,098557$ & 0,055346 & $-0,231091$ & \\
\hline 313 & 15 & 415 & 0,106293 & 0,042466 & 0,917503 & 0,003618 & $-0,029346$ & 0,097236 & $-0,326944$ & \\
\hline 413 & 4 & 49 & 0,012663 & 0,012329 & 0,026756 & 0,00105 & $-0,010394$ & 0,011584 & $-0,074506$ & \\
\hline 114 & 17 & 444 & 0,113712 & 0,047945 & 0,86361 & 0,004085 & $-0,032418$ & 0,104023 & $-0,339638$ & \\
\hline 214 & 32 & 220 & 0,056408 & 0,089041 & $-0,45648$ & 0,007587 & $-0,053429$ & 0,051602 & $-0,220672$ & \\
\hline 314 & 21 & 645 & 0,165132 & 0,058904 & 1,030833 & 0,005019 & $-0,038337$ & 0,151062 & $-0,411913$ & \\
\hline \multirow[t]{4}{*}{$4 \backslash 4$} & 1 & 138 & 0,035431 & 0,00411 & 2,154266 & 0,00035 & $-0,004020$ & 0,032412 & $-0,160353$ & \\
\hline & 364 & 3908 & & 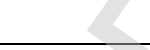 & 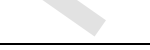 & & $-0,607068$ & & $-3,495433$ & \\
\hline & & & & 2 & & & 0,607068 & & 3,495433 & \\
\hline & & & & 2 & 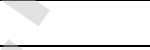 & & 0,051726 & & 3,197601 & 3,249327 \\
\hline
\end{tabular}




\begin{tabular}{|c|c|c|c|c|c|c|c|c|c|c|}
\hline HYRJYR & BADS & GOODS & $f(x / 1)$ & $f(x / 0)$ & $w(x)$ & & & ( & & \\
\hline 010 & 68 & 204 & 0,052315 & 0,187671 & $-1,27741$ & 0,015991 & $-0,095411$ & 0,047858 & $-0,209861$ & \\
\hline $0 \backslash 1$ & 71 & 262 & 0,067153 & 0,19589 & $-1,07059$ & 0,016691 & $-0,098557$ & 0,061431 & $-0,247253$ & \\
\hline 012 & 17 & 119 & 0,03057 & 0,047945 & $-0,45002$ & 0,004085 & $-0,032418$ & 0,027966 & $-0,144309$ & \\
\hline 013 & 39 & 206 & 0,052827 & 0,108219 & $-0,71714$ & 0,009221 & $-0,062341$ & 0,048326 & $-0,211235$ & \\
\hline 110 & 31 & 311 & 0,079688 & 0,086301 & $-0,07973$ & 0,007353 & $-0,052116$ & 0,072898 & $-0,275407$ & \\
\hline $1 \backslash 1$ & 22 & 494 & 0,126503 & 0,061644 & 0,718892 & 0,005252 & $-0,039776$ & 0,115724 & $-0,360045$ & \\
\hline 112 & 13 & 242 & 0,062036 & 0,036986 & 0,517173 & 0,003151 & $-0,026188$ & 0,05675 & $-0,234903$ & \\
\hline 113 & 23 & 656 & 0,167946 & 0,064384 & 0,958783 & 0,005486 & $-0,041199$ & 0,153636 & $-0,415187$ & \\
\hline 210 & 19 & 249 & 0,063827 & 0,053425 & 0,177905 & 0,004552 & $-0,035412$ & 0,058389 & $-0,239286$ & \\
\hline $2 \backslash 1$ & 26 & 327 & 0,083781 & 0,072603 & 0,143204 & 0,006186 & $-0,045386$ & 0,076642 & $-0,284015$ & \\
\hline 212 & 15 & 156 & 0,040036 & 0,042466 & $-0,05892$ & 0,003618 & $-0,029346$ & 0,036625 & $-0,174737$ & \\
\hline 213 & 15 & 440 & 0,112689 & 0,042466 & 0,975931 & 0,003618 & $-0,029346$ & 0,103087 & $-0,337926$ & \\
\hline 310 & 0 & 34 & 0,008826 & 0,00137 & 1,862967 & 0,000117 & $-0,001525$ & 0,008074 & $-0,056133$ & \\
\hline $3 \backslash 1$ & 4 & 51 & 0,013175 & 0,012329 & 0,066365 & 0,00105 & $-0,010394$ & 0,012052 & $-0,076827$ & \\
\hline 312 & 1 & 41 & 0,010617 & 0,00411 & 0,949089 & 0,00035 & $-0,004020$ & 0,009712 & $-0,064934$ & \\
\hline \multirow[t]{4}{*}{313} & 0 & 116 & 0,029803 & 0,00137 & 3,079899 & 0,000117 & $-0,001525$ & 0,027264 & $-0,141686$ & \\
\hline & 364 & 3908 & & & 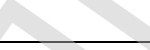 & & $-0,604960$ & & $-3,473744$ & \\
\hline & & & & & & & 0,604960 & & 3,473744 & \\
\hline & & & & & & & 0,051546 & & 3,17776 & 3,229307 \\
\hline
\end{tabular}




\begin{tabular}{|c|c|c|c|c|c|c|c|c|c|c|}
\hline HYRJYR & BADS & תחסר & $f(x / 1)$ & $f(x / 0)$ & $w(x)$ & & & 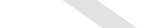 & & \\
\hline 010 & 72 & 213 & 0,054618 & 0,19863 & $-1,29109$ & 0,016924 & $-0,099596$ & 0,049964 & $-0,215992$ & \\
\hline $0 \backslash 1$ & 65 & 270 & 0,069199 & 0,179452 & $-0,95292$ & 0,01529 & $-0,092220$ & 0,063303 & $-0,252046$ & \\
\hline 012 & 23 & 111 & 0,028524 & 0,064384 & $-0,81412$ & 0,005486 & $-0,041199$ & 0,026094 & $-0,137256$ & \\
\hline 013 & 39 & 225 & 0,057687 & 0,108219 & $-0,62912$ & 0,009221 & $-0,062341$ & 0,052772 & $-0,223969$ & \\
\hline $1 \backslash 0$ & 31 & 329 & 0,084293 & 0,086301 & $-0,02355$ & 0,007353 & $-0,052116$ & 0,07711 & $-0,285072$ & \\
\hline $1 \backslash 1$ & 20 & 464 & 0,118828 & 0,056164 & 0,749397 & 0,004786 & $-0,036883$ & 0,108703 & $-0,348017$ & \\
\hline $1 \backslash 2$ & 10 & 260 & 0,066641 & 0,028767 & 0,840088 & 0,002451 & $-0,021257$ & 0,060963 & $-0,246042$ & \\
\hline 113 & 25 & 646 & 0,165388 & 0,069863 & 0,861755 & 0,005953 & $-0,044004$ & 0,151296 & $-0,412214$ & \\
\hline 210 & 25 & 234 & 0,05999 & 0,069863 & $-0,15236$ & 0,005953 & $-0,044004$ & 0,054878 & $-0,229809$ & \\
\hline $2 \backslash 1$ & 22 & 324 & 0,083014 & 0,061644 & 0,297631 & 0,005252 & $-0,039776$ & 0,07594 & $-0,282421$ & \\
\hline 212 & 14 & 145 & 0,037222 & 0,039726 & $-0,06511$ & 0,003385 & $-0,027779$ & 0,03405 & $-0,166036$ & \\
\hline 213 & 16 & 431 & 0,110386 & 0,045205 & 0,892768 & 0,003852 & $-0,030892$ & 0,100981 & $-0,334029$ & \\
\hline 310 & 0 & 38 & 0,009849 & 0,00137 & 1,972666 & 0,000117 & $-0,001525$ & 0,00901 & $-0,061216$ & \\
\hline $3 \backslash 1$ & 1 & 56 & 0,014454 & 0,00411 & 1,257636 & 0,00035 & $-0,004020$ & 0,013222 & $-0,082519$ & \\
\hline 312 & 0 & 34 & 0,008826 & 0,00137 & 1,862967 & 0,000117 & $-0,001525$ & 0,008074 & $-0,056133$ & \\
\hline \multirow[t]{4}{*}{313} & 1 & 128 & 0,032873 & 0,00411 & 2,079324 & 0,00035 & $-0,004020$ & 0,030072 & $-0,152027$ & \\
\hline & 364 & 3908 & & & 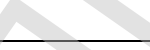 & & $-0,603157$ & & $-3,484797$ & \\
\hline & & & & & 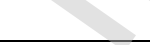 & & 0,603157 & & 3,484797 & \\
\hline & & & & & & & 0,051393 & & 3,187872 & 3,239264 \\
\hline
\end{tabular}




\begin{tabular}{|c|c|c|c|c|c|c|c|c|c|c|}
\hline HYRJYR & RADS & תחת & $f(x / 1)$ & $f(y / 0)$ & & & & & & \\
\hline $0 \backslash 0$ & 45 & 123 & 0,031594 & 0,124658 & $-1,37261$ & 0,010622 & $-0,069644$ & 0,028902 & $-0,147766$ & \\
\hline $0 \backslash 1$ & 80 & 347 & 0,088897 & 0,220548 & $-0,90863$ & 0,018792 & $-0,107748$ & 0,081323 & $-0,294405$ & \\
\hline 012 & 28 & 130 & 0,033384 & 0,078082 & $-0,84967$ & 0,006653 & $-0,048113$ & 0,03054 & $-0,153712$ & \\
\hline 013 & 38 & 219 & 0,056152 & 0,105479 & $-0,63045$ & 0,008987 & $-0,061096$ & 0,051368 & $-0,220008$ & \\
\hline $1 \backslash 0$ & 18 & 210 & 0,05385 & 0,050685 & 0,060575 & 0,004319 & $-0,033924$ & 0,049262 & $-0,213963$ & \\
\hline $1 \backslash 1$ & 26 & 548 & 0,140317 & 0,072603 & 0,658903 & 0,006186 & $-0,045386$ & 0,128361 & $-0,380170$ & \\
\hline $1 \backslash 2$ & 16 & 301 & 0,07713 & 0,045205 & 0,53427 & 0,003852 & $-0,030892$ & 0,070558 & $-0,269887$ & \\
\hline 113 & 25 & 625 & 0,160015 & 0,069863 & 0,828733 & 0,005953 & $-0,044004$ & 0,146381 & $-0,405797$ & \\
\hline $2 \backslash 0$ & 19 & 156 & 0,040036 & 0,053425 & $-0,2885$ & 0,004552 & $-0,035412$ & 0,036625 & $-0,174737$ & \\
\hline $2 \backslash 1$ & 32 & 399 & 0,1022 & 0,089041 & 0,137834 & 0,007587 & $-0,053429$ & 0,093492 & $-0,319650$ & \\
\hline 212 & 14 & 178 & 0,045664 & 0,039726 & 0,1393 & 0,003385 & $-0,027779$ & 0,041773 & $-0,191374$ & \\
\hline $2 \backslash 3$ & 18 & 426 & 0,109107 & 0,050685 & 0,766702 & 0,004319 & $-0,033924$ & 0,099811 & $-0,331837$ & \\
\hline $3 \backslash 0$ & 0 & 22 & 0,005756 & 0,00137 & 1,435523 & 0,000117 & $-0,001525$ & 0,005266 & $-0,039856$ & \\
\hline $3 \backslash 1$ & 3 & 64 & 0,0165 & 0,009589 & 0,542763 & 0,000817 & $-0,008381$ & 0,015094 & $-0,091319$ & \\
\hline 312 & 1 & 35 & 0,009082 & 0,00411 & 0,792928 & 0,00035 & $-0,004020$ & 0,008308 & $-0,057418$ & \\
\hline \multirow[t]{4}{*}{313} & 1 & 125 & 0,032105 & 0,00411 & 2,055701 & 0,00035 & $-0,004020$ & 0,02937 & $-0,149478$ & \\
\hline & 364 & 3908 & & & 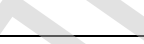 & & $-0,609297$ & & $-3,441378$ & \\
\hline & & & & & & & 0,609297 & & 3,441378 & \\
\hline & & & & & & & 0,051916 & & 3,148152 & 3,200068 \\
\hline
\end{tabular}




\begin{tabular}{|c|c|c|c|c|c|c|c|c|c|c|}
\hline \multicolumn{11}{|c|}{ 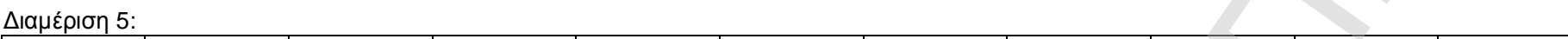 } \\
\hline HYRJYR & BADS & GOODS & $f(x / 1)$ & $f(x / 0)$ & $w(x)$ & & & 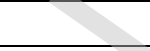 & & \\
\hline 010 & 69 & 207 & 0,053083 & 0,190411 & $-1,27733$ & 0,016224 & $-0,096464$ & 0,04856 & $-0,211919$ & \\
\hline $0 \backslash 1$ & 62 & 243 & 0,062292 & 0,171233 & $-1,01119$ & 0,01459 & $-0,088983$ & 0,056984 & $-0,235533$ & \\
\hline 012 & 30 & 133 & 0,034152 & 0,083562 & $-0,89476$ & 0,00712 & $-0,050793$ & 0,031242 & $-0,156222$ & \\
\hline 013 & 40 & 226 & 0,057943 & 0,110959 & $-0,6497$ & 0,009454 & $-0,063579$ & 0,053006 & $-0,224624$ & \\
\hline 110 & 27 & 298 & 0,076362 & 0,075342 & 0,013444 & 0,00642 & $-0,046756$ & 0,069856 & $-0,268209$ & \\
\hline $1 \backslash 1$ & 17 & 412 & 0,105526 & 0,047945 & 0,788896 & 0,004085 & $-0,032418$ & 0,096534 & $-0,325592$ & \\
\hline 112 & 13 & 291 & 0,074572 & 0,036986 & 0,701211 & 0,003151 & $-0,026188$ & 0,068218 & $-0,264255$ & \\
\hline 113 & 24 & 639 & 0,163597 & 0,067123 & 0,890874 & 0,005719 & $-0,042609$ & 0,149657 & $-0,410101$ & \\
\hline 210 & 28 & 265 & 0,06792 & 0,078082 & $-0,13943$ & 0,006653 & $-0,048113$ & 0,062133 & $-0,249060$ & \\
\hline $2 \backslash 1$ & 25 & 310 & 0,079432 & 0,069863 & 0,128366 & 0,005953 & $-0,044004$ & 0,072664 & $-0,274860$ & \\
\hline 212 & 11 & 177 & 0,045408 & 0,031507 & 0,365484 & 0,002685 & $-0,022929$ & 0,041539 & $-0,190639$ & \\
\hline 213 & 12 & 451 & 0,115503 & 0,034247 & 1,215707 & 0,002918 & $-0,024572$ & 0,105661 & $-0,342605$ & \\
\hline 310 & 0 & 32 & 0,008314 & 0,00137 & 1,803248 & 0,000117 & $-0,001525$ & 0,007606 & $-0,053534$ & \\
\hline $3 \backslash 1$ & 4 & 49 & 0,012663 & 0,012329 & 0,026756 & 0,00105 & $-0,010394$ & 0,011584 & $-0,074506$ & \\
\hline 312 & 1 & 41 & 0,010617 & 0,00411 & 0,949089 & 0,00035 & $-0,004020$ & 0,009712 & $-0,064934$ & \\
\hline \multirow[t]{4}{*}{313} & 1 & 134 & 0,034408 & 0,00411 & 2,12496 & 0,00035 & $-0,004020$ & 0,031476 & $-0,157053$ & \\
\hline & 364 & 3908 & & & & & $-0,607366$ & & $-3,503646$ & \\
\hline & & & & & & & 0,607366 & & 3,503646 & \\
\hline & & & & & & & 0,051751 & & 3,205114 & 3,256865 \\
\hline
\end{tabular}




\begin{tabular}{|c|c|c|c|c|c|c|c|c|c|c|}
\hline HYRIMAR & BADS & GOODS & $f(x / 1)$ & $f(x / 0)$ & $w(x)$ & & & & & \\
\hline $1 \backslash 1$ & 30 & 381 & 0,097595 & 0,083562 & 0,155245 & 0,00712 & $-0,050793$ & 0,08928 & $-0,311186$ & \\
\hline 211 & 117 & 225 & 0,057687 & 0,321918 & $-1,71926$ & 0,027429 & $-0,142307$ & 0,052772 & $-0,223969$ & \\
\hline $3 \backslash 1$ & 19 & 338 & 0,086595 & 0,053425 & 0,48297 & 0,004552 & $-0,035412$ & 0,079217 & $-0,289779$ & \\
\hline $4 \backslash 1$ & 0 & 21 & 0,0055 & 0,00137 & 1,390061 & 0,000117 & $-0,001525$ & 0,005031 & $-0,038414$ & \\
\hline 112 & 42 & 54 & 0,013942 & 0,116438 & $-2,12244$ & 0,009921 & $-0,066028$ & 0,012754 & $-0,080261$ & \\
\hline 212 & 31 & 44 & 0,011384 & 0,086301 & $-2,02564$ & 0,007353 & $-0,052116$ & 0,010414 & $-0,068580$ & \\
\hline 312 & 46 & 59 & 0,015221 & 0,127397 & $-2,12462$ & 0,010855 & $-0,070834$ & 0,013924 & $-0,085861$ & \\
\hline 412 & 6 & 25 & 0,006523 & 0,017808 & $-1,00426$ & 0,001517 & $-0,014209$ & 0,005968 & $-0,044092$ & \\
\hline 113 & 7 & 759 & 0,194295 & 0,020548 & 2,246618 & 0,001751 & $-0,016033$ & 0,17774 & $-0,442957$ & \\
\hline 213 & 50 & 529 & 0,135457 & 0,138356 & $-0,02118$ & 0,011789 & $-0,075524$ & 0,123915 & $-0,373303$ & \\
\hline 313 & 16 & 1270 & 0,325019 & 0,045205 & 1,972666 & 0,003852 & $-0,030892$ & 0,297326 & $-0,520285$ & \\
\hline \multirow[t]{4}{*}{413} & 0 & 203 & 0,052059 & 0,00137 & 3,637674 & 0,000117 & $-0,001525$ & 0,047624 & $-0,209171$ & \\
\hline & 364 & 3908 & & & & & $-0,557200$ & & $-2,687859$ & \\
\hline & & & & 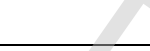 & 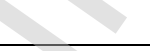 & & 0,557200 & & 2,687859 & \\
\hline & & & & & $\theta$ & & 0,047477 & & 2,458837 & 2,506314 \\
\hline
\end{tabular}




\begin{tabular}{|c|c|c|c|c|c|c|c|c|c|c|}
\hline HYRMAR & PADC & sons & $f(y / 1)$ & $f(x / 0)$ & 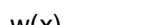 & & & & & \\
\hline 010 & 32 & 43 & 0.011128 & 0.089041 & -2.07962 & 0007587 & -0053429 & 0,01018 & -0067372 & \\
\hline $0 \backslash 1$ & 116 & 240 & 0.061525 & 0319178 & -164631 & 0.027196 & -0.141431 & 0.056282 & -0.233638 & \\
\hline $0 \backslash 2$ & 47 & 508 & 0,130084 & 0,130137 & $-0,0004$ & 0,011088 & $-0,072017$ & 0,119 & $-0,365446$ & \\
\hline 110 & 52 & 70 & 0,018035 & 0,143836 & $-2,07634$ & 0,012256 & $-0,077828$ & 0,016499 & $-0,097697$ & \\
\hline $1 \backslash 1$ & 22 & 348 & 0,089153 & 0,061644 & 0,368983 & 0,005252 & $-0,039776$ & 0,081557 & $-0,294914$ & \\
\hline 112 & 15 & 1285 & 0,328856 & 0,042466 & 2,046923 & 0,003618 & $-0,029346$ & 0,300836 & $-0,521334$ & \\
\hline 210 & 38 & 54 & 0,013942 & 0,105479 & $-2,0236$ & 0,008987 & $-0,061096$ & 0,012754 & $-0,080261$ & \\
\hline $2 \backslash 1$ & 29 & 383 & 0,098107 & 0,080822 & 0,19381 & 0,006887 & $-0,049459$ & 0,089748 & $-0,312141$ & \\
\hline 212 & 8 & 735 & 0,188156 & 0,023288 & 2,089345 & 0,001984 & $-0,017813$ & 0,172124 & $-0,436933$ & \\
\hline 310 & 5 & 25 & 0,006523 & 0,015068 & $-0,83721$ & 0,001284 & $-0,012332$ & 0,005968 & $-0,044092$ & \\
\hline $3 \backslash 1$ & 0 & 13 & 0,003454 & 0,00137 & 0,924697 & 0,000117 & $-0,001525$ & 0,003159 & $-0,026242$ & \\
\hline 312 & 0 & 204 & 0,052315 & 0,00137 & 3,642576 & 0,000117 & $-0,001525$ & 0,047858 & $-0,209861$ & \\
\hline & 364 & 3908 & & & & $P$ & $-0,557578$ & & $-2,689929$ & \\
\hline & & & & & & 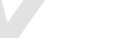 & 0,557578 & & 2,689929 & \\
\hline & & & & & 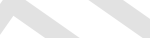 & & 0,047509 & & 2,460731 & 2,50824 \\
\hline
\end{tabular}




\begin{tabular}{|c|c|c|c|c|c|c|c|c|c|c|}
\hline HYRMAR & RADS & sחת & $f(x / 1)$ & $f(x / 0)$ & $w(\mathrm{x})$ & & & & & \\
\hline $0 \backslash 0$ & 31 & 41 & 0,010617 & 0.086301 & -2.09543 & 0.007353 & -0.052116 & 0,009712 & -0.064934 & \\
\hline $0 \backslash 1$ & 121 & 245 & 0,062804 & 0.332877 & -1.66776 & 0.028363 & -0.145782 & 0.057453 & -0.236790 & \\
\hline 012 & 47 & 533 & 0,13648 & 0,130137 & 0,04759 & 0,011088 & $-0,072017$ & 0,124851 & $-0,374768$ & \\
\hline 110 & 44 & 66 & 0,017012 & 0,121918 & $-1,96943$ & 0,010388 & $-0,068447$ & 0,015562 & $-0,093465$ & \\
\hline $1 \backslash 1$ & 23 & 343 & 0,087874 & 0,064384 & 0,311047 & 0,005486 & $-0,041199$ & 0,080387 & $-0,292358$ & \\
\hline 112 & 19 & 1290 & 0,330136 & 0,053425 & 1,821231 & 0,004552 & $-0,035412$ & 0,302006 & $-0,521670$ & \\
\hline 210 & 41 & 41 & 0,010617 & 0,113699 & $-2,37114$ & 0,009688 & $-0,064808$ & 0,009712 & $-0,064934$ & \\
\hline 2\1 & 32 & 382 & 0,097851 & 0,089041 & 0,094349 & 0,007587 & $-0,053429$ & 0,089514 & $-0,311664$ & \\
\hline 212 & 4 & 711 & 0,182016 & 0,012329 & 2,692159 & 0,00105 & $-0,010394$ & 0,166507 & $-0,430645$ & \\
\hline 310 & 2 & 26 & 0,006779 & 0,006849 & $-0,01029$ & 0,000584 & $-0,006269$ & 0,006202 & $-0,045477$ & \\
\hline $3 \backslash 1$ & 0 & 25 & 0,006523 & 0,00137 & 1,560686 & 0,000117 & $-0,001525$ & 0,005968 & $-0,044092$ & \\
\hline \multirow[t]{4}{*}{312} & 0 & 205 & 0,052571 & 0,00137 & 3,647454 & 0,000117 & $-0,001525$ & 0,048092 & $-0,210549$ & \\
\hline & 364 & 3908 & & & & 2 & $-0,552923$ & & $-2,691346$ & \\
\hline & & & & & 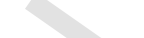 & 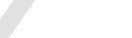 & 0,552923 & & 2,691346 & \\
\hline & & & & & . & & 0,047112 & & 2,462028 & 2,50914 \\
\hline
\end{tabular}




\begin{tabular}{|c|c|c|c|c|c|c|c|c|c|c|}
\hline HYRMAR & RARS & Sחת & $f(x / 1)$ & $f(x / 0)$ & w) & & & & & \\
\hline $0 \backslash 0$ & 27 & 48 & 0.012407 & 0.075342 & -180376 & 0,00642 & -0.046756 & 0.01135 & -0.073335 & \\
\hline $0 \backslash 1$ & 119 & 242 & 0.062036 & 0.327397 & -166345 & 0.027896 & -0.144050 & 0.05675 & -0.234903 & \\
\hline $0 \backslash 2$ & 45 & 529 & 0,135457 & 0,124658 & 0,083081 & 0,010622 & $-0,069644$ & 0,123915 & $-0,373303$ & \\
\hline 110 & 45 & 60 & 0,015477 & 0,124658 & $-2,08621$ & 0,010622 & $-0,069644$ & 0,014158 & $-0,086964$ & \\
\hline $1 \backslash 1$ & 23 & 347 & 0,088897 & 0,064384 & 0,322625 & 0,005486 & $-0,041199$ & 0,081323 & $-0,294405$ & \\
\hline 112 & 17 & 1277 & 0,32681 & 0,047945 & 1,91932 & 0,004085 & $-0,032418$ & 0,298964 & $-0,520782$ & \\
\hline 210 & 42 & 51 & 0,013175 & 0,116438 & $-2,17906$ & 0,009921 & $-0,066028$ & 0,012052 & $-0,076827$ & \\
\hline $2 \backslash 1$ & 33 & 373 & 0,095549 & 0,091781 & 0,040233 & 0,00782 & $-0,054731$ & 0,087407 & $-0,307333$ & \\
\hline 212 & 8 & 735 & 0,188156 & 0,023288 & 2,089345 & 0,001984 & $-0,017813$ & 0,172124 & $-0,436933$ & \\
\hline 310 & 5 & 25 & 0,006523 & 0,015068 & $-0,83721$ & 0,001284 & $-0,012332$ & 0,005968 & $-0,044092$ & \\
\hline $3 \backslash 1$ & 0 & 21 & 0,0055 & 0,00137 & 1,390061 & 0,000117 & $-0,001525$ & 0,005031 & $-0,038414$ & \\
\hline 312 & 0 & 200 & 0,051292 & 0,00137 & 3,622822 & 0,000117 & $-0,001525$ & 0,046922 & $-0,207093$ & \\
\hline & 364 & 3908 & & & & 2 & $-0,557666$ & & $-2,694384$ & \\
\hline & & & & & 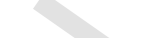 & 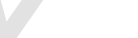 & 0,557666 & & 2,694384 & \\
\hline & & & & & 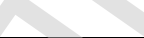 & & 0,047516 & & 2,464807 & 2,512323 \\
\hline
\end{tabular}




\begin{tabular}{|c|c|c|c|c|c|c|c|c|c|c|}
\hline HYRMAR & BADS & GOODS & $f(x / 1)$ & $f(x / 0)$ & $w(x)$ & & & 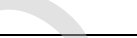 & & \\
\hline 010 & 35 & 40 & 0,010361 & 0,09726 & $-2,23937$ & 0,008287 & $-0,057305$ & 0,009478 & $-0,063703$ & \\
\hline $0 \backslash 1$ & 119 & 232 & 0,059478 & 0,327397 & $-1,70557$ & 0,027896 & $-0,144050$ & 0,05441 & $-0,228522$ & \\
\hline 012 & 47 & 537 & 0,137503 & 0,130137 & 0,05506 & 0,011088 & $-0,072017$ & 0,125787 & $-0,376222$ & \\
\hline 110 & 43 & 56 & 0,014454 & 0,119178 & $-2,10966$ & 0,010155 & $-0,067241$ & 0,013222 & $-0,082519$ & \\
\hline $1 \backslash 1$ & 21 & 328 & 0,084037 & 0,058904 & 0,355344 & 0,005019 & $-0,038337$ & 0,076876 & $-0,284544$ & \\
\hline 112 & 17 & 1256 & 0,321438 & 0,047945 & 1,902745 & 0,004085 & $-0,032418$ & 0,294049 & $-0,519253$ & \\
\hline 210 & 39 & 55 & 0,014198 & 0,108219 & $-2,03106$ & 0,009221 & $-0,062341$ & 0,012988 & $-0,081393$ & \\
\hline 211 & 32 & 381 & 0,097595 & 0,089041 & 0,091731 & 0,007587 & $-0,053429$ & 0,08928 & $-0,311186$ & \\
\hline 212 & 5 & 767 & 0,196342 & 0,015068 & 2,567251 & 0,001284 & $-0,012332$ & 0,179612 & $-0,444907$ & \\
\hline 310 & 6 & 21 & 0,0055 & 0,017808 & $-1,17489$ & 0,001517 & $-0,014209$ & 0,005031 & $-0,038414$ & \\
\hline $3 \backslash 1$ & 0 & 20 & 0,005244 & 0,00137 & 1,342433 & 0,000117 & $-0,001525$ & 0,004797 & $-0,036957$ & \\
\hline 312 & 0 & 215 & 0,055129 & 0,00137 & 3,694969 & 0,000117 & $-0,001525$ & 0,050432 & $-0,217337$ & \\
\hline & 364 & 3908 & & & & 2 & $-0,556730$ & & $-2,684958$ & \\
\hline & & & & & 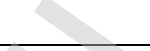 & 7 & 0,556730 & & 2,684958 & \\
\hline & & & & & $x+y$ & & 0,047437 & & 2,456183 & 2,50362 \\
\hline
\end{tabular}




\begin{tabular}{|c|c|c|c|c|c|c|c|c|c|c|}
\hline HYR\TRM & BADS & GOODS & $f(x / 1)$ & $f(x / 0)$ & $w(x)$ & & & & & \\
\hline $1 \backslash 1$ & 10 & 113 & 0,029036 & 0,028767 & 0,009288 & 0,002451 & $-0,021257$ & 0,026562 & $-0,139037$ & \\
\hline $2 \backslash 1$ & 38 & 84 & 0,021617 & 0,105479 & $-1,58505$ & 0,008987 & $-0,061096$ & 0,019775 & $-0,111930$ & \\
\hline $3 \backslash 1$ & 7 & 179 & 0,04592 & 0,020548 & 0,804133 & 0,001751 & $-0,016033$ & 0,042007 & $-0,192108$ & \\
\hline $4 \backslash 1$ & 0 & 27 & 0,007035 & 0,00137 & 1,636194 & 0,000117 & $-0,001525$ & 0,006436 & $-0,046849$ & \\
\hline 112 & 10 & 307 & 0,078665 & 0,028767 & 1,00596 & 0,002451 & $-0,021257$ & 0,071962 & $-0,273212$ & \\
\hline 212 & 15 & 211 & 0,054106 & 0,042466 & 0,242245 & 0,003618 & $-0,029346$ & 0,049496 & $-0,214641$ & \\
\hline 312 & 11 & 393 & 0,100665 & 0,031507 & 1,161595 & 0,002685 & $-0,022929$ & 0,092088 & $-0,316860$ & \\
\hline 412 & 1 & 62 & 0,015989 & 0,00411 & 1,358562 & 0,00035 & $-0,004020$ & 0,014626 & $-0,089152$ & \\
\hline 113 & 59 & 774 & 0,198133 & 0,163014 & 0,195102 & 0,01389 & $-0,085697$ & 0,18125 & $-0,446591$ & \\
\hline 213 & 145 & 503 & 0,128805 & 0,39863 & $-1,12973$ & 0,033966 & $-0,165745$ & 0,11783 & $-0,363532$ & \\
\hline 313 & 63 & 1095 & 0,280251 & 0,173973 & 0,476787 & 0,014824 & $-0,090067$ & 0,256372 & $-0,503435$ & \\
\hline \multirow[t]{4}{*}{413} & 5 & 160 & 0,041059 & 0,015068 & 1,002406 & 0,001284 & $-0,012332$ & 0,037561 & $-0,177836$ & \\
\hline & 364 & 3908 & & & & & $-0,531305$ & & $-2,875183$ & \\
\hline & & & & & & & 0,531305 & & 2,875183 & \\
\hline & & & & & & & 0,045270 & & 2,6302 & 2,675470 \\
\hline
\end{tabular}




\begin{tabular}{|c|c|c|c|c|c|c|c|c|c|c|}
\hline & & & & & & & & & & \\
\hline HYRTRM & BADS & GOODS & $f(x / 1)$ & $f(x / 0)$ & $w(x)$ & & & 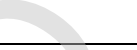 & & \\
\hline $0 \backslash 0$ & 14 & 203 & 0,052059 & 0,039726 & 0,270378 & 0,003385 & $-0,027779$ & 0,047624 & $-0,209171$ & \\
\hline $0 \backslash 1$ & 150 & 508 & 0,130084 & 0,412329 & $-1,15364$ & 0,035133 & $-0,169728$ & 0,119 & $-0,365446$ & \\
\hline $0 \backslash 2$ & 31 & 80 & 0,020594 & 0,086301 & $-1,43287$ & 0,007353 & $-0,052116$ & 0,018839 & $-0,107949$ & \\
\hline 110 & 10 & 398 & 0,101944 & 0,028767 & 1,265193 & 0,002451 & $-0,021257$ & 0,093258 & $-0,319187$ & \\
\hline $1 \backslash 1$ & 73 & 1132 & 0,289716 & 0,20137 & 0,363758 & 0,017158 & $-0,100631$ & 0,26503 & $-0,507737$ & \\
\hline 112 & 6 & 173 & 0,044385 & 0,017808 & 0,913236 & 0,001517 & $-0,014209$ & 0,040603 & $-0,187678$ & \\
\hline $2 \backslash 0$ & 7 & 301 & 0,07713 & 0,020548 & 1,322727 & 0,001751 & $-0,016033$ & 0,070558 & $-0,269887$ & \\
\hline $2 \backslash 1$ & 58 & 764 & 0,195574 & 0,160274 & 0,199056 & 0,013656 & $-0,084591$ & 0,17891 & $-0,444179$ & \\
\hline $2 \backslash 2$ & 10 & 107 & 0,027501 & 0,028767 & $-0,04502$ & 0,002451 & $-0,021257$ & 0,025157 & $-0,133658$ & \\
\hline $3 \backslash 0$ & 0 & 59 & 0,015221 & 0,00137 & 2,407984 & 0,000117 & $-0,001525$ & 0,013924 & $-0,085861$ & \\
\hline $3 \backslash 1$ & 5 & 159 & 0,040803 & 0,015068 & 0,996156 & 0,001284 & $-0,012332$ & 0,037327 & $-0,177064$ & \\
\hline 312 & 0 & 24 & 0,006268 & 0,00137 & 1,520681 & 0,000117 & $-0,001525$ & 0,005734 & $-0,042694$ & \\
\hline & 364 & 3908 & & & & $P$ & $-0,522984$ & & $-2,850513$ & \\
\hline & & & & & & 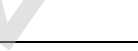 & 0,522984 & & 2,850513 & \\
\hline & & & & & 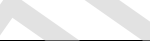 & & 0,044561 & & 2,607632 & 2,652193 \\
\hline
\end{tabular}




\begin{tabular}{|c|c|c|c|c|c|c|c|c|c|c|}
\hline \multicolumn{11}{|c|}{ 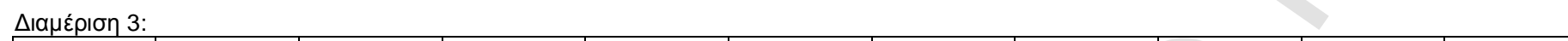 } \\
\hline HYRTRM & BADS & GOODS & $f(x / 1)$ & $f(x / 0)$ & $w(x)$ & & & 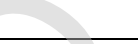 & & \\
\hline 010 & 12 & 191 & 0,04899 & 0,034247 & 0,35802 & 0,002918 & $-0,024572$ & 0,044815 & $-0,200767$ & \\
\hline $0 \backslash 1$ & 150 & 551 & 0,141085 & 0,412329 & $-1,07246$ & 0,035133 & $-0,169728$ & 0,129063 & $-0,381234$ & \\
\hline 012 & 37 & 77 & 0,019826 & 0,10274 & $-1,6452$ & 0,008754 & $-0,059841$ & 0,018137 & $-0,104920$ & \\
\hline 110 & 11 & 392 & 0,100409 & 0,031507 & 1,15905 & 0,002685 & $-0,022929$ & 0,091854 & $-0,316392$ & \\
\hline $1 \backslash 1$ & 69 & 1138 & 0,291251 & 0,190411 & 0,425001 & 0,016224 & $-0,096464$ & 0,266435 & $-0,508396$ & \\
\hline 112 & 6 & 169 & 0,043361 & 0,017808 & 0,889911 & 0,001517 & $-0,014209$ & 0,039667 & $-0,184686$ & \\
\hline 210 & 4 & 287 & 0,073548 & 0,012329 & 1,786006 & 0,00105 & $-0,010394$ & 0,067281 & $-0,261970$ & \\
\hline $2 \backslash 1$ & 64 & 744 & 0,190458 & 0,176712 & 0,074908 & 0,015057 & $-0,091146$ & 0,17423 & $-0,439222$ & \\
\hline 212 & 9 & 103 & 0,026477 & 0,026027 & 0,01714 & 0,002218 & $-0,019553$ & 0,024221 & $-0,130010$ & \\
\hline 310 & 1 & 58 & 0,014965 & 0,00411 & 1,292422 & 0,00035 & $-0,004020$ & 0,01369 & $-0,084753$ & \\
\hline $3 \backslash 1$ & 1 & 171 & 0,043873 & 0,00411 & 2,367979 & 0,00035 & $-0,004020$ & 0,040135 & $-0,186186$ & \\
\hline 312 & 0 & 27 & 0,007035 & 0,00137 & 1,636194 & 0,000117 & $-0,001525$ & 0,006436 & $-0,046849$ & \\
\hline & 364 & 3908 & & & & 2 & $-0,518401$ & & $-2,845384$ & \\
\hline & & & & & & 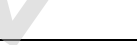 & 0,518401 & & 2,845384 & \\
\hline & & & & & & & 0,044171 & & 2,602941 & 2,647111 \\
\hline
\end{tabular}




\begin{tabular}{|c|c|c|c|c|c|c|c|c|c|c|}
\hline Ion 4 & & & & & & & & & & \\
\hline HYRTRM & BADS & GOODS & $f(x / 1)$ & $f(x / 0)$ & $w(x)$ & & & 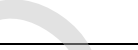 & & \\
\hline $0 \backslash 0$ & 15 & 185 & 0,047455 & 0,042466 & 0,111075 & 0,003618 & $-0,029346$ & 0,043411 & $-0,196470$ & \\
\hline $0 \backslash 1$ & 146 & 557 & 0,14262 & 0,40137 & $-1,0347$ & 0,034199 & $-0,166546$ & 0,130468 & $-0,383345$ & \\
\hline 012 & 30 & 77 & 0,019826 & 0,083562 & $-1,43859$ & 0,00712 & $-0,050793$ & 0,018137 & $-0,104920$ & \\
\hline $1 \backslash 0$ & 10 & 380 & 0,097339 & 0,028767 & 1,218971 & 0,002451 & $-0,021257$ & 0,089046 & $-0,310708$ & \\
\hline $1 \backslash 1$ & 67 & 1142 & 0,292274 & 0,184932 & 0,457707 & 0,015757 & $-0,094352$ & 0,267371 & $-0,508830$ & \\
\hline 112 & 8 & 162 & 0,041571 & 0,023288 & 0,579472 & 0,001984 & $-0,017813$ & 0,038029 & $-0,179372$ & \\
\hline 210 & 5 & 285 & 0,073037 & 0,015068 & 1,578354 & 0,001284 & $-0,012332$ & 0,066813 & $-0,260821$ & \\
\hline $2 \backslash 1$ & 67 & 776 & 0,198644 & 0,184932 & 0,07153 & 0,015757 & $-0,094352$ & 0,181718 & $-0,447068$ & \\
\hline 212 & 11 & 98 & 0,025198 & 0,031507 & $-0,22343$ & 0,002685 & $-0,022929$ & 0,023051 & $-0,125376$ & \\
\hline 310 & 1 & 52 & 0,013431 & 0,00411 & 1,184209 & 0,00035 & $-0,004020$ & 0,012286 & $-0,077978$ & \\
\hline $3 \backslash 1$ & 4 & 167 & 0,04285 & 0,012329 & 1,245766 & 0,00105 & $-0,010394$ & 0,039199 & $-0,183178$ & \\
\hline 312 & 0 & 27 & 0,007035 & 0,00137 & 1,636194 & 0,000117 & $-0,001525$ & 0,006436 & $-0,046849$ & \\
\hline & 364 & 3908 & & & & 2 & $-0,525660$ & & $-2,824914$ & \\
\hline & & & & & & 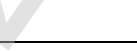 & 0,525660 & & 2,824914 & \\
\hline & & & & & 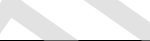 & & 0,044789 & & 2,584215 & 2,629004 \\
\hline
\end{tabular}




\begin{tabular}{|c|c|c|c|c|c|c|c|c|c|c|}
\hline \multicolumn{11}{|c|}{ 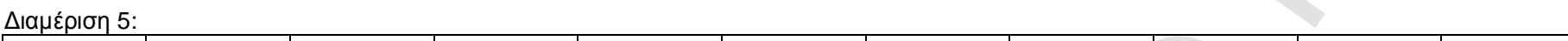 } \\
\hline HYRTRM & BADS & GOODS & $f(x / 1)$ & $f(x / 0)$ & $w(x)$ & & & 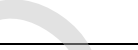 & & \\
\hline 010 & 17 & 189 & 0,048478 & 0,047945 & 0,011049 & 0,004085 & $-0,032418$ & 0,044347 & $-0,199341$ & \\
\hline $0 \backslash 1$ & 144 & 540 & 0,138271 & 0,39589 & $-1,05192$ & 0,033732 & $-0,164941$ & 0,126489 & $-0,377306$ & \\
\hline 012 & 40 & 80 & 0,020594 & 0,110959 & $-1,68418$ & 0,009454 & $-0,063579$ & 0,018839 & $-0,107949$ & \\
\hline 110 & 6 & 346 & 0,088642 & 0,017808 & 1,604941 & 0,001517 & $-0,014209$ & 0,081089 & $-0,293894$ & \\
\hline $1 \backslash 1$ & 68 & 1131 & 0,28946 & 0,187671 & 0,433326 & 0,015991 & $-0,095411$ & 0,264796 & $-0,507627$ & \\
\hline 112 & 7 & 163 & 0,041827 & 0,020548 & 0,71077 & 0,001751 & $-0,016033$ & 0,038263 & $-0,180138$ & \\
\hline 210 & 6 & 271 & 0,069455 & 0,017808 & 1,36102 & 0,001517 & $-0,014209$ & 0,063537 & $-0,252640$ & \\
\hline $2 \backslash 1$ & 60 & 821 & 0,210156 & 0,165753 & 0,237349 & 0,014123 & $-0,086798$ & 0,192249 & $-0,457352$ & \\
\hline 212 & 10 & 111 & 0,028524 & 0,028767 & $-0,00849$ & 0,002451 & $-0,021257$ & 0,026094 & $-0,137256$ & \\
\hline 310 & 1 & 59 & 0,015221 & 0,00411 & 1,309372 & 0,00035 & $-0,004020$ & 0,013924 & $-0,085861$ & \\
\hline $3 \backslash 1$ & 5 & 174 & 0,044641 & 0,015068 & 1,086037 & 0,001284 & $-0,012332$ & 0,040837 & $-0,188421$ & \\
\hline \multirow[t]{4}{*}{312} & 0 & 23 & 0,006012 & 0,00137 & 1,479008 & 0,000117 & $-0,001525$ & 0,0055 & $-0,041282$ & \\
\hline & 364 & 3908 & & & & 2 & $-0,526732$ & & $-2,829067$ & \\
\hline & & & & & & 2 & 0,526732 & & 2,829067 & \\
\hline & & & & & 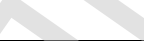 & & 0,044881 & & 2,588014 & 2,632894 \\
\hline
\end{tabular}




\begin{tabular}{|c|c|c|c|c|c|c|c|c|c|c|}
\hline HOMEIINC & BADS & GOODS & $f(x / 1)$ & $f(x / 0)$ & $w(x)$ & & & D. & & \\
\hline $1 \backslash 1$ & 11 & 130 & 0,033384 & 0,031507 & 0,057887 & 0,002685 & $-0,022929$ & 0,03054 & $-0,153712$ & \\
\hline $2 \backslash 1$ & 42 & 128 & 0,032873 & 0,116438 & $-1,26471$ & 0,009921 & $-0,066028$ & 0,030072 & $-0,152027$ & \\
\hline $3 \backslash 1$ & 33 & 457 & 0,117038 & 0,091781 & 0,243092 & 0,00782 & $-0,054731$ & 0,107065 & $-0,345118$ & \\
\hline 112 & 38 & 153 & 0,039268 & 0,105479 & $-0,9881$ & 0,008987 & $-0,061096$ & 0,035922 & $-0,172391$ & \\
\hline 212 & 67 & 143 & 0,03671 & 0,184932 & $-1,61693$ & 0,015757 & $-0,094352$ & 0,033582 & $-0,164424$ & \\
\hline 312 & 52 & 348 & 0,089153 & 0,143836 & $-0,47831$ & 0,012256 & $-0,077828$ & 0,081557 & $-0,294914$ & \\
\hline 113 & 16 & 264 & 0,067664 & 0,045205 & 0,403341 & 0,003852 & $-0,030892$ & 0,061899 & $-0,248459$ & \\
\hline 213 & 43 & 374 & 0,095805 & 0,119178 & $-0,21831$ & 0,010155 & $-0,067241$ & 0,087641 & $-0,307818$ & \\
\hline 313 & 56 & 1382 & 0,353671 & 0,154795 & 0,826269 & 0,013189 & $-0,082361$ & 0,323536 & $-0,526717$ & \\
\hline 114 & 0 & 52 & 0,013431 & 0,00137 & 2,282821 & 0,000117 & $-0,001525$ & 0,012286 & $-0,077978$ & \\
\hline 214 & 2 & 97 & 0,024942 & 0,006849 & 1,292422 & 0,000584 & $-0,006269$ & 0,022817 & $-0,124439$ & \\
\hline \multirow[t]{4}{*}{$3 \backslash 4$} & 4 & 380 & 0,097339 & 0,012329 & 2,066269 & 0,00105 & $-0,010394$ & 0,089046 & $-0,310708$ & \\
\hline & 364 & 3908 & & & & & $-0,575648$ & & $-2,878705$ & \\
\hline & & & & & & & 0,575648 & & 2,878705 & \\
\hline & & & & & & & 0,049049 & & 2,633422 & 2,68247 \\
\hline
\end{tabular}




\begin{tabular}{|c|c|c|c|c|c|c|c|c|c|c|}
\hline HOMEINC & BADS & GOODS & $f(x / 1)$ & $f(x / 0)$ & $w(x)$ & & & 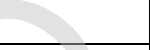 & & \\
\hline $0 \backslash 0$ & 40 & 159 & 0,040803 & 0,110959 & $-1,0004$ & 0,009454 & $-0,063579$ & 0,037327 & $-0,177064$ & \\
\hline $0 \backslash 1$ & 10 & 131 & 0,03364 & 0,028767 & 0,156492 & 0,002451 & $-0,021257$ & 0,030774 & $-0,154551$ & \\
\hline 012 & 15 & 270 & 0,069199 & 0,042466 & 0,488293 & 0,003618 & $-0,029346$ & 0,063303 & $-0,252046$ & \\
\hline $0 \backslash 3$ & 0 & 51 & 0,013175 & 0,00137 & 2,263589 & 0,000117 & $-0,001525$ & 0,012052 & $-0,076827$ & \\
\hline 110 & 73 & 137 & 0,035175 & 0,20137 & $-1,7448$ & 0,017158 & $-0,100631$ & 0,032178 & $-0,159532$ & \\
\hline $1 \backslash 1$ & 45 & 141 & 0,036199 & 0,124658 & $-1,23655$ & 0,010622 & $-0,069644$ & 0,033114 & $-0,162803$ & \\
\hline 112 & 40 & 369 & 0,094525 & 0,110959 & $-0,16029$ & 0,009454 & $-0,063579$ & 0,086471 & $-0,305385$ & \\
\hline 113 & 1 & 98 & 0,025198 & 0,00411 & 1,813452 & 0,00035 & $-0,004020$ & 0,023051 & $-0,125376$ & \\
\hline 210 & 48 & 343 & 0,087874 & 0,132877 & $-0,41352$ & 0,011322 & $-0,073193$ & 0,080387 & $-0,292358$ & \\
\hline $2 \backslash 1$ & 37 & 438 & 0,112177 & 0,10274 & 0,087879 & 0,008754 & $-0,059841$ & 0,102619 & $-0,337065$ & \\
\hline 212 & 52 & 1390 & 0,355718 & 0,143836 & 0,905466 & 0,012256 & $-0,077828$ & 0,325408 & $-0,527056$ & \\
\hline 213 & 3 & 381 & 0,097595 & 0,009589 & 2,320208 & 0,000817 & $-0,008381$ & 0,08928 & $-0,311186$ & \\
\hline & 364 & 3908 & & & & 2 & $-0,572824$ & & $-2,881251$ & \\
\hline & & & & & & 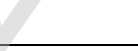 & 0,572824 & & 2,881251 & \\
\hline & & & & & 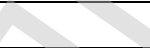 & & 0,048808 & & 2,635751 & 2,684559 \\
\hline
\end{tabular}




\begin{tabular}{|c|c|c|c|c|c|c|c|c|c|c|}
\hline HOMEINC & & & & & & & & & & \\
\hline $0 \backslash 0$ & 44 & & & 010 & 57 & 0010388 & 0068147 & & 0171727 & \\
\hline $0 \backslash 1$ & 12 & 142 & 0036454 & 0034247 & 0062474 & 0002918 & -0024572 & 0033348 & -0163615 & \\
\hline 012 & 15 & 259 & 0,066385 & 0,042466 & 0,446777 & 0,003618 & $-0,029346$ & 0,060729 & $-0,245434$ & \\
\hline 013 & 0 & 50 & 0,012919 & 0,00137 & 2,243981 & 0,000117 & $-0,001525$ & 0,011818 & $-0,075670$ & \\
\hline 110 & 79 & 137 & 0,035175 & 0,217808 & $-1,82327$ & 0,018559 & $-0,106745$ & 0,032178 & $-0,159532$ & \\
\hline $1 \backslash 1$ & 43 & 139 & 0,035687 & 0,119178 & $-1,20584$ & 0,010155 & $-0,067241$ & 0,032646 & $-0,161172$ & \\
\hline 112 & 38 & 361 & 0,092479 & 0,105479 & $-0,13154$ & 0,008987 & $-0,061096$ & 0,084599 & $-0,301445$ & \\
\hline 113 & 2 & 95 & 0,024431 & 0,006849 & 1,271696 & 0,000584 & $-0,006269$ & 0,022349 & $-0,122555$ & \\
\hline 210 & 43 & 343 & 0,087874 & 0,119178 & $-0,30471$ & 0,010155 & $-0,067241$ & 0,080387 & $-0,292358$ & \\
\hline $2 \backslash 1$ & 35 & 427 & 0,109363 & 0,09726 & 0,117282 & 0,008287 & $-0,057305$ & 0,100045 & $-0,332277$ & \\
\hline 212 & 49 & 1416 & 0,362369 & 0,135616 & 0,982832 & 0,011555 & $-0,074362$ & 0,331493 & $-0,528052$ & \\
\hline \multirow[t]{4}{*}{213} & 4 & 383 & 0,098107 & 0,012329 & 2,074123 & 0,00105 & $-0,010394$ & 0,089748 & $-0,312141$ & \\
\hline & 364 & 3908 & & & & 8 & $-0,574544$ & & $-2,868986$ & \\
\hline & & & & & & & 0,574544 & & 2,868986 & \\
\hline & & & & & 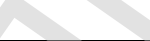 & & 0,048955 & & 2,624532 & 2,673486 \\
\hline
\end{tabular}




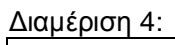

\begin{tabular}{|c|c|c|c|c|c|c|c|c|c|c|}
\hline HOMEINC & BADS & GOODS & $f(x / 1)$ & $f(x / 0)$ & $w(x)$ & & & 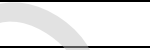 & & \\
\hline 010 & 40 & 159 & 0,040803 & 0,110959 & $-1,0004$ & 0,009454 & $-0,063579$ & 0,037327 & $-0,177064$ & \\
\hline $0 \backslash 1$ & 12 & 143 & 0,03671 & 0,034247 & 0,069467 & 0,002918 & $-0,024572$ & 0,033582 & $-0,164424$ & \\
\hline 012 & 14 & 253 & 0,06485 & 0,039726 & 0,490076 & 0,003385 & $-0,027779$ & 0,059325 & $-0,241761$ & \\
\hline 013 & 0 & 56 & 0,014454 & 0,00137 & 2,356248 & 0,000117 & $-0,001525$ & 0,013222 & $-0,082519$ & \\
\hline 110 & 78 & 137 & 0,035175 & 0,215068 & $-1,81061$ & 0,018325 & $-0,105737$ & 0,032178 & $-0,159532$ & \\
\hline $1 \backslash 1$ & 44 & 136 & 0,034919 & 0,121918 & $-1,2503$ & 0,010388 & $-0,068447$ & 0,031944 & $-0,158708$ & \\
\hline 112 & 39 & 371 & 0,095037 & 0,108219 & $-0,12989$ & 0,009221 & $-0,062341$ & 0,086939 & $-0,306361$ & \\
\hline 113 & 2 & 95 & 0,024431 & 0,006849 & 1,271696 & 0,000584 & $-0,006269$ & 0,022349 & $-0,122555$ & \\
\hline 210 & 49 & 338 & 0,086595 & 0,135616 & $-0,44859$ & 0,011555 & $-0,074362$ & 0,079217 & $-0,289779$ & \\
\hline 211 & 33 & 433 & 0,110898 & 0,091781 & 0,189207 & 0,00782 & $-0,054731$ & 0,101449 & $-0,334900$ & \\
\hline 212 & 49 & 1410 & 0,360834 & 0,135616 & 0,978587 & 0,011555 & $-0,074362$ & 0,330089 & $-0,527836$ & \\
\hline \multirow[t]{4}{*}{213} & 4 & 377 & 0,096572 & 0,012329 & 2,058354 & 0,00105 & $-0,010394$ & 0,088343 & $-0,309267$ & \\
\hline & 364 & 3908 & & & & 2 & $-0,574097$ & & $-2,874706$ & \\
\hline & & & & & & 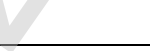 & 0,574097 & & 2,874706 & \\
\hline & & & & & 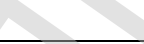 & & 0,048917 & & 2,629764 & 2,67868 \\
\hline
\end{tabular}




\begin{tabular}{|c|c|c|c|c|c|c|c|c|c|c|}
\hline HОMЕINC & & & & & & & & & & \\
\hline $0 \backslash 0$ & & & & 011000 & 114157 & & & 0 & & \\
\hline & & & & & & & & & & \\
\hline 011 & 13 & 135 & 0,034664 & 0,036986 & $-0,06486$ & 0,003151 & $-0,026188$ & $0,031 / 1$ & $-0,15 / 882$ & \\
\hline 012 & 16 & 274 & 0,070223 & 0,045205 & 0,440451 & 0,003852 & $-0,030892$ & 0,064239 & $-0,254413$ & \\
\hline 013 & 0 & 53 & 0,013686 & 0,00137 & 2,301689 & 0,000117 & $-0,001525$ & 0,01252 & $-0,079123$ & \\
\hline 110 & 75 & 156 & 0,040036 & 0,206849 & $-1,64222$ & 0,017625 & $-0,102686$ & 0,036625 & $-0,174737$ & \\
\hline $1 \backslash 1$ & 42 & 128 & 0,032873 & 0,116438 & $-1,26471$ & 0,009921 & $-0,066028$ & 0,030072 & $-0,152027$ & \\
\hline 112 & 36 & 357 & 0,091456 & 0,1 & $-0,08932$ & 0,008521 & $-0,058578$ & 0,083663 & $-0,299452$ & \\
\hline 113 & 1 & 97 & 0,024942 & 0,00411 & 1,803248 & 0,00035 & $-0,004020$ & 0,022817 & $-0,124439$ & \\
\hline 210 & 51 & 342 & 0,087618 & 0,141096 & $-0,47645$ & 0,012022 & $-0,076680$ & 0,080153 & $-0,291844$ & \\
\hline $2 \backslash 1$ & 36 & 446 & 0,114224 & 0,1 & 0,132988 & 0,008521 & $-0,058578$ & 0,104491 & $-0,340489$ & \\
\hline 212 & 53 & 1400 & 0,358276 & 0,146575 & 0,893763 & 0,012489 & $-0,078971$ & 0,327749 & $-0,527458$ & \\
\hline 213 & 1 & 382 & 0,097851 & 0,00411 & 3,170124 & 0,00035 & $-0,004020$ & 0,089514 & $-0,311664$ & \\
\hline & 364 & 3908 & & & & 2 & $-0,571744$ & & $-2,873882$ & \\
\hline & & & & & & 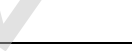 & 0,571744 & & 2,873882 & \\
\hline & & & & & + & & 0,048716 & & 2,62901 & 2,677726 \\
\hline
\end{tabular}




\begin{tabular}{|c|c|c|c|c|c|c|c|c|c|c|}
\hline HOME\JOB & BADS & GOODS & $f(x / 1)$ & $f(x / 0)$ & $w(x)$ & & & 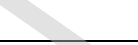 & & \\
\hline $1 \backslash 1$ & 15 & 122 & 0,031338 & 0,042466 & $-0,30387$ & 0,003618 & $-0,029346$ & 0,028668 & $-0,146906$ & \\
\hline $2 \backslash 1$ & 30 & 116 & 0,029803 & 0,083562 & $-1,03097$ & 0,00712 & $-0,050793$ & 0,027264 & $-0,141686$ & \\
\hline $3 \backslash 1$ & 13 & 436 & 0,111665 & 0,036986 & 1,104959 & 0,003151 & $-0,026188$ & 0,102151 & $-0,336202$ & \\
\hline 112 & 6 & 14 & 0,003709 & 0,017808 & $-1,56879$ & 0,001517 & $-0,014209$ & 0,003393 & $-0,027836$ & \\
\hline 212 & 37 & 52 & 0,013431 & 0,10274 & $-2,03467$ & 0,008754 & $-0,059841$ & 0,012286 & $-0,077978$ & \\
\hline 312 & 62 & 382 & 0,097851 & 0,171233 & $-0,55958$ & 0,01459 & $-0,088983$ & 0,089514 & $-0,311664$ & \\
\hline 113 & 13 & 111 & 0,028524 & 0,036986 & $-0,2598$ & 0,003151 & $-0,026188$ & 0,026094 & $-0,137256$ & \\
\hline 213 & 29 & 189 & 0,048478 & 0,080822 & $-0,51114$ & 0,006887 & $-0,049459$ & 0,044347 & $-0,199341$ & \\
\hline 313 & 28 & 604 & 0,154643 & 0,078082 & 0,683358 & 0,006653 & $-0,048113$ & 0,141467 & $-0,399143$ & \\
\hline $1 \backslash 4$ & 4 & 72 & 0,018547 & 0,012329 & 0,40837 & 0,00105 & $-0,010394$ & 0,016967 & $-0,099783$ & \\
\hline $2 \backslash 4$ & 10 & 60 & 0,015477 & 0,028767 & $-0,61987$ & 0,002451 & $-0,021257$ & 0,014158 & $-0,086964$ & \\
\hline $3 \backslash 4$ & 4 & 113 & 0,029036 & 0,012329 & 0,856586 & 0,00105 & $-0,010394$ & 0,026562 & $-0,139037$ & \\
\hline 115 & 27 & 235 & 0,060246 & 0,075342 & $-0,22361$ & 0,00642 & $-0,046756$ & 0,055112 & $-0,230451$ & \\
\hline 215 & 40 & 256 & 0,065618 & 0,110959 & $-0,52531$ & 0,009454 & $-0,063579$ & 0,060027 & $-0,243604$ & \\
\hline 315 & 31 & 927 & 0,237273 & 0,086301 & 1,011366 & 0,007353 & $-0,052116$ & 0,217056 & $-0,478361$ & \\
\hline 116 & 0 & 45 & 0,01164 & 0,00137 & 2,13972 & 0,000117 & $-0,001525$ & 0,010648 & $-0,069779$ & \\
\hline 216 & 8 & 69 & 0,017779 & 0,023288 & $-0,26988$ & 0,001984 & $-0,017813$ & 0,016265 & $-0,096646$ & \\
\hline \multirow[t]{4}{*}{316} & 7 & 105 & 0,026989 & 0,020548 & 0,272668 & 0,001751 & $-0,016033$ & 0,024689 & $-0,131840$ & \\
\hline & 364 & 3908 & & 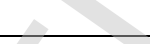 & & & $-0,632989$ & & $-3,354478$ & \\
\hline & & & & & & & 0,632989 & & 3,354478 & \\
\hline & & & & 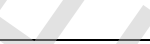 & & & 0,053934 & & 3,068656 & 3,12259 \\
\hline
\end{tabular}




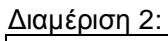

\begin{tabular}{|c|c|c|c|c|c|c|c|c|c|c|}
\hline HOMEJOB & BADS & GOODS & $f(x / 1)$ & $f(x / 0)$ & $w(x)$ & & & 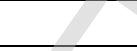 & 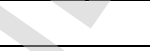 & \\
\hline 010 & 6 & 14 & 0,003709 & 0,017808 & $-1,56879$ & 0,001517 & $-0,014209$ & 0,003393 & $-0,027836$ & \\
\hline 012 & 18 & 123 & 0,031594 & 0,050685 & $-0,47267$ & 0,004319 & $-0,033924$ & 0,028902 & $-0,147766$ & \\
\hline 013 & 10 & 116 & 0,029803 & 0,028767 & 0,035377 & 0,002451 & $-0,021257$ & 0,027264 & $-0,141686$ & \\
\hline 014 & 2 & 81 & 0,020849 & 0,006849 & 1,113173 & 0,000584 & $-0,006269$ & 0,019073 & $-0,108950$ & \\
\hline 015 & 27 & 230 & 0,058966 & 0,075342 & $-0,24507$ & 0,00642 & $-0,046756$ & 0,053942 & $-0,227228$ & \\
\hline 016 & 2 & 47 & 0,012151 & 0,006849 & 0,573299 & 0,000584 & $-0,006269$ & 0,011116 & $-0,072157$ & \\
\hline 110 & 33 & 58 & 0,014965 & 0,091781 & $-1,81366$ & 0,00782 & $-0,054731$ & 0,01369 & $-0,084753$ & \\
\hline 112 & 33 & 116 & 0,029803 & 0,091781 & $-1,12479$ & 0,00782 & $-0,054731$ & 0,027264 & $-0,141686$ & \\
\hline 113 & 34 & 172 & 0,044129 & 0,094521 & $-0,7617$ & 0,008054 & $-0,056023$ & 0,040369 & $-0,186933$ & \\
\hline 114 & 11 & 58 & 0,014965 & 0,031507 & $-0,74446$ & 0,002685 & $-0,022929$ & 0,01369 & $-0,084753$ & \\
\hline 115 & 38 & 272 & 0,069711 & 0,105479 & $-0,41416$ & 0,008987 & $-0,061096$ & 0,063771 & $-0,253232$ & \\
\hline 116 & 10 & 69 & 0,017779 & 0,028767 & $-0,48119$ & 0,002451 & $-0,021257$ & 0,016265 & $-0,096646$ & \\
\hline 210 & 61 & 396 & 0,101433 & 0,168493 & $-0,5075$ & 0,014357 & $-0,087893$ & 0,09279 & $-0,318259$ & \\
\hline 212 & 12 & 438 & 0,112177 & 0,034247 & 1,186492 & 0,002918 & $-0,024572$ & 0,102619 & $-0,337065$ & \\
\hline 213 & 25 & 582 & 0,149015 & 0,069863 & 0,757511 & 0,005953 & $-0,044004$ & 0,136318 & $-0,391908$ & \\
\hline 214 & 4 & 115 & 0,029547 & 0,012329 & 0,874054 & 0,00105 & $-0,010394$ & 0,02703 & $-0,140806$ & \\
\hline 215 & 32 & 924 & 0,236506 & 0,089041 & 0,976873 & 0,007587 & $-0,053429$ & 0,216354 & $-0,477825$ & \\
\hline \multirow[t]{4}{*}{216} & 6 & 97 & 0,024942 & 0,017808 & 0,336911 & 0,001517 & $-0,014209$ & 0,022817 & $-0,124439$ & \\
\hline & 364 & 3908 & & & 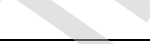 & & $-0,633952$ & & $-3,363927$ & \\
\hline & & & & 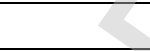 & & & 0,633952 & & 3,363927 & \\
\hline & & & & 2 & & & 0,054017 & & 3,0773 & 3,131317 \\
\hline
\end{tabular}




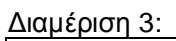

\begin{tabular}{|c|c|c|c|c|c|c|c|c|c|c|}
\hline HOMEJOB & BADS & GOODS & $f(x / 1)$ & $f(x / 0)$ & $w(x)$ & & & 8 & 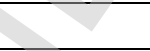 & \\
\hline 010 & 5 & 11 & 0,002942 & 0,015068 & $-1,63354$ & 0,001284 & $-0,012332$ & 0,002691 & $-0,022977$ & \\
\hline 012 & 22 & 110 & 0,028268 & 0,061644 & $-0,77964$ & 0,005252 & $-0,039776$ & 0,025859 & $-0,136361$ & \\
\hline 013 & 15 & 137 & 0,035175 & 0,042466 & $-0,18836$ & 0,003618 & $-0,029346$ & 0,032178 & $-0,159532$ & \\
\hline $0 \backslash 4$ & 4 & 77 & 0,019826 & 0,012329 & 0,475061 & 0,00105 & $-0,010394$ & 0,018137 & $-0,104920$ & \\
\hline 015 & 23 & 222 & 0,05692 & 0,064384 & $-0,12321$ & 0,005486 & $-0,041199$ & 0,05207 & $-0,221995$ & \\
\hline 016 & 2 & 50 & 0,012919 & 0,006849 & 0,634543 & 0,000584 & $-0,006269$ & 0,011818 & $-0,075670$ & \\
\hline 110 & 34 & 46 & 0,011896 & 0,094521 & $-2,07265$ & 0,008054 & $-0,056023$ & 0,010882 & $-0,070972$ & \\
\hline 112 & 32 & 120 & 0,030826 & 0,089041 & $-1,06073$ & 0,007587 & $-0,053429$ & 0,0282 & $-0,145177$ & \\
\hline 113 & 41 & 196 & 0,050269 & 0,113699 & $-0,81617$ & 0,009688 & $-0,064808$ & 0,045985 & $-0,204299$ & \\
\hline $1 \backslash 4$ & 13 & 59 & 0,015221 & 0,036986 & $-0,88785$ & 0,003151 & $-0,026188$ & 0,013924 & $-0,085861$ & \\
\hline 115 & 34 & 250 & 0,064083 & 0,094521 & $-0,38864$ & 0,008054 & $-0,056023$ & 0,058623 & $-0,239907$ & \\
\hline 116 & 8 & 61 & 0,015733 & 0,023288 & $-0,39217$ & 0,001984 & $-0,017813$ & 0,014392 & $-0,088061$ & \\
\hline 210 & 61 & 390 & 0,099898 & 0,168493 & $-0,52275$ & 0,014357 & $-0,087893$ & 0,091386 & $-0,315453$ & \\
\hline 212 & 13 & 424 & 0,108596 & 0,036986 & 1,077083 & 0,003151 & $-0,026188$ & 0,099343 & $-0,330954$ & \\
\hline 213 & 26 & 646 & 0,165388 & 0,072603 & 0,823289 & 0,006186 & $-0,045386$ & 0,151296 & $-0,412214$ & \\
\hline 214 & 3 & 120 & 0,030826 & 0,009589 & 1,167747 & 0,000817 & $-0,008381$ & 0,0282 & $-0,145177$ & \\
\hline 215 & 23 & 879 & 0,224994 & 0,064384 & 1,251214 & 0,005486 & $-0,041199$ & 0,205823 & $-0,469384$ & \\
\hline \multirow[t]{4}{*}{216} & 5 & 110 & 0,028268 & 0,015068 & 0,629128 & 0,001284 & $-0,012332$ & 0,025859 & $-0,136361$ & \\
\hline & 364 & 3908 & & & 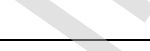 & & $-0,634980$ & & $-3,365275$ & \\
\hline & & & & - & & & 0,634980 & & 3,365275 & \\
\hline & & & & $P$ & & & 0,054104 & & 3,078533 & 3,132637 \\
\hline
\end{tabular}




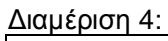

\begin{tabular}{|c|c|c|c|c|c|c|c|c|c|c|}
\hline HOMEJOB & BADS & GOODS & $f(x / 1)$ & $f(x / 0)$ & $w(x)$ & & & 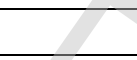 & & \\
\hline 010 & 6 & 14 & 0,003709 & 0,017808 & $-1,56879$ & 0,001517 & $-0,014209$ & 0,003393 & $-0,027836$ & \\
\hline 012 & 23 & 141 & 0,036199 & 0,064384 & $-0,57584$ & 0,005486 & $-0,041199$ & 0,033114 & $-0,162803$ & \\
\hline 013 & 11 & 118 & 0,030315 & 0,031507 & $-0,03857$ & 0,002685 & $-0,022929$ & 0,027732 & $-0,143437$ & \\
\hline 014 & 5 & 87 & 0,022384 & 0,015068 & 0,395751 & 0,001284 & $-0,012332$ & 0,020477 & $-0,114873$ & \\
\hline 015 & 19 & 204 & 0,052315 & 0,053425 & $-0,02099$ & 0,004552 & $-0,035412$ & 0,047858 & $-0,209861$ & \\
\hline 016 & 2 & 47 & 0,012151 & 0,006849 & 0,573299 & 0,000584 & $-0,006269$ & 0,011116 & $-0,072157$ & \\
\hline 110 & 33 & 55 & 0,014198 & 0,091781 & $-1,8663$ & 0,00782 & $-0,054731$ & 0,012988 & $-0,081393$ & \\
\hline 112 & 32 & 133 & 0,034152 & 0,089041 & $-0,95828$ & 0,007587 & $-0,053429$ & 0,031242 & $-0,156222$ & \\
\hline 113 & 42 & 175 & 0,044896 & 0,116438 & $-0,953$ & 0,009921 & $-0,066028$ & 0,041071 & $-0,189162$ & \\
\hline 114 & 12 & 57 & 0,01471 & 0,034247 & $-0,84508$ & 0,002918 & $-0,024572$ & 0,013456 & $-0,083639$ & \\
\hline 115 & 36 & 254 & 0,065106 & 0,1 & $-0,42915$ & 0,008521 & $-0,058578$ & 0,059559 & $-0,242377$ & \\
\hline 116 & 8 & 65 & 0,016756 & 0,023288 & $-0,32916$ & 0,001984 & $-0,017813$ & 0,015328 & $-0,092395$ & \\
\hline 210 & 58 & 396 & 0,101433 & 0,160274 & $-0,45749$ & 0,013656 & $-0,084591$ & 0,09279 & $-0,318259$ & \\
\hline 212 & 9 & 490 & 0,12548 & 0,026027 & 1,572994 & 0,002218 & $-0,019553$ & 0,114788 & $-0,358478$ & \\
\hline 213 & 30 & 585 & 0,149783 & 0,083562 & 0,5836 & 0,00712 & $-0,050793$ & 0,13702 & $-0,392911$ & \\
\hline 214 & 1 & 109 & 0,028012 & 0,00411 & 1,91932 & 0,00035 & $-0,004020$ & 0,025625 & $-0,135463$ & \\
\hline 215 & 29 & 869 & 0,222435 & 0,080822 & 1,012389 & 0,006887 & $-0,049459$ & 0,203483 & $-0,467404$ & \\
\hline \multirow[t]{4}{*}{216} & 8 & 109 & 0,028012 & 0,023288 & 0,184719 & 0,001984 & $-0,017813$ & 0,025625 & $-0,135463$ & \\
\hline & 364 & 3908 & & & 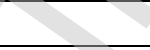 & & $-0,633730$ & & $-3,384131$ & \\
\hline & & & & & & & 0,633730 & & 3,384131 & \\
\hline & & & & $P$ & 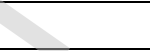 & & 0,053998 & & 3,095783 & 3,14978 \\
\hline
\end{tabular}




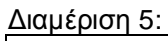

\begin{tabular}{|c|c|c|c|c|c|c|c|c|c|c|}
\hline HOMEJOB & BADS & GOODS & $f(x / 1)$ & $f(x / 0)$ & $w(x)$ & & & 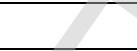 & & \\
\hline 010 & 5 & 11 & 0,002942 & 0,015068 & $-1,63354$ & 0,001284 & $-0,012332$ & 0,002691 & $-0,022977$ & \\
\hline 012 & 18 & 118 & 0,030315 & 0,050685 & $-0,514$ & 0,004319 & $-0,033924$ & 0,027732 & $-0,143437$ & \\
\hline 013 & 15 & 112 & 0,02878 & 0,042466 & $-0,38903$ & 0,003618 & $-0,029346$ & 0,026328 & $-0,138148$ & \\
\hline $0 \backslash 4$ & 5 & 75 & 0,019314 & 0,015068 & 0,248245 & 0,001284 & $-0,012332$ & 0,017669 & $-0,102879$ & \\
\hline 015 & 24 & 237 & 0,060757 & 0,067123 & $-0,09965$ & 0,005719 & $-0,042609$ & 0,05558 & $-0,231730$ & \\
\hline 016 & 2 & 47 & 0,012151 & 0,006849 & 0,573299 & 0,000584 & $-0,006269$ & 0,011116 & $-0,072157$ & \\
\hline 110 & 31 & 49 & 0,012663 & 0,086301 & $-1,91915$ & 0,007353 & $-0,052116$ & 0,011584 & $-0,074506$ & \\
\hline 112 & 32 & 126 & 0,032361 & 0,089041 & $-1,01214$ & 0,007587 & $-0,053429$ & 0,029604 & $-0,150330$ & \\
\hline 113 & 37 & 181 & 0,046431 & 0,10274 & $-0,79422$ & 0,008754 & $-0,059841$ & 0,042475 & $-0,193569$ & \\
\hline 114 & 10 & 54 & 0,013942 & 0,028767 & $-0,72431$ & 0,002451 & $-0,021257$ & 0,012754 & $-0,080261$ & \\
\hline 115 & 34 & 260 & 0,066641 & 0,094521 & $-0,3495$ & 0,008054 & $-0,056023$ & 0,060963 & $-0,246042$ & \\
\hline 116 & 10 & 68 & 0,017524 & 0,028767 & $-0,49568$ & 0,002451 & $-0,021257$ & 0,016031 & $-0,095591$ & \\
\hline 210 & 58 & 404 & 0,103479 & 0,160274 & $-0,43751$ & 0,013656 & $-0,084591$ & 0,094662 & $-0,321952$ & \\
\hline 212 & 13 & 440 & 0,112689 & 0,036986 & 1,114081 & 0,003151 & $-0,026188$ & 0,103087 & $-0,337926$ & \\
\hline 213 & 31 & 581 & 0,148759 & 0,086301 & 0,544484 & 0,007353 & $-0,052116$ & 0,136084 & $-0,391572$ & \\
\hline 214 & 4 & 111 & 0,028524 & 0,012329 & 0,838808 & 0,00105 & $-0,010394$ & 0,026094 & $-0,137256$ & \\
\hline 215 & 29 & 923 & 0,23625 & 0,080822 & 1,072641 & 0,006887 & $-0,049459$ & 0,21612 & $-0,477646$ & \\
\hline \multirow[t]{4}{*}{216} & 6 & 111 & 0,028524 & 0,017808 & 0,471083 & 0,001517 & $-0,014209$ & 0,026094 & $-0,137256$ & \\
\hline & 364 & 3908 & & & 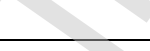 & & $-0,637693$ & & $-3,355234$ & \\
\hline & & & & 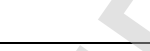 & & & 0,637693 & & 3,355234 & \\
\hline & & & & 2 & & & 0,054335 & & 3,069348 & 3,123684 \\
\hline
\end{tabular}




\begin{tabular}{|c|c|c|c|c|c|c|c|c|c|c|}
\hline \multicolumn{11}{|l|}{ 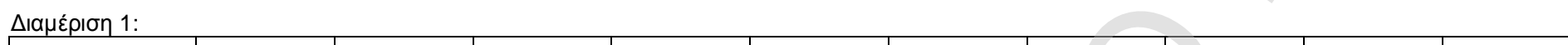 } \\
\hline HOME\JYR & BADS & GOODS & $f(x / 1)$ & $f(x / 0)$ & $w(x)$ & & & . & & \\
\hline $1 \backslash 1$ & 6 & 91 & 0,023408 & 0,017808 & 0,273397 & 0,001517 & $-0,014209$ & 0,021413 & $-0,118743$ & \\
\hline $2 \backslash 1$ & 20 & 133 & 0,034152 & 0,056164 & $-0,49746$ & 0,004786 & $-0,036883$ & 0,031242 & $-0,156222$ & \\
\hline $3 \backslash 1$ & 30 & 427 & 0,109363 & 0,083562 & 0,269088 & 0,00712 & $-0,050793$ & 0,100045 & $-0,332277$ & \\
\hline 112 & 31 & 205 & 0,052571 & 0,086301 & $-0,49568$ & 0,007353 & $-0,052116$ & 0,048092 & $-0,210549$ & \\
\hline $2 \backslash 2$ & 56 & 181 & 0,046431 & 0,154795 & $-1,20412$ & 0,013189 & $-0,082361$ & 0,042475 & $-0,193569$ & \\
\hline 312 & 34 & 416 & 0,106549 & 0,094521 & 0,119788 & 0,008054 & $-0,056023$ & 0,09747 & $-0,327393$ & \\
\hline 113 & 24 & 217 & 0,055641 & 0,067123 & $-0,18761$ & 0,005719 & $-0,042609$ & 0,0509 & $-0,218676$ & \\
\hline 213 & 59 & 213 & 0,054618 & 0,163014 & $-1,09348$ & 0,01389 & $-0,085697$ & 0,049964 & $-0,215992$ & \\
\hline 313 & 33 & 578 & 0,147992 & 0,091781 & 0,477754 & 0,00782 & $-0,054731$ & 0,135382 & $-0,390563$ & \\
\hline $1 \backslash 4$ & 4 & 86 & 0,022128 & 0,012329 & 0,584928 & 0,00105 & $-0,010394$ & 0,020243 & $-0,113896$ & \\
\hline $2 \backslash 4$ & 19 & 215 & 0,055129 & 0,053425 & 0,031407 & 0,004552 & $-0,035412$ & 0,050432 & $-0,217337$ & \\
\hline \multirow[t]{4}{*}{$3 \backslash 4$} & 48 & 1146 & 0,293298 & 0,132877 & 0,791766 & 0,011322 & $-0,073193$ & 0,268307 & $-0,509258$ & \\
\hline & 364 & 3908 & & & & & $-0,594421$ & & $-3,004473$ & \\
\hline & & & & & & & 0,594421 & & 3,004473 & \\
\hline & & & & 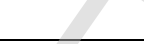 & & & 0,050648 & & 2,748474 & 2,799122 \\
\hline
\end{tabular}




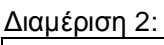

\begin{tabular}{|c|c|c|c|c|c|c|c|c|c|c|}
\hline HOMEJYR & BADS & GOODS & $f(x / 1)$ & $f(x / 0)$ & $w(x)$ & & & 8 & & \\
\hline 010 & 34 & 207 & 0,053083 & 0,094521 & $-0,57697$ & 0,008054 & $-0,056023$ & 0,04856 & $-0,211919$ & \\
\hline $0 \backslash 1$ & 24 & 234 & 0,05999 & 0,067123 & $-0,11236$ & 0,005719 & $-0,042609$ & 0,054878 & $-0,229809$ & \\
\hline 012 & 4 & 76 & 0,01957 & 0,012329 & 0,462074 & 0,00105 & $-0,010394$ & 0,017903 & $-0,103902$ & \\
\hline 013 & 3 & 94 & 0,024175 & 0,009589 & 0,924697 & 0,000817 & $-0,008381$ & 0,022115 & $-0,121607$ & \\
\hline 110 & 56 & 176 & 0,045152 & 0,154795 & $-1,23206$ & 0,013189 & $-0,082361$ & 0,041305 & $-0,189901$ & \\
\hline $1 \backslash 1$ & 63 & 261 & 0,066897 & 0,173973 & $-0,95575$ & 0,014824 & $-0,090067$ & 0,061197 & $-0,246648$ & \\
\hline 112 & 16 & 99 & 0,025454 & 0,045205 & $-0,57434$ & 0,003852 & $-0,030892$ & 0,023285 & $-0,126309$ & \\
\hline 113 & 24 & 209 & 0,053594 & 0,067123 & $-0,22509$ & 0,005719 & $-0,042609$ & 0,049028 & $-0,213283$ & \\
\hline 210 & 28 & 415 & 0,106293 & 0,078082 & 0,308439 & 0,006653 & $-0,048113$ & 0,097236 & $-0,326944$ & \\
\hline $2 \backslash 1$ & 36 & 639 & 0,163597 & 0,1 & 0,492235 & 0,008521 & $-0,058578$ & 0,149657 & $-0,410101$ & \\
\hline 212 & 26 & 383 & 0,098107 & 0,072603 & 0,301055 & 0,006186 & $-0,045386$ & 0,089748 & $-0,312141$ & \\
\hline \multirow[t]{4}{*}{213} & 50 & 1115 & 0,285367 & 0,138356 & 0,723945 & 0,011789 & $-0,075524$ & 0,261052 & $-0,505812$ & \\
\hline & 364 & 3908 & & & & 2 & $-0,590937$ & & $-2,998376$ & \\
\hline & & & & & & 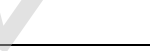 & 0,590937 & & 2,998376 & \\
\hline & & & & & 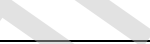 & & 0,050351 & & 2,742897 & 2,793248 \\
\hline
\end{tabular}




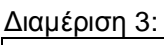

\begin{tabular}{|l|r|r|r|r|r|r|r|r|l|l|}
\hline HOMEJYR & BADS & GOODS & $\mathrm{f}(\mathrm{x} / 1)$ & $\mathrm{f}(\mathrm{x} / 0)$ & $\mathrm{w}(\mathrm{x})$ & & & & \\
\hline $0 \backslash 0$ & 37 & 208 & 0,053338 & 0,10274 & $-0,65554$ & 0,008754 & $-0,059841$ & 0,048794 & $-0,212602$ & \\
\hline $0 \backslash 1$ & 23 & 240 & 0,061525 & 0,064384 & $-0,04542$ & 0,005486 & $-0,041199$ & 0,056282 & $-0,233638$ & \\
\hline 012 & 5 & 74 & 0,019059 & 0,015068 & 0,234912 & 0,001284 & $-0,012332$ & 0,017435 & $-0,101852$ & \\
\hline 013 & 6 & 85 & 0,021873 & 0,017808 & 0,205575 & 0,001517 & $-0,014209$ & 0,020009 & $-0,112915$ & \\
\hline 110 & 57 & 177 & 0,045408 & 0,157534 & $-1,24395$ & 0,013423 & $-0,083479$ & 0,041539 & $-0,190639$ & \\
\hline $1 \backslash 1$ & 61 & 254 & 0,065106 & 0,168493 & $-0,95088$ & 0,014357 & $-0,087893$ & 0,059559 & $-0,242377$ & \\
\hline 112 & 17 & 102 & 0,026222 & 0,047945 & $-0,60348$ & 0,004085 & $-0,032418$ & 0,023987 & $-0,129090$ & \\
\hline 113 & 27 & 199 & 0,051036 & 0,075342 & $-0,38951$ & 0,00642 & $-0,046756$ & 0,046687 & $-0,206397$ & \\
\hline $2 \backslash 0$ & 34 & 429 & 0,109875 & 0,094521 & 0,150523 & 0,008054 & $-0,056023$ & 0,100513 & $-0,333154$ & \\
\hline $2 \backslash 1$ & 24 & 620 & 0,158736 & 0,067123 & 0,860713 & 0,005719 & $-0,042609$ & 0,145211 & $-0,404235$ & \\
\hline $2 \backslash 2$ & 25 & 374 & 0,095805 & 0,069863 & 0,315774 & 0,005953 & $-0,044004$ & 0,087641 & $-0,307818$ & \\
\hline 213 & 48 & 1146 & 0,293298 & 0,132877 & 0,791766 & 0,011322 & $-0,073193$ & 0,268307 & $-0,509258$ & \\
\hline & 364 & 3908 & & & & & $-0,593956$ & & $-2,983974$ & \\
\hline & & & & & & & 0,593956 & & 2,983974 & \\
\hline & & & & & & & 0,050609 & & 2,729722 & 2,78033 \\
\hline
\end{tabular}




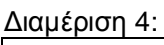

\begin{tabular}{|l|r|r|r|r|r|r|r|r|l|l|}
\hline HOMEJYR & BADS & GOODS & $\mathrm{f}(\mathrm{x} / 1)$ & $\mathrm{f}(\mathrm{x} / 0)$ & $\mathrm{w}(\mathrm{x})$ & & & & \\
\hline $0 \backslash 0$ & 26 & 129 & 0,033129 & 0,072603 & $-0,7846$ & 0,006186 & $-0,045386$ & 0,030306 & $-0,152871$ & \\
\hline $0 \backslash 1$ & 26 & 316 & 0,080967 & 0,072603 & 0,109039 & 0,006186 & $-0,045386$ & 0,074068 & $-0,278126$ & \\
\hline 012 & 8 & 85 & 0,021873 & 0,023288 & $-0,06269$ & 0,001984 & $-0,017813$ & 0,020009 & $-0,112915$ & \\
\hline 013 & 6 & 81 & 0,020849 & 0,017808 & 0,157661 & 0,001517 & $-0,014209$ & 0,019073 & $-0,108950$ & \\
\hline 110 & 38 & 105 & 0,026989 & 0,105479 & $-1,36309$ & 0,008987 & $-0,061096$ & 0,024689 & $-0,131840$ & \\
\hline $1 \backslash 1$ & 75 & 301 & 0,07713 & 0,206849 & $-0,9865$ & 0,017625 & $-0,102686$ & 0,070558 & $-0,269887$ & \\
\hline 112 & 27 & 135 & 0,034664 & 0,075342 & $-0,77635$ & 0,00642 & $-0,046756$ & 0,03171 & $-0,157882$ & \\
\hline 113 & 23 & 198 & 0,05078 & 0,064384 & $-0,23735$ & 0,005486 & $-0,041199$ & 0,046453 & $-0,205699$ & \\
\hline $2 \backslash 0$ & 18 & 277 & 0,07099 & 0,050685 & 0,336911 & 0,004319 & $-0,033924$ & 0,064941 & $-0,256175$ & \\
\hline $2 \backslash 1$ & 40 & 741 & 0,18969 & 0,110959 & 0,536234 & 0,009454 & $-0,063579$ & 0,173528 & $-0,438463$ & \\
\hline $2 \backslash 2$ & 24 & 424 & 0,108596 & 0,067123 & 0,481099 & 0,005719 & $-0,042609$ & 0,099343 & $-0,330954$ & \\
\hline 213 & 53 & 1116 & 0,285623 & 0,146575 & 0,667133 & 0,012489 & $-0,078971$ & 0,261286 & $-0,505928$ \\
\hline & 364 & 3908 & & & & & $-0,593614$ & & $-2,949691$ & \\
\hline & & & & & & & 0,593614 & & 2,949691 & \\
\hline & & & & & & & 0,050579 & & 2,698359 & 2,748939 \\
\hline
\end{tabular}




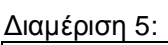

\begin{tabular}{|l|r|r|r|l|l|l|l|l|l|l|}
\hline HOMEJYR & BADS & GOODS & $\mathrm{f}(\mathrm{x} / 1)$ & $\mathrm{f}(\mathrm{x} / 0)$ & $\mathrm{w}(\mathrm{x})$ & & & & \\
\hline 010 & 32 & 199 & 0,051036 & 0,089041 & $-0,55657$ & 0,007587 & $-0,053429$ & 0,046687 & $-0,206397$ & \\
\hline $0 \backslash 1$ & 23 & 219 & 0,056152 & 0,064384 & $-0,13679$ & 0,005486 & $-0,041199$ & 0,051368 & $-0,220008$ & \\
\hline 012 & 9 & 88 & 0,02264 & 0,026027 & $-0,13943$ & 0,002218 & $-0,019553$ & 0,020711 & $-0,115846$ & \\
\hline 013 & 5 & 94 & 0,024175 & 0,015068 & 0,472712 & 0,001284 & $-0,012332$ & 0,022115 & $-0,121607$ & \\
\hline 110 & 57 & 177 & 0,045408 & 0,157534 & $-1,24395$ & 0,013423 & $-0,083479$ & 0,041539 & $-0,190639$ & \\
\hline $1 \backslash 1$ & 54 & 221 & 0,056664 & 0,149315 & $-0,96892$ & 0,012723 & $-0,080107$ & 0,051836 & $-0,221335$ & \\
\hline 112 & 20 & 125 & 0,032105 & 0,056164 & $-0,55926$ & 0,004786 & $-0,036883$ & 0,02937 & $-0,149478$ & \\
\hline 113 & 23 & 215 & 0,055129 & 0,064384 & $-0,15518$ & 0,005486 & $-0,041199$ & 0,050432 & $-0,217337$ & \\
\hline 210 & 35 & 426 & 0,109107 & 0,09726 & 0,11494 & 0,008287 & $-0,057305$ & 0,099811 & $-0,331837$ & \\
\hline $2 \backslash 1$ & 31 & 574 & 0,146969 & 0,086301 & 0,532373 & 0,007353 & $-0,052116$ & 0,134446 & $-0,389208$ & \\
\hline $2 \backslash 2$ & 26 & 429 & 0,109875 & 0,072603 & 0,414337 & 0,006186 & $-0,045386$ & 0,100513 & $-0,333154$ & \\
\hline 213 & 49 & 1141 & 0,292018 & 0,135616 & 0,766986 & 0,011555 & $-0,074362$ & 0,267137 & $-0,508722$ & \\
\hline & 364 & 3908 & & & & & $-0,597351$ & & $-3,005568$ & \\
\hline & & & & & & & 0,597351 & & 3,005568 & \\
\hline & & & & & & & 0,050898 & & 2,749475 & 2,800373 \\
\hline
\end{tabular}




\begin{tabular}{|c|c|c|c|c|c|c|c|c|c|c|}
\hline HOMEIMAR & BADS & GOODS & $f(x / 1)$ & $f(x / 0)$ & $w(x)$ & & & 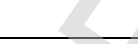 & 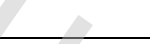 & \\
\hline $1 \backslash 1$ & 51 & 420 & 0,107572 & 0,141096 & $-0,27128$ & 0,012022 & $-0,076680$ & 0,098406 & $-0,329180$ & \\
\hline 211 & 85 & 223 & 0,057176 & 0,234247 & $-1,41024$ & 0,019959 & $-0,112706$ & 0,052304 & $-0,222655$ & \\
\hline 311 & 30 & 322 & 0,082502 & 0,083562 & $-0,01276$ & 0,00712 & $-0,050793$ & 0,075472 & $-0,281354$ & \\
\hline 112 & 10 & 13 & 0,003454 & 0,028767 & $-2,11983$ & 0,002451 & $-0,021257$ & 0,003159 & $-0,026242$ & \\
\hline 212 & 30 & 49 & 0,012663 & 0,083562 & $-1,88689$ & 0,00712 & $-0,050793$ & 0,011584 & $-0,074506$ & \\
\hline 312 & 85 & 120 & 0,030826 & 0,234247 & $-2,02801$ & 0,019959 & $-0,112706$ & 0,0282 & $-0,145177$ & \\
\hline 113 & 4 & 166 & 0,042594 & 0,012329 & 1,239778 & 0,00105 & $-0,010394$ & 0,038965 & $-0,182421$ & \\
\hline 213 & 39 & 470 & 0,120363 & 0,108219 & 0,106356 & 0,009221 & $-0,062341$ & 0,110108 & $-0,350474$ & \\
\hline \multirow[t]{4}{*}{313} & 30 & 2125 & 0,543745 & 0,083562 & 1,872896 & 0,00712 & $-0,050793$ & 0,497415 & $-0,501135$ & \\
\hline & 364 & 3908 & & & & & $-0,548463$ & & $-2,113142$ & \\
\hline & & & & & & + & 0,548463 & & 2,113142 & \\
\hline & & & & & & & 0,046732 & & 1,93309 & \\
\hline
\end{tabular}

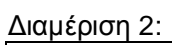

\begin{tabular}{|c|c|c|c|c|c|c|c|c|c|c|}
\hline HOMEMAR & BADS & GOODS & $f(x / 1)$ & $f(x / 0)$ & $w(x)$ & 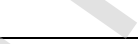 & & & & \\
\hline $0 \backslash 0$ & 11 & 15 & 0,003965 & 0,031507 & $-2,07265$ & 0,002685 & $-0,022929$ & 0,003627 & $-0,029406$ & \\
\hline $0 \backslash 1$ & 49 & 440 & 0,112689 & 0,135616 & $-0,1852$ & 0,011555 & $-0,074362$ & 0,103087 & $-0,337926$ & \\
\hline 012 & 5 & 156 & 0,040036 & 0,015068 & 0,977168 & 0,001284 & $-0,012332$ & 0,036625 & $-0,174737$ & \\
\hline $1 \backslash 0$ & 32 & 54 & 0,013942 & 0,089041 & $-1,85418$ & 0,007587 & $-0,053429$ & 0,012754 & $-0,080261$ & \\
\hline $1 \backslash 1$ & 89 & 233 & 0,059734 & 0,245205 & $-1,4122$ & 0,020893 & $-0,116600$ & 0,054644 & $-0,229166$ & \\
\hline 112 & 38 & 458 & 0,117293 & 0,105479 & 0,106163 & 0,008987 & $-0,061096$ & 0,107299 & $-0,345535$ & \\
\hline $2 \backslash 0$ & 84 & 123 & 0,031594 & 0,231507 & $-1,99165$ & 0,019726 & $-0,111722$ & 0,028902 & $-0,147766$ & \\
\hline $2 \backslash 1$ & 29 & 311 & 0,079688 & 0,080822 & $-0,01413$ & 0,006887 & $-0,049459$ & 0,072898 & $-0,275407$ & \\
\hline \multirow[t]{4}{*}{212} & 27 & 2118 & 0,541954 & 0,075342 & 1,973138 & 0,00642 & $-0,046756$ & 0,495777 & $-0,501844$ & \\
\hline & 364 & 3908 & & 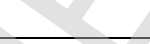 & & & $-0,548686$ & & $-2,122048$ & \\
\hline & & & & +4 & & & 0,548686 & & 2,122048 & \\
\hline & & & & 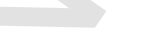 & & & 0,046751 & & 1,941237 & 1,987988 \\
\hline
\end{tabular}




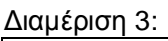

\begin{tabular}{|c|c|c|c|c|c|c|c|c|c|c|}
\hline HOMEMAR & BADS & GOODS & $f(x / 1)$ & $f(x / 0)$ & $w(x)$ & & & & 2 & \\
\hline 010 & 9 & 16 & 0,004221 & 0,026027 & $-1,81907$ & 0,002218 & $-0,019553$ & 0,003861 & $-0,030955$ & \\
\hline $0 \backslash 1$ & 56 & 437 & 0,111921 & 0,154795 & $-0,3243$ & 0,013189 & $-0,082361$ & 0,102385 & $-0,336634$ & \\
\hline 012 & 6 & 154 & 0,039524 & 0,017808 & 0,797252 & 0,001517 & $-0,014209$ & 0,036156 & $-0,173175$ & \\
\hline 110 & 31 & 48 & 0,012407 & 0,086301 & $-1,93956$ & 0,007353 & $-0,052116$ & 0,01135 & $-0,073335$ & \\
\hline $1 \backslash 1$ & 94 & 241 & 0,061781 & 0,258904 & $-1,43287$ & 0,02206 & $-0,121384$ & 0,056516 & $-0,234271$ & \\
\hline 112 & 37 & 443 & 0,113456 & 0,10274 & 0,099217 & 0,008754 & $-0,059841$ & 0,103789 & $-0,339211$ & \\
\hline 210 & 78 & 110 & 0,028268 & 0,215068 & $-2,02922$ & 0,018325 & $-0,105737$ & 0,025859 & $-0,136361$ & \\
\hline $2 \backslash 1$ & 26 & 317 & 0,081223 & 0,072603 & 0,112194 & 0,006186 & $-0,045386$ & 0,074302 & $-0,278667$ & \\
\hline \multirow[t]{4}{*}{212} & 27 & 2142 & 0,548094 & 0,075342 & 1,984403 & 0,00642 & $-0,046756$ & 0,501393 & $-0,499380$ & \\
\hline & 364 & 3908 & & & & & $-0,547343$ & & $-2,101989$ & \\
\hline & & & & & & & 0,547343 & & 2,101989 & \\
\hline & & & & & & & 0,046637 & & 1,922887 & 1,969524 \\
\hline
\end{tabular}

\begin{tabular}{|c|c|c|c|c|c|c|c|c|c|c|}
\hline HOMEMAR & BADS & GOODS & $f(x / 1)$ & $f(x / 0)$ & $w(x)$ & 2 & & & & \\
\hline $0 \backslash 0$ & 8 & 14 & 0,003709 & 0,023288 & $-1,83706$ & 0,001984 & $-0,017813$ & 0,003393 & $-0,027836$ & \\
\hline $0 \backslash 1$ & 53 & 443 & 0,113456 & 0,146575 & $-0,25612$ & 0,012489 & $-0,078971$ & 0,103789 & $-0,339211$ & \\
\hline 012 & 5 & 154 & 0,039524 & 0,015068 & 0,964306 & 0,001284 & $-0,012332$ & 0,036156 & $-0,173175$ & \\
\hline 110 & 29 & 48 & 0,012407 & 0,080822 & $-1,87397$ & 0,006887 & $-0,049459$ & 0,01135 & $-0,073335$ & \\
\hline $1 \backslash 1$ & 96 & 225 & 0,057687 & 0,264384 & $-1,52236$ & 0,022527 & $-0,123273$ & 0,052772 & $-0,223969$ & \\
\hline 112 & 38 & 466 & 0,11934 & 0,105479 & 0,12346 & 0,008987 & $-0,061096$ & 0,109172 & $-0,348839$ & \\
\hline 210 & 82 & 122 & 0,031338 & 0,226027 & $-1,97583$ & 0,019259 & $-0,109744$ & 0,028668 & $-0,146906$ & \\
\hline $2 \backslash 1$ & 26 & 315 & 0,080711 & 0,072603 & 0,105874 & 0,006186 & $-0,045386$ & 0,073834 & $-0,277584$ & \\
\hline \multirow[t]{4}{*}{212} & 27 & 2121 & 0,542722 & 0,075342 & 1,974553 & 0,00642 & $-0,046756$ & 0,496479 & $-0,501541$ & \\
\hline & 364 & 3908 & & 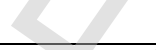 & 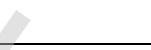 & & $-0,544830$ & & $-2,112396$ & \\
\hline & & & & & & & 0,544830 & & 2,112396 & \\
\hline & & & & $x_{y}$ & & & 0,046423 & & 1,932407 & 1,97883 \\
\hline
\end{tabular}




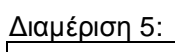

\begin{tabular}{|c|c|c|c|c|c|c|c|c|c|c|}
\hline HOMEMAR & BADS & GOODS & $f(x / 1)$ & $f(x / 0)$ & $w(x)$ & & & & 2 & 8 \\
\hline 010 & 10 & 14 & 0,003709 & 0,028767 & $-2,04837$ & 0,002451 & $-0,021257$ & 0,003393 & $-0,027836$ & \\
\hline $0 \backslash 1$ & 55 & 428 & 0,109619 & 0,152055 & $-0,32723$ & 0,012956 & $-0,081237$ & 0,100279 & $-0,332716$ & \\
\hline 012 & 4 & 158 & 0,040547 & 0,012329 & 1,190538 & 0,00105 & $-0,010394$ & 0,037093 & $-0,176291$ & \\
\hline 110 & 30 & 45 & 0,01164 & 0,083562 & $-1,97115$ & 0,00712 & $-0,050793$ & 0,010648 & $-0,069779$ & \\
\hline $1 \backslash 1$ & 88 & 218 & 0,055897 & 0,242466 & $-1,46736$ & 0,02066 & $-0,115632$ & 0,051134 & $-0,219343$ & \\
\hline 112 & 36 & 475 & 0,121642 & 0,1 & 0,195915 & 0,008521 & $-0,058578$ & 0,111278 & $-0,352501$ & \\
\hline 210 & 83 & 113 & 0,029036 & 0,228767 & $-2,06418$ & 0,019492 & $-0,110735$ & 0,026562 & $-0,139037$ & \\
\hline $2 \backslash 1$ & 29 & 315 & 0,080711 & 0,080822 & $-0,00137$ & 0,006887 & $-0,049459$ & 0,073834 & $-0,277584$ & \\
\hline \multirow[t]{4}{*}{212} & 29 & 2142 & 0,548094 & 0,080822 & 1,914199 & 0,006887 & $-0,049459$ & 0,501393 & $-0,499380$ & \\
\hline & 364 & 3908 & & & & & $-0,547545$ & & $-2,094468$ & \\
\hline & & & & & & & 0,547545 & & 2,094468 & \\
\hline & & & & & & & 0,046654 & & 1,916007 & 1,962661 \\
\hline
\end{tabular}

45) H(HOME TRM)

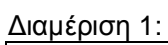

\begin{tabular}{|c|c|c|c|c|c|c|c|c|c|c|}
\hline HOMEITRM & BADS & GOODS & $f(x / 1)$ & $f(x / 0)$ & $w(x)$ & 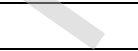 & & & & \\
\hline $1 \backslash 1$ & 5 & 42 & 0,010872 & 0,015068 & $-0,32638$ & 0,001284 & $-0,012332$ & 0,009946 & $-0,066157$ & \\
\hline $2 \backslash 1$ & 15 & 56 & 0,014454 & 0,042466 & $-1,07774$ & 0,003618 & $-0,029346$ & 0,013222 & $-0,082519$ & \\
\hline $3 \backslash 1$ & 35 & 305 & 0,078153 & 0,09726 & $-0,21872$ & 0,008287 & $-0,057305$ & 0,071494 & $-0,272108$ & \\
\hline 112 & 8 & 193 & 0,049501 & 0,023288 & 0,754072 & 0,001984 & $-0,017813$ & 0,045283 & $-0,202185$ & \\
\hline 212 & 18 & 211 & 0,054106 & 0,050685 & 0,065315 & 0,004319 & $-0,033924$ & 0,049496 & $-0,214641$ & \\
\hline 312 & 11 & 569 & 0,145689 & 0,031507 & 1,531272 & 0,002685 & $-0,022929$ & 0,133276 & $-0,387501$ & \\
\hline 113 & 52 & 364 & 0,093246 & 0,143836 & $-0,43343$ & 0,012256 & $-0,077828$ & 0,085301 & $-0,302929$ & \\
\hline 213 & 121 & 475 & 0,121642 & 0,332877 & $-1,00669$ & 0,028363 & $-0,145782$ & 0,111278 & $-0,352501$ & \\
\hline \multirow[t]{4}{*}{313} & 99 & 1693 & 0,433231 & 0,272603 & 0,463256 & 0,023227 & $-0,126079$ & 0,396317 & $-0,529191$ & \\
\hline & 364 & 3908 & & 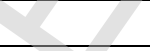 & & & $-0,523339$ & & $-2,409733$ & \\
\hline & & & & 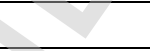 & & & 0,523339 & & 2,409733 & \\
\hline & & & & 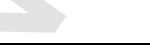 & & & 0,044592 & & 2,204409 & 2,249001 \\
\hline
\end{tabular}




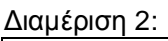

\begin{tabular}{|c|c|c|c|c|c|c|c|c|c|c|}
\hline HOMETRM & BADS & GOODS & $f(x / 1)$ & $f(x / 0)$ & $w(x)$ & & & & 2 & \\
\hline 010 & 6 & 204 & 0,052315 & 0,017808 & 1,077626 & 0,001517 & $-0,014209$ & 0,047858 & $-0,209861$ & \\
\hline $0 \backslash 1$ & 57 & 364 & 0,093246 & 0,157534 & $-0,5244$ & 0,013423 & $-0,083479$ & 0,085301 & $-0,302929$ & \\
\hline $0 \backslash 2$ & 2 & 43 & 0,011128 & 0,006849 & 0,485331 & 0,000584 & $-0,006269$ & 0,01018 & $-0,067372$ & \\
\hline 110 & 18 & 205 & 0,052571 & 0,050685 & 0,036536 & 0,004319 & $-0,033924$ & 0,048092 & $-0,210549$ & \\
\hline $1 \backslash 1$ & 129 & 489 & 0,125224 & 0,354795 & $-1,04144$ & 0,030231 & $-0,152600$ & 0,114554 & $-0,358084$ & \\
\hline 112 & 12 & 51 & 0,013175 & 0,034247 & $-0,95529$ & 0,002918 & $-0,024572$ & 0,012052 & $-0,076827$ & \\
\hline 210 & 7 & 552 & 0,14134 & 0,020548 & 1,928411 & 0,001751 & $-0,016033$ & 0,129297 & $-0,381587$ & \\
\hline $2 \backslash 1$ & 100 & 1710 & 0,43758 & 0,275342 & 0,463244 & 0,023461 & $-0,127008$ & 0,400296 & $-0,528735$ & \\
\hline \multirow[t]{4}{*}{212} & 33 & 290 & 0,074316 & 0,091781 & $-0,21108$ & 0,00782 & $-0,054731$ & 0,067984 & $-0,263686$ & \\
\hline & 364 & 3908 & & & & & $-0,512825$ & & $-2,399631$ & \\
\hline & & & & & & & 0,512825 & & 2,399631 & \\
\hline & & & & & & & 0,043696 & & 2,195168 & 2,238864 \\
\hline
\end{tabular}

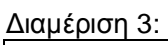

\begin{tabular}{|c|c|c|c|c|c|c|c|c|c|c|}
\hline HOMETRM & BADS & GOODS & $f(x / 1)$ & $f(x / 0)$ & $w(x)$ & 2 & & & & \\
\hline 010 & 6 & 196 & 0,050269 & 0,017808 & 1,037721 & 0,001517 & $-0,014209$ & 0,045985 & $-0,204299$ & \\
\hline $0 \backslash 1$ & 61 & 376 & 0,096316 & 0,168493 & $-0,55926$ & 0,014357 & $-0,087893$ & 0,088109 & $-0,308785$ & \\
\hline 012 & 4 & 35 & 0,009082 & 0,012329 & $-0,30568$ & 0,00105 & $-0,010394$ & 0,008308 & $-0,057418$ & \\
\hline 110 & 15 & 199 & 0,051036 & 0,042466 & 0,183835 & 0,003618 & $-0,029346$ & 0,046687 & $-0,206397$ & \\
\hline $1 \backslash 1$ & 130 & 481 & 0,123177 & 0,357534 & $-1,06561$ & 0,030464 & $-0,153440$ & 0,112682 & $-0,354911$ & \\
\hline 112 & 17 & 52 & 0,013431 & 0,047945 & $-1,27253$ & 0,004085 & $-0,032418$ & 0,012286 & $-0,077978$ & \\
\hline 210 & 7 & 533 & 0,13648 & 0,020548 & 1,893417 & 0,001751 & $-0,016033$ & 0,124851 & $-0,374768$ & \\
\hline $2 \backslash 1$ & 93 & 1747 & 0,447045 & 0,256164 & 0,556841 & 0,021827 & $-0,120435$ & 0,408954 & $-0,527546$ & \\
\hline \multirow[t]{4}{*}{212} & 31 & 289 & 0,07406 & 0,086301 & $-0,15297$ & 0,007353 & $-0,052116$ & 0,06775 & $-0,263115$ & \\
\hline & 364 & 3908 & & 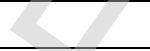 & 8 & & $-0,516285$ & & $-2,375217$ & \\
\hline & & & & & & & 0,516285 & & 2,375217 & \\
\hline & & & & 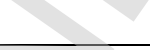 & & & 0,043991 & & 2,172834 & 2,216824 \\
\hline
\end{tabular}




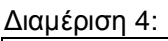

\begin{tabular}{|c|c|c|c|c|c|c|c|c|c|c|}
\hline HOMETRM & BADS & GOODS & $f(x / 1)$ & $f(x / 0)$ & $w(x)$ & & & & 2 & \\
\hline 010 & 8 & 195 & 0,050013 & 0,023288 & 0,764355 & 0,001984 & $-0,017813$ & 0,045751 & $-0,203596$ & \\
\hline $0 \backslash 1$ & 54 & 372 & 0,095293 & 0,149315 & $-0,4491$ & 0,012723 & $-0,080107$ & 0,087173 & $-0,306848$ & \\
\hline $0 \backslash 2$ & 4 & 44 & 0,011384 & 0,012329 & $-0,07973$ & 0,00105 & $-0,010394$ & 0,010414 & $-0,068580$ & \\
\hline 110 & 17 & 202 & 0,051804 & 0,047945 & 0,077399 & 0,004085 & $-0,032418$ & 0,04739 & $-0,208480$ & \\
\hline $1 \backslash 1$ & 132 & 492 & 0,125991 & 0,363014 & $-1,05823$ & 0,030931 & $-0,155113$ & 0,115256 & $-0,359263$ & \\
\hline 112 & 14 & 45 & 0,01164 & 0,039726 & $-1,22758$ & 0,003385 & $-0,027779$ & 0,010648 & $-0,069779$ & \\
\hline 210 & 6 & 505 & 0,129317 & 0,017808 & 1,982606 & 0,001517 & $-0,014209$ & 0,118298 & $-0,364300$ & \\
\hline $2 \backslash 1$ & 98 & 1778 & 0,454976 & 0,269863 & 0,52233 & 0,022994 & $-0,125147$ & 0,416209 & $-0,526346$ & \\
\hline \multirow[t]{4}{*}{212} & 31 & 275 & 0,070478 & 0,086301 & $-0,20254$ & 0,007353 & $-0,052116$ & 0,064473 & $-0,255002$ & \\
\hline & 364 & 3908 & & & & & $-0,515096$ & & $-2,362193$ & \\
\hline & & & & & & & 0,515096 & & 2,362193 & \\
\hline & & & & & & & 0,043889 & & 2,16092 & 2,204809 \\
\hline
\end{tabular}

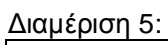

\begin{tabular}{|c|c|c|c|c|c|c|c|c|c|c|}
\hline HOMETRM & BADS & GOODS & $f(x / 1)$ & $f(x / 0)$ & $w(x)$ & 2 & & & & \\
\hline 010 & 8 & 182 & 0,046687 & 0,023288 & 0,695544 & 0,001984 & $-0,017813$ & 0,042709 & $-0,194297$ & \\
\hline $0 \backslash 1$ & 56 & 378 & 0,096828 & 0,154795 & $-0,46916$ & 0,013189 & $-0,082361$ & 0,088578 & $-0,309748$ & \\
\hline 012 & 5 & 40 & 0,010361 & 0,015068 & $-0,37459$ & 0,001284 & $-0,012332$ & 0,009478 & $-0,063703$ & \\
\hline 110 & 17 & 179 & 0,04592 & 0,047945 & $-0,04317$ & 0,004085 & $-0,032418$ & 0,042007 & $-0,192108$ & \\
\hline $1 \backslash 1$ & 123 & 514 & 0,131619 & 0,338356 & $-0,94419$ & 0,02883 & $-0,147502$ & 0,120405 & $-0,367720$ & \\
\hline 112 & 14 & 45 & 0,01164 & 0,039726 & $-1,22758$ & 0,003385 & $-0,027779$ & 0,010648 & $-0,069779$ & \\
\hline 210 & 5 & 504 & 0,129061 & 0,015068 & 2,14768 & 0,001284 & $-0,012332$ & 0,118064 & $-0,363916$ & \\
\hline $2 \backslash 1$ & 98 & 1774 & 0,453952 & 0,269863 & 0,520078 & 0,022994 & $-0,125147$ & 0,415273 & $-0,526511$ & \\
\hline \multirow[t]{4}{*}{212} & 38 & 292 & 0,074827 & 0,105479 & $-0,34333$ & 0,008987 & $-0,061096$ & 0,068452 & $-0,264824$ & \\
\hline & 364 & 3908 & & 20 & 8 & & $-0,518780$ & & $-2,352607$ & \\
\hline & & & & & & & 0,518780 & & 2,352607 & \\
\hline & & & & 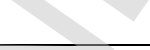 & & & 0,044203 & & 2,15215 & 2,196354 \\
\hline
\end{tabular}




\begin{tabular}{|c|c|c|c|c|c|c|c|c|c|c|}
\hline INCIJOB & BADS & GOODS & $f(x / 1)$ & $f(x / 0)$ & $w(x)$ & & & & z & \\
\hline $1 \backslash 1$ & 20 & 186 & 0,04771 & 0,056164 & $-0,16313$ & 0,004786 & $-0,036883$ & 0,043645 & $-0,197190$ & \\
\hline $2 \backslash 1$ & 28 & 155 & 0,03978 & 0,078082 & $-0,6744$ & 0,006653 & $-0,048113$ & 0,036391 & $-0,173957$ & \\
\hline $3 \backslash 1$ & 10 & 287 & 0,073548 & 0,028767 & 0,938708 & 0,002451 & $-0,021257$ & 0,067281 & $-0,261970$ & \\
\hline $4 \backslash 1$ & 0 & 46 & 0,011896 & 0,00137 & 2,16146 & 0,000117 & $-0,001525$ & 0,010882 & $-0,070972$ & \\
\hline 112 & 22 & 57 & 0,01471 & 0,061644 & $-1,43287$ & 0,005252 & $-0,039776$ & 0,013456 & $-0,083639$ & \\
\hline 212 & 33 & 54 & 0,013942 & 0,091781 & $-1,88448$ & 0,00782 & $-0,054731$ & 0,012754 & $-0,080261$ & \\
\hline 312 & 45 & 262 & 0,067153 & 0,124658 & $-0,6186$ & 0,010622 & $-0,069644$ & 0,061431 & $-0,247253$ & \\
\hline 412 & 5 & 75 & 0,019314 & 0,015068 & 0,248245 & 0,001284 & $-0,012332$ & 0,017669 & $-0,102879$ & \\
\hline 113 & 17 & 163 & 0,041827 & 0,047945 & $-0,13653$ & 0,004085 & $-0,032418$ & 0,038263 & $-0,180138$ & \\
\hline 213 & 34 & 178 & 0,045664 & 0,094521 & $-0,72751$ & 0,008054 & $-0,056023$ & 0,041773 & $-0,191374$ & \\
\hline 313 & 19 & 388 & 0,099386 & 0,053425 & 0,620739 & 0,004552 & $-0,035412$ & 0,090918 & $-0,314511$ & \\
\hline 413 & 0 & 175 & 0,044896 & 0,00137 & 3,489647 & 0,000117 & $-0,001525$ & 0,041071 & $-0,189162$ & \\
\hline $1 \backslash 4$ & 6 & 69 & 0,017779 & 0,017808 & $-0,00161$ & 0,001517 & $-0,014209$ & 0,016265 & $-0,096646$ & \\
\hline $2 \backslash 4$ & 7 & 89 & 0,022896 & 0,020548 & 0,108196 & 0,001751 & $-0,016033$ & 0,020945 & $-0,116816$ & \\
\hline $3 \backslash 4$ & 5 & 74 & 0,019059 & 0,015068 & 0,234912 & 0,001284 & $-0,012332$ & 0,017435 & $-0,101852$ & \\
\hline $4 \backslash 4$ & 0 & 13 & 0,003454 & 0,00137 & 0,924697 & 0,000117 & $-0,001525$ & 0,003159 & $-0,026242$ & \\
\hline 115 & 20 & 220 & 0,056408 & 0,056164 & 0,004333 & 0,004786 & $-0,036883$ & 0,051602 & $-0,220672$ & \\
\hline 215 & 50 & 159 & 0,040803 & 0,138356 & $-1,22107$ & 0,011789 & $-0,075524$ & 0,037327 & $-0,177064$ & \\
\hline 315 & 27 & 841 & 0,215272 & 0,075342 & 1,04986 & 0,00642 & $-0,046756$ & 0,19693 & $-0,461652$ & \\
\hline 415 & 1 & 198 & 0,05078 & 0,00411 & 2,514184 & 0,00035 & $-0,004020$ & 0,046453 & $-0,205699$ & \\
\hline 116 & 1 & 20 & 0,005244 & 0,00411 & 0,24382 & 0,00035 & $-0,004020$ & 0,004797 & $-0,036957$ & \\
\hline 216 & 5 & 9 & 0,00243 & 0,015068 & $-1,8246$ & 0,001284 & $-0,012332$ & 0,002223 & $-0,019593$ & \\
\hline 316 & 9 & 168 & 0,043106 & 0,026027 & 0,504504 & 0,002218 & $-0,019553$ & 0,039433 & $-0,183933$ & \\
\hline \multirow[t]{4}{*}{416} & 0 & 22 & 0,005756 & 0,00137 & 1,435523 & 0,000117 & $-0,001525$ & 0,005266 & $-0,039856$ & \\
\hline & 364 & 3908 & & 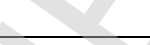 & & & $-0,654350$ & & $-3,780288$ & \\
\hline & & & & 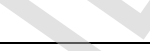 & & & 0,654350 & & 3,780288 & \\
\hline & & & & 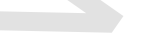 & & & 0,055755 & & 3,458185 & 3,513939 \\
\hline
\end{tabular}




\begin{tabular}{|c|c|c|c|c|c|c|c|c|c|c|}
\hline INCJOB & BADS & GOODS & $f(x / 1)$ & $f(x / 0)$ & $w(x)$ & & & & 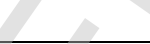 & $\mathrm{z}$ \\
\hline 010 & 28 & 54 & 0,013942 & 0,078082 & $-1,72284$ & 0,006653 & $-0,048113$ & 0,012754 & $-0,080261$ & \\
\hline 012 & 35 & 152 & 0,039013 & 0,09726 & $-0,91351$ & 0,008287 & $-0,057305$ & 0,035688 & $-0,171604$ & \\
\hline 013 & 39 & 170 & 0,043617 & 0,108219 & $-0,9087$ & 0,009221 & $-0,062341$ & 0,039901 & $-0,185437$ & \\
\hline 014 & 5 & 82 & 0,021105 & 0,015068 & 0,336911 & 0,001284 & $-0,012332$ & 0,019307 & $-0,109948$ & \\
\hline 015 & 46 & 171 & 0,043873 & 0,127397 & $-1,06601$ & 0,010855 & $-0,070834$ & 0,040135 & $-0,186186$ & \\
\hline 016 & 8 & 10 & 0,002686 & 0,023288 & $-2,15983$ & 0,001984 & $-0,017813$ & 0,002457 & $-0,021301$ & \\
\hline 110 & 23 & 64 & 0,0165 & 0,064384 & $-1,36147$ & 0,005486 & $-0,041199$ & 0,015094 & $-0,091319$ & \\
\hline 112 & 19 & 187 & 0,047966 & 0,053425 & $-0,10778$ & 0,004552 & $-0,035412$ & 0,043879 & $-0,197909$ & \\
\hline 113 & 15 & 160 & 0,041059 & 0,042466 & $-0,03369$ & 0,003618 & $-0,029346$ & 0,037561 & $-0,177836$ & \\
\hline $1 \backslash 4$ & 7 & 67 & 0,017268 & 0,020548 & $-0,17391$ & 0,001751 & $-0,016033$ & 0,015797 & $-0,094530$ & \\
\hline 115 & 24 & 213 & 0,054618 & 0,067123 & $-0,20618$ & 0,005719 & $-0,042609$ & 0,049964 & $-0,215992$ & \\
\hline 116 & 4 & 19 & 0,004988 & 0,012329 & $-0,9048$ & 0,00105 & $-0,010394$ & 0,004563 & $-0,035484$ & \\
\hline 210 & 46 & 275 & 0,070478 & 0,127397 & $-0,592$ & 0,010855 & $-0,070834$ & 0,064473 & $-0,255002$ & \\
\hline 212 & 9 & 293 & 0,075083 & 0,026027 & 1,059446 & 0,002218 & $-0,019553$ & 0,068686 & $-0,265391$ & \\
\hline 213 & 15 & 379 & 0,097084 & 0,042466 & 0,826875 & 0,003618 & $-0,029346$ & 0,088812 & $-0,310228$ & \\
\hline 214 & 5 & 92 & 0,023663 & 0,015068 & 0,451321 & 0,001284 & $-0,012332$ & 0,021647 & $-0,119702$ & \\
\hline 215 & 26 & 827 & 0,211691 & 0,072603 & 1,070125 & 0,006186 & $-0,045386$ & 0,193654 & $-0,458659$ & \\
\hline 216 & 6 & 163 & 0,041827 & 0,017808 & 0,853871 & 0,001517 & $-0,014209$ & 0,038263 & $-0,180138$ & \\
\hline 310 & 3 & 75 & 0,019314 & 0,009589 & 0,70023 & 0,000817 & $-0,008381$ & 0,017669 & $-0,102879$ & \\
\hline 312 & 0 & 45 & 0,01164 & 0,00137 & 2,13972 & 0,000117 & $-0,001525$ & 0,010648 & $-0,069779$ & \\
\hline 313 & 0 & 161 & 0,041315 & 0,00137 & 3,406513 & 0,000117 & $-0,001525$ & 0,037795 & $-0,178605$ & \\
\hline $3 \backslash 4$ & 0 & 13 & 0,003454 & 0,00137 & 0,924697 & 0,000117 & $-0,001525$ & 0,003159 & $-0,026242$ & \\
\hline 315 & 1 & 215 & 0,055129 & 0,00411 & 2,596356 & 0,00035 & $-0,004020$ & 0,050432 & $-0,217337$ & \\
\hline \multirow[t]{4}{*}{316} & 0 & 21 & 0,0055 & 0,00137 & 1,390061 & 0,000117 & $-0,001525$ & 0,005031 & $-0,038414$ & \\
\hline & 364 & 3908 & & 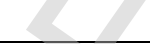 & 8 & & $-0,653895$ & & $-3,790182$ & \\
\hline & & & & 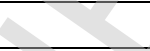 & 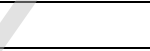 & & 0,653895 & & 3,790182 & \\
\hline & & & & & & & 0,055716 & & 3,467236 & 3,522951 \\
\hline
\end{tabular}




\begin{tabular}{|c|c|c|c|c|c|c|c|c|c|c|}
\hline INCJOB & BADS & GOODS & $f(x / 1)$ & $f(x / 0)$ & $w(x)$ & & & & 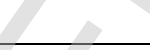 & $\theta$ \\
\hline 010 & 30 & 53 & 0,013686 & 0,083562 & $-1,80918$ & 0,00712 & $-0,050793$ & 0,01252 & $-0,079123$ & \\
\hline 012 & 36 & 144 & 0,036966 & 0,1 & $-0,99517$ & 0,008521 & $-0,058578$ & 0,033816 & $-0,165231$ & \\
\hline 013 & 45 & 197 & 0,050524 & 0,124658 & $-0,90311$ & 0,010622 & $-0,069644$ & 0,046219 & $-0,205000$ & \\
\hline 014 & 8 & 86 & 0,022128 & 0,023288 & $-0,05106$ & 0,001984 & $-0,017813$ & 0,020243 & $-0,113896$ & \\
\hline 015 & 40 & 148 & 0,037989 & 0,110959 & $-1,07186$ & 0,009454 & $-0,063579$ & 0,034752 & $-0,168436$ & \\
\hline 016 & 7 & 8 & 0,002174 & 0,020548 & $-2,24598$ & 0,001751 & $-0,016033$ & 0,001989 & $-0,017850$ & \\
\hline 110 & 23 & 61 & 0,015733 & 0,064384 & $-1,4091$ & 0,005486 & $-0,041199$ & 0,014392 & $-0,088061$ & \\
\hline 112 & 22 & 174 & 0,044641 & 0,061644 & $-0,32273$ & 0,005252 & $-0,039776$ & 0,040837 & $-0,188421$ & \\
\hline 113 & 18 & 180 & 0,046175 & 0,050685 & $-0,09318$ & 0,004319 & $-0,033924$ & 0,042241 & $-0,192839$ & \\
\hline 114 & 8 & 68 & 0,017524 & 0,023288 & $-0,28437$ & 0,001984 & $-0,017813$ & 0,016031 & $-0,095591$ & \\
\hline 115 & 16 & 203 & 0,052059 & 0,045205 & 0,141166 & 0,003852 & $-0,030892$ & 0,047624 & $-0,209171$ & \\
\hline 116 & 3 & 22 & 0,005756 & 0,009589 & $-0,51039$ & 0,000817 & $-0,008381$ & 0,005266 & $-0,039856$ & \\
\hline 210 & 42 & 260 & 0,066641 & 0,116438 & $-0,55804$ & 0,009921 & $-0,066028$ & 0,060963 & $-0,246042$ & \\
\hline 212 & 9 & 294 & 0,075339 & 0,026027 & 1,062848 & 0,002218 & $-0,019553$ & 0,06892 & $-0,265957$ & \\
\hline 213 & 19 & 429 & 0,109875 & 0,053425 & 0,721068 & 0,004552 & $-0,035412$ & 0,100513 & $-0,333154$ & \\
\hline 214 & 4 & 86 & 0,022128 & 0,012329 & 0,584928 & 0,00105 & $-0,010394$ & 0,020243 & $-0,113896$ & \\
\hline 215 & 23 & 794 & 0,203249 & 0,064384 & 1,149573 & 0,005486 & $-0,041199$ & 0,185931 & $-0,451284$ & \\
\hline 216 & 5 & 173 & 0,044385 & 0,015068 & 1,08029 & 0,001284 & $-0,012332$ & 0,040603 & $-0,187678$ & \\
\hline 310 & 5 & 73 & 0,018803 & 0,015068 & 0,221398 & 0,001284 & $-0,012332$ & 0,017201 & $-0,100820$ & \\
\hline 312 & 0 & 42 & 0,010872 & 0,00137 & 2,071512 & 0,000117 & $-0,001525$ & 0,009946 & $-0,066157$ & \\
\hline 313 & 0 & 173 & 0,044385 & 0,00137 & 3,478185 & 0,000117 & $-0,001525$ & 0,040603 & $-0,187678$ & \\
\hline 314 & 0 & 16 & 0,004221 & 0,00137 & 1,125368 & 0,000117 & $-0,001525$ & 0,003861 & $-0,030955$ & \\
\hline 315 & 1 & 206 & 0,052827 & 0,00411 & 2,553696 & 0,00035 & $-0,004020$ & 0,048326 & $-0,211235$ & \\
\hline \multirow[t]{4}{*}{316} & 0 & 18 & 0,004733 & 0,00137 & 1,239778 & 0,000117 & $-0,001525$ & 0,004329 & $-0,033993$ & \\
\hline & 364 & 3908 & & & s & & $-0,655796$ & & $-3,792322$ & \\
\hline & & & & & & & 0,655796 & & 3,792322 & \\
\hline & & & & - & & & 0,055878 & & 3,469193 & 3,525071 \\
\hline
\end{tabular}




\begin{tabular}{|c|c|c|c|c|c|c|c|c|c|c|}
\hline INCJOB & BADS & GOODS & $f(x / 1)$ & $f(x / 0)$ & $w(x)$ & & & & 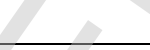 & $\theta$ \\
\hline 010 & 29 & 56 & 0,014454 & 0,080822 & $-1,72129$ & 0,006887 & $-0,049459$ & 0,013222 & $-0,082519$ & \\
\hline 012 & 37 & 171 & 0,043873 & 0,10274 & $-0,8509$ & 0,008754 & $-0,059841$ & 0,040135 & $-0,186186$ & \\
\hline 013 & 47 & 171 & 0,043873 & 0,130137 & $-1,08729$ & 0,011088 & $-0,072017$ & 0,040135 & $-0,186186$ & \\
\hline $0 \backslash 4$ & 7 & 91 & 0,023408 & 0,020548 & 0,130296 & 0,001751 & $-0,016033$ & 0,021413 & $-0,118743$ & \\
\hline 015 & 40 & 137 & 0,035175 & 0,110959 & $-1,14882$ & 0,009454 & $-0,063579$ & 0,032178 & $-0,159532$ & \\
\hline 016 & 7 & 8 & 0,002174 & 0,020548 & $-2,24598$ & 0,001751 & $-0,016033$ & 0,001989 & $-0,017850$ & \\
\hline 110 & 24 & 62 & 0,015989 & 0,067123 & $-1,43465$ & 0,005719 & $-0,042609$ & 0,014626 & $-0,089152$ & \\
\hline 112 & 19 & 209 & 0,053594 & 0,053425 & 0,00317 & 0,004552 & $-0,035412$ & 0,049028 & $-0,213283$ & \\
\hline 113 & 15 & 143 & 0,03671 & 0,042466 & $-0,14564$ & 0,003618 & $-0,029346$ & 0,033582 & $-0,164424$ & \\
\hline 114 & 6 & 72 & 0,018547 & 0,017808 & 0,040645 & 0,001517 & $-0,014209$ & 0,016967 & $-0,099783$ & \\
\hline 115 & 21 & 200 & 0,051292 & 0,058904 & $-0,13838$ & 0,005019 & $-0,038337$ & 0,046922 & $-0,207093$ & \\
\hline 116 & 4 & 26 & 0,006779 & 0,012329 & $-0,59807$ & 0,00105 & $-0,010394$ & 0,006202 & $-0,045477$ & \\
\hline 210 & 39 & 275 & 0,070478 & 0,108219 & $-0,42885$ & 0,009221 & $-0,062341$ & 0,064473 & $-0,255002$ & \\
\hline 212 & 8 & 335 & 0,085828 & 0,023288 & 1,304416 & 0,001984 & $-0,017813$ & 0,078515 & $-0,288219$ & \\
\hline 213 & 21 & 393 & 0,100665 & 0,058904 & 0,535889 & 0,005019 & $-0,038337$ & 0,092088 & $-0,316860$ & \\
\hline 214 & 5 & 78 & 0,020082 & 0,015068 & 0,287211 & 0,001284 & $-0,012332$ & 0,018371 & $-0,105934$ & \\
\hline 215 & 22 & 784 & 0,200691 & 0,061644 & 1,180392 & 0,005252 & $-0,039776$ & 0,183591 & $-0,448959$ & \\
\hline 216 & 7 & 169 & 0,043361 & 0,020548 & 0,74681 & 0,001751 & $-0,016033$ & 0,039667 & $-0,184686$ & \\
\hline 310 & 5 & 72 & 0,018547 & 0,015068 & 0,207699 & 0,001284 & $-0,012332$ & 0,016967 & $-0,099783$ & \\
\hline 312 & 0 & 49 & 0,012663 & 0,00137 & 2,22398 & 0,000117 & $-0,001525$ & 0,011584 & $-0,074506$ & \\
\hline 313 & 0 & 171 & 0,043873 & 0,00137 & 3,466591 & 0,000117 & $-0,001525$ & 0,040135 & $-0,186186$ & \\
\hline 314 & 0 & 12 & 0,003198 & 0,00137 & 0,847736 & 0,000117 & $-0,001525$ & 0,002925 & $-0,024623$ & \\
\hline 315 & 1 & 206 & 0,052827 & 0,00411 & 2,553696 & 0,00035 & $-0,004020$ & 0,048326 & $-0,211235$ & \\
\hline \multirow[t]{4}{*}{316} & 0 & 18 & 0,004733 & 0,00137 & 1,239778 & 0,000117 & $-0,001525$ & 0,004329 & $-0,033993$ & \\
\hline & 364 & 3908 & & & 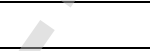 & & $-0,656355$ & & $-3,800212$ & \\
\hline & & & & & & & 0,656355 & & 3,800212 & \\
\hline & & & & 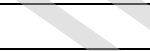 & & & 0,055925 & & 3,476411 & 3,532337 \\
\hline
\end{tabular}




\begin{tabular}{|c|c|c|c|c|c|c|c|c|c|c|}
\hline INCJOB & BADS & GOODS & $f(x / 1)$ & $f(x / 0)$ & $w(x)$ & & & & 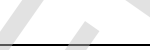 & 8 \\
\hline $0 \backslash 0$ & 30 & 65 & 0,016756 & 0,083562 & $-1,60682$ & 0,00712 & $-0,050793$ & 0,015328 & $-0,092395$ & \\
\hline 012 & 33 & 153 & 0,039268 & 0,091781 & $-0,84898$ & 0,00782 & $-0,054731$ & 0,035922 & $-0,172391$ & \\
\hline $0 \backslash 3$ & 46 & 167 & 0,04285 & 0,127397 & $-1,08961$ & 0,010855 & $-0,070834$ & 0,039199 & $-0,183178$ & \\
\hline $0 \backslash 4$ & 9 & 84 & 0,021617 & 0,026027 & $-0,18568$ & 0,002218 & $-0,019553$ & 0,019775 & $-0,111930$ & \\
\hline $0 \backslash 5$ & 42 & 155 & 0,03978 & 0,116438 & $-1,074$ & 0,009921 & $-0,066028$ & 0,036391 & $-0,173957$ & \\
\hline 016 & 6 & 12 & 0,003198 & 0,017808 & $-1,71721$ & 0,001517 & $-0,014209$ & 0,002925 & $-0,024623$ & \\
\hline $1 \backslash 0$ & 23 & 62 & 0,015989 & 0,064384 & $-1,39297$ & 0,005486 & $-0,041199$ & 0,014626 & $-0,089152$ & \\
\hline $1 \backslash 2$ & 21 & 179 & 0,04592 & 0,058904 & $-0,24902$ & 0,005019 & $-0,038337$ & 0,042007 & $-0,192108$ & \\
\hline $1 \backslash 3$ & 18 & 159 & 0,040803 & 0,050685 & $-0,21687$ & 0,004319 & $-0,033924$ & 0,037327 & $-0,177064$ & \\
\hline $1 \backslash 4$ & 5 & 56 & 0,014454 & 0,015068 & $-0,04165$ & 0,001284 & $-0,012332$ & 0,013222 & $-0,082519$ & \\
\hline $1 \backslash 5$ & 21 & 232 & 0,059478 & 0,058904 & 0,009698 & 0,005019 & $-0,038337$ & 0,05441 & $-0,228522$ & \\
\hline 116 & 3 & 21 & 0,0055 & 0,009589 & $-0,55585$ & 0,000817 & $-0,008381$ & 0,005031 & $-0,038414$ & \\
\hline 210 & 39 & 269 & 0,068943 & 0,108219 & $-0,45087$ & 0,009221 & $-0,062341$ & 0,063069 & $-0,251452$ & \\
\hline 212 & 9 & 308 & 0,07892 & 0,026027 & 1,109291 & 0,002218 & $-0,019553$ & 0,072196 & $-0,273763$ & \\
\hline 213 & 19 & 372 & 0,095293 & 0,053425 & 0,578683 & 0,004552 & $-0,035412$ & 0,087173 & $-0,306848$ & \\
\hline $2 \backslash 4$ & 5 & 86 & 0,022128 & 0,015068 & 0,384257 & 0,001284 & $-0,012332$ & 0,020243 & $-0,113896$ & \\
\hline $2 \backslash 5$ & 24 & 824 & 0,210924 & 0,067123 & 1,144965 & 0,005719 & $-0,042609$ & 0,192952 & $-0,458007$ & \\
\hline 216 & 9 & 172 & 0,044129 & 0,026027 & 0,527966 & 0,002218 & $-0,019553$ & 0,040369 & $-0,186933$ & \\
\hline 310 & 2 & 68 & 0,017524 & 0,006849 & 0,939404 & 0,000584 & $-0,006269$ & 0,016031 & $-0,095591$ & \\
\hline 312 & 0 & 44 & 0,011384 & 0,00137 & 2,117497 & 0,000117 & $-0,001525$ & 0,010414 & $-0,068580$ & \\
\hline 313 & 0 & 176 & 0,045152 & 0,00137 & 3,495329 & 0,000117 & $-0,001525$ & 0,041305 & $-0,189901$ & \\
\hline $3 \backslash 4$ & 0 & 14 & 0,003709 & 0,00137 & 0,996156 & 0,000117 & $-0,001525$ & 0,003393 & $-0,027836$ & \\
\hline 315 & 0 & 209 & 0,053594 & 0,00137 & 3,666731 & 0,000117 & $-0,001525$ & 0,049028 & $-0,213283$ & \\
\hline \multirow[t]{4}{*}{316} & 0 & 21 & 0,0055 & 0,00137 & 1,390061 & 0,000117 & $-0,001525$ & 0,005031 & $-0,038414$ & \\
\hline & 364 & 3908 & & 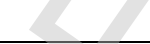 & 8 & & $-0,654352$ & & $-3,790754$ & \\
\hline & & & & + & 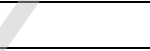 & & 0,654352 & & 3,790754 & \\
\hline & & & & + & & & 0,055755 & & 3,467759 & 3,523514 \\
\hline
\end{tabular}




\begin{tabular}{|c|c|c|c|c|c|c|c|c|c|c|}
\hline INCIJYR & BADS & GOODS & $f(x / 1)$ & $f(x / 0)$ & $w(x)$ & & & - & & \\
\hline $1 \backslash 1$ & 10 & 134 & 0,034408 & 0,028767 & 0,179049 & 0,002451 & $-0,021257$ & 0,031476 & $-0,157053$ & \\
\hline $2 \backslash 1$ & 24 & 78 & 0,020082 & 0,067123 & $-1,20671$ & 0,005719 & $-0,042609$ & 0,018371 & $-0,105934$ & \\
\hline $3 \backslash 1$ & 21 & 355 & 0,090944 & 0,058904 & 0,434333 & 0,005019 & $-0,038337$ & 0,083195 & $-0,298450$ & \\
\hline $4 \backslash 1$ & 1 & 84 & 0,021617 & 0,00411 & 1,660147 & 0,00035 & $-0,004020$ & 0,019775 & $-0,111930$ & \\
\hline 112 & 34 & 177 & 0,045408 & 0,094521 & $-0,73313$ & 0,008054 & $-0,056023$ & 0,041539 & $-0,190639$ & \\
\hline 212 & 57 & 228 & 0,058455 & 0,157534 & $-0,99139$ & 0,013423 & $-0,083479$ & 0,053474 & $-0,225929$ & \\
\hline 312 & 28 & 322 & 0,082502 & 0,078082 & 0,05506 & 0,006653 & $-0,048113$ & 0,075472 & $-0,281354$ & \\
\hline 412 & 2 & 75 & 0,019314 & 0,006849 & 1,036702 & 0,000584 & $-0,006269$ & 0,017669 & $-0,102879$ & \\
\hline 113 & 33 & 242 & 0,062036 & 0,091781 & $-0,39168$ & 0,00782 & $-0,054731$ & 0,05675 & $-0,234903$ & \\
\hline 213 & 47 & 210 & 0,05385 & 0,130137 & $-0,88238$ & 0,011088 & $-0,072017$ & 0,049262 & $-0,213963$ & \\
\hline 313 & 35 & 458 & 0,117293 & 0,09726 & 0,187288 & 0,008287 & $-0,057305$ & 0,107299 & $-0,345535$ & \\
\hline 413 & 1 & 98 & 0,025198 & 0,00411 & 1,813452 & 0,00035 & $-0,004020$ & 0,023051 & $-0,125376$ & \\
\hline 114 & 9 & 162 & 0,041571 & 0,026027 & 0,468247 & 0,002218 & $-0,019553$ & 0,038029 & $-0,179372$ & \\
\hline 214 & 29 & 128 & 0,032873 & 0,080822 & $-0,8996$ & 0,006887 & $-0,049459$ & 0,030072 & $-0,152027$ & \\
\hline $3 \backslash 4$ & 31 & 885 & 0,226529 & 0,086301 & 0,965025 & 0,007353 & $-0,052116$ & 0,207227 & $-0,470554$ & \\
\hline \multirow[t]{4}{*}{$4 \backslash 4$} & 2 & 272 & 0,069711 & 0,006849 & 2,320208 & 0,000584 & $-0,006269$ & 0,063771 & $-0,253232$ & \\
\hline & 364 & 3908 & & 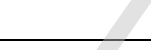 & 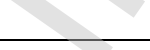 & & $-0,615577$ & & $-3,449128$ & \\
\hline & & & & 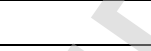 & & & 0,615577 & & 3,449128 & \\
\hline & & & & $P$ & & & 0,052451 & & 3,155242 & 3,207693 \\
\hline
\end{tabular}




\begin{tabular}{|c|c|c|c|c|c|c|c|c|c|c|}
\hline INCJYR & BADS & GOODS & $f(x / 1)$ & $f(x / 0)$ & $w(x)$ & & & ( & & \\
\hline 010 & 60 & 220 & 0,056408 & 0,165753 & $-1,07789$ & 0,014123 & $-0,086798$ & 0,051602 & $-0,220672$ & \\
\hline $0 \backslash 1$ & 44 & 231 & 0,059222 & 0,121918 & $-0,72205$ & 0,010388 & $-0,068447$ & 0,054176 & $-0,227876$ & \\
\hline 012 & 21 & 64 & 0,0165 & 0,058904 & $-1,27253$ & 0,005019 & $-0,038337$ & 0,015094 & $-0,091319$ & \\
\hline 013 & 36 & 124 & 0,03185 & 0,1 & $-1,14415$ & 0,008521 & $-0,058578$ & 0,029136 & $-0,148624$ & \\
\hline $1 \backslash 0$ & 30 & 177 & 0,045408 & 0,083562 & $-0,6099$ & 0,00712 & $-0,050793$ & 0,041539 & $-0,190639$ & \\
\hline $1 \backslash 1$ & 42 & 250 & 0,064083 & 0,116438 & $-0,59718$ & 0,009921 & $-0,066028$ & 0,058623 & $-0,239907$ & \\
\hline 112 & 10 & 115 & 0,029547 & 0,028767 & 0,026756 & 0,002451 & $-0,021257$ & 0,02703 & $-0,140806$ & \\
\hline 113 & 10 & 168 & 0,043106 & 0,028767 & 0,404421 & 0,002451 & $-0,021257$ & 0,039433 & $-0,183933$ & \\
\hline 210 & 28 & 328 & 0,084037 & 0,078082 & 0,073493 & 0,006653 & $-0,048113$ & 0,076876 & $-0,284544$ & \\
\hline $2 \backslash 1$ & 36 & 542 & 0,138782 & 0,1 & 0,327736 & 0,008521 & $-0,058578$ & 0,126957 & $-0,378026$ & \\
\hline 212 & 14 & 311 & 0,079688 & 0,039726 & 0,696111 & 0,003385 & $-0,027779$ & 0,072898 & $-0,275407$ & \\
\hline 213 & 29 & 848 & 0,217063 & 0,080822 & 0,98794 & 0,006887 & $-0,049459$ & 0,198568 & $-0,463119$ & \\
\hline 310 & 0 & 73 & 0,018803 & 0,00137 & 2,619293 & 0,000117 & $-0,001525$ & 0,017201 & $-0,100820$ & \\
\hline $3 \backslash 1$ & 1 & 111 & 0,028524 & 0,00411 & 1,93742 & 0,00035 & $-0,004020$ & 0,026094 & $-0,137256$ & \\
\hline 312 & 1 & 68 & 0,017524 & 0,00411 & 1,450229 & 0,00035 & $-0,004020$ & 0,016031 & $-0,095591$ & \\
\hline \multirow[t]{4}{*}{313} & 2 & 278 & 0,071246 & 0,006849 & 2,341988 & 0,000584 & $-0,006269$ & 0,065175 & $-0,256760$ & \\
\hline & 364 & 3908 & & & 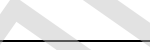 & & $-0,611258$ & & $-3,435297$ & \\
\hline & & & & & 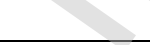 & & 0,611258 & & 3,435297 & \\
\hline & & & & & & & 0,052083 & & 3,14259 & 3,194672 \\
\hline
\end{tabular}




\begin{tabular}{|c|c|c|c|c|c|c|c|c|c|c|}
\hline \multicolumn{11}{|c|}{ 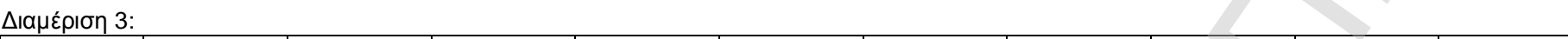 } \\
\hline INCJYR & BADS & GOODS & $f(x / 1)$ & $f(x / 0)$ & $w(x)$ & & & & & \\
\hline $0 \backslash 0$ & 65 & 211 & 0,054106 & 0,179452 & $-1,19896$ & 0,01529 & $-0,092220$ & 0,049496 & $-0,214641$ & \\
\hline $0 \backslash 1$ & 45 & 231 & 0,059222 & 0,124658 & $-0,74427$ & 0,010622 & $-0,069644$ & 0,054176 & $-0,227876$ & \\
\hline 012 & 22 & 65 & 0,016756 & 0,061644 & $-1,3026$ & 0,005252 & $-0,039776$ & 0,015328 & $-0,092395$ & \\
\hline 013 & 34 & 129 & 0,033129 & 0,094521 & $-1,04842$ & 0,008054 & $-0,056023$ & 0,030306 & $-0,152871$ & \\
\hline 110 & 33 & 182 & 0,046687 & 0,091781 & $-0,67593$ & 0,00782 & $-0,054731$ & 0,042709 & $-0,194297$ & \\
\hline $1 \backslash 1$ & 34 & 256 & 0,065618 & 0,094521 & $-0,36497$ & 0,008054 & $-0,056023$ & 0,060027 & $-0,243604$ & \\
\hline 112 & 9 & 115 & 0,029547 & 0,026027 & 0,126839 & 0,002218 & $-0,019553$ & 0,02703 & $-0,140806$ & \\
\hline 113 & 14 & 155 & 0,03978 & 0,039726 & 0,001358 & 0,003385 & $-0,027779$ & 0,036391 & $-0,173957$ & \\
\hline 210 & 28 & 340 & 0,087107 & 0,078082 & 0,109372 & 0,006653 & $-0,048113$ & 0,079685 & $-0,290813$ & \\
\hline $2 \backslash 1$ & 28 & 523 & 0,133922 & 0,078082 & 0,539493 & 0,006653 & $-0,048113$ & 0,122511 & $-0,371088$ & \\
\hline 212 & 15 & 307 & 0,078665 & 0,042466 & 0,616496 & 0,003618 & $-0,029346$ & 0,071962 & $-0,273212$ & \\
\hline 213 & 31 & 866 & 0,221668 & 0,086301 & 0,943335 & 0,007353 & $-0,052116$ & 0,202781 & $-0,466803$ & \\
\hline 310 & 2 & 81 & 0,020849 & 0,006849 & 1,113173 & 0,000584 & $-0,006269$ & 0,019073 & $-0,108950$ & \\
\hline $3 \backslash 1$ & 1 & 104 & 0,026733 & 0,00411 & 1,872582 & 0,00035 & $-0,004020$ & 0,024455 & $-0,130927$ & \\
\hline 312 & 1 & 63 & 0,016245 & 0,00411 & 1,374435 & 0,00035 & $-0,004020$ & 0,01486 & $-0,090238$ & \\
\hline \multirow[t]{4}{*}{313} & 2 & 280 & 0,071757 & 0,006849 & 2,349143 & 0,000584 & $-0,006269$ & 0,065643 & $-0,257926$ & \\
\hline & 364 & 3908 & & & 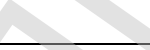 & & $-0,614015$ & & $-3,430403$ & \\
\hline & & & & & 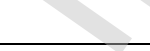 & & 0,614015 & & 3,430403 & \\
\hline & & & & & & & 0,052318 & & 3,138112 & 3,19043 \\
\hline
\end{tabular}




\begin{tabular}{|c|c|c|c|c|c|c|c|c|c|c|}
\hline INCJYR & BADS & GOOS & $f(x / 1)$ & $f(x / 0)$ & $w(x)$ & & & & & \\
\hline 010 & 45 & 135 & 0.034664 & 0.124658 & $-1,27988$ & 0.010622 & $-0,069644$ & 0,03171 & $-0,157882$ & \\
\hline $0 \backslash 1$ & 58 & 306 & 0,078409 & 0,160274 & $-0,71495$ & 0,013656 & $-0,084591$ & 0.071728 & $-0,272661$ & \\
\hline 012 & 27 & 72 & 0,018547 & 0,075342 & $-1,40174$ & 0,00642 & $-0,046756$ & 0,016967 & $-0,099783$ & \\
\hline 013 & 37 & 121 & 0,031082 & 0,10274 & $-1,19557$ & 0,008754 & $-0,059841$ & 0,028434 & $-0,146043$ & \\
\hline $1 \backslash 0$ & 16 & 111 & 0,028524 & 0,045205 & $-0,46048$ & 0,003852 & $-0,030892$ & 0,026094 & $-0,137256$ & \\
\hline $1 \backslash 1$ & 49 & 318 & 0,081479 & 0,135616 & $-0,50949$ & 0,011555 & $-0,074362$ & 0,074536 & $-0,279206$ & \\
\hline 112 & 11 & 130 & 0,033384 & 0,031507 & 0,057887 & 0,002685 & $-0,022929$ & 0,03054 & $-0,153712$ & \\
\hline 113 & 13 & 153 & 0,039268 & 0,036986 & 0,059871 & 0,003151 & $-0,026188$ & 0,035922 & $-0,172391$ & \\
\hline 210 & 19 & 211 & 0,054106 & 0,053425 & 0,012671 & 0,004552 & $-0,035412$ & 0,049496 & $-0,214641$ & \\
\hline $2 \backslash 1$ & 33 & 604 & 0,154643 & 0,091781 & 0,521717 & 0,00782 & $-0,054731$ & 0,141467 & $-0,399143$ & \\
\hline 212 & 20 & 356 & 0,0912 & 0,056164 & 0,48477 & 0,004786 & $-0,036883$ & 0,083429 & $-0,298952$ & \\
\hline 213 & 30 & 863 & 0,2209 & 0,083562 & 0,972128 & 0,00712 & $-0,050793$ & 0,202078 & $-0,466198$ & \\
\hline 310 & 2 & 54 & 0,013942 & 0,006849 & 0,71077 & 0,000584 & $-0,006269$ & 0,012754 & $-0,080261$ & \\
\hline $3 \backslash 1$ & 1 & 130 & 0,033384 & 0,00411 & 2,094769 & 0,00035 & $-0,004020$ & 0,03054 & $-0,153712$ & \\
\hline 312 & 1 & 86 & 0,022128 & 0,00411 & 1,68354 & 0,00035 & $-0,004020$ & 0,020243 & $-0,113896$ & \\
\hline 313 & 2 & 258 & 0,066129 & 0,006849 & 2,267465 & 0,000584 & $-0,006269$ & 0,060495 & $-0,244825$ & \\
\hline & 364 & 3908 & & & 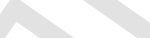 & & $-0,613601$ & & $-3,390562$ & \\
\hline & & & & & ( & & 0,613601 & & 3,390562 & \\
\hline & & & & & & & 0,052282 & & 3,101666 & 3,153948 \\
\hline
\end{tabular}




\begin{tabular}{|c|c|c|c|c|c|c|c|c|c|c|}
\hline \multicolumn{11}{|c|}{ 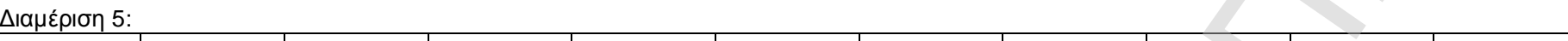 } \\
\hline INCJYR & BADS & GOODS & $f(x / 1)$ & $f(x / 0)$ & $w(x)$ & & & 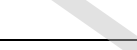 & & \\
\hline 010 & 62 & 213 & 0,054618 & 0,171233 & $-1,14267$ & 0,01459 & $-0,088983$ & 0,049964 & $-0,215992$ & \\
\hline $0 \backslash 1$ & 43 & 228 & 0,058455 & 0,119178 & $-0,71236$ & 0,010155 & $-0,067241$ & 0,053474 & $-0,225929$ & \\
\hline 012 & 25 & 68 & 0,017524 & 0,069863 & $-1,38298$ & 0,005953 & $-0,044004$ & 0,016031 & $-0,095591$ & \\
\hline 013 & 36 & 127 & 0,032617 & 0,1 & $-1,12034$ & 0,008521 & $-0,058578$ & 0,029838 & $-0,151180$ & \\
\hline 110 & 33 & 185 & 0,047455 & 0,091781 & $-0,65963$ & 0,00782 & $-0,054731$ & 0,043411 & $-0,196470$ & \\
\hline $1 \backslash 1$ & 33 & 226 & 0,057943 & 0,091781 & $-0,45994$ & 0,00782 & $-0,054731$ & 0,053006 & $-0,224624$ & \\
\hline 112 & 11 & 139 & 0,035687 & 0,031507 & 0,124578 & 0,002685 & $-0,022929$ & 0,032646 & $-0,161172$ & \\
\hline 113 & 14 & 159 & 0,040803 & 0,039726 & 0,026756 & 0,003385 & $-0,027779$ & 0,037327 & $-0,177064$ & \\
\hline 210 & 27 & 325 & 0,083269 & 0,075342 & 0,100037 & 0,00642 & $-0,046756$ & 0,076174 & $-0,282953$ & \\
\hline $2 \backslash 1$ & 32 & 457 & 0,117038 & 0,089041 & 0,273397 & 0,007587 & $-0,053429$ & 0,107065 & $-0,345118$ & \\
\hline 212 & 19 & 345 & 0,088386 & 0,053425 & 0,503439 & 0,004552 & $-0,035412$ & 0,080855 & $-0,293383$ & \\
\hline 213 & 27 & 904 & 0,231389 & 0,075342 & 1,122057 & 0,00642 & $-0,046756$ & 0,211673 & $-0,474167$ & \\
\hline 310 & 2 & 79 & 0,020338 & 0,006849 & 1,088327 & 0,000584 & $-0,006269$ & 0,018605 & $-0,106944$ & \\
\hline $3 \backslash 1$ & 0 & 103 & 0,026477 & 0,00137 & 2,961579 & 0,000117 & $-0,001525$ & 0,024221 & $-0,130010$ & \\
\hline 312 & 0 & 90 & 0,023152 & 0,00137 & 2,827358 & 0,000117 & $-0,001525$ & 0,021179 & $-0,117781$ & \\
\hline \multirow[t]{4}{*}{313} & 0 & 260 & 0,066641 & 0,00137 & 3,884611 & 0,000117 & $-0,001525$ & 0,060963 & $-0,246042$ & \\
\hline & 364 & 3908 & & & & & $-0,612172$ & & $-3,444421$ & \\
\hline & & & & & & & 0,612172 & & 3,444421 & \\
\hline & & & & & & & 0,052161 & & 3,150935 & 3,203096 \\
\hline
\end{tabular}




\begin{tabular}{|c|c|c|c|c|c|c|c|c|c|c|}
\hline INCIMAR & BADS & GOODS & $f(x / 1)$ & $f(x / 0)$ & $w(x)$ & & & - & & \\
\hline $1 \backslash 1$ & 46 & 227 & 0,058199 & 0,127397 & $-0,78344$ & 0,010855 & $-0,070834$ & 0,05324 & $-0,225277$ & \\
\hline $2 \backslash 1$ & 82 & 237 & 0,060757 & 0,226027 & $-1,31377$ & 0,019259 & $-0,109744$ & 0,05558 & $-0,231730$ & \\
\hline $3 \backslash 1$ & 38 & 430 & 0,11013 & 0,105479 & 0,04315 & 0,008987 & $-0,061096$ & 0,100747 & $-0,333592$ & \\
\hline $4 \backslash 1$ & 0 & 71 & 0,018291 & 0,00137 & 2,591705 & 0,000117 & $-0,001525$ & 0,016733 & $-0,098742$ & \\
\hline 112 & 29 & 24 & 0,006268 & 0,080822 & $-2,55686$ & 0,006887 & $-0,049459$ & 0,005734 & $-0,042694$ & \\
\hline 212 & 37 & 28 & 0,007291 & 0,10274 & $-2,64558$ & 0,008754 & $-0,059841$ & 0,00667 & $-0,048209$ & \\
\hline 312 & 54 & 96 & 0,024687 & 0,149315 & $-1,7998$ & 0,012723 & $-0,080107$ & 0,022583 & $-0,123499$ & \\
\hline 412 & 5 & 34 & 0,008826 & 0,015068 & $-0,53493$ & 0,001284 & $-0,012332$ & 0,008074 & $-0,056133$ & \\
\hline 113 & 11 & 464 & 0,118828 & 0,031507 & 1,327475 & 0,002685 & $-0,022929$ & 0,108703 & $-0,348017$ & \\
\hline 213 & 38 & 379 & 0,097084 & 0,105479 & $-0,08294$ & 0,008987 & $-0,061096$ & 0,088812 & $-0,310228$ & \\
\hline 313 & 23 & 1494 & 0,382323 & 0,064384 & 1,781407 & 0,005486 & $-0,041199$ & 0,349747 & $-0,530082$ & \\
\hline \multirow[t]{4}{*}{413} & 1 & 424 & 0,108596 & 0,00411 & 3,274307 & 0,00035 & $-0,004020$ & 0,099343 & $-0,330954$ & \\
\hline & 364 & 3908 & & & $\Delta$ & & $-0,574182$ & & $-2,679159$ & \\
\hline & & & & & & & 0,574182 & & 2,679159 & \\
\hline & & & & 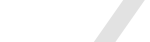 & + & & 0,048924 & & 2,450879 & 2,499802 \\
\hline
\end{tabular}




\begin{tabular}{|c|c|c|c|c|c|c|c|c|c|c|}
\hline INCMAR & Cחמק & 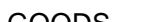 & $f(x / 1)$ & & (n) & & & & & \\
\hline $0 \backslash 0$ & 37 & 29 & 0,007547 & 0,10274 & -2.61109 & 0,008754 & -0.059841 & 0.006904 & -0.049557 & \\
\hline $0 \backslash 1$ & 86 & 251 & 0,064339 & 0,236986 & $-1,30384$ & 0,020193 & $-0,113685$ & 0,058857 & $-0,240527$ & \\
\hline $0 \backslash 2$ & 38 & 359 & 0,091967 & 0,105479 & $-0,13708$ & 0,008987 & $-0,061096$ & 0,084131 & $-0,300450$ & \\
\hline $1 \backslash 0$ & 32 & 29 & 0,007547 & 0,089041 & $-2,46799$ & 0,007587 & $-0,053429$ & 0,006904 & $-0,049557$ & \\
\hline $1 \backslash 1$ & 47 & 226 & 0,057943 & 0,130137 & $-0,80912$ & 0,011088 & $-0,072017$ & 0,053006 & $-0,224624$ & \\
\hline 112 & 13 & 455 & 0,116526 & 0,036986 & 1,147567 & 0,003151 & $-0,026188$ & 0,106597 & $-0,344283$ & \\
\hline 210 & 55 & 98 & 0,025198 & 0,152055 & $-1,79747$ & 0,012956 & $-0,081237$ & 0,023051 & $-0,125376$ & \\
\hline $2 \backslash 1$ & 34 & 428 & 0,109619 & 0,094521 & 0,148192 & 0,008054 & $-0,056023$ & 0,100279 & $-0,332716$ & \\
\hline 212 & 18 & 1503 & 0,384625 & 0,050685 & 2,026641 & 0,004319 & $-0,033924$ & 0,351853 & $-0,530227$ & \\
\hline 310 & 3 & 36 & 0,009337 & 0,009589 & $-0,02659$ & 0,000817 & $-0,008381$ & 0,008542 & $-0,058693$ & \\
\hline $3 \backslash 1$ & 0 & 79 & 0,020338 & 0,00137 & 2,697765 & 0,000117 & $-0,001525$ & 0,018605 & $-0,106944$ & \\
\hline 312 & 1 & 415 & 0,106293 & 0,00411 & 3,252878 & 0,00035 & $-0,004020$ & 0,097236 & $-0,326944$ & \\
\hline & 364 & 3908 & & & & 2 & $-0,571365$ & & $-2,689898$ & \\
\hline & & & & & 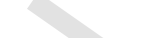 & 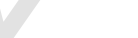 & 0,571365 & & 2,689898 & \\
\hline & & & & & 1 & & 0,048684 & & 2,460702 & 2,509386 \\
\hline
\end{tabular}




\begin{tabular}{|c|c|c|c|c|c|c|c|c|c|c|}
\hline \multicolumn{11}{|c|}{ } \\
\hline INCMAR & BADS & GOODS & $f(x / 1)$ & $f(x / 0)$ & $w(x)$ & & & 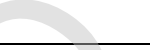 & & \\
\hline $0 \backslash 0$ & 32 & 27 & 0,007035 & 0,089041 & $-2,53819$ & 0,007587 & $-0,053429$ & 0,006436 & $-0,046849$ & \\
\hline $0 \backslash 1$ & 97 & 244 & 0,062548 & 0,267123 & $-1,45178$ & 0,022761 & $-0,124211$ & 0,057219 & $-0,236162$ & \\
\hline $0 \backslash 2$ & 37 & 365 & 0,093502 & 0,10274 & $-0,09421$ & 0,008754 & $-0,059841$ & 0,085535 & $-0,303422$ & \\
\hline 110 & 32 & 28 & 0,007291 & 0,089041 & $-2,50248$ & 0,007587 & $-0,053429$ & 0,00667 & $-0,048209$ & \\
\hline $1 \backslash 1$ & 48 & 247 & 0,063315 & 0,132877 & $-0,74129$ & 0,011322 & $-0,073193$ & 0,057921 & $-0,238041$ & \\
\hline 112 & 10 & 433 & 0,110898 & 0,028767 & 1,349377 & 0,002451 & $-0,021257$ & 0,101449 & $-0,334900$ & \\
\hline $2 \backslash 0$ & 49 & 87 & 0,022384 & 0,135616 & $-1,80147$ & 0,011555 & $-0,074362$ & 0,020477 & $-0,114873$ & \\
\hline $2 \backslash 1$ & 31 & 432 & 0,110642 & 0,086301 & 0,248455 & 0,007353 & $-0,052116$ & 0,101215 & $-0,334465$ & \\
\hline $2 \backslash 2$ & 22 & 1517 & 0,388207 & 0,061644 & 1,840165 & 0,005252 & $-0,039776$ & 0,355129 & $-0,530415$ & \\
\hline 310 & 5 & 32 & 0,008314 & 0,015068 & $-0,59465$ & 0,001284 & $-0,012332$ & 0,007606 & $-0,053534$ & \\
\hline $3 \backslash 1$ & 0 & 72 & 0,018547 & 0,00137 & 2,605594 & 0,000117 & $-0,001525$ & 0,016967 & $-0,099783$ & \\
\hline \multirow[t]{4}{*}{312} & 1 & 424 & 0,108596 & 0,00411 & 3,274307 & 0,00035 & $-0,004020$ & 0,099343 & $-0,330954$ & \\
\hline & 364 & 3908 & & & & 2 & $-0,569491$ & & $-2,671610$ & \\
\hline & & & & & 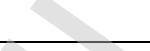 & 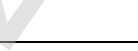 & 0,569491 & & 2,671610 & \\
\hline & & & & & $\Delta$ & & 0,048524 & & 2,443972 & 2,492496 \\
\hline
\end{tabular}




\begin{tabular}{|c|c|c|c|c|c|c|c|c|c|c|}
\hline INCMAR & Cחמק & 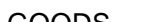 & $f(x / 1)$ & $f(x)(0)$ & (n) & & & & & \\
\hline $0 \backslash 0$ & 36 & 23 & 0,006012 & 0,1 & $-2,81145$ & 0,008521 & -0.058578 & 0.0055 & -0.041282 & \\
\hline $0 \backslash 1$ & 92 & 240 & 0,061525 & 0,253425 & $-1,41563$ & 0,021593 & $-0,119482$ & 0,056282 & $-0,233638$ & \\
\hline $0 \backslash 2$ & 39 & 371 & 0,095037 & 0,108219 & $-0,12989$ & 0,009221 & $-0,062341$ & 0,086939 & $-0,306361$ & \\
\hline $1 \backslash 0$ & 30 & 26 & 0,006779 & 0,083562 & $-2,51172$ & 0,00712 & $-0,050793$ & 0,006202 & $-0,045477$ & \\
\hline $1 \backslash 1$ & 48 & 250 & 0,064083 & 0,132877 & $-0,72924$ & 0,011322 & $-0,073193$ & 0,058623 & $-0,239907$ & \\
\hline 112 & 11 & 436 & 0,111665 & 0,031507 & 1,265302 & 0,002685 & $-0,022929$ & 0,102151 & $-0,336202$ & \\
\hline 210 & 48 & 100 & 0,02571 & 0,132877 & $-1,64255$ & 0,011322 & $-0,073193$ & 0,023519 & $-0,127240$ & \\
\hline $2 \backslash 1$ & 35 & 417 & 0,106805 & 0,09726 & 0,093612 & 0,008287 & $-0,057305$ & 0,097704 & $-0,327841$ & \\
\hline 212 & 19 & 1517 & 0,388207 & 0,053425 & 1,983266 & 0,004552 & $-0,035412$ & 0,355129 & $-0,530415$ & \\
\hline 310 & 5 & 35 & 0,009082 & 0,015068 & $-0,50635$ & 0,001284 & $-0,012332$ & 0,008308 & $-0,057418$ & \\
\hline $3 \backslash 1$ & 0 & 76 & 0,01957 & 0,00137 & 2,659298 & 0,000117 & $-0,001525$ & 0,017903 & $-0,103902$ & \\
\hline 312 & 1 & 417 & 0,106805 & 0,00411 & 3,25768 & 0,00035 & $-0,004020$ & 0,097704 & $-0,327841$ & \\
\hline & 364 & 3908 & & & & 2 & $-0,571103$ & & $-2,677522$ & \\
\hline & & & & & 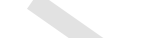 & 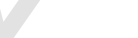 & 0,571103 & & 2,677522 & \\
\hline & & & & & 1 & & 0,048661 & & 2,449381 & 2,498043 \\
\hline
\end{tabular}




\begin{tabular}{|c|c|c|c|c|c|c|c|c|c|c|}
\hline INCMAR & BADS & GOODS & $f(x / 1)$ & $f(x / 0)$ & $w(x)$ & & & & & \\
\hline 010 & 39 & 21 & 0,0055 & 0,108219 & $-2,97939$ & 0,009221 & $-0,062341$ & 0,005031 & $-0,038414$ & \\
\hline $0 \backslash 1$ & 89 & 234 & 0,05999 & 0,245205 & $-1,40792$ & 0,020893 & $-0,116600$ & 0,054878 & $-0,229809$ & \\
\hline 012 & 38 & 381 & 0,097595 & 0,105479 & $-0,07769$ & 0,008987 & $-0,061096$ & 0,08928 & $-0,311186$ & \\
\hline 110 & 33 & 30 & 0,007803 & 0,091781 & $-2,46496$ & 0,00782 & $-0,054731$ & 0,007138 & $-0,050894$ & \\
\hline $1 \backslash 1$ & 49 & 228 & 0,058455 & 0,135616 & $-0,84158$ & 0,011555 & $-0,074362$ & 0,053474 & $-0,225929$ & \\
\hline 112 & 9 & 451 & 0,115503 & 0,026027 & 1,490144 & 0,002218 & $-0,019553$ & 0,105661 & $-0,342605$ & \\
\hline 210 & 49 & 89 & 0,022896 & 0,135616 & $-1,77887$ & 0,011555 & $-0,074362$ & 0,020945 & $-0,116816$ & \\
\hline $2 \backslash 1$ & 34 & 421 & 0,107828 & 0,094521 & 0,131721 & 0,008054 & $-0,056023$ & 0,09864 & $-0,329625$ & \\
\hline 212 & 22 & 1521 & 0,38923 & 0,061644 & 1,842797 & 0,005252 & $-0,039776$ & 0,356065 & $-0,530461$ & \\
\hline 310 & 2 & 32 & 0,008314 & 0,006849 & 0,19381 & 0,000584 & $-0,006269$ & 0,007606 & $-0,053534$ & \\
\hline $3 \backslash 1$ & 0 & 78 & 0,020082 & 0,00137 & 2,685106 & 0,000117 & $-0,001525$ & 0,018371 & $-0,105934$ & \\
\hline 312 & 0 & 422 & 0,108084 & 0,00137 & 4,368197 & 0,000117 & $-0,001525$ & 0,098875 & $-0,330069$ & \\
\hline & 364 & 3908 & & & & 2 & $-0,568163$ & & $-2,665276$ & \\
\hline & & & & & & 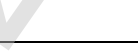 & 0,568163 & & 2,665276 & \\
\hline & & & & & $\Delta-\sqrt{x}$ & & 0,048411 & & 2,438179 & 2,486589 \\
\hline
\end{tabular}




\begin{tabular}{|c|c|c|c|c|c|c|c|c|c|c|}
\hline INCITRM & BADS & Gחת & $f(x / 1)$ & $f(x / 0)$ & $w(x)$ & & & & & \\
\hline $1 \backslash 1$ & & & & & & & & & & \\
\hline & & r & 0,010000 & $0,004 \angle 4 T$ & $-0,041<0$ & $0,00<910$ & $-0,0<40 / 2$ & 0,010455 & $-0,05105 i$ & \\
\hline $2 \backslash 1$ & 18 & 16 & 0,004221 & 0,050685 & $-2,48555$ & 0,004319 & $-0,033924$ & 0,003861 & $-0,030955$ & \\
\hline $3 \backslash 1$ & 24 & 259 & 0,066385 & 0,067123 & $-0,01106$ & 0,005719 & $-0,042609$ & 0,060729 & $-0,245434$ & \\
\hline $4 \backslash 1$ & 1 & 58 & 0,014965 & 0,00411 & 1,292422 & 0,00035 & $-0,004020$ & 0,01369 & $-0,084753$ & \\
\hline 112 & 7 & 172 & 0,044129 & 0,020548 & 0,764355 & 0,001751 & $-0,016033$ & 0,040369 & $-0,186933$ & \\
\hline 212 & 21 & 254 & 0,065106 & 0,058904 & 0,100108 & 0,005019 & $-0,038337$ & 0,059559 & $-0,242377$ & \\
\hline 312 & 9 & 406 & 0,103991 & 0,026027 & 1,385153 & 0,002218 & $-0,019553$ & 0,09513 & $-0,322867$ & \\
\hline 412 & 0 & 141 & 0,036199 & 0,00137 & 3,274307 & 0,000117 & $-0,001525$ & 0,033114 & $-0,162803$ & \\
\hline 113 & 67 & 473 & 0,121131 & 0,184932 & $-0,42312$ & 0,015757 & $-0,094352$ & 0,11081 & $-0,351693$ & \\
\hline 213 & 118 & 374 & 0,095805 & 0,324658 & $-1,22046$ & 0,027663 & $-0,143180$ & 0,087641 & $-0,307818$ & \\
\hline 313 & 82 & 1355 & 0,346764 & 0,226027 & 0,427988 & 0,019259 & $-0,109744$ & 0,317218 & $-0,525457$ & \\
\hline \multirow[t]{4}{*}{413} & 5 & 330 & 0,084548 & 0,015068 & 1,724719 & 0,001284 & $-0,012332$ & 0,077344 & $-0,285599$ & \\
\hline & 364 & 3908 & & & $\Delta$ & 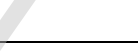 & $-0,540180$ & & $-2,844385$ & \\
\hline & & & & & & & 0,540180 & & 2,844385 & \\
\hline & & & & . & 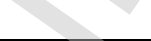 & & 0,046027 & & 2,602026 & 2,648053 \\
\hline
\end{tabular}




\begin{tabular}{|c|c|c|c|c|c|c|c|c|c|c|}
\hline INCTRM & בחתמי & sחסc & & & m(x) & & & & & \\
\hline $0 \backslash 0$ & 18 & 260 & 0066641 & 0050685 & 0273693 & 0004319 & -0033924 & 0 060963 & 0246042 & \\
\hline $0 \backslash 1$ & 124 & 365 & 0.093502 & 0.341096 & -1.29418 & 0.029063 & -0.148359 & 0.085535 & -0.303422 & \\
\hline $0 \backslash 2$ & 19 & 14 & 0,003709 & 0,053425 & $-2,66741$ & 0,004552 & $-0,035412$ & 0,003393 & $-0,027836$ & \\
\hline $1 \backslash 0$ & 5 & 162 & 0,041571 & 0,015068 & 1,01479 & 0,001284 & $-0,012332$ & 0,038029 & $-0,179372$ & \\
\hline $1 \backslash 1$ & 76 & 480 & 0,122921 & 0,209589 & $-0,5336$ & 0,017858 & $-0,103708$ & 0,112448 & $-0,354511$ & \\
\hline 112 & 11 & 68 & 0,017524 & 0,031507 & $-0,58665$ & 0,002685 & $-0,022929$ & 0,016031 & $-0,095591$ & \\
\hline $2 \backslash 0$ & 8 & 400 & 0,102456 & 0,023288 & 1,481508 & 0,001984 & $-0,017813$ & 0,093726 & $-0,320112$ & \\
\hline $2 \backslash 1$ & 82 & 1377 & 0,352392 & 0,226027 & 0,444088 & 0,019259 & $-0,109744$ & 0,322366 & $-0,526497$ & \\
\hline 212 & 17 & 252 & 0,064595 & 0,047945 & 0,298071 & 0,004085 & $-0,032418$ & 0,059091 & $-0,241145$ & \\
\hline 310 & 0 & 139 & 0,035687 & 0,00137 & 3,260072 & 0,000117 & $-0,001525$ & 0,032646 & $-0,161172$ & \\
\hline $3 \backslash 1$ & 4 & 341 & 0,087362 & 0,012329 & 1,958131 & 0,00105 & $-0,010394$ & 0,079919 & $-0,291329$ & \\
\hline 312 & 0 & 50 & 0,012919 & 0,00137 & 2,243981 & 0,000117 & $-0,001525$ & 0,011818 & $-0,075670$ & \\
\hline & 364 & 3908 & & & & 2 & $-0,530082$ & & $-2,822700$ & \\
\hline & & & & & & & 0,530082 & & 2,822700 & \\
\hline & & & & & & & 0,045166 & & 2,582189 & 2,627355 \\
\hline
\end{tabular}




\begin{tabular}{|c|c|c|c|c|c|c|c|c|c|c|}
\hline \multicolumn{11}{|c|}{ 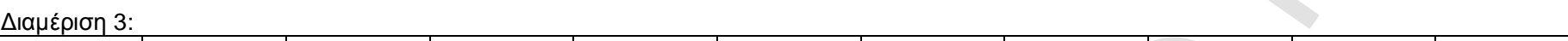 } \\
\hline INCTRM & BADS & GOODS & $f(x / 1)$ & $f(x / 0)$ & $w(x)$ & & & 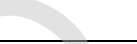 & & \\
\hline $0 \backslash 0$ & 18 & 264 & 0,067664 & 0,050685 & 0,288931 & 0,004319 & $-0,033924$ & 0,061899 & $-0,248459$ & \\
\hline $0 \backslash 1$ & 132 & 357 & 0,091456 & 0,363014 & $-1,37859$ & 0,030931 & $-0,155113$ & 0,083663 & $-0,299452$ & \\
\hline 012 & 16 & 15 & 0,003965 & 0,045205 & $-2,43366$ & 0,003852 & $-0,030892$ & 0,003627 & $-0,029406$ & \\
\hline 110 & 5 & 152 & 0,039013 & 0,015068 & 0,951277 & 0,001284 & $-0,012332$ & 0,035688 & $-0,171604$ & \\
\hline $1 \backslash 1$ & 72 & 493 & 0,126247 & 0,19863 & $-0,4532$ & 0,016924 & $-0,099596$ & 0,11549 & $-0,359655$ & \\
\hline 112 & 13 & 63 & 0,016245 & 0,036986 & $-0,82279$ & 0,003151 & $-0,026188$ & 0,01486 & $-0,090238$ & \\
\hline 210 & 5 & 378 & 0,096828 & 0,015068 & 1,860328 & 0,001284 & $-0,012332$ & 0,088578 & $-0,309748$ & \\
\hline $2 \backslash 1$ & 75 & 1411 & 0,36109 & 0,206849 & 0,557136 & 0,017625 & $-0,102686$ & 0,330323 & $-0,527873$ & \\
\hline 212 & 22 & 247 & 0,063315 & 0,061644 & 0,026756 & 0,005252 & $-0,039776$ & 0,057921 & $-0,238041$ & \\
\hline 310 & 0 & 134 & 0,034408 & 0,00137 & 3,223572 & 0,000117 & $-0,001525$ & 0,031476 & $-0,157053$ & \\
\hline $3 \backslash 1$ & 5 & 343 & 0,087874 & 0,015068 & 1,7633 & 0,001284 & $-0,012332$ & 0,080387 & $-0,292358$ & \\
\hline \multirow[t]{4}{*}{312} & 1 & 51 & 0,013175 & 0,00411 & 1,164977 & 0,00035 & $-0,004020$ & 0,012052 & $-0,076827$ & \\
\hline & 364 & 3908 & & & & 2 & $-0,530717$ & & $-2,800715$ & \\
\hline & & & & & & 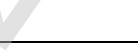 & 0,530717 & & 2,800715 & \\
\hline & & & & & & & 0,04522 & & 2,562077 & 2,607297 \\
\hline
\end{tabular}




\begin{tabular}{|c|c|c|c|c|c|c|c|c|c|c|}
\hline 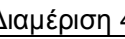 & & & & & & & & & & \\
\hline INCTRM & BADS & GOODS & $f(x / 1)$ & $f(x / 0)$ & $w(x)$ & & & 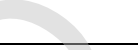 & & \\
\hline $0 \backslash 0$ & 18 & 250 & 0,064083 & 0,050685 & 0,234549 & 0,004319 & $-0,033924$ & 0,058623 & $-0,239907$ & \\
\hline $0 \backslash 1$ & 132 & 371 & 0,095037 & 0,363014 & $-1,34017$ & 0,030931 & $-0,155113$ & 0,086939 & $-0,306361$ & \\
\hline 012 & 17 & 13 & 0,003454 & 0,047945 & $-2,63065$ & 0,004085 & $-0,032418$ & 0,003159 & $-0,026242$ & \\
\hline $1 \backslash 0$ & 8 & 158 & 0,040547 & 0,023288 & 0,554549 & 0,001984 & $-0,017813$ & 0,037093 & $-0,176291$ & \\
\hline $1 \backslash 1$ & 71 & 498 & 0,127526 & 0,19589 & $-0,42923$ & 0,016691 & $-0,098557$ & 0,11666 & $-0,361602$ & \\
\hline 112 & 10 & 56 & 0,014454 & 0,028767 & $-0,68827$ & 0,002451 & $-0,021257$ & 0,013222 & $-0,082519$ & \\
\hline 210 & 5 & 362 & 0,092735 & 0,015068 & 1,817137 & 0,001284 & $-0,012332$ & 0,084833 & $-0,301941$ & \\
\hline $2 \backslash 1$ & 76 & 1428 & 0,365439 & 0,209589 & 0,55595 & 0,017858 & $-0,103708$ & 0,334301 & $-0,528456$ & \\
\hline 212 & 21 & 244 & 0,062548 & 0,058904 & 0,060023 & 0,005019 & $-0,038337$ & 0,057219 & $-0,236162$ & \\
\hline 310 & 0 & 132 & 0,033896 & 0,00137 & 3,20859 & 0,000117 & $-0,001525$ & 0,031008 & $-0,155388$ & \\
\hline $3 \backslash 1$ & 5 & 345 & 0,088386 & 0,015068 & 1,769105 & 0,001284 & $-0,012332$ & 0,080855 & $-0,293383$ & \\
\hline 312 & 1 & 51 & 0,013175 & 0,00411 & 1,164977 & 0,00035 & $-0,004020$ & 0,012052 & $-0,076827$ & \\
\hline & 364 & 3908 & & & & 2 & $-0,531335$ & & $-2,785079$ & \\
\hline & & & & & & 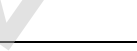 & 0,531335 & & 2,785079 & \\
\hline & & & & & 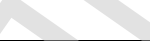 & & 0,045273 & & 2,547773 & 2,593046 \\
\hline
\end{tabular}




\begin{tabular}{|c|c|c|c|c|c|c|c|c|c|c|}
\hline \multicolumn{11}{|c|}{$\Delta ı \alpha \mu \varepsilon ́ p ı \sigma \eta ~ 5:$} \\
\hline INCTRM & BADS & GOODS & $f(x / 1)$ & $f(x / 0)$ & $w(x)$ & & & - & & \\
\hline $0 \backslash 0$ & 19 & 235 & 0,060246 & 0,053425 & 0,120157 & 0,004552 & $-0,035412$ & 0,055112 & $-0,230451$ & \\
\hline $0 \backslash 1$ & 125 & 387 & 0,09913 & 0,343836 & $-1,24373$ & 0,029297 & $-0,149212$ & 0,090684 & $-0,314039$ & \\
\hline 012 & 22 & 14 & 0,003709 & 0,061644 & $-2,81051$ & 0,005252 & $-0,039776$ & 0,003393 & $-0,027836$ & \\
\hline 110 & 6 & 154 & 0,039524 & 0,017808 & 0,797252 & 0,001517 & $-0,014209$ & 0,036156 & $-0,173175$ & \\
\hline $1 \backslash 1$ & 71 & 485 & 0,124201 & 0,19589 & $-0,45566$ & 0,016691 & $-0,098557$ & 0,113618 & $-0,356503$ & \\
\hline 112 & 14 & 70 & 0,018035 & 0,039726 & $-0,78968$ & 0,003385 & $-0,027779$ & 0,016499 & $-0,097697$ & \\
\hline 210 & 5 & 359 & 0,091967 & 0,015068 & 1,808827 & 0,001284 & $-0,012332$ & 0,084131 & $-0,300450$ & \\
\hline $2 \backslash 1$ & 80 & 1431 & 0,366206 & 0,220548 & 0,507081 & 0,018792 & $-0,107748$ & 0,335003 & $-0,528552$ & \\
\hline 212 & 20 & 241 & 0,061781 & 0,056164 & 0,095305 & 0,004786 & $-0,036883$ & 0,056516 & $-0,234271$ & \\
\hline 310 & 0 & 117 & 0,030059 & 0,00137 & 3,088446 & 0,000117 & $-0,001525$ & 0,027498 & $-0,142563$ & \\
\hline $3 \backslash 1$ & 1 & 363 & 0,092991 & 0,00411 & 3,119175 & 0,00035 & $-0,004020$ & 0,085067 & $-0,302435$ & \\
\hline \multirow[t]{4}{*}{312} & 1 & 52 & 0,013431 & 0,00411 & 1,184209 & 0,00035 & $-0,004020$ & 0,012286 & $-0,077978$ & \\
\hline & 364 & 3908 & & & & 2 & $-0,531472$ & & $-2,785951$ & \\
\hline & & & & & + & 8 & 0,531472 & & 2,785951 & \\
\hline & & & & & 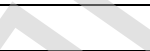 & & 0,045285 & & 2,548571 & 2,593856 \\
\hline
\end{tabular}




\begin{tabular}{|c|c|c|c|c|c|c|c|c|c|c|}
\hline IOR IYR & סחים & & & & & & & & & \\
\hline & & & & & & & & & & \\
\hline $1 \backslash 1$ & 11 & 121 & 0,031082 & 0,031507 & $-0,01357$ & 0,002685 & $-0,022929$ & 0,028434 & $-0,146043$ & \\
\hline $2 \backslash 1$ & 17 & 64 & 0,0165 & 0,047945 & $-1,06668$ & 0,004085 & $-0,032418$ & 0,015094 & $-0,091319$ & \\
\hline $3 \backslash 1$ & 9 & 154 & 0,039524 & 0,026027 & 0,417763 & 0,002218 & $-0,019553$ & 0,036156 & $-0,173175$ & \\
\hline $4 \backslash 1$ & 2 & 32 & 0,008314 & 0,006849 & 0,19381 & 0,000584 & $-0,006269$ & 0,007606 & $-0,053534$ & \\
\hline $5 \backslash 1$ & 12 & 233 & 0,059734 & 0,034247 & 0,556314 & 0,002918 & $-0,024572$ & 0,054644 & $-0,229166$ & \\
\hline $6 \backslash 1$ & 5 & 47 & 0,012151 & 0,015068 & $-0,21516$ & 0,001284 & $-0,012332$ & 0,011116 & $-0,072157$ & \\
\hline 112 & 26 & 152 & 0,039013 & 0,072603 & $-0,62112$ & 0,006186 & $-0,045386$ & 0,035688 & $-0,171604$ & \\
\hline 212 & 36 & 148 & 0,037989 & 0,1 & $-0,96787$ & 0,008521 & $-0,058578$ & 0,034752 & $-0,168436$ & \\
\hline 312 & 17 & 163 & 0,041827 & 0,047945 & $-0,13653$ & 0,004085 & $-0,032418$ & 0,038263 & $-0,180138$ & \\
\hline 412 & 9 & 86 & 0,022128 & 0,026027 & $-0,16229$ & 0,002218 & $-0,019553$ & 0,020243 & $-0,113896$ & \\
\hline $5 \backslash 2$ & 33 & 240 & 0,061525 & 0,091781 & $-0,39996$ & 0,00782 & $-0,054731$ & 0,056282 & $-0,233638$ & \\
\hline 612 & 0 & 13 & 0,003454 & 0,00137 & 0,924697 & 0,000117 & $-0,001525$ & 0,003159 & $-0,026242$ & \\
\hline 113 & 14 & 173 & 0,044385 & 0,039726 & 0,110889 & 0,003385 & $-0,027779$ & 0,040603 & $-0,187678$ & \\
\hline 213 & 39 & 126 & 0,032361 & 0,108219 & $-1,2072$ & 0,009221 & $-0,062341$ & 0,029604 & $-0,150330$ & \\
\hline 313 & 25 & 248 & 0,063571 & 0,069863 & $-0,09438$ & 0,005953 & $-0,044004$ & 0,058155 & $-0,238664$ & \\
\hline 413 & 7 & 86 & 0,022128 & 0,020548 & 0,074102 & 0,001751 & $-0,016033$ & 0,020243 & $-0,113896$ & \\
\hline 513 & 28 & 316 & 0,080967 & 0,078082 & 0,03628 & 0,006653 & $-0,048113$ & 0,074068 & $-0,278126$ & \\
\hline 613 & 3 & 59 & 0,015221 & 0,009589 & 0,462074 & 0,000817 & $-0,008381$ & 0,013924 & $-0,085861$ & \\
\hline $1 \backslash 4$ & 7 & 228 & 0,058455 & 0,020548 & 1,045494 & 0,001751 & $-0,016033$ & 0,053474 & $-0,225929$ & \\
\hline $2 \backslash 4$ & 13 & 110 & 0,028268 & 0,036986 & $-0,26881$ & 0,003151 & $-0,026188$ & 0,025859 & $-0,136361$ & \\
\hline $3 \backslash 4$ & 19 & 339 & 0,086851 & 0,053425 & 0,48592 & 0,004552 & $-0,035412$ & 0,079451 & $-0,290297$ & \\
\hline $4 \backslash 4$ & 0 & 41 & 0,010617 & 0,00137 & 2,047701 & 0,000117 & $-0,001525$ & 0,009712 & $-0,064934$ & \\
\hline $5 \backslash 4$ & 25 & 629 & 0,161039 & 0,069863 & 0,835108 & 0,005953 & $-0,044004$ & 0,147317 & $-0,407038$ & \\
\hline $6 \backslash 4$ & 7 & 100 & 0,02571 & 0,020548 & 0,224115 & 0,001751 & $-0,016033$ & 0,023519 & $-0,127240$ & \\
\hline & 364 & 3908 & & 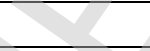 & & & $-0,676111$ & & $-3,965701$ & \\
\hline & & & & & & & 0,676111 & & 3,965701 & \\
\hline & & & & -2 & & & 0,057609 & & 3,627799 & 3,685408 \\
\hline
\end{tabular}




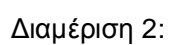

\begin{tabular}{|c|c|c|c|c|c|c|c|c|c|c|}
\hline JOBJYR & BADS & GOODS & $f(x / 1)$ & $f(x / 0)$ & $w(x)$ & & & & & 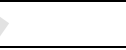 \\
\hline 010 & 30 & 147 & 0,037733 & 0,083562 & $-0,79504$ & 0,00712 & $-0,050793$ & 0,034518 & $-0,167638$ & \\
\hline 011 & 37 & 147 & 0,037733 & 0,10274 & $-1,00165$ & 0,008754 & $-0,059841$ & 0,034518 & $-0,167638$ & \\
\hline 012 & 15 & 63 & 0,016245 & 0,042466 & $-0,96094$ & 0,003618 & $-0,029346$ & 0,01486 & $-0,090238$ & \\
\hline 013 & 18 & 111 & 0,028524 & 0,050685 & $-0,57489$ & 0,004319 & $-0,033924$ & 0,026094 & $-0,137256$ & \\
\hline 210 & 27 & 158 & 0,040547 & 0,075342 & $-0,61957$ & 0,00642 & $-0,046756$ & 0,037093 & $-0,176291$ & \\
\hline $2 \backslash 1$ & 19 & 214 & 0,054873 & 0,053425 & 0,026756 & 0,004552 & $-0,035412$ & 0,050198 & $-0,216665$ & \\
\hline 212 & 10 & 104 & 0,026733 & 0,028767 & $-0,07333$ & 0,002451 & $-0,021257$ & 0,024455 & $-0,130927$ & \\
\hline 213 & 7 & 201 & 0,051548 & 0,020548 & 0,919747 & 0,001751 & $-0,016033$ & 0,047156 & $-0,207788$ & \\
\hline 310 & 17 & 142 & 0,036454 & 0,047945 & $-0,274$ & 0,004085 & $-0,032418$ & 0,033348 & $-0,163615$ & \\
\hline $3 \backslash 1$ & 23 & 261 & 0,066897 & 0,064384 & 0,038294 & 0,005486 & $-0,041199$ & 0,061197 & $-0,246648$ & \\
\hline 312 & 9 & 138 & 0,035431 & 0,026027 & 0,308439 & 0,002218 & $-0,019553$ & 0,032412 & $-0,160353$ & \\
\hline 313 & 20 & 329 & 0,084293 & 0,056164 & 0,406012 & 0,004786 & $-0,036883$ & 0,07711 & $-0,285072$ & \\
\hline 410 & 10 & 90 & 0,023152 & 0,028767 & $-0,21716$ & 0,002451 & $-0,021257$ & 0,021179 & $-0,117781$ & \\
\hline $4 \backslash 1$ & 6 & 94 & 0,024175 & 0,017808 & 0,305658 & 0,001517 & $-0,014209$ & 0,022115 & $-0,121607$ & \\
\hline 412 & 1 & 25 & 0,006523 & 0,00411 & 0,462074 & 0,00035 & $-0,004020$ & 0,005968 & $-0,044092$ & \\
\hline 413 & 0 & 45 & 0,01164 & 0,00137 & 2,13972 & 0,000117 & $-0,001525$ & 0,010648 & $-0,069779$ & \\
\hline 510 & 34 & 248 & 0,063571 & 0,094521 & $-0,39666$ & 0,008054 & $-0,056023$ & 0,058155 & $-0,238664$ & \\
\hline $5 \backslash 1$ & 30 & 355 & 0,090944 & 0,083562 & 0,084659 & 0,00712 & $-0,050793$ & 0,083195 & $-0,298450$ & \\
\hline 512 & 9 & 193 & 0,049501 & 0,026027 & 0,642846 & 0,002218 & $-0,019553$ & 0,045283 & $-0,202185$ & \\
\hline 513 & 24 & 630 & 0,161294 & 0,067123 & 0,876701 & 0,005719 & $-0,042609$ & 0,147551 & $-0,407346$ & \\
\hline 610 & 0 & 13 & 0,003454 & 0,00137 & 0,924697 & 0,000117 & $-0,001525$ & 0,003159 & $-0,026242$ & \\
\hline $6 \backslash 1$ & 8 & 63 & 0,016245 & 0,023288 & $-0,36017$ & 0,001984 & $-0,017813$ & 0,01486 & $-0,090238$ & \\
\hline 612 & 2 & 35 & 0,009082 & 0,006849 & 0,282102 & 0,000584 & $-0,006269$ & 0,008308 & $-0,057418$ & \\
\hline \multirow[t]{4}{*}{613} & 8 & 102 & 0,026222 & 0,023288 & 0,118657 & 0,001984 & $-0,017813$ & 0,023987 & $-0,129090$ & \\
\hline & 364 & 3908 & & 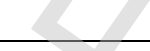 & 2 & & $-0,676824$ & & $-3,953022$ & \\
\hline & & & & & & & 0,676824 & & 3,953022 & \\
\hline & & & & 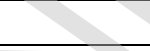 & & & 0,057669 & & 3,6162 & 3,67387 \\
\hline
\end{tabular}




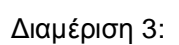

\begin{tabular}{|c|c|c|c|c|c|c|c|c|c|c|}
\hline JOBJYR & BADS & GOODS & $f(x / 1)$ & $f(x / 0)$ & $w(x)$ & & & & & 8 \\
\hline 010 & 36 & 140 & 0,035943 & 0,1 & $-1,02324$ & 0,008521 & $-0,058578$ & 0,03288 & $-0,161989$ & \\
\hline $0 \backslash 1$ & 27 & 129 & 0,033129 & 0,075342 & $-0,82164$ & 0,00642 & $-0,046756$ & 0,030306 & $-0,152871$ & \\
\hline 012 & 16 & 59 & 0,015221 & 0,045205 & $-1,08852$ & 0,003852 & $-0,030892$ & 0,013924 & $-0,085861$ & \\
\hline 013 & 21 & 119 & 0,03057 & 0,058904 & $-0,65588$ & 0,005019 & $-0,038337$ & 0,027966 & $-0,144309$ & \\
\hline 210 & 29 & 145 & 0,037222 & 0,080822 & $-0,77535$ & 0,006887 & $-0,049459$ & 0,03405 & $-0,166036$ & \\
\hline $2 \backslash 1$ & 19 & 203 & 0,052059 & 0,053425 & $-0,02589$ & 0,004552 & $-0,035412$ & 0,047624 & $-0,209171$ & \\
\hline 212 & 9 & 100 & 0,02571 & 0,026027 & $-0,01227$ & 0,002218 & $-0,019553$ & 0,023519 & $-0,127240$ & \\
\hline 213 & 10 & 206 & 0,052827 & 0,028767 & 0,607786 & 0,002451 & $-0,021257$ & 0,048326 & $-0,211235$ & \\
\hline 310 & 25 & 181 & 0,046431 & 0,069863 & $-0,40856$ & 0,005953 & $-0,044004$ & 0,042475 & $-0,193569$ & \\
\hline $3 \backslash 1$ & 23 & 283 & 0,072525 & 0,064384 & 0,119072 & 0,005486 & $-0,041199$ & 0,066345 & $-0,259667$ & \\
\hline 312 & 11 & 163 & 0,041827 & 0,031507 & 0,283326 & 0,002685 & $-0,022929$ & 0,038263 & $-0,180138$ & \\
\hline 313 & 23 & 352 & 0,090177 & 0,064384 & 0,336911 & 0,005486 & $-0,041199$ & 0,082493 & $-0,296940$ & \\
\hline 410 & 11 & 93 & 0,023919 & 0,031507 & $-0,27553$ & 0,002685 & $-0,022929$ & 0,021881 & $-0,120656$ & \\
\hline $4 \backslash 1$ & 8 & 93 & 0,023919 & 0,023288 & 0,026756 & 0,001984 & $-0,017813$ & 0,021881 & $-0,120656$ & \\
\hline 412 & 1 & 26 & 0,006779 & 0,00411 & 0,50054 & 0,00035 & $-0,004020$ & 0,006202 & $-0,045477$ & \\
\hline 413 & 0 & 44 & 0,011384 & 0,00137 & 2,117497 & 0,000117 & $-0,001525$ & 0,010414 & $-0,068580$ & \\
\hline 510 & 27 & 240 & 0,061525 & 0,075342 & $-0,20261$ & 0,00642 & $-0,046756$ & 0,056282 & $-0,233638$ & \\
\hline $5 \backslash 1$ & 26 & 341 & 0,087362 & 0,072603 & 0,185063 & 0,006186 & $-0,045386$ & 0,079919 & $-0,291329$ & \\
\hline 512 & 7 & 164 & 0,042082 & 0,020548 & 0,716868 & 0,001751 & $-0,016033$ & 0,038497 & $-0,180901$ & \\
\hline 513 & 20 & 606 & 0,155155 & 0,056164 & 1,01614 & 0,004786 & $-0,036883$ & 0,141935 & $-0,399788$ & \\
\hline 610 & 0 & 15 & 0,003965 & 0,00137 & 1,062848 & 0,000117 & $-0,001525$ & 0,003627 & $-0,029406$ & \\
\hline $6 \backslash 1$ & 5 & 65 & 0,016756 & 0,015068 & 0,106163 & 0,001284 & $-0,012332$ & 0,015328 & $-0,092395$ & \\
\hline 612 & 3 & 38 & 0,009849 & 0,009589 & 0,026756 & 0,000817 & $-0,008381$ & 0,00901 & $-0,061216$ & \\
\hline \multirow[t]{4}{*}{613} & 7 & 103 & 0,026477 & 0,020548 & 0,253529 & 0,001751 & $-0,016033$ & 0,024221 & $-0,130010$ & \\
\hline & 364 & 3908 & & & 2 & & $-0,679192$ & & $-3,963075$ & \\
\hline & & & & & & & 0,679192 & & 3,963075 & \\
\hline & & & & 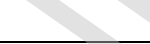 & & & 0,057871 & & 3,625397 & 3,683268 \\
\hline
\end{tabular}




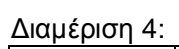

\begin{tabular}{|c|c|c|c|c|c|c|c|c|c|c|}
\hline JOBJYR & BADS & GOODS & $f(x / 1)$ & $f(x / 0)$ & $w(x)$ & & & & & 8 \\
\hline 010 & 20 & 117 & 0,030059 & 0,056164 & $-0,62513$ & 0,004786 & $-0,036883$ & 0,027498 & $-0,142563$ & \\
\hline $0 \backslash 1$ & 44 & 164 & 0,042082 & 0,121918 & $-1,06372$ & 0,010388 & $-0,068447$ & 0,038497 & $-0,180901$ & \\
\hline 012 & 15 & 71 & 0,018291 & 0,042466 & $-0,84228$ & 0,003618 & $-0,029346$ & 0,016733 & $-0,098742$ & \\
\hline 013 & 18 & 113 & 0,029036 & 0,050685 & $-0,55711$ & 0,004319 & $-0,033924$ & 0,026562 & $-0,139037$ & \\
\hline 210 & 20 & 100 & 0,02571 & 0,056164 & $-0,78141$ & 0,004786 & $-0,036883$ & 0,023519 & $-0,127240$ & \\
\hline $2 \backslash 1$ & 20 & 276 & 0,070734 & 0,056164 & 0,230646 & 0,004786 & $-0,036883$ & 0,064707 & $-0,255589$ & \\
\hline 212 & 13 & 151 & 0,038757 & 0,036986 & 0,046756 & 0,003151 & $-0,026188$ & 0,035454 & $-0,170815$ & \\
\hline 213 & 11 & 237 & 0,060757 & 0,031507 & 0,656681 & 0,002685 & $-0,022929$ & 0,05558 & $-0,231730$ & \\
\hline 310 & 14 & 82 & 0,021105 & 0,039726 & $-0,63249$ & 0,003385 & $-0,027779$ & 0,019307 & $-0,109948$ & \\
\hline $3 \backslash 1$ & 32 & 335 & 0,085828 & 0,089041 & $-0,03676$ & 0,007587 & $-0,053429$ & 0,078515 & $-0,288219$ & \\
\hline 312 & 13 & 153 & 0,039268 & 0,036986 & 0,059871 & 0,003151 & $-0,026188$ & 0,035922 & $-0,172391$ & \\
\hline 313 & 24 & 308 & 0,07892 & 0,067123 & 0,161909 & 0,005719 & $-0,042609$ & 0,072196 & $-0,273763$ & \\
\hline 410 & 9 & 56 & 0,014454 & 0,026027 & $-0,58819$ & 0,002218 & $-0,019553$ & 0,013222 & $-0,082519$ & \\
\hline $4 \backslash 1$ & 9 & 130 & 0,033384 & 0,026027 & 0,248942 & 0,002218 & $-0,019553$ & 0,03054 & $-0,153712$ & \\
\hline 412 & 0 & 31 & 0,008058 & 0,00137 & 1,771995 & 0,000117 & $-0,001525$ & 0,007372 & $-0,052220$ & \\
\hline 413 & 0 & 36 & 0,009337 & 0,00137 & 1,91932 & 0,000117 & $-0,001525$ & 0,008542 & $-0,058693$ & \\
\hline 510 & 19 & 150 & 0,038501 & 0,053425 & $-0,32759$ & 0,004552 & $-0,035412$ & 0,03522 & $-0,170025$ & \\
\hline $5 \backslash 1$ & 30 & 383 & 0,098107 & 0,083562 & 0,160473 & 0,00712 & $-0,050793$ & 0,089748 & $-0,312141$ & \\
\hline 512 & 12 & 195 & 0,050013 & 0,034247 & 0,378692 & 0,002918 & $-0,024572$ & 0,045751 & $-0,203596$ & \\
\hline 513 & 23 & 599 & 0,153364 & 0,064384 & 0,867956 & 0,005486 & $-0,041199$ & 0,140296 & $-0,397523$ & \\
\hline 610 & 0 & 6 & 0,001663 & 0,00137 & 0,19381 & 0,000117 & $-0,001525$ & 0,001521 & $-0,014239$ & \\
\hline $6 \backslash 1$ & 6 & 70 & 0,018035 & 0,017808 & 0,012671 & 0,001517 & $-0,014209$ & 0,016499 & $-0,097697$ & \\
\hline 612 & 6 & 43 & 0,011128 & 0,017808 & $-0,47018$ & 0,001517 & $-0,014209$ & 0,01018 & $-0,067372$ & \\
\hline \multirow[t]{4}{*}{613} & 6 & 102 & 0,026222 & 0,017808 & 0,386921 & 0,001517 & $-0,014209$ & 0,023987 & $-0,129090$ & \\
\hline & 364 & 3908 & & 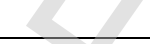 & 2 & & $-0,679769$ & & $-3,929762$ & \\
\hline & & & & & & & 0,679769 & & 3,929762 & \\
\hline & & & & 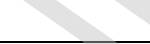 & & & 0,05792 & & 3,594922 & 3,652843 \\
\hline
\end{tabular}




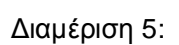

\begin{tabular}{|c|c|c|c|c|c|c|c|c|c|c|}
\hline JOBJYR & BADS & GOODS & $f(x / 1)$ & $f(x / 0)$ & $w(x)$ & & & & 7 & 8 \\
\hline 010 & 34 & 151 & 0,038757 & 0,094521 & $-0,89151$ & 0,008054 & $-0,056023$ & 0,035454 & $-0,170815$ & \\
\hline $0 \backslash 1$ & 33 & 132 & 0,033896 & 0,091781 & $-0,9961$ & 0,00782 & $-0,054731$ & 0,031008 & $-0,155388$ & \\
\hline 012 & 13 & 70 & 0,018035 & 0,036986 & $-0,71822$ & 0,003151 & $-0,026188$ & 0,016499 & $-0,097697$ & \\
\hline 013 & 14 & 111 & 0,028524 & 0,039726 & $-0,33126$ & 0,003385 & $-0,027779$ & 0,026094 & $-0,137256$ & \\
\hline 210 & 30 & 159 & 0,040803 & 0,083562 & $-0,71682$ & 0,00712 & $-0,050793$ & 0,037327 & $-0,177064$ & \\
\hline $2 \backslash 1$ & 15 & 178 & 0,045664 & 0,042466 & 0,072609 & 0,003618 & $-0,029346$ & 0,041773 & $-0,191374$ & \\
\hline 212 & 9 & 126 & 0,032361 & 0,026027 & 0,217811 & 0,002218 & $-0,019553$ & 0,029604 & $-0,150330$ & \\
\hline 213 & 9 & 221 & 0,056664 & 0,026027 & 0,777991 & 0,002218 & $-0,019553$ & 0,051836 & $-0,221335$ & \\
\hline 310 & 22 & 158 & 0,040547 & 0,061644 & $-0,4189$ & 0,005252 & $-0,039776$ & 0,037093 & $-0,176291$ & \\
\hline $3 \backslash 1$ & 24 & 238 & 0,061013 & 0,067123 & $-0,09544$ & 0,005719 & $-0,042609$ & 0,055814 & $-0,232367$ & \\
\hline 312 & 11 & 147 & 0,037733 & 0,031507 & 0,180342 & 0,002685 & $-0,022929$ & 0,034518 & $-0,167638$ & \\
\hline 313 & 26 & 331 & 0,084804 & 0,072603 & 0,155344 & 0,006186 & $-0,045386$ & 0,077578 & $-0,286125$ & \\
\hline 410 & 12 & 80 & 0,020594 & 0,034247 & $-0,50861$ & 0,002918 & $-0,024572$ & 0,018839 & $-0,107949$ & \\
\hline $4 \backslash 1$ & 5 & 87 & 0,022384 & 0,015068 & 0,395751 & 0,001284 & $-0,012332$ & 0,020477 & $-0,114873$ & \\
\hline 412 & 2 & 31 & 0,008058 & 0,006849 & 0,162557 & 0,000584 & $-0,006269$ & 0,007372 & $-0,052220$ & \\
\hline 413 & 0 & 42 & 0,010872 & 0,00137 & 2,071512 & 0,000117 & $-0,001525$ & 0,009946 & $-0,066157$ & \\
\hline 510 & 26 & 237 & 0,060757 & 0,072603 & $-0,17812$ & 0,006186 & $-0,045386$ & 0,05558 & $-0,231730$ & \\
\hline $5 \backslash 1$ & 26 & 323 & 0,082758 & 0,072603 & 0,130915 & 0,006186 & $-0,045386$ & 0,075706 & $-0,281888$ & \\
\hline 512 & 15 & 224 & 0,057432 & 0,042466 & 0,301896 & 0,003618 & $-0,029346$ & 0,052538 & $-0,223313$ & \\
\hline 513 & 20 & 636 & 0,162829 & 0,056164 & 1,06442 & 0,004786 & $-0,036883$ & 0,148955 & $-0,409187$ & \\
\hline 610 & 0 & 17 & 0,004477 & 0,00137 & 1,184209 & 0,000117 & $-0,001525$ & 0,004095 & $-0,032484$ & \\
\hline $6 \backslash 1$ & 5 & 56 & 0,014454 & 0,015068 & $-0,04165$ & 0,001284 & $-0,012332$ & 0,013222 & $-0,082519$ & \\
\hline 612 & 5 & 44 & 0,011384 & 0,015068 & $-0,2804$ & 0,001284 & $-0,012332$ & 0,010414 & $-0,068580$ & \\
\hline \multirow[t]{4}{*}{613} & 8 & 109 & 0,028012 & 0,023288 & 0,184719 & 0,001984 & $-0,017813$ & 0,025625 & $-0,135463$ & \\
\hline & 364 & 3908 & & 2 & 2 & & $-0,680368$ & & $-3,970043$ & \\
\hline & & & & & & & 0,680368 & & 3,970043 & \\
\hline & & & & 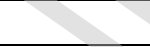 & & & 0,057971 & & 3,631772 & 3,689743 \\
\hline
\end{tabular}




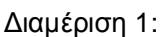

\begin{tabular}{|c|c|c|c|c|c|c|c|c|c|c|}
\hline JOB\MAR & BADS & GOODS & $f(x / 1)$ & $f(x / 0)$ & $w(x)$ & & & & & \\
\hline $1 \backslash 1$ & 40 & 159 & 0,040803 & 0,110959 & $-1,0004$ & 0,009454 & $-0,063579$ & 0,037327 & $-0,177064$ & \\
\hline $2 \backslash 1$ & 4 & 24 & 0,006268 & 0,012329 & $-0,67654$ & 0,00105 & $-0,010394$ & 0,005734 & $-0,042694$ & \\
\hline $3 \backslash 1$ & 39 & 203 & 0,052059 & 0,108219 & $-0,73177$ & 0,009221 & $-0,062341$ & 0,047624 & $-0,209171$ & \\
\hline $4 \backslash 1$ & 13 & 112 & 0,02878 & 0,036986 & $-0,25088$ & 0,003151 & $-0,026188$ & 0,026328 & $-0,138148$ & \\
\hline $5 \backslash 1$ & 60 & 386 & 0,098874 & 0,165753 & $-0,51665$ & 0,014123 & $-0,086798$ & 0,09045 & $-0,313566$ & \\
\hline $6 \backslash 1$ & 10 & 81 & 0,020849 & 0,028767 & $-0,32191$ & 0,002451 & $-0,021257$ & 0,019073 & $-0,108950$ & \\
\hline 112 & 9 & 30 & 0,007803 & 0,026027 & $-1,2047$ & 0,002218 & $-0,019553$ & 0,007138 & $-0,050894$ & \\
\hline 212 & 83 & 19 & 0,004988 & 0,228767 & $-3,82557$ & 0,019492 & $-0,110735$ & 0,004563 & $-0,035484$ & \\
\hline 312 & 16 & 42 & 0,010872 & 0,045205 & $-1,425$ & 0,003852 & $-0,030892$ & 0,009946 & $-0,066157$ & \\
\hline 412 & 2 & 9 & 0,00243 & 0,006849 & $-1,03614$ & 0,000584 & $-0,006269$ & 0,002223 & $-0,019593$ & \\
\hline 512 & 12 & 77 & 0,019826 & 0,034247 & $-0,54659$ & 0,002918 & $-0,024572$ & 0,018137 & $-0,104920$ & \\
\hline 612 & 3 & 5 & 0,001407 & 0,009589 & $-1,91915$ & 0,000817 & $-0,008381$ & 0,001287 & $-0,012358$ & \\
\hline 113 & 9 & 485 & 0,124201 & 0,026027 & 1,562748 & 0,002218 & $-0,019553$ & 0,113618 & $-0,356503$ & \\
\hline 213 & 18 & 405 & 0,103735 & 0,050685 & 0,716211 & 0,004319 & $-0,033924$ & 0,094896 & $-0,322410$ & \\
\hline 313 & 15 & 659 & 0,168713 & 0,042466 & 1,379502 & 0,003618 & $-0,029346$ & 0,154338 & $-0,416070$ & \\
\hline 413 & 3 & 124 & 0,03185 & 0,009589 & 1,200403 & 0,000817 & $-0,008381$ & 0,029136 & $-0,148624$ & \\
\hline 513 & 26 & 955 & 0,244436 & 0,072603 & 1,213951 & 0,006186 & $-0,045386$ & 0,223609 & $-0,483207$ & \\
\hline \multirow[t]{4}{*}{613} & 2 & 133 & 0,034152 & 0,006849 & 1,606671 & 0,000584 & $-0,006269$ & 0,031242 & $-0,156222$ & \\
\hline & 364 & 3908 & & & & & $-0,613819$ & & $-3,162037$ & \\
\hline & & & & . & 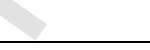 & & 0,613819 & & 3,162037 & \\
\hline & & & & 2 & & & 0,052301 & & 2,892612 & 2,944913 \\
\hline
\end{tabular}




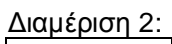

\begin{tabular}{|c|c|c|c|c|c|c|c|c|c|c|}
\hline JOBMAR & BADS & GOODS & $f(x / 1)$ & $f(x / 0)$ & $w(x)$ & & & 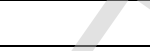 & 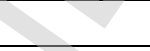 & \\
\hline 010 & 83 & 21 & 0,0055 & 0,228767 & $-3,72793$ & 0,019492 & $-0,110735$ & 0,005031 & $-0,038414$ & \\
\hline $0 \backslash 1$ & 3 & 19 & 0,004988 & 0,009589 & $-0,65349$ & 0,000817 & $-0,008381$ & 0,004563 & $-0,035484$ & \\
\hline 012 & 14 & 428 & 0,109619 & 0,039726 & 1,015003 & 0,003385 & $-0,027779$ & 0,100279 & $-0,332716$ & \\
\hline 210 & 9 & 33 & 0,00857 & 0,026027 & $-1,11089$ & 0,002218 & $-0,019553$ & 0,00784 & $-0,054839$ & \\
\hline $2 \backslash 1$ & 46 & 176 & 0,045152 & 0,127397 & $-1,03727$ & 0,010855 & $-0,070834$ & 0,041305 & $-0,189901$ & \\
\hline 212 & 8 & 468 & 0,119852 & 0,023288 & 1,63833 & 0,001984 & $-0,017813$ & 0,10964 & $-0,349658$ & \\
\hline 310 & 16 & 40 & 0,010361 & 0,045205 & $-1,4732$ & 0,003852 & $-0,030892$ & 0,009478 & $-0,063703$ & \\
\hline $3 \backslash 1$ & 37 & 202 & 0,051804 & 0,10274 & $-0,68474$ & 0,008754 & $-0,059841$ & 0,04739 & $-0,208480$ & \\
\hline 312 & 16 & 628 & 0,160783 & 0,045205 & 1,268836 & 0,003852 & $-0,030892$ & 0,147083 & $-0,406728$ & \\
\hline 410 & 3 & 9 & 0,00243 & 0,009589 & $-1,37261$ & 0,000817 & $-0,008381$ & 0,002223 & $-0,019593$ & \\
\hline $4 \backslash 1$ & 11 & 121 & 0,031082 & 0,031507 & $-0,01357$ & 0,002685 & $-0,022929$ & 0,028434 & $-0,146043$ & \\
\hline 412 & 3 & 124 & 0,03185 & 0,009589 & 1,200403 & 0,000817 & $-0,008381$ & 0,029136 & $-0,148624$ & \\
\hline 510 & 14 & 83 & 0,021361 & 0,039726 & $-0,62044$ & 0,003385 & $-0,027779$ & 0,019541 & $-0,110941$ & \\
\hline $5 \backslash 1$ & 58 & 384 & 0,098363 & 0,160274 & $-0,48822$ & 0,013656 & $-0,084591$ & 0,089982 & $-0,312617$ & \\
\hline 512 & 25 & 959 & 0,245459 & 0,069863 & 1,256594 & 0,005953 & $-0,044004$ & 0,224545 & $-0,483877$ & \\
\hline 610 & 2 & 6 & 0,001663 & 0,006849 & $-1,41563$ & 0,000584 & $-0,006269$ & 0,001521 & $-0,014239$ & \\
\hline $6 \backslash 1$ & 12 & 82 & 0,021105 & 0,034247 & $-0,48407$ & 0,002918 & $-0,024572$ & 0,019307 & $-0,109948$ & \\
\hline \multirow[t]{4}{*}{612} & 4 & 125 & 0,032105 & 0,012329 & 0,957089 & 0,00105 & $-0,010394$ & 0,02937 & $-0,149478$ & \\
\hline & 364 & 3908 & & & & & $-0,614020$ & & $-3,175283$ & \\
\hline & & & & & & & 0,614020 & & 3,175283 & \\
\hline & & & & 2 & 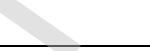 & & 0,052318 & & 2,90473 & 2,957048 \\
\hline
\end{tabular}




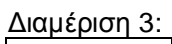

\begin{tabular}{|c|c|c|c|c|c|c|c|c|c|c|}
\hline JOBMAR & BADS & GOODS & $f(x / 1)$ & $f(x / 0)$ & $w(x)$ & & & 8 & 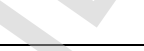 & \\
\hline 010 & 82 & 13 & 0,003454 & 0,226027 & $-4,18125$ & 0,019259 & $-0,109744$ & 0,003159 & $-0,026242$ & \\
\hline $0 \backslash 1$ & 4 & 20 & 0,005244 & 0,012329 & $-0,85479$ & 0,00105 & $-0,010394$ & 0,004797 & $-0,036957$ & \\
\hline 012 & 14 & 414 & 0,106037 & 0,039726 & 0,981785 & 0,003385 & $-0,027779$ & 0,097002 & $-0,326494$ & \\
\hline 210 & 7 & 28 & 0,007291 & 0,020548 & $-1,03614$ & 0,001751 & $-0,016033$ & 0,00667 & $-0,048209$ & \\
\hline $2 \backslash 1$ & 49 & 160 & 0,041059 & 0,135616 & $-1,19482$ & 0,011555 & $-0,074362$ & 0,037561 & $-0,177836$ & \\
\hline 212 & 11 & 466 & 0,11934 & 0,031507 & 1,331771 & 0,002685 & $-0,022929$ & 0,109172 & $-0,348839$ & \\
\hline 310 & 18 & 48 & 0,012407 & 0,050685 & $-1,40735$ & 0,004319 & $-0,033924$ & 0,01135 & $-0,073335$ & \\
\hline $3 \backslash 1$ & 45 & 242 & 0,062036 & 0,124658 & $-0,69785$ & 0,010622 & $-0,069644$ & 0,05675 & $-0,234903$ & \\
\hline 312 & 19 & 689 & 0,176388 & 0,053425 & 1,194413 & 0,004552 & $-0,035412$ & 0,161359 & $-0,424640$ & \\
\hline 410 & 2 & 10 & 0,002686 & 0,006849 & $-0,93605$ & 0,000584 & $-0,006269$ & 0,002457 & $-0,021301$ & \\
\hline $4 \backslash 1$ & 15 & 124 & 0,03185 & 0,042466 & $-0,28767$ & 0,003618 & $-0,029346$ & 0,029136 & $-0,148624$ & \\
\hline 412 & 3 & 122 & 0,031338 & 0,009589 & 1,184209 & 0,000817 & $-0,008381$ & 0,028668 & $-0,146906$ & \\
\hline 510 & 7 & 70 & 0,018035 & 0,020548 & $-0,13043$ & 0,001751 & $-0,016033$ & 0,016499 & $-0,097697$ & \\
\hline $5 \backslash 1$ & 53 & 361 & 0,092479 & 0,146575 & $-0,46056$ & 0,012489 & $-0,078971$ & 0,084599 & $-0,301445$ & \\
\hline 512 & 20 & 920 & 0,235482 & 0,056164 & 1,433353 & 0,004786 & $-0,036883$ & 0,215418 & $-0,477105$ & \\
\hline 610 & 2 & 5 & 0,001407 & 0,006849 & $-1,58268$ & 0,000584 & $-0,006269$ & 0,001287 & $-0,012358$ & \\
\hline $6 \backslash 1$ & 10 & 88 & 0,02264 & 0,028767 & $-0,23951$ & 0,002451 & $-0,021257$ & 0,020711 & $-0,115846$ & \\
\hline \multirow[t]{4}{*}{612} & 3 & 128 & 0,032873 & 0,009589 & 1,232026 & 0,000817 & $-0,008381$ & 0,030072 & $-0,152027$ & \\
\hline & 364 & 3908 & & & 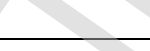 & & $-0,612012$ & & $-3,170764$ & \\
\hline & & & & 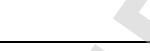 & & & 0,612012 & & 3,170764 & \\
\hline & & & & $P$ & 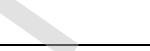 & & 0,052147 & & 2,900596 & 2,952743 \\
\hline
\end{tabular}




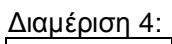

\begin{tabular}{|c|c|c|c|c|c|c|c|c|c|}
\hline JOBMAR & BADS & GOODS & $f(x / 1)$ & $f(x / 0)$ & $w(x)$ & & & 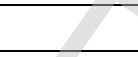 & \\
\hline 010 & 83 & 22 & 0,005756 & 0,228767 & $-3,68247$ & 0,019492 & $-0,110735$ & 0,005266 & $-0,039856$ \\
\hline $0 \backslash 1$ & 3 & 22 & 0,005756 & 0,009589 & $-0,51039$ & 0,000817 & $-0,008381$ & 0,005266 & $-0,039856$ \\
\hline 012 & 11 & 421 & 0,107828 & 0,031507 & 1,230333 & 0,002685 & $-0,022929$ & 0,09864 & $-0,329625$ \\
\hline 210 & 7 & 35 & 0,009082 & 0,020548 & $-0,81651$ & 0,001751 & $-0,016033$ & 0,008308 & $-0,057418$ \\
\hline $2 \backslash 1$ & 47 & 184 & 0,047199 & 0,130137 & $-1,01422$ & 0,011088 & $-0,072017$ & 0,043177 & $-0,195747$ \\
\hline 212 & 10 & 545 & 0,13955 & 0,028767 & 1,579188 & 0,002451 & $-0,021257$ & 0,127659 & $-0,379101$ \\
\hline 310 & 16 & 36 & 0,009337 & 0,045205 & $-1,57719$ & 0,003852 & $-0,030892$ & 0,008542 & $-0,058693$ \\
\hline $3 \backslash 1$ & 46 & 215 & 0,055129 & 0,127397 & $-0,83763$ & 0,010855 & $-0,070834$ & 0,050432 & $-0,217337$ \\
\hline 312 & 21 & 627 & 0,160527 & 0,058904 & 1,002551 & 0,005019 & $-0,038337$ & 0,146849 & $-0,406419$ \\
\hline 410 & 2 & 8 & 0,002174 & 0,006849 & $-1,14736$ & 0,000584 & $-0,006269$ & 0,001989 & $-0,017850$ \\
\hline $4 \backslash 1$ & 14 & 123 & 0,031594 & 0,039726 & $-0,22905$ & 0,003385 & $-0,027779$ & 0,028902 & $-0,147766$ \\
\hline 412 & 2 & 122 & 0,031338 & 0,006849 & 1,520681 & 0,000584 & $-0,006269$ & 0,028668 & $-0,146906$ \\
\hline $5 \backslash 0$ & 9 & 78 & 0,020082 & 0,026027 & $-0,25933$ & 0,002218 & $-0,019553$ & 0,018371 & $-0,105934$ \\
\hline $5 \backslash 1$ & 53 & 357 & 0,091456 & 0,146575 & $-0,47169$ & 0,012489 & $-0,078971$ & 0,083663 & $-0,299452$ \\
\hline 512 & 22 & 892 & 0,228319 & 0,061644 & 1,309372 & 0,005252 & $-0,039776$ & 0,208865 & $-0,471901$ \\
\hline 610 & 2 & 5 & 0,001407 & 0,006849 & $-1,58268$ & 0,000584 & $-0,006269$ & 0,001287 & $-0,012358$ \\
\hline $6 \backslash 1$ & 12 & 82 & 0,021105 & 0,034247 & $-0,48407$ & 0,002918 & $-0,024572$ & 0,019307 & $-0,109948$ \\
\hline \multirow[t]{4}{*}{612} & 4 & 134 & 0,034408 & 0,012329 & 1,026347 & 0,00105 & $-0,010394$ & 0,031476 & $-0,157053$ \\
\hline & 364 & 3908 & & & 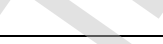 & & $-0,611269$ & & $-3,193219$ \\
\hline & & & & 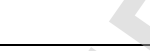 & & & 0,611269 & & 3,193219 \\
\hline & & & & 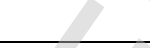 & & & 0,052084 & & 2,921138 \\
\hline
\end{tabular}




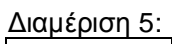

\begin{tabular}{|c|c|c|c|c|c|c|c|c|c|c|}
\hline JOBMAR & BADS & GOODS & $f(x / 1)$ & $f(x / 0)$ & $w(x)$ & & & 8 & $x+y$ & \\
\hline $0 \backslash 0$ & 77 & 17 & 0,004477 & 0,212329 & $-3,85922$ & 0,018092 & $-0,104724$ & 0,004095 & $-0,032484$ & \\
\hline $0 \backslash 1$ & 2 & 19 & 0,004988 & 0,006849 & $-0,31702$ & 0,000584 & $-0,006269$ & 0,004563 & $-0,035484$ & \\
\hline 012 & 15 & 428 & 0,109619 & 0,042466 & 0,948311 & 0,003618 & $-0,029346$ & 0,100279 & $-0,332716$ & \\
\hline 210 & 9 & 30 & 0,007803 & 0,026027 & $-1,2047$ & 0,002218 & $-0,019553$ & 0,007138 & $-0,050894$ & \\
\hline 211 & 47 & 164 & 0,042082 & 0,130137 & $-1,12896$ & 0,011088 & $-0,072017$ & 0,038497 & $-0,180901$ & \\
\hline 212 & 7 & 490 & 0,12548 & 0,020548 & 1,809383 & 0,001751 & $-0,016033$ & 0,114788 & $-0,358478$ & \\
\hline 310 & 19 & 36 & 0,009337 & 0,053425 & $-1,74424$ & 0,004552 & $-0,035412$ & 0,008542 & $-0,058693$ & \\
\hline $3 \backslash 1$ & 42 & 205 & 0,052571 & 0,116438 & $-0,7952$ & 0,009921 & $-0,066028$ & 0,048092 & $-0,210549$ & \\
\hline 312 & 22 & 633 & 0,162062 & 0,061644 & 0,966605 & 0,005252 & $-0,039776$ & 0,148253 & $-0,408269$ & \\
\hline 410 & 3 & 8 & 0,002174 & 0,009589 & $-1,48384$ & 0,000817 & $-0,008381$ & 0,001989 & $-0,017850$ & \\
\hline $4 \backslash 1$ & 15 & 112 & 0,02878 & 0,042466 & $-0,38903$ & 0,003618 & $-0,029346$ & 0,026328 & $-0,138148$ & \\
\hline 412 & 1 & 120 & 0,030826 & 0,00411 & 2,015045 & 0,00035 & $-0,004020$ & 0,0282 & $-0,145177$ & \\
\hline 510 & 12 & 78 & 0,020082 & 0,034247 & $-0,53377$ & 0,002918 & $-0,024572$ & 0,018371 & $-0,105934$ & \\
\hline $5 \backslash 1$ & 54 & 378 & 0,096828 & 0,149315 & $-0,43312$ & 0,012723 & $-0,080107$ & 0,088578 & $-0,309748$ & \\
\hline 512 & 21 & 964 & 0,246738 & 0,058904 & 1,432417 & 0,005019 & $-0,038337$ & 0,225715 & $-0,484706$ & \\
\hline 610 & 3 & 3 & 0,000895 & 0,009589 & $-2,37114$ & 0,000817 & $-0,008381$ & 0,000819 & $-0,008399$ & \\
\hline $6 \backslash 1$ & 12 & 83 & 0,021361 & 0,034247 & $-0,47202$ & 0,002918 & $-0,024572$ & 0,019541 & $-0,110941$ & \\
\hline \multirow[t]{4}{*}{612} & 3 & 140 & 0,035943 & 0,009589 & 1,321305 & 0,000817 & $-0,008381$ & 0,03288 & $-0,161989$ & \\
\hline & 364 & 3908 & & & & & $-0,615256$ & & $-3,151358$ & \\
\hline & & & & 8 & 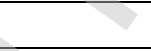 & & 0,615256 & & 3,151358 & \\
\hline & & & & 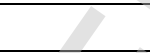 & & & 0,052423 & & 2,882844 & 2,935267 \\
\hline
\end{tabular}




\begin{tabular}{|c|c|c|c|c|c|c|c|c|c|c|}
\hline $\mathrm{IOB} \backslash \mathrm{TRM}$ & BADS & Sחת & $f(x / 1)$ & & $w(y)$ & & & & & \\
\hline $1 \backslash 1$ & 8 & 62 & 0015989 & 023288 & 37604 & 0001984 & $2+>$ & 0014626 & $\therefore$ & \\
\hline $2 \ 1$ & 18 & 44 & 0.011384 & 0.050685 & $-1,49342$ & 0.004319 & -0.033924 & 0.010414 & $-0,068580$ & \\
\hline $3 \backslash 1$ & 11 & 74 & 0.019059 & 0.031507 & -0.50269 & 0.002685 & -0.022929 & 0.017435 & $-0,101852$ & \\
\hline $4 \backslash 1$ & 3 & 19 & 0,004988 & 0,009589 & $-0,65349$ & 0,000817 & $-0,008381$ & 0,004563 & $-0,035484$ & \\
\hline $5 \backslash 1$ & 12 & 174 & 0,044641 & 0,034247 & 0,265057 & 0,002918 & $-0,024572$ & 0,040837 & $-0,188421$ & \\
\hline $6 \backslash 1$ & 3 & 30 & 0,007803 & 0,009589 & $-0,20618$ & 0,000817 & $-0,008381$ & 0,007138 & $-0,050894$ & \\
\hline 112 & 5 & 183 & 0,046943 & 0,015068 & 1,136327 & 0,001284 & $-0,012332$ & 0,042943 & $-0,195023$ & \\
\hline 212 & 16 & 77 & 0,019826 & 0,045205 & $-0,82422$ & 0,003852 & $-0,030892$ & 0,018137 & $-0,104920$ & \\
\hline 312 & 8 & 261 & 0,066897 & 0,023288 & 1,055229 & 0,001984 & $-0,017813$ & 0,061197 & $-0,246648$ & \\
\hline 412 & 1 & 75 & 0,019314 & 0,00411 & 1,547528 & 0,00035 & $-0,004020$ & 0,017669 & $-0,102879$ & \\
\hline 512 & 7 & 334 & 0,085572 & 0,020548 & 1,426594 & 0,001751 & $-0,016033$ & 0,078281 & $-0,287697$ & \\
\hline 612 & 0 & 43 & 0,011128 & 0,00137 & 2,094769 & 0,000117 & $-0,001525$ & 0,01018 & $-0,067372$ & \\
\hline 113 & 45 & 429 & 0,109875 & 0,124658 & $-0,12623$ & 0,010622 & $-0,069644$ & 0,100513 & $-0,333154$ & \\
\hline 213 & 71 & 327 & 0,083781 & 0,19589 & $-0,84935$ & 0,016691 & $-0,098557$ & 0,076642 & $-0,284015$ & \\
\hline 313 & 51 & 569 & 0,145689 & 0,141096 & 0,032037 & 0,012022 & $-0,076680$ & 0,133276 & $-0,387501$ & \\
\hline 413 & 14 & 151 & 0,038757 & 0,039726 & $-0,0247$ & 0,003385 & $-0,027779$ & 0,035454 & $-0,170815$ & \\
\hline 513 & 79 & 910 & 0,232924 & 0,217808 & 0,067097 & 0,018559 & $-0,106745$ & 0,213077 & $-0,475280$ & \\
\hline 613 & 12 & 146 & 0,037478 & 0,034247 & 0,090157 & 0,002918 & $-0,024572$ & 0,034284 & $-0,166838$ & \\
\hline & 364 & 3908 & & D & & & $-0,602591$ & & $-3,356525$ & \\
\hline & & & & & 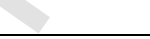 & & 0,602591 & & 3,356525 & \\
\hline & & & & & 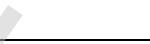 & & 0,051344 & & 3,070529 & 3,121873 \\
\hline
\end{tabular}




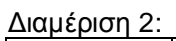

\begin{tabular}{|c|c|c|c|c|c|c|c|c|c|c|}
\hline JOBTRM & BADS & GOODS & $f(x / 1)$ & $f(x / 0)$ & $w(x)$ & & & 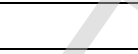 & 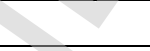 & \\
\hline $0 \backslash 0$ & 13 & 87 & 0,022384 & 0,036986 & $-0,50219$ & 0,003151 & $-0,026188$ & 0,020477 & $-0,114873$ & \\
\hline $0 \backslash 1$ & 72 & 332 & 0,08506 & 0,19863 & $-0,84809$ & 0,016924 & $-0,099596$ & 0,077812 & $-0,286650$ & \\
\hline 012 & 15 & 49 & 0,012663 & 0,042466 & $-1,21001$ & 0,003618 & $-0,029346$ & 0,011584 & $-0,074506$ & \\
\hline 210 & 4 & 178 & 0,045664 & 0,012329 & 1,309372 & 0,00105 & $-0,010394$ & 0,041773 & $-0,191374$ & \\
\hline $2 \backslash 1$ & 53 & 436 & 0,111665 & 0,146575 & $-0,27203$ & 0,012489 & $-0,078971$ & 0,102151 & $-0,336202$ & \\
\hline 212 & 6 & 63 & 0,016245 & 0,017808 & $-0,0919$ & 0,001517 & $-0,014209$ & 0,01486 & $-0,090238$ & \\
\hline 310 & 7 & 245 & 0,062804 & 0,020548 & 1,117254 & 0,001751 & $-0,016033$ & 0,057453 & $-0,236790$ & \\
\hline $3 \backslash 1$ & 54 & 557 & 0,14262 & 0,149315 & $-0,04588$ & 0,012723 & $-0,080107$ & 0,130468 & $-0,383345$ & \\
\hline 312 & 8 & 68 & 0,017524 & 0,023288 & $-0,28437$ & 0,001984 & $-0,017813$ & 0,016031 & $-0,095591$ & \\
\hline 410 & 1 & 75 & 0,019314 & 0,00411 & 1,547528 & 0,00035 & $-0,004020$ & 0,017669 & $-0,102879$ & \\
\hline $4 \backslash 1$ & 14 & 161 & 0,041315 & 0,039726 & 0,039217 & 0,003385 & $-0,027779$ & 0,037795 & $-0,178605$ & \\
\hline 412 & 2 & 18 & 0,004733 & 0,006849 & $-0,36966$ & 0,000584 & $-0,006269$ & 0,004329 & $-0,033993$ & \\
\hline 510 & 5 & 331 & 0,084804 & 0,015068 & 1,72774 & 0,001284 & $-0,012332$ & 0,077578 & $-0,286125$ & \\
\hline $5 \backslash 1$ & 80 & 933 & 0,238808 & 0,220548 & 0,079544 & 0,018792 & $-0,107748$ & 0,21846 & $-0,479423$ & \\
\hline 512 & 12 & 162 & 0,041571 & 0,034247 & 0,19381 & 0,002918 & $-0,024572$ & 0,038029 & $-0,179372$ & \\
\hline 610 & 1 & 45 & 0,01164 & 0,00411 & 1,041108 & 0,00035 & $-0,004020$ & 0,010648 & $-0,069779$ & \\
\hline $6 \backslash 1$ & 13 & 144 & 0,036966 & 0,036986 & $-0,00055$ & 0,003151 & $-0,026188$ & 0,033816 & $-0,165231$ & \\
\hline \multirow[t]{4}{*}{$6 \backslash 2$} & 4 & 24 & 0,006268 & 0,012329 & $-0,67654$ & 0,00105 & $-0,010394$ & 0,005734 & $-0,042694$ & \\
\hline & 364 & 3908 & & & 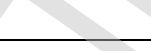 & & $-0,595981$ & & $-3,347669$ & \\
\hline & & & & 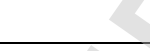 & & & 0,595981 & & 3,347669 & \\
\hline & & & & 2 & & & 0,050781 & & 3,062428 & 3,113209 \\
\hline
\end{tabular}




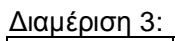

\begin{tabular}{|c|c|c|c|c|c|c|c|c|c|c|}
\hline JOBTRM & BADS & GOODS & $f(x / 1)$ & $f(x / 0)$ & $w(x)$ & & & 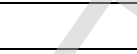 & & \\
\hline 010 & 9 & 72 & 0,018547 & 0,026027 & $-0,33884$ & 0,002218 & $-0,019553$ & 0,016967 & $-0,099783$ & \\
\hline $0 \backslash 1$ & 75 & 330 & 0,084548 & 0,206849 & $-0,89467$ & 0,017625 & $-0,102686$ & 0,077344 & $-0,285599$ & \\
\hline 012 & 16 & 45 & 0,01164 & 0,045205 & $-1,35679$ & 0,003852 & $-0,030892$ & 0,010648 & $-0,069779$ & \\
\hline 210 & 5 & 166 & 0,042594 & 0,015068 & 1,039108 & 0,001284 & $-0,012332$ & 0,038965 & $-0,182421$ & \\
\hline $2 \backslash 1$ & 54 & 436 & 0,111665 & 0,149315 & $-0,29055$ & 0,012723 & $-0,080107$ & 0,102151 & $-0,336202$ & \\
\hline 212 & 8 & 52 & 0,013431 & 0,023288 & $-0,55039$ & 0,001984 & $-0,017813$ & 0,012286 & $-0,077978$ & \\
\hline 310 & 7 & 268 & 0,068688 & 0,020548 & 1,206808 & 0,001751 & $-0,016033$ & 0,062835 & $-0,250856$ & \\
\hline $3 \backslash 1$ & 60 & 626 & 0,160271 & 0,165753 & $-0,03363$ & 0,014123 & $-0,086798$ & 0,146615 & $-0,406108$ & \\
\hline 312 & 15 & 85 & 0,021873 & 0,042466 & $-0,66346$ & 0,003618 & $-0,029346$ & 0,020009 & $-0,112915$ & \\
\hline 410 & 1 & 84 & 0,021617 & 0,00411 & 1,660147 & 0,00035 & $-0,004020$ & 0,019775 & $-0,111930$ & \\
\hline $4 \backslash 1$ & 17 & 155 & 0,03978 & 0,047945 & $-0,18669$ & 0,004085 & $-0,032418$ & 0,036391 & $-0,173957$ & \\
\hline 412 & 2 & 17 & 0,004477 & 0,006849 & $-0,42523$ & 0,000584 & $-0,006269$ & 0,004095 & $-0,032484$ & \\
\hline 510 & 5 & 295 & 0,075595 & 0,015068 & 1,612781 & 0,001284 & $-0,012332$ & 0,069154 & $-0,266522$ & \\
\hline $5 \backslash 1$ & 68 & 900 & 0,230366 & 0,187671 & 0,204977 & 0,015991 & $-0,095411$ & 0,210737 & $-0,473418$ & \\
\hline 512 & 7 & 156 & 0,040036 & 0,020548 & 0,667013 & 0,001751 & $-0,016033$ & 0,036625 & $-0,174737$ & \\
\hline 610 & 1 & 43 & 0,011128 & 0,00411 & 0,996156 & 0,00035 & $-0,004020$ & 0,01018 & $-0,067372$ & \\
\hline $6 \backslash 1$ & 10 & 157 & 0,040292 & 0,028767 & 0,336911 & 0,002451 & $-0,021257$ & 0,036859 & $-0,175515$ & \\
\hline \multirow[t]{4}{*}{612} & 4 & 21 & 0,0055 & 0,012329 & $-0,80716$ & 0,00105 & $-0,010394$ & 0,005031 & $-0,038414$ & \\
\hline & 364 & 3908 & & & 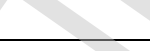 & & $-0,597716$ & & $-3,335990$ & \\
\hline & & & & & & & 0,597716 & & 3,335990 & \\
\hline & & & & 2 & & & 0,050929 & & 3,051743 & 3,102672 \\
\hline
\end{tabular}




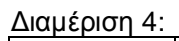

\begin{tabular}{|c|c|c|c|c|c|c|c|c|c|c|}
\hline JOBTRM & BADS & GOODS & $f(x / 1)$ & $f(x / 0)$ & $w(x)$ & & & 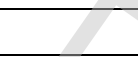 & 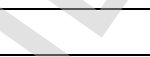 & \\
\hline 010 & 11 & 79 & 0,020338 & 0,031507 & $-0,43773$ & 0,002685 & $-0,022929$ & 0,018605 & $-0,106944$ & \\
\hline $0 \backslash 1$ & 70 & 348 & 0,089153 & 0,193151 & $-0,77311$ & 0,016458 & $-0,097513$ & 0,081557 & $-0,294914$ & \\
\hline 012 & 16 & 38 & 0,009849 & 0,045205 & $-1,52384$ & 0,003852 & $-0,030892$ & 0,00901 & $-0,061216$ & \\
\hline 210 & 5 & 189 & 0,048478 & 0,015068 & 1,168501 & 0,001284 & $-0,012332$ & 0,044347 & $-0,199341$ & \\
\hline $2 \backslash 1$ & 55 & 507 & 0,129829 & 0,152055 & $-0,15803$ & 0,012956 & $-0,081237$ & 0,118766 & $-0,365064$ & \\
\hline 212 & 4 & 68 & 0,017524 & 0,012329 & 0,351617 & 0,00105 & $-0,010394$ & 0,016031 & $-0,095591$ & \\
\hline 310 & 9 & 230 & 0,058966 & 0,026027 & 0,81782 & 0,002218 & $-0,019553$ & 0,053942 & $-0,227228$ & \\
\hline $3 \backslash 1$ & 61 & 585 & 0,149783 & 0,168493 & $-0,11771$ & 0,014357 & $-0,087893$ & 0,13702 & $-0,392911$ & \\
\hline 312 & 13 & 63 & 0,016245 & 0,036986 & $-0,82279$ & 0,003151 & $-0,026188$ & 0,01486 & $-0,090238$ & \\
\hline 410 & 0 & 77 & 0,019826 & 0,00137 & 2,672286 & 0,000117 & $-0,001525$ & 0,018137 & $-0,104920$ & \\
\hline $4 \backslash 1$ & 15 & 160 & 0,041059 & 0,042466 & $-0,03369$ & 0,003618 & $-0,029346$ & 0,037561 & $-0,177836$ & \\
\hline 412 & 3 & 16 & 0,004221 & 0,009589 & $-0,82054$ & 0,000817 & $-0,008381$ & 0,003861 & $-0,030955$ & \\
\hline 510 & 5 & 282 & 0,072269 & 0,015068 & 1,567791 & 0,001284 & $-0,012332$ & 0,066111 & $-0,259088$ & \\
\hline $5 \backslash 1$ & 70 & 890 & 0,227808 & 0,193151 & 0,165031 & 0,016458 & $-0,097513$ & 0,208397 & $-0,471518$ & \\
\hline 512 & 9 & 155 & 0,03978 & 0,026027 & 0,424214 & 0,002218 & $-0,019553$ & 0,036391 & $-0,173957$ & \\
\hline 610 & 1 & 45 & 0,01164 & 0,00411 & 1,041108 & 0,00035 & $-0,004020$ & 0,010648 & $-0,069779$ & \\
\hline $6 \backslash 1$ & 13 & 152 & 0,039013 & 0,036986 & 0,053335 & 0,003151 & $-0,026188$ & 0,035688 & $-0,171604$ & \\
\hline \multirow[t]{4}{*}{612} & 4 & 24 & 0,006268 & 0,012329 & $-0,67654$ & 0,00105 & $-0,010394$ & 0,005734 & $-0,042694$ & \\
\hline & 364 & 3908 & & & 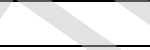 & & $-0,598184$ & & $-3,335798$ & \\
\hline & & & & & & & 0,598184 & & 3,335798 & \\
\hline & & & & 2 & 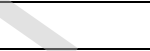 & & 0,050969 & & 3,051568 & 3,102537 \\
\hline
\end{tabular}




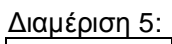

\begin{tabular}{|c|c|c|c|c|c|c|c|c|c|c|}
\hline JOBTRM & BADS & GOODS & $f(x / 1)$ & $f(x / 0)$ & $w(x)$ & & & 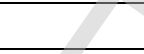 & & \\
\hline 010 & 8 & 73 & 0,018803 & 0,023288 & $-0,21392$ & 0,001984 & $-0,017813$ & 0,017201 & $-0,100820$ & \\
\hline $0 \backslash 1$ & 71 & 351 & 0,089921 & 0,19589 & $-0,77863$ & 0,016691 & $-0,098557$ & 0,082259 & $-0,296435$ & \\
\hline 012 & 15 & 40 & 0,010361 & 0,042466 & $-1,41068$ & 0,003618 & $-0,029346$ & 0,009478 & $-0,063703$ & \\
\hline 210 & 5 & 155 & 0,03978 & 0,015068 & 0,970758 & 0,001284 & $-0,012332$ & 0,036391 & $-0,173957$ & \\
\hline $2 \backslash 1$ & 51 & 465 & 0,119084 & 0,141096 & $-0,16961$ & 0,012022 & $-0,076680$ & 0,108937 & $-0,348429$ & \\
\hline 212 & 7 & 64 & 0,0165 & 0,020548 & $-0,21938$ & 0,001751 & $-0,016033$ & 0,015094 & $-0,091319$ & \\
\hline 310 & 9 & 230 & 0,058966 & 0,026027 & 0,81782 & 0,002218 & $-0,019553$ & 0,053942 & $-0,227228$ & \\
\hline $3 \backslash 1$ & 60 & 568 & 0,145434 & 0,165753 & $-0,13078$ & 0,014123 & $-0,086798$ & 0,133042 & $-0,387158$ & \\
\hline 312 & 14 & 76 & 0,01957 & 0,039726 & $-0,708$ & 0,003385 & $-0,027779$ & 0,017903 & $-0,103902$ & \\
\hline 410 & 1 & 71 & 0,018291 & 0,00411 & 1,493093 & 0,00035 & $-0,004020$ & 0,016733 & $-0,098742$ & \\
\hline $4 \backslash 1$ & 16 & 156 & 0,040036 & 0,045205 & $-0,12144$ & 0,003852 & $-0,030892$ & 0,036625 & $-0,174737$ & \\
\hline 412 & 2 & 13 & 0,003454 & 0,006849 & $-0,68474$ & 0,000584 & $-0,006269$ & 0,003159 & $-0,026242$ & \\
\hline 510 & 6 & 289 & 0,07406 & 0,017808 & 1,425214 & 0,001517 & $-0,014209$ & 0,06775 & $-0,263115$ & \\
\hline $5 \backslash 1$ & 67 & 972 & 0,248785 & 0,184932 & 0,296603 & 0,015757 & $-0,094352$ & 0,227587 & $-0,486014$ & \\
\hline 512 & 14 & 159 & 0,040803 & 0,039726 & 0,026756 & 0,003385 & $-0,027779$ & 0,037327 & $-0,177064$ & \\
\hline 610 & 1 & 47 & 0,012151 & 0,00411 & 1,084125 & 0,00035 & $-0,004020$ & 0,011116 & $-0,072157$ & \\
\hline $6 \backslash 1$ & 12 & 154 & 0,039524 & 0,034247 & 0,143326 & 0,002918 & $-0,024572$ & 0,036156 & $-0,173175$ & \\
\hline \multirow[t]{4}{*}{612} & 5 & 25 & 0,006523 & 0,015068 & $-0,83721$ & 0,001284 & $-0,012332$ & 0,005968 & $-0,044092$ & \\
\hline & 364 & 3908 & & & 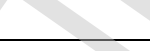 & & $-0,603337$ & & $-3,308290$ & \\
\hline & & & & & & & 0,603337 & & 3,308290 & \\
\hline & & & & 2 & & & 0,051408 & & 3,026404 & 3,077812 \\
\hline
\end{tabular}




\begin{tabular}{|c|c|c|c|c|c|c|c|c|c|c|}
\hline JYRIMAR & BADS & GOODS & $f(x / 1)$ & $f(x / 0)$ & $w(x)$ & & & & & \\
\hline $1 \backslash 1$ & 24 & 152 & 0,039013 & 0,067123 & $-0,54265$ & 0,005719 & $-0,042609$ & 0,035688 & $-0,171604$ & \\
\hline $2 \backslash 1$ & 69 & 330 & 0,084548 & 0,190411 & $-0,81186$ & 0,016224 & $-0,096464$ & 0,077344 & $-0,285599$ & \\
\hline $3 \backslash 1$ & 54 & 352 & 0,090177 & 0,149315 & $-0,50429$ & 0,012723 & $-0,080107$ & 0,082493 & $-0,296940$ & \\
\hline $4 \backslash 1$ & 19 & 131 & 0,03364 & 0,053425 & $-0,46255$ & 0,004552 & $-0,035412$ & 0,030774 & $-0,154551$ & \\
\hline 112 & 22 & 29 & 0,007547 & 0,061644 & $-2,10026$ & 0,005252 & $-0,039776$ & 0,006904 & $-0,049557$ & \\
\hline 212 & 40 & 39 & 0,010105 & 0,110959 & $-2,39614$ & 0,009454 & $-0,063579$ & 0,009244 & $-0,062464$ & \\
\hline 312 & 37 & 30 & 0,007803 & 0,10274 & $-2,57775$ & 0,008754 & $-0,059841$ & 0,007138 & $-0,050894$ & \\
\hline 412 & 26 & 84 & 0,021617 & 0,072603 & $-1,21153$ & 0,006186 & $-0,045386$ & 0,019775 & $-0,111930$ & \\
\hline 113 & 10 & 470 & 0,120363 & 0,028767 & 1,431281 & 0,002451 & $-0,021257$ & 0,110108 & $-0,350474$ & \\
\hline 213 & 12 & 433 & 0,110898 & 0,034247 & 1,175024 & 0,002918 & $-0,024572$ & 0,101449 & $-0,334900$ & \\
\hline 313 & 25 & 626 & 0,160271 & 0,069863 & 0,830331 & 0,005953 & $-0,044004$ & 0,146615 & $-0,406108$ & \\
\hline \multirow[t]{4}{*}{413} & 26 & 1232 & 0,315298 & 0,072603 & 1,468516 & 0,006186 & $-0,045386$ & 0,288433 & $-0,517360$ & \\
\hline & 364 & 3908 & & & 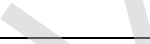 & & $-0,598393$ & & $-2,792382$ & \\
\hline & & & & & & & 0,598393 & & 2,792382 & \\
\hline & & & & & & & 0,050987 & & 2,554454 & 2,605441 \\
\hline
\end{tabular}




\begin{tabular}{|c|c|c|c|c|c|c|c|c|c|c|}
\hline \multicolumn{11}{|c|}{ 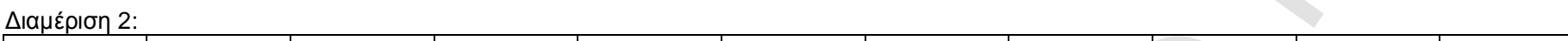 } \\
\hline JYRMAR & BADS & GOODS & $f(x / 1)$ & $f(x / 0)$ & $w(x)$ & & & 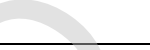 & & \\
\hline 010 & 34 & 39 & 0,010105 & 0,094521 & $-2,2358$ & 0,008054 & $-0,056023$ & 0,009244 & $-0,062464$ & \\
\hline $0 \backslash 1$ & 70 & 343 & 0,087874 & 0,193151 & $-0,78757$ & 0,016458 & $-0,097513$ & 0,080387 & $-0,292358$ & \\
\hline 012 & 14 & 416 & 0,106549 & 0,039726 & 0,986598 & 0,003385 & $-0,027779$ & 0,09747 & $-0,327393$ & \\
\hline 110 & 42 & 41 & 0,010617 & 0,116438 & $-2,39495$ & 0,009921 & $-0,066028$ & 0,009712 & $-0,064934$ & \\
\hline $1 \backslash 1$ & 54 & 394 & 0,100921 & 0,149315 & $-0,39172$ & 0,012723 & $-0,080107$ & 0,092322 & $-0,317327$ & \\
\hline 112 & 27 & 699 & 0,178946 & 0,075342 & 0,86504 & 0,00642 & $-0,046756$ & 0,163699 & $-0,427399$ & \\
\hline 210 & 19 & 27 & 0,007035 & 0,053425 & $-2,02737$ & 0,004552 & $-0,035412$ & 0,006436 & $-0,046849$ & \\
\hline 211 & 22 & 120 & 0,030826 & 0,061644 & $-0,69301$ & 0,005252 & $-0,039776$ & 0,0282 & $-0,145177$ & \\
\hline 212 & 5 & 411 & 0,10527 & 0,015068 & 1,943921 & 0,001284 & $-0,012332$ & 0,0963 & $-0,325140$ & \\
\hline 310 & 32 & 85 & 0,021873 & 0,089041 & $-1,40386$ & 0,007587 & $-0,053429$ & 0,020009 & $-0,112915$ & \\
\hline $3 \backslash 1$ & 21 & 127 & 0,032617 & 0,058904 & $-0,59108$ & 0,005019 & $-0,038337$ & 0,029838 & $-0,151180$ & \\
\hline \multirow[t]{4}{*}{312} & 24 & 1206 & 0,308647 & 0,067123 & 1,525666 & 0,005719 & $-0,042609$ & 0,282348 & $-0,515131$ & \\
\hline & 364 & 3908 & & & & 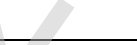 & $-0,596100$ & & $-2,788267$ & \\
\hline & & & & & & 2 & 0,596100 & & 2,788267 & \\
\hline & & & & & 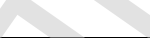 & & 0,050791 & & 2,55069 & 2,601481 \\
\hline
\end{tabular}




\begin{tabular}{|c|c|c|c|c|c|c|c|c|c|c|}
\hline \multicolumn{11}{|c|}{ 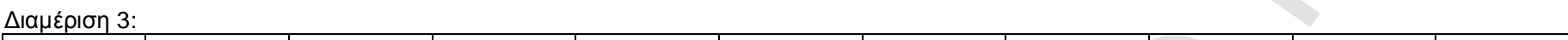 } \\
\hline JYRMAR & BADS & GOODS & $f(x / 1)$ & $f(x / 0)$ & $w(x)$ & & & - & & \\
\hline 010 & 40 & 34 & 0,008826 & 0,110959 & $-2,53148$ & 0,009454 & $-0,063579$ & 0,008074 & $-0,056133$ & \\
\hline $0 \backslash 1$ & 77 & 338 & 0,086595 & 0,212329 & $-0,89689$ & 0,018092 & $-0,104724$ & 0,079217 & $-0,289779$ & \\
\hline 012 & 11 & 442 & 0,1132 & 0,031507 & 1,278954 & 0,002685 & $-0,022929$ & 0,103555 & $-0,338783$ & \\
\hline 110 & 28 & 40 & 0,010361 & 0,078082 & $-2,01974$ & 0,006653 & $-0,048113$ & 0,009478 & $-0,063703$ & \\
\hline $1 \backslash 1$ & 57 & 410 & 0,105014 & 0,157534 & $-0,40555$ & 0,013423 & $-0,083479$ & 0,096066 & $-0,324687$ & \\
\hline 112 & 23 & 664 & 0,169992 & 0,064384 & 0,970895 & 0,005486 & $-0,041199$ & 0,155508 & $-0,417529$ & \\
\hline 210 & 18 & 17 & 0,004477 & 0,050685 & $-2,42671$ & 0,004319 & $-0,033924$ & 0,004095 & $-0,032484$ & \\
\hline $2 \backslash 1$ & 22 & 119 & 0,03057 & 0,061644 & $-0,70134$ & 0,005252 & $-0,039776$ & 0,027966 & $-0,144309$ & \\
\hline 212 & 7 & 414 & 0,106037 & 0,020548 & 1,64103 & 0,001751 & $-0,016033$ & 0,097002 & $-0,326494$ & \\
\hline 310 & 32 & 83 & 0,021361 & 0,089041 & $-1,42753$ & 0,007587 & $-0,053429$ & 0,019541 & $-0,110941$ & \\
\hline $3 \backslash 1$ & 20 & 128 & 0,032873 & 0,056164 & $-0,53564$ & 0,004786 & $-0,036883$ & 0,030072 & $-0,152027$ & \\
\hline \multirow[t]{4}{*}{312} & 29 & 1219 & 0,311972 & 0,080822 & 1,350666 & 0,006887 & $-0,049459$ & 0,28539 & $-0,516269$ & \\
\hline & 364 & 3908 & & & & 2 & $-0,593527$ & & $-2,773137$ & \\
\hline & & & & & & 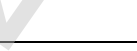 & 0,593527 & & 2,773137 & \\
\hline & & & & & 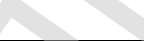 & & 0,050572 & & 2,536849 & 2,587421 \\
\hline
\end{tabular}




\begin{tabular}{|c|c|c|c|c|c|c|c|c|c|c|}
\hline JYRMAR & BADS & GOODS & $f(x / 1)$ & $f(x / 0)$ & $w(x)$ & & & 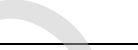 & & \\
\hline 010 & 22 & 22 & 0,005756 & 0,061644 & $-2,37114$ & 0,005252 & $-0,039776$ & 0,005266 & $-0,039856$ & \\
\hline $0 \backslash 1$ & 53 & 196 & 0,050269 & 0,146575 & $-1,07016$ & 0,012489 & $-0,078971$ & 0,045985 & $-0,204299$ & \\
\hline 012 & 7 & 293 & 0,075083 & 0,020548 & 1,295835 & 0,001751 & $-0,016033$ & 0,068686 & $-0,265391$ & \\
\hline 110 & 45 & 52 & 0,013431 & 0,124658 & $-2,22804$ & 0,010622 & $-0,069644$ & 0,012286 & $-0,077978$ & \\
\hline $1 \backslash 1$ & 74 & 525 & 0,134433 & 0,20411 & $-0,41759$ & 0,017391 & $-0,101661$ & 0,122979 & $-0,371829$ & \\
\hline 112 & 22 & 781 & 0,199923 & 0,061644 & 1,17656 & 0,005252 & $-0,039776$ & 0,182889 & $-0,448253$ & \\
\hline 210 & 22 & 23 & 0,006012 & 0,061644 & $-2,32765$ & 0,005252 & $-0,039776$ & 0,0055 & $-0,041282$ & \\
\hline $2 \backslash 1$ & 28 & 148 & 0,037989 & 0,078082 & $-0,72046$ & 0,006653 & $-0,048113$ & 0,034752 & $-0,168436$ & \\
\hline 212 & 9 & 473 & 0,121131 & 0,026027 & 1,537721 & 0,002218 & $-0,019553$ & 0,11081 & $-0,351693$ & \\
\hline 310 & 30 & 87 & 0,022384 & 0,083562 & $-1,31723$ & 0,00712 & $-0,050793$ & 0,020477 & $-0,114873$ & \\
\hline $3 \backslash 1$ & 20 & 114 & 0,029291 & 0,056164 & $-0,65099$ & 0,004786 & $-0,036883$ & 0,026796 & $-0,139923$ & \\
\hline 312 & 32 & 1194 & 0,305577 & 0,089041 & 1,233103 & 0,007587 & $-0,053429$ & 0,27954 & $-0,514039$ & \\
\hline & 364 & 3908 & & & & 2 & $-0,594407$ & & $-2,737850$ & \\
\hline & & & & & 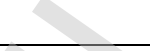 & 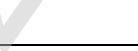 & 0,594407 & & 2,737850 & \\
\hline & & & & & 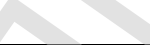 & & 0,050647 & & 2,504569 & 2,555216 \\
\hline
\end{tabular}




\begin{tabular}{|c|c|c|c|c|c|c|c|c|c|c|}
\hline \multicolumn{11}{|c|}{ 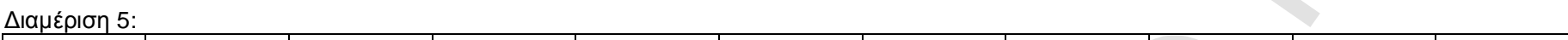 } \\
\hline JYRMAR & BADS & GOODS & $f(x / 1)$ & $f(x / 0)$ & $w(x)$ & & & 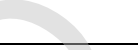 & & \\
\hline 010 & 40 & 37 & 0,009593 & 0,110959 & $-2,4481$ & 0,009454 & $-0,063579$ & 0,008776 & $-0,059959$ & \\
\hline $0 \backslash 1$ & 73 & 327 & 0,083781 & 0,20137 & $-0,87694$ & 0,017158 & $-0,100631$ & 0,076642 & $-0,284015$ & \\
\hline 012 & 11 & 438 & 0,112177 & 0,031507 & 1,269873 & 0,002685 & $-0,022929$ & 0,102619 & $-0,337065$ & \\
\hline 110 & 35 & 30 & 0,007803 & 0,09726 & $-2,52295$ & 0,008287 & $-0,057305$ & 0,007138 & $-0,050894$ & \\
\hline $1 \backslash 1$ & 53 & 355 & 0,090944 & 0,146575 & $-0,4773$ & 0,012489 & $-0,078971$ & 0,083195 & $-0,298450$ & \\
\hline 112 & 20 & 629 & 0,161039 & 0,056164 & 1,053361 & 0,004786 & $-0,036883$ & 0,147317 & $-0,407038$ & \\
\hline 210 & 20 & 24 & 0,006268 & 0,056164 & $-2,19289$ & 0,004786 & $-0,036883$ & 0,005734 & $-0,042694$ & \\
\hline $2 \backslash 1$ & 26 & 147 & 0,037733 & 0,072603 & $-0,65446$ & 0,006186 & $-0,045386$ & 0,034518 & $-0,167638$ & \\
\hline 212 & 9 & 471 & 0,120619 & 0,026027 & 1,533488 & 0,002218 & $-0,019553$ & 0,110342 & $-0,350881$ & \\
\hline 310 & 28 & 81 & 0,020849 & 0,078082 & $-1,32044$ & 0,006653 & $-0,048113$ & 0,019073 & $-0,108950$ & \\
\hline $3 \backslash 1$ & 20 & 132 & 0,033896 & 0,056164 & $-0,50498$ & 0,004786 & $-0,036883$ & 0,031008 & $-0,155388$ & \\
\hline \multirow[t]{4}{*}{312} & 29 & 1237 & 0,316577 & 0,080822 & 1,365319 & 0,006887 & $-0,049459$ & 0,289603 & $-0,517767$ & \\
\hline & 364 & 3908 & & & & 2 & $-0,596574$ & & $-2,780739$ & \\
\hline & & & & & & 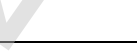 & 0,596574 & & 2,780739 & \\
\hline & & & & & 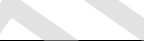 & & 0,050832 & & 2,543803 & 2,594635 \\
\hline
\end{tabular}




\begin{tabular}{|c|c|c|c|c|c|c|c|c|c|c|}
\hline \multicolumn{11}{|c|}{ 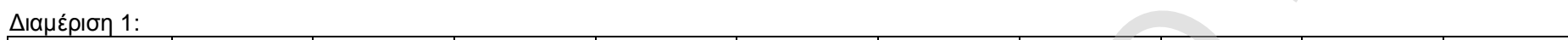 } \\
\hline JYRITRM & BADS & GOODS & $f(x / 1)$ & $f(x / 0)$ & $w(x)$ & & & & & \\
\hline $1 \backslash 1$ & 8 & 59 & 0,015221 & 0,023288 & $-0,42523$ & 0,001984 & $-0,017813$ & 0,013924 & $-0,085861$ & \\
\hline 211 & 9 & 77 & 0,019826 & 0,026027 & $-0,27215$ & 0,002218 & $-0,019553$ & 0,018137 & $-0,104920$ & \\
\hline 311 & 19 & 96 & 0,024687 & 0,053425 & $-0,77201$ & 0,004552 & $-0,035412$ & 0,022583 & $-0,123499$ & \\
\hline 411 & 19 & 171 & 0,043873 & 0,053425 & $-0,19697$ & 0,004552 & $-0,035412$ & 0,040135 & $-0,186186$ & \\
\hline 112 & 3 & 167 & 0,04285 & 0,009589 & 1,497081 & 0,000817 & $-0,008381$ & 0,039199 & $-0,183178$ & \\
\hline 212 & 14 & 237 & 0,060757 & 0,039726 & 0,424879 & 0,003385 & $-0,027779$ & 0,05558 & $-0,231730$ & \\
\hline 312 & 15 & 277 & 0,07099 & 0,042466 & 0,513841 & 0,003618 & $-0,029346$ & 0,064941 & $-0,256175$ & \\
\hline 412 & 5 & 292 & 0,074827 & 0,015068 & 1,602577 & 0,001284 & $-0,012332$ & 0,068452 & $-0,264824$ & \\
\hline 113 & 45 & 425 & 0,108851 & 0,124658 & $-0,13559$ & 0,010622 & $-0,069644$ & 0,099577 & $-0,331396$ & \\
\hline 213 & 98 & 488 & 0,124968 & 0,269863 & $-0,76986$ & 0,022994 & $-0,125147$ & 0,11432 & $-0,357690$ & \\
\hline 313 & 82 & 635 & 0,162574 & 0,226027 & $-0,32953$ & 0,019259 & $-0,109744$ & 0,148721 & $-0,408882$ & \\
\hline \multirow[t]{4}{*}{413} & 47 & 984 & 0,251855 & 0,130137 & 0,660265 & 0,011088 & $-0,072017$ & 0,230395 & $-0,487935$ & \\
\hline & 364 & 3908 & & & $\Delta$ & & $-0,562580$ & & $-3,022274$ & \\
\hline & & & & & & & 0,562580 & & 3,022274 & \\
\hline & & & & 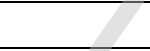 & 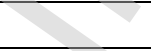 & & 0,047935 & & 2,764759 & 2,812694 \\
\hline
\end{tabular}




\begin{tabular}{|c|c|c|c|c|c|c|c|c|c|c|}
\hline JYRTRM & אחמק & 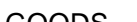 & & & (2) & & & & & \\
\hline 010 & 12 & 237 & 0060757 & 0034247 & 573299 & 0002918 & -02457? & 005558 & ח 231730 & \\
\hline $0 \backslash 1$ & 100 & 492 & 0.125991 & 0,275342 & -0.7818 & 0.023461 & -0.127008 & 0.115256 & $-0,359263$ & \\
\hline 012 & 6 & 69 & 0,017779 & 0,017808 & $-0,00161$ & 0,001517 & $-0,014209$ & 0,016265 & $-0,096646$ & \\
\hline 110 & 13 & 302 & 0,077386 & 0,036986 & 0,738252 & 0,003151 & $-0,026188$ & 0,070792 & $-0,270444$ & \\
\hline $1 \backslash 1$ & 94 & 723 & 0,185086 & 0,258904 & $-0,33564$ & 0,02206 & $-0,121384$ & 0,169315 & $-0,433822$ & \\
\hline 112 & 16 & 109 & 0,028012 & 0,045205 & $-0,47858$ & 0,003852 & $-0,030892$ & 0,025625 & $-0,135463$ & \\
\hline 210 & 1 & 131 & 0,03364 & 0,00411 & 2,102402 & 0,00035 & $-0,004020$ & 0,030774 & $-0,154551$ & \\
\hline $2 \backslash 1$ & 36 & 373 & 0,095549 & 0,1 & $-0,04553$ & 0,008521 & $-0,058578$ & 0,087407 & $-0,307333$ & \\
\hline 212 & 9 & 54 & 0,013942 & 0,026027 & $-0,62423$ & 0,002218 & $-0,019553$ & 0,012754 & $-0,080261$ & \\
\hline 310 & 5 & 291 & 0,074572 & 0,015068 & 1,599152 & 0,001284 & $-0,012332$ & 0,068218 & $-0,264255$ & \\
\hline $3 \backslash 1$ & 56 & 975 & 0,249552 & 0,154795 & 0,47757 & 0,013189 & $-0,082361$ & 0,228289 & $-0,486499$ & \\
\hline \multirow[t]{4}{*}{312} & 16 & 152 & 0,039013 & 0,045205 & $-0,14734$ & 0,003852 & $-0,030892$ & 0,035688 & $-0,171604$ & \\
\hline & 364 & 3908 & & & & 2 & $-0,551989$ & & $-2,991873$ & \\
\hline & & & & & & & 0,551989 & & 2,991873 & \\
\hline & & & & & 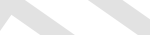 & & 0,047033 & & 2,736947 & 2,78398 \\
\hline
\end{tabular}




\begin{tabular}{|c|c|c|c|c|c|c|c|c|c|c|}
\hline \multicolumn{11}{|c|}{ 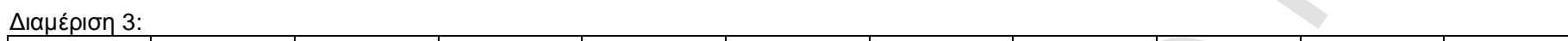 } \\
\hline JYRTRM & BADS & GOODS & $f(x / 1)$ & $f(x / 0)$ & $w(x)$ & & & 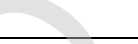 & & \\
\hline 010 & 10 & 230 & 0,058966 & 0,028767 & 0,717736 & 0,002451 & $-0,021257$ & 0,053942 & $-0,227228$ & \\
\hline $0 \backslash 1$ & 108 & 513 & 0,131364 & 0,29726 & $-0,81664$ & 0,025328 & $-0,134319$ & 0,120171 & $-0,367343$ & \\
\hline $0 \backslash 2$ & 10 & 71 & 0,018291 & 0,028767 & $-0,45282$ & 0,002451 & $-0,021257$ & 0,016733 & $-0,098742$ & \\
\hline 110 & 12 & 296 & 0,075851 & 0,034247 & 0,795179 & 0,002918 & $-0,024572$ & 0,069388 & $-0,267085$ & \\
\hline $1 \backslash 1$ & 83 & 722 & 0,18483 & 0,228767 & $-0,21327$ & 0,019492 & $-0,110735$ & 0,169081 & $-0,433560$ & \\
\hline 112 & 13 & 96 & 0,024687 & 0,036986 & $-0,40429$ & 0,003151 & $-0,026188$ & 0,022583 & $-0,123499$ & \\
\hline 210 & 2 & 130 & 0,033384 & 0,006849 & 1,583943 & 0,000584 & $-0,006269$ & 0,03054 & $-0,153712$ & \\
\hline $2\lfloor 1$ & 35 & 368 & 0,09427 & 0,09726 & $-0,03123$ & 0,008287 & $-0,057305$ & 0,086237 & $-0,304896$ & \\
\hline 212 & 10 & 52 & 0,013431 & 0,028767 & $-0,7617$ & 0,002451 & $-0,021257$ & 0,012286 & $-0,077978$ & \\
\hline 310 & 4 & 272 & 0,069711 & 0,012329 & 1,732422 & 0,00105 & $-0,010394$ & 0,063771 & $-0,253232$ & \\
\hline $3 \backslash 1$ & 58 & 1001 & 0,256204 & 0,160274 & 0,469088 & 0,013656 & $-0,084591$ & 0,234374 & $-0,490572$ & \\
\hline 312 & 19 & 157 & 0,040292 & 0,053425 & $-0,28213$ & 0,004552 & $-0,035412$ & 0,036859 & $-0,175515$ & \\
\hline & 364 & 3908 & & & & 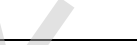 & $-0,553556$ & & $-2,973363$ & \\
\hline & & & & & & 2 & 0,553556 & & 2,973363 & \\
\hline & & & & & & & 0,047166 & & 2,720014 & 2,767181 \\
\hline
\end{tabular}




\begin{tabular}{|c|c|c|c|c|c|c|c|c|c|c|}
\hline & & & & & & & & & & \\
\hline JYRTRM & BADS & GOODS & $f(x / 1)$ & $f(x / 0)$ & $w(x)$ & & & 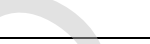 & & \\
\hline 010 & 9 & 140 & 0,035943 & 0,026027 & 0,322776 & 0,002218 & $-0,019553$ & 0,03288 & $-0,161989$ & \\
\hline $0 \backslash 1$ & 68 & 328 & 0,084037 & 0,187671 & $-0,80344$ & 0,015991 & $-0,095411$ & 0,076876 & $-0,284544$ & \\
\hline $0 \backslash 2$ & 5 & 43 & 0,011128 & 0,015068 & $-0,30313$ & 0,001284 & $-0,012332$ & 0,01018 & $-0,067372$ & \\
\hline 110 & 16 & 361 & 0,092479 & 0,045205 & 0,715762 & 0,003852 & $-0,030892$ & 0,084599 & $-0,301445$ & \\
\hline $1 \backslash 1$ & 107 & 881 & 0,225505 & 0,294521 & $-0,26701$ & 0,025095 & $-0,133416$ & 0,206291 & $-0,469775$ & \\
\hline 112 & 18 & 116 & 0,029803 & 0,050685 & $-0,53102$ & 0,004319 & $-0,033924$ & 0,027264 & $-0,141686$ & \\
\hline 210 & 3 & 148 & 0,037989 & 0,009589 & 1,376682 & 0,000817 & $-0,008381$ & 0,034752 & $-0,168436$ & \\
\hline $2\lfloor 1$ & 49 & 434 & 0,111154 & 0,135616 & $-0,19892$ & 0,011555 & $-0,074362$ & 0,101683 & $-0,335335$ & \\
\hline 212 & 7 & 62 & 0,015989 & 0,020548 & $-0,25088$ & 0,001751 & $-0,016033$ & 0,014626 & $-0,089152$ & \\
\hline 310 & 3 & 253 & 0,06485 & 0,009589 & 1,911461 & 0,000817 & $-0,008381$ & 0,059325 & $-0,241761$ & \\
\hline $3 \backslash 1$ & 60 & 999 & 0,255692 & 0,165753 & 0,433472 & 0,014123 & $-0,086798$ & 0,233906 & $-0,490266$ & \\
\hline 312 & 19 & 143 & 0,03671 & 0,053425 & $-0,37522$ & 0,004552 & $-0,035412$ & 0,033582 & $-0,164424$ & \\
\hline & 364 & 3908 & & & & 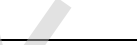 & $-0,554895$ & & $-2,916185$ & \\
\hline & & & & & & 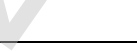 & 0,554895 & & 2,916185 & \\
\hline & & & & & & & 0,04728 & & 2,667709 & 2,714989 \\
\hline
\end{tabular}




\begin{tabular}{|c|c|c|c|c|c|c|c|c|c|c|}
\hline & Cחמק & 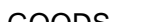 & & & (n) & & & & & \\
\hline $0 \backslash 0$ & 13 & 210 & 0.05385 & 0,036986 & 0,375656 & 0,003151 & -0.026188 & 0,049262 & -0213963 & \\
\hline $0 \backslash 1$ & 101 & 524 & 0,134178 & 0,278082 & $-0,72875$ & 0,023694 & $-0,127933$ & 0,122745 & $-0,371458$ & \\
\hline $0 \backslash 2$ & 10 & 68 & 0,017524 & 0,028767 & $-0,49568$ & 0,002451 & $-0,021257$ & 0,016031 & $-0,095591$ & \\
\hline $1 \backslash 0$ & 11 & 264 & 0,067664 & 0,031507 & 0,764355 & 0,002685 & $-0,022929$ & 0,061899 & $-0,248459$ & \\
\hline $1 \backslash 1$ & 79 & 661 & 0,169225 & 0,217808 & $-0,25239$ & 0,018559 & $-0,106745$ & 0,154806 & $-0,416655$ & \\
\hline 112 & 18 & 89 & 0,022896 & 0,050685 & $-0,79467$ & 0,004319 & $-0,033924$ & 0,020945 & $-0,116816$ & \\
\hline 210 & 3 & 145 & 0,037222 & 0,009589 & 1,356274 & 0,000817 & $-0,008381$ & 0,03405 & $-0,166036$ & \\
\hline $2 \backslash 1$ & 42 & 430 & 0,11013 & 0,116438 & $-0,0557$ & 0,009921 & $-0,066028$ & 0,100747 & $-0,333592$ & \\
\hline 212 & 10 & 67 & 0,017268 & 0,028767 & $-0,51039$ & 0,002451 & $-0,021257$ & 0,015797 & $-0,094530$ & \\
\hline 310 & 3 & 246 & 0,06306 & 0,009589 & 1,88346 & 0,000817 & $-0,008381$ & 0,057687 & $-0,237416$ & \\
\hline $3 \backslash 1$ & 55 & 1051 & 0,268995 & 0,152055 & 0,57045 & 0,012956 & $-0,081237$ & 0,246075 & $-0,497768$ & \\
\hline 312 & 19 & 153 & 0,039268 & 0,053425 & $-0,30785$ & 0,004552 & $-0,035412$ & 0,035922 & $-0,172391$ & \\
\hline & 364 & 3908 & & & & 2 & $-0,559671$ & & $-2,964674$ & \\
\hline & & & & & & 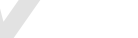 & 0,559671 & & 2,964674 & \\
\hline & & & & & 1 & & 0,047687 & & 2,712066 & 2,759753 \\
\hline
\end{tabular}




\begin{tabular}{|c|c|c|c|c|c|c|c|c|c|}
\hline MARITRM & BADS & GOODS & $f(x / 1)$ & $f(x / 0)$ & $w(x)$ & & & 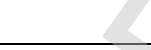 & 2 \\
\hline $1 \backslash 1$ & 16 & 75 & 0,019314 & 0,045205 & $-0,85037$ & 0,003852 & $-0,030892$ & 0,017669 & $-0,102879$ \\
\hline 211 & 20 & 25 & 0,006523 & 0,056164 & $-2,15289$ & 0,004786 & $-0,036883$ & 0,005968 & $-0,044092$ \\
\hline $3 \backslash 1$ & 19 & 303 & 0,077641 & 0,053425 & 0,373828 & 0,004552 & $-0,035412$ & 0,071026 & $-0,271000$ \\
\hline 112 & 13 & 322 & 0,082502 & 0,036986 & 0,802274 & 0,003151 & $-0,026188$ & 0,075472 & $-0,281354$ \\
\hline $2 \backslash 2$ & 20 & 46 & 0,011896 & 0,056164 & $-1,55211$ & 0,004786 & $-0,036883$ & 0,010882 & $-0,070972$ \\
\hline 312 & 4 & 605 & 0,154899 & 0,012329 & 2,530838 & 0,00105 & $-0,010394$ & 0,141701 & $-0,399466$ \\
\hline $1 \backslash 3$ & 137 & 568 & 0,145434 & 0,376712 & $-0,95176$ & 0,032098 & $-0,159251$ & 0,133042 & $-0,387158$ \\
\hline $2 \backslash 3$ & 85 & 111 & 0,028524 & 0,234247 & $-2,10563$ & 0,019959 & $-0,112706$ & 0,026094 & $-0,137256$ \\
\hline \multirow[t]{4}{*}{313} & 50 & 1853 & 0,474162 & 0,138356 & 1,231718 & 0,011789 & $-0,075524$ & 0,433761 & $-0,522694$ \\
\hline & 364 & 3908 & & & & & $-0,524132$ & & $-2,216871$ \\
\hline & & & & & & 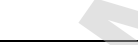 & 0,524132 & & 2,216871 \\
\hline & & & & & & & 0,044659 & & 2,02798 \\
\hline
\end{tabular}

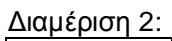

\begin{tabular}{|l|r|r|r|r|r|r|r|r|r|r|}
\hline MARTRM & \multicolumn{1}{l|}{ BADS } & GOODS & $\mathrm{f}(\mathrm{x} / 1)$ & $\mathrm{f}(\mathrm{x} / 0)$ & $\mathrm{w}(\mathrm{x})$ & & & & \\
\hline 010 & 15 & 48 & 0,012407 & 0,042466 & $-1,23042$ & 0,003618 & $-0,029346$ & 0,01135 & $-0,073335$ & \\
\hline $0 \backslash 1$ & 94 & 120 & 0,030826 & 0,258904 & $-2,12809$ & 0,02206 & $-0,121384$ & 0,0282 & $-0,145177$ & \\
\hline 012 & 18 & 24 & 0,006268 & 0,050685 & $-2,09024$ & 0,004319 & $-0,033924$ & 0,005734 & $-0,042694$ & \\
\hline 110 & 13 & 337 & 0,086339 & 0,036986 & 0,847736 & 0,003151 & $-0,026188$ & 0,078983 & $-0,289260$ & \\
\hline $1 \backslash 1$ & 141 & 578 & 0,147992 & 0,387671 & $-0,963$ & 0,033032 & $-0,162517$ & 0,135382 & $-0,390563$ & \\
\hline 112 & 13 & 69 & 0,017779 & 0,036986 & $-0,7325$ & 0,003151 & $-0,026188$ & 0,016265 & $-0,096646$ & \\
\hline 210 & 3 & 576 & 0,14748 & 0,009589 & 2,733073 & 0,000817 & $-0,008381$ & 0,134914 & $-0,389886$ & \\
\hline $2 \backslash 1$ & 51 & 1865 & 0,477232 & 0,141096 & 1,218563 & 0,012022 & $-0,076680$ & 0,436569 & $-0,522014$ & \\
\hline 212 & 16 & 291 & 0,074572 & 0,045205 & 0,50054 & 0,003852 & $-0,030892$ & 0,068218 & $-0,264255$ & \\
\hline & 364 & 3908 & & & & & $-0,515500$ & & $-2,213829$ & \\
\hline & & & & & & & 0,515500 & & 2,213829 & \\
\hline & & & & & & & 0,043924 & & 2,025198 & 2,069121 \\
\hline
\end{tabular}




\begin{tabular}{|c|c|c|c|c|c|c|c|c|c|c|}
\hline MARTRM & BADS & GOODS & $f(x / 1)$ & $f(x / 0)$ & $w(x)$ & & & & 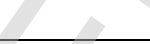 & 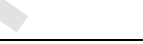 \\
\hline 010 & 13 & 39 & 0,010105 & 0,036986 & $-1,29753$ & 0,003151 & $-0,026188$ & 0,009244 & $-0,062464$ & \\
\hline $0 \backslash 1$ & 87 & 109 & 0,028012 & 0,239726 & $-2,14685$ & 0,020426 & $-0,114661$ & 0,025625 & $-0,135463$ & \\
\hline 012 & 18 & 26 & 0,006779 & 0,050685 & $-2,01177$ & 0,004319 & $-0,033924$ & 0,006202 & $-0,045477$ & \\
\hline 110 & 12 & 331 & 0,084804 & 0,034247 & 0,90676 & 0,002918 & $-0,024572$ & 0,077578 & $-0,286125$ & \\
\hline $1 \backslash 1$ & 148 & 606 & 0,155155 & 0,406849 & $-0,96402$ & 0,034666 & $-0,168142$ & 0,141935 & $-0,399788$ & \\
\hline 112 & 16 & 58 & 0,014965 & 0,045205 & $-1,10547$ & 0,003852 & $-0,030892$ & 0,01369 & $-0,084753$ & \\
\hline 210 & 3 & 558 & 0,142875 & 0,009589 & 2,701352 & 0,000817 & $-0,008381$ & 0,130702 & $-0,383694$ & \\
\hline $2 \backslash 1$ & 49 & 1889 & 0,483372 & 0,135616 & 1,270955 & 0,011555 & $-0,074362$ & 0,442186 & $-0,520575$ & \\
\hline \multirow[t]{4}{*}{212} & 18 & 292 & 0,074827 & 0,050685 & 0,389554 & 0,004319 & $-0,033924$ & 0,068452 & $-0,264824$ & \\
\hline & 364 & 3908 & & & & & $-0,515045$ & 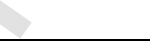 & $-2,183162$ & \\
\hline & & & & & & & 0,515045 & & 2,183162 & \\
\hline & & & & & & & 0,043885 & & 1,997143 & 2,041028 \\
\hline
\end{tabular}

\begin{tabular}{|c|c|c|c|c|c|c|c|c|c|c|}
\hline MARTRM & BADS & GOODS & $f(x / 1)$ & $f(x / 0)$ & $w(x)$ & 8 & & & & \\
\hline 010 & 14 & 43 & 0,011128 & 0,039726 & $-1,27253$ & 0,003385 & $-0,027779$ & 0,01018 & $-0,067372$ & \\
\hline $0 \backslash 1$ & 88 & 116 & 0,029803 & 0,242466 & $-2,09625$ & 0,02066 & $-0,115632$ & 0,027264 & $-0,141686$ & \\
\hline $0 \backslash 2$ & 17 & 25 & 0,006523 & 0,047945 & $-1,99466$ & 0,004085 & $-0,032418$ & 0,005968 & $-0,044092$ & \\
\hline 110 & 15 & 324 & 0,083014 & 0,042466 & 0,670306 & 0,003618 & $-0,029346$ & 0,07594 & $-0,282421$ & \\
\hline $1 \backslash 1$ & 146 & 593 & 0,151829 & 0,40137 & $-0,97213$ & 0,034199 & $-0,166546$ & 0,138892 & $-0,395560$ & \\
\hline 112 & 14 & 66 & 0,017012 & 0,039726 & $-0,84809$ & 0,003385 & $-0,027779$ & 0,015562 & $-0,093465$ & \\
\hline 210 & 2 & 535 & 0,136992 & 0,006849 & 2,995771 & 0,000584 & $-0,006269$ & 0,125319 & $-0,375496$ & \\
\hline $2 \backslash 1$ & 50 & 1933 & 0,494628 & 0,138356 & 1,273974 & 0,011789 & $-0,075524$ & 0,452483 & $-0,517670$ & \\
\hline \multirow[t]{4}{*}{$2 \backslash 2$} & 18 & 273 & 0,069967 & 0,050685 & 0,322391 & 0,004319 & $-0,033924$ & 0,064005 & $-0,253823$ & \\
\hline & 364 & 3908 & & & 2 & & $-0,515218$ & & $-2,171586$ & \\
\hline & & & & & & & 0,515218 & & 2,171586 & \\
\hline & & & & & & & 0,0439 & & 1,986554 & 2,030453 \\
\hline
\end{tabular}




\begin{tabular}{|c|c|c|c|c|c|c|c|c|c|c|}
\hline \multicolumn{11}{|l|}{ 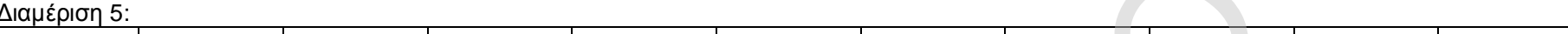 } \\
\hline MARTRM & BADS & GOODS & $f(x / 1)$ & $f(x / 0)$ & $w(x)$ & & & & & \\
\hline $0 \backslash 0$ & 11 & 36 & 0,009337 & 0,031507 & $-1,21617$ & 0,002685 & $-0,022929$ & 0,008542 & $-0,058693$ & \\
\hline $0 \backslash 1$ & 93 & 116 & 0,029803 & 0,256164 & $-2,15121$ & 0,021827 & $-0,120435$ & 0,027264 & $-0,141686$ & \\
\hline 012 & 19 & 20 & 0,005244 & 0,053425 & $-2,32113$ & 0,004552 & $-0,035412$ & 0,004797 & $-0,036957$ & \\
\hline 110 & 15 & 301 & 0,07713 & 0,042466 & 0,59679 & 0,003618 & $-0,029346$ & 0,070558 & $-0,269887$ & \\
\hline $1 \backslash 1$ & 140 & 590 & 0,151062 & 0,384932 & $-0,93538$ & 0,032798 & $-0,161704$ & 0,13819 & $-0,394571$ & \\
\hline 112 & 17 & 70 & 0,018035 & 0,047945 & $-0,97773$ & 0,004085 & $-0,032418$ & 0,016499 & $-0,097697$ & \\
\hline 210 & 4 & 528 & 0,135201 & 0,012329 & 2,394826 & 0,00105 & $-0,010394$ & 0,123681 & $-0,372936$ & \\
\hline $2 \backslash 1$ & 44 & 1960 & 0,501535 & 0,121918 & 1,414326 & 0,010388 & $-0,068447$ & 0,458801 & $-0,515720$ & \\
\hline \multirow[t]{4}{*}{212} & 21 & 287 & 0,073548 & 0,058904 & 0,22203 & 0,005019 & $-0,038337$ & 0,067281 & $-0,261970$ & \\
\hline & 364 & 3908 & & & 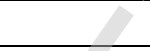 & $\nabla$ & $-0,519422$ & & $-2,150116$ & \\
\hline & & & & & & & 0,519422 & & 2,150116 & \\
\hline & & & & & - & & 0,044258 & & 1,966913 & 2,011171 \\
\hline
\end{tabular}

